\title{
EXPERIMENT DATA REPORT FOR SEMISCALE MOD-1 TEST S-28-2 (STEAM GENERATOR TUBE RUPTURE TEST)
}

\author{
MORRIS L. PATTON \\ KENNETH E. SACKETT
}

October 1977

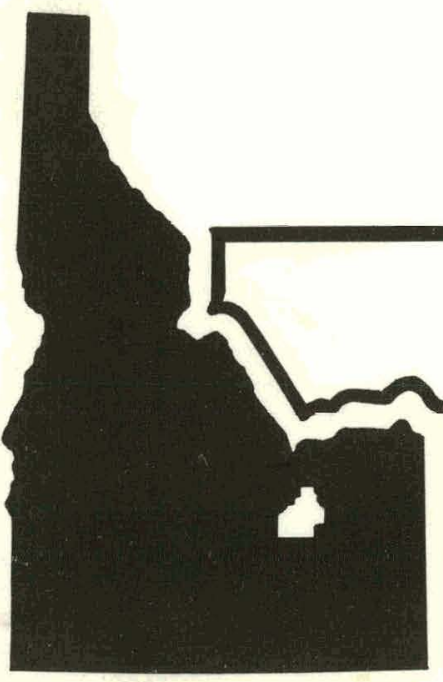

$\prod_{\forall} E G_{\Sigma} G$ Idaho, Inc.

IDAHO NATIONAL ENGINEERING LABORATORY

ENERGY RESEARCH AND DEVELOPMENT ADMINISTRATION

IDAHO OPERATIONS OFFICE UNDER CONTRACT EY-76-C-07-1570 


\section{DISCLAIMER}

This report was prepared as an account of work sponsored by an agency of the United States Government. Neither the United States Government nor any agency Thereof, nor any of their employees, makes any warranty, express or implied, or assumes any legal liability or responsibility for the accuracy, completeness, or usefulness of any information, apparatus, product, or process disclosed, or represents that its use would not infringe privately owned rights. Reference herein to any specific commercial product, process, or service by trade name, trademark, manufacturer, or otherwise does not necessarily constitute or imply its endorsement, recommendation, or favoring by the United States Government or any agency thereof. The views and opinions of authors expressed herein do not necessarily state or reflect those of the United States Government or any agency thereof. 


\section{DISCLAIMER}

Portions of this document may be illegible in electronic image products. Images are produced from the best available original document. 
Printed in the United States of America Available from

National Technical Information Service

U.S. Department of Commerce 5285 Port Royal Road

Springfield, Virginia 22161

Price: Printed Copy $\$ 11.00$; Microfiche $\$ 3.00$

"The NRC will make available data tapes and operational computer codes on research programs dealing with postulated loss-of-coolant accidents in light water reactors. Persons requesting this information must reimburse the NRC contractors for their expenses in preparing copies of the data tapes and the operational computer codes. Requests should be submitted to the Research Applications Branch, Office of Nuclear Regulatory Research, Nuclear Regulatory Commission, Washington, D.C. 20555."

\section{NOTICE}

This report was prepared as an account of work sponsored by the United States Government. Neither the United States nor the Energy Research and Development Administration, nor the Nuclear Regulatory Commission, nor any of their employees, nor any of their contractors, subcontractors, or their employees, makes any warranty, express or implied, or assumes any legal liability or responsibility for the accuracy, completeness or usefulness of any information, apparatus, product or process disclosed, or represents that its use would not infringe privately owned rights. 
TREE-NUREG-1149

EXPERMMNT DATA REPORT FOR SEMISCALE MOD-1

TEST S-28-2

(STEAM GENERATOR TUBE RUPTURE TEST SERIES)

Approved:

6
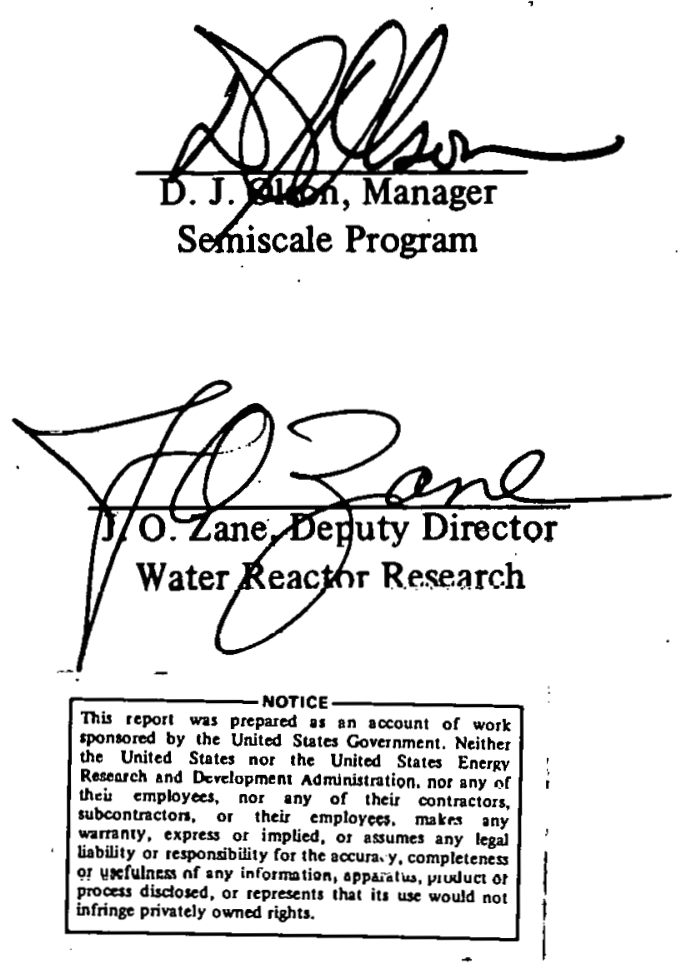
EXPERIMENT DATA REPORT FOR SEMISCALE MOD-1

TEST S-28-2

(STEAM GENERATOR TUBE RUPTURE TEST SERIES)

by

Morris L. Patton

Kenneth E. Sackett

EG\&G IDAHO, INC.

October 1977

PREPARED FOR THE

U.S. NUCLEAR REGULATORY COMMISSION

AND

ENERGY RESEARCH AND DEVELOPMENT ADMINISTRATION

IDAHO OPERATIONS OFFIC.F.

UNDER CONTRACT NO. EY-76-C-07-1570 


\begin{abstract}
Recorded test data are presented for Test S-28-2 of the Semiscale Mod-1 steam generator tube rupture test series. These tests are among several Semiscale Mod-1 experiments conducted to investigate the thermal and hydraulic phenomena accompanying a hypothesized loss-of-coolant accident in a pressurized water reactor (PWR) system.

Test S-28-2 was conducted from initial conditions of $15936 \mathrm{kPa}$ and $558 \mathrm{~K}$ to investigate the response of the Semiscale Mod-1 system to a depressurization and reflood transient following a simulated double-ended offset shear of the broken loop cold leg piping. During the test, cooling water was injected into the cold leg of the intact and broken loops to simulate emergency core coolant injection in a. PWR. For Test S-28-2, accumulator injection into the intact loop hot leg was provided to simulate the rupture of six steam generator tubes.
\end{abstract}

The purpose of this report is to make available the uninterpreted data from Test S-28-2 for future data analysis and test reporting activities. The data, presented in the form of graphs in engineering units, have been analyzed only to the extent necessary to ensure that they are reasonable and cunsislerit. 


\section{SUMMARY}

Test S-28-2 was performed as part of the Semiscale Mod-1 portion of the Semiscale Program conducted by EG\&G Idaho, Inc. for the United States Government. This test is part of the steam generator tube rupture test series (Test Series 28) performed to investigate the response of the Mod-1 system to the rupture of steam generator tubes during a hypothesized loss-of-coolant accident (LOCA). The test objective specific to Test S-28-2 was to define a lower limit on the range of steam generator tube ruptures over which high peak cladding temperatures can occur. Hardware configuration and test parameters were selected to yield a system response that simulates the response of a pressurized water reactor during a hypothesized LOCA with subsequent refill and reflood.

Test S-28-2 utilized the Semiscale Mod-1 system equipped with a pressure vessel with a 40-rod electrically heated core; an intact loop with pump, steam generator, and pressurizer; a broken loop with simulated pump, simulated steam generator, and rupture assemblies; and a pressure suppression system with header; pressure suppression tank, and heated steam supply system. High and low pressure coolant injection pumps and a coolant injection accumulator were provided for each system loop. An additional injection accumulator was provided for the intact loop hot leg. Intact loop hot leg injection flow rate was set to simulate the rupture of six steam generator tubes. In addition, for Test S-28-2, four heater rods were intentionally unpowered to simulate the effects of control rod guide tubes and the power in three heater rods was increased to produce a slightly peaked power profile.

The test was conducted from initial conditions of $15936 \mathrm{kPa}$ and $558 \mathrm{~K}$ (at the intact loop cold leg vessel inlet) with a simulated full size (200\%) double-ended offset shear of the cold leg broken loop piping at an initial core power level of $1418 \mathrm{~kW}$ and an initial core inlet flow rate $9.84 \mathrm{l} / \mathrm{s}$. The instantaneous offset shear of the broken loop cold leg piping was simulated by simultaneous (within $10 \mathrm{~ms}$ ) actuation of the rupture assemblies. After initiation of blowdown, power to the heated core was reduced to simulate the predicted heat flux response of nuclear fuel rods during a LOCA. Blowdown was accompanied by simulated emergency core coolant injected into the cold legs of the intact and broken loops and followed by injection into the intact loop hot leg to simulate steam generator tube ruptures.

Test S-28-2 was generally conducted as specified. Conditions which did not conform to the specified test configuration were considered acceptable for analysis purposes within the test objectives. The instrumentation used generally functioned as intended. Of 230 measurements taken, 229 produced usable data. 


\section{CONTENTS}

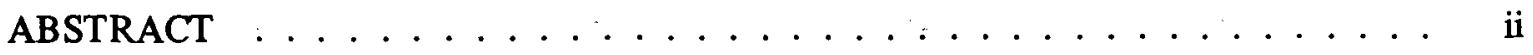

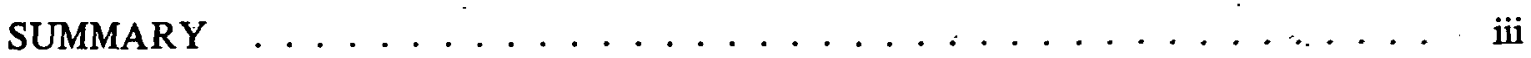

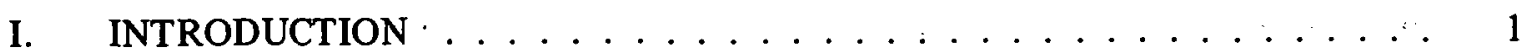

II. SYSTEM, PROCEDURES, CONDITIONS, AND

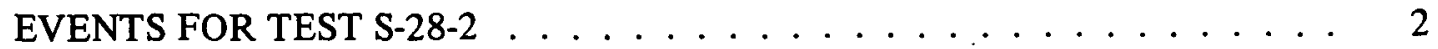

1. SYSTEM CONFIGURATION AND
TEST PROCEDURES $\ldots \ldots \ldots \ldots \ldots \ldots$

2. INITIAL TEST CONDITIONS AND
SEQUENCE OF EVENTS .................. 5

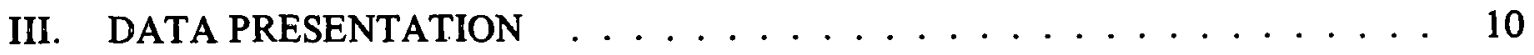

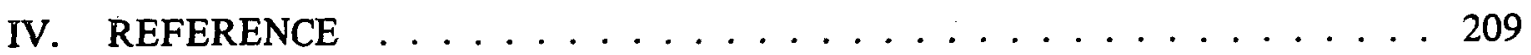

APPENDIX A - DATA ACQUISITION SYSTEM CAPABILITIES . . . . . . 211

APPENDIX B - POSTTEST ADJUSTMENTS TO DATA FROM

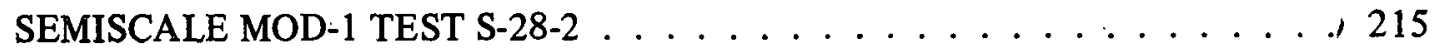

1. PRESSURE MEASUREMENTS . . . . . . . . . . . . . . . . 217

2. DIFFERENTIAL PRESSURE MEASUREMENTS . . . . . . . . 218

3. MOMENTUM FLUX MEASUREMENTS . . . . . . . . . . . . . 220

4. DENSITY MEASUREMENTS . . . . . . . . . . . . . . 222

APPENDIX C - SELECTED DATA WITH ESTIMATED TOTAL ERROR

BANDS FROM SEMISCALE MOD-1 TEST S-28-2 . . . . . . . . . . . . . 227

FIGURES

1. Semiscale Mod-1 system for cold leg break

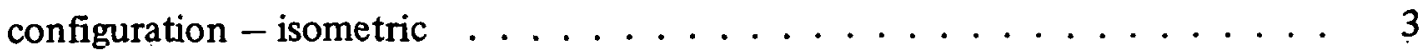


2. Semiscale Mod-1 system for cold leg break configuration - schematic $\ldots \ldots \ldots \ldots \ldots$

3. Semiscale Mod-1 system and instrumentation

for cold leg break configuration - isometric . . . . . . . . . . . . . . 11

4. Semiscale Mod-1 system and instrumentation for cold leg break configuration - schematic ............. 12

5. Semiscale Mod-1 pressure vessel - cross section showing instrumentation . . . . . . . . . . . . . 13

6. Semiscale Mod-1 pressure vessel - isometric showing instrumentation . . . . . . . . . . . . . . . . . 14

7. Semiscale Mod-1 pressure vessel - penetrations and instrumentation .................... 15

8. Semiscale Mod-1 heated core - plan view . . . . . . . . . . . 16

9. Fluid temperature in intact loop hot leg (TFU-1 and RBU-2), from -20 to $350 \mathrm{~s}$

10. Fluid temperature in in tact loop hot leg

(TFU-1 and RBU-2), from -6 to $42 \mathrm{~s} \ldots \ldots \ldots . \ldots 28$

11. Fluid temperature in intact loop hot leg (TFU-6),

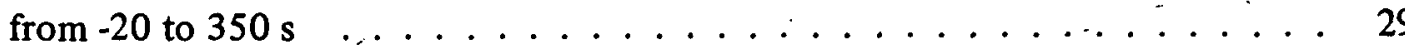

12. Fluid temperature in intact loop hot leg (TFU-6),

from -6 to $42 \mathrm{~s}$

13. Fluid temperature in intact loop cold leg

(TFU-7 and TFU-10), from -20 to $350 \mathrm{~s} \ldots \ldots . \ldots . \ldots . \ldots$

14. Fluid temperature in intact loop cold leg (TFU-7 and TFU-10), from -6 to $42 \mathrm{~s}$

15. Fluid temperature in intact loop cold leg (RBU-14A and TFU-14B), from -20 to $350 \mathrm{~s}$

16. Fluid temperature in intact loop cold leg (RBU-14A and TFU-14B), from -6 to $42 \mathrm{~s} \ldots \ldots \ldots \ldots$

17. Fluid temperature in intact loop cold leg (TFU-15), from -20 to $350 \mathrm{~s}$ 
18. Fluid temperature in intact loop cold leg.(TFU-15),

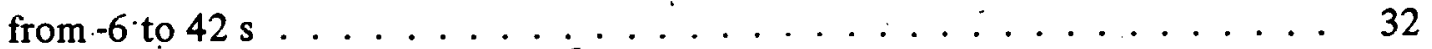

19. Fluid temperature in broken loop, vèssel side.

(TFB-20 and TFB-23), from -20 to $350 \mathrm{~s}$

20. Fluid temperature in broken loop, vessel side

(TFB-20 and TFB-23), from -6 to $42 \mathrm{~s}$

21. Fluid temperature in broken loop, pump side

(TFB-30, TFB-37, and TFB-42), from -20 to $350 \mathrm{~s}$

22. Fluid temperature in broken loop, pump side

(TFB-30, TFB-37, and TГB-42), from -6. to $12 \mathrm{~g} \ldots \ldots \ldots$

23. Fluid temperature in inlet annulus (TFV-ANN-4A and TFV-ANN-4M), from -20 to $350 \mathrm{~s} \ldots \ldots \ldots 35$

24. Fluid temperature in inlet annulus (TFV-ANN-4A and TFV-ANN-4M), from -6 to $42 \mathrm{~s} \ldots \ldots \ldots$

25: Fluid temperature in downcomer annulus (TFV-ANN-35A, TFV-ANN-70A, TFV-ANN-1 15A, and TFV-ANN-156A), from -20 to $350 \mathrm{~s}$

26. Fluid temperature in downcomer annulus (TFV-ANN-35A, TFV-ANN-70A, TFV-ANN-115A, and TFV-ANN-156A), from -6 to $42 \mathrm{~s} \ldots \ldots \ldots \ldots \ldots$

27. Fluid temperature in upper plenum (TFV-UP+13), from -20 to $350 \mathrm{~s}$

28. Fluid temperature in upper plenum (TFV-UP+13), from -6 to $42 \mathrm{~s} \ldots \ldots \ldots \ldots \ldots \ldots$

29: Fluid temperature in lower plenum (TFV-LP-2, TFV-LP-4, and TFV-LP-7), from -20 to $350 \mathrm{~s}$

30. Fluid temperature in lower plenum (TFV-LP-2, TFV-LP-4, and TFV-LP-7), from -6 to $42 \mathrm{~s}$

31. Fluid temperature in corc inlet (TFV-CORE-IN), from -20 to $350 \mathrm{~s}$

32. Fluid temperature in core inlet (TFV-CORE-IN), from -6 to $42 \mathrm{~s} \ldots \ldots \ldots \ldots \ldots$ 
33. Fluid temperature in core, Grid Spacer 5

(TFG-5CD-45), from -20 to $350 \mathrm{~s} \ldots \ldots \ldots \ldots$. . . . . . . 40

34. Fluid temperature in core, Grid Spacer 5

(TFG-5CD-45), from -6 to $42 \mathrm{~s}$

35. Fluid temperature in core, Grid Spacer 6

(TFG-6CD-45), from-20 to $350 \mathrm{~s} \ldots \ldots \ldots \ldots . \ldots \ldots$

36. Fluid temperature in core, Grid Spacer 6

(TFG-6CD-45), from -6 to $42 \mathrm{~s}$

37. Fluid temperature in core, Grid Spacer 10 (TFG-10AB-45), from -20 to $350 \mathrm{~s} \ldots \ldots \ldots \ldots \ldots \ldots \ldots \ldots$

38. Fluid temperature in core, Grid Spacer 10

(TFG-10AB-45), from -6 to $42 \mathrm{~s} \ldots \ldots \ldots \ldots \ldots$

39. Fluid temperature in intact loop coolant injection line (TFU-ECC-14), from -20 to $350 \mathrm{~s} \ldots \ldots \ldots \ldots \ldots$

40. Fluid temperature in intact loop coolant injection line (TFU-ECC-14), from -6 to $42 \mathrm{~s} \ldots \ldots \ldots \ldots \ldots \ldots$

41. Fluid temperature in broken loop coolant injection line (TFB-ECC-42), from -20 to $350 \mathrm{~s} \ldots \ldots \ldots \ldots \ldots$

42. Fluid temperature in broken loop coolant injection line (TFB-ECC-42), from -6 to $42 \mathrm{~s} \ldots \ldots \ldots \ldots \ldots$

43. Fluid temperature in steam generator, feedwater line (TFU-SGFW), from -20 to $350 \mathrm{~s} \ldots \ldots \ldots \ldots \ldots \ldots$

44. Fluid temperature in steam generator, feedwater line (TFU-SGFW), from -6 to $42 \mathrm{~s} \ldots \ldots \ldots \ldots \ldots$. . . . . . . . 45

45. Fluid temperature in steam generator, steam dome (TFU-SGSD), from -20 to $350 \mathrm{~s}$

46. Fluid temperature in steam generator, steam dome (TFU-SGSD), from -6 to $42 \mathrm{~s}$

47. Fluid temperature in steam generator, secondary side (TFU-SG1, TFU-SG2, and TFU-SG3), from -20 to $350 \mathrm{~s}$

48. Fluid temperature in steam generator, secondary side (TFU-SG1, TFU-SG2, and TFU-SG3), from -6 to $42 \mathrm{~s} \ldots \ldots \ldots \ldots \ldots$ 
49. Fluid temperature in steam generator rupture system accumulator (TFU-SGS3), from -20 to $350 \mathrm{~s} \ldots \ldots \ldots$. . . . . . . 48

50. Fluid temperature in steam generator rupture system accumulator (TFU-SGS3), from -6 to $42 \mathrm{~s} \ldots \ldots \ldots . \ldots . \ldots 48$

51. Fluid temperature in steam generator rupture system injection line (TFU-SGS), from -20 to $350 \mathrm{~s} \ldots$. . . . . . . . . . . . 49

52. Fluid temperature in steam generator rupture system injection line (TFU-SGS), from -6 to $42 \mathrm{~s} \ldots \ldots . \ldots . \ldots 49$

53. Fluid temperature in steam generator rupture system (TFU-SGS-D), from -20 to $350 \mathrm{~s} \ldots \ldots \ldots \ldots$. . . . . . . . . . 50

54. : Fluid temperature in steam generator rupture system (TFU-SGS-D), from -6 to $42 \mathrm{~s} \ldots \ldots \ldots \ldots$. . . . . . . . . . . 50

55. Fluid temperature in pressurizer surge line (TFU-PRIZE), from -20 to $350 \mathrm{~s} \ldots \ldots \ldots \ldots \ldots$. . . . . . . . . . . . .

56. Fluid temperature in pressurizer surge line (TFU-PRIZE), from -6 to $42 \mathrm{~s} \ldots \ldots \ldots \ldots \ldots \ldots$

57. Fluid temperature in pressure suppression tank (TF-PSS-33 and TF-PSS-130), from -20 to $350 \mathrm{~s} \ldots \ldots \ldots$. . . . . . . 52

58. Fluid temperature in pressure suppression tank (TF-PSS-33 and TF-PSS-130), from -6 to $42 \mathrm{~s} \ldots \ldots . \ldots . \ldots 52$

59. Material temperature in intact loop ('IMU-I'1'16), from -20 to $350 \mathrm{~s} \ldots \ldots \ldots \ldots 3$

60. Material temperature in intact loop (TMU-1T16), from -6 to $42 \mathrm{~s} \ldots \ldots \ldots \ldots 53$

61. Material temperature in broken loop (TMB-20B 16 and TMB-30B 16), from $2010350 \mathrm{~s} \ldots \ldots \ldots . \ldots \ldots$

62. Material temperature in broken loop (TMB-20B16 and TMB-30B 16), from -6 to $42 \mathrm{~s} \ldots \ldots \ldots 54$

63. Material temperature in vessel filler (TMV-FI-115A and TMV-FI-156A), from -20 to $350 \mathrm{~s} \ldots \ldots \ldots \ldots$ 
64. Material temperature in vessel filler (TMV-FI-115A and TMV-FI-156A), from -6 to $42 \mathrm{~s} \ldots \ldots \ldots \ldots 5$

65. Material temperature in vessel filler (TMV-FO-1 56A), from -20 to $350 \mathrm{~s} \ldots \ldots \ldots 56$

66. Material temperature in vessel filler (TMV-FO-156A), from -6 to $42 \mathrm{~s} \ldots \ldots \ldots 56$

67. Material temperature in vessel filler (TIV-FO-35A and TIV-FO-115A), from -20 to $350 \mathrm{~s} \ldots \ldots \ldots$. . . . . . . . 57

68. Material temperature in vessel filler (TIV-FO-35A and TIV-F.O-115A), from -6 to $42 \mathrm{~s} \ldots \ldots \ldots$. . . . . . . . . 57

69. Material temperature in core barrel inner diameter (TMV-CI-70A), from -20 to $350 \mathrm{~s} \ldots \ldots \ldots$. . . . . . . . . 58

70. Material temperature in core barrel inner diameter (TMV-CI-70A), from -6 to 42

71. Material temperature in core barrel inner diameter (TMV-CO-70A and TMV-CO-1 15A), from -20 to $350 \mathrm{~s} \ldots \ldots 59$

72. Material temperature in core barrel inner diameter (TMV-CO-70A and TMV-CO-115A), from -6 to $42 \mathrm{~s}$

73. Material temperature in core housing filler (TMV-HF-115W, TMV-HF-127W, and TMV-HF-138W), from -20 to $350 \mathrm{~s}$

74. Material temperature in core housing filler (TMV-HF-115W, TMV-HF-127W, and TMV-HF-138W), from -6 to $42 \mathrm{~s} \ldots \ldots$. . . . . . . . 60

75. Material temperature in steam generator (TMU-SG1, TMU-SG2, and TMU-SG3), from -20 to $350 \mathrm{~s} \ldots \ldots$. . . . . . . . . 61

76. Material temperature in steam generator (TMU-SG1, TMU-SG2, and TMU-SG3), from $=6$ to $42 \mathrm{~s}$

77. Core heatcr tcmperature, Rod D-4 (TH-D4-14 and TH-D4-29), from -20 to $350 \mathrm{~s} \ldots \ldots \ldots \ldots$

78. Core heater temperature, Rod D-4 (TH-D4-14 and TH-D4-29), from -6 to $42 \mathrm{~s}$

79. Core heater temperature, Rod D-5 (TH-D5-29), from -20 to $350 \mathrm{~s}$ 
80. Core heater temperature, Rod D-5 (TH-D5-29),

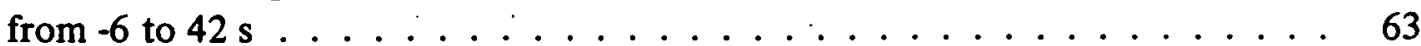

81. Core heater temperature, Rod E-4 (TH-E4-09, TH-E4-27, and TH-E4-55), from -20 to $350 \mathrm{~s} \ldots \ldots . \ldots . . \ldots 64$

82. Core heater temperature, Rod E-4 (TH-E4-09, TH-E4-27, and TH-E4-55), from -6 to $42 \mathrm{~s}$

83. Core heater temperature, Rod E-5 (TH-E5-20

and TH-E5-25), from -20 to $350 \mathrm{~s} \ldots \ldots \ldots 65$

84. Core heater temperature, Rod E-5 (TH-E5-20

and TH-E5-25), from -6 to $42 \mathrm{~s} \ldots \ldots \ldots 5$

85. Core heater temperature, Rod A-4 (TH-A4-09,

TH-A4-29, and TH-A4-39), from -20 to $350 \mathrm{~s} \ldots \ldots 66$

86. Core heater temperature, Rod A-4 (TH-A4-09,

TH-A4-29, and TH-A4-39), from -6 to $42 \mathrm{~s} \ldots \ldots 66$

87. Core heater temperature, Rod A-5 (TH-A5-29

and TH-A5-45), from -20 to $350 \mathrm{~s} \ldots \ldots \ldots 7$

88. Core heater temperature, Rod A-5 (TH-A5-29

and TH-A5-45), from -6 to $42 \mathrm{~s} \ldots \ldots \ldots 67$

89. Core heater temperature, Rod B-3

(TH-B3-32), from -20 to $350 \mathrm{~s} \ldots \ldots \ldots 8$

90. Core heater temperature, Rod B-3

(TH-B3-32), from -6 to $42 \mathrm{~s} \ldots \ldots \ldots \ldots$

91. Core heater temperature, Rod B-5 (TH-B5-29

and TH-B5-33), from -20 to $350 \mathrm{~s} \ldots \ldots \ldots 9 . \ldots \ldots$

92. Core heater temperature, Rod B-5 (TH-B5-29

and TH-B 5-3.3), from -6 to $42 \& \ldots \ldots$. . . . . . . . . . . 69

93. Core heater temperature, Rod B-6

(TH-B6-29), from -20 to $350 \mathrm{~s} \ldots \ldots \ldots$. . . . . . . . . .

94. Core heater temperature, Rod B-6

(TH-B6-29), from -6 to $42 \mathrm{~s} \ldots \ldots \ldots \ldots$. . . . . . . . . . 
95. Core heater temperature, Rod C-2

(TH-C2-38), from -20 to 350 s . . . . . . . . . . . . . . 71

96. Core heater temperature, Rod C-2

(TH-C2-38), from -6 to $42 \mathrm{~s} \ldots \ldots \ldots$. . . . . . . . . . 71

97. Core heater temperature, Rod C-4 (TH-C4-20,

TH-C4-26, and TH-C4-53), from -20 to $350 \mathrm{~s} \ldots \ldots . \ldots . . . . . . .72$

98. Core heater temperature, Rod C-4 (TH-C4-20,

TH-C4-26, and TH-C4-53), from -6 to $42 \mathrm{~s}$

99. Core heater temperature, Rod C-5

(TH-C5-28), from -20 to $350 \mathrm{~s} \ldots \ldots \ldots 73$

100. Core heater temperature, Rod C-5

(TH-C5-28), from -6 to $42 \mathrm{~s}$

101. Core heater temperature, Rod C-6

(TH-C6-53), from -20 to $350 \mathrm{~s}$

102. Core heater temperature, Rod C-6

(TH-C6-53), from -6 to $42 \mathrm{~s}$

103. Core heater temperature, Rod C-7 (TH-C7-07

and $\mathrm{TH}-\mathrm{C} 7-15)$, from -20 to $350 \mathrm{~s}$

104. Core heater temperature, Rod C-7 (TH-C7-07

and TH-C7-15), from -6 to $42 \mathrm{~s}$

105. Core heater temperature, Rod D-1

(TH-D 1-21), from -20 to $350 \mathrm{~s}$

106. Core heater temperature, Rod D-1

(TH-D1-21), from -6 to $42 \mathrm{~s}$

107. Core heater temperature, Rod D-2 (TH-D2-14

and $\mathrm{TH}-\mathrm{D} 2-61)$, from -20 to $350 \mathrm{~s}$

108. Core heater temperature, Rod D-2 (TH-D2-14 and TH-D2-61), from -6 to $42 \mathrm{~s}$

109. Core heater temperature, Rod D-3 (TH-D3-29 and TH-D3-39), from -20 to $350 \mathrm{~s}$ 
110. Core heater temperature, Rod D-3 (TH-D3-29 and TH-D3-39), from -6 to $42 \mathrm{~s} \ldots \ldots \ldots \ldots \ldots \ldots$

111. Core heater temperature, Rod D-6

(TH-D6-25), from -20 to $350 \mathrm{~s}$

112. Core heater temperature, Rod D-6

(TH-D6-25), from -6 to $42 \mathrm{~s} \ldots \ldots \ldots \ldots \ldots$. . . . . . . . . . . . . . . .

113. Core heater temperature, Rod D-7

(TH-D7-20), from -20 to $350 \mathrm{~s} \ldots \ldots \ldots \ldots$. . . . . . . 80

114. Core heater temperature, Rod D-7

(TH-D7-20), from -6 to $42 \mathrm{~s} \ldots \ldots \ldots \ldots \ldots \ldots \ldots$

115. Core heater temperature, Rod D-8

(TH-D8-26), from -20 to $350 \mathrm{~s} \ldots \ldots \ldots \ldots \ldots$. . . . . . . 81

116. Core heater temperature, Rod D-8

(TH-D8-26), from -6 to $42 \mathrm{~s} \ldots \ldots \ldots \ldots \ldots \ldots \ldots$

117. Core heater temperature, Rod E-1

(TH-E1-33), from -20 to $350 \mathrm{~s}$

118. Core heater temperature, Rod E-1

(TH-E1-33), from -6 to $42 \mathrm{~s}$

119. Core heater temperature, Rod E-2 (TH-E2-20

and TH-E2-33), from -20 to $350 \mathrm{~s} \ldots \ldots \ldots \ldots \ldots$

120. Core heater temperature, Rod E-2 (TH-E2-20 and TH-E2-33), from -6 to $42 \mathrm{~s}$

121. Core heater temperature, Rod E-3 (TH-E3-05,

TH-E3-20, and TH-E3-24), from -20 to $350 \mathrm{~s}$

122. Core heater temperature, Rod E-3 (TH-E3-05,

TH-E3-20, and 'TH-E3-24), from -6 to $42 \mathrm{~s}$

84

123. Core heater temperature, Rod E-6 (TH-E6-08,

TH-E6-28, and TH-E6-37), from -20 to $350 \mathrm{~s}$

124. Core heatcr temperature, Rod E-6 (TH-E6=08,

TH-E6-28, and TH-E6-37), from -6 to $42 \mathrm{~s}$

125. Core heater temperature, Rod E-7

(TH-E7-44), from -20 to $350 \mathrm{~s}$ 
126. Core heater temperature, Rod E-7 (TH-E7-44), from -6 to $42 \mathrm{~s} \ldots \ldots \ldots$. . . . . . . . 86

127. Core heater temperature, Rod E-8 (TH-E8-14 and TH-E8-29), from -20 to $350 \mathrm{~s} \ldots \ldots \ldots$. . . . . . . . . 87

128. Core heater temperature, Rod E-8 (TH-E8-14 and TH-E8-29), from -6 to $42 \mathrm{~s}$

129. Core heater temperature, Rod F-2 (TH-F2-07, TH-F2-22, and TH-F2-25), from -20 to $350 \mathrm{~s}$

130. Core heater temperature, Rod F-2 (TH-F2-07, TH-F2-22, and TH-F2-25), from -6 to $42 \mathrm{~s}$

131. Core heater temperature, Rod F-4 (TH-F4-14, TH-F4-29, and TH-F4-44), from -20 to $350 \mathrm{~s}$

132. Core heater temperature, Rod F-4 (TH-F4-14, TH-F4-29, and TH-F4-44), from -6 to $42 \mathrm{~s}$

133. Core heater tcmperature, Rod F-5 (TH-F5-20, TH-F5-26, TH-F5-33, and TH-F5-53), from -20 to $350 \mathrm{~s}$

134. Core heater temperature, Rod F-5 (TH-F5-20, TH-F5-26, TH-F5-33, and TH-F5-53), from -6 to $42 \mathrm{~s}$

135. Core heater temperature, Rod G-3 (TH-G3-13), from -20 to 350 s

136. Core heater temperature, Rod G-3 (TH-G3-13), from -6 to $42 \mathrm{~s}$

137. Core heater temperature, Rod G-4 (TH-G4-29, TH-G4-33, and TH-G4-38), from -20 to $350 \mathrm{~s}$

138. Corc heater temperature, Rod G-4 (TH-G4-29, TH-G4-33, and TH-G4-38), from -6 to $42 \mathrm{~s}$

139. Core heater temperature, Rod G-5 (l'H-G5-14 and TH-G5-24), from -20 to $350 \mathrm{~s}$

140. Core heater temperature, Rod G-5 (TH-G5-14 and TH-G5-24), from -6 to $42 \mathrm{~s}$ 
141. Core heater temperature, Rod H-5,

(TH-H5-32), from -20 to $350 \mathrm{~s}$

142. Core heater temperature, Rod H-5,

(TH-H5-32), from -6 to $42 \mathrm{~s} \ldots \ldots \ldots$. . . . . . . . . . . 94

143. Pressure in intact loop (PU-13

and PU-15L), from -20 to $350 \mathrm{~s} \ldots \ldots \ldots$. . . . . . . . . 95 .

144. Pressure in intact loop (PU-13

and PU-15L), from -6 to $42 \mathrm{~s}$

145. Pressure in broken loop, vessel side (PB-21

and PB-23), from -20 to $350 \mathrm{~s} \ldots \ldots$. . . . . . . . . . . 96

146. Pressure in broken loop, vessel side (PB-21

and PB-23), from -6 to $42 \mathrm{~s} \ldots \ldots \ldots$. . . . . . . . . . 96

147. Pressure in broken loop, vessel side

(PB-CN4), from -20 to $350 \mathrm{~s} \ldots \ldots \ldots \ldots$

148. Pressure in broken loop, vessel side

(PB-CN4), from -6 to $42 \mathrm{~s}$

149. Pressure in broken loop, pump side (PB-37

and $\mathrm{PB}-42)$, from -20 to $350 \mathrm{~s} \ldots \ldots \ldots \ldots$

150. Pressure in broken loop, pump side (PB-37

and $\mathrm{PB}-42$ ), from -6 to $42 \mathrm{~s}$

151. Pressure in broken loop, vessel side

(PB-HN1), from -20 to $350 \mathrm{~s}$

99

152. Pressure in broken loop, vessel side (PB-HN1), from -6 to $42 \mathrm{~s} \ldots \ldots \ldots \ldots$

153. Pressure in vessel (PV-UP+10 and

PV-LP-166), fruini 20 to $350 \mathrm{~s}$

154. Pressure in vessel (PV-UP+10 and

PV-LP-166), from -6 to $42 \mathrm{~s}$

155. Pressure in intact loop accumulator

(PU-ACC1), from -20 to $350 \mathrm{~s}$ 
156. Pressure in intact loop accumulator

(PU-ACC1), from -6 to $42 \mathrm{~s} \ldots \ldots \ldots 10 \ldots \ldots$

157. Pressure in broken loop accumulator (PB-ACC2), from -20 to $350 \mathrm{~s} \ldots \ldots \ldots 102$

158. Pressure in broken loop accumulator (PB-ACC2), from -6 to $42 \mathrm{~s} \ldots \ldots \ldots \ldots 2$

159. Pressure in steam generator, secondary side (PU-SGSD), from -20 to $350 \mathrm{~s} \ldots \ldots \ldots$. . . . . . . . . . . 103

160. Pressure in steam generator, secondary side (PU-SGSD), from -6 to $42 \mathrm{~s} \ldots \ldots \ldots \ldots . \ldots \ldots$

161. Pressure in steam generator, secondary side (PU-SGS3), from -20 to $350 \mathrm{~s} \ldots \ldots \ldots$. . . . . . . . . 104

162. Pressure in steam generator, secondary side

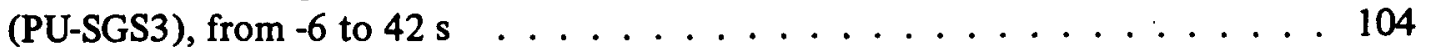

163. Pressure in pressurizer (PU-PRIZE),
from -20 to $350 \mathrm{~s} \ldots \ldots \ldots \ldots \ldots \ldots \ldots \ldots \ldots \ldots \ldots \ldots$

164. Pressure in pressurizer (PU-PRIZE), from -6 to $42 \mathrm{~s} \ldots \ldots \ldots \ldots \ldots$

165. Pressure in pressure suppression tank (P-PSS), from -20 to $350 \mathrm{~s} \ldots \ldots \ldots \ldots$. . . . . . . . . . . 106

166. Pressure in pressure suppression tank (P-PSS), from -6 to $42 \mathrm{~s} \ldots \ldots \ldots \ldots \ldots$

167. Differential pressure in intact loop (DPU-3-7), from -20 to $350 \mathrm{~s}$ 107

168. Differential pressure in intact loop (DPU-3-7), from -6 to $42 \mathrm{~s}$

169. Differential pressure in intact loop (DPU-6-SGIP), from -20 to $350 \mathrm{~s}$

170. Differential pressure in intact loop (DPU-6-SGIP), from -6 to $42 \mathrm{~s}$

171. Differential pressure in intact loop (DPU-SGOP-7), from -20 to $350 \mathrm{~s}$ 
172. Differential pressure in intact loop (DPU-SGOP-7), from -6 to $42 \mathrm{~s}$

173. Differential pressure in intact loop (DPU-7-10), from -20 to $350 \mathrm{~s}$

174. Differential pressure in intact loop (DPU-7-10), from -6 to $42 \mathrm{~s}$.

175. Differential pressure in intact loop. (DPU-12-10), from -20 to $350 \mathrm{~s}$

176. Differential pressure in intact loop (DPU-12-10), from -6 to 428

177. Differential pressure in intact loop, low range (DPU-12-10L), from -20 to $350 \mathrm{~s}$

178. Differential pressure in intact loop, low range (DPU-12-10L), from -6 to $42 \mathrm{~s}$

179. Differential pressure in in tact loop (DPU-12-15), from -20 to $350 \mathrm{~s}$

180. Differential pressure in intact loop (DPU-12-15), from -6 to $42 \mathrm{~s}$

181. Differential pressure in intact loop (DPU-15-3), from -20 to $350 \mathrm{~s}$

182. Differential pressure in intact loop (DPIJ-15-3), from -6 to $42 \mathrm{~s}$

183. Differential pressure in intact loop, low range (DPU-15-3L), from -20 to $350 \mathrm{~s}$

184. Differential pressure in intact loop, low range (DPU $15-3 \mathrm{~L}$ ), from -6 to $12 \mathrm{E}$

185. Differential pressure in intact loop (DPU-PRESLL), from -20 to $350 \mathrm{~s}$

186. Differential pressure in intact loop (DPU-PRESLL), from -6 to $42 \mathrm{~s}$ 
187. Differential pressure in intact loop (DPU-PR-4), from -20 to $350 \mathrm{~s}$

188. Differential pressure in in tact loop (DPU-PR-4), from -6 to $42 \mathrm{~s}$

189. Differential pressure in broken loop (DPB-21-IANN), from -20 to $350 \mathrm{~s}$

190. Differential pressure in broken loop (DPB-21-IANN), from -6 to $42 \mathrm{~s}$

191. Differential pressure in broken loop (DPB-23-CN1), from -20 to $350 \mathrm{~s}$

192. Differential pressure in broken loop (DPB-23-CN1), from -6 to $42 \mathrm{~s}$

193. Differential pressure in broken loop (DPB-CN1-24), from -20 to 350 s

194. Differential pressure in broken loop (DPB-CN1-24), from -6 to $42 \mathrm{~s}$

195. Differential pressure in broken loop

(DPB-30-43), from -20 to $350 \mathrm{~s}$

196. Differential pressure in broken loop (DPB-30-43), from -6 to $42 \mathrm{~s}$

197. Differential pressure in broken loop (DPB-32U-36L), from -20 to $350 \mathrm{~s}$

198. Differential pressure in broken loop (DPB-32U-36L), from -6 to $42 \mathrm{~s}$

199. Differential pressure in broken loop (DPB-36L-37), from -20 to $350 \mathrm{~s}$

200. Differential pressure in broken loop (DPB-36L-37), from -6 to $42 \mathrm{~s}$

201. Differential pressure in broken loop (DPB-38-40), from -20 to $350 \mathrm{~s}$

202. Differential pressure in broken loop (DPB-38-40), from -6 to $42 \mathrm{~s}$ 
203. Differential pressure in broken loop

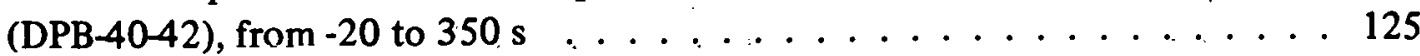

204. Differential pressure in broken loop

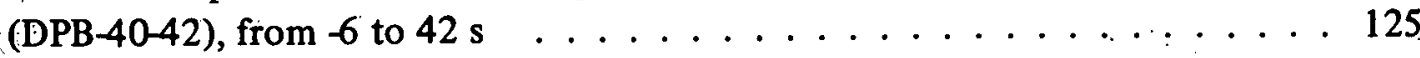

205. Differential pressure in broken loop

(DPB-42-HN1), from -20 to $350 \mathrm{~s} \ldots \ldots \ldots \ldots \ldots \ldots \ldots$

206. Differential pressure in broken loop

(DPB-42-HN1), from -6 to $42 \mathrm{~s}$

207. Differential pressure in broken loop

(DPB-HN1-43), from -20 to $350 \mathrm{~s} \ldots \ldots \ldots \ldots \ldots \ldots$

208. Differential pressure in broken loop

(DPB-HNI-43), from -6 to $42 \mathrm{~s}$

209. Differential pressure in vessel

(DPV-UP-IANN), from -20 to $350 \mathrm{~s} \ldots \ldots \ldots \ldots \ldots \ldots \ldots$

210. Differential pressure in vessel

(DPV-UP-IANN), from -6 to $42 \mathrm{~s} \ldots \ldots \ldots \ldots \ldots \ldots$

211. Differential pressure in vessel

(DPV-0-9GQ); from -20 to $350 \mathrm{~s} \ldots \ldots \ldots \ldots \ldots \ldots$

212. Differentiäl pressure in vessel

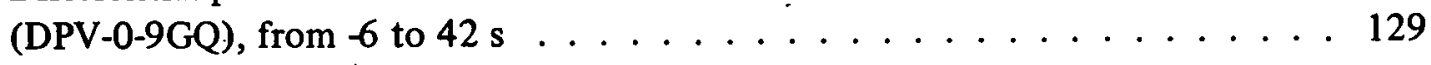

213. Differential pressure in vessel

(DPV-9-26QQ), from -20 to $350 \mathrm{~s} \ldots \ldots \ldots \ldots \ldots \ldots \ldots \ldots$

214. Differential pressure in vessel

(DPV-9-26QQ), from -6 to $42 \mathrm{~s} \ldots \ldots \ldots \ldots \ldots \ldots \ldots$

215. Differential pressure in vessel

(DPV-9-166QQ), from $-2 n$ tn $350 \mathrm{~s} \ldots \ldots \ldots \ldots \ldots \ldots \ldots$

216. Differential pressure in vessel

(DPV-9-166QQ), from -6 to $42 \mathrm{~s} \ldots \ldots \ldots \ldots \ldots$. . . . . . . . . .

217. Differential pressure in vessel

(DPV-26-55QM), from -20 to $350 \mathrm{~s} \ldots \ldots \ldots \ldots \ldots \ldots \ldots \ldots \ldots \ldots \ldots$ 
218. Differential pressure in vessel

(DPV-26-55QM), from -6 to $42 \mathrm{~s} \ldots \ldots \ldots 132$

219. Differential pressure in vessel

(DPV-55-1 10MM), from -20 to $350 \mathrm{~s} \ldots \ldots \ldots \ldots$

220. Differential pressure in vessel

(DPV-55-1 10MM), from -6 to $42 \mathrm{~s} \ldots \ldots \ldots$. . . . . . . . . . . . . . . 33

221. Differential pressure in vessel

(DPV-1 10-156MQ), from -20 to $350 \mathrm{~s} \ldots \ldots \ldots$. . . . . . . . . . .

222. Differential pressure in vessel

(DPV-1 10-1 56MQ), from -6 to $42 \mathrm{~s} \ldots \ldots \ldots 134$

223. Differential pressure in vessel

(DPV-156-173QQ), from -20 to $350 \mathrm{~s} \ldots \ldots 135$

224. Differential pressure in vessel

(DPV-156-173QQ), from -6 to $42 \mathrm{~s} \ldots \ldots \ldots$. . . . . . . . . 135

225. Differential pressure in vessel

(DPV-166Q+10), from -20 to $350 \mathrm{~s} \ldots \ldots 136$

226. Differential pressure in vessel

(DPV-166Q+10), from -6 to $42 \mathrm{~s} \ldots \ldots \ldots \ldots$

227. Differential pressure in intact

loop accumulator (DPU-ACC1-TB), from -20 to $350 \mathrm{~s} \ldots \ldots . . \ldots 137$

228. Differential pressure in intact loop accumulator (DPU-ACC. 1-TR), from 6 to $42 \mathrm{~s} \ldots \ldots 137$

229. Differential pressure in broken loop accumulator (DPB-ACC2-TB), from -20 to 350 s f . . . . . . . . . 138

230. Differential pressure in broken loop accumulator (DPB-ACC2-TB), from -6 to $42 \mathrm{~s} \ldots \ldots \ldots \ldots$

231. Differential prcssure in steam generatoi' secondary (DPU-SG-SEC), from -20 to $350 \mathrm{~s} \ldots \ldots 139$

232. Differential pressure in steam generator secondary (DPU-SG-SEC), from -6 to $42 \mathrm{~s} \ldots \ldots$. . . . . . . . . . . 139 
233. Differential pressure across steam generator outlet orifice (DPU-SG-DISC), from -20 to $350 \mathrm{~s}$. . . . . . . . . . . . . . . . . 140

234. Differential pressure across steam generator outlet orifice (DPU-SG-DISC), from -6 to $42 \mathrm{~s} \ldots \ldots \ldots$. . . . . . . . . 140

235. Differential pressure between simulated rupture injection line and Spool 6 (DPU-SGS-6), from -20 to $350 \mathrm{~s}$

236. Differential pressure between simulated rupture injection line and Spool 6 (DPU-SGS-6), from -6 to $42 \mathrm{~s}$

237. Differential pressure in intact loop accumulator (DPU-SGS3-TB), from -20 to $350 \mathrm{~s}$

238. Differential pressure in intact loop accumulator (DPU-SGS3-TB), from -6 to $42 \mathrm{~s} \ldots \ldots \ldots 142$

239. Differential pressure in pressure suppression tank (DP-PSS-TB), from -20 to $350 \mathrm{~s}$

240. Differential pressure in pressure suppression tank (DP-PSS-TB), from -6 to $42 \mathrm{~s}$

241. Volumetric flow in intact loop (FTU-1 aud FTU-9), from -20 to 350 . . . . . . . . . . . . . . . . . . . 144

242. Volumetric flow in intact loop (FTU-1 and FTU-9), from -6 to $42 \mathrm{~s}$

243. Volumetric flow in intact loop (FTU-13 and FTU-15), from -20 to $350 \mathrm{~s} \ldots \ldots \ldots \ldots \ldots$

244. Volumetric flow in intact loop (FTU-13 and FTU-15), from -6 to $42 \mathrm{~s}$

245. Volumetric flow in broken loop (FTB-21), from -20 to $350 \mathrm{~s}$ 146

246. Volumetric flow in broken loop (FTB-21), from -6 to $42 \mathrm{~s}$

247. Volumetric flow in broken loop (FTB-30 and FTB-37), from -20 tó $350 \mathrm{~s}$

248. Volumetric flow in broken loop (FTB-30 and FTB-37), from -6 to $42 \mathrm{~s}$ 
249. Volumetric flow in core entrance

(FTV-CORE-IN), from -20 to $350 \mathrm{~s} \ldots \ldots \ldots$. . . . . . . . . . 148

250. Volumetric flow in core entrance (FTV-CORE-IN), from -6 to $42 \mathrm{~s}$

251. Volumetric flow in intact loop high pressure injection line (FTU-HPIS), from -20 to $350 \mathrm{~s}$

252. Volumetric flow in in tact loop high pressure injection line (FTU-HPIS), from -6 to $42 \mathrm{~s} \ldots \ldots$. . . . . . . . . . . . . 149

253. Volumetric flow in broken loop high pressure injection line (FTB-HPIS), from -20 to $350 \mathrm{~s}$

254. Volumetric flow in broken loop high pressure injection line (FTB-HPIS), from -6 to $42 \mathrm{~s} \ldots \ldots \ldots 150$

255. Volumetric flow in in tact loop low pressure injection line (FTU-LPIS), from -20 to $350 \mathrm{~s}$

256. Volumetric flow in intact loop low pressure injection line (FTU-LPIS), from -6 to $42 \mathrm{~s}$

257. Volumetric flow in broken loop low pressure injection line (FTB-LPIS), from -20 to $350 \mathrm{~s}$

258. Volumetric flow in broken loop low pressure injection line (FTB-LPIS), from -6 to 42

259. Volumetric flow in in tact loop accumulator discharge line (FTU-ACC1), from -20 to $350 \mathrm{~s}$

260. Volumetric flow in in tact loop accumulator discharge line (FTU-ACC1), from -6 to 42 .

261. Volumetric flow in broken loop accumulator discharge line (FTB-ACC2), from -20 to $350 \mathrm{~s}$

262. Volumetric flow in broken loop accumulator discharge line (FTB-ACC2), from -6 to $42 \mathrm{~s} \ldots \ldots \ldots 154$

263. Volumetric flow in intact loop steam generator (FTU-SGS), from -20 to $350 \mathrm{~s}$ 
264. Volumetric flow in in tact loop steam generator

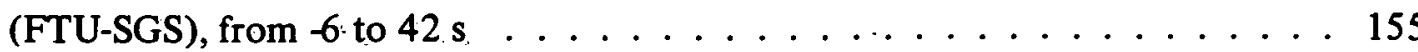

265. Volumetric flow from pressurizer

(FTU-PRIZE), from -20 to $350 \mathrm{~s} \ldots \ldots \ldots \ldots \ldots \ldots$

266. Volumetric flow from pressurizer (FTU-PRIZE), from -6 to $42 \mathrm{~s} \ldots \ldots \ldots \ldots \ldots \ldots \ldots$

267. Fluid velocity in vessel (FTV $-40 \mathrm{~A}$

and FTV-40M), from -20 to $350 \mathrm{~s} \ldots \ldots \ldots \ldots . \ldots \ldots$

268. Fluid velocity in vessel (FTV-40A and FTV-40M), from -6 to $42 \mathrm{~s} \ldots \ldots \ldots \ldots \ldots . \ldots \ldots$

26. Momentum flux in intact loop (FDU-1), from -20 to $350 \mathrm{~s}$

270. Momentum flux in intact loop (FDU-1), from -6 to $42 \mathrm{~s} \ldots \ldots \ldots \ldots \ldots \ldots \ldots \ldots \ldots \ldots$

271. Momentum flux in intact loop (FDU-5), from -20 to 350 s . . . . . . . . . . . . . . . . . 159

272. Momentum flux in intact loop (FDU-5), from -6 to 42 s . . . . . . . . . . . . . . . . . . . . . . . 159

273. Momentum flux in intact loop (FDU-10), from -20 to $350 \mathrm{~s} \ldots \ldots \ldots \ldots \ldots \ldots \ldots \ldots$

274. Momentum flux in intact loop (FDU-10), from -6 to $42 \mathrm{~s} \ldots \ldots \ldots \ldots \ldots \ldots \ldots \ldots$

275. Momentum flux in intact loop (FDU-13), from -20 to $350 \mathrm{~s}$

276. Momentum flux in intact loop (FDU-13), fruili -6 to $42 \mathrm{~s}$

277. Momentum flux in intact loop (FDU-15),

278. Momentum flux in intact loop (FDU-15), from -6 to $42 \mathrm{~s} \ldots \ldots \ldots \ldots$ 
279. Momentum flux in broken loop (FDB-21), from -20 to $350 \mathrm{~s}$

280. Momentum flux in broken loop (FDB-21), from -6 to $42 \mathrm{~s}$

281. Momentum flux in broken loop (FDB-23), from -20 to $350 \mathrm{~s}$

282. Momentum flux in broken loop (FDB-23), from -6 to $42 \mathrm{~s}$

283. Momentum flux in broken loop (FDB-30), from -20 to $350 \mathrm{~s}$

284. Momentum flux in broken loop (FDB-30), from -6 to $42 \mathrm{~s}$

285. Momentum flux in broken loop (FDB-37), from -20 to $350 \mathrm{~s}$

286. Momentum flux in broken loop (FDB-37), from -6 to $42 \mathrm{~s}$

287. Momentum flux in broken loop (FDB-42), from -20 to $350 \mathrm{~s}$

288. Momentum flux in broken loop (FDB-42), from -6 to $42 \mathrm{~s}$

289. Momentum flux in core entrance (FDV-CORE-IN), from -20 to $350 \mathrm{~s}$

290. Momentum flux in core entrance (FDV-CORE-IN), from -6 to $42 \mathrm{~s}$

291. Density in intact loop (GU-1T and GU-1B), from -20 to $350 \mathrm{~s}$

292. Density in intact loop (GU-1T and GU-1B), from -6 to 42

293. Density in intact loop (GU-1C), from -20 to $350 \mathrm{~s} \ldots \ldots$. . . . . . . . 170

294. Density in intact loop (GU-1C), from -6 to $42 \mathrm{~s} \ldots \ldots \ldots$ 
295. Density in intact loop (GU-5VR

and GU-10VR), from -20 to $350 \mathrm{~s} \ldots \ldots \ldots \ldots \ldots \ldots$

296. Density in intact loop (GU-5VR

and GU-10VR), from -6 to 42 s . . . . . . . . . . . . . 171

297. Density in intact loop (GU-13:VR), from -20 to $350 \mathrm{~s} \ldots \ldots \ldots \ldots$

298. Density in intact loop (GU-13VR), from -6 to $42 \mathrm{~s} \ldots \ldots \ldots \ldots$

299. Density in intact loop (GU-15T

and GU-15B), from -20 to 350 s . . . . . . . . . . . . 173

300. Density in intact loop (GU-15T

and $\mathrm{GU}-15 \mathrm{~B})$, frum $-\mathrm{G}$ to $42 \mathrm{~s} \ldots \ldots \ldots \ldots \ldots \ldots$

301. Density in intact loop (GU-1 sc), from -20 to $350 \mathrm{~s} \ldots \ldots \ldots 171$

302. Density in intact loop (GU-15C), from -6 to $42 \mathrm{~s} \ldots \ldots \ldots$

303. Density in broken loop (GB-21T

and GB-21B), from -20 to $350 \mathrm{~s} \ldots \ldots \ldots \ldots \ldots \ldots$

304. Density in broken loop (GB-21T

and GB-21B), from -6 to $42 . \mathrm{s} \ldots \ldots \ldots \ldots \ldots \ldots$

305. Density in broken loop (GB-21C), from -20 to $350 \mathrm{~s} \ldots \ldots \ldots$

306. Density in broken loop (GB-21C), from -6 to $42 \mathrm{~s} \ldots \ldots \ldots \ldots$

307. Density in broken loop (GB-23VR), from -20 to $350 \mathrm{~s} \ldots \ldots \ldots$

308. Density in broken loop (GB-23VR), from -6 to $42 \mathrm{~s} \ldots \ldots \ldots \ldots$

309. Density in broken loop (GB-30T

and GB-30B), from -20 to $350 \mathrm{~s} \ldots \ldots \ldots \ldots \ldots \ldots \ldots \ldots$

310. Density in broken loop (GB-30T and GB-30B), from -6 to $42 \mathrm{~s} \ldots \ldots \ldots \ldots \ldots$

311. Density in broken loop (GB-30C), from -20 to $350 \mathrm{~s} \ldots \ldots \ldots$

312. Density in broken loop (GB-30C), from -6 to $42 \mathrm{~s} \ldots \ldots \ldots \ldots$

313. Density in broken loop (GB-37HZ), from -20 to $350 \mathrm{~s} \ldots \ldots \ldots$ 
314. Density in broken loop (GB-37HZ), from -6 to $42 \mathrm{~s} \ldots \ldots \ldots$

315. Density in broken loop (GB-42VR), from -20 to $350 \mathrm{~s} \ldots \ldots \ldots 1$

316. Density in broken loop (GB-42VR), from -6 to $42 \mathrm{~s} \ldots \ldots \ldots 181$

317. Density in vessel (GV-COR-150HZ), from -20 to $350 \mathrm{~s} \ldots \ldots \ldots$

318. Density in vessel (GV-COR-150HZ), from -6 to $42 \mathrm{~s} \ldots \ldots \ldots 2$

319. Density in vessel (GVLP-165HZ and GVLP-172HZ), from -20 to $350 \mathrm{~s} \ldots \ldots \ldots \ldots$

320. Density in vessel (GVLP-165HZ and GVLP-172HZ), from -6 to $42 \mathrm{~s} \ldots \ldots \ldots 183$

321. Density in pressurizer (GU-PRIZE), from -20 to $350 \mathrm{~s} \ldots \ldots \ldots$. . . . . . 184

322. Density in pressurizer (GU-PRIZE), from -6 to $42 \mathrm{~s} \ldots \ldots \ldots$

323. Mass flow in intact loop (FDU-1, GU-1C), from -20 to $350 \mathrm{~s} \ldots \ldots \ldots \ldots$. . . . . . . . . . . . . . . .

324. Mass flow in intact loop (FDU-1, GU-1C), from -6 to $42 \mathrm{~s}$

325. Mass flow in intact loop (FTU-1, GU-1C), from -20 to $350 \mathrm{~s} \ldots \ldots \ldots \ldots$. . . . . . . . . . . 186

326. Mass flow in intact loop (FTU-1, GU-1C), from -6 to $42 \mathrm{~s} \ldots \ldots \ldots \ldots$. . . . . . . . . . . . . . . . .

327. Mass flow in intact loop (FDU-5, GU-5VR), from -20 to $350 \mathrm{~s}$

328. Mass flow in intact loop (FDU-5, GU-5VR), from -6 to $42 \mathrm{~s} \ldots \ldots \ldots \ldots$. . . . . . . . . . . . . . .

329. Mass flow in intact loop (FTU-9, GU-10VR), from -20 to $350 \mathrm{~s}$

330. Mass flow in intact loop (FTU-9, GU-10VR), from -6 to $42 \mathrm{~s}$

331. Mass flow in intact loop (FDU-10, GU-10VR), from -20 to $350 \mathrm{~s}$ 
332. Mass flow in intact loop (FDU-1.0,

GU-10VR), from -6 to $42 \mathrm{~s}: \ldots \ldots \ldots$. . . . . . . . . . . . . . . . .

333. Mass flow in intact loop (FDU-13, GU-13VR), from -20 to $350 \mathrm{~s}$. . . . . . . . . . . . . 190

334. Mass flow in intact loop (FDU-13, GU-13VR), from -6 to $42 \mathrm{~s}$

335. Mass flow in intact loop (FTU-13,

GU-13VR), from -20 to $350 \mathrm{~s}$

336. Mass flow in intact loop (FTU-13, GIJ-13VR), from -6 to $42 \mathrm{~s}$

337. Mass flow in intact loop (FDU-15,

GU-15C), from -20 to $350 \mathrm{~s}$

338. Mass flow in intact loop (FDU-15,

GU-15C), from -6 to $42 \mathrm{~s}$

339. Mass flow in intact loop (FTU-15, GU-15C), from -20 to $350 \mathrm{~s} \ldots \ldots \ldots$. . . . . . . . . . . 193

340. Mass flow in intact loop (FTU-15, GU-15C), from -6 to $42 \mathrm{~s}$

341. Mass flow in broken loop (FDB-21,

GB-21C), from -20 to 350 s

342. Mass flow in broken loop (FDB-21, GB-21C), from -6 to $42 \mathrm{~s}$

343. Mass flow in broken loop (FTB-21, GB-21C), from -20 to $350 \mathrm{~s}$

344. Màss flow in broken loop (FTB-21,

GD-21C), from -6 to 12 s

345. Mass flow in broken loop (FDB-23, GB-23VR), from -20 to $350 \mathrm{~s}$ 196

346. Mass flow in broken loop (FDB-23,

GB-23VR), from -6 to $42 \mathrm{~s}$ 
347. Mass flow in broken loop (FDB-30, GB-30C), from -6 to $42 \mathrm{~s}$

348. Mass flow in broken loop (FDB-30,

GB-30C), from -6 to $42 \mathrm{~s}$

349. Mass flow in broken loop (FTB-30,

GB-30C), from -20 to $350 \mathrm{~s}$

350. Mass flow in broken loop (FTB-30, GB-30C), from -6 to $42 \mathrm{~s}$

351. Mass flow in broken loop (FDB-37,

GB-37HZ), from -20 to $350 \mathrm{~s}$

352. Mass flow in broken loop (FDB-37,

GB-37HZ), from -6 to $42 \mathrm{~s}$

353. Mass flow in broken loop (FTB-37, GB-37HZ), from -20 to $350 \mathrm{~s}$

354. Mass flow in broken loop (FTB-37, GB-37HZ), from -6 to $42 \mathrm{~s}$

355. Mass flow in vessel (FDV-CORE-IN,

GV-COR-150HZ), from -20 to $350 \mathrm{~s} \ldots \ldots \ldots$. . . . . . . . . . . . .

356. Mass flow in vessel (FDV-CORE-IN, GV-COR-150HZ), from -6 to $42 \mathrm{~s} \ldots \ldots \ldots$. . . . . . . . . . . . .

357: Mass flow in vessel (FTV-CORE-IN,

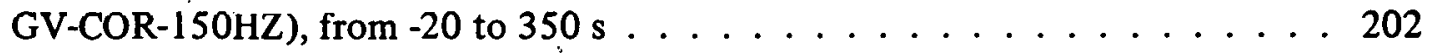

358. Mass flow in vessel (FTV-CORE-IN, GV-COR-150HZ), from -6 to $42 \mathrm{~s} \ldots \ldots . . . . . . . . . .202$

359. Mass flow in pressurizer (FTU-PRIZE, GU-PRIZE), from -20 to 350 s . . . . . . . . . . . . . . . 203

360. Mass flow in pressurizer (FTU-PRIZE, GU-PRIZE), from 6 to $42 \mathrm{~s} \ldots \ldots . \ldots 203$

361. Core heater rod total power (PWRCOR T-1 and PWRCOR T-2), from -20 to $350 \mathrm{~s}$ 
362. Core heater rod total power (PWRCOR T-1 and

PWRCOR T-2), from -6 to $42 \mathrm{~s}$. . . . . . . . . . . . . . . . 204

363. Core heater voltage (VOLTCOR-T), from -20 to $350 \mathrm{~s} \ldots \ldots 205$

364. Core heater voltage (VOLTCOR-T), from -6 to $42 \mathrm{~s} \ldots \ldots \ldots$

365. Core heater total current (AMPCOR-T), from -20 to $350 \mathrm{~s} \ldots \ldots$

366. Core heater total current (AMPCOR-T), from -6 to $42 \mathrm{~s} \ldots \ldots$. . . . . . 206

367. Primary pump current (PUMPU-CUR), from -20 to $350 \mathrm{~s} \ldots \ldots$. . . . . . 207

368. Primary pump current (PUMPU-CUR), from -6 to $42 \mathrm{~s} \ldots \ldots$. . . . . . 207

369. Primary pump speed (PUMPU-RPM), from -20 to $350 \mathrm{~s} \ldots \ldots$. . . . . . 208

370. Primary pump speed (PUMPU-RPM), from -6 to $42 \mathrm{~s} \ldots \ldots$. . . . . . 208

B-1. Geometry used for processing of density

data obtained from two-beam gamma densitometers . . . . . . . . . . 224

C-1. Fluid temperature in broken loop (TFB-23) . . . . . . . . . . . 234

C-2. Fluid temperature in downcomer annulus (TFV-ANN-35A) . . . . . . . . 235

C-3, Fluid temperature in lower plenum (TFV-LP-8) . . . . . . . . 235

C-4. Fluid temperature in pressurizer surge line (TFU-PRIZE) $\ldots \ldots . \ldots \ldots$

C-5. Material temperature in intact loop (TMU-1T16) . . . . . . . . 236

r.6. Material temperature in vessel filler (TMV-CI-70A) . . . . . . . . 237

C-7. Core heater temperature, Rod E-4 (TH-E4-09) . . . . . . . . . . . 237

C-8. Core heater temperature, Rod E-4 (TH-E4-27) . . . . . . . . . . 238

C-9. Core heater temperature, Rod E-4 (TH-E4-55) . . . . . . . . . . . 238

C-10. Pressure in intact loop, Spool 13 (PU-13) . . . . . . . . . . . . 239

C-11. Pressure in broken loop, Spool $23(\mathrm{~PB}-23) \ldots \ldots . \ldots . \ldots . . \ldots 239$

C-12. Differential pressure in intact loop (DPU-7-10) . . . . . . . . 240 
C-13. Differential pressure in intact loop (DPU-12-10) . . . . . . . . . . 240

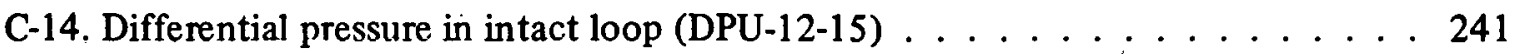

C-15. Volumetric flow in intact loop (FTU-1) $\ldots \ldots \ldots \ldots \ldots \ldots$

C-16. Volumetric flow in intact loop (FTU-15) $\ldots \ldots \ldots \ldots . \ldots 242$

C-17. Volumetric flow in intact loop high pressure injection line (FTU-HPIS) . . . . . . . . . . . . . . 242

C-18. Volumetric flow in intact loop low pressure injection line (FTU-LPIS) $\ldots \ldots \ldots \ldots \ldots \ldots \ldots \ldots$

C-19. Volumetric flow in intact loop accumulator discharge line (FTU-ACC1) . . . . . . . . . . . . . . 243

C-20. Fluid velocity in vessel (FTV-40A) $\ldots \ldots \ldots \ldots \ldots$

C-21. Fluid velocity in vessel (FTV-40M) $\ldots \ldots \ldots \ldots \ldots \ldots$

C-22. Density in intact loop (GU-1T) $\ldots \ldots \ldots \ldots \ldots \ldots \ldots$

C-23. Density in intact loop (GU-1B) $\ldots \ldots \ldots \ldots \ldots \ldots \ldots$

C-24. Density in intact loop (GU-1C) $\ldots \ldots \ldots \ldots \ldots \ldots \ldots$

C-25. Density in in tact loop (GU-10VR) $\ldots \ldots \ldots \ldots \ldots \ldots \ldots$

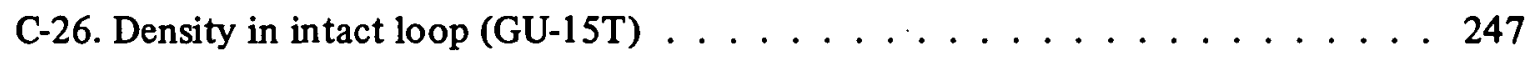

C-27. Density in intact loop (GU-15B) $\ldots \ldots \ldots \ldots \ldots \ldots \ldots$

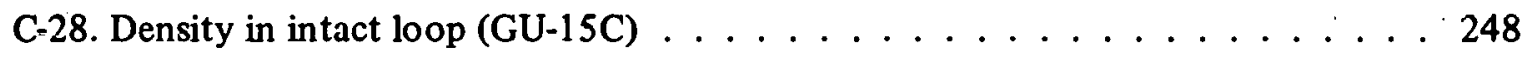

C-29. Density in broken loop (GB-23VR) $\ldots \ldots \ldots \ldots \ldots \ldots \ldots \ldots$

C-30. Density in vessel (GV-COR-150HZ) $\ldots \ldots \ldots \ldots \ldots \ldots$

C-31. Density in vessel (GVLP-165HZ) $\ldots \ldots \ldots \ldots \ldots \ldots \ldots$

C-32. Density in vessel (GVLP-172HZ) $\ldots \ldots \ldots \ldots \ldots \ldots \ldots \ldots$

C-33. Mass flow in intact loop (FTU-1, GU-1C) $\ldots \ldots \ldots \ldots \ldots$

C-34. Mass flow in intact loop (FTU-1, GU-1C) $\ldots \ldots \ldots \ldots \ldots$ 
C-35. Mass flow in intact loop (FDU-5, GU-5VR) . . . . . . . . . . 251

C-36. Mass flow in intact loop (FTU-13, GU-13VR) . . . . . . . . . 252

C-37..Mass flow in intact loop (FTU-15,.GU-15C) . . . . . . . . 252

.C-38. Mass flow in broken loop (FDB-21, GB-21C) . . . . . . . . . 253

C-39. Mass flow in broken loop (FTB-21, GB-21C) . . . . . . . . 253

C-40. Mass flow in vessel (FTV-CORE-IN, GV-COR-150HZ) . . . . . . . . . 254

\section{TABLES}

I. Cundiliuns at Dlowdown Initiation $\ldots \ldots \ldots$

II. Primary Coolant Temperature Distribution

Prior to Rupture . . . . . . . . . . . . . . . . . . . 7

III. Water Chemistry Prior to Blowdown ............... 8

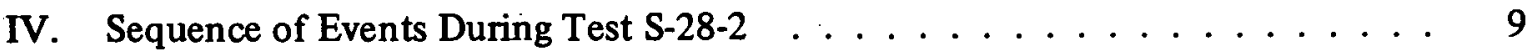

V. Data Presentation for Semiscale Mod-1 Test S-28-2 . . . . . . . . . . 17

B-I. Constants for Pressure Measurement

Corrections (Test S-28-2) . . . . . . . . . . . . . . 218

B-II. Constants for Differential Pressure

Measurement Corrections (Test S-28-2) _ . . . . . . . . . . . 220

B-III. Constants for Momentum Flux

Measurement Corrections (Test S-28-2) . . . . . . . . . . . . 221

B-IV. Constants for Density Measurement Conversions

to Engineering Units (Test S-28-2) . . . . . . . . . . . . . . . 223

C-I. Random Error Variance . . . . . . . . . . . . . . . . . . . 229

C.-II. General Measurement Engineering Error

Sources and Error Values (Test 2-28-2) . . . . . . . . . . . . 255

C-III. Time Periods when Flow Regime Errors were Applied (Test S-28-2) f . . . . . . . . . . . . . . 260 


\section{EXPERIMENT DATA REPORT FOR SEMISCALE MOD-1}

TEST S-28-2

(STEAM GENERATOR TUBE RUPTURE TEST)

\section{INTRODUCTION}

The Semiscale Mod-1 experiments represent the current phase of the Semiscale Program conducted by EG\&G Idaho, Inc. for the United States Government. The program, which is sponsored by the Nuclear Regulatory Commission through the Energy Research and Development Administration, is part of the overall program designed to investigate the response of a pressurized water reactor system to a hypothesized loss-of-coolant accident (LOCA). The underlying objectives of the Semiscale Program are to quantify the physical processes controlling system behavior during a LOCA and to provide an experimental data base for assessing reactor safety evaluation models. The Semiscale Mod-1 Program has the further objective of providing support to other experimental programs in the form of instrumentation assessment, optimization of test series, selection of test parameters, and evaluation of test results.

Test S-28-2 was conducted June 9, 1977 in the Semiscale Mod-1 system as part of the steam generator tube rupture test series (Test Series 28). This series was designed to obtain thermal-hydraulic response data from blowdown, refill, and reflood transients in a simulated nuclear reactor with a heated core to study system response during a LOCA with steam generator tube ruptures. The test objective specific to Test S-28-2 was to define a lower limit on the range of steam generator tube ruptures over which high peak cladding temperatures can occur.

The purpose of this report is to present the test data in an uninterpreted but readily usable form for use by the nuclear community in advance of detailed analysis and interpretation. Section II briefly déscribes the system configuration, procedures, initial test conditions, and events that are applicable to Test S-28-2; Section III presents the data graphs and provides comments and supporting information necessary for interpretation of the data. A description of the overall Semiscale Program and test series, a more detailed description of the Semiscale Mod-1 system, and a description of the measurement and data processing techniques and uncertainties can be found in Reference 1. Appendix A also provides additional in formation on the data acquisition system capabilities. 


\section{SYSTEM, PROCEDURES, CONDITIONS, AND EVENTS FOR TEST S-28-2}

The following system configuration, procedures, initial test conditions, and events are specific to Test S-28-2 as indicated.

\section{SYSTEM CONFIGURATION AND TEST PROCEDURES}

The Semiscale Mod-1 system used for this test consisted of a pressure vessel with internals, including a 40-rod core with 36 electrically heated rods; an intact loop with steam generator, pump, and pressurizer; a broken loup with simulated stcam gcnorator, simulated pump, and two rupture assemblies; coolant injection acciumulators for the cold legs of the intact and broken loops, and a heated accumulator for the intact loop hot leg; high and low pressure injection pumps for each of the system loops; and a pressure suppression system with a suppression tank, header, and heated steam supply system. For Tcst S-28-2, the volume of the lower plenum was reduced to $0.014980 \mathrm{~m}^{3}$ by the addition of a metal filler piece. Semiscale Mod-1 experimental system configuration information is described further in Reference 1. Figures 1 and 2 show the system configuration for Test S-28-2.

For Test S-28-2, 33 rods of the 40-rod electrically heated core were operated at a peak power density of approximately $37.7 \mathrm{~kW} / \mathrm{m}$, three rods (Rods D-4, E-4, and E-5) were operated at a peak power density of $39.7 \mathrm{~kW} / \mathrm{m}$ to yield a slightly peaked power profile, and four rods (Rods C-3, D-5, F-3, and F-6) were unpowered to simulate the effect of control rod guide tubes. 'I'he resulting total core power was appruximately $1418 \mathrm{~kW}$.

In preparation for the test, the accumulators for the intact and broken loop cold legs were filled with treated demineralized water, drained to the spccificd initial levels, and pressurized with nitrogen to $4223 \mathrm{kPa}$. The accumulator for the intact loop hol leg was filled with treated demineralized water, drained to the specified initial level, heated to $541 \mathrm{~K}$, and pressurized to $7584 \mathrm{kPa}$. The system was filled with treated demineralized water and vented at strategic points to ensure a liquid full system. Prior to warmup the system was pressurized to check for leakage, system instrumentation was checked, and transducer readings were initialized. Warmup to initial test conditions was accomplished with the heaters in the vessel core. Heatup of the broken loop piping was accomplished with bypass lines which served to allow circulation through the broken loop. During warmup, the purification and sampling systems were valved into the primary system to maintain water chemistry requirements and to provide a water sample at system conditions for subsequent analysis. At 55.6-K-temperature intervals during warmup, detector readings were sampled to allow the integrity of the measurement instrimentation and the operability of the data acquisition system to be checked. 


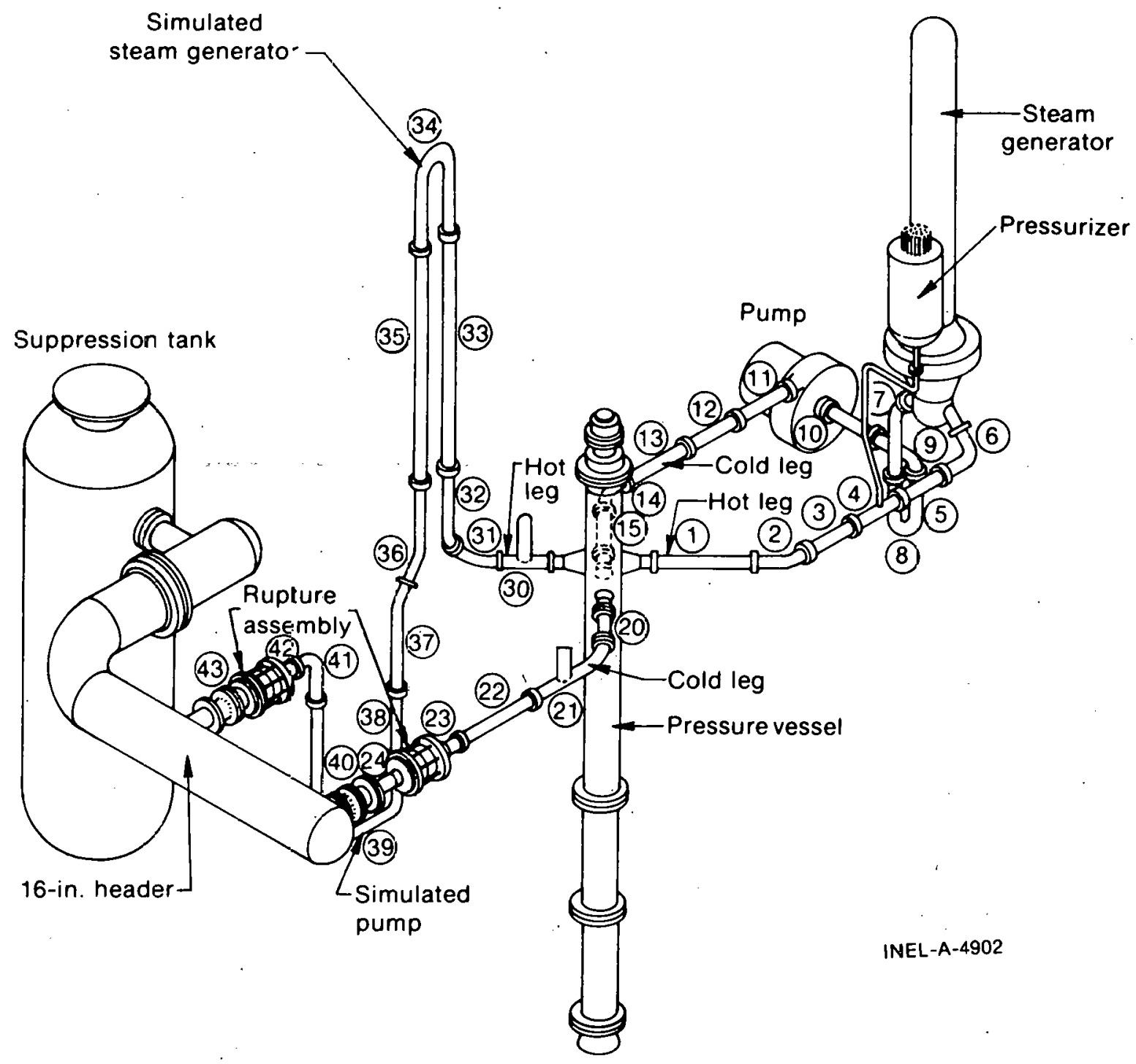

Fig. I Semiscale Mod-1 system for cold leg break configuration -isometric. 


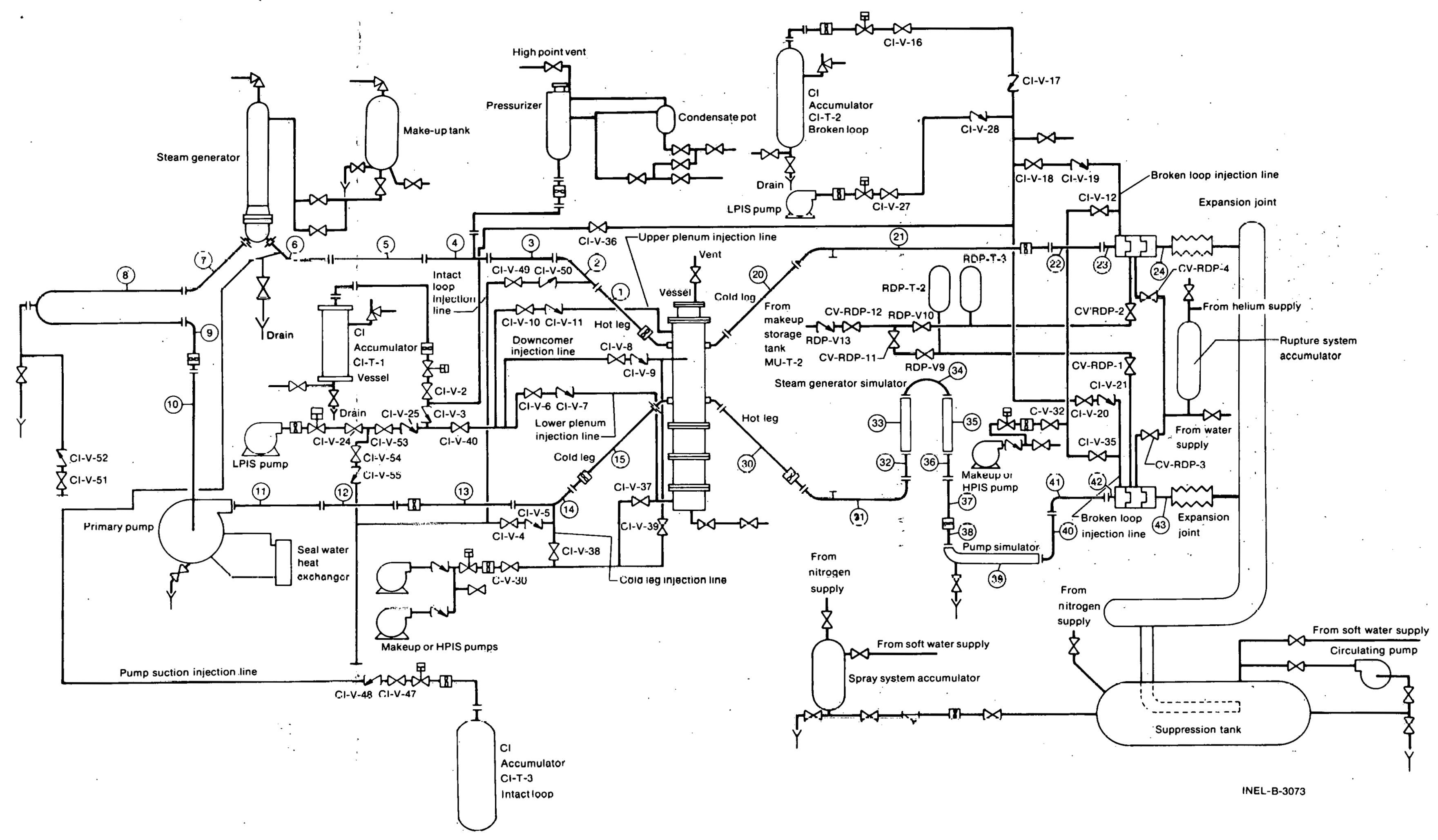

Fig. 2 Semiscale Mod-1 system for cold leg break configuration -schematic. 
Prior to the initial core power level being established, the pressure suppression system was pressurized to $241 \mathrm{kPa}$ with saturated steam from the steam supply system. After the core power was increased to $1418 \mathrm{~kW}$, initial test conditions were held for $521 \mathrm{~s}$ to establish equilibrium in the system. At the end of this period all auxiliary systems including the bypass lines were isolated to prevent blowdown through those systems.

The system was successfully subjected to a simulated double-ended cold leg break through two rupture assemblies and two blowdown nozzles, each having a break area of $0.000243 \mathrm{~m}^{2}$. Pressure to operate the rupture assemblies and initiate blowdown was taken from an accumulator system filled with water and pressurized to $15513 \mathrm{kPa}$ with gaseous nitrogen. Immediately (within $0.02 \mathrm{~s}$ ) after initiation of blowdown, the lines to the accumulator were again isolated. The effluent was ejected from the primary system into the pressure suppression system which was vented to maintain a constant pressure of $241 \mathrm{kPa}$. At blowdown, power to the primary coolant circulation pump was reduced and the pump was allowed to coast down to a speed of $1475 \mathrm{rpm}$ which was maintained for the duration of the test. During the blowdown transient, power to the electrically heated core was automatically controlled to simulate the thermal response of nuclear heated fuel rods.

For Test S-28-2, the coolant injection systems were arranged to discharge into both system loops at the cold leg injection points (Spool 14 and Spool 42). The high pressure coolant injection pumps were started immediately after initiation of blowdown (0 s) and continued to the end of the test $(640 \mathrm{~s})$. Low pressure coolant injection was initiated $28 \mathrm{~s}$ after blowdown at a system pressure of $1034 \mathrm{kPa}$ and continued until test termination (640 s). Coolant injection from the intact loop accumulator (CI-T-1) started $17 \mathrm{~s}$ after blowdown, and nitrogen discharge began $64 \mathrm{~s}$ after rupture and was terminated $88 \mathrm{~s}$ after blowdown. The broken loop accumulator (CI-T-2) began injection $4 \mathrm{~s}$ after blowdown, nitrogen discharge began $28 \mathrm{~s}$ after rupture, and was terminated $67 \mathrm{~s}$ after blowdown. The steam generator rupture system accumulator (CI-T-3) began injection at $40 \mathrm{~s}$ at a system pressure of $680 \mathrm{kPa}$ and continued to test termination.

\section{INITIAL TEST CONDITIONS AND SEQUENCE OF EVENTS}

Conditions in the Semiscale Mod-1 system at initiation of blowdown are given in Tables I and II; the primary system water chemistry prior to blowdown is given in Table III; and the sequence of events relative to rupture is given in Table IV. 
TABLE I

CONDITIONS AT BLOWDOWN INITIATION

Test $\mathrm{S}-28-2$

Measured $[\mathrm{a}]$

1.418

Specified

Core power (MW)

Intact loop cold. leg fluid temperature $(K)$

558

$1.43 \pm 0.01$

Hot leg to cold leg temperature differential (K)

.36

15936

.10 .6

$557.6 \pm 1$

Prcssurizer pressure (k.Pa)

Pressurizer liquid mass $(\mathrm{kg})^{[\mathrm{b}]}$

Steam generator feedwater temperature $(K)$

495

$497+17$

Steam generator liquid level (from bottom of tube sheet) $(\mathrm{cm})$

292

$294.6 \pm 5$

Broken loop fluid temperature (pump-side) (K)

Broken loop fluid temperature

(vessel-side) (K)

558

Not Specified

Intact loop cold leg flow $(\ell / s)$

9.84

【c」

Pressure suppresslun lank waler level $(\mathrm{cm})$

121

$121+1$

Pressure suppression tank pressure (kPa)

228

$241.3+6.9$

Pressure suppression tank water temperature $(K)$

290

[a] Measured initial conditions are taken from digital scan read just prior to blowdown. Those measured conditions which did not meet the specified initial conditions were considered acceptable for analysis purposes within the test objectives.

[b] Pressurizer liquid mass including surge line.

[c] Flow is not specified, since it must be adjusted to achieve the required differential temperature across the core. 


\section{TABLE II}

PRIMARY COOLANT TEMPERATURE DISTRIBUTION PRIOR TO RUPTURE[a]

Test S-28-2

\begin{tabular}{llc}
\hline & \multicolumn{1}{c}{ Test S-28-2 } \\
\cline { 3 - 3 } & Detector & Temperature (K) \\
$\begin{array}{l}\text { Vessel lower plenum (upper portion } \\
\text { above filler block) }\end{array}$ & TF.-LP-7 & 560 \\
Intact loop hot leg (near vessel) & RBU-2 & 594 \\
Intact loop cold leg (near pump inlet) & TFU-10 & 560 \\
Intact loop cold leg (near vessel). & RBU-14A & 558 \\
Broken loop cold leg (near nozzle) & TFB-23 & 558 \\
Broken loop hot leg (near vessel) & TFB-30 & 592 \\
Broken loop cold leg (near nozzle) & TFB-42 & 589 \\
\hline [a] Data taken from digital scan 0.5 s before blowdown. \\
\hline
\end{tabular}




\section{TABLE III}

WATER CHEMISTRY PRIOR TO BLOWDOWN ${ }^{\text {[a] }}$

Test S-28-2

$\mathrm{pH}$

10.80

Conductivity $(\mu \mathrm{mho} / \mathrm{cm})$

158.0

Lithium $(\mu / m \ell)$

4.2

Chlorides (ppm)

$<0.1$

Fluorides $(\mu / m \ell)^{[b]}$

$<0.4$

Oxygen $(\mathrm{cc} / \ell)$

0.2

lotal gas (cc/l)

115.5

Suspended solids $(\mu / \mathrm{ml})$

24.6

[a] Water sample taken at a system pressure of approximately $15603 \mathrm{kPa}$ and a system temperature of approximately $555 \mathrm{~K}$ (cold leg).

[b] Present analytical methods prevent accurate determination of fluorides at concentrations of less than $0.4 \mathrm{ppm}$. 
TABLE IV

SEQUENCE OF EVENTS DURING TEST S-28-2 [a.]

Time Relative

Event

Core power level established

Bypass lines valued out of system

Blowdown initiated

Pump power reduced

High pressure injection system pumps started $[b]$

ECC accumulators valved in [b]

Steam generator feedwater and discharge valves crosêd

Core power decay transient started

Low pressure injection system pumps started [b]

Core power tripped off [c] to Rupture (s)

$-521$

$-2.5$

i

0

0

0

1

640

[a] A time-controlled sequencer was used to control critical events during the test.

[b] Injection from ECC accumulators and high and low pressure injection system pumps does not start until systcm prossure drops below accumulator or pump pressure, respectively.

[c] Core power tripped manually at termination of test. 


\section{DATA PRESENTATION}

The data from Semiscalc Mod-1 Test S-28-2 are presented with brief comment. Processing analysis has been performed only to the extent necessary to obtain appropriate engineering units and to ensure that the data are reasonable and consistent. In all cases, in converting transducer output to engineering units, a homogeneous fluid was assumed. Further interpretation and analysis should consider that sudden decompression processes such as those occurring during blowdown may have subjected the measurement devices to nonhomogeneous fluid conditions.

The performance of the system during Test S-28-2 was monitored by 230 detectors. The data obtained were recorded on both digital and analog data acquisition systems. The digital system was used to process the data presented in this report. The analog system was used to provide better resolution capability (needed as input to various data analysis codes). The long term data ( -32 to $640 \mathrm{~s}$ ) presented in this report were recorded at an effective sample rate of 1.369 points per second. Long term plots are given for -20 to $350 \mathrm{~s}$ rather than for the full $640 \mathrm{~s}$ recording time to provide better resolution. Short term data ( -6 to $42 \mathrm{~s}$ ) were recorded at an effective sample rate of 19.17 points per second.

The data are presented, in some instances, in the form of composite graphs to facilitate comparison of the values of given variables at several locations. The scales selected for the graphs do not reflect the obtainable resolution of the data. (The data processing techniques are described further in Appendix A and Reference 1.)

Figures 3 through 8 and Table $\mathrm{V}$ provide supporting information for interpretation of the data graphs shown in Figures 9 through, 370 and provide relative locations of all detectors used during Test S-28-2. Table $V$ groups the medsurements accurding to mcasurement type; identifies the specific measurement location and the range of the detector and actual recording range of the data acquisition system; provides brief comments regarding the data; and references the measurements and comments to the corresponding figure. Figures 9 through 370 present all the blowdown and reflood data obtained. Time zero on the graphs is the time of rupture initiation. Appendix A provides information explaining the data acquisition system capabilities. Appendix B provides information explaining posttest data processing for data conversion into engineering units and data adjustments. Presented in Appendix $\mathrm{C}$ is an analysis of selected data which provide a guide to the uncertainty associated with data measurements in the Semiscale Mod-1 system. 


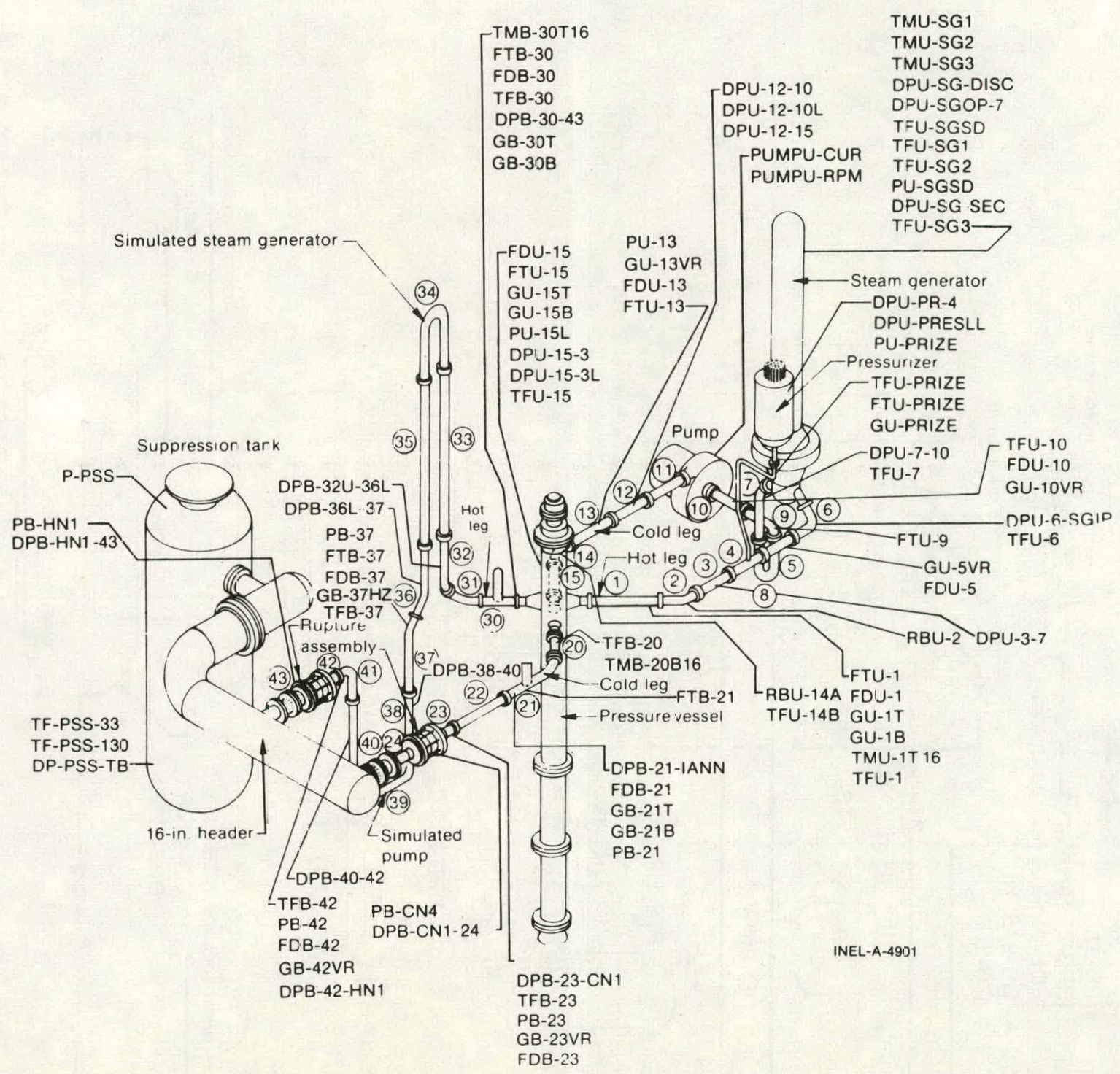

Fig. 3 Semiscale Mod-1 system and instrumentation for cold leg break configuration -- isometric. 


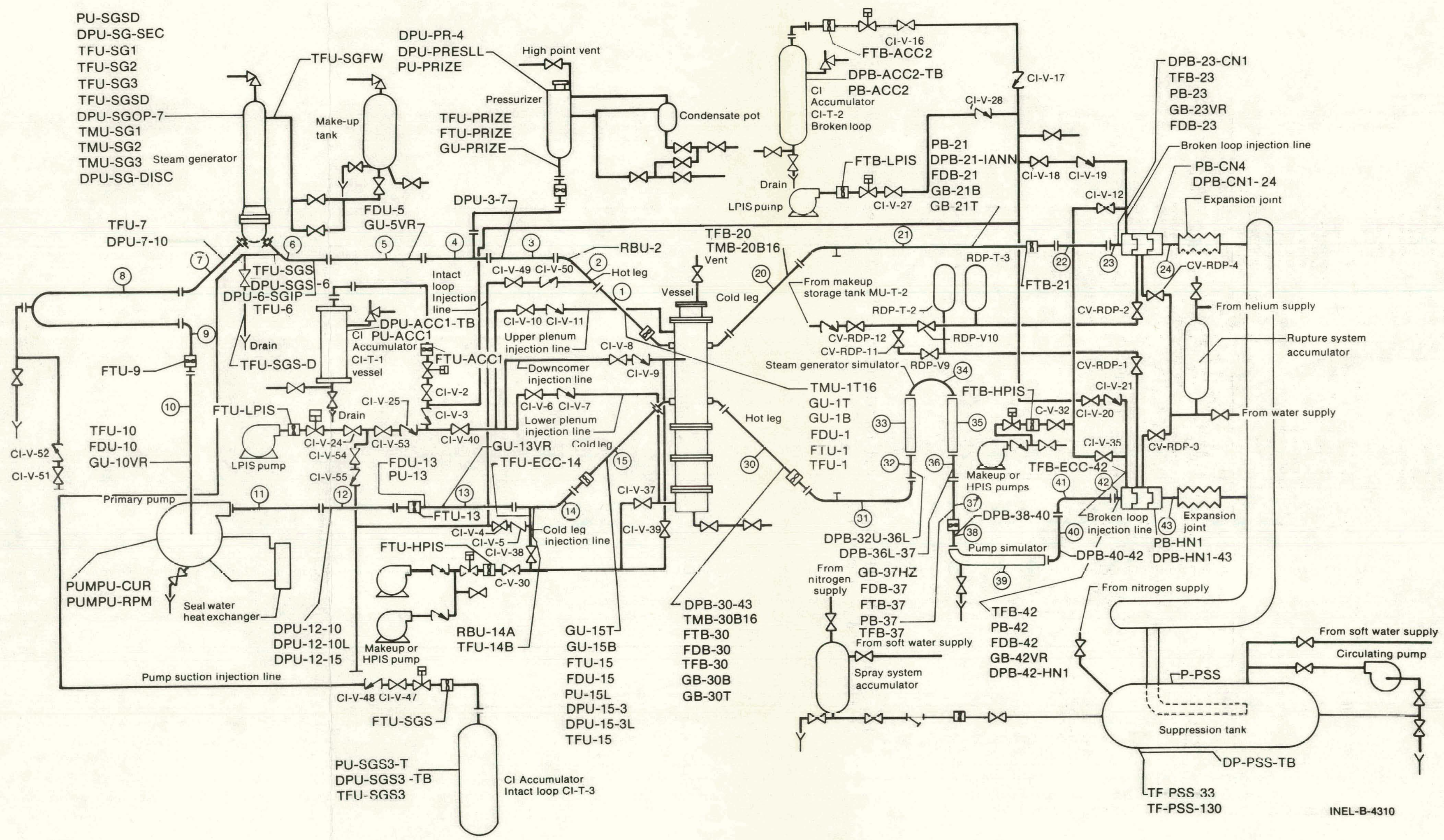

Fig. 4 Semiscale Mod-1 system and instrumentation for cold leg break configuration -- schematic. 


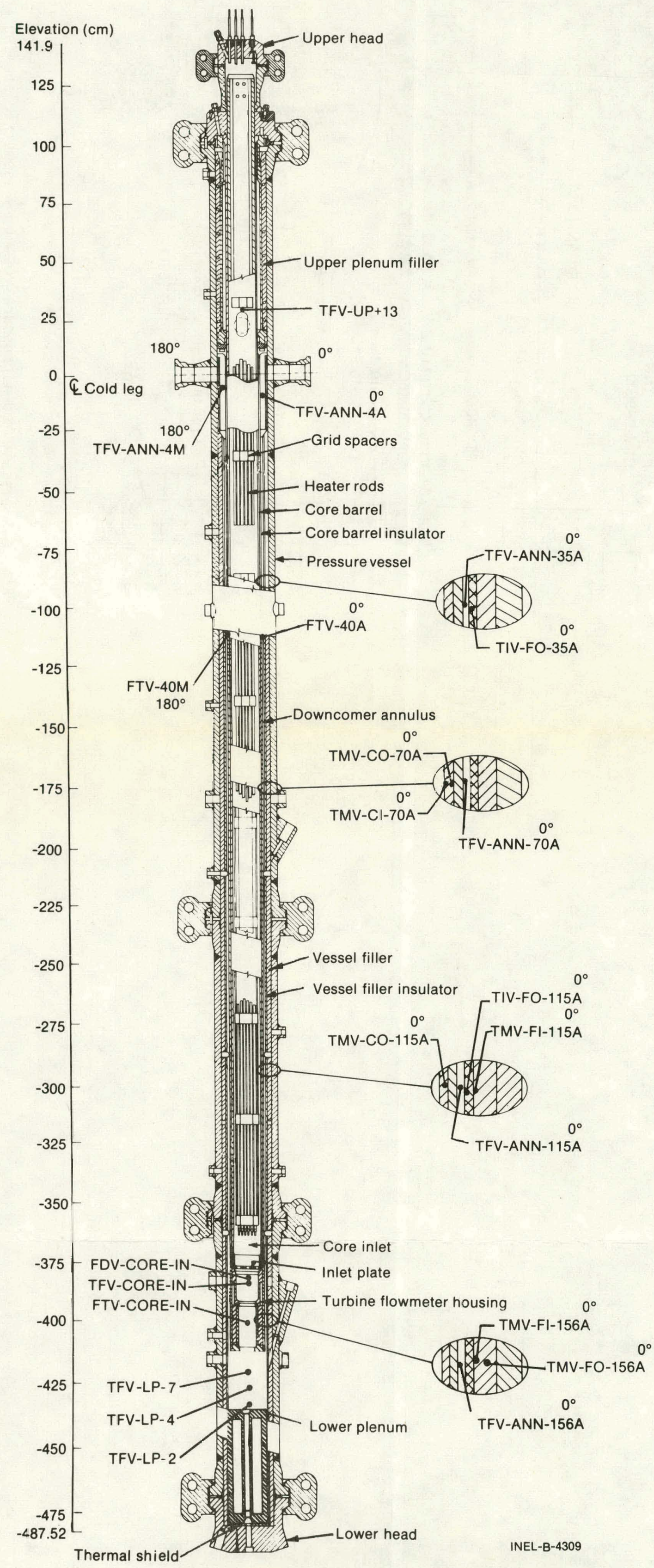

Fig. 5 Semiscale Mod-1 pressure vessel -- cross section showing instrumentation. 
Distance from

cold leg $\&(\mathrm{~cm})$

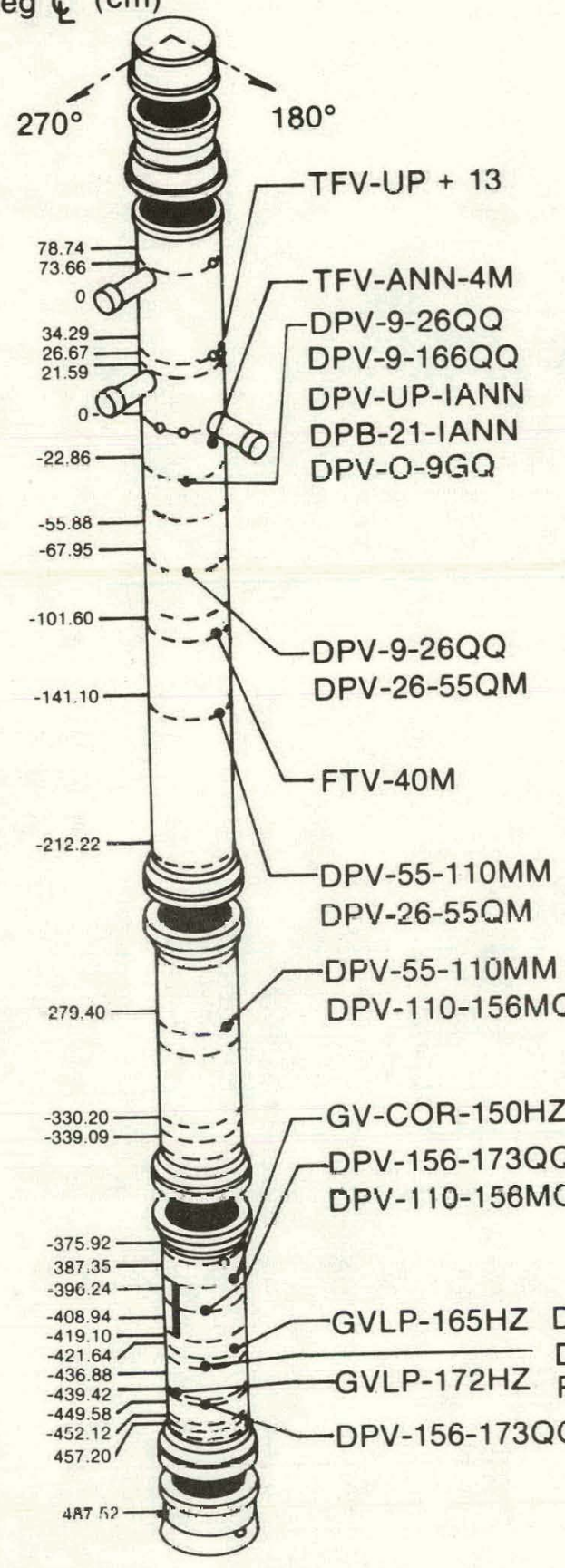

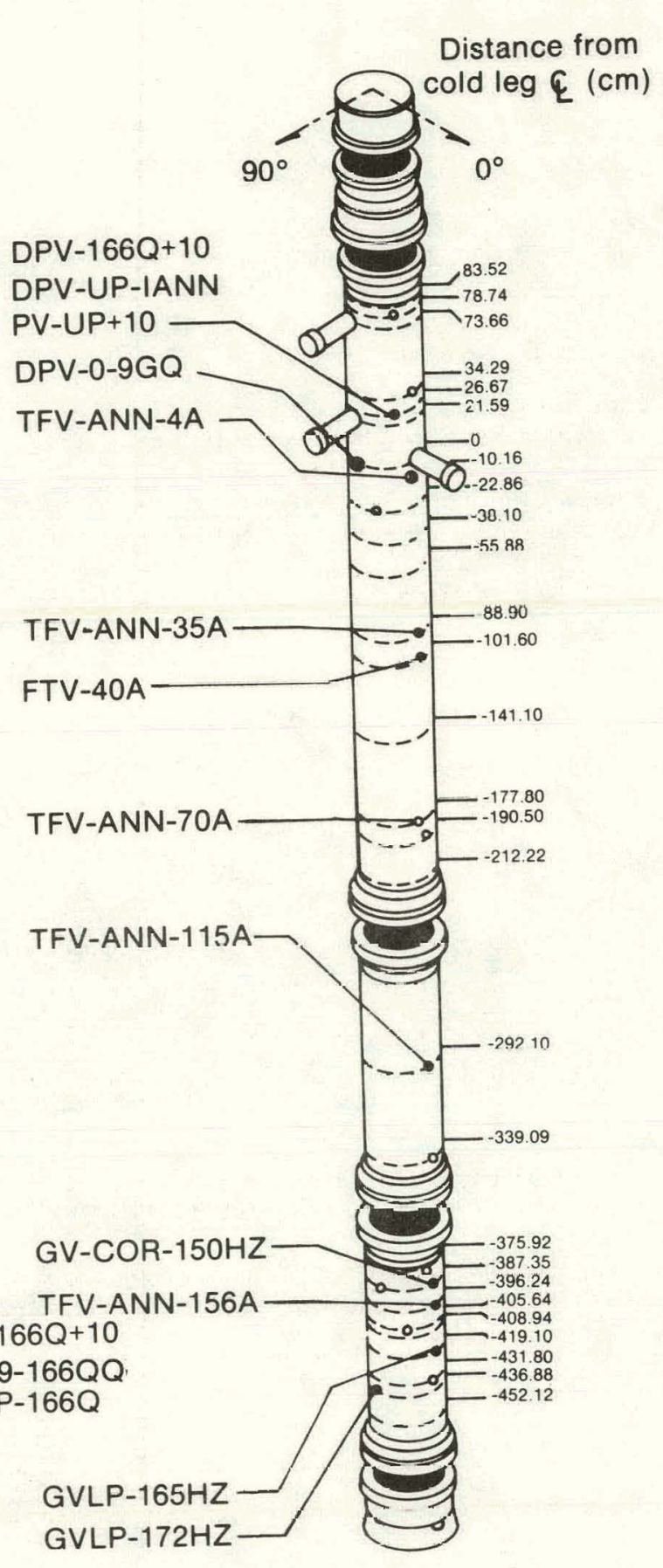

INEL-A-4307

Fig. 6 Semiscale Mod-1 pressurc ve'sel -- isometric showing instrumentation. 


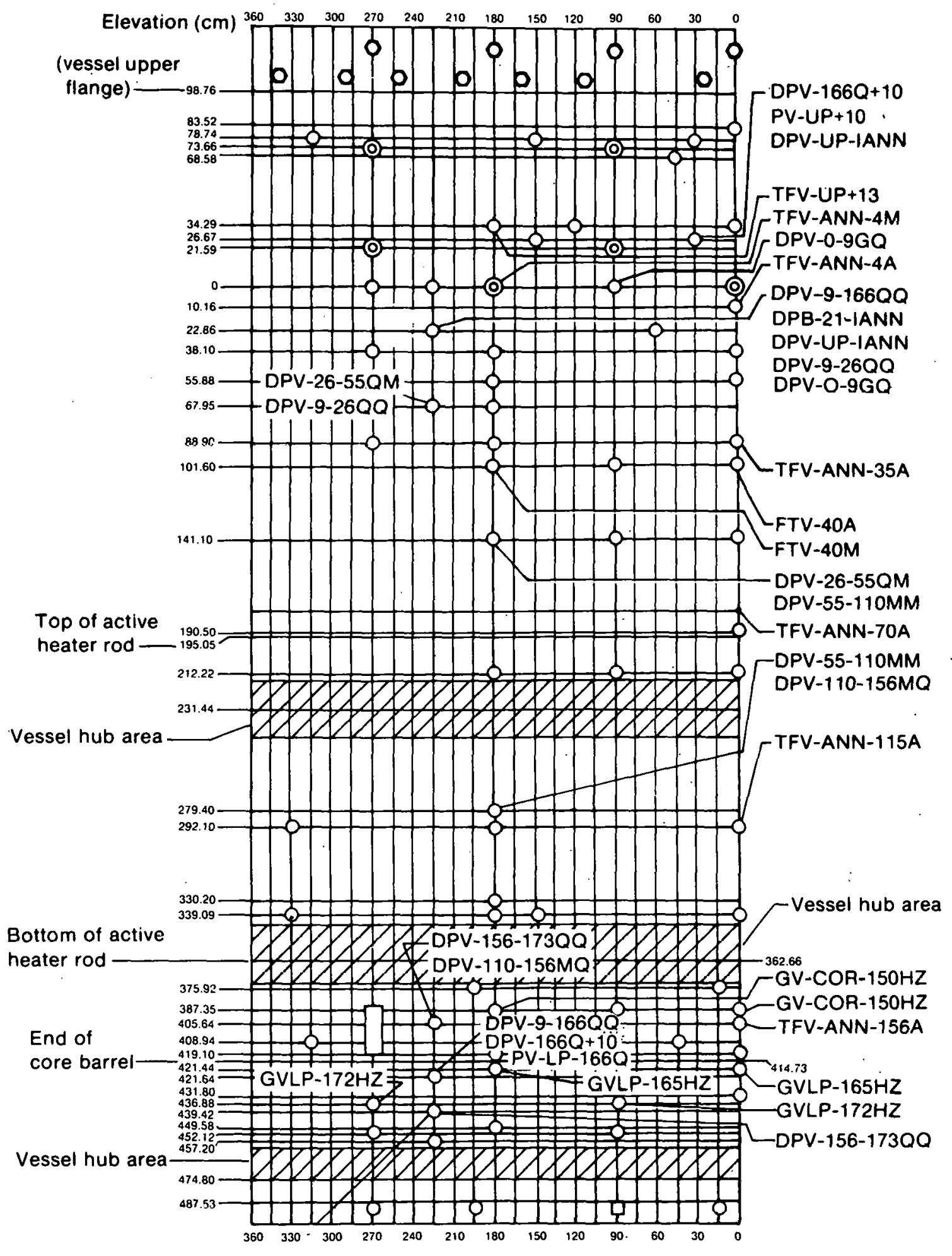

INEL-A-4306

Fig. 7 Semiscale Mod-1 pressure vessel -- penetrations and instrumentation. 

A $\cdot B$
C D
E
$\mathbf{F} \quad \mathbf{G}$
$H$

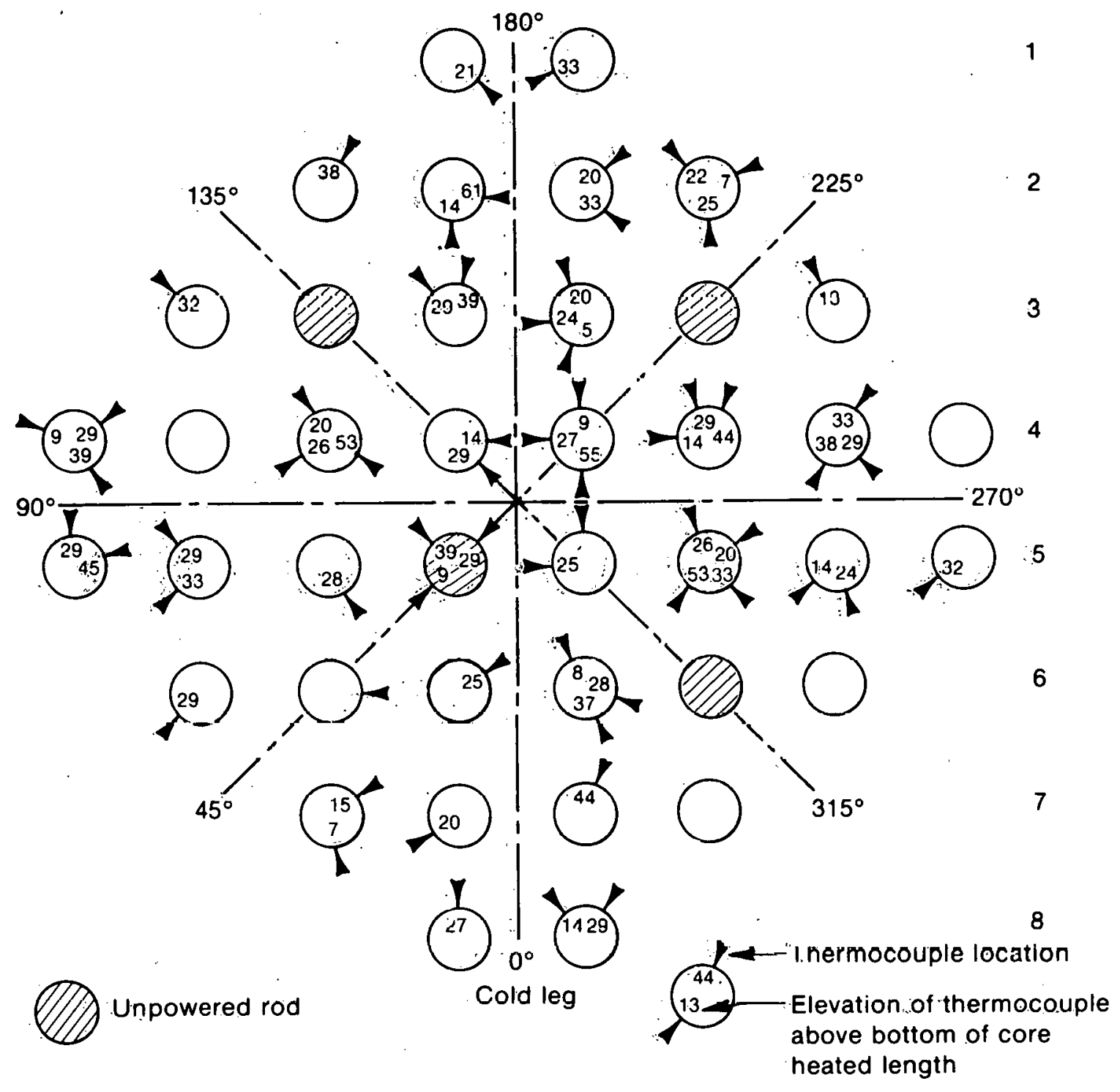

INEL-A-4308

Fig. 8 Somiscale Mod-1 heated core -- plan view. 
TABLE $V$

DATA PRESENTATION FOR SEMISCALE MOD-1 TEST S-28-2

\begin{tabular}{|c|c|c|c|c|c|}
\hline \multirow[b]{2}{*}{ Measurement } & \multirow[b]{2}{*}{ Location and Comfients ${ }^{[a]}$} & \multicolumn{2}{|c|}{$\operatorname{Range}^{[a]}$} & \multirow[b]{2}{*}{ Figure $^{[\mathrm{a}]}$} & \multirow[b]{2}{*}{ Measurement Corments ${ }^{[b]}$} \\
\hline & & Detector & $\begin{array}{l}\text { Data Acquisition } \\
\text { System }\end{array}$ & & \\
\hline FLUID TEMPERATURE & $\begin{array}{l}\text { Chromel-Alumel thernocouples unless } \\
\text { specified otherwise. }\end{array}$ & & & & \\
\hline Intaet Loop & & 0 to $1533 \mathrm{~K}$ & 0 to $584 \mathrm{~K}$ & & \\
\hline TFU-1 & $\begin{array}{l}\text { Hot leg, Spool } 1,54 \mathrm{~cm} \text { from vessel } \\
\text { center. }\end{array}$ & & 0 to $820 \mathrm{~K}$ & 9,10 & . \\
\hline RBU-2 & $\begin{array}{l}\text { Hot } 1 \text { eg, Spool } 2,117 \mathrm{~cm} \text { from vessel } \\
\text { center (platinum resistance bulb). }\end{array}$ & 0 to $811 \mathrm{~K}$ & 0 to $811 \mathrm{~K}$ & 9,10 & \\
\hline TFU-6 & $\begin{array}{l}\text { Hot leg. Spool } 6,290 \mathrm{~cm} \text { from vessel } \\
\text { center. }\end{array}$ & & & 11,12 & \\
\hline TFU-7 & $\begin{array}{l}\text { Cold leg, Spool } 7,624 \mathrm{~cm} \text { from vessel } \\
\text { center. }\end{array}$ & & & 13,14 & \\
\hline TFU-10 & $\begin{array}{l}\text { Cold leg, Spool } 10,367 \mathrm{~cm} \text { from } \\
\text { vessel center. }\end{array}$ & & & 13,14 & \\
\hline RBU-14A & $\begin{array}{l}\text { Cold leg, Spool } 14,109 \mathrm{~cm} \text { from } \\
\text { vessel center, upstream of cold leg } \\
\text { injection port (platinum resistance } \\
\text { bulb). }\end{array}$ & 0 to $811 \mathrm{~K}$ & 0 to $811 \mathrm{~K}$ & 15,16 & \\
\hline $\begin{array}{l}\text { TFU-14B } \\
.\end{array}$ & $\begin{array}{l}\text { Cold leg, Spool } 14,99 \mathrm{~cm} \text { from } \\
\text { vessel center, downstream of cold } \\
\text { leg injection port. }\end{array}$ & & & 15,16 & \\
\hline TFU-15 & $\begin{array}{l}\text { Cold leg, Spool } 15,54 \mathrm{~cm} \text { from } \\
\text { vessel center. }\end{array}$ & & . & 17,18 & $\cdot$ \\
\hline Broken Loop & $\cdot$ & 0 to $1533 \mathrm{~K}$ & 0 to $820 \mathrm{~K}$ & & \\
\hline$T F B-20$ & $\begin{array}{l}\text { Coid leg. Spool } 20,52 \mathrm{~cm} \text { from } \\
\text { vessel center. }\end{array}$ & . & & 19,20 & \\
\hline TFR-P3 & $\begin{array}{l}\text { Cold leg. Spool } 23,232 \mathrm{~cm} \text { from } \\
\text { vessel center, upstrean of yessel- } \\
\text { side nozzle. }\end{array}$ & & & 19,20 & \\
\hline$T F B-30$ & $\begin{array}{l}\text { Mot } 1 \mathrm{eg}, \text { Spool } 30,40 \mathrm{~cm} \text { from } \\
\text { vessel center. }\end{array}$ & & & $? .1, ? ?$ & \\
\hline TFB-37 & $\begin{array}{l}\text { Cold leg, Spool } 37,703 \mathrm{~cm} \text { from } \\
\text { vessel center a long hot leg, dis- } \\
\text { charge of simulated steam generator. }\end{array}$ & & & 21,22 & \\
\hline TFB-42 & $\begin{array}{l}\text { Cold leg, } 5 \text { pool } 42,1054 \mathrm{~cm} \text { from } \\
\text { vessel center along hot leg, upstream } \\
\text { of pump-side nozzle. }\end{array}$ & . & & 21,22 & \\
\hline$\underline{\text { Inlet Annulus }}$ & $\begin{array}{l}10 \mathrm{~cm} \text { below cold leg centerline, } \\
0.5 \mathrm{~cm} \text { from vessel wall, Type } \mathrm{J} \\
\text { iron-constantan thermocouples. }\end{array}$ & 0 to $1033 \mathrm{~K}$ & 0 to $701 \mathrm{~K}$ & & \\
\hline TFV-ANN-4A & $0^{\circ}$. & & & 23,24 & \\
\hline TFV-ANN-AM & $180^{\circ}$. & & & 23,24 & \\
\hline Downcomer Annulus & $\begin{array}{l}\text { Centered in annulus, Type } \mathcal{J} \text { iron- } \\
\text { constantan thermocouples. }\end{array}$ & 0 to $1033 \mathrm{~K}$ & 0 to $701 \mathrm{~K}$ & & \\
\hline TFV-ANN-35R & $\begin{array}{l}89 \mathrm{~cm} \text { be low cold leg renteritine, } \\
0^{\circ} \text {. }\end{array}$ & & & 25,26 & \\
\hline TFV-ANN-70A & $\begin{array}{l}178 \mathrm{~cm} \text { below cold leg centeríine, } \\
0^{\circ} \text {. }\end{array}$ & & & 25,26 & \\
\hline TFY-ANN-11SA & $\begin{array}{l}29 \hat{\mathrm{em}} \text { below cold leg centerline, } \\
0^{\circ} \text {. }\end{array}$ & & & 25,26 & \\
\hline TFV-ANN- $156 \mathrm{~A}$ & $396 \mathrm{~cm}$ below cold leg centerline, & & & 25,26 & \\
\hline Upper Plenum & & 0 to $1533 \mathrm{~K}$ & 0 to $820 \mathrm{~K}$ & & \\
\hline$T F V-U P+13$ & $\begin{array}{l}\text { In upper plenum, } 34 \mathrm{cal} \text { above } \\
\text { cold leg centeritine at } 180^{\circ} \text {. }\end{array}$ & & & $21,28 \dot{8}$ & \\
\hline Lower Plenum & $\begin{array}{l}\text { On fluid thernocouple rack, } 2.54 \mathrm{~cm} \\
\text { iram vesse' center, } 49^{\circ} \text {. }\end{array}$ & .0 to $1533 \mathrm{~K}$ & 0 to $820 \mathrm{~K}$ & & \\
\hline TFV-LP-2 & $\begin{array}{l}5 \mathrm{~cm} \text { above top of lower plenum filler } \\
\text { block. }\end{array}$ & & & 29,30 & \\
\hline$T F V-L P-4$ & $\begin{array}{l}10 \mathrm{~cm} \text { above top of lower plenum filler } \\
\text { block. }\end{array}$ & & & 29,30 & \\
\hline$T F V-L P-7$ & $\begin{array}{l}18 \mathrm{~cm} \text { above top of lower plenum filler } \\
\text { block. }\end{array}$ & & & 29,30 & \\
\hline \multicolumn{6}{|l|}{ Core. } \\
\hline TFV-CORE-IN & $\begin{array}{l}\text { In core flow mixer box, } 381 \mathrm{~cm} \\
\text { below cold leg centerline (a } \\
\text { part of FOV-CORE-IK). }\end{array}$ & 0 to $1533 \mathrm{~K}$ & 0 to $820 \mathrm{~K}$ & 31,32 & $\begin{array}{l}\text { Data acquisition system saturated } \\
\text { intermittently between } t=32 \\
\text { and } t=60 \mathrm{~s} \text {. }\end{array}$ \\
\hline
\end{tabular}


TABLE V. (continued)

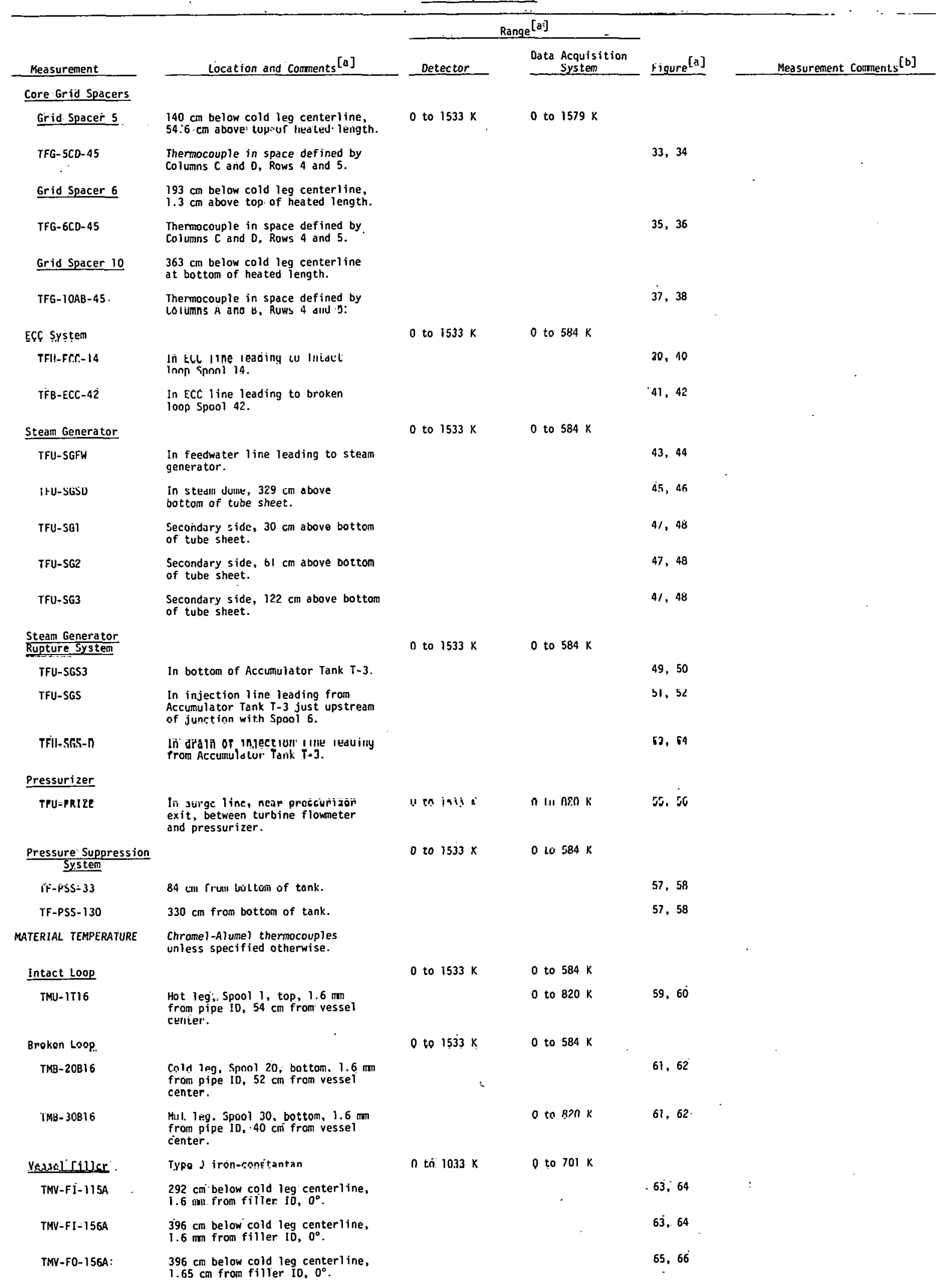


TABLE V (continued)

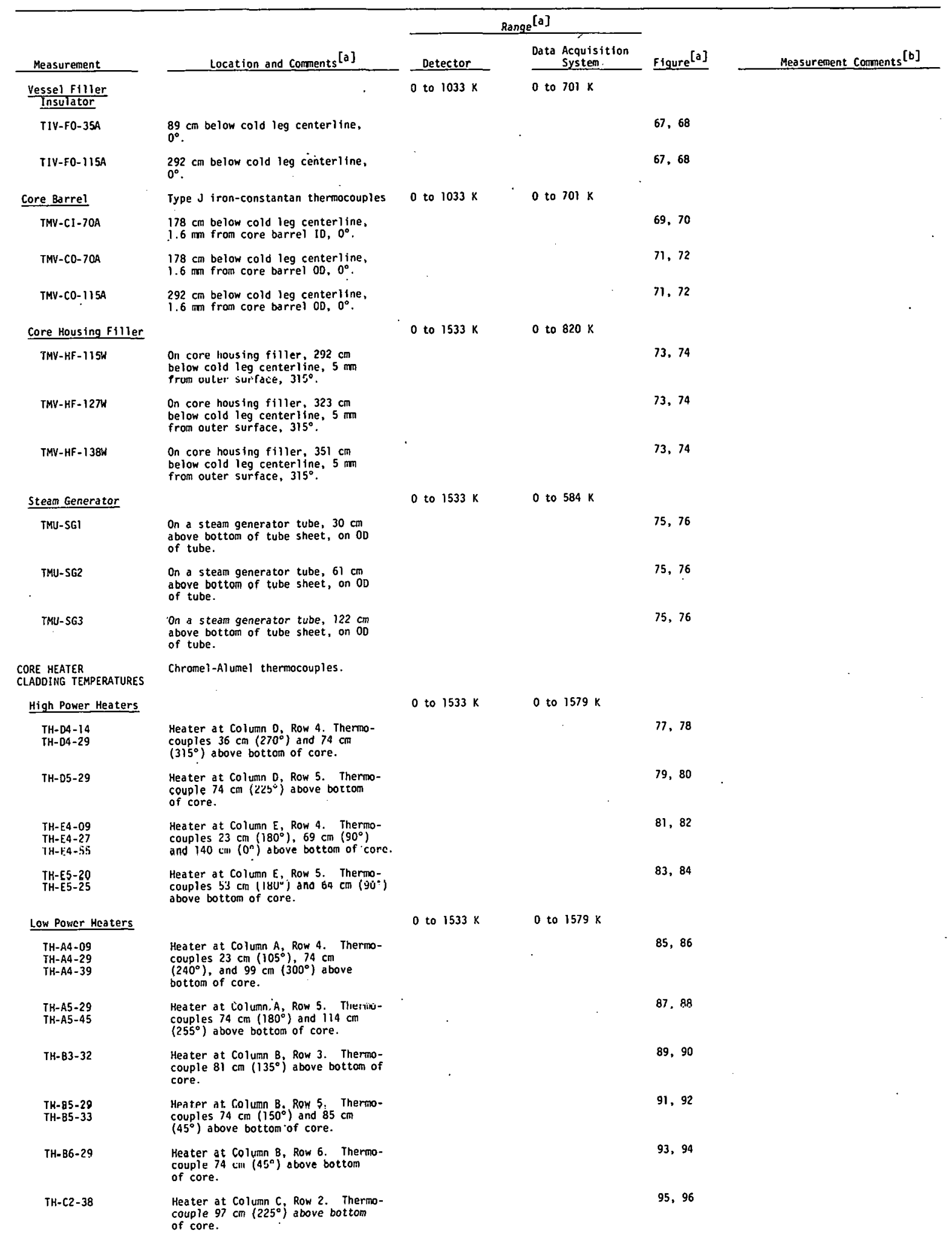




\begin{tabular}{|c|c|c|c|c|c|}
\hline \multirow[b]{2}{*}{ Measurement } & \multirow[b]{2}{*}{ Location and Compents ${ }^{[a]}$} & \multicolumn{2}{|c|}{ Range $^{[\text {A] }}$} & \multirow[b]{2}{*}{ Figure ${ }^{[a]}$} & \multirow[b]{2}{*}{ Measurement Corments ${ }^{[\mathrm{b}]}$} \\
\hline & & Detector & $\begin{array}{c}\text { Oata Acquisition } \\
\quad \text { System } \\
\end{array}$ & & \\
\hline \multicolumn{2}{|c|}{ Low Power Heaters (cont inued) } & & . & & \\
\hline $\begin{array}{l}T H-C 4-20 \\
T H-C 4-26 \\
T H-C 4-53\end{array}$ & $\begin{array}{l}\text { Heater at Column } \mathrm{c}, \text { Row } 3 \text {. Thermo- } \\
\text { couples } 51 \mathrm{~cm}\left(150^{\circ}\right), 66 \mathrm{~cm}\left(75^{\circ}\right) \text {, } \\
\text { and } 135 \mathrm{~cm}\left(300^{\circ}\right) \text { above bottom } \\
\text { of core. }\end{array}$ & & - & 97,98 & \\
\hline TH-C5-28 & $\begin{array}{l}\text { Heater at Column Co Row 5. Thermo- } \\
\text { couple } 71 \mathrm{~cm}\left(315^{\circ}\right) \text { above bottom of } \\
\text { core. }\end{array}$ & & & 99,100 & \\
\hline$T H-C 6-53$ & $\begin{array}{l}\text { Heater at column C, Row 6. Thermo- } \\
\text { couple } 135 \mathrm{~cm}\left(270^{\circ}\right) \text { above bottom } \\
\text { of core. }\end{array}$ & & & 701,102 & \\
\hline $\begin{array}{l}\mathrm{TH}-\mathrm{C} 7-07 \\
\mathrm{TH}-\mathrm{CT}-15\end{array}$ & 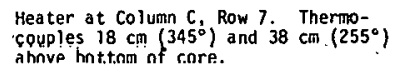 & & & 103,104 & , \\
\hline TH-D1-21 & $\begin{array}{l}\text { Heater at column o , Row 1. Thermo- } \\
\text { couple } 53 \mathrm{~cm}\left(330^{\circ}\right) \text { above bottam of } \\
\text { coro. }\end{array}$ & & & $105,-106$ & - \\
\hline $\begin{array}{l}\mathrm{TH}-02-14 \\
\mathrm{TH}-02-61\end{array}$ & $\begin{array}{l}\text { Heater at column }{ }^{0}, \text { Row } 2 . \text { Therno- } \\
\text { couples } 36 \mathrm{~cm}\left(0^{\circ}\right) \text { and } 155 \mathrm{~cm}\left(270^{\circ}\right) \\
\text { above bottom of core. }\end{array}$ & & & 107,108 & . \\
\hline $\begin{array}{l}T_{H} \mathrm{H}-03-29 \\
\mathrm{TH}-03-39\end{array}$ & $\begin{array}{l}\text { Heater at column } D_{0} \text {, Row } 3 . \text { Thermo- } \\
\text { couples } 74 \mathrm{~cm}\left(150^{\circ}\right) \text { and } 99 \mathrm{~cm} \\
\left(210^{\circ}\right) \text { a bove bottom of core. }\end{array}$ & & & 109,110 & \\
\hline TH-06-25 & $\begin{array}{l}\text { Heater at column o Row } 6 \text {. Thermo- } \\
\text { couple } 64 \mathrm{~cm}\left(255^{\circ}\right) \text { above bottom of } \\
\text { core. }\end{array}$ & & . & 111,112 & \\
\hline$T H=07-20$ & $\begin{array}{l}\text { Heater at Column o, Row } 7 \text { Thermo- } \\
\text { couple } 51 \mathrm{~cm}\left(60^{\circ}\right) \text { above bottom of } \\
\text { core. }\end{array}$ & & & 113,114 & \\
\hline TH-D8-26 & $\begin{array}{l}\text { Heater at column 0, Row 8. Thermo- } \\
\text { couplc } 69 \mathrm{~cm}\left(180^{\circ}\right) \text { above bottom of } \\
\text { core. }\end{array}$ & & & 115,116 & \\
\hline TH-E1-33 & $\begin{array}{l}\text { Heater at Column } 4 \text {, Row } 1 \text {. Thermo- } \\
\text { couple } 84 \mathrm{~cm}\left(50^{\circ}\right) \text { above bottom of } \\
\text { curre. }\end{array}$ & & & 117,118 & \\
\hline $\begin{array}{l}T H-E 2-20 \\
T H 1-[2=-93\end{array}$ & 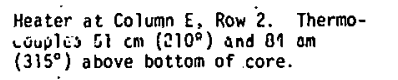 & & & 119,120 & \\
\hline $\begin{array}{l}T H-E 3-05 \\
T H-E 3-20 \\
T H \\
T H 3\end{array}$ & 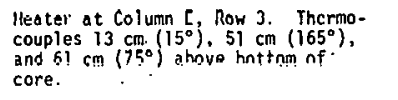 & & & 121,122 & . \\
\hline $\begin{array}{l}T H-E 6-08 \\
T H-E 6-28 \\
T H-E 6-37\end{array}$ & $\begin{array}{l}\text { Heater at Column E, Row 6. Thermo- } \\
\text { couples } 20 \mathrm{~cm}\left(150^{\circ}\right), 71 \mathrm{~cm}\left(285^{\circ}\right) \text {, } \\
\text { and } 94 \mathrm{~cm}\left(330^{\circ}\right) \text { above bottom } \\
\text { of core. }\end{array}$ & & & 123,124 & \\
\hline$T H-E \overline{7}-\dot{4} \dot{4}$ & $\begin{array}{l}\text { Hedter al. Culuun E, Row } 1 \text {. Therio- } \\
\text { couple } 112 \mathrm{~cm}\left(195^{\circ}\right) \text { above bottom } \\
\text { of core. }\end{array}$ & & & 120,120 & \\
\hline $\begin{array}{l}T H-E 8-14 \\
T H-E 8-29\end{array}$ & 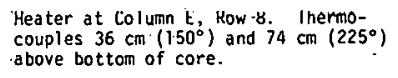 & & . & $\cdot 127,128$ & \\
\hline $\begin{array}{l}T H-F 2-07 \\
T H-F 2-22 \\
T H-F 2=25\end{array}$ & $\begin{array}{l}\text { Heater at Column } \mathrm{F} \text {, Row } 2 \text {. Thermo- } \\
\text { couples } 18 \mathrm{~cm}\left(255^{\circ}\right), 56 \mathrm{~cm}\left(105^{\circ}\right) \text {, } \\
\text { and } 64 \mathrm{~cm}\left(0^{\circ}\right) \text { a bove bottom. of core. }\end{array}$ & & & 129,130 & \\
\hline $\begin{array}{l}T H-F 4-14 \\
T H-F 4-29 \\
T H-F 4-44\end{array}$ & $\begin{array}{l}\text { Heater at column } \mathrm{F}, \text { Row } 4 \text {. Thermo- } \\
\text { couples } 36 \mathrm{~cm}\left(90^{\circ}\right), 74 \mathrm{cml}\left(165^{\circ}\right) \text {, } \\
\text { and } 112 \mathrm{~cm}\left(210^{\circ}\right) \text { above bottom of } \\
\text { core. }\end{array}$ & & & 131,132 & \\
\hline $\begin{array}{l}\text { TH-PA-10 } \\
\text { TH-F5-26 } \\
T H-F 5-33 \\
T H-F 5-53\end{array}$ & 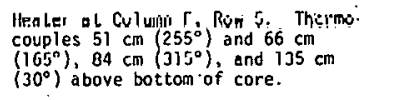 & & & 133,134 & . \\
\hline$T 11-63-13$ & $\begin{array}{l}\text { Heater at col lumn G, Row } 3 \text {. Thermo- } \\
\text { couples } 33 \mathrm{~cm}\left(150^{\circ}\right) \text { above bottom of } \\
\text { corre. }\end{array}$ & & & 135.136 & \\
\hline $\begin{array}{l}T H-64-29 \\
\text { IH } 6-13 \\
T H=64-38\end{array}$ & 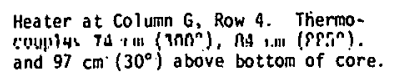 & & & $.137,138$ & \\
\hline $\begin{array}{l}\cdot T H-65-14 \\
T H-65-24\end{array}$ & $\begin{array}{l}\text { Heater at column 6, Row H. Inermo- } \\
\text { couples } 36 \mathrm{~cm}\left(45^{\circ}\right) \text {. and } 61 \mathrm{~cm}\left(330^{\circ}\right) \\
\text { above bot tom of core. }\end{array}$ & & & 139,740 & \\
\hline TH-H5-32 & $\begin{array}{l}\text { Heater at column H, Row's. Therma- } \\
\text { couple } 81 \mathrm{~cm}\left(45^{\circ}\right)^{\prime} \text { ) above bottom of } \\
\text { core. }\end{array}$ & & & 141,142 & \\
\hline
\end{tabular}




\begin{tabular}{|c|c|c|c|c|c|}
\hline \multirow[b]{2}{*}{ Measurement } & \multirow[b]{2}{*}{ Location and Comments ${ }^{[a]}$} & \multicolumn{2}{|c|}{ Range[a] } & \multirow[b]{2}{*}{ Figure ${ }^{[a]}$} & \multirow[b]{2}{*}{ Measurement Corments ${ }^{[b]}$} \\
\hline & & Detector & $\begin{array}{l}\text { Data Acquisition } \\
\text { Systera }\end{array}$ & & \\
\hline \multicolumn{6}{|l|}{ PRESSURE } \\
\hline Intact Loop & & $\begin{array}{l}0 \text { to } 20 \\
\mathrm{kPa}\end{array}$ & & & \\
\hline PU-13 & $\begin{array}{l}\text { Cold leg, Spool 13, } 138 \mathrm{~cm} \text { from } \\
\text { vessel center. }\end{array}$ & & 0 to $31614 \mathrm{kPa}$ & 143,144 & . \\
\hline PU-15L & $\begin{array}{l}\text { Cold leg, Spool } 15,55 \mathrm{~cm} \text { from } \\
\text { vessel center, to atrosphere } \\
\text { (low range). }\end{array}$ & 0 to $3447 \mathrm{kPa}$ & 0 to $3561 \mathrm{kPa}$ & 143,144 & $\begin{array}{l}\text { Data acquisition system saturated } \\
\text { between } t=18 \text { and } t=20 \mathrm{~s} \text {. }\end{array}$ \\
\hline Broken Loop & & $\begin{array}{l}0 \text { to } 17237 \\
\mathrm{xPd}\end{array}$ & & & \\
\hline PB-21 & $\begin{array}{l}\text { Cold leg, Spool } 21,112 \mathrm{~cm} \text { from } \\
\text { vessel center. }\end{array}$ & $\begin{array}{l}0 \text { to } 20684 \\
\mathrm{kPa}\end{array}$ & 0 to $29666 \mathrm{kPa}$ & 145,146 & \\
\hline $\mathrm{PB}-23$ & $\begin{array}{l}\text { Cold leg, } 5 p 00123,235 \mathrm{~cm} \text { from } \\
\text { vessel center, upstream of nozzle } \\
\text { (tee off DP tap). }\end{array}$ & & 0 to $21480 \mathrm{kPa}$ & 145,146 & . \\
\hline$P B-C N A$ & $\begin{array}{l}\text { Cold leg, Spool 23, vessel-side } \\
\text { nozzle, nozzle divergent. certinn. } \\
257 \mathrm{~cm} \text { from vessel center along } \\
\text { cold leg, } 0^{\circ} \text {. }\end{array}$ & $\begin{array}{l}0 \text { to } 20684 \\
\mathrm{kPa}\end{array}$ & 0 to $30223 \mathrm{kPa}$ & 147,148 & . \\
\hline P8-37 & $\begin{array}{l}\text { Cold leg. Spool } 37,718 \mathrm{~cm} \text { from } \\
\text { vessel center along hot leg. }\end{array}$ & & 0 to $21675 \mathrm{kPa}$ & 149,150 & \\
\hline$P B=42$ & $\begin{array}{l}\text { Cold leg, Spool } 42,1057 \mathrm{~cm} \text { from } \\
\text { vessel center along hot leg, up- } \\
\text { stream of pump-side nozzle (tee } \\
\text { off op tap). }\end{array}$ & & 0 to $20359 \mathrm{kPa}$ & 149,150 & \\
\hline PB-HNI & $\begin{array}{l}\text { Pump-side nozzle, nozzle throat, } \\
1066 \mathrm{~cm} \text { from vessel center along } \\
\text { hot leg (tee off or top) }\end{array}$ & & 0 to $20654 \mathrm{kPa}$ & 151,152 & 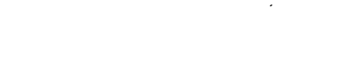 \\
\hline \multicolumn{6}{|l|}{ Yessel } \\
\hline PV-UP+10 & $\begin{array}{l}\text { In upper plenum, } 25 \mathrm{~cm} \text { above cold } \\
\text { leg centerline, mounted on standoff, } \\
30^{\circ} \text {. }\end{array}$ & $\begin{array}{l}0 \mathrm{Lu} 17237 \\
\mathrm{kPa}\end{array}$ & 0 to $21201 \mathrm{kPa}$ & 153,154 & . \\
\hline PV-LP-166 & $\begin{array}{l}\text { In upper part of lower plenum, } 422 \\
\text { cm below cold leg centerline, } \\
\text { mounted on standoff, } 225^{\circ} \text {. }\end{array}$ & $\begin{array}{l}0 \text { to } 20684 \\
\mathrm{xPa}\end{array}$ & 0 to $34769 \mathrm{kPa}$ & 153,154 & \\
\hline ECC System & & 0 to $6895 \mathrm{kPa}$ & & & \\
\hline PU-ACCI & In intact loop accumulator. & & 0 to $8629 \mathrm{kPa}$ & 155,156 & \\
\hline PB-ACC2 & In broken loop accumulator." & & 0 to $8368 \mathrm{kPa}$ & 157,158 & \\
\hline Steam Generator & & & & & \\
\hline PU-SGSD & Secondary side stcam dome. & $\begin{array}{l}0 \text { to } 20684 \\
k \text { po }\end{array}$ & 0 to $13357 \mathrm{kPa}$ & 159,160 & \\
\hline \multicolumn{6}{|l|}{$\begin{array}{l}\text { Steam Generator } \\
\text { Rupture System }\end{array}$} \\
\hline$P U-5 G S 3$ & $\begin{array}{l}\text { In vessel, simulating steam } \\
\text { generator tube rupture. (Vessel } \\
\text { occumulator,) }\end{array}$ & 0 to $6895 \mathrm{kPa}$ & 0 to $8368 \mathrm{kPa}$ & 161,162 & . \\
\hline \multicolumn{6}{|l|}{ Pressurizer } \\
\hline PU-PRIZE & Pressurizer steam dome. & $\begin{array}{l}0 \text { to } 17237 \\
\mathrm{kPa}\end{array}$ & 0 tu $22113 \mathrm{kPa}$ & 163,164 & \\
\hline$\frac{\text { Pressure Suppression }}{\text { System }}$ & . & $\cdot$ & & & \\
\hline p-pse & Suppression tank top. & 0 th $1724 \mathrm{kPa}$ & 0 to $2173 \mathrm{kPa}$ & 165,166 & \\
\hline DIFFERENTIAL PRESSURE & $\begin{array}{l}\text { Elevation difference between } \\
\text { transducer taps is zero unless } \\
\text { otherwise specified. }\end{array}$ & & & & \\
\hline \multicolumn{6}{|l|}{ Intact loop } \\
\hline DPU-3-7 & $\begin{array}{l}\text { Hot leg Spool 3, } 158 \mathrm{~cm} \text { from vessel } \\
\text { cenler iu culd ley } 3 \text { pusl } 7,507 \text { iñ } \\
\text { from vessel center, Spool } 3 \text { tap is } \\
46 \mathrm{~cm} \text { above Spool } 7 \text { tap. }\end{array}$ & $\begin{array}{l} \pm 1270 \mathrm{~cm} \\
\text { riater }\end{array}$ & $\pm 170 \mathrm{kPa}$ & 167,168 & \\
\hline$Q P U=6-5 G 1 P$ & $\begin{array}{l}\text { Hut leg. Spool 6. } 290 \mathrm{~cm} \text { from } \\
\text { vessel center to steam generator } \\
\text { inlet plenum } 368 \mathrm{~cm} \text { from vessel } \\
\text { center. Spool } 6 \text { tap is } 41 \mathrm{~cm} \\
\text { below SGIP tap. }\end{array}$ & $\begin{array}{l}+2032 \mathrm{~cm} \\
\text { water }\end{array}$ & $\pm 268 \mathrm{kPa}$ & 169.170 & \\
\hline
\end{tabular}


TABLE V (continued)

\begin{tabular}{|c|c|c|c|c|c|c|}
\hline \multirow[b]{2}{*}{ Measurement } & \multirow[b]{2}{*}{ Location and Coments ${ }^{[a]}$} & \multicolumn{3}{|c|}{$\operatorname{Range}^{[a]}$} & \multirow[b]{2}{*}{ Figure ${ }^{[a]}$} & \multirow[b]{2}{*}{ Measurement Comments ${ }^{[b]}$} \\
\hline & & Detector & $\begin{array}{r}\text { Data A } \\
\text { S } \\
\end{array}$ & $\begin{array}{l}\text { Acquisition } \\
\text { System }\end{array}$ & & \\
\hline \multicolumn{7}{|c|}{ Intact Loop (continued) } \\
\hline DPU $\div 560 P-7$ & $\begin{array}{l}\text { From steam generator outlet plenum } \\
683 \mathrm{~cm} \text { from vessel center along } \\
\text { cold leg to cold leg Spool } 7,587 \mathrm{~cm} \\
\text { from vessel center, including orifice. } \\
\text { Spool } 7 \text { tap is } 89 \mathrm{~cm} \text { below } 560 p \text { tap. }\end{array}$ & $\frac{+1270 \mathrm{~cm}}{\text { water }}$ & $\pm 169 k$ & $\mathrm{kPa}$ & 171,172 & \\
\hline DPU-7-10 & $\begin{array}{l}\text { Steam generator outlet to pump } \\
\text { inlet, cold leg Spool } 7,587 \mathrm{~cm} \\
\text { from vessel center, to cold leg } \\
\text { Spool } 10,359 \mathrm{~cm} \text { from vessel } \\
\text { center. }\end{array}$ & $\underset{\text { water }}{+127 \mathrm{~cm}}$ & \pm 17.2 & $\mathrm{KPa}$ & 173,174 & \\
\hline DPU-12-10 & $\begin{array}{l}\text { Pump outlet to pump inlet, cold } \\
\text { leg Spool } 12 \text {, } 192 \mathrm{~cm} \text { from vessel } \\
\text { center, to cold leg Spool } 10,359 \\
\text { m fram vessel center. Spool } 10 \\
\text { tap is } 25 \mathrm{~cm} \text { below Spool } 12 \text { tap. }\end{array}$ & $\pm 345 \times \mathrm{Pa}$ & $\pm 343 k$ & $\mathrm{KPa}$ & 175,176 & \\
\hline DPU-12-iOL & 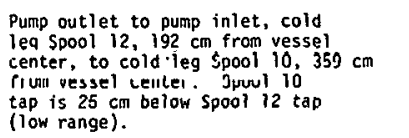 & $\begin{array}{l}+254 \mathrm{~cm} \\
\text { water }\end{array}$ & \pm 33.2 & $\mathrm{kPa}$ & $: 177,178$ & . \\
\hline OPU-12-15 & $\begin{array}{l}\text { Cold leg } 5 \text { pool } 12,192 \mathrm{~cm} \text { from } \\
\text { vessel center, to cold ieg } \$ \text { pool } \\
15,55 \mathrm{~cm} \text { from vessel center. }\end{array}$ & $\begin{array}{l} \pm 254 \mathrm{~cm} \\
\text { water }\end{array}$ & \pm 33.9 & $\mathrm{kPa}$ & 179,180 & $\begin{array}{l}\text { Data acquisition system } \\
\text { satura ted intermittentiy } \\
\text { between } t=1 \text { and } t=3 \mathrm{~s} \text {. }\end{array}$ \\
\hline DPU-15-3 & $\begin{array}{l}\text { Cold leg to hot leg; cold leg Spool } \\
15,55 \mathrm{~cm} \text { from vessel center, to } \\
\text { hot leg Spool } 3,158 \mathrm{~cm} \text { from vessel } \\
\text { center. Spool is tap is } 22 \mathrm{~cm} \\
\text { beluw Spuol } 3 \text { lap. }\end{array}$ & $\begin{array}{l}+1270 \mathrm{~cm} \\
\text { water }\end{array}$ & $\pm 169 k$ & $k P_{a}$ & $181, .182$ & $\cdot$ \\
\hline DPU-15-3L & $\begin{array}{l}\text { Cold leg to hot leg, cold leg } 5 p o o l \\
15,55 \mathrm{~cm} \text { from vessel center, to } \\
\text { hot leg Spool } 3,158 \mathrm{~cm} \text { from vessel } \\
\text { center. Spool is tap is } 22 \mathrm{~cm} \text { below } \\
\text { Spuol } 3 \text { tap (low range). }\end{array}$ & $\underset{\text { water }}{+254 \mathrm{~cm}}$ & \pm 33.5 & $\mathrm{KPa}$ & 183,184 & $\because$ \\
\hline DPU-PRESLL & $\begin{array}{l}\text { Pressurizer water level. Eleva- } \\
\text { tion difference between taps is } \\
135 \mathrm{~cm} \text {. Lower tap is } 9 \mathrm{~cm} \text { above } \\
\text { presssurizer exit. }\end{array}$ & $\begin{array}{l}+127 \mathrm{~cm} \\
\text { water }\end{array}$ & \pm 17.1 & $\mathrm{kPa}$ & 185,186 & : \\
\hline OPU-PR-4 & $\begin{array}{l}\text { Pressurizer bottom to Spool } 4 \text {. Eleva- } \\
\text { llur differ eille leL Lweell lays is } \\
157 \mathrm{~cm} \text {. Spool } 4 \text { tap is } 140 \mathrm{~cm} \text { below } \\
\text { pressurizer exit. }\end{array}$ & $\pm 3447 \mathrm{kPa}$ & \pm 3456 & $\mathrm{kPa}$ & 187,188 & $\begin{array}{l}\text { Data acquisition system } \\
\text { caturated botupen t=0 and } \\
t=13 \mathrm{~s} \text {. }\end{array}$ \\
\hline \multicolumn{7}{|l|}{ Bnoknn Inop } \\
\hline DPB-21-IANN & $\begin{array}{l}\text { Cold icg Spool } 21,112 \mathrm{~cm} \text { from } \\
\text { vessel center, to vessel inlet } \\
\text { annulus, } 23 \mathrm{~cm} \text { below cold leg } \\
\text { centerline at } 225^{\circ} \text {. Inlet annulus } \\
\text { tap is } 23 \mathrm{~cm} \text { below Spool } 21 \text { tap. }\end{array}$ & $\begin{array}{l}+254 \mathrm{~cm} \\
\text { water }\end{array}$ & \pm 3.3 .6 & $\mathrm{KPa}$ & 189,190 & \\
\hline DPB-23-CN1 & $\begin{array}{l}\text { Cold leg, } 5 \text { pool } 23,235 \mathrm{~cm} \text { from } \\
\text { veissel dexter to vessel }-51 \text { ade } \\
\text { nozzle throat, } 245 \mathrm{~cm} \text { from vessel } \\
\text { center. }\end{array}$ & 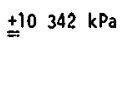 & \pm 1384 & $43 \mathrm{kPa}$ & 191,192 & \\
\hline$O P B-C N 1-24$ & $\begin{array}{l}\text { Vessel-side nozzle throat, } 245 \mathrm{~cm} \\
\text { from vessel center to Spool } 24,264 \mathrm{~cm} \\
\text { from yesse! centerr. }\end{array}$ & $\pm 344 \% \mathrm{kPa}$ & \pm 6961. & $\mathrm{kHa}$ & $\cdot 193,194$ & \\
\hline$D P B-30-43$ & $\begin{array}{l}\text { Across entire broken loop hot leg } \\
\text { including rupture assembly; hot leg } \\
\text { Spool } 30,45 \text { cm from vesse } \text {, to cold } \\
\text { leg Spool } 43 \text {, } 1086 \mathrm{~cm} \text { from vessel } \\
\text { ccntcr along hot.1cg. }\end{array}$ & $\pm 3447 \mathrm{kPa}$ & \pm 6973 & $\mathrm{kPa}$ & 195,196 & \\
\hline$D P B-32 U-36 L$ & 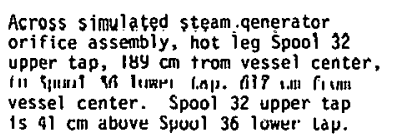 & $+1379 \mathrm{kPa}$ & \pm 1388 & $\mathrm{kPa}$ & 197,198 & \\
\hline DPB-36L-37 & $\begin{array}{l}\text { Across nozzle assembly. Spool } 36 \\
\text { lower tap, } 617 \mathrm{~cm} \text { from vesscl } \\
\text { cullter alutis liot leg to spool } 37, \\
718 \mathrm{~cm} \text { from vessel center alang } \\
\text { hot leg. Spool. } 37 \text { top is } 102 \mathrm{~cm} \\
\text { below Spool } 36 \text { lower tap. }\end{array}$ & $\pm 345 \mathrm{kPa}$ & $\pm 346 \mathrm{k}$ & $\mathrm{kPa}$ & 199,200 & \\
\hline$D P B-38-40$ & $\begin{array}{l}\text { Across simulated pump, cold leg } \\
\text { Spool } 38,776 \mathrm{~cm} \text { from vessel center } \\
\text { along hot leg, to cold leg Spool } 40 \text {, } \\
929 \text { from vessel center along hot } \\
\text { leg. }\end{array}$ & $\pm 6895 \mathrm{kPa}$ & \pm 9227 & $\mathrm{kPa}$ & 201,202 & \\
\hline
\end{tabular}


JABLE y (continued)

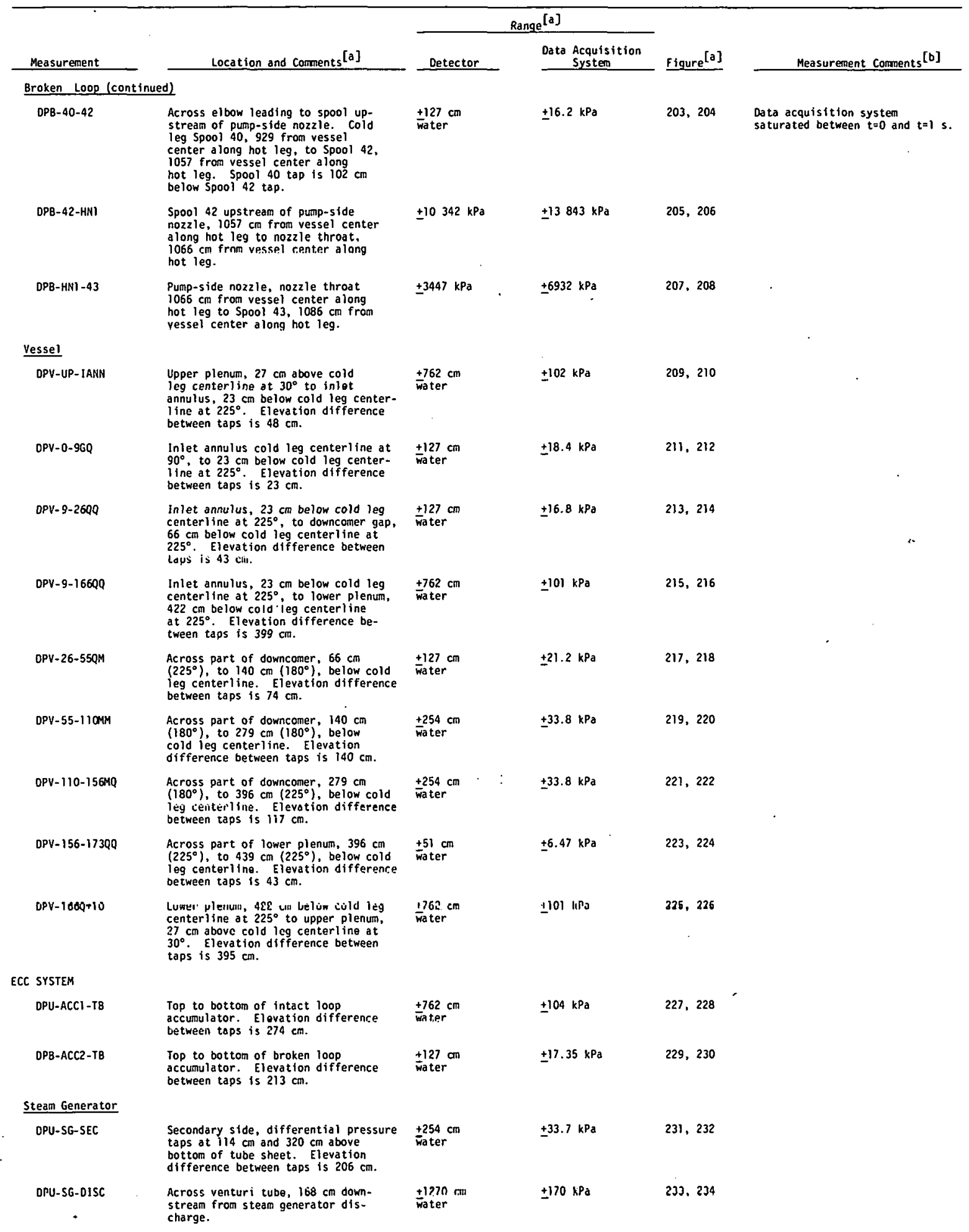


TABLE $\vee$ (continucd)

\begin{tabular}{|c|c|c|c|c|c|}
\hline \multirow[b]{2}{*}{ Measurement } & \multirow[b]{2}{*}{ Location and Comments ${ }^{[\mathrm{a}]}$} & \multicolumn{2}{|c|}{ Range $^{[a]}$} & \multirow[b]{2}{*}{ Figure $^{[\mathrm{a}]}$} & \multirow[b]{2}{*}{ Measurement Cominents $[\mathrm{b}]$} \\
\hline & & Detector & $\begin{array}{l}\text { Data Acquisition } \\
\text { System } \\
\end{array}$ & & \\
\hline \multicolumn{6}{|l|}{$\begin{array}{l}\text { Steam Generator } \\
\text { Rupture System }\end{array}$} \\
\hline OPU-SGS-6 & $\begin{array}{l}\text { From instrumented spool piece } \\
\text { in simulated tube rupture injection } \\
\text { line to hot leg- Spool } 6,318 \mathrm{~cm} \text { from } \\
\text { vessel center. }\end{array}$ & $\pm 6895 \mathrm{kPa}$ & $\pm 9230 \mathrm{kPa}$ & 235,236 & \\
\hline DPU-SGS3-TB & $\begin{array}{l}\text { Top to bottom of stean generator } \\
\text { rupture system accumulator tank. }\end{array}$ & $\begin{array}{l}+1270 \mathrm{~cm} \\
\text { water }\end{array}$ & $\pm 108 \mathrm{kPa}$ & 237,238 & \\
\hline \multicolumn{6}{|l|}{$\frac{\text { Pressure Suppression }}{\text { System }}$} \\
\hline DP-PSS-TB & $\begin{array}{l}\text { Top to bottom of pressure } \\
\text { suppression tank. Elevation } \\
\text { difference between taps } \\
\text { io } 338 \text { em. }\end{array}$ & $\begin{array}{l}\frac{4762 \mathrm{~cm}}{\text { water }} \\
\text { wat }\end{array}$ & $\pm 103 \mathrm{kPa}$ & 239,240 & \\
\hline VOLUMETRIC FLOW RATE & Turbine flowmeter, bidiréctional: & r & & & 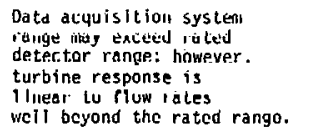 \\
\hline \multicolumn{6}{|c|}{ 3-in. Schedule 160 pipe. } \\
\hline FTU-1 & $\begin{array}{l}\text { Hot leg, Spooi } 1,42 \mathrm{~cm} \text { from } \\
\text { vessel center. }\end{array}$ & $\begin{array}{l} \pm 1.26 \text { to } \\
\pm 25.2 \mathrm{l} / \mathrm{s}\end{array}$ & \pm 0 to $75.7 \mathrm{R} / \mathrm{s}$ & 241,242 & \\
\hline FTU-9 & $\begin{array}{l}\text { rold leg, Spnoi 9. } 393 \mathrm{~cm} \text { from } \\
\text { vessel center. }\end{array}$ & $\begin{array}{l} \pm 5.05 \text { to } \\
. \pm 50.5 \mathrm{l} / \mathrm{s}\end{array}$ & $\pm 75.7 \mathrm{l} / \mathrm{s}$ & 241,242 & \\
\hline FTU-13 & $\begin{array}{l}\text { Culd ley, Spool } 13,103 \mathrm{~cm} \text { from } \\
\text { vesseî́ centèr. }\end{array}$ & $\begin{array}{l}11.26 \text { to } \\
\frac{11}{+50.5} \mathrm{l} / \mathrm{s}\end{array}$ & $175.7 \mathrm{R} / \mathrm{s}$ & 243,244 & \\
\hline FTU-15 & $\begin{array}{l}\text { Cold leg, Spool } 15,42 \mathrm{~cm} \text { from } \\
\text { vessel eenter. }\end{array}$ & $\begin{array}{l}+1.26 \text { to } \\
\pm=00.5 \mathrm{R} / \mathrm{s}\end{array}$ & $\pm 63.1 \mathrm{l} / \mathrm{s}$ & 243,244 & \\
\hline Broken Loop & Schedule 160 pipe. & & & & \\
\hline FT8-21 & $\begin{array}{l}\text { Culd ley, spool } 21, .140 \mathrm{~cm} \text { fram } \\
\text { vessel center; } 3-\text { in. pipe. }\end{array}$ & $\begin{array}{l}11.26 \text { to } \\
\pm 25.2 \mathrm{l} / \mathrm{s}\end{array}$ & $\pm 75.7 \mathrm{R} / \mathrm{s}$ & 245,246 & \\
\hline FTB-30 & $\begin{array}{l}\text { Hot leg, Spool } 30,63 \mathrm{~cm} \text { from } \\
\text { vessel center: } 3-\text { in. plpe. }\end{array}$ & $\begin{array}{l} \pm 1.26 \text { to } \\
\pm 25.2 \mathrm{l} / \mathrm{s}\end{array}$ & $\pm 50.5 \mathrm{l} / \mathrm{s}$ & 247,248 & \\
\hline FT8-37 & $\begin{array}{l}\text { Cold leg, Spool } 37,739 \mathrm{~cm} \text { from } \\
\text { vessel center à lónğ hot leg: }\langle-1 \mathrm{n} \text {. } \\
\text { pipe. }\end{array}$ & 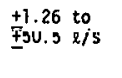 & $\pm 50.5 \ell / \mathrm{s}$ & 247,248 & \\
\hline \multicolumn{6}{|l|}{ Core } \\
\hline FTV-CORE-IN & $\begin{array}{l}\text { Entrance to core, } 401 \mathrm{~cm} \text { below } \\
\text { cold leg centerline. }\end{array}$ & $\begin{array}{l} \pm 1.26 \text { to } \\
\pm 12.6 \mathrm{k} / \mathrm{s}\end{array}$ & $\pm 56.78 t / \mathrm{s}$ & 249,250 & \\
\hline \multicolumn{6}{|l|}{ ECC System } \\
\hline FTU-HPIS & $\begin{array}{l}\text { In line immediately after HPIS } \\
\text { pump for intact lonp, } 1 / 7-\text { in. } \\
\text { line. }\end{array}$ & $\begin{array}{l}+0.047 \text { to } \\
\pm 0.47 \mathrm{e} / \mathrm{s}\end{array}$ & $\pm 0.315 \mathrm{2} / \mathrm{s}$ & 251,252 & \\
\hline FTB-HPIS & $\begin{array}{l}\text { In line irmediately after HPIS } \\
\text { pump for broken loop, } 1 / 2-i n \text {. line. }\end{array}$ & $\begin{array}{l} \pm 0.0126 \text { to } \\
\pm 0.63 \mathrm{l} / \mathrm{s}\end{array}$ & $\pm 0.315 \mathrm{t} / \mathrm{s}$ & 253,254 & \\
\hline FTU-LPIS & $\begin{array}{l}\text { In line leading fram LPIS pump } \\
\text { for intact loop, } 1 / 2-i n \text {. line. }\end{array}$ & $\begin{array}{l}+0.047 \text { to } \\
+0.47 \mathrm{e} / \mathrm{s}\end{array}$ & $\pm 0.315 \mathrm{l} / \mathrm{s}$ & 255,256 & \\
\hline FTB-LPIS & $\begin{array}{l}\text { In } 1 \text { ine leading from LPIS pump } \\
\text { for broken } 100 p, 3 / 4-i n \text {. IIne. }\end{array}$ & $\begin{array}{l}+0.047 \text { to } \\
\underline{+0} .47 \mathrm{l} / \mathrm{s}\end{array}$ & $\pm 0.1262 / \mathrm{s}$ & 257,258 & \\
\hline FTU-ACCI & $\begin{array}{l}\text { In line leading from intact loop } \\
\text { accumiator, l-in. line. }\end{array}$ & $\begin{array}{l}+0.315 \text { to } \\
\pm 3.15 \mathrm{Q} / \mathrm{s}\end{array}$ & $\pm 4.42 \mathrm{l} / \mathrm{s}$ & 259,260 & $\begin{array}{l}\text { Data acquisition system } \\
\text { saturated from } t=72 \text { and } t=88 \mathrm{~s} \text {. }\end{array}$ \\
\hline FTB-ACC? & $\begin{array}{l}\text { In line leading from broken loop } \\
\text { ascumulator, } 1-i n .1 \text { ine. }\end{array}$ & $\begin{array}{l}+0.126 \text { to } \\
\pm 1.26 \mathrm{p} / \mathrm{s}\end{array}$ & $\pm 1.39 \mathrm{~g} / \mathrm{s}$ & 261,262 & $\begin{array}{l}\text { Data acquisition system } \\
\text { saturated from } t=33 \text { and } t=48 \text { s. }\end{array}$ \\
\hline \multicolumn{6}{|l|}{$\begin{array}{l}\text { Stean Generator. } \\
\text { Rupluie Eystem }\end{array}$} \\
\hline FTU-SGS & $\begin{array}{l}\text { In simulated tube rupture infection } \\
\text { line. }\end{array}$ & $\begin{array}{l}+0.0126 \text { to } \\
\pm 0.631 \mathrm{o} / \mathrm{s}\end{array}$ & $\pm 0.315 \mathrm{R} / \mathrm{s}$ & $263 \cdot 264$ & \\
\hline Pressur1zar & $1-1 / 2-\ln$, Lurling & & & & \\
\hline FTU-PRIZE & Surge itne. & $\begin{array}{l} \pm 0.315 \text { to } \\
\pm 3.15 \mathrm{l} / \mathrm{s}\end{array}$ & $\pm 6.31 \mathrm{2} / \mathrm{s}$ & 265,266 & \\
\hline
\end{tabular}




\begin{tabular}{|c|c|c|c|c|c|}
\hline \multirow[b]{2}{*}{ Measurement } & \multirow[b]{2}{*}{ Location and Comnents ${ }^{[a]}$} & \multicolumn{2}{|c|}{ Range $^{[a]}$} & \multirow[b]{2}{*}{ Figure $^{[\mathrm{a}]}$} & \multirow[b]{2}{*}{ Measurement Comments ${ }^{[\mathrm{b}]}$} \\
\hline & & Detector & $\begin{array}{l}\text { Data Acquisition } \\
\text { System } \\
\end{array}$ & & \\
\hline FLUID YELOCITY & Turbine flowmeter, bidirectional. & & & & \\
\hline \multicolumn{6}{|l|}{ Downcomer } \\
\hline FTV-40A & $\begin{array}{l}102 \mathrm{~cm} \text { below cold leg centerline, } \\
0^{\circ} \text {. }\end{array}$ & $\begin{array}{l} \pm 0.762 \text { to } \\
\pm 15.24 \mathrm{~m} / \mathrm{s}\end{array}$ & $\pm 15.24 \mathrm{~m} / \mathrm{s}$ & 267,268 & $\begin{array}{l}\text { Data acquisition system saturated } \\
\text { intermittently at } t=56 \mathrm{~s} \text {. }\end{array}$ \\
\hline FTV-40M & $\begin{array}{l}102 \mathrm{~cm} \text { below cold leg centerline, } \\
180^{\circ} \text {. }\end{array}$ & $\begin{array}{l}+0.762 \text { to } \\
\pm 15.24 \mathrm{~m} / \mathrm{s}\end{array}$ & $\pm 15.24 \mathrm{~m} / \mathrm{s}$ & 267,268 & $\begin{array}{l}\text { Data acquisition system saturated } \\
\text { intermittentiy between } t=30 \text { and } \\
t=60 \mathrm{~s} \text {. }\end{array}$ \\
\hline MOMENTUM FLUX & Drag dise, bidirectional. & & & & $\begin{array}{l}\text { Drag disc data may exhibit } \\
\text { significant temperature } \\
\text { dependence. Drag disc data } \\
\text { should be used only for short- } \\
\text { term transient response. }\end{array}$ \\
\hline Intact Loop & 3-in. pipe. & & & & \\
\hline FDU-1 & $\begin{array}{l}\text { Hul lèy, Sjool } 1,60 \mathrm{~cm} \text { from } \\
\text { vessel center; target size } \\
2.22 \mathrm{~cm} \text {. }\end{array}$ & $\begin{array}{l} \pm 298 \text { to } \pm 17 \quad 113 \\
\mathrm{~kg} / \mathrm{m}-\mathrm{s}^{2}\end{array}$ & $\begin{array}{l} \pm 76090 \\
\mathrm{~kg} / \mathrm{m}-\mathrm{s}^{2}\end{array}$ & 269.270 & \\
\hline FDU-5 & $\begin{array}{l}\text { Hot leg, Spool } 5,256 \mathrm{~cm} \text { from } \\
\text { vessel center; target size } \\
2.54 \mathrm{~cm} \text {. }\end{array}$ & $\begin{array}{l} \pm 1.49 \text { to }+2976 \\
\mathrm{~kg} / \mathrm{m}-\mathrm{s}^{2}\end{array}$ & $\frac{+6994}{\mathrm{~kg} / \mathrm{m}-\mathrm{s}^{2}}$ & 271,272 & - \\
\hline FDU-10 & $\begin{array}{l}\text { Cold leg, Spool } 10,349 \mathrm{~cm} \text { from } \\
\text { vessel center; target size } 2.22 \mathrm{~cm} \text {. }\end{array}$ & $\begin{array}{l} \pm 298 \text { to } \pm 154769 \\
\mathrm{~kg} / \mathrm{m}-\mathrm{s}^{2}\end{array}$ & $\begin{array}{l} \pm 35344 \\
\mathrm{~kg} / \mathrm{m}-\mathrm{s}^{2}\end{array}$ & 273,274 & \\
\hline FOU-13 & $\begin{array}{l}\text { Cold leg, spool 13, } 138 \mathrm{~cm} \text { from } \\
\text { vessel center; target size } 2.22 \mathrm{~cm} \text {. }\end{array}$ & $\begin{array}{l} \pm 298 \text { to }+22025 \\
\mathrm{~kg} / \mathrm{m}-\mathrm{s}^{2}\end{array}$ & $\begin{array}{l} \pm 28528 \\
\mathrm{~kg} / \mathrm{m}-\mathrm{s}^{2}\end{array}$ & 275,276 & \\
\hline FOU $=15$ & $\begin{array}{l}\text { Cold leg, Spool } 15,60 \mathrm{~cm} \text { from } \\
\text { vessel center; target size } 2.22 \mathrm{~cm} \text {. }\end{array}$ & $\begin{array}{l} \pm 298 \text { to } \\
\mathrm{kg} / \mathrm{m}-\mathrm{s}^{2}\end{array}$ & $\begin{array}{l}+39124 \\
\mathrm{~kg} / \mathrm{m}-\mathrm{s}^{2}\end{array}$ & 277,278 & . \\
\hline \multicolumn{6}{|l|}{ Broken Loop } \\
\hline FOB-21 & $\begin{array}{l}\text { Cold leg, Spool } 21,134 \mathrm{~cm} \text { from } \\
\text { vessel center, } 3-\text { in. pipe; target } \\
\text { size } 1.67 \mathrm{~cm} \text {. }\end{array}$ & $\underset{\mathrm{kg} / \mathrm{mi}-\mathrm{s}^{2}}{+298 \text { to }} \pm 104915$ & $\begin{array}{l} \pm 66774 \\
\mathrm{~kg} / \mathrm{m}-\mathrm{s}^{2}\end{array}$ & 279,280 & . \\
\hline FDB-23 & $\begin{array}{l}\text { Cold leg, Spool 23, } 238 \mathrm{~cm} \text { from } \\
\text { vessel center, upstream of vessel } \\
\text { side nozzle, z-in., pipe; target } \\
\text { size } 1.03 \mathrm{~cm} \text {. }\end{array}$ & $\begin{array}{l} \pm 298 \text { to } \\
\mathrm{kg} / \mathrm{m}-\mathrm{s}^{2}\end{array}$ & $\begin{array}{l} \pm 155320 \\
\mathrm{~kg} / \mathrm{m}-\mathrm{s}^{2}\end{array}$ & 281,282 & $\begin{array}{l}\text { Data acquisition system } \\
\text { saturated between } t=0 \text { and } t=2 \mathrm{~s} \text {. }\end{array}$ \\
\hline FDB-30 & $\begin{array}{l}\text { Hot leg, Spool } 30,52 \mathrm{~cm} \text { from } \\
\text { vessel center, 3-in. pipe; target } \\
\text { size } 1.67 \mathrm{~cm} \text {. }\end{array}$ & $\begin{array}{l} \pm 298 \text { to } \\
\mathrm{kg} / \mathrm{m}-\mathrm{s}^{2}\end{array}$ & $\begin{array}{l}+67280 \\
\mathrm{~kg} / \mathrm{m}-\mathrm{s}^{2}\end{array}$ & 283,284 & \\
\hline FDB -37 & $\begin{array}{l}\text { Cold leg, Spool } 37,725 \mathrm{~cm} \text { from } \\
\text { vessel center along hot leg, steam } \\
\text { generator outlet, vertical pipe, } \\
\text { 2-in. pipe; target size } 1.03 \mathrm{~cm} \text {. }\end{array}$ & $\begin{array}{l} \pm 298 \text { to } \pm 180067 \\
\mathrm{~kg} / \mathrm{m}-\mathrm{s}^{2}\end{array}$ & $\begin{array}{l} \pm 374272 \\
\mathrm{~kg} / \mathrm{m}-\mathrm{s}^{2}\end{array}$ & 285,286 & $\checkmark$ \\
\hline $\mathrm{FDB}+42$ & $\begin{array}{l}\text { C.nld leg. Sponl } 42,1057 \mathrm{~cm} \text { from } \\
\text { vessel center along hot leg, up- } \\
\text { stream of pump-side nozzle, down- } \\
\text { stream of injection point, } 2-\text { in. } \\
\text { pipe; target size } 1.03 \mathrm{~cm} \text {. }\end{array}$ & $\begin{array}{l} \pm 298 \text { to } \pm 172627 \\
\mathrm{~kg} / \mathrm{m}-\mathrm{s}^{2}\end{array}$ & $\begin{array}{l} \pm 165320 \\
\mathrm{~kg} / \mathrm{mi}-\mathrm{s}^{2}\end{array}$ & 287,288 & \\
\hline \multicolumn{6}{|l|}{ Vessel } \\
\hline FnV_rnRF-IN & $\begin{array}{l}\text { In rnre flow mixer } \operatorname{mox} .381 \mathrm{~cm} \\
\text { below cold leg centeri ine; target } \\
\text { size } 2.54 \mathrm{~cm} \text {. }\end{array}$ & $\begin{array}{l} \pm 1.44 \text { to } \pm 2977 \\
\mathrm{~kg} / \mathrm{m}-\mathrm{s}^{2}\end{array}$ & $\begin{array}{l}+1725 \\
\mathrm{~kg} / \mathrm{m}-\mathrm{s}^{2}\end{array}$ & 289,290 & \\
\hline \multicolumn{6}{|l|}{ DENSITY } \\
\hline Intact Loop & & $\begin{array}{l}1.6 \text { to } 3600 \\
\mathrm{~kg} / \mathrm{m}^{3}\end{array}$ & $\begin{array}{l}0 \text { to } 1600 \\
\mathrm{~kg} / \mathrm{m}^{3}\end{array}$ & & . \\
\hline $\begin{array}{l}\text { GII-1T } \\
\text { GU-18 } \\
\text { GU-1C }\end{array}$ & $\begin{array}{l}\text { Hot leg. Spool } 1,77 \mathrm{~cm} \text { from } \\
\text { vessel center. T' (top) ranges } \\
270 \text { to } 360^{\circ} .8 \text { (bottom) ranges } \\
30 \text { to } 330^{\circ} \text {. C, mathematical } \\
\text { composite of T and } \mathrm{B} \text {. }\end{array}$ & & & $\begin{array}{l}291,292 \\
293,294\end{array}$ & . \\
\hline GU-5VR & $\begin{array}{l}\text { Hot leg, Spool } 5,246 \mathrm{~cm} \text { from } \\
\text { vessel center, vertical. }\end{array}$ & & & 295,296 & \\
\hline QU- 10VR & $\begin{array}{l}\text { Cold leg, spool } 10,350 \text { em from } \\
\text { vessel center, vertical. }\end{array}$ & & & 295,296 & $\cdot$ \\
\hline GU-13VR & $\begin{array}{l}\text { Cold leg, Spool } 13,142 \mathrm{~cm} \text { from } \\
\text { vessel center, vertical. }\end{array}$ & & & 297,298 & \\
\hline $\begin{array}{l}\text { GU-15T } \\
G U-158 \\
G U-15 C\end{array}$ & $\begin{array}{l}\text { Cold leg, Spool } 15,77 \mathrm{~cm} \text { from vessel } \\
\text { center. T (top) ranges } 270 \text { to } 360^{\circ} \text {. } \\
\text { B (bottom) ranges } 30 \text { to } 330^{\circ} \text {. C, } \\
\text { mathematical composite of } T \text { and } B \text {. }\end{array}$ & & & $\begin{array}{l}299,300 \\
301,302\end{array}$ & $\begin{array}{l}\text { Data acquisition system } \\
\text { saturated between } t=28 \text { and } t=65 \mathrm{~s} \text {. }\end{array}$ \\
\hline
\end{tabular}


TABLE $\bigvee$ (cọnt inued)

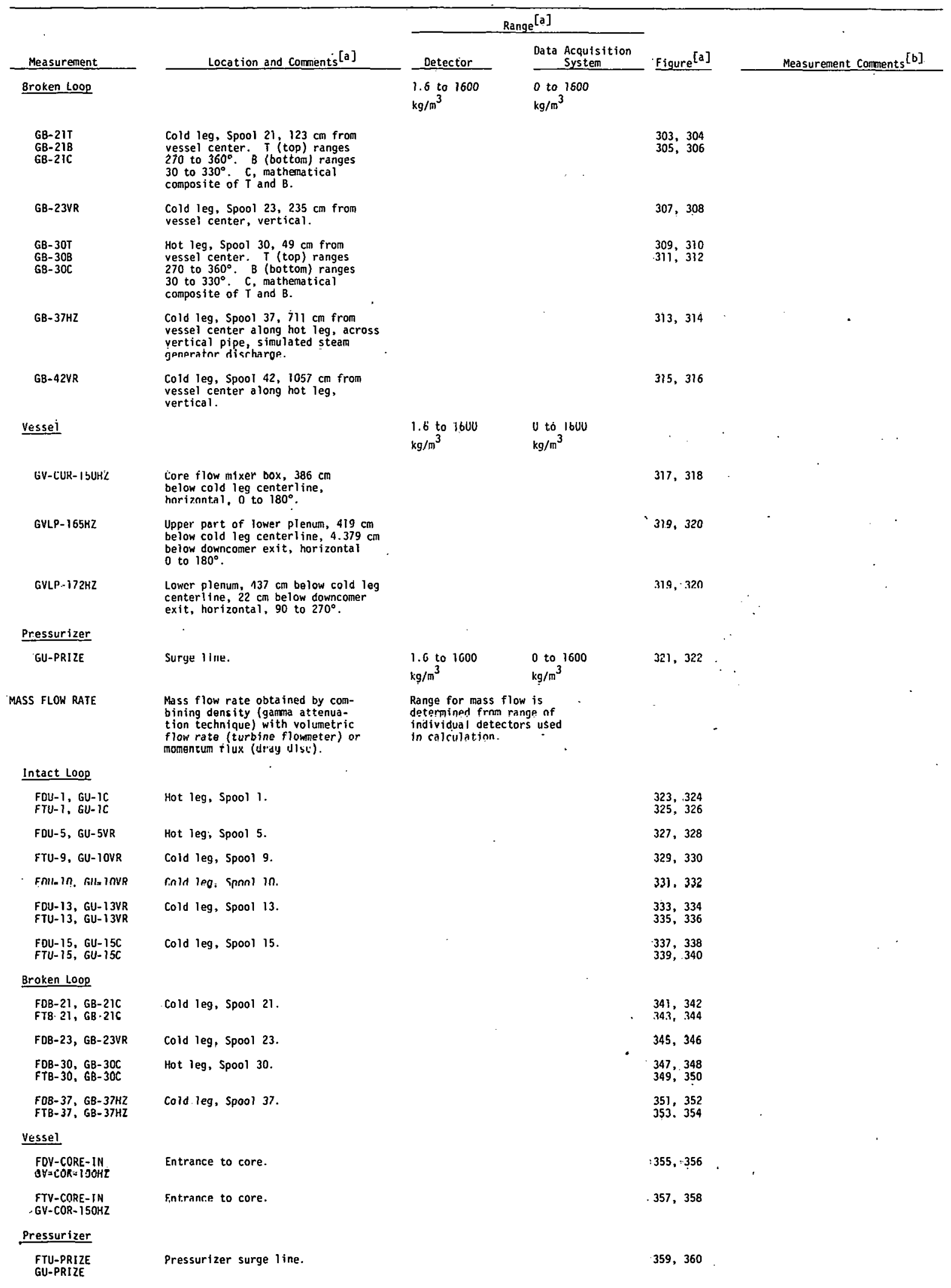




\begin{tabular}{|c|c|c|c|c|c|}
\hline \multirow[b]{2}{*}{ Measurement } & \multirow[b]{2}{*}{ Location and Comments $[\mathrm{a}]$} & \multicolumn{2}{|c|}{$\operatorname{Range}^{[\mathrm{a}]}$} & \multirow[b]{2}{*}{ Figure $^{[a]}$} & \multirow[b]{2}{*}{ Measurement Comments $[\mathrm{b}]$} \\
\hline & & Detector & $\begin{array}{c}\text { Data Acquisition } \\
\text { System } \\
\end{array}$ & & \\
\hline \multicolumn{6}{|c|}{ CORE CHARACTERISTICS } \\
\hline PWRCOR T-1 & Core power. & 0 to $1600 \mathrm{kH}$ & 0 to $2076 \mathrm{kH}$ & 361,362 & \\
\hline PWRCOR T-2 & Core power. & 0 to $1600 \mathrm{~kW}$ & 0 to $2602 \mathrm{kH}$ & $36 \%, 362$ & \\
\hline VOLICOR-I & Core voltage. & & 0 to $200 \mathrm{Vdc}$ & 363,364 & \\
\hline AMPCOR-T & Core current. & 0 to $10000 \mathrm{~A}$ & 0 to $9645 \mathrm{~A}$ & 365,366 & \\
\hline \multicolumn{5}{|c|}{ PUMP CHARACTERISTICS } & \\
\hline PUMPU-CUR & Pump current. & 0 to $25 \mathrm{~A}$ & 0 to $44 \mathrm{~A}$ & 367,368 & \\
\hline PUMPU-RPM & Pump speed. & 0 to $3600 \mathrm{rpm}$ & 0 to $3600 \mathrm{rpm}$ & 369,370 & \\
\hline
\end{tabular}

[a] Statements at the beginning of a measurement category regarding location and camments, range, and figure apply to all subsequent measurements within the given category unless specified otherwise.

[b] Detectors which were subjected to overrange conditions during portions of the test were capable of withstanding these conditions without change in operating or measuring characteristics when the physical conditions were again within the detector range. 


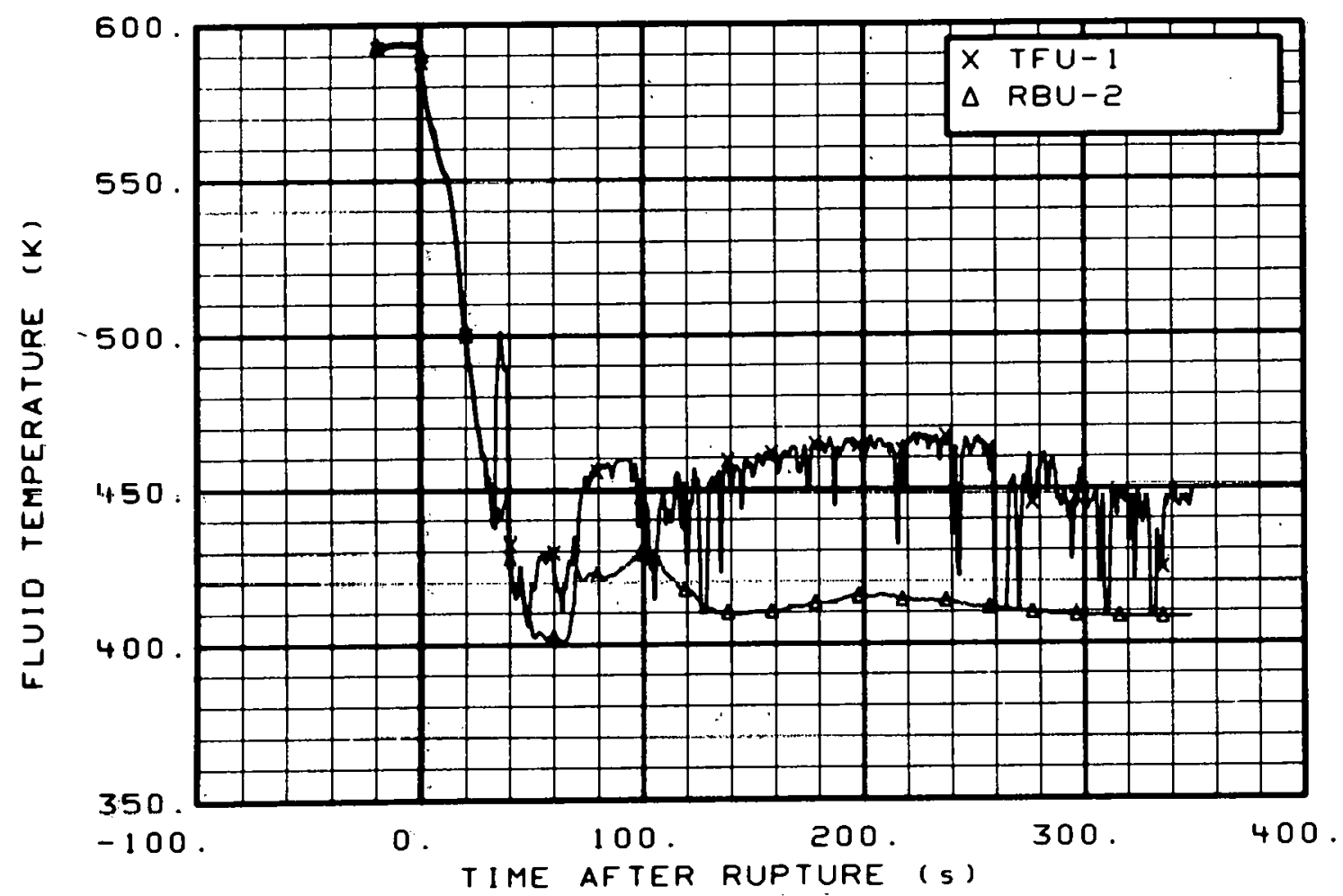

Fig. 9 Fluid temperature in intact loop hot leg (TFU-1 and RBU-2), from -20 to $350 \mathrm{~s}$.

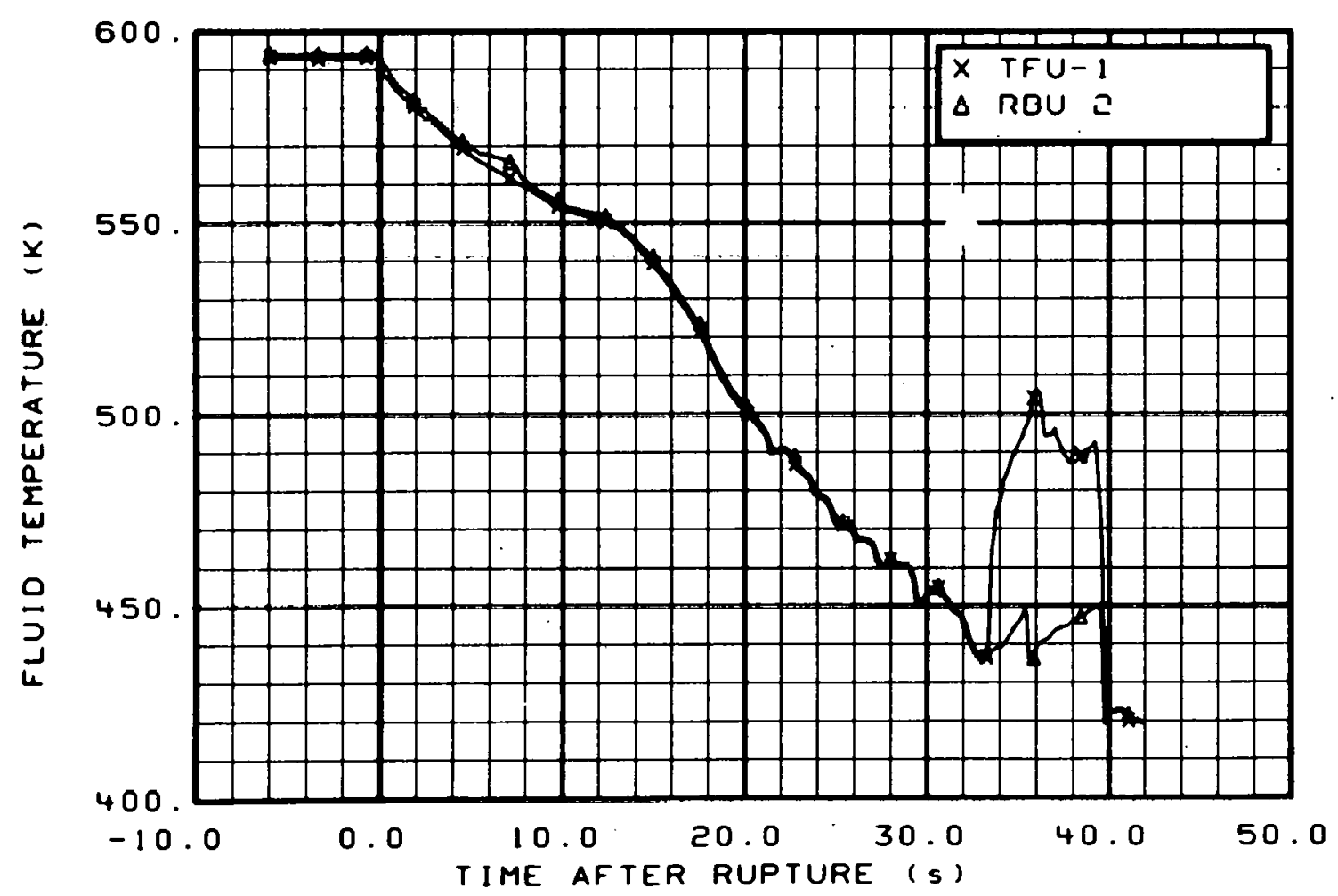

Fig. 10 Fluid temperature in intact loop hot leg (TFU-1 and RBU-2), from -6 to $42 \mathrm{~s}$. 


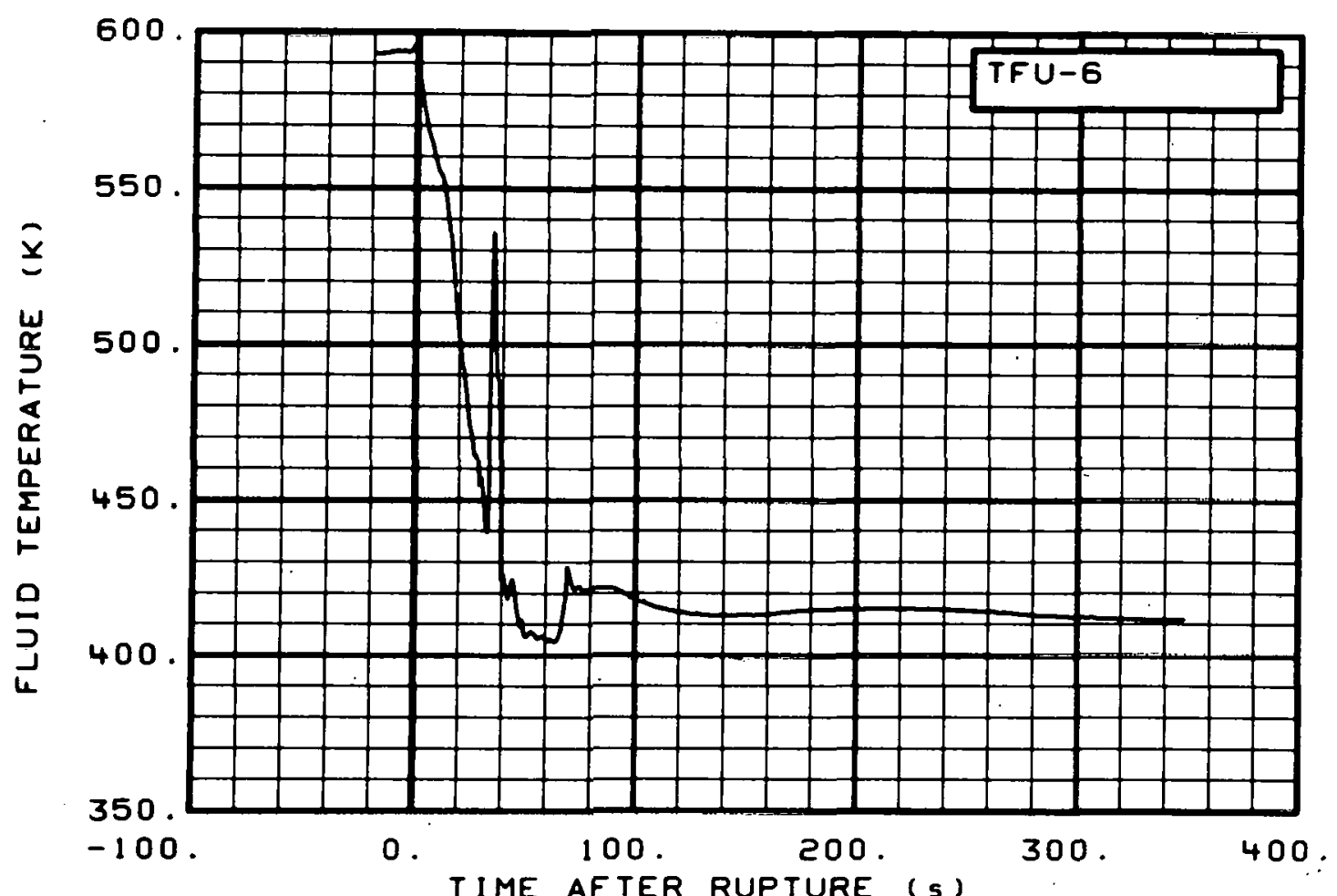

Fig. 11 Fluid temperature in intact loop hot leg (TFU-6), from -20 to $350 \mathrm{~s}$.

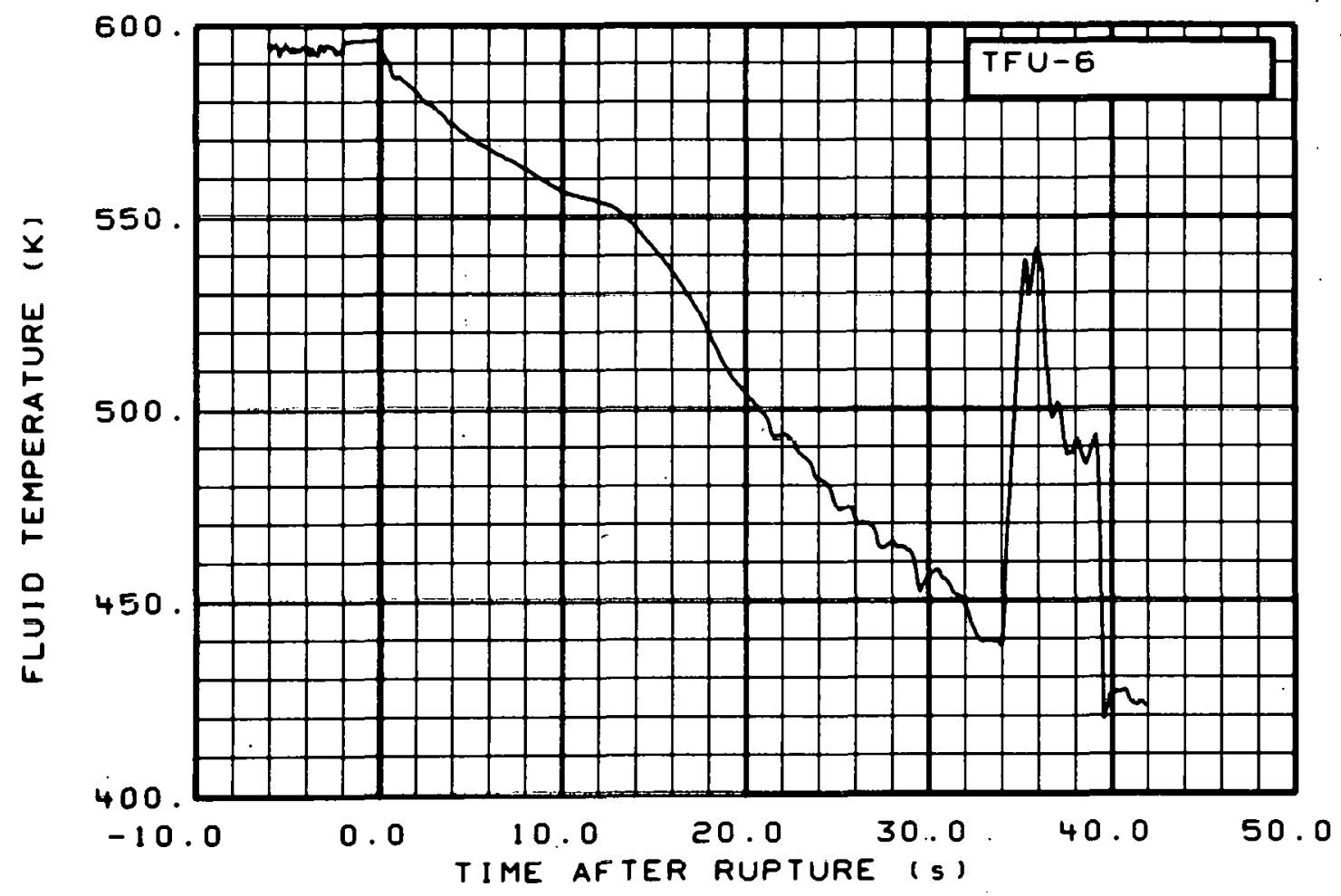

Fig. 12 Fluid temperature in intact loop hot leg (TFU-6), from -6 to $42 \mathrm{~s}$. 


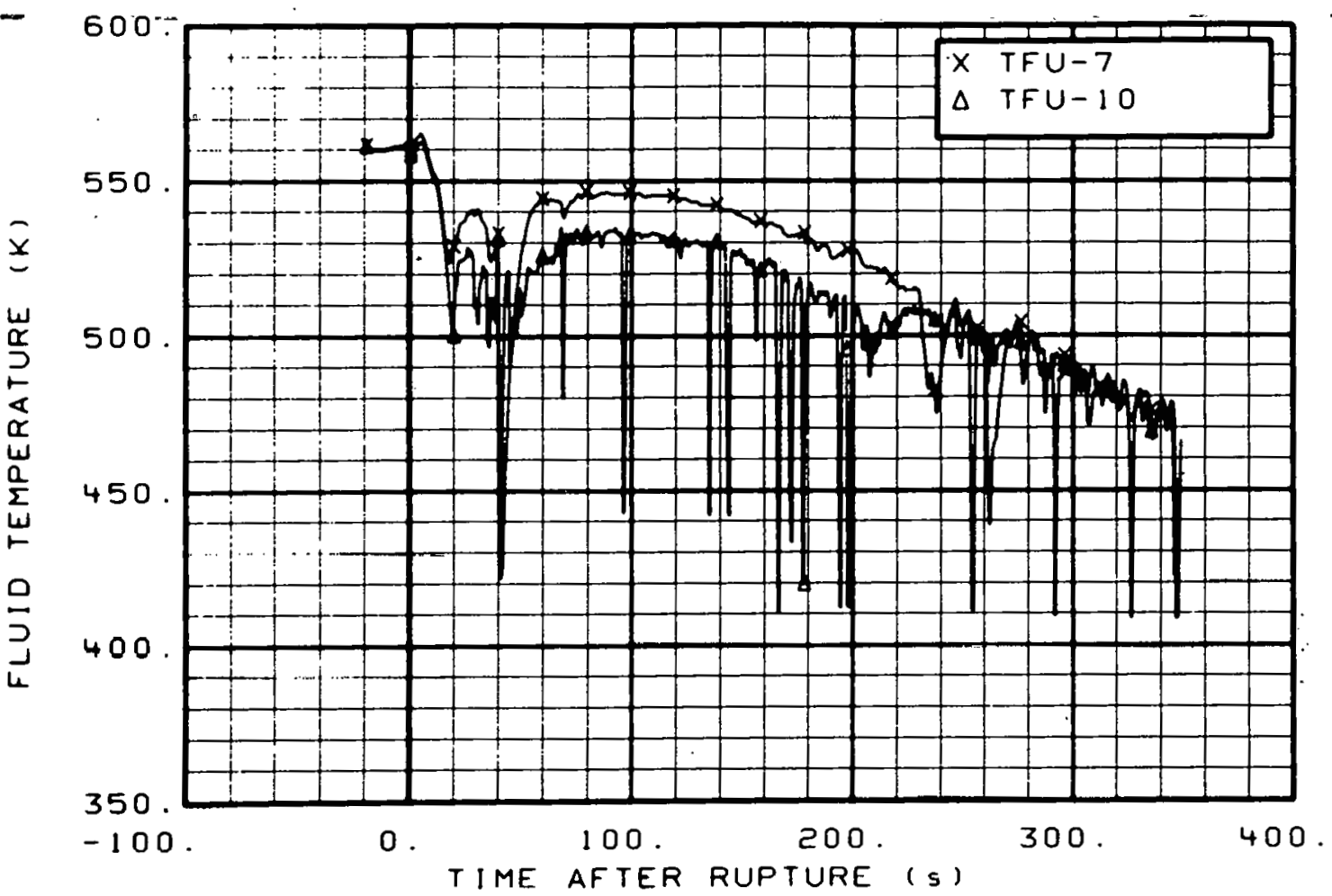

Fig. 13 Fluid temperature in intact loop cold leg (TFU-7 and TFU-10), from -20 to $350 \mathrm{~s}$.

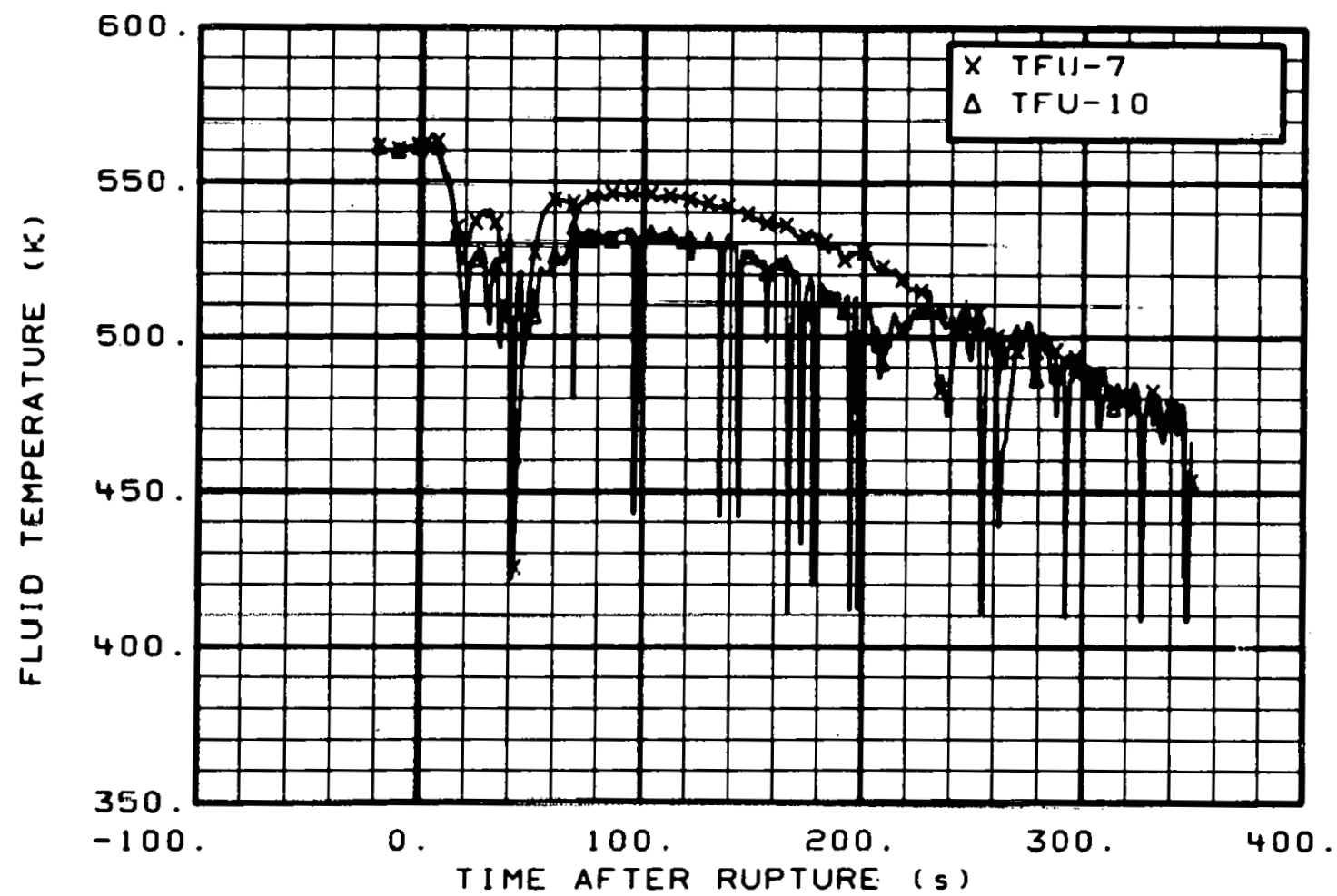

Fig. 14 Fluid temperature in intact loop cold leg (TFU-7 and TFU-10), from -6 to $42 \mathrm{~s}$. 


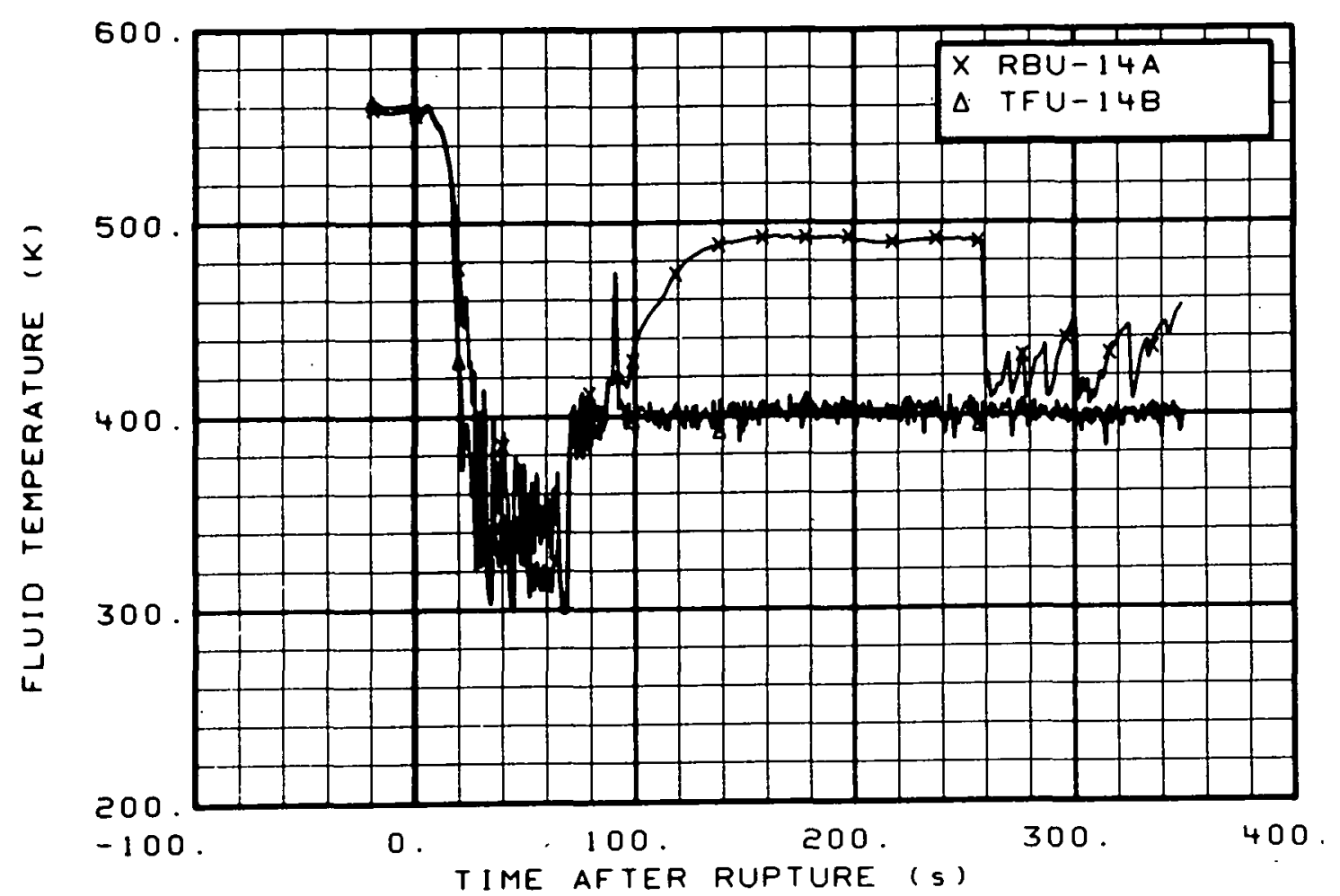

Fig. 15 Fluid temperature in intact loop cold leg (RBU-14A and TFU-14B), from -20 to $350 \mathrm{~s}$.

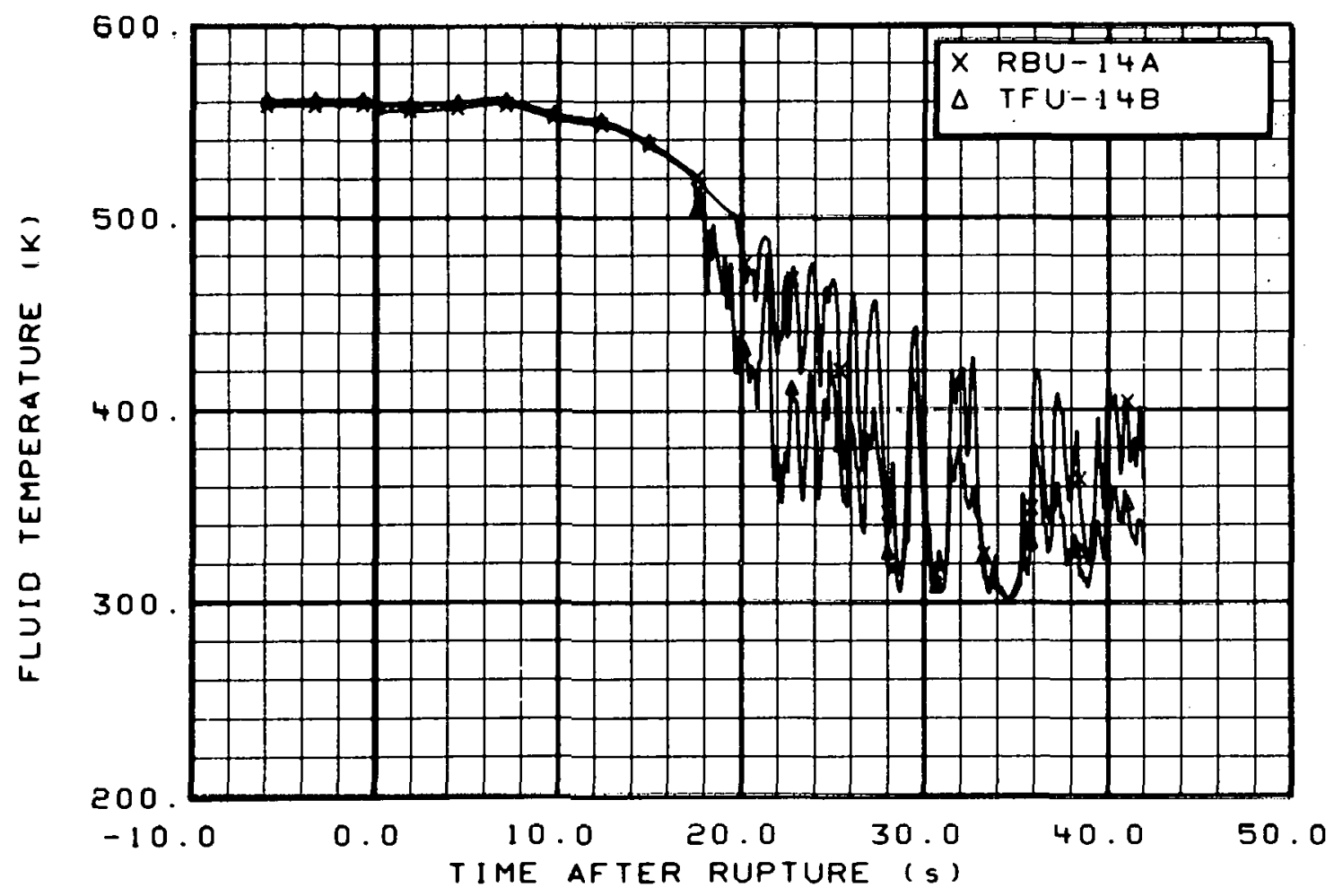

Fig. 16 Fluid temperature in intact loop cold leg (RBU-14A and TFU-14B), from -6 to $42 \mathrm{~s}$. 


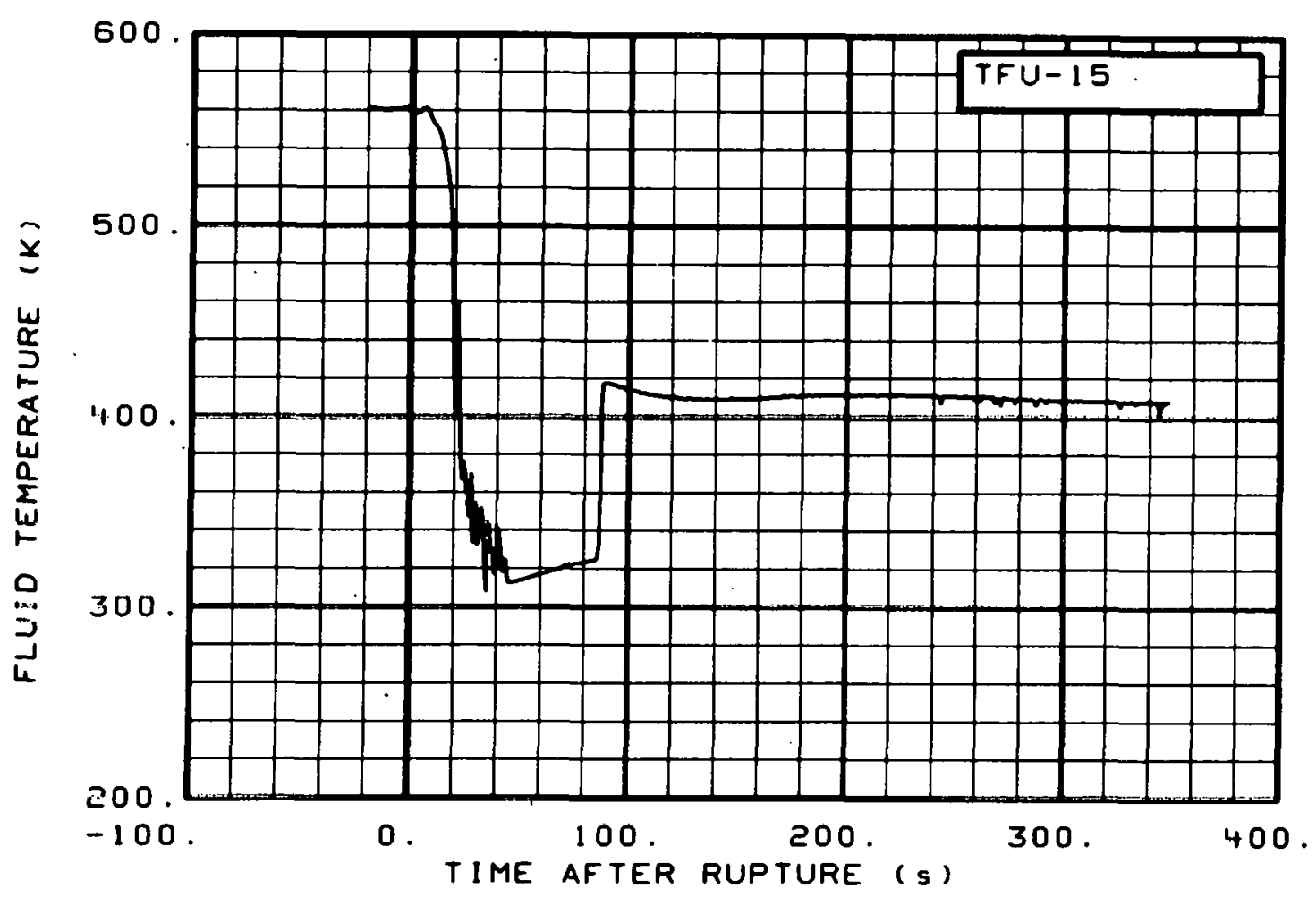

Fig. 17 Fluid temperature in intact loop cold leg (TFU-15), from -20 to $350 \mathrm{~s}$.

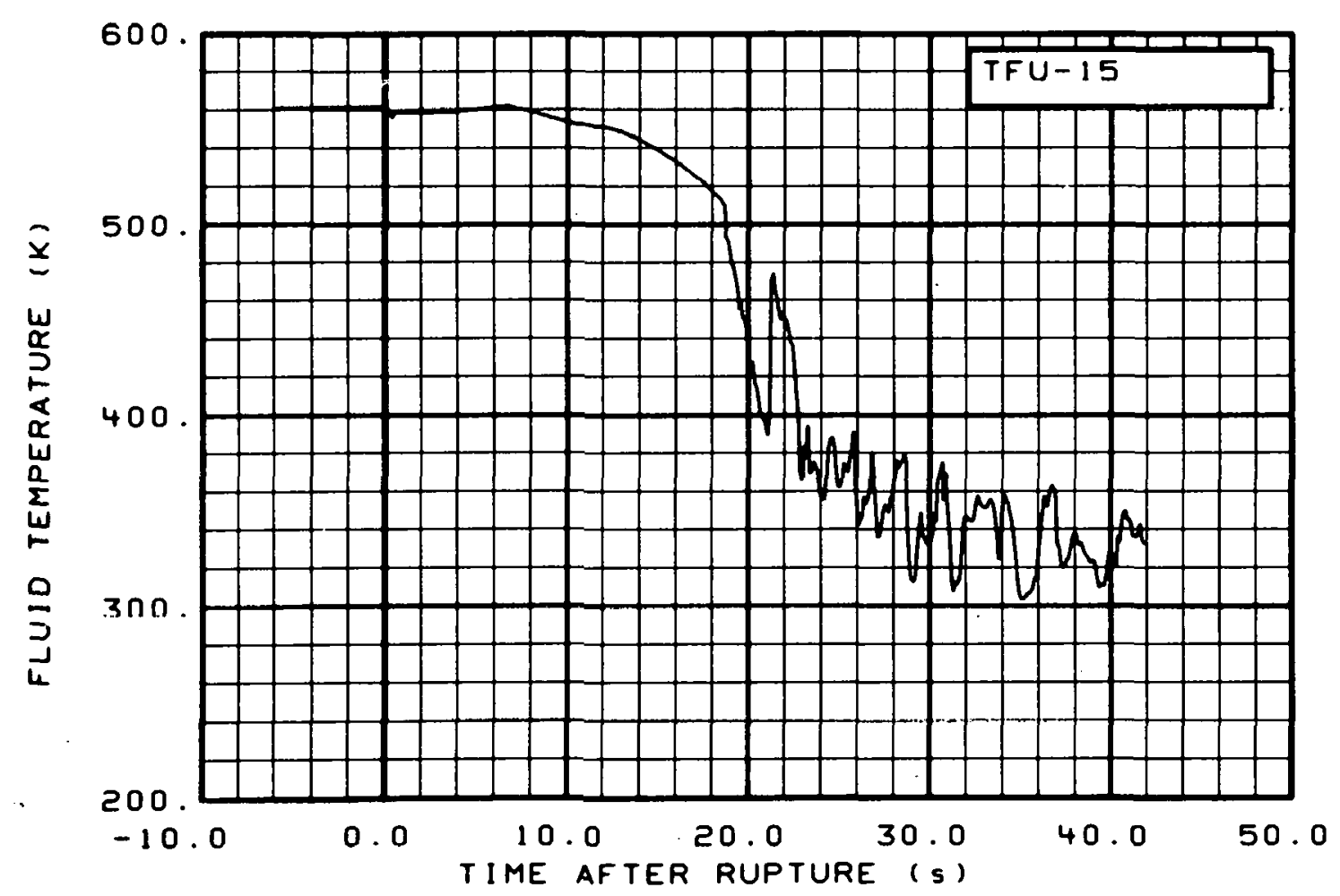

Fig. 18 Fluid temperature in intact loop cold leg (TFU-15), from -6 to $42 \mathrm{~s}$. 


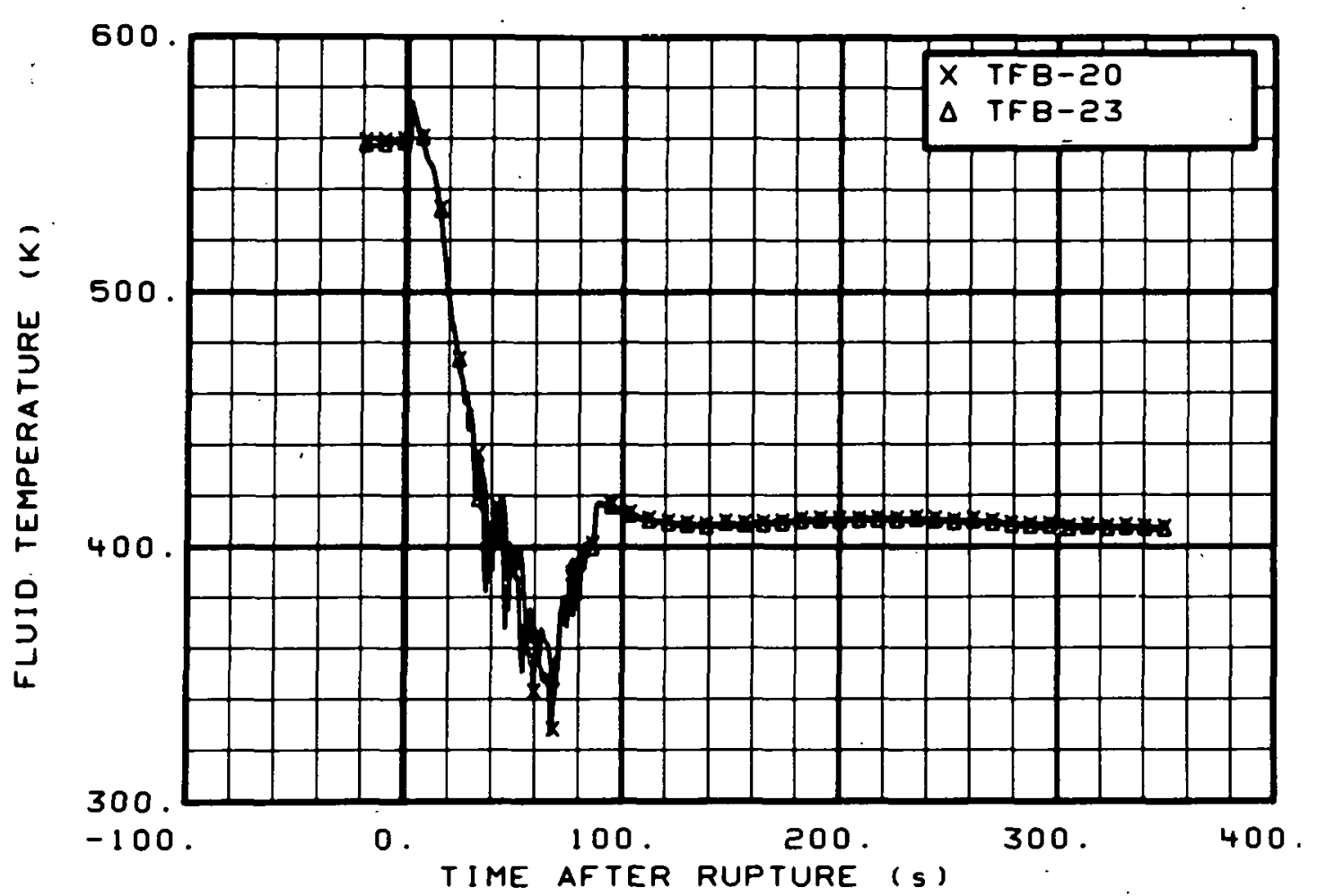

Fig. 19 Fluid temperature in broken loop, vessel side (TFB-20 and TFB-23), from -20 to $350 \mathrm{~s}$.

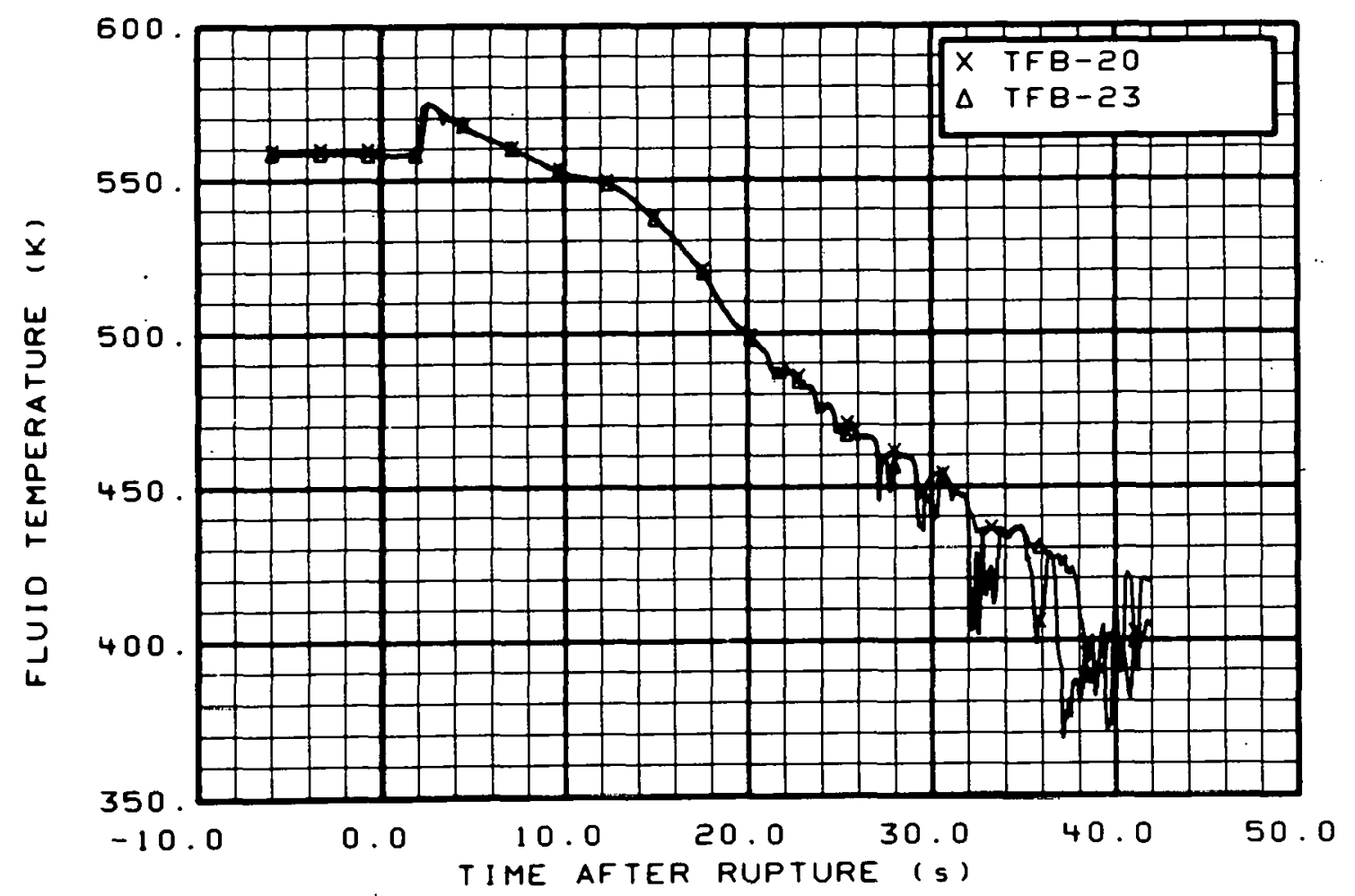

Fig. 20 Fluid temperature in broken loop, vessel side (TFB-20 and TFB-23), from -6 to $42 \mathrm{~s}$. 


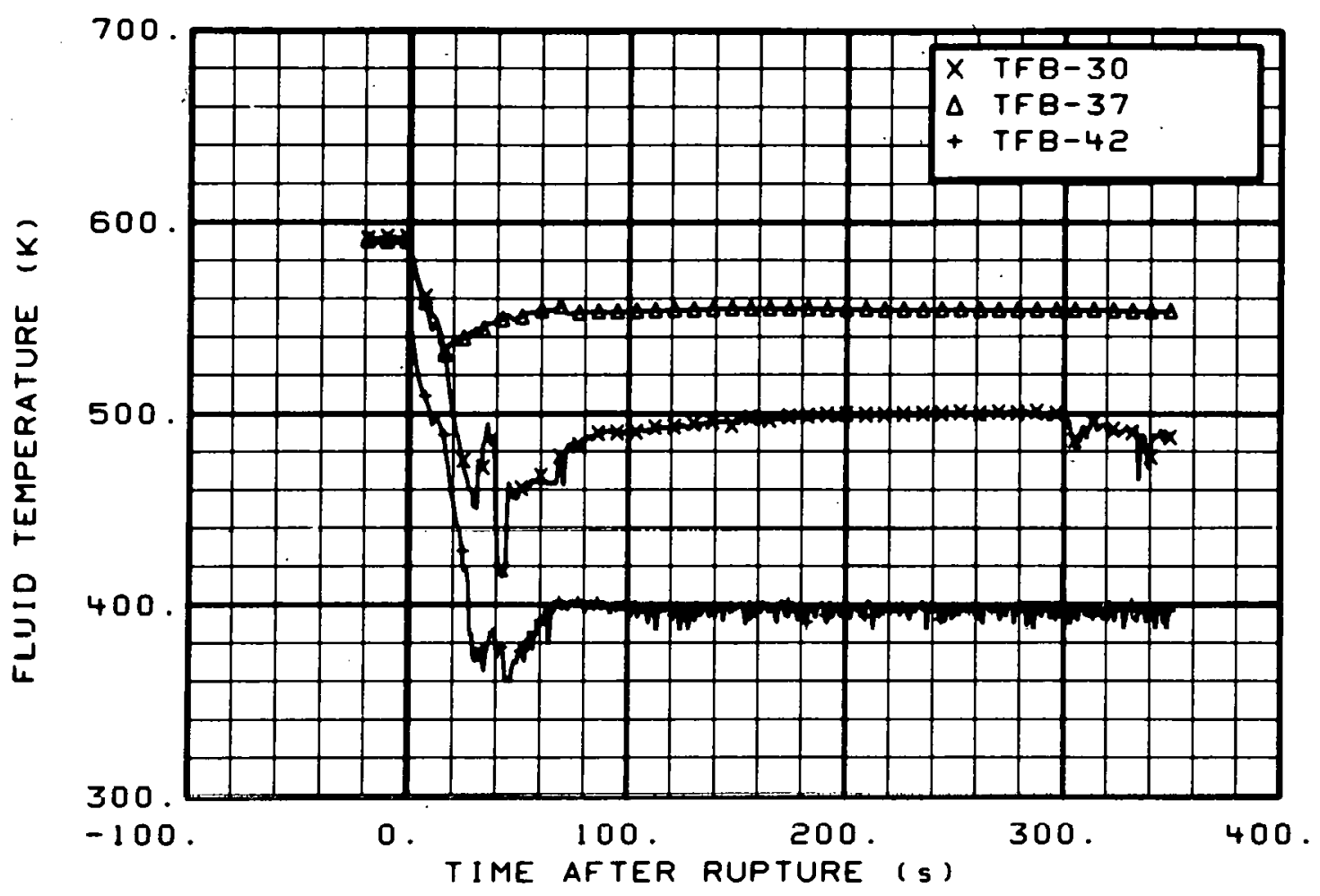

Fig. 21 Fluid temperature in broken loop, pump side (TFB-30, TFB-37, and TFB-42), from -20 to $350 \mathrm{~s}$.

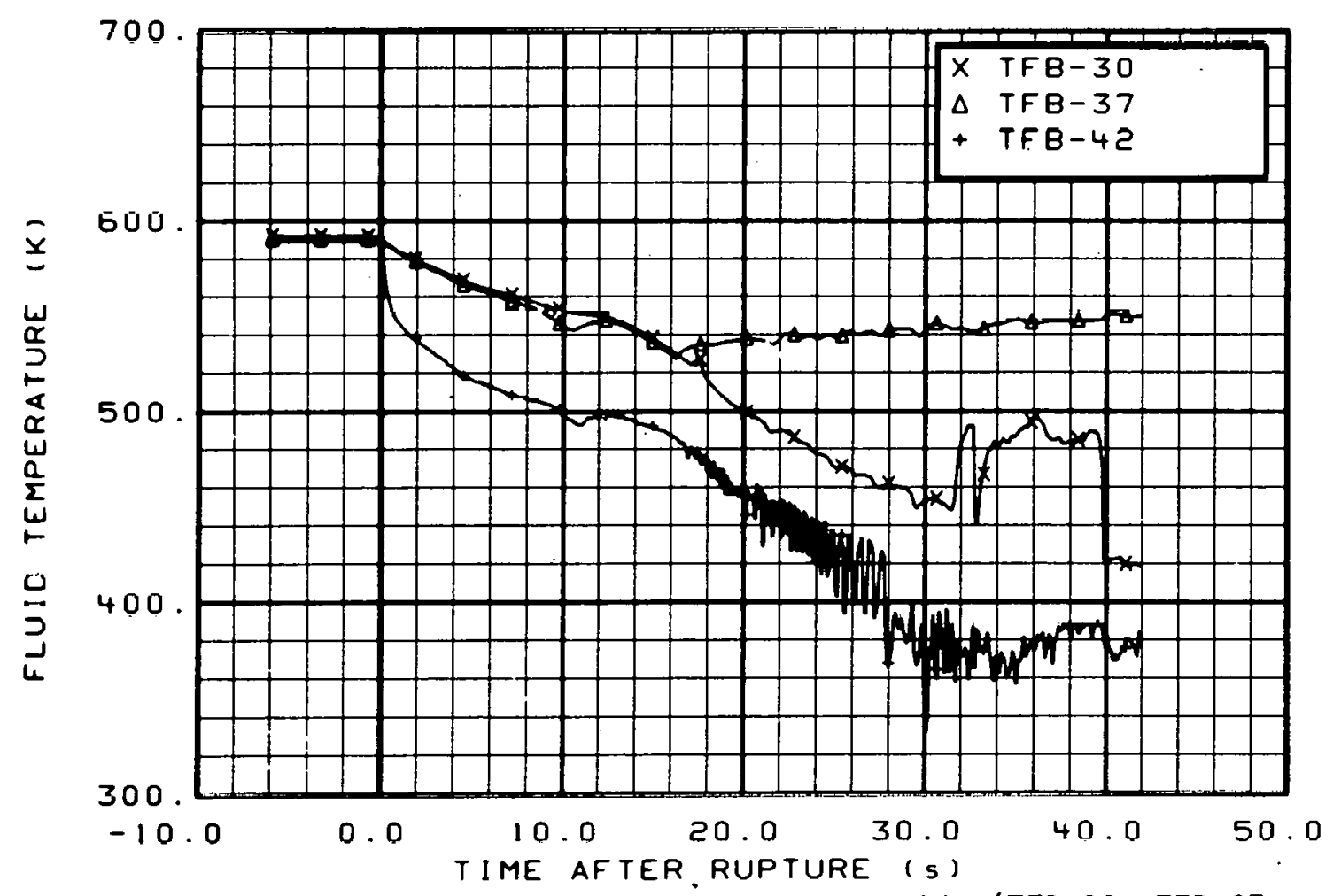

Fig. 22 Fluid temperature in broken toop, pump side (TFB-30, TFB-37, and TFB-42), from -6 to $42 \mathrm{~s}$. 


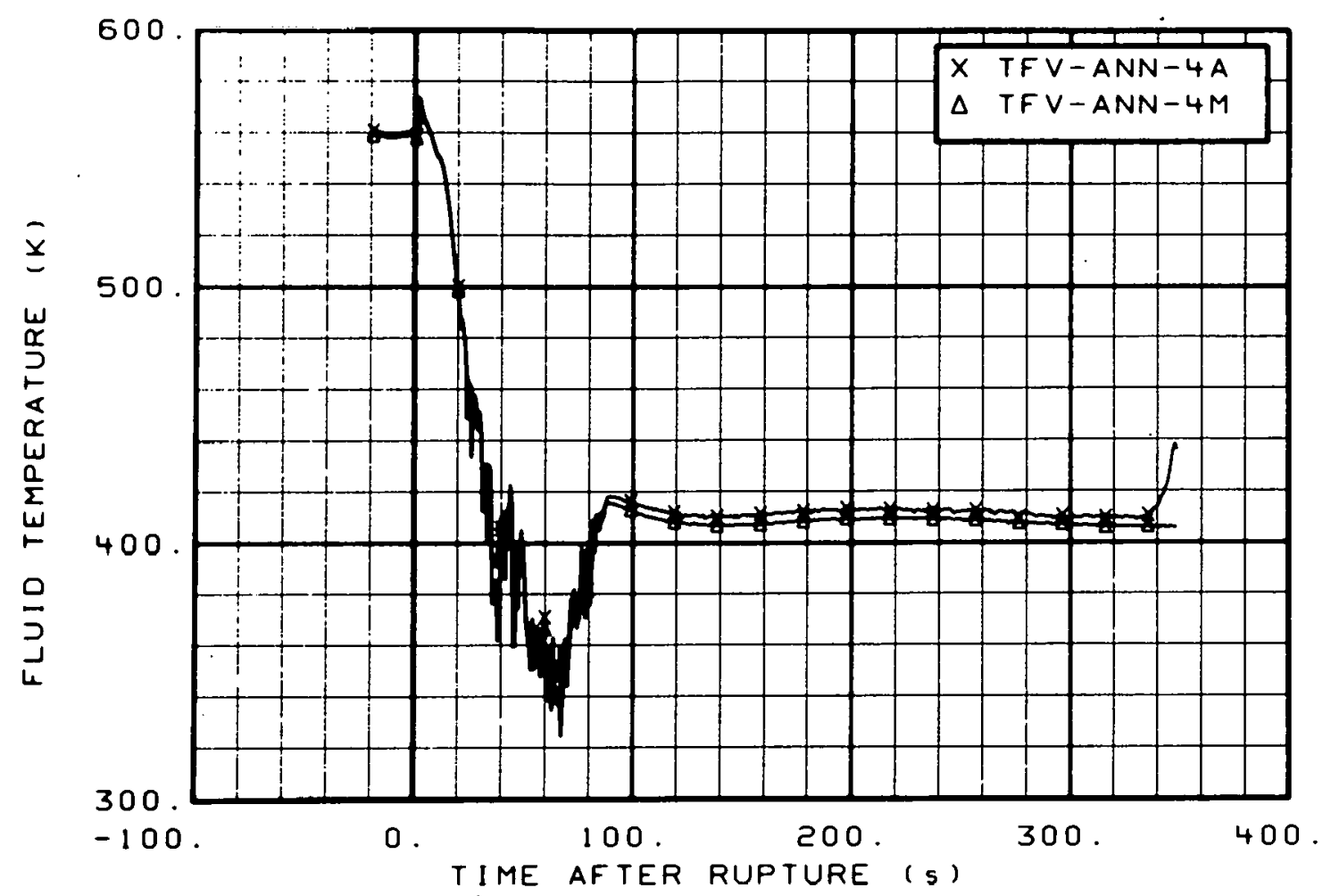

Fig. 23 Fluid temperature in inlet annulus (TFV-ANN-4A and TFV-ANN-4M), from -20 to $350 \mathrm{~s}$.

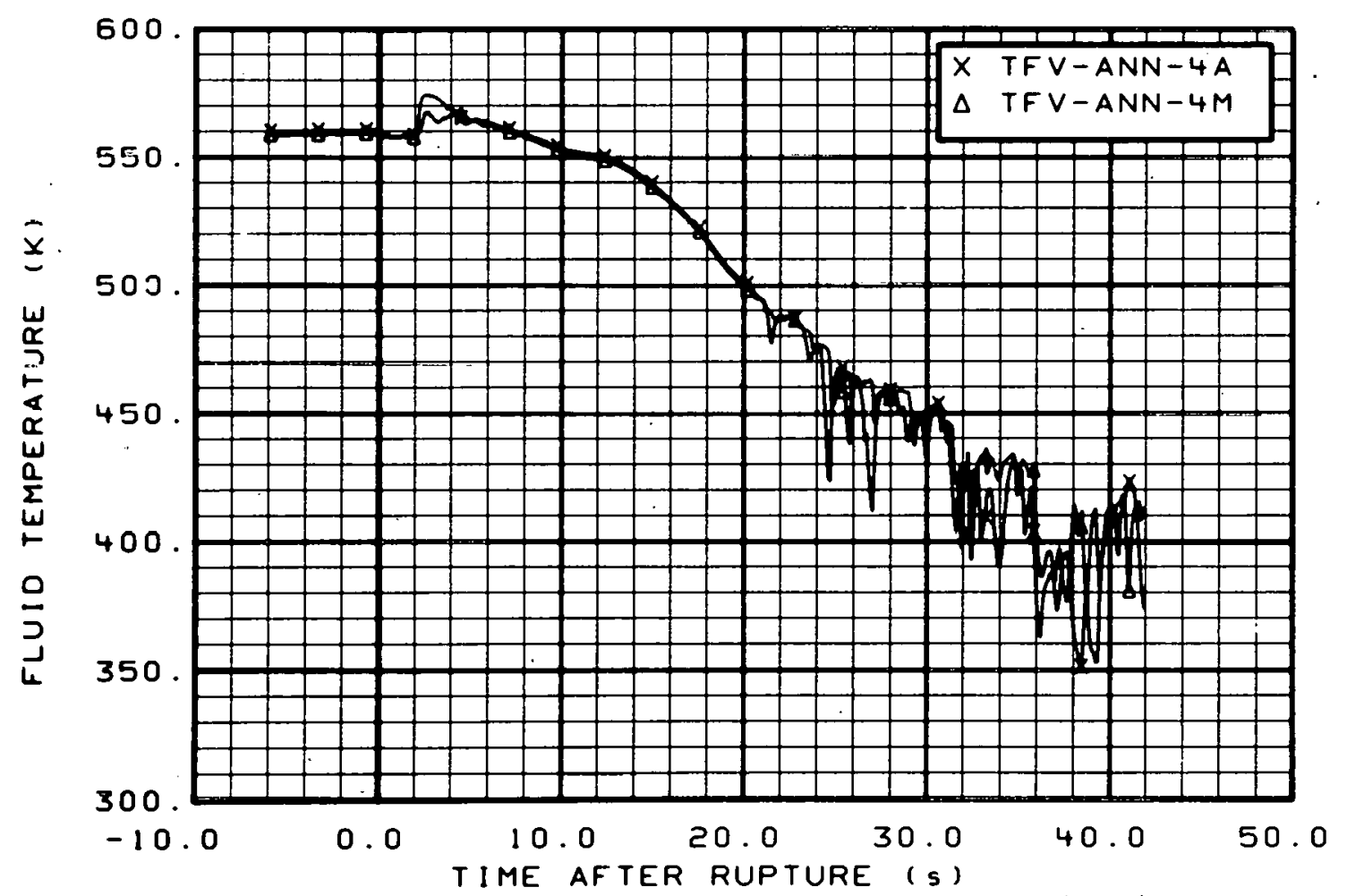

Fig. 24 Fluid temperature in inlet annulus (TFV-ANN-4A and TFV-ANN-4M), from -6 to $42 \mathrm{~s}$. 


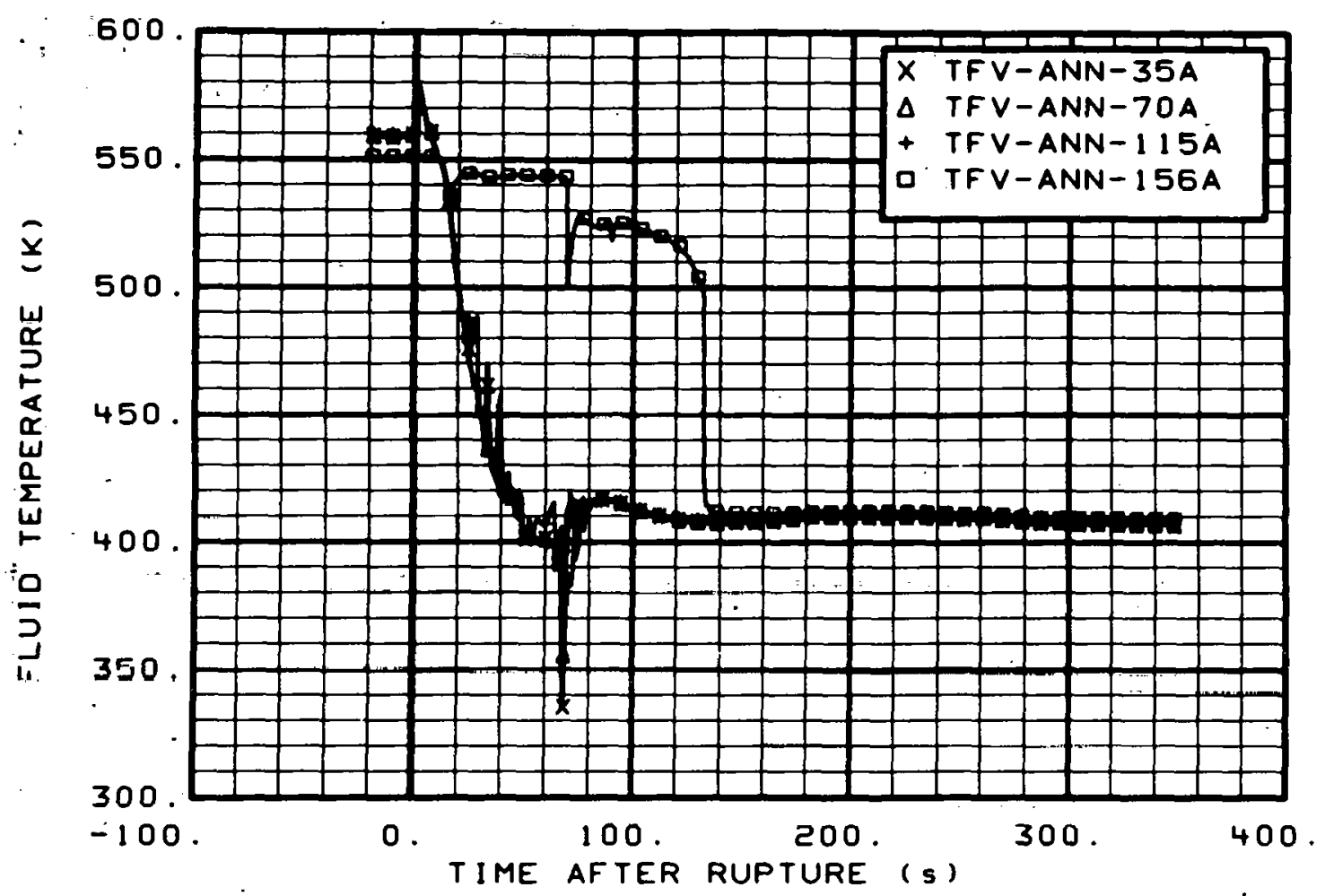

Fig. 25 Fluid temperature in downcomer annulus (TFV-ANN-35A, TFV-ANN-70A, TFV-ANN-115A, and TFV-ANN-156A), from -20 to $350 \mathrm{~s}$.

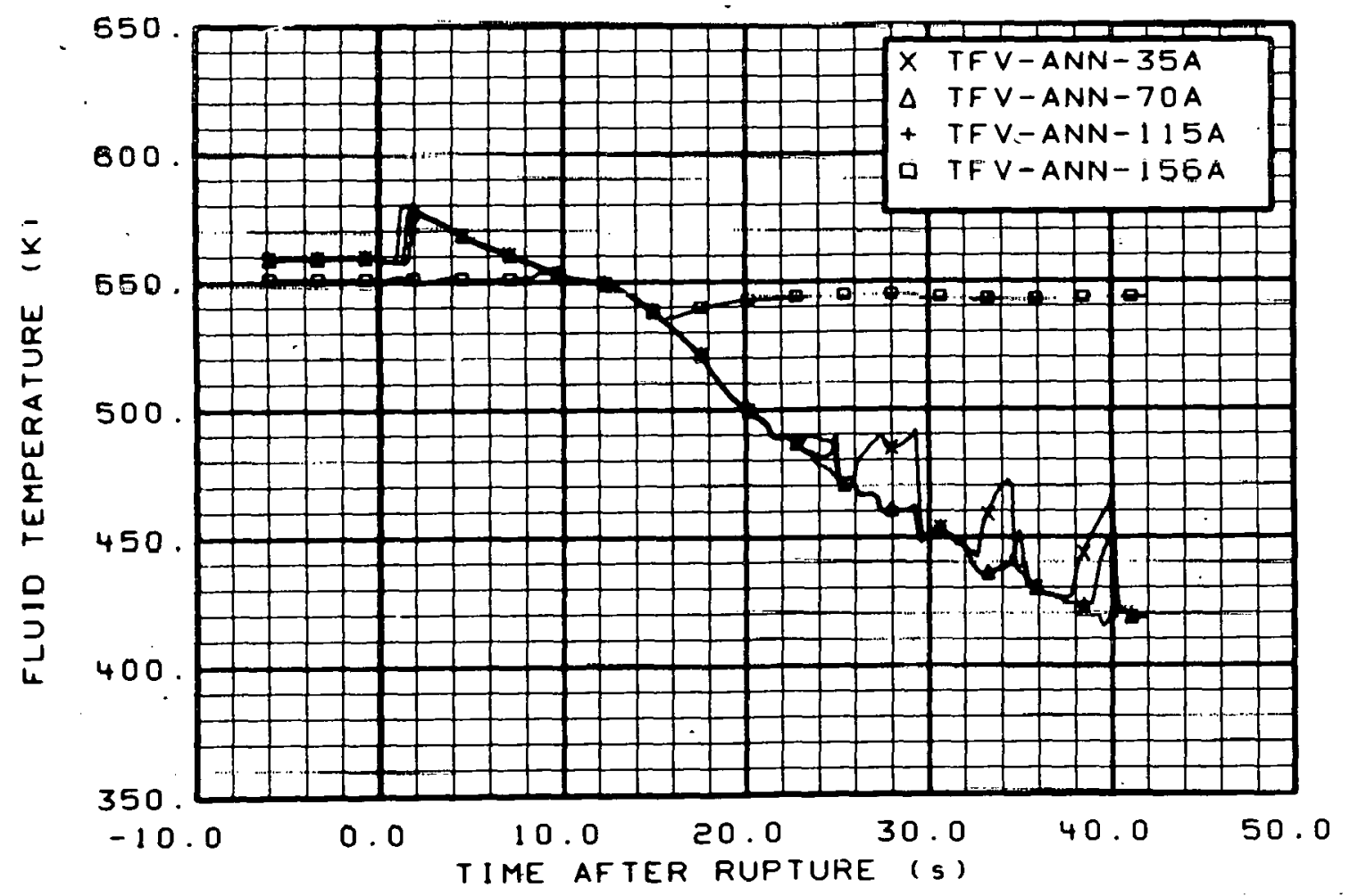

Fig. 26 Fluid temperature in downcomer annulus (TFV-ANN-35A, TFV-ANN-70A, TFV-ANN-115A, and TFV-ANN-156A), from -6 to $42 \mathrm{~s}$. 


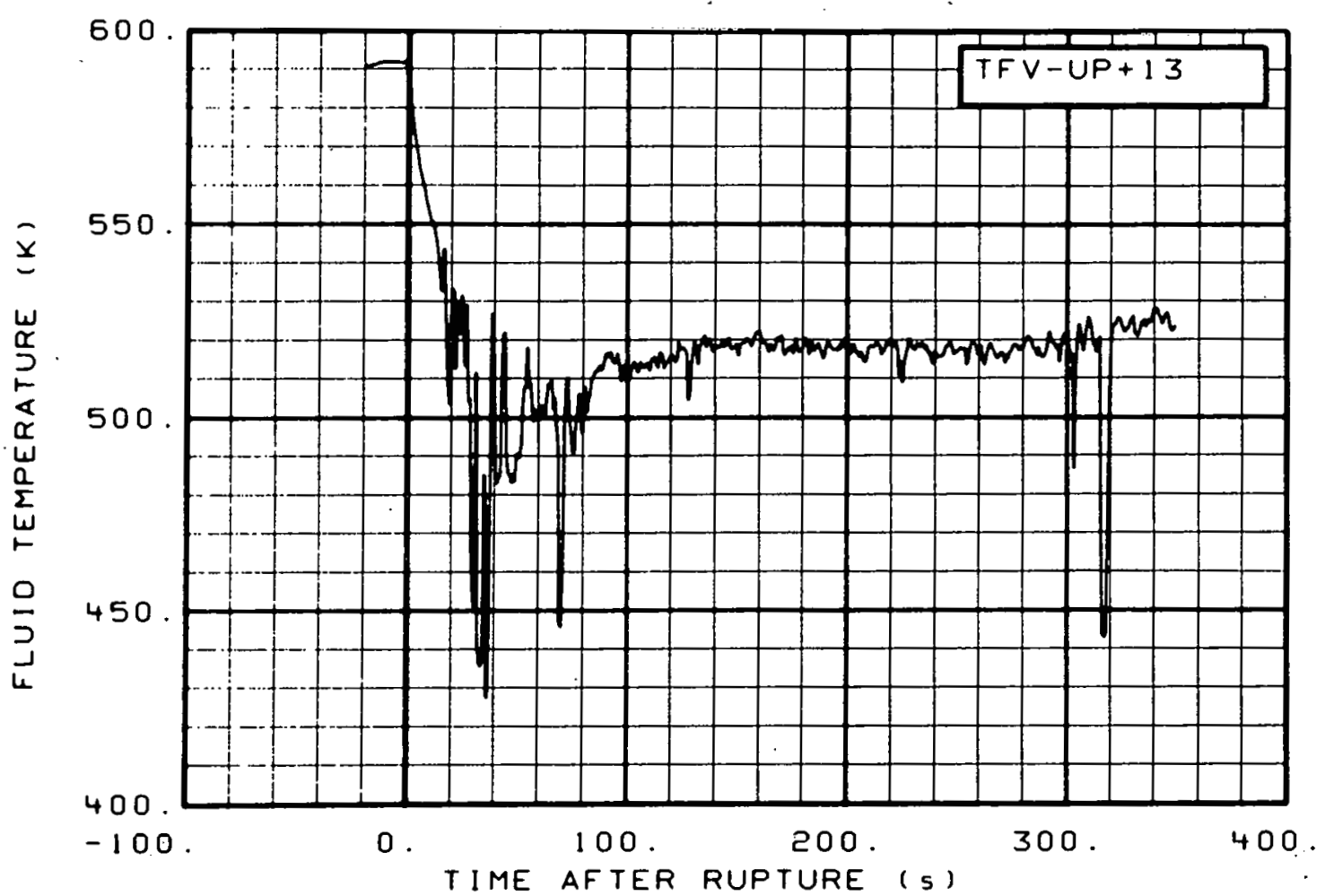

Fig. 27 Fluid temperature in upper plenum (TFV-UP+13), from -20 to $350 \mathrm{~s}$.

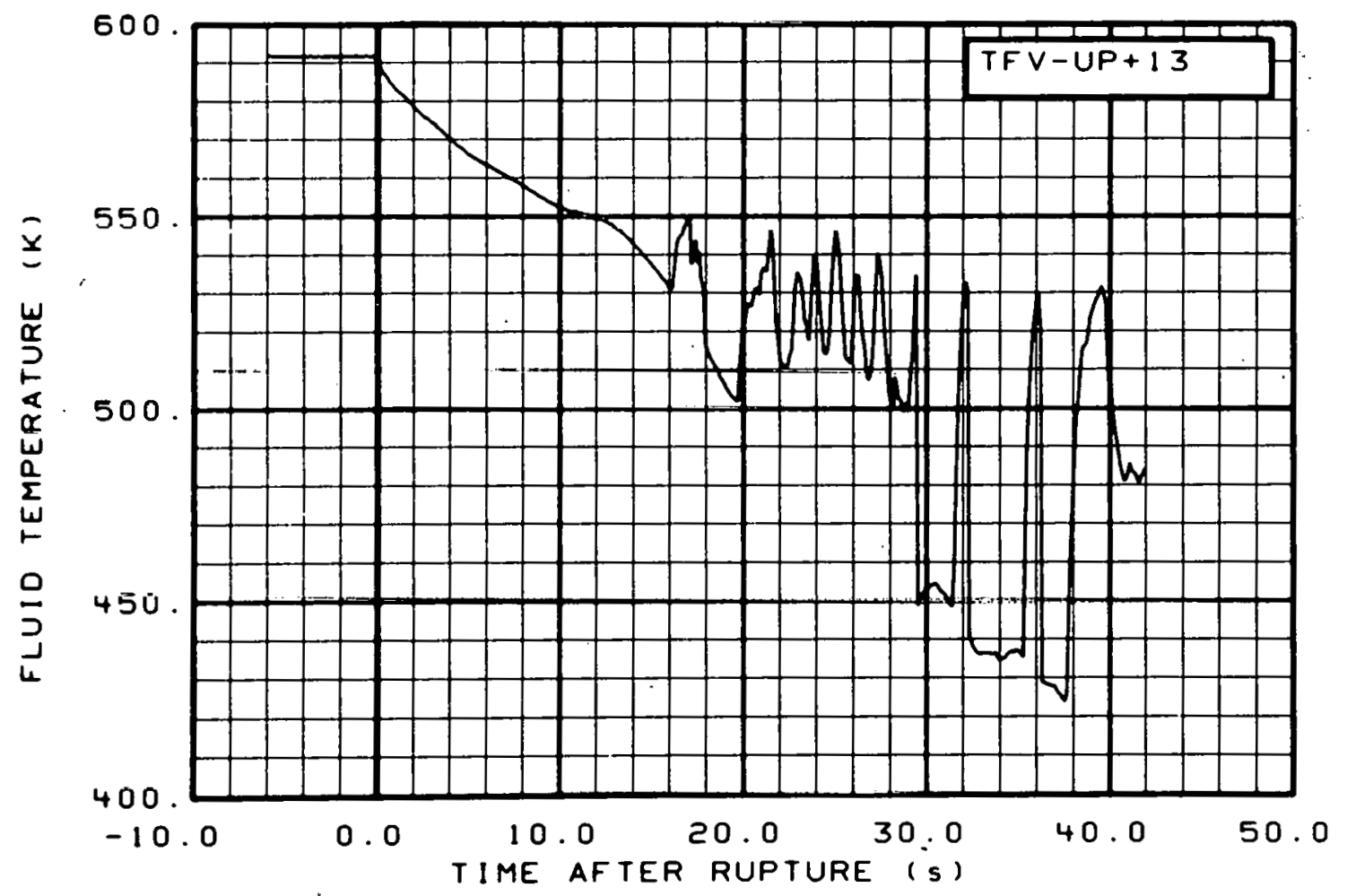

Fig. 28 Fluid temperature in upper plenum (TFV-UP+13), from -6 to $42 \mathrm{~s}$. 


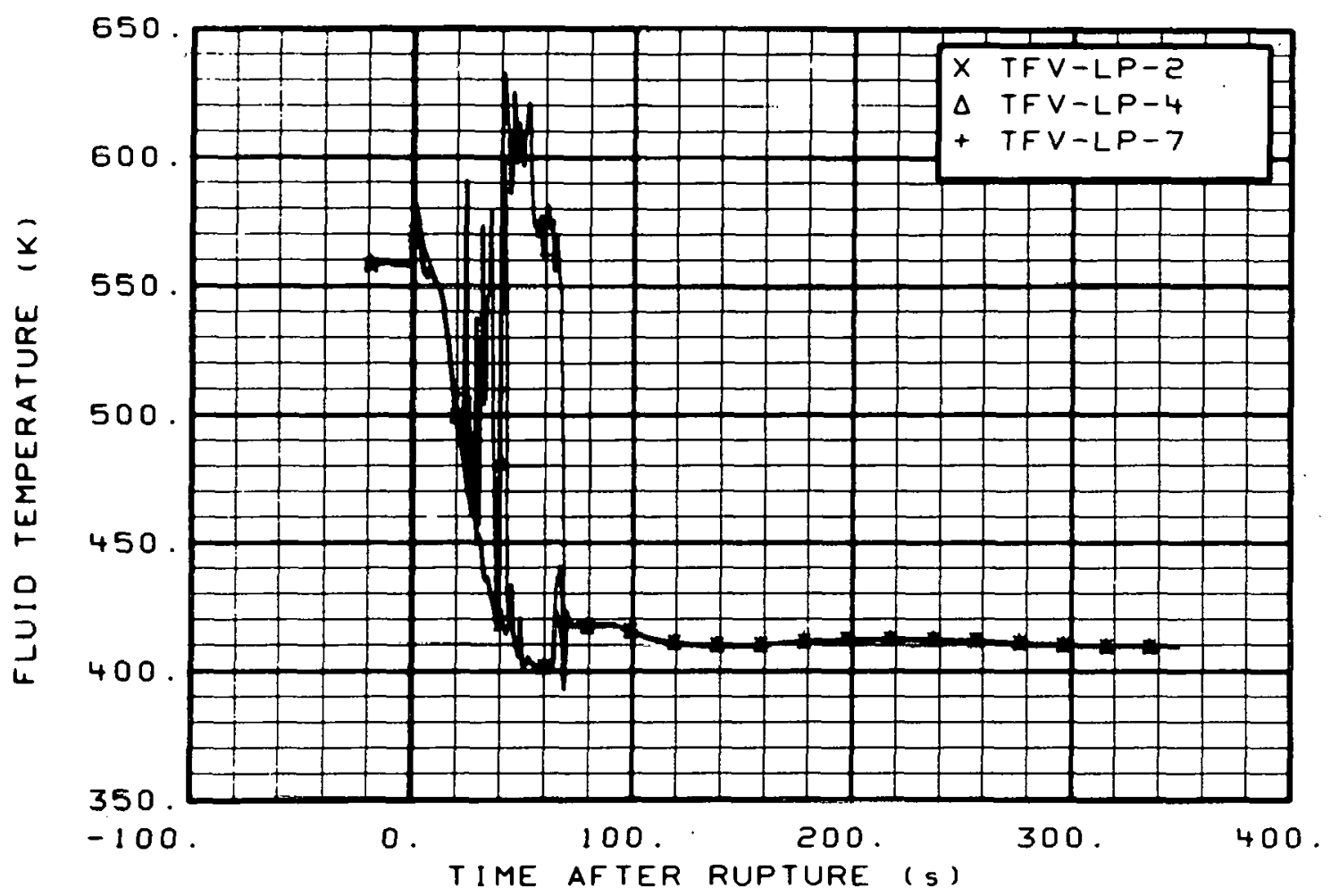

Fig. 29 Fluid temperature in lower plenum (TFV-LP-2, TFV-LP-4, and TFV-LP-7), from -20 to $350 \mathrm{~s}$.

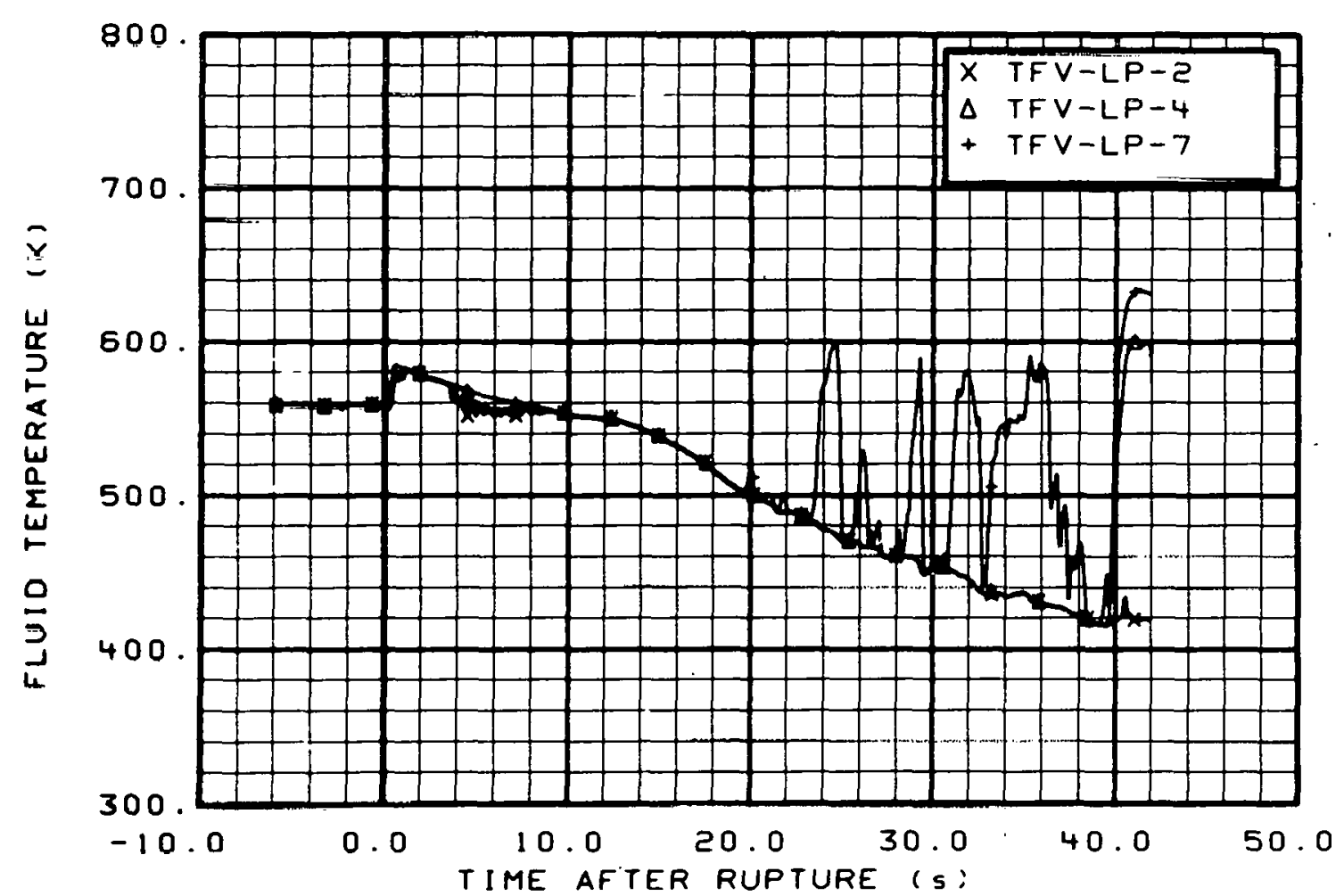

Fig. 30 Fluid temperature in lower plenum (TFV-LP-2, TFV-LP-4, and TFV-LP-7), from. -6 to $42 \mathrm{~s}$. 


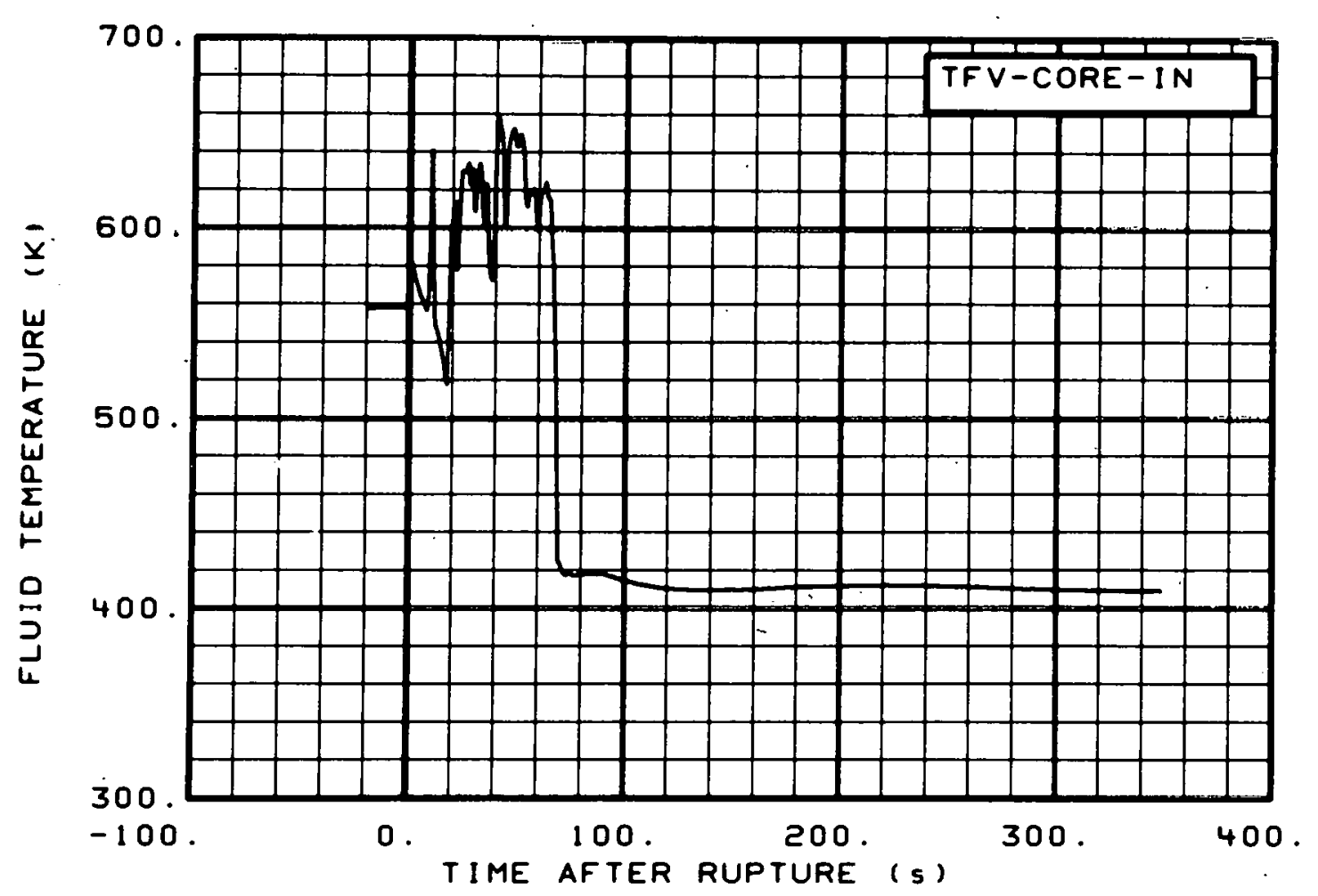

Fig. 31 Fluid temperature in core inlet (TFV-CORE-IN), from -20 to $350 \mathrm{~s}$.

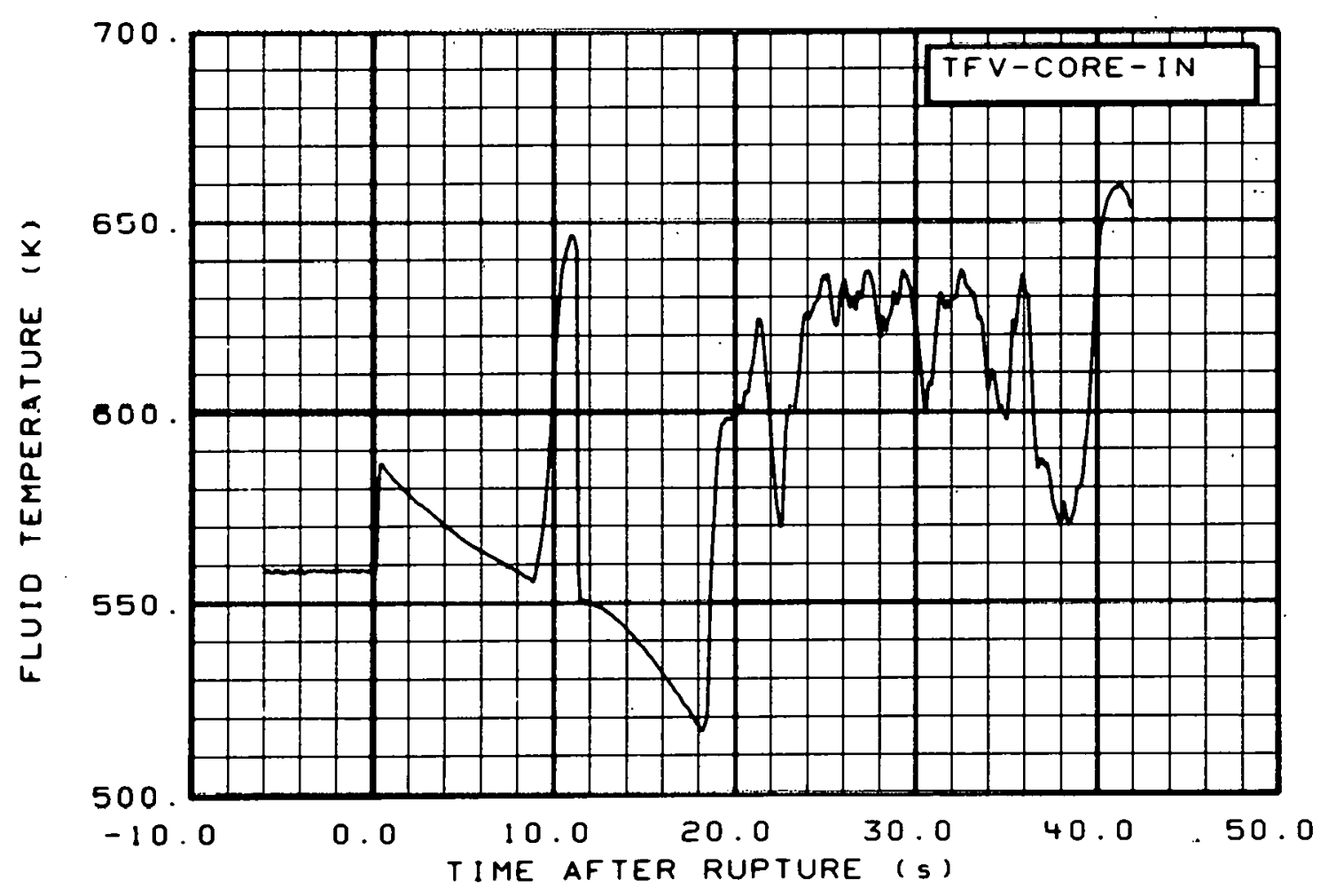

Fig. 32 Fluid temperature in core inlet (TFV-CORE-IN), from -6 to $42 \mathrm{~s}$. 


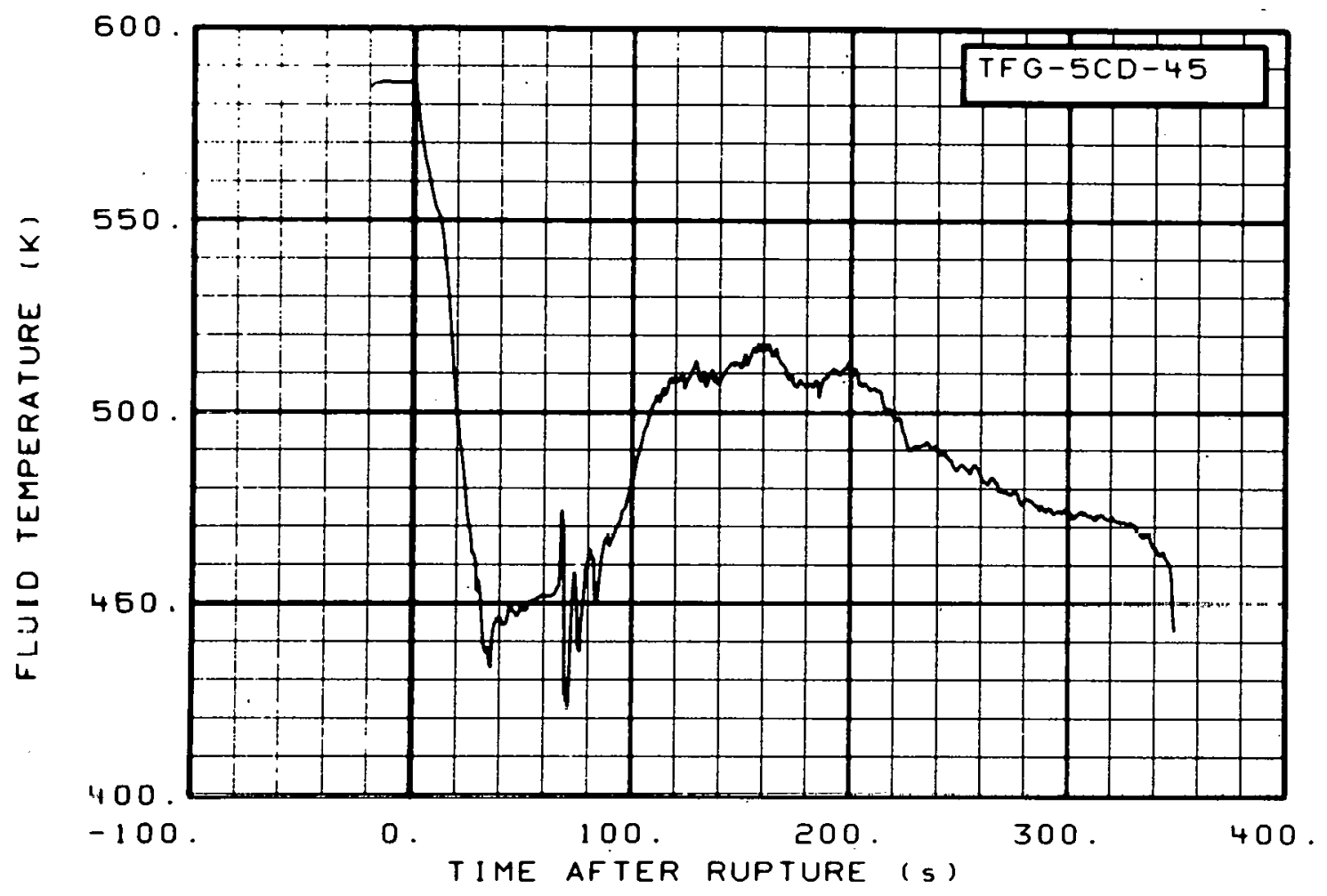

Fig. 33 Fluid temperature in core, Grid Spacer 5 (TFG-5CD-45), from -20 to $350 \mathrm{~s}$.

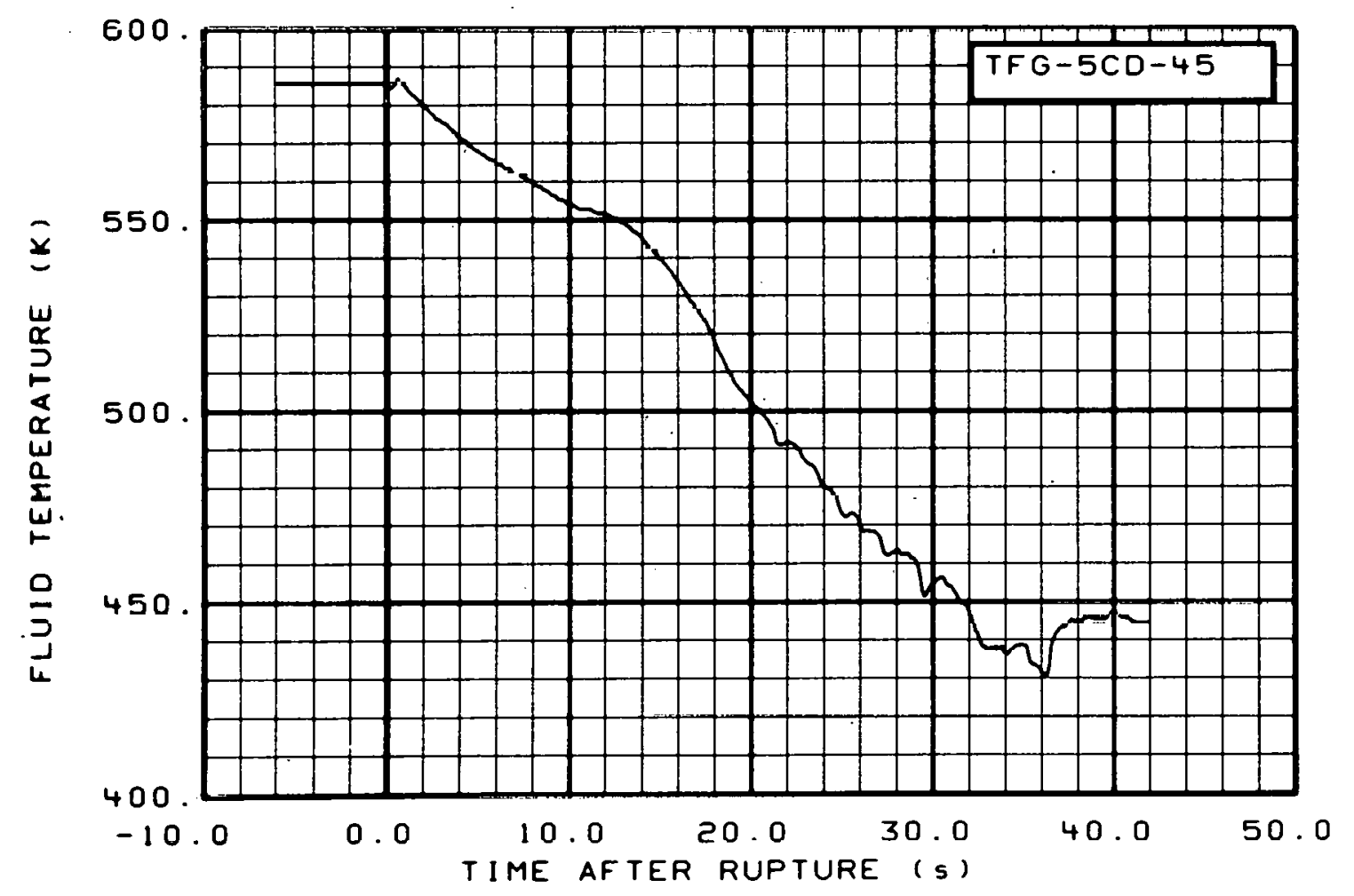

Fig. 34 Fluid temperature in core, Grid Spacer 5 (TFG-5CD-45), from -6 to $42 \mathrm{~s}$. 


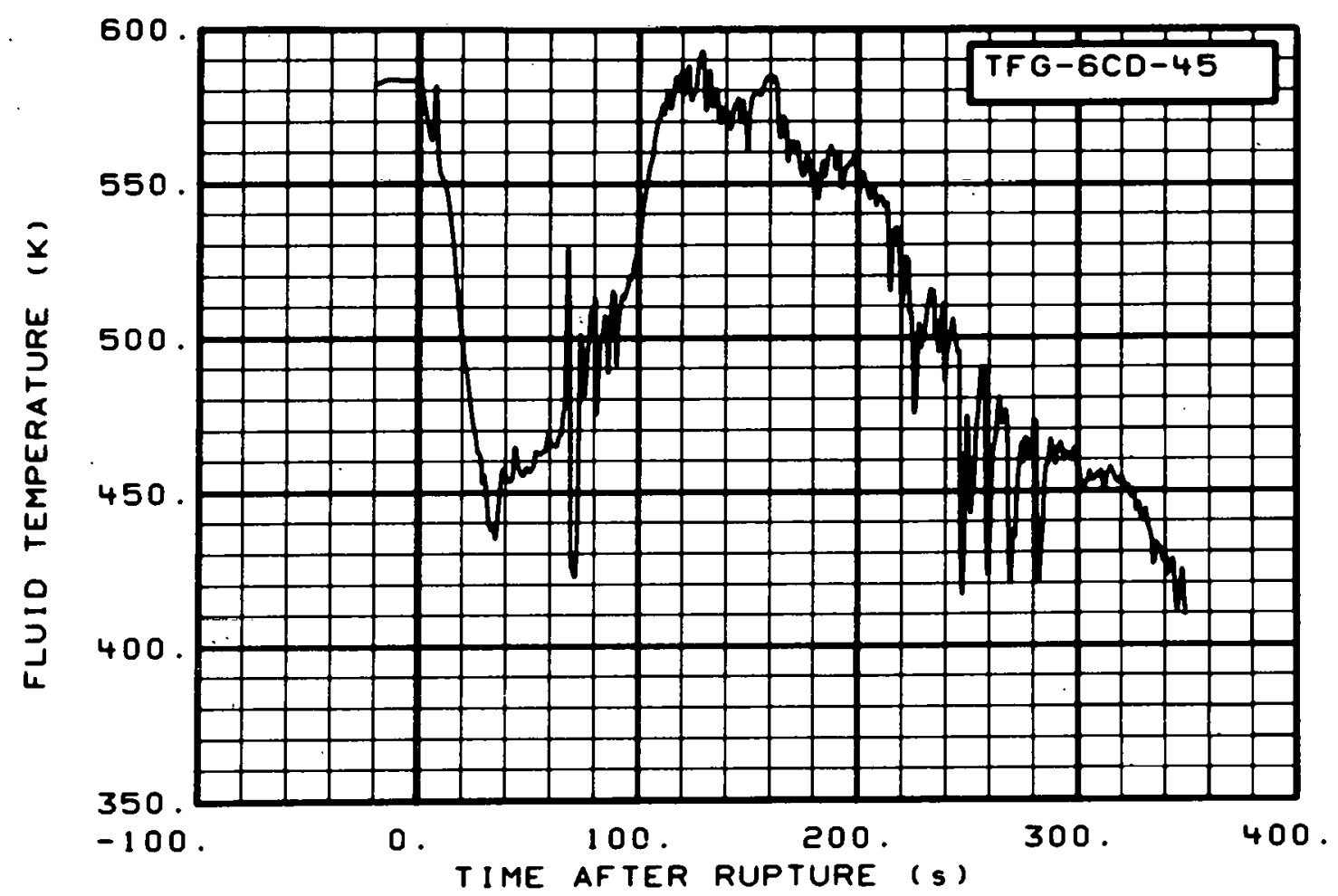

Fig. 35 Fluid temperature in core, Grid Spacer 6 (TFG-6CD-45), from -20 to $350 \mathrm{~s}$.

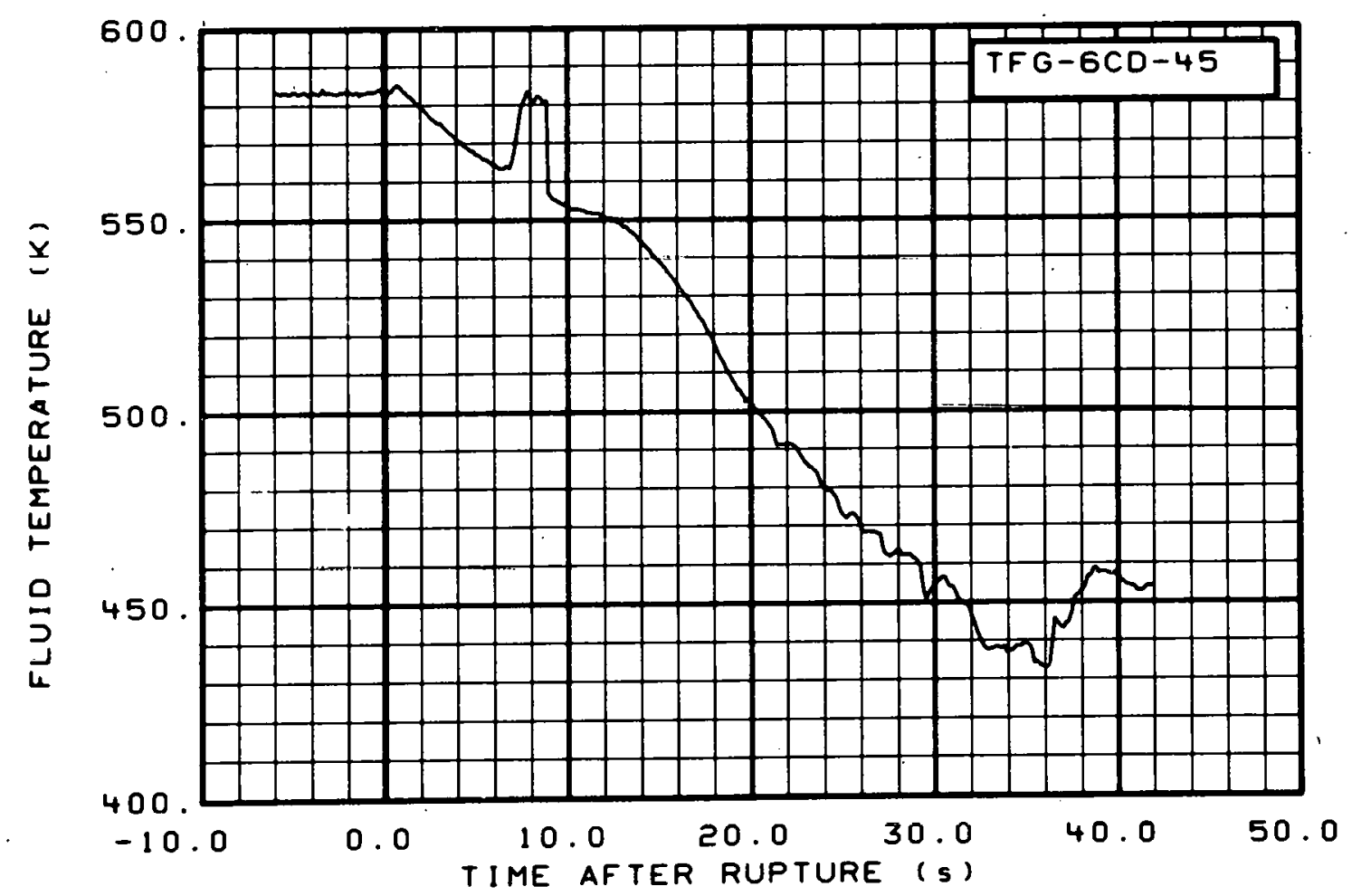

Fig. 36 Fluid temperature in core, Grid Spacer 6 (TFG-6CD-45), from -6 to $42 \mathrm{~s}$. 


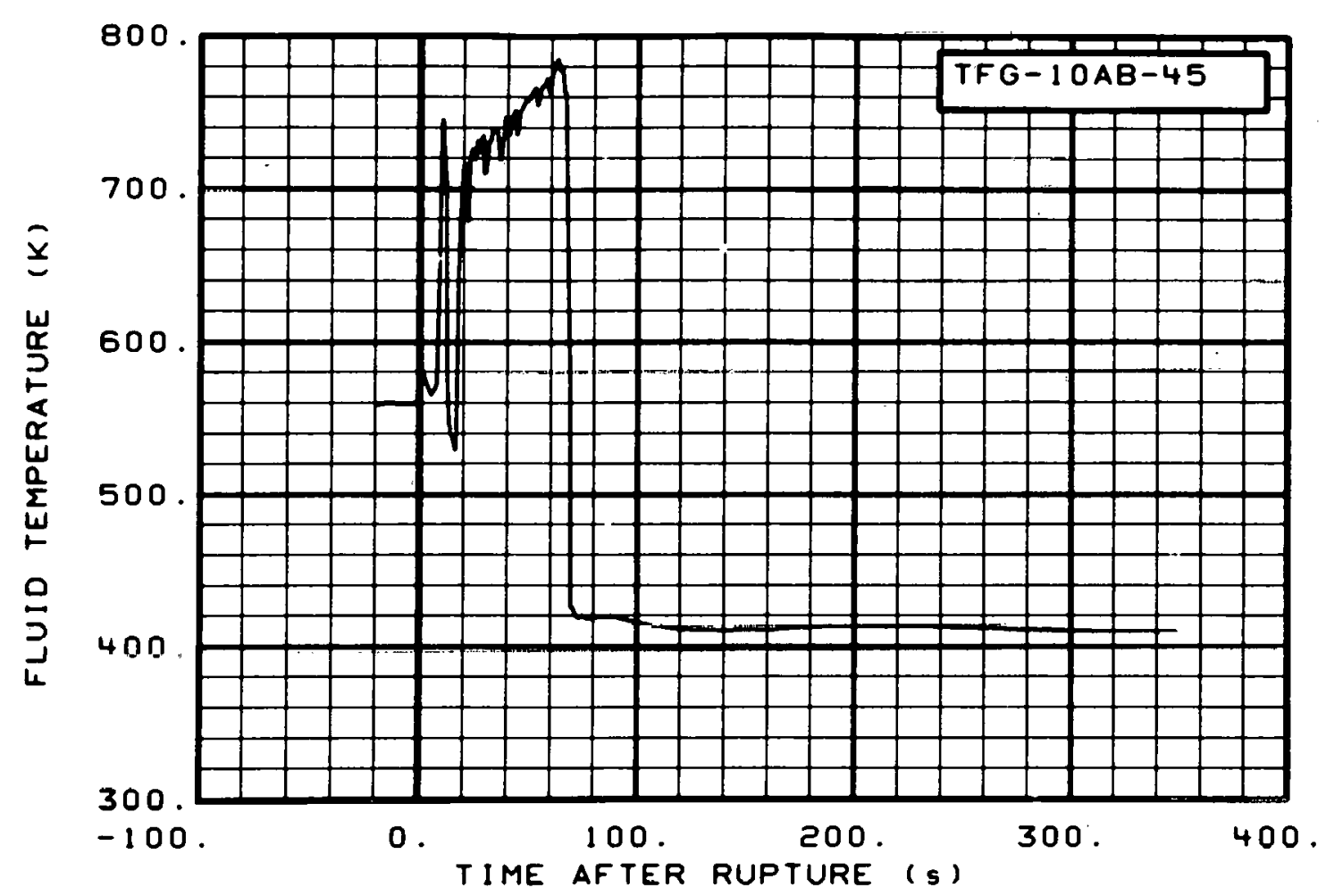

Fig. 37 Fluid temperature in core, Grid Spacer 10 (TFG-10AB-45), from -20 to $350 \mathrm{~s}$.

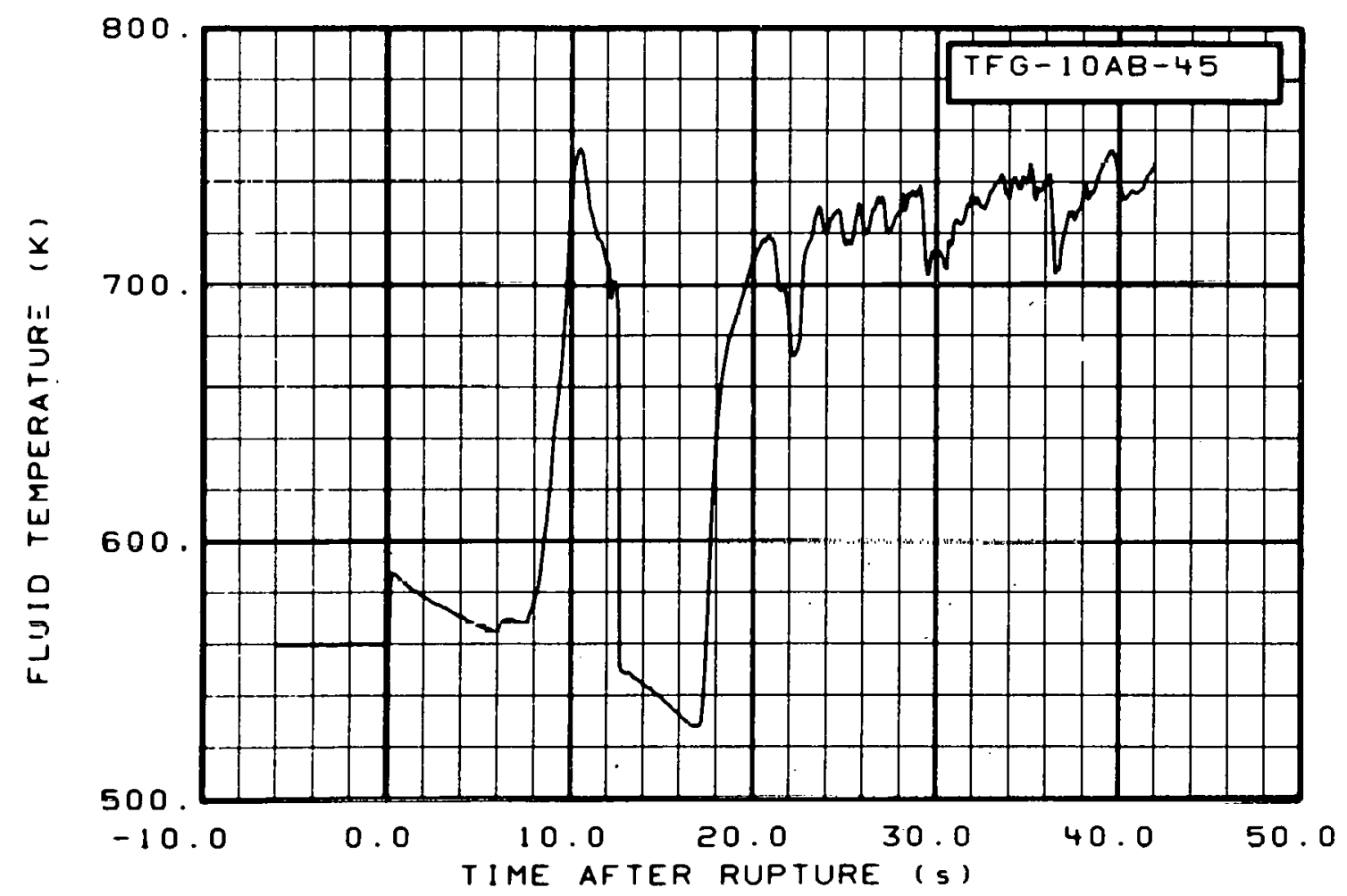

Fig. 38 Fluid temperature in core, Grid Spacer 10 (TFG-10AB-45), from -6 to $42 \mathrm{~s}$. 


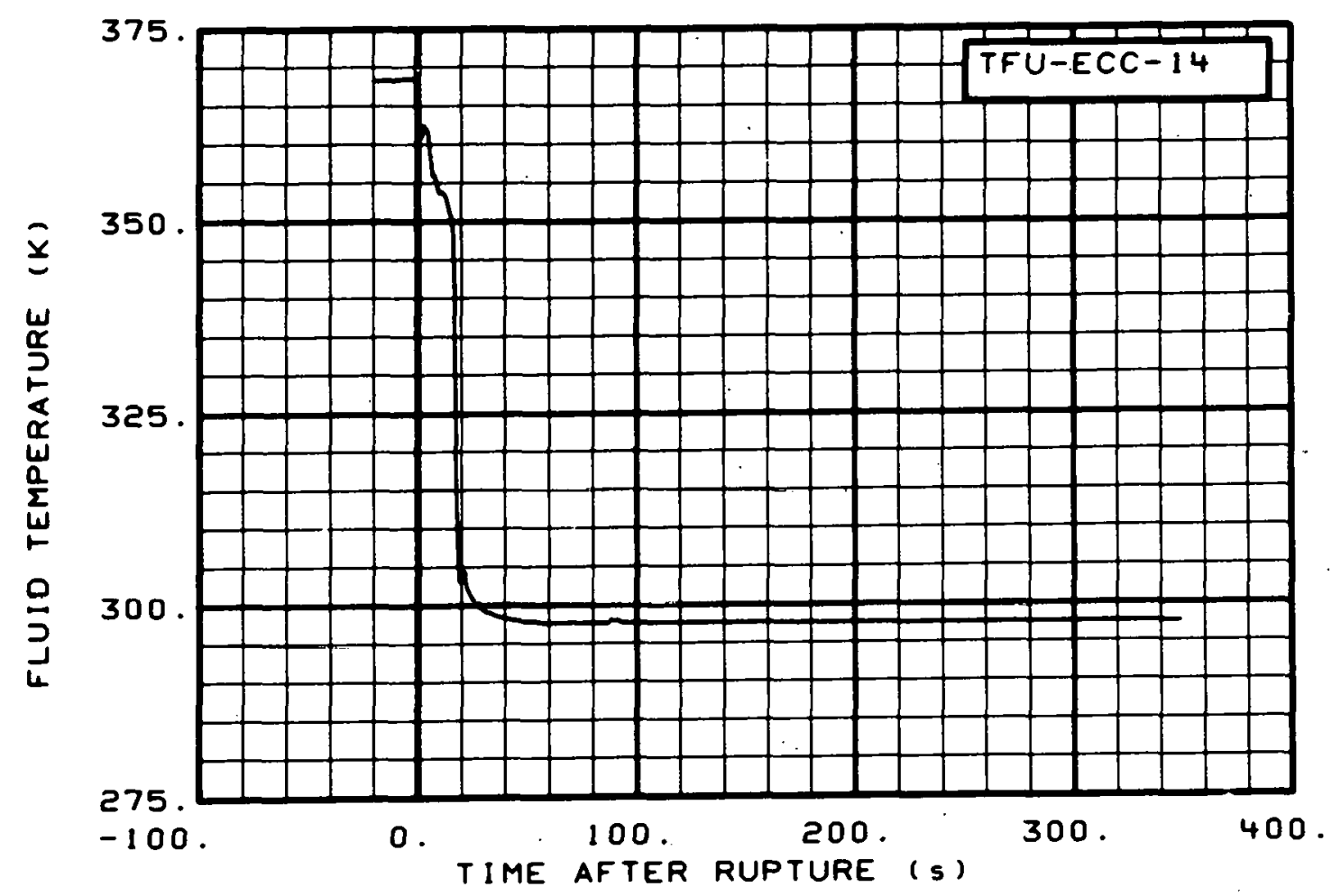

Fig. 39 Fluid temperature in intact loop coolant injection line (TFU-ECC14), from -20 to $350 \mathrm{~s}$.

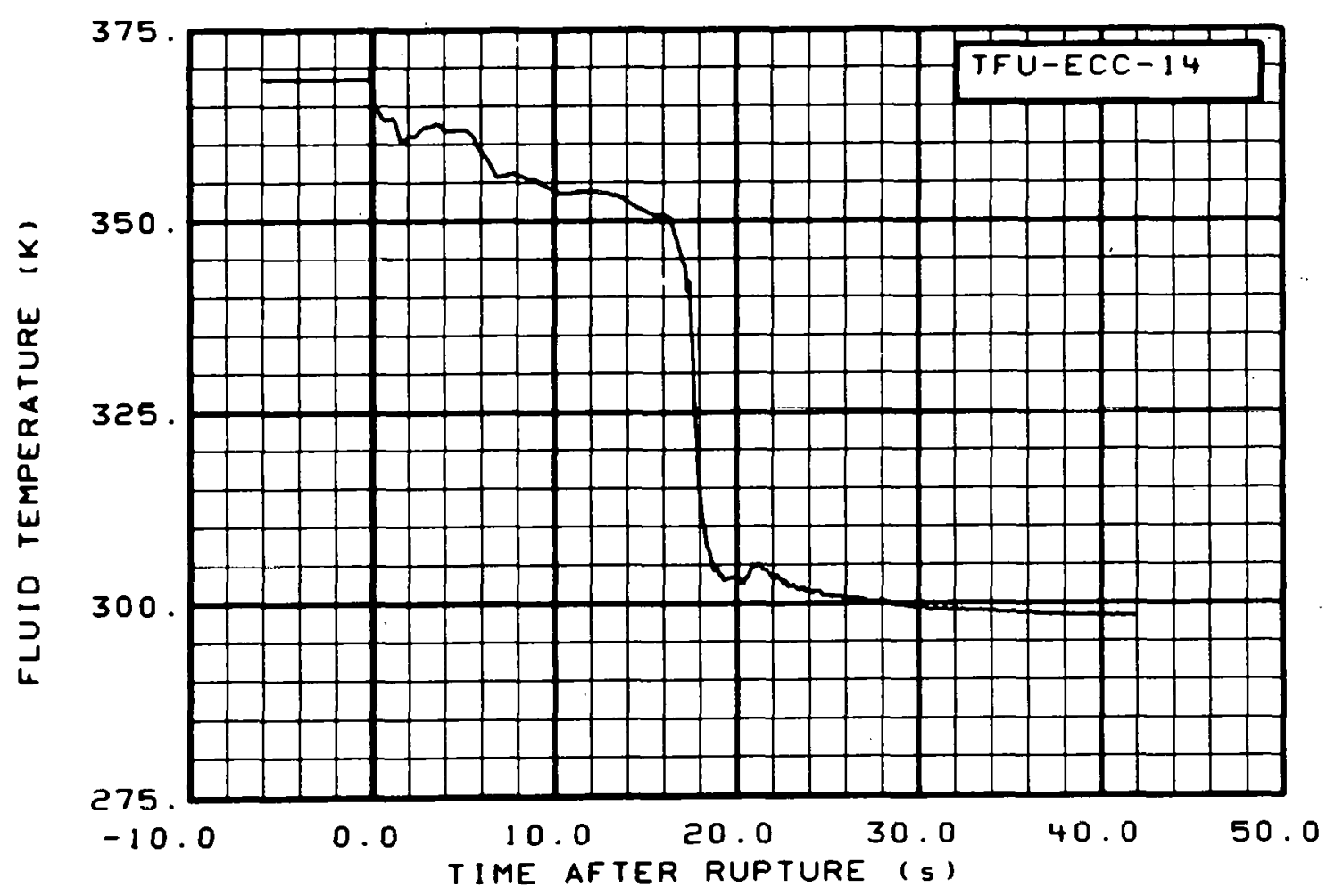

Fig. 40 Fluid temperature in intact loop coolant injection line (TFU-ECC14), from -6 to $42 \mathrm{s.}$ 


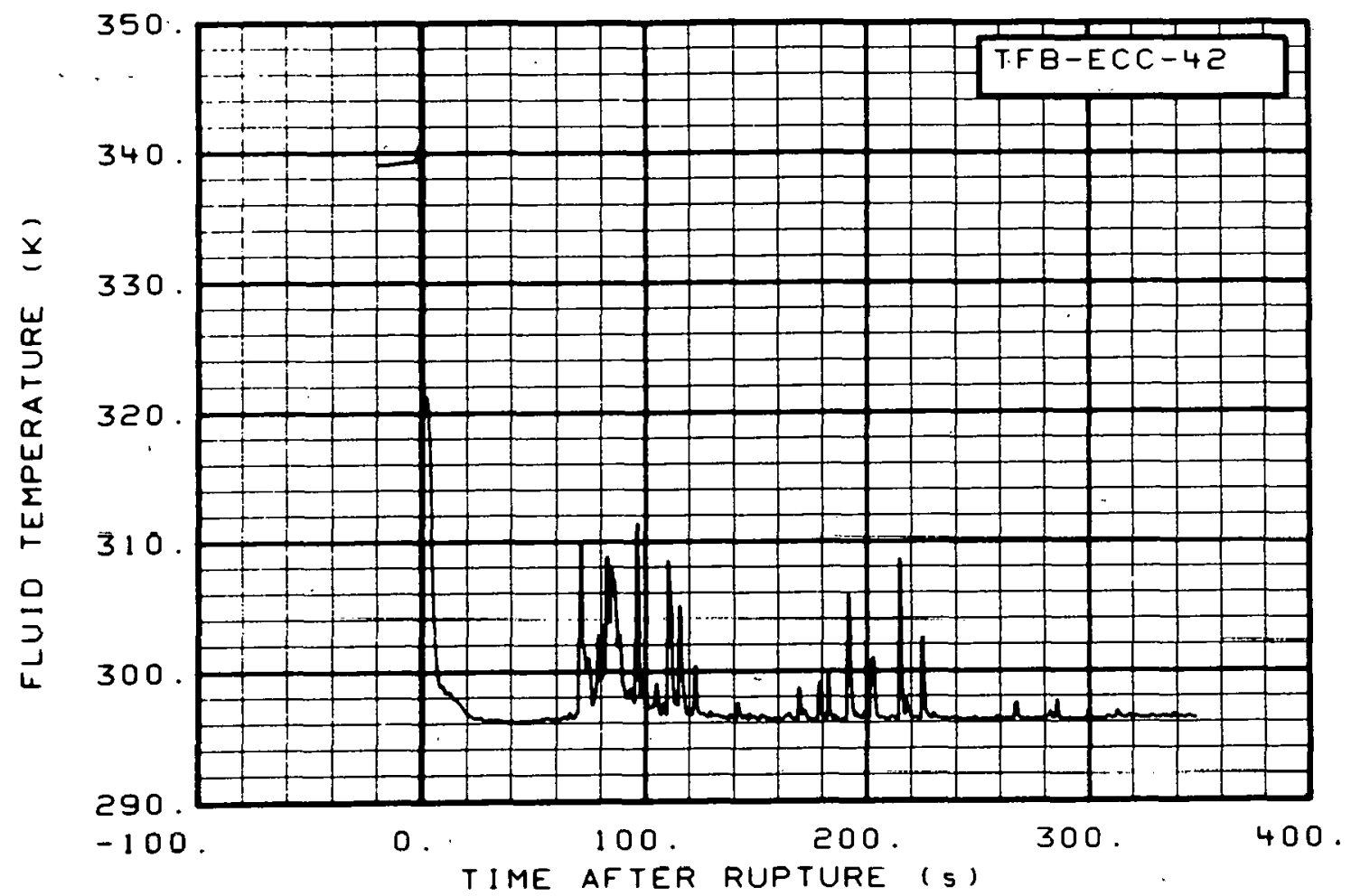

Fig. 41 Fluid temperature in broken loop coolant injection line (TFB-ECC42), from -20 to $350 \mathrm{~s}$.

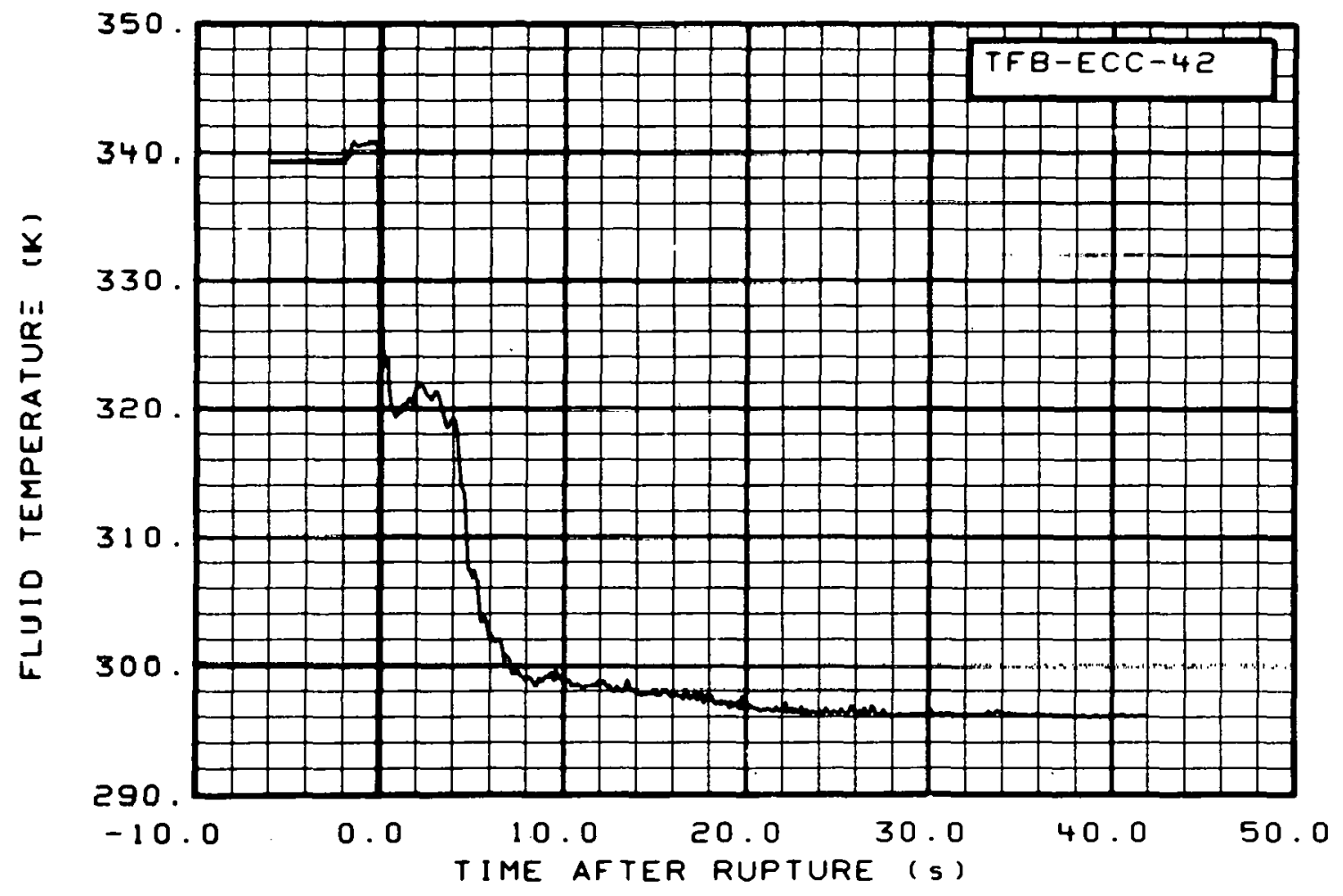

Fig. 42 Fluid temperature in broken loop coolant injection line (TFB-ECC42), from -6 to $42 \mathrm{~s}$. 


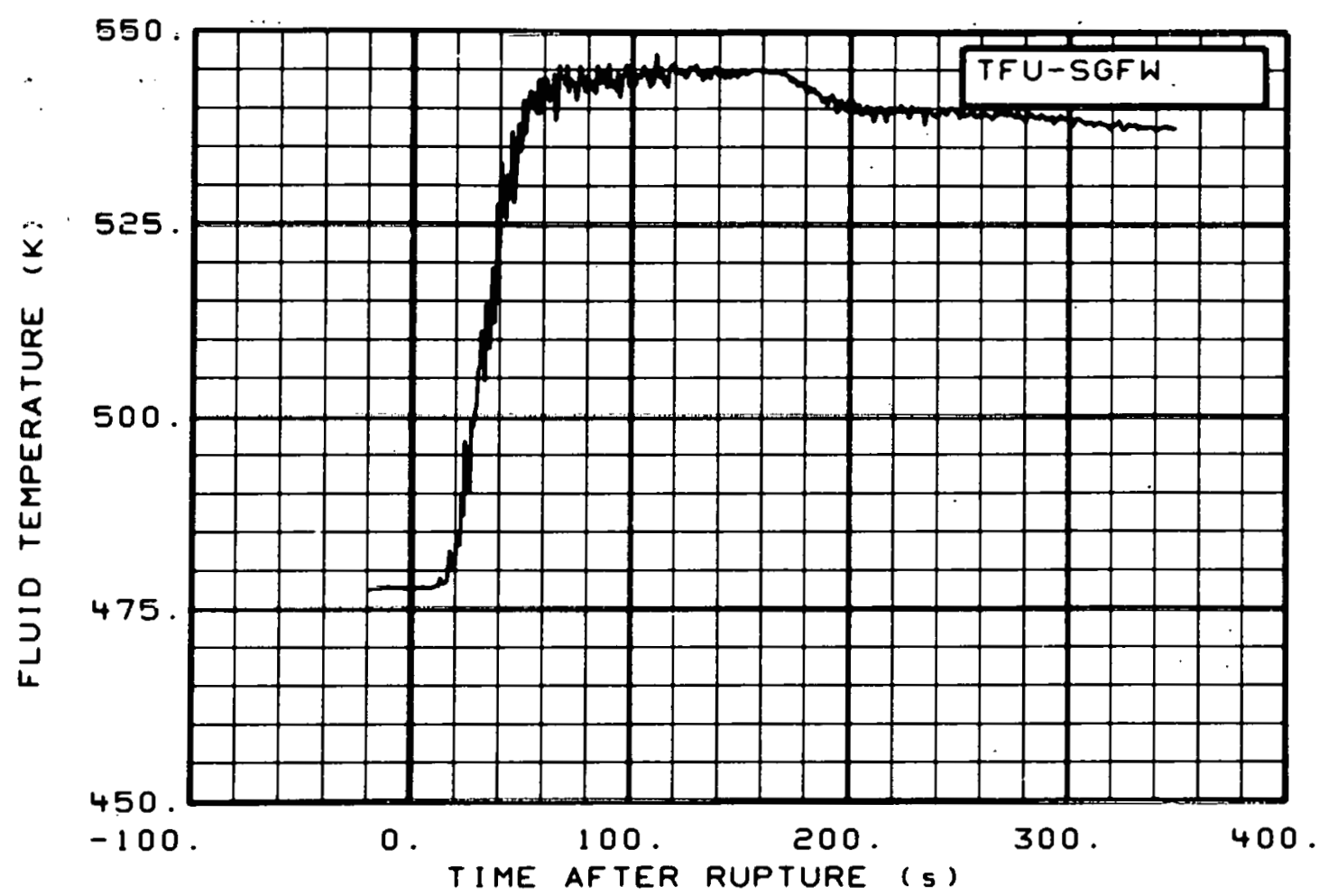

Fig. 43 Fluid temperature in steam generator, feedwater line (TFU-SGFW), from -20 to $350 \mathrm{~s}$.

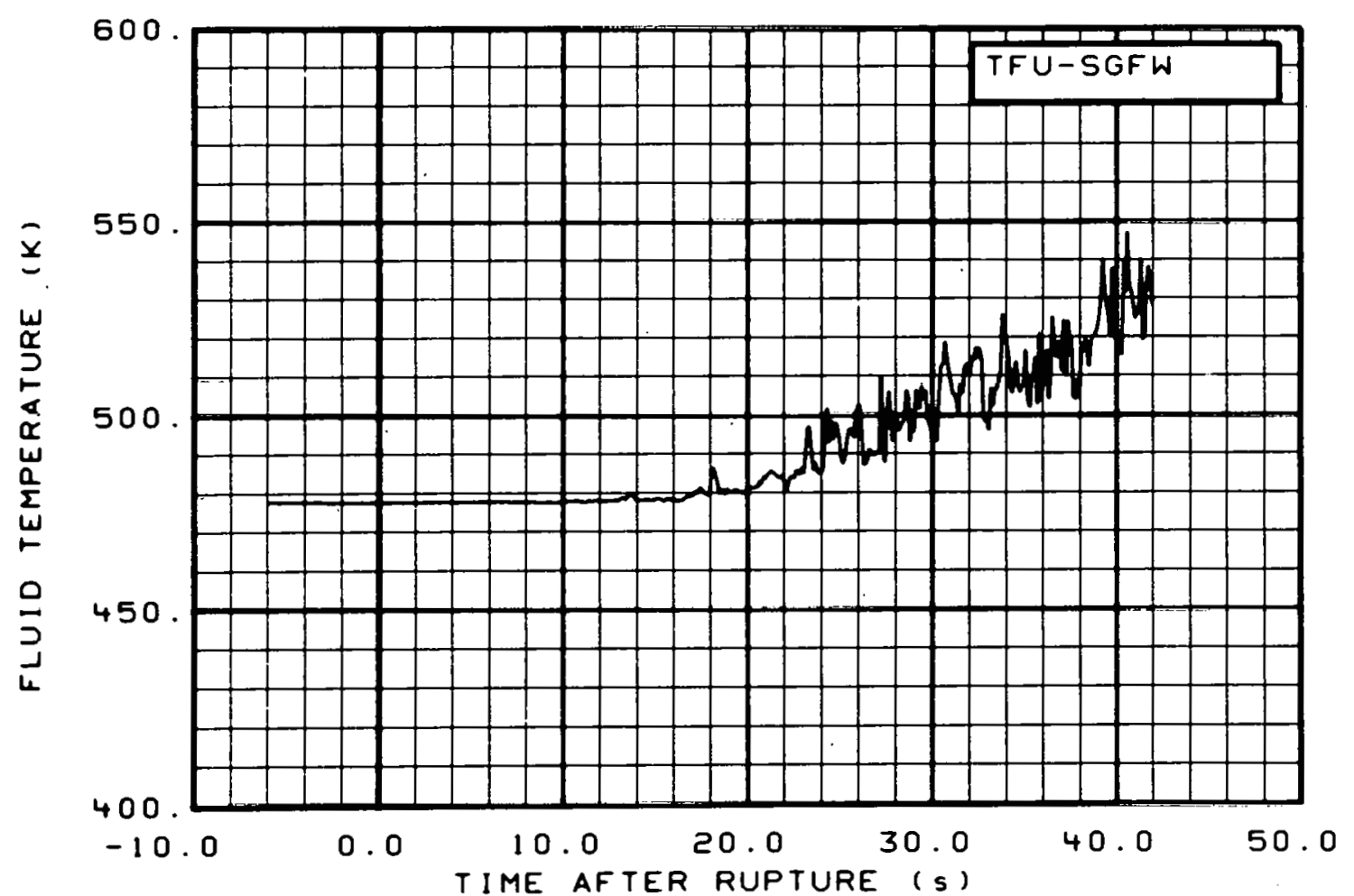

Fig. 44 Fluid temperature in steam generator, feedwater line (TFU-SGFW), from -6 to $42 \mathrm{~s}$. 


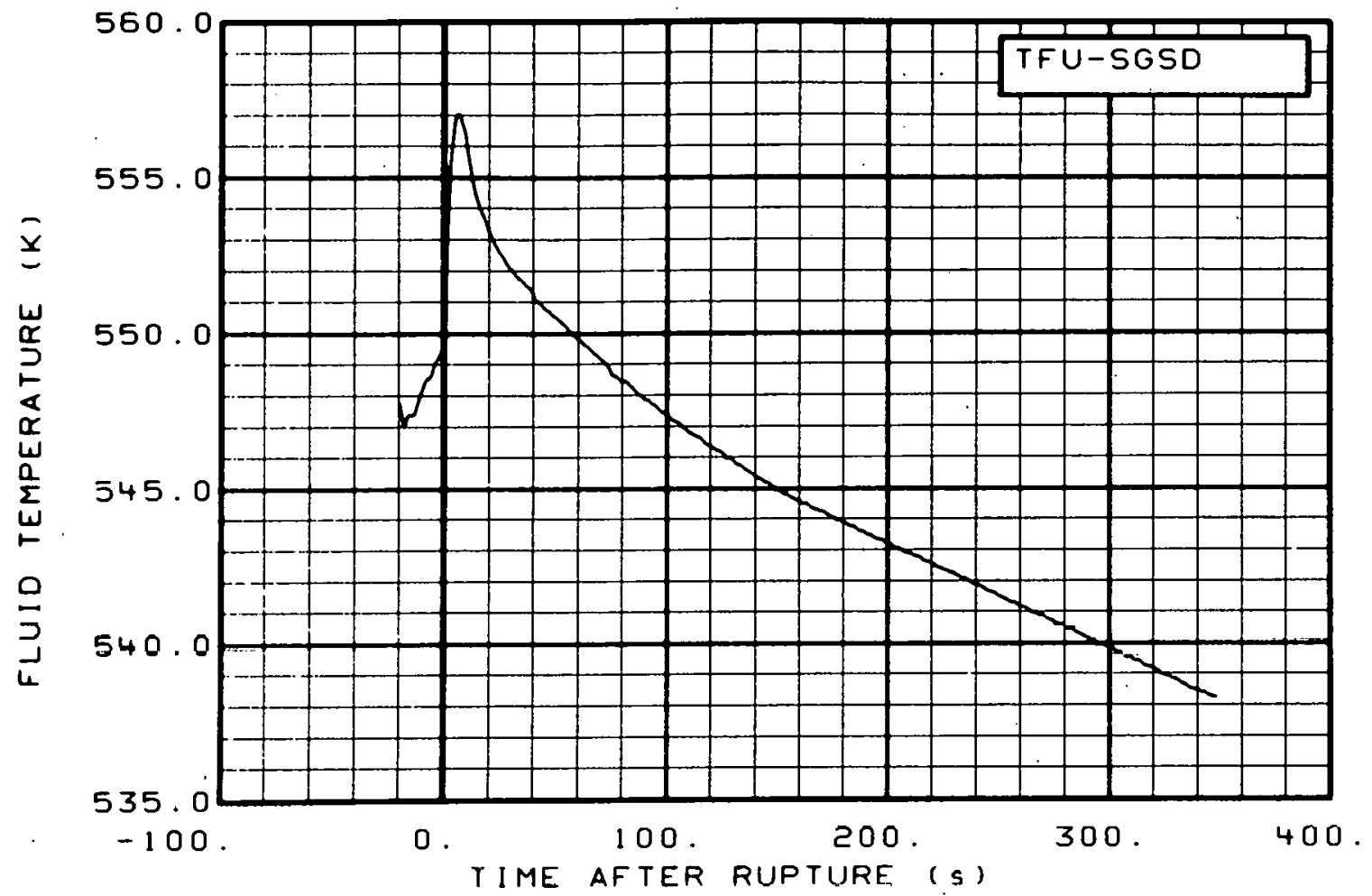

Fig. 45 Fluid temperature in steam generator, steam dome (TFU-SGSD), from -20 to $350 \mathrm{~s}$.

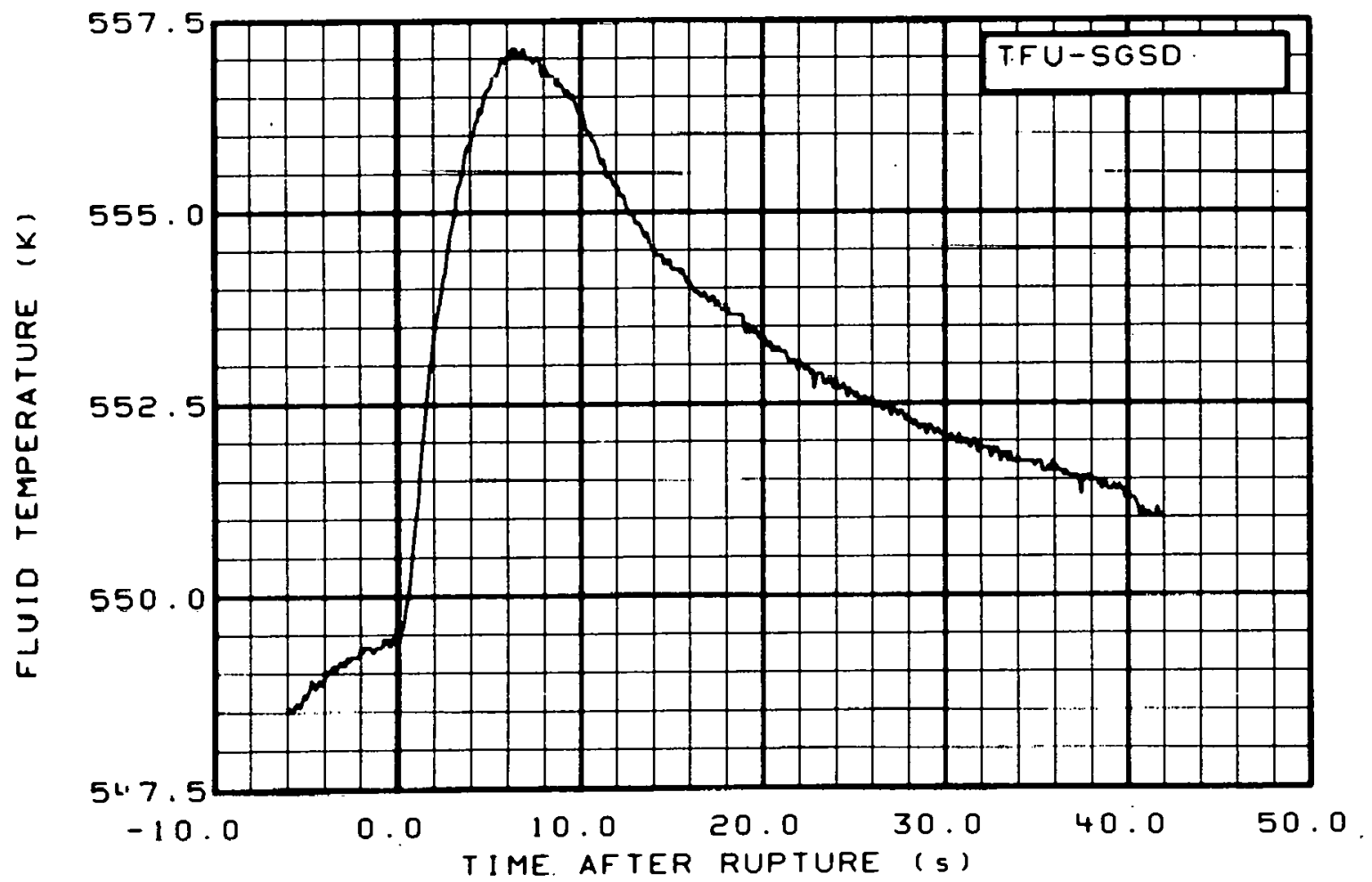

Fig. 46 Fluid temperature in steam generator, steam dome (TFU-SGSD), fromil -6 to $42 \mathrm{~s}$. 


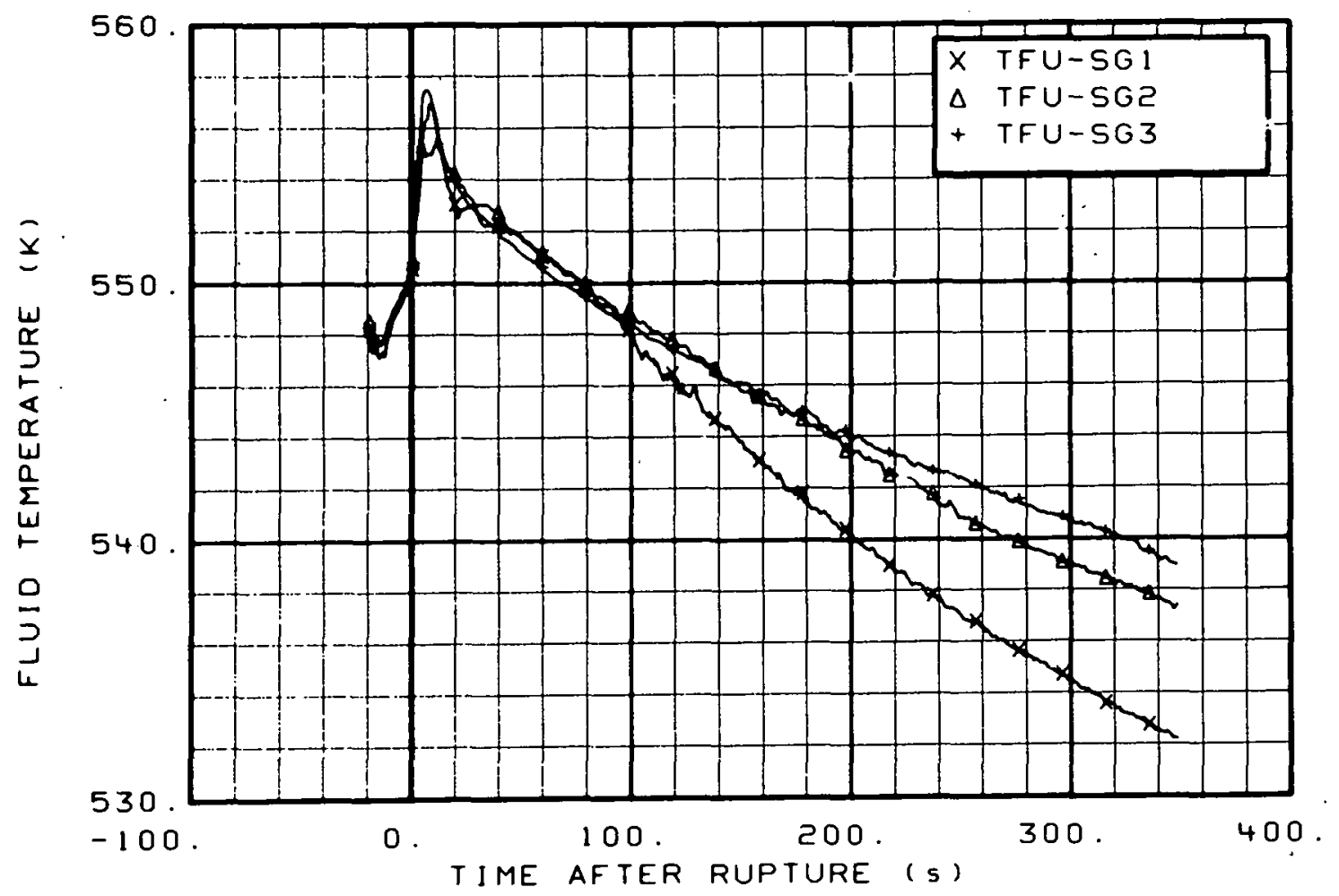

Fig. 47 Fluid temperature in steam generator, secondary side (TFU-SGi), TFU-SG2, and TFU-SG3), from -20 to $350 \mathrm{~s}$.

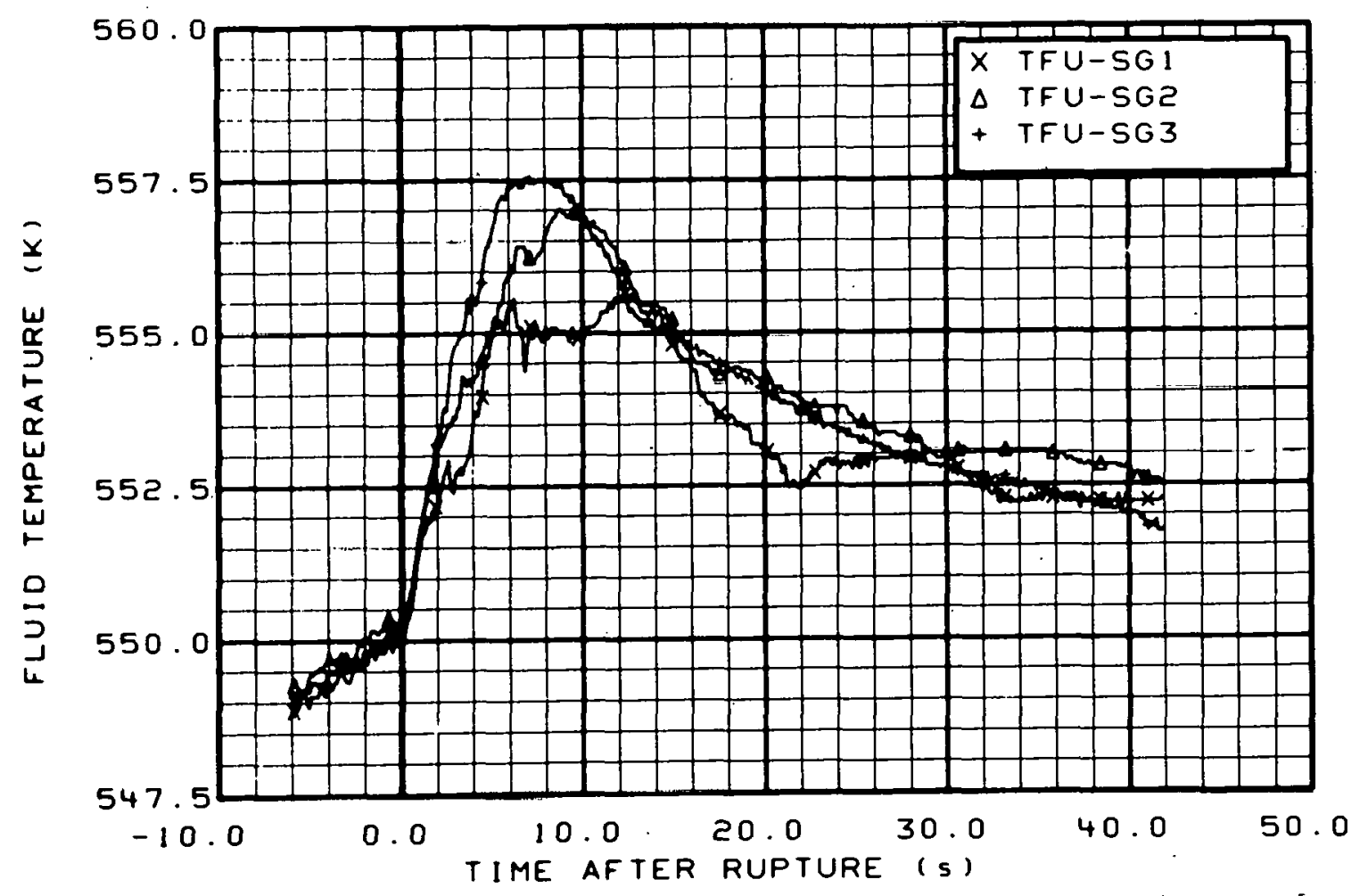

Fig. 48 Fluid temperature in steam generator, secondary side (TFU-SGI, TFU-SG2, and TFU-SG3), from. -6 to $42 \mathrm{~s}$. 


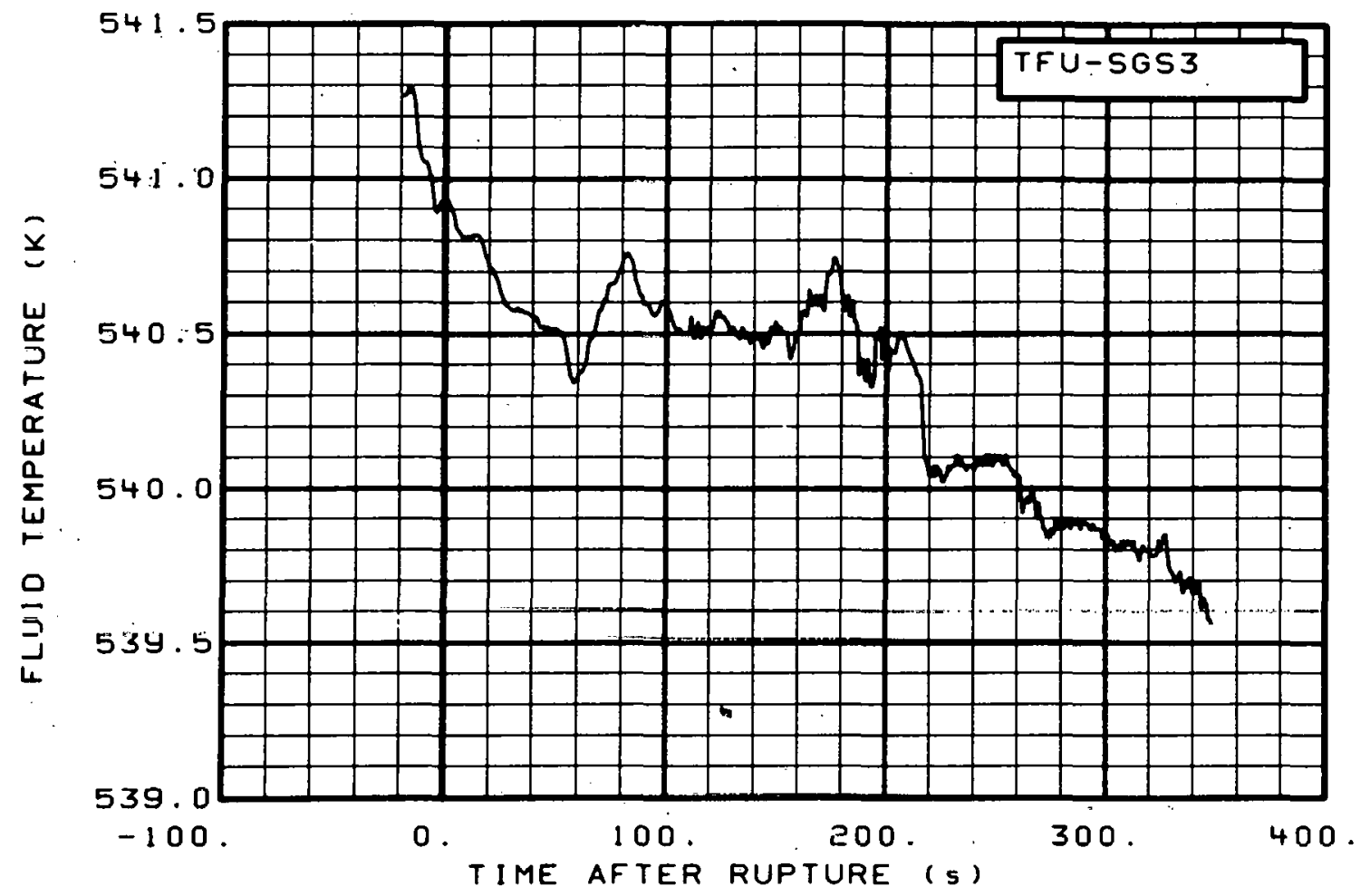

Fig. 49. Fluid temperature in steam generator rupture system accumulator (TFU-SGS3), from -20 to $350 \mathrm{~s}$.

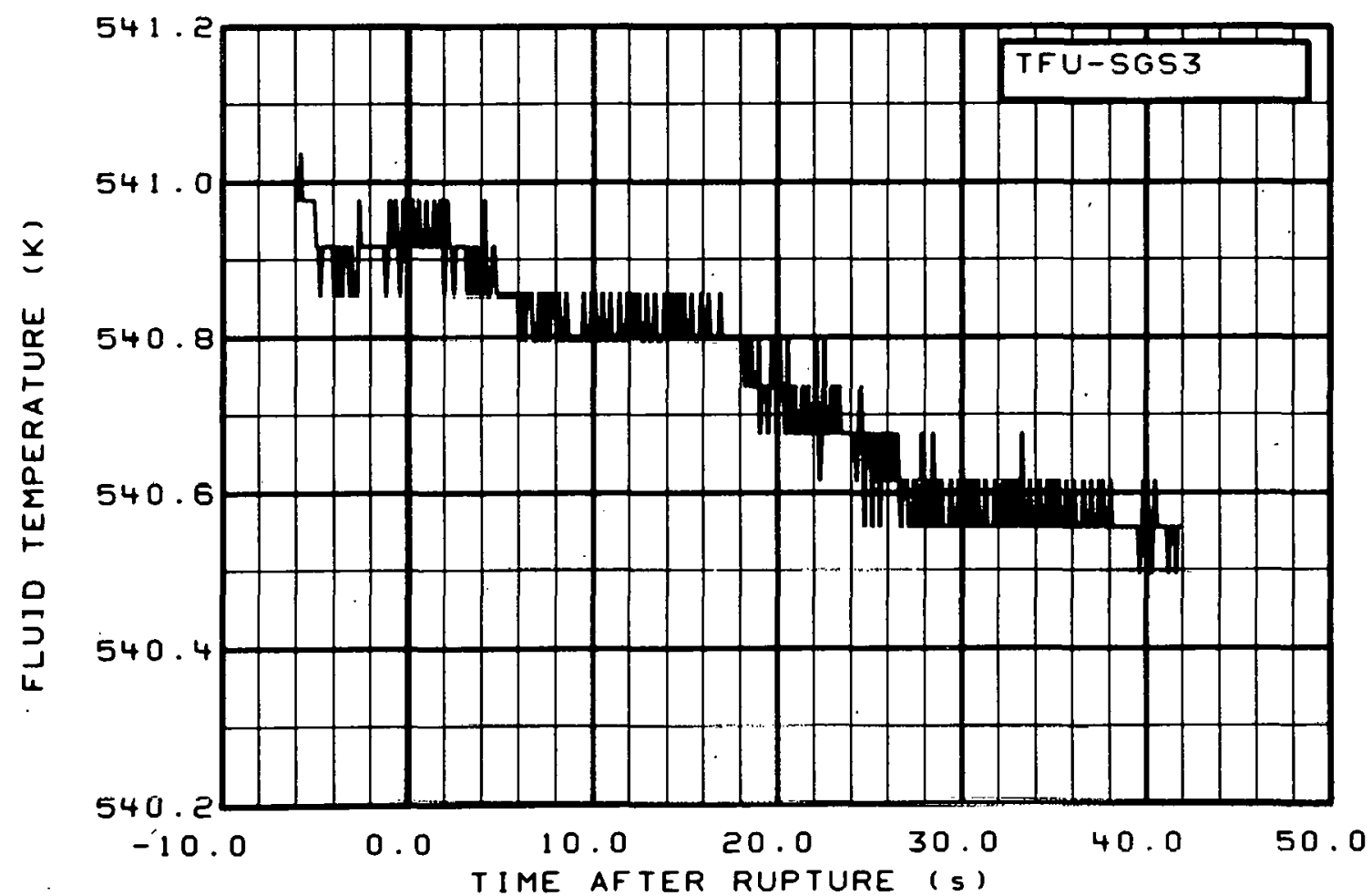

Fig. 50 Fluid temperature in steam generator rupture system accumulator (TFU-SGS3), from -6 to $42 \mathrm{~s}$. 


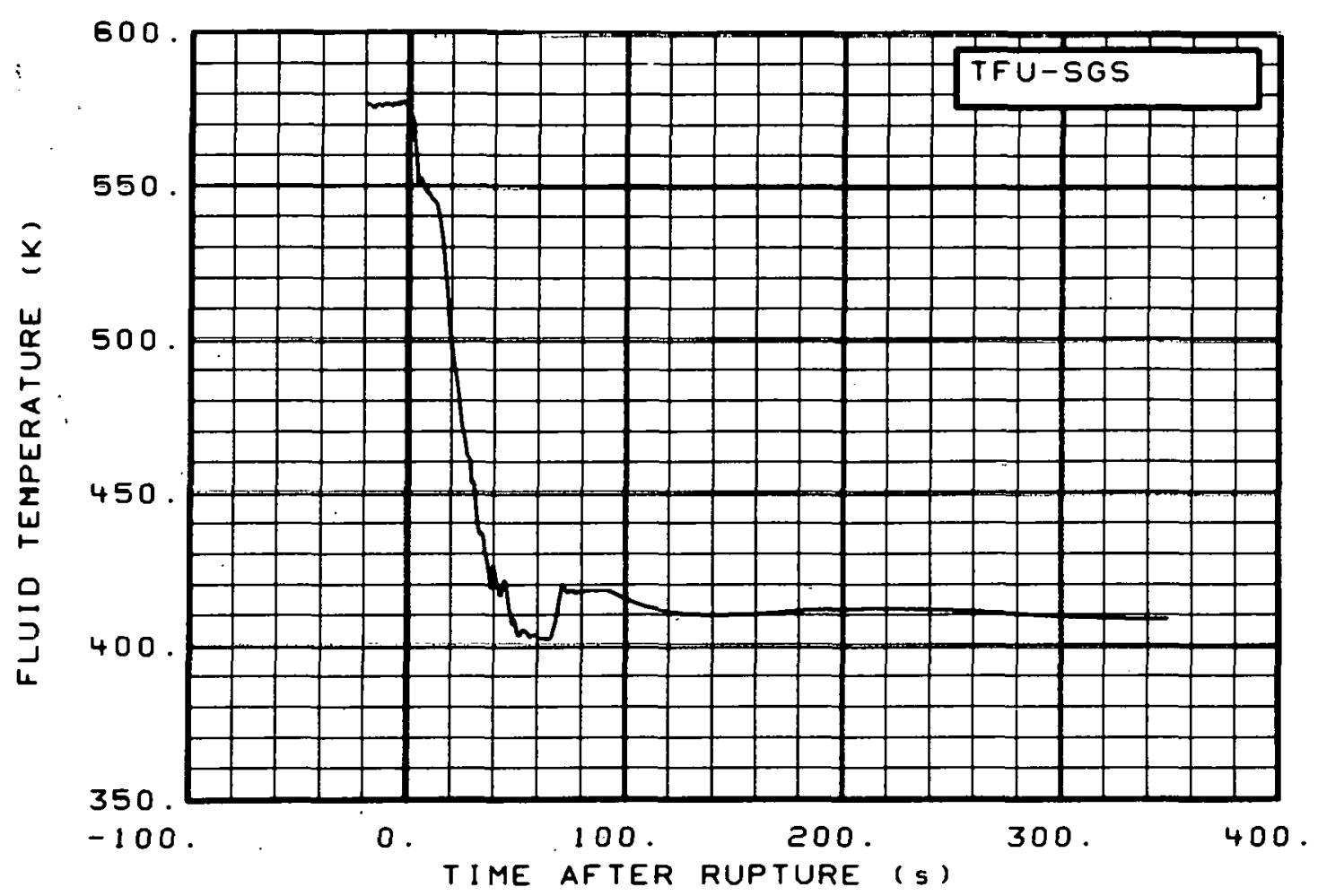

Fig. 51 Fluid temperature in steam generator rupture system injection line (TFU-SGS), from -20 to $350 \mathrm{~s}$.

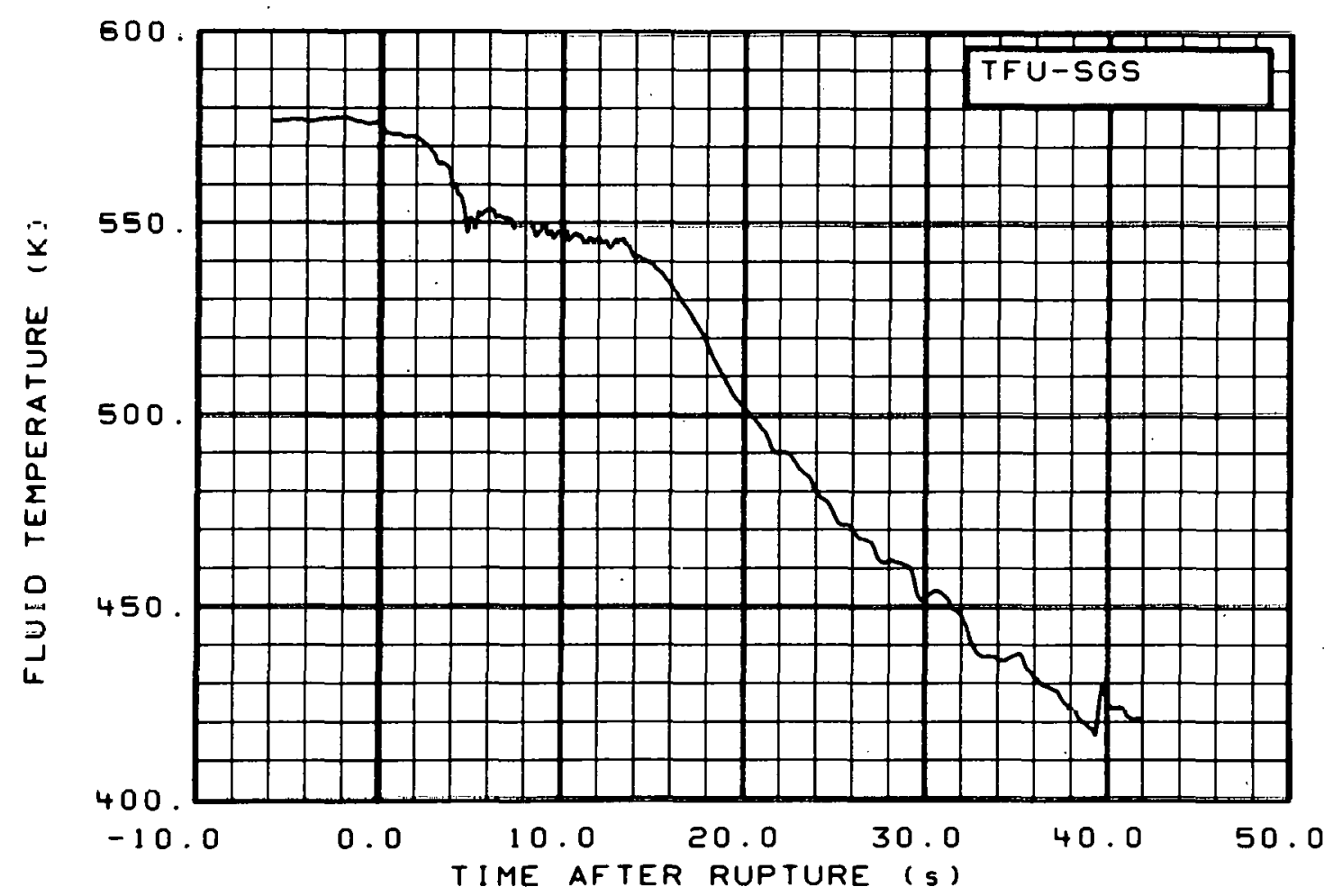

Fig. 52 Fluid temperature in steam generator rupture system injection line (TFU-SGS), from -6 to $42 \mathrm{~s}$. 


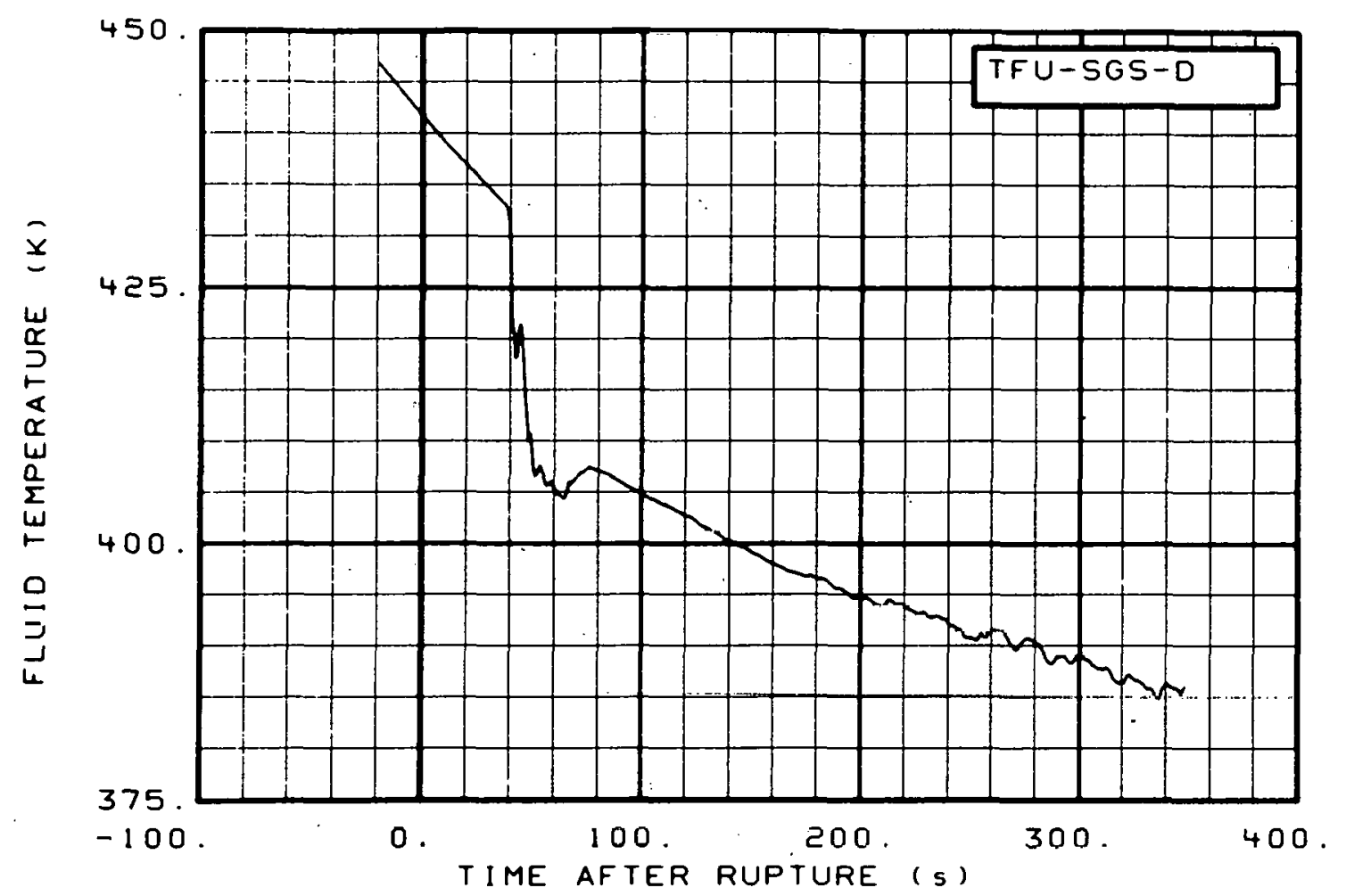

Fig. 53 Fluid temperature in steam generator rupture system (TFU-SGS-D), from -20 to $350 \mathrm{~s}$.

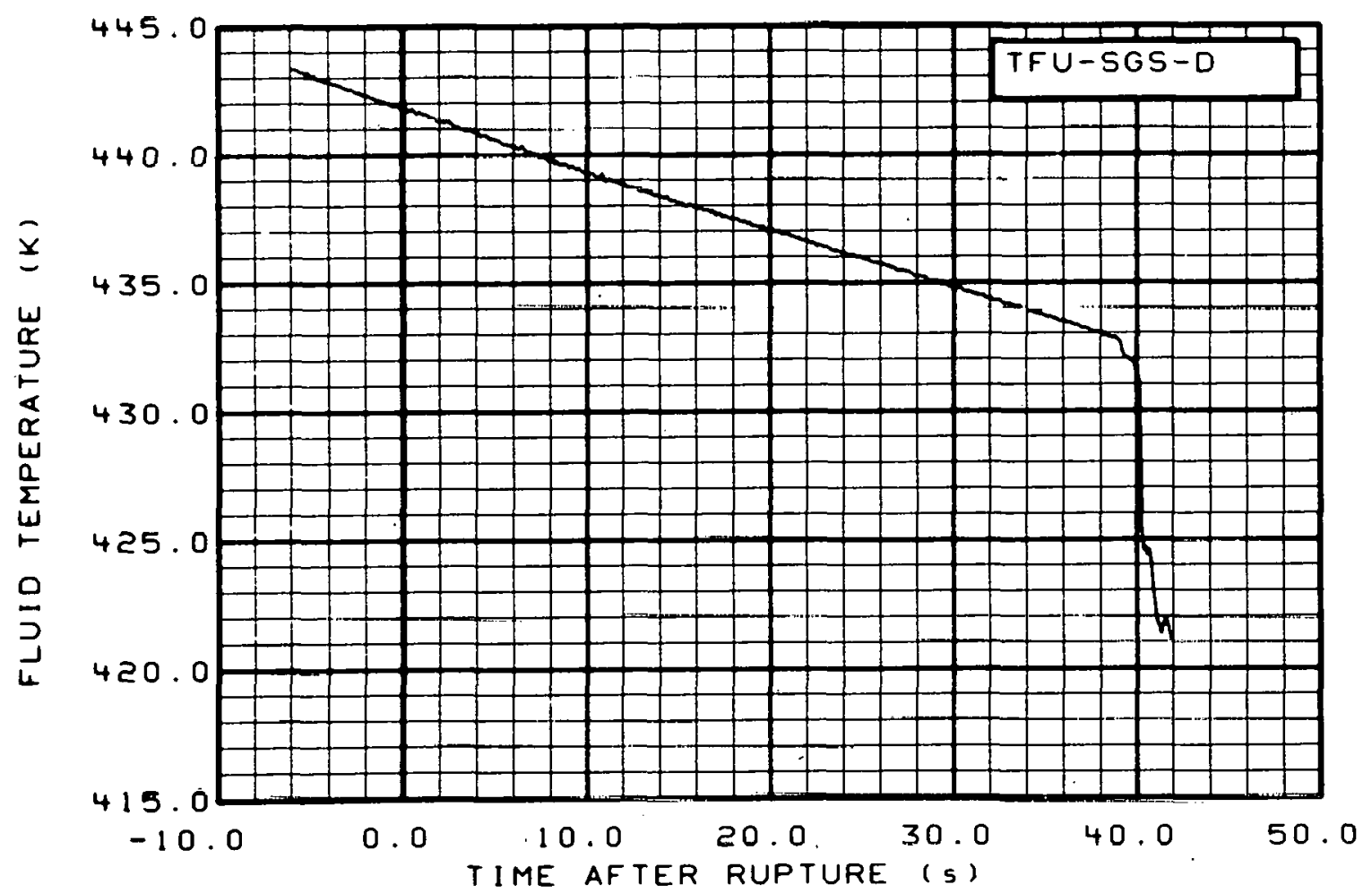

Fig. 54 Fluid temperature in steam generator rupture system (TFU-SGS-D), from -6 to $42 \mathrm{~s}$. 


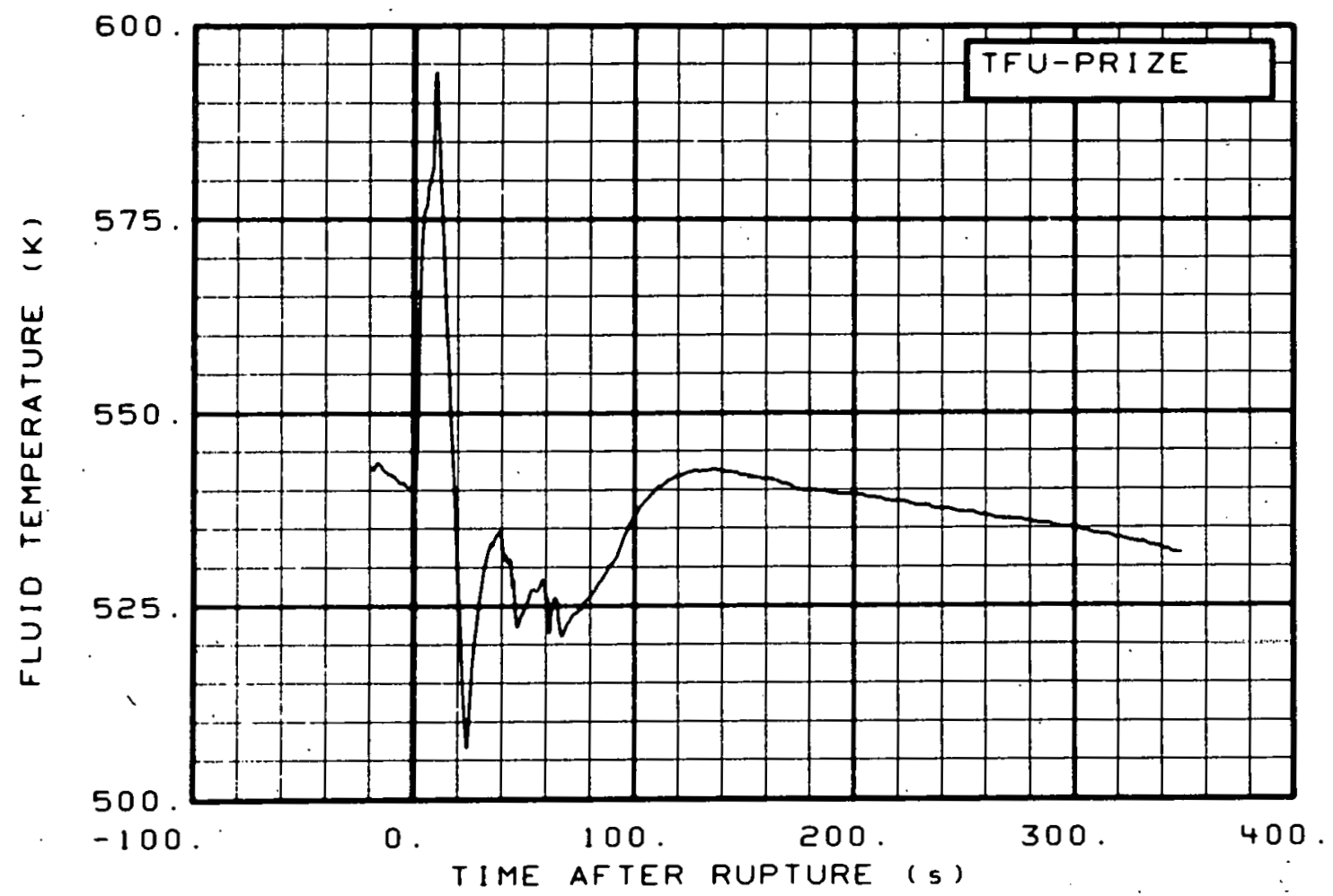

Fig. 55 Fluid temperature in pressurizer surge. 1 ine (TFU-PRIZE), from -20 to $350 \mathrm{~s}$.

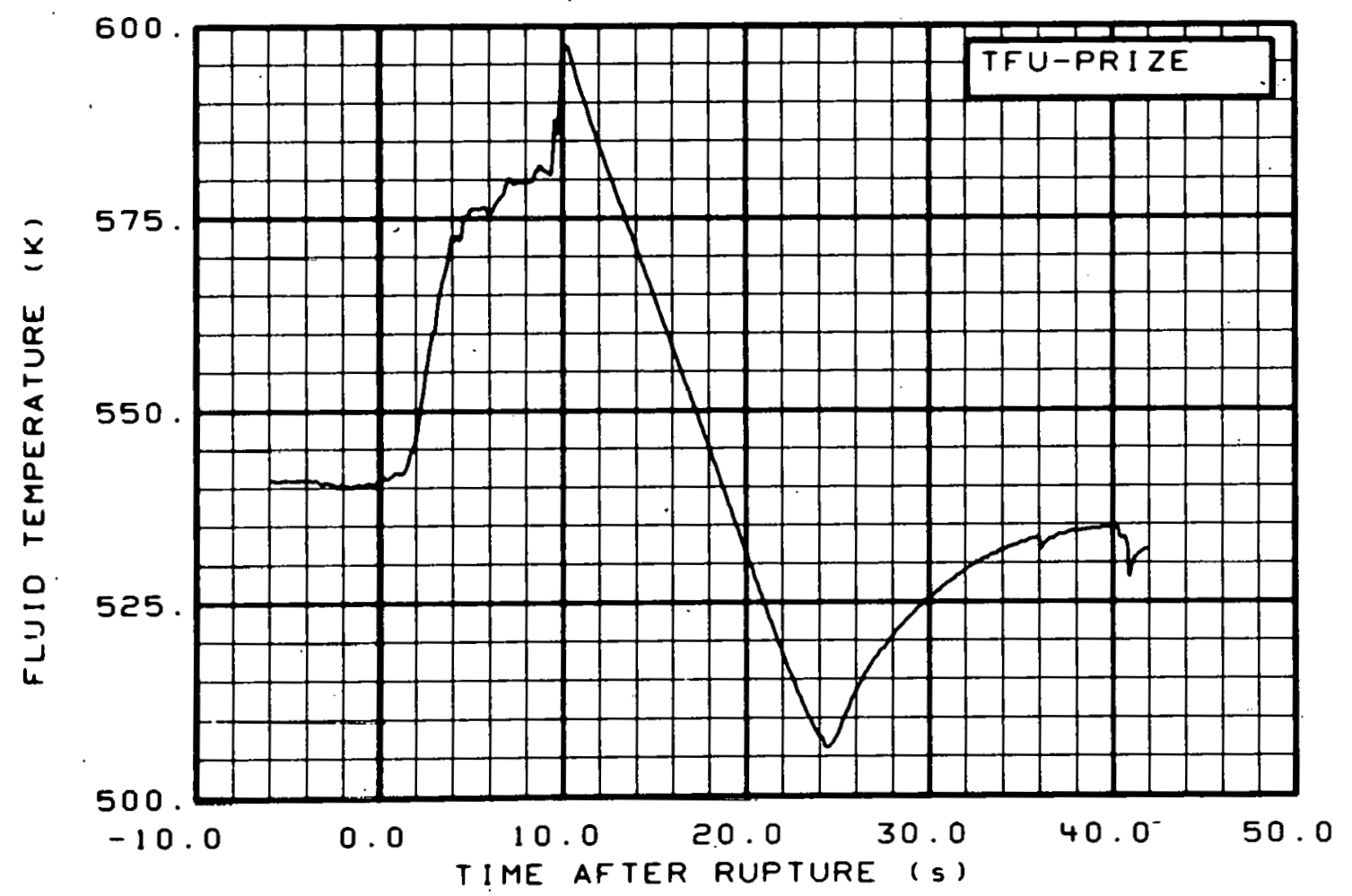

Fig. 56 Fluid temperature in pressurizer surge line (TFU-PRIZE), from -6 to $42 \mathrm{~s}$. 


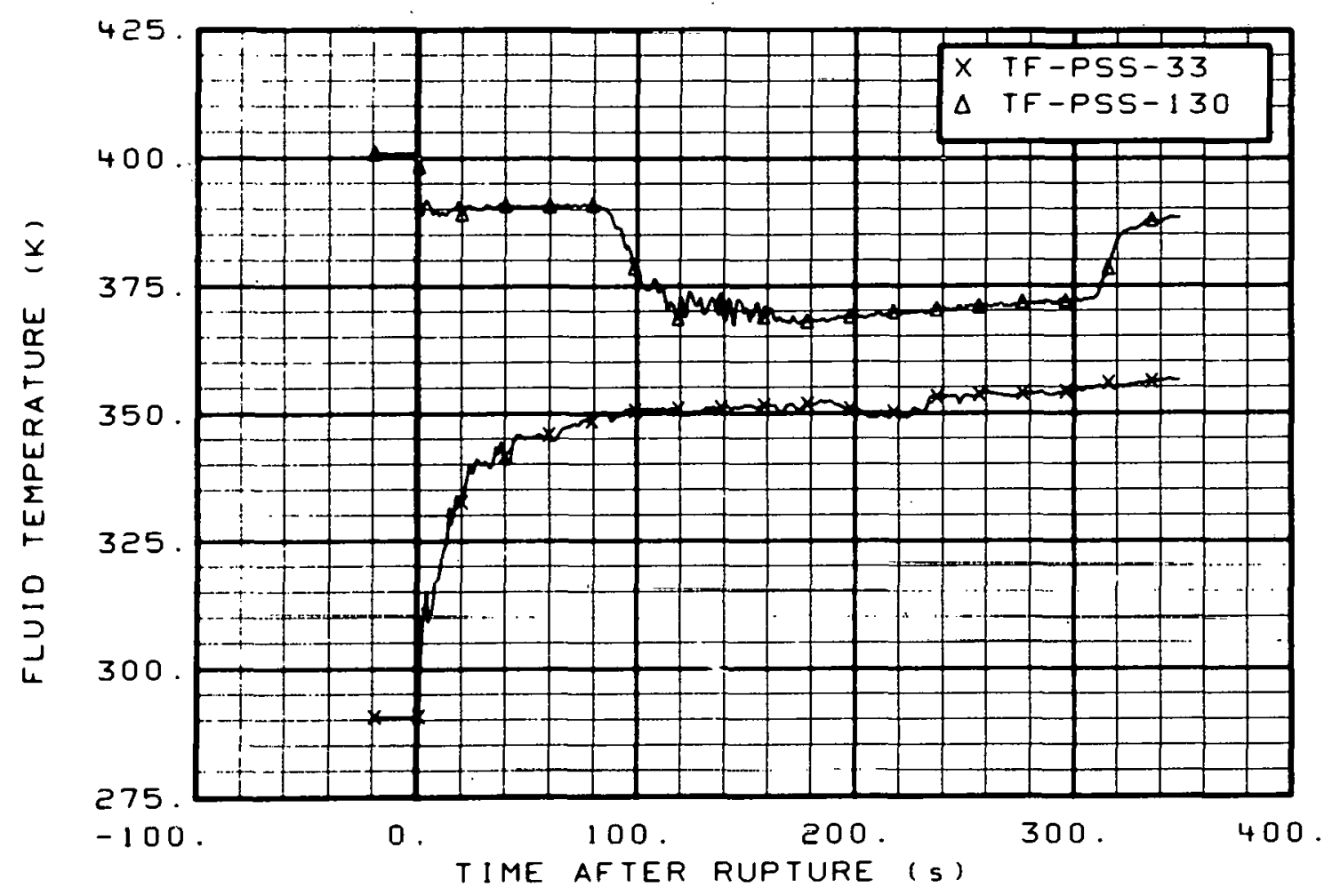

Fig. 57 Fluid temperature in pressure suppression tank (TF-PSS-33 and TF-PSS-130), from -20 to $350 \mathrm{~s}$.

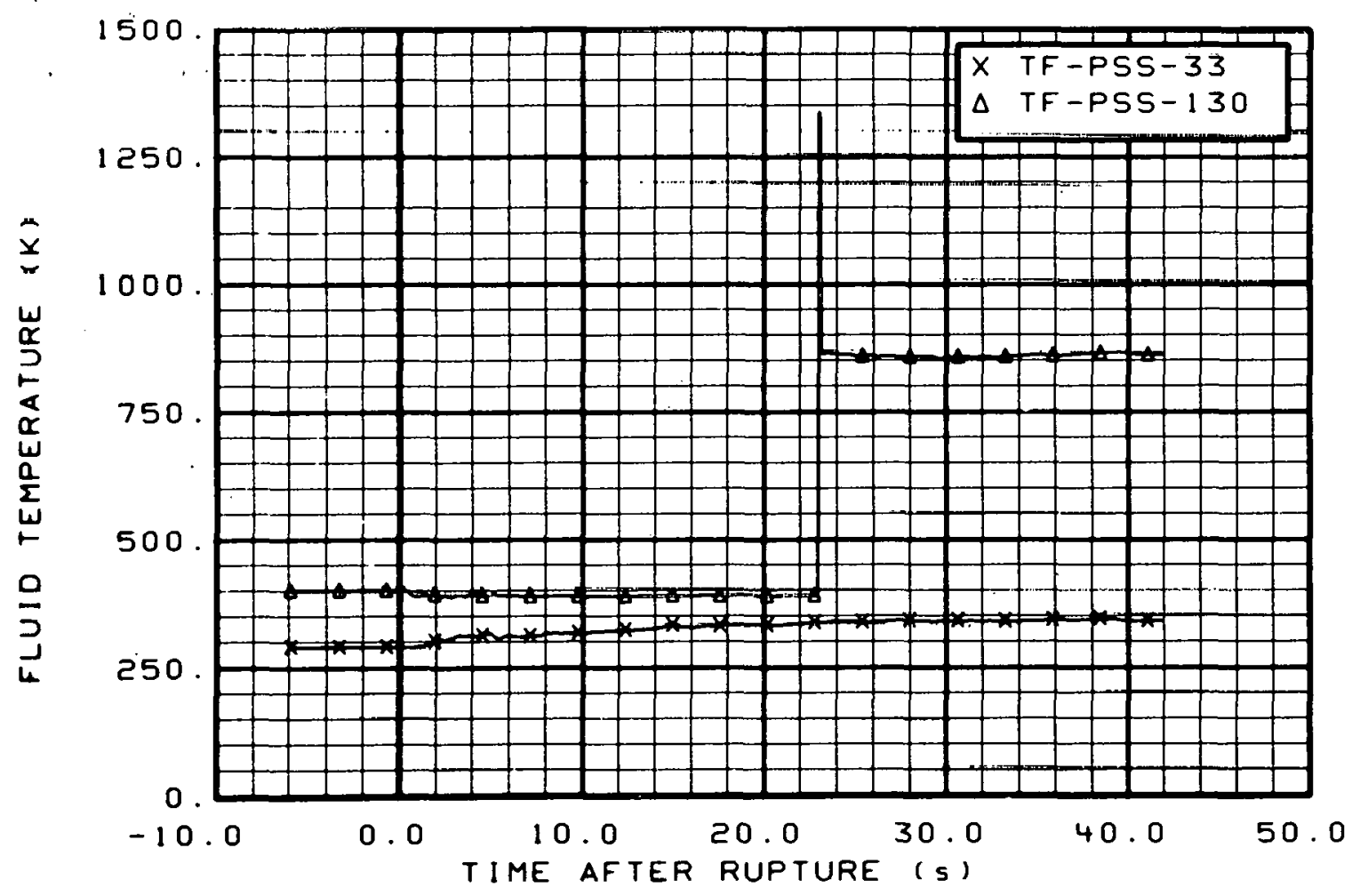

Fig. 58 Fluid temperature in pressure suppression tank (TF-PSS-33 and TF-PSS-130), from -6 to $42 \mathrm{~s}$. 


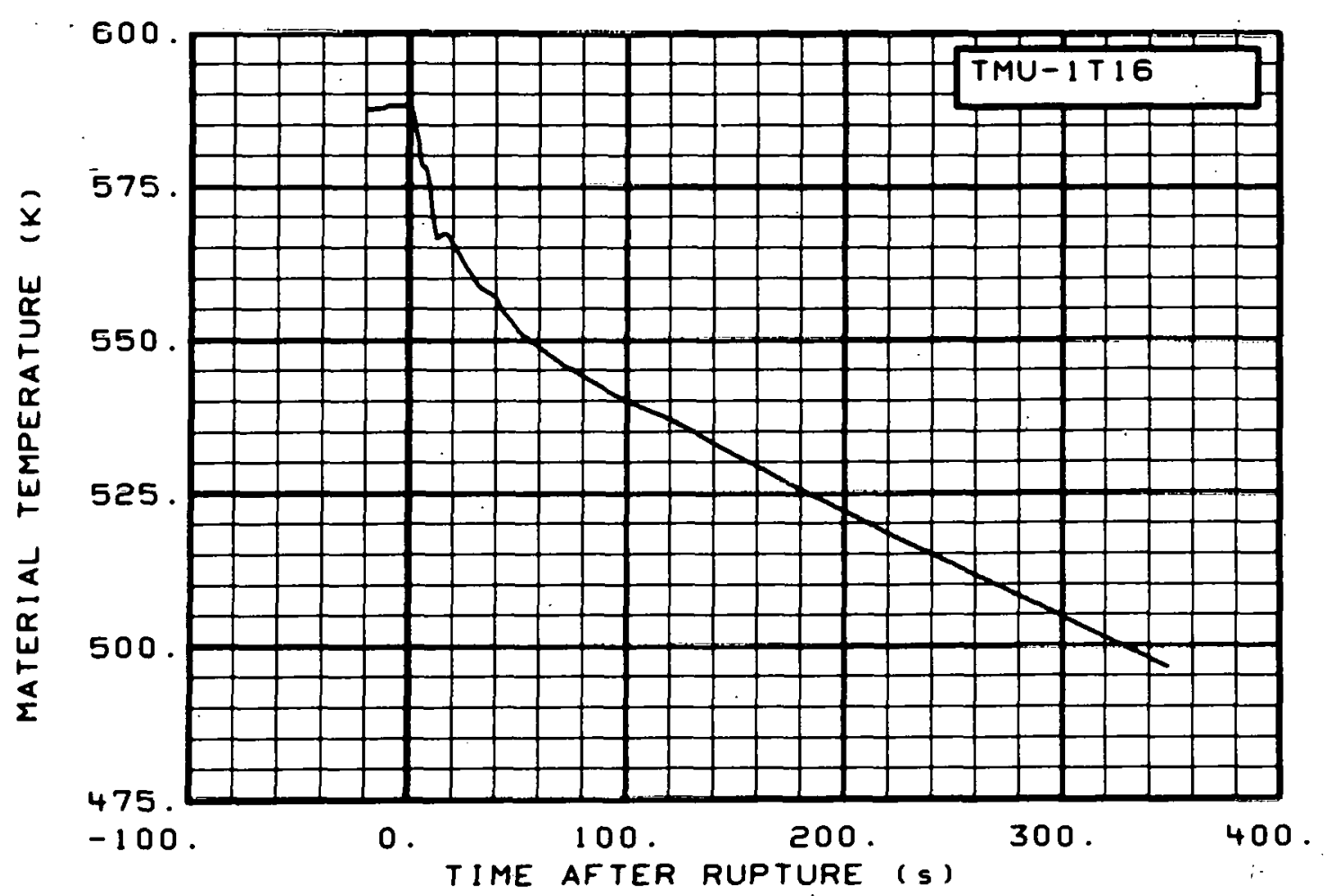

Fig. 59 Material temperature in intact loop (TMU-1T16), from -20 to $350 \mathrm{~s}$.

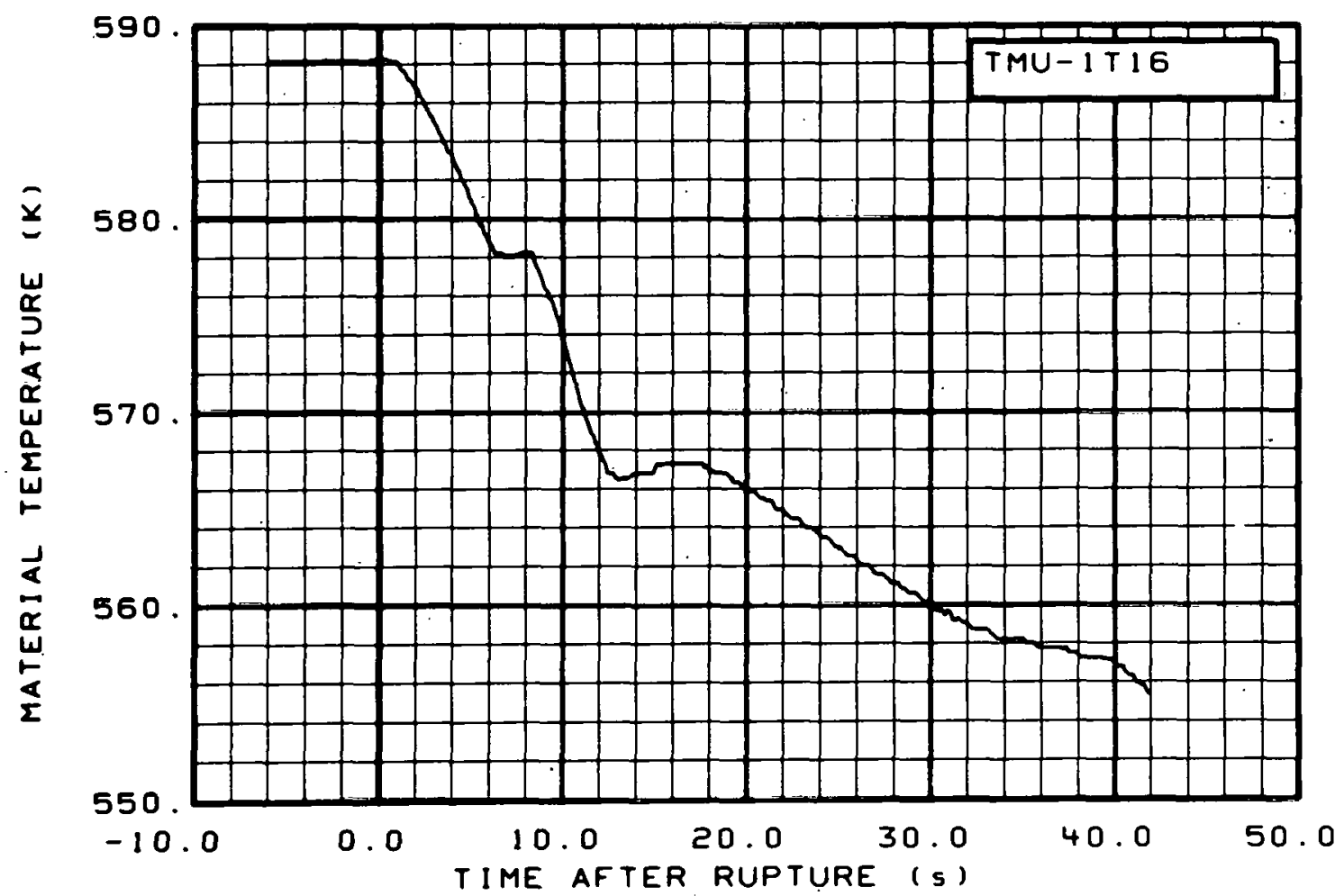

Fig. 60 Material temperature in intact loop (TMU-1T16), from -6 to $42 \mathrm{~s}$. 


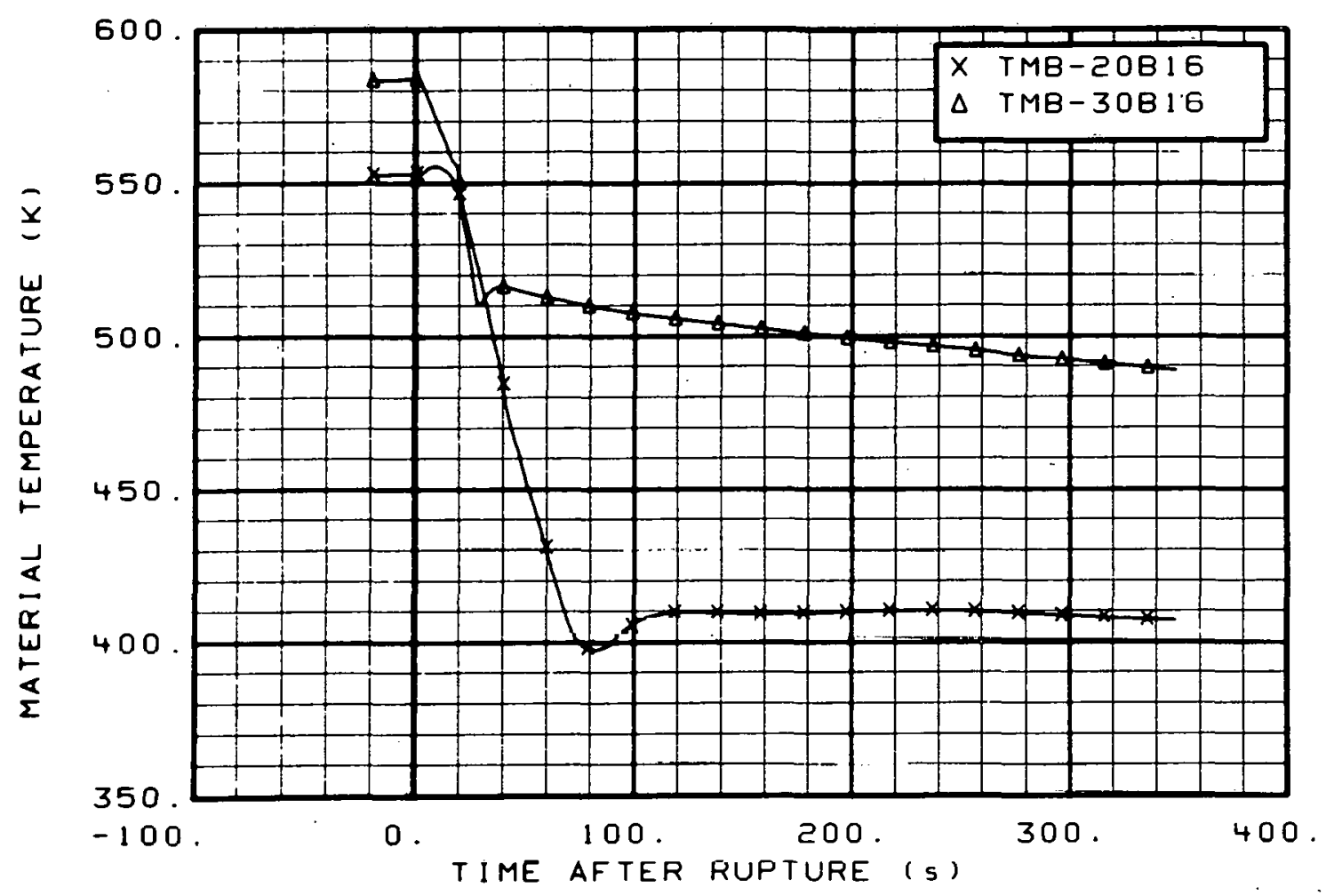

Fig. 61 Material temperature in broken loop (TMB-20B16 and TMB-30B16), from -20 to $350 \mathrm{~s}$.

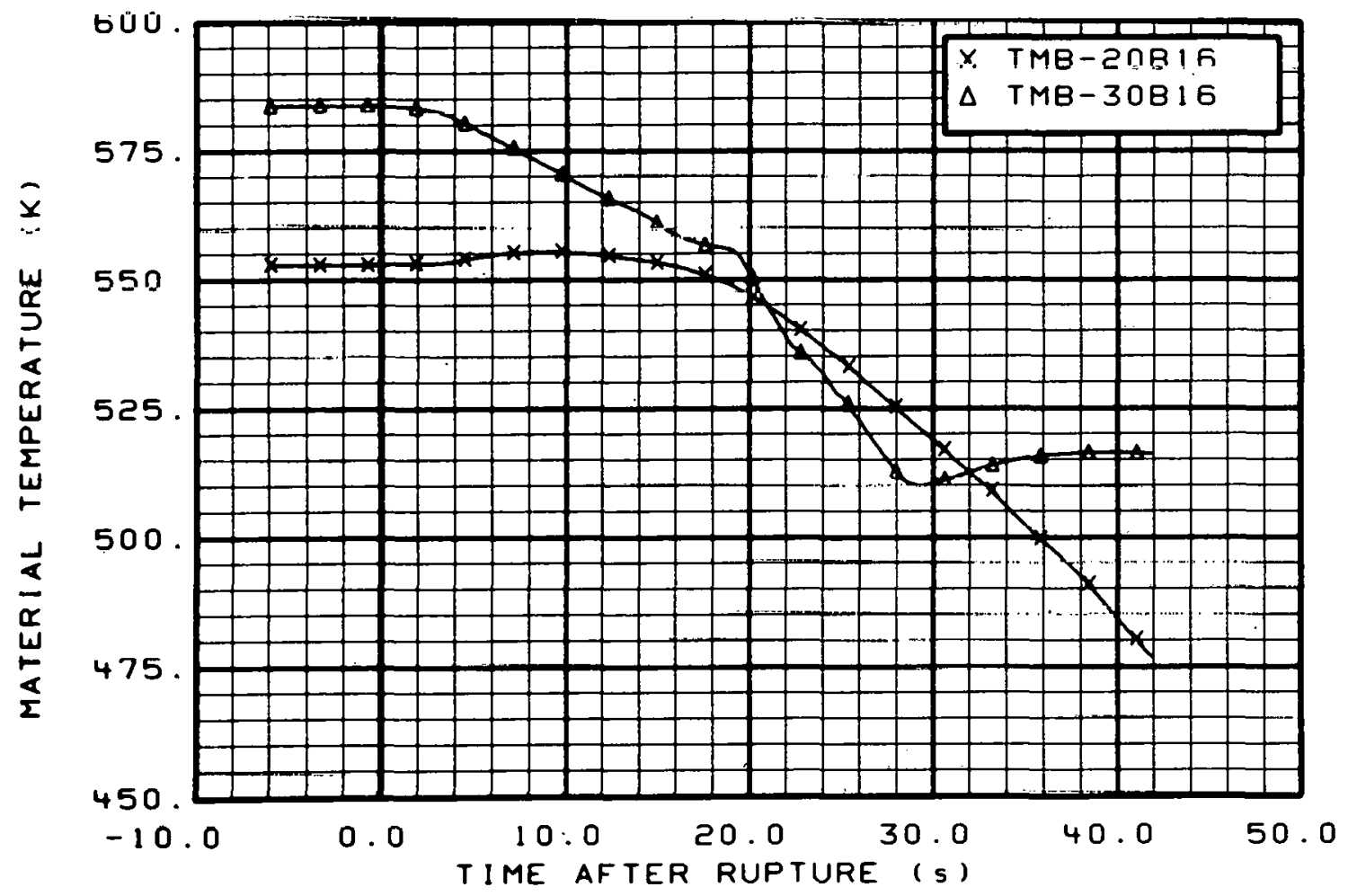

Fig. 62 Material temperature in broken loop (TMB-20B16 and TMB-30B16), from -6 to $42 \mathrm{~s}$. 


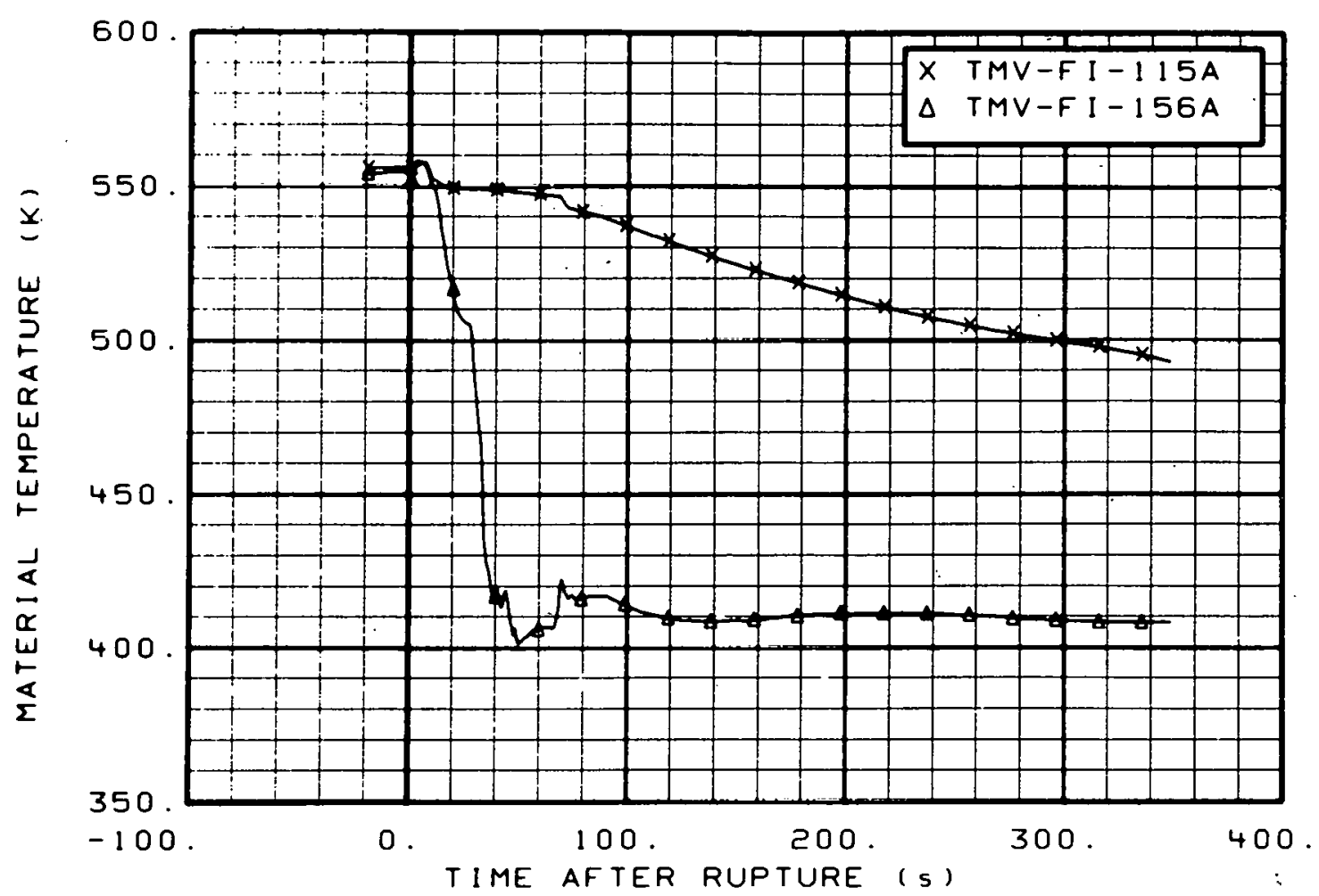

Fig. 63 Material temperature in vessel filler (TMV-FI-115A and TMV-FI$156 \mathrm{~A}$ ), from -20 to $350 \mathrm{~s}$.

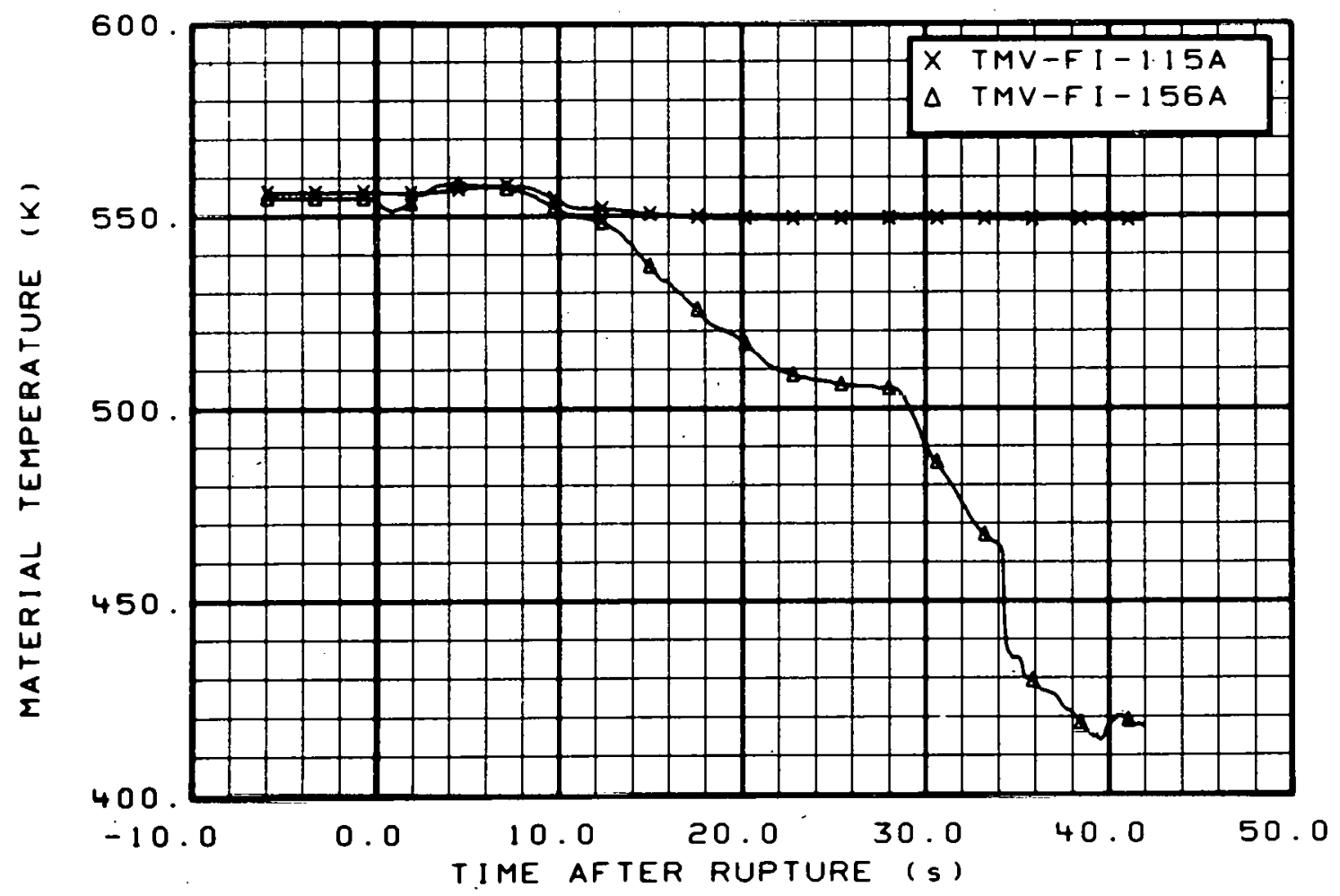

Fig. 64 Material temperature in vessel filler (TMV-FI-115A and TMV-FI$156 \mathrm{~A}$ ), from -6 to $42 \mathrm{~s}$. 


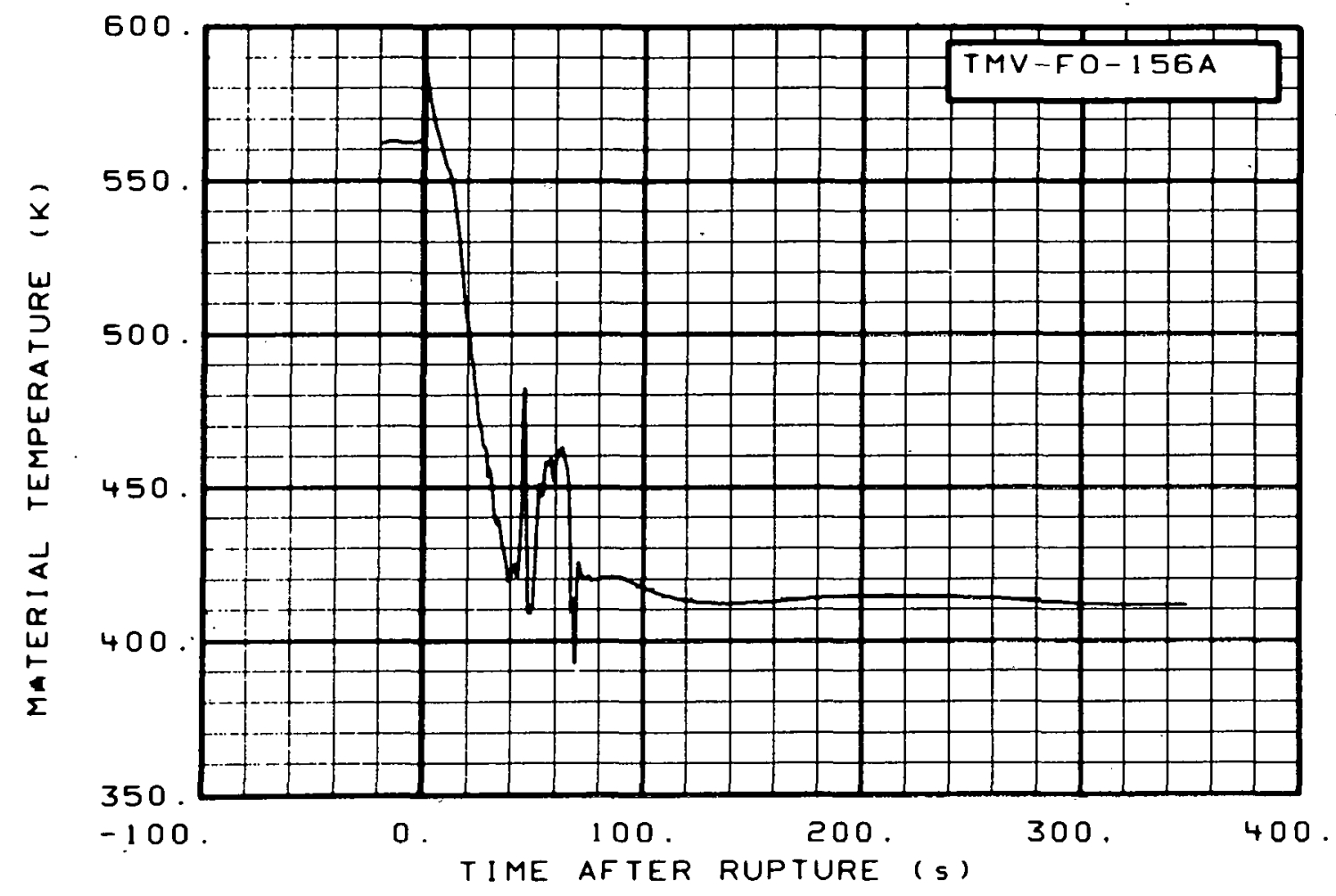

Fig. 65 Material temperature in vessel filler (TMV-F0-156A), from -20 to $350 \mathrm{~s}$.

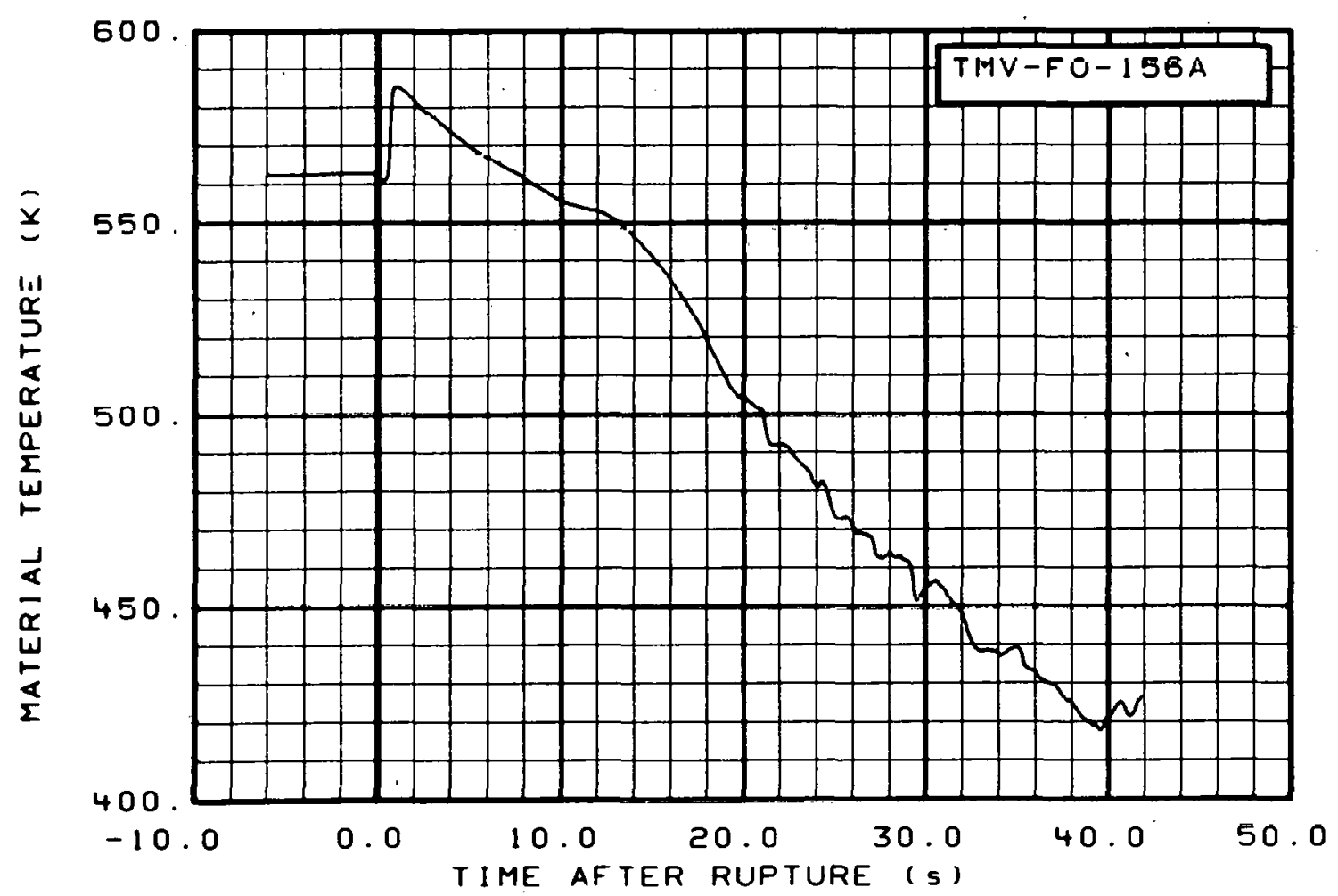

Fig. 66 Material temperature in vessel filler. (TMV-FO-156A), from -6 to $42 \mathrm{~s}$. 


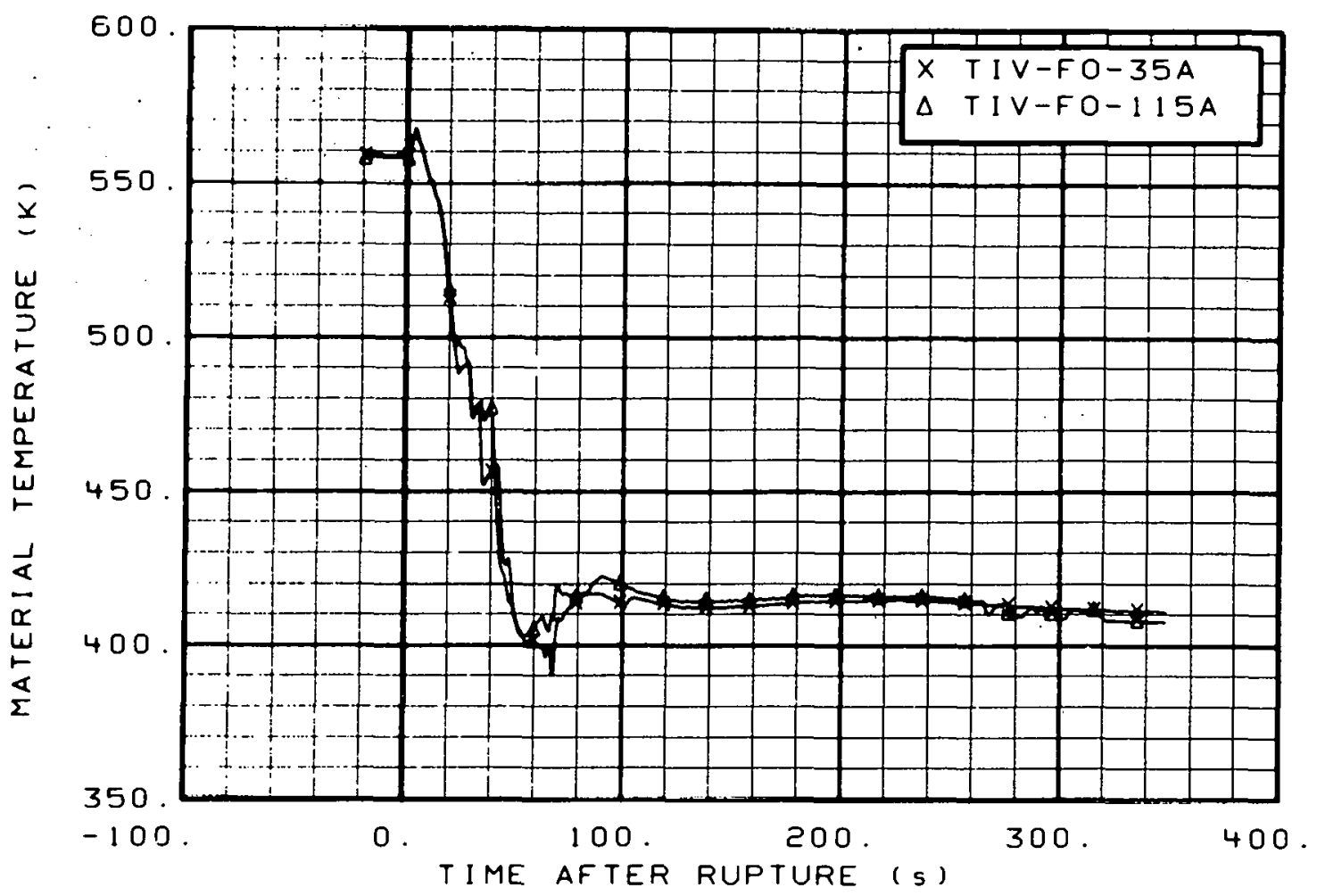

Fig. 67 Material temperature in vessel filler (TIV-F0-35A and TIV-F0$115 \mathrm{~A}$ ), from -20 to $350 \mathrm{~s}$.

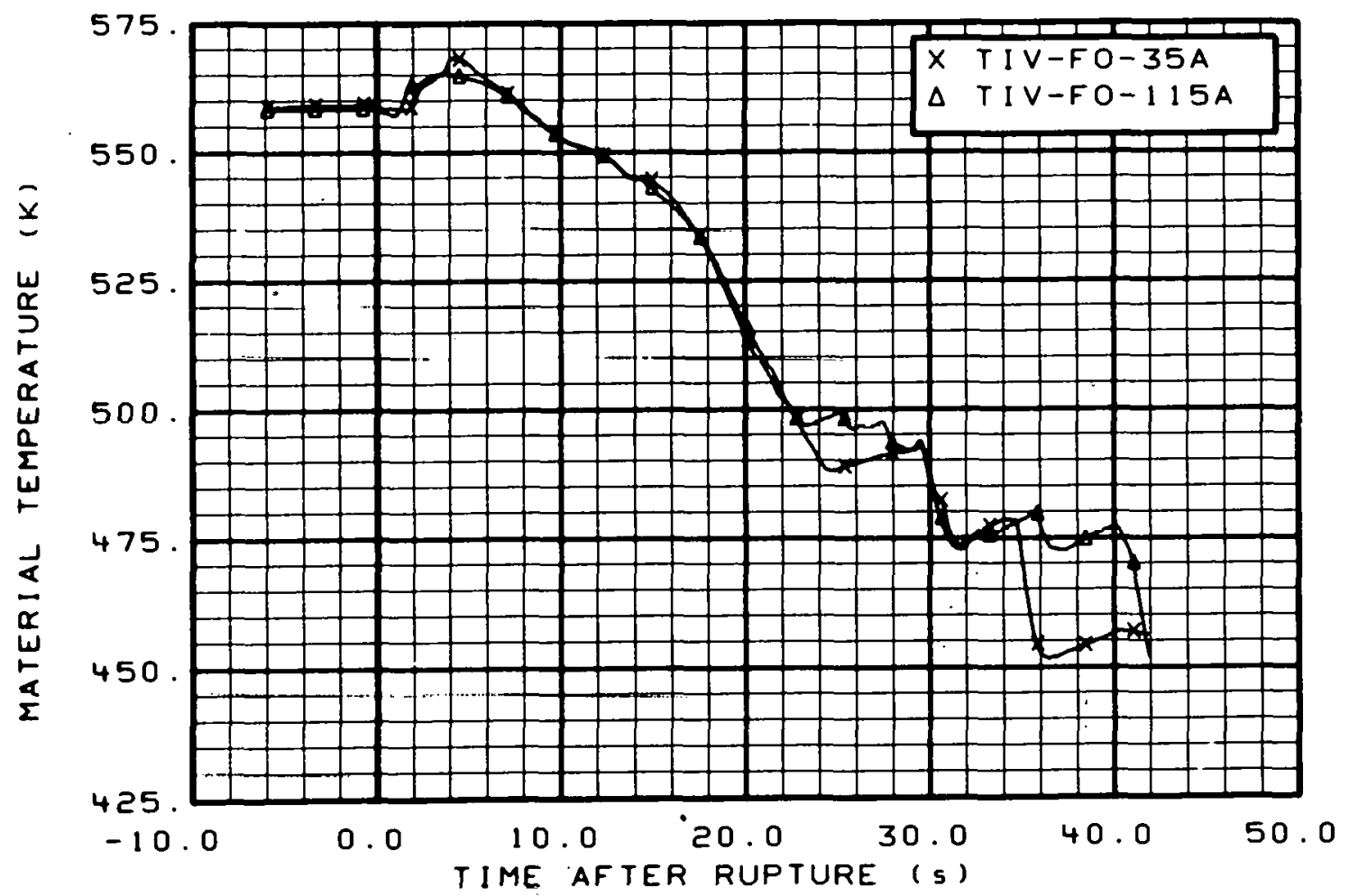

Fig. 68 Material temperature in vessel filler (TIV-F0-35A and TIV-FO$115 \mathrm{~A})$, from -6 to $42 \mathrm{~s}$. 


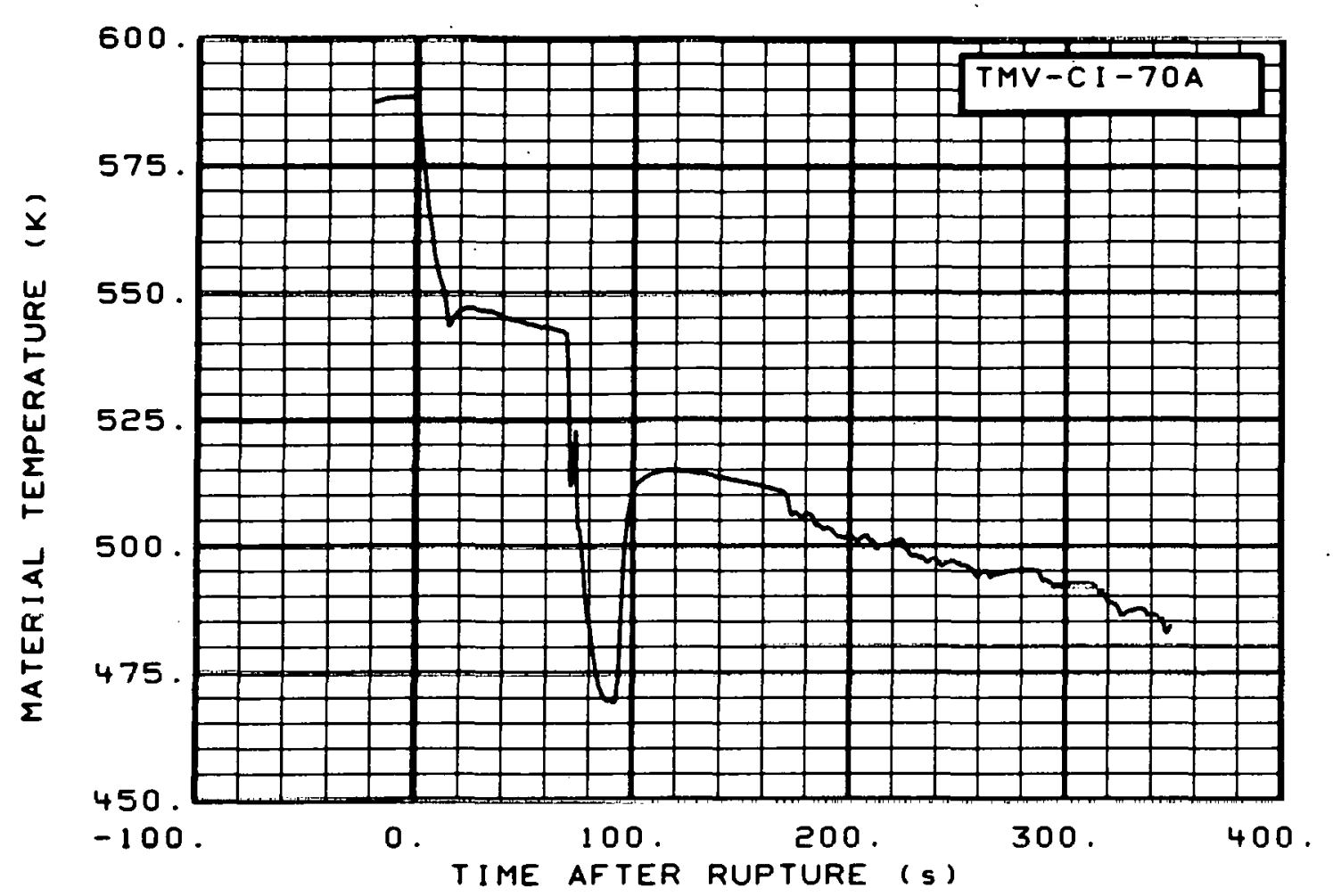

Fig. 69 Material temperature in core barrel inner diameter (TMV-CI-70A), from -20 to $350 \mathrm{~s}$.

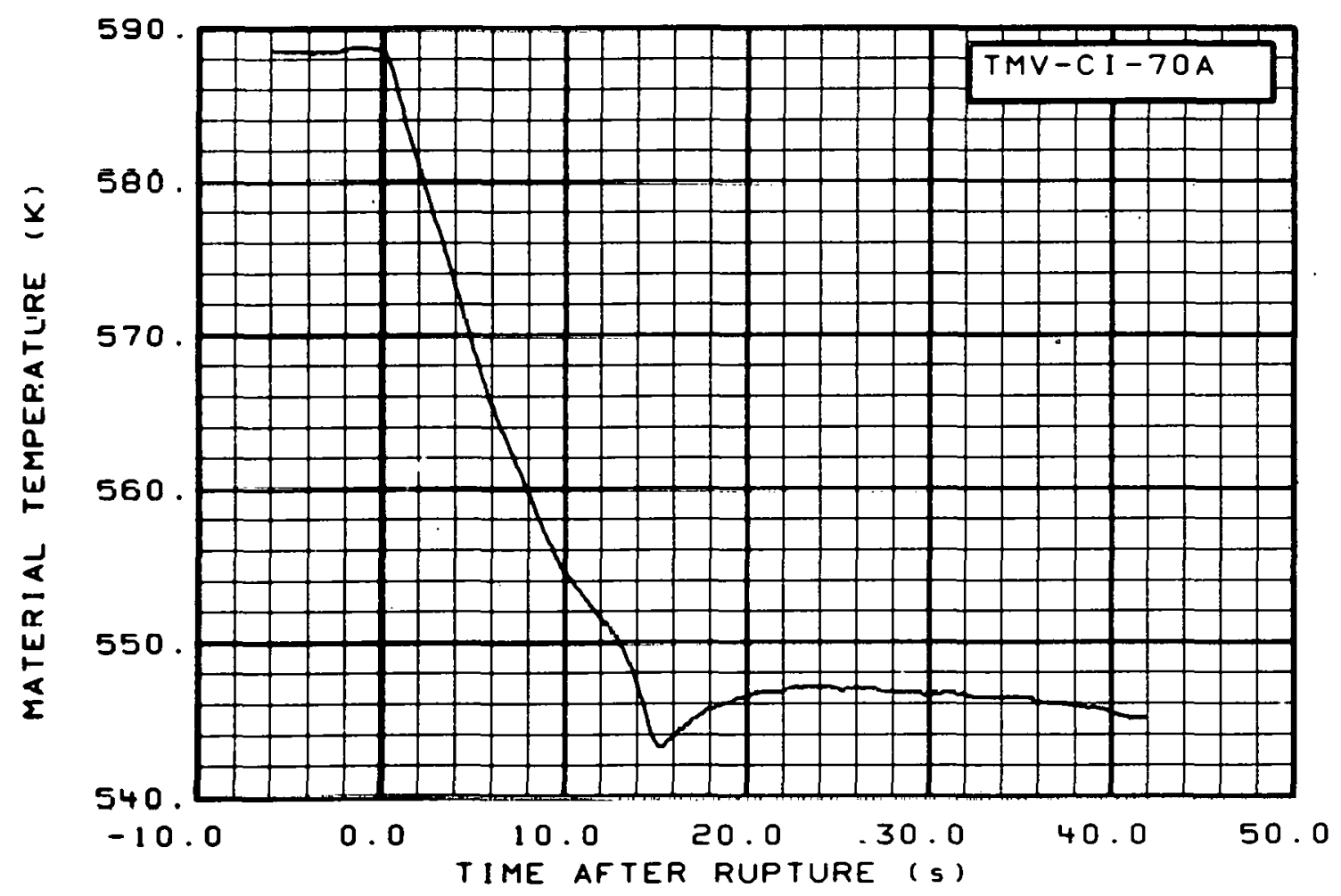

Fig. 70 Material temperature in core barrel inner diameter (TMV-CI-70A), from -6 to $42 \mathrm{~s}$. 


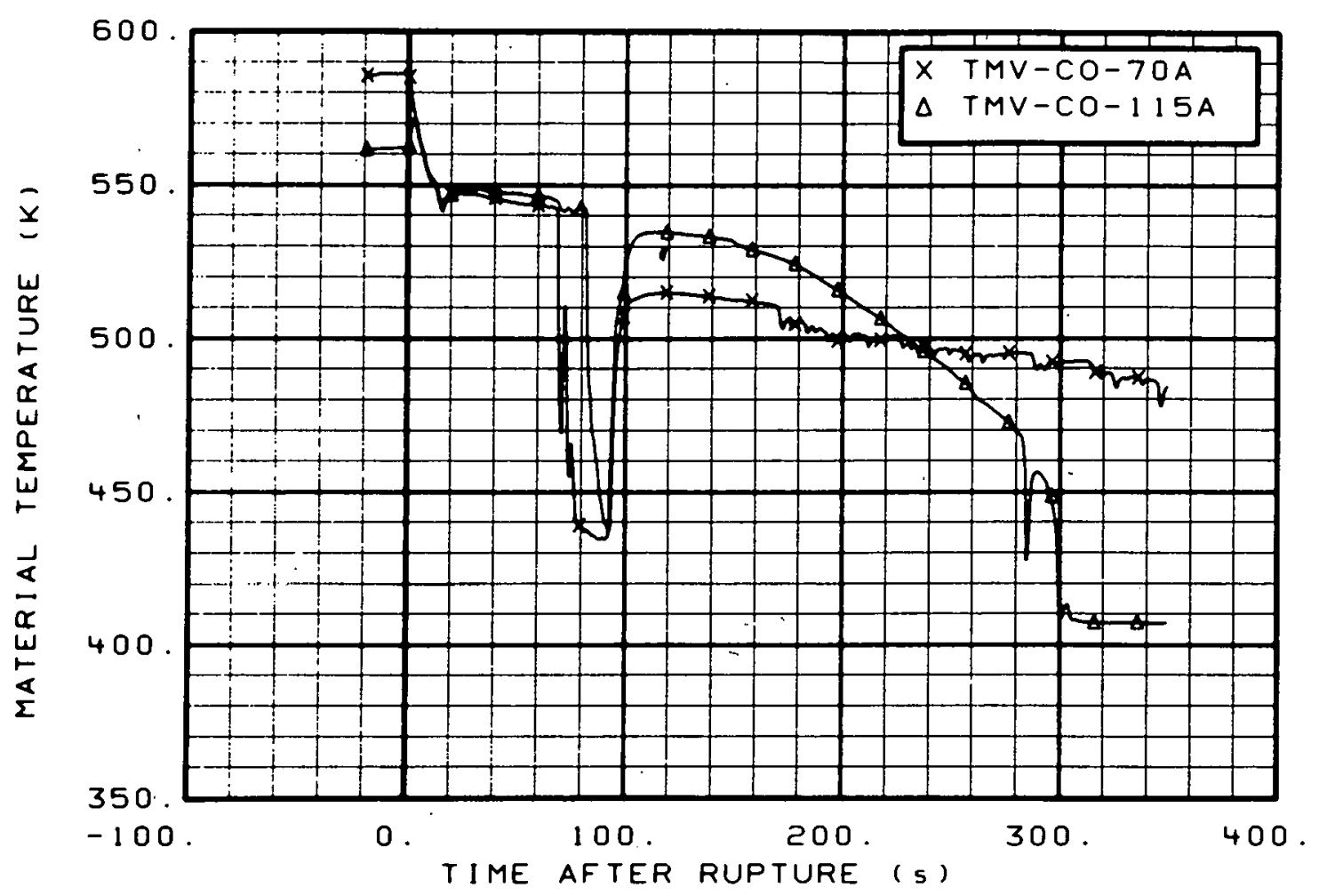

Fig. 71 Material temperature in core barrel inner diameter (TMV-CO-70A and TMV-CO-115A), from -20 to $350 \mathrm{~s}$.

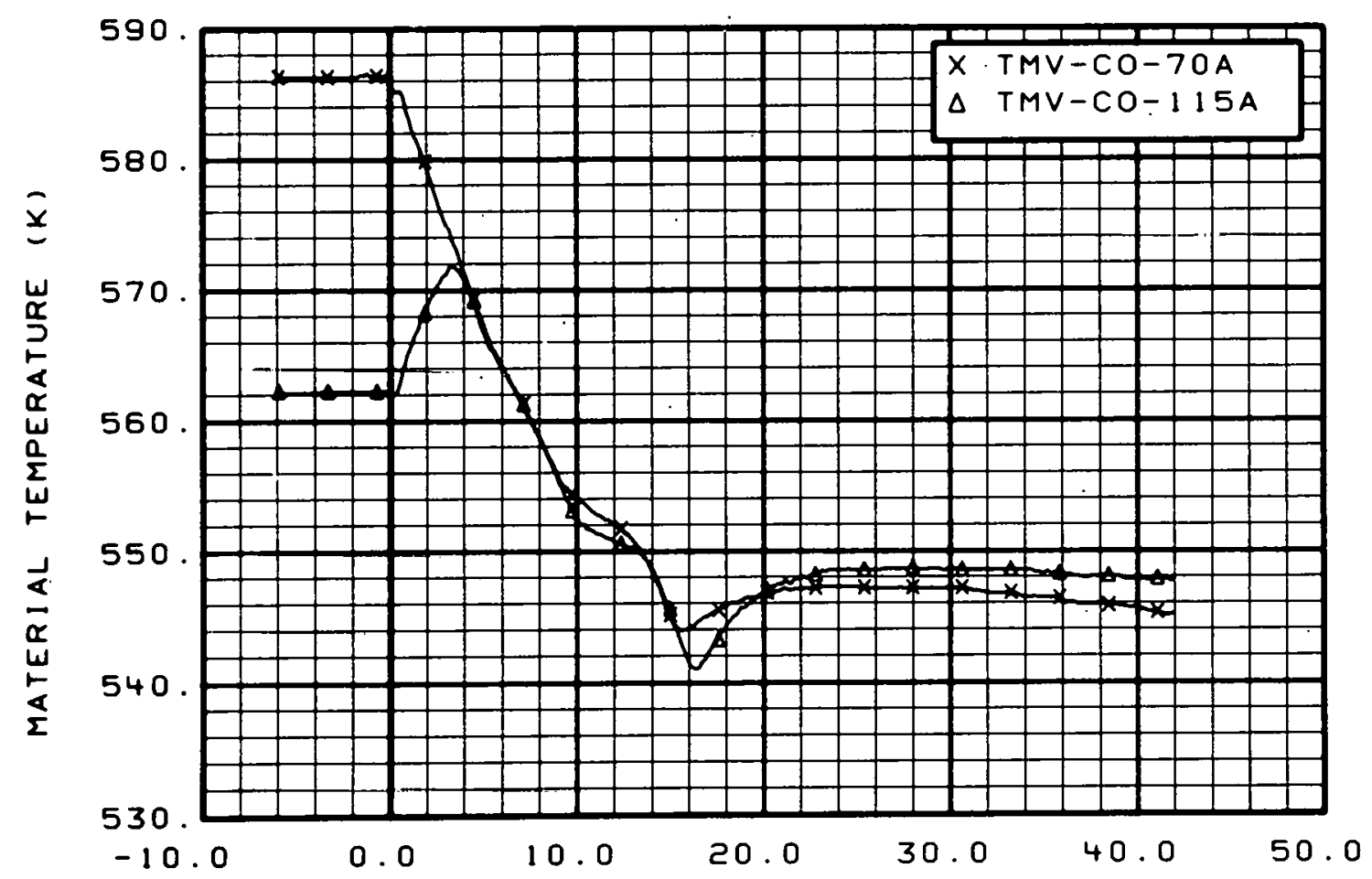

Fig. 72 Material temperature in core barrel inner diameter (TMV-C0-70A and TMV-C0-115A), from -6 to $42 \mathrm{~s}$. 


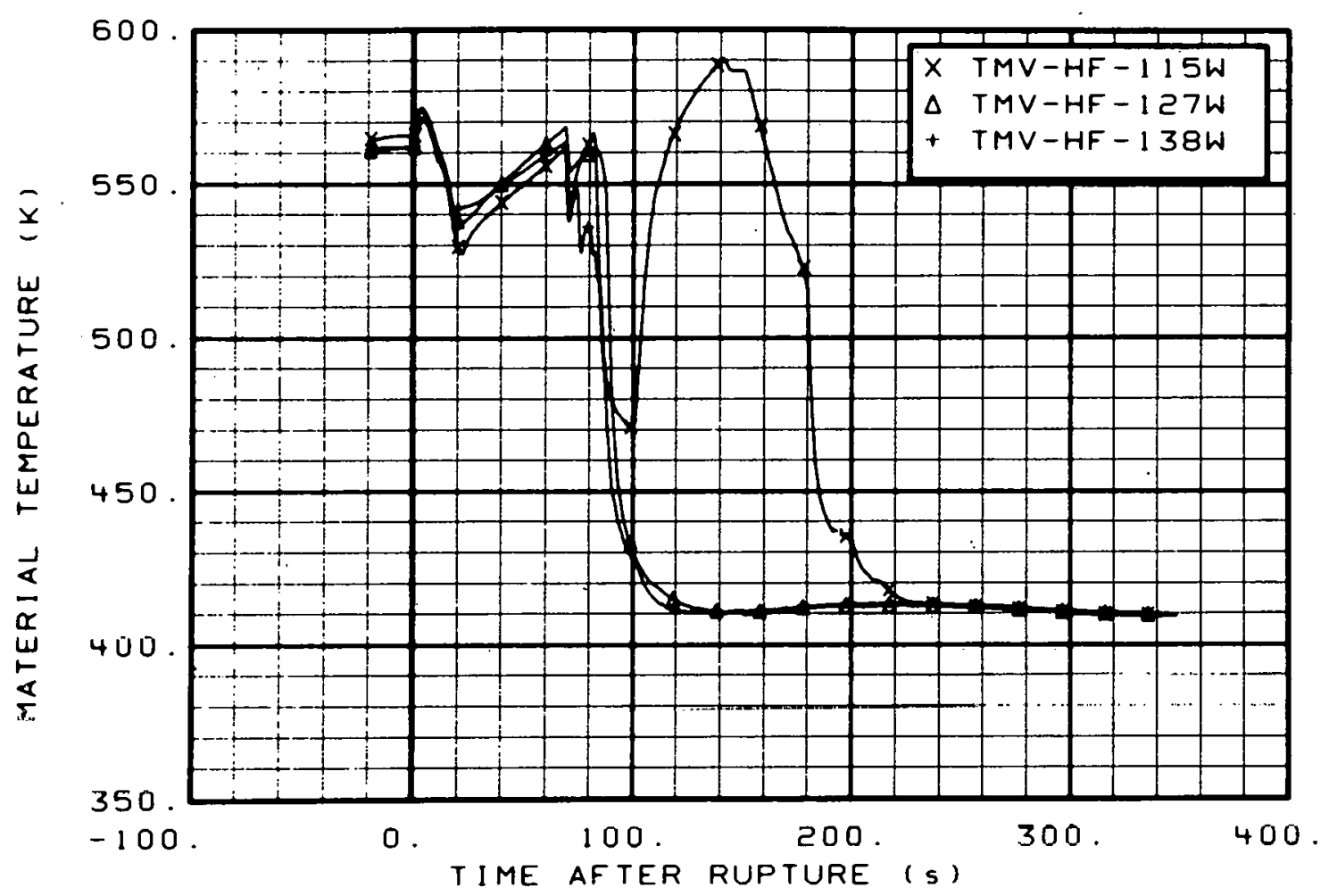

Fig. 73 Material temperature in core housing filler (TMV-HF-115W, TMV-HF$127 \mathrm{~W}$, and TMV-HF-138W), from -20 to $350 \mathrm{~s}$.

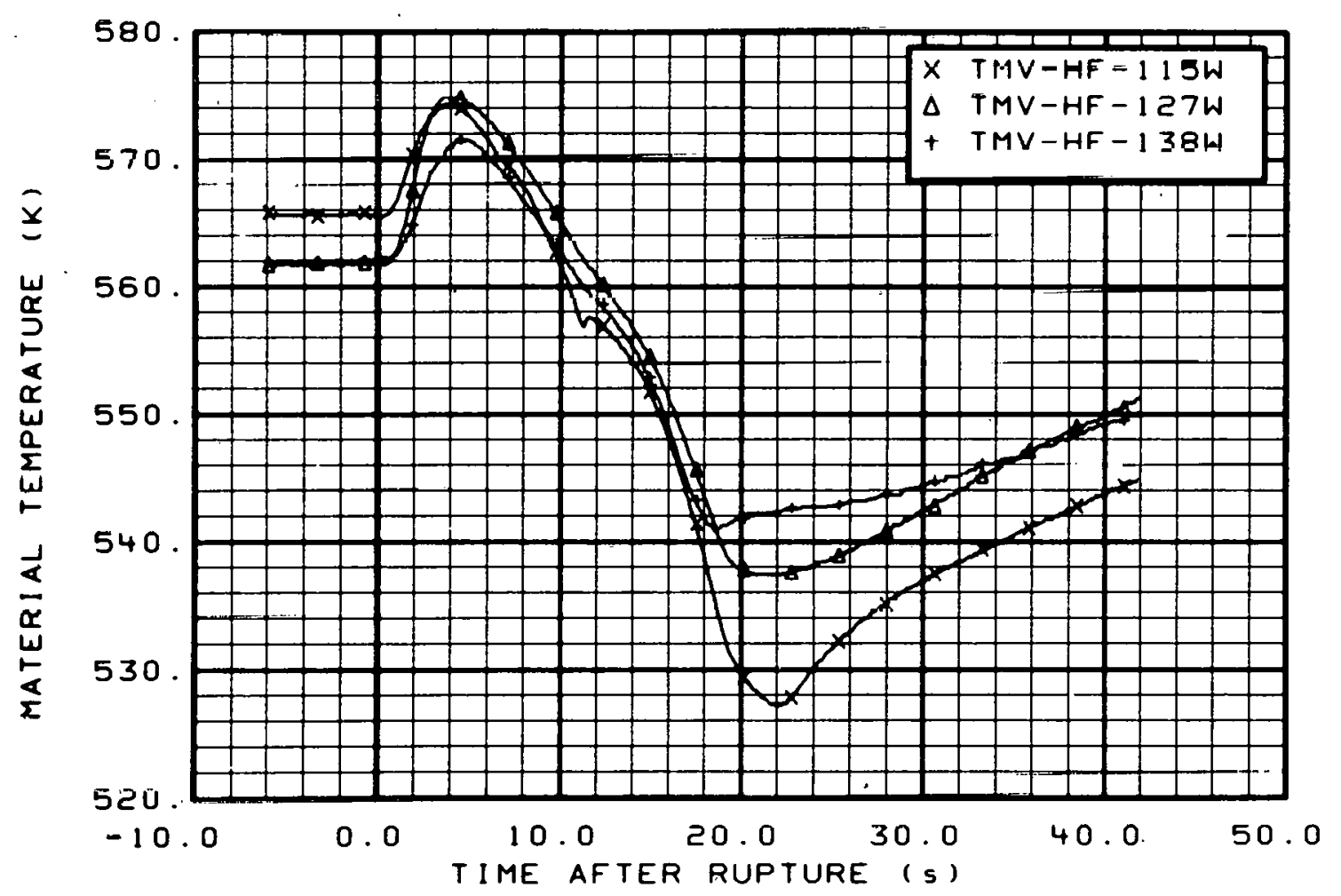

Fig. 74 Material temperature in core housing filler (TMV-HF-115W, TMV-HF$127 \mathrm{~W}$, and TMV-HF-138W), from -6 to $42 \mathrm{~s}$. 


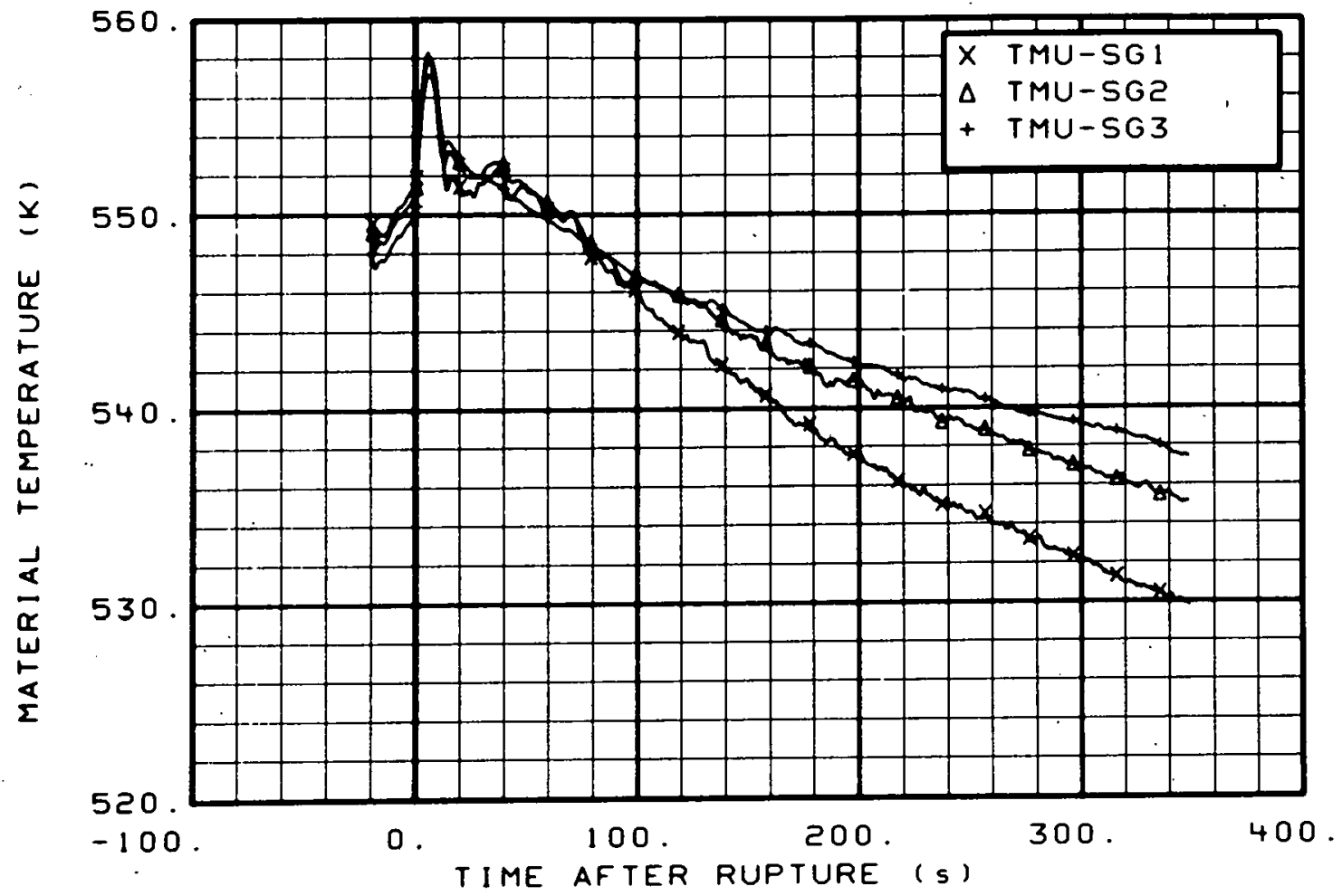

Fig. 75 Material temperature in steam generator (TMU-SG1, TMU-SG2, and TMU-SG3), from -20 to $350 \mathrm{~s}$.

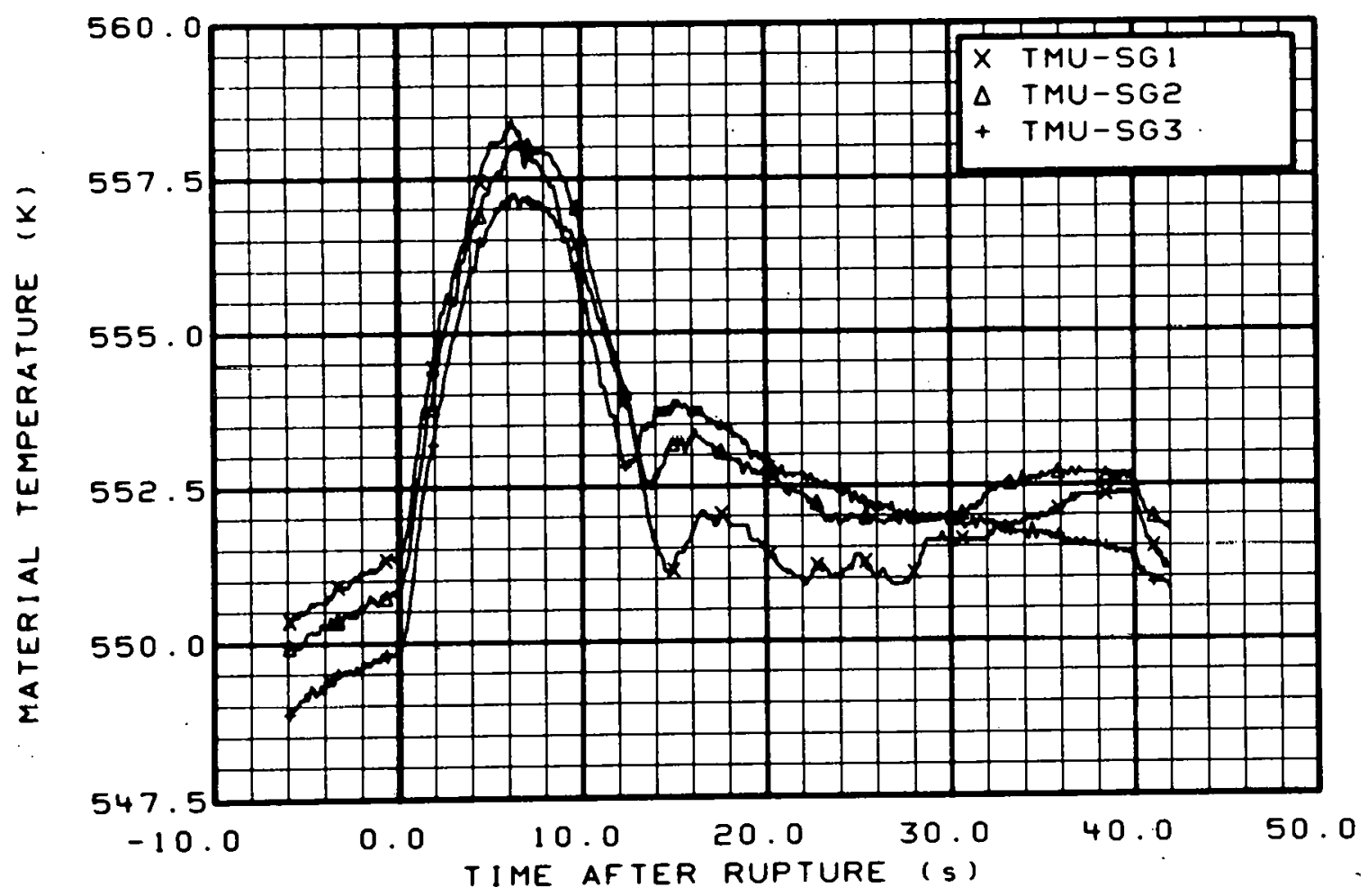

Fig. 76 Material temperature in steam generator (TMU-SGI, TMU-SG2, and TMU-SG3), from -6 to $42 \mathrm{~s}$. 


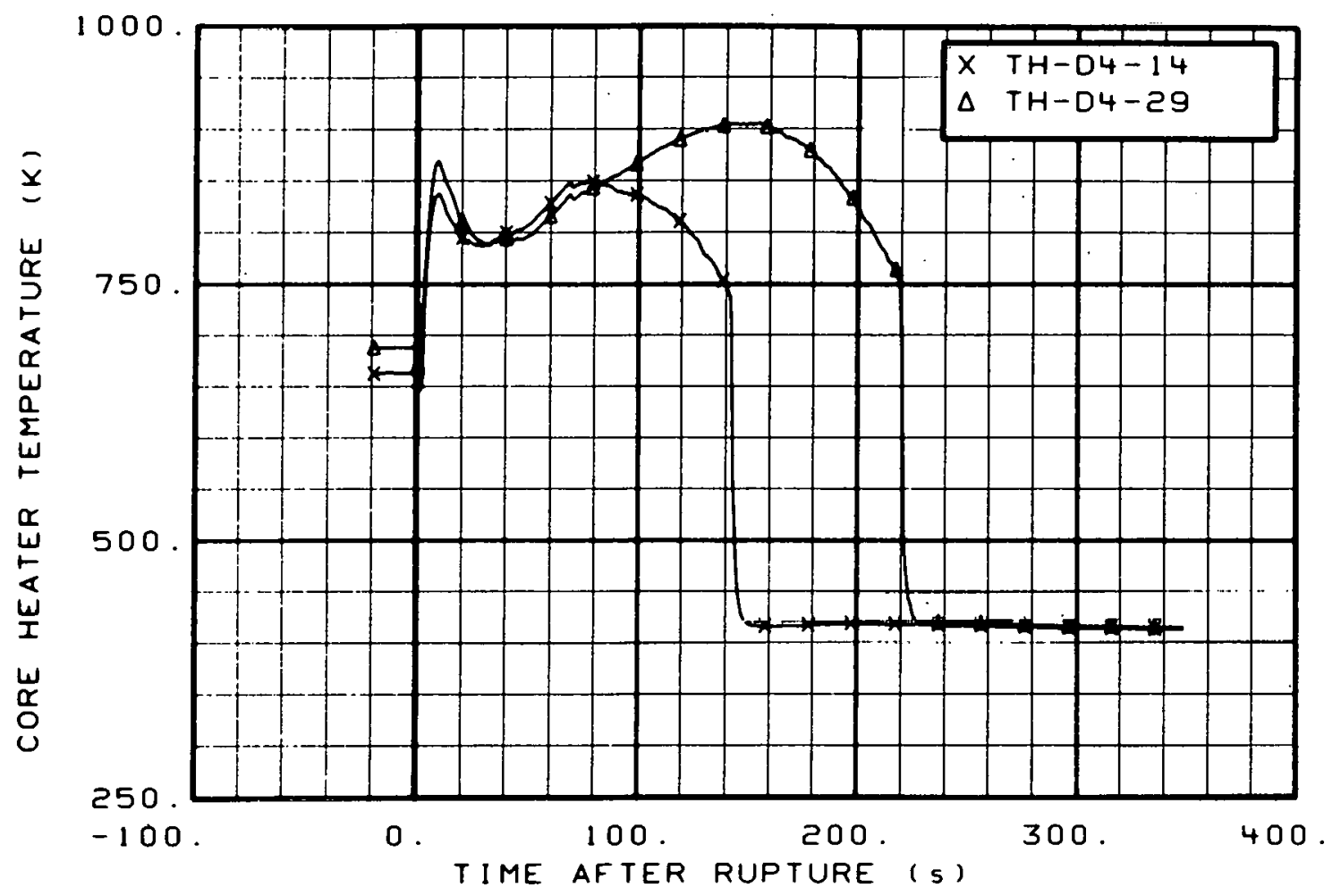

Fig. 77 Core heater temperature, Rod D-4 (TH-D4-14 and TH-D4-29), from -20 to $350 \mathrm{~s}$.

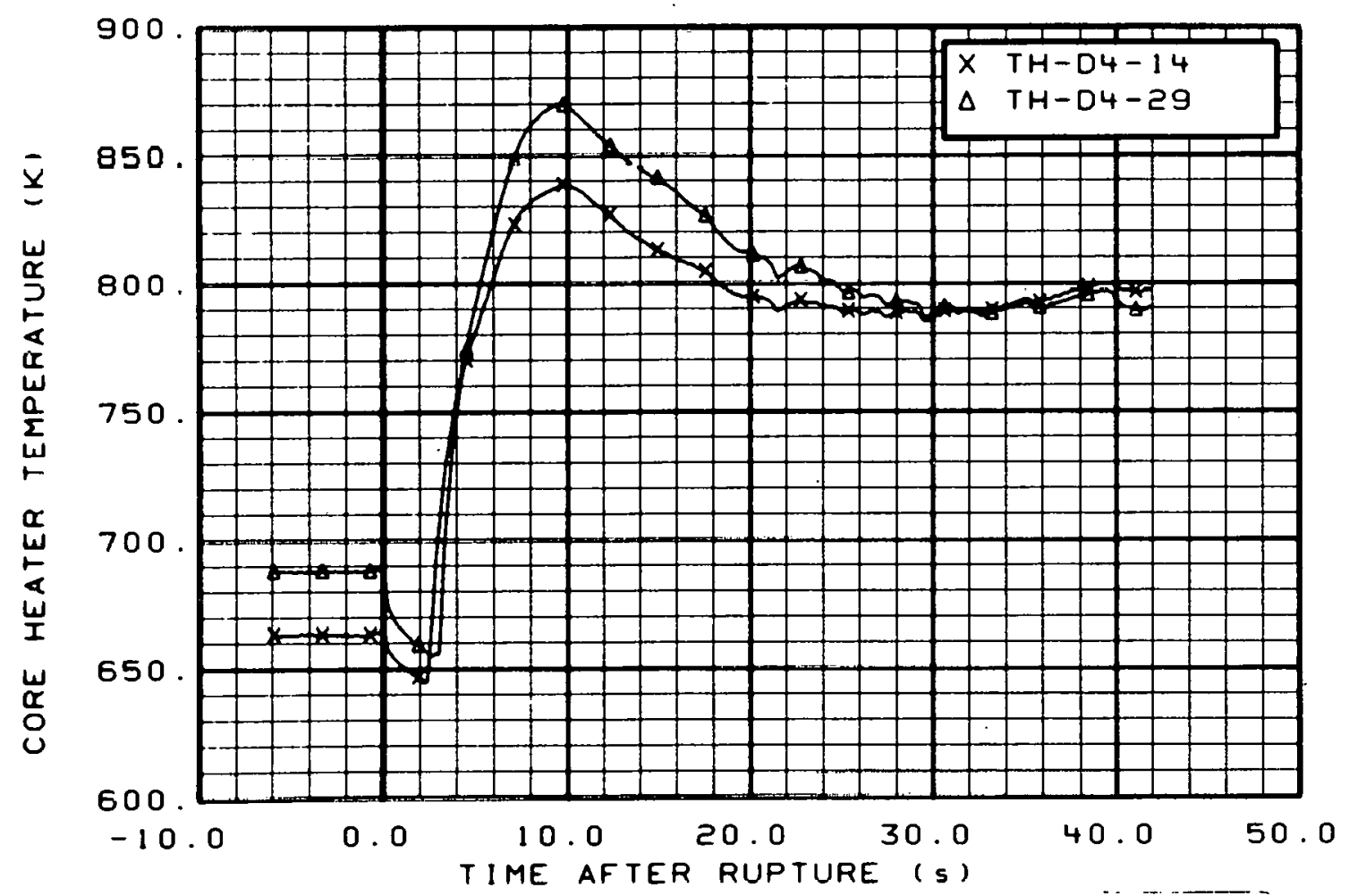

Fig. 78 Core heater temperature, Rod $D=4$ (TH-D4-14 and TH-D4-29), from -6 to $42 \mathrm{~s}$. 


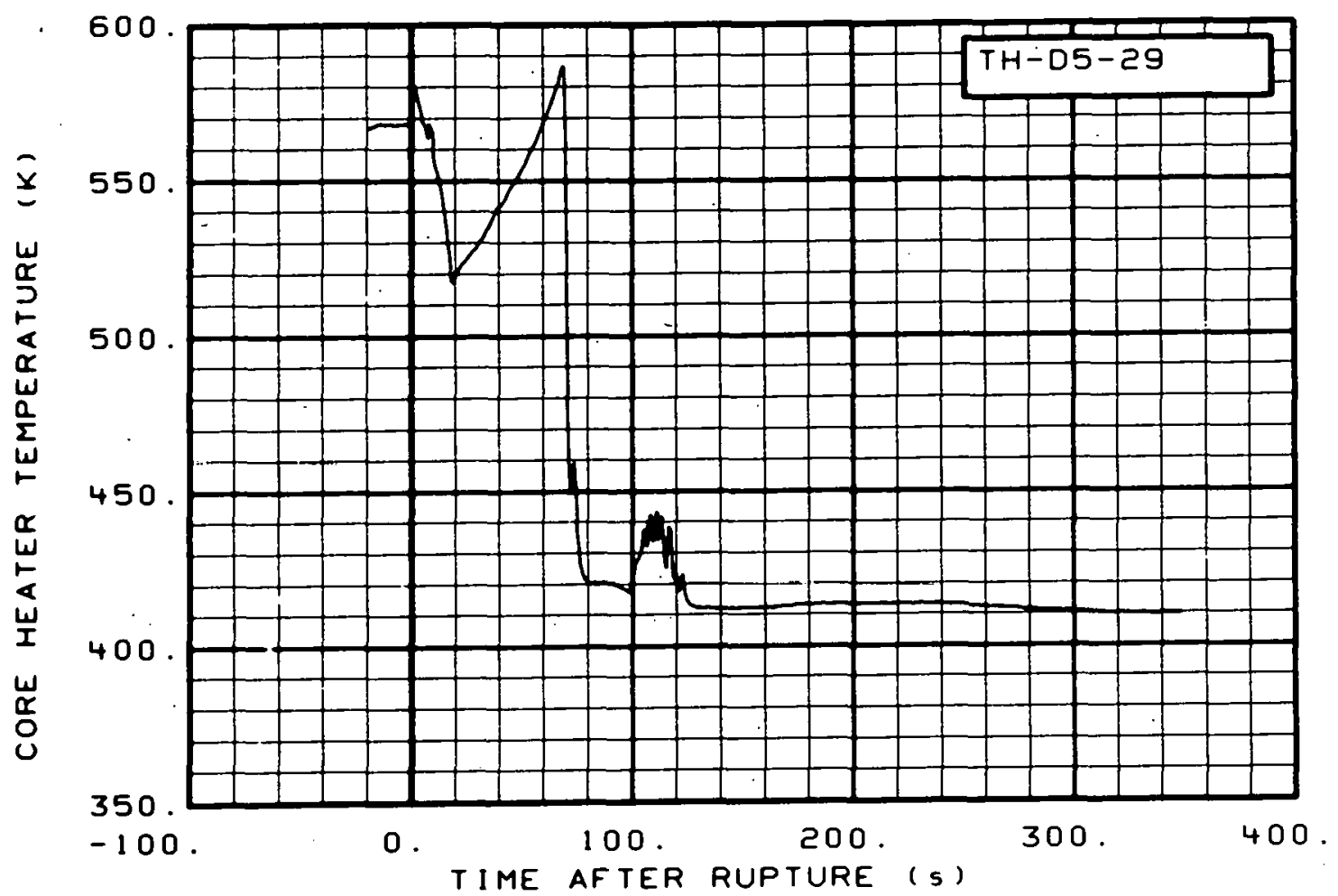

Fig. 79 Core heater temperature, Rod D-5 (TH-D5-29), from -20 to $350 \mathrm{~s}$.

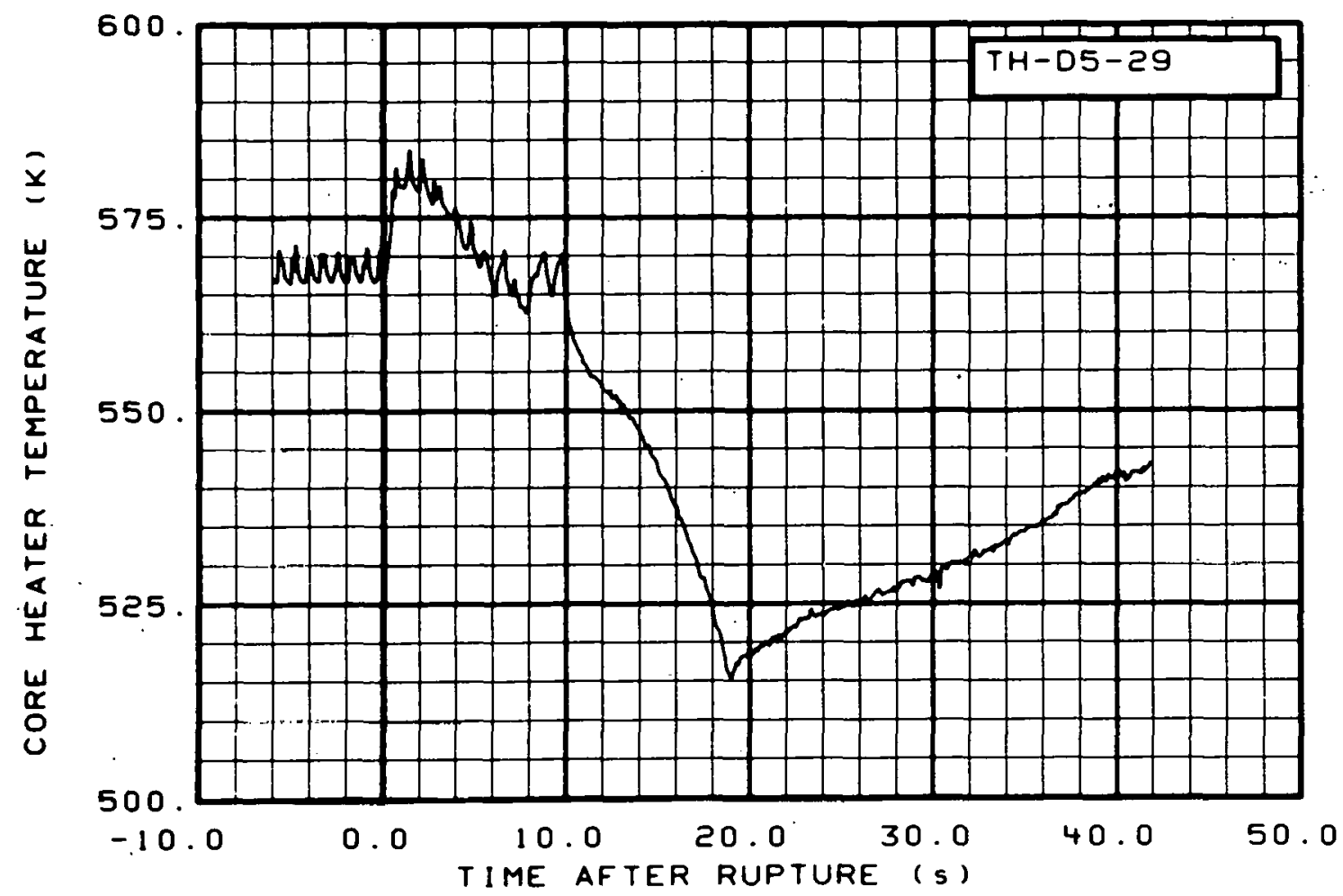

Fig. 80 Core heater temperature, Rod D-5 (TH-D5-29), from -6 to $42 \mathrm{~s}$. 


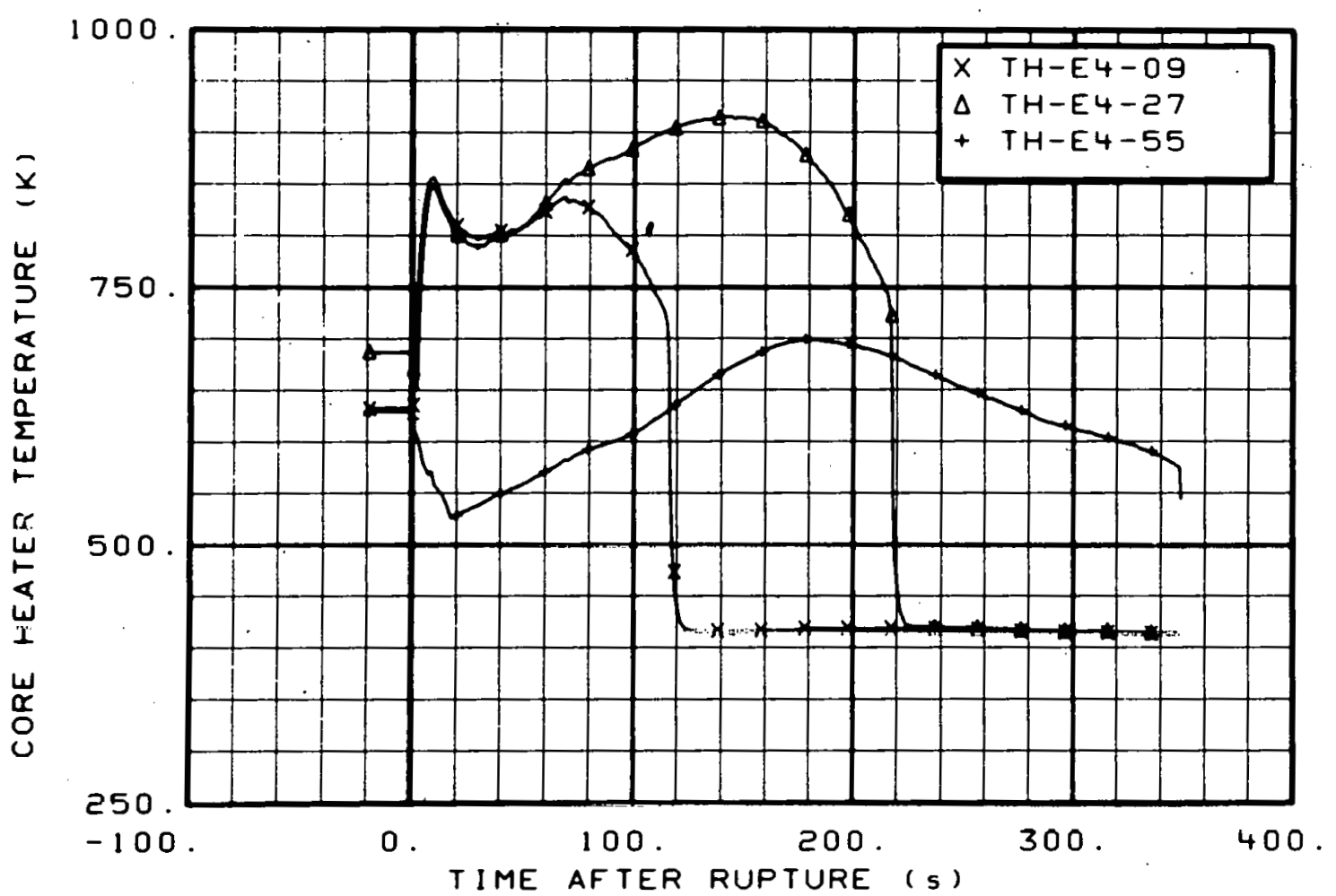

Fig. 81 Core heater temperature, Rod E-4 (TH-E4-09, TH-E4-27, and TH-E4-55), from -20 to $350 \mathrm{~s}$.

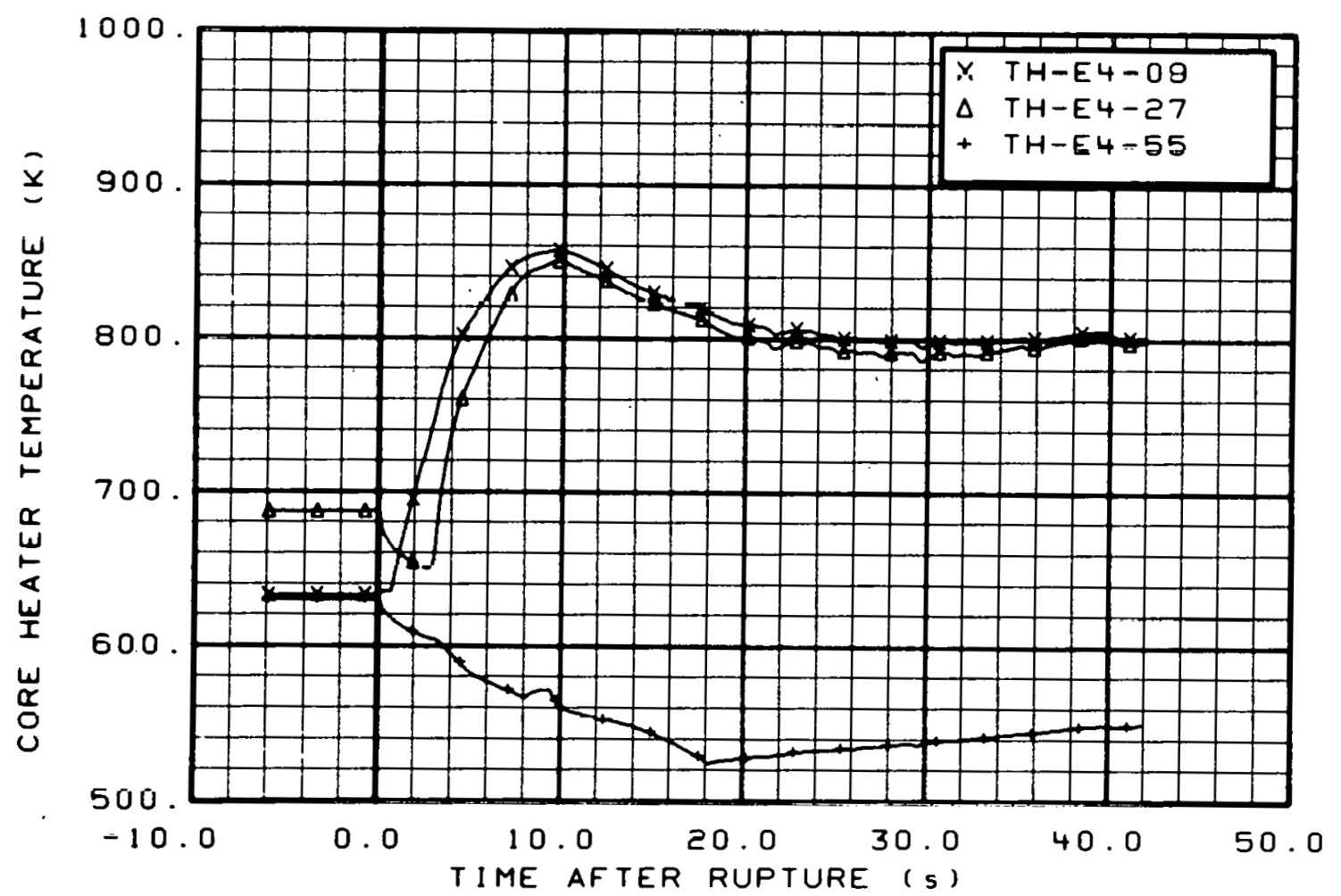

Fig. 82 Core heater temperature, Rod E-4 (TH-E4-09, TH-E4-27, and TH-E4-55), from -6 to $42 \mathrm{~s}$. 


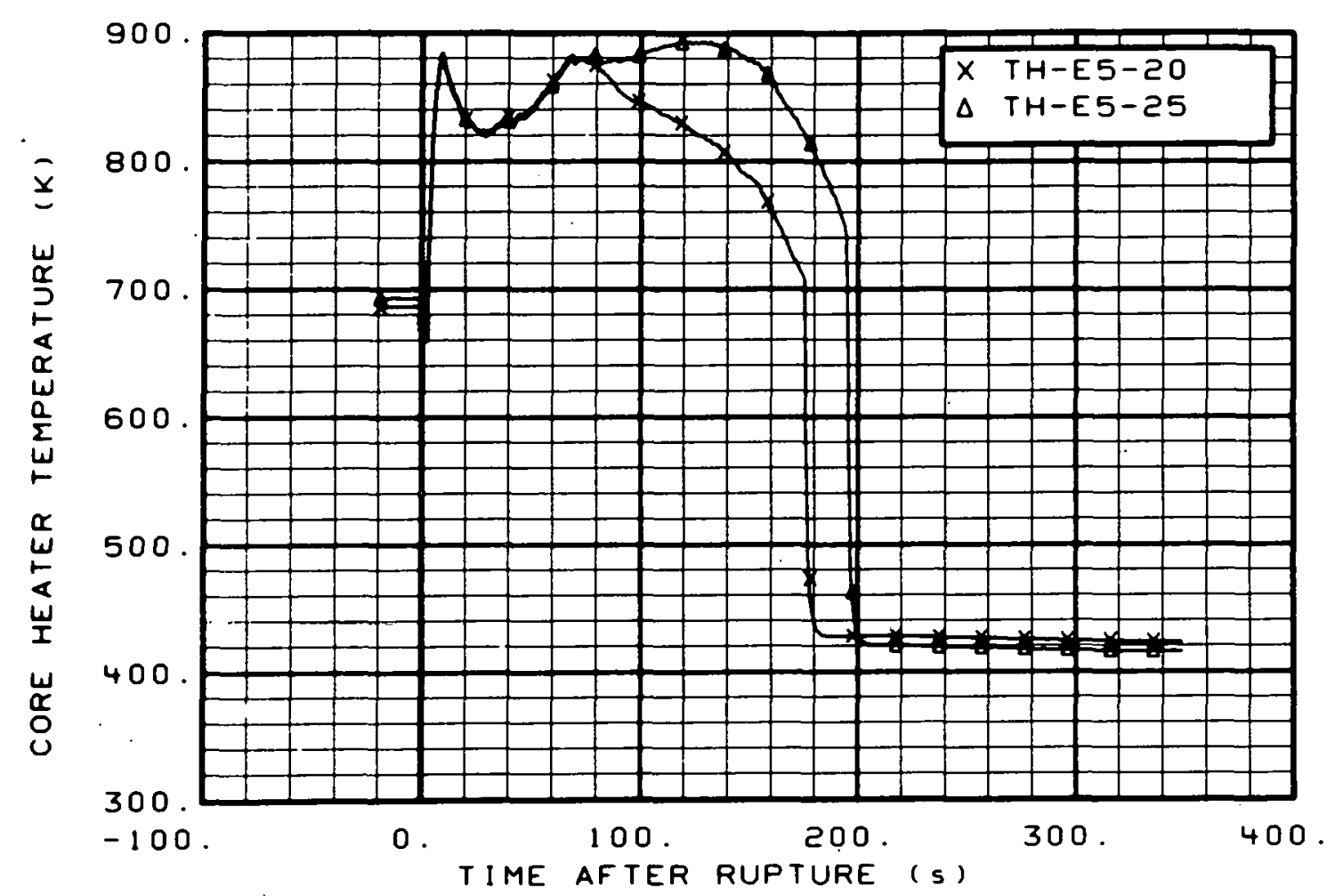

Fig. 83 Core heater temperature, Rod E-5 (TH-E5-20 and TH-E5-25), from -20 to $350 \mathrm{~s}$.

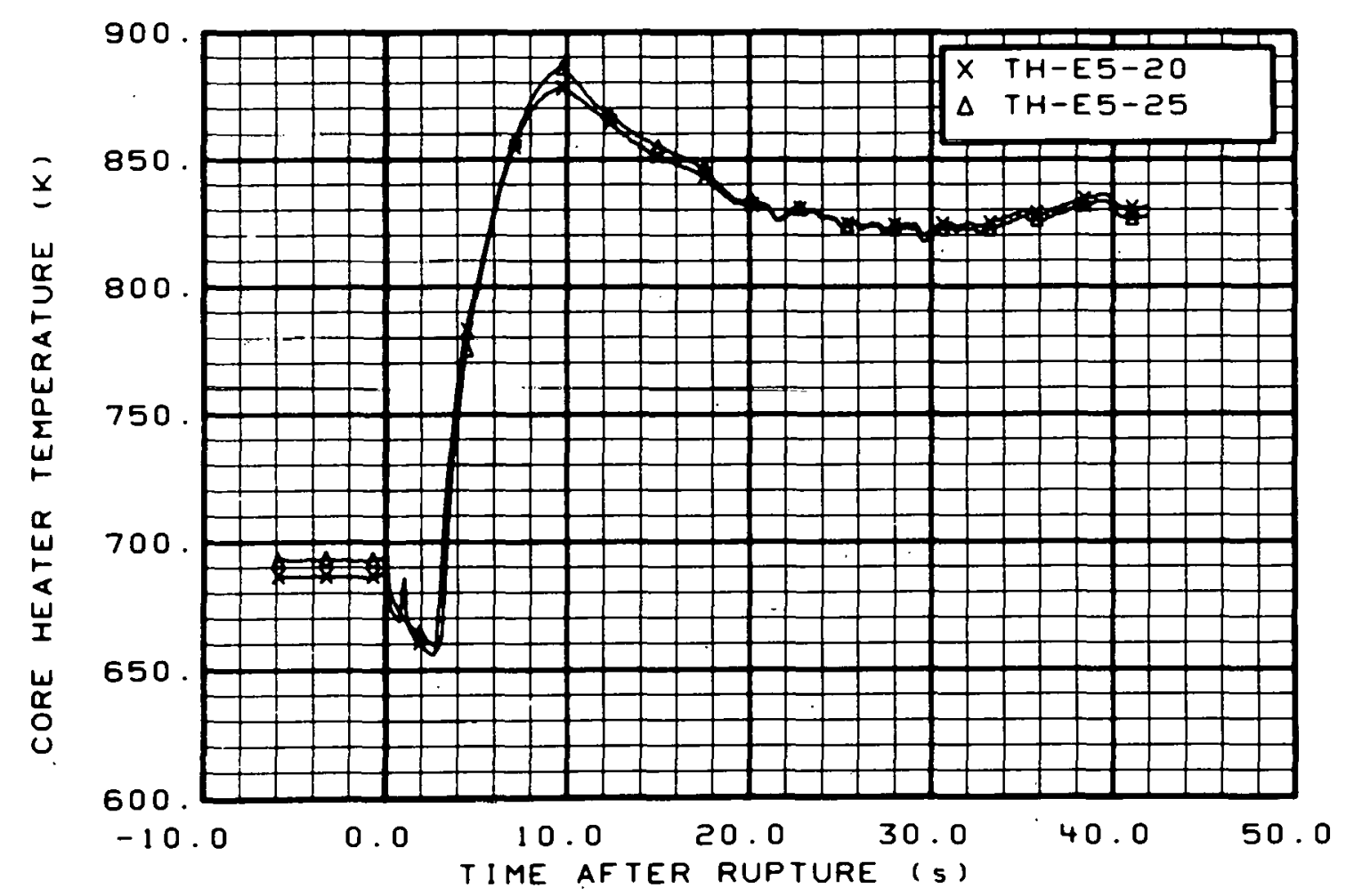

Fig. 84 Core heater temperature, Rod E-5 (TH-E5-20 and TH-E5-25), from -6 to $42 \mathrm{~s}$. 


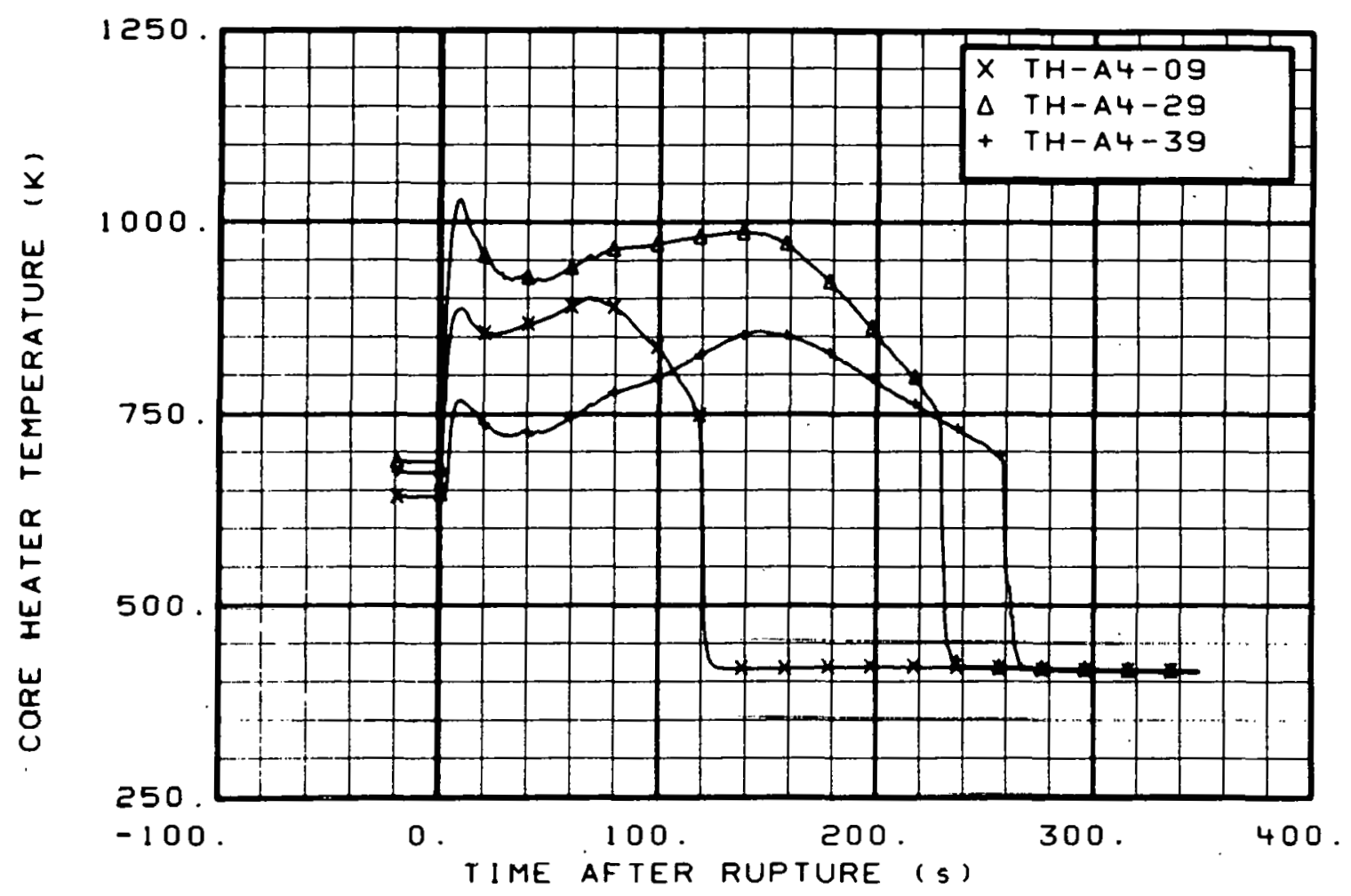

Fig. 85 Core heater temperature, Rod A-4 (TH-A4-09, TH-A4-29, and $\mathrm{TH}-\mathrm{A} 4-39$ ), from -20 to $350 \mathrm{~s}$.

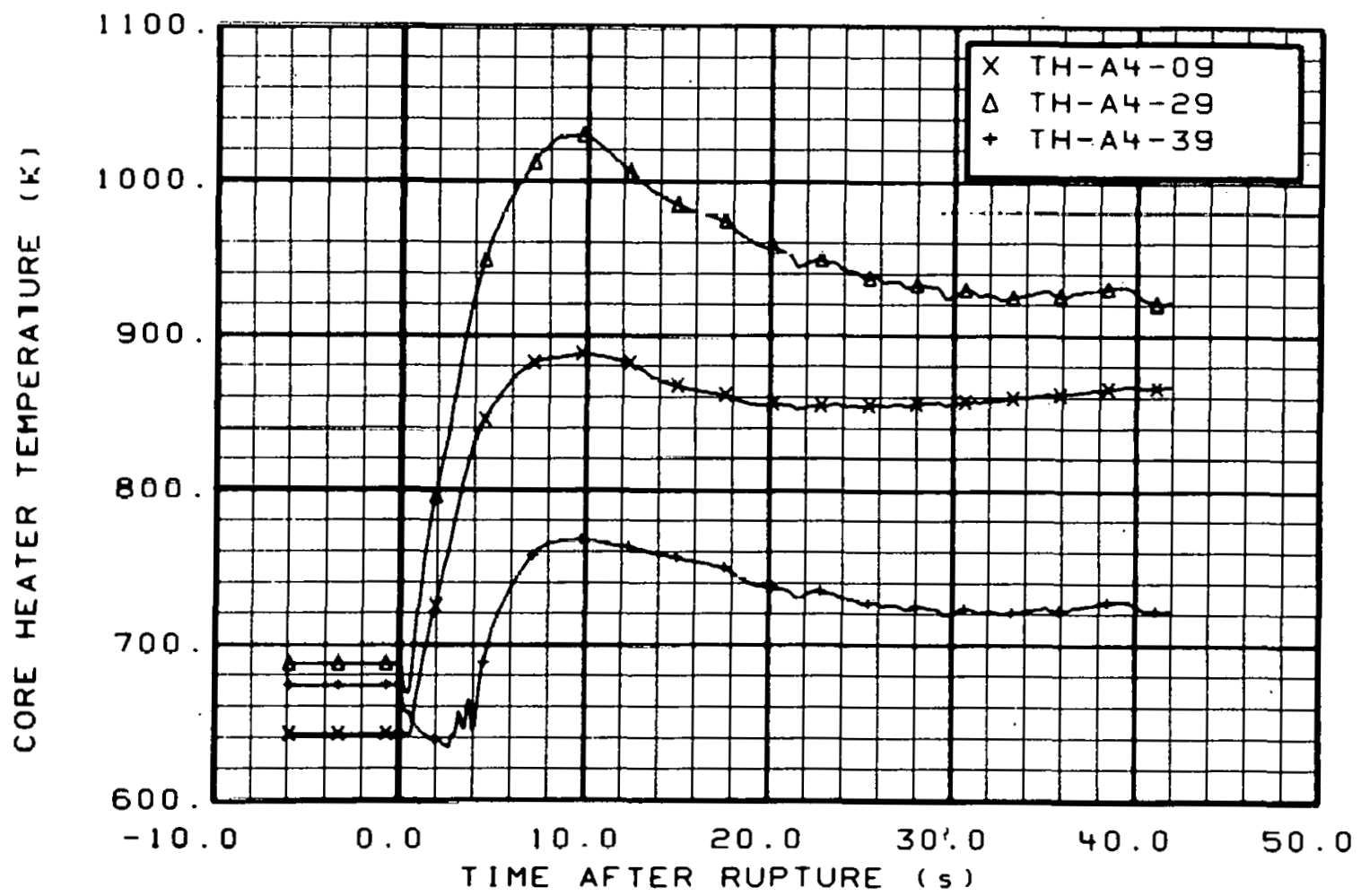

Fig. 86 Core heater temperature, Rod A-4 (TH-A4-09, TH-A4-29, and TH-A4-39), from -6 to $42 \mathrm{~s}$. 


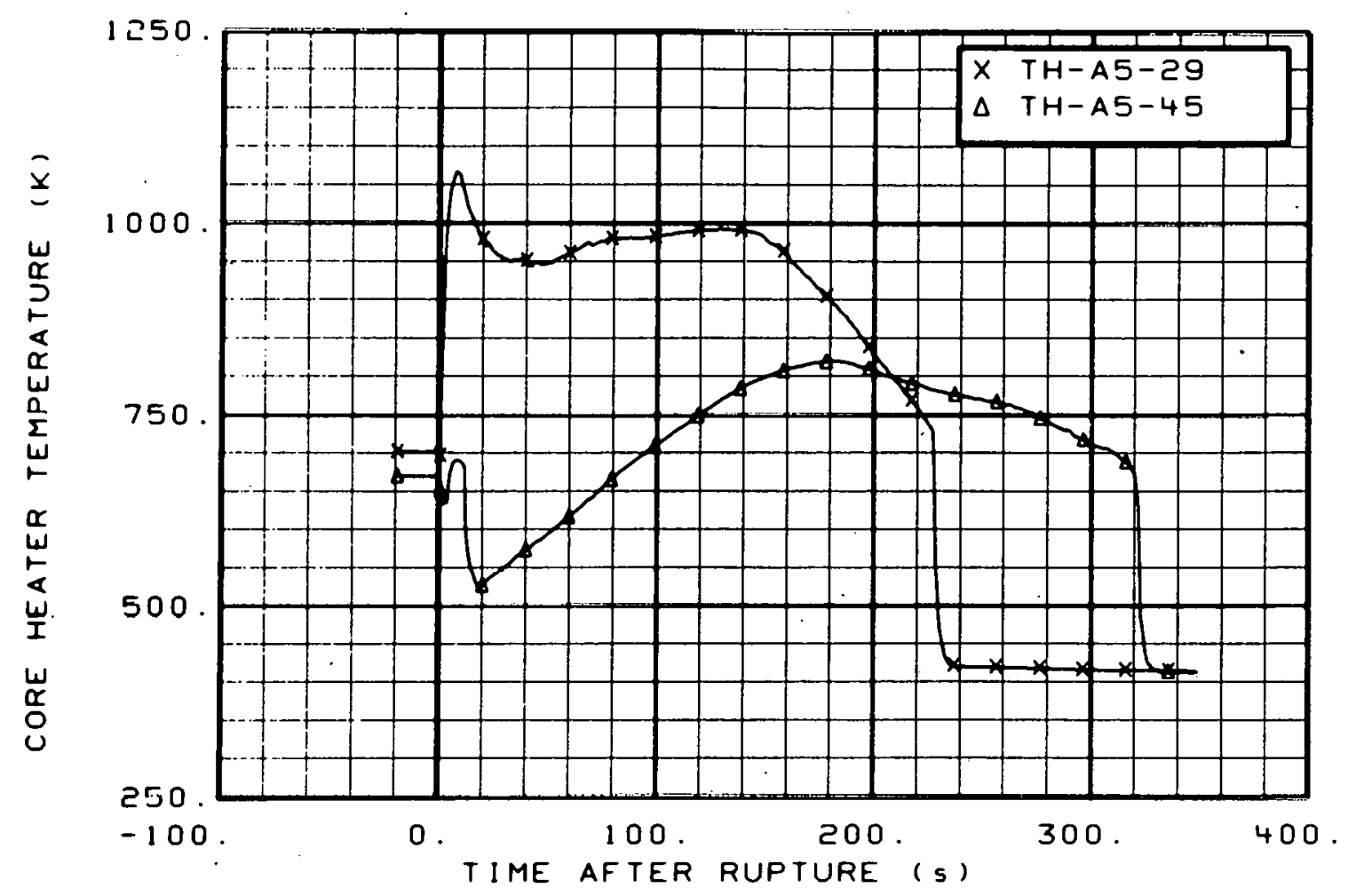

Fig. 87 Core heater temperature, Rod A-5 (TH-A5-29 and TH-A5-45), from -20 to $350 \mathrm{~s}$.

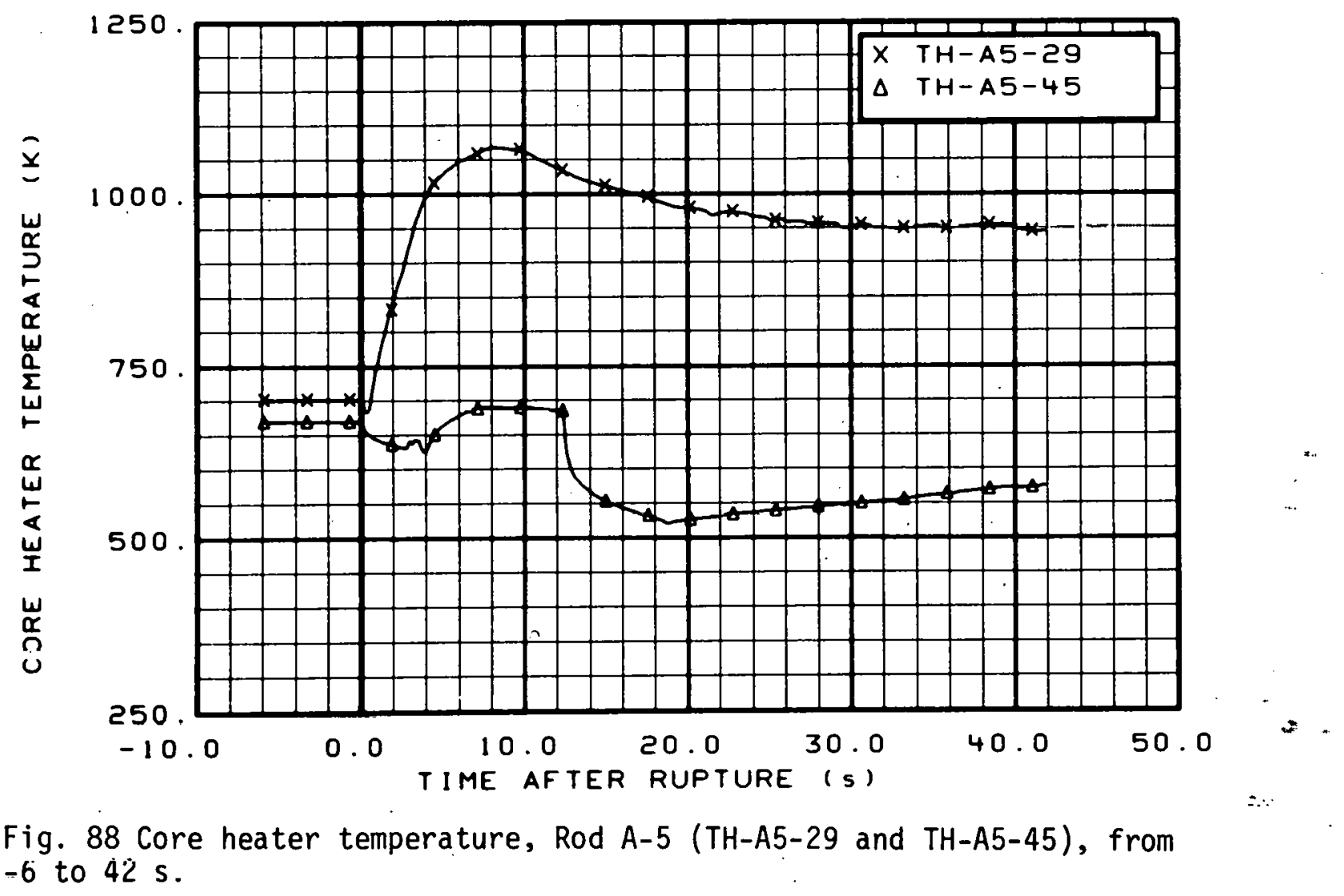




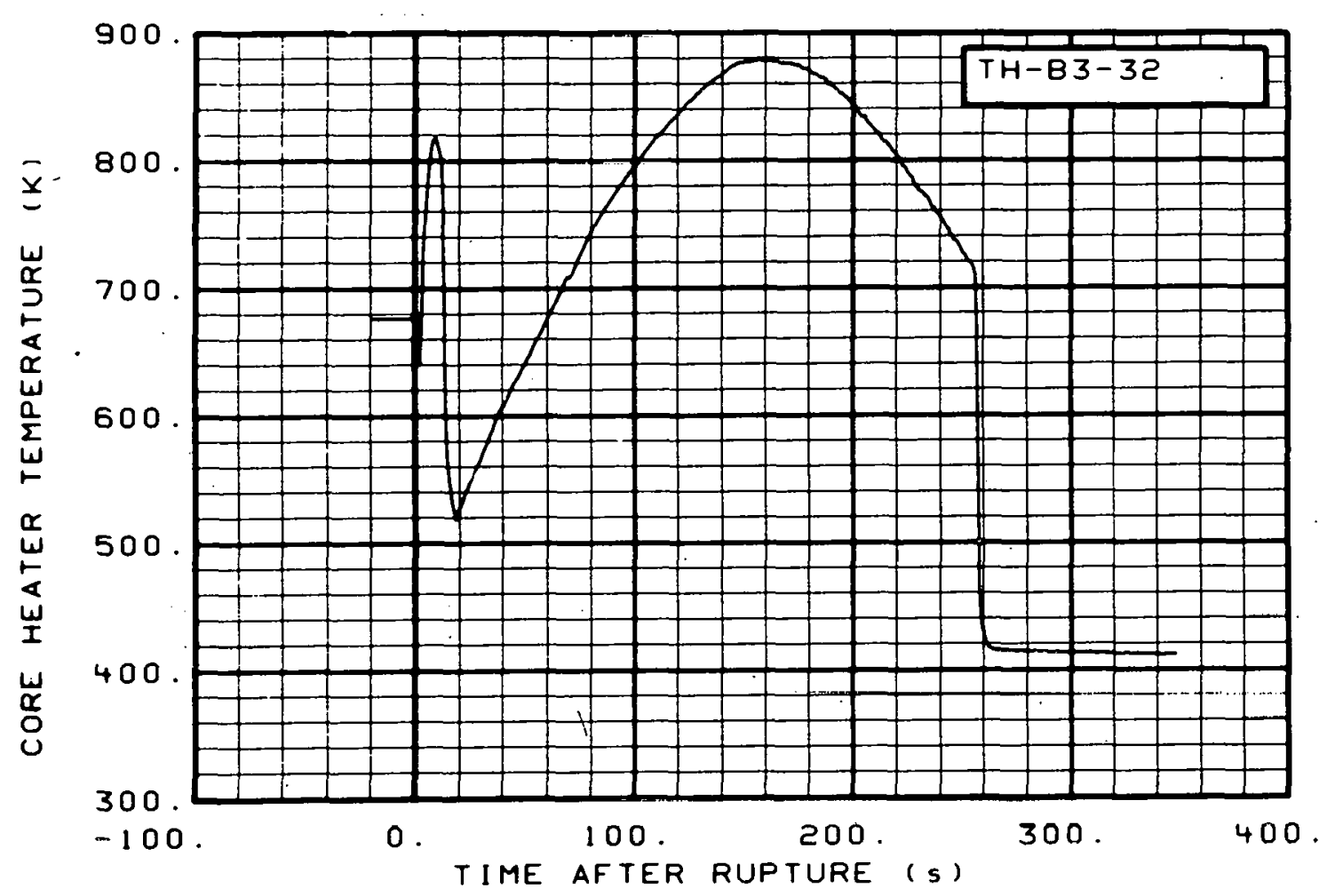

Fig. 89 Core heater temperature, Rod B-3 (TH-B3-32), from -20 to $350 \mathrm{~s}$.

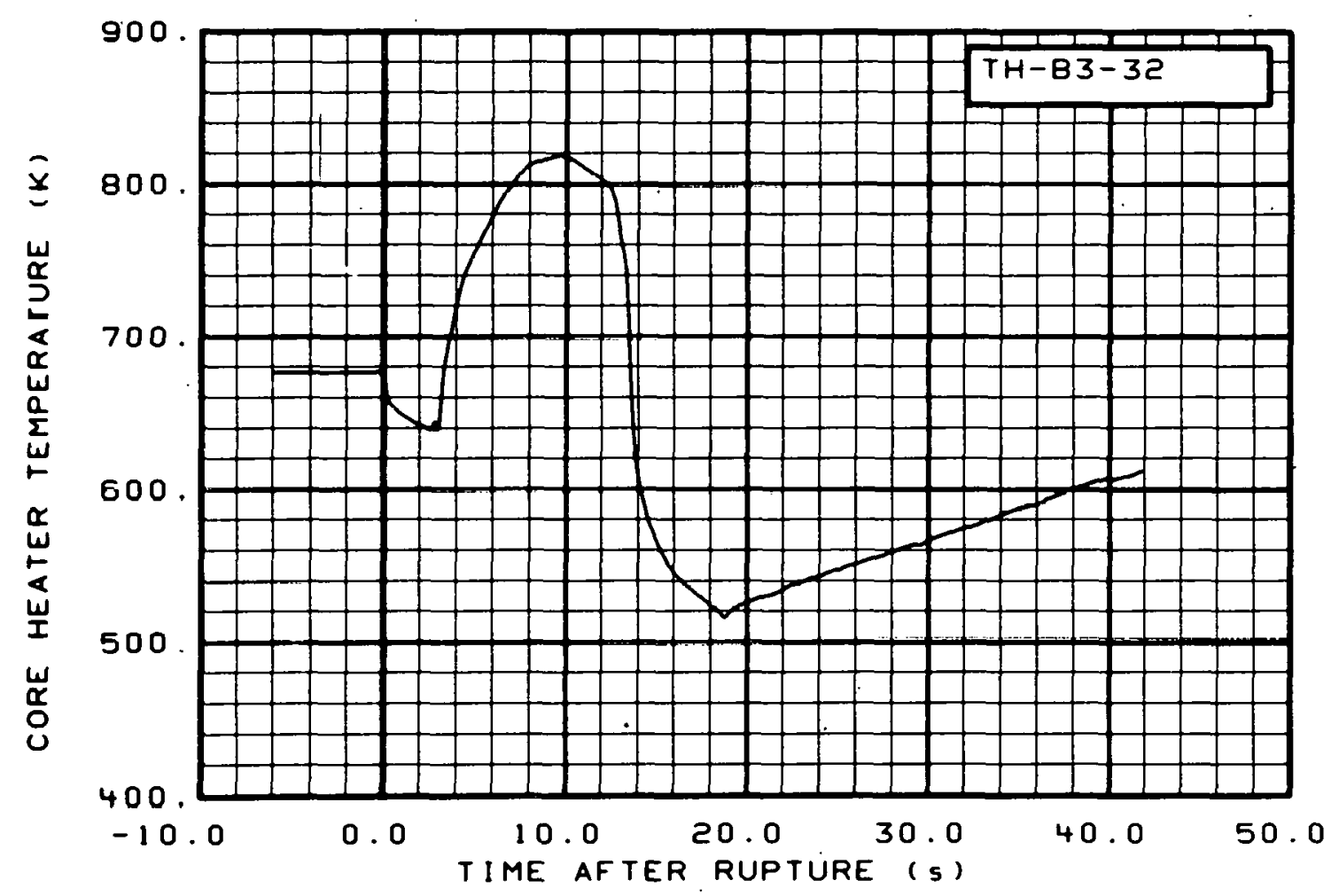

Fig. 90 Core heater temperature, Rod B-3 (TH-B3-32), from -6 to $42 \mathrm{~s}$. 


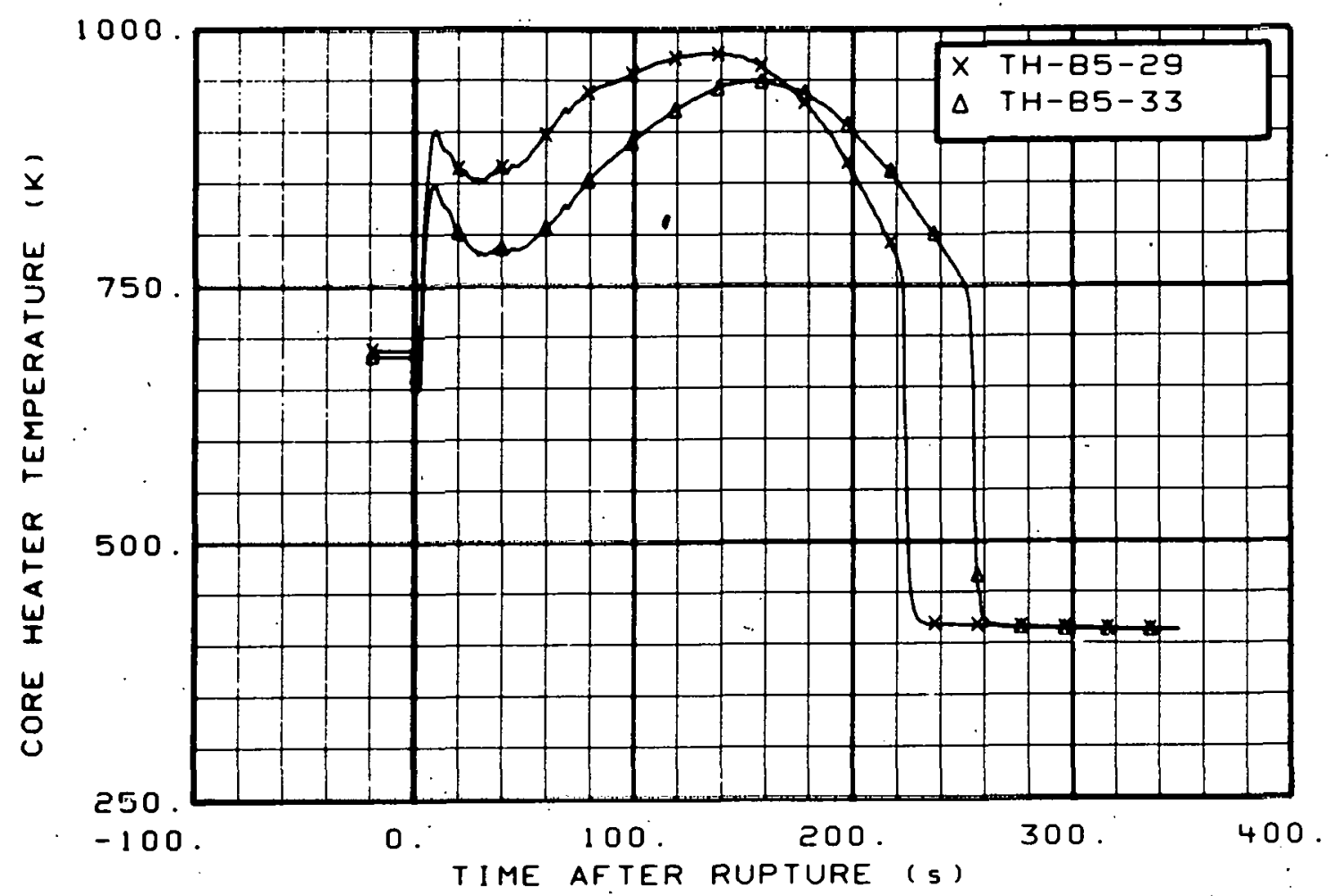

Fig. 91 Core heater temperature, Rod B-5 (TH-B5-29 and TH-B5-33), from -20 to $350 \mathrm{~s}$.

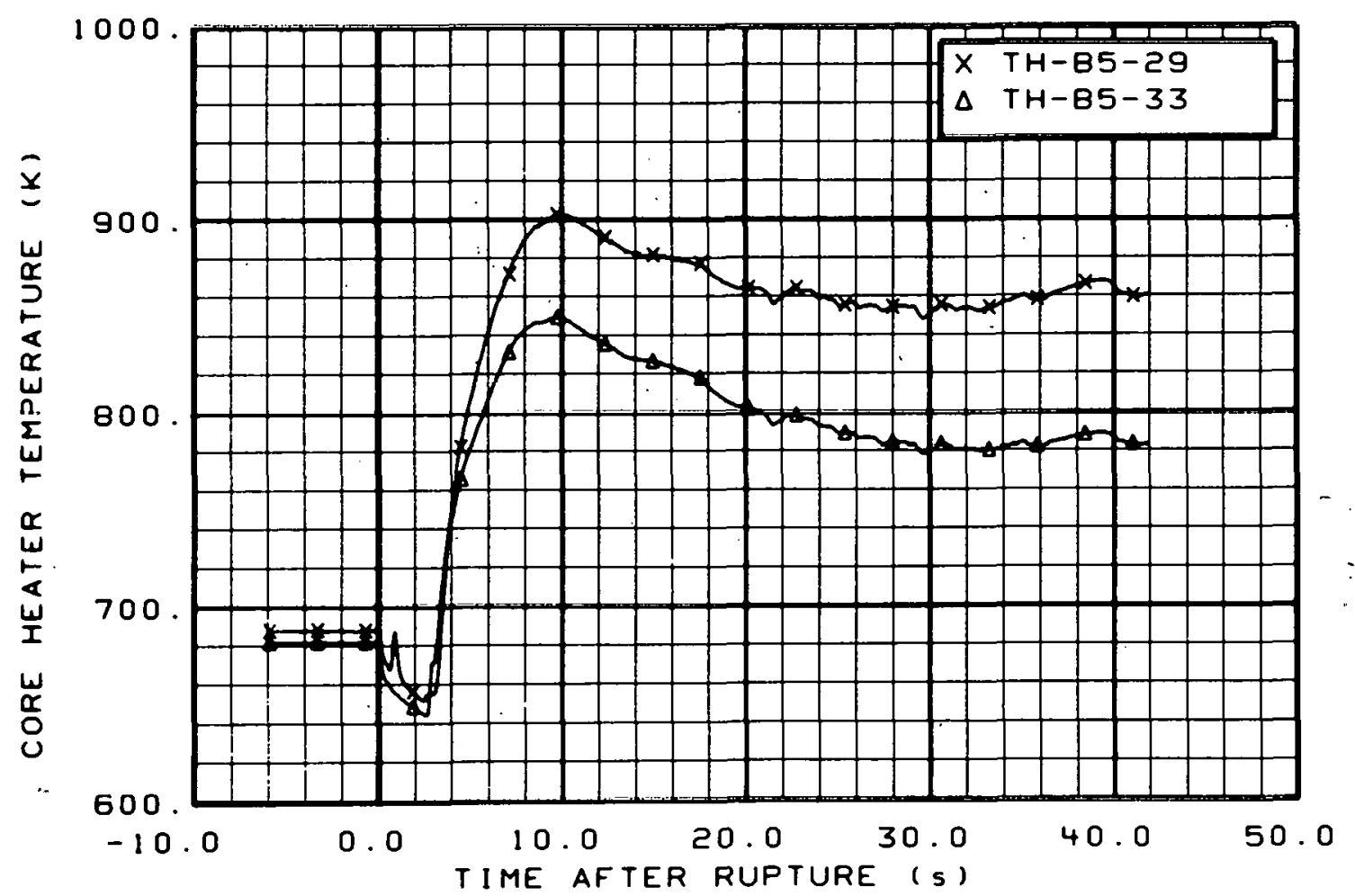

Fig. 92 Core heater temperature, Rod B-5 (TH-B5-29 and TH-B5-33), from -6 to $42 \mathrm{~s}$. 


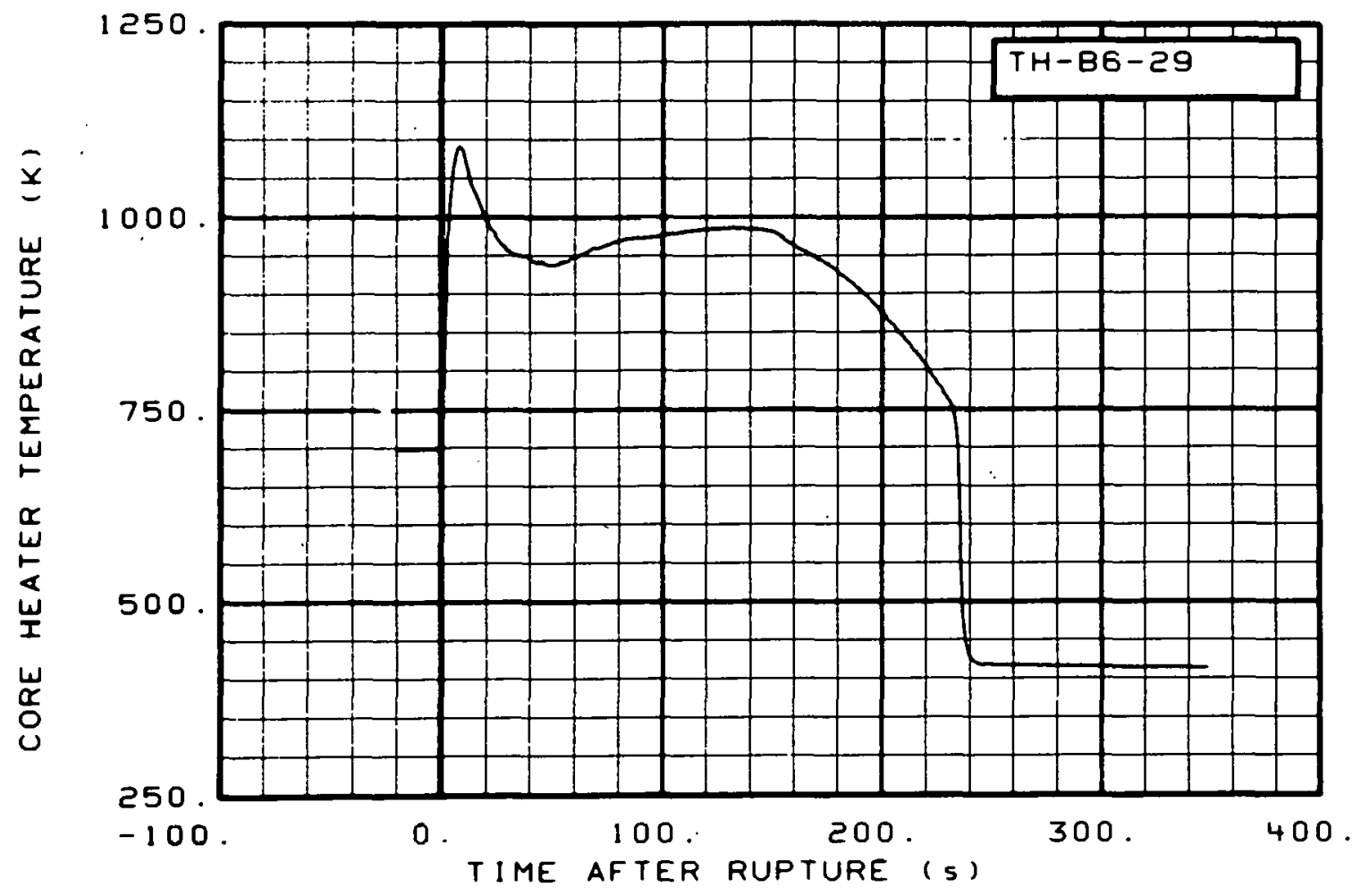

Fig. 93 Core heater temperature, Rod B-6 (TH-B6-29), from -20 to $350 \cdot \mathrm{s}$.

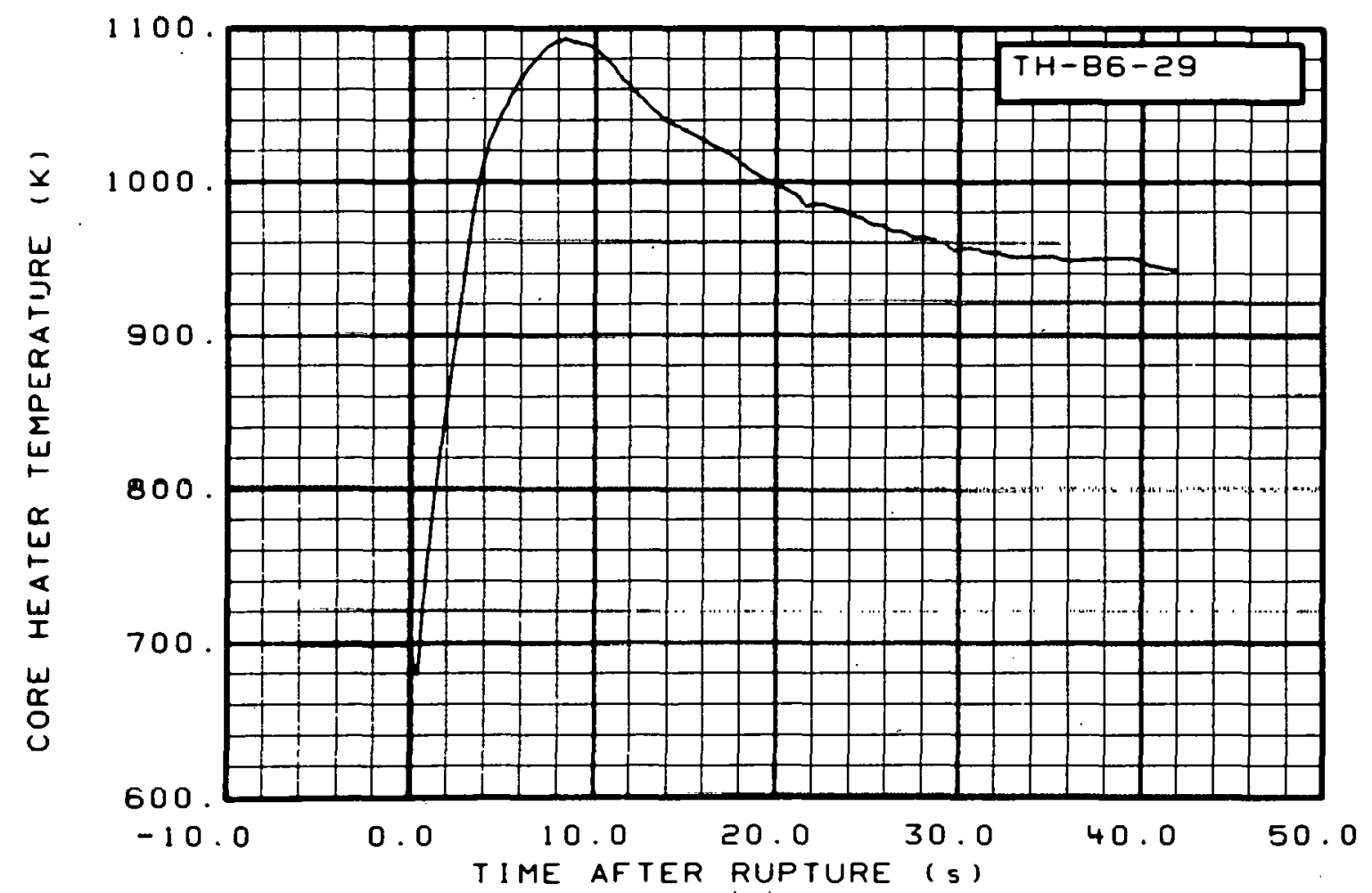

Fig. 94 Core heater temperature, Rod B-6 (TH-B6-29), from -6 to $42 \mathrm{~s}$. 


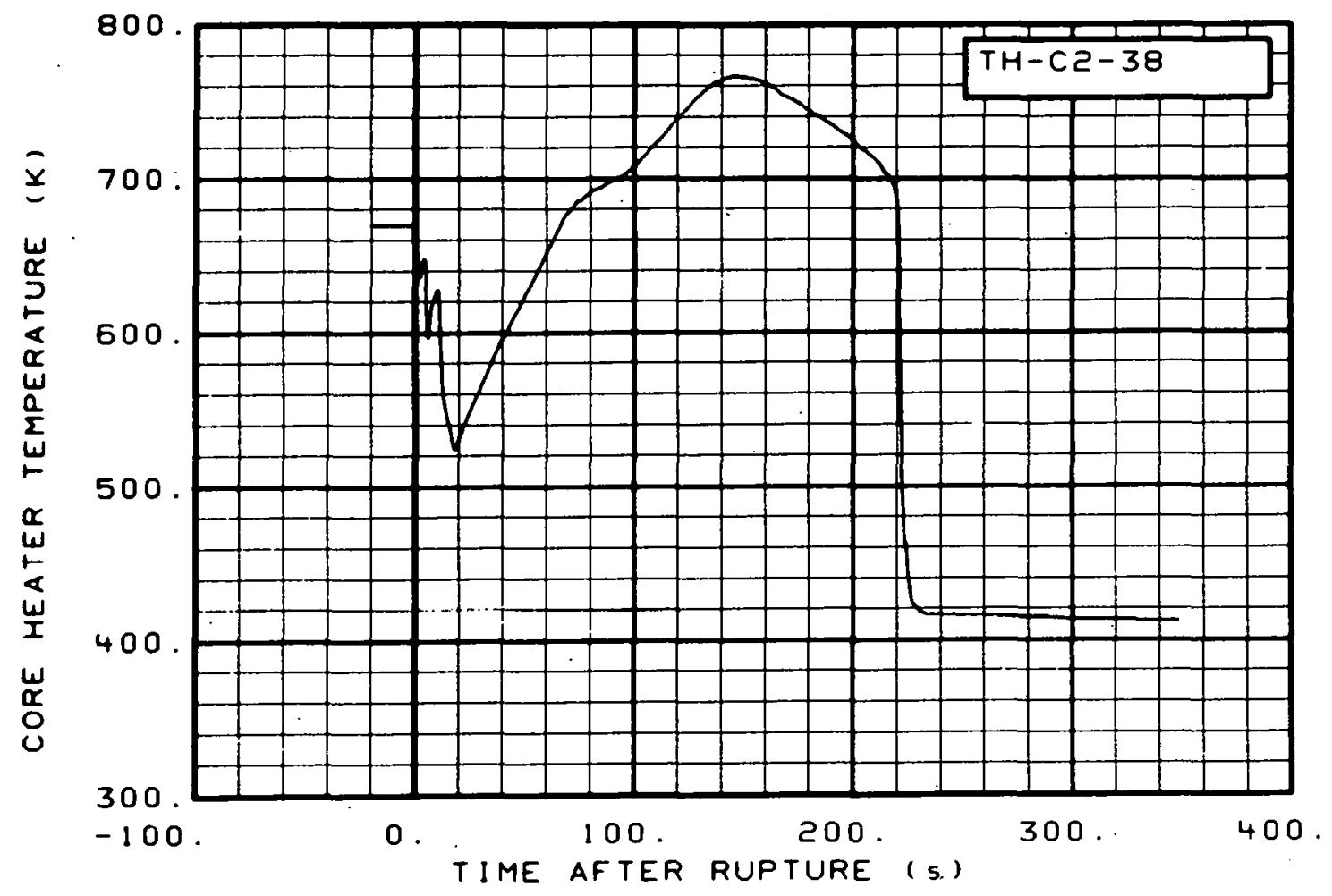

Fig. 95 Core heater temperature, Rod C-2 (TH-C2-38), from -20 to $350 \mathrm{~s}$.

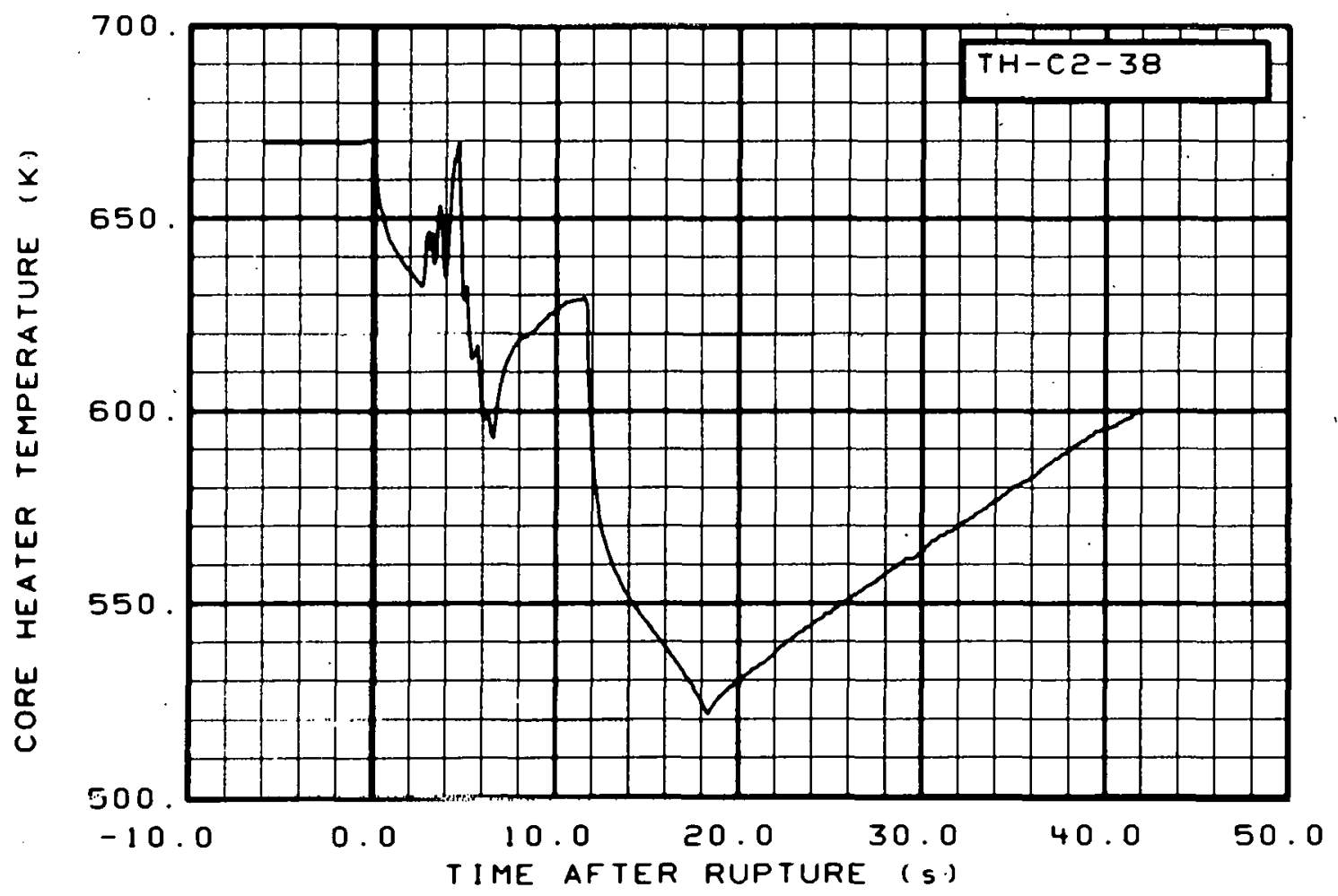

Fig. 96 Core heater temperature, Rod C-2 (TH-C2-38), from -6 to 4 ? $\mathrm{s}$. 


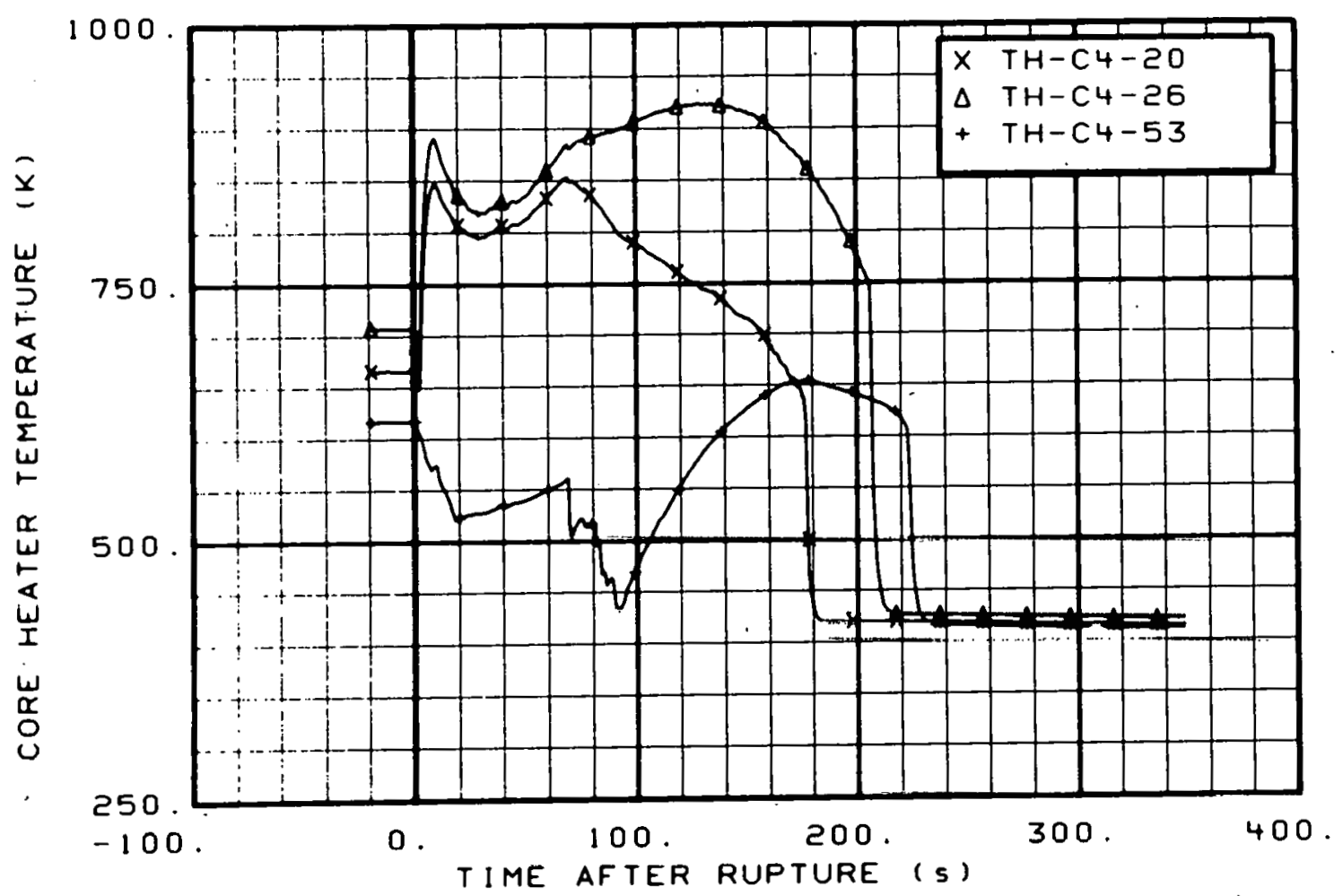

Fig. 97 Core heater temperature, Rod C-4 (TH-C4-20, TH-C4-26, and $\mathrm{TH}-(4-53)$, from -20 to $350 \mathrm{~s}$.

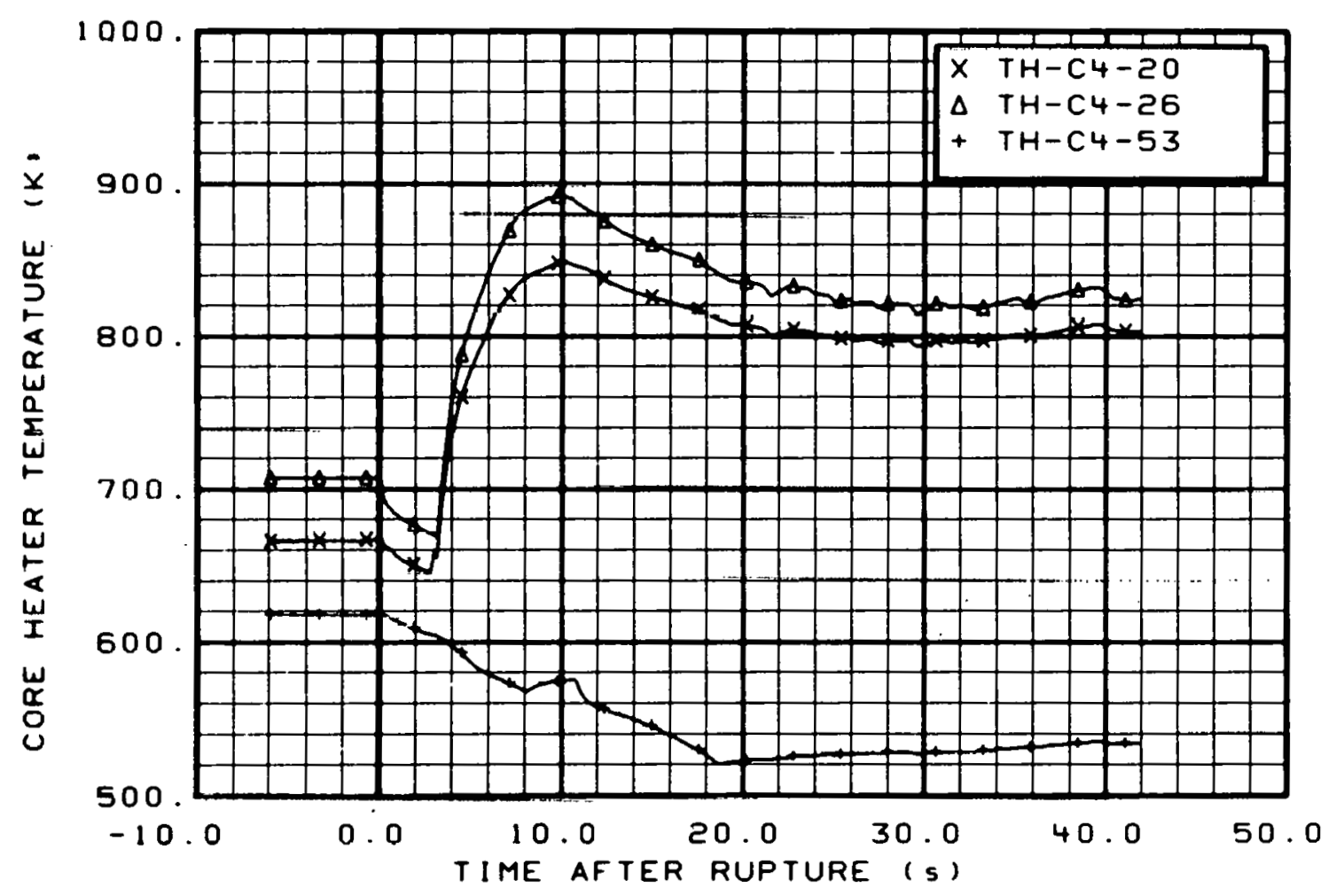

Fig. 98 Core heater temperature, Rod C-4 (TH-C4-20, TH-C4-26, and $\mathrm{TH}-(4-53)$, from -6 to $42 \mathrm{~s}$. 


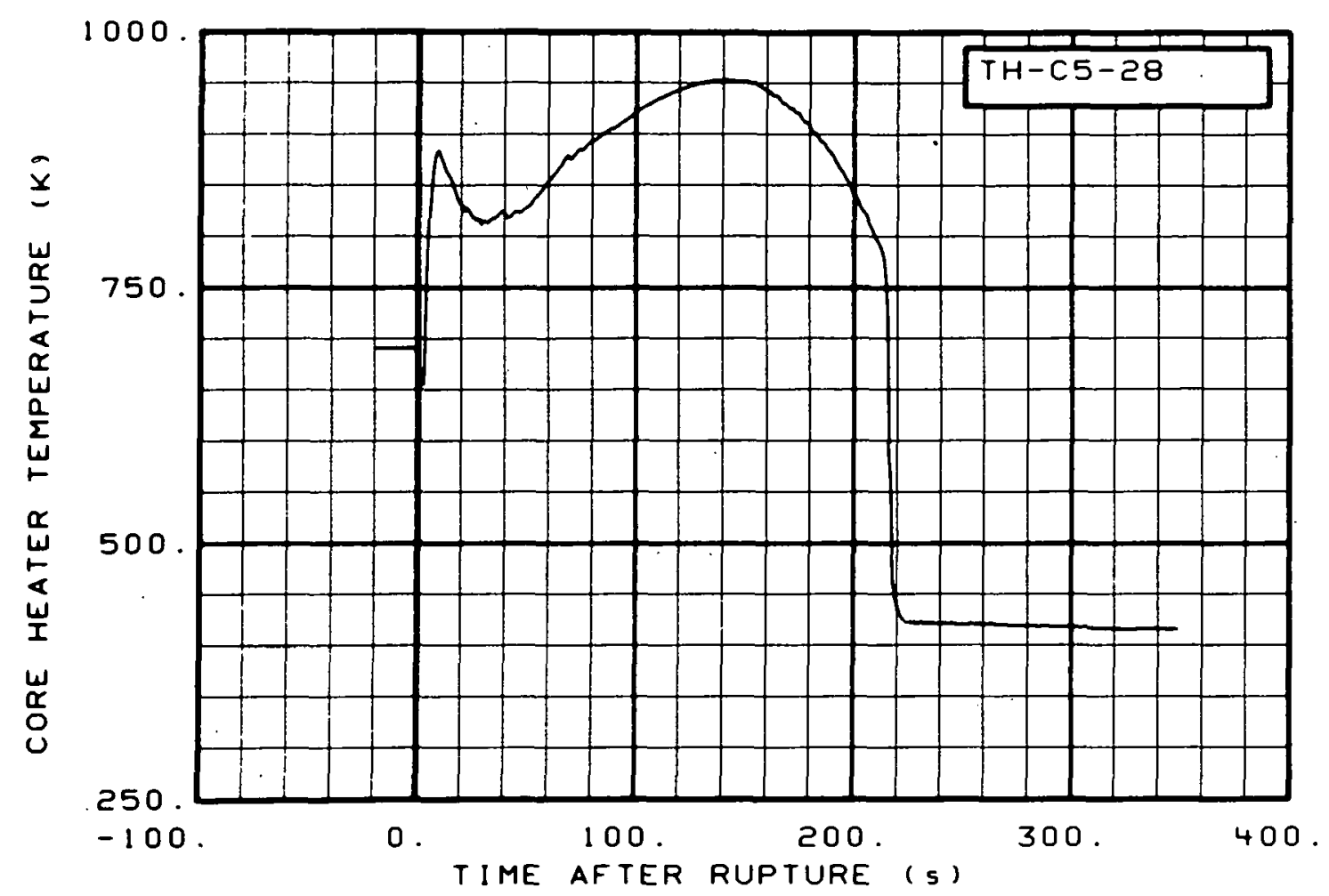

Fig. 99 Core heater temperature, Rod $\mathrm{C}-5(\mathrm{TH}-\mathrm{C} 5-28)$, from -20 to $350 \mathrm{~s}$.

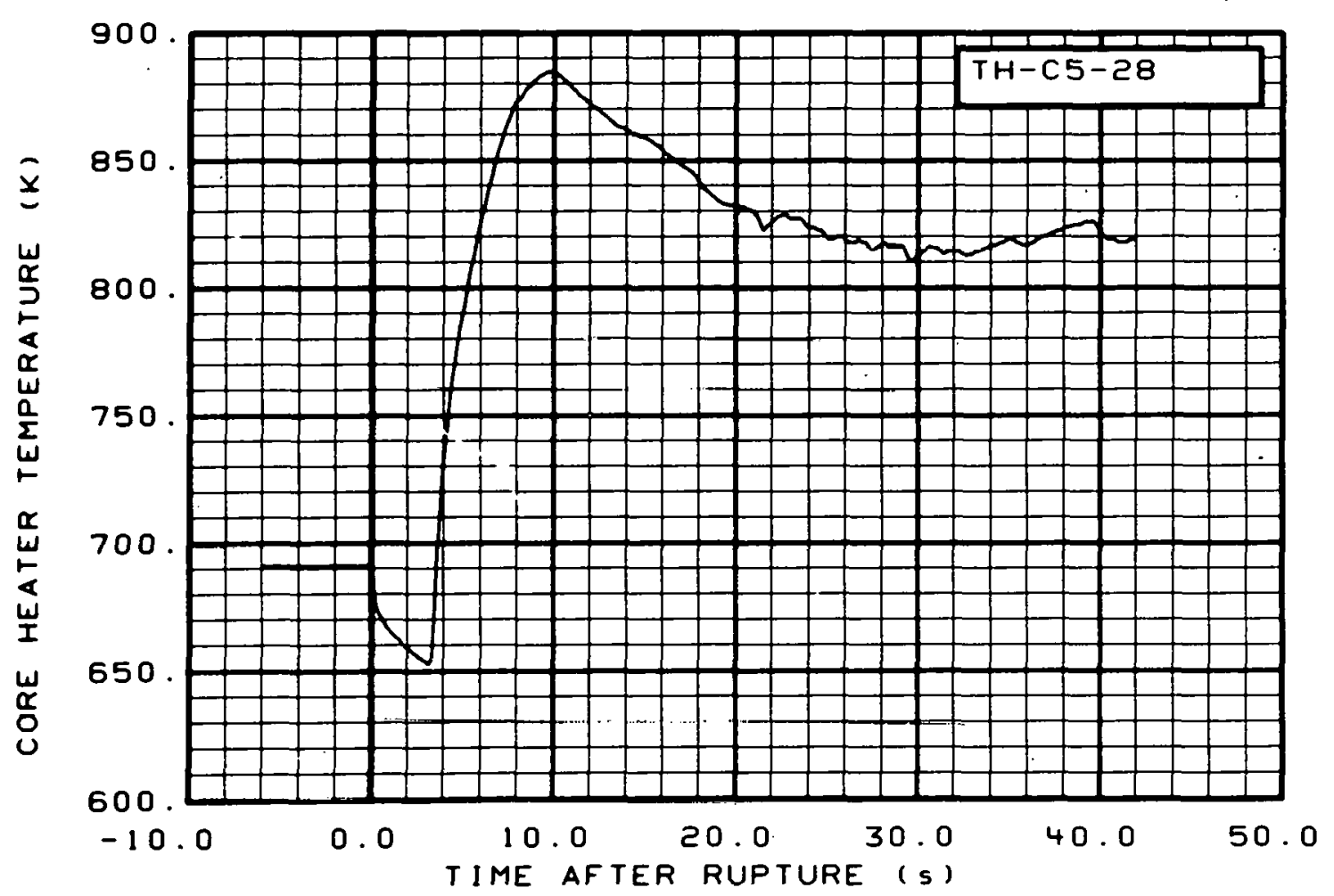

Fig. 100 Core heater temperature, Rod $\mathrm{C}-5(\mathrm{TH}-\mathrm{C} 5-28)$, from -6 to $42 \mathrm{~s}$. 


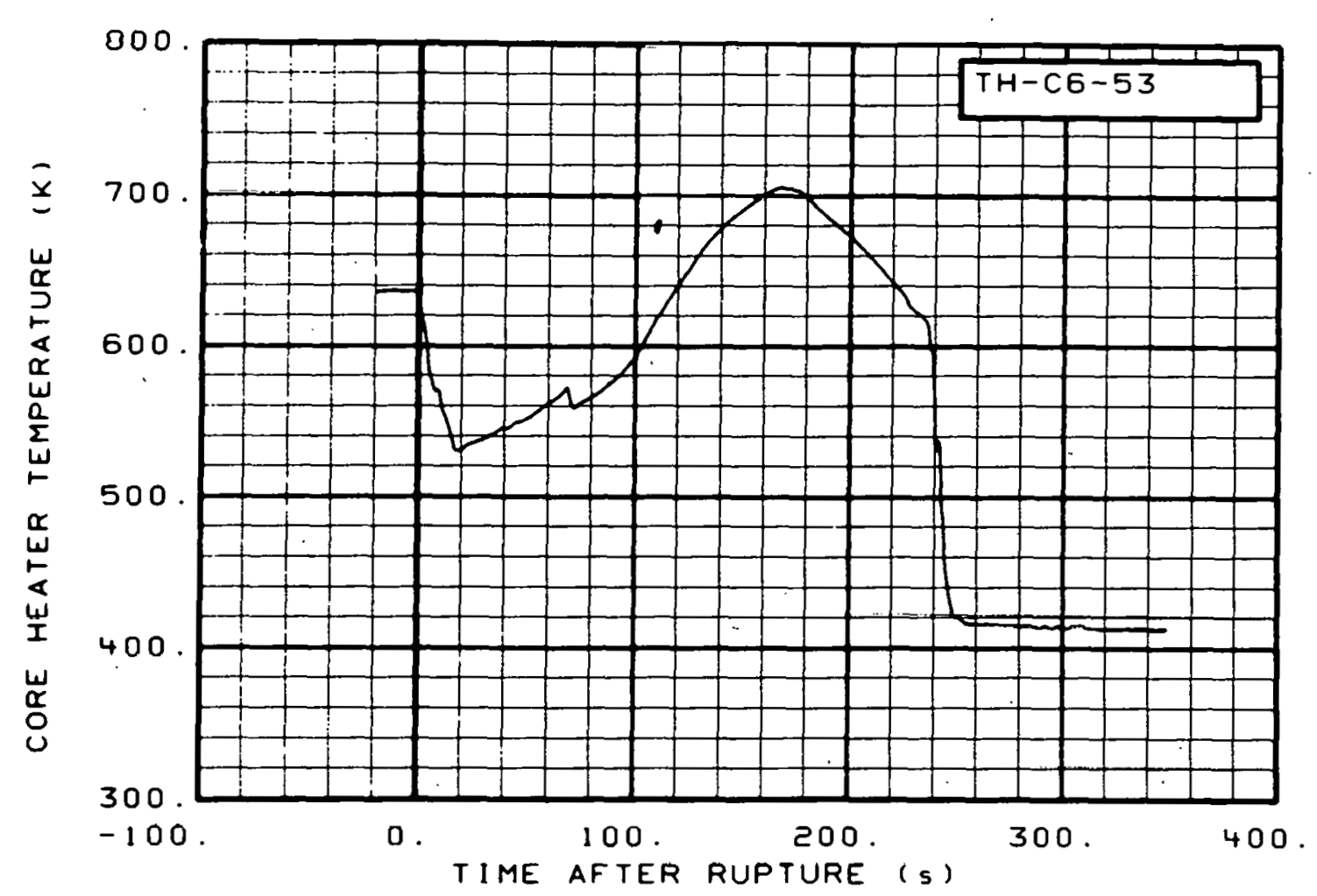

Fig. 101 Core heater temperature, Rod C-6 (TH-C6-53), from -20 to $350 \mathrm{~s}$.

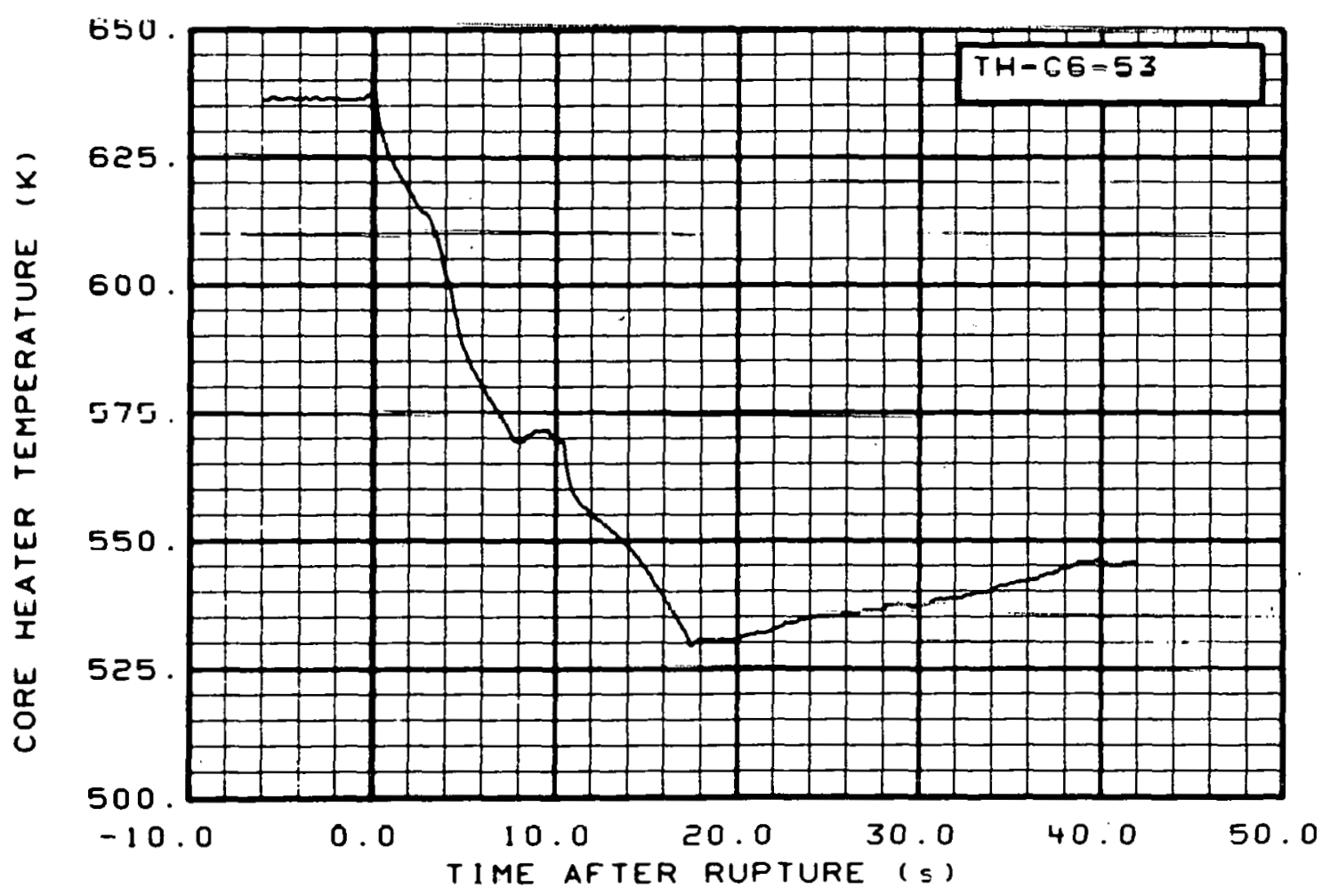

Fig. 102 Core heater temperature, Rod $\mathrm{C}-6(\mathrm{TH}-\mathrm{C} 6-53)$, from -6 to $42 \mathrm{~s}$. 


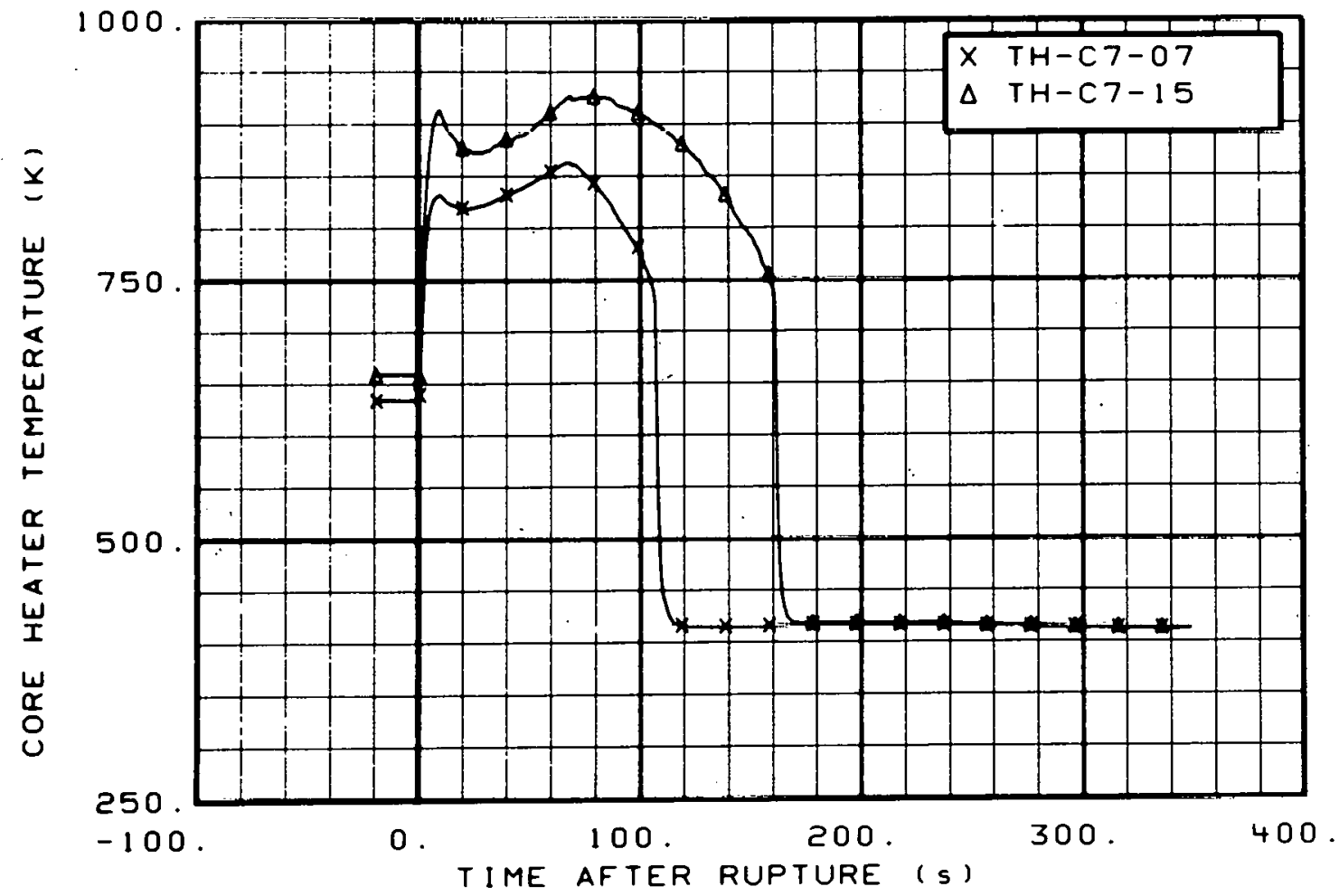

Fig. 103 Core heater temperature, Rod $\mathrm{C}-7$ (TH-C7-07 and $\mathrm{TH}-\mathrm{C} 7-15)$, from -20 to $350 \mathrm{~s}$.

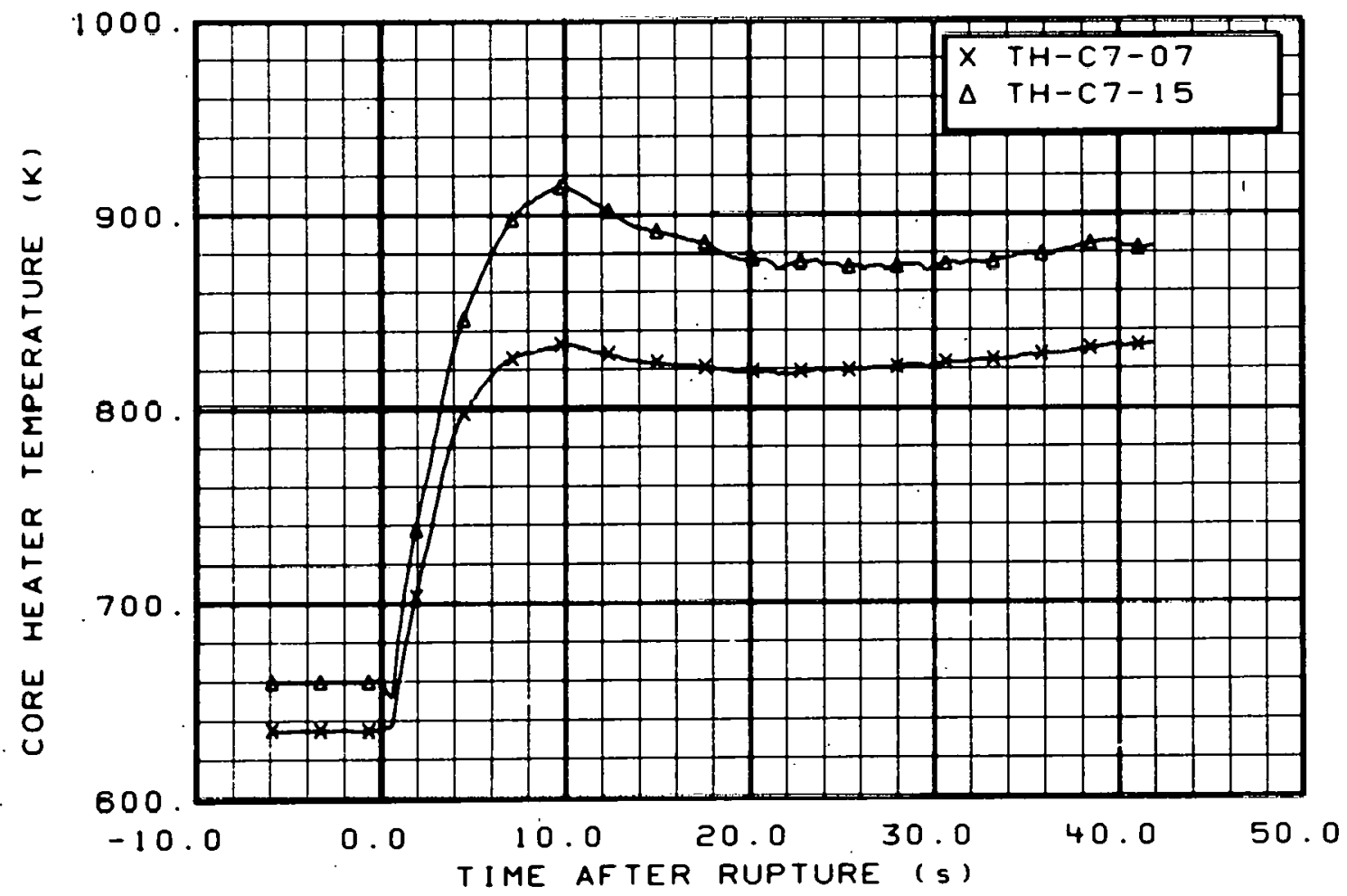

Fig. 104 Core heater temperature, Rod $\mathrm{C}-7$ (TH-C7-07 and TH-C7-15), from -6 to $42 \mathrm{~s}$. 


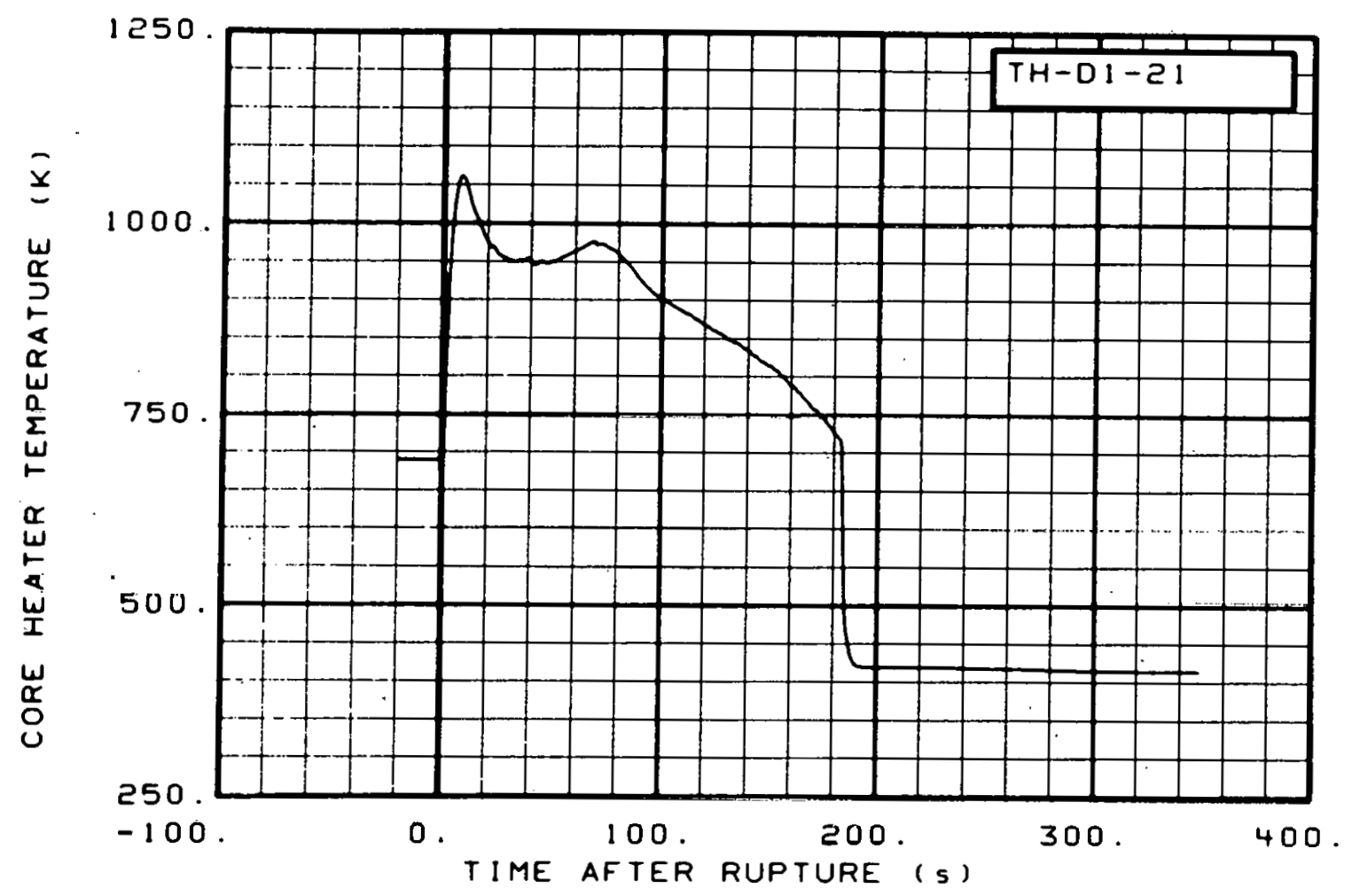

Fig. 105 Core heater temperature, Rod D-1 (TH-D1-21), from -20 to $350 \mathrm{~s}$.

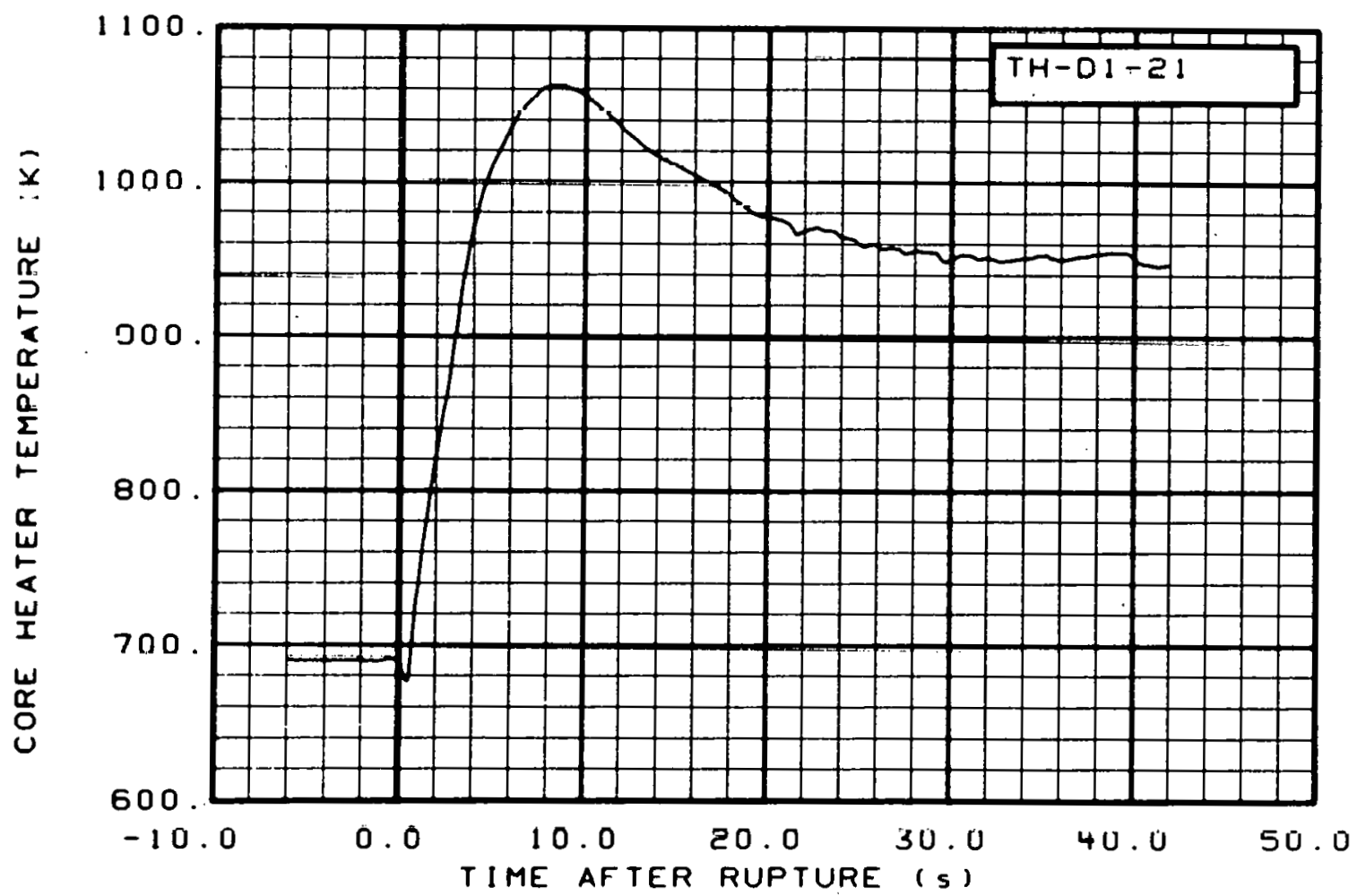

Fig. 106 Core heater temperature, Rod D-1 (TH-DT-21), from -6 to $42 \mathrm{~s}$. 


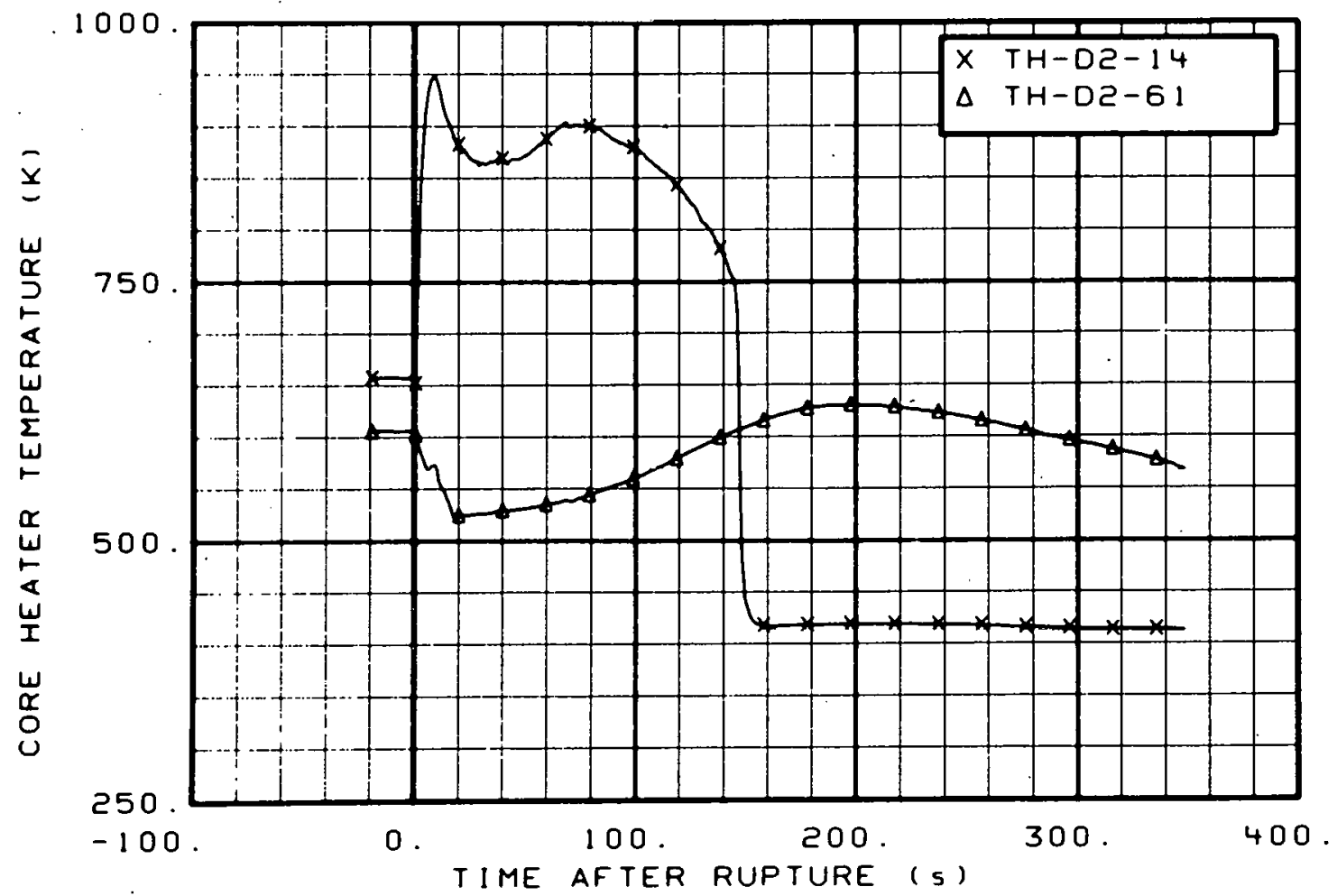

Fig. 107 Core heater temperature, Rod D-2 (TH-D2-14 and TH-D2-61), from -20 to $350 \mathrm{~s}$.

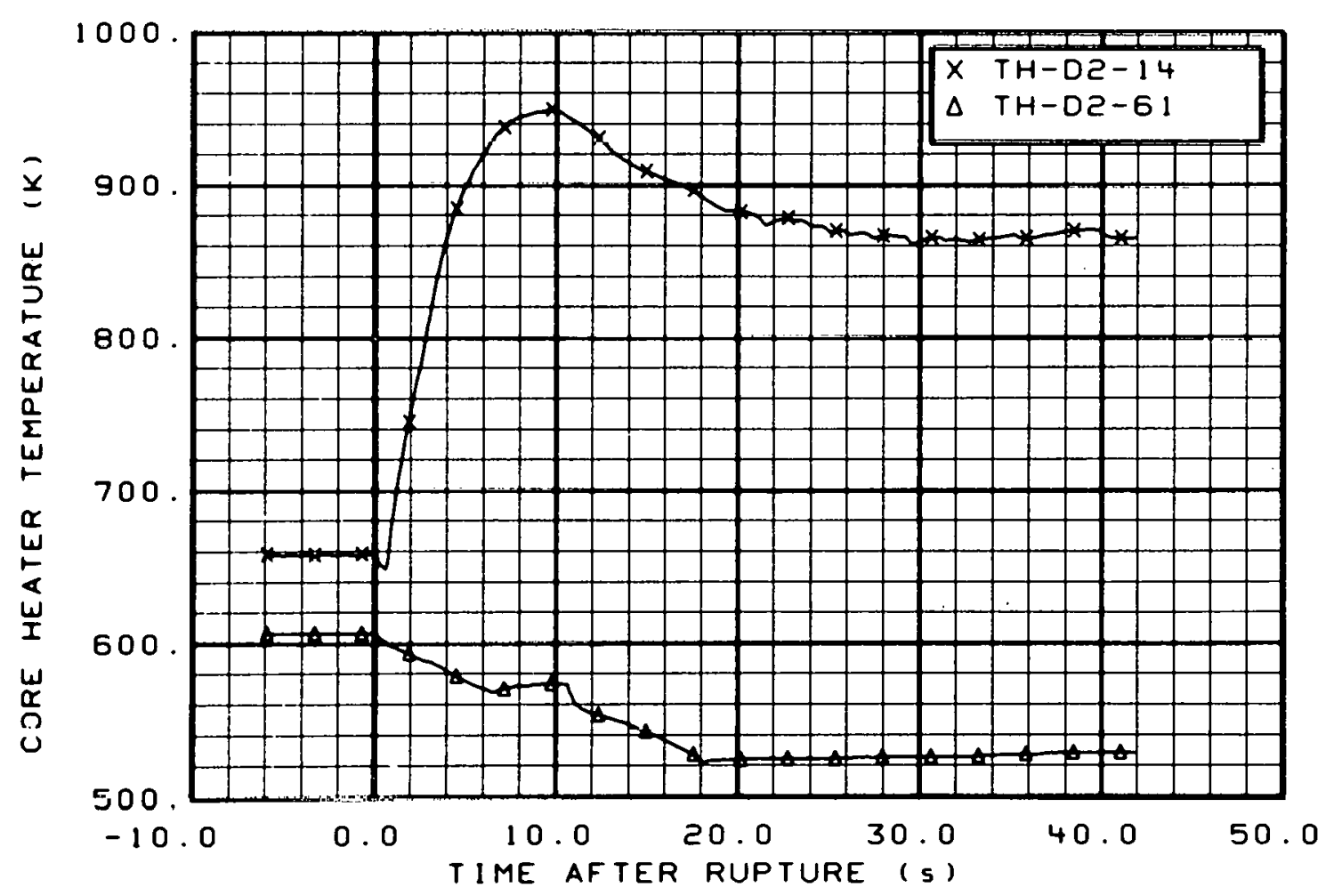

Fig. 108 Core heater temperature, Rod D-2 (TH-D2-14 and TH-D2-61), from -6 to $42 \mathrm{~s}$. 


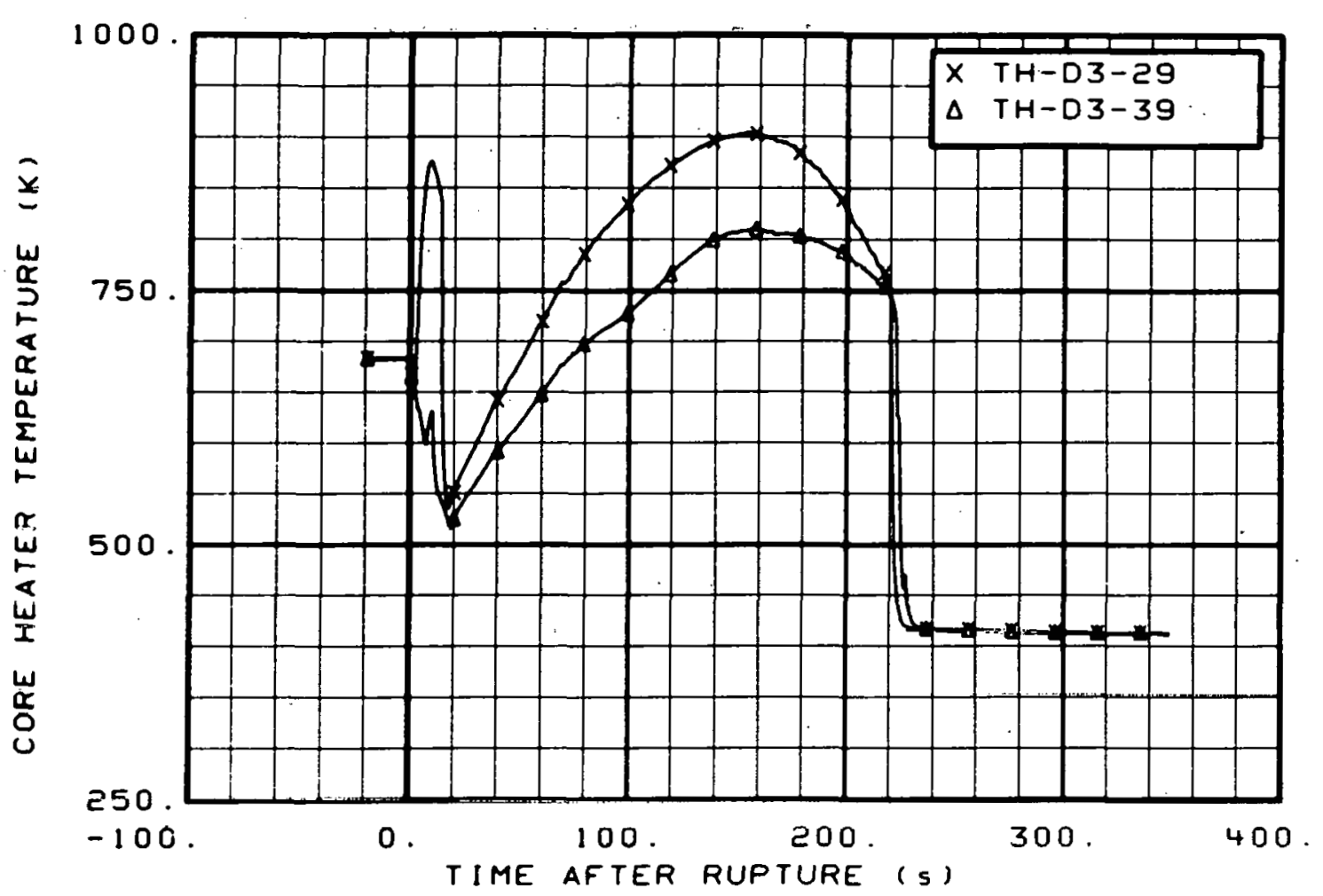

Fig. 109 Core heater temperature, Rod D-3 (TH-D3-29 and TH-D3-39), from -20 to $350 \mathrm{~s}$.

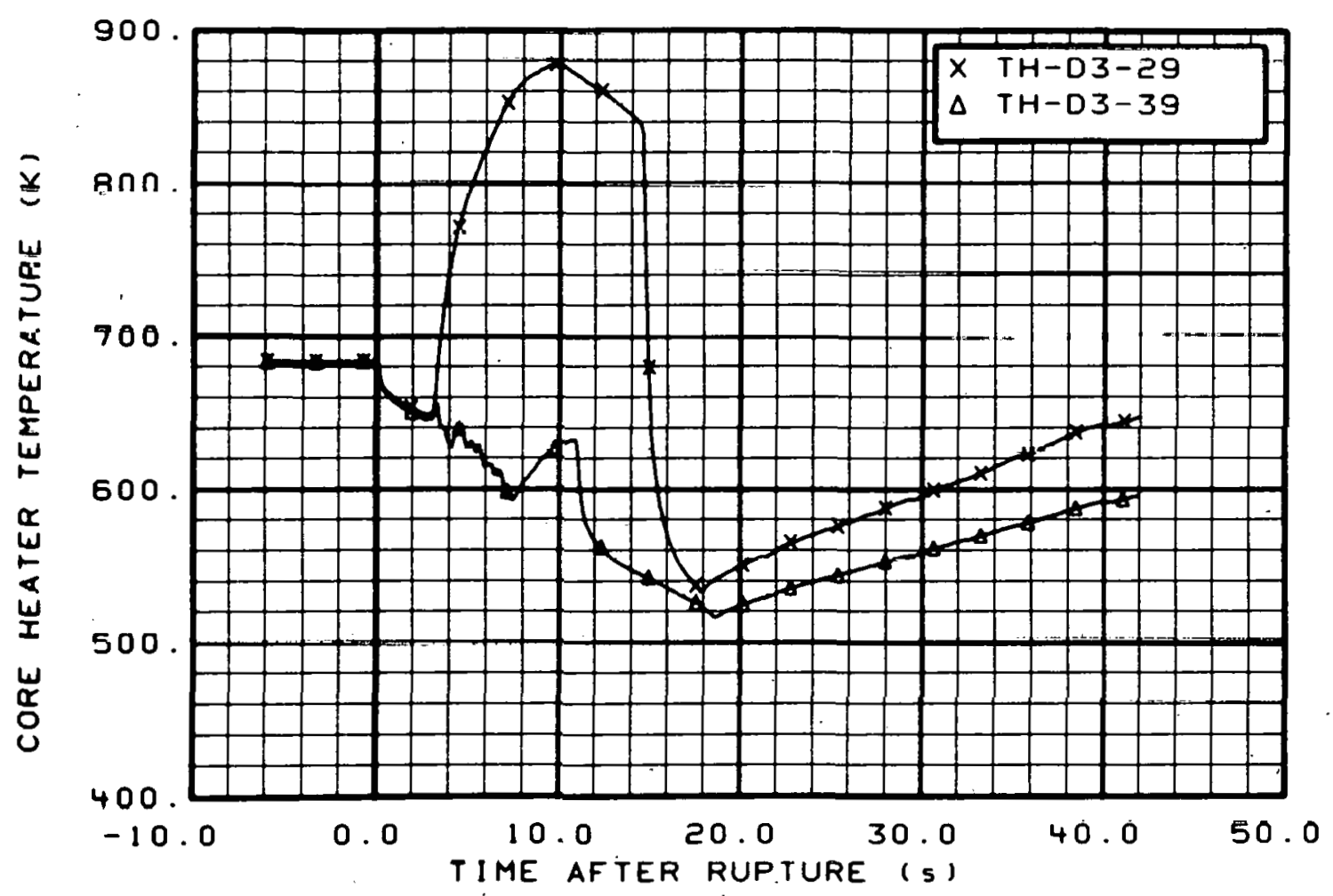

Fig. 110 Core heater temperature, Rod D-3 (TH-D3-29 and TH-D3-39), from -6 to $42 \mathrm{~s}$. 


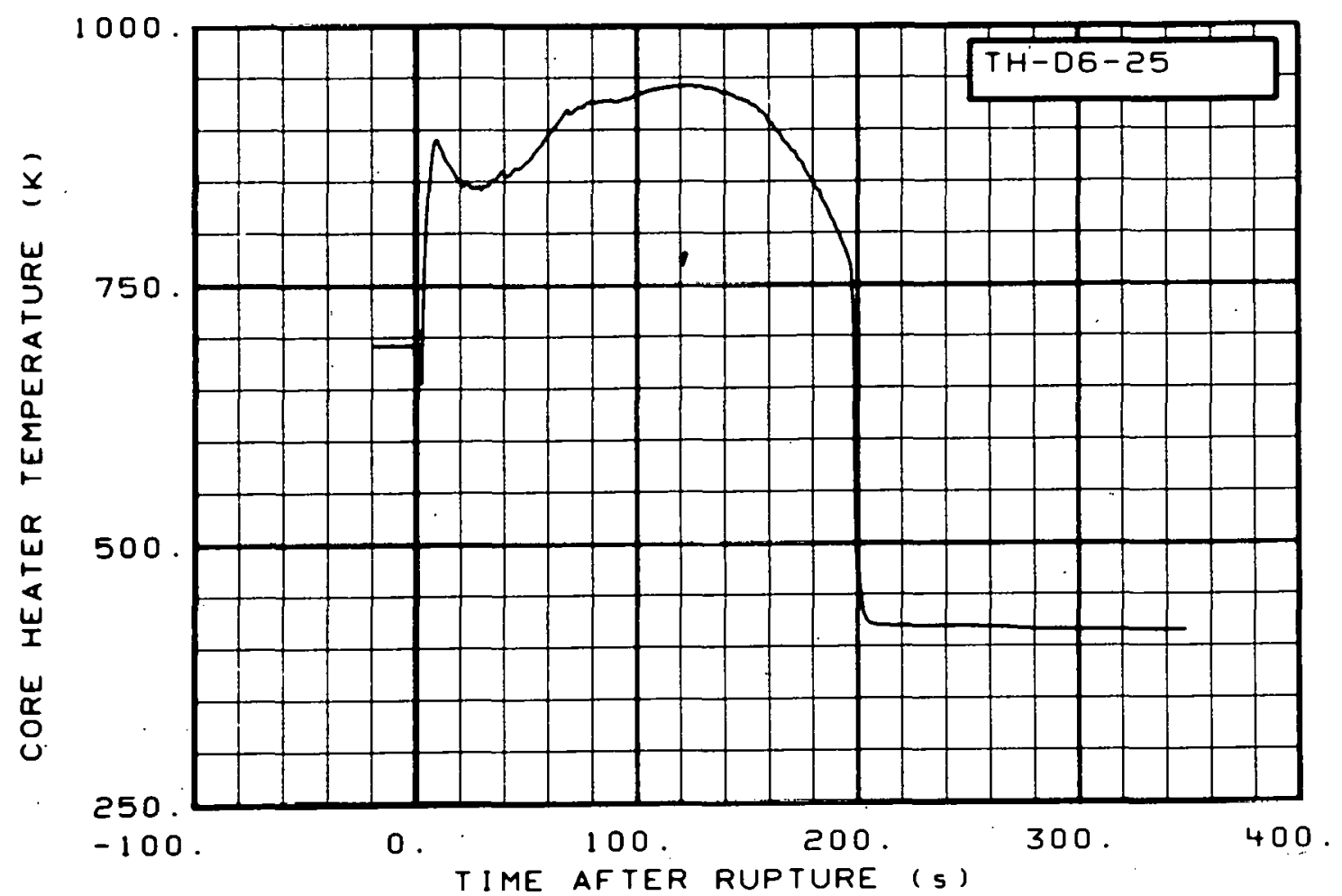

Fig. 111 Core heater temperature, Rod D-6 (TH-D6-25), from -20 to $350 \mathrm{~s}$.

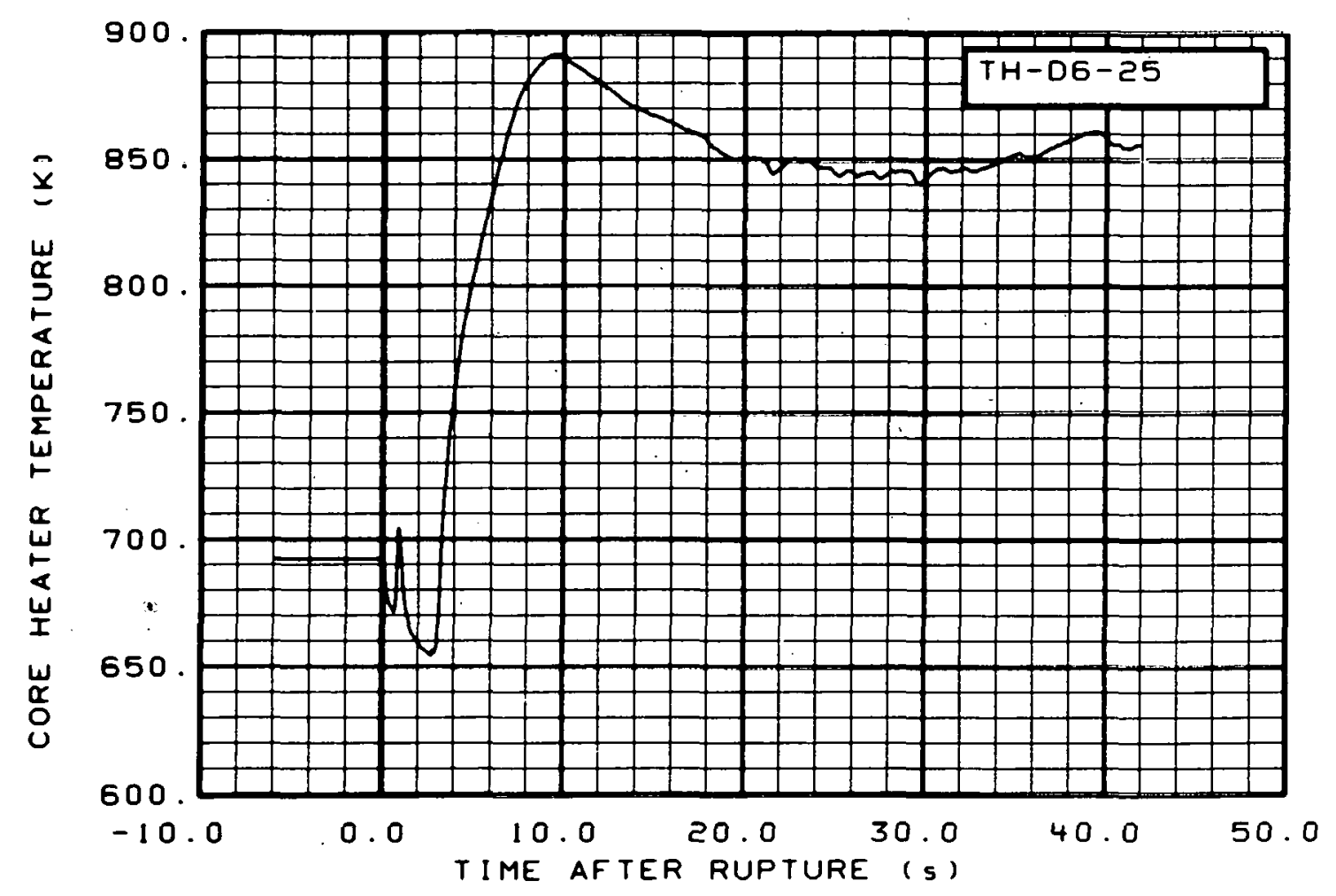

Fig. 112 Core heater temperature, Rod D-6 (TH-D6-25), from -6 to $42 \mathrm{~s}$. 


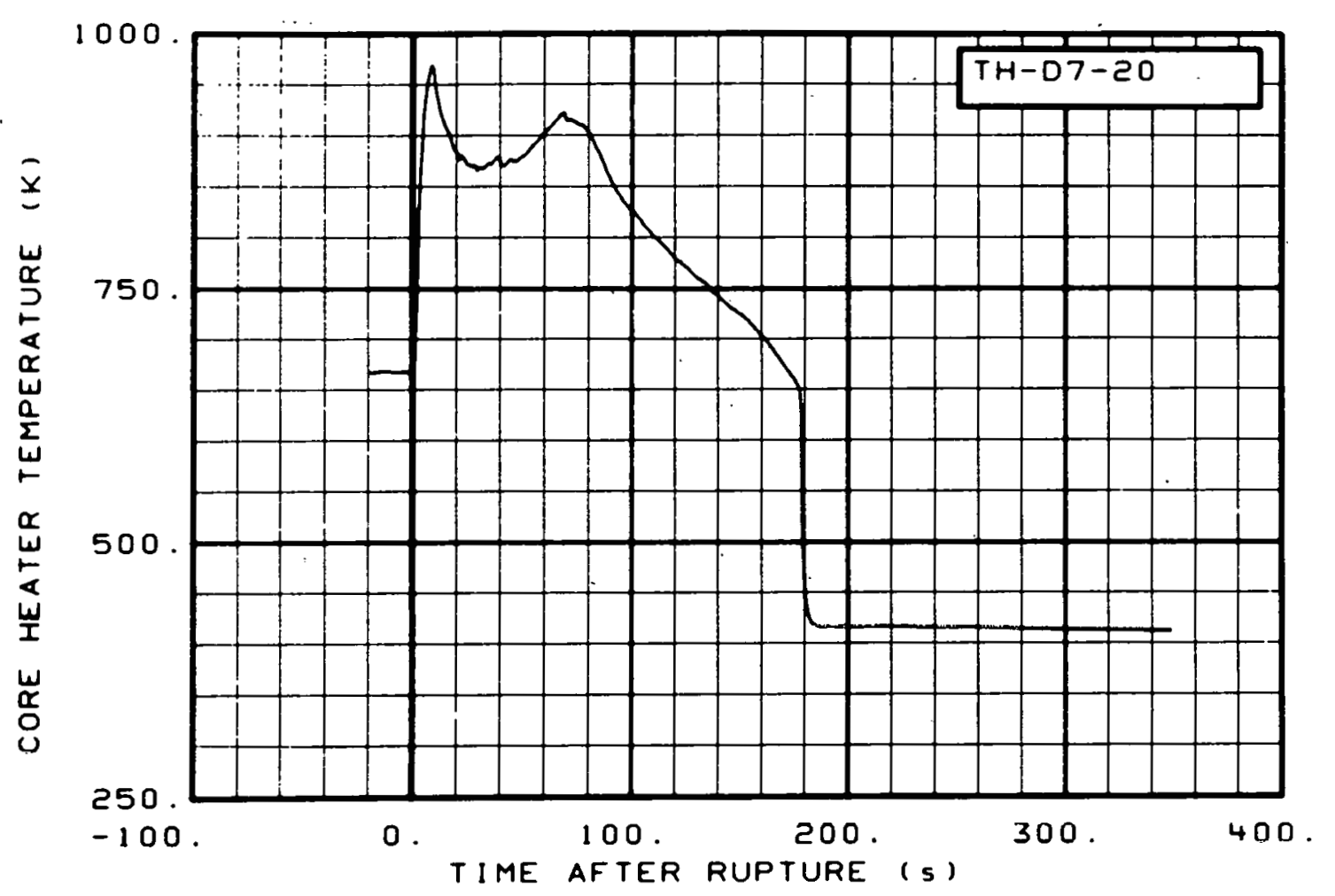

Fig. 113 Core heater temperature, Rod D-7 (TH-D7-20), from -20 to $350 \mathrm{~s}$.

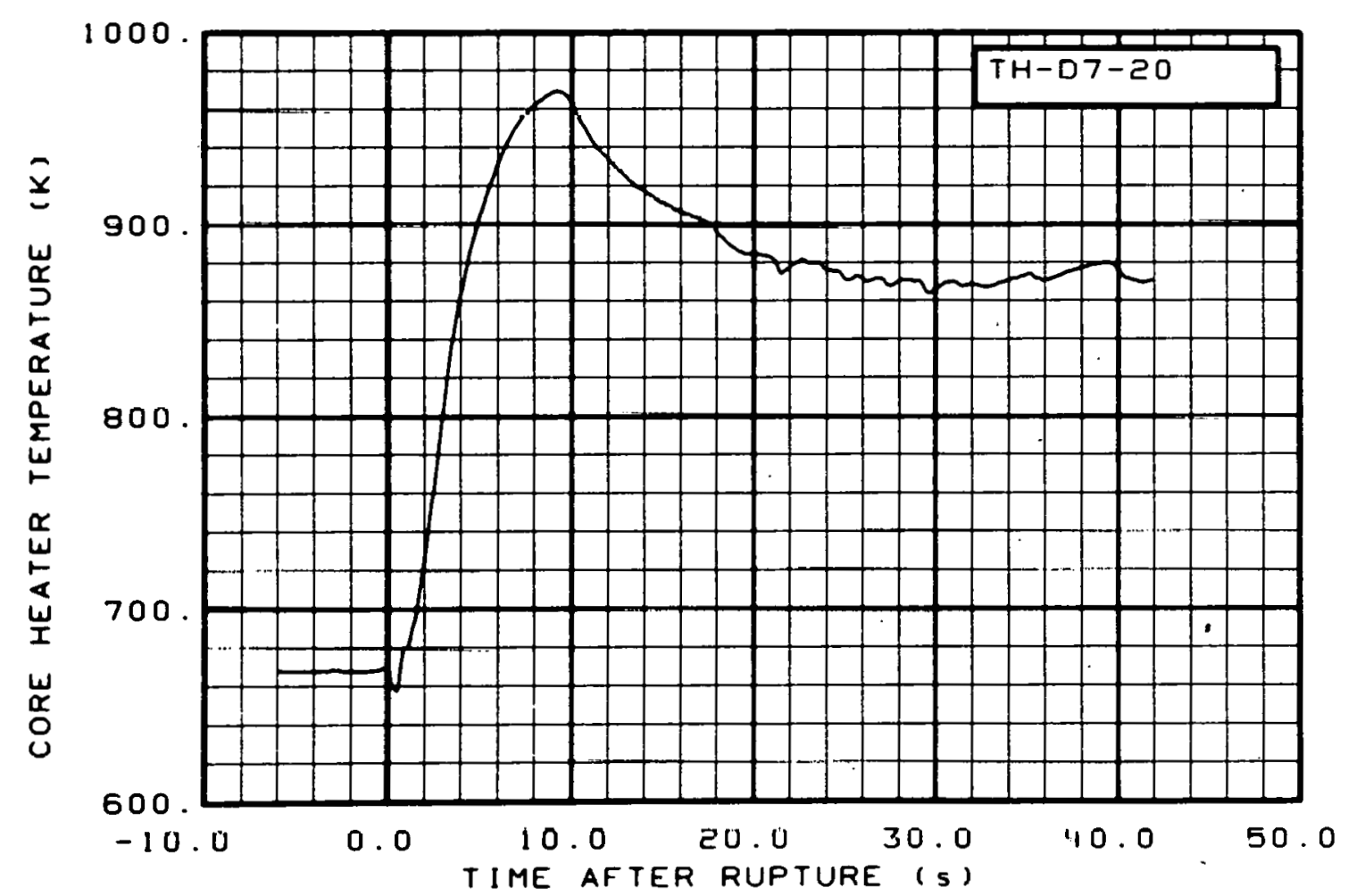

Fig. 114 Core heater temperature, Rod D-7 (TH-D7-20), from -6 to $42 \mathrm{~s}$. 


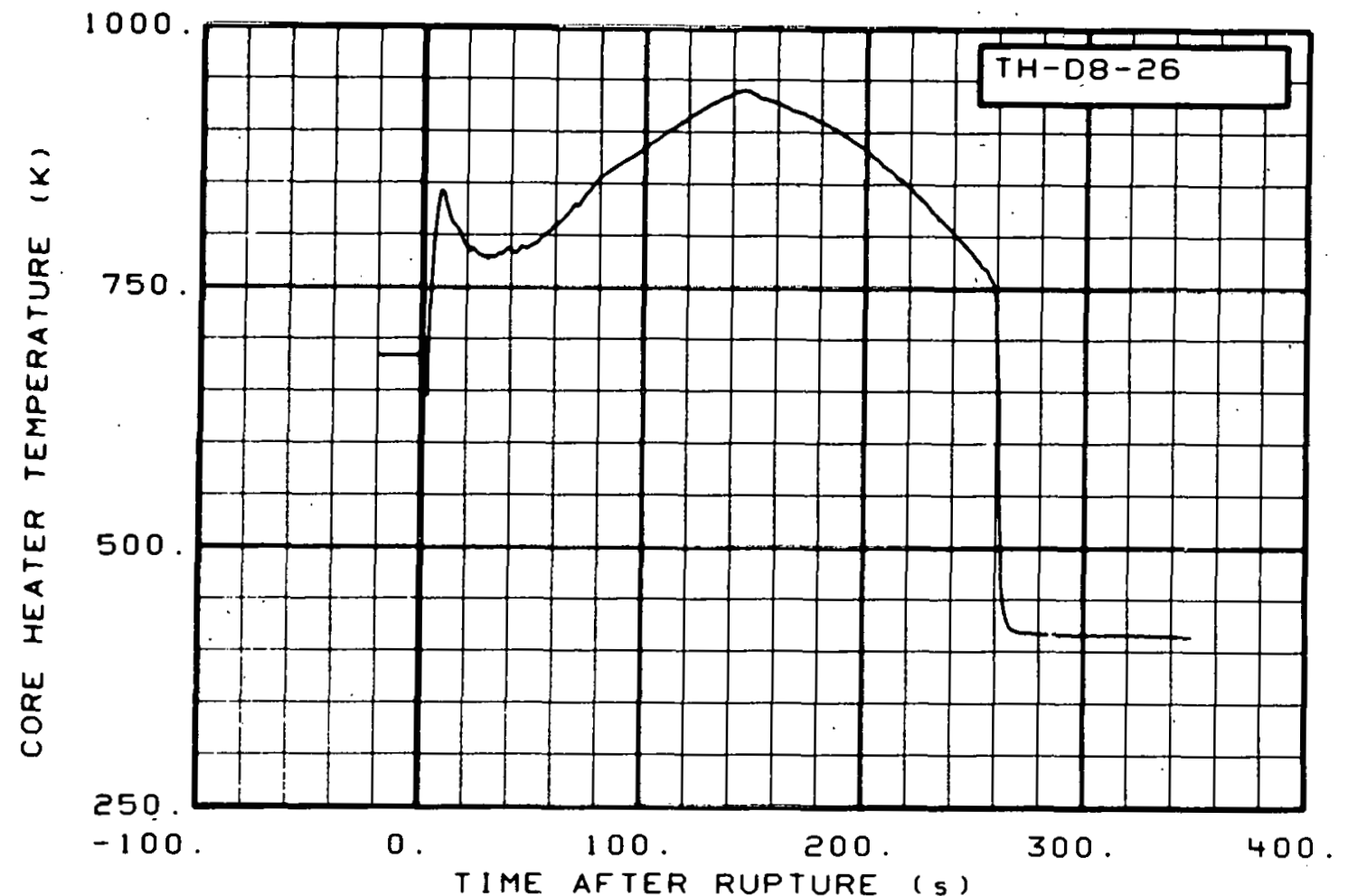

Fig. 115 Core heater temperature, Rod D-8 (TH-D8-26), from -20 to $350 \mathrm{~s}$.

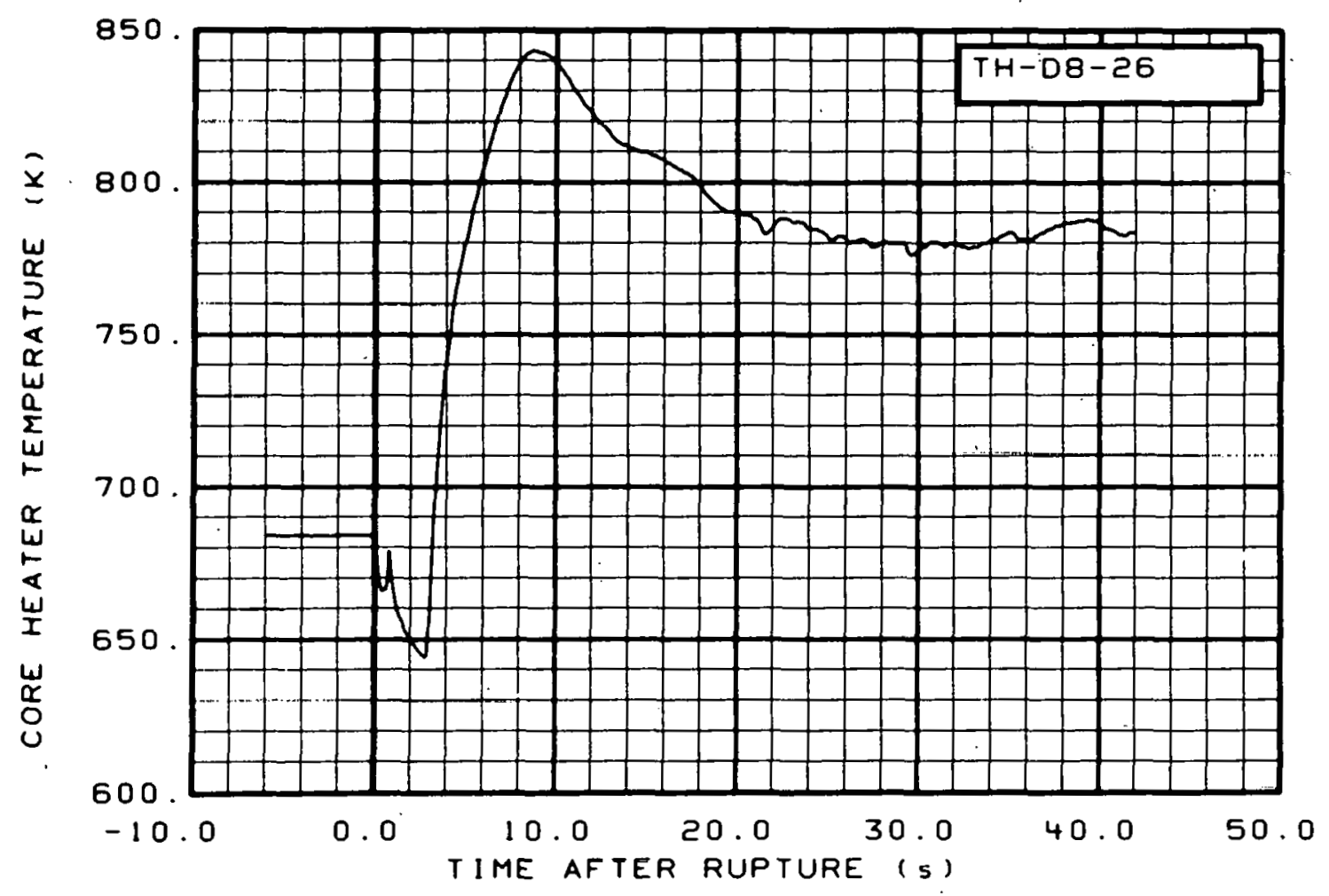

Fig. 116 Core heater temperature, Rod D-8 (TH-D8-26), from -6 to $42 \mathrm{~s}$. 


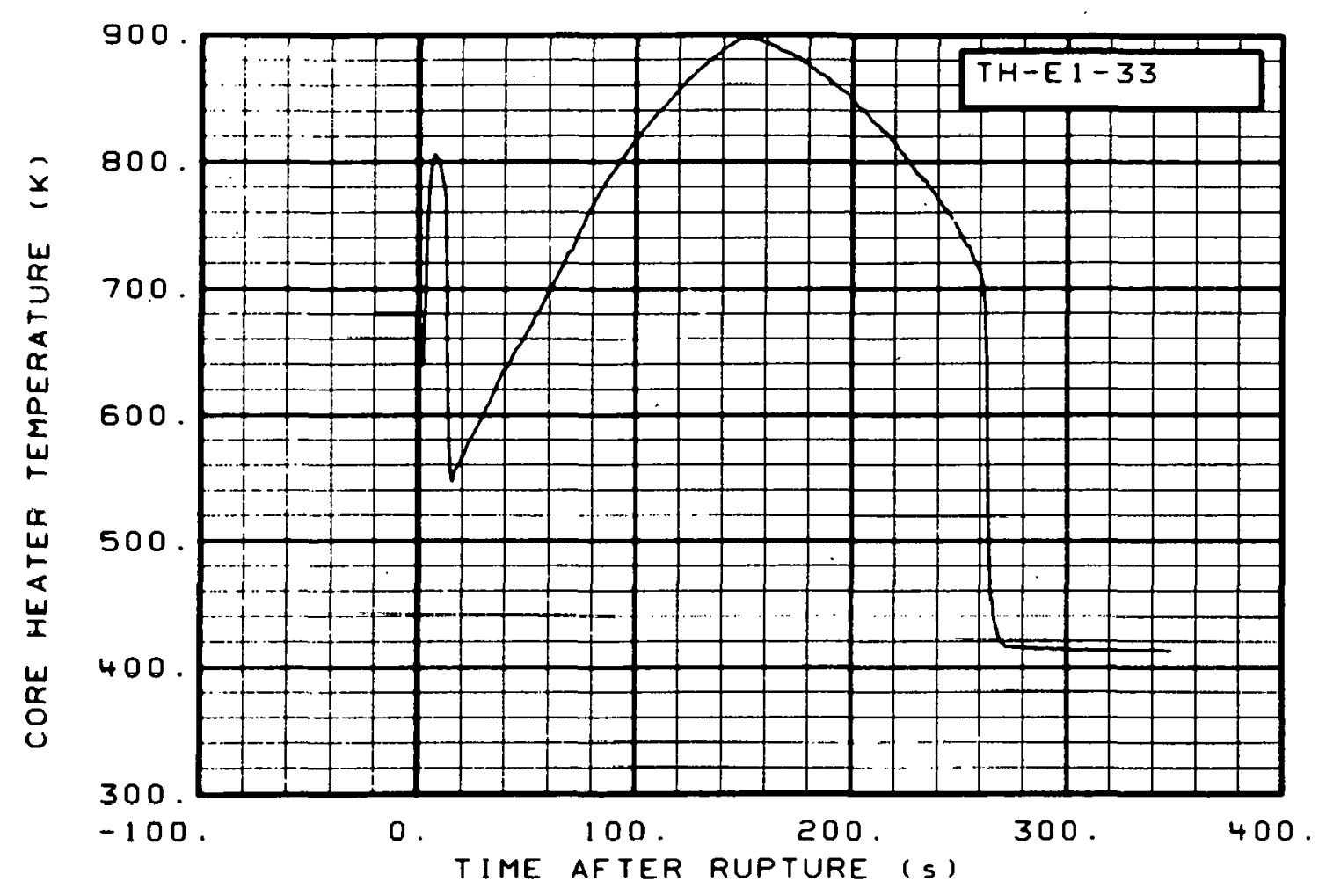

Fig. 117 Core heater temperature, Rod E-1 (TH-EI-33), from -20 to $350 \mathrm{~s}$.

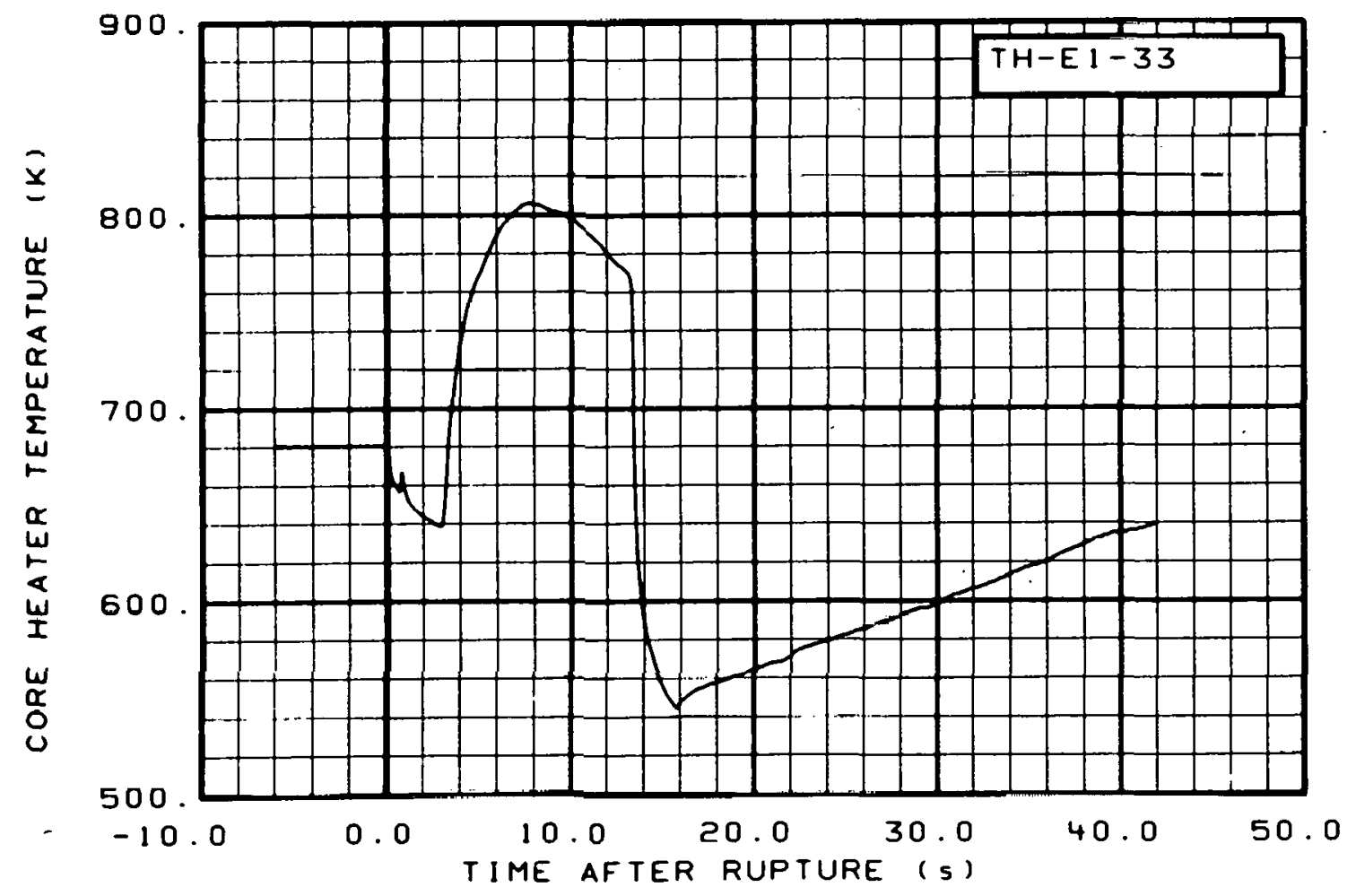

Fig. 118 Core heater temperature, Rod E-1 (TH-ET-33), from -6 to $42 \mathrm{~s}$. 


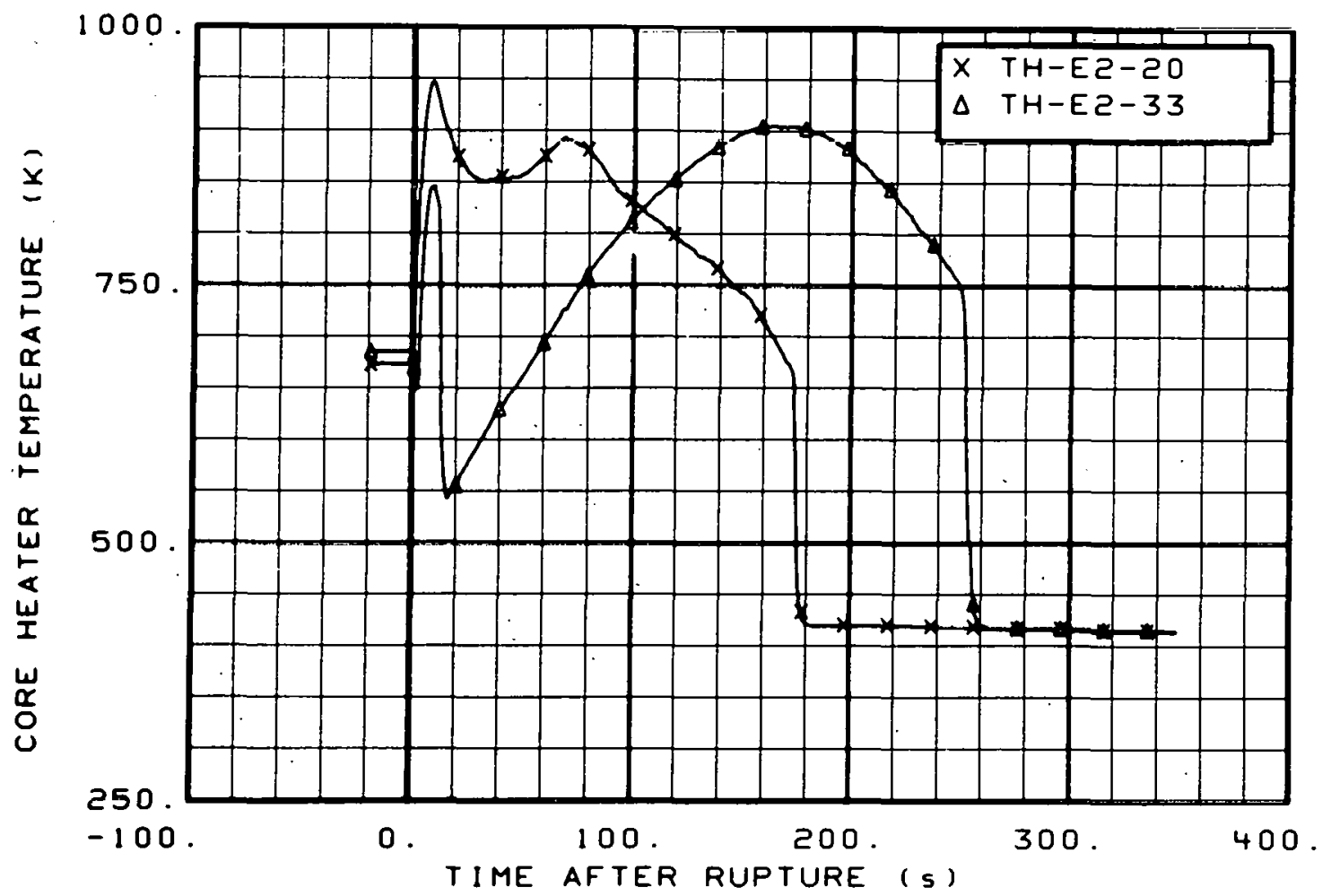

Fig. 119 Core heater temperature, Rod E-2 (TH-E2-20 and TH-E2-33), from -20 to $350 \mathrm{~s}$.

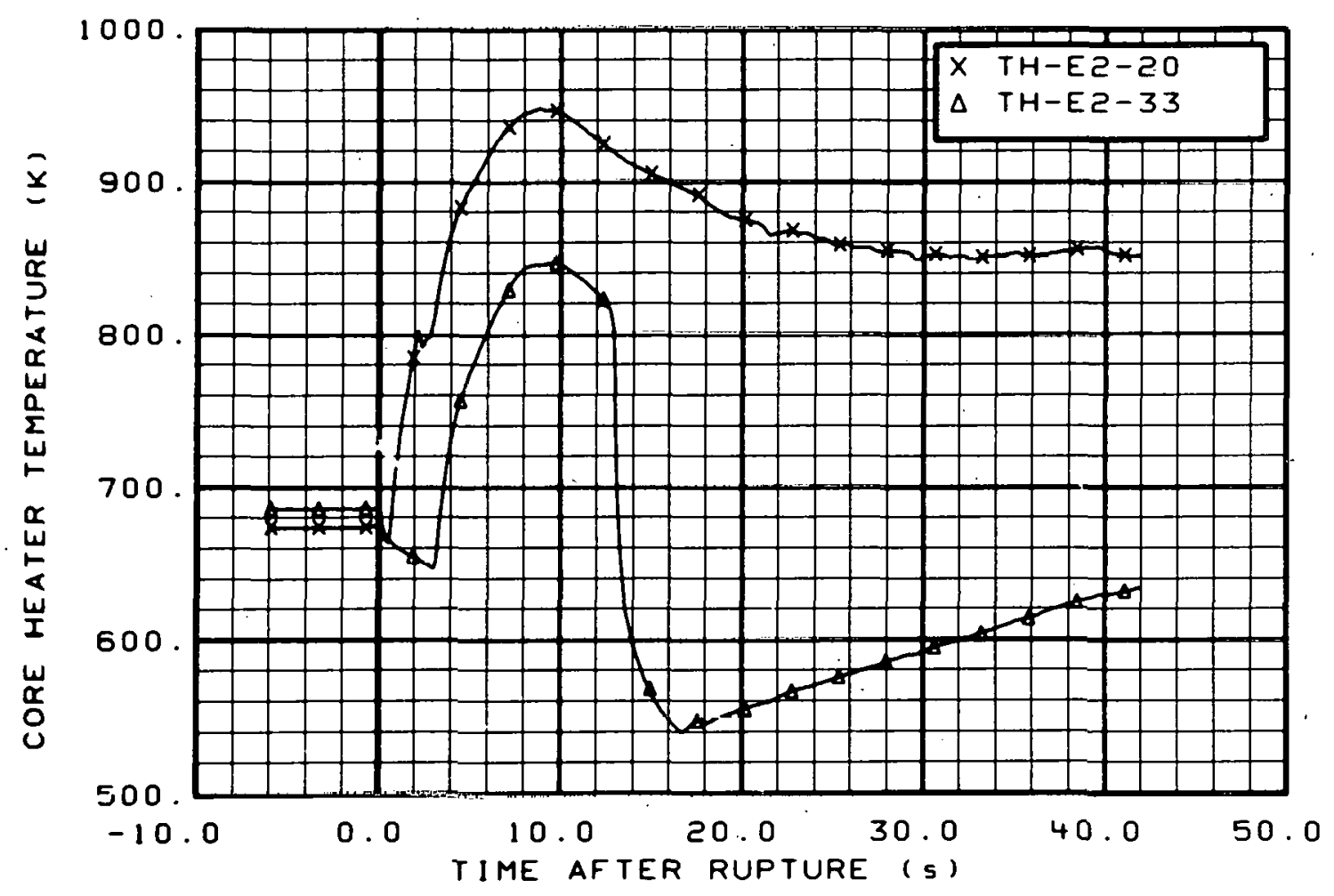

l.ig. 120 Core heater temperature, Rod E-2 (TH-E2-20 and TH-E2-33), from -6 to 42.5 . 


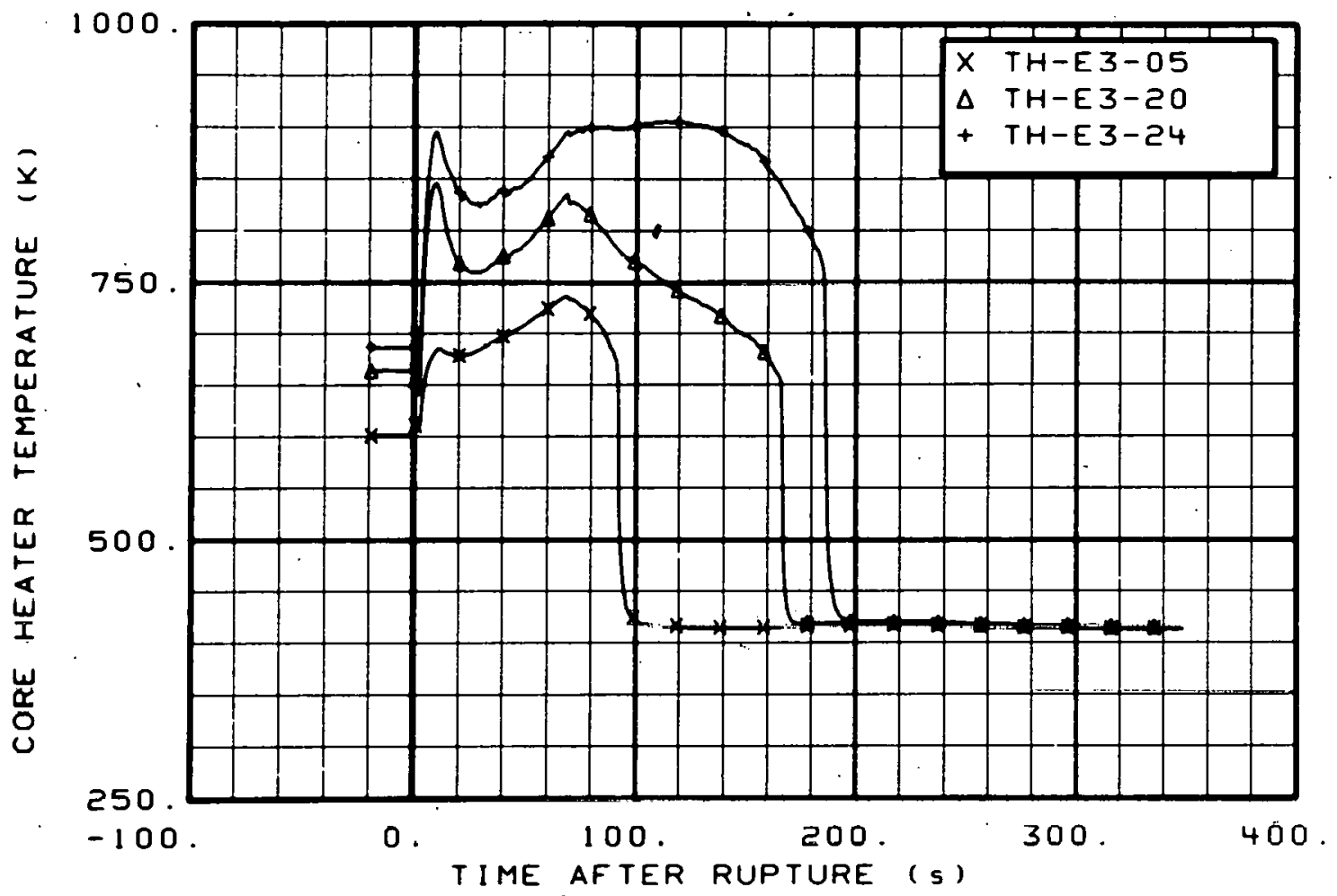

Fig. 121 Core heater temperature, Rod E-3 (TH-E3-05, TH-E3-20, and TH-E3-24), from -20 to $350 \mathrm{~s}$.

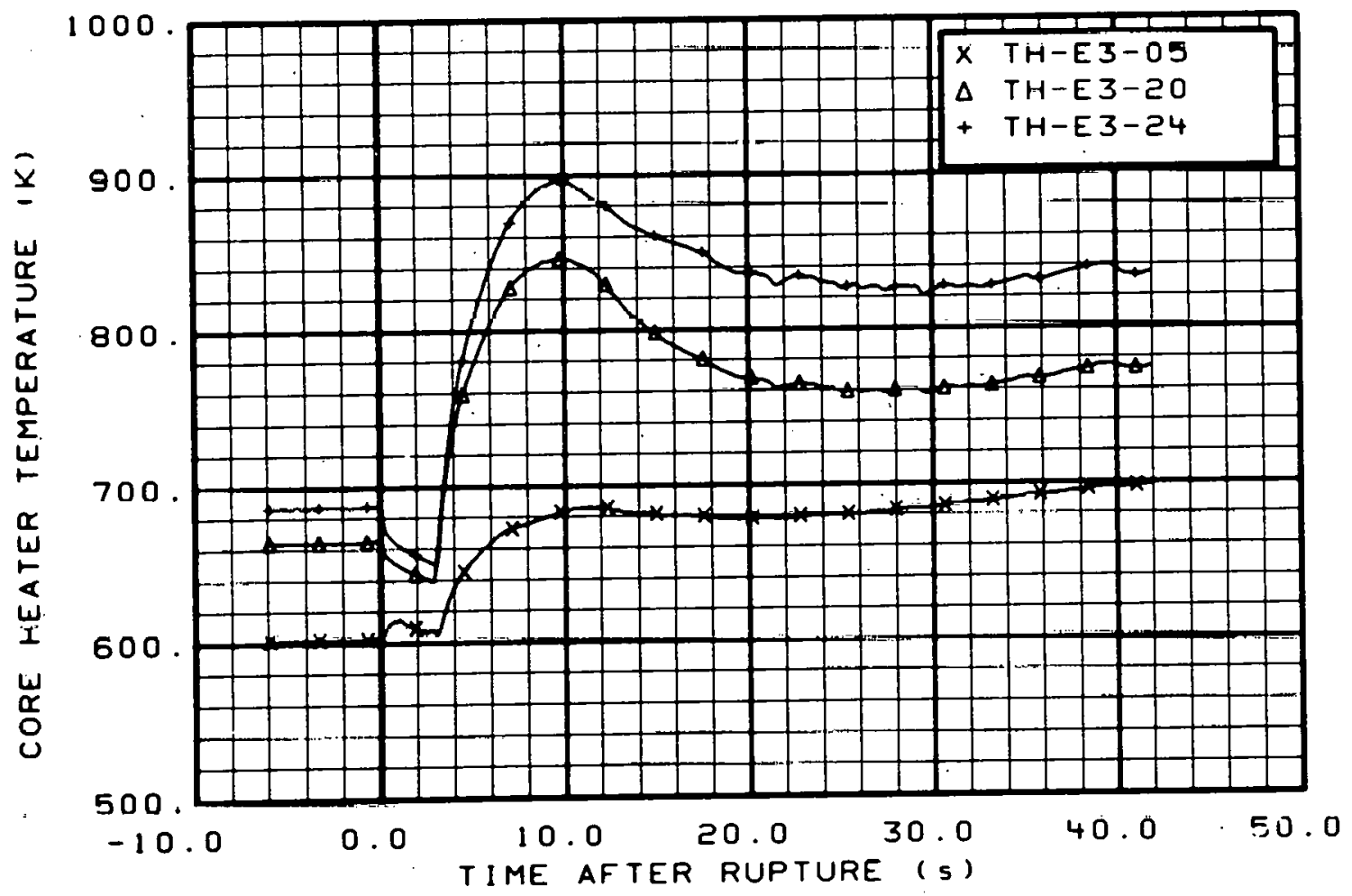

Fig. 122 Core heater temperature, Rod E-3 (TH-E3-05, TH-E3-20, and TH-E3-24), from -6 to $42 \mathrm{~s}$. 


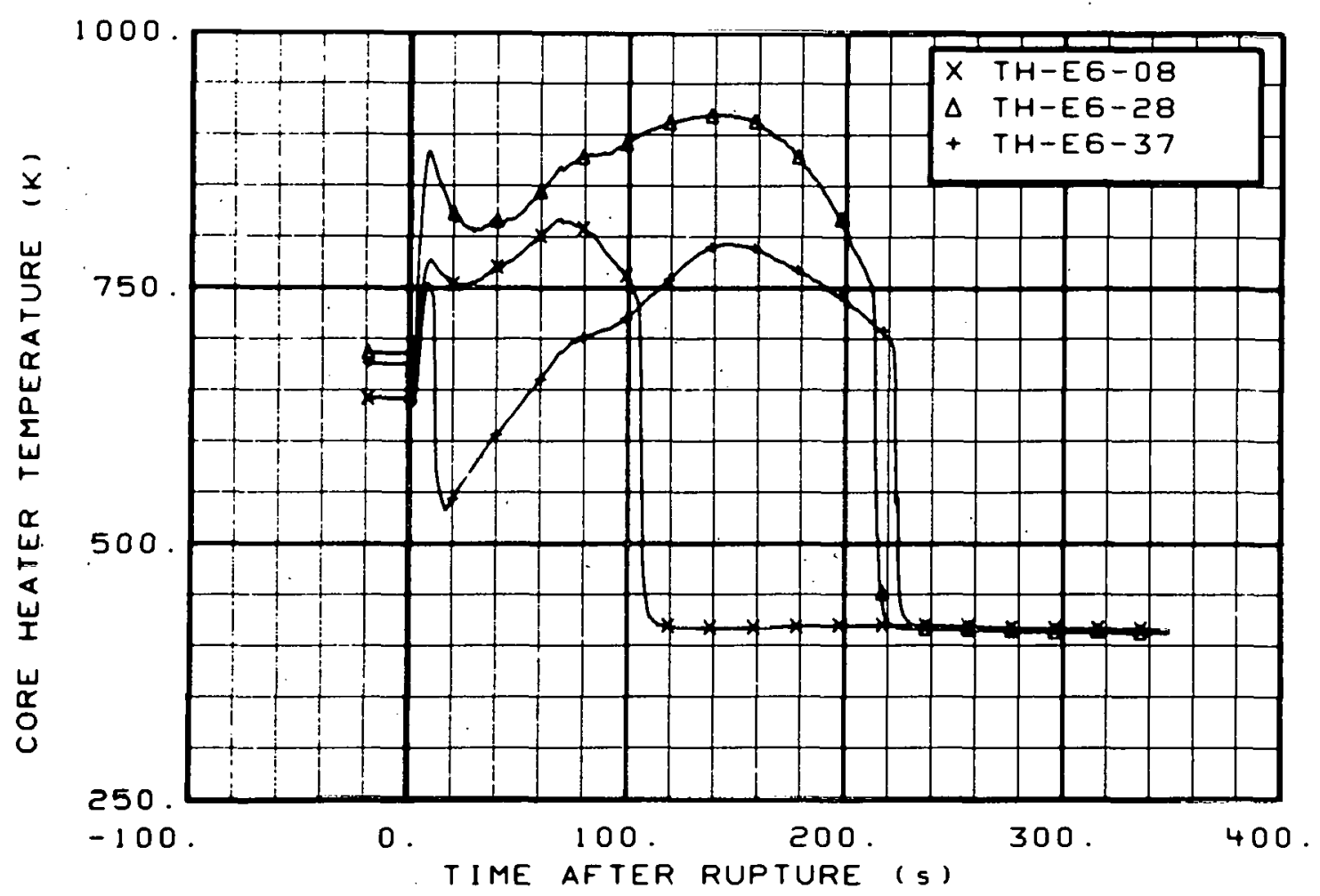

Fig. 123 Core heater temperature, Rod E-6 (TH-E6-08, TH-E6-28, and TH-E6-37), from -20 to $350 \mathrm{~s}$.

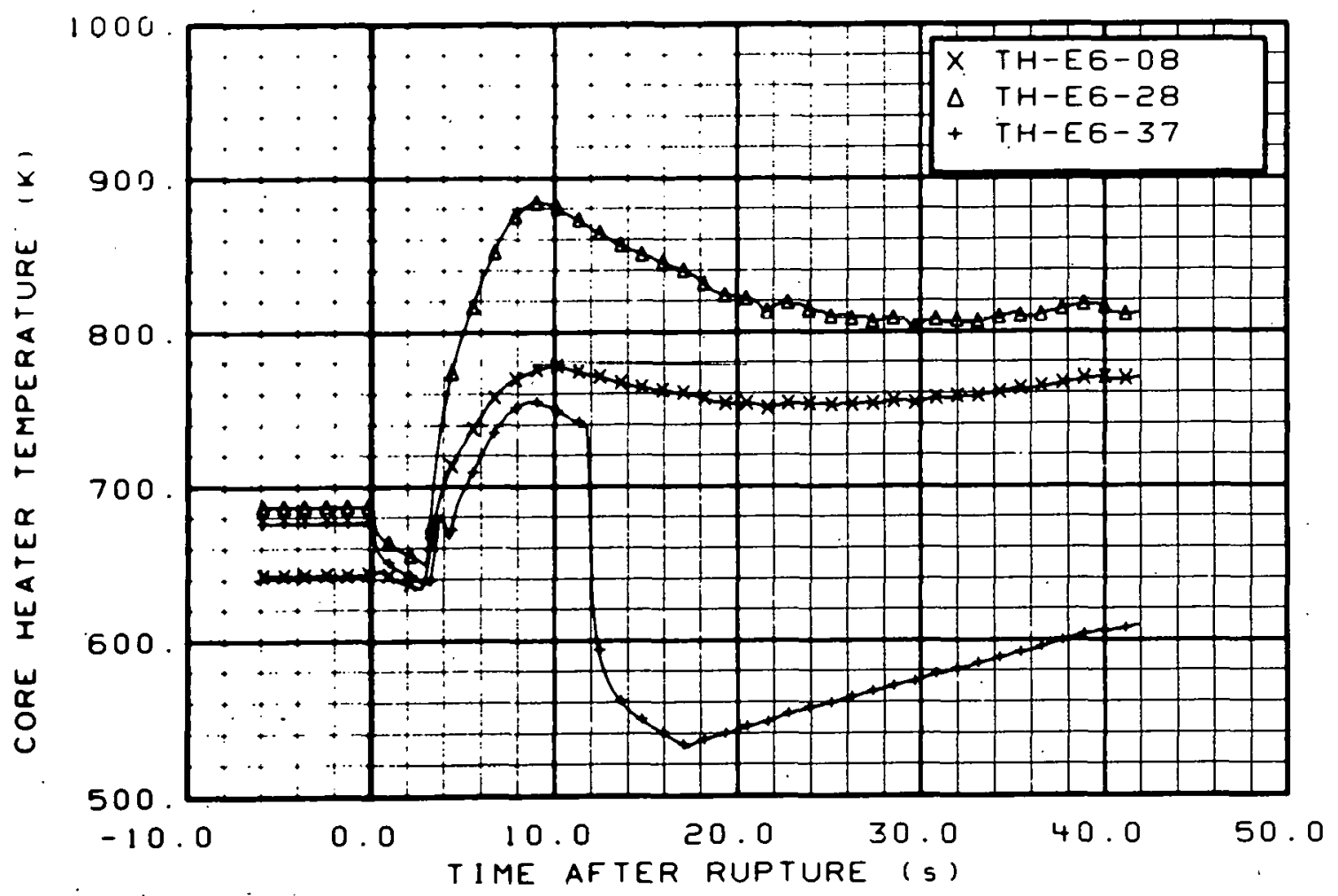

Fig. 124 Core heater temperature, Rod E-6 (TH-E6-08, TH-E6-28, and $\mathrm{TH}-\mathrm{E} 6-37)$, from -6 to $42 \mathrm{~s}$. 


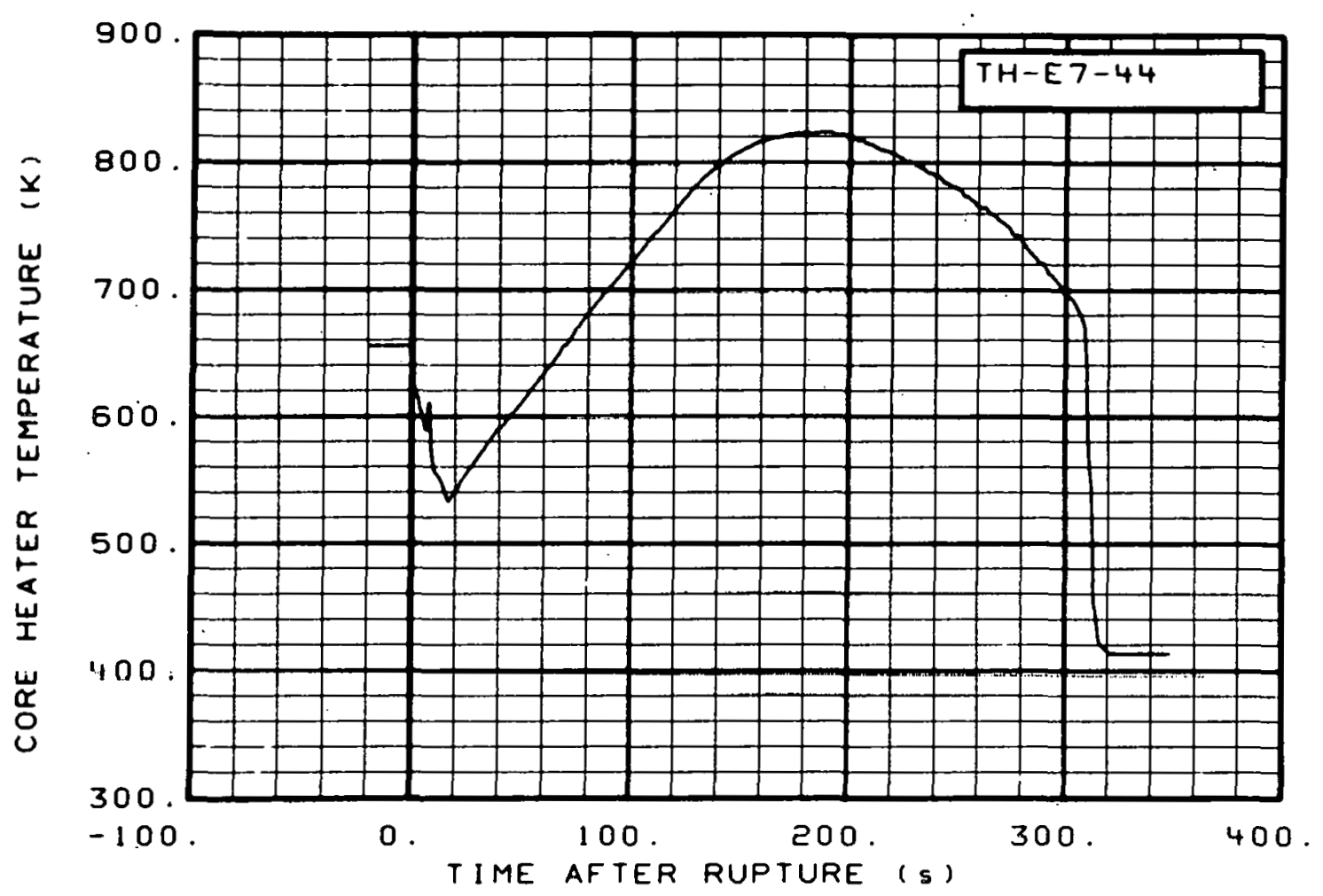

Fig. 125 Core heater temperature, Rod E-7 (TH-E7-44), from -20 to $350 \mathrm{~s}$.

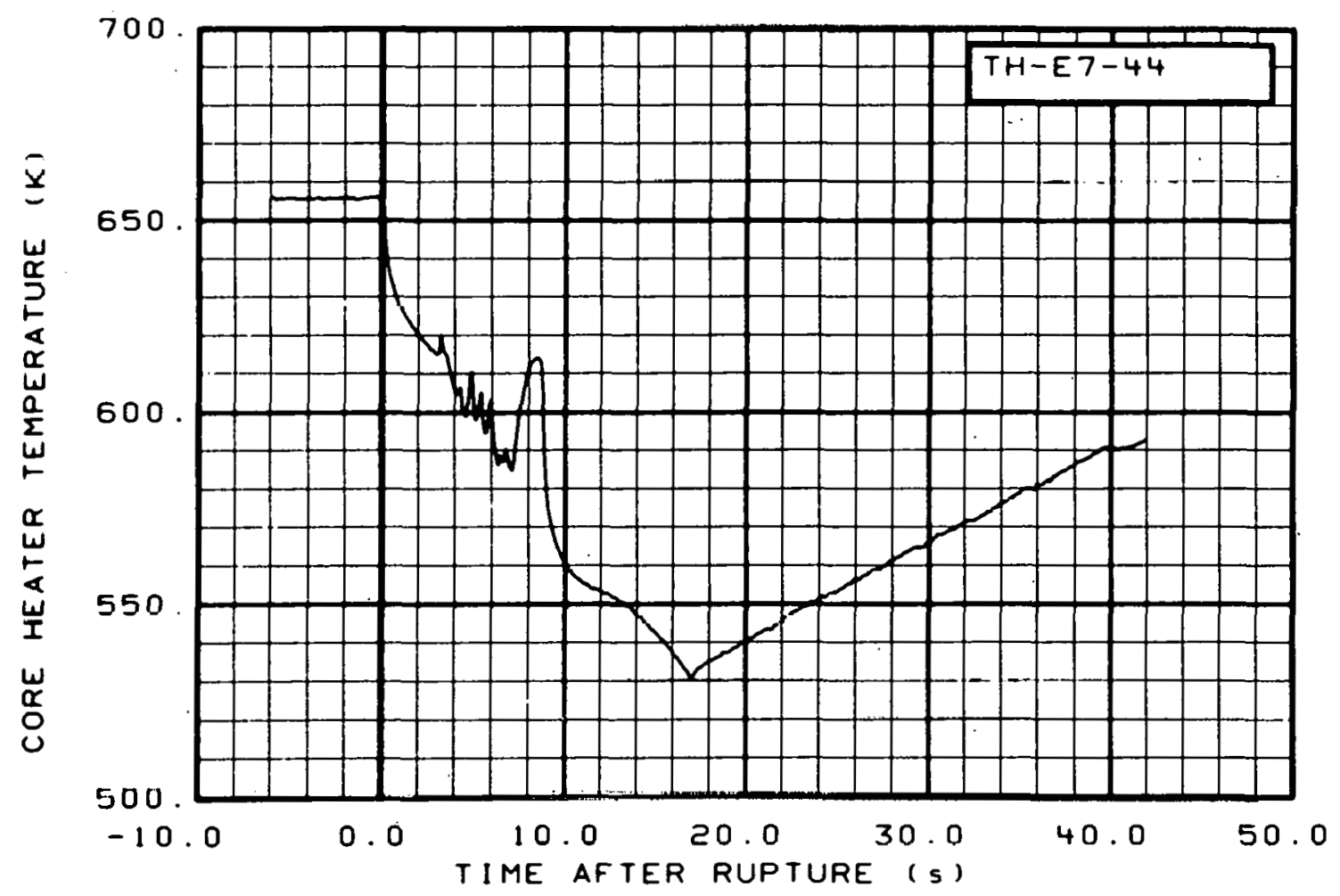

Fig. 126 Core heater temperature, Rod E-7 (TH-E7-44), from -6 to $42 \mathrm{~s}$. 


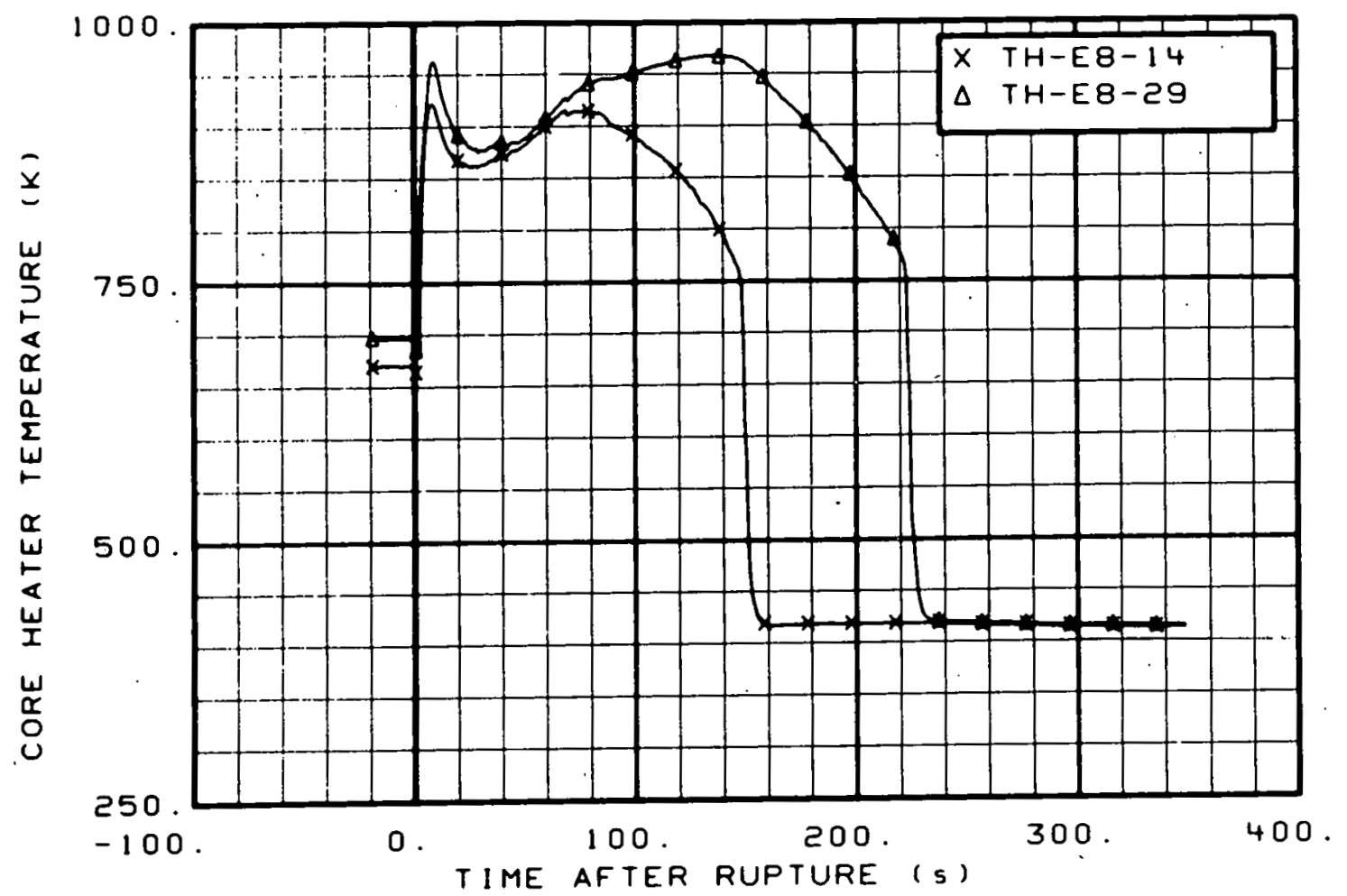

Fig. 127 Core heater temperature, Rod E-8 (TH-E8-14 and TH-E8-29), from -20 to $350 \mathrm{~s}$.

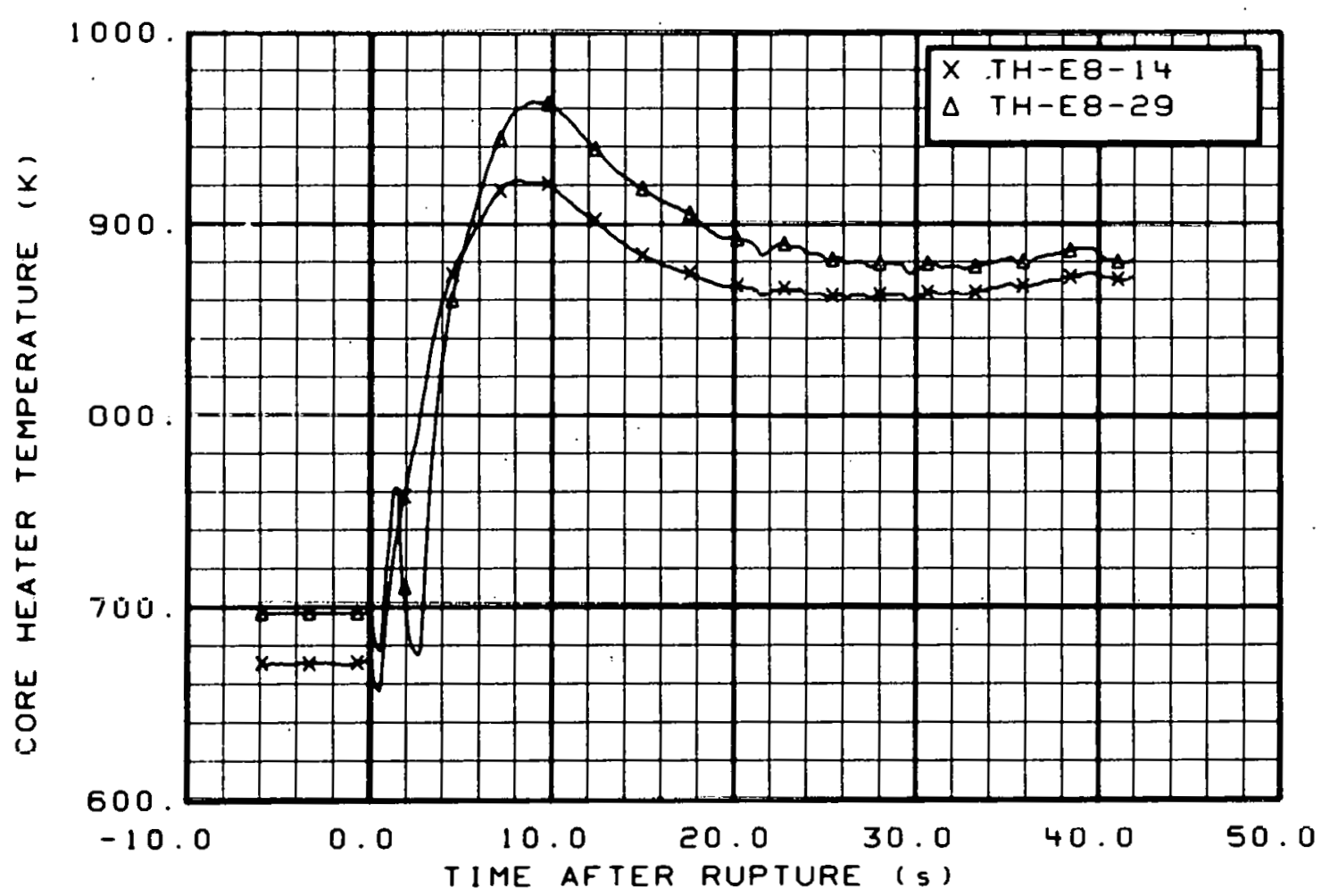

Fig. 128 Core heater temperature, Rod E-8 (TH-E8-14 and TH-E8-29), from -6 to $42 \mathrm{~s}$. 


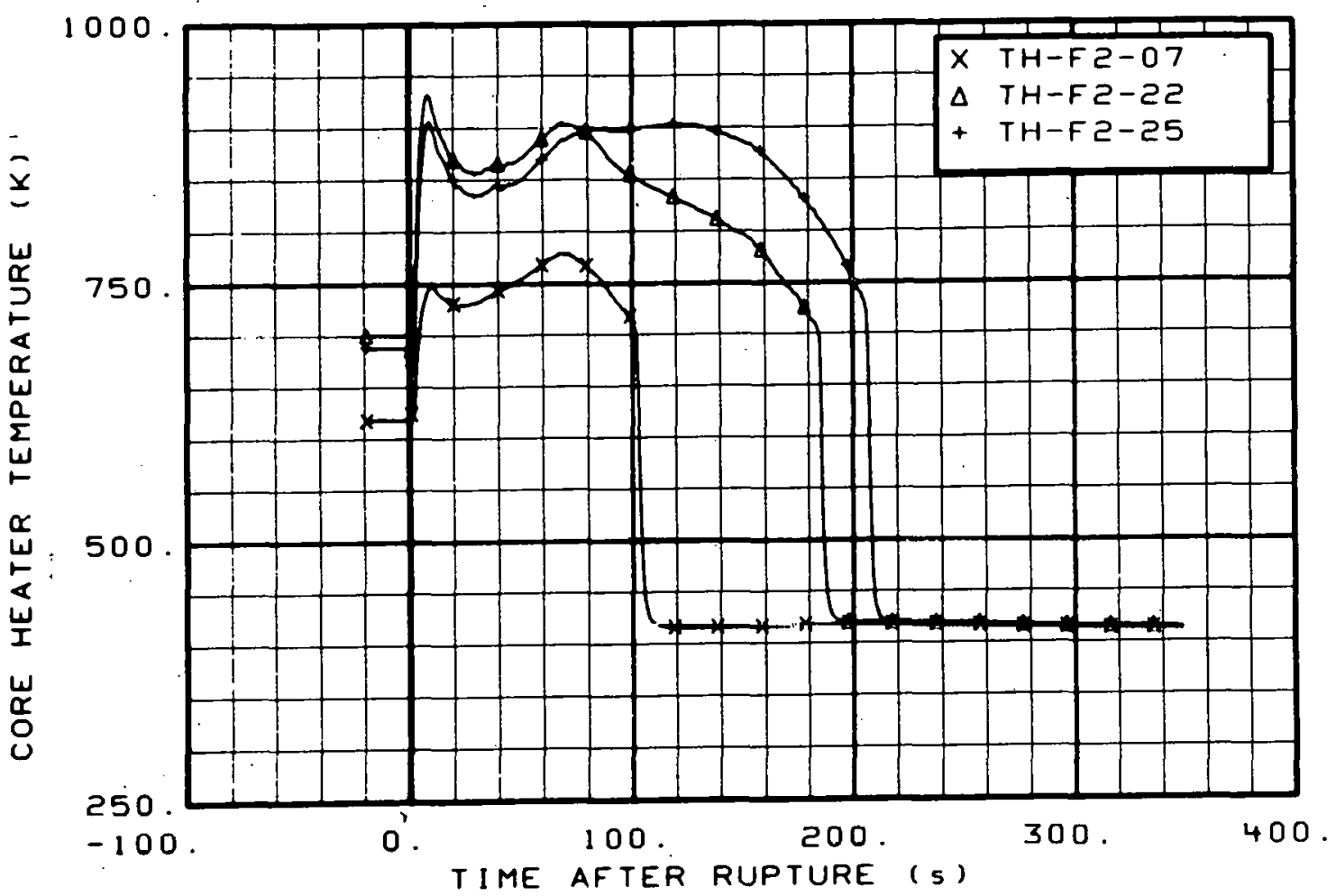

Fig. 129 Core heater temperature, Rod F-2 (TH-F2-07, TH-F2-22, and TH-F2-25), from -20 to $350 \mathrm{~s}$.

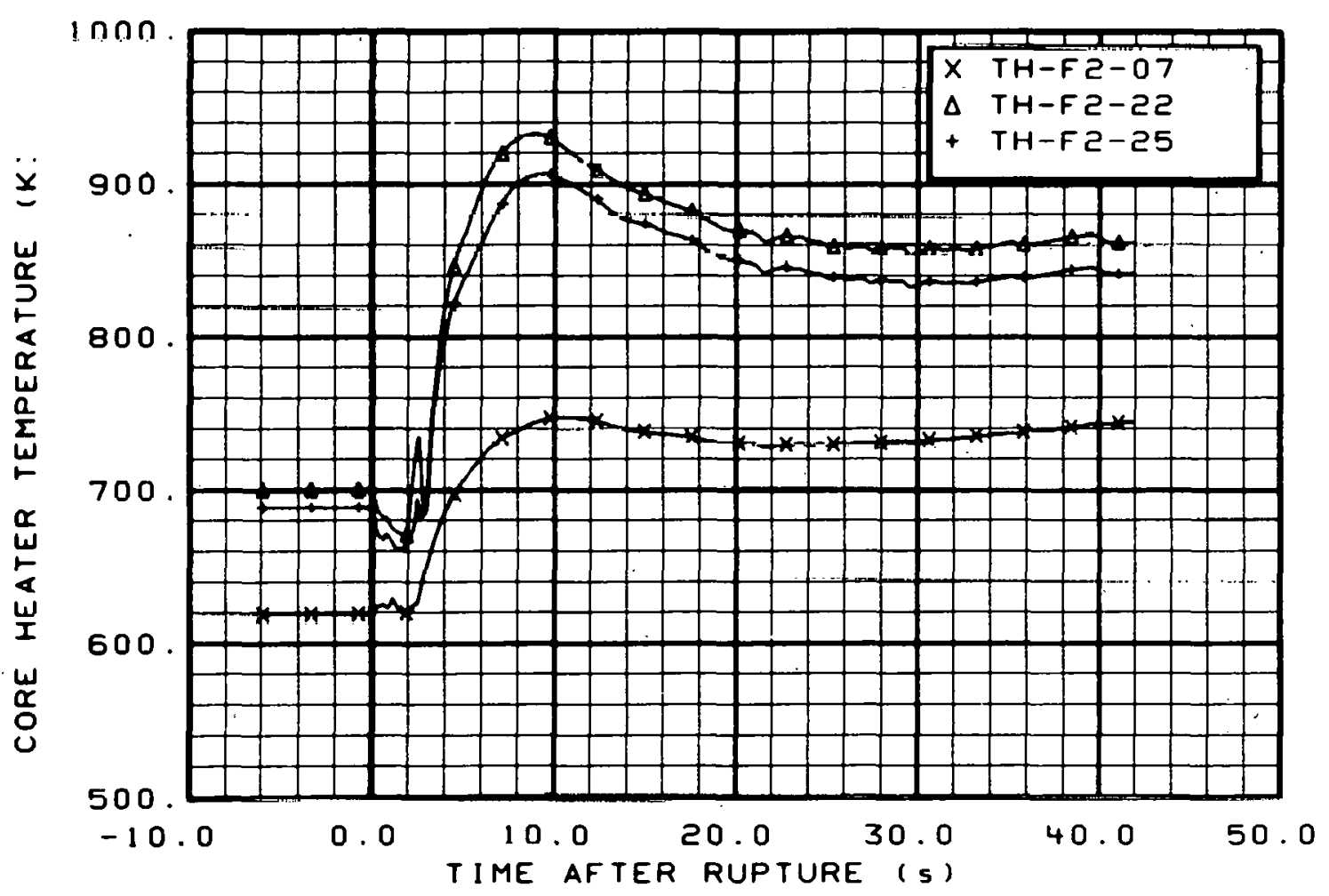

Fig. 130 Core heater temperature, Rod F-2 (TH-F2-07, TH-F2-22, and $\mathrm{TH}-\mathrm{F} 2-25$ ), from -6 to $42 \mathrm{~s}$. 


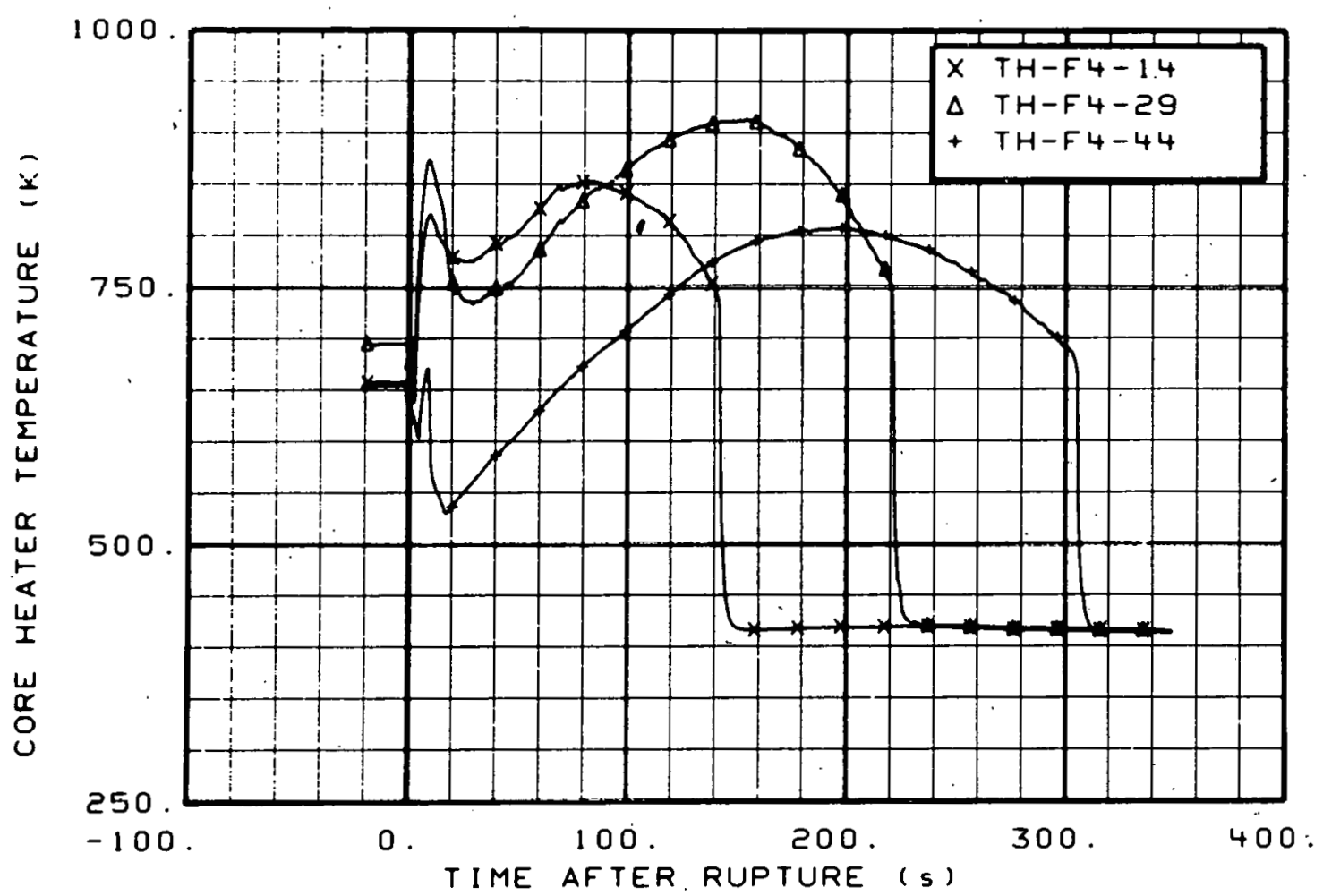

Fig. 131 Core heater temperature, Rod F-4 (TH-F4-14, TH-F4-29, and . $\mathrm{TH}-\mathrm{F} 4-44$ ), from -20 to $350 \mathrm{~s}$.

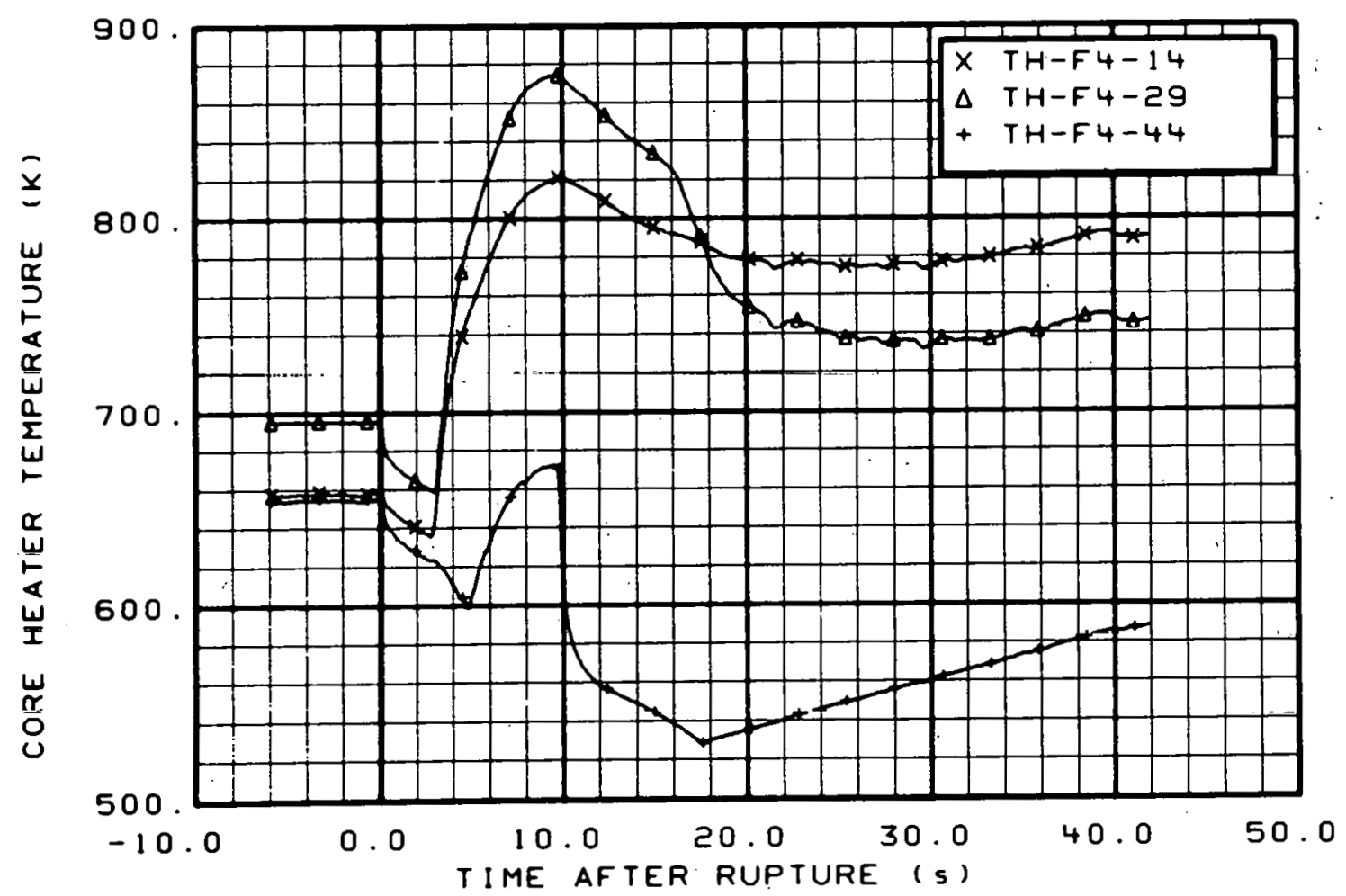

Fig. 132 Core heater temperature, Rod F-4 (TH-F4-14, TH-F4-29, and $\mathrm{TH}-\mathrm{F} 4-44$ ), from -6 to $42 \mathrm{~s}$. 


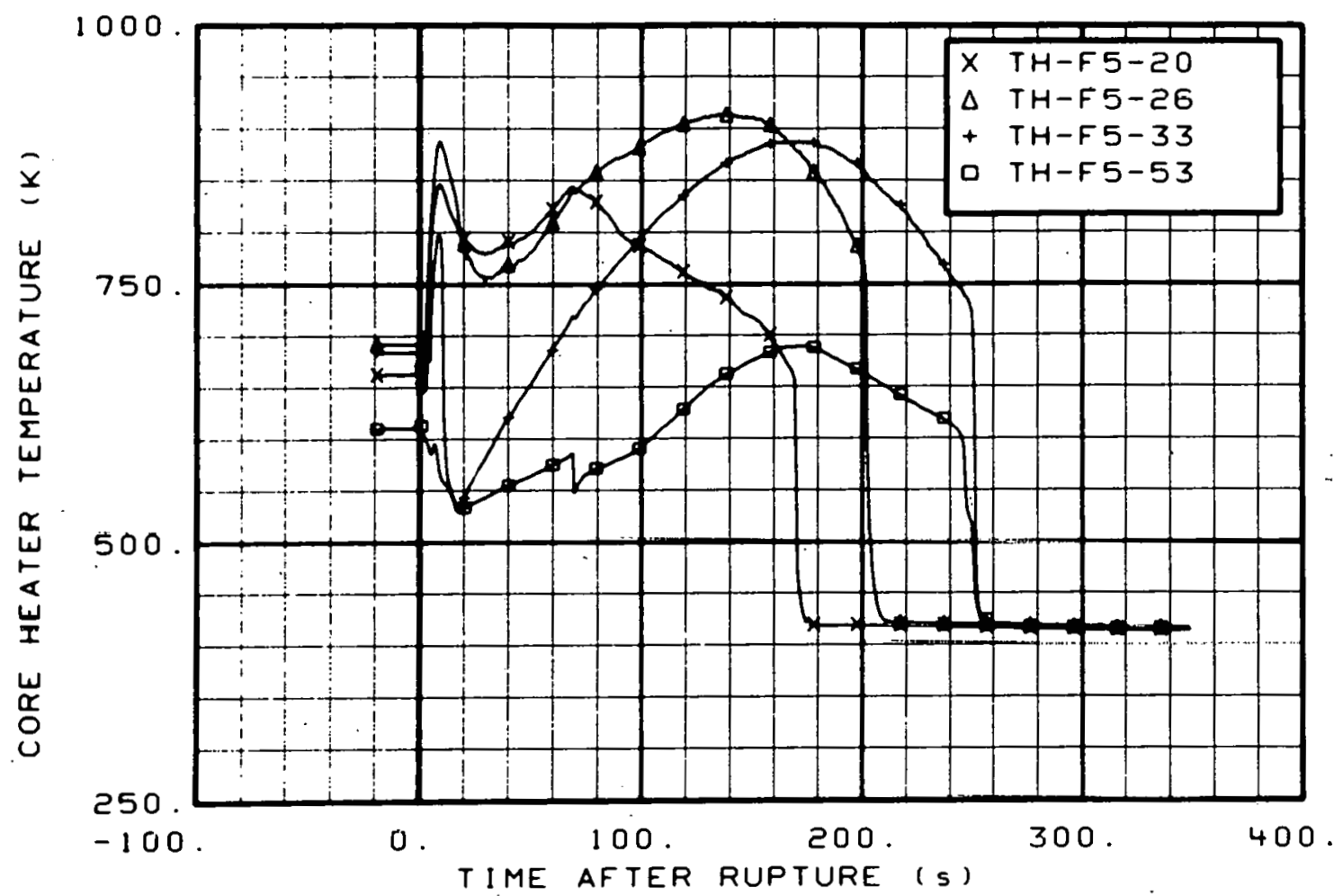

Fig. 133 Core heater temperature, Rod F-5 (TH-F5-20, TH-F5-26, TH-F5-33, and $\mathrm{TH}-\mathrm{F} 5-53$ ), from -20 to $350 \mathrm{~s}$.

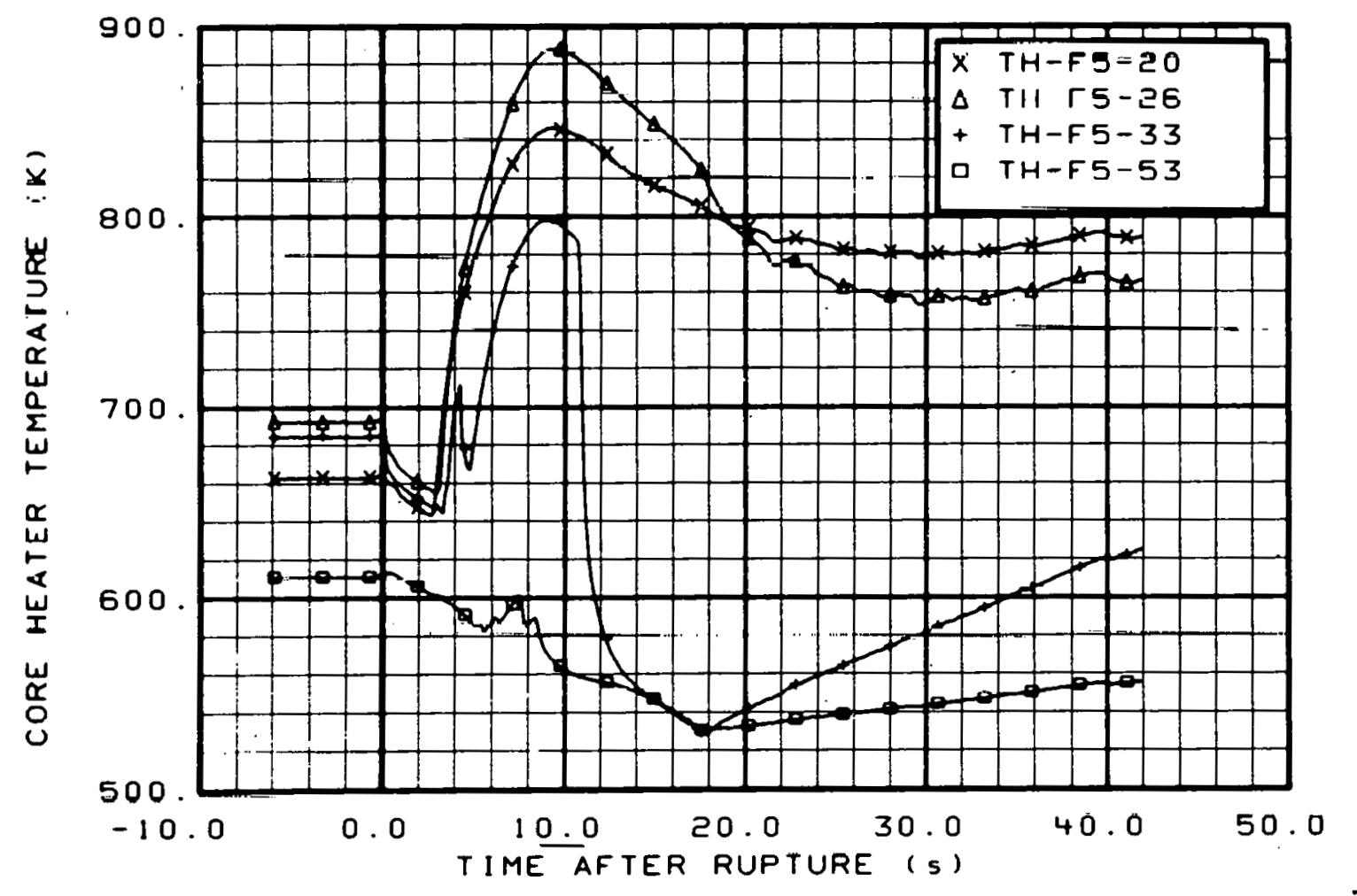

Fig. 134 Core heater temperature, Rod F-5 (TH-F5-20, TH-F5-26, TH-F5-33, and TH-F5-53), from -6 to $42 \mathrm{~s}$. 


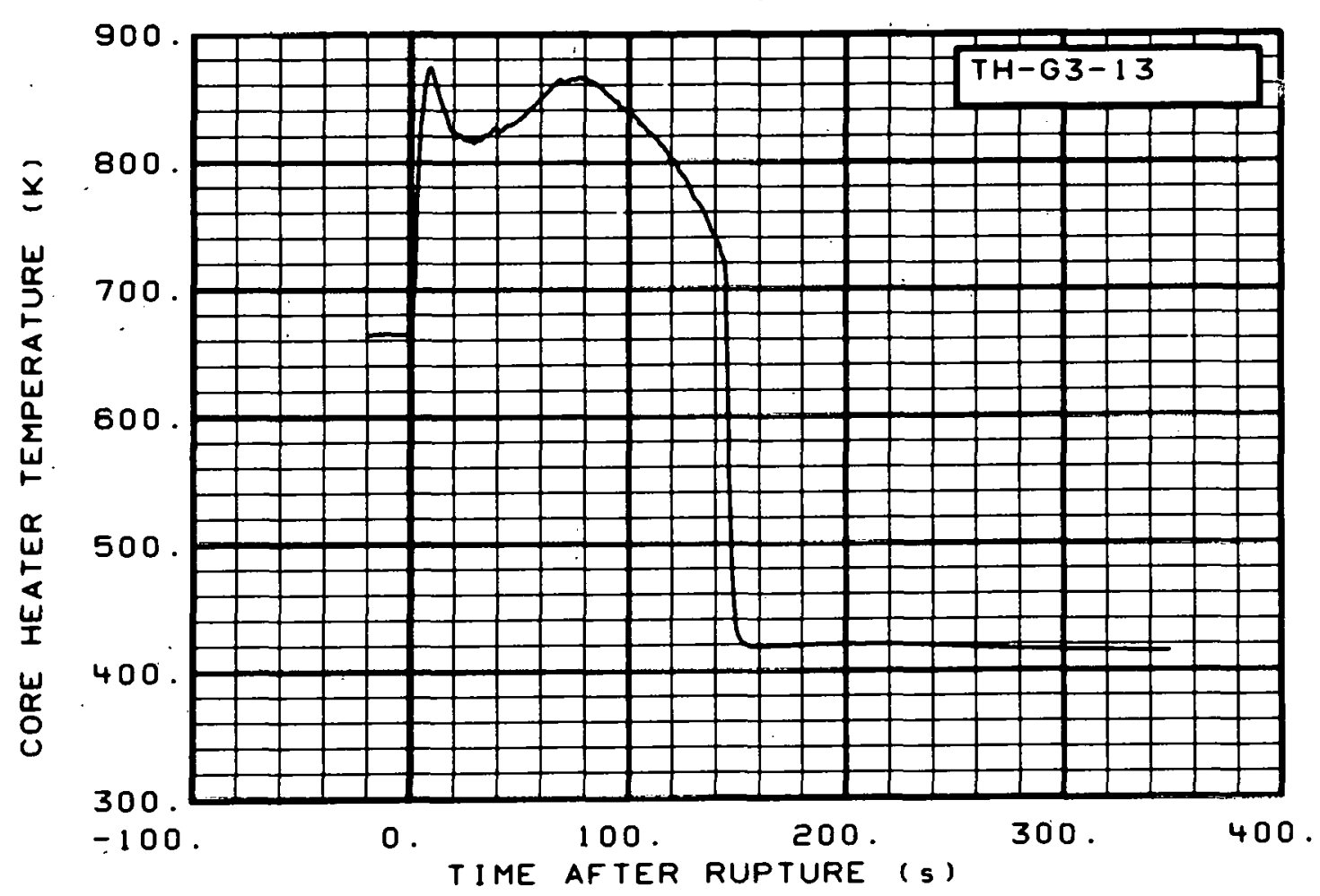

Fig. 135 Core heater temperature, Rod G-3 (TH-G3-13), from -20 to $350 \mathrm{~s}$.

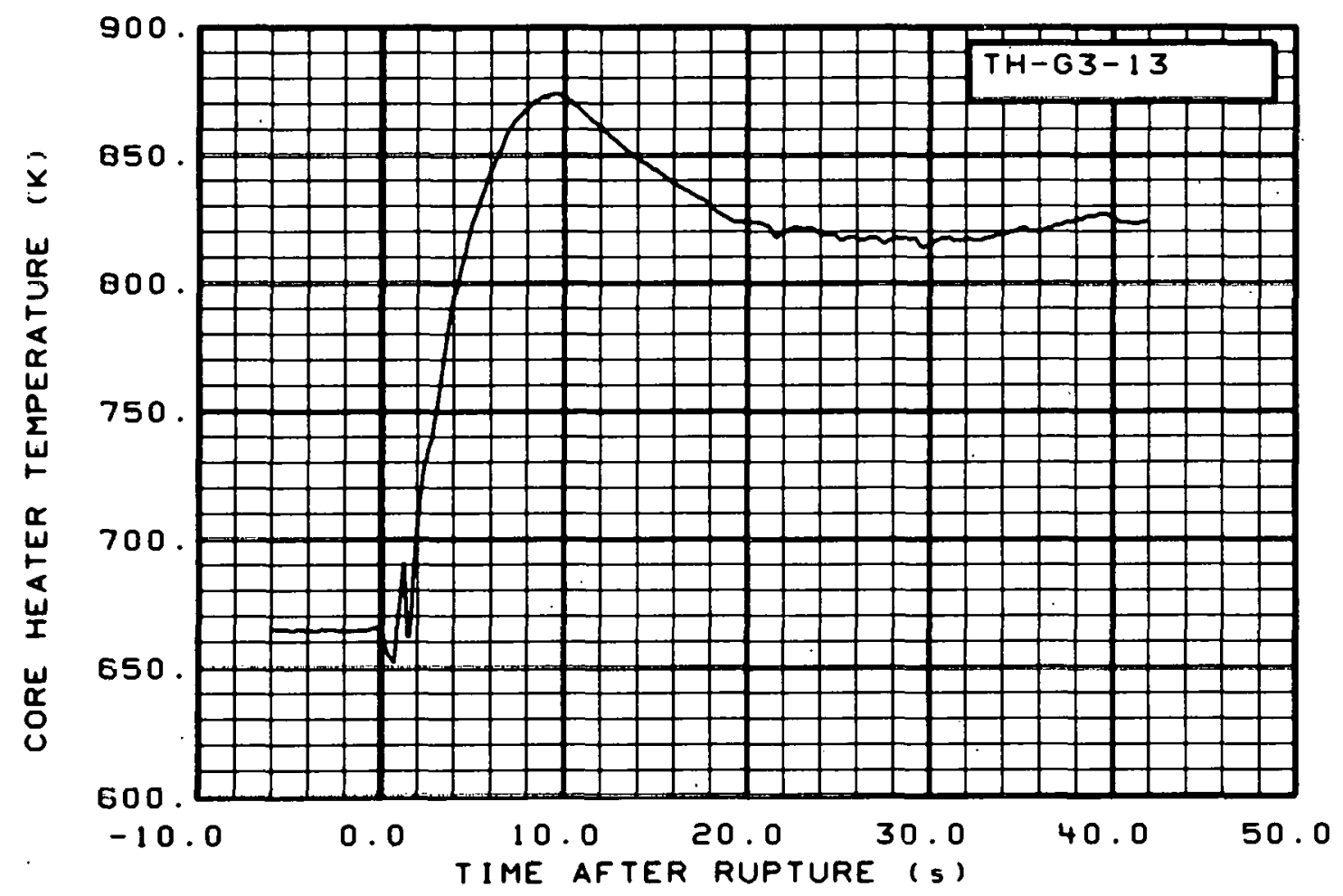

Fig. 136 Core heater temperature, Rod G-3 (TH-G3-13), from -6 to $42 \mathrm{~s}$. 


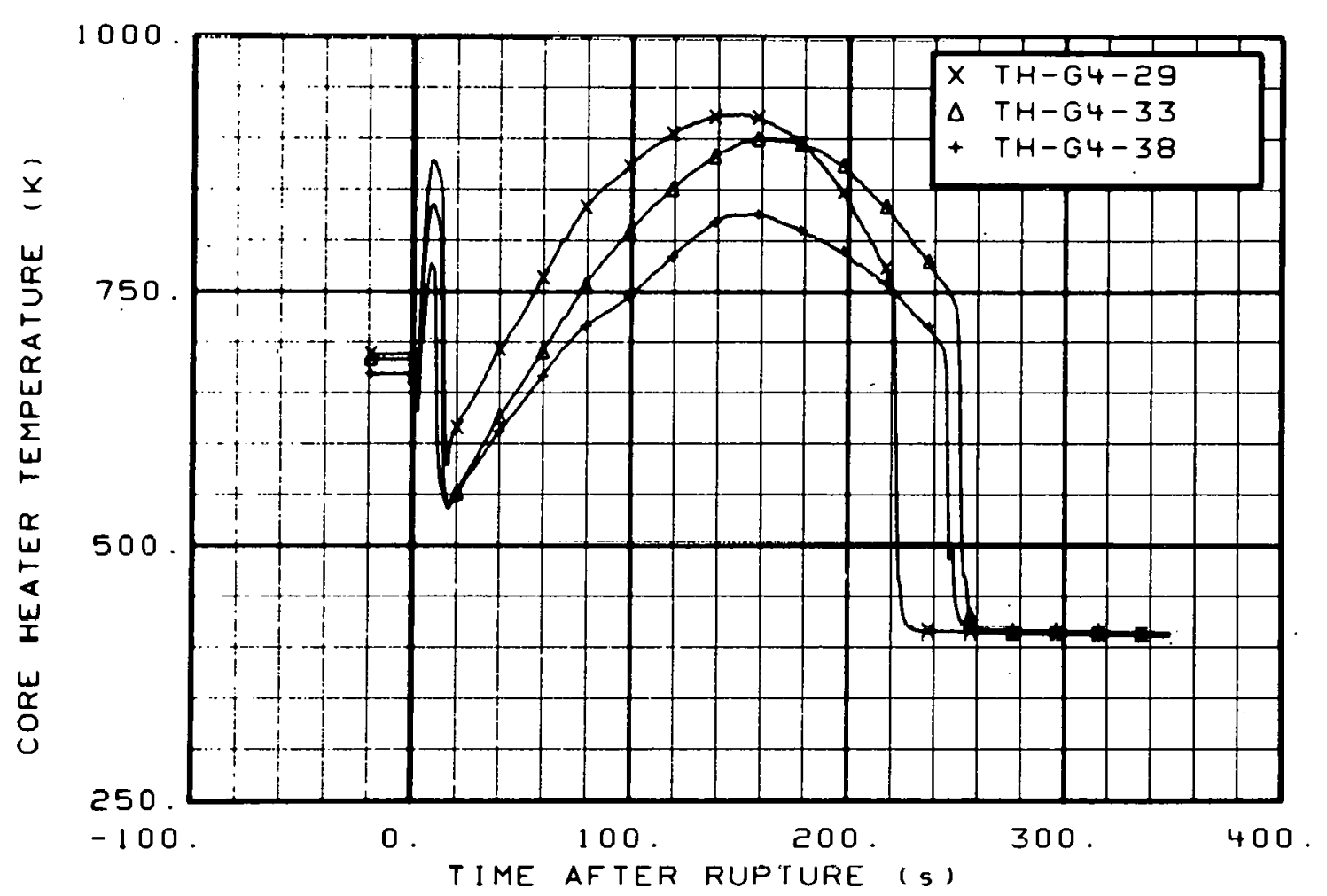

Fig. 137 Core heater temperature, Rod G-4 (TH-G4-29, TH-G4-33, and TH-G4-38), from -20 to $350 \mathrm{~s}$.

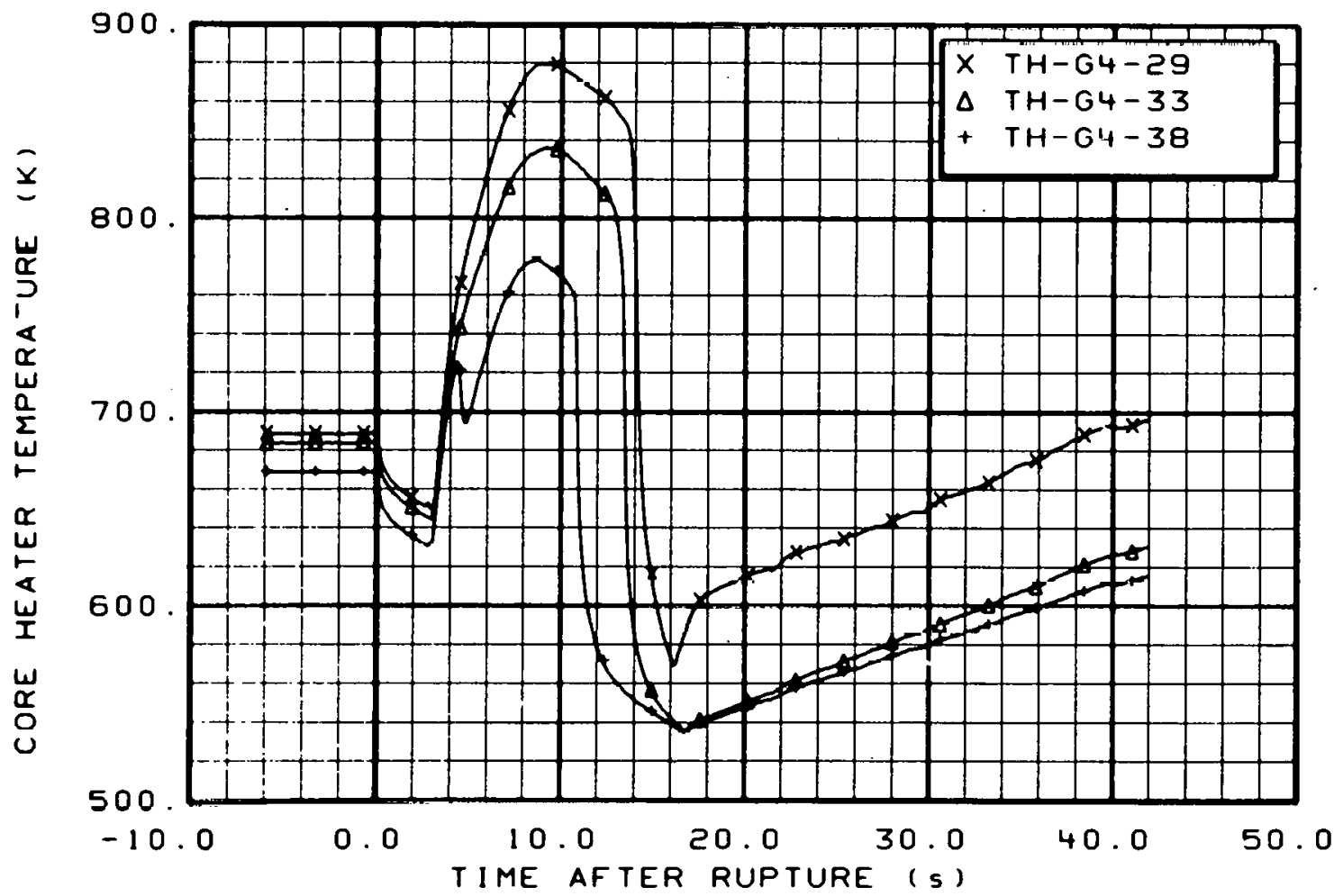

Fig. 138 Core heater temperature, Rod G-4 (TH-G4-29, TH-G4-33, and $\mathrm{TH}-\mathrm{G} 4-38$ ), from -6 to $42 \mathrm{~s}$. 


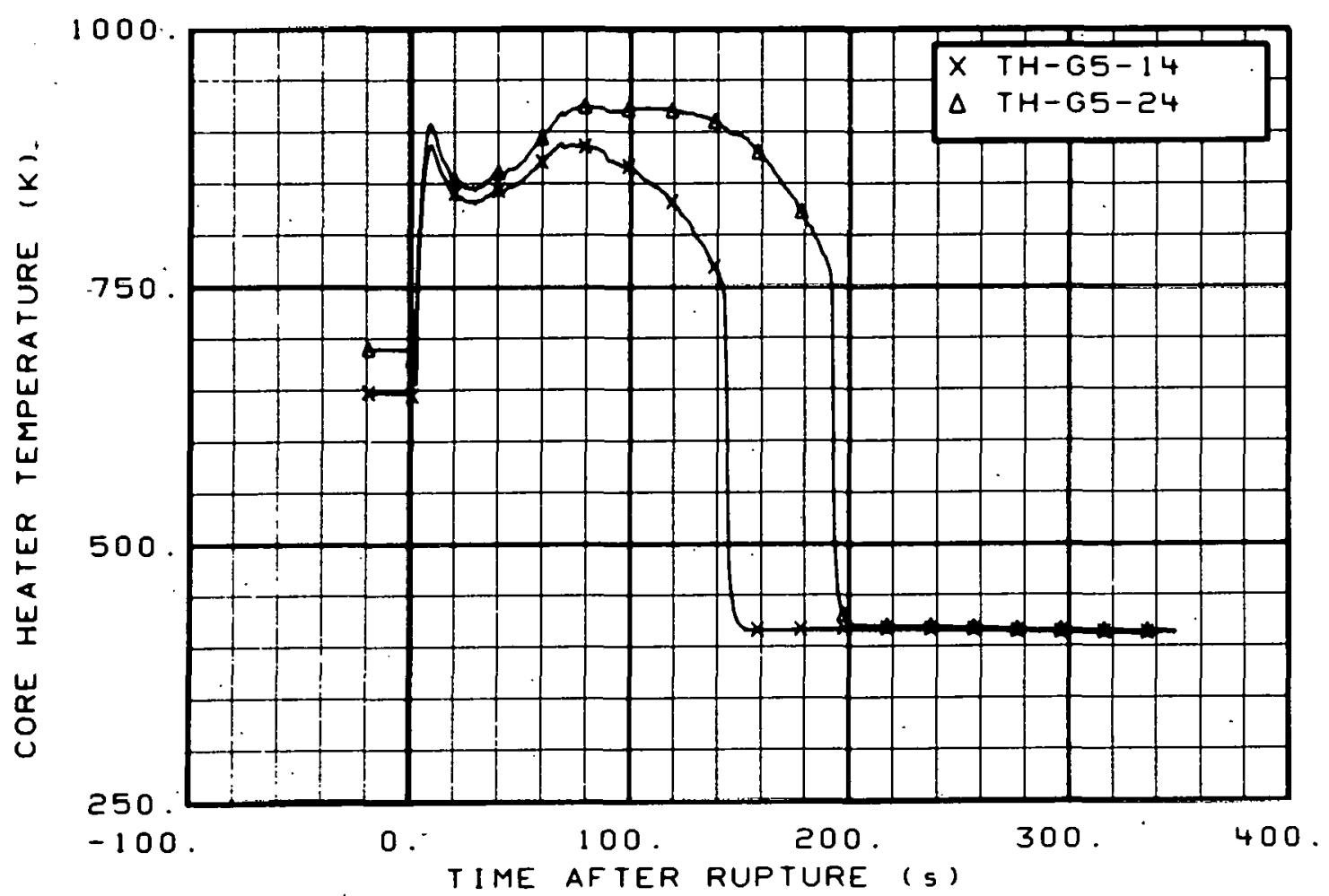

Fig. 139 Core heater temperature, Rod G-5 (TH-G5-14 and TH-G5-24), from -20 to $350 \mathrm{~s}$.

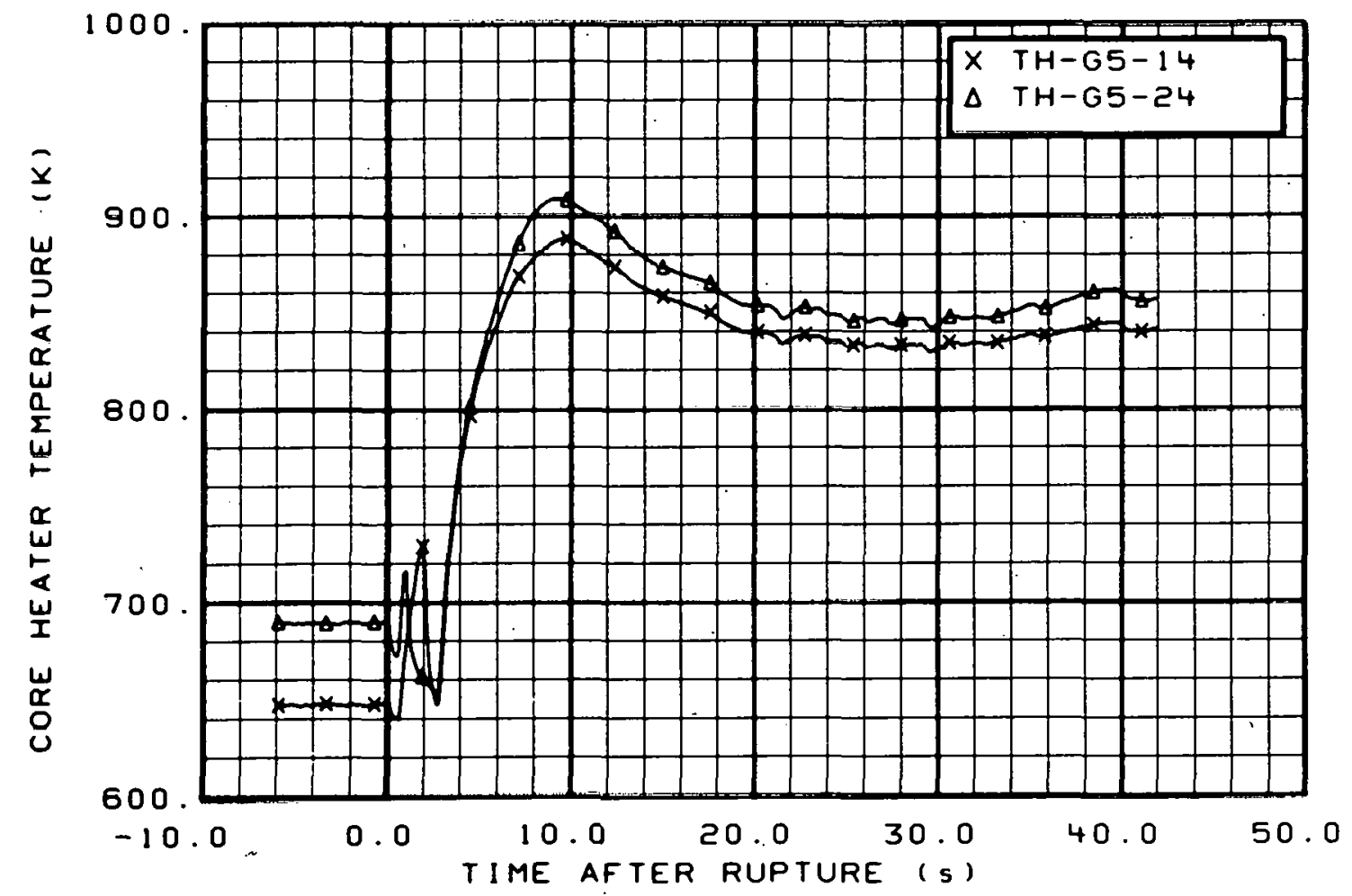

Fig. 140 Core heater temperature, Rod G-5 (TH-G5-14 and TH-G5-24), from -6 to $42 \mathrm{~s}$. 


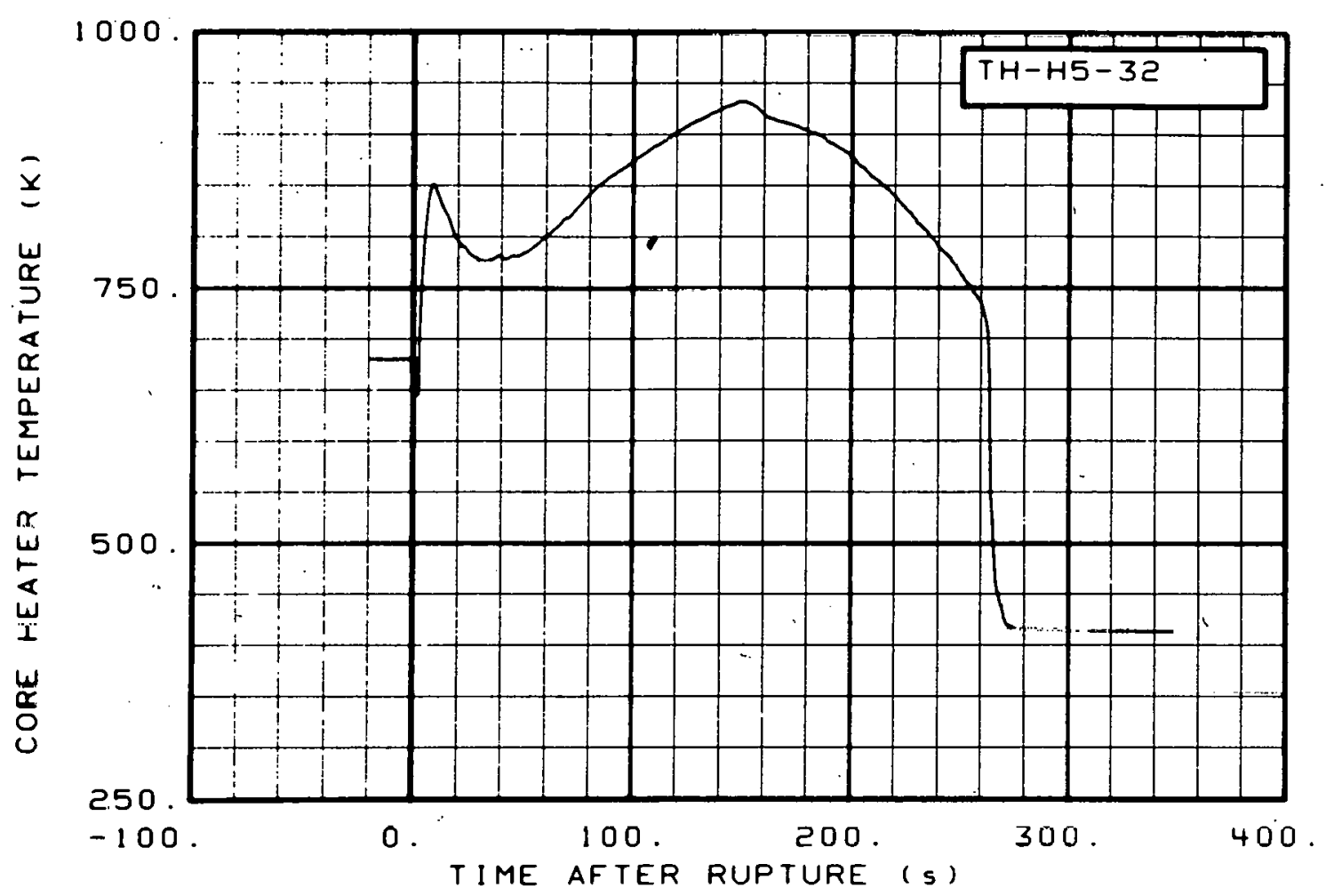

Fig. 141 Core heater temperature, Rod $\mathrm{H}-5$ ( $\mathrm{TH}-\mathrm{H} 5-32)$, from -20 to $350 \mathrm{~s}$.

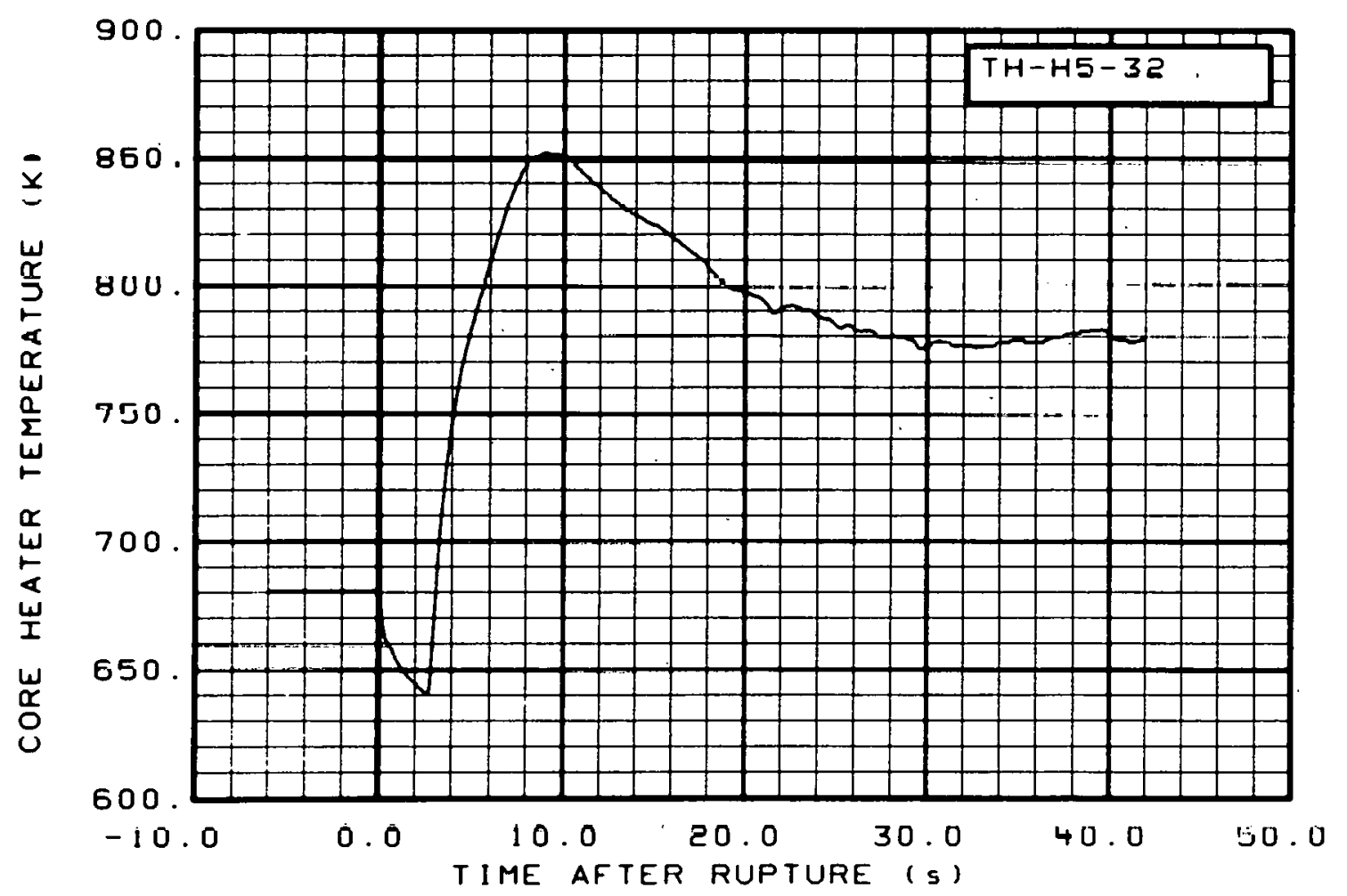

Fig. 142 Core heater temperature, Rod H-5 (TH-H5-32), from -6 to $42 \mathrm{~s}$. 


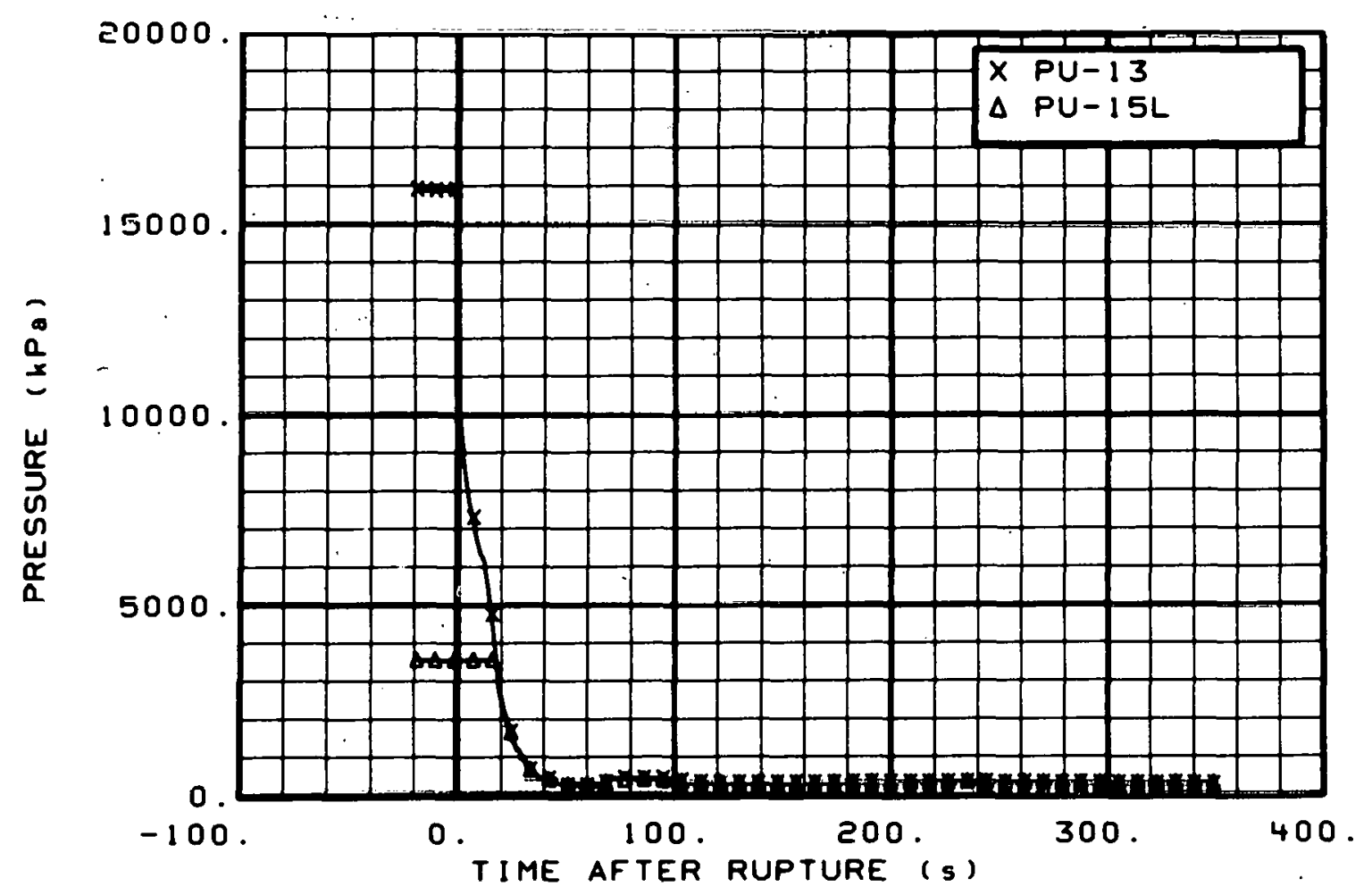

Fig. 143 Pressure in intact loop (PU-13 and PU-15L), from -20 to $350 \mathrm{~s}$.

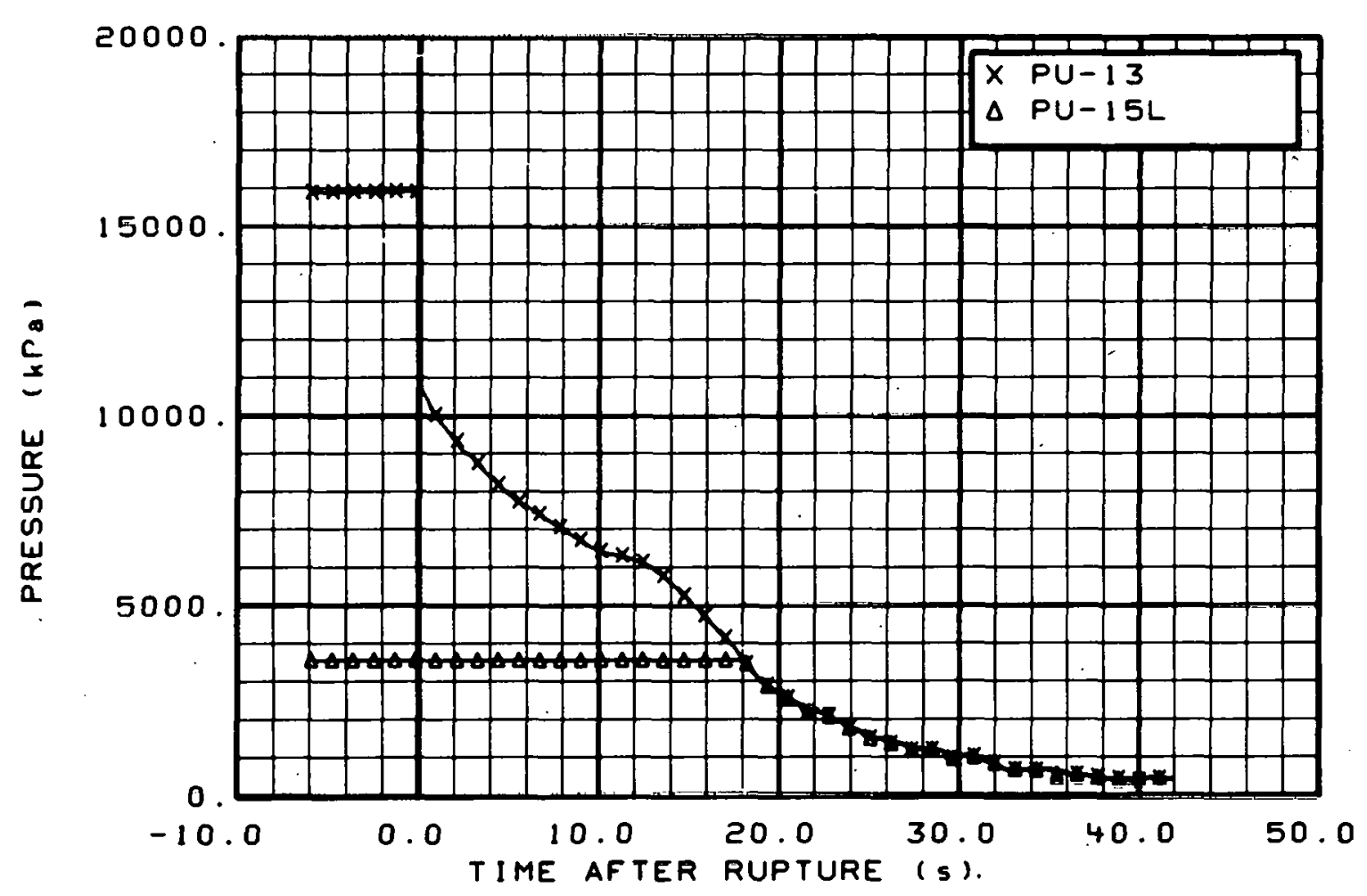

Fig. 144 Pressure in intact loop (PU-13 and PU-15L), from -6 to $42 \mathrm{~s}$. 


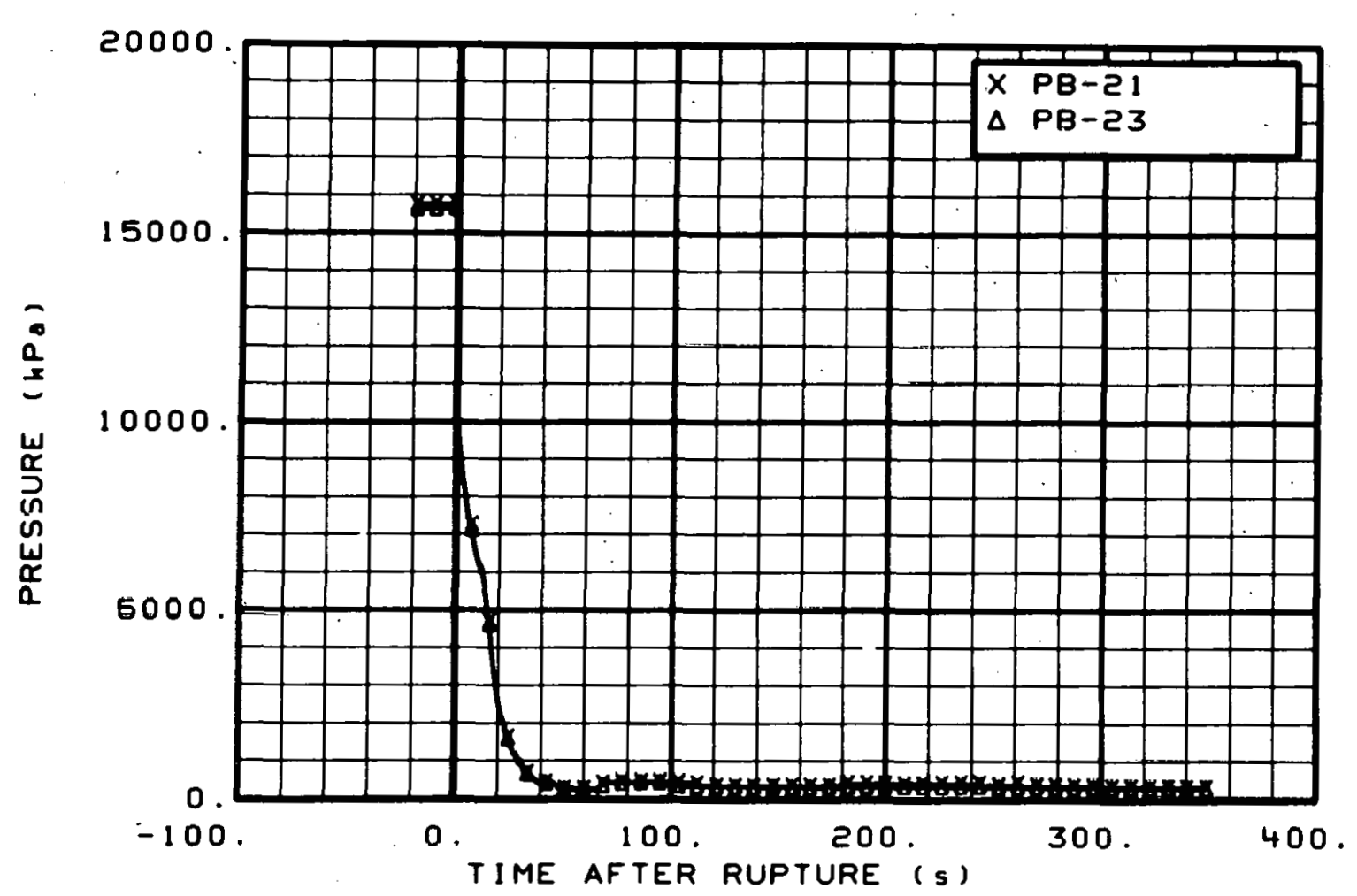

Fig. 145 Pressure in broken loop, vessel side (PB-21 and $P B-23)$, from -20 to $350 \mathrm{~s}$.

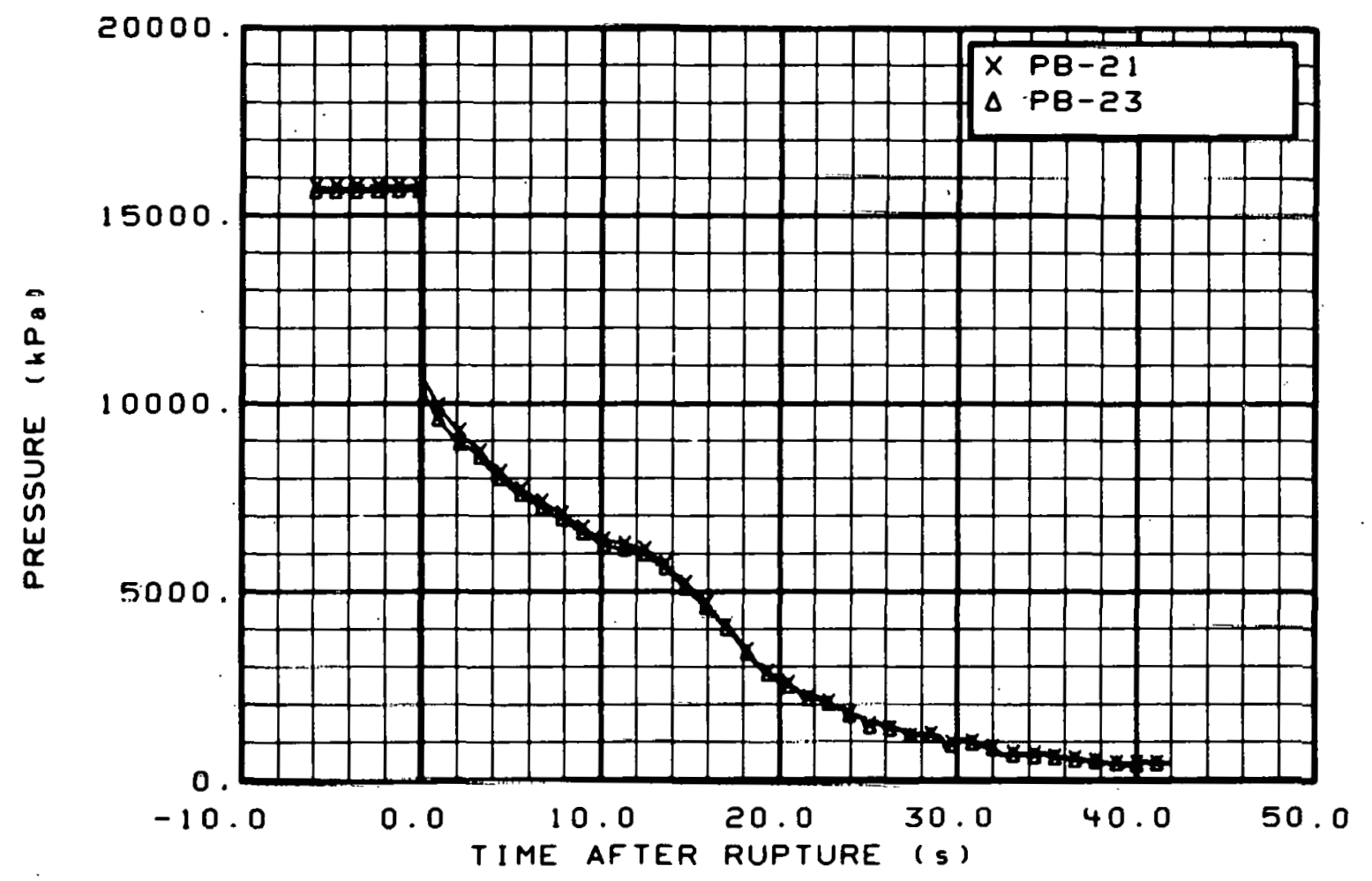

Fig. 146 Pressure in broken 1oop, vessel side (PB-21 and PB-23), from -6 to $42 \mathrm{~s}$. 


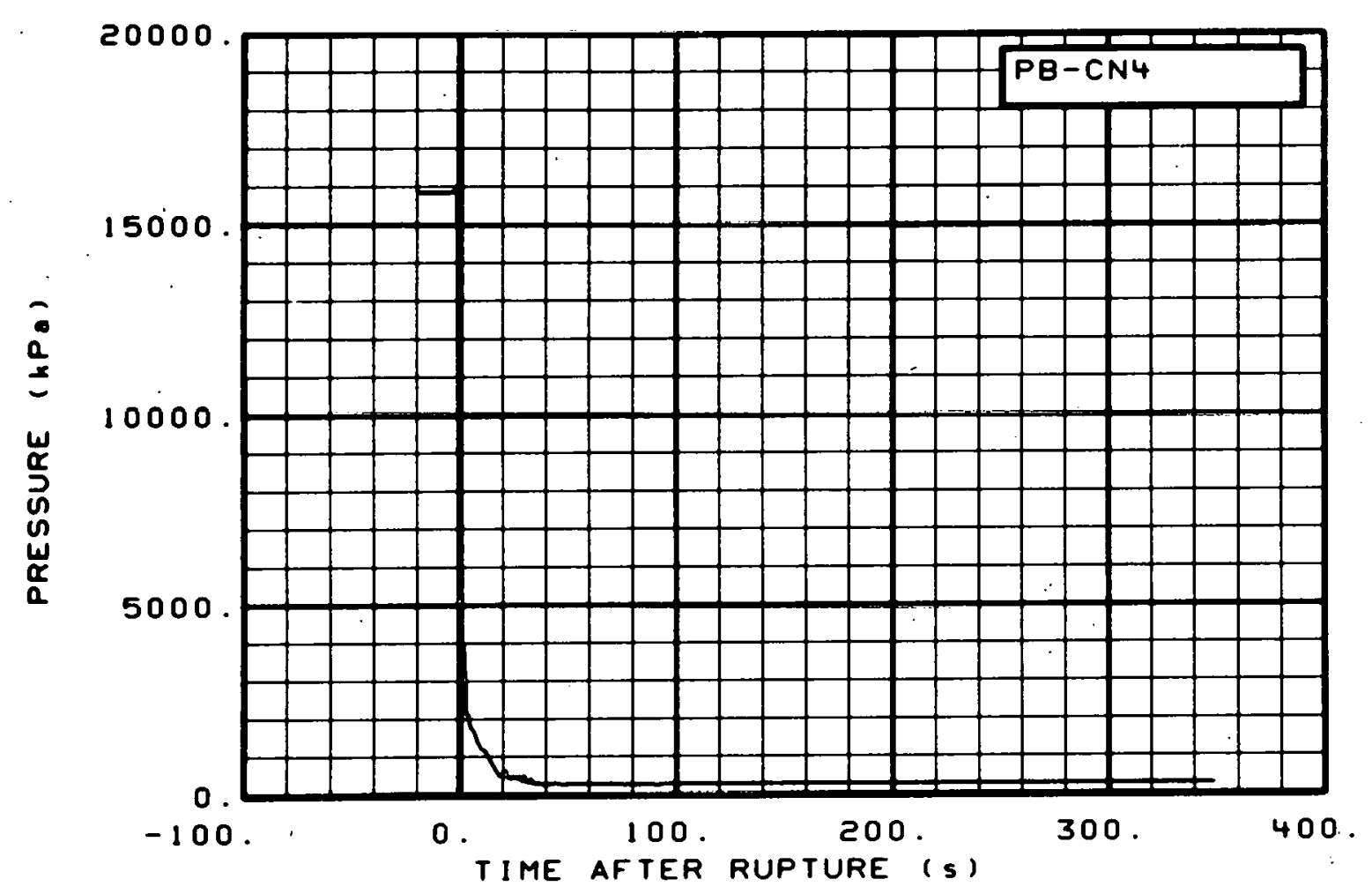

Fig. 147 Pressure in broken loop, vessel side (PB-CN4), from -20 to $350 \mathrm{~s}$.

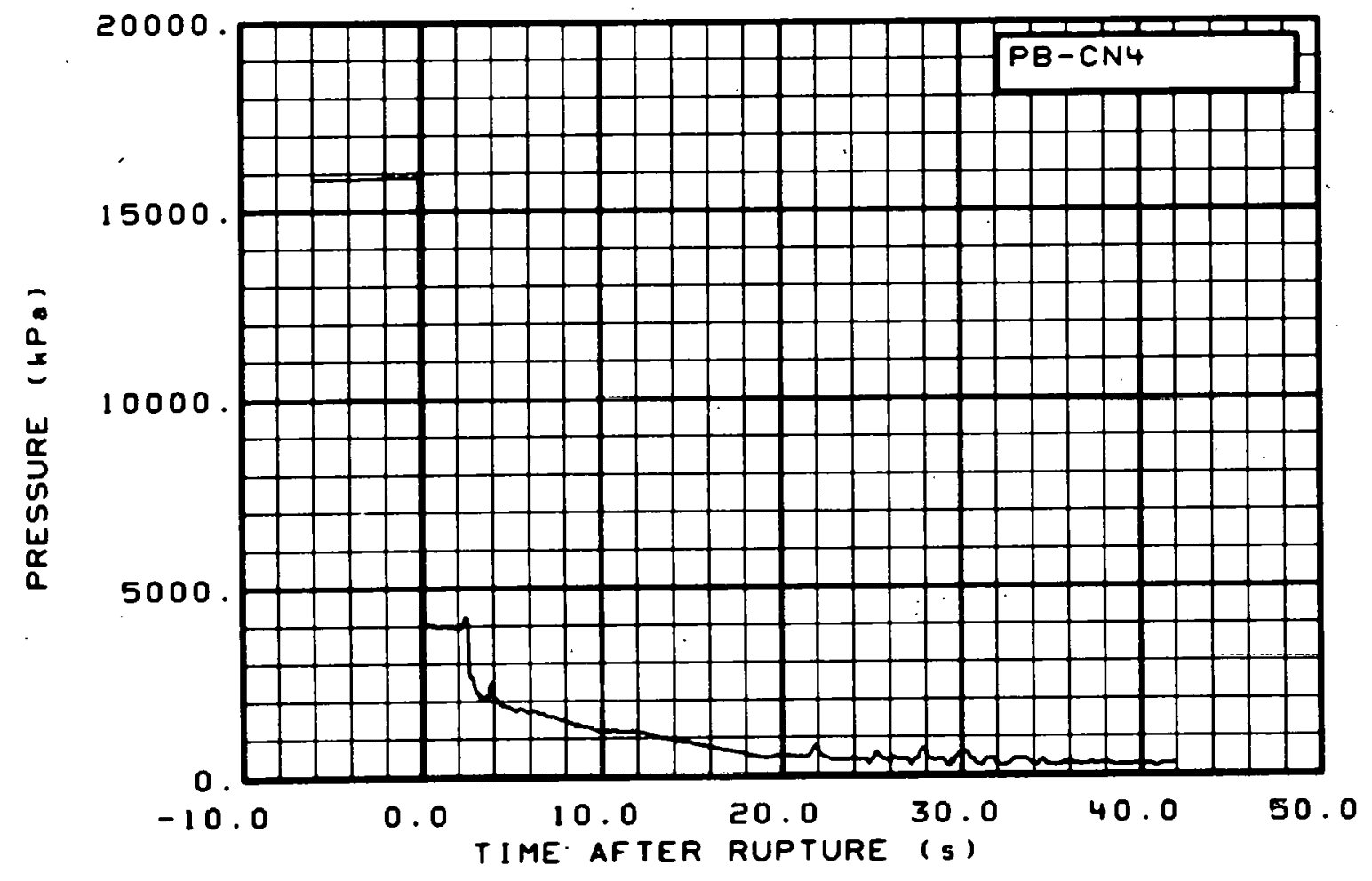

Fig. 148 Pressure in broken loop, vessel side (PB-CN4), from -6 to $42 \mathrm{~s}$. 


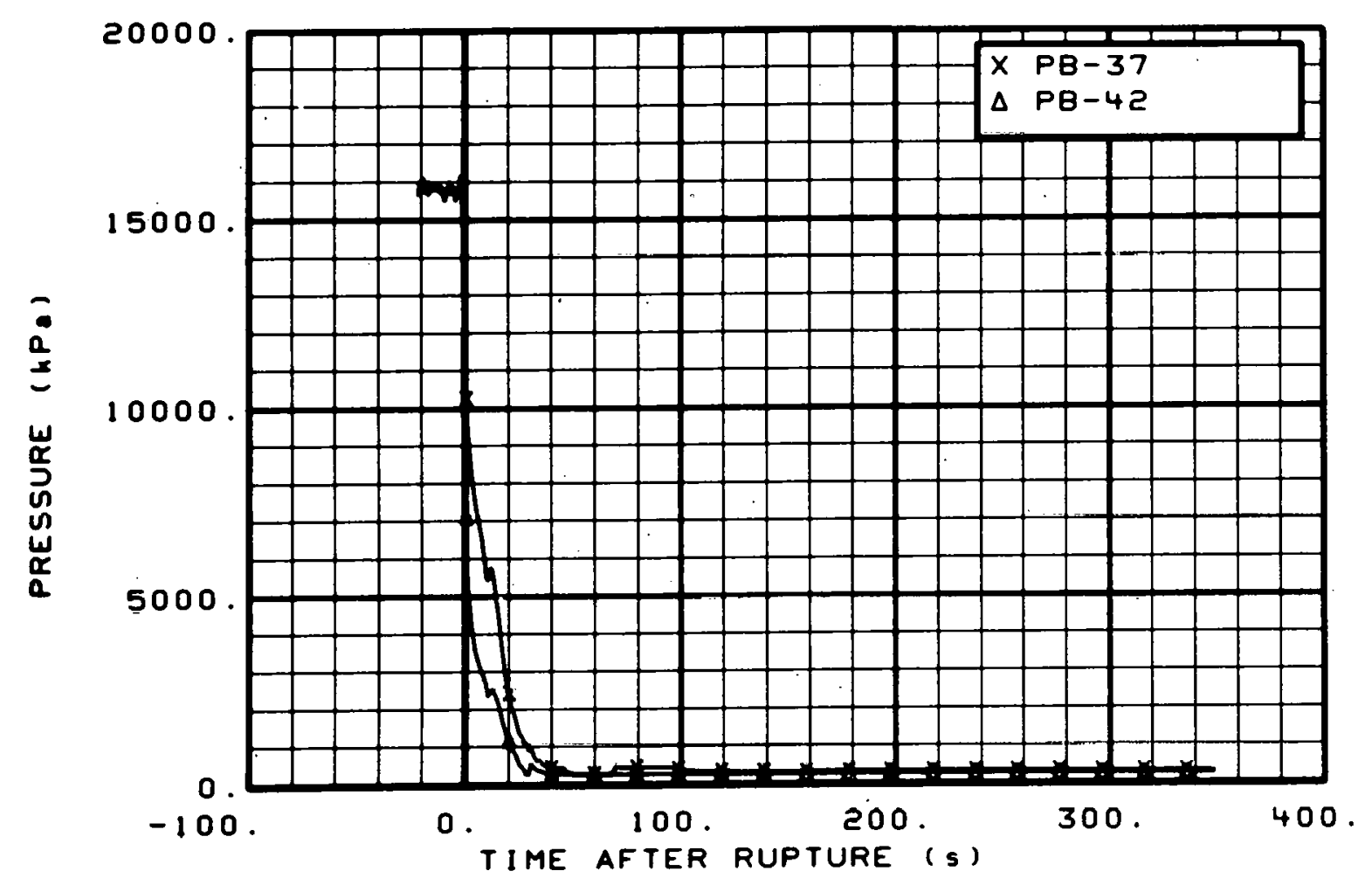

Fig. 149 Pressure in broken 10op, pump side (PB-37 and PB-42), from -20 to $350 \mathrm{~s}$.

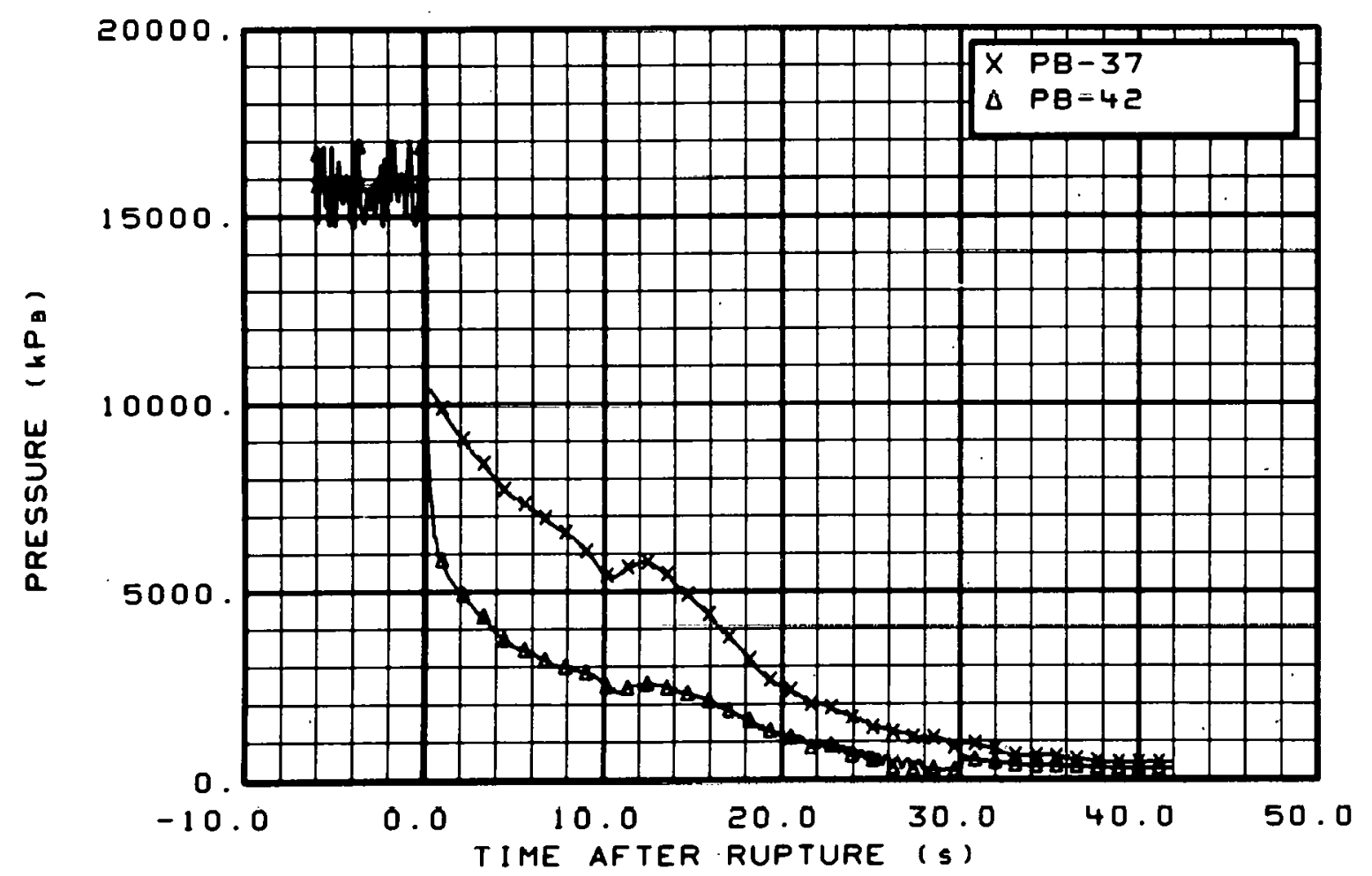

Fig. 150 Pressure in broken loop, pump side (PB-37 and PB-42), from -6 to $42 \mathrm{~s}$. 


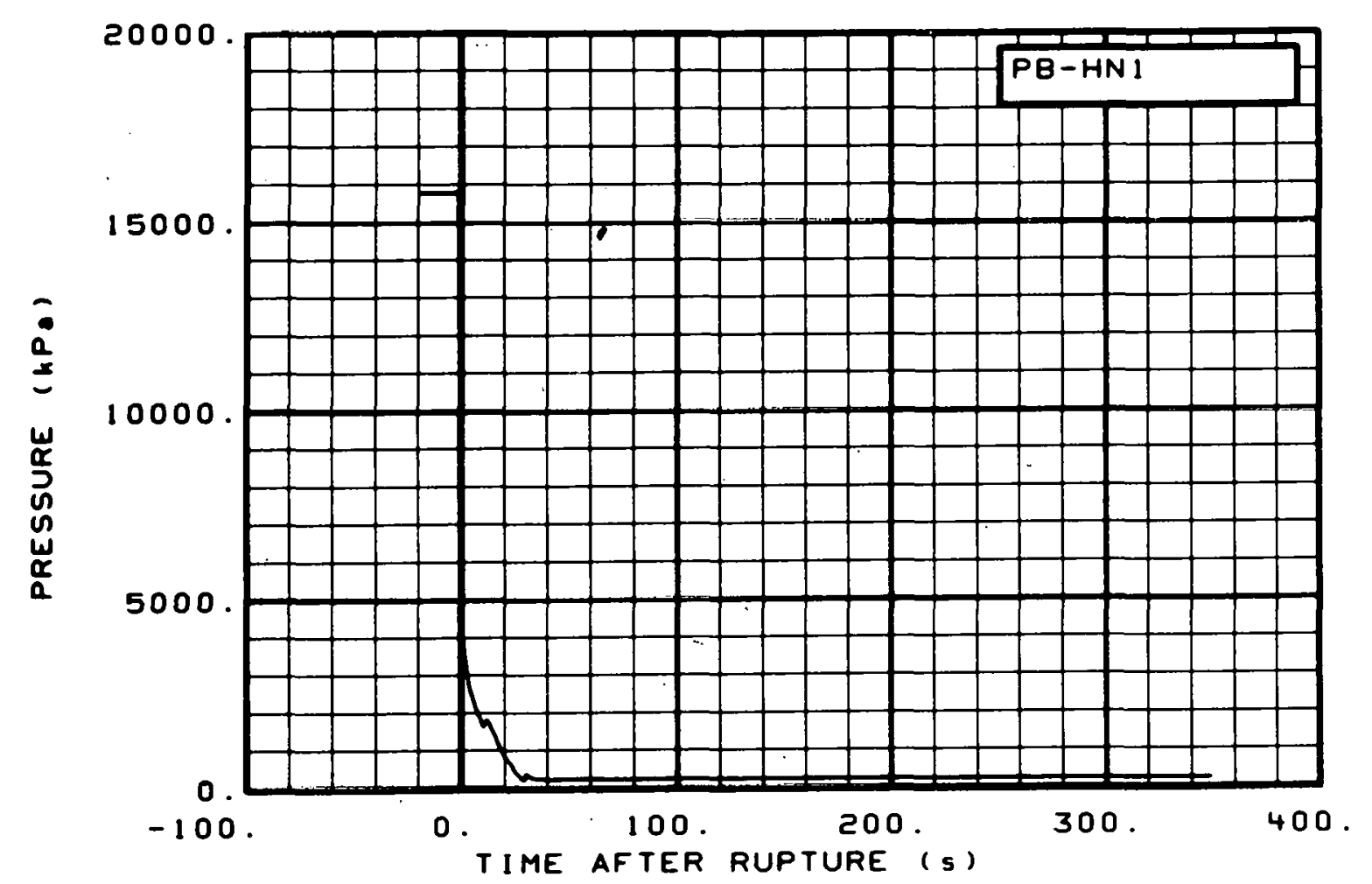

Fig. 151 Pressure in broken loop, vessel side (PB-HN1), from -20 to $350 \mathrm{~s}$.

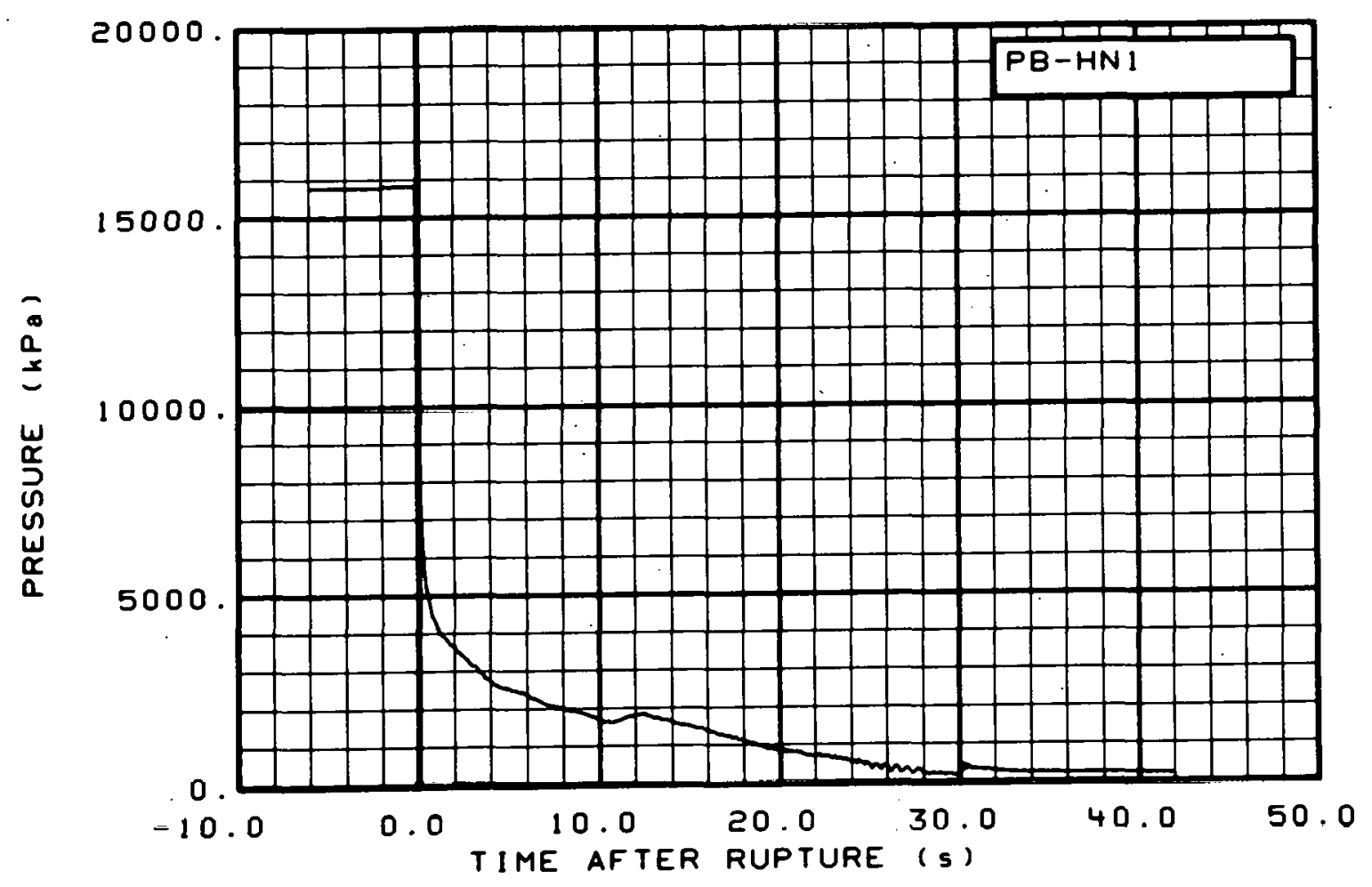

Fig. 152 Pressure in broken loop, vessel side (PB-HN1), from -6 to $42 \mathrm{~s}$. 


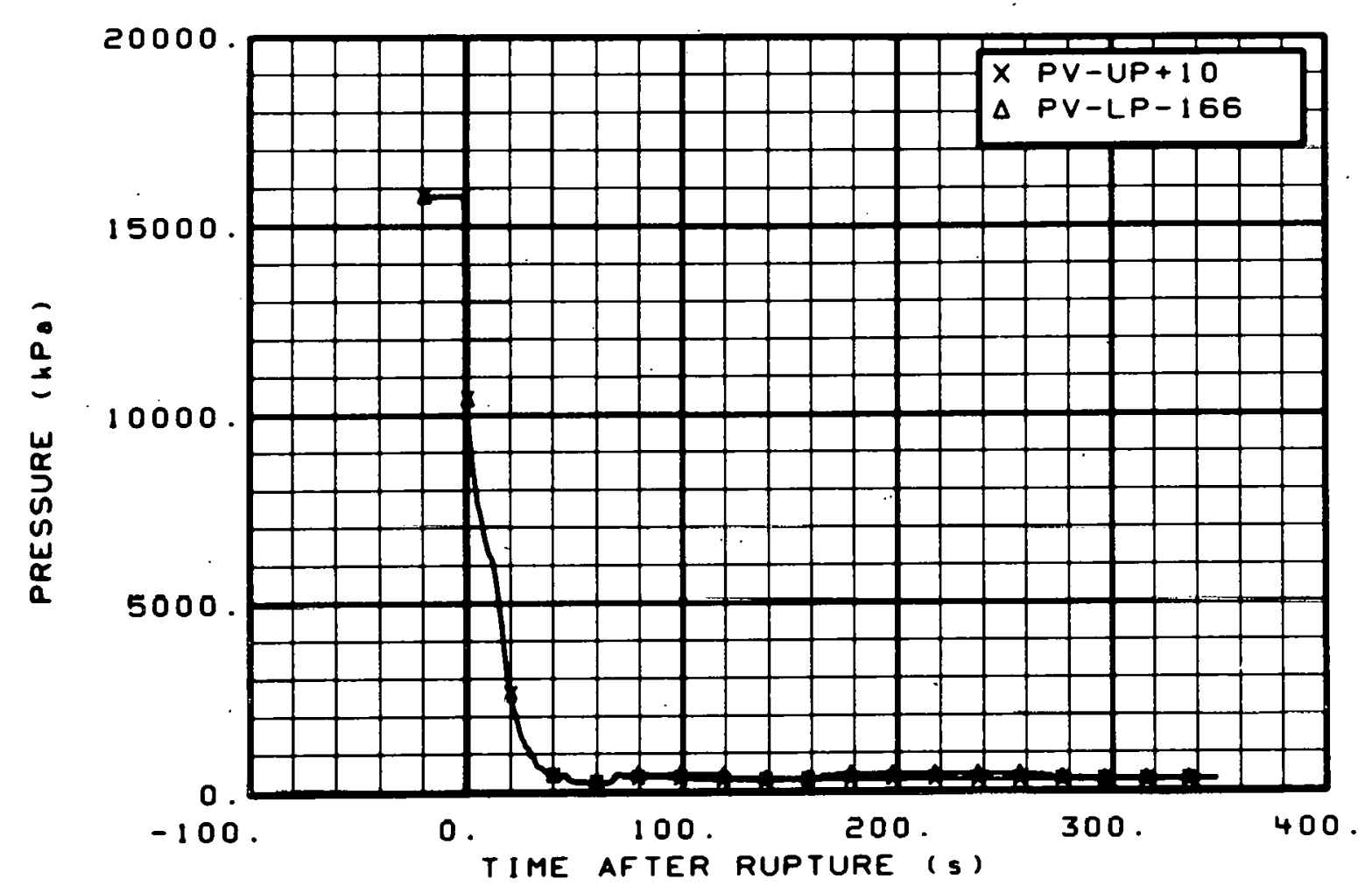

Fig. 153 Pressure in vessel (PV-UP+10 and PV-LP-166), from -20 to $350 \mathrm{~s}$.

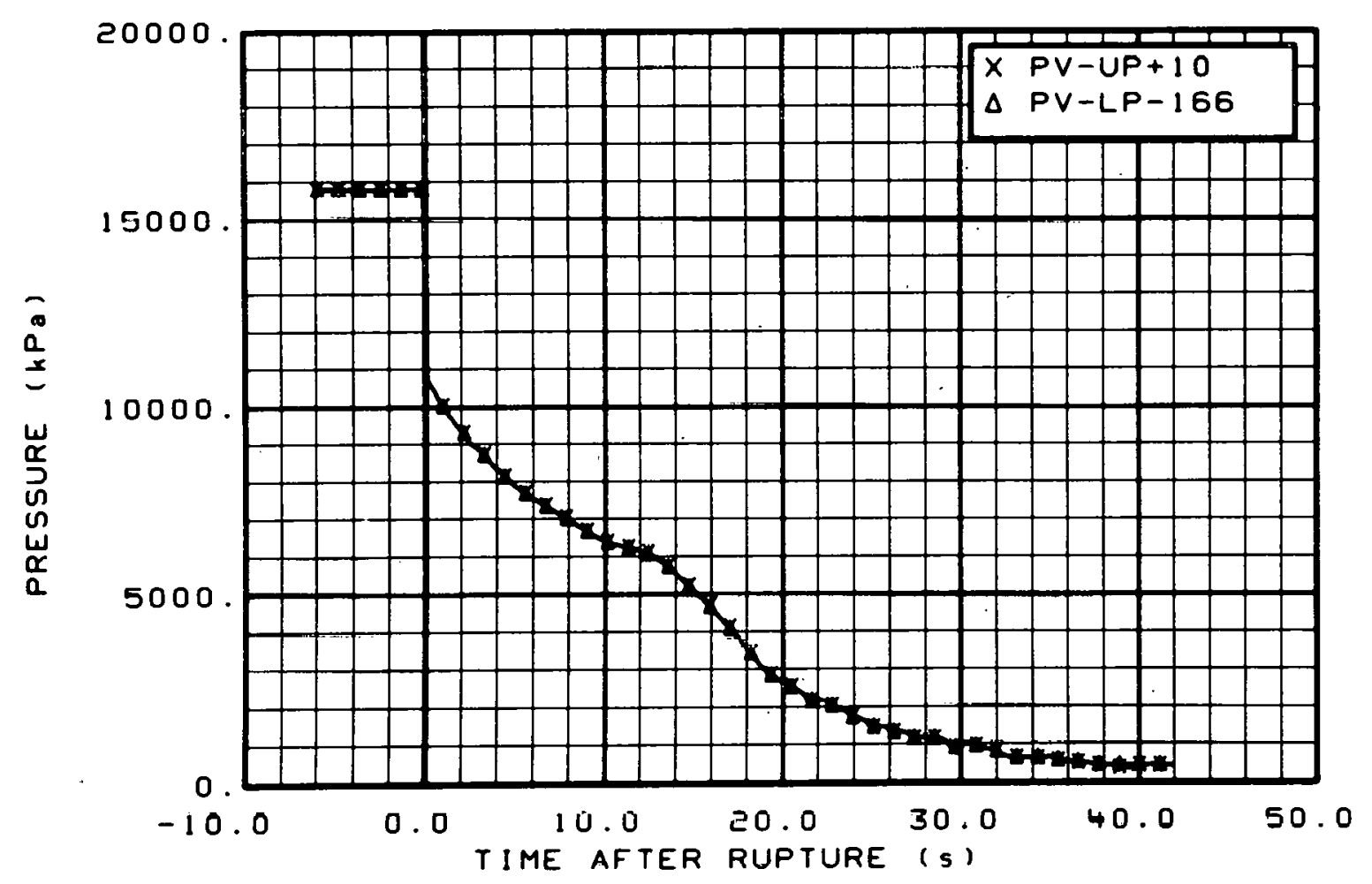

Fig. 154 Pressure in vessel (PV-UP+10 and PV-LP-166), from -6 to $42 \mathrm{~s}$. 


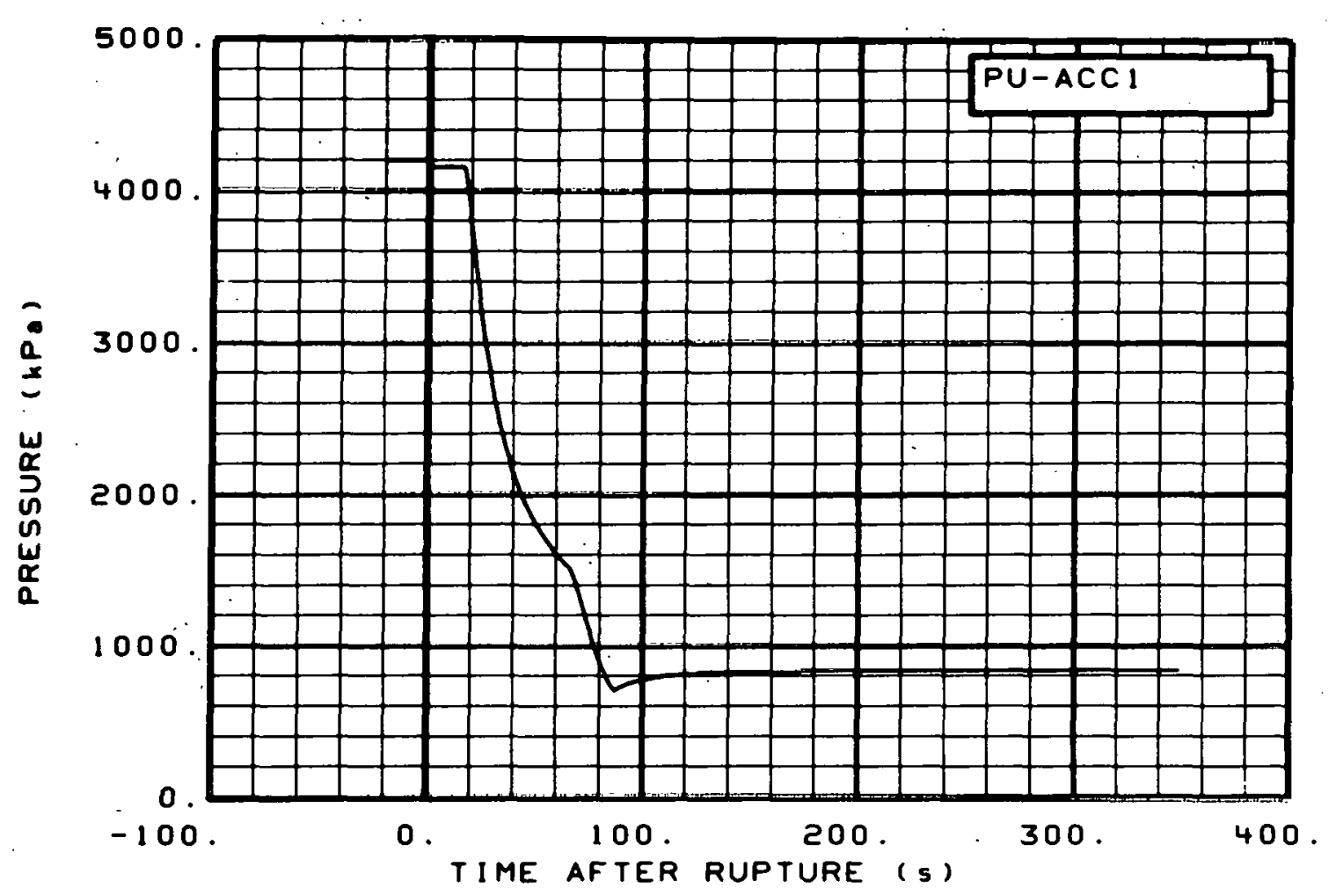

Fig. 155 Pressure in intact loop accumulator (PU-ACC1), from -20 to $350 \mathrm{~s}$.

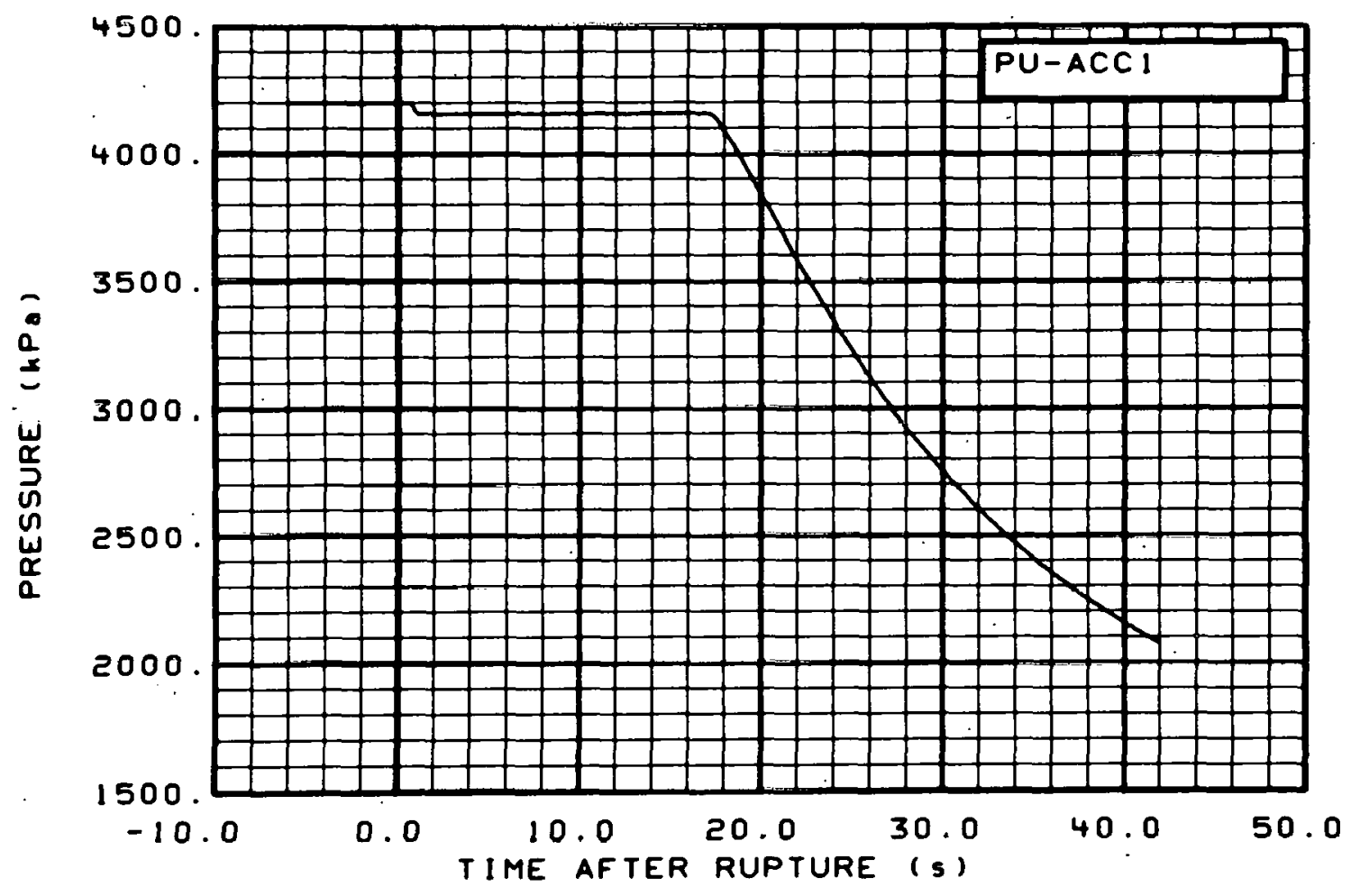

Fig. 156 Pressure in intact loop accumulator (PU-ACCl), from -6 to $42 \mathrm{~s}$. 


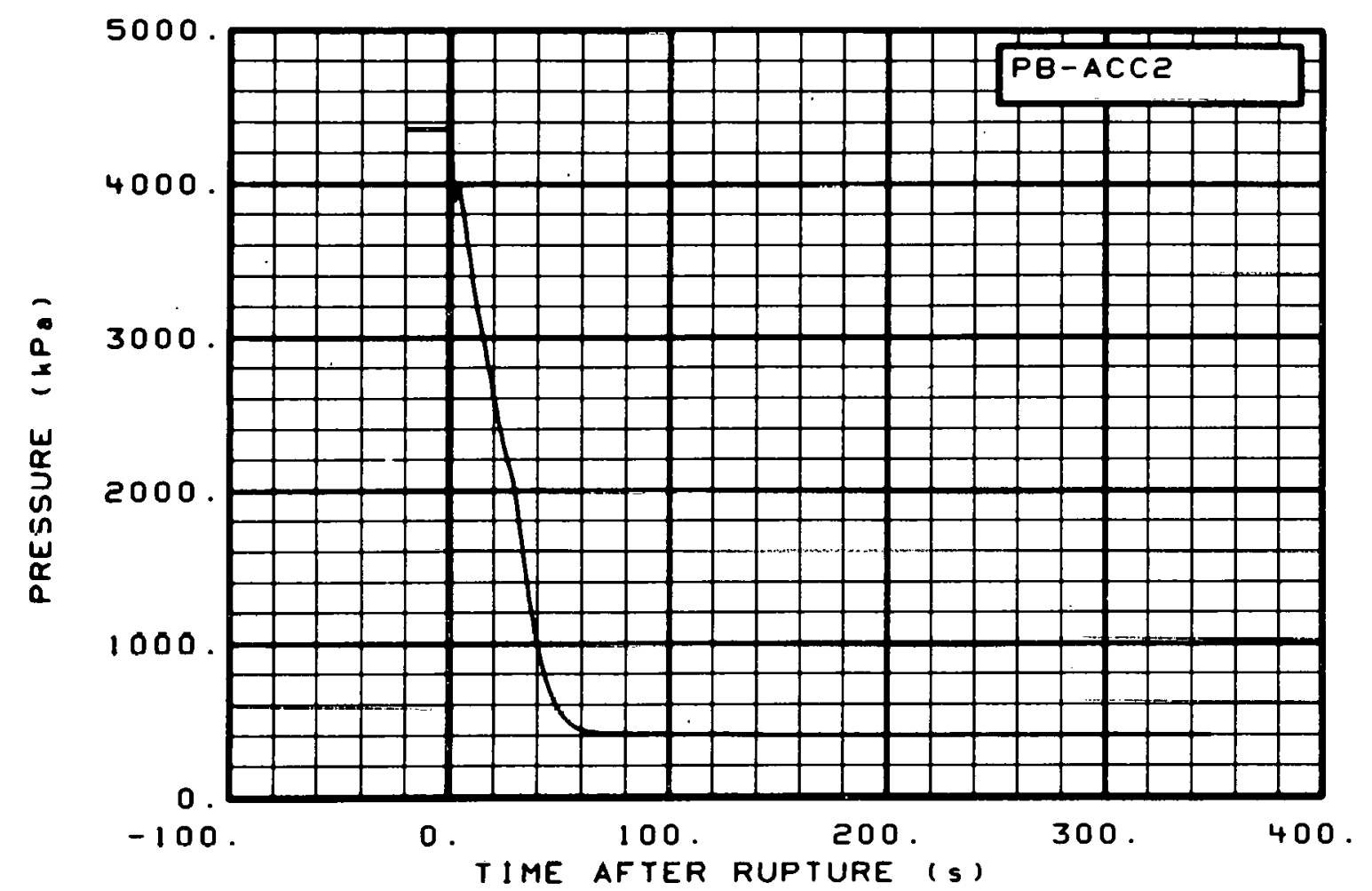

Fig. 157 Pressure in broken loop accumulator (PB-ACC2), from -20 to $350 \mathrm{~s}$.

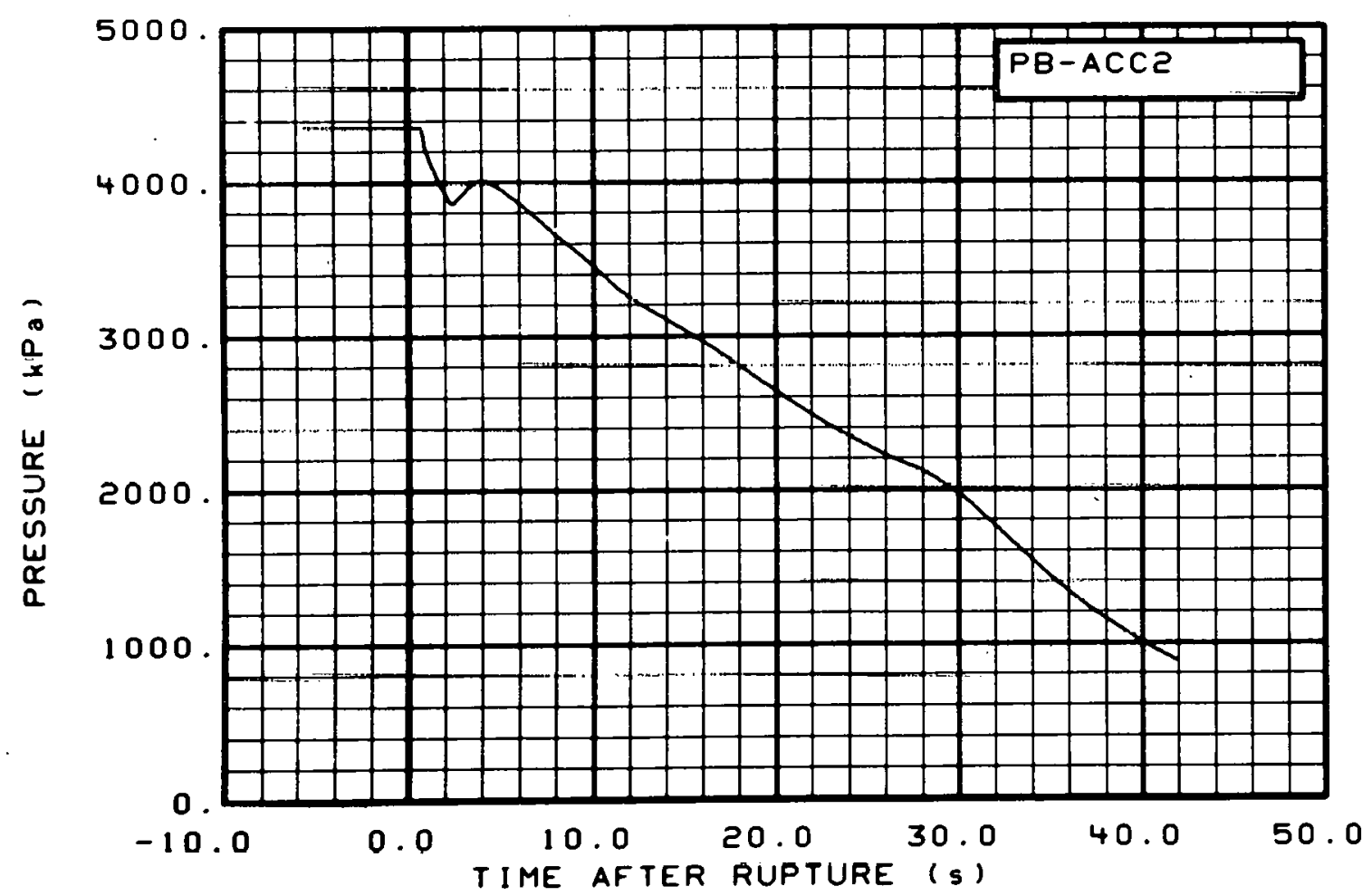

Fig. 158 Pressure in broken loop accumulator (PB-ACC2), from -6 to $42 \mathrm{~s}$. 


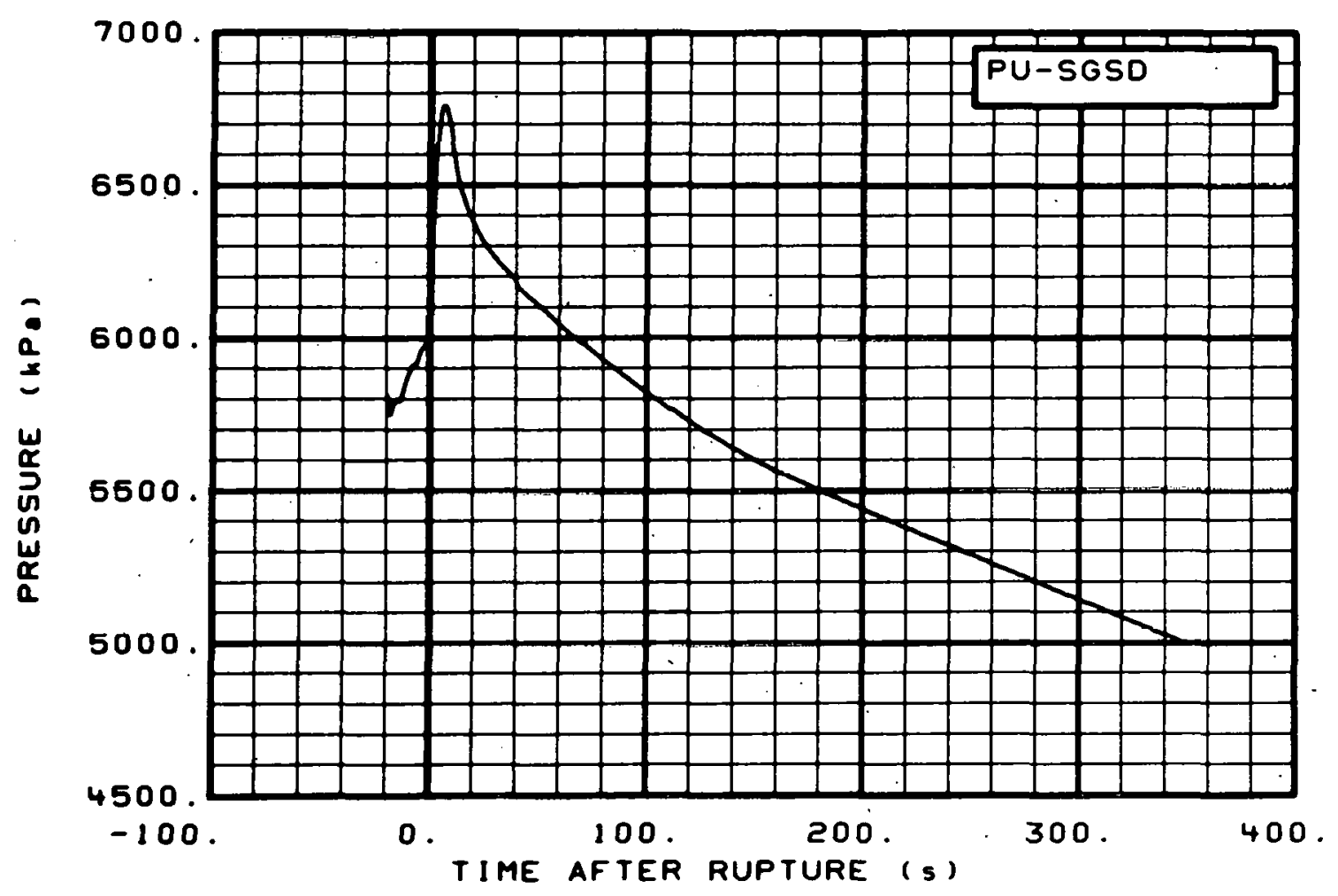

Fig. 159 Pressure in steam generator, secondary side (PU-SGSD), from -20 to $350 \mathrm{~s}$.

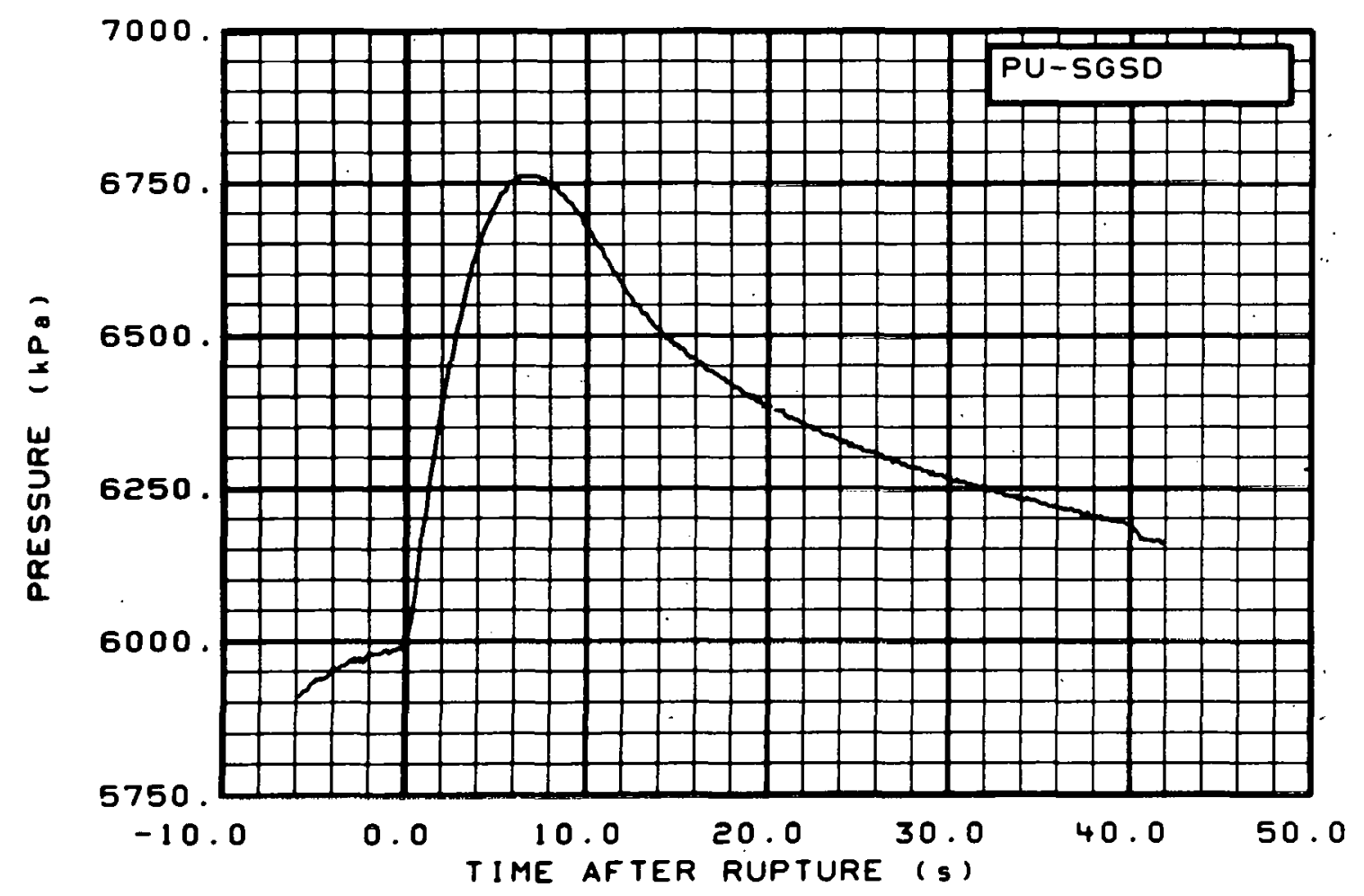

Fig. 160 Pressure in steam generator, secondary side (PU-SGSD), from -6 to 42 s. 


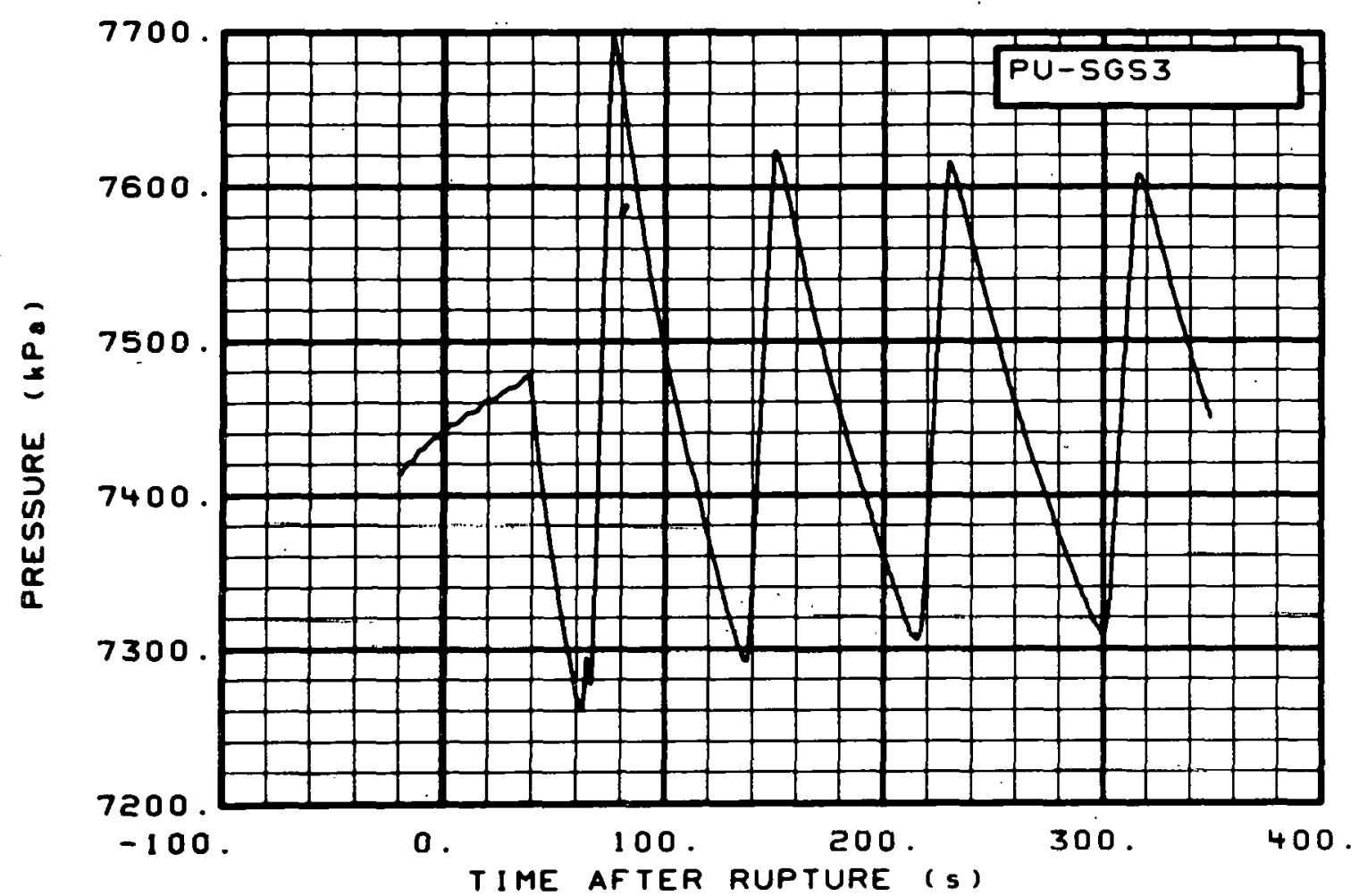

Fig. 161 Pressure in steam generator, secondary side (PU-SGS3), from -20 to $350 \mathrm{~s}$,

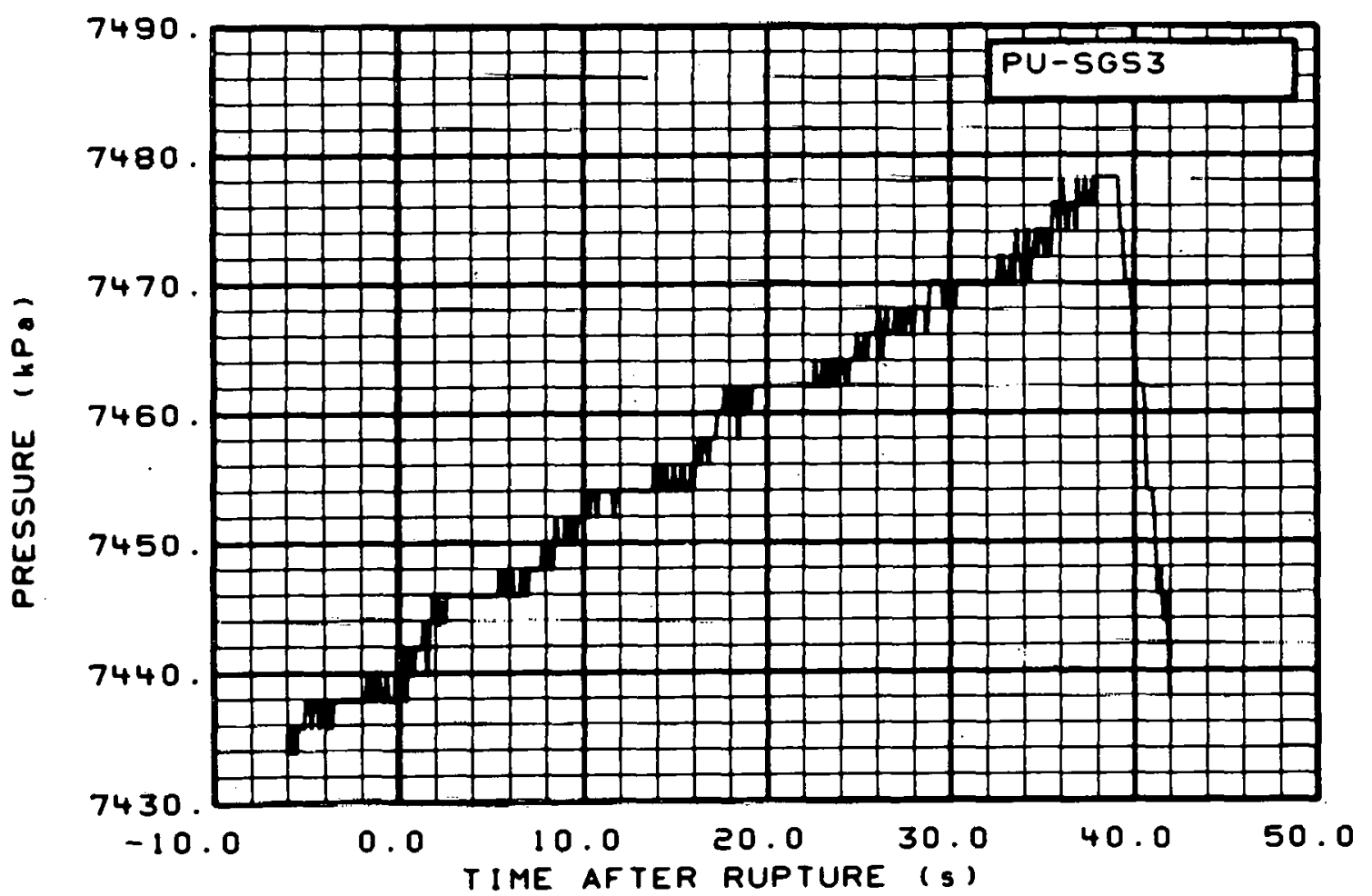

Fig. 162 Pressure in steam generator, secondary side (PU-SGS3), from -6 to $42 \mathrm{~s}$. 


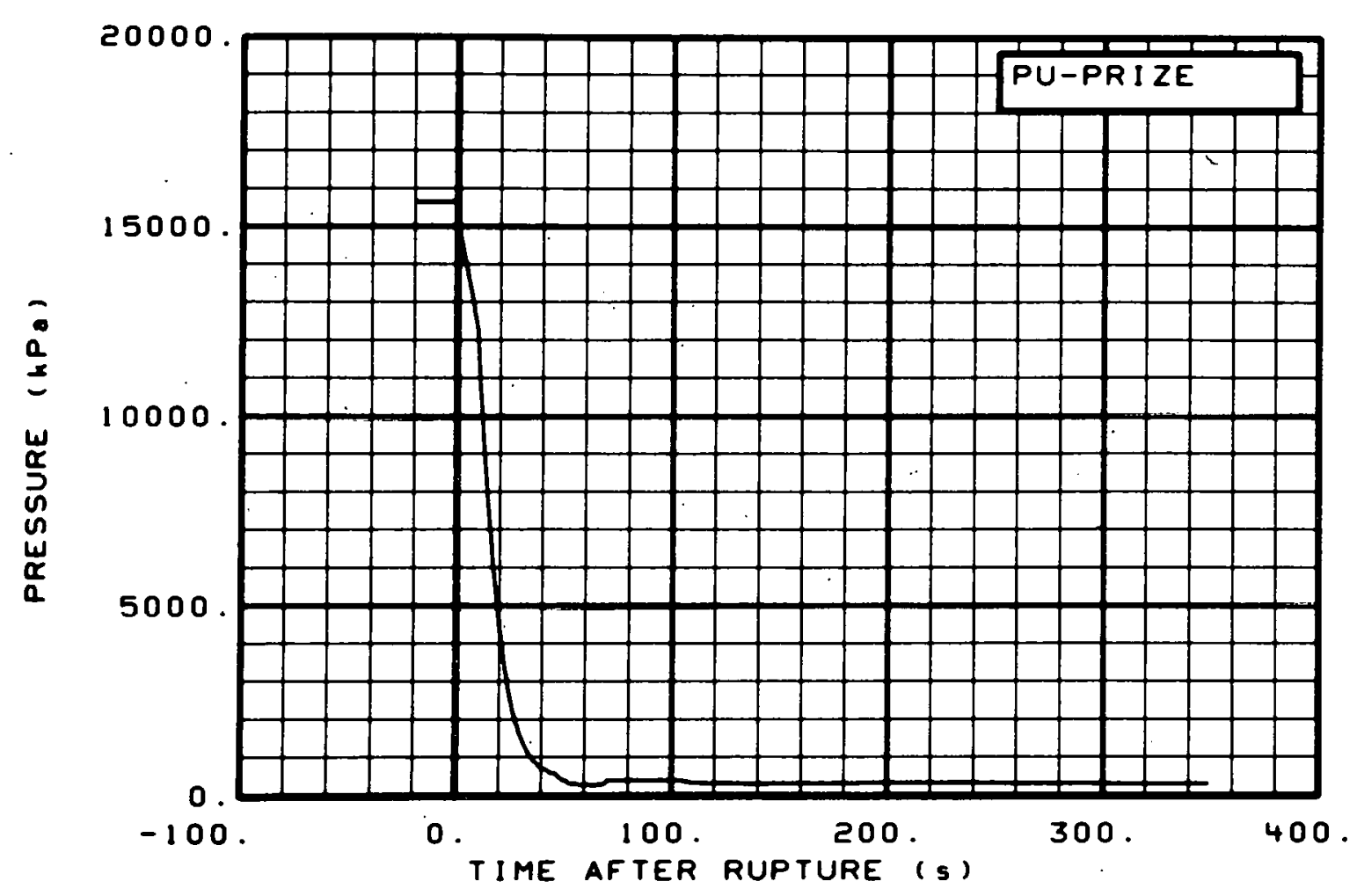

Fig. 163 Pressure in pressurizer (PU-PRIZE), from -20 to $350 \mathrm{~s}$.

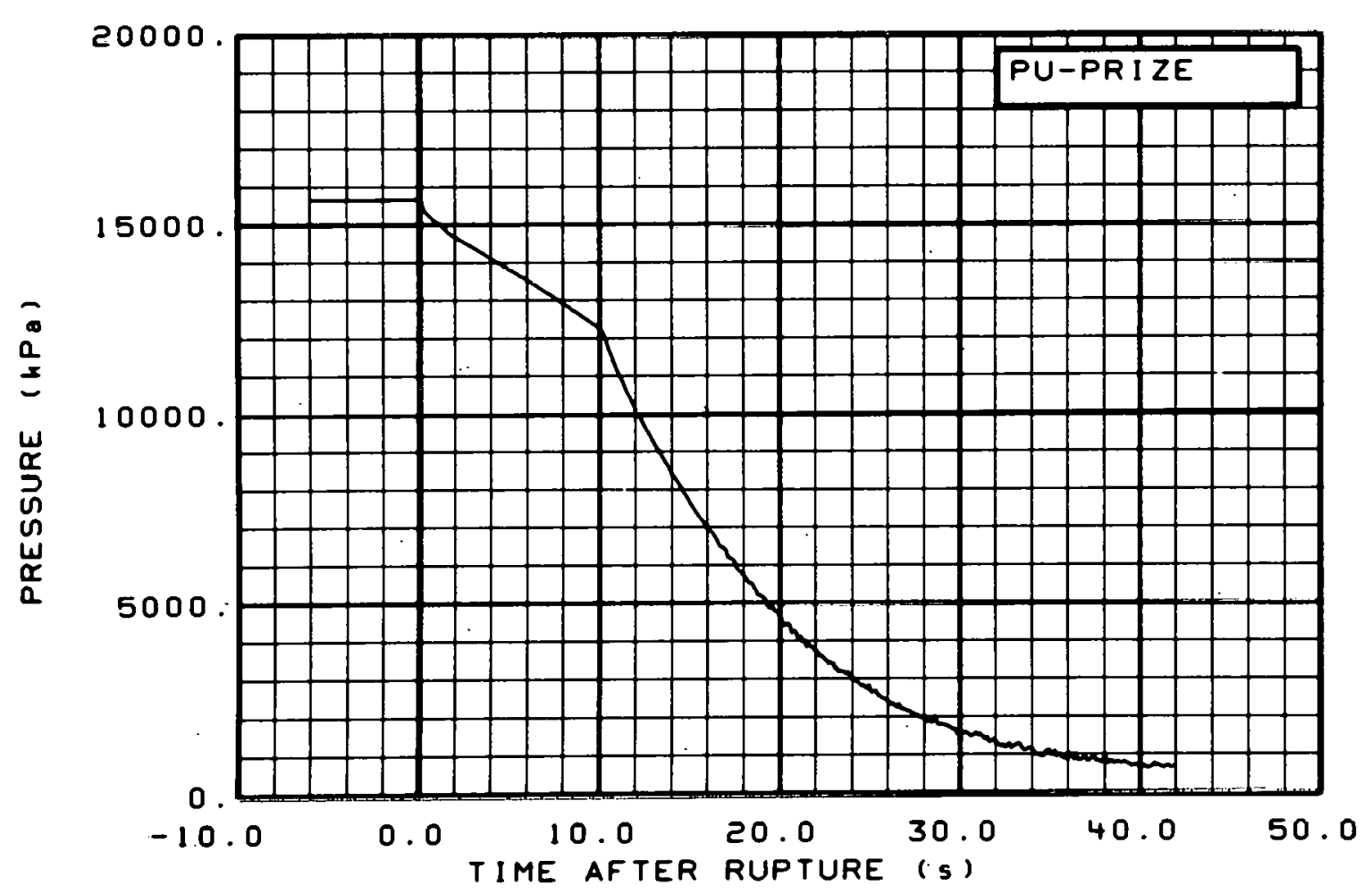

Fig. 164 Pressure in pressurizer (PU-PRIZE), from -6 to $42 \mathrm{~s}$. 


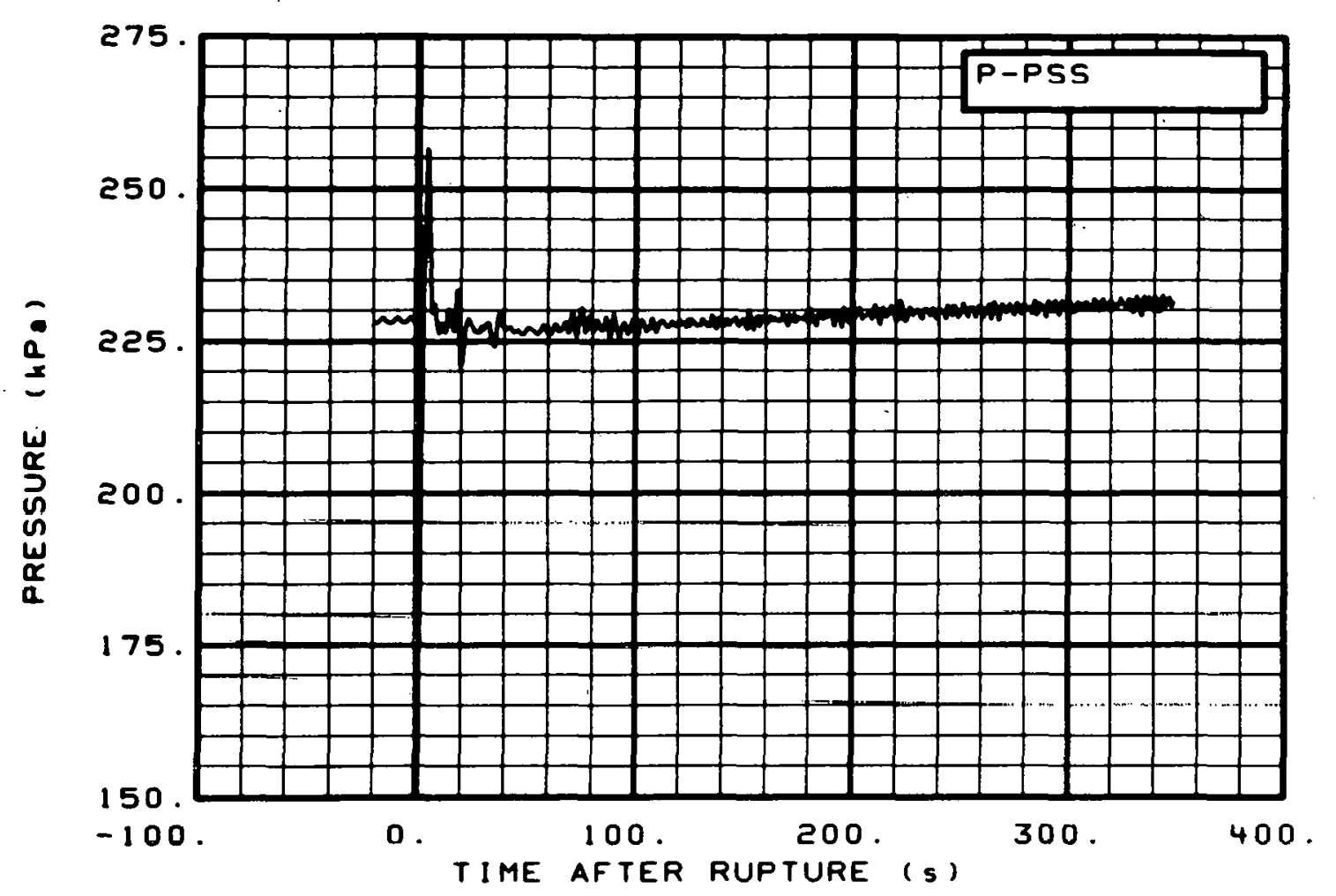

Fig. 165 Pressure in pressure suppression tank (P-PSS), from -20 to $350 \mathrm{~s}$.

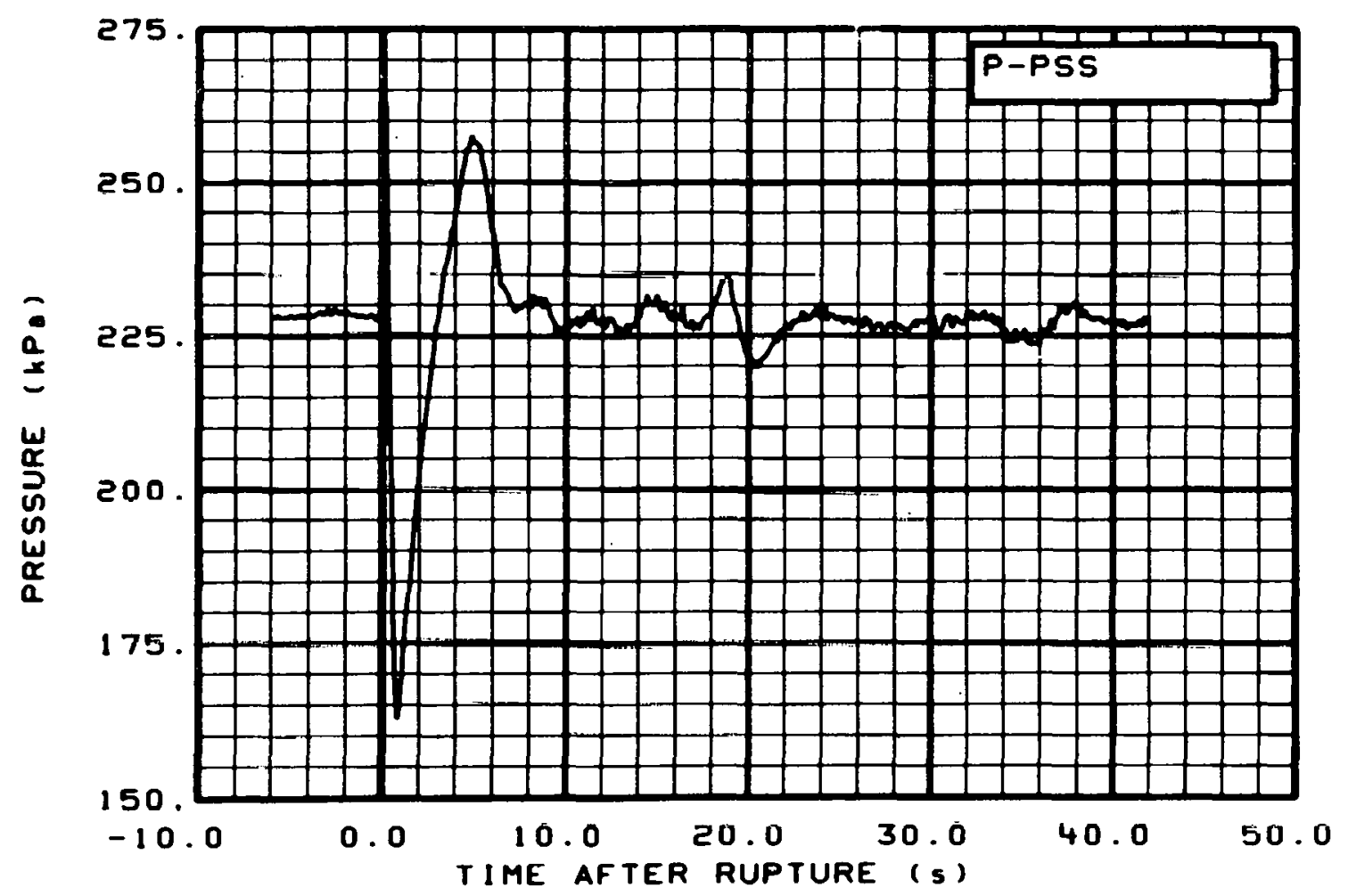

Fig. 166 Pressure in pressure suppression tank (P-PSS), from -6 to $42 \mathrm{~s}$. 


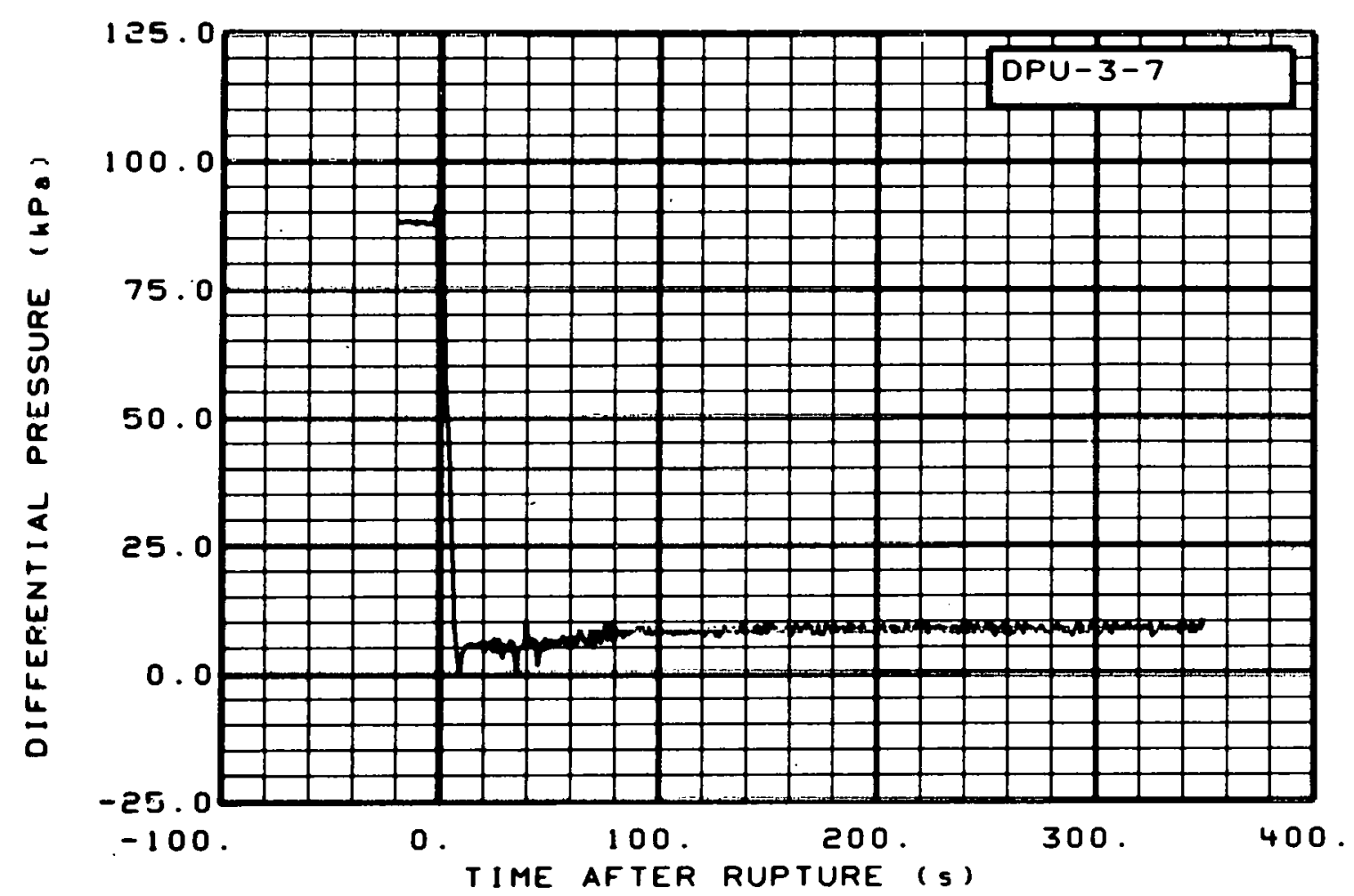

Fig. 167 Differential pressure in intact loop (DPU-3-7), from -20 to $350 \mathrm{~s}$.

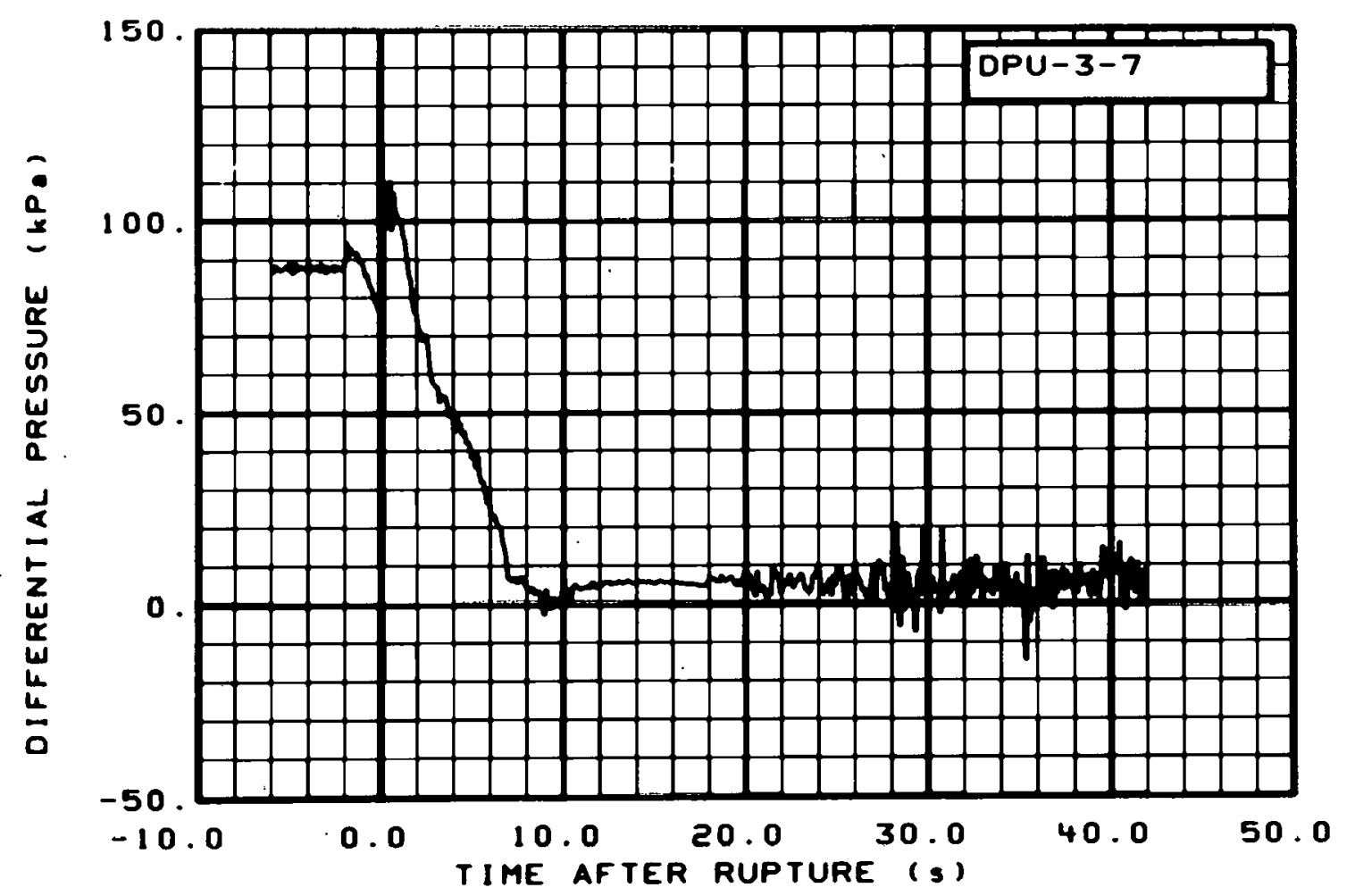

Fig. 168 Differential pressure in intact loop (DPU-3-7), from -6 to $42 \mathrm{~s}$. 


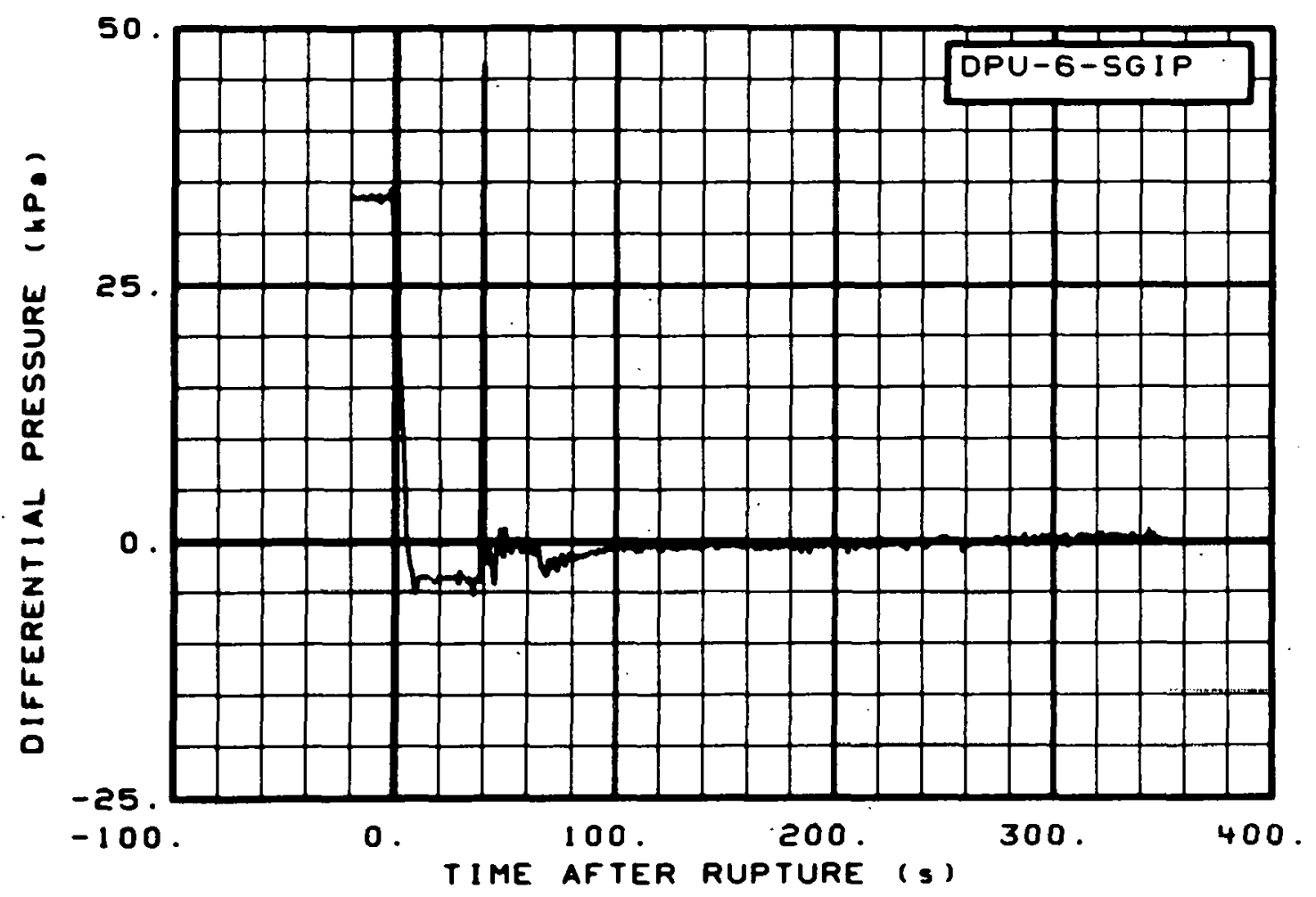

Fig. 169 Differential pressure in intact loop (DPU-6-SGIP), from -20 to $350 \mathrm{~s}$.

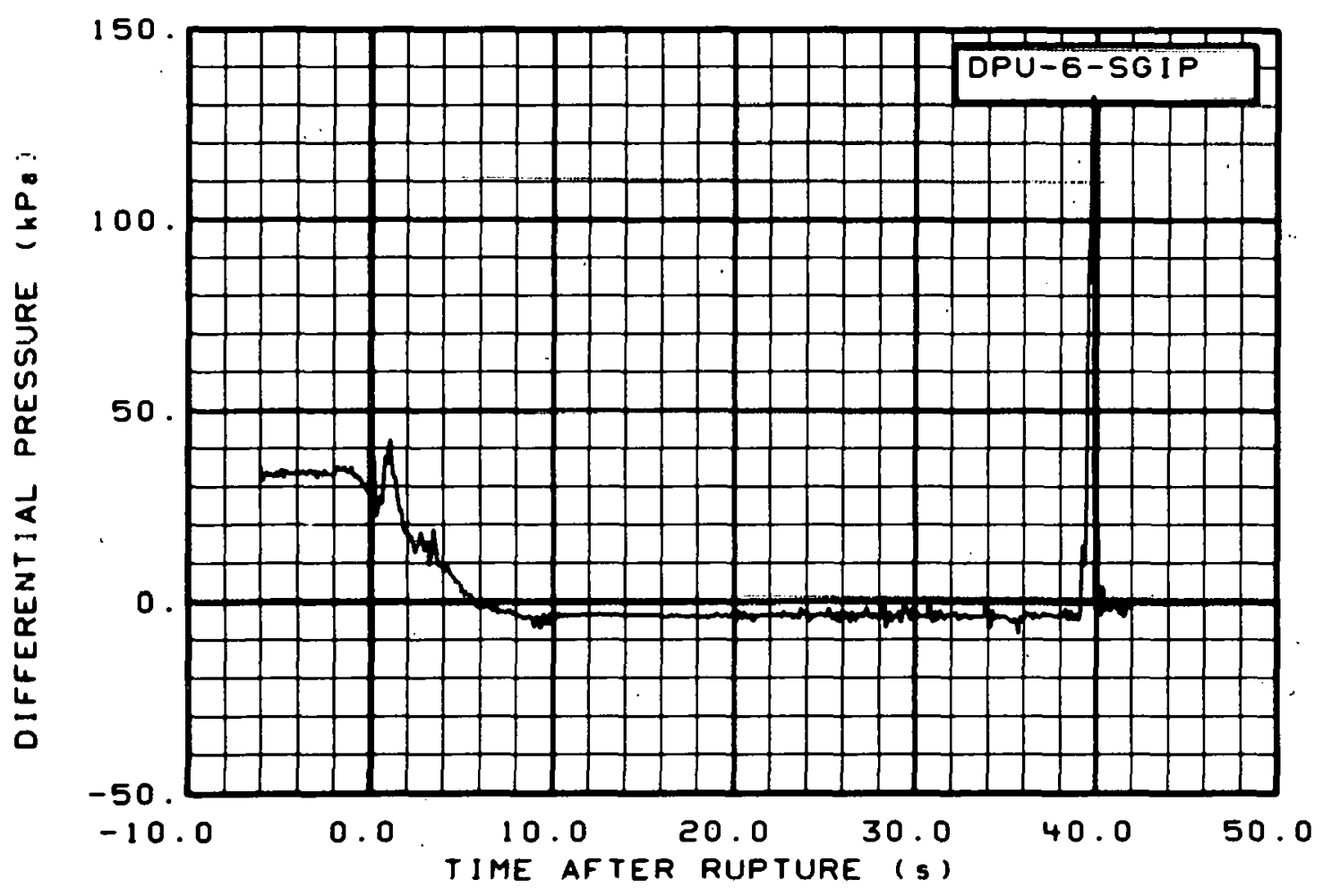

Fig. 170 Differential pressure in intact loop (DPU-6-SGIP), from -6 to $42 \mathrm{~s}$. 


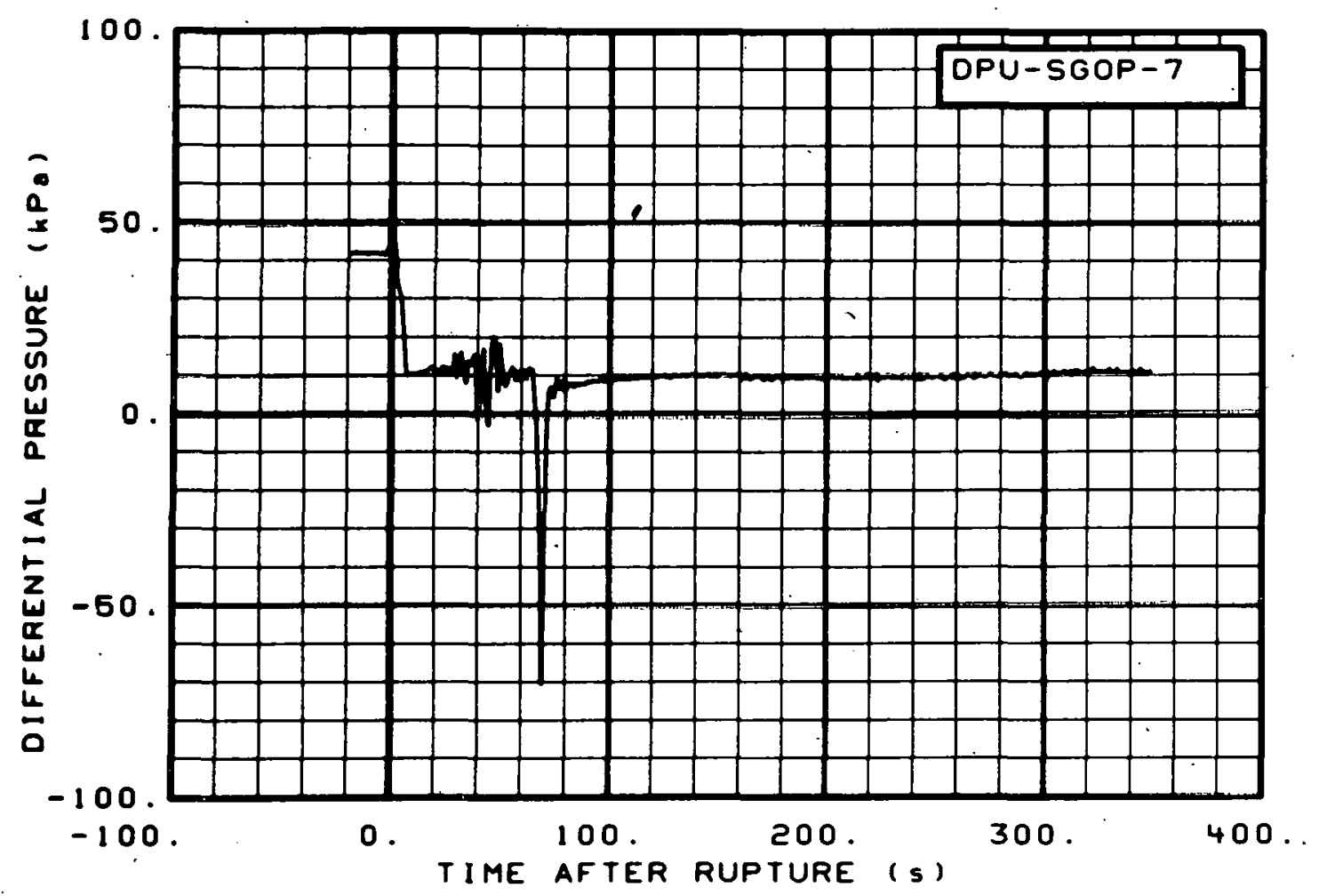

Fig. 171 Differential pressure in intact 100p (DPU-SGOP-7), from -20 to $350 \mathrm{~s}$.

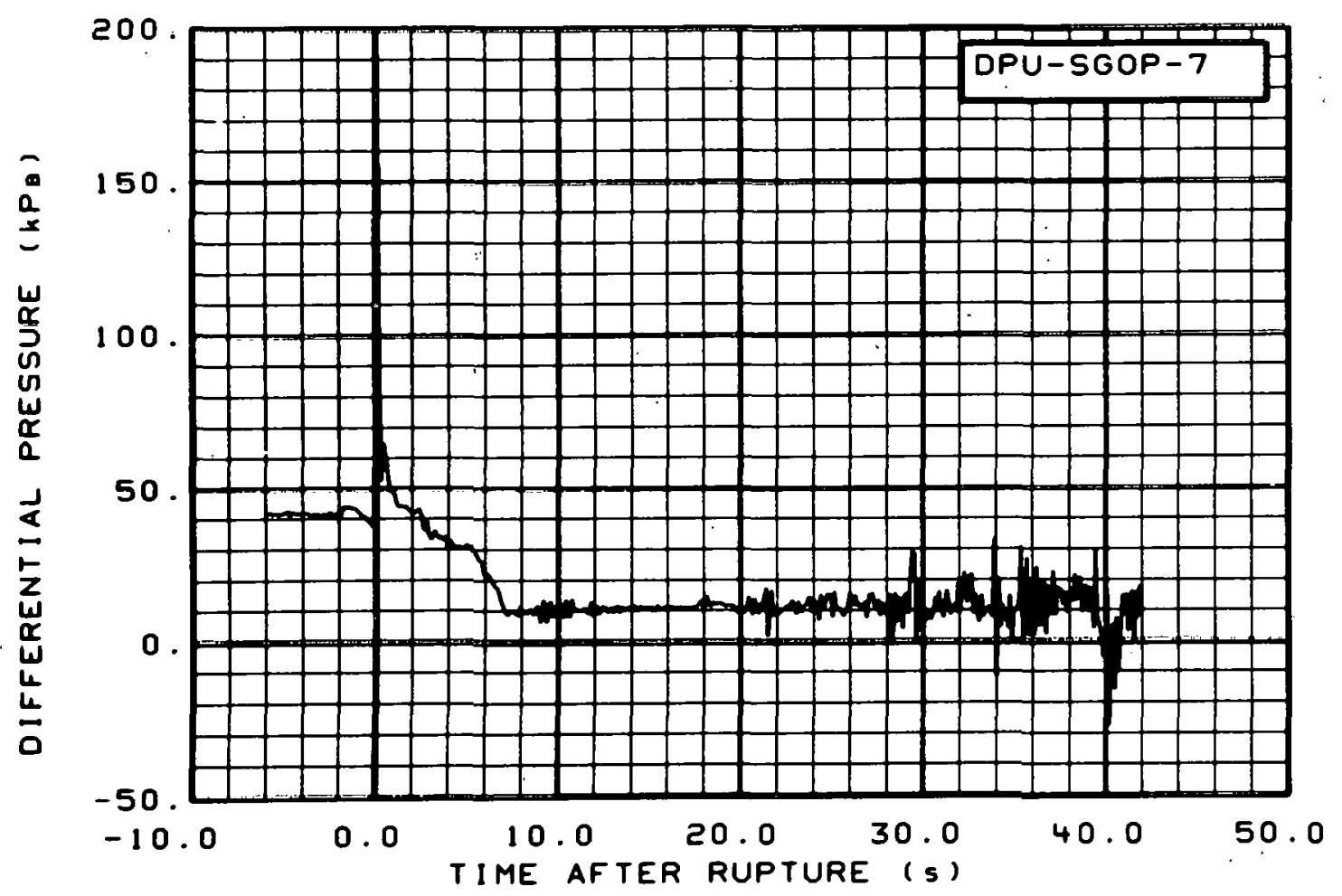

Fig. 172 Differential pressure in intact loop (DPU-SGOP-7), from -6 to $42 \mathrm{~s}$. 


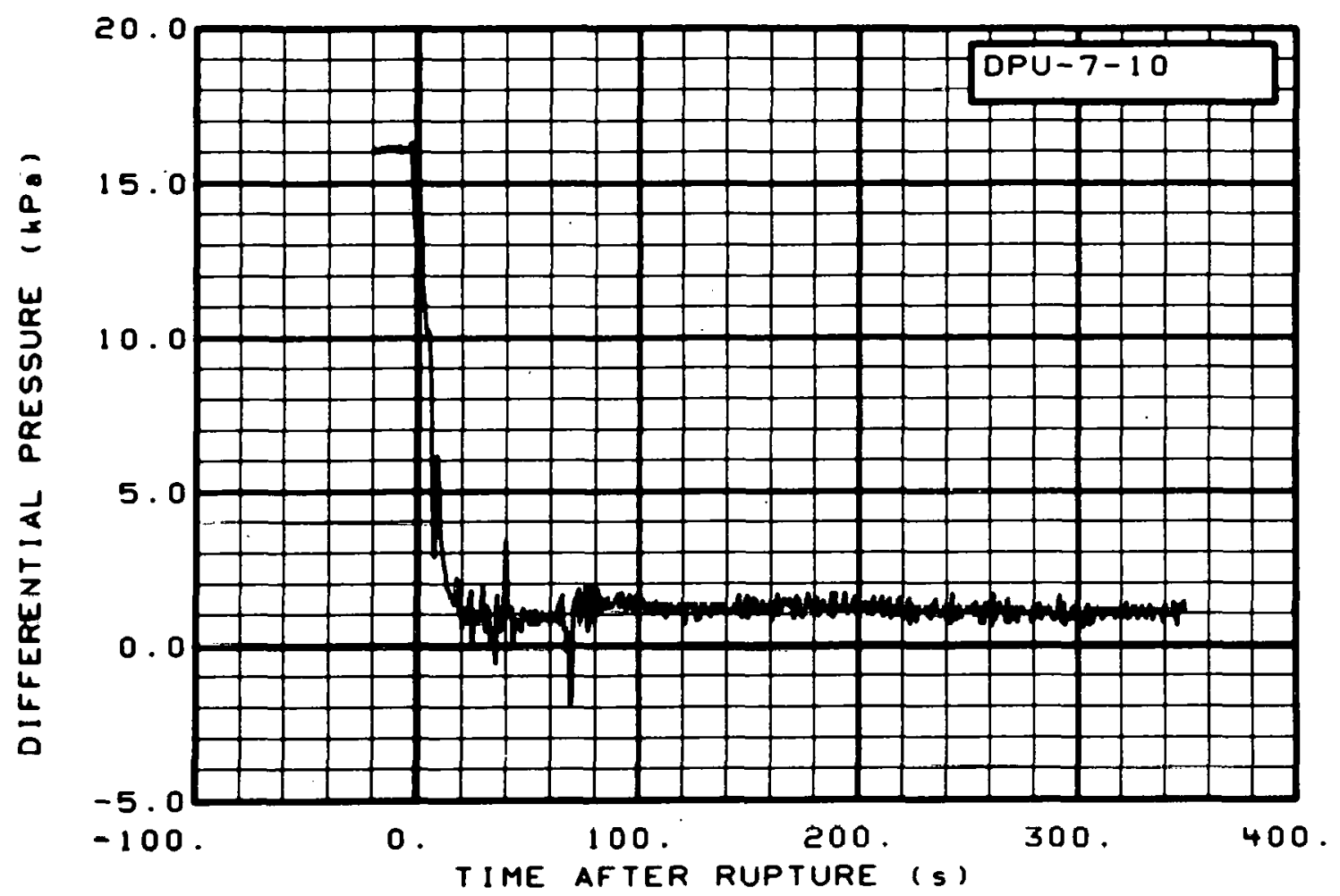

Fig. 173 Differential pressure in intact 100p (DPU-7-10), from -20 to $350 \mathrm{~s}$.

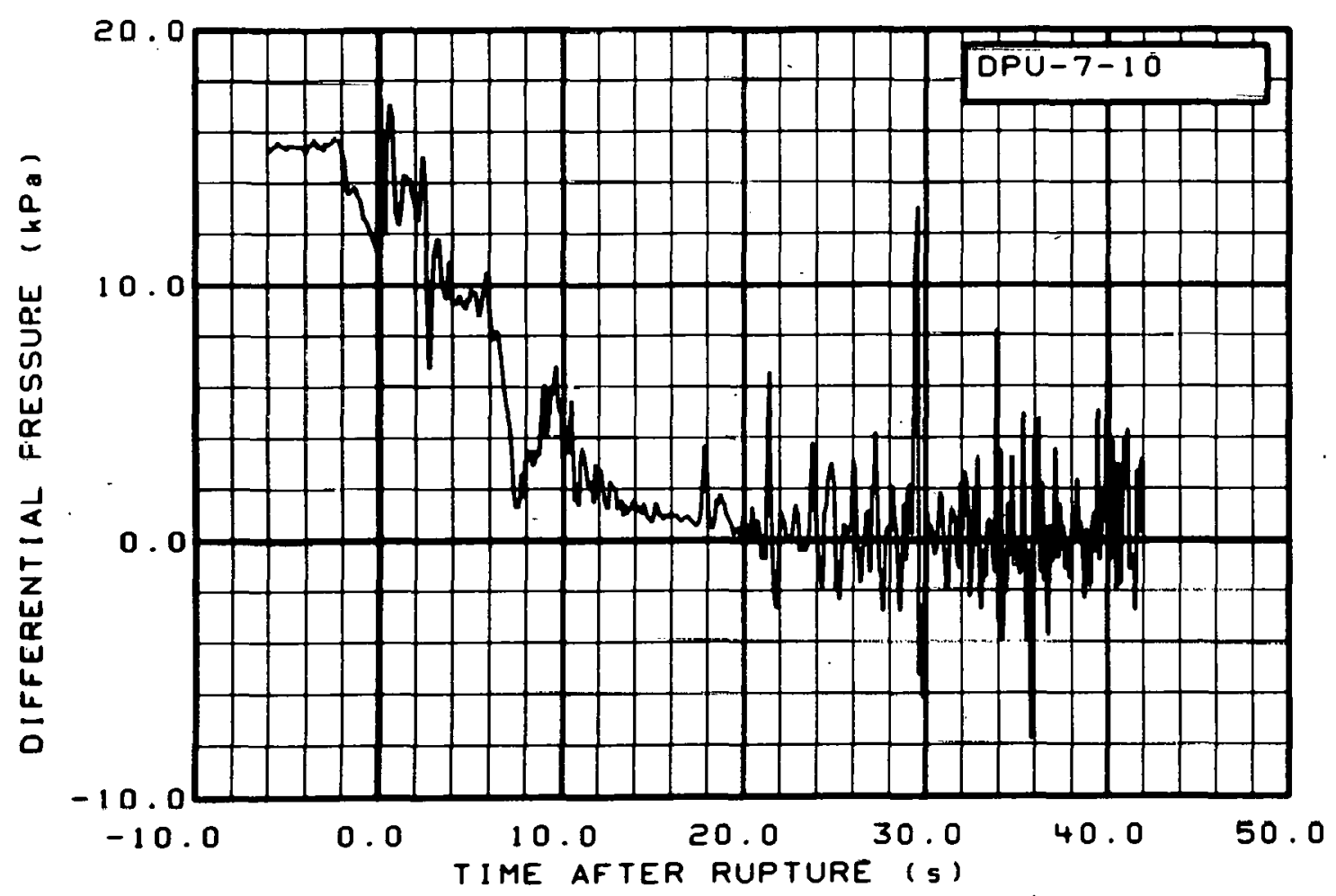

Fig. 174 Differential pressure in intact loop (DPU-7-10), from -6 to $42 \mathrm{~s}$. 


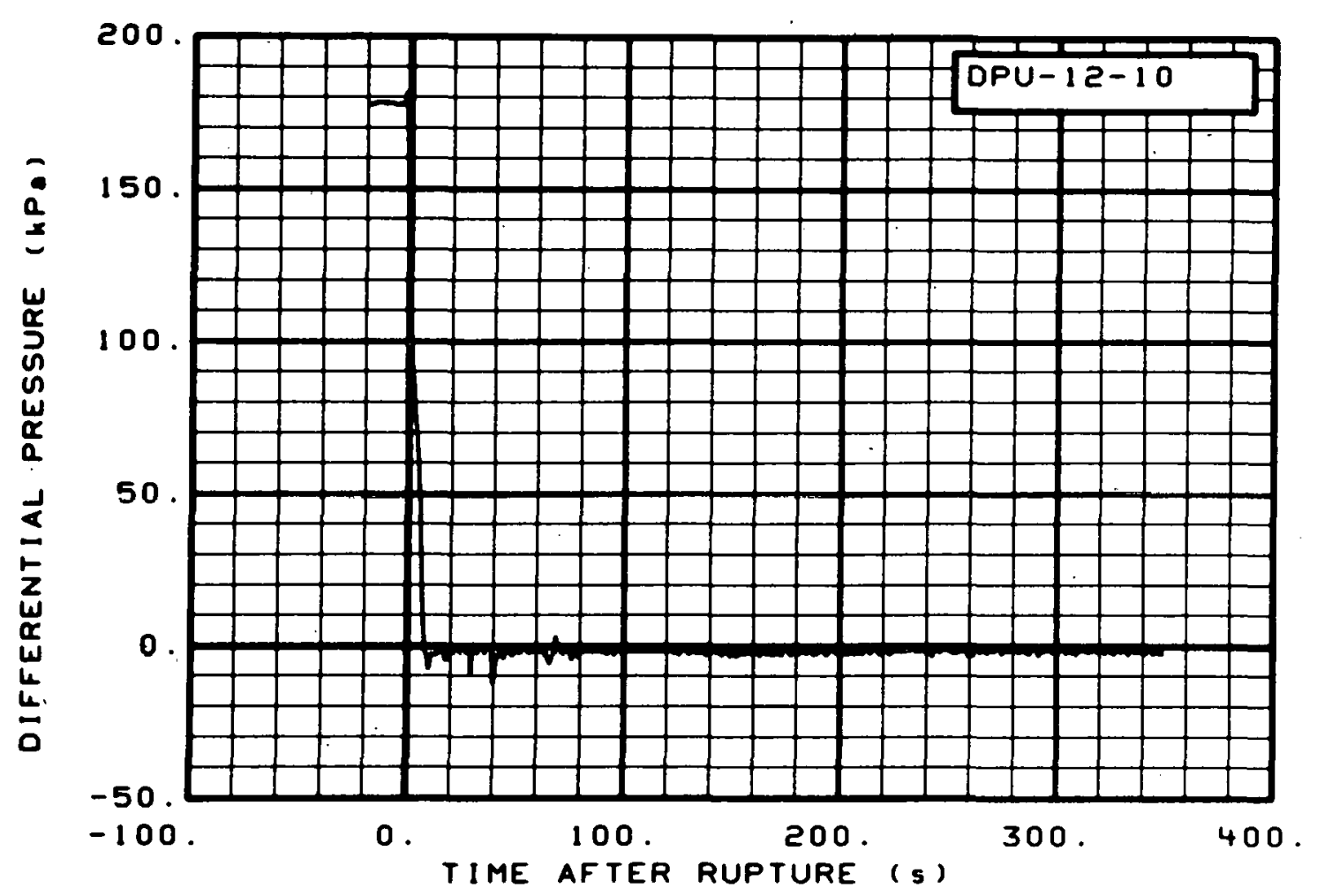

Fig. 175 Differential pressure in intact loop (DPU-12-10), from -20 to $350 \mathrm{~s}$.

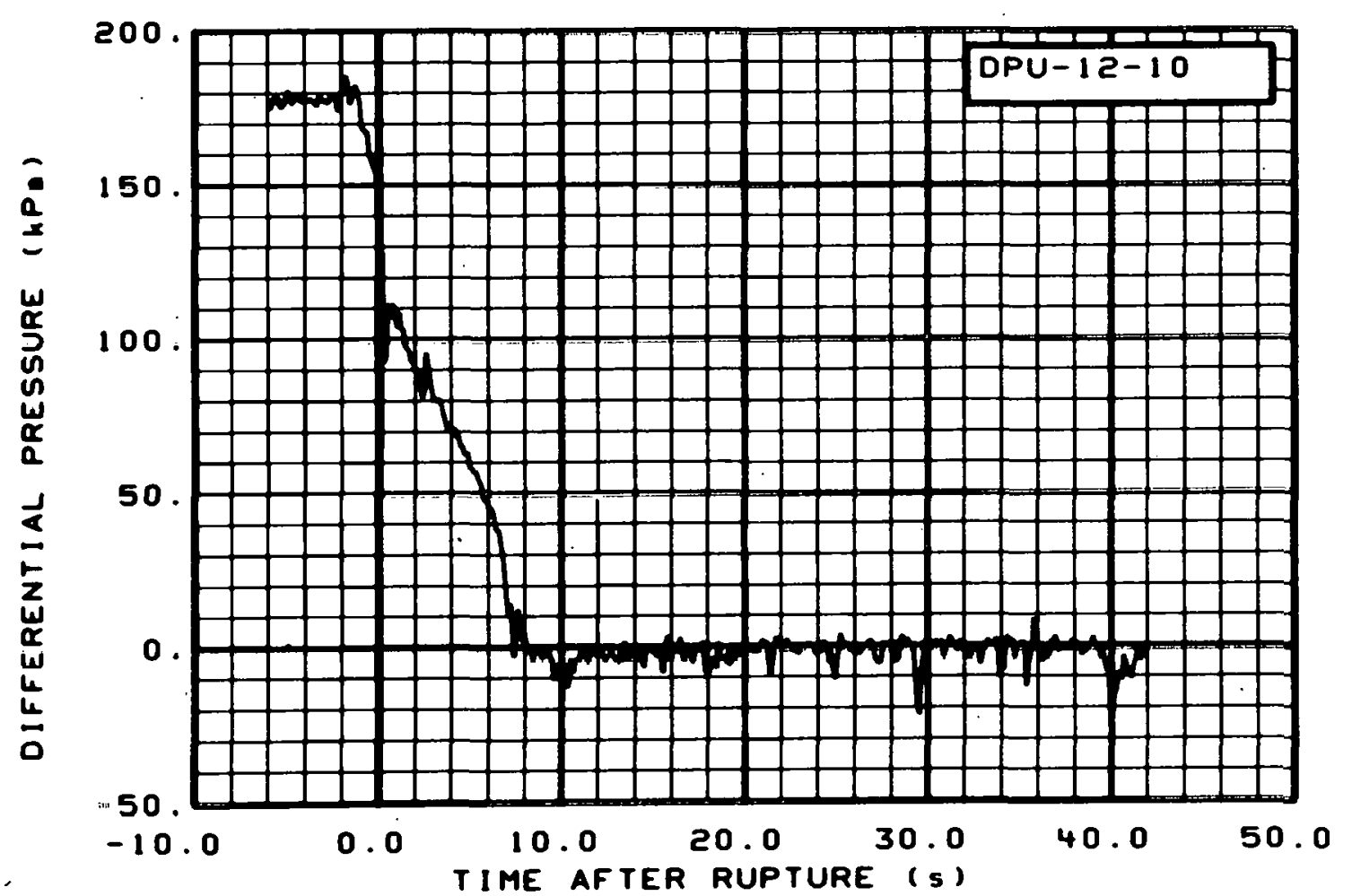

Fig. 176 Differential pressure in intact loop (DPU-12-10), from -6 to $42 \mathrm{~s}$. 


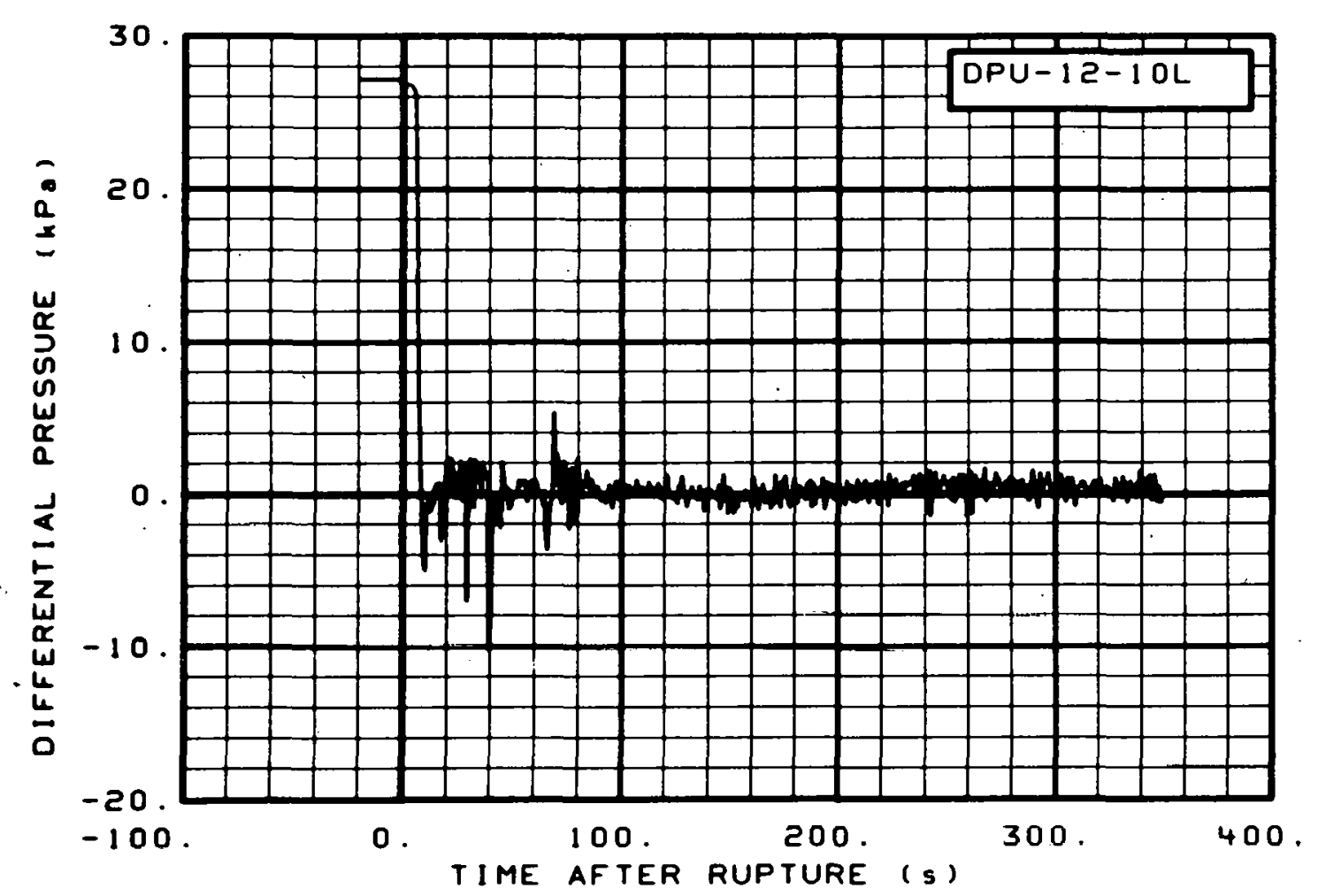

Fig. 177 Differential pressure in intact loop, low range (DPU-12-10L), from -20 to $350 \mathrm{~s}$.

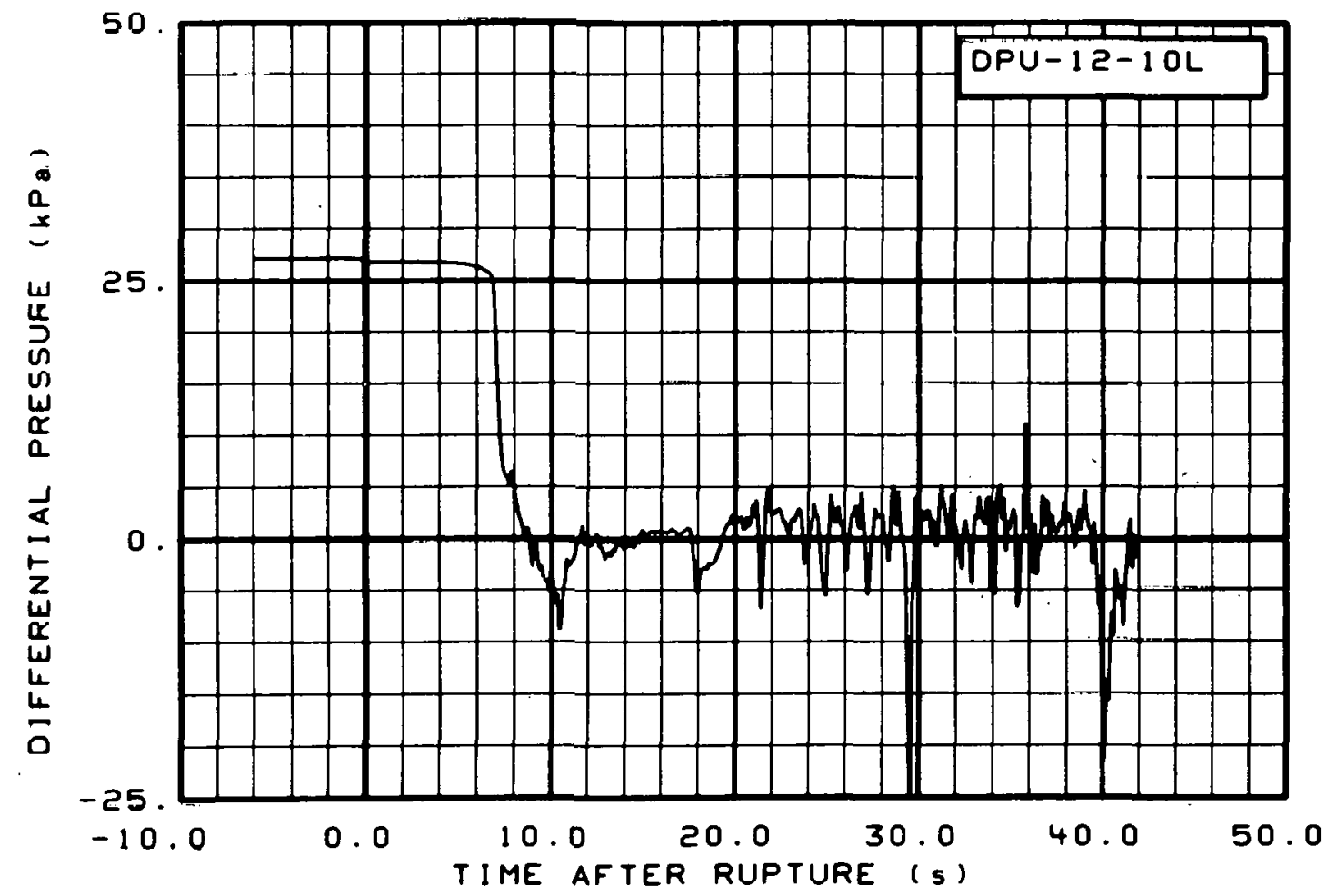

Fig. 178 Differential pressure in intact loop, low range (DPU-12-10L), from -6 to $42 \mathrm{~s}$. 


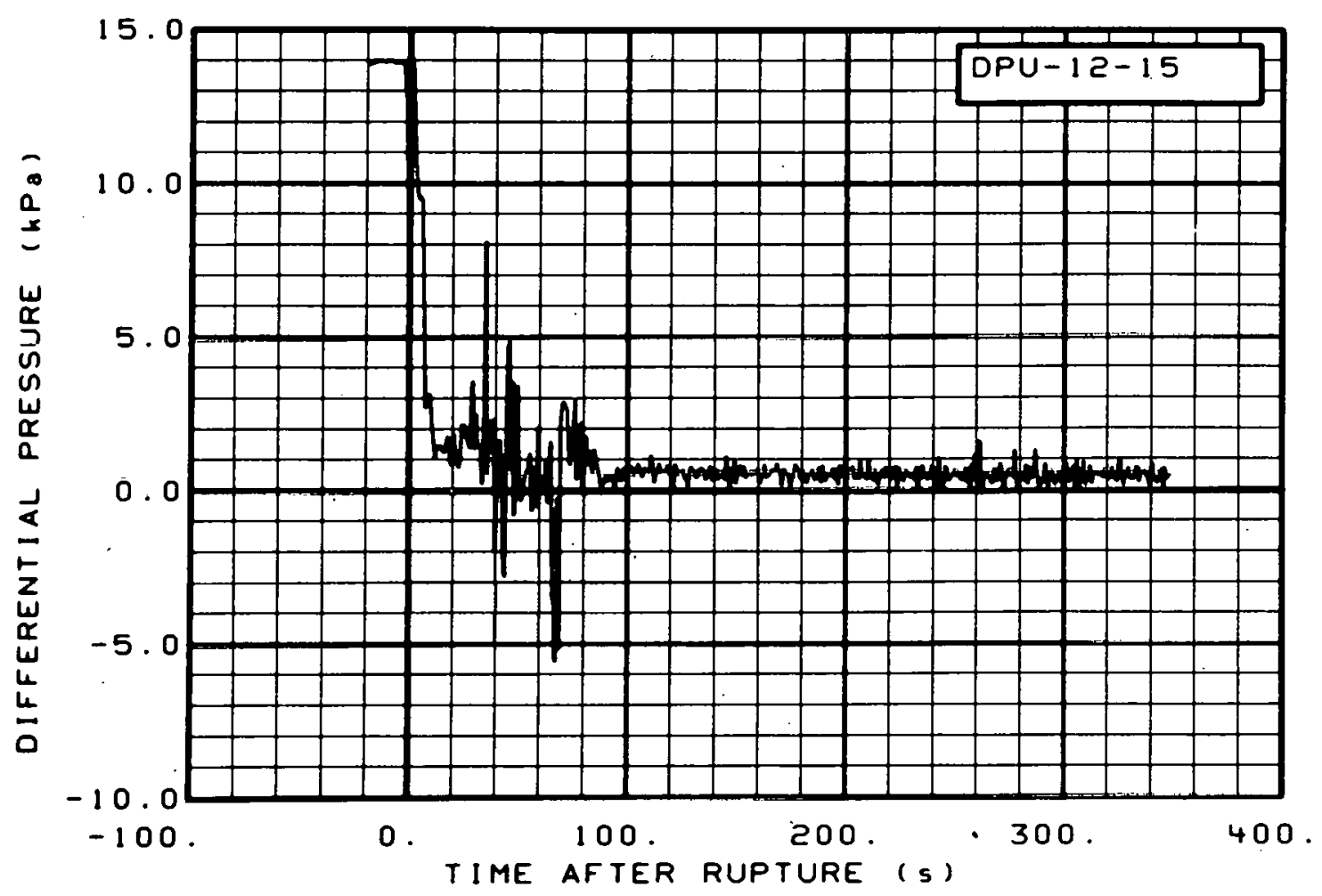

Fig. 179 Differential pressure in intact 10op (DPU-12-15), from -20 to $350 \mathrm{~s}$.

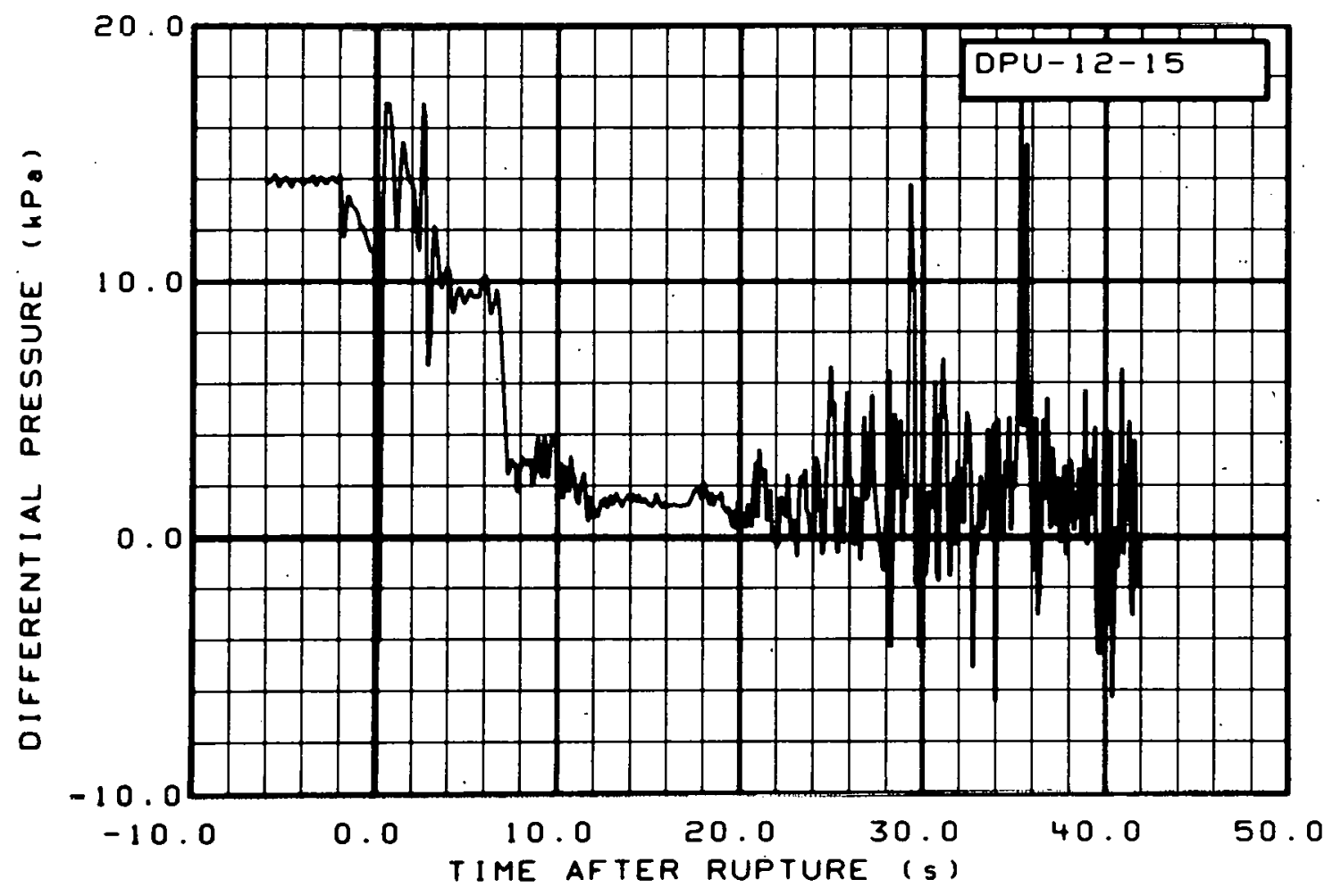

Fig. 180 Differential pressure in intact loop (DPU-12-15), from -6 to. 42 s. 


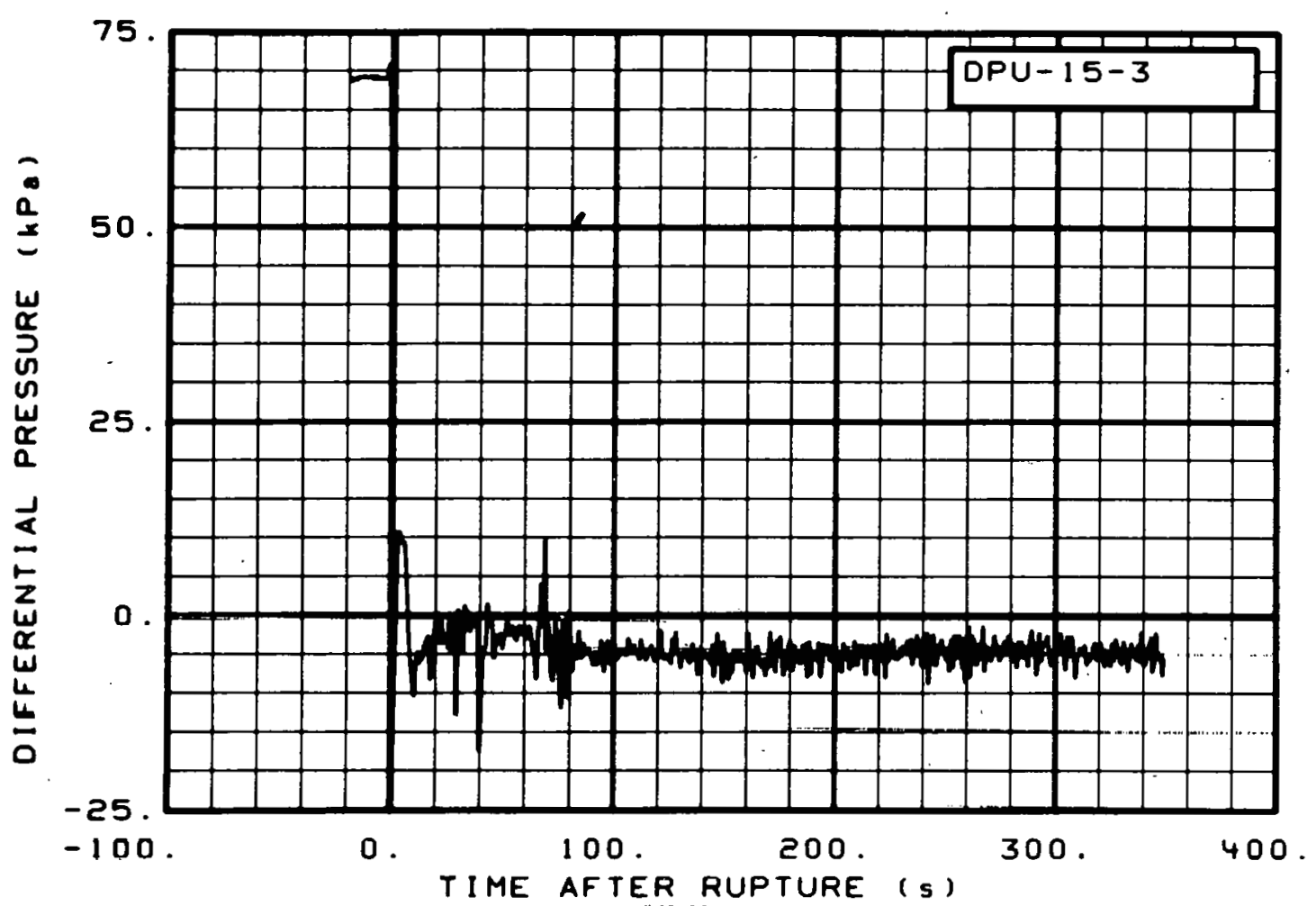

Fig. 181 Differential pressure in intact 100p (OPU-15-3), from -20 to $350 \mathrm{~s}$.

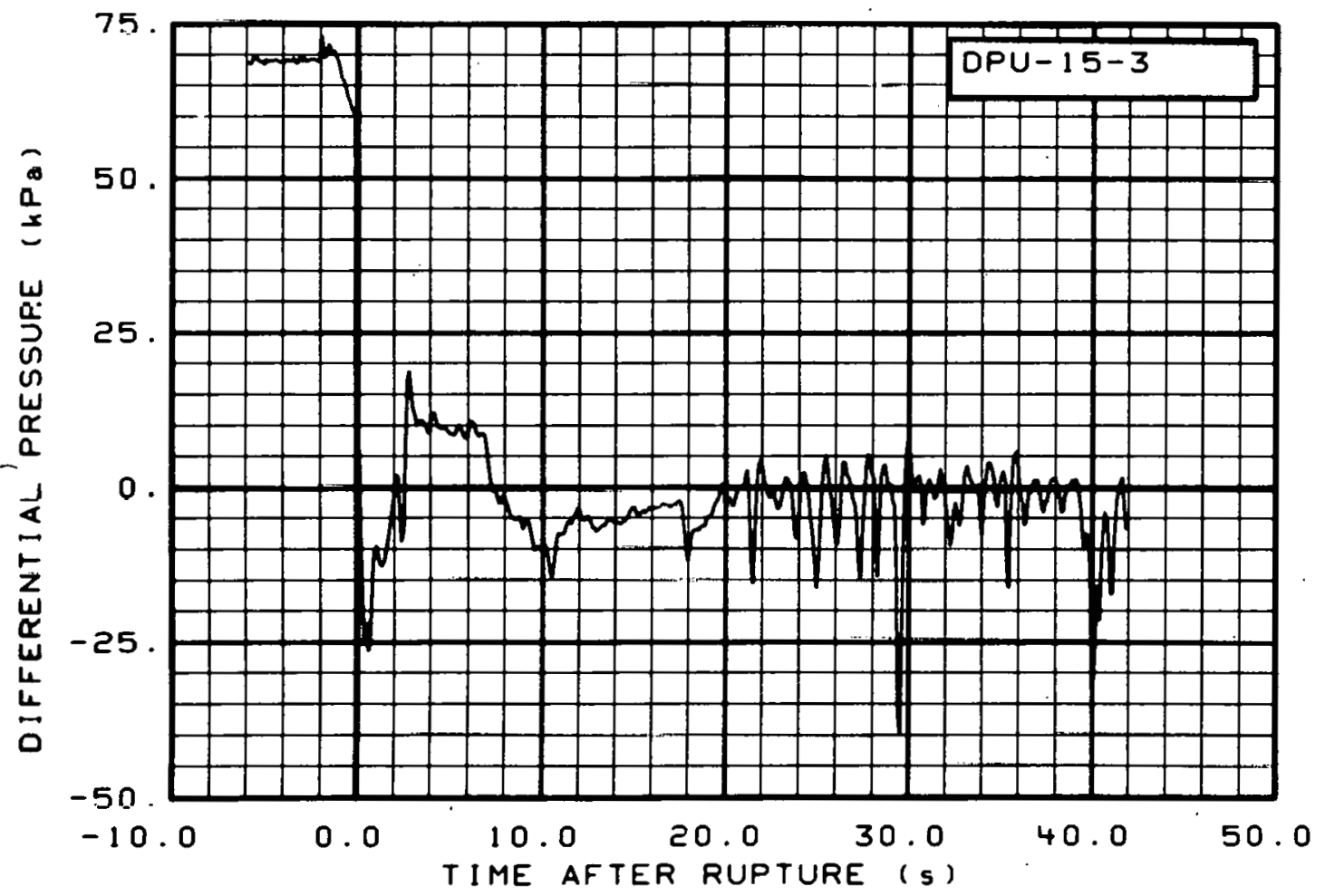

Fig. 182 Differential pressure in intact loop (DPU-15-3), from -6 to $42 \mathrm{~s}$. 


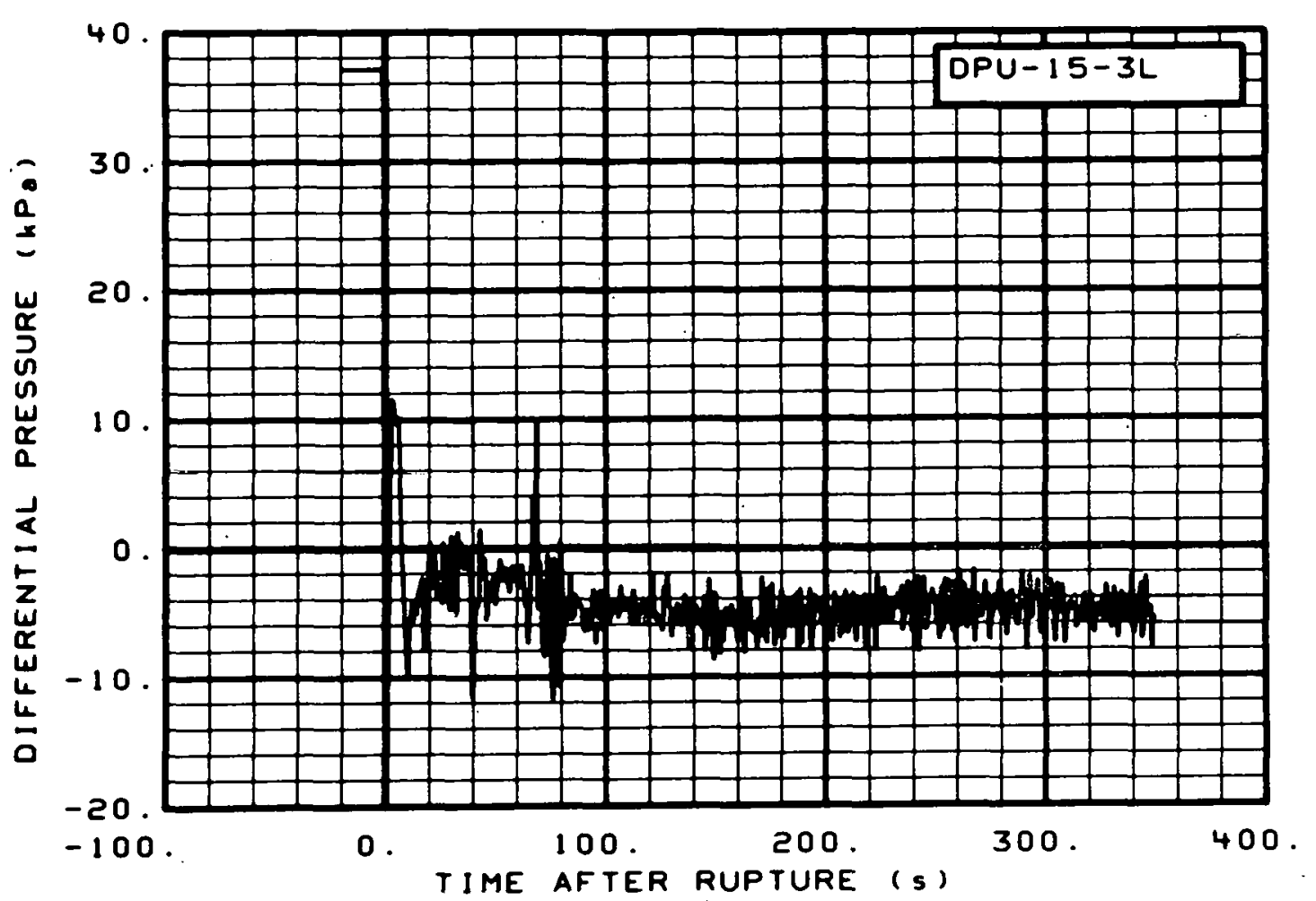

Fig. 183 Differential pressure in intact loop, low range (DPU-15-3L), from -20 to $350 \mathrm{~s}$.

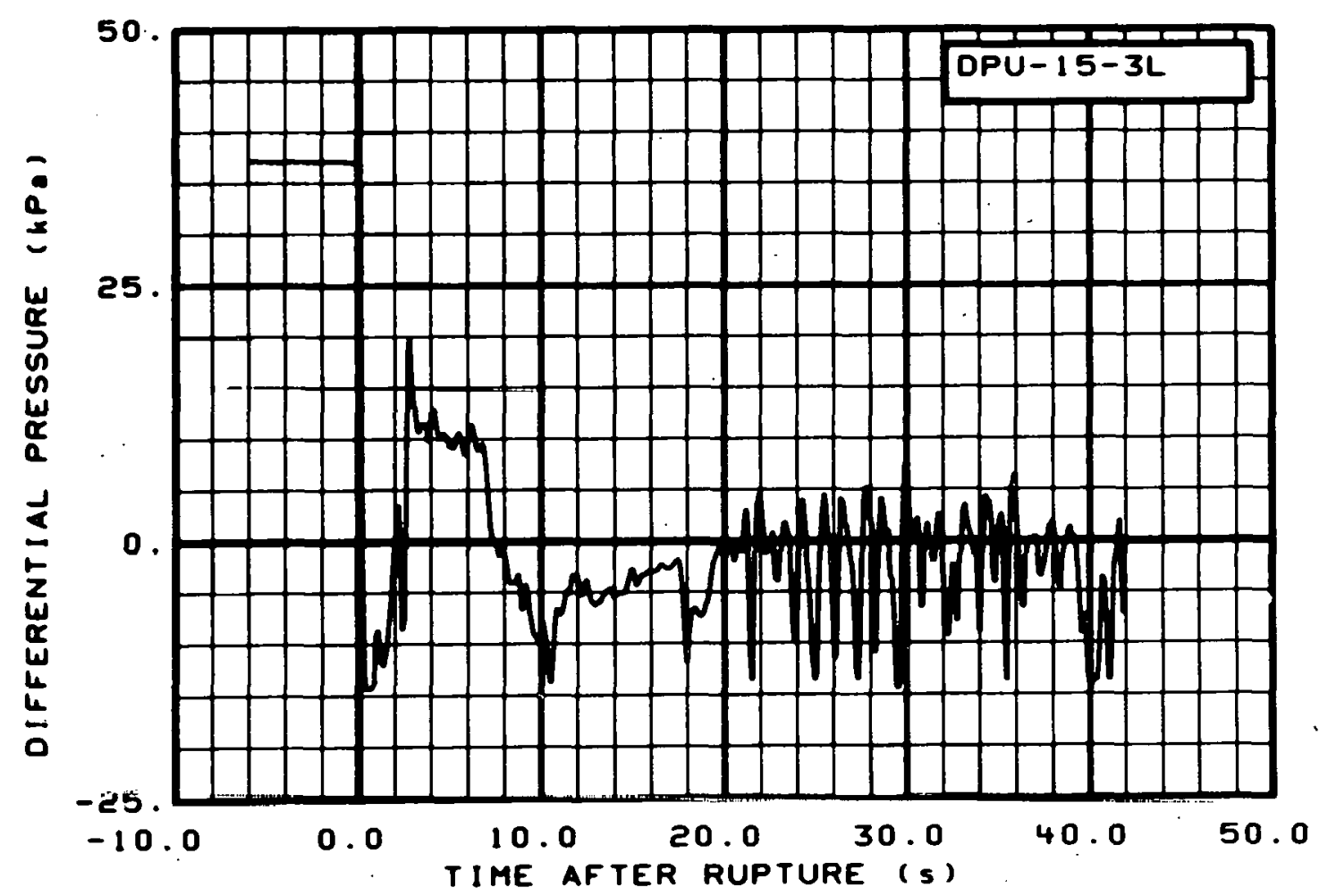

Fig. 184 Differential pressure in intact loop, low range (DPU-15-3L), from -6 to $42 \mathrm{~s}$. 


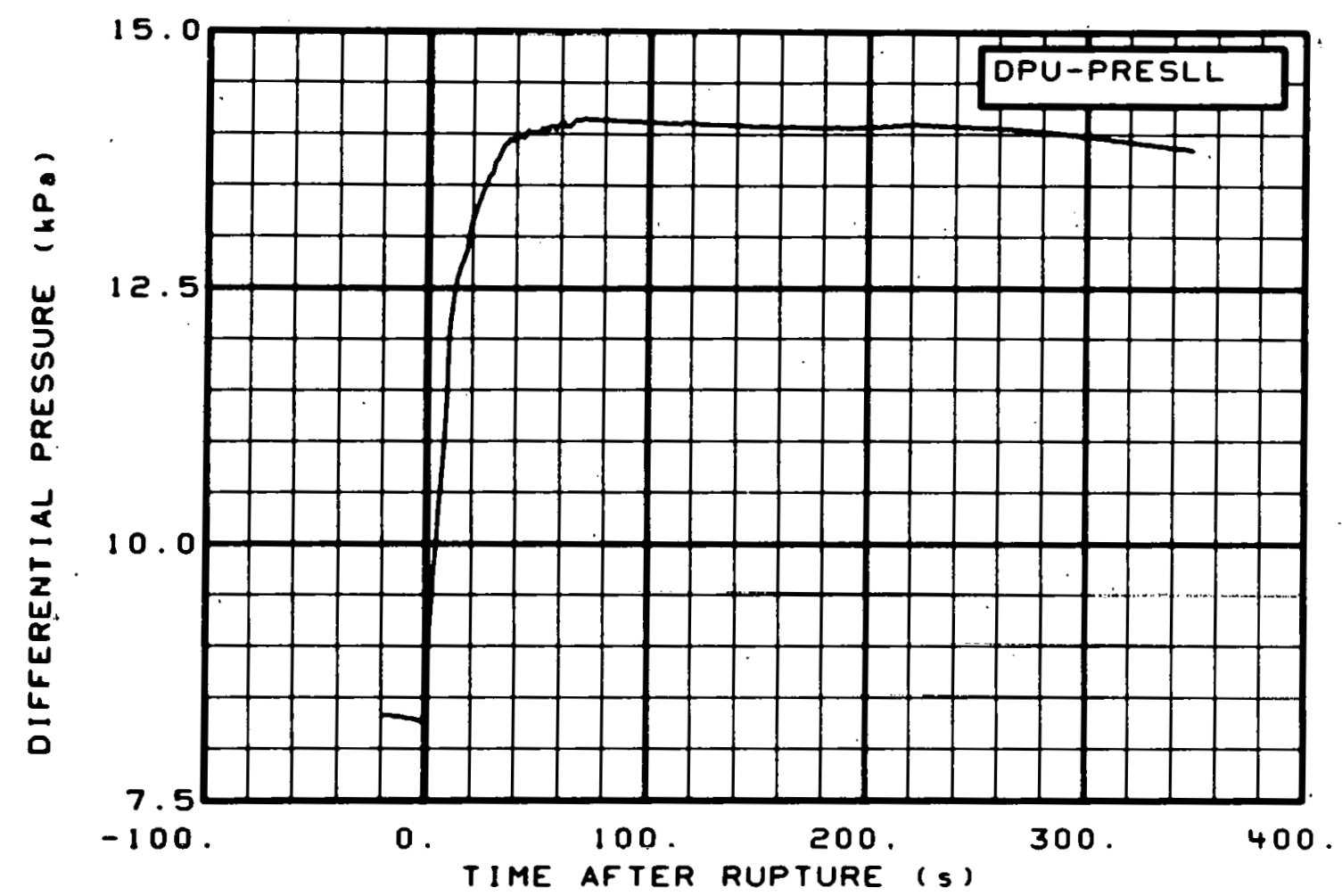

Fig. 185 Differential pressure in intact loop (DPU-PRESLL), from -20 to $350 \mathrm{~s}$.

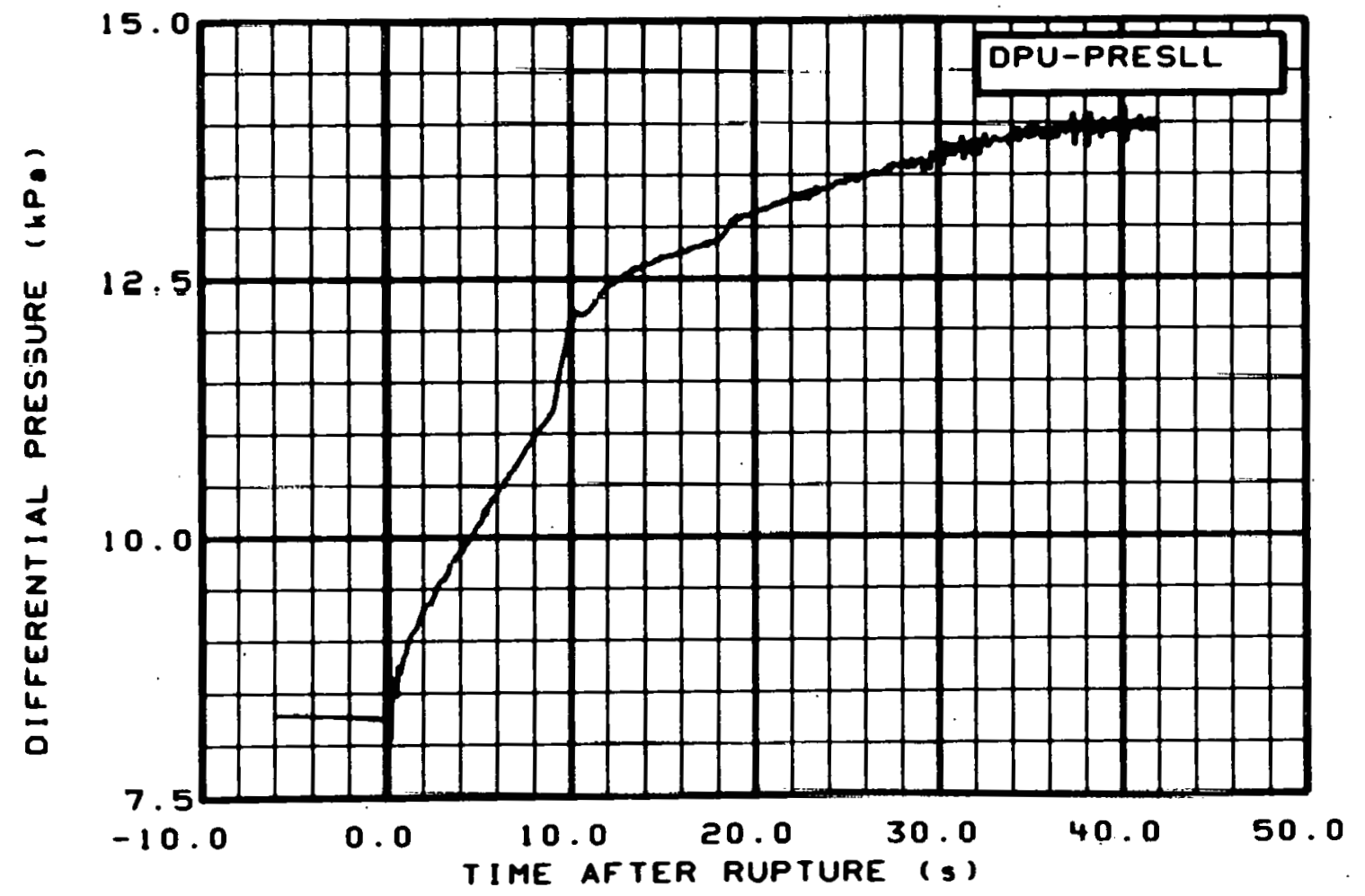

Fig. 186 Differential pressure in intact loop (DPU-PRESLL), from -6 to $42 \mathrm{~s}$. 


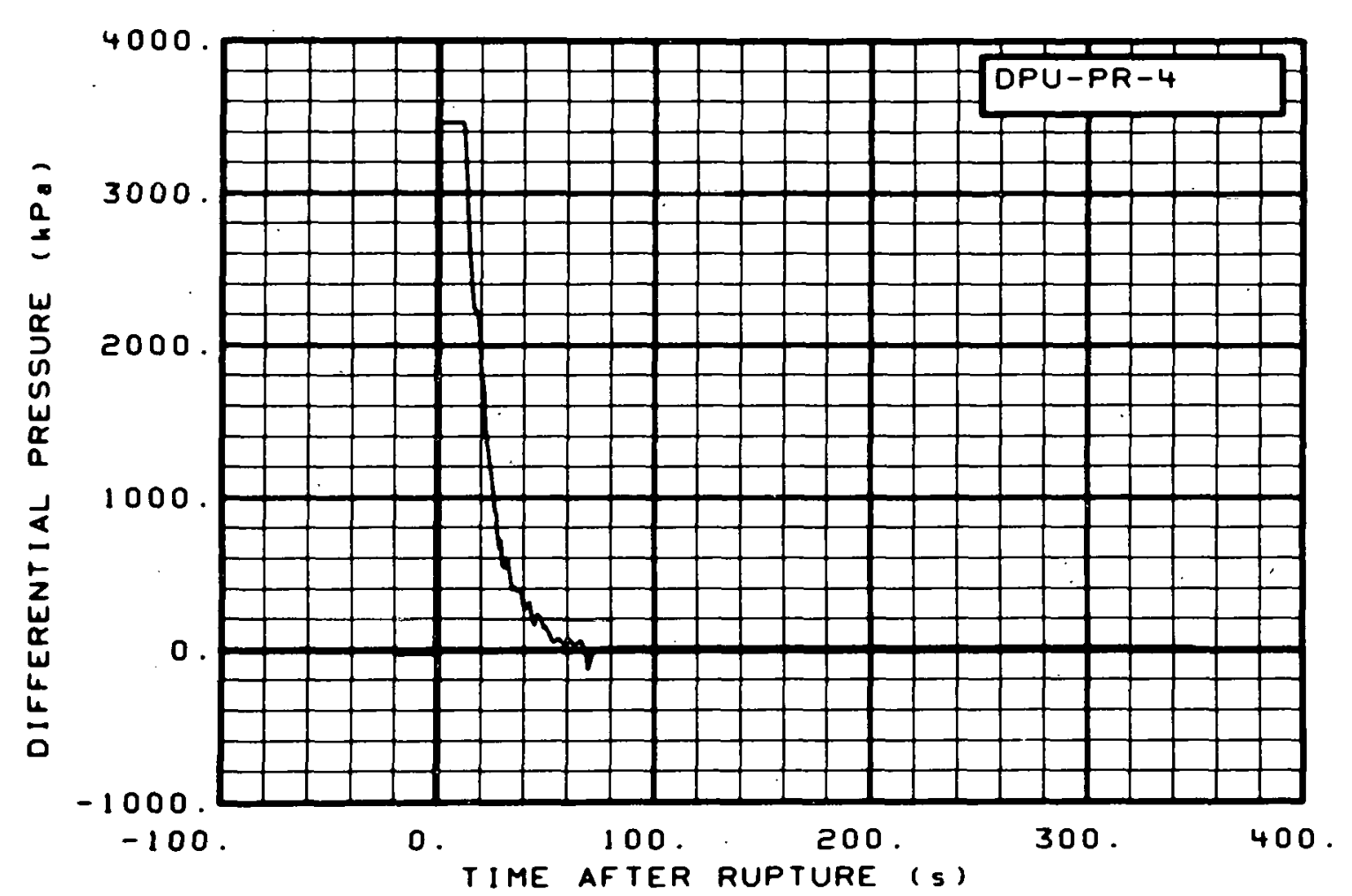

Fig. 187 Differential pressure in intact loop (DPU-PR-4), from -20 to $350 \mathrm{~s}$.

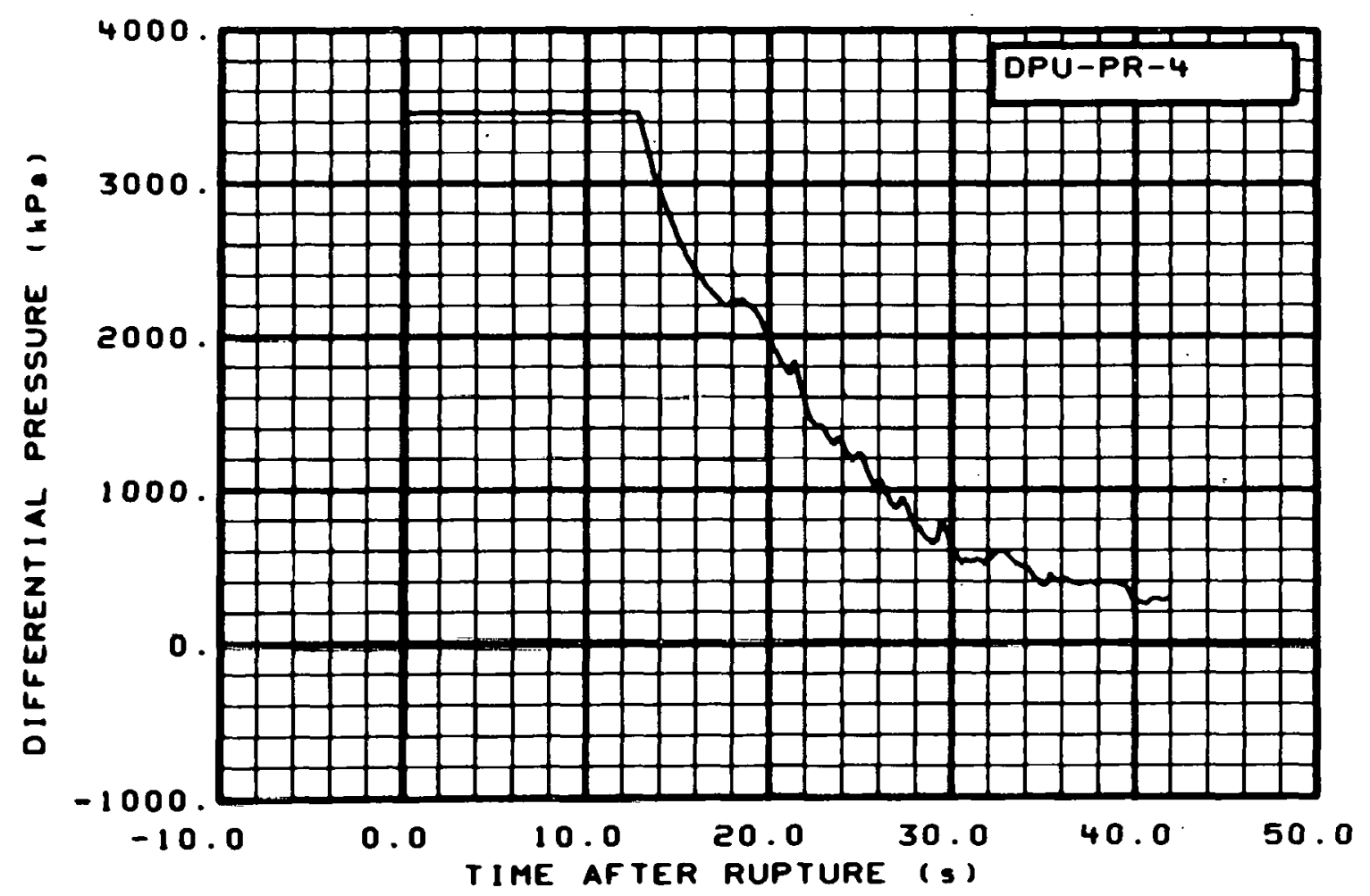

Fig. 188 Differential pressure in intact loop (DPU-PR-4), from -6 to $42 \mathrm{~s}$. 


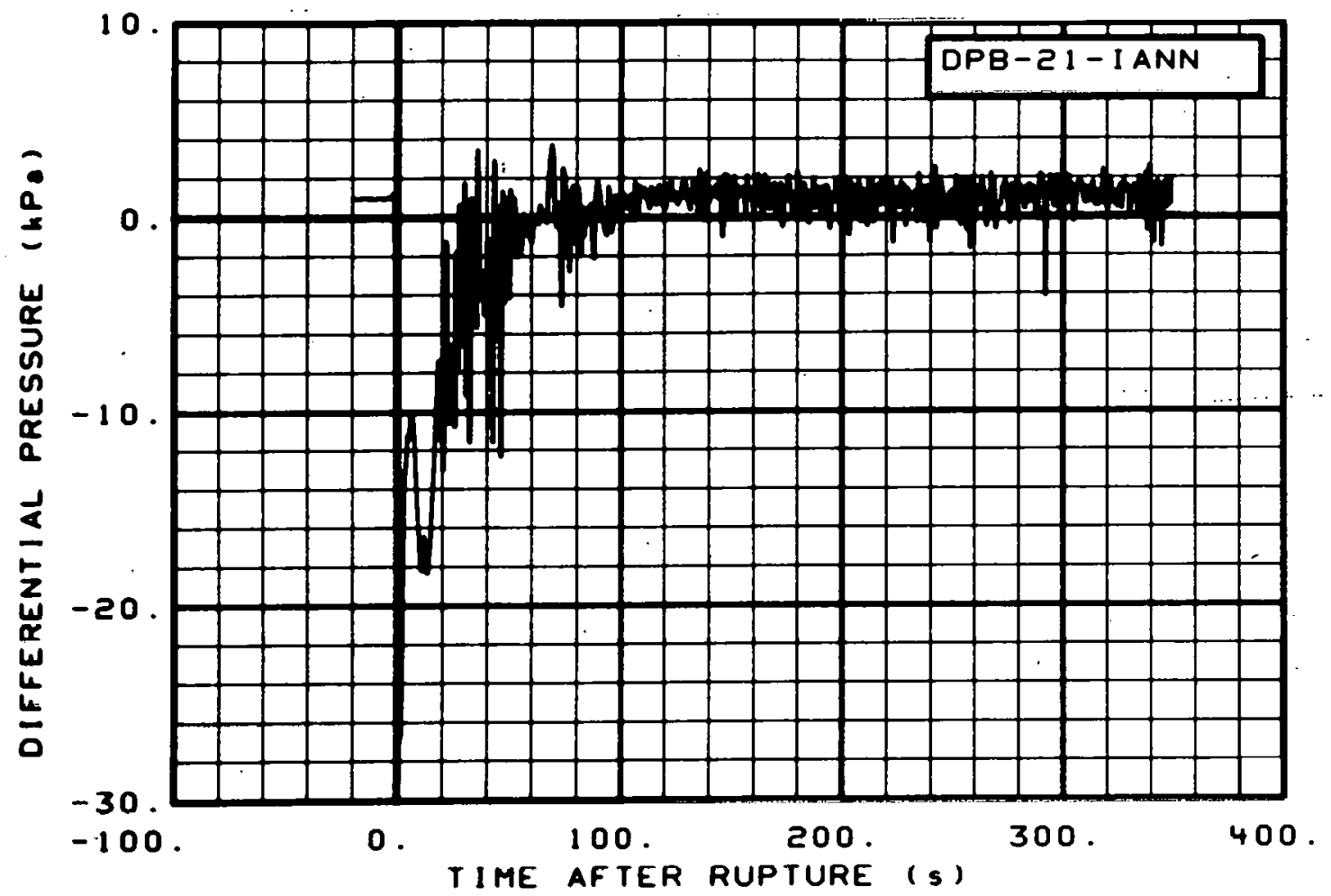

Fig. 189 Differential pressure in broken loop (DPB-21-IANN), from -20 to $350 \mathrm{~s}$.

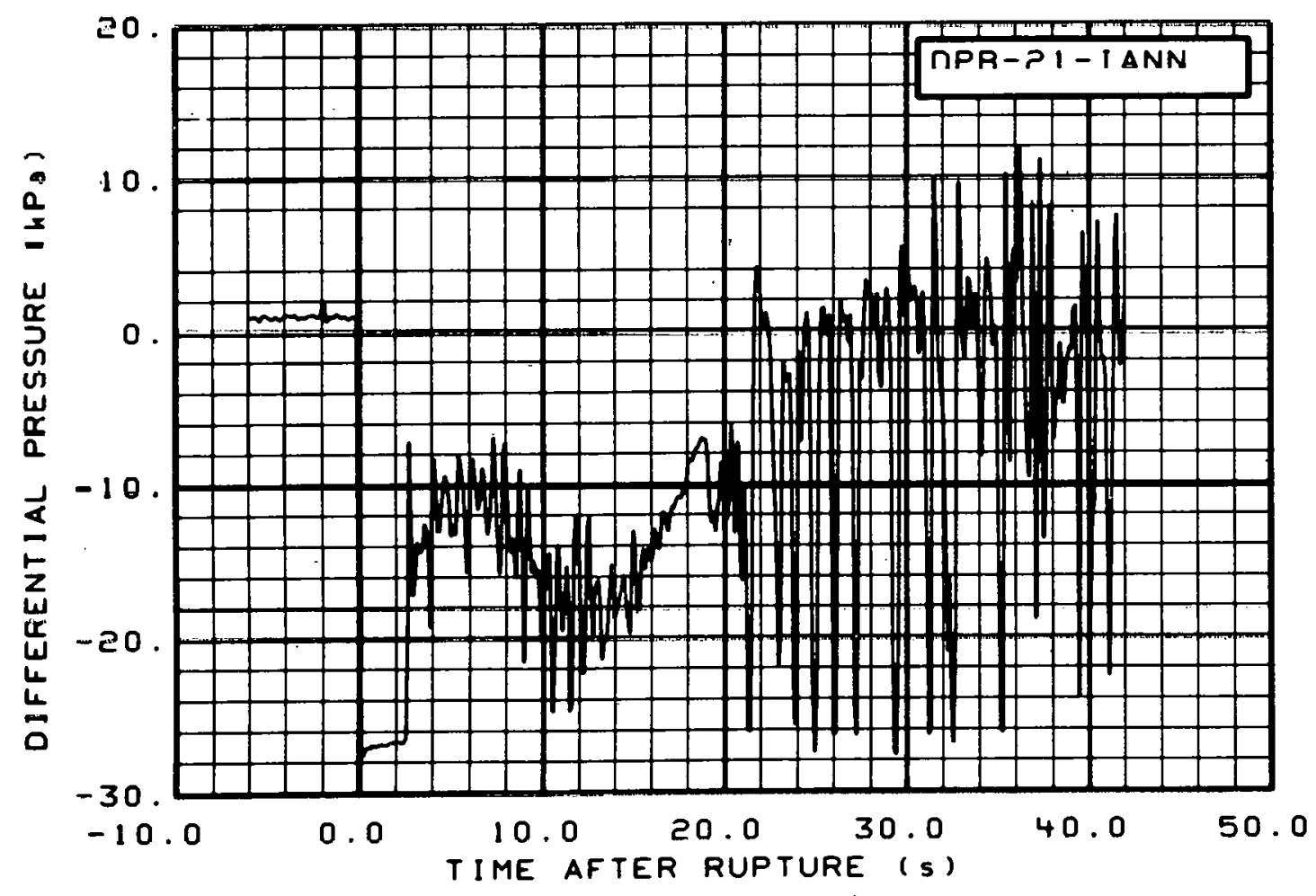

Fig. 190 Differential pressure in broken loop (DPB-21-IANN), from -6 to 42 s. 


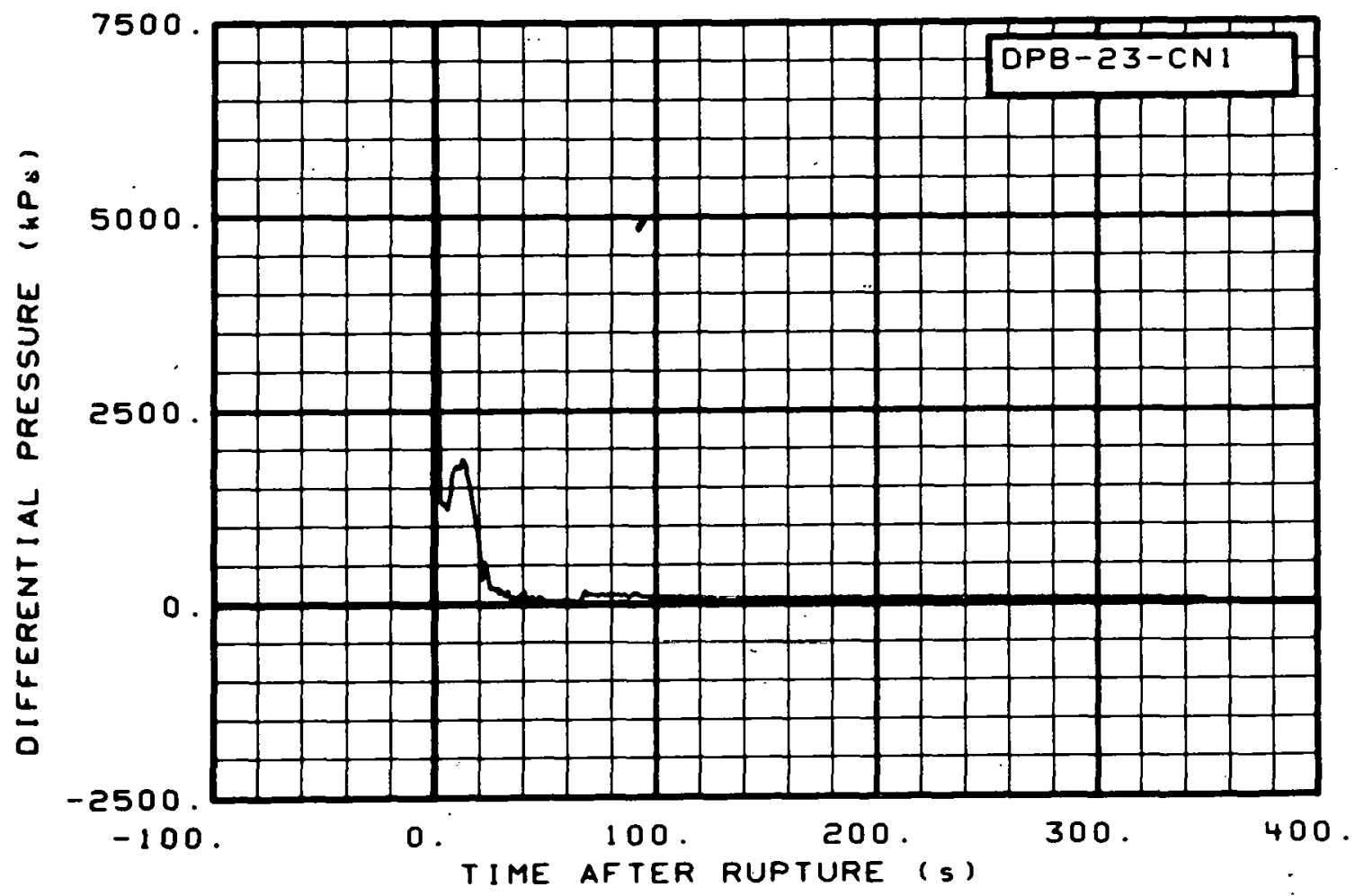

Fig. 191 Differential pressure in broken loop (DPB-23-CNI), from -20 to $350 \mathrm{~s}$.

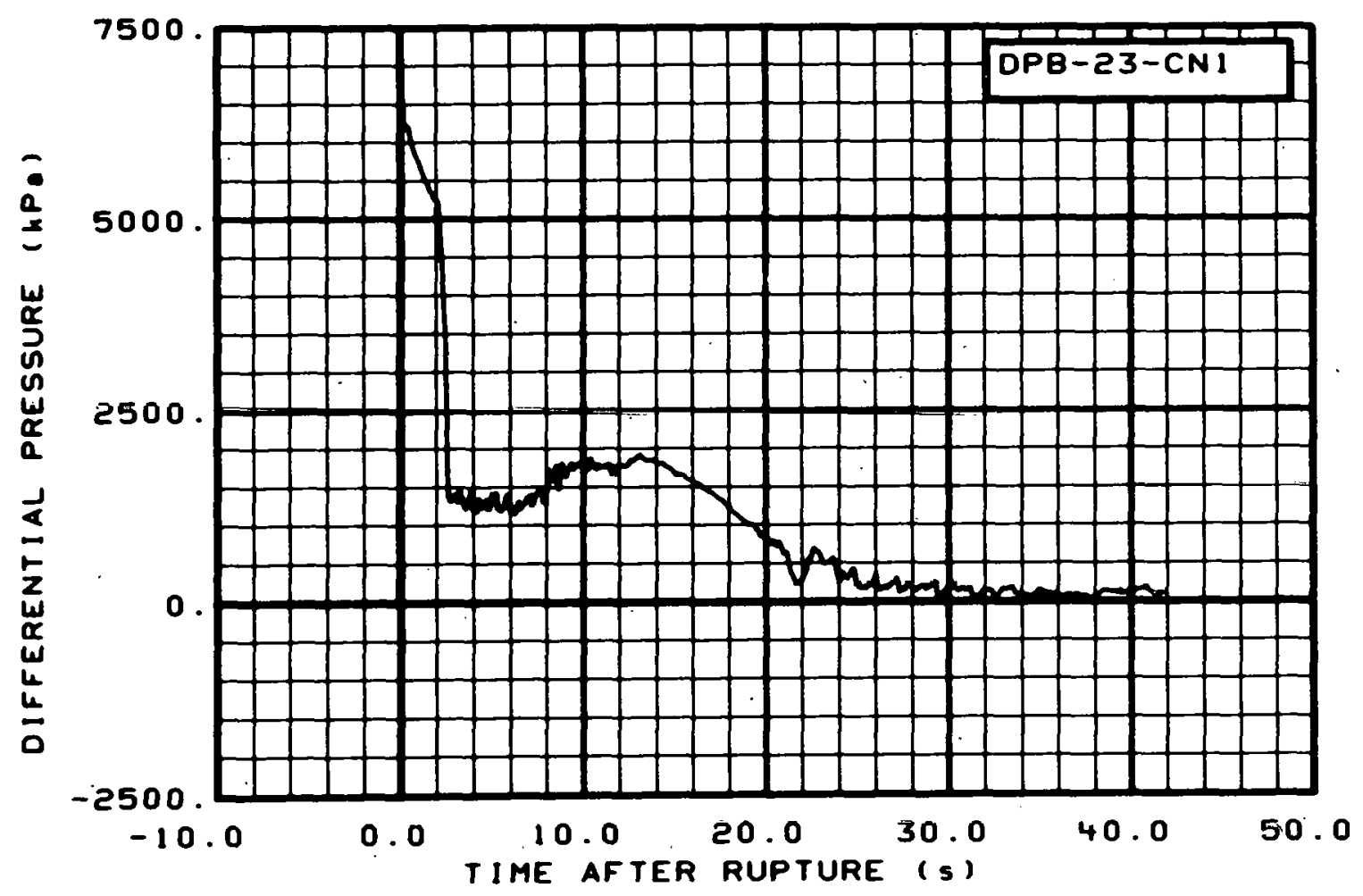

Fig. 192 Differential pressure in broken loop (DPB-23-CN1), from -6 to $42 \mathrm{~s}$. 


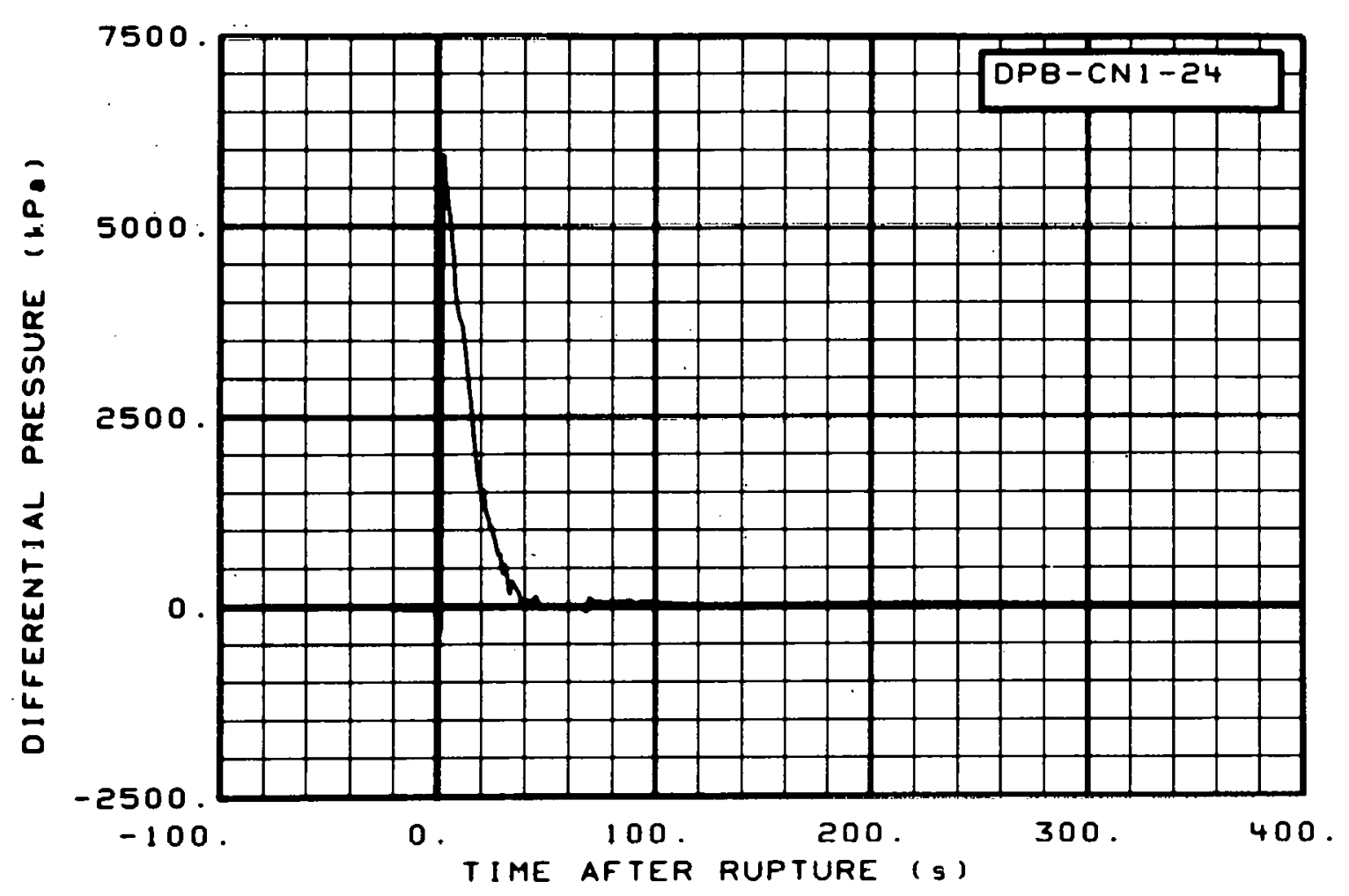

Fig. 193 Differential pressure in broken loop (DPB-CN1-24), from -20 to $350 \mathrm{~s}$.

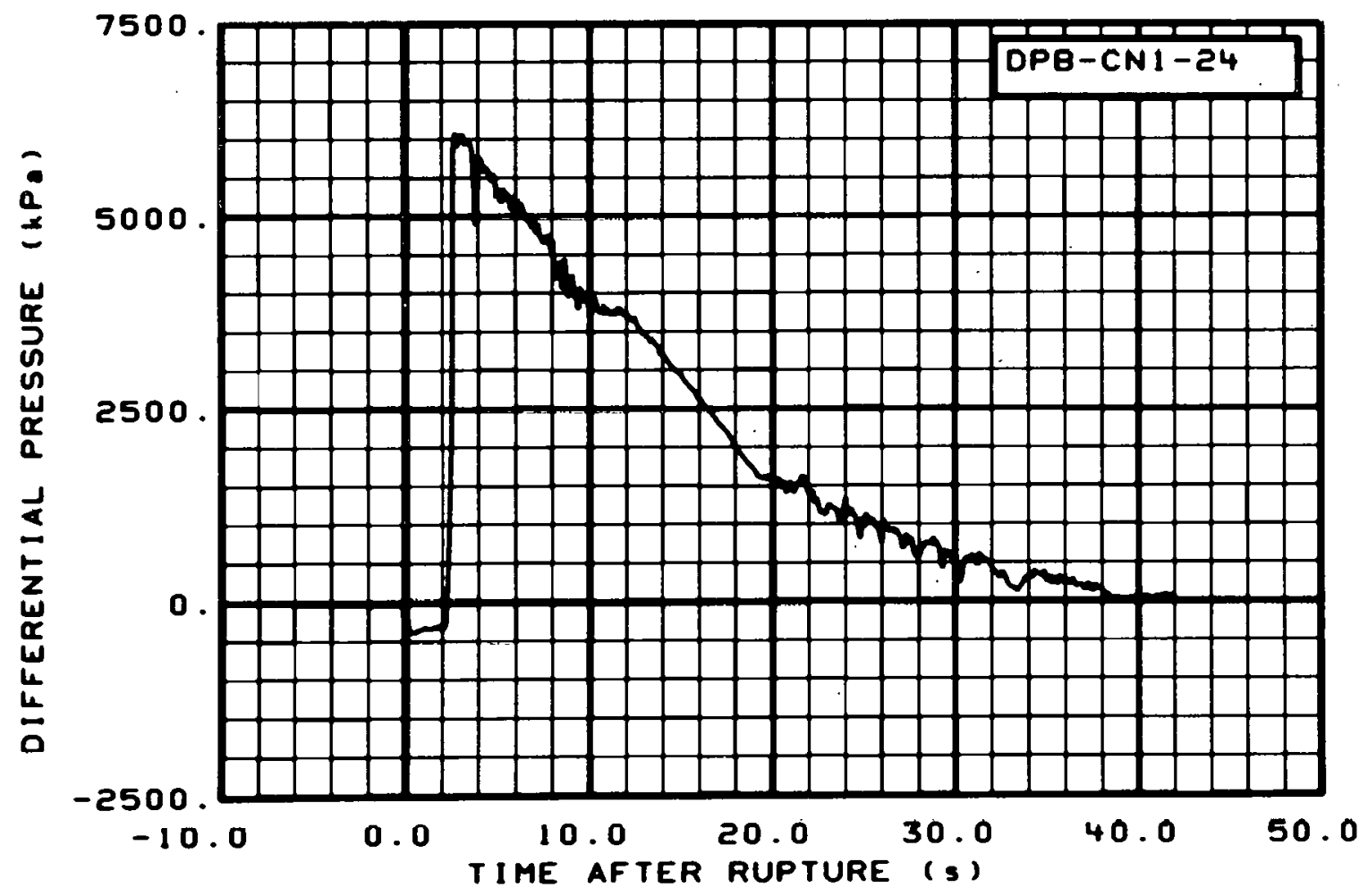

Fig. 194 Differential pressure in broken loop (DPB-CNT-24), from -6 to $42 \mathrm{~s}$. 


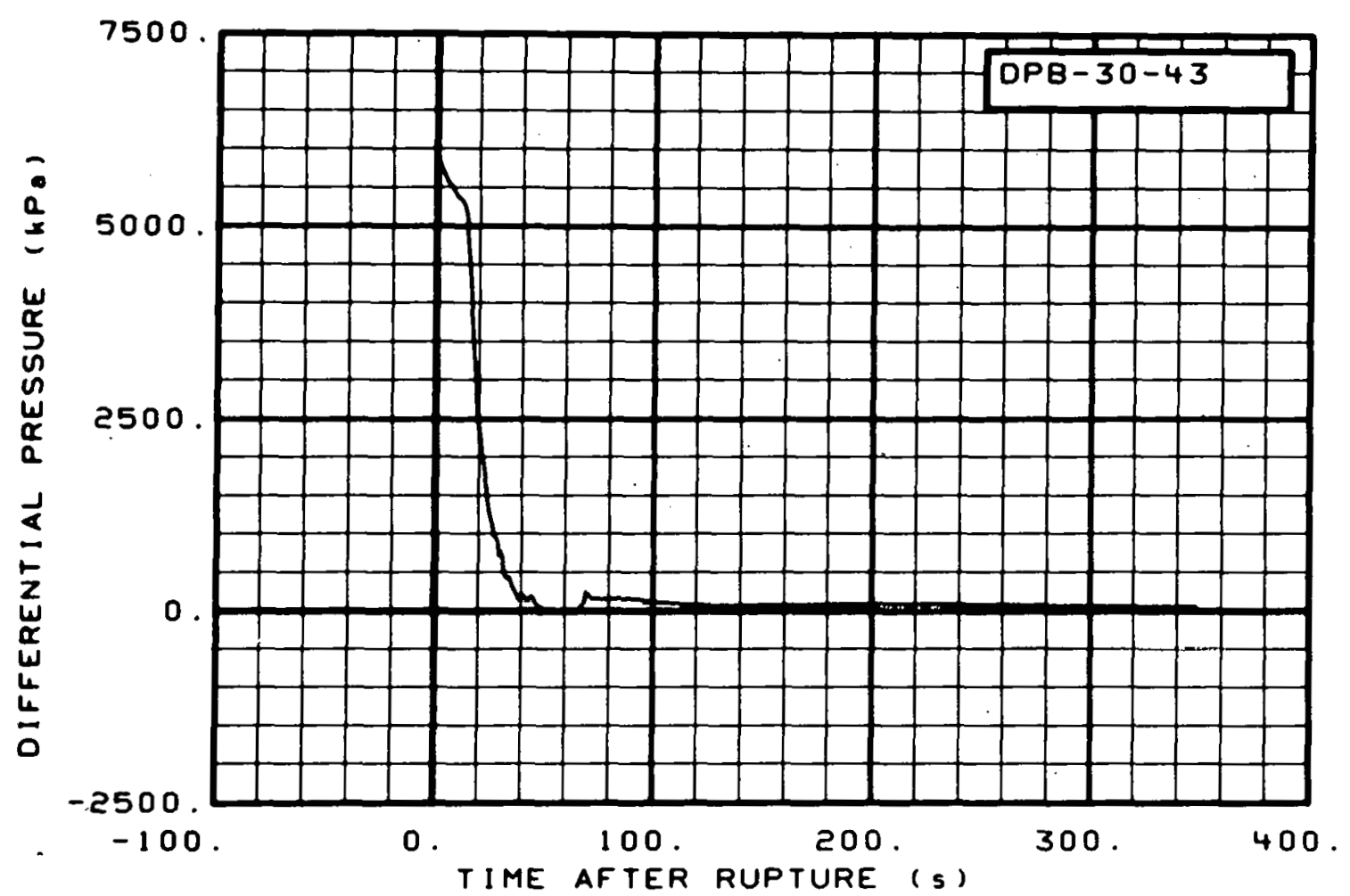

Fig. 195 Differential pressure in broken loop (DPB-30-43), from -20 to $350 \mathrm{~s}$.

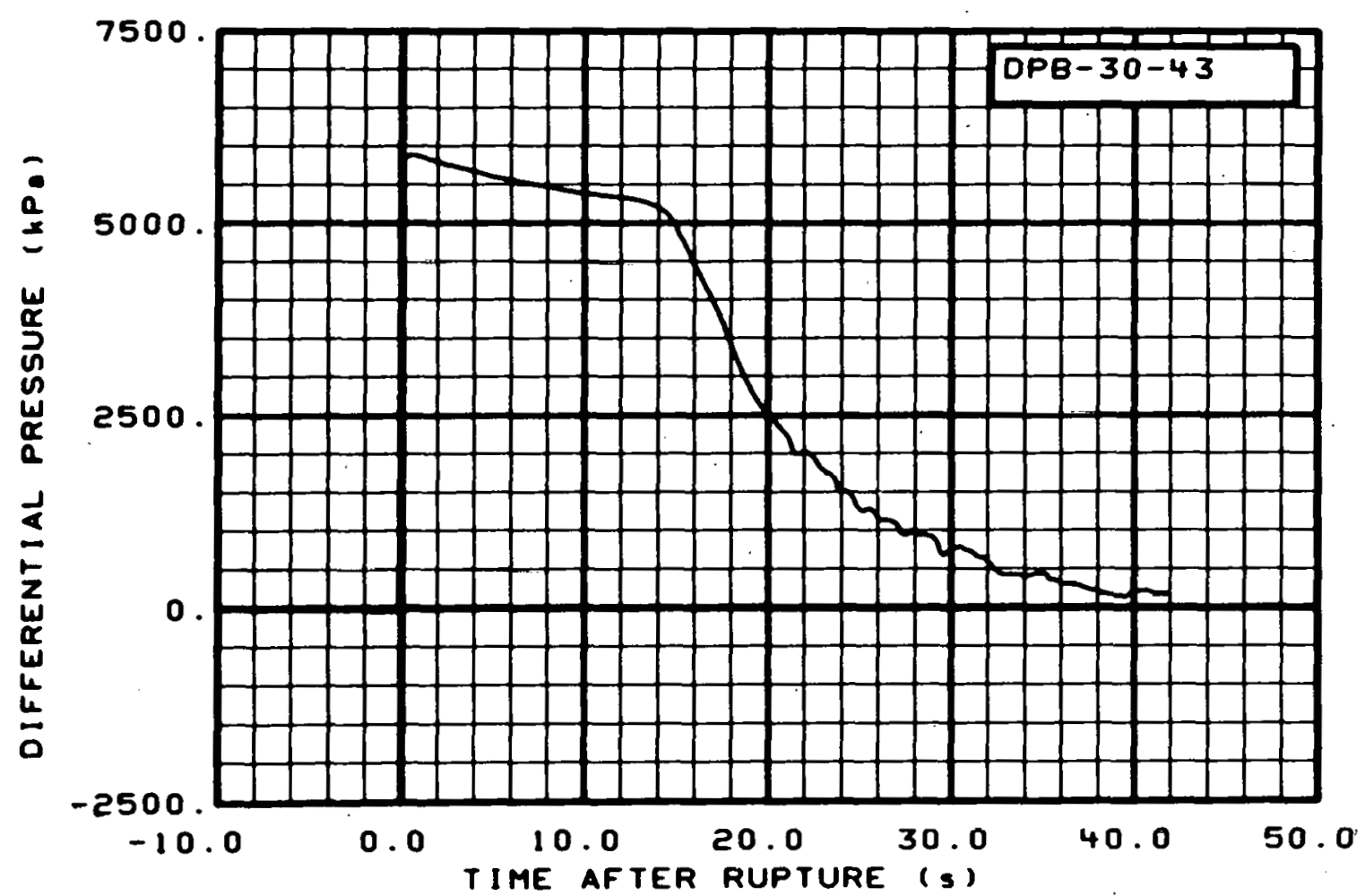

Fig. 196 Differential pressure in broken loop (DPB-30-43), from -6 to $42 \mathrm{~s}$. 


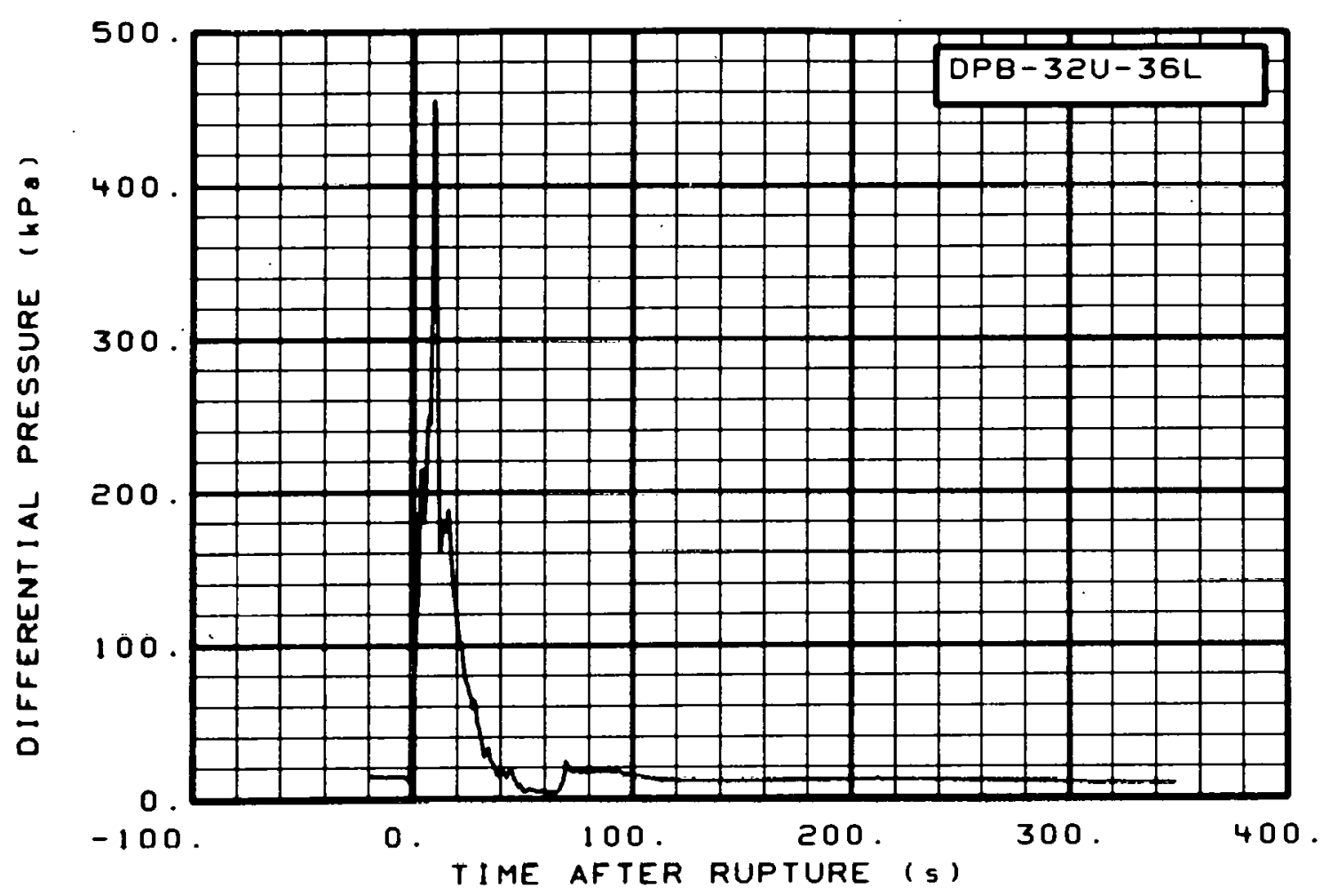

Fig. 197 Differential pressure in broken loop (DPB-32U-36L), from -20 to $350 \mathrm{~s}$.

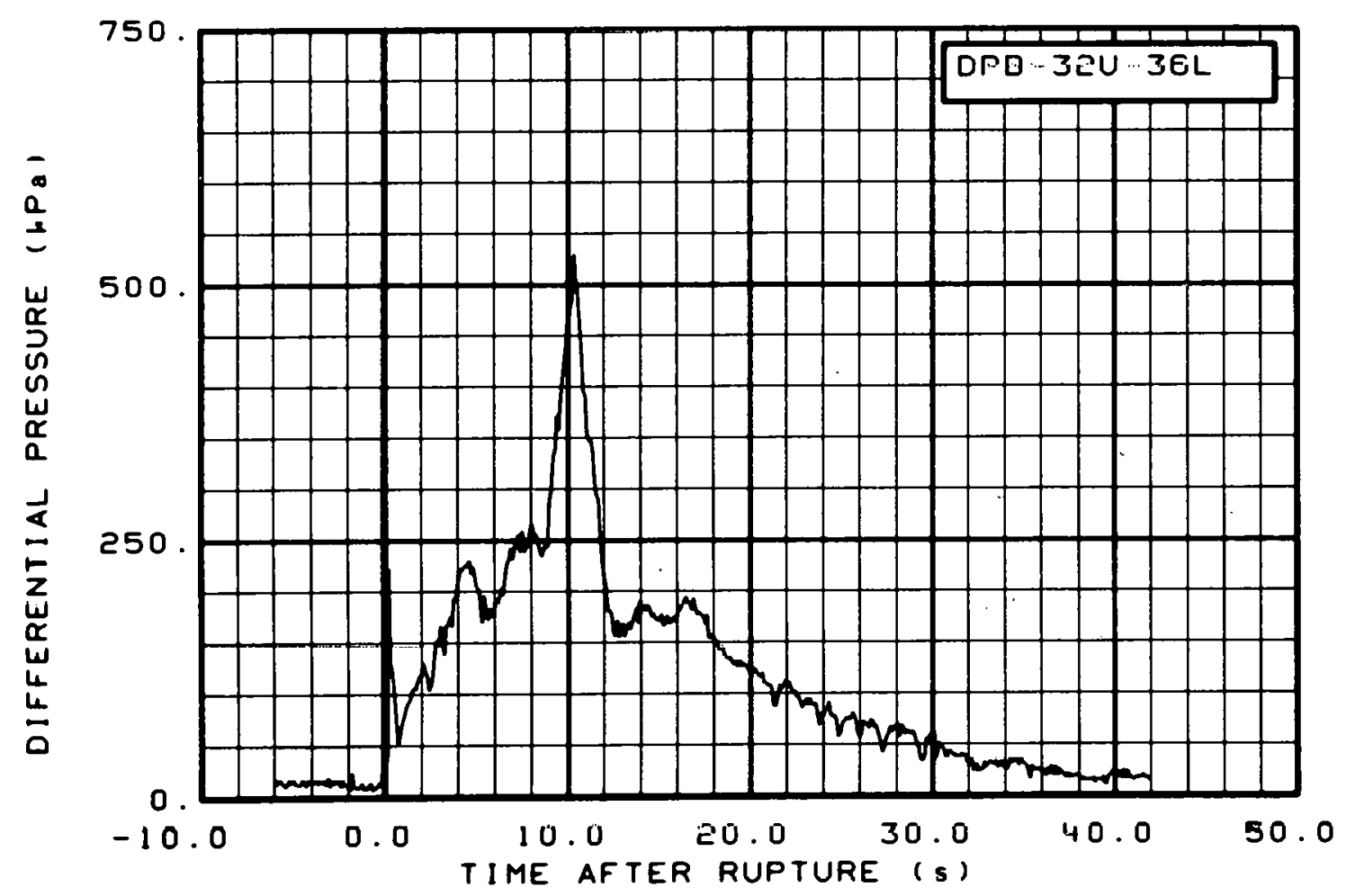

Fig. 198. Differential pressure in broken loop (DPB-32U-36L), from -6 to $42 \mathrm{~s}$. 


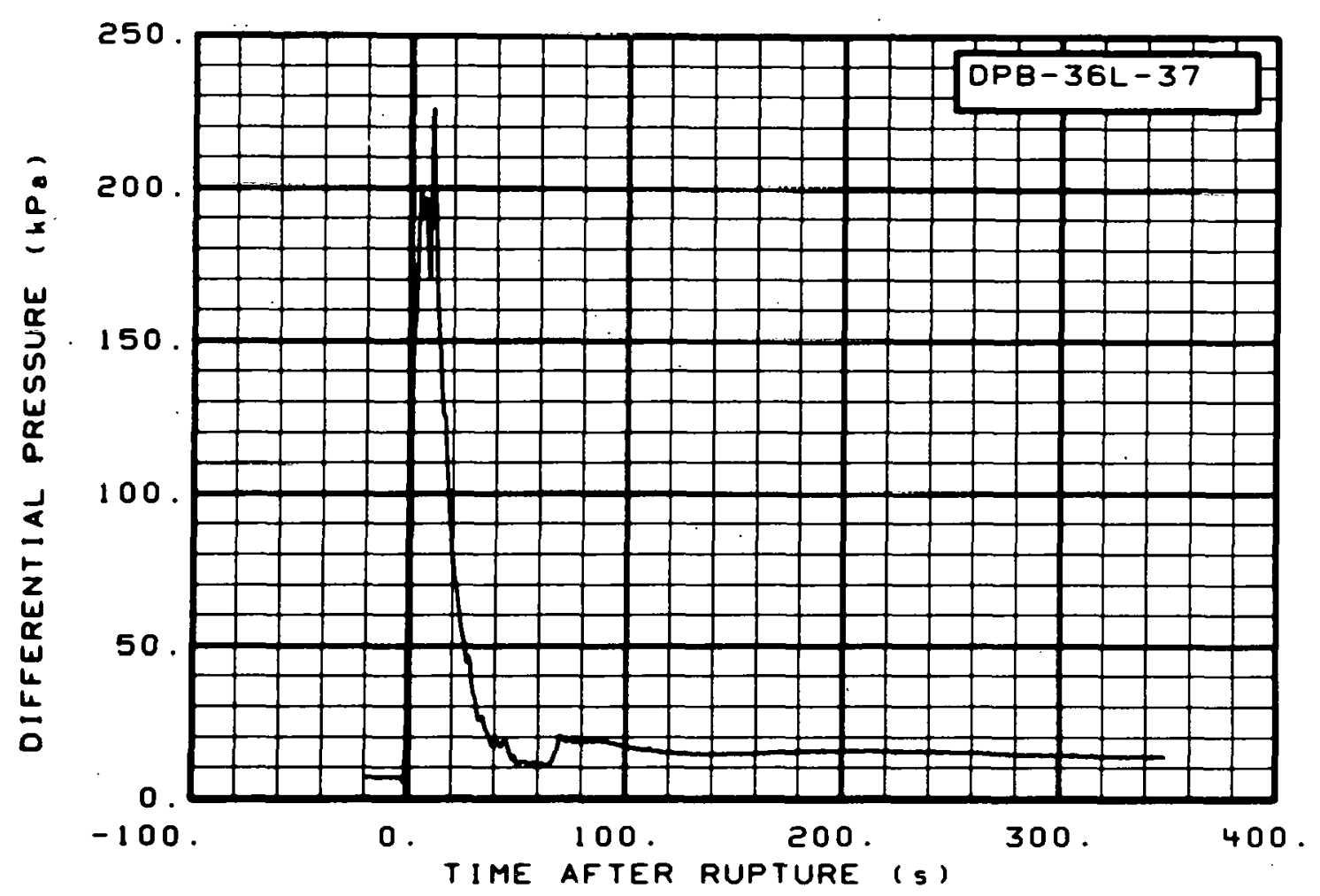

Fig. 199 Differential pressure in broken loop (DPB-36L-37), from -20 to $350 \mathrm{~s}$.

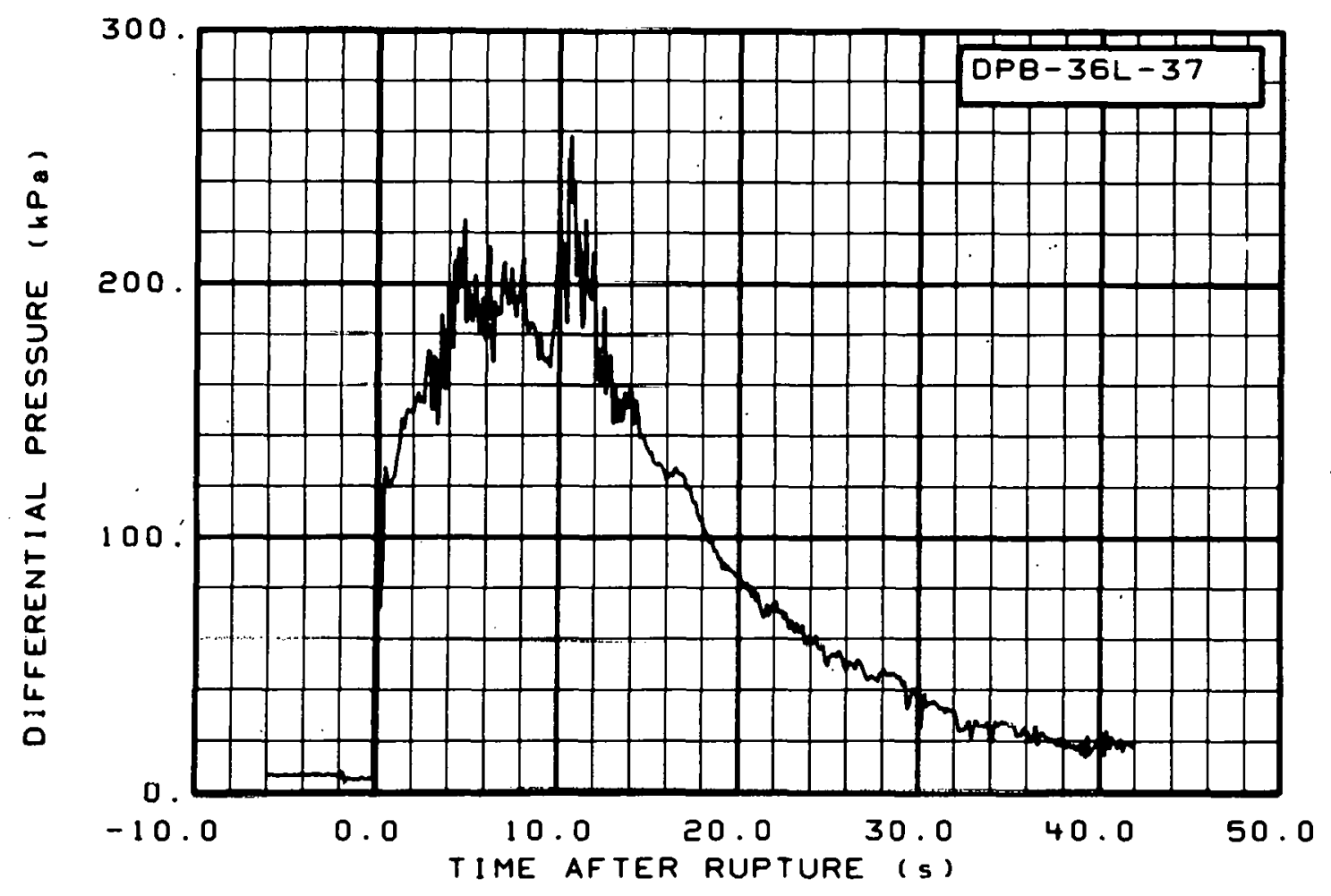

Fig. 200 Differential pressure in broken loop (DPB-36L-37), from -6 to $42 \mathrm{~s}$. 


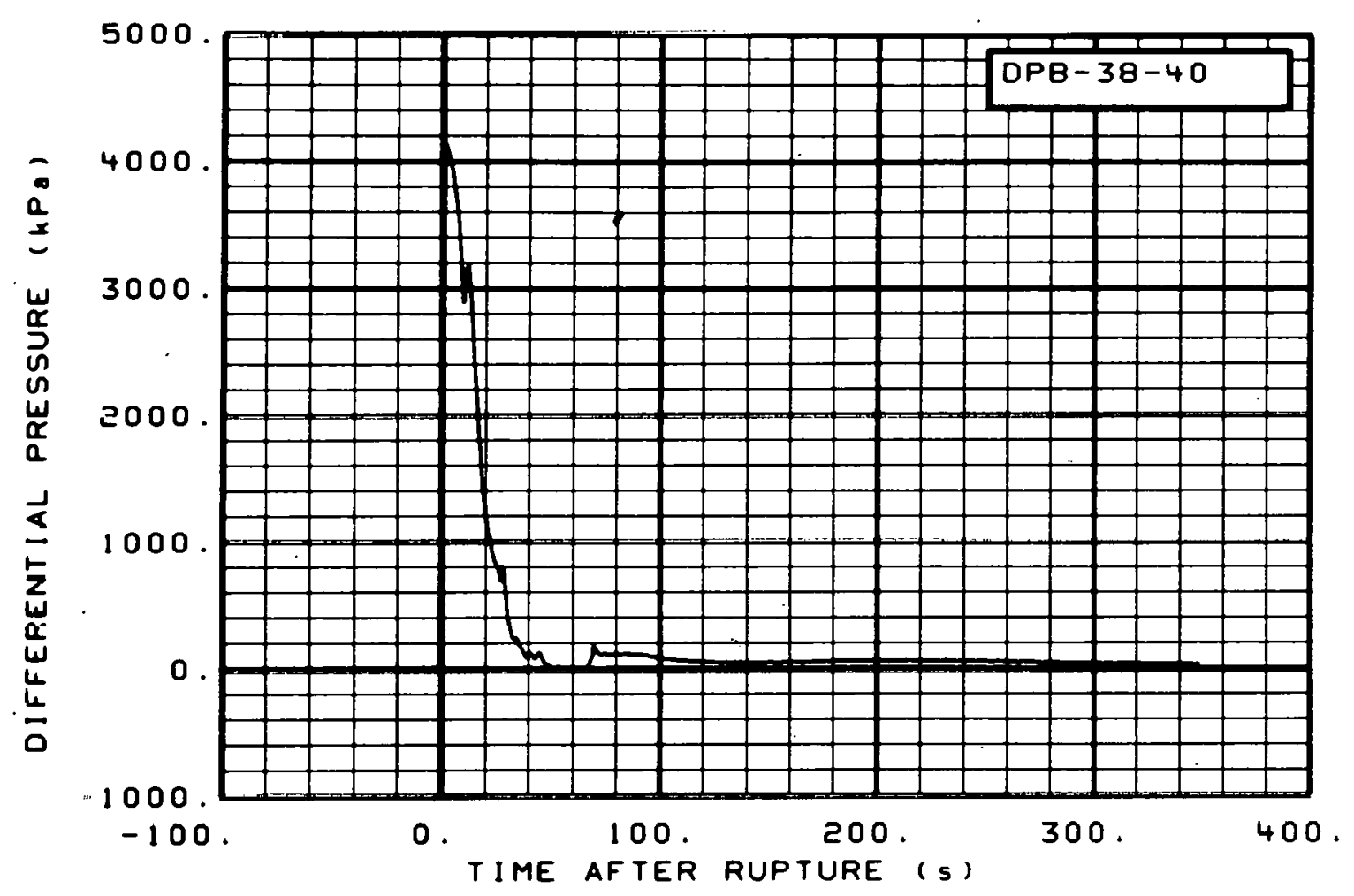

Fig. 201 Differential pressure in broken loop (DPB-38-40), from -20 to $350 \mathrm{~s}$.

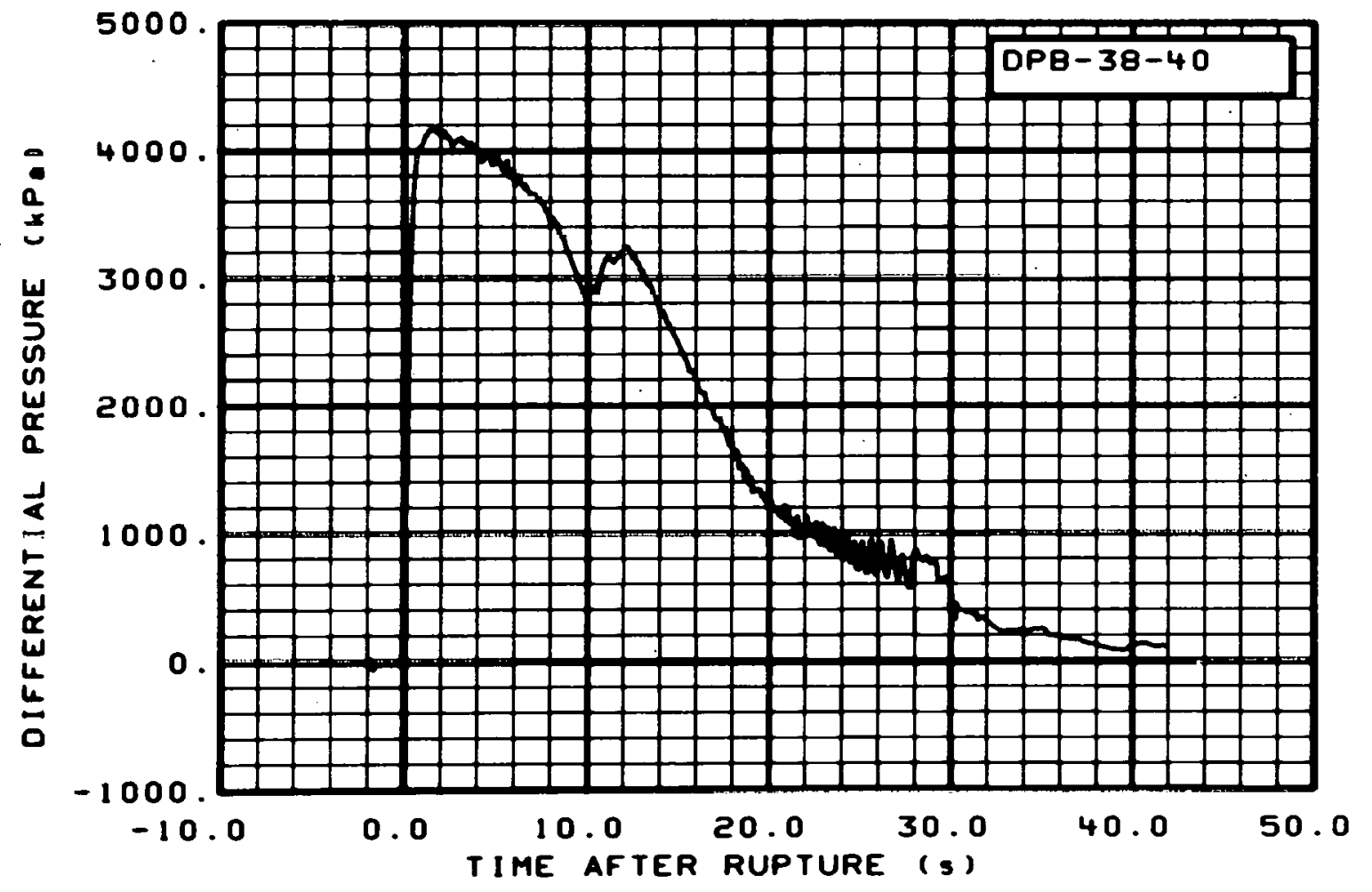

Fig. 202 Differential pressure in broken loop (DPB-38-40), from -6 to $42 \mathrm{~s}$. 


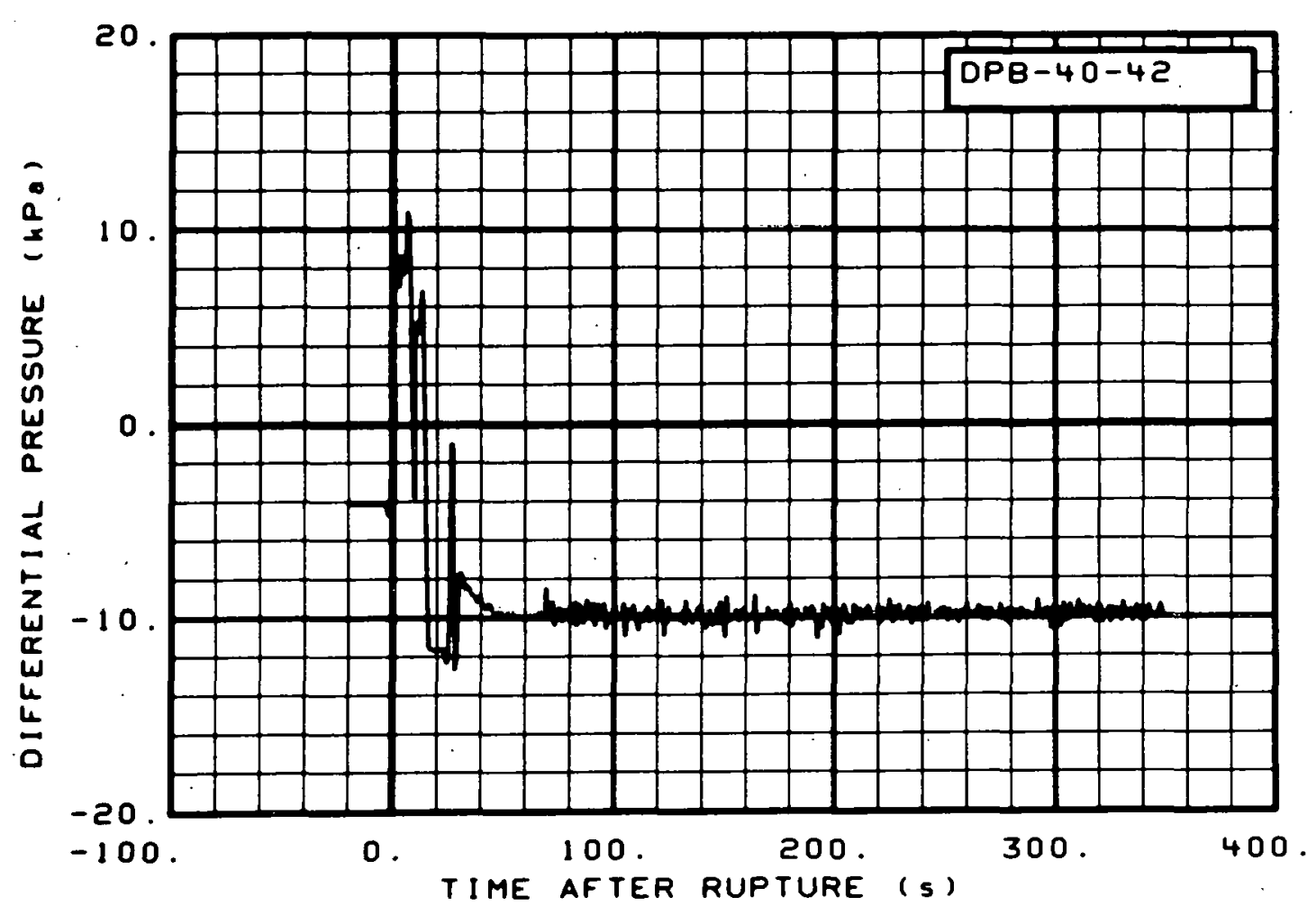

Fig. 203 Differential pressure in broken loop (DPB-40-42), from -20 to $350 \mathrm{~s}$.

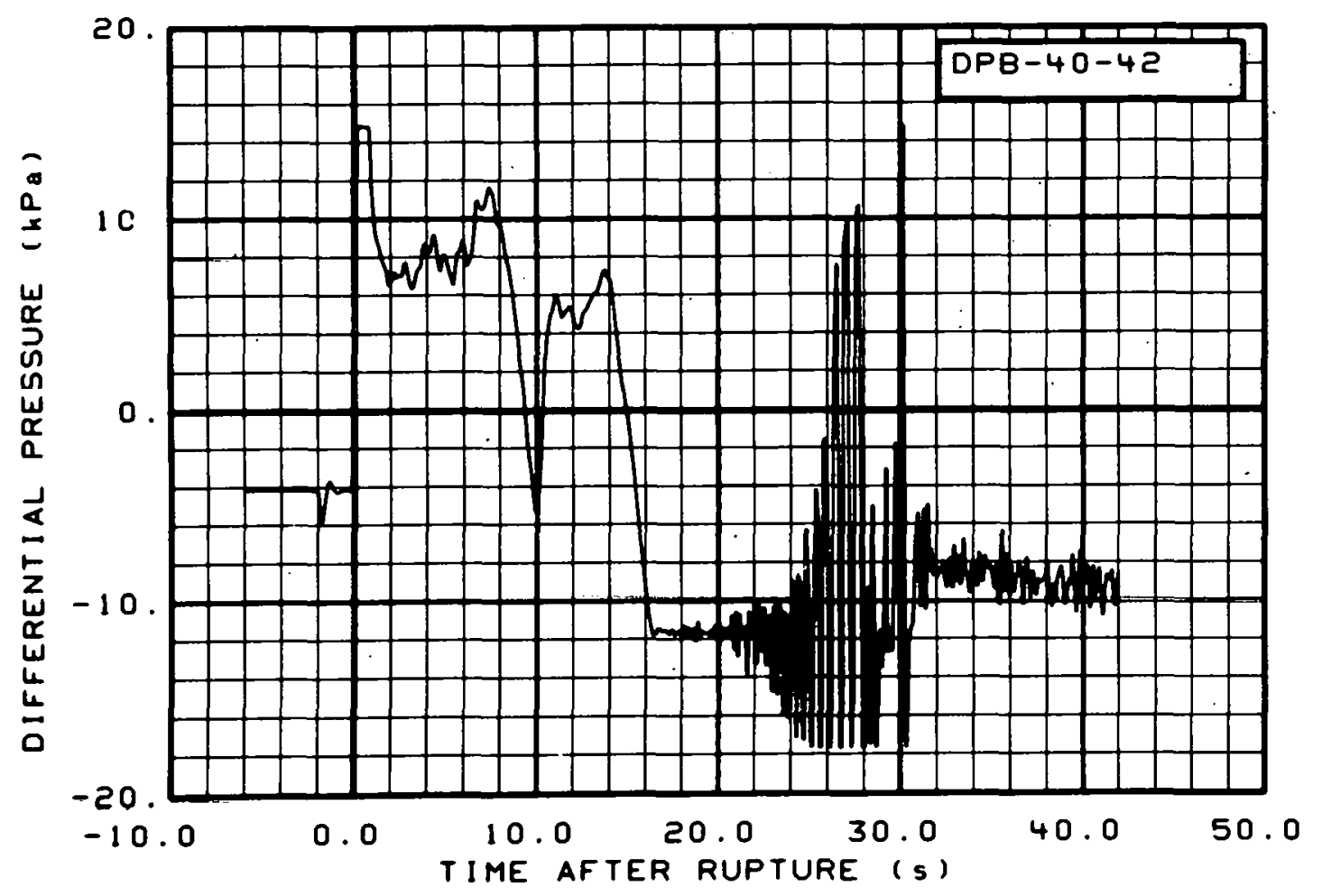

Fig. 204 Differential pressure in broken loop (DPB-40-42), from -6 to $42 \mathrm{~s}$. 


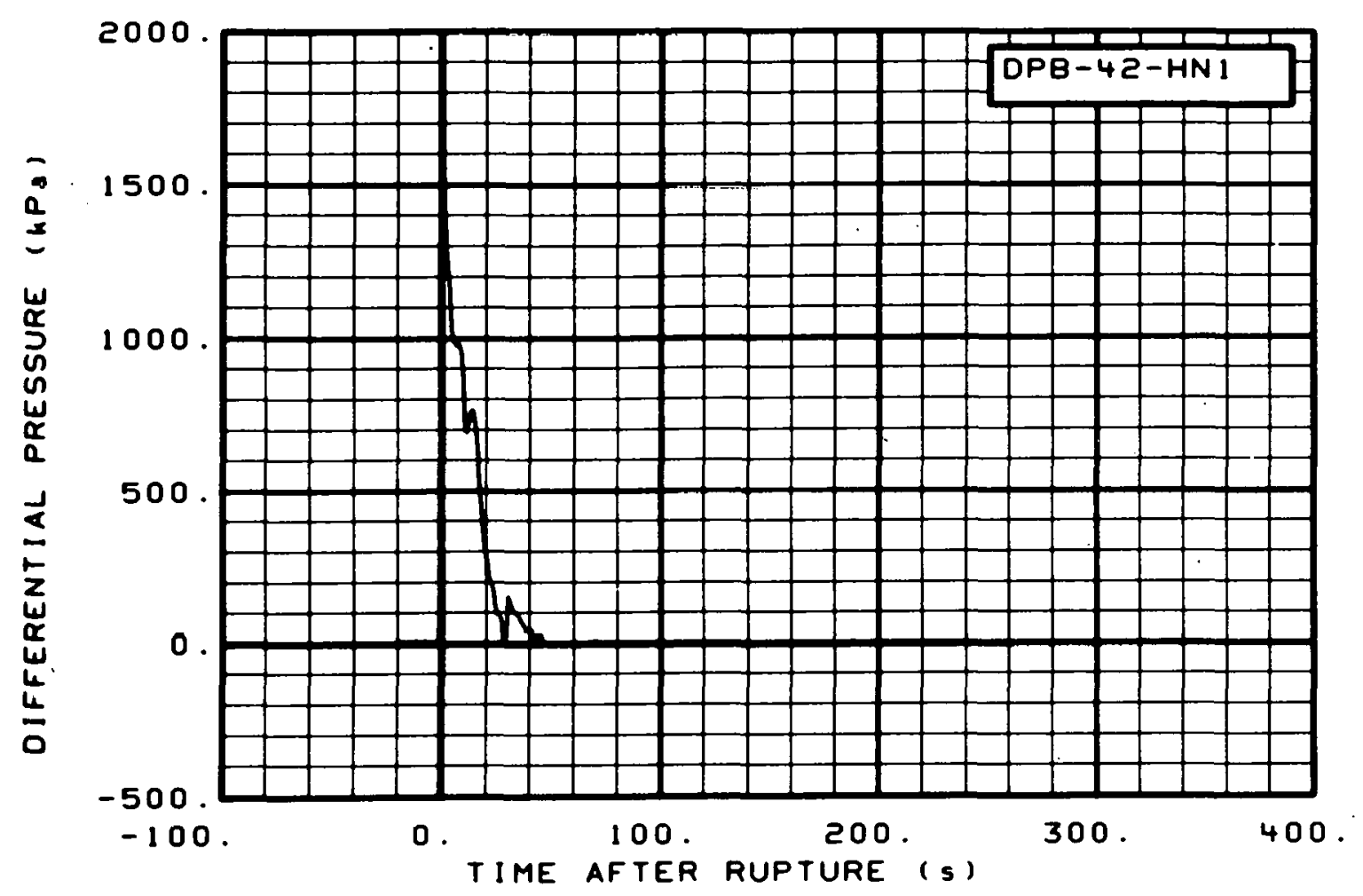

Fig. 205 Differential pressure in broken loop (DPB-42-HN1), from -20 to $350 \mathrm{~s}$.

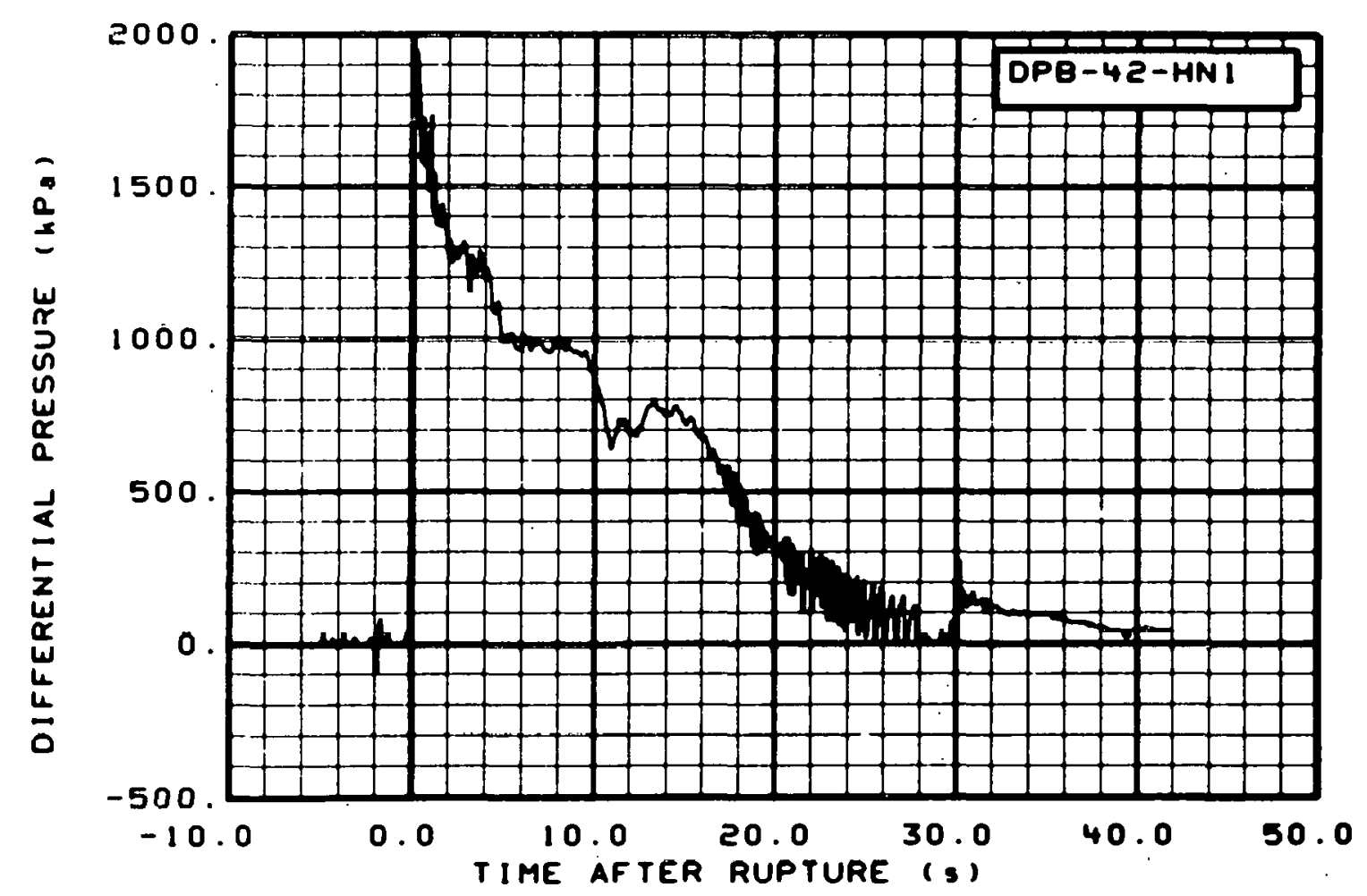

Fig. 206 Differential pressure in broken loop (DPB-42-HN1), from -6 to $42 \mathrm{~s}$. 


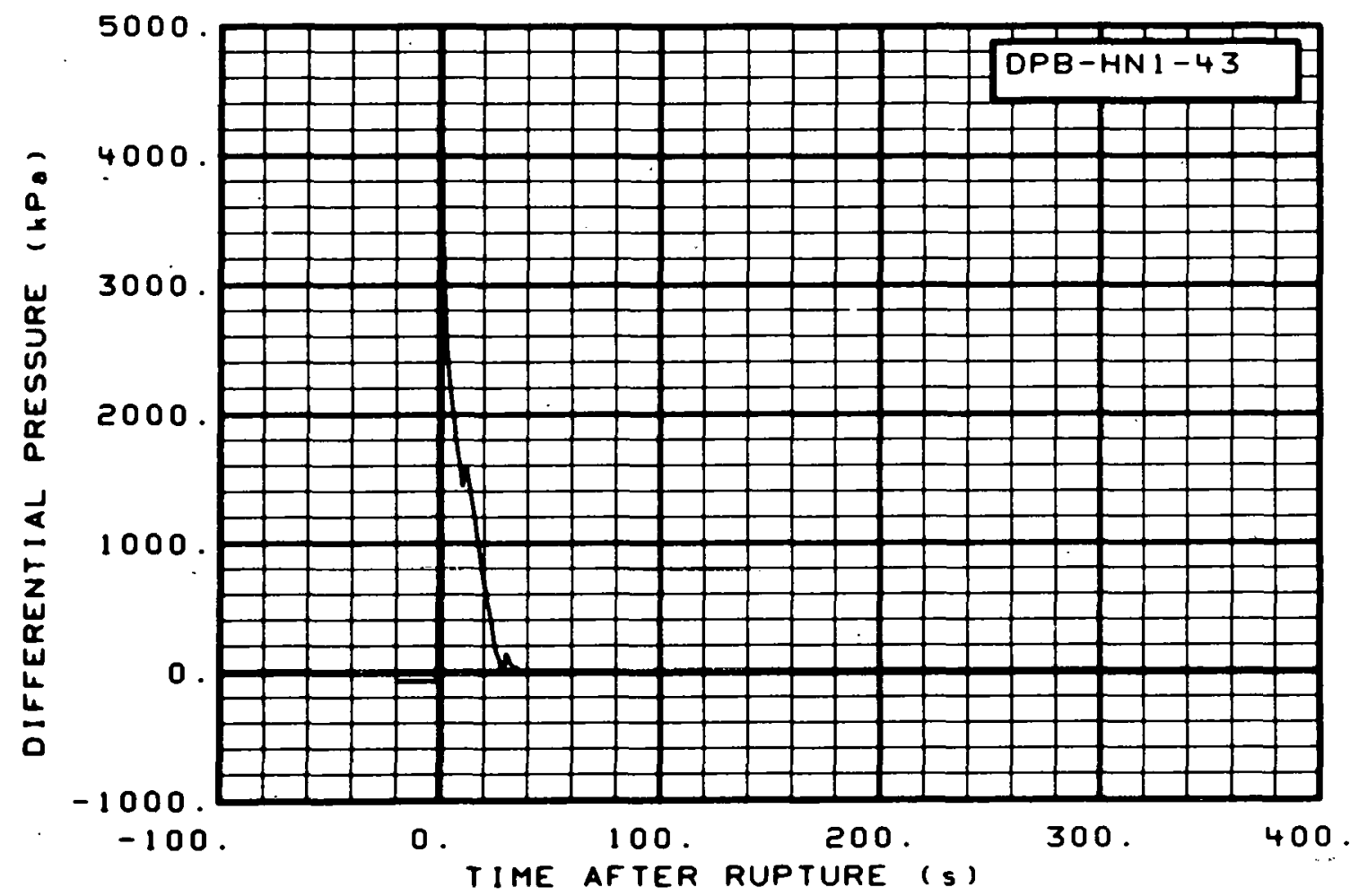

Fig. 207 Differential pressure in broken loop (DPB-HN1-43), from -20 to $350 \mathrm{~s}$.

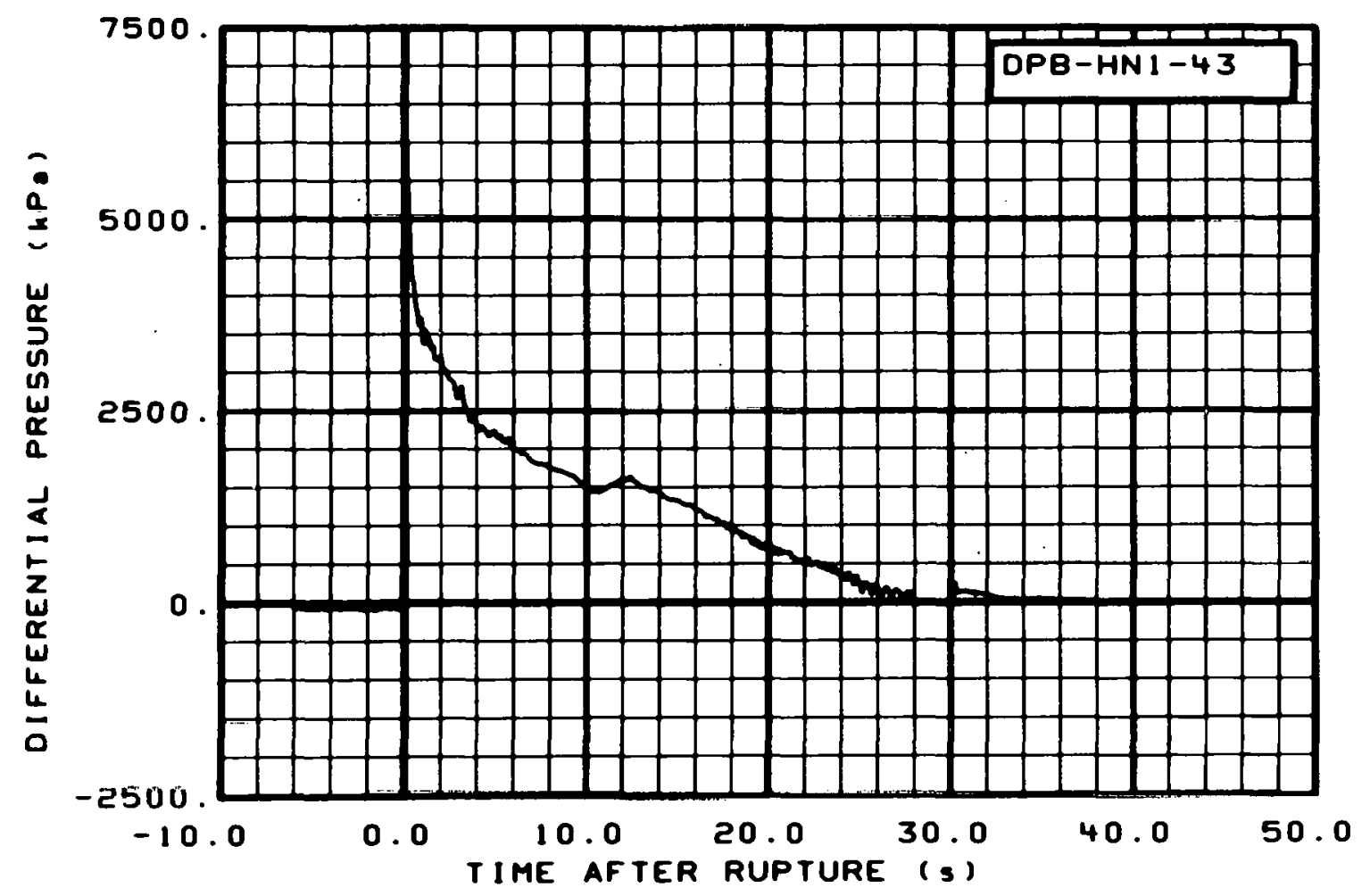

Fig. 208 Differential pressure in broken loop (DPB-HN1-43), from -6 to $42 \mathrm{~s}$. 


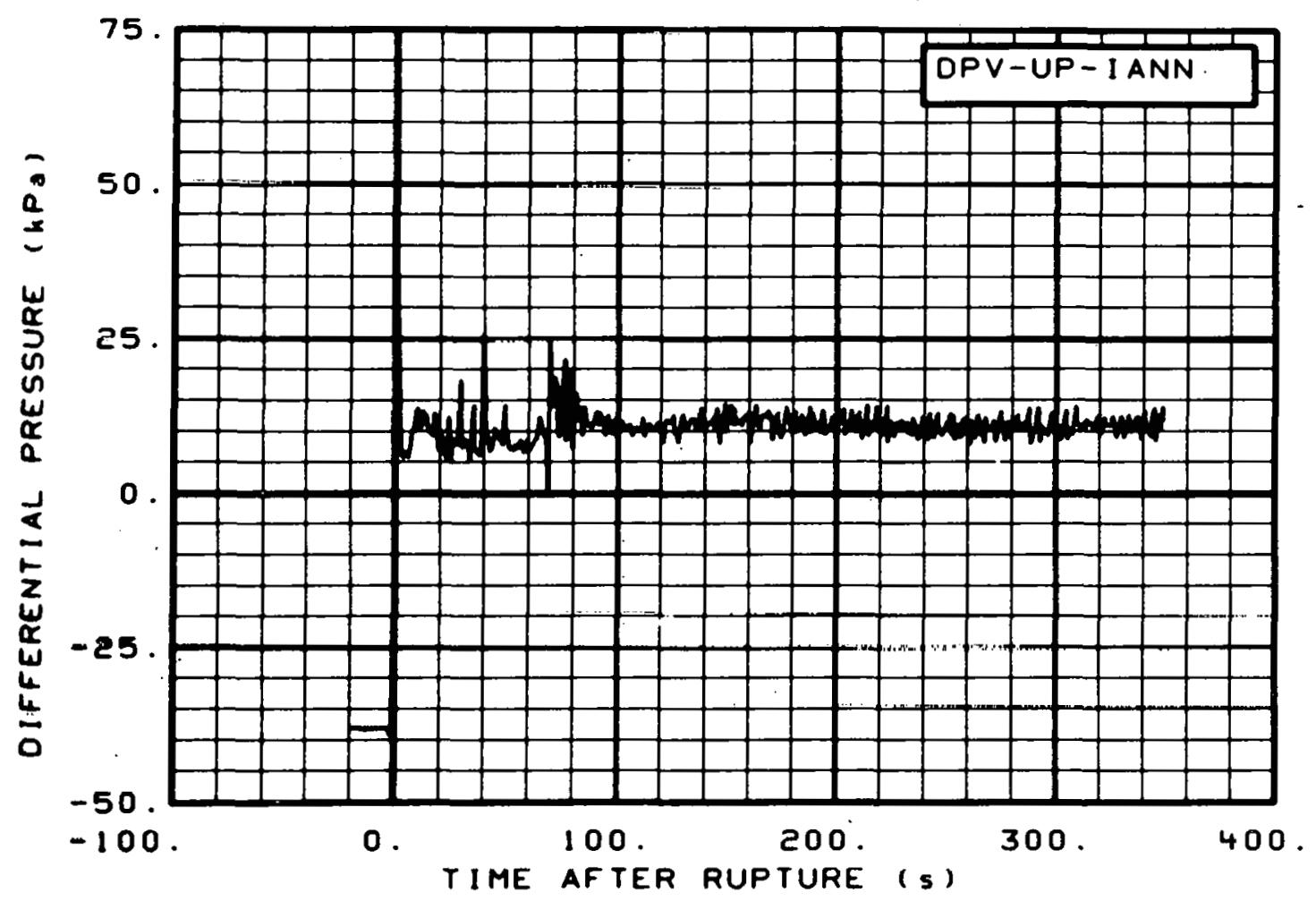

Fig. 209 Differential pressure in vessel (DPV-UP-IANN), from -20 to $350 \mathrm{~s}$.

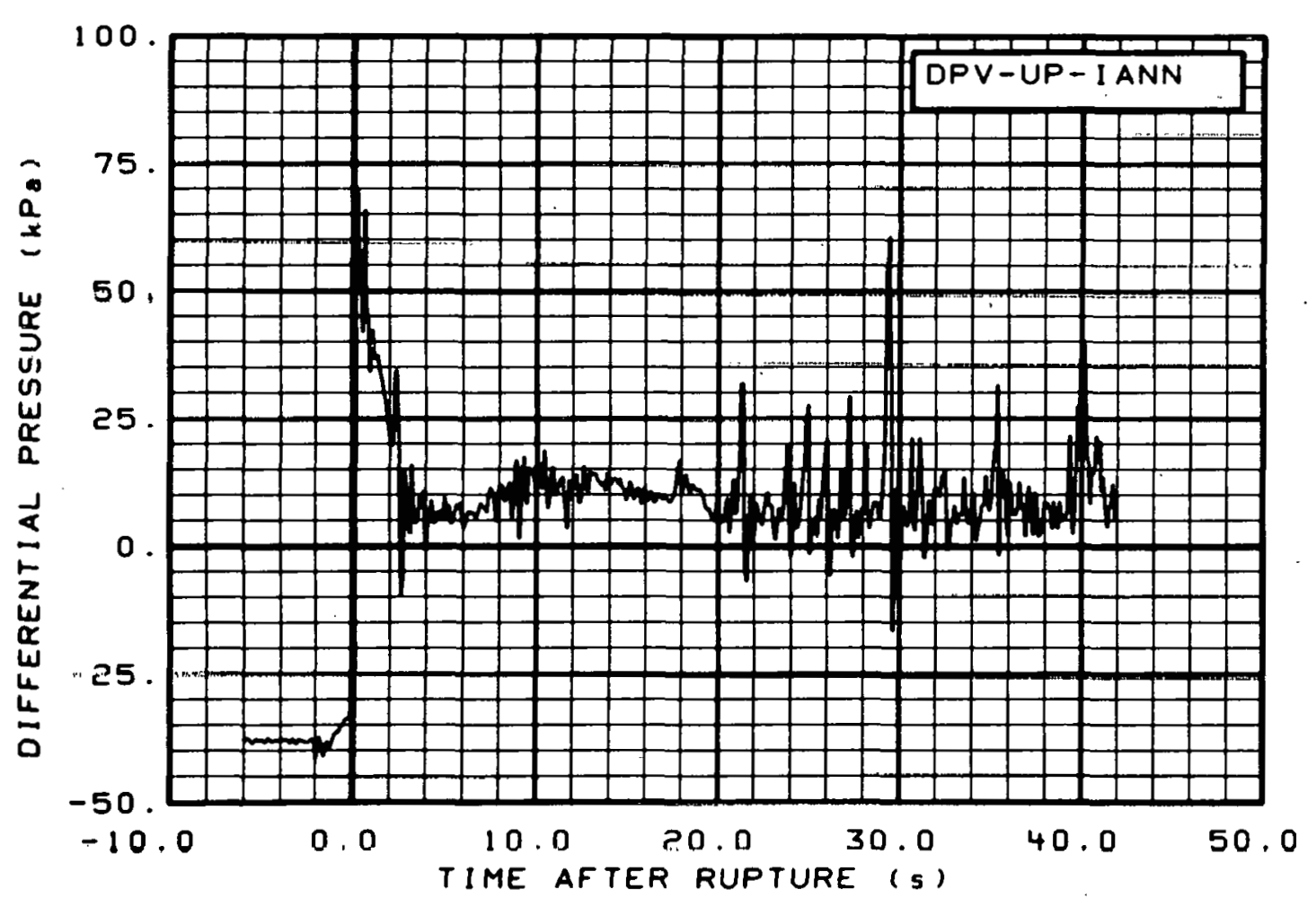

Fig. 210 Differential pressure in vessel (DPV-UP-IANN), from -6 to $42 \mathrm{~s}$. 


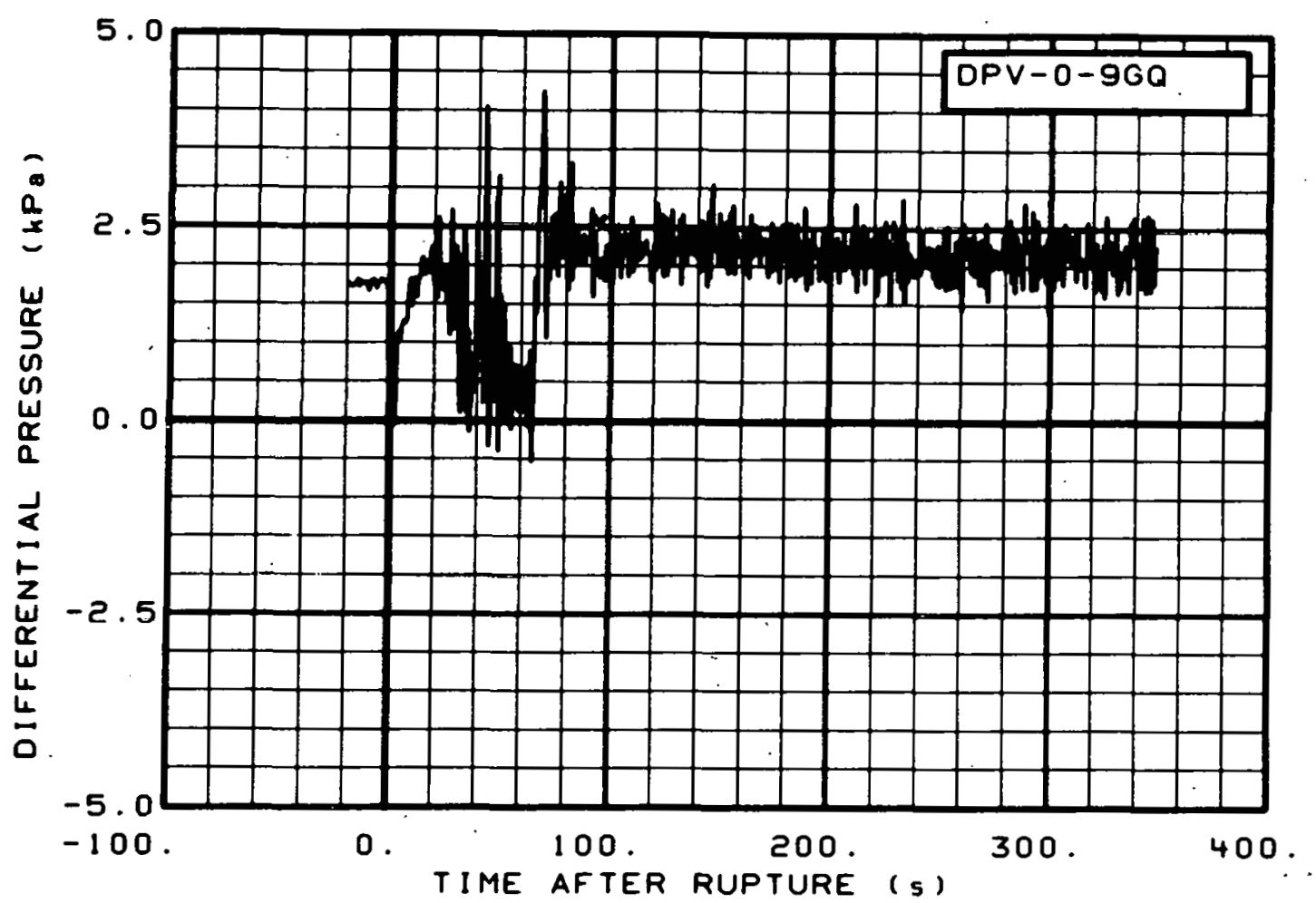

Fig. 211 Differential pressure in vessel (DPV-0-9GQ), from -20 to $350 \mathrm{~s}$.

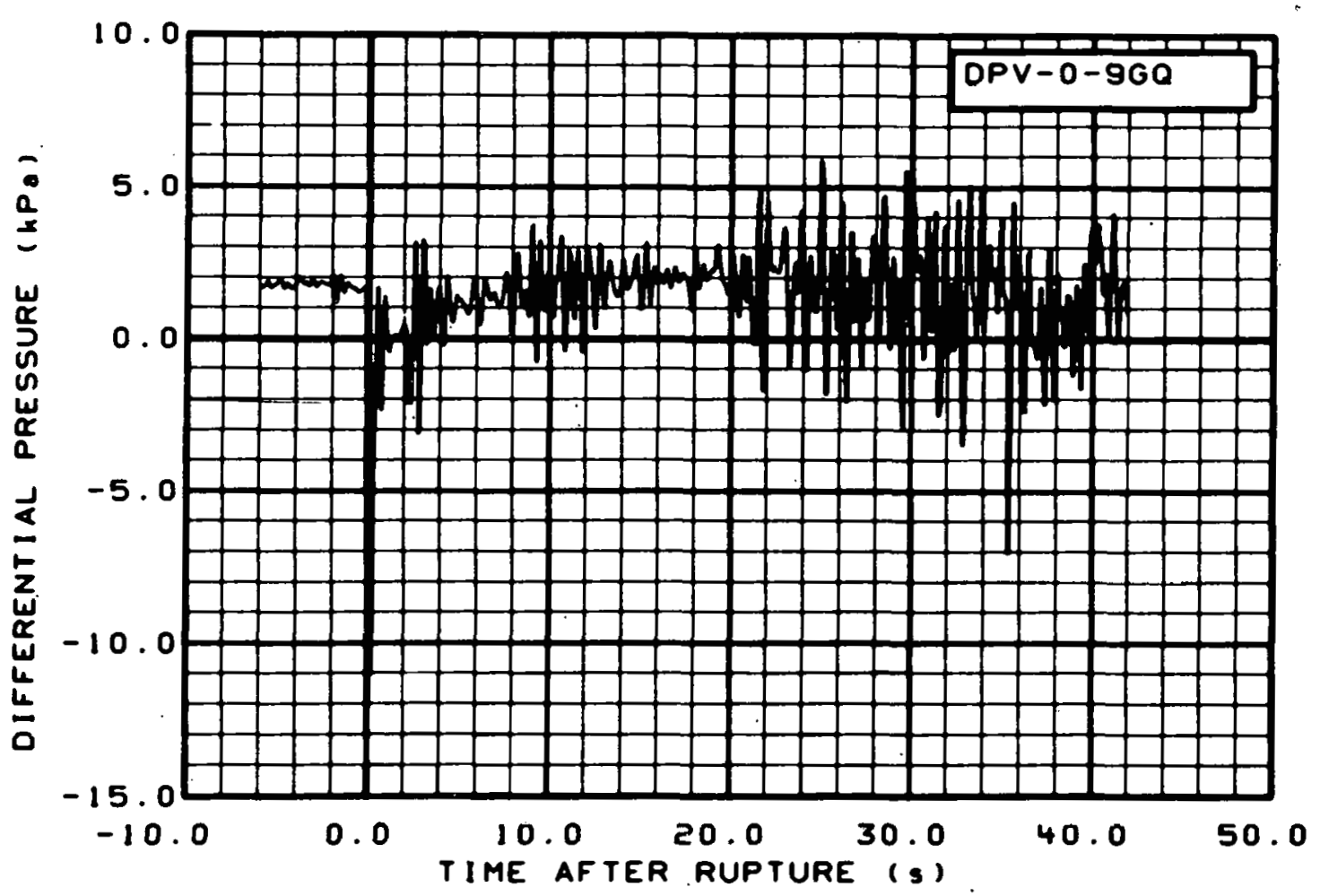

Fig. 212 Differential pressure in vessel (DPV-0-9GQ), from -6 to $42 \mathrm{~s}$. 


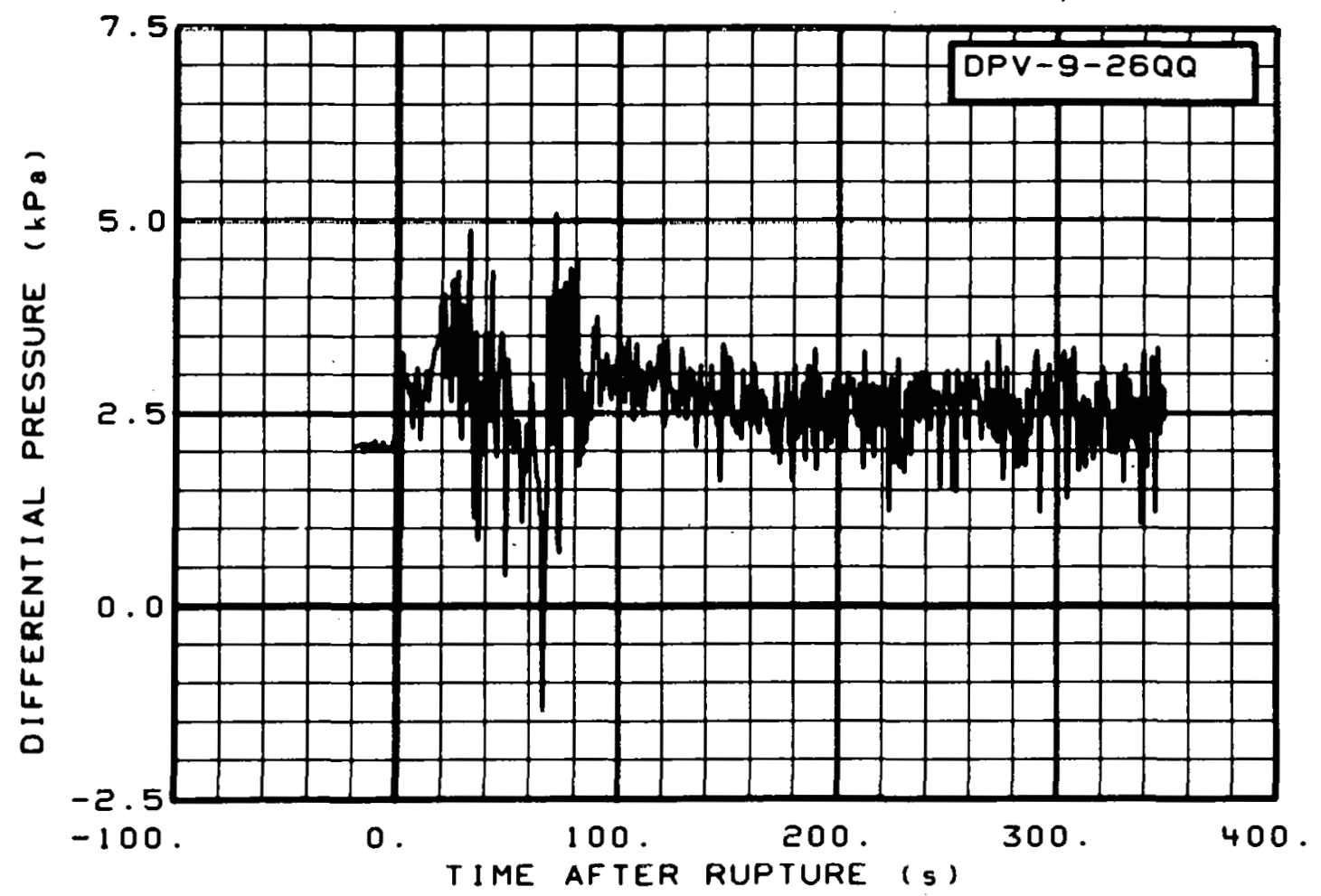

Fig. 213 Differential pressure in vessel (DPV-9-26QQ), from -20 to $350 \mathrm{~s}$.

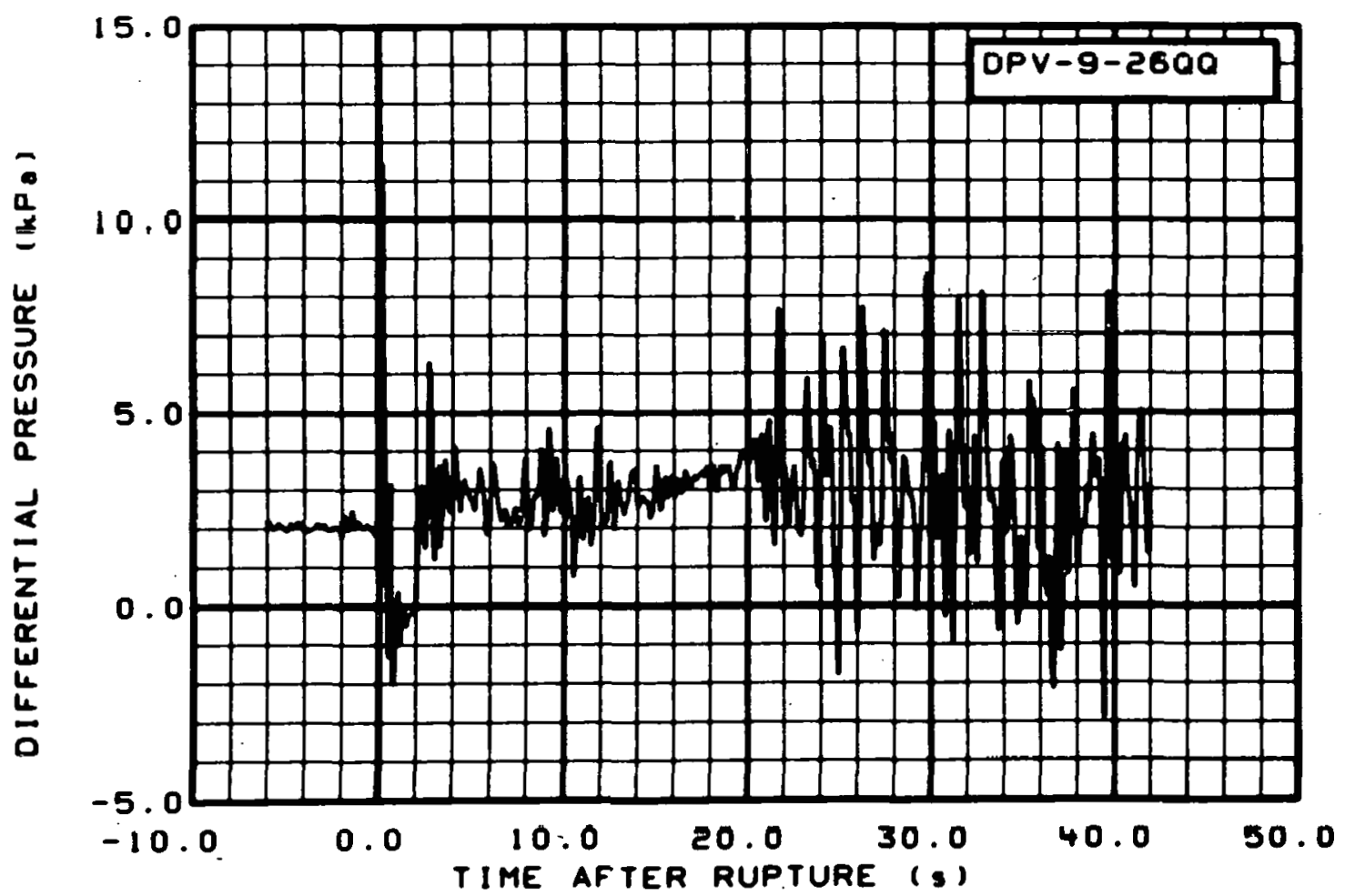

Fig. 214 Differential pressure in vessel (DPV-9-26QQ), from -6 to $42 \mathrm{~s}$. 


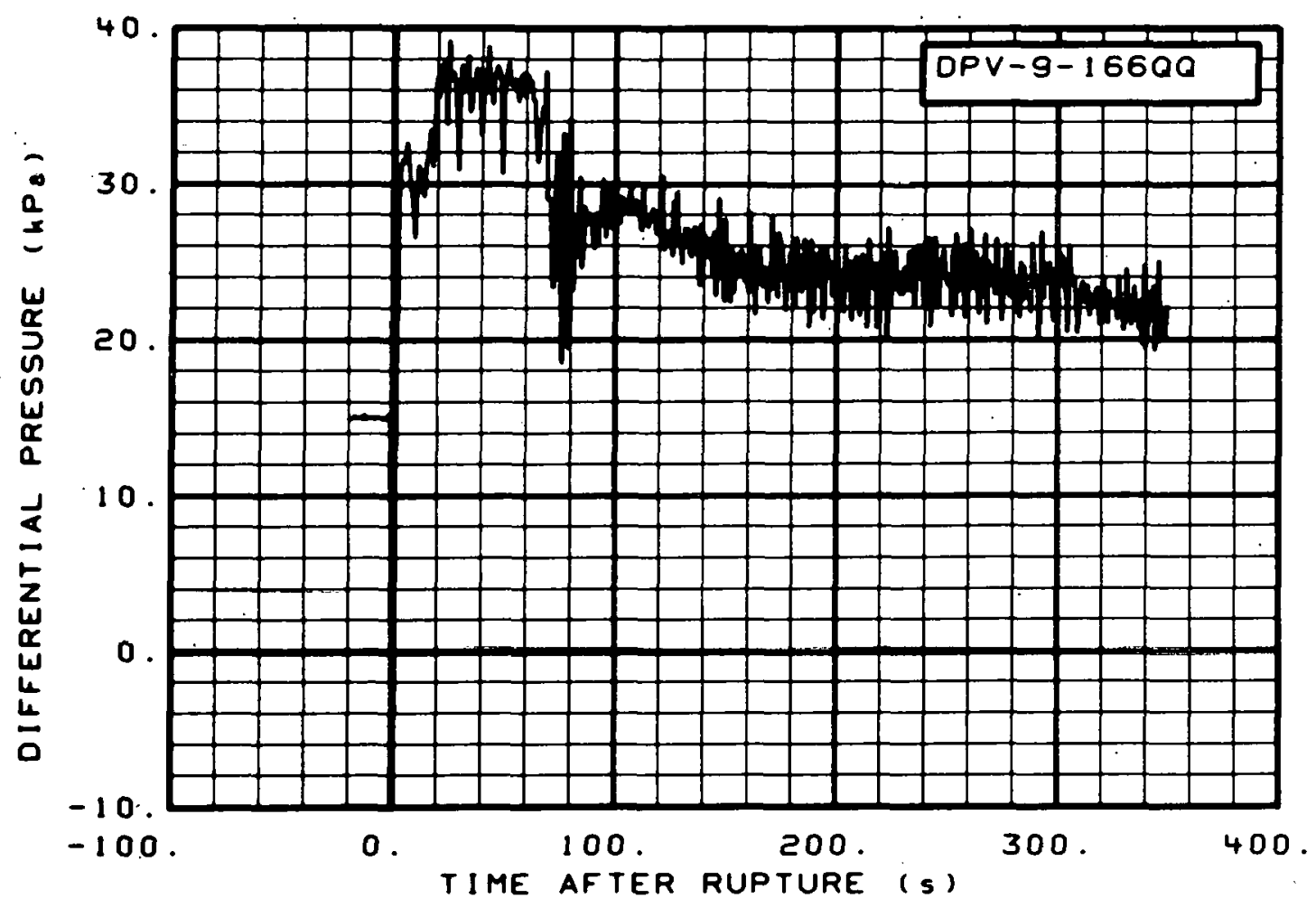

Fig. 215 Differential pressure in vessel (DPV-9-166QQ), from -20 to $350 \mathrm{~s}$.

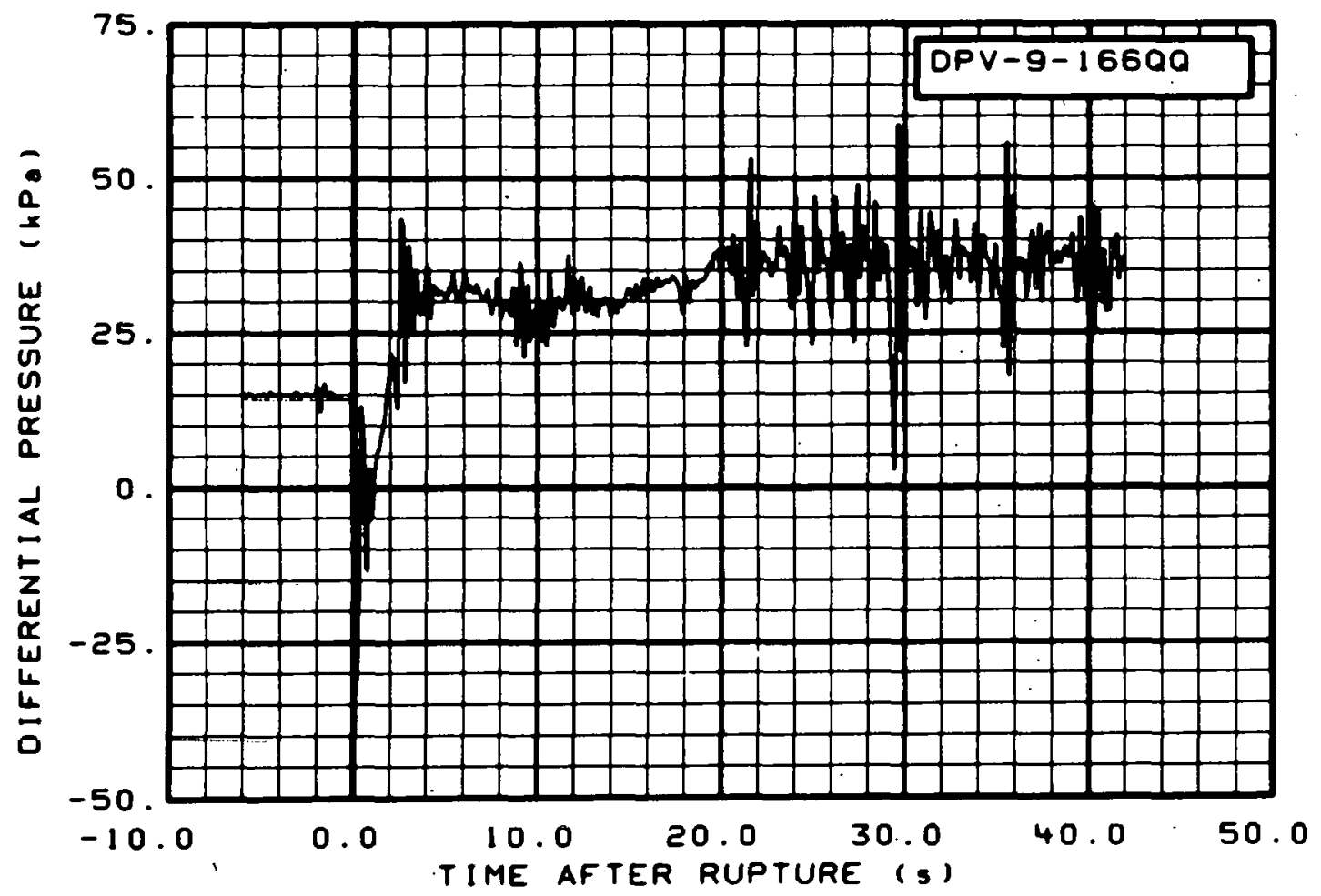

Fig. 216 Differential pressure in vessel (DPV-9-166QQ), from -6 to $42 \mathrm{~s}$. 


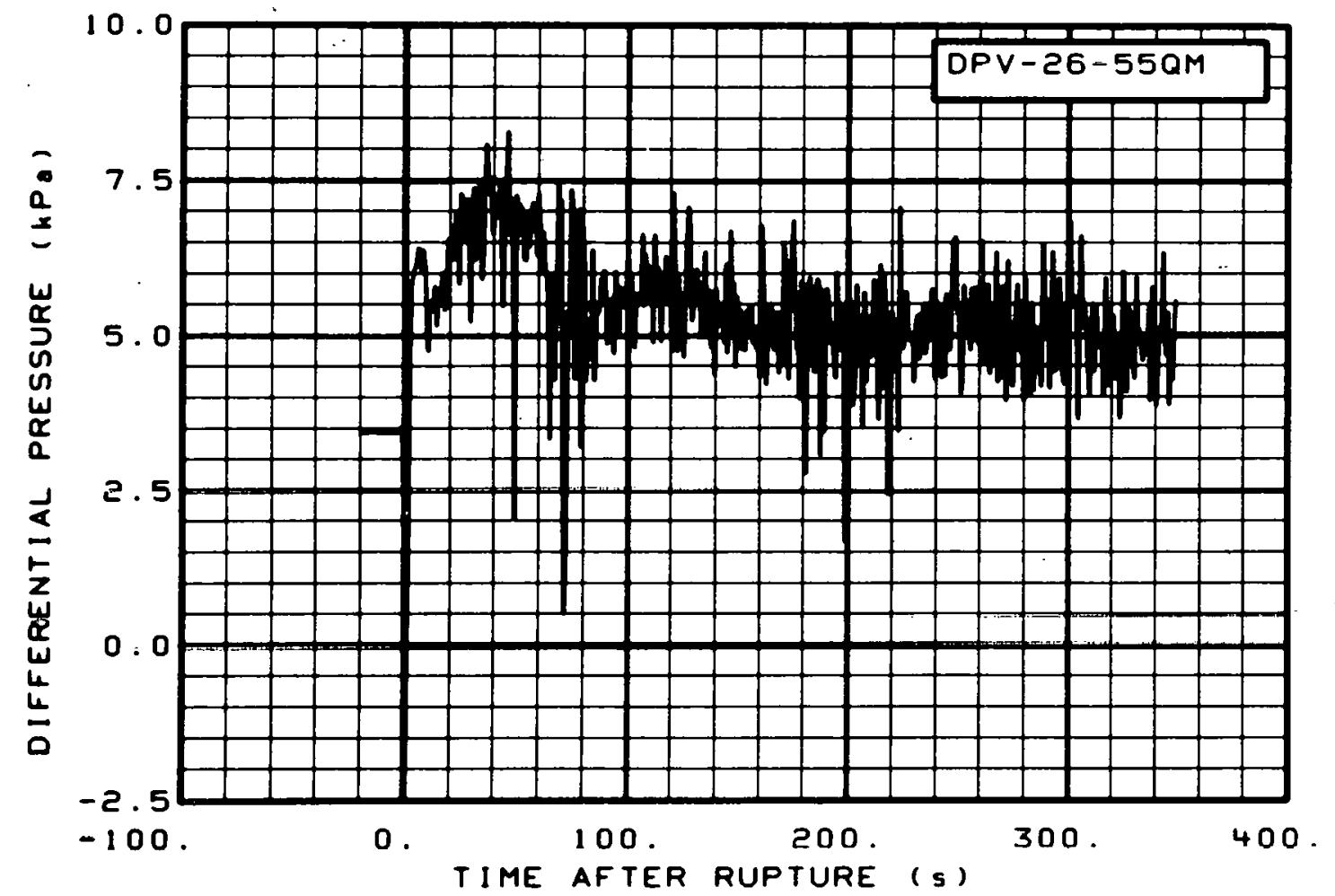

Fig. 217 Differential pressure in vessel (DPV-26-550M), from -20 to $35 \overline{0} \mathrm{~s}$.

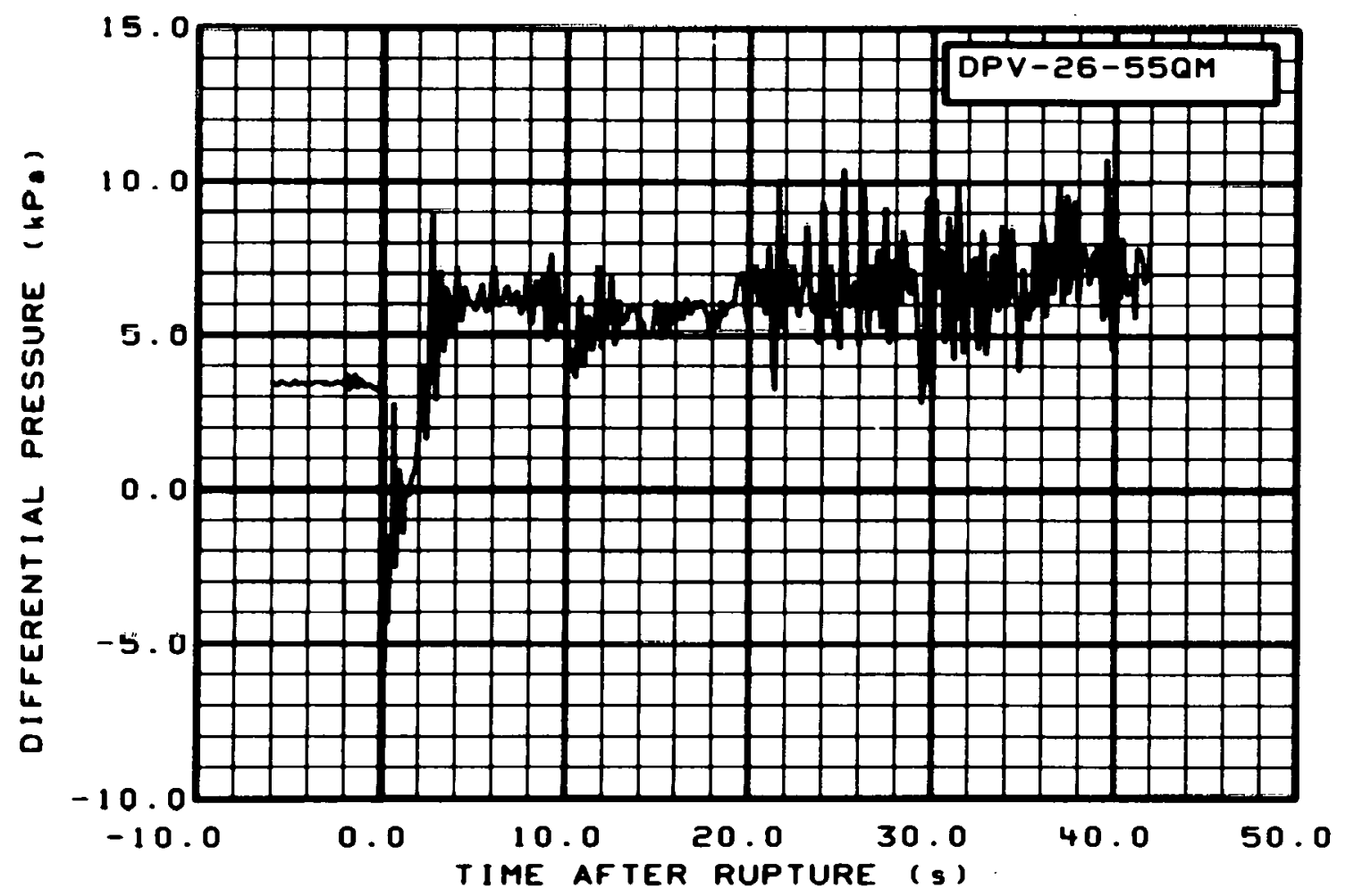

Fig. 218 Differential pressure in vessel (DPV-26-55QM), from -6 to $42 \mathrm{~s}$. 


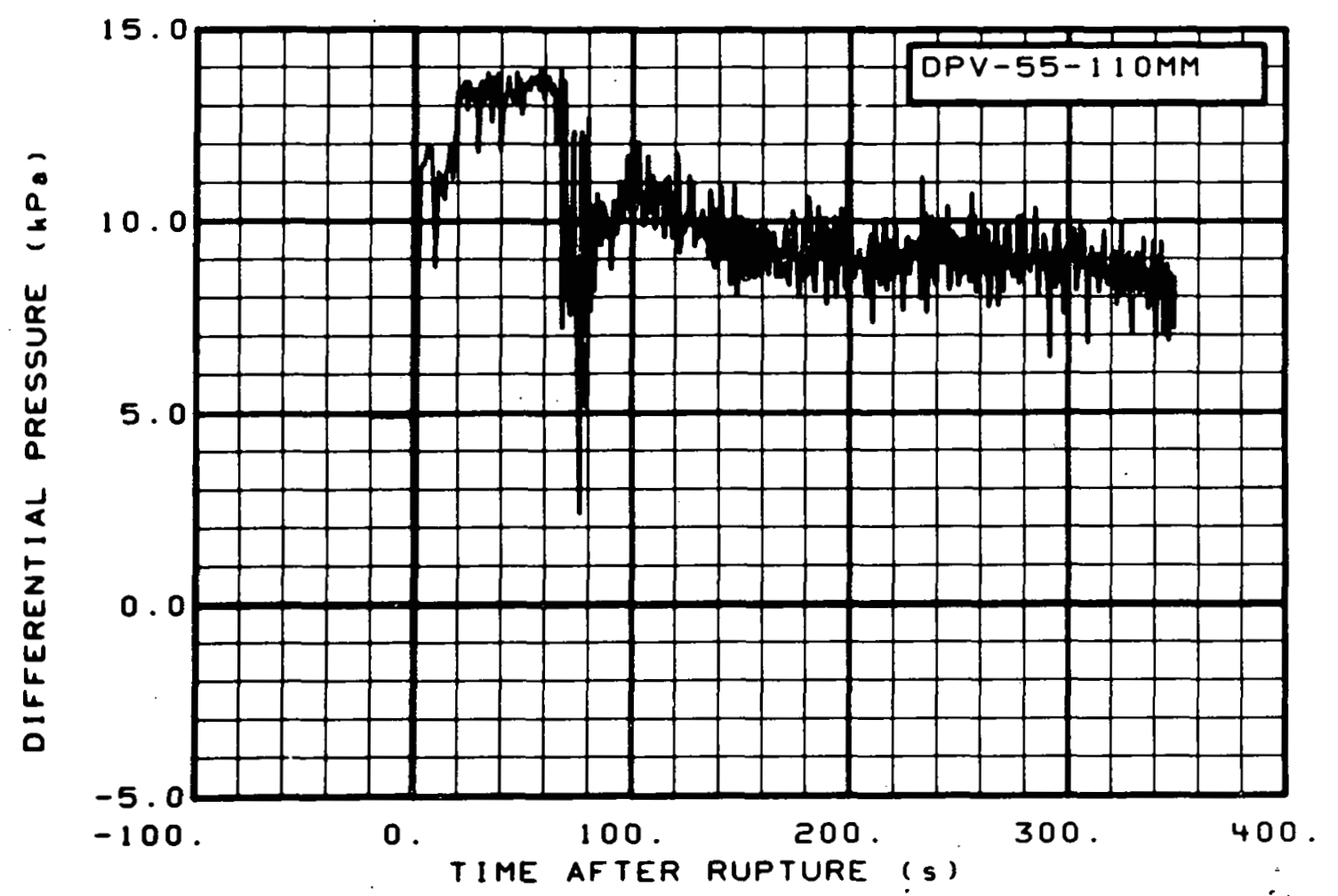

Fig. 219 Differential pressure in vessel (DPV-55-110MM), from -20 to $350 \mathrm{~s}$.

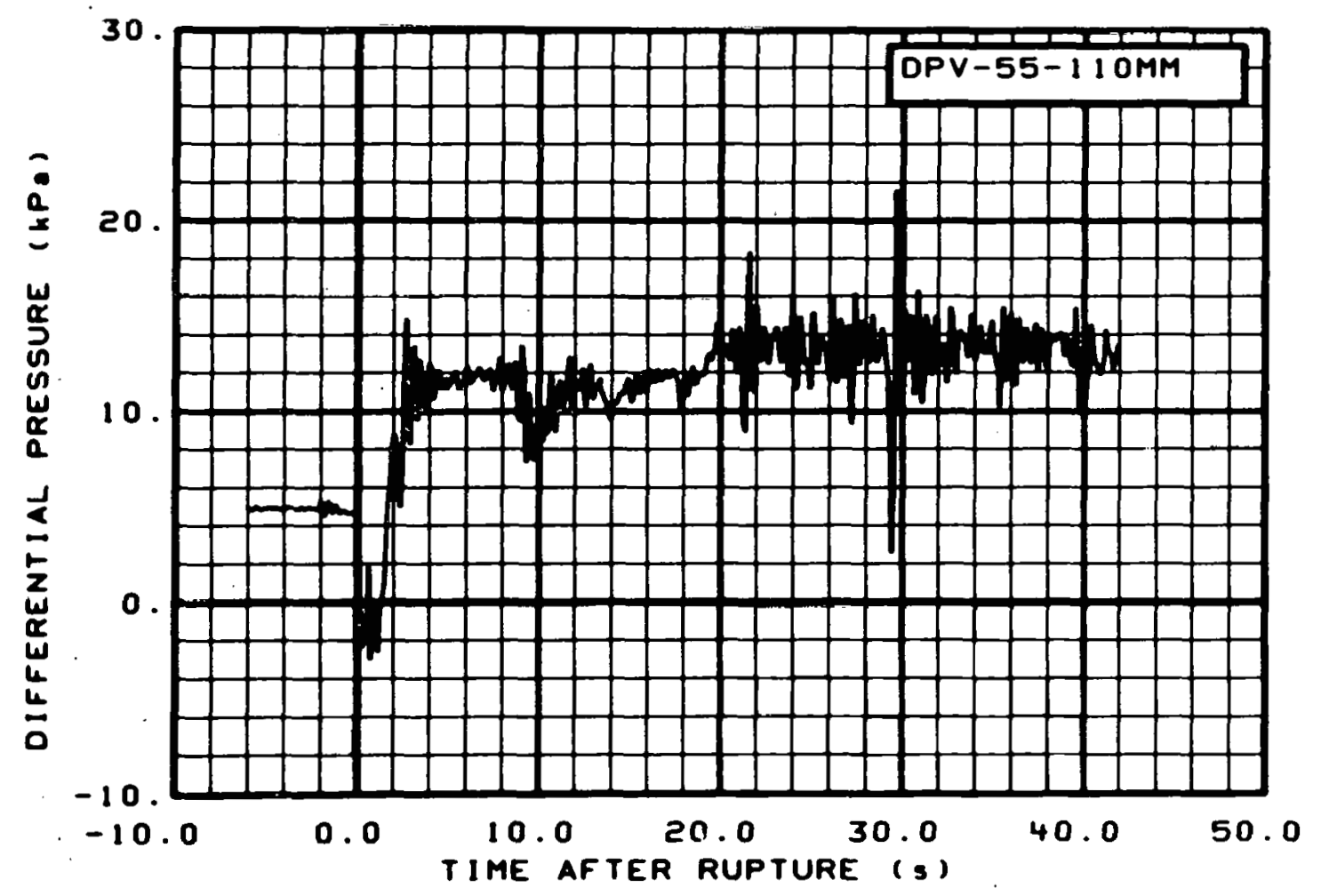

Fig. 220 Differential pressure in vessel (DPV-55-110MM), from -6 to $42 \mathrm{~s}$. 


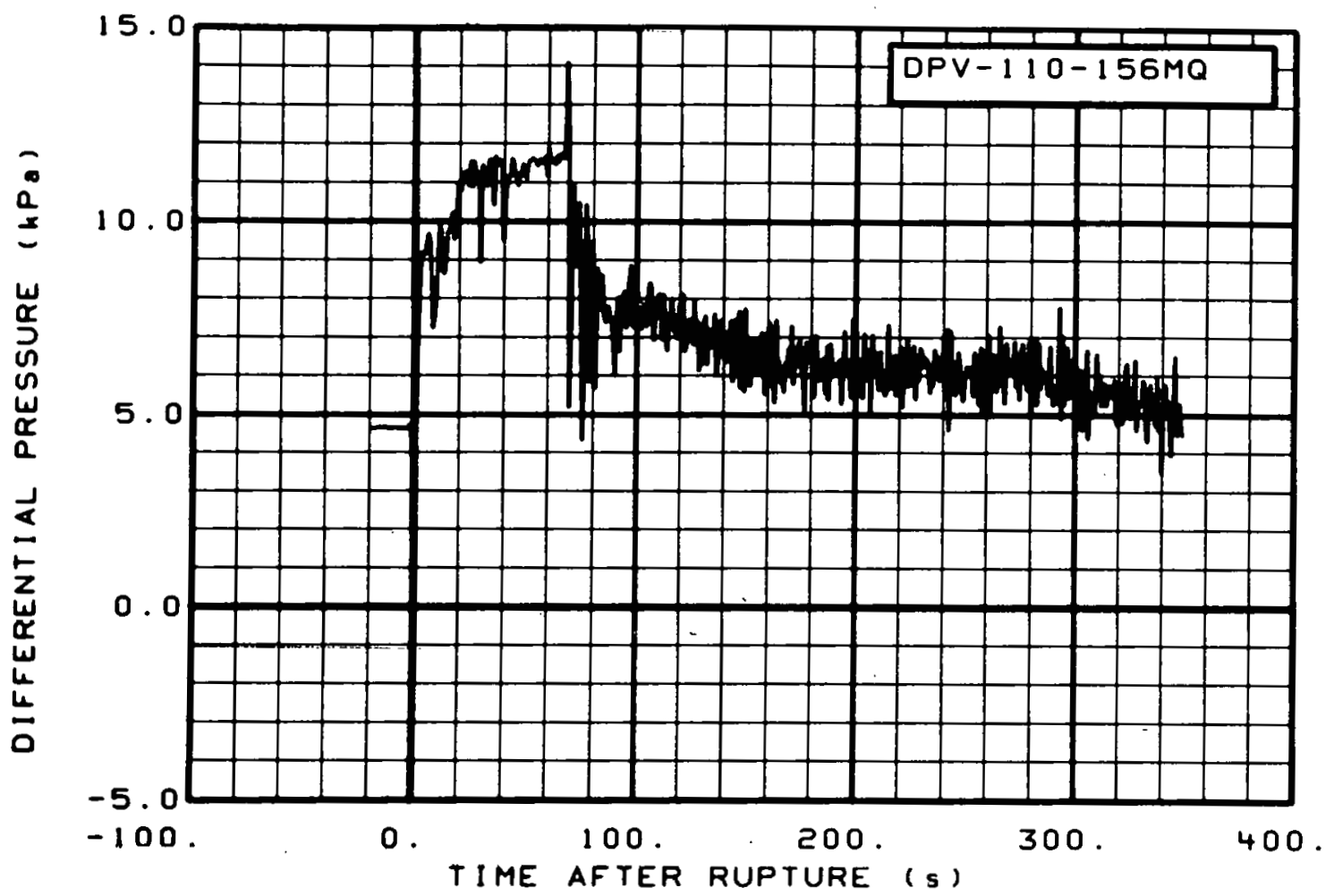

Fig. 221 Differential pressure in vessel (DPV-110-156MQ), from -20 to $350 \mathrm{~s}$.

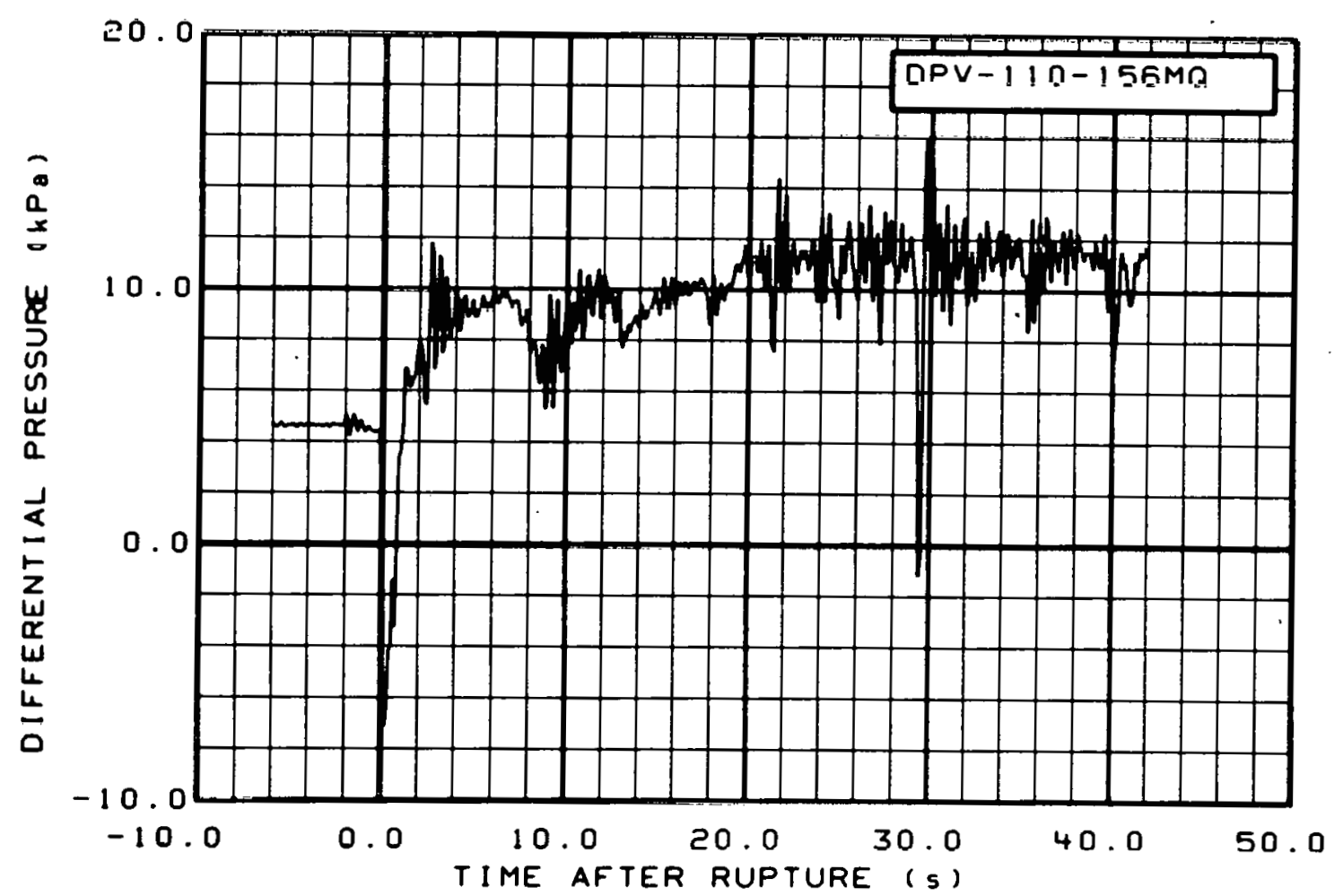

Fig. 222 Differential pressure in vessel (DPV-110-156MQ), from -6 to $42 \mathrm{~s}$. 


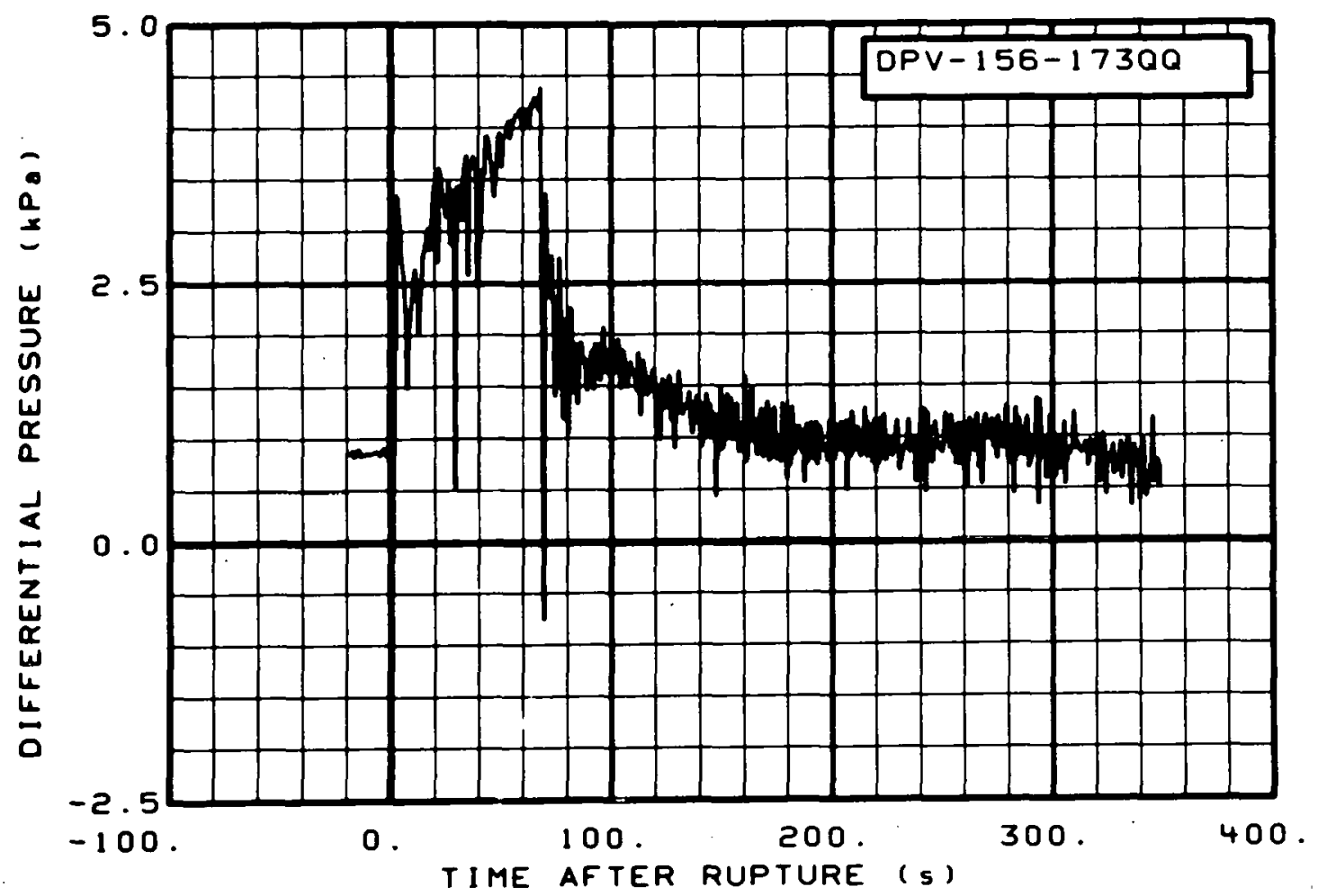

Fig. 223 Differential pressure in vessel (DPV-156-173QQ), from -20 to $350 \mathrm{~s}$.

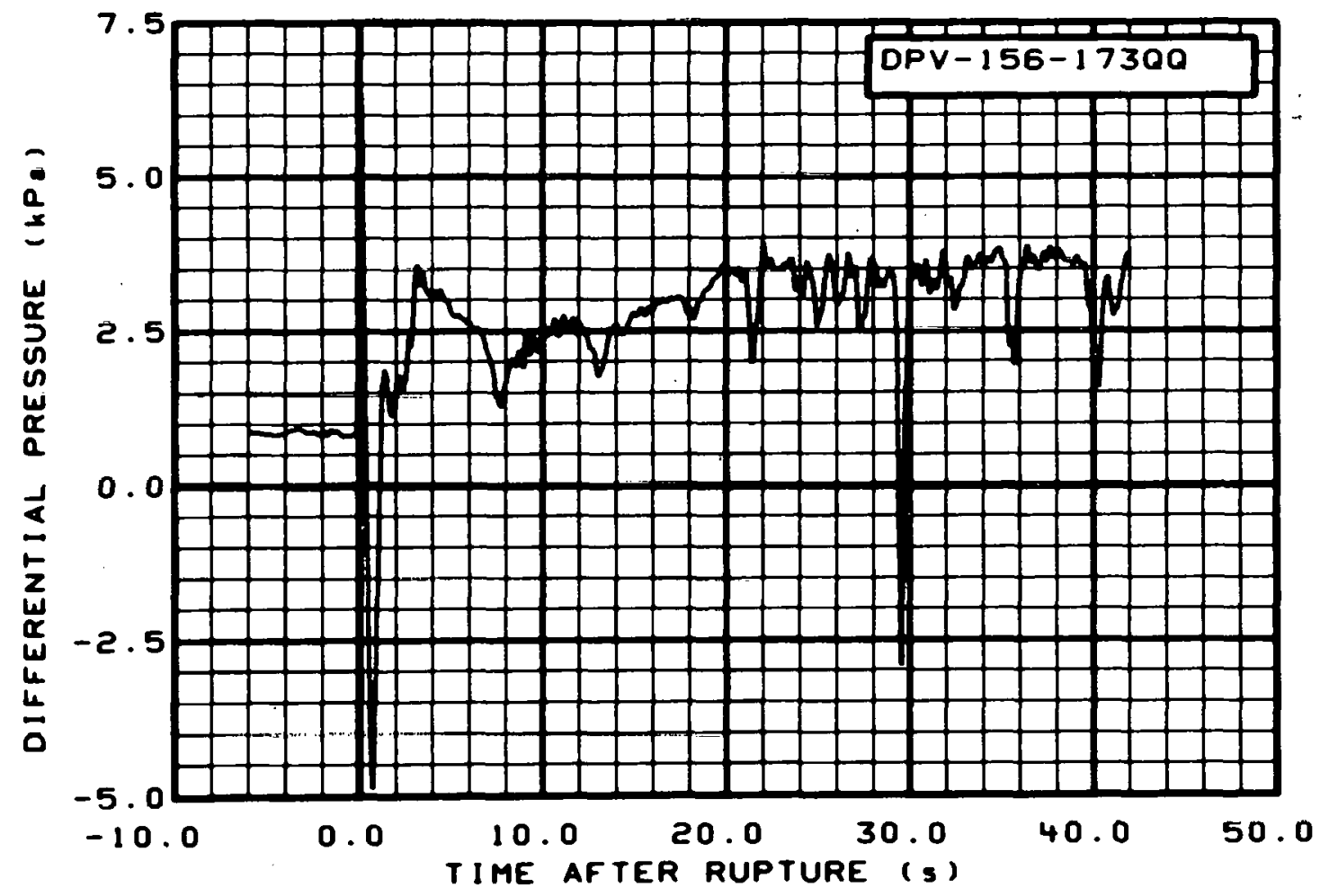

Fig. 224 Differential pressure in vessel (DPV-156-173QQ), from -6 to $42 \mathrm{~s}$. 


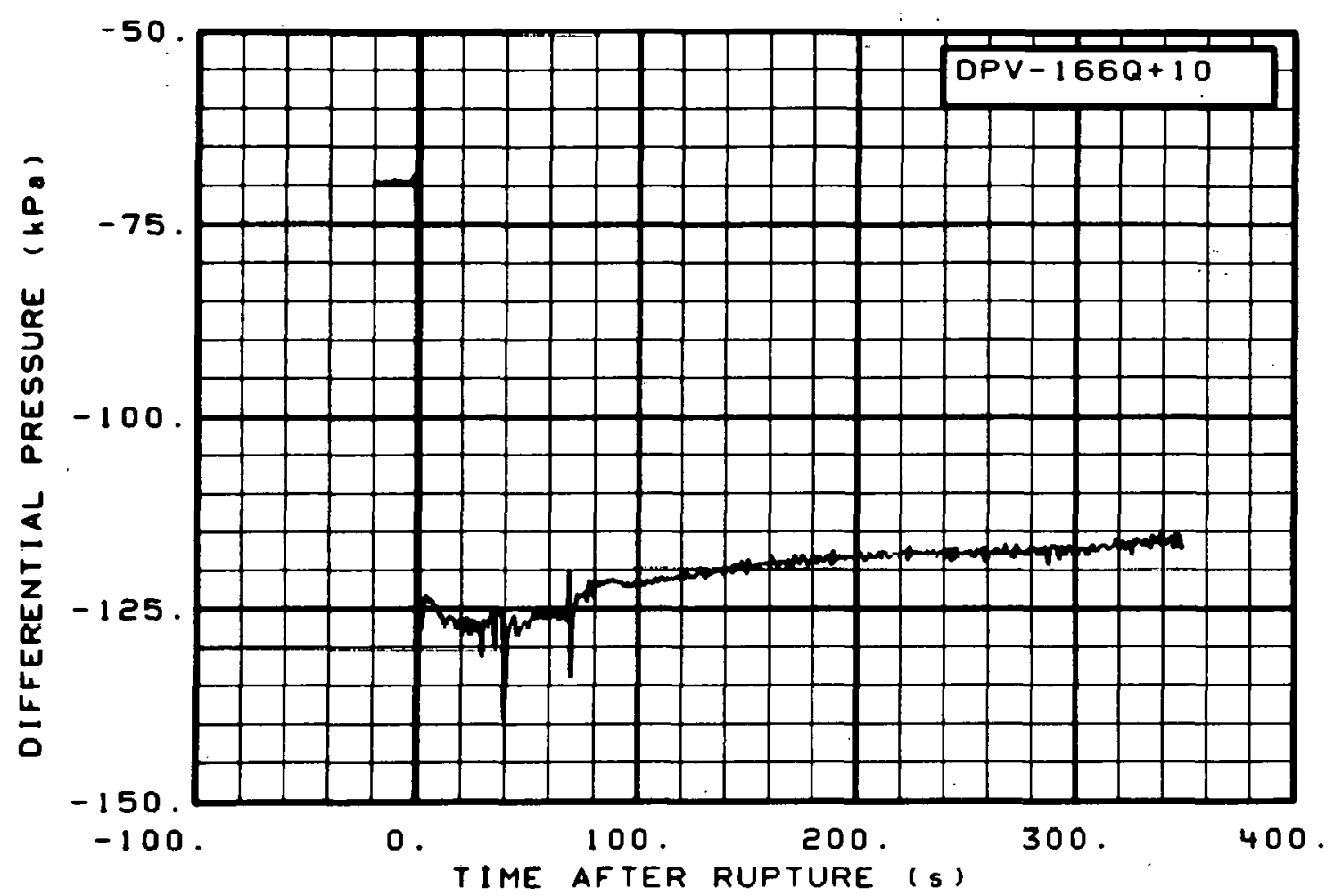

Fig. 225 Differential pressure in vessel (DPV-1660+10), from -20 to $350 \mathrm{~s}$.

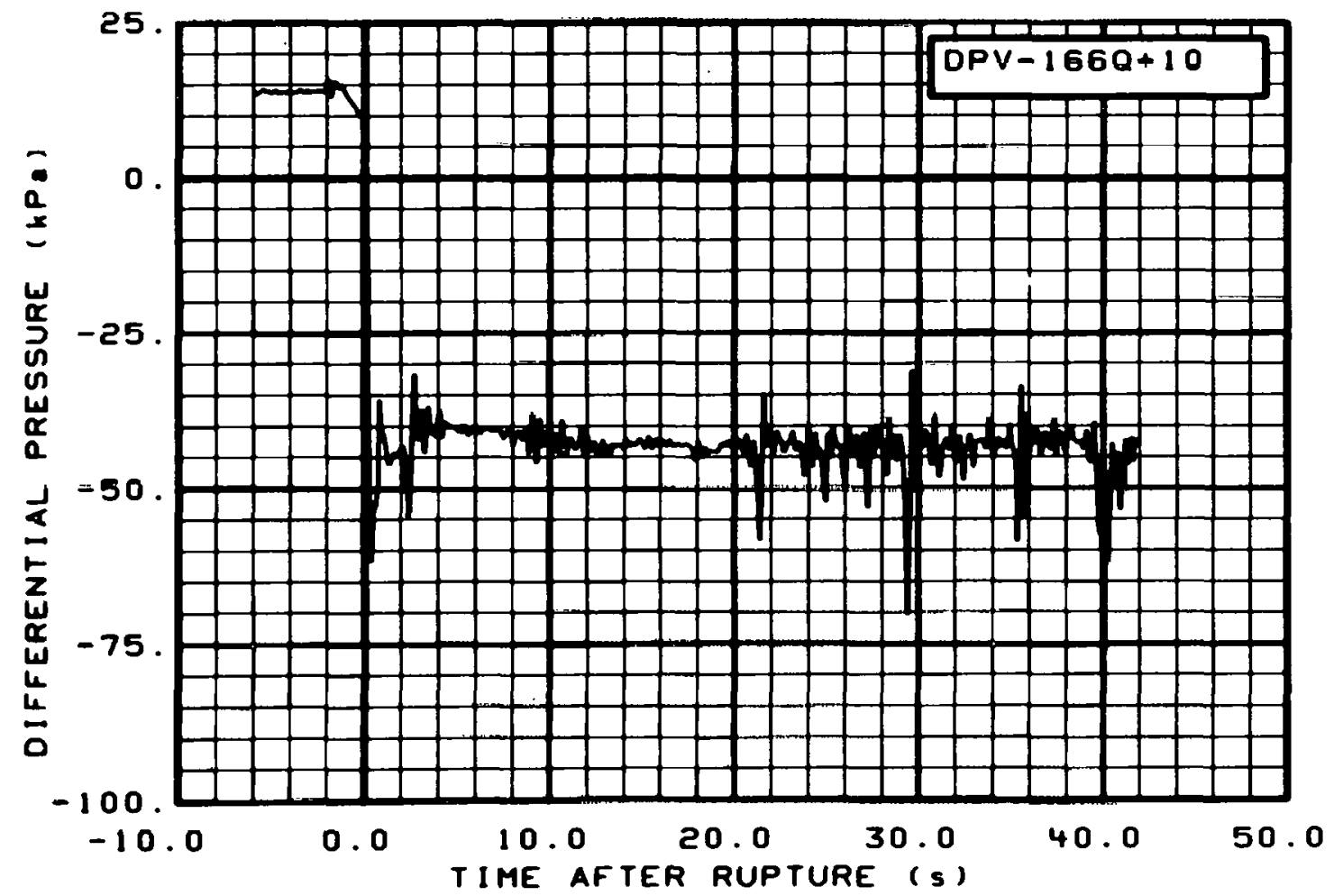

Fig. 226 Differential pressure in vessel (DPV-166Q+10), from -6 to $42 \mathrm{~s}$. 


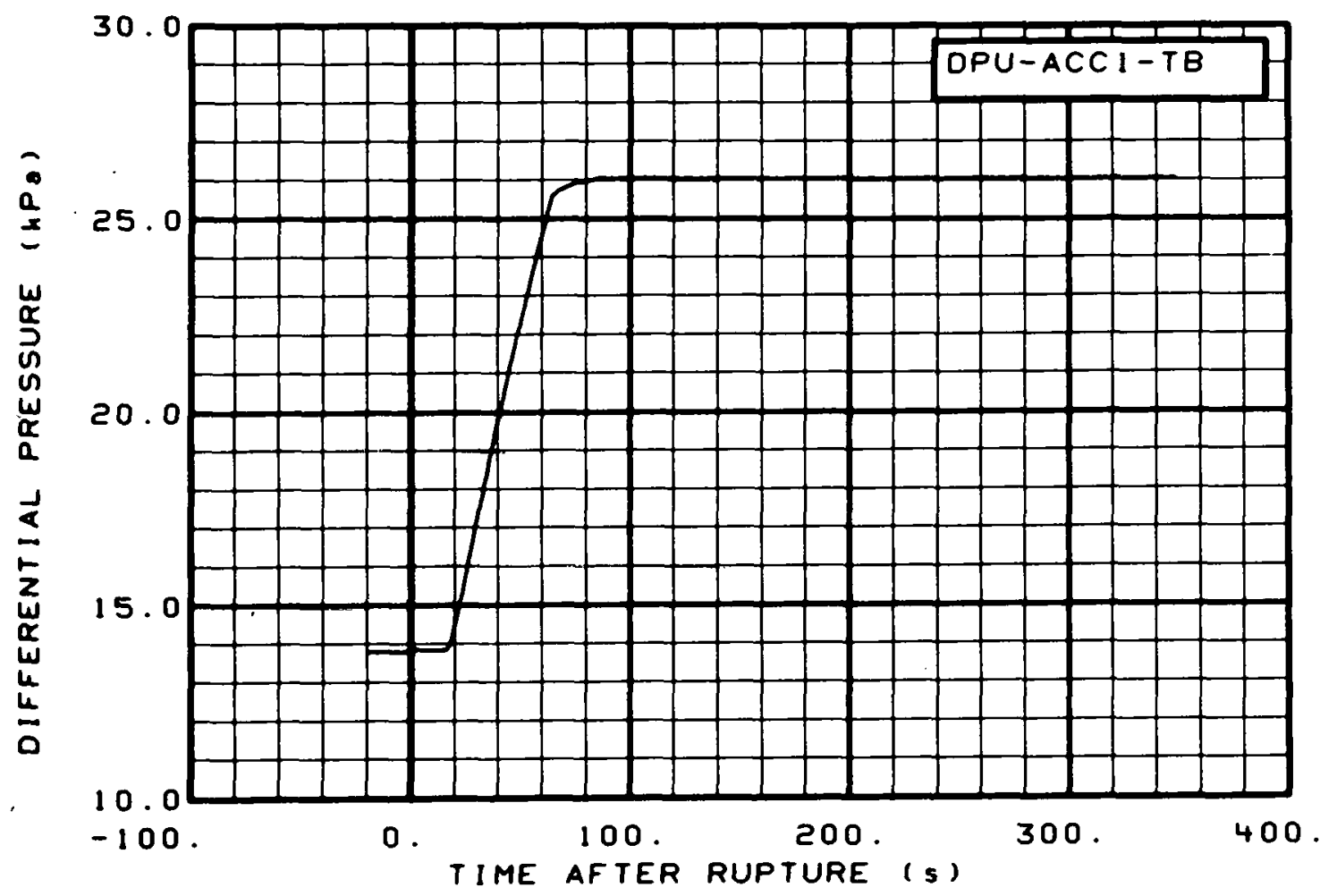

Fig. 227 Differential pressure in intact loop accumulator (DPU-ACC1-TB), from -20 to $350 \mathrm{~s}$.

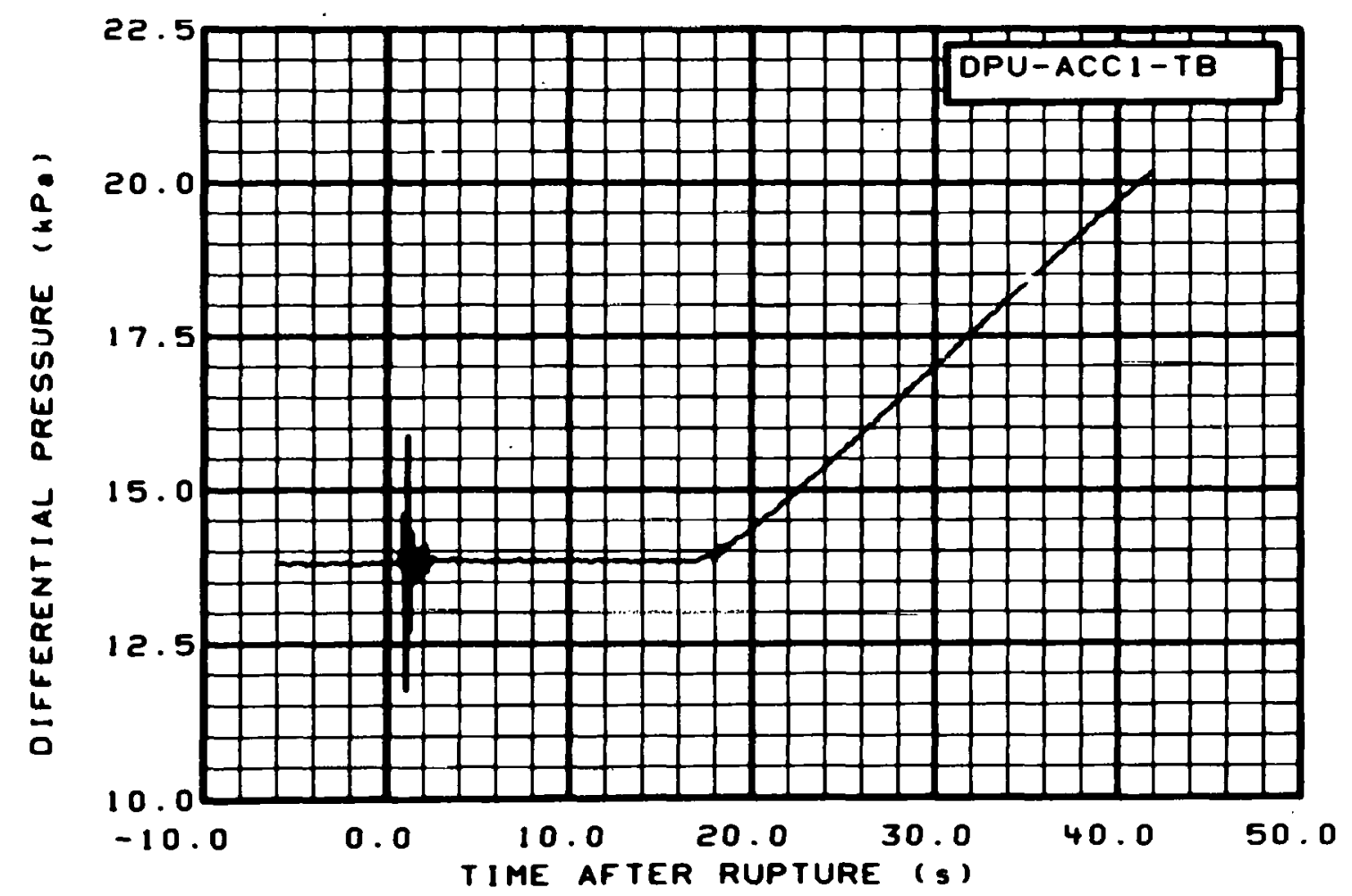

Fig. 228 Differential pressure in intact loop accumulator (DPU-ACCI-TB), from -6 to $42 \mathrm{~s}$. 


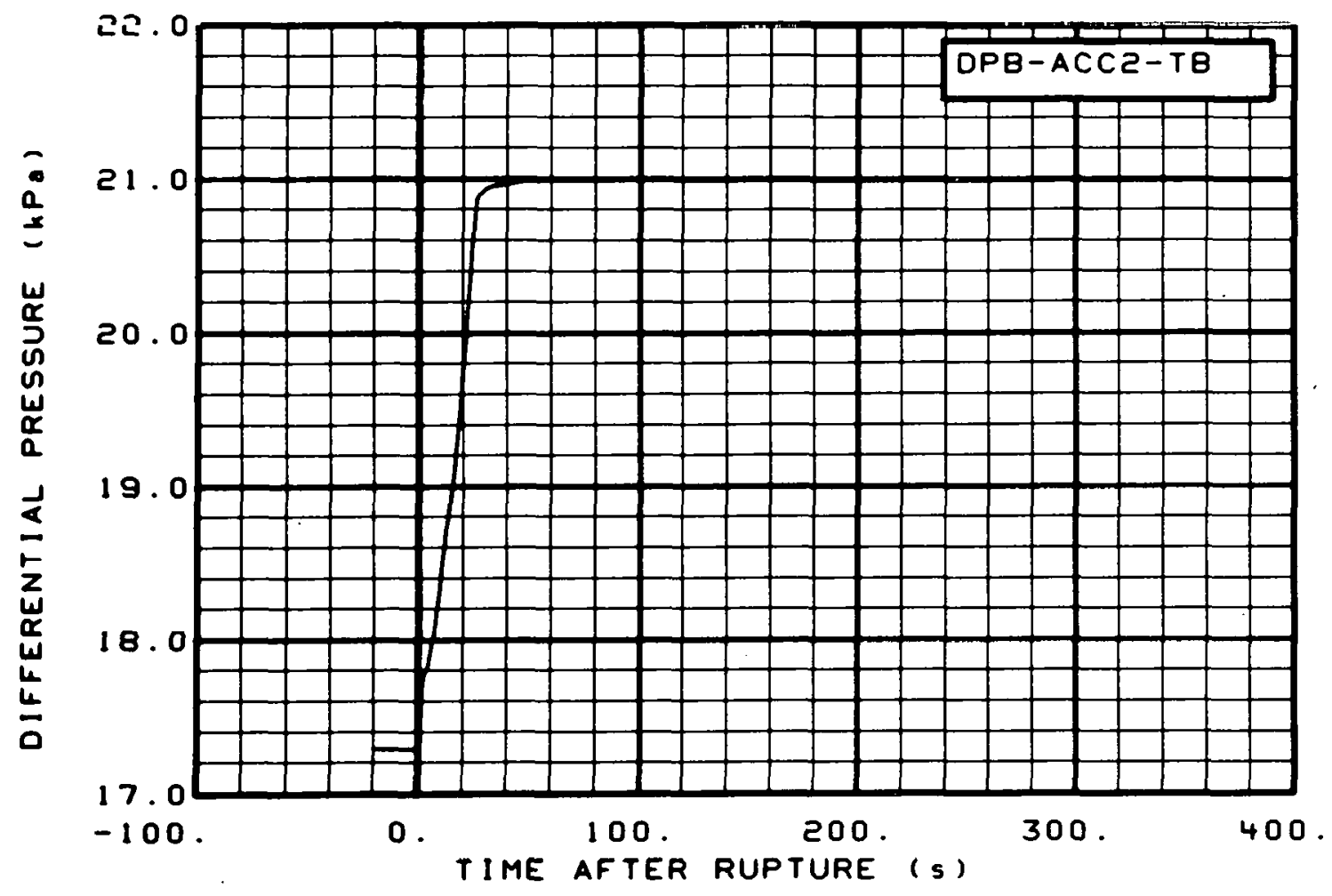

Fig. 229 Differential pressure in broken loop accumulator (DPB-ACC2-TB), from -20 to $350 \mathrm{~s}$.

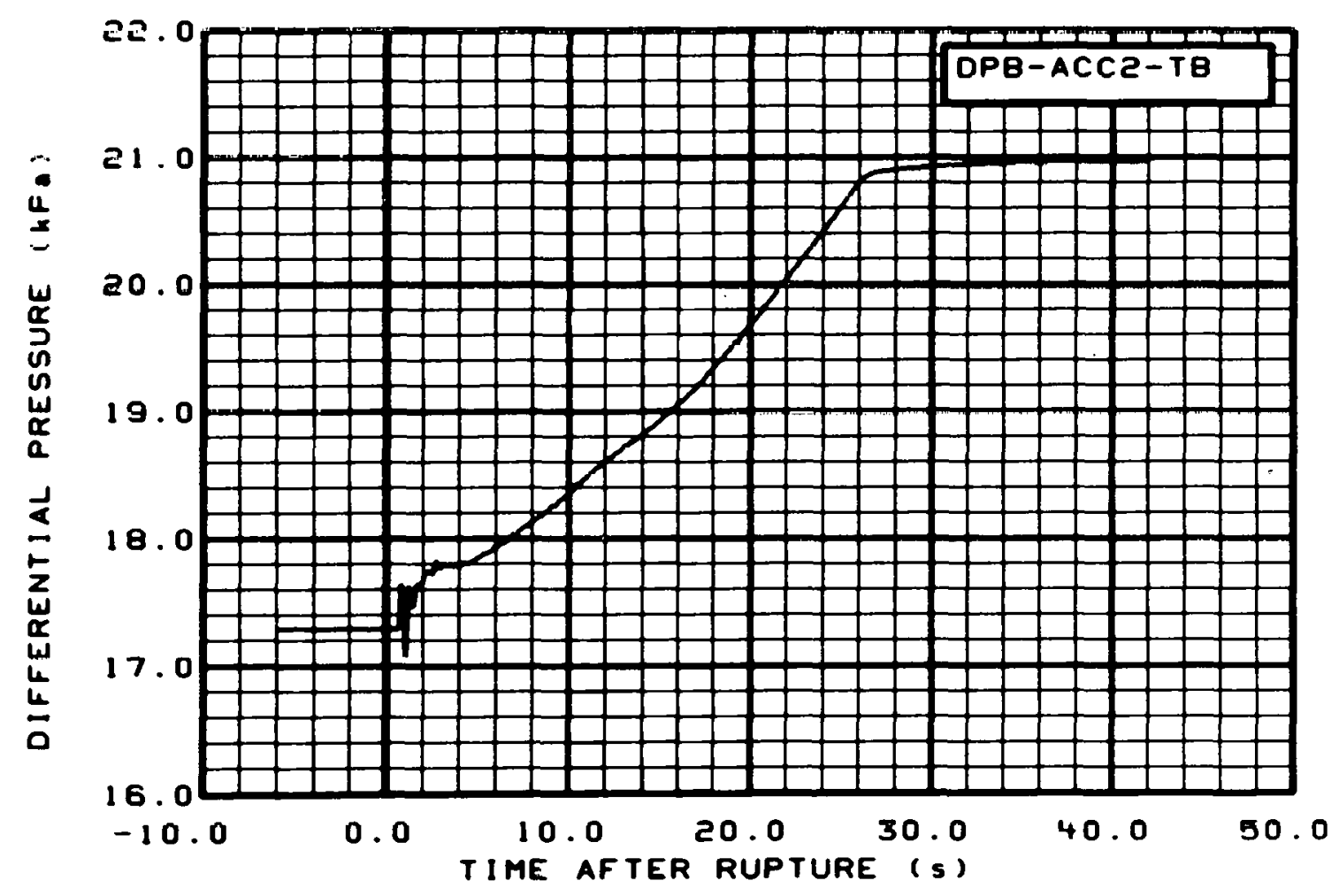

Fig. 230 Differential pressure in broken loop accumulator (DPB-ACC2-TB), from -6 to $42 \mathrm{~s}$. 


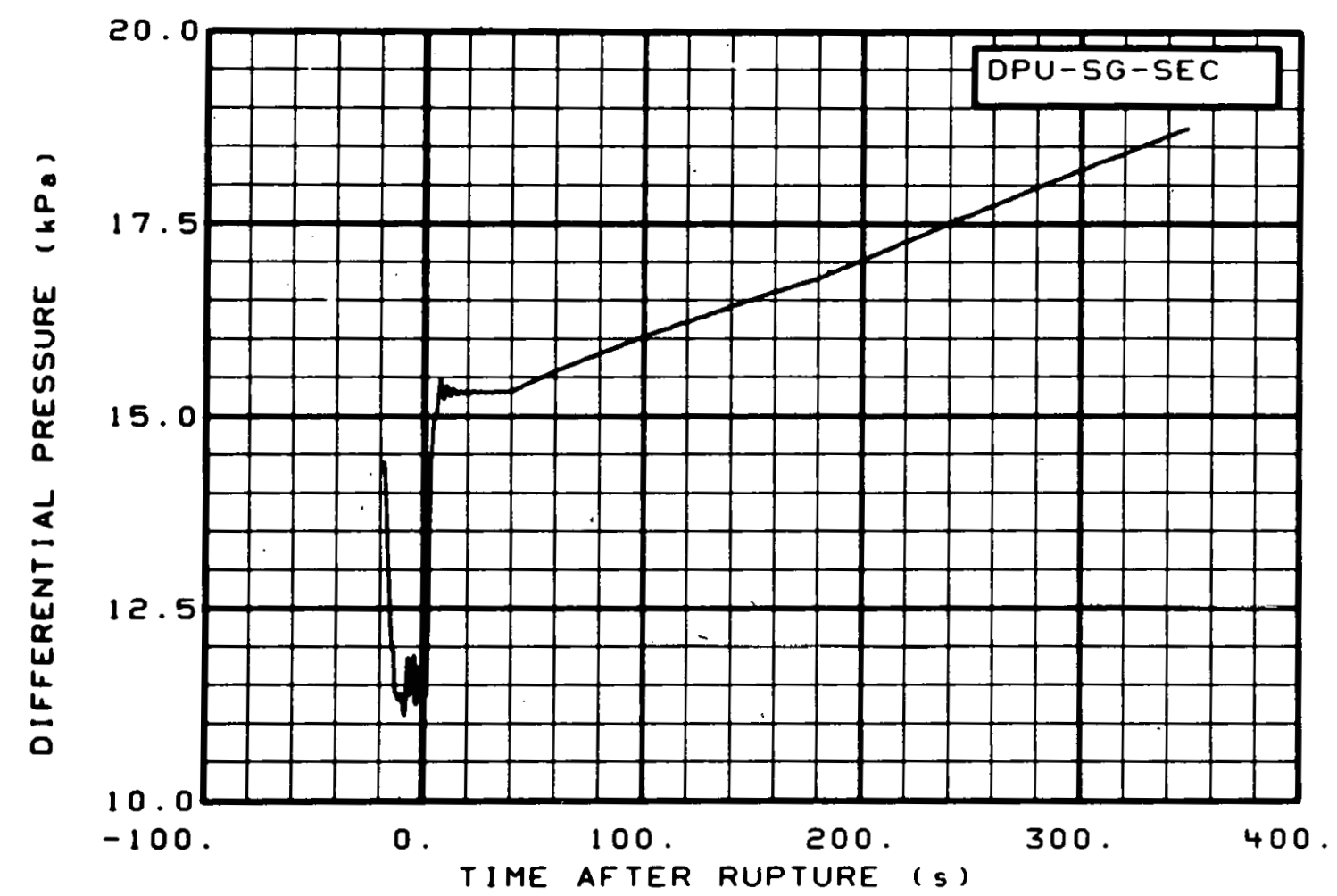

Fig. 231 Differential pressure in steam generator secondary (DPU-SG-SEC), from -20 to $350 \mathrm{~s}$.

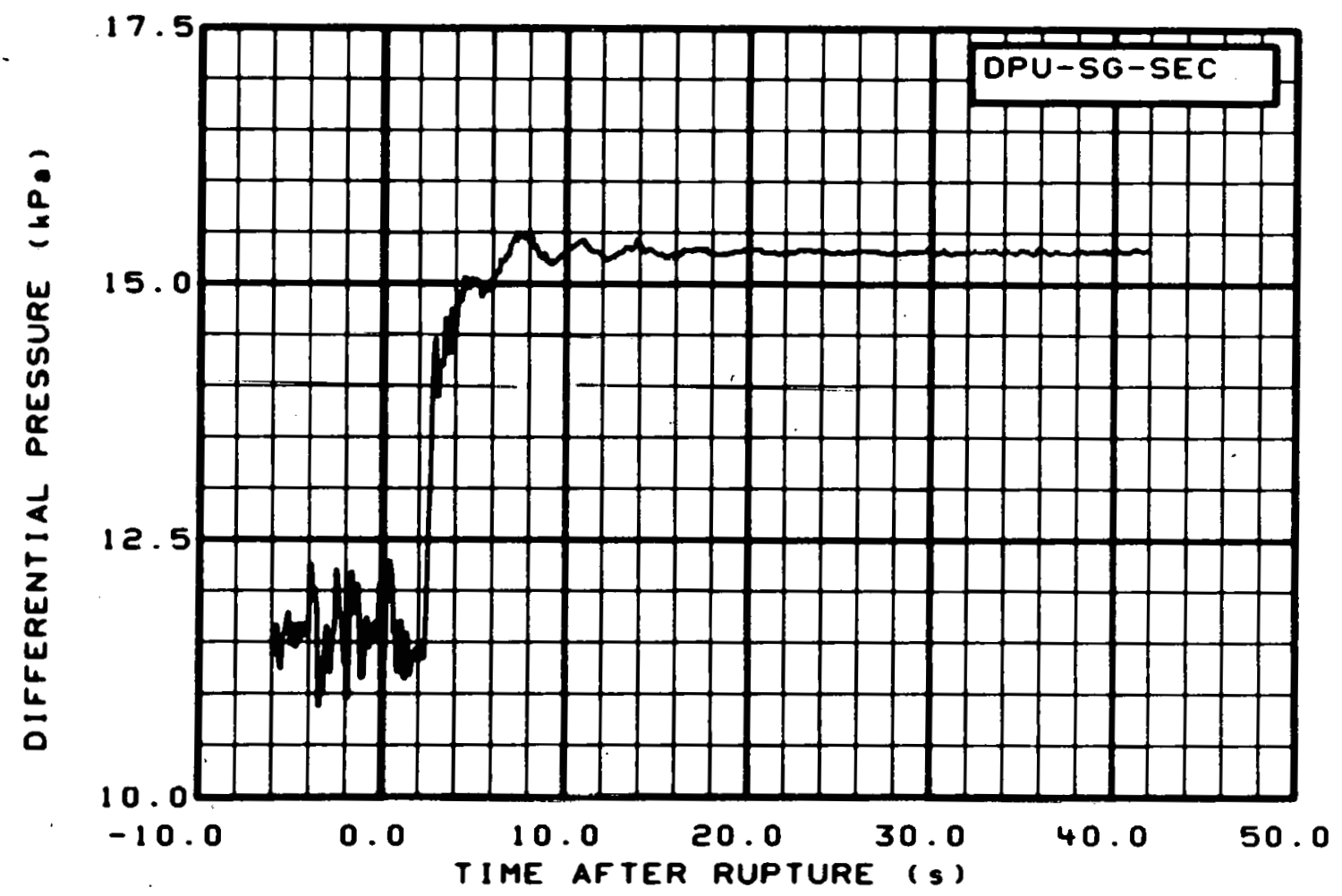

Fig. 232 Differential pressure in steam generator secondary (DPU-SG-SEC), from -6 to $42 \mathrm{~s}$. 


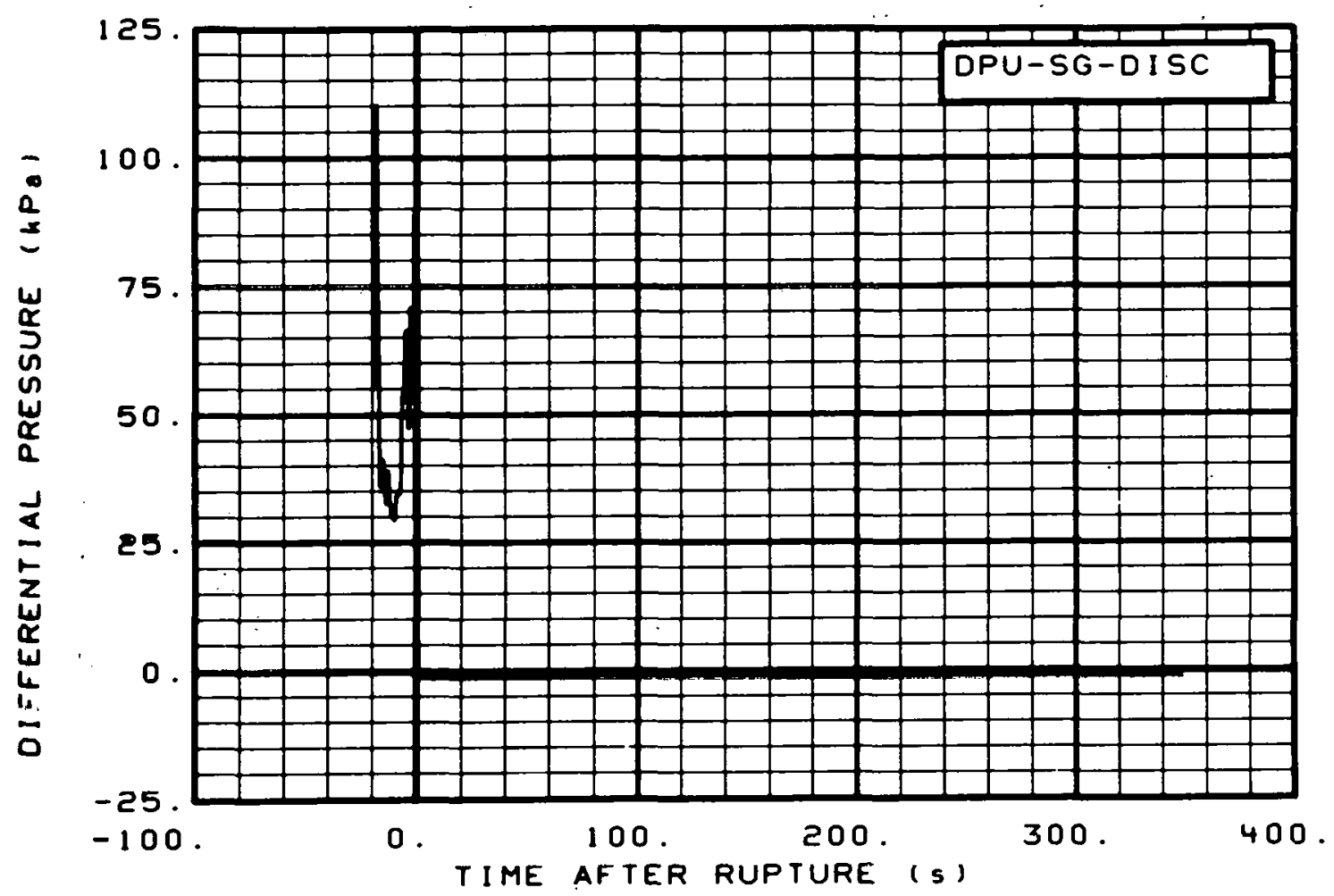

Fig. 233 Differential pressure across steam generator outlet orifice (DPU-SG-DISC), from -20 to $350 \mathrm{~s}$.

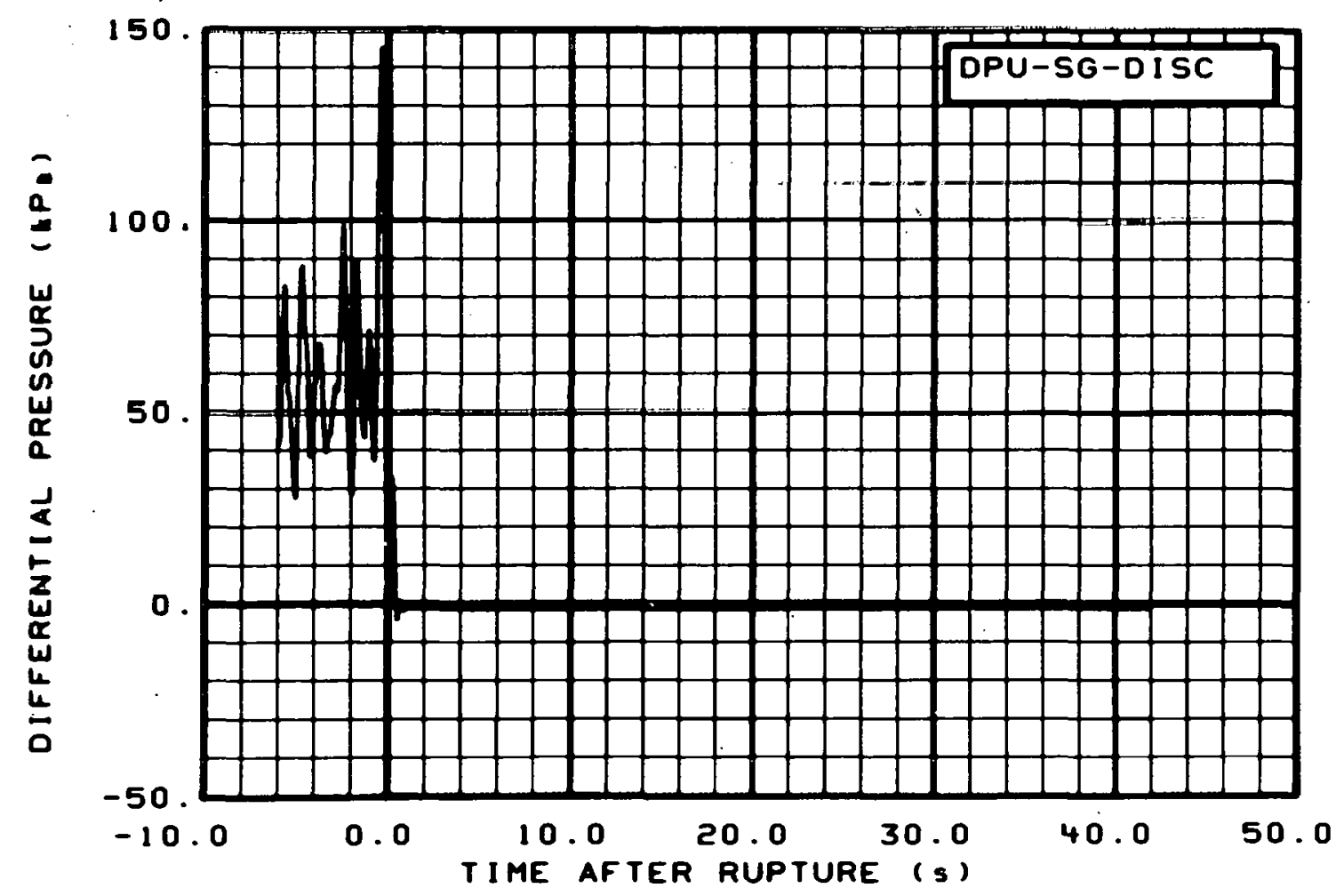

Fig. 234 Differential pressure across steam generator outlet orifice (DPU-SG-DISC), from -6 to $42 \mathrm{~s}$. 


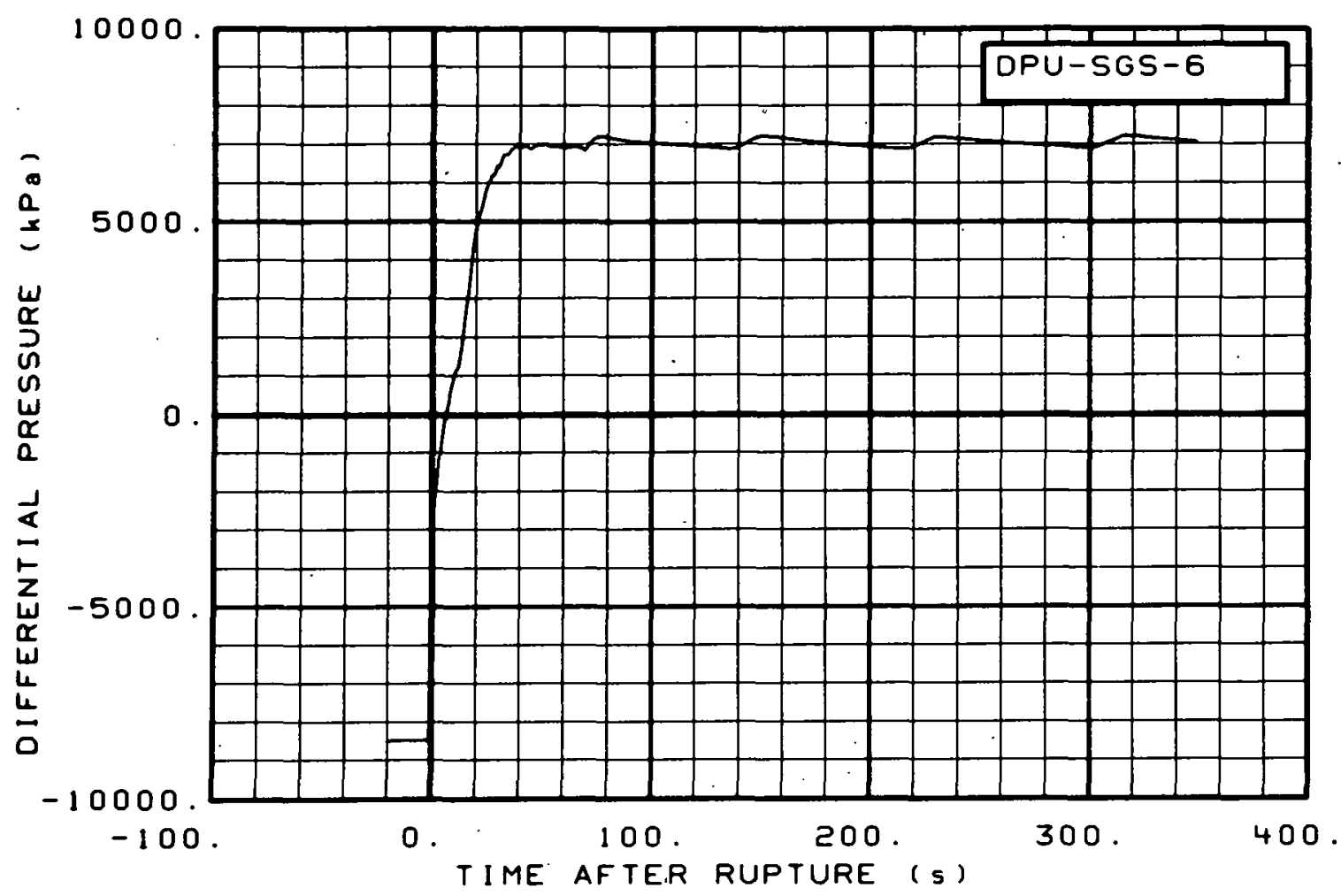

Fig. 235 Differential pressure between simulated rupture injection line and Spool 6 (DPU-SGS-6), from -20 to $350 \mathrm{~s}$.

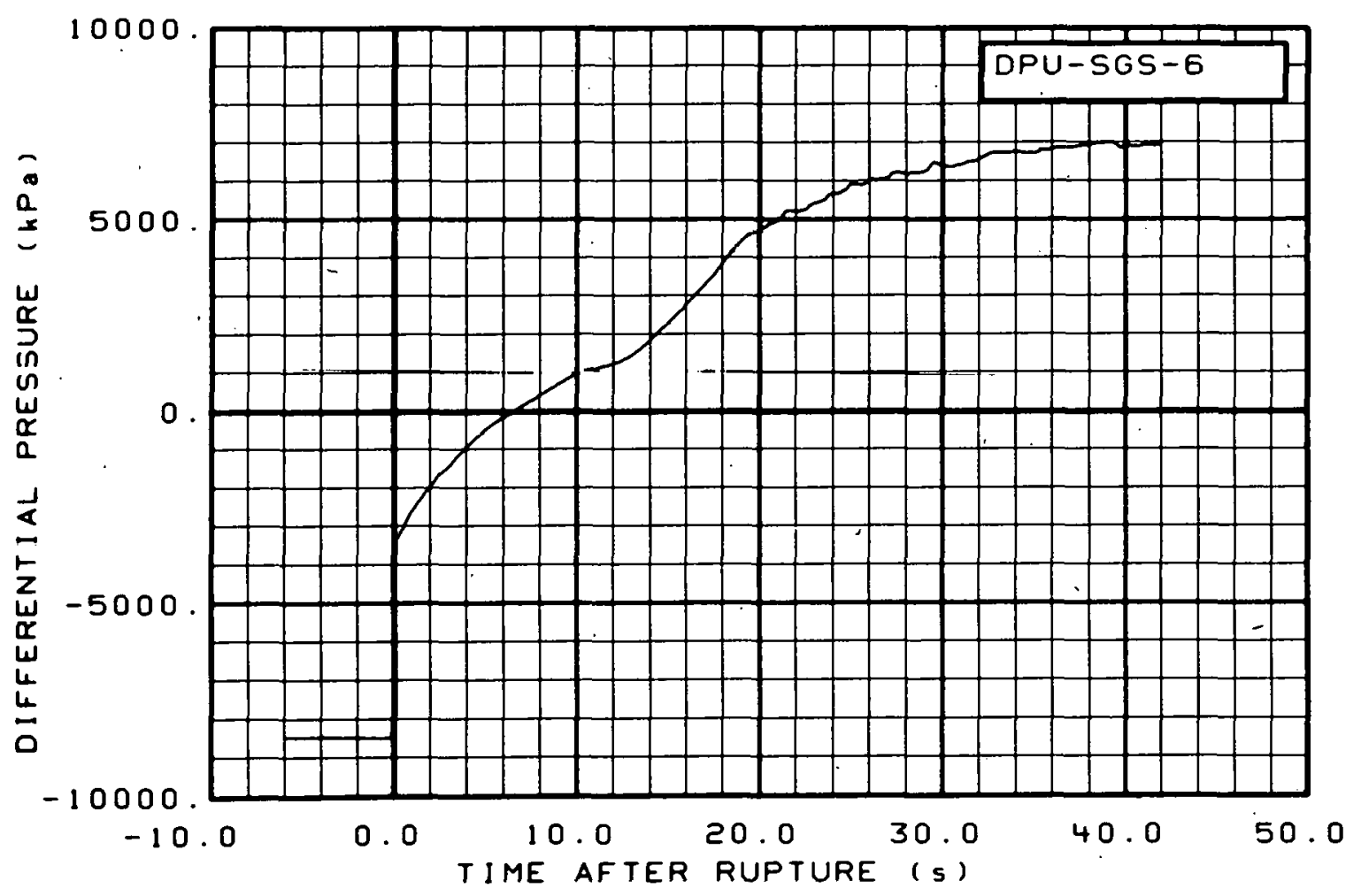

Fig. 236 Differential pressure hetween simulated rupture injection line and Spool 6 (DPU-SGS-6), from -6 to $42 \mathrm{~s}$. 


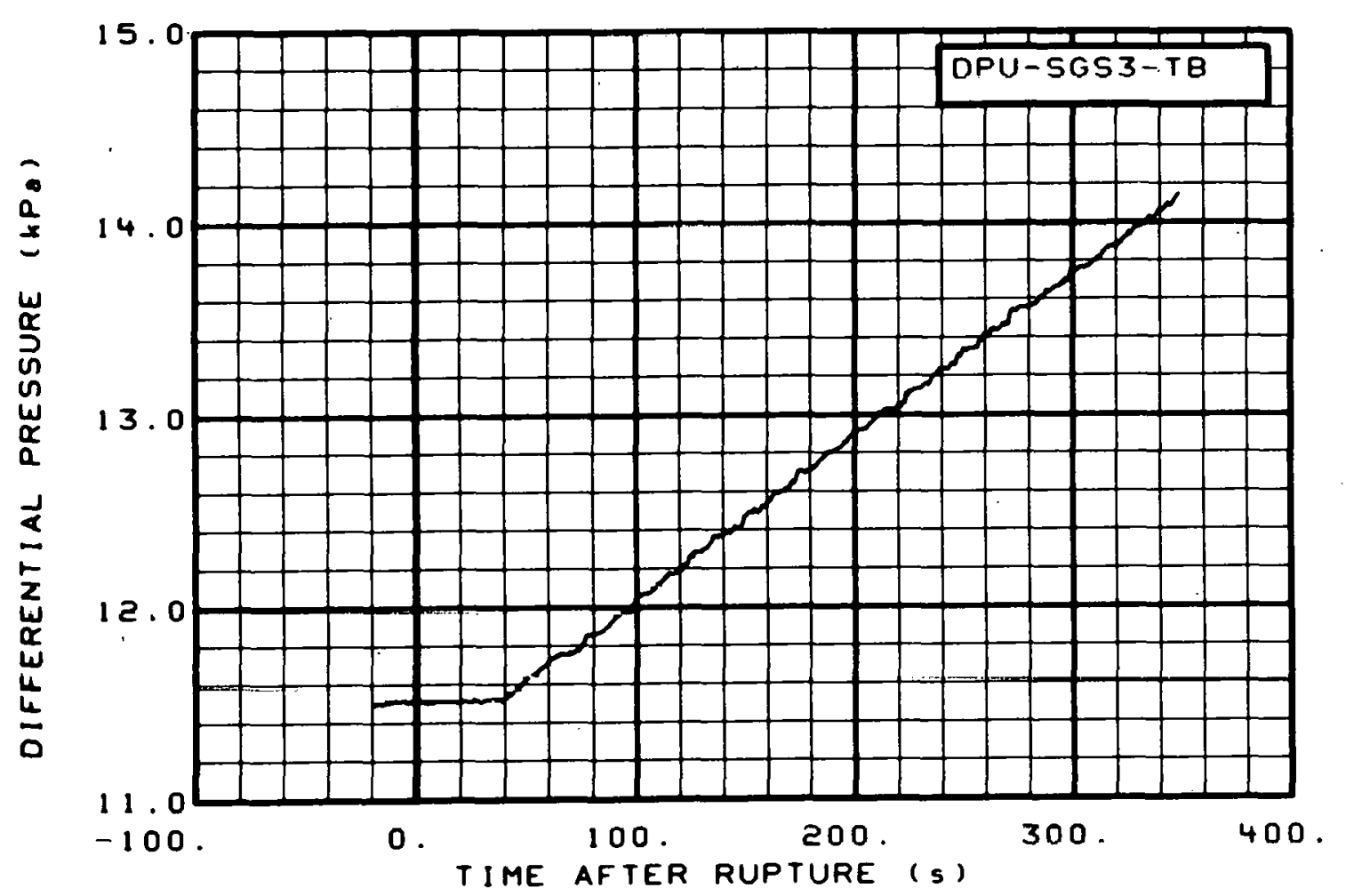

Fig. 237 Differential pressure in intact loop accumulator (DPU-SGS3-TB), from -20 to $350 \mathrm{~s}$.

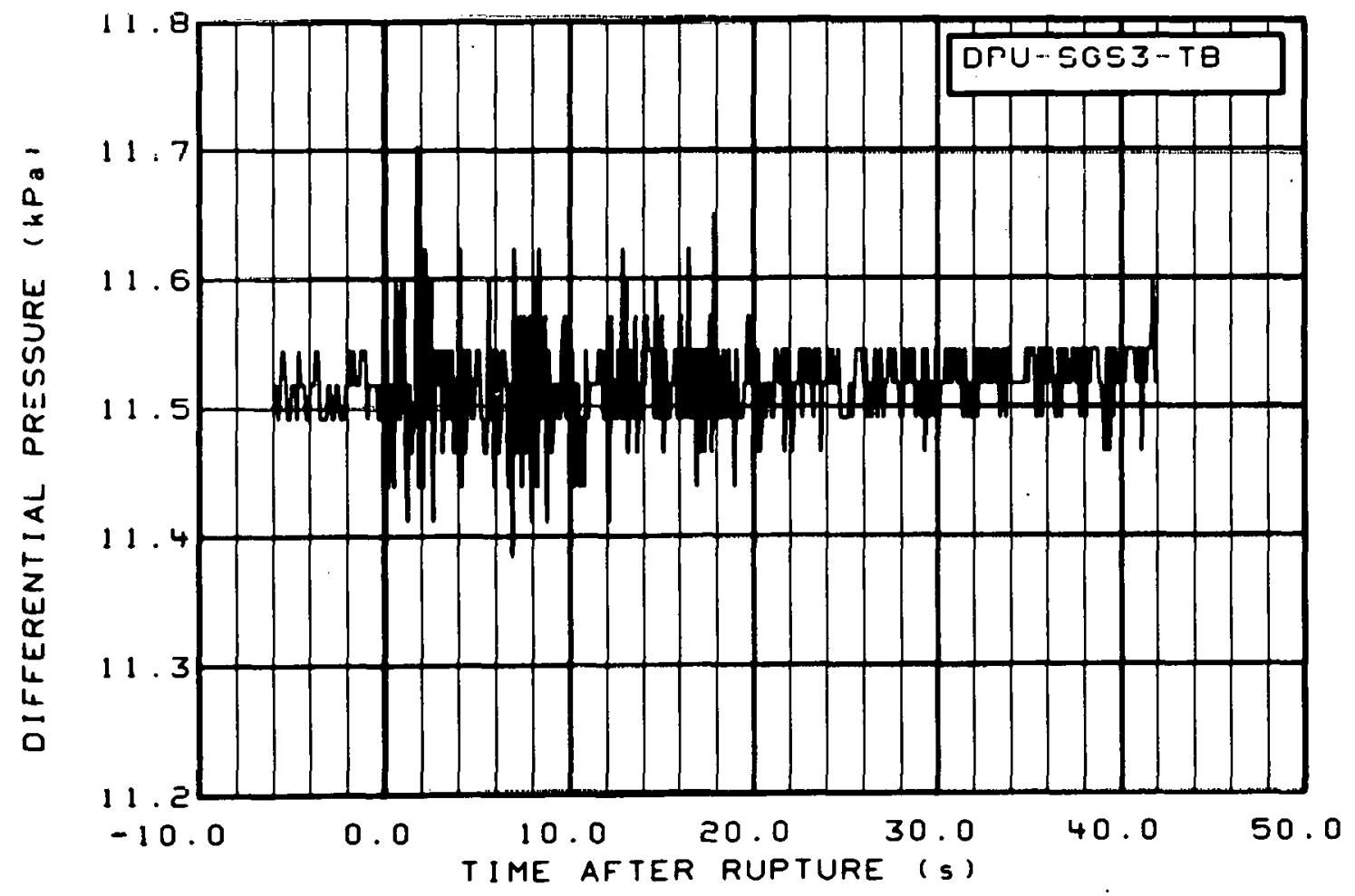

Fig. 238 Differential pressure in intact loop accumulator (DPU-SGS3-TB), from -6 to $42 \mathrm{~s}$. 


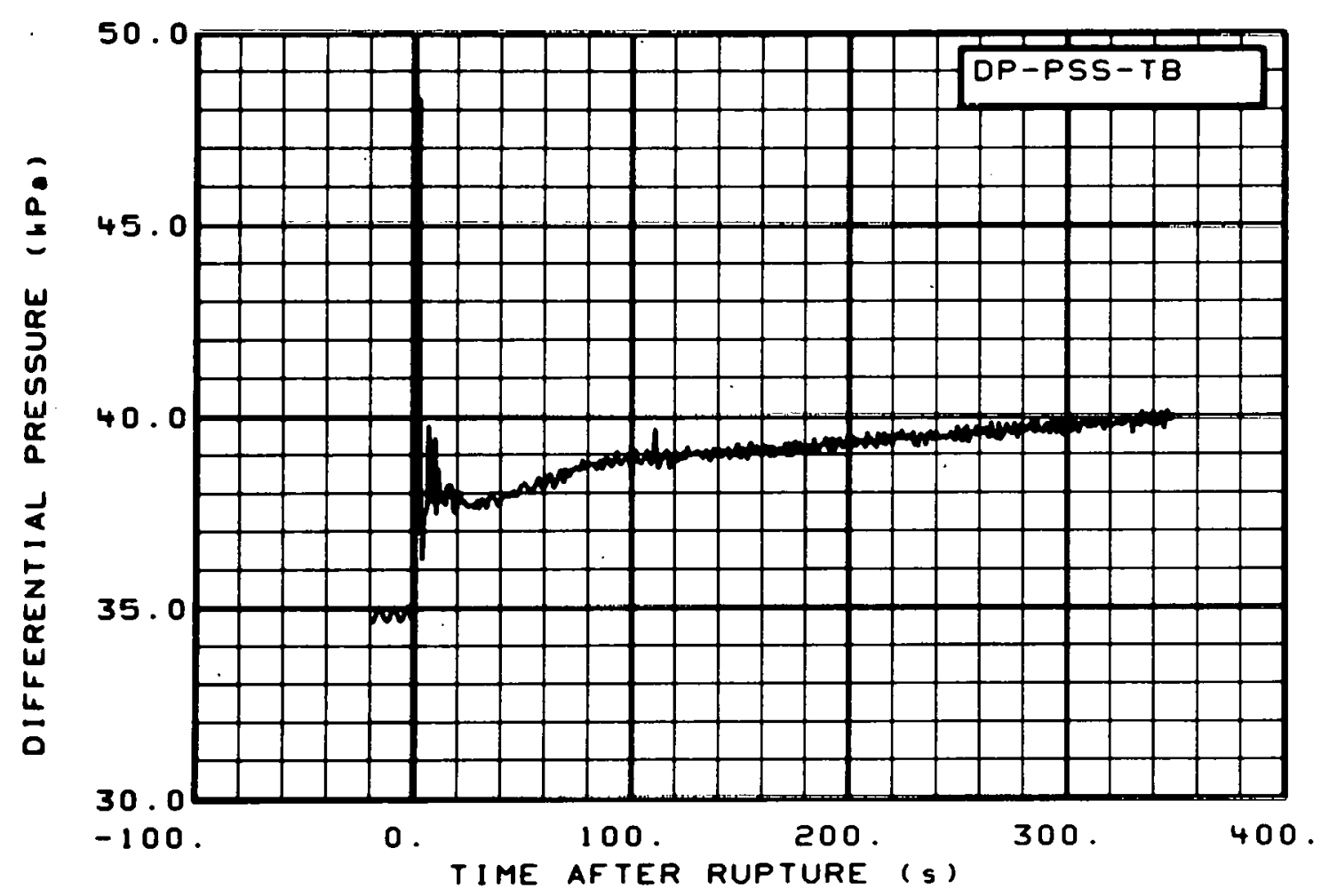

Fig. 239 Differential pressure in pressure suppression tank (DP-PSS-TB), from -20 to $350 \mathrm{~s}$.

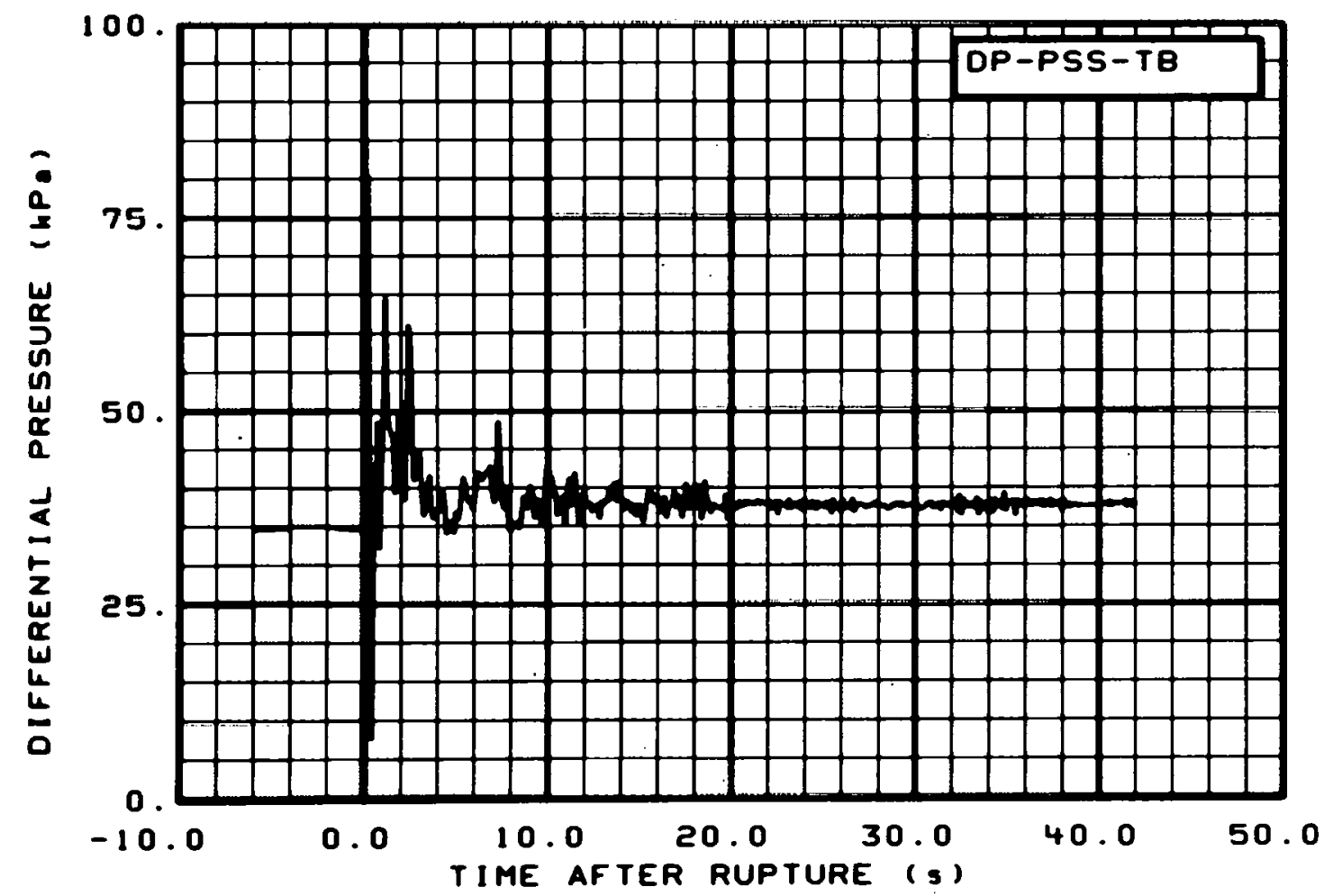

Fig. 240 Differential pressure in pressure suppression tank (DP-PSS-TB), from -6 to $42 \mathrm{~s}$. 


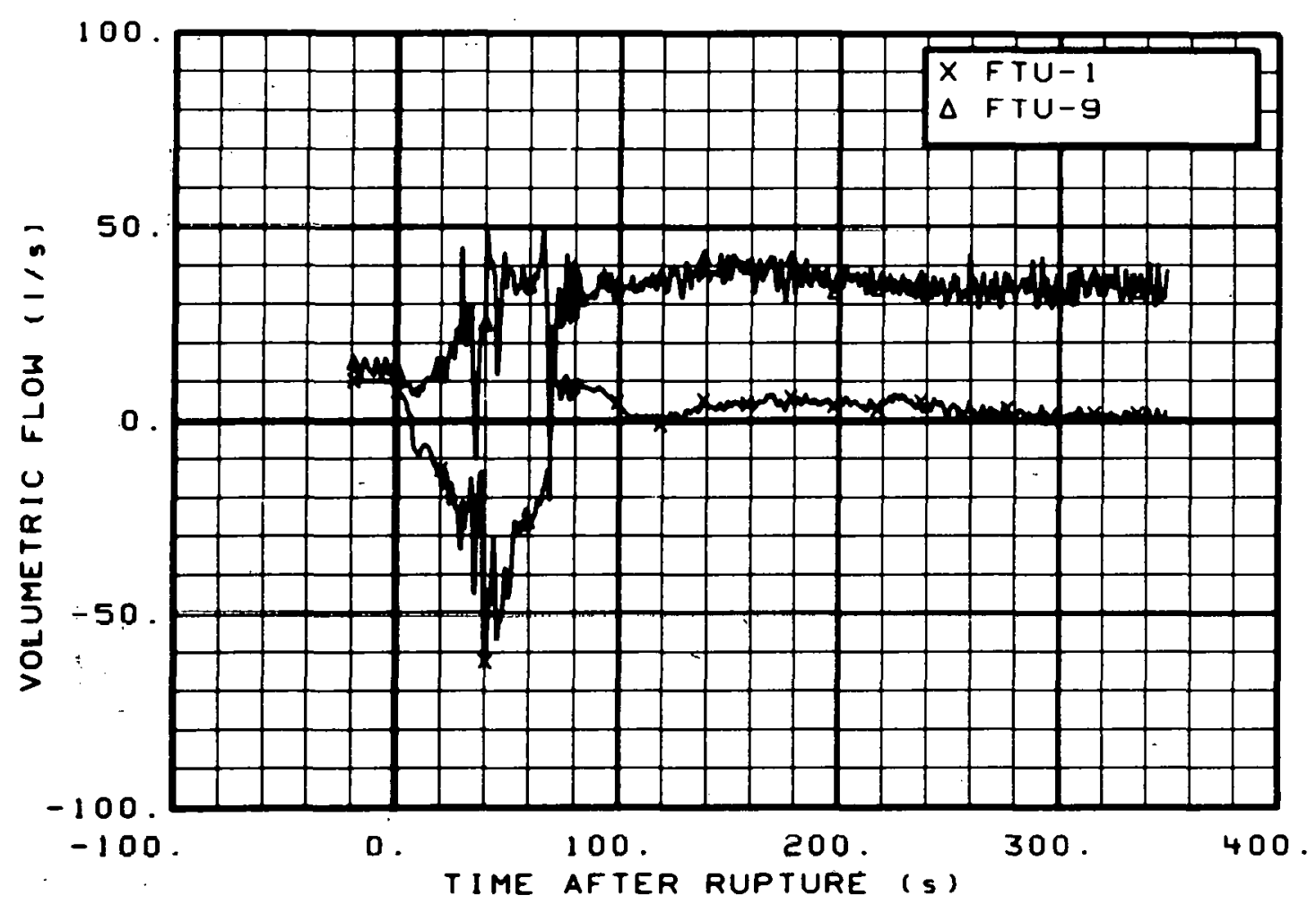

Fig. 241 Volumetric flow in intact loop (FTU-1 and FTU-9), from -20 to $350 \mathrm{~s}$.

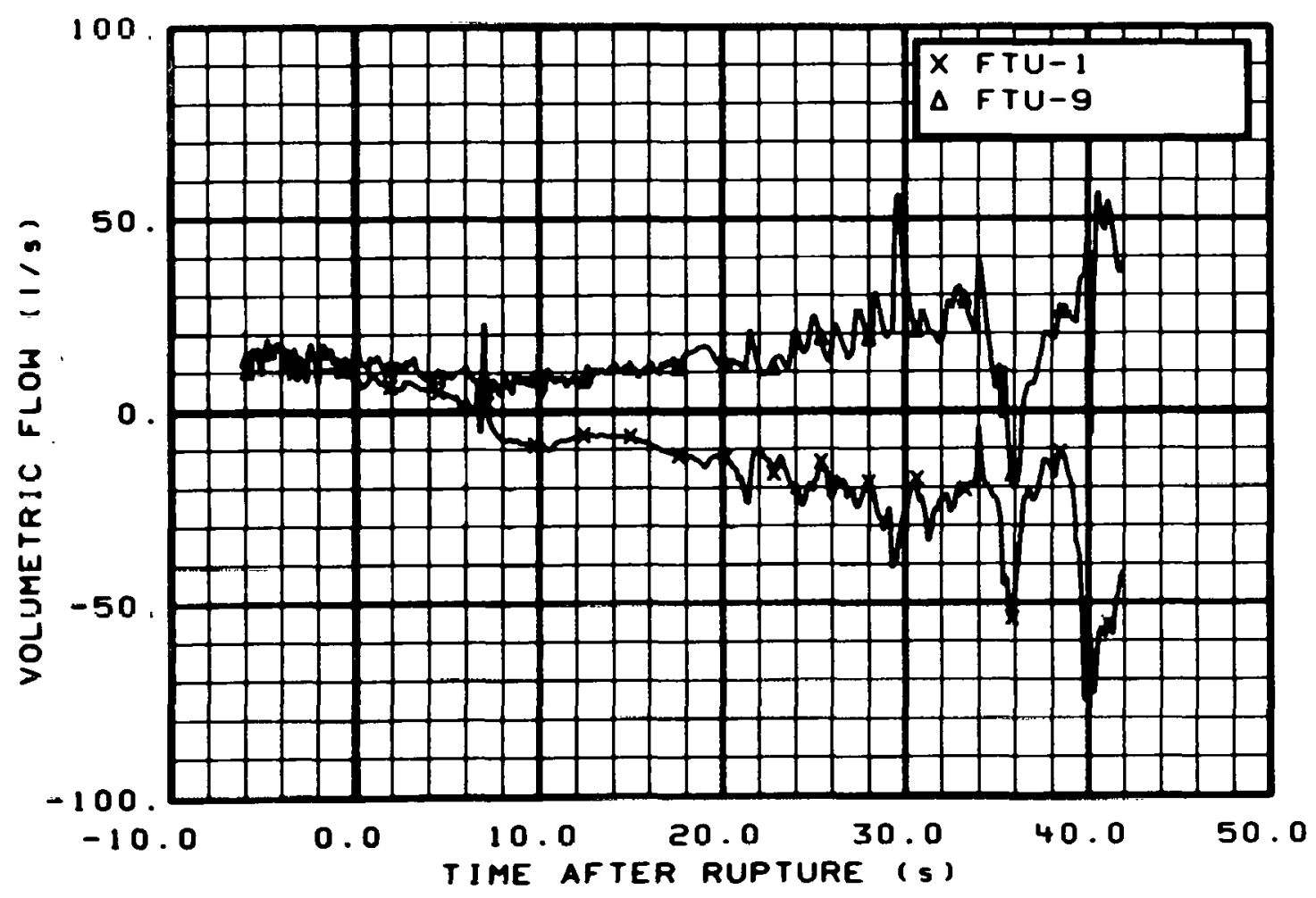

Fig. 242 Volumetric flow in intact loop (FTU-1 and FTU-9), from -6 to $42 \mathrm{~s}$. 


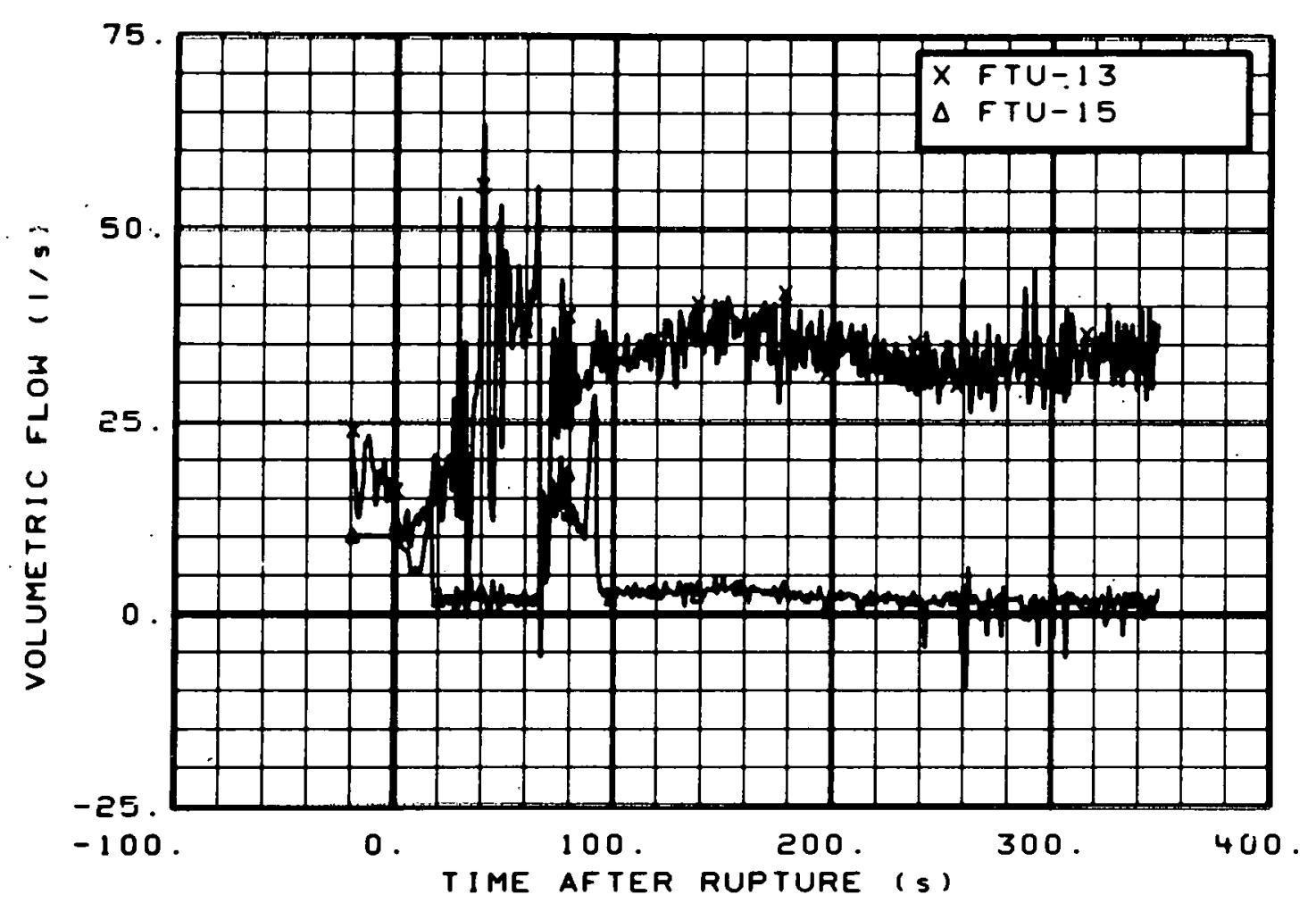

Fig. 243 Volumetric flow in intact loop (FTU-13 and FTU-15), from -20 to $350 \mathrm{~s}$.

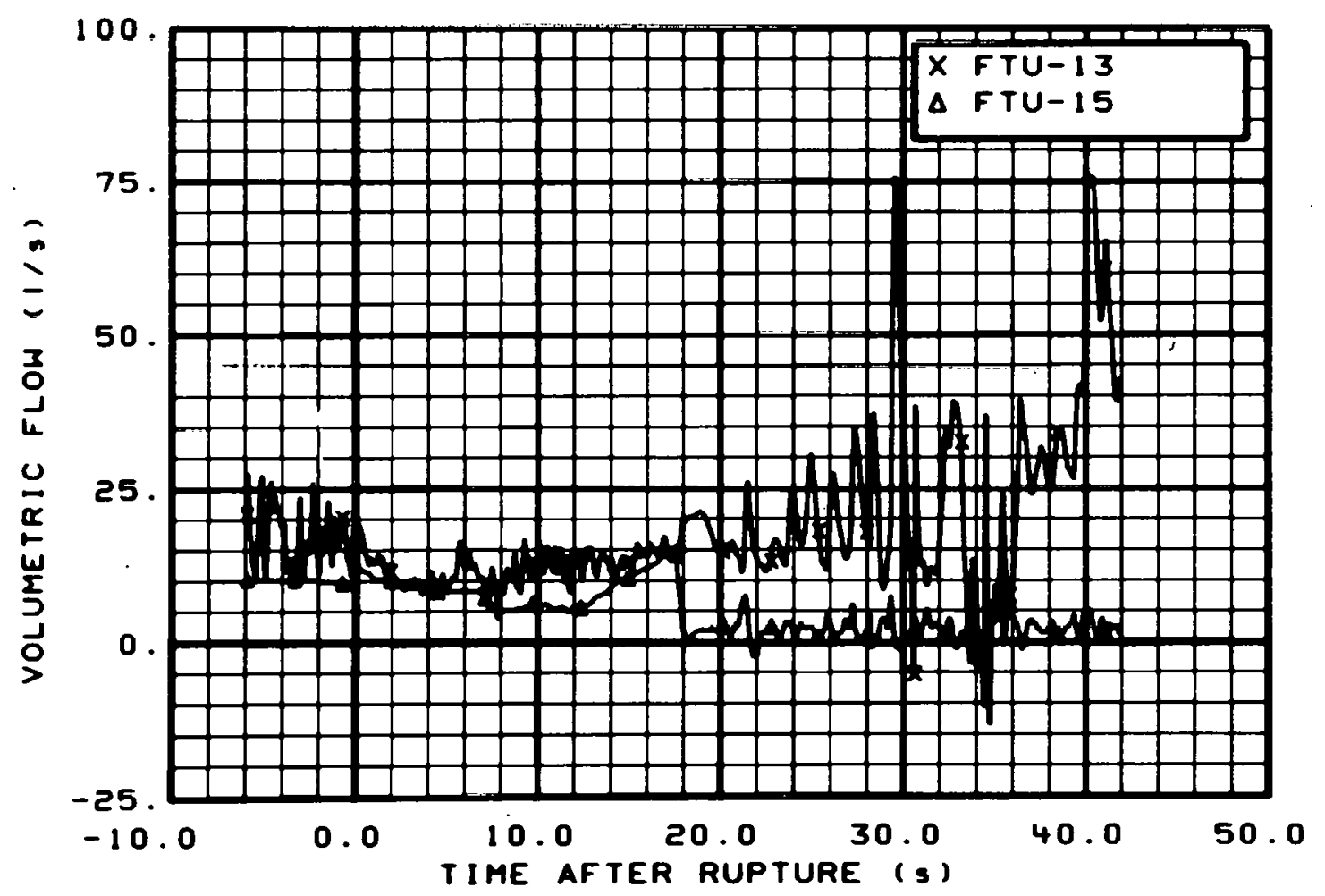

Fig. 244 Volumetric flow in intact loop (FTU-13 and FTU-15), from -6 to $42 \mathrm{~s}$. 


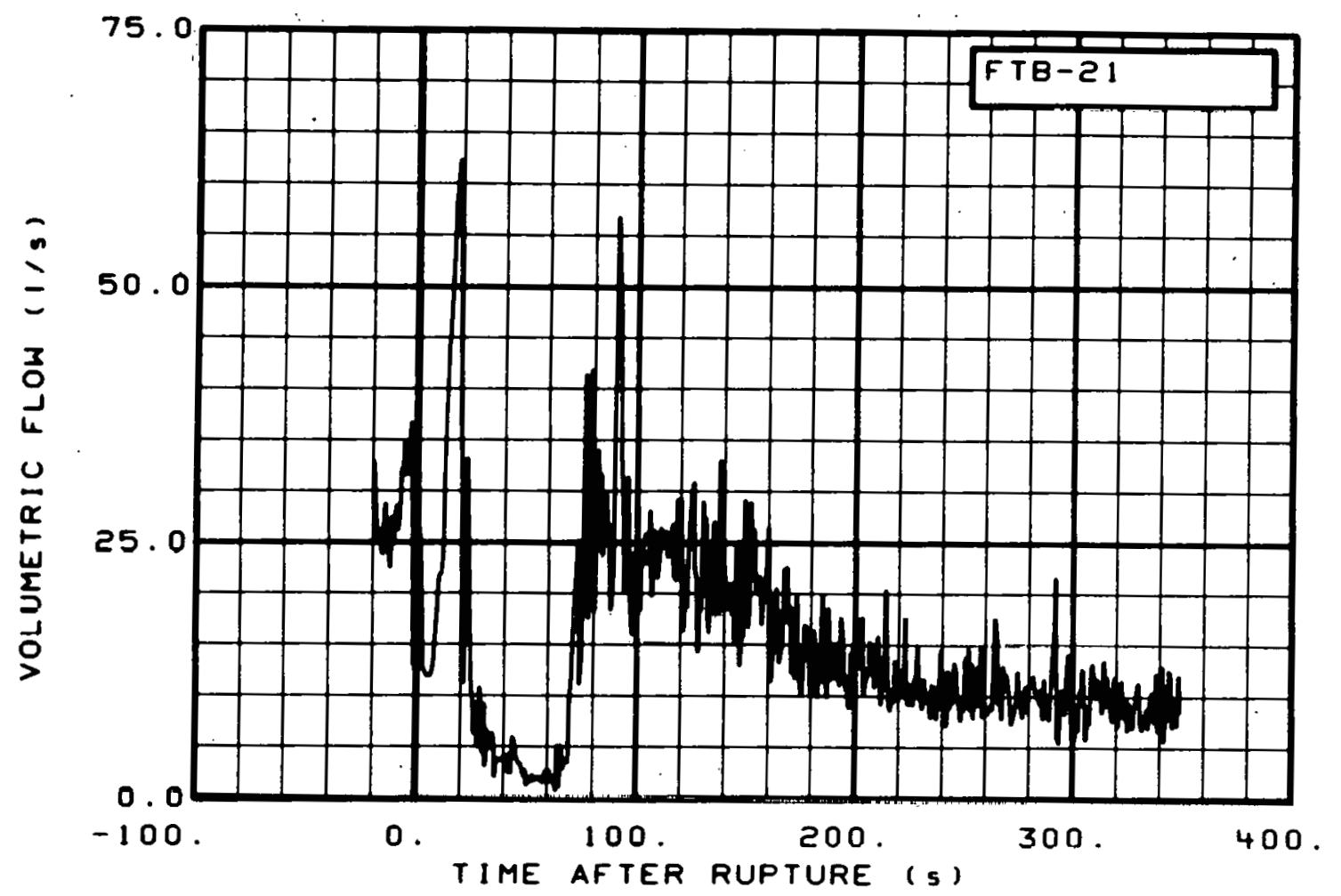

Fig. 245 Volumetric flow in broken loop (FTB-21), from -20 to $350 \mathrm{~s}$.

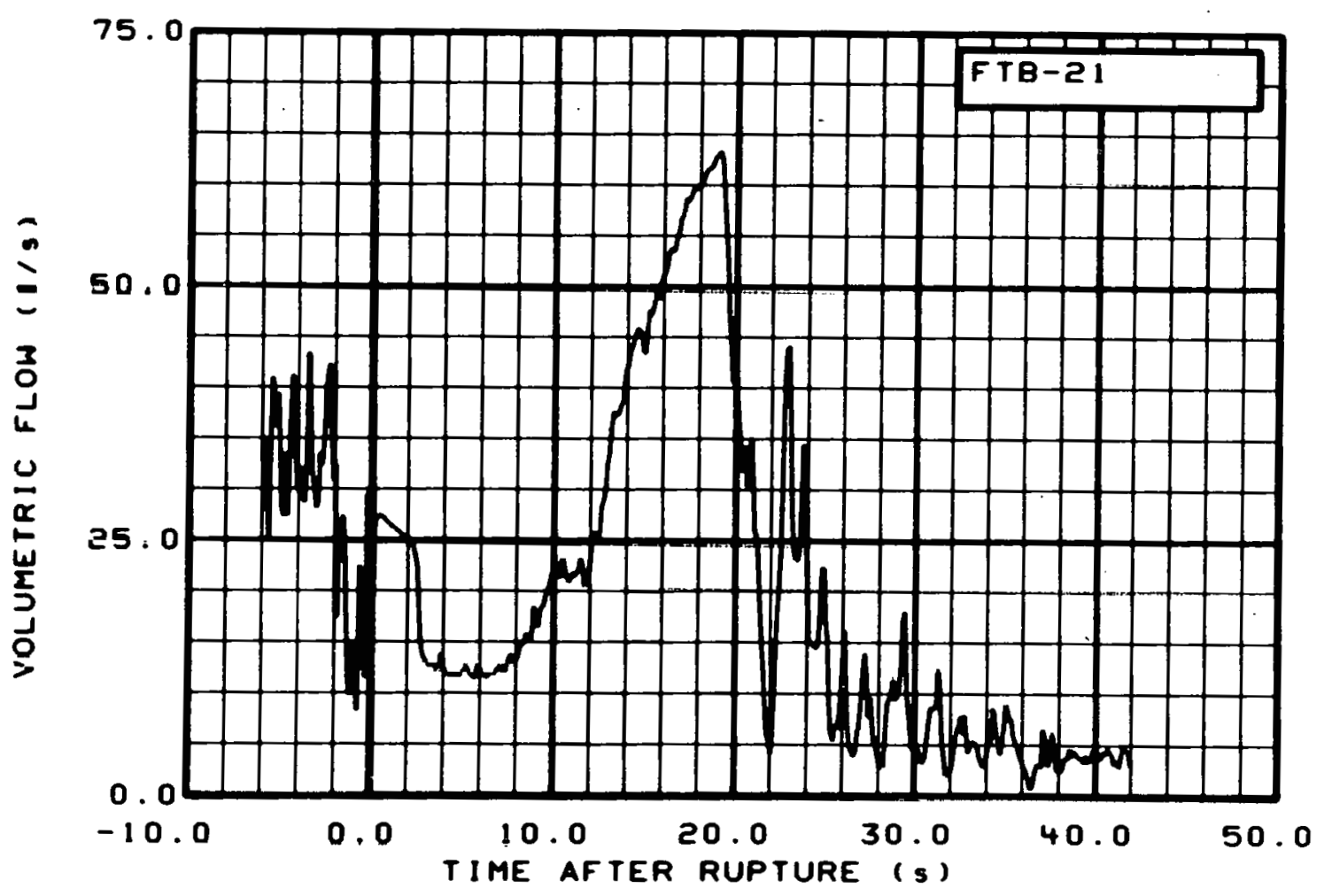

Fig. 246 Volumetric flow in broken loop (FTB-21), from -6 to $42 \mathrm{~s}$. 


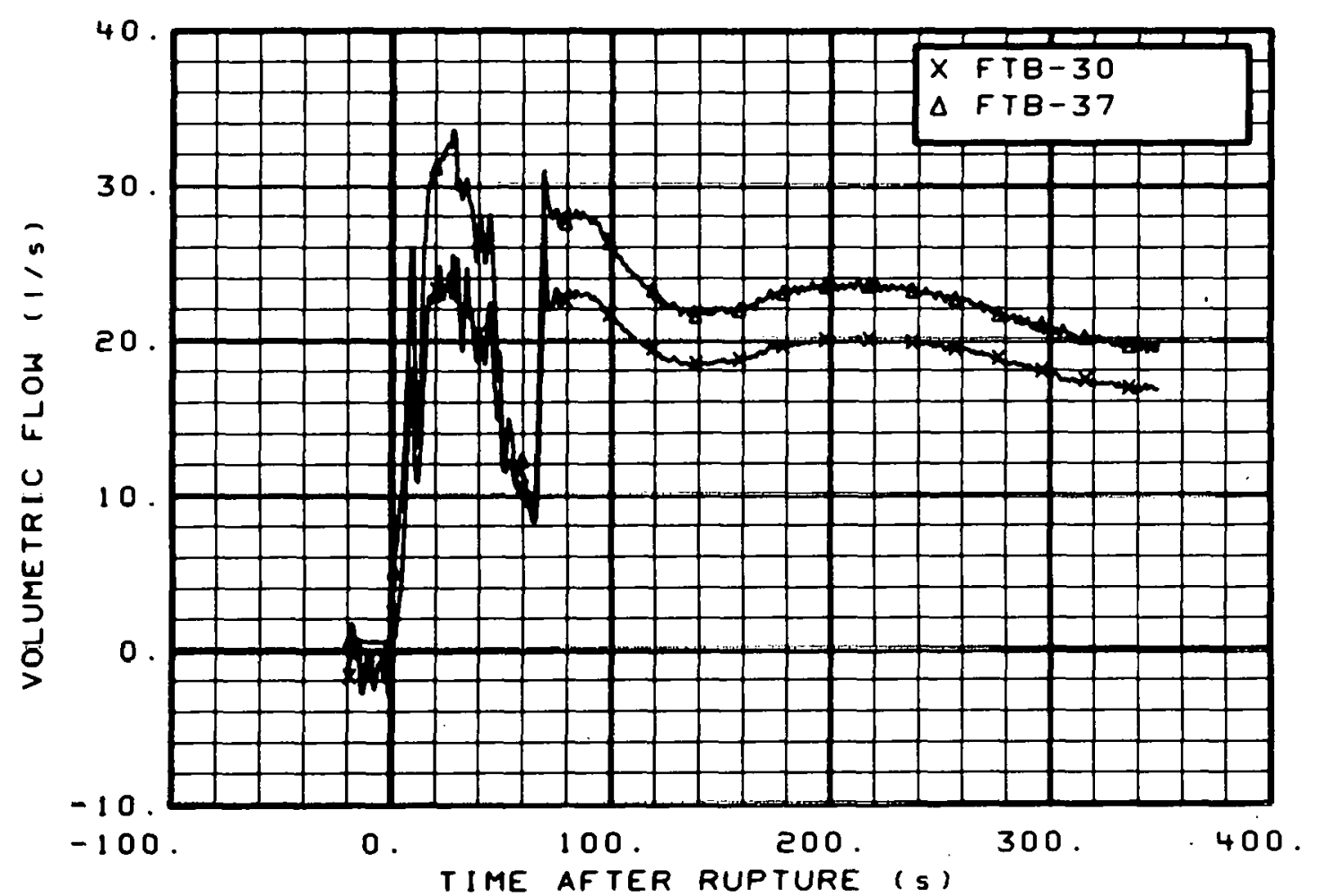

Fig. 247 Volumetric flow in broken loop (FTB-30 and FTB-37), from -20 to $350 \mathrm{~s}$.

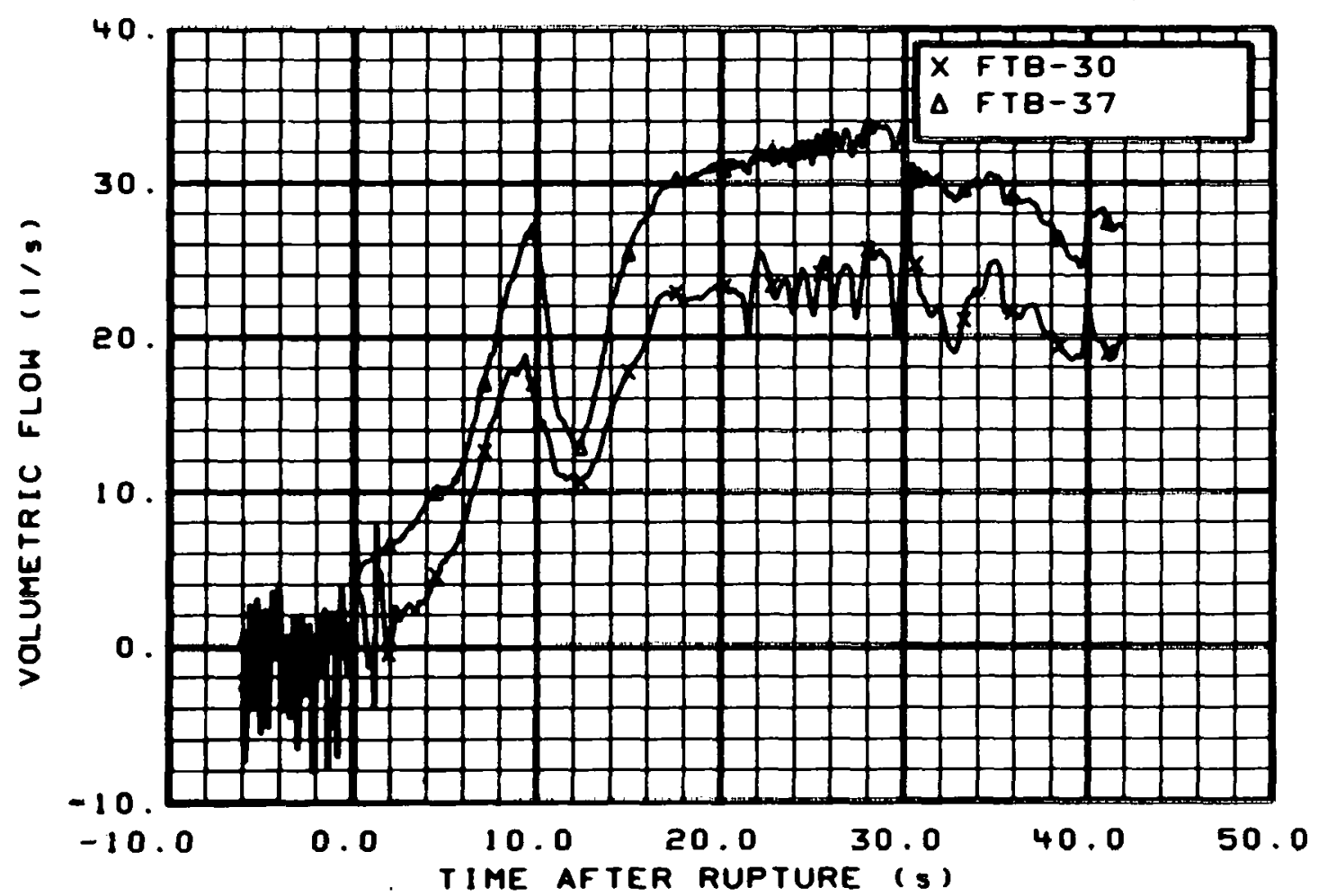

Fig. 248 Volumetric flow in broken loop (FTB-30 and FTB-37), from -6 to $42 \mathrm{~s}$. 


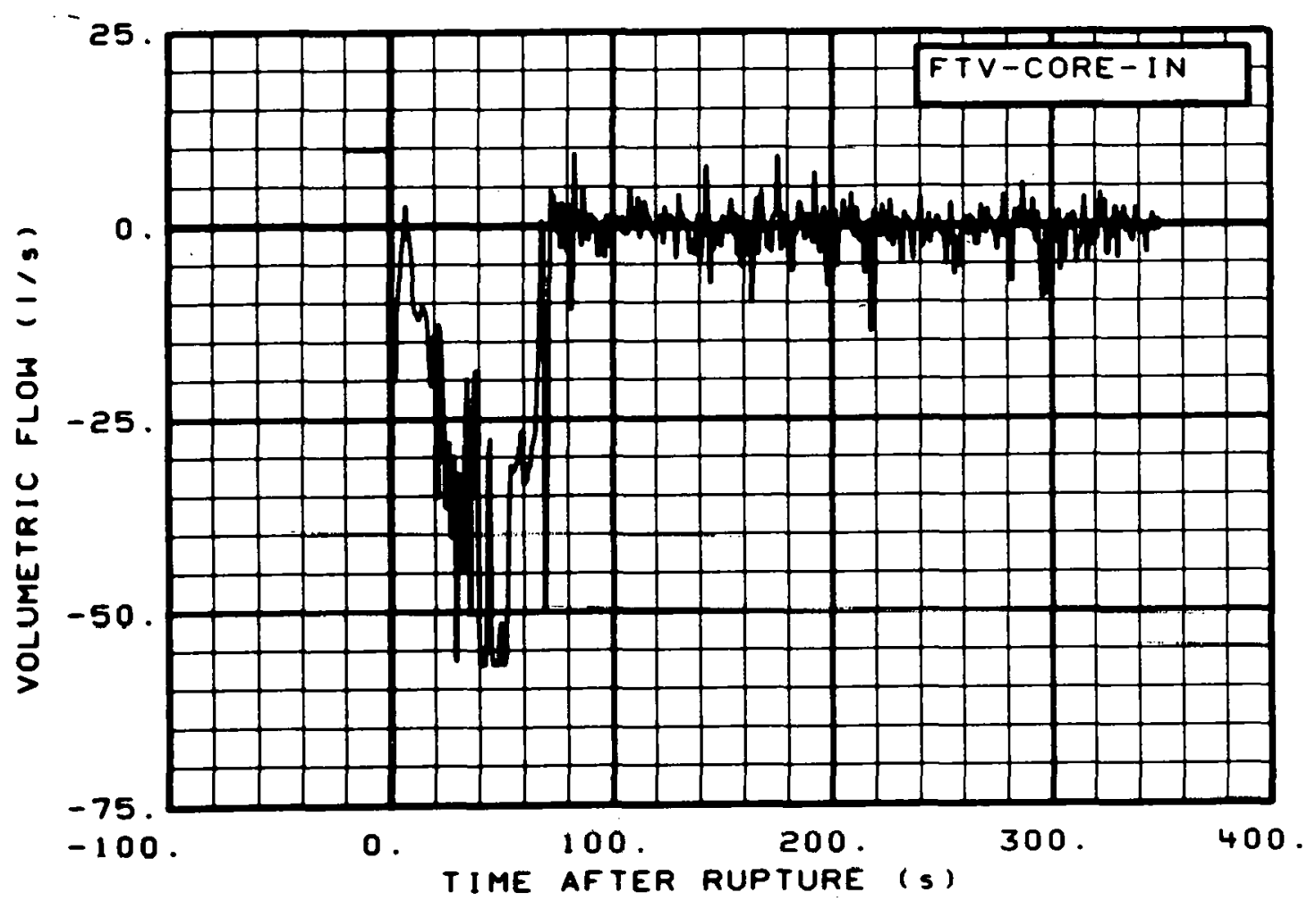

Fig. 249 Volumetric flow in core entrance (FTV-CORE-IN), from -20 to $350 \mathrm{~s}$.

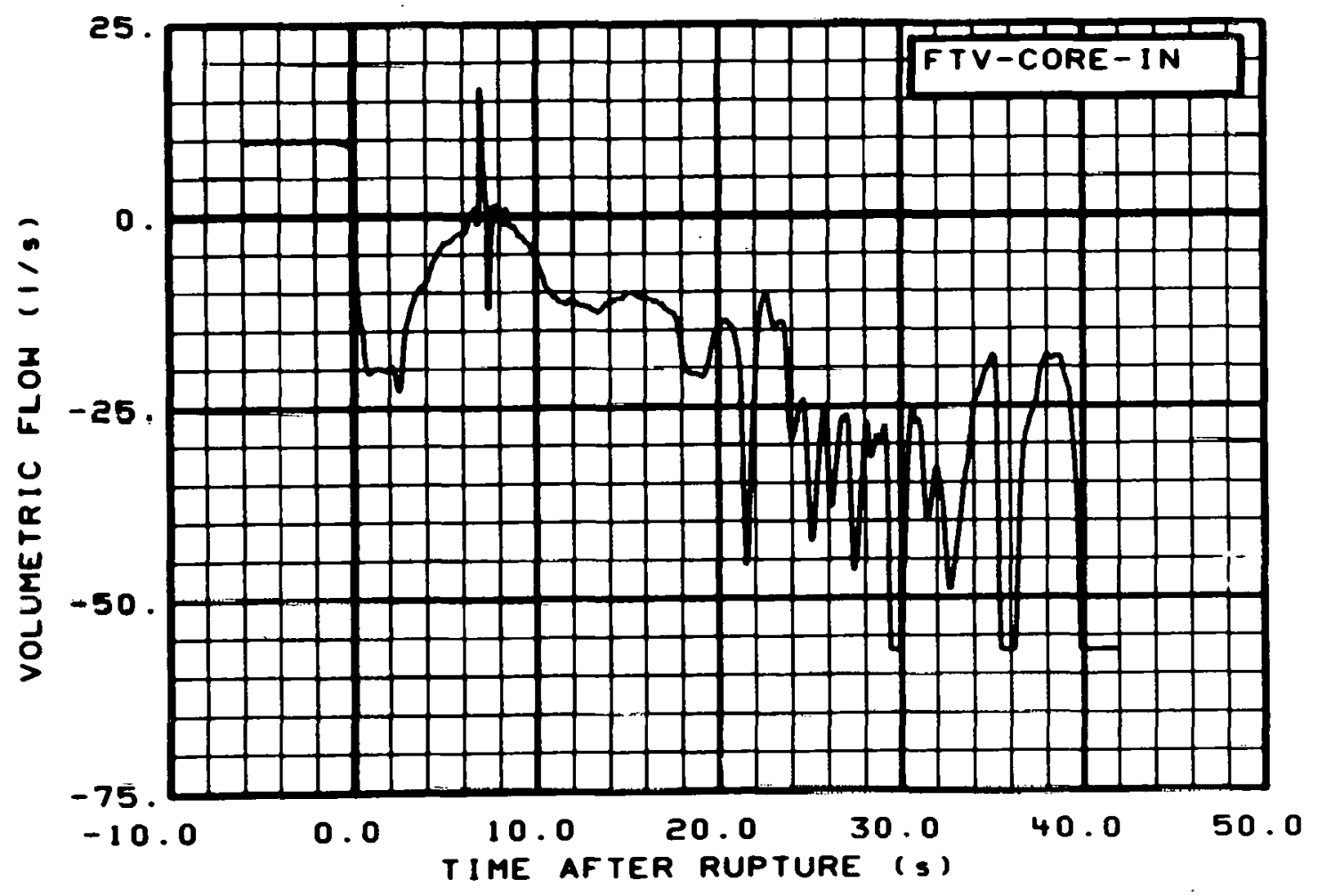

Fig. 250 Volumetric flow in core entrance (FTV-CORE-IN), from -6 to $42 \mathrm{~s}$. 


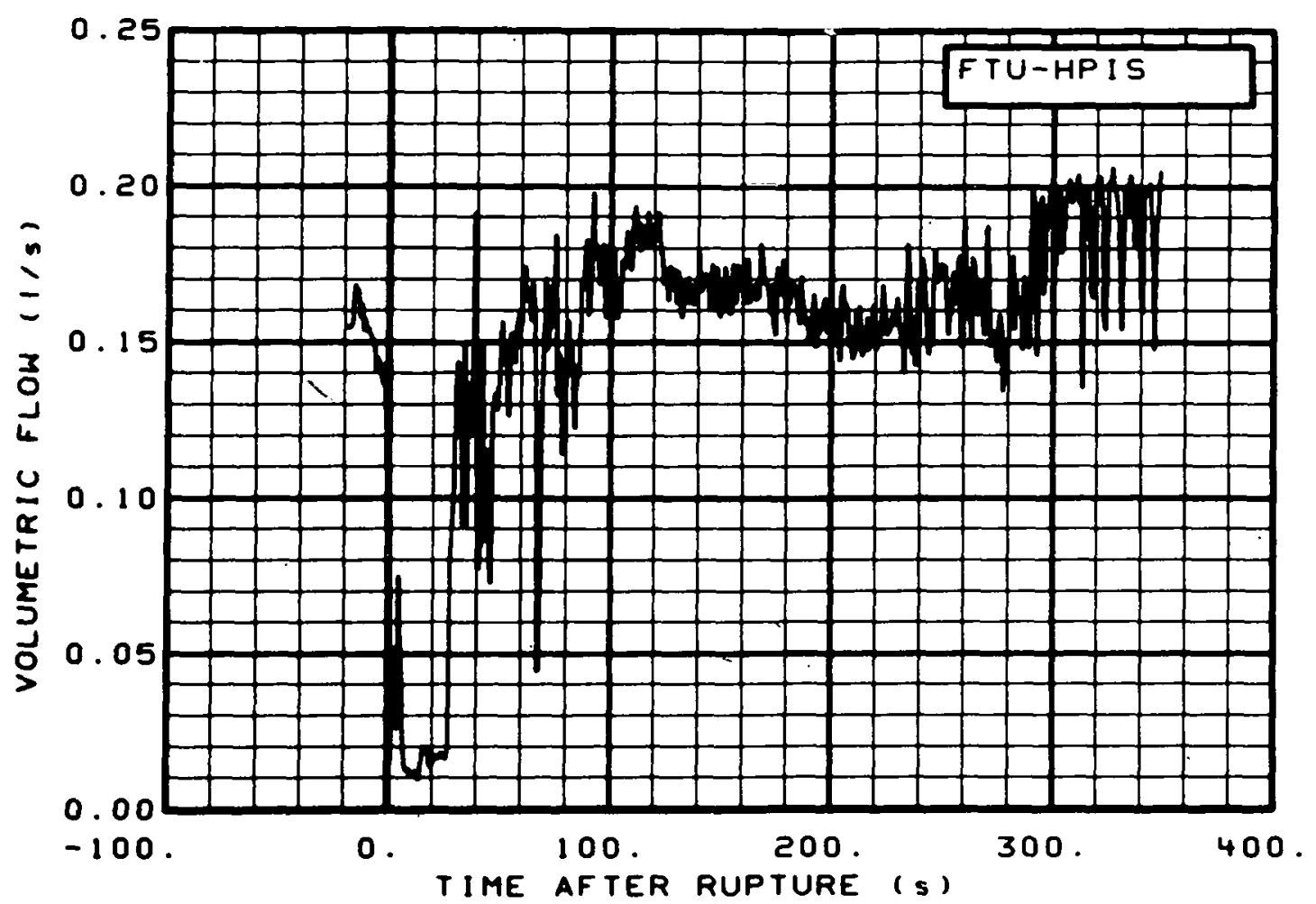

Fig. 251 Volumetric flow in intact loop high pressure injection line (FTU-HPIS), from -20 to. $350 \mathrm{~s}$.

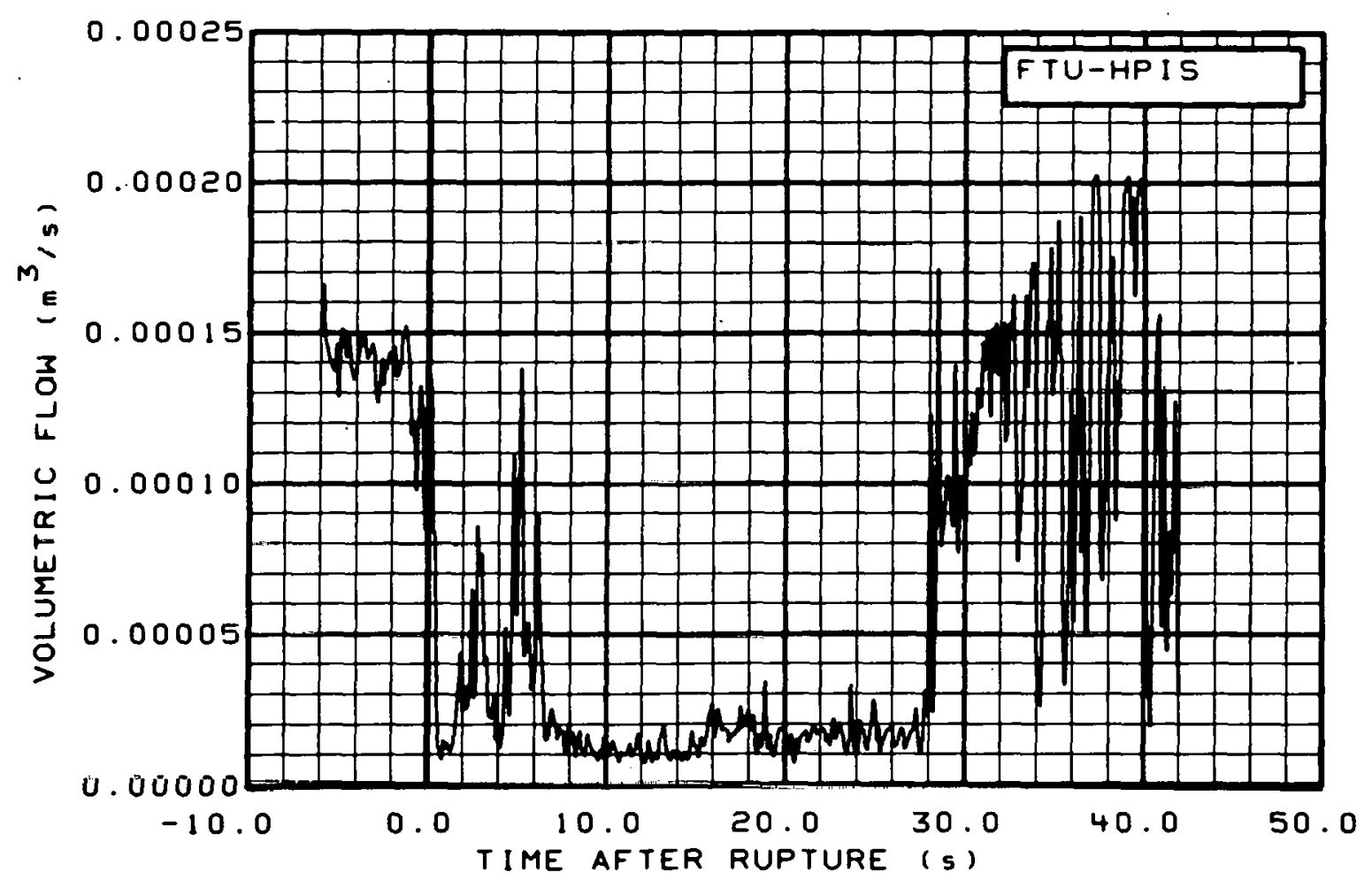

Fig. 252 Volumetric flow in intact loop high pressure injection line (FTU-HPIS), from -6 to $42 \mathrm{~s}$. 


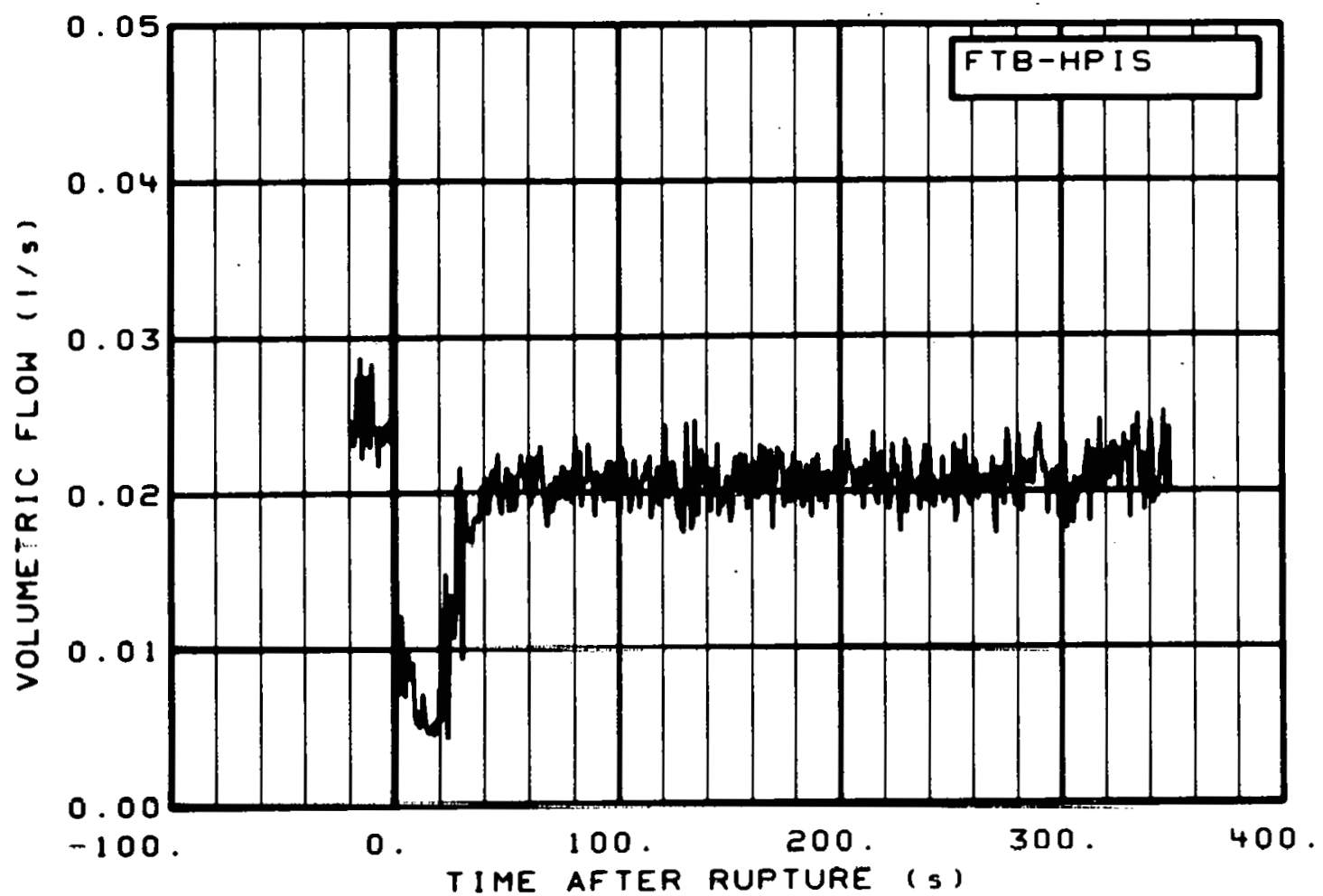

Fig. 253 Volumetric flow in broken loop high pressure injection line (FTB-HPIS), from -20 to $350 \mathrm{~s}$.

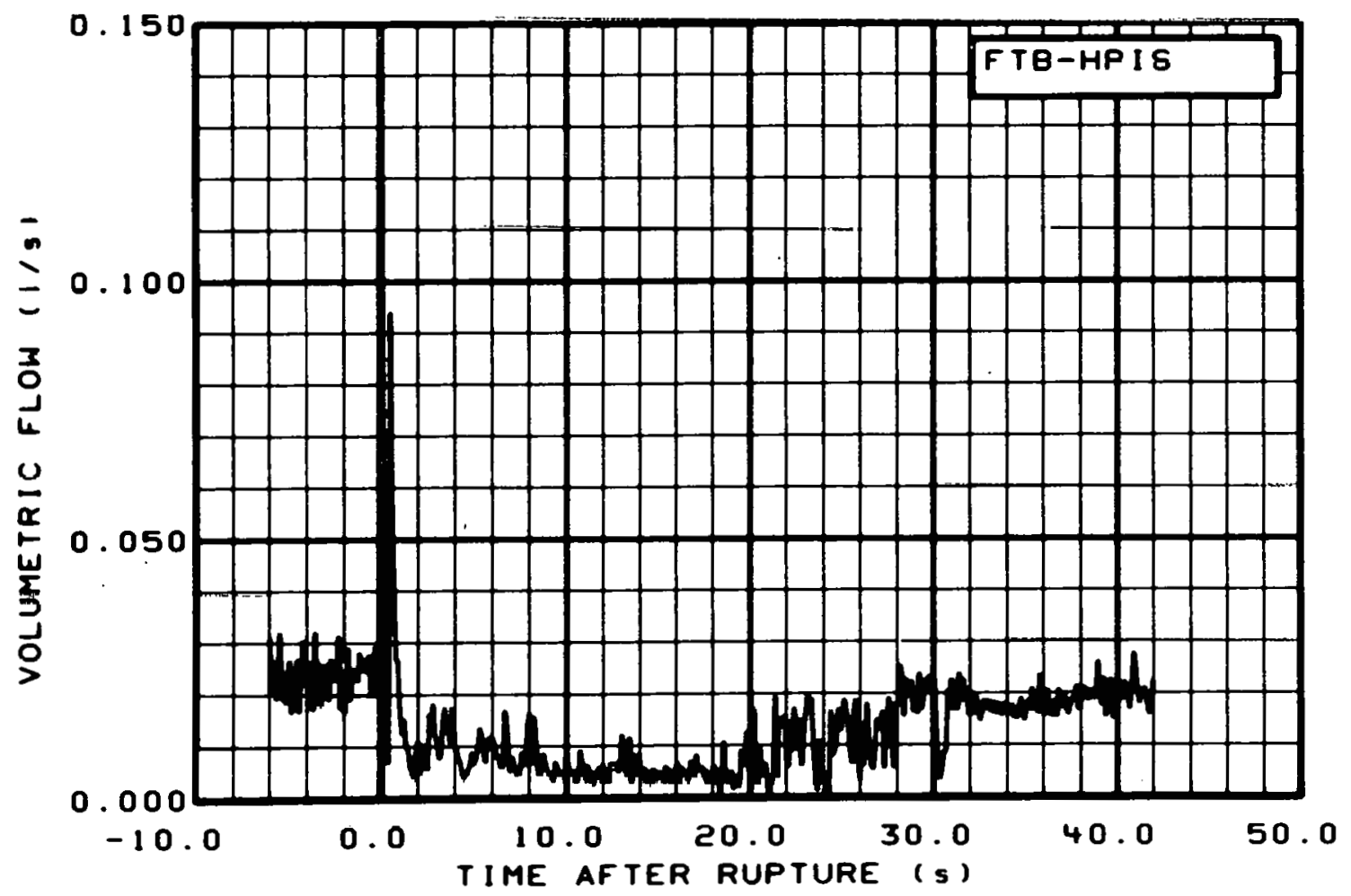

Fig. 254 Volumetric flow in broken loop high pressure injection line (FTB-HPIS), from -6 to $42 \mathrm{~s}$. 


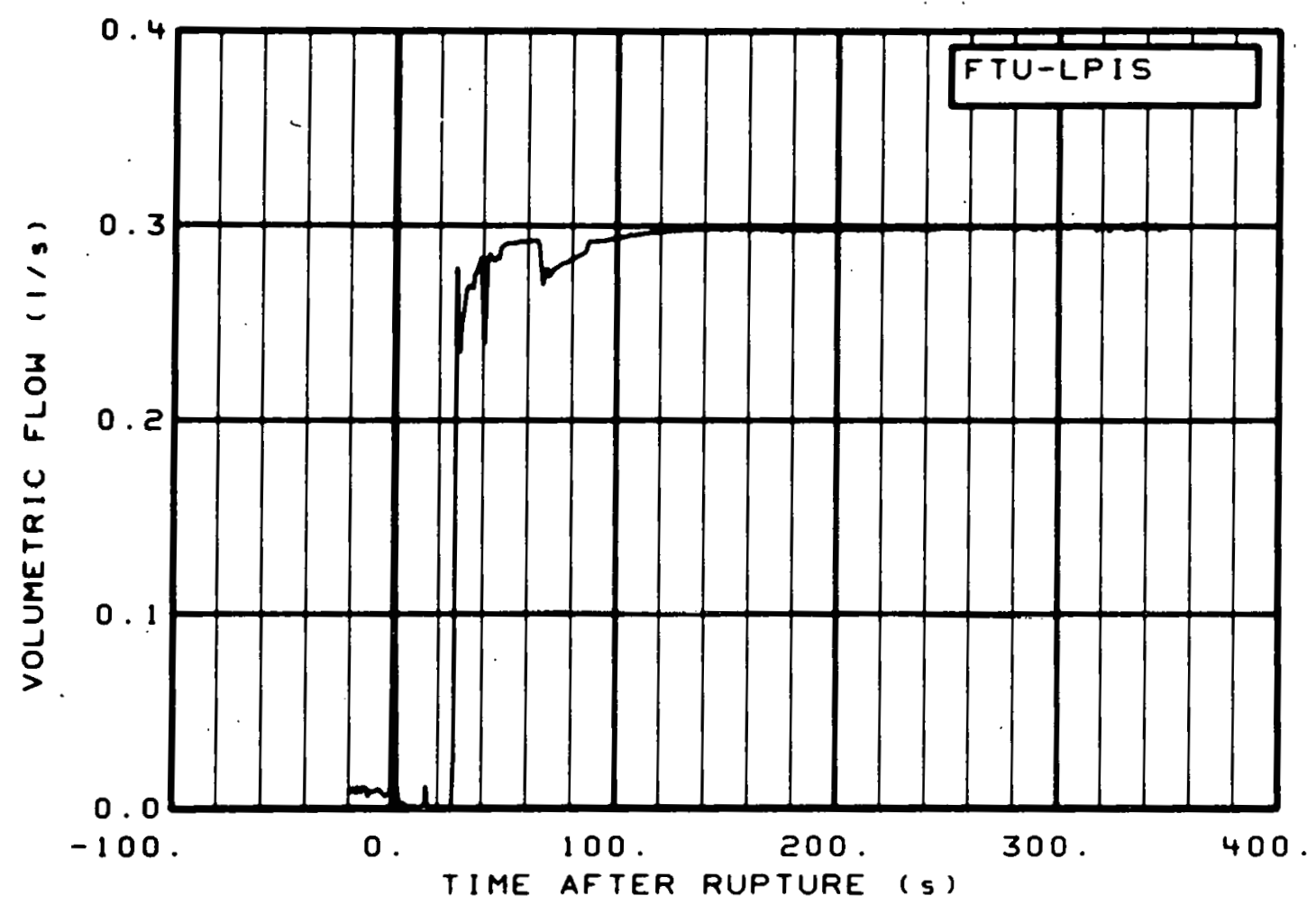

Fig. 255 Volumetric flow in intact loop low pressure injection line (FTU-LPIS), from -20 to $350 \mathrm{~s}$.

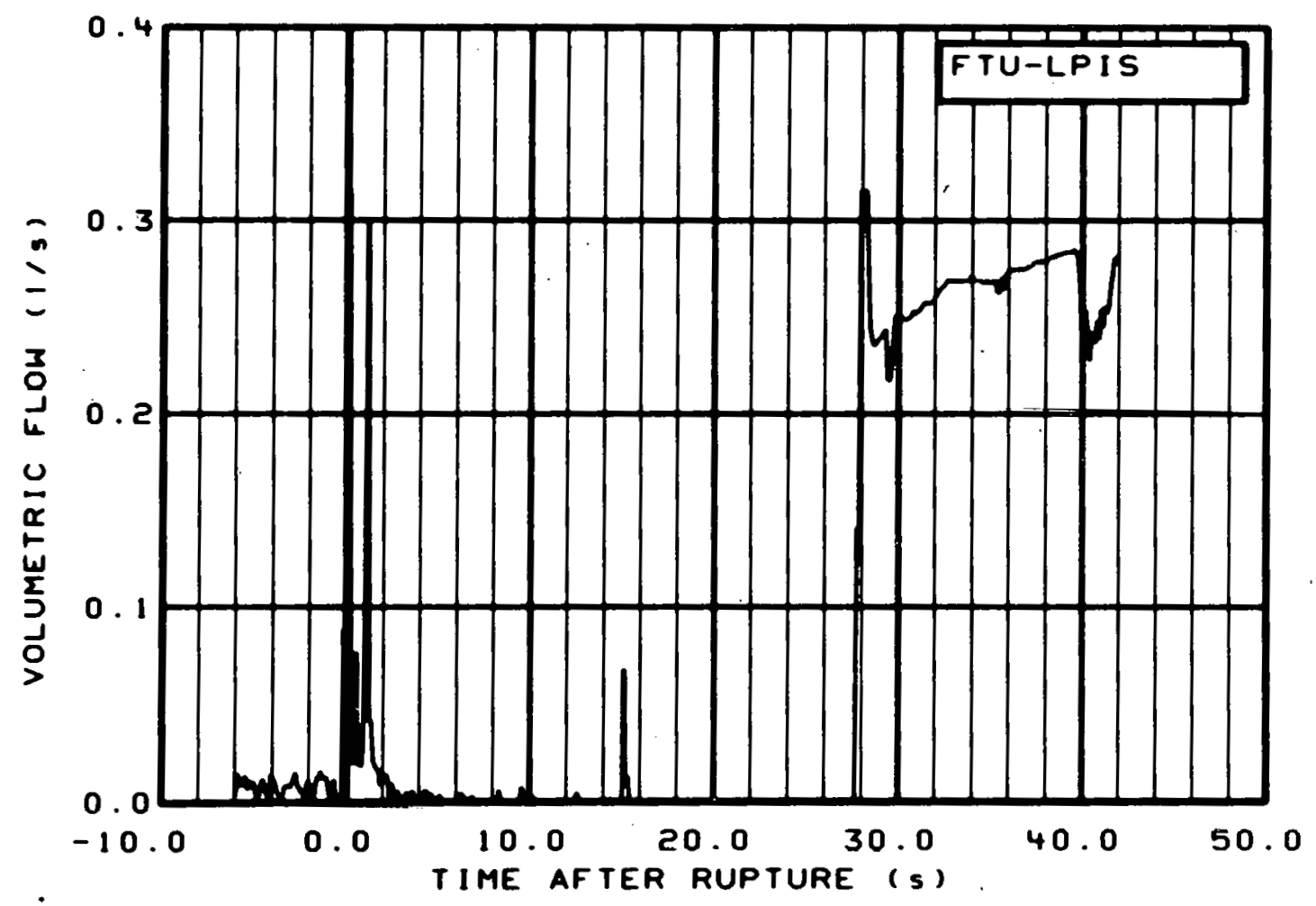

Fig. 256 Volumetric flow in intact loop low pressure injection line (FTU-LFIS), from -6 to $42 \mathrm{~s}$. 


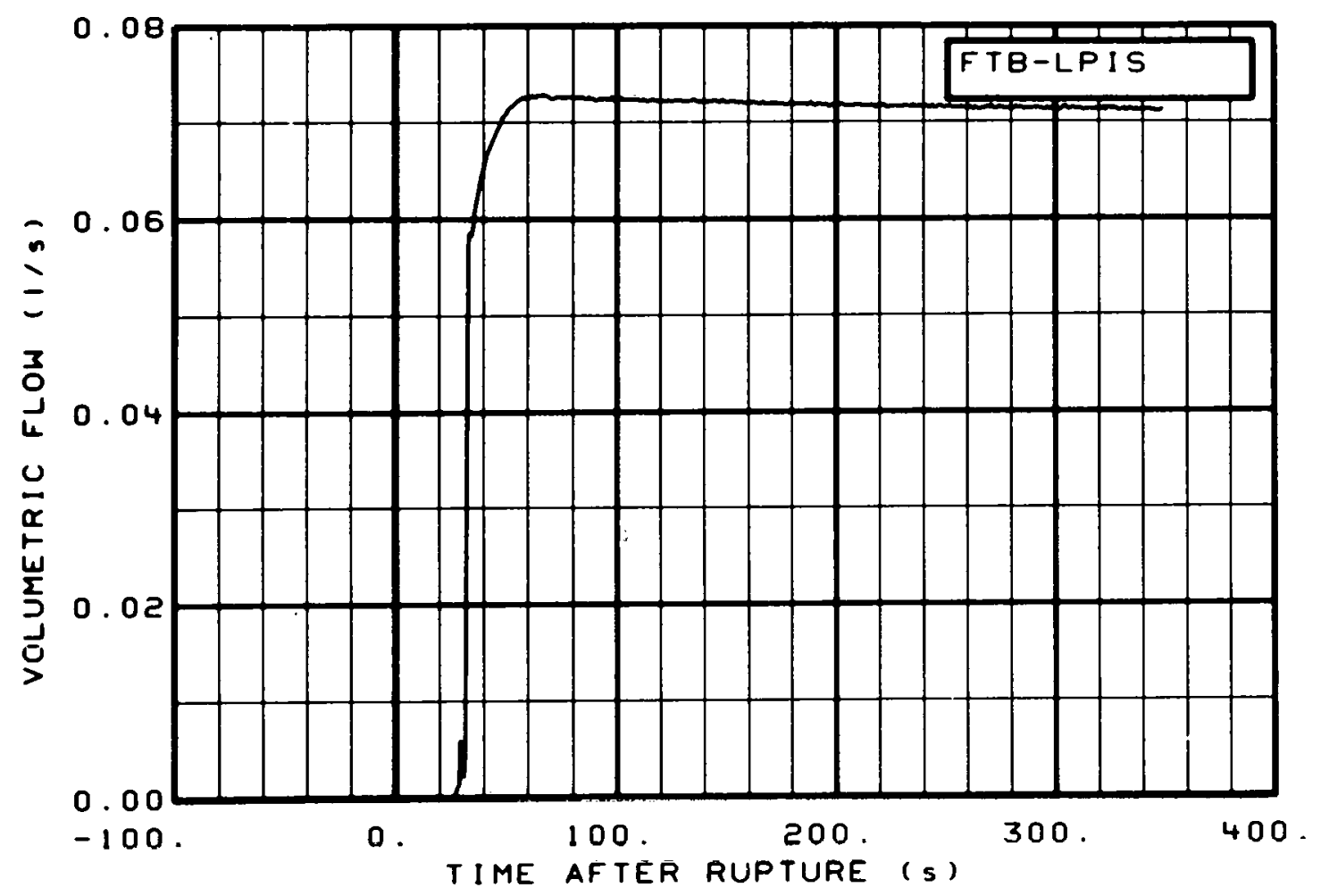

Fig. 257 Volumetric flow in broken loop low pressure injection line (FTB-LPIS), from -20 to $350 \mathrm{~s}$.

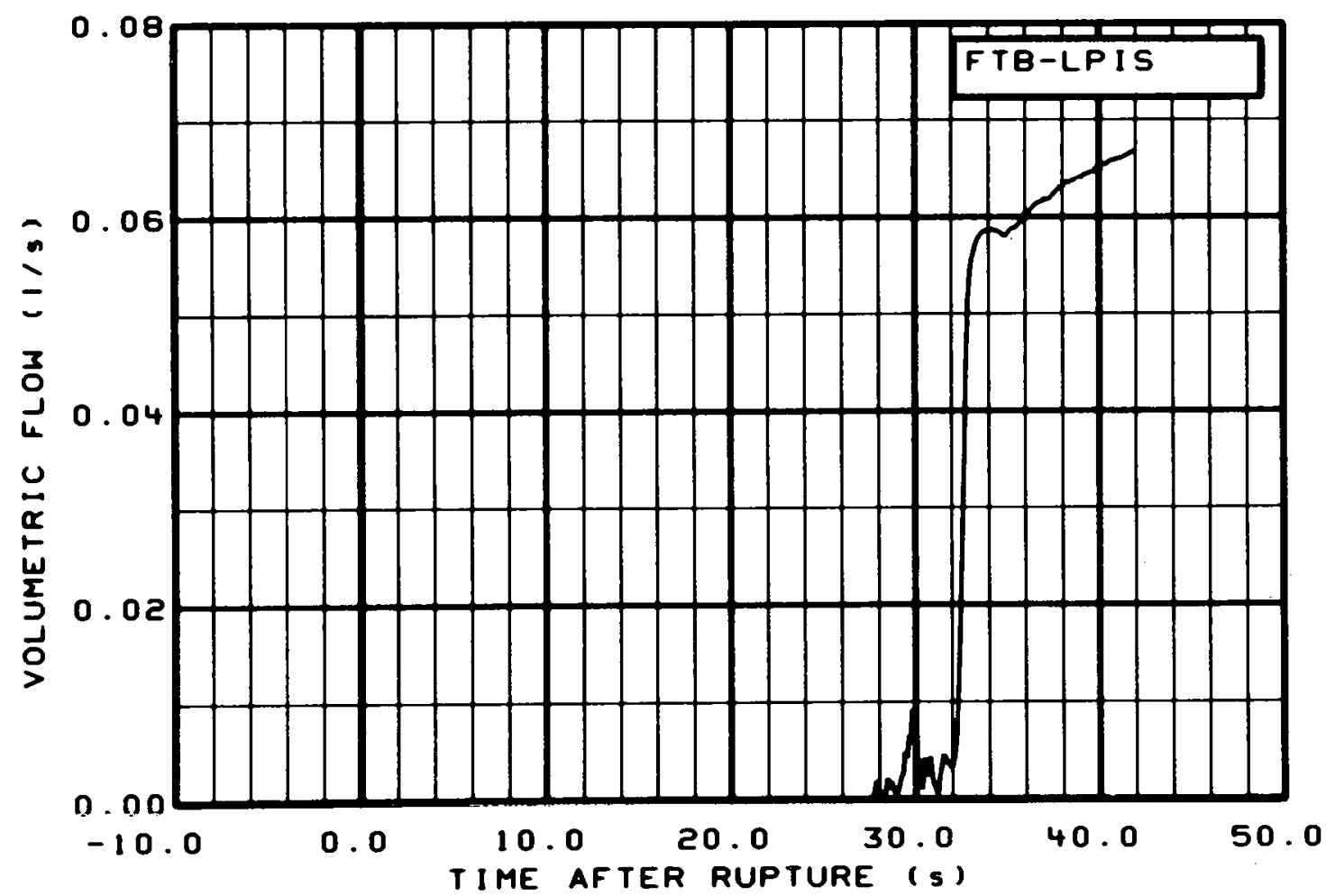

Fig. 258 Volumetric flow in broken loop low pressure injection line (FTB-LPIS), from -6 to $42 \mathrm{~s}$. 


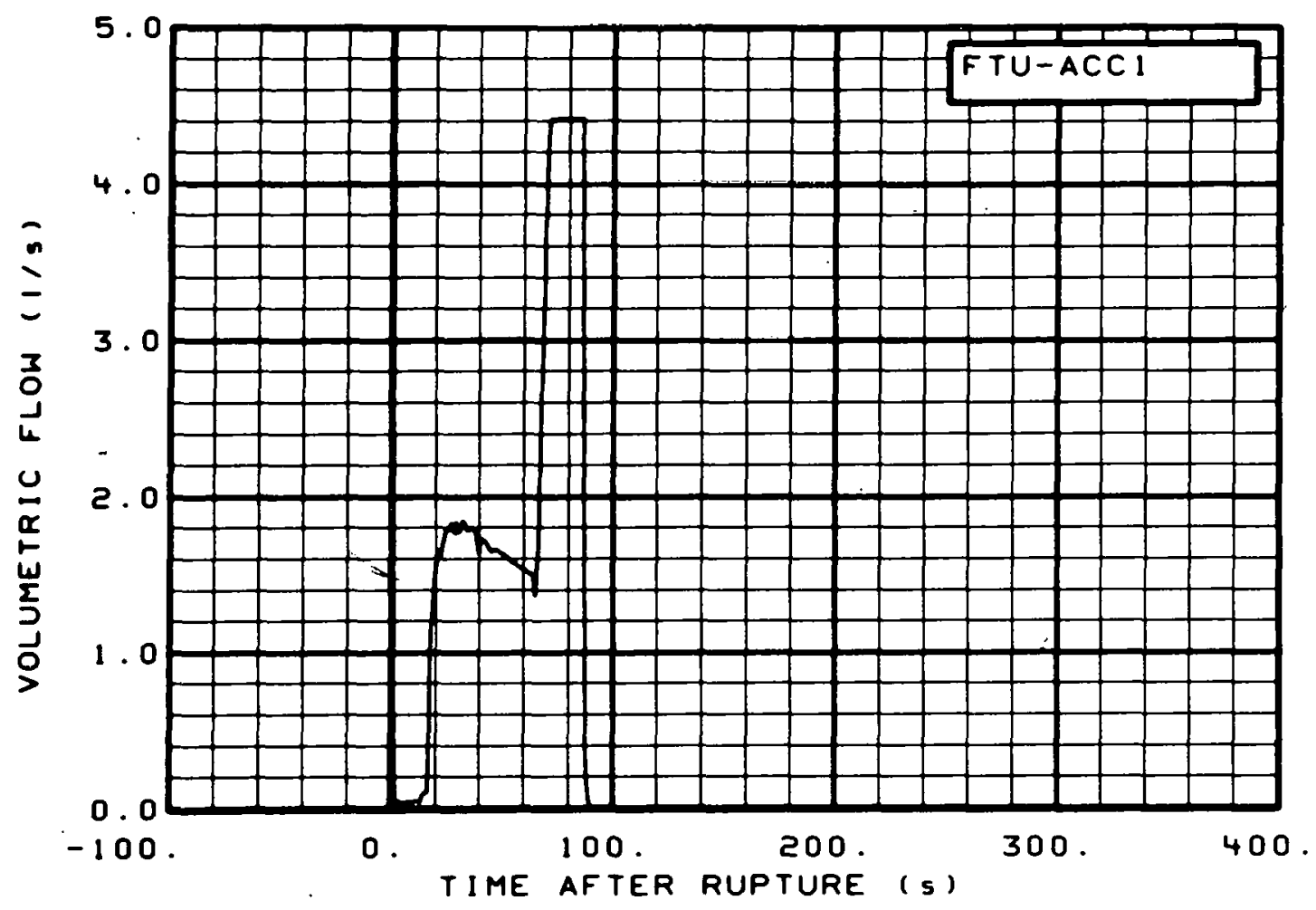

Fig. 259 Volumetric flow in intact loop accumulator discharge line (FT.U-ACCl), from -20 to $350 \mathrm{~s}$.

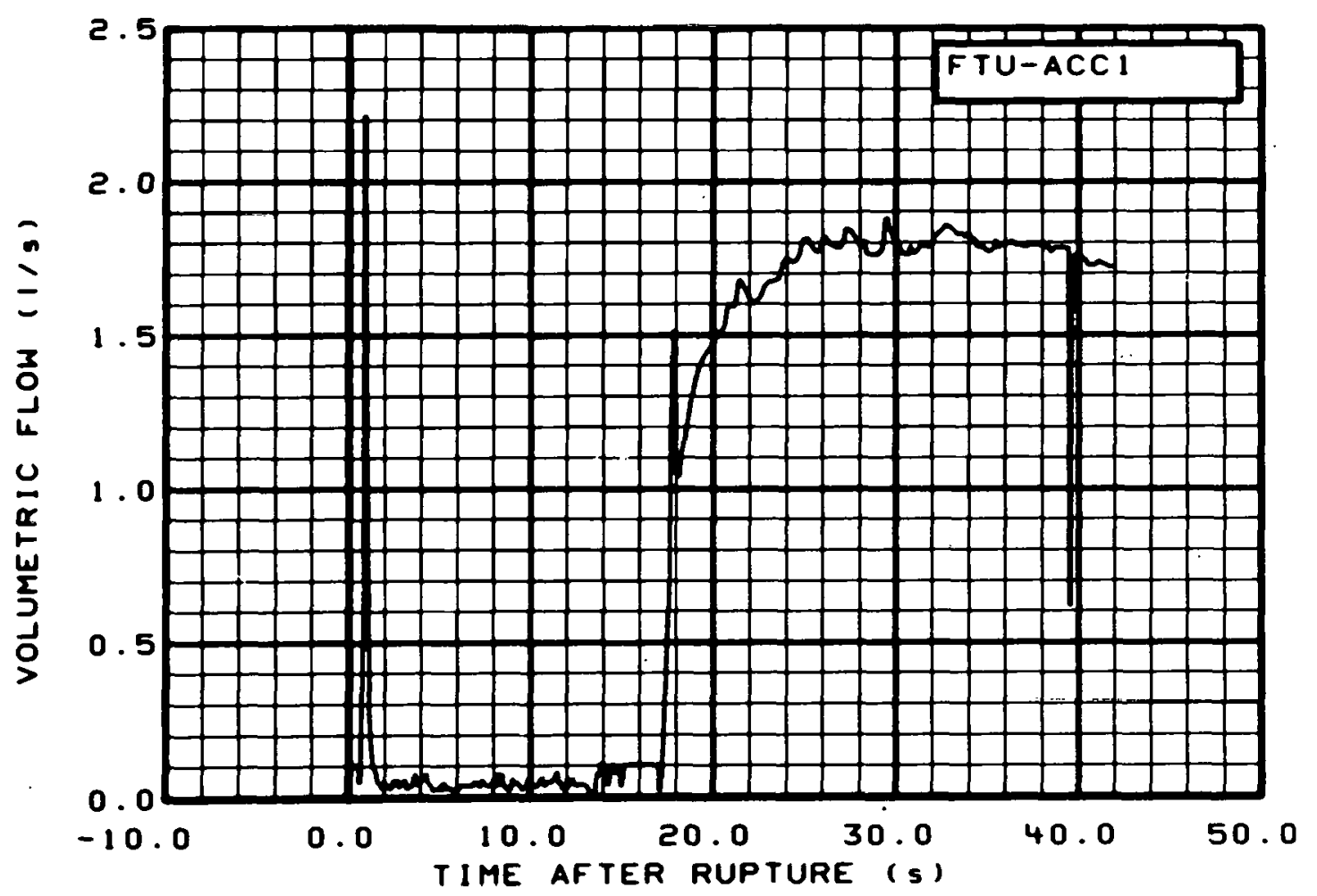

Fig. 260 Volumetric flow in intact loop accumulator discharge line (FTU-ACC1), from -6 to $42 \mathrm{~s}$. 


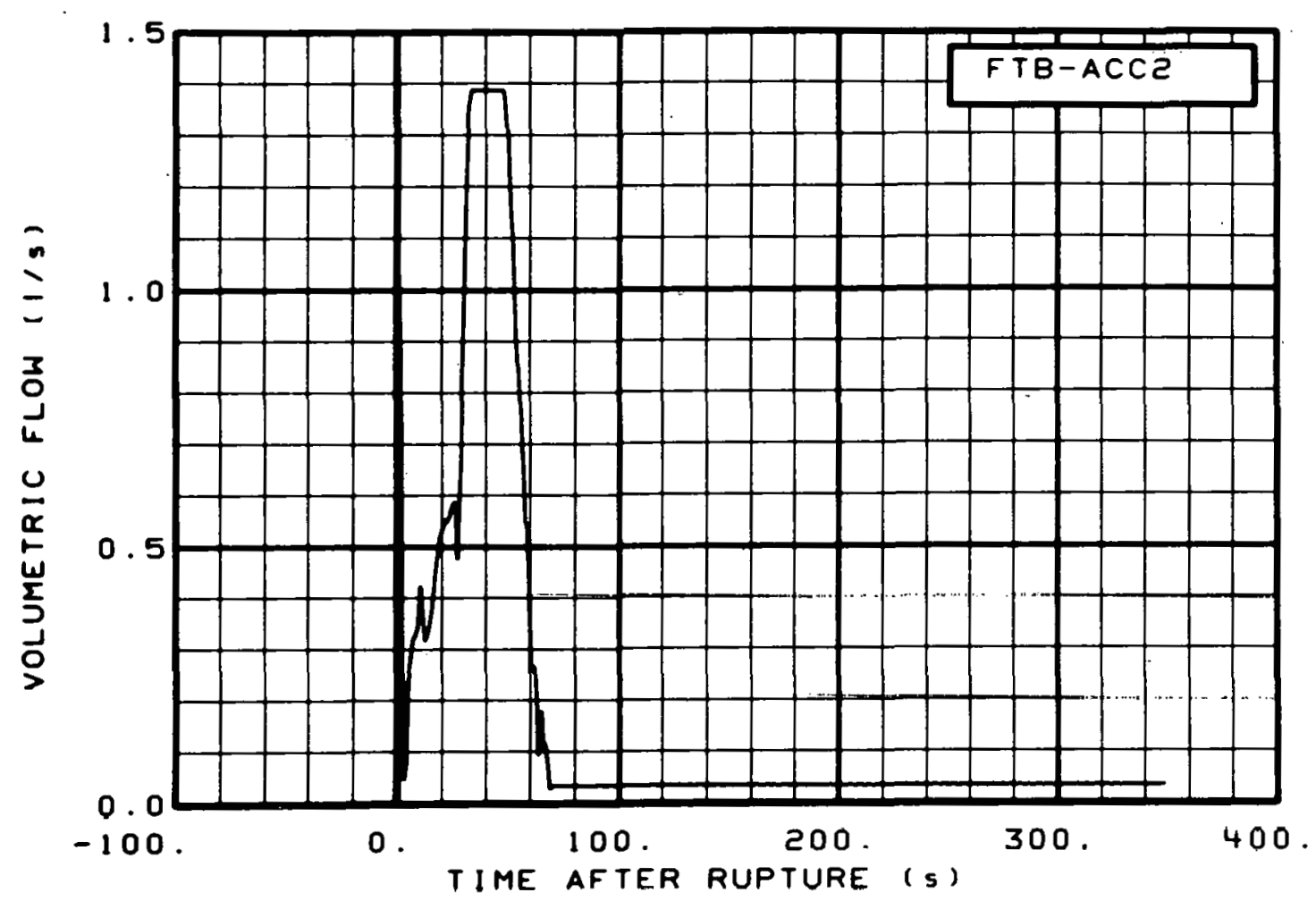

Fig. 261 Volumetric flow in broken loop accurrulator discharge line (FTB-ACC2), from -20 to $350 \mathrm{~s}$.

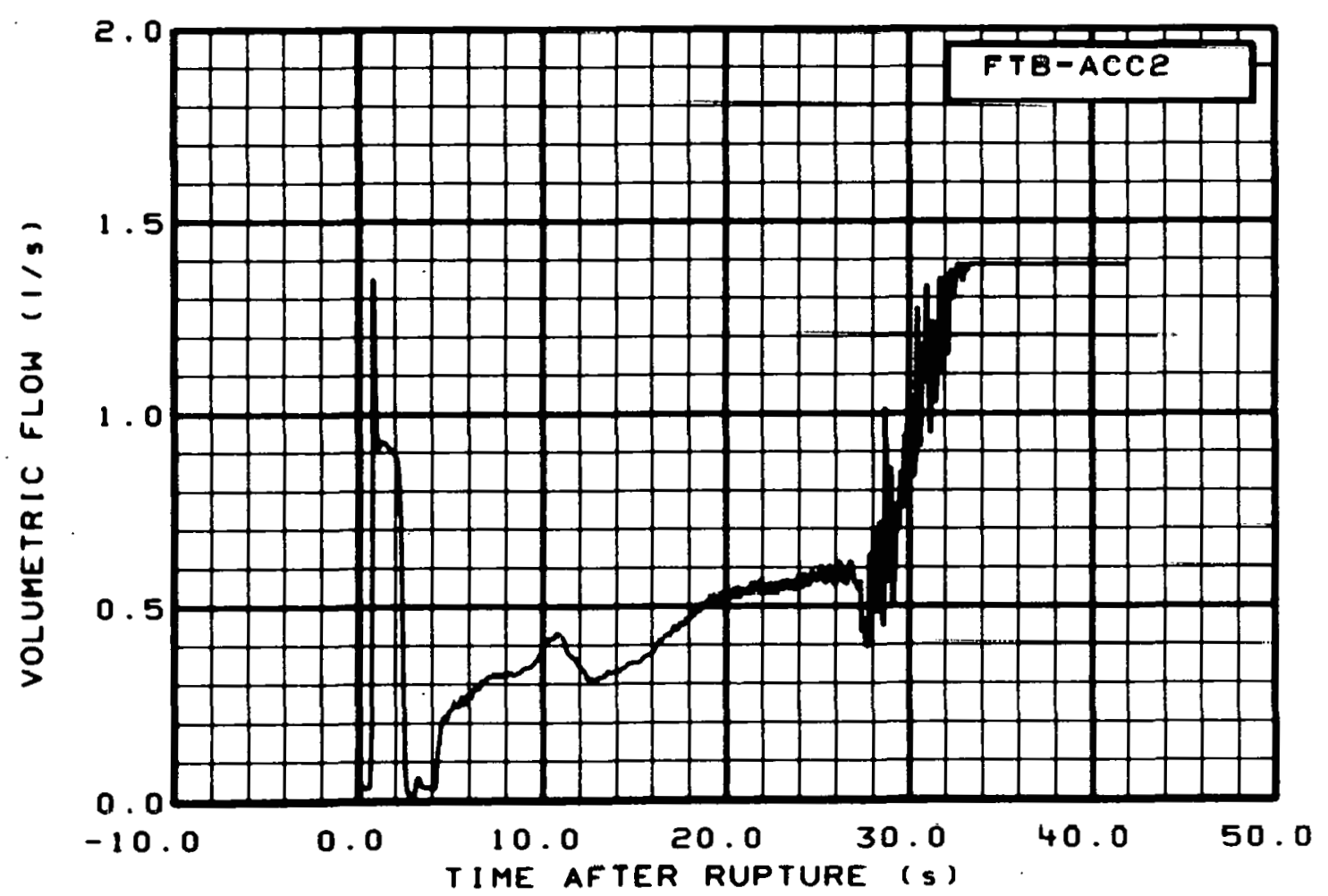

Fig. 262 Volumetric flow in broken loop accumulator discharge line (FTB-ACC2), from -6 to $42 \mathrm{~s}$. 


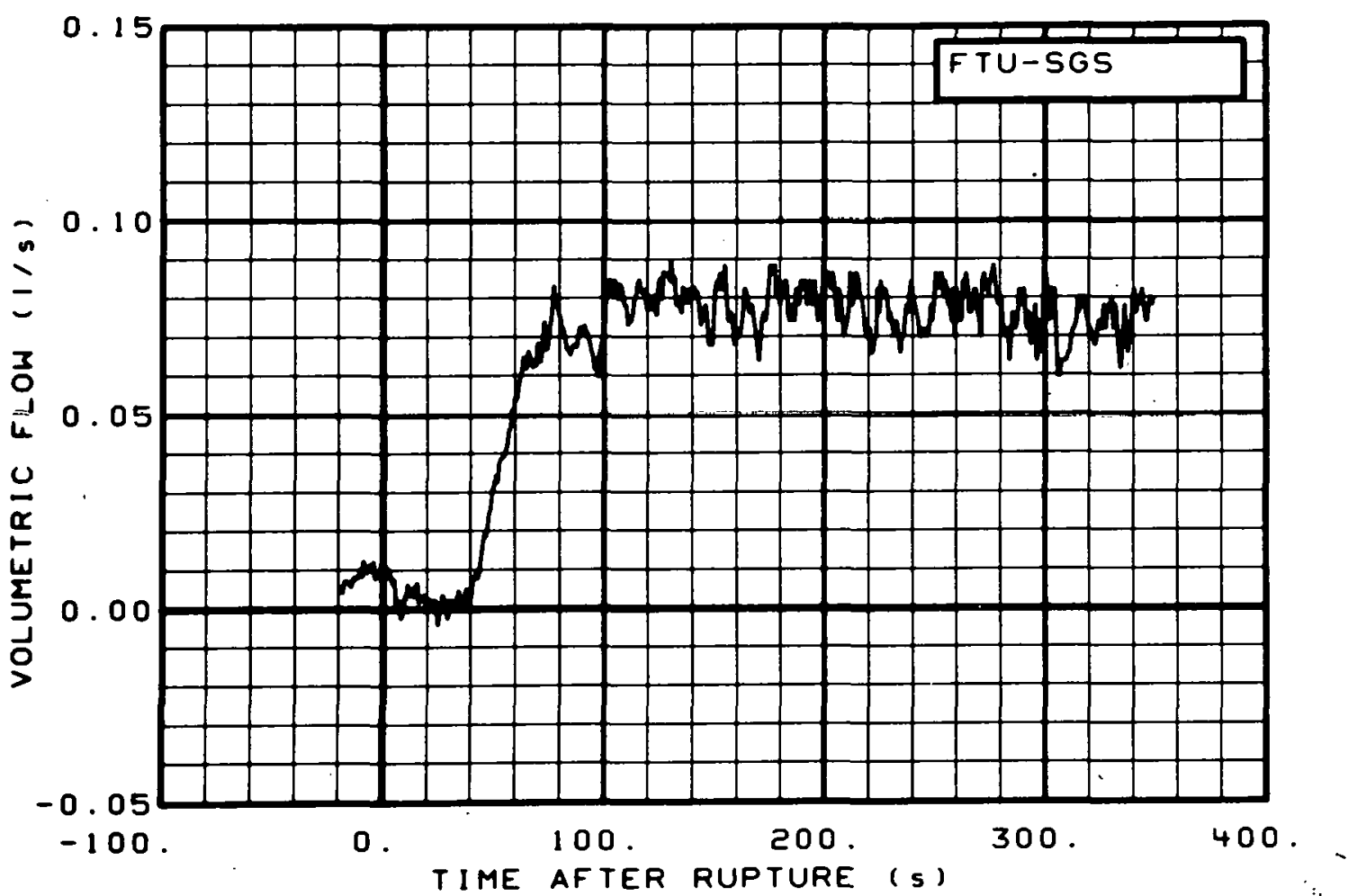

Fig. 263 Volumetric flow in intact loop steam generator (FTU-SGS), from -20 to $350 \mathrm{~s}$.

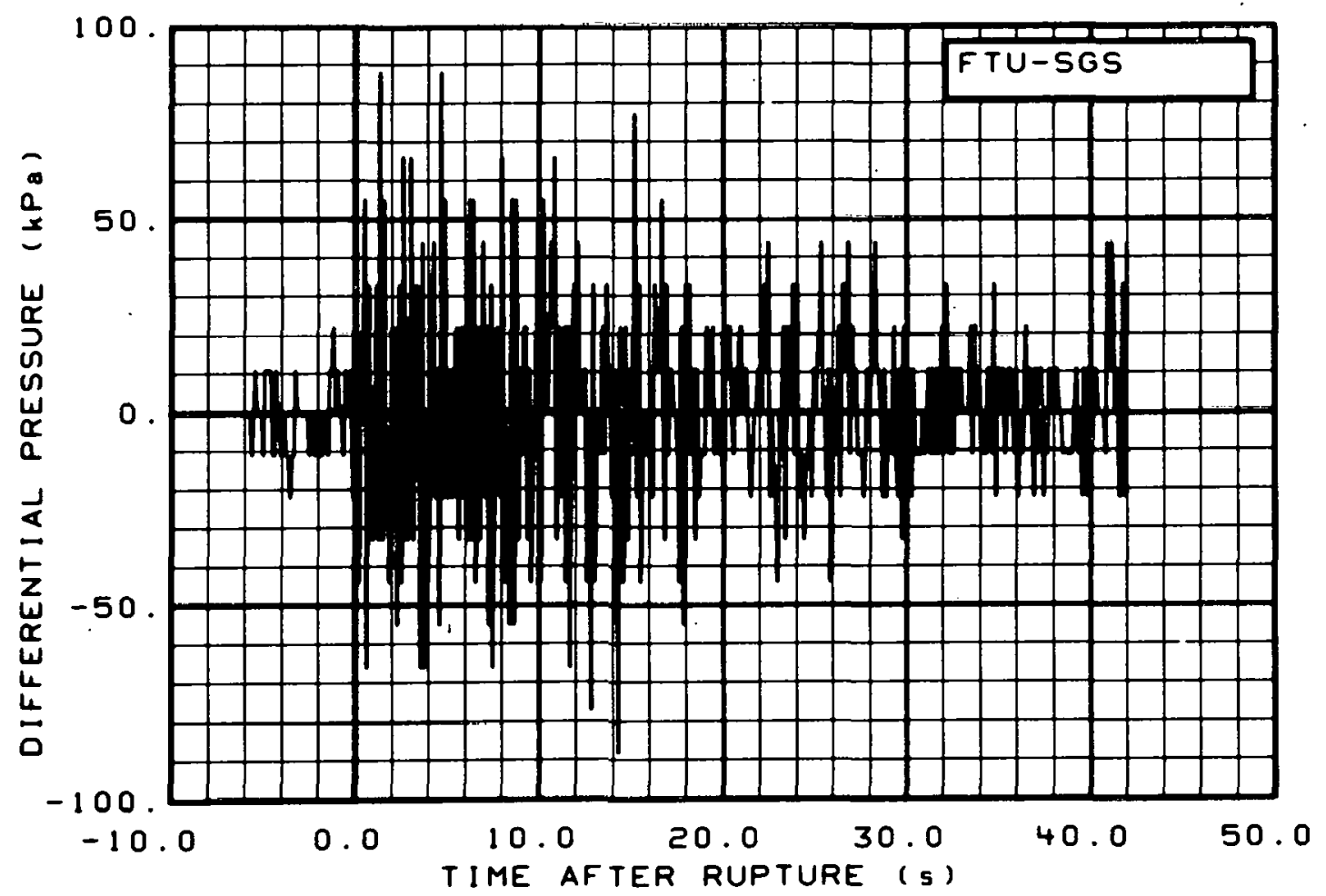

Fig. 264 Volumetric flow in intact loop steam generator (FTU-SGS), from -6 to $42 \mathrm{~s}$. 


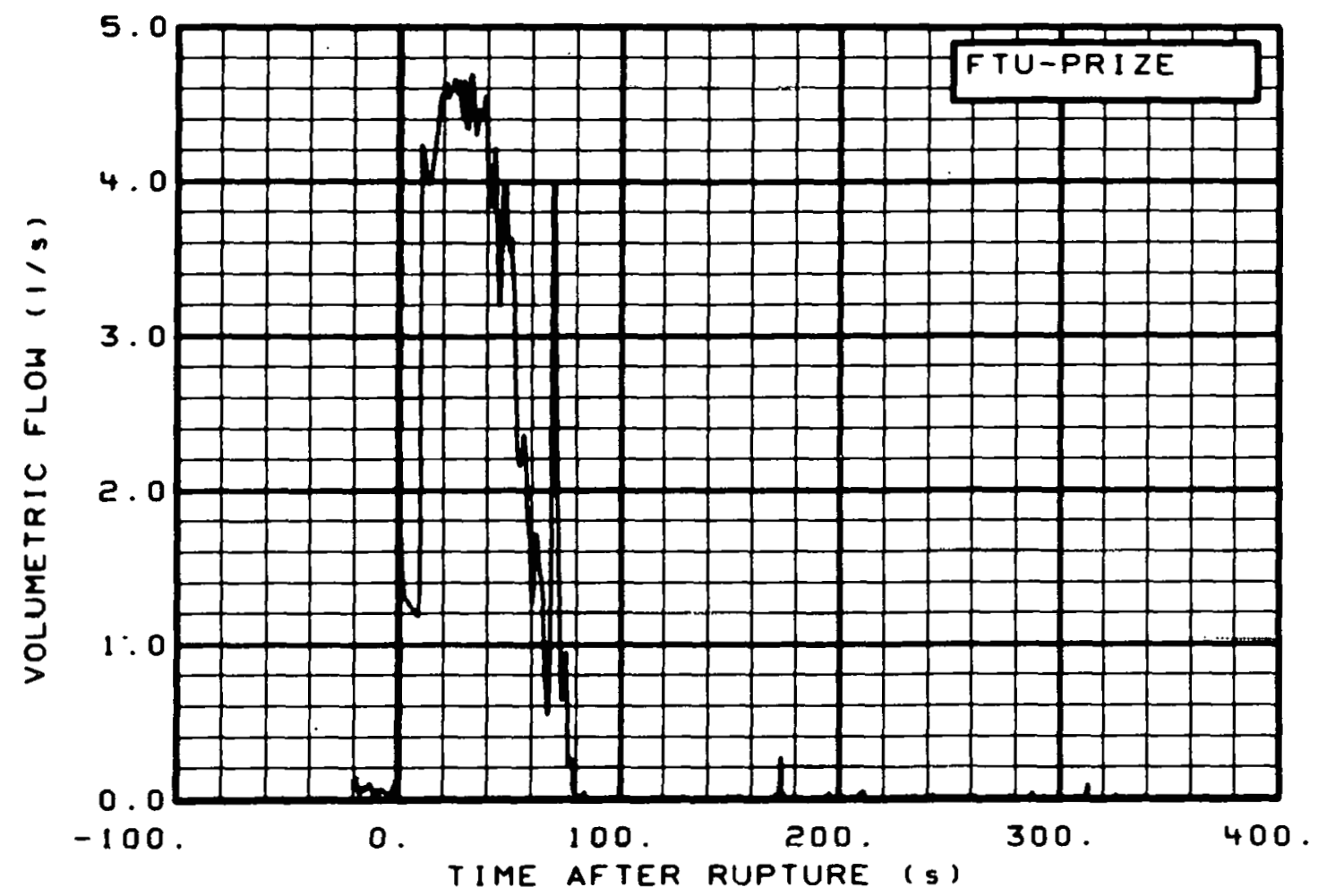

Fig. 265 Volumetric flow from pressurizer (FTU-PRIZE), from -20 to $350 \mathrm{~s}$.

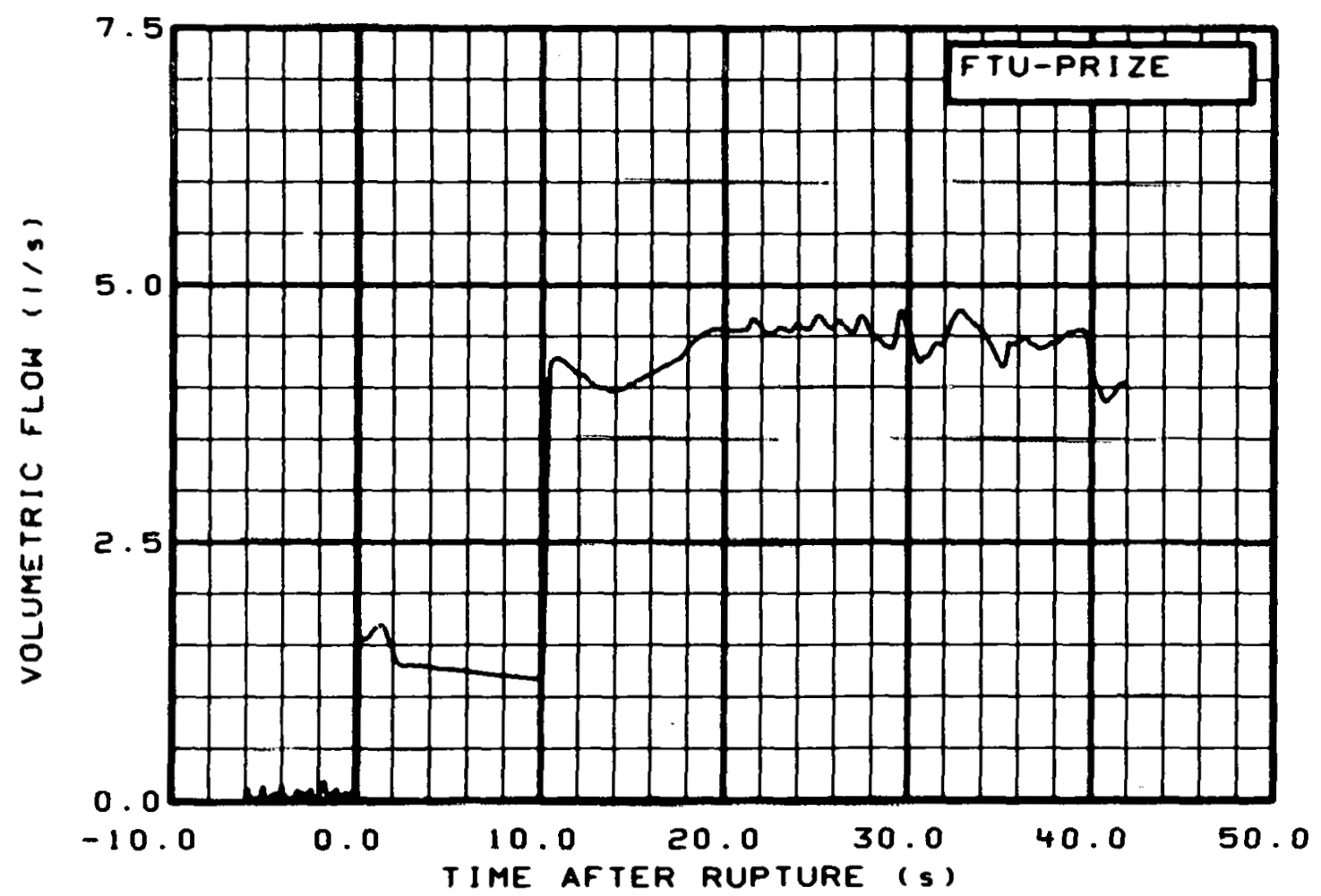

Fig. 266 Volumetric flow from pressurizer (FTU-PRIZE), from -6 to $42 \mathrm{~s}$. 


$$
\frac{r}{m^{2}}
$$




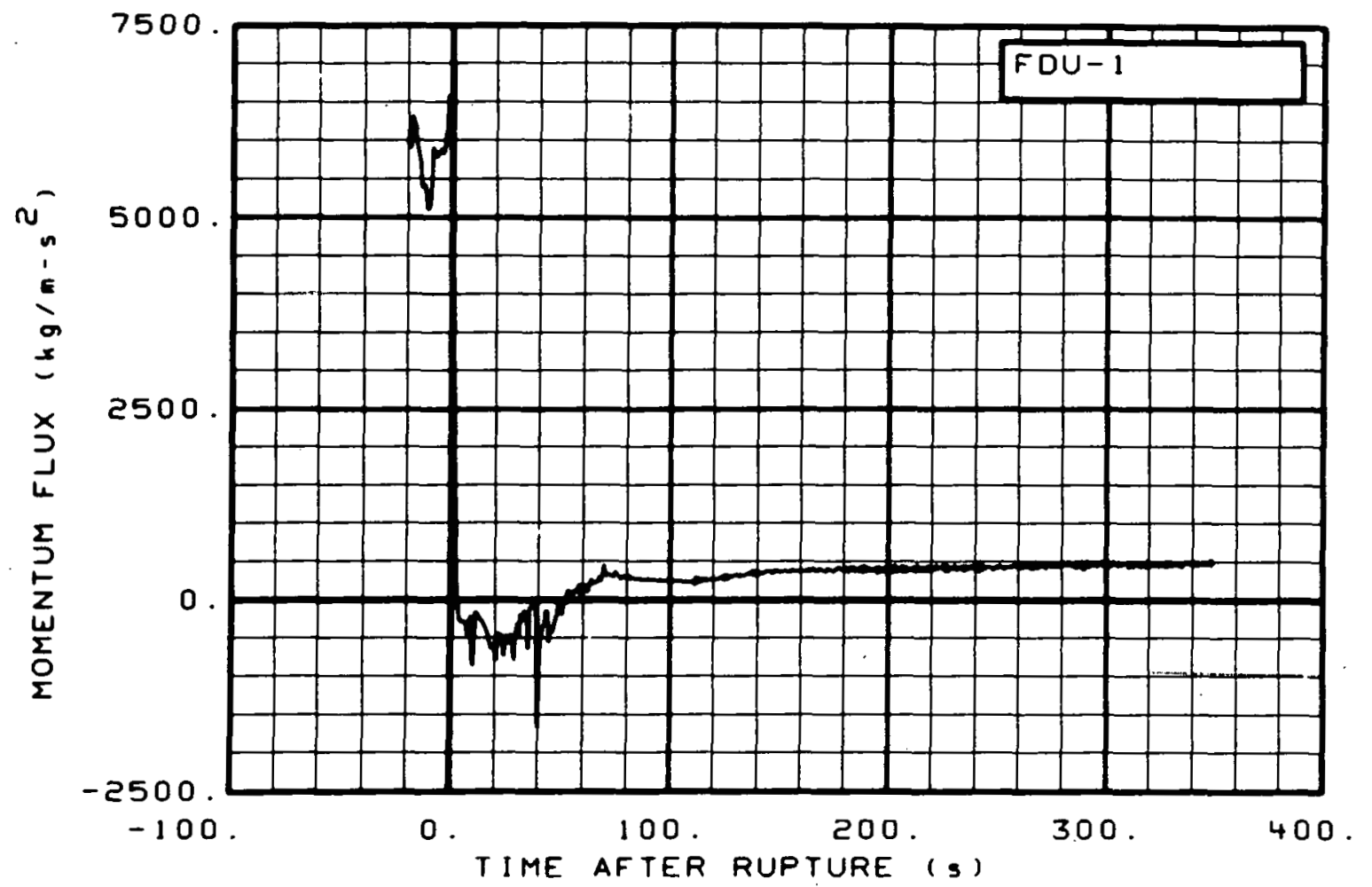

Fig. 269 Momentum flux in intact loop (FDU-1), from -20 to $350 \mathrm{~s}$.

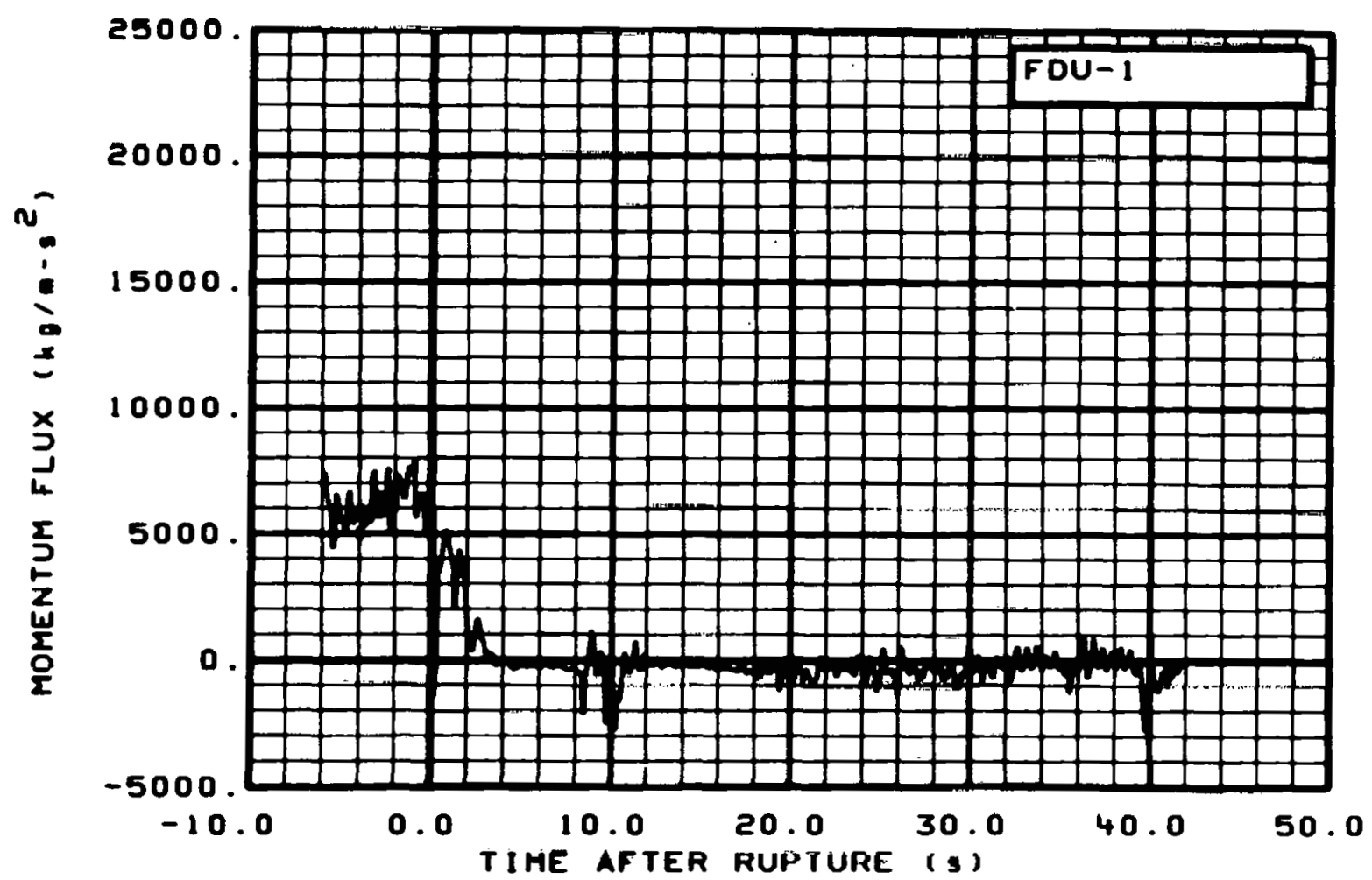

Fig. 270 Momentum flux in intact loop (FDU-1), from -6 to $42 \mathrm{~s}$. 


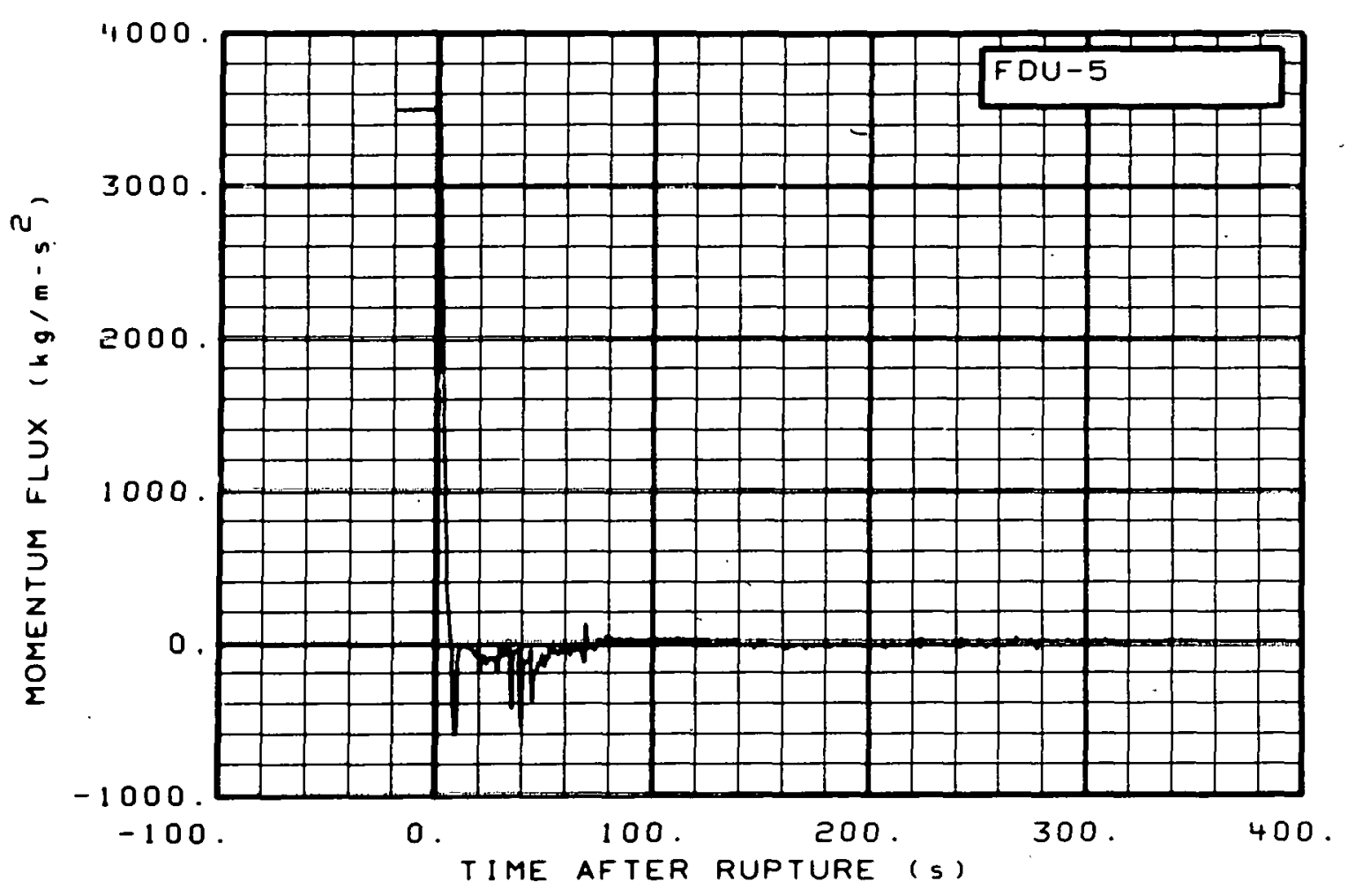

Fig. 271 Momentum flux in intact loop (FDU-5), from -20 to $350 \mathrm{~s}$.

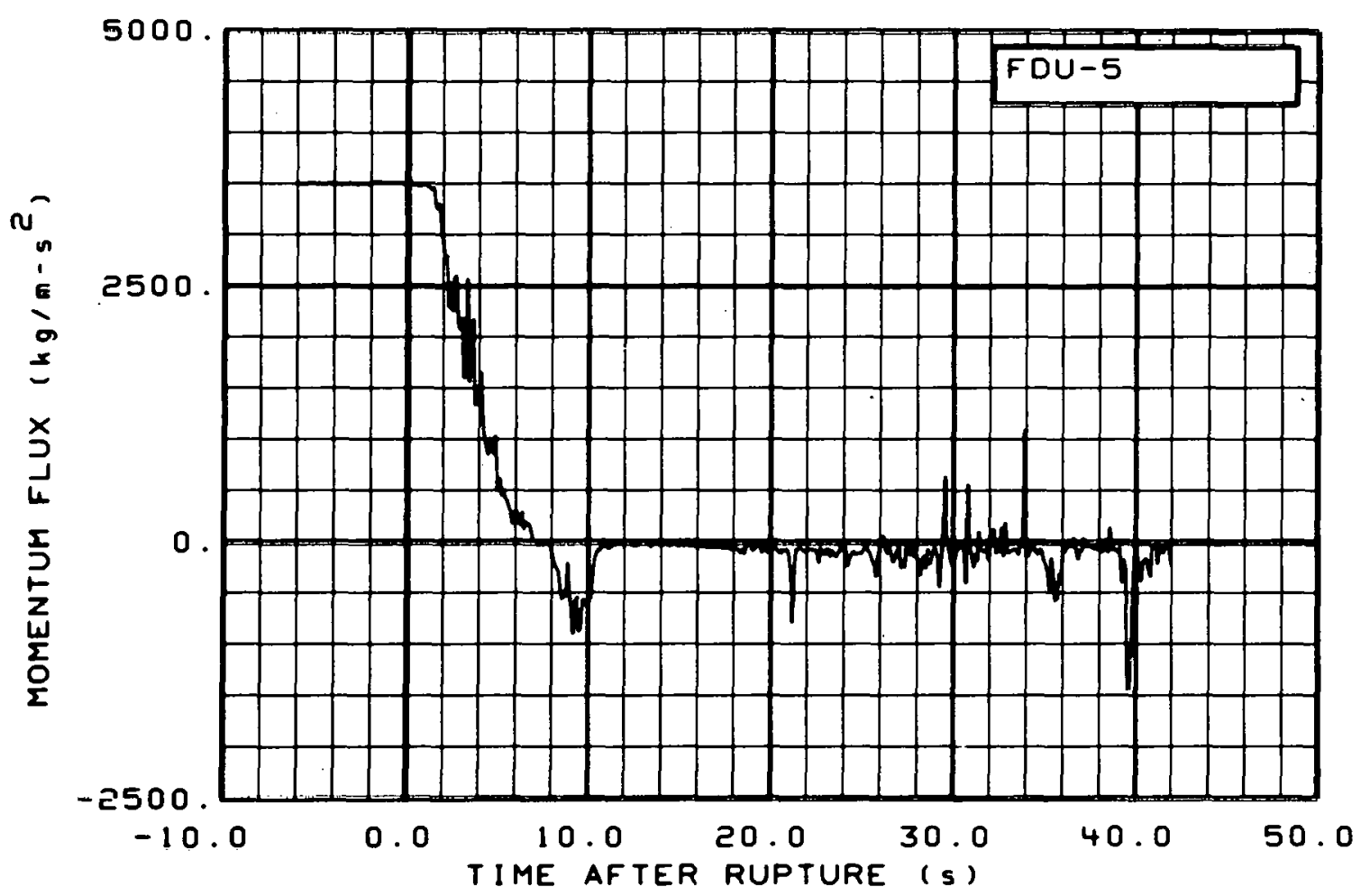

Fig. 272 Momentum flux in intact loop (FDU-5), from -6 to $42 \mathrm{~s}$. 


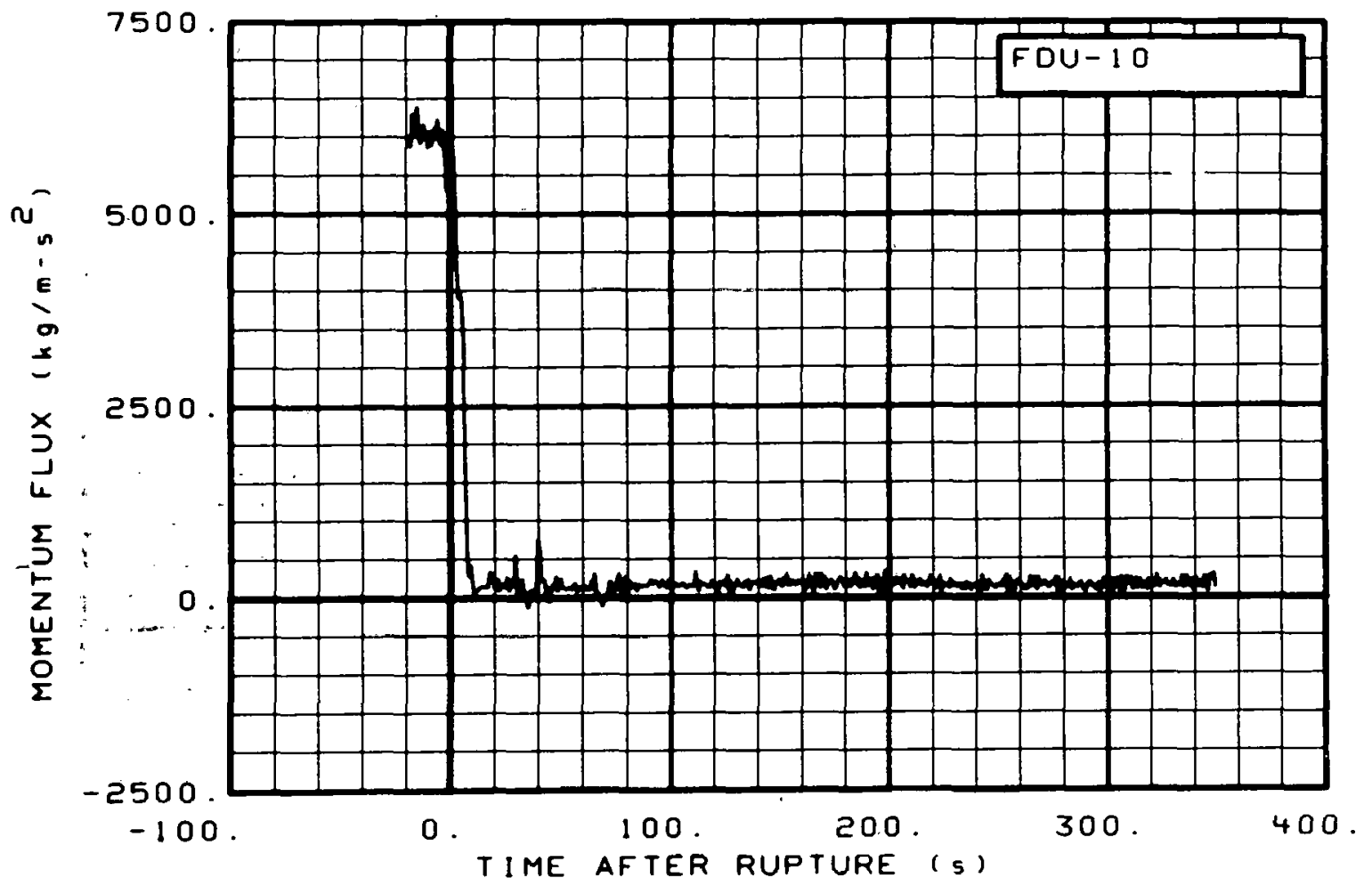

Fig. 273 Momentum flux in intact loop (FDU-10), from -20 to $350 \mathrm{~s}$.

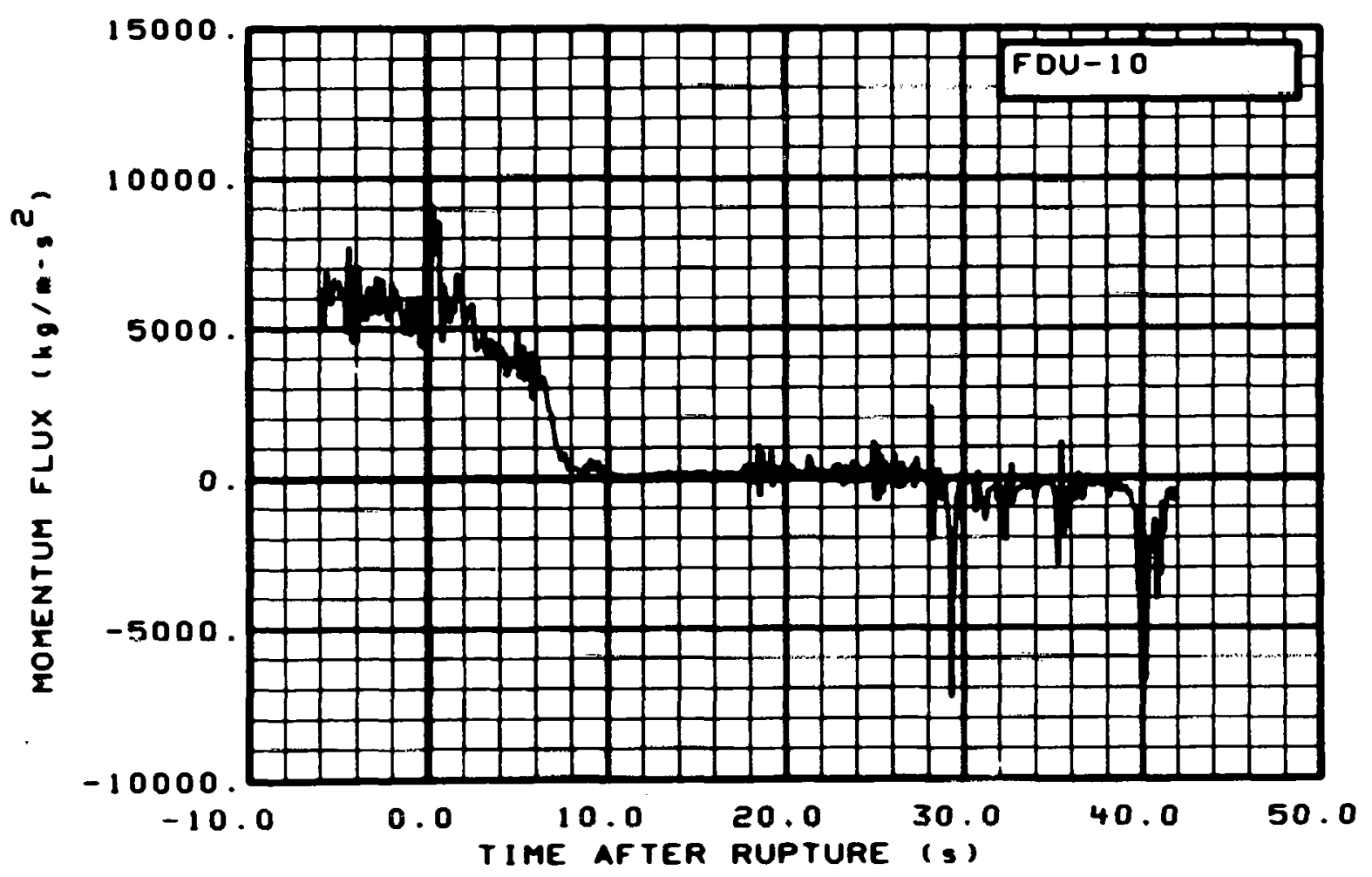

Fig. 274 Momentum flux in intact loop (FDU-10), from -6 to $42 \mathrm{~s}$. 


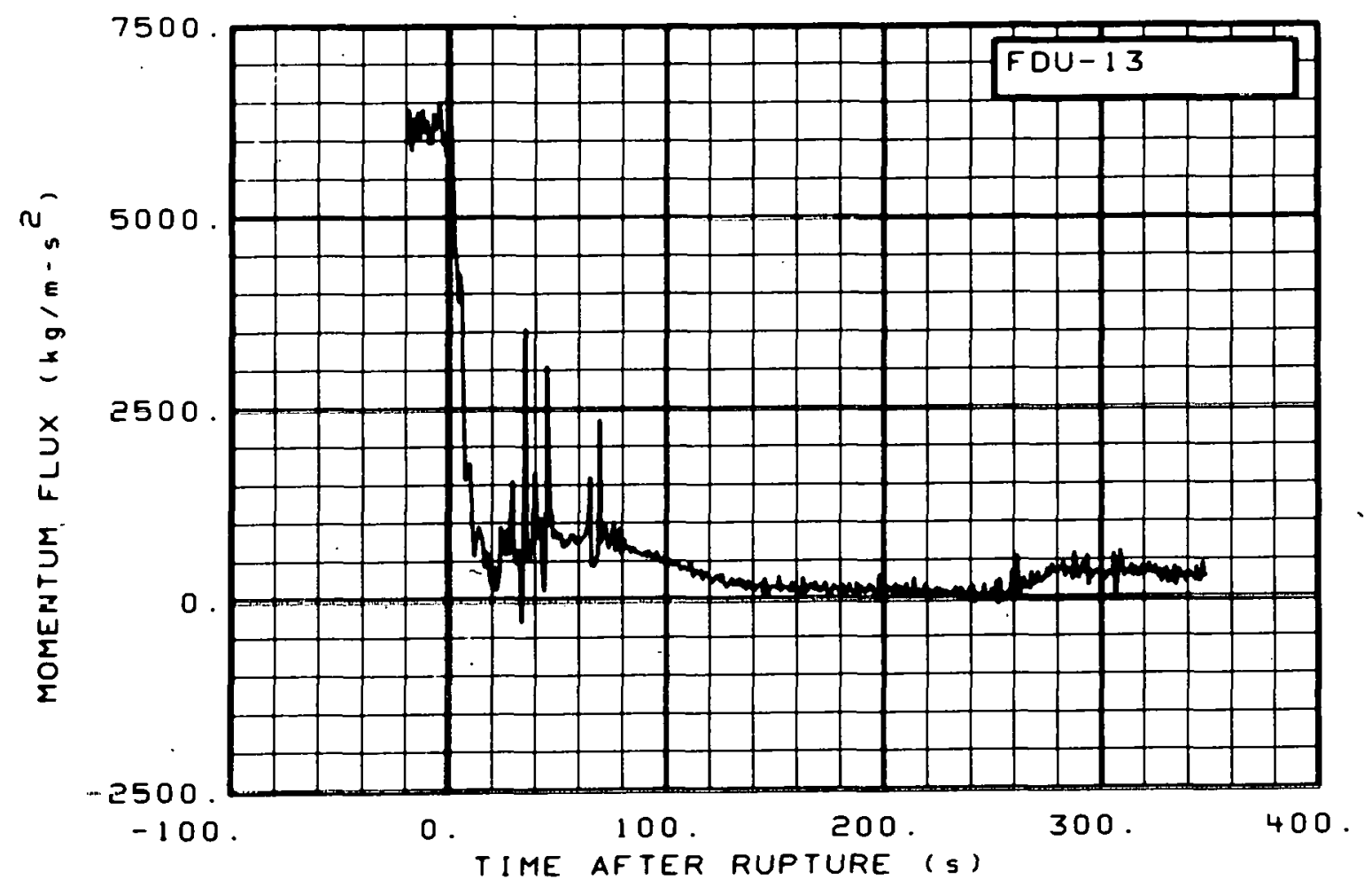

Fig. 275 Momentum flux in intact loop (FDU-13), from -20 to $350 \mathrm{~s}$.

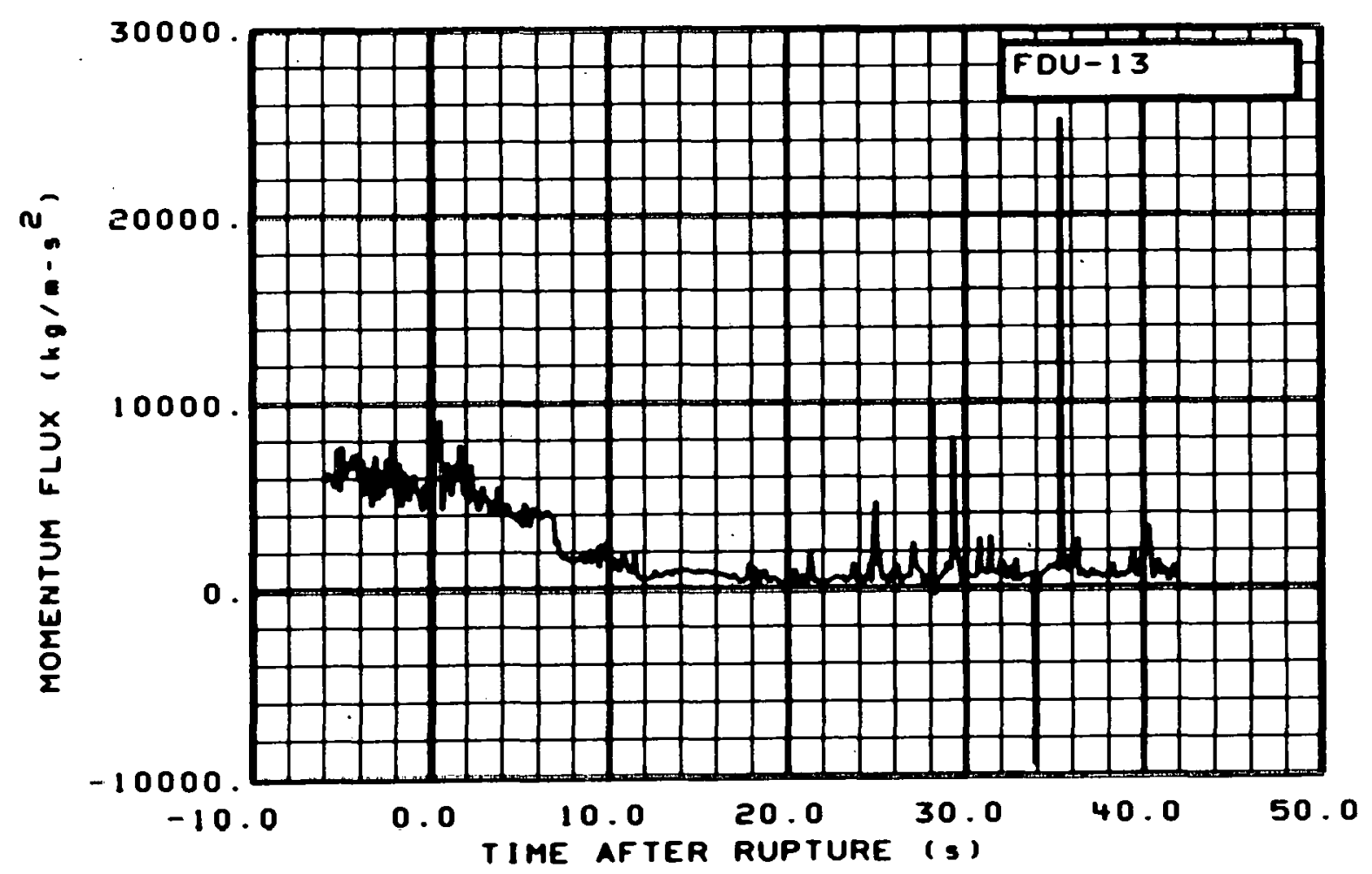

Fig. 276 Momentum flux in intact loop (FDU-13), from -6 to $42 \mathrm{~s}$. 


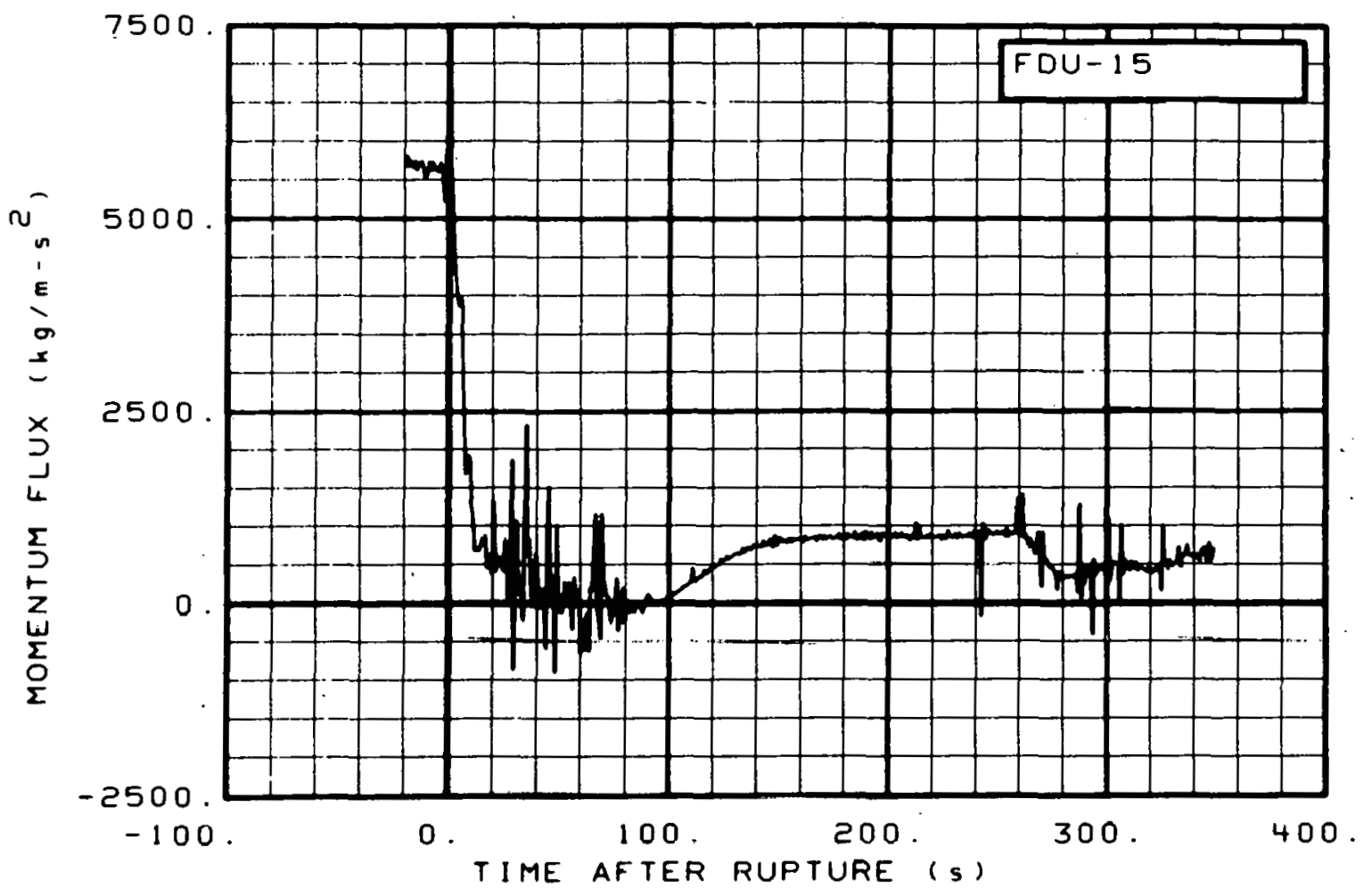

Fig. 277 Momentum flux in intact loop (FDU-15), from -20 to $350 \mathrm{~s}$.

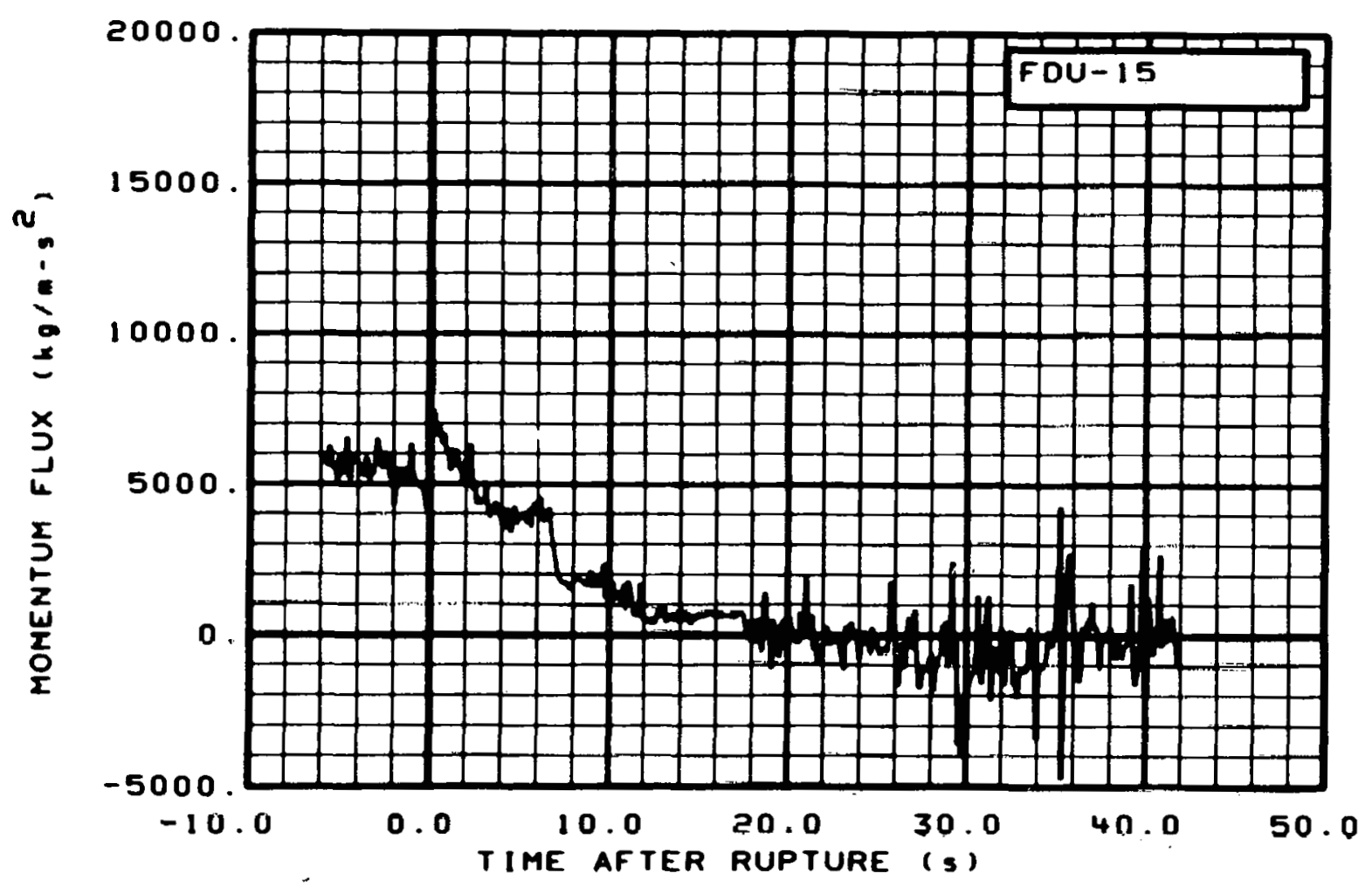

Fig. 278 Momentum flux in intact loop (FDU-15), from -6 to $42 \mathrm{~s}$. 


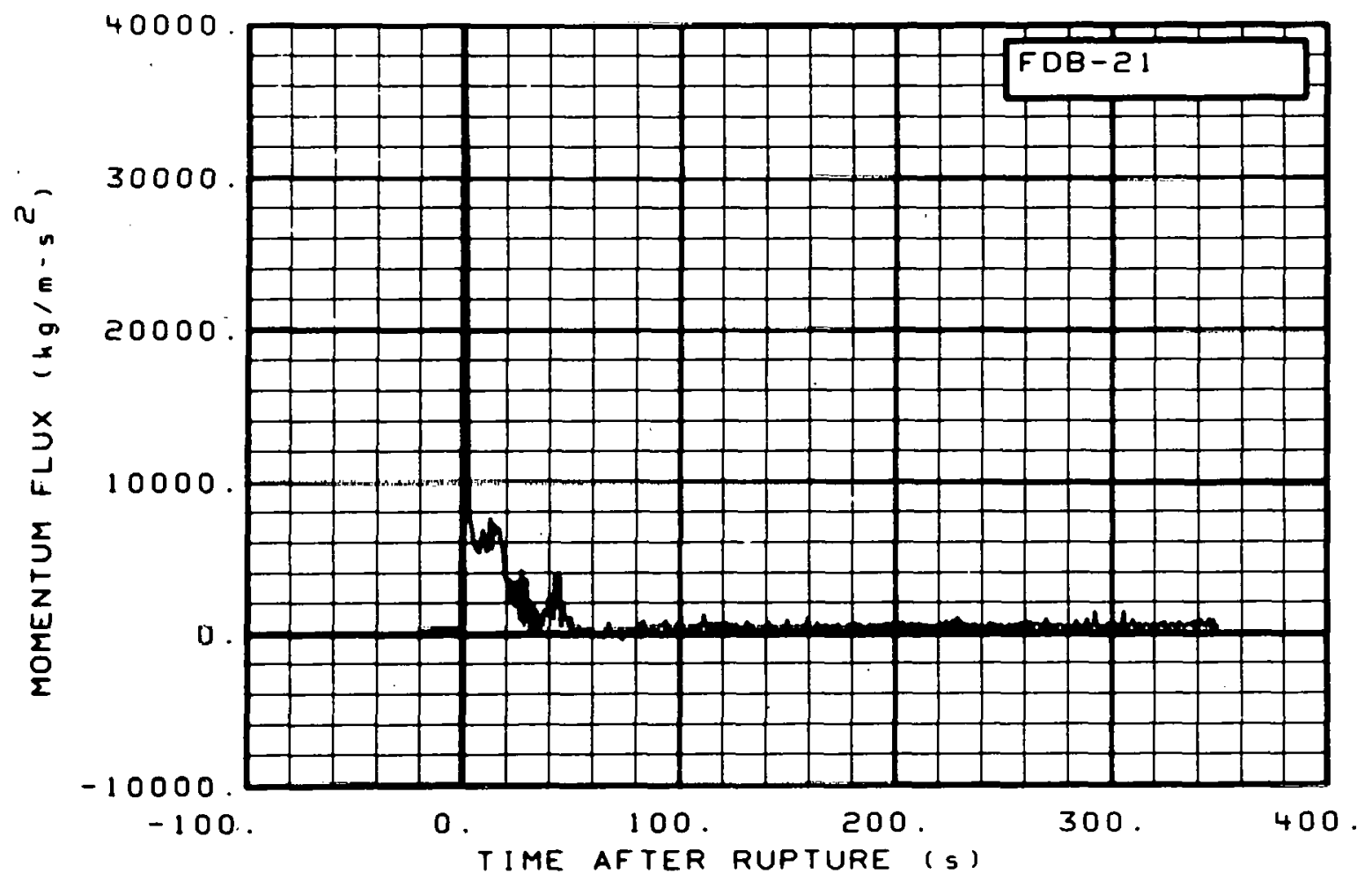

Fig. 279 Momentum flux in broken loop (FDB-21), from -20 to $350 \mathrm{~s}$.

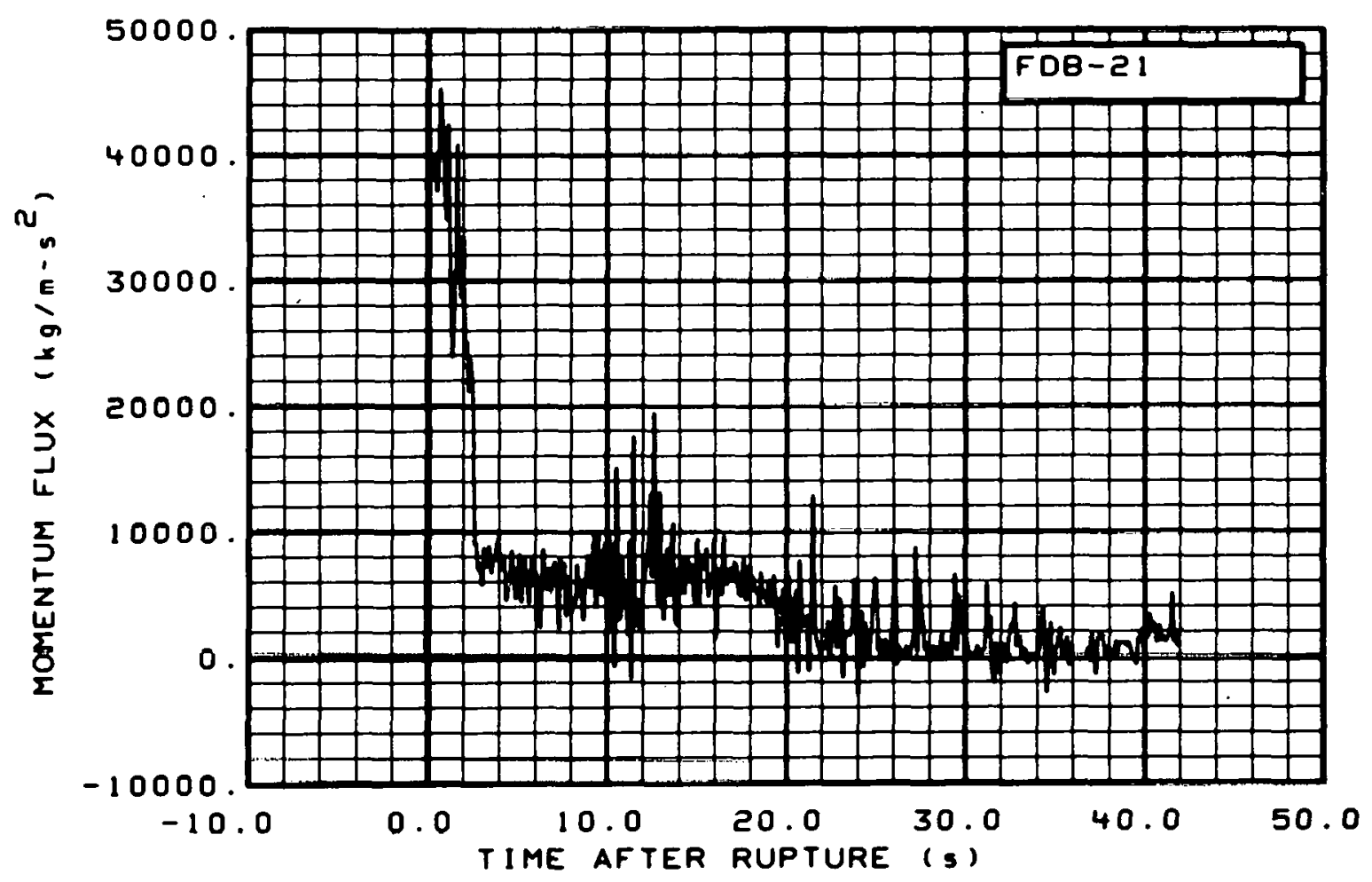

Fig. 280 Momentum flux in broken loop (FDB-21), from -6 to $42 \mathrm{~s}$. 


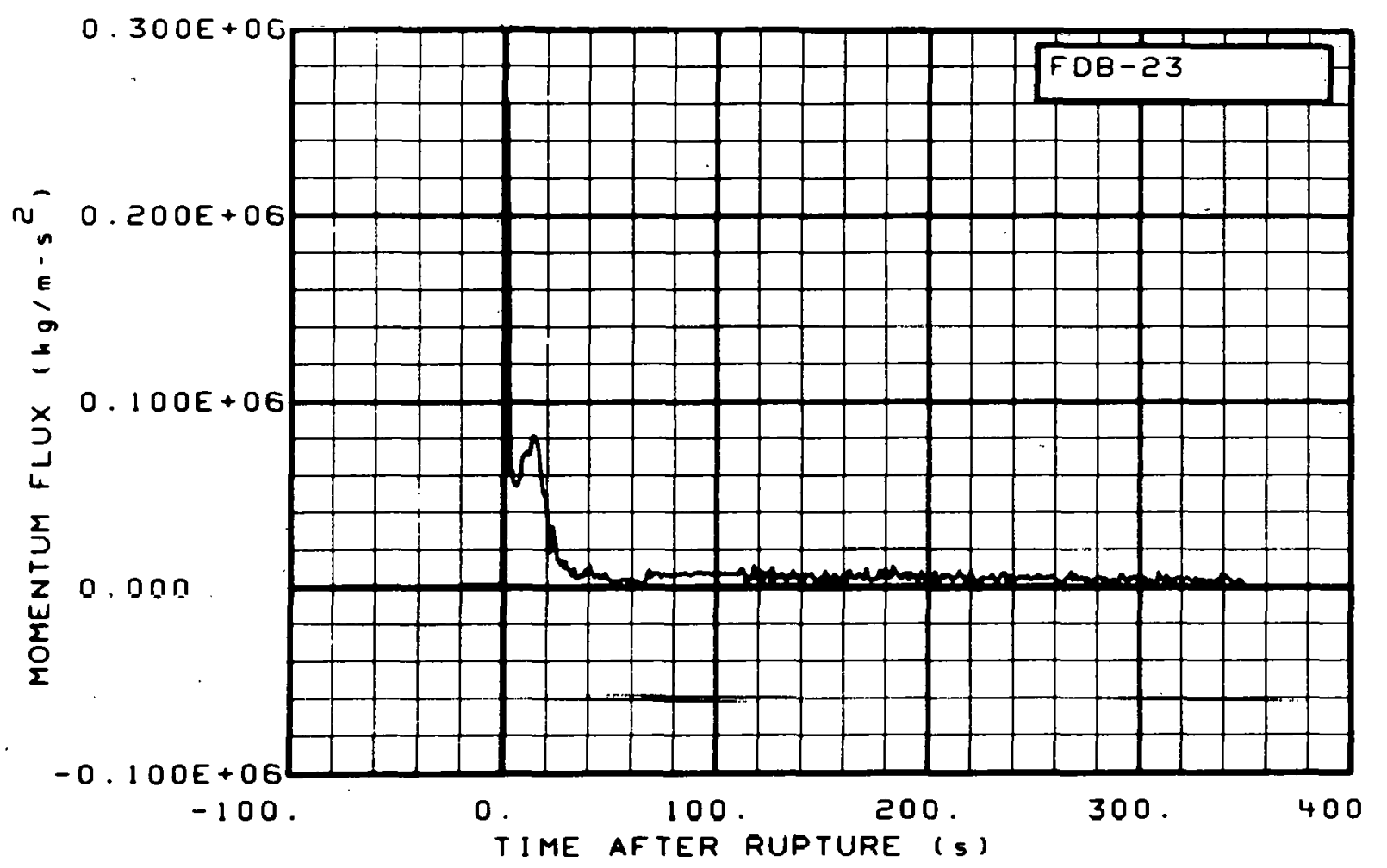

Fig. 281 Momentum flux in broken loop (FDB-23), from -20 to $350 \mathrm{~s}$.

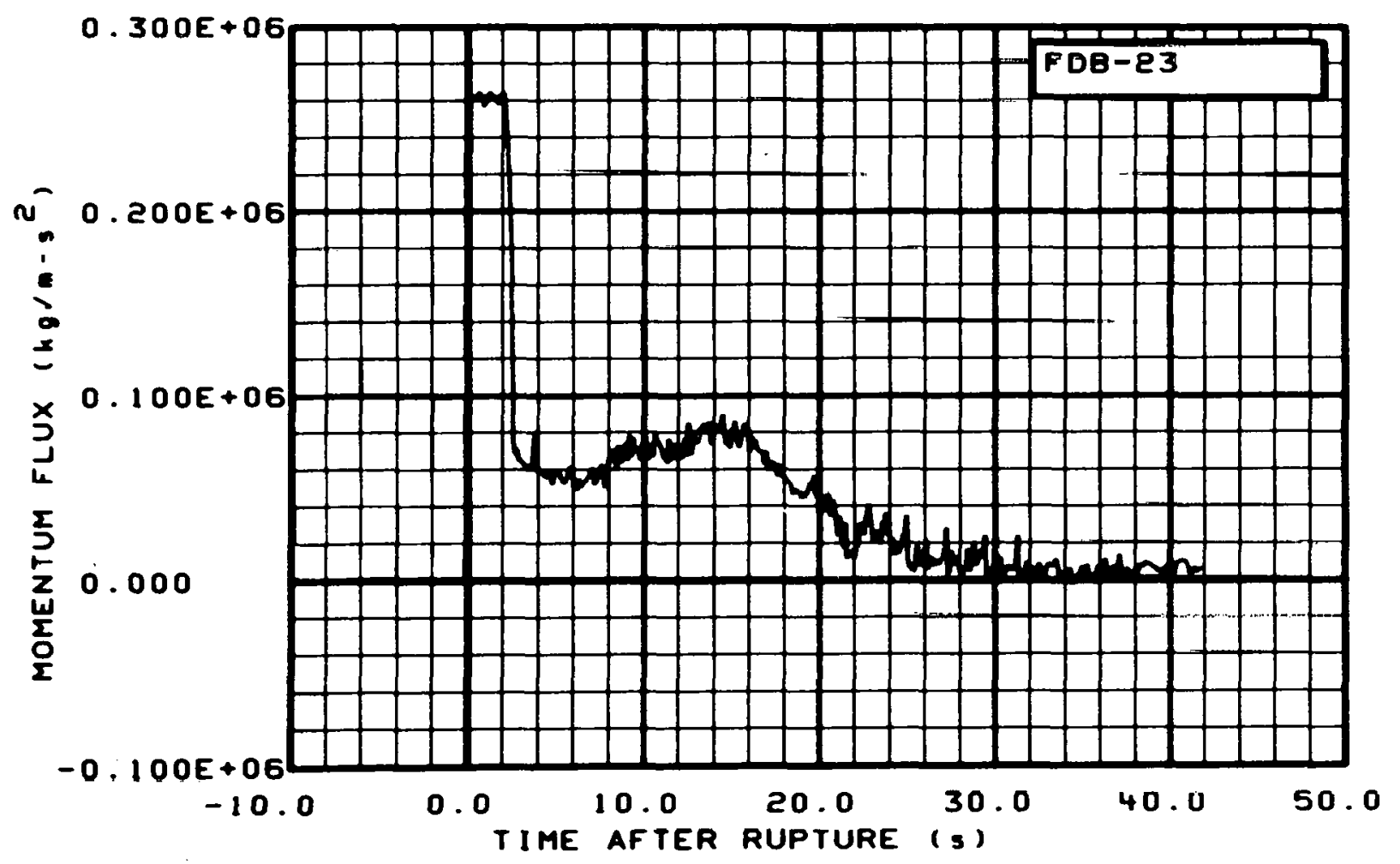

Fig. 282 Momentum flux in broken loop (FDB-23), from -6 to $42 \mathrm{~s}$. 


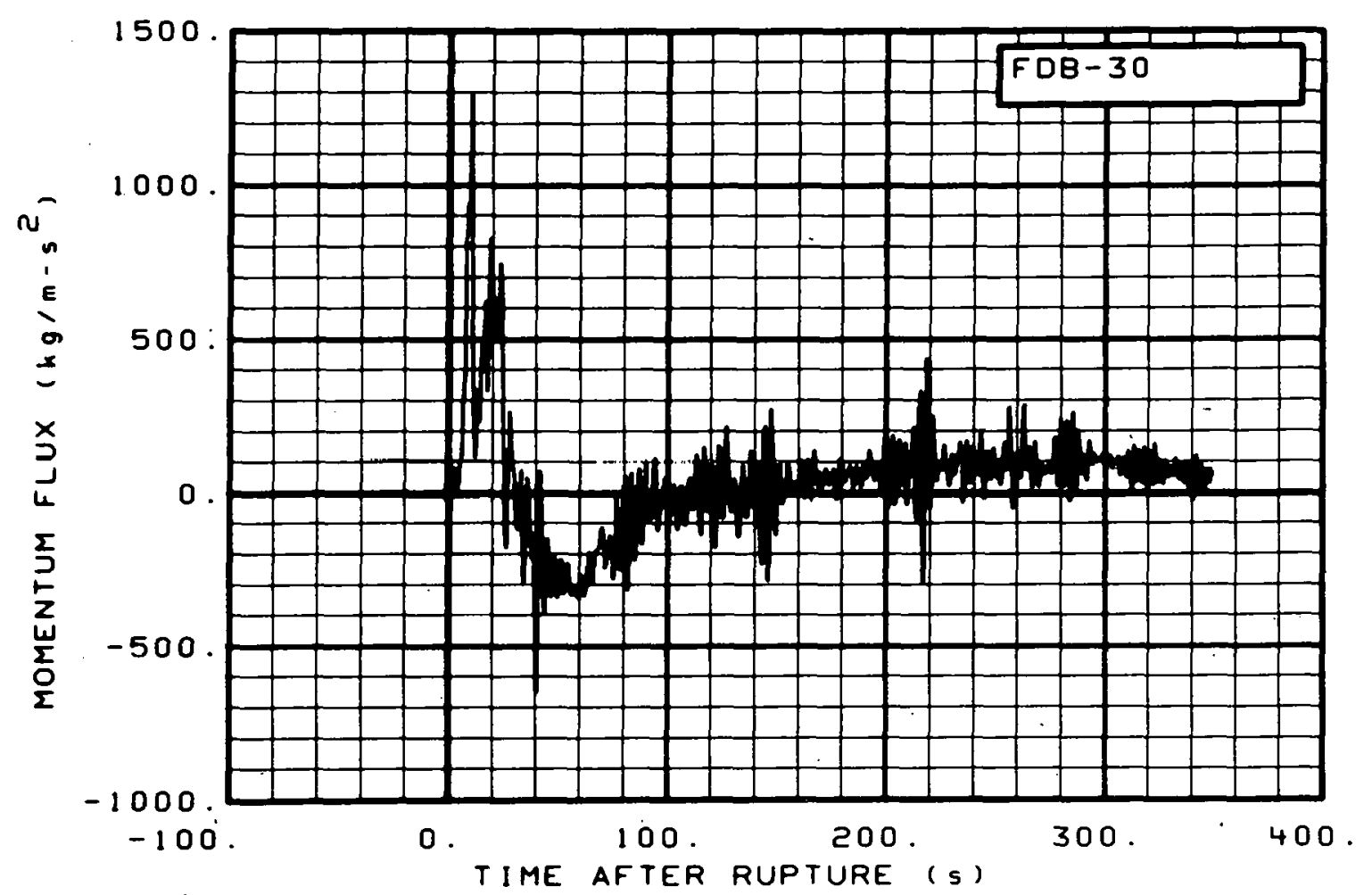

Fig. 283 Momentum flux in broken loop (FDB-30), from -20 to $350 \mathrm{~s}$.

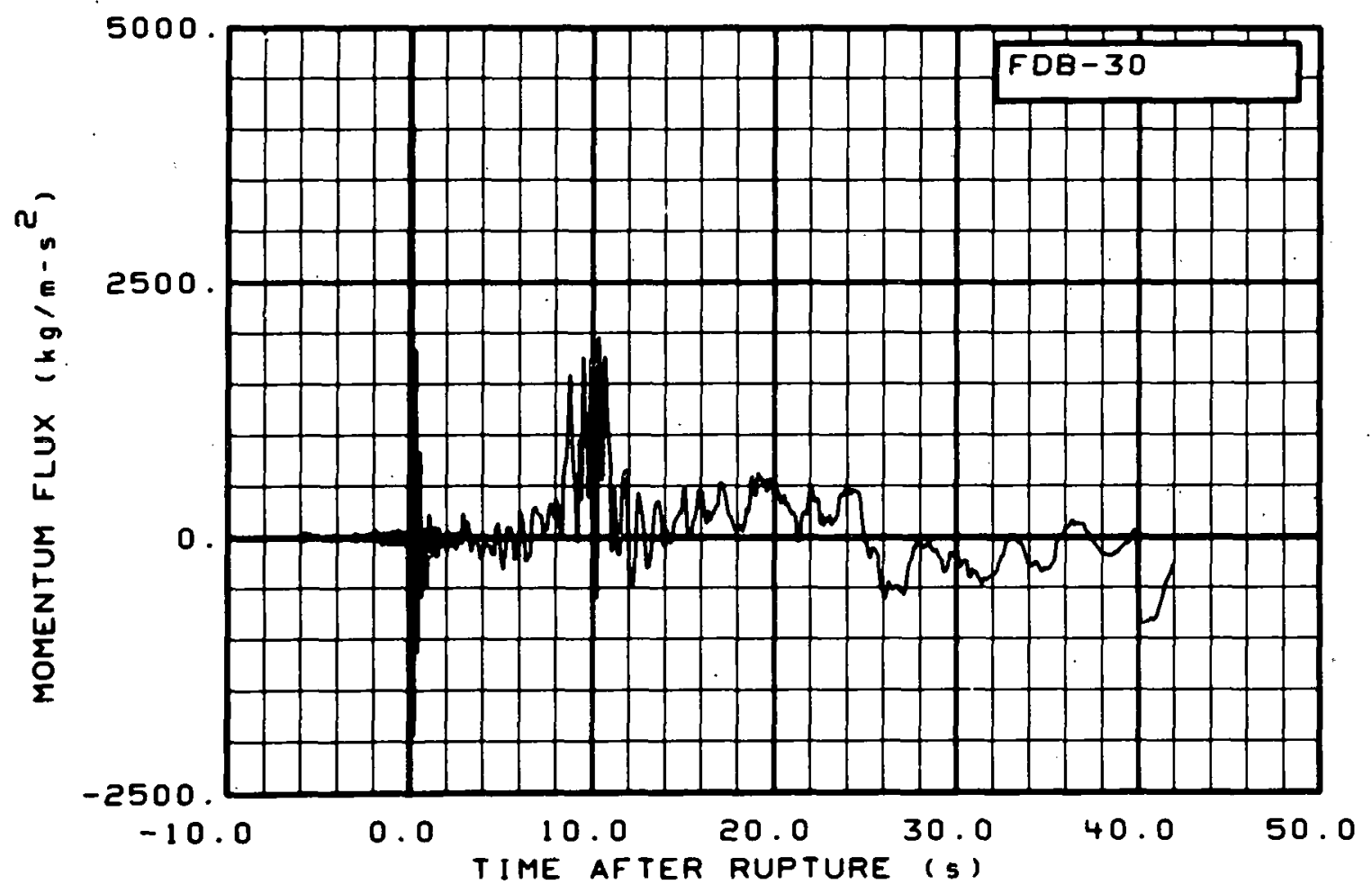

Fig. 284 Momentum flux in broken loop (FDB-30), from . -6 to $42 \mathrm{~s}$. 


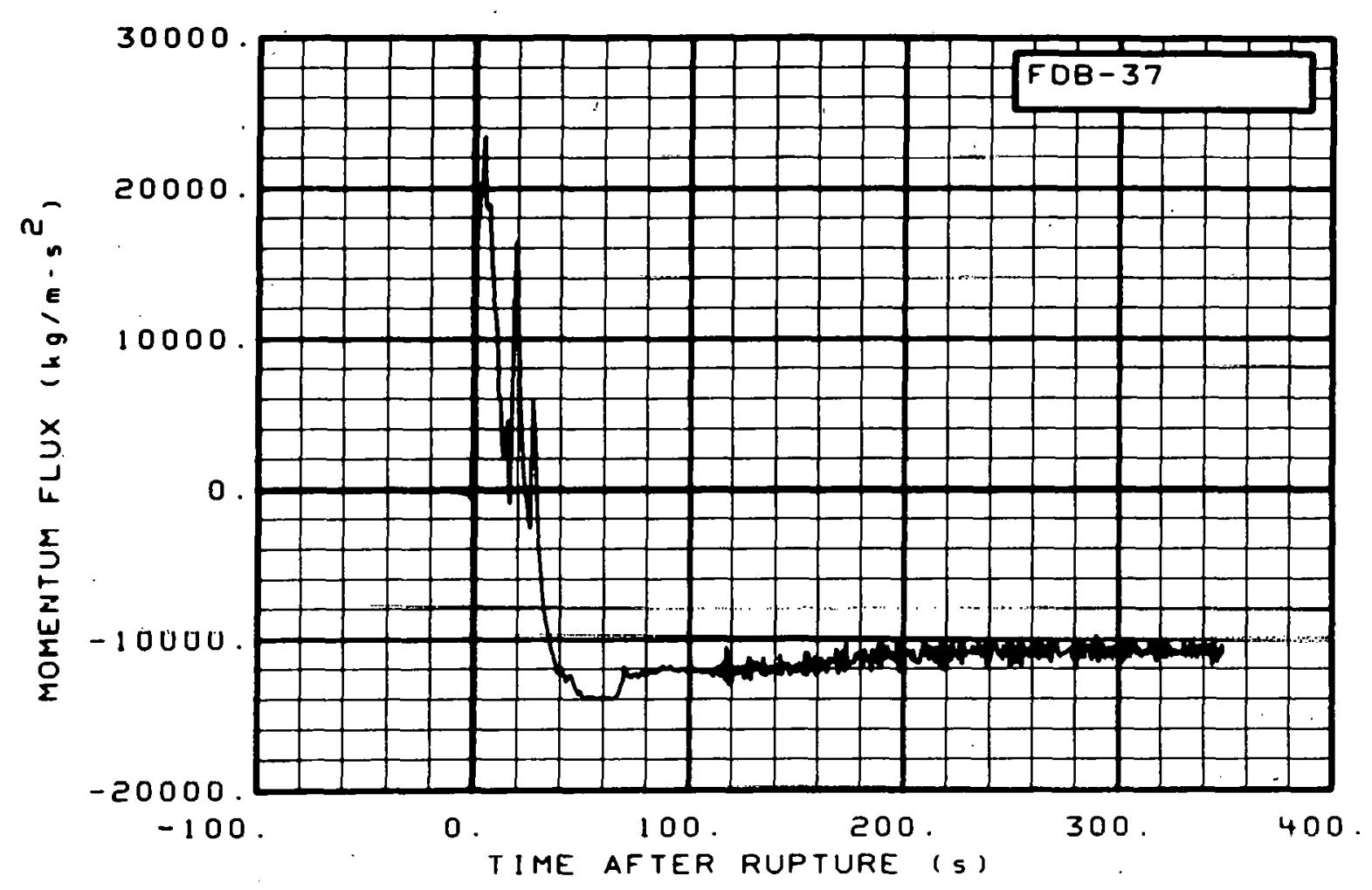

Fig. 285 Momentum flux in broken loop (FDB-37), from -20 to $350 \mathrm{~s}$.

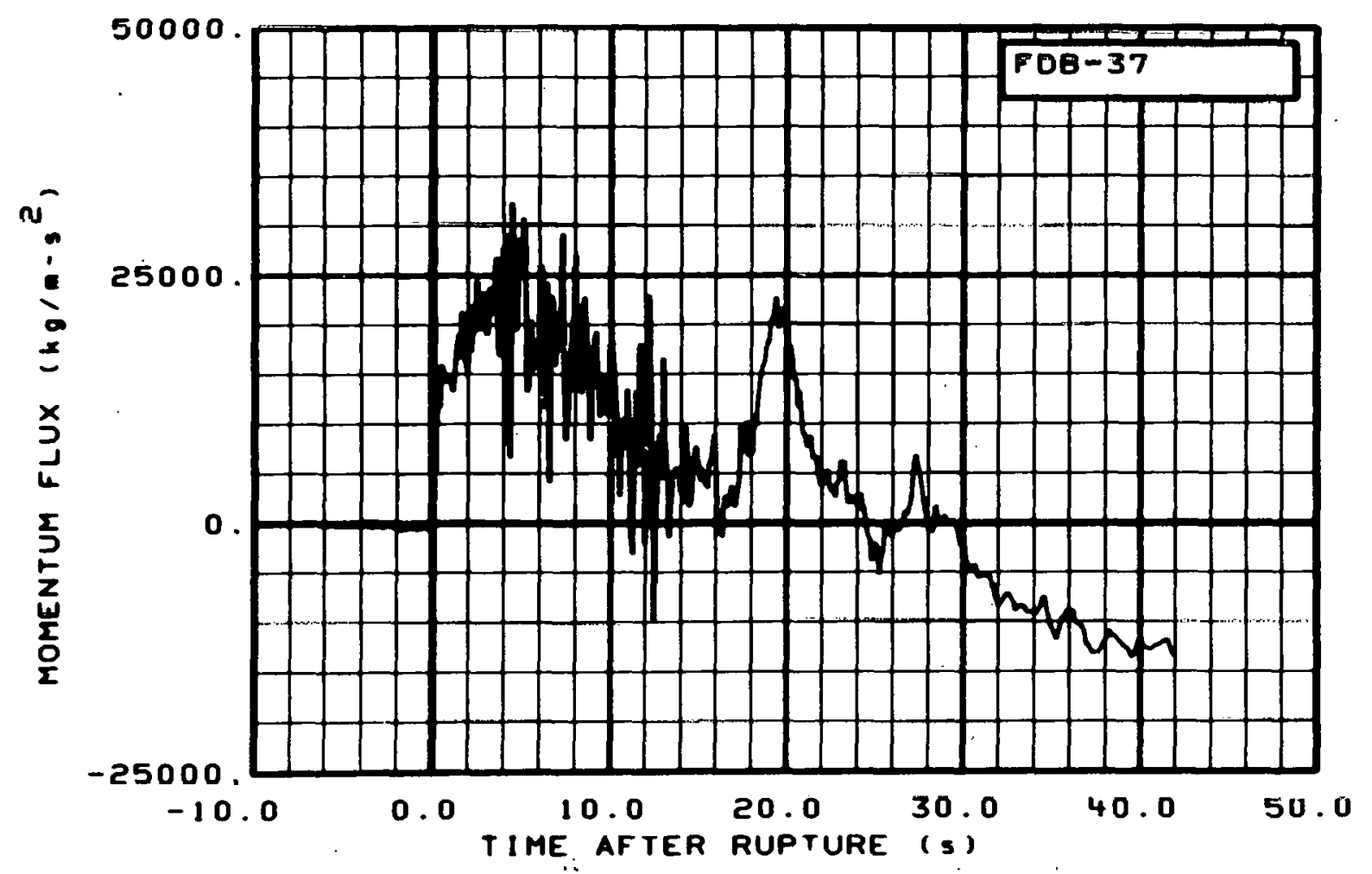

Fig. 286 Momentum flux in broken loop (FDB-37), from -6 to $42 \mathrm{~s}$. 


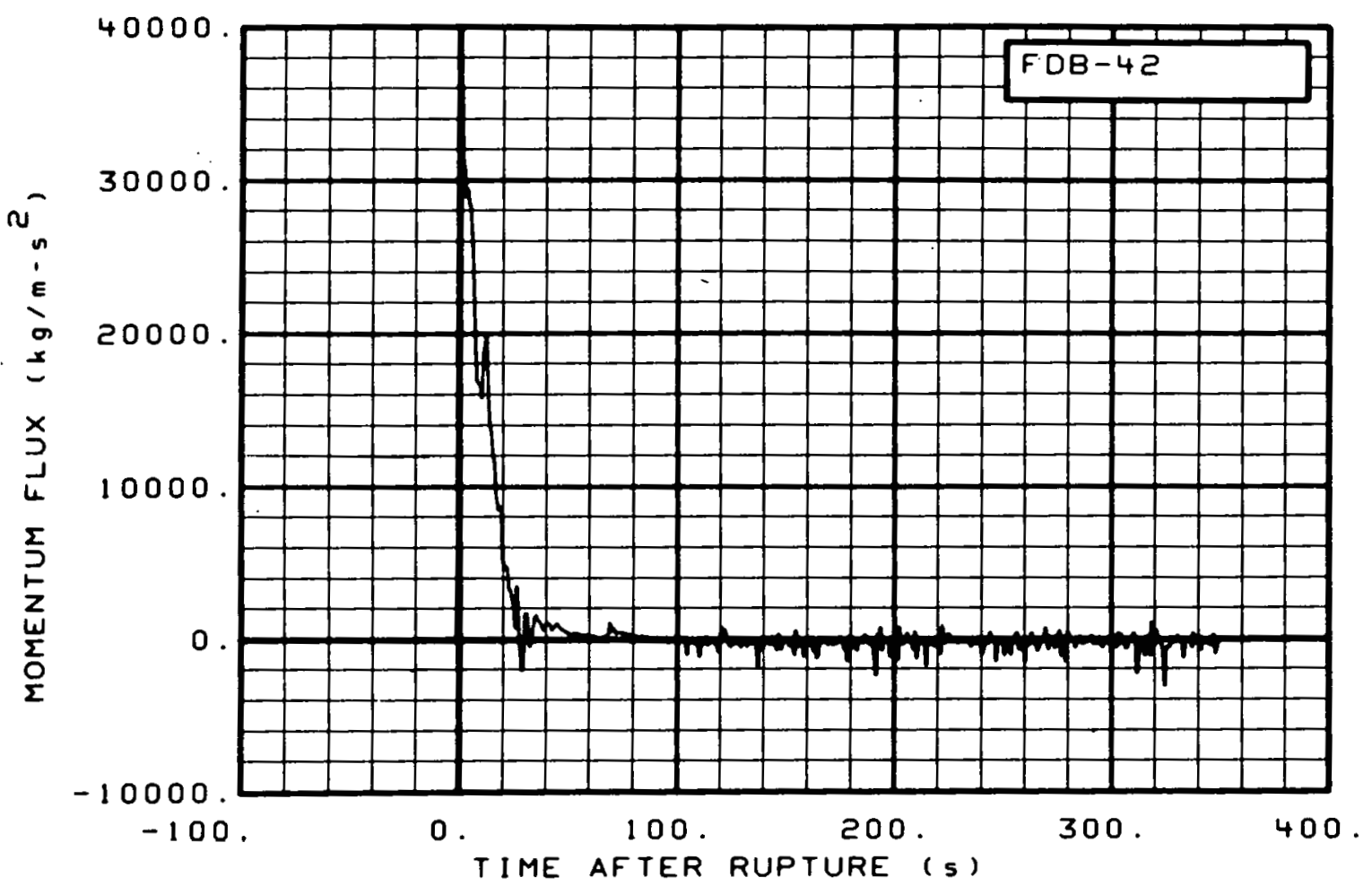

Fig. 287 Momentum flux in broken loop (FDB-42), from -20 to $350 \mathrm{~s}$.

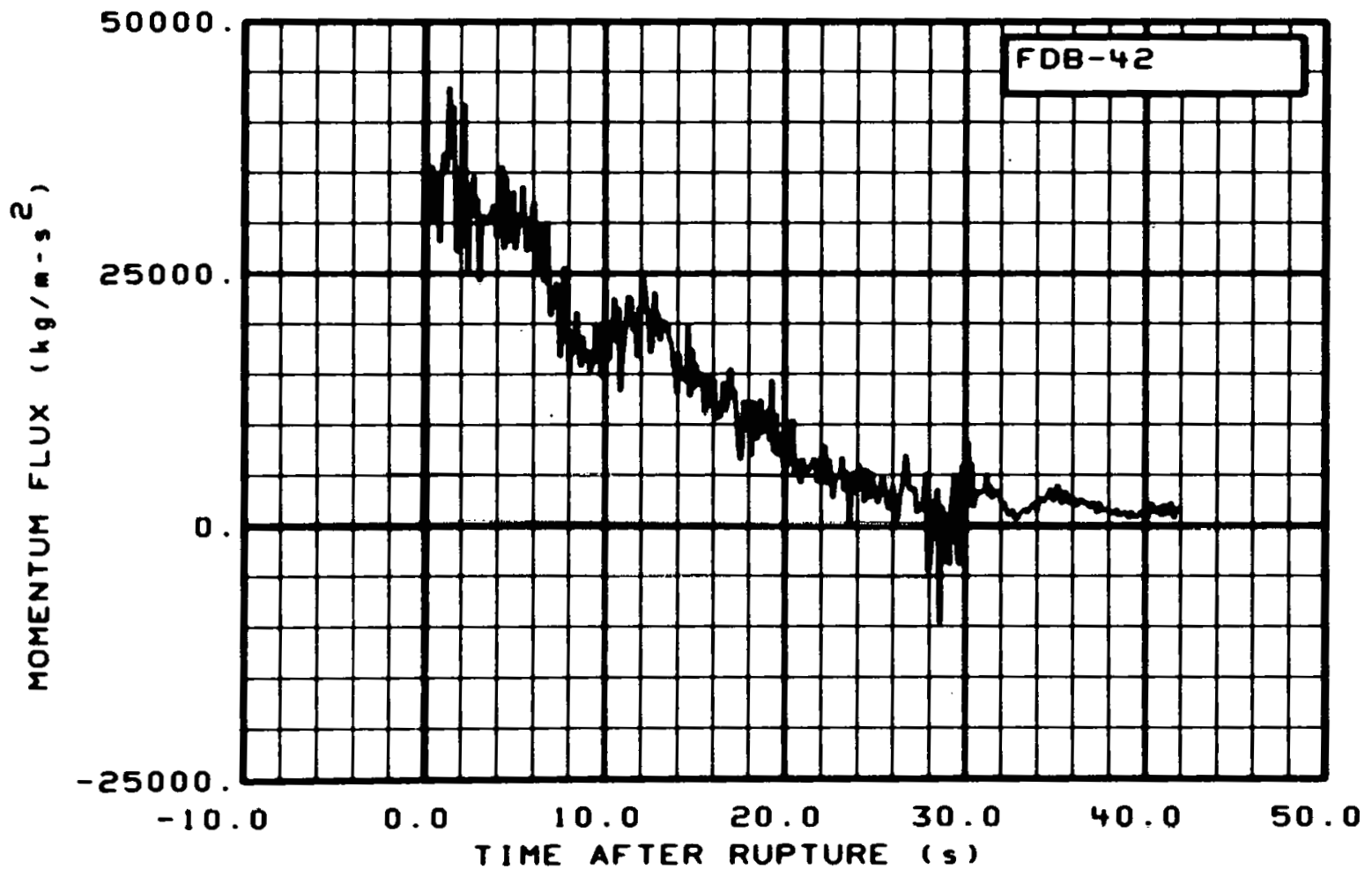

Fig. 288 Momentum flux in broken loop (FDB-42), from -6 to $42 \mathrm{~s}$. 


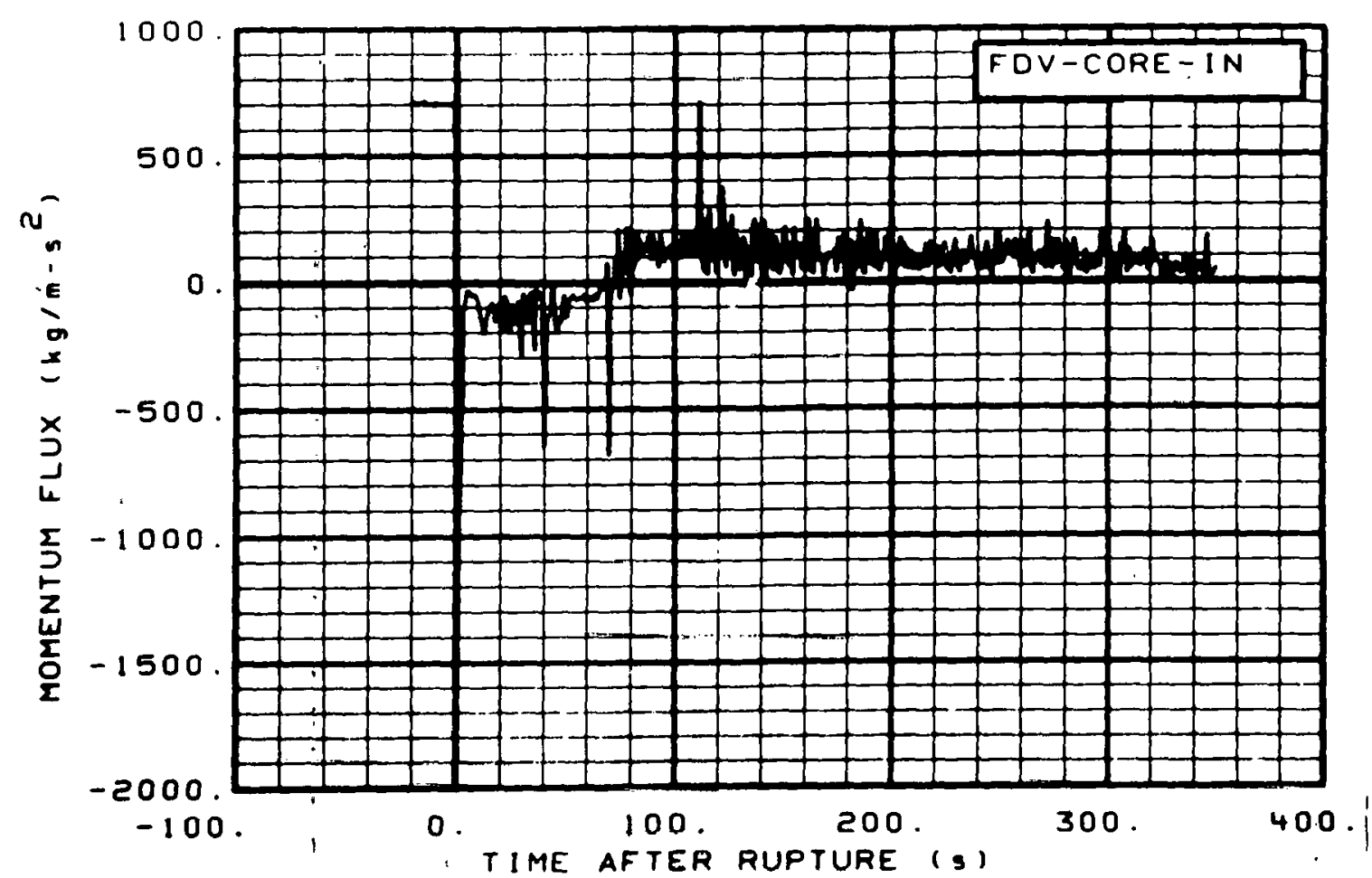

Fig. 289 Momentum flux in core entrance (FDV-CORE-IN), from -20 to $350 \mathrm{~s}$.

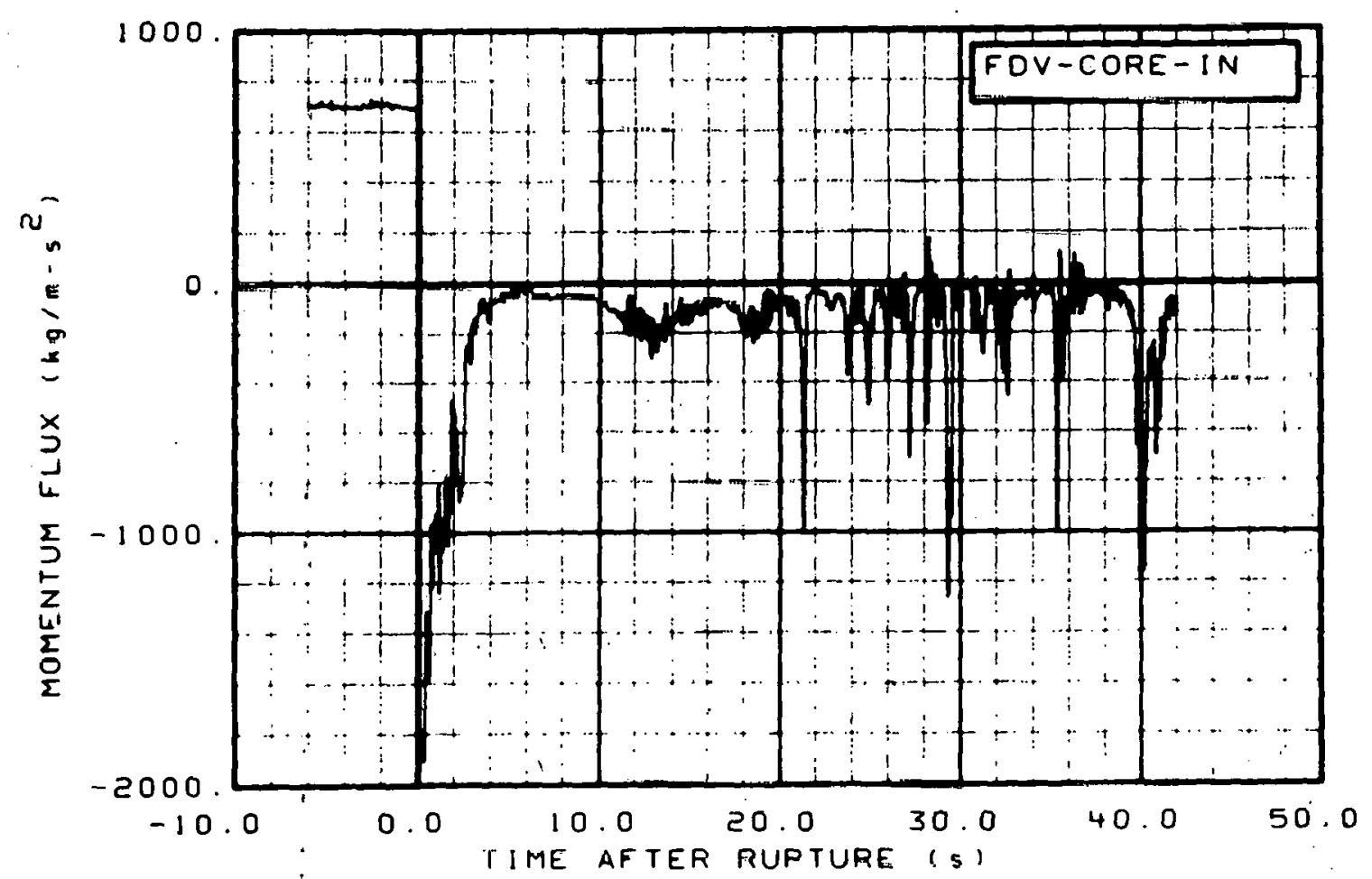

Fig. 290 Momentum flux in core entrance (FDV-CORE-IN), from -6 to $42 \mathrm{~s}$. 


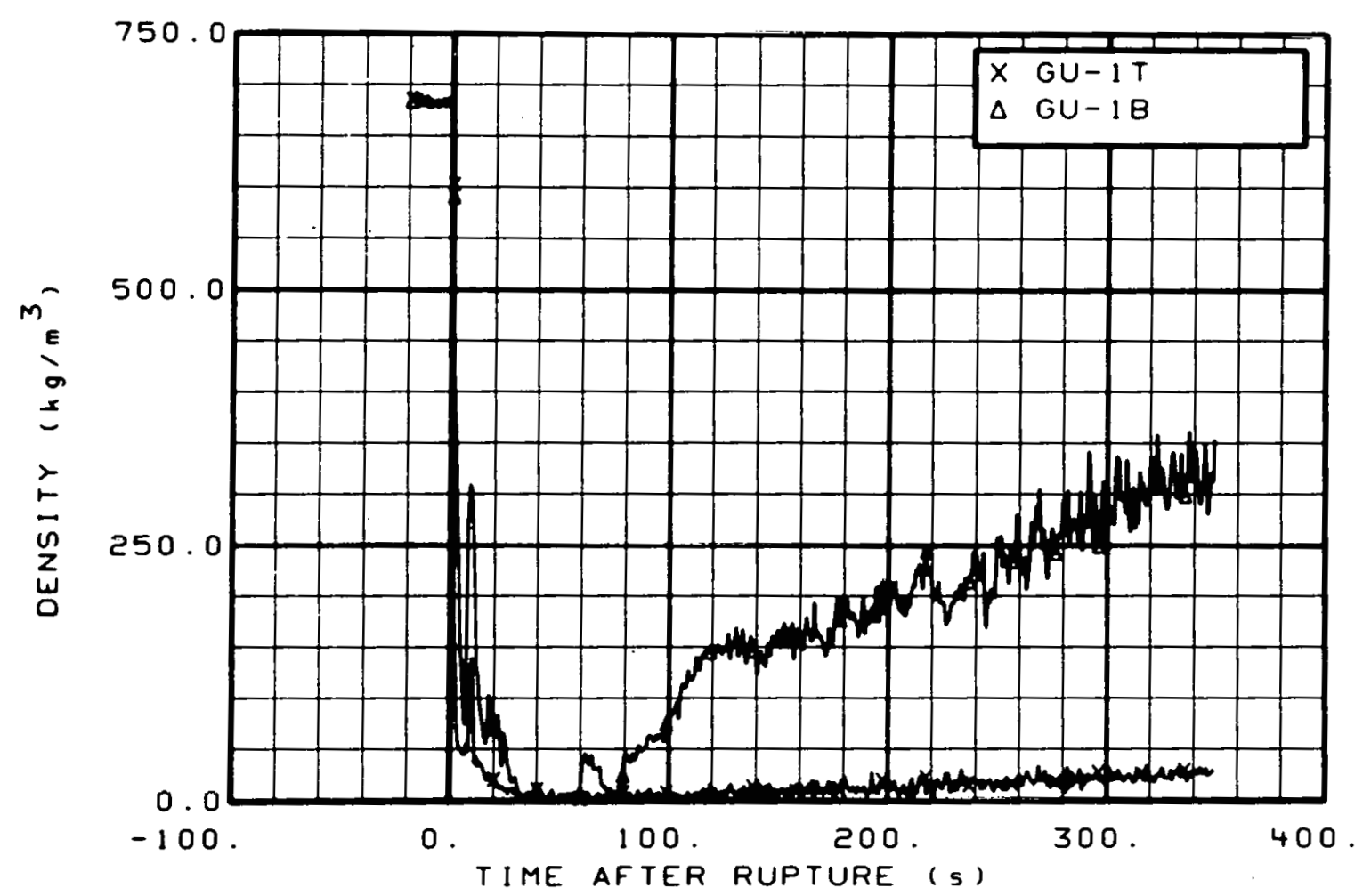

Fig. 291 Density in intact loop (GU-1T and GU-1B), from -20 to $350 \mathrm{~s}$.

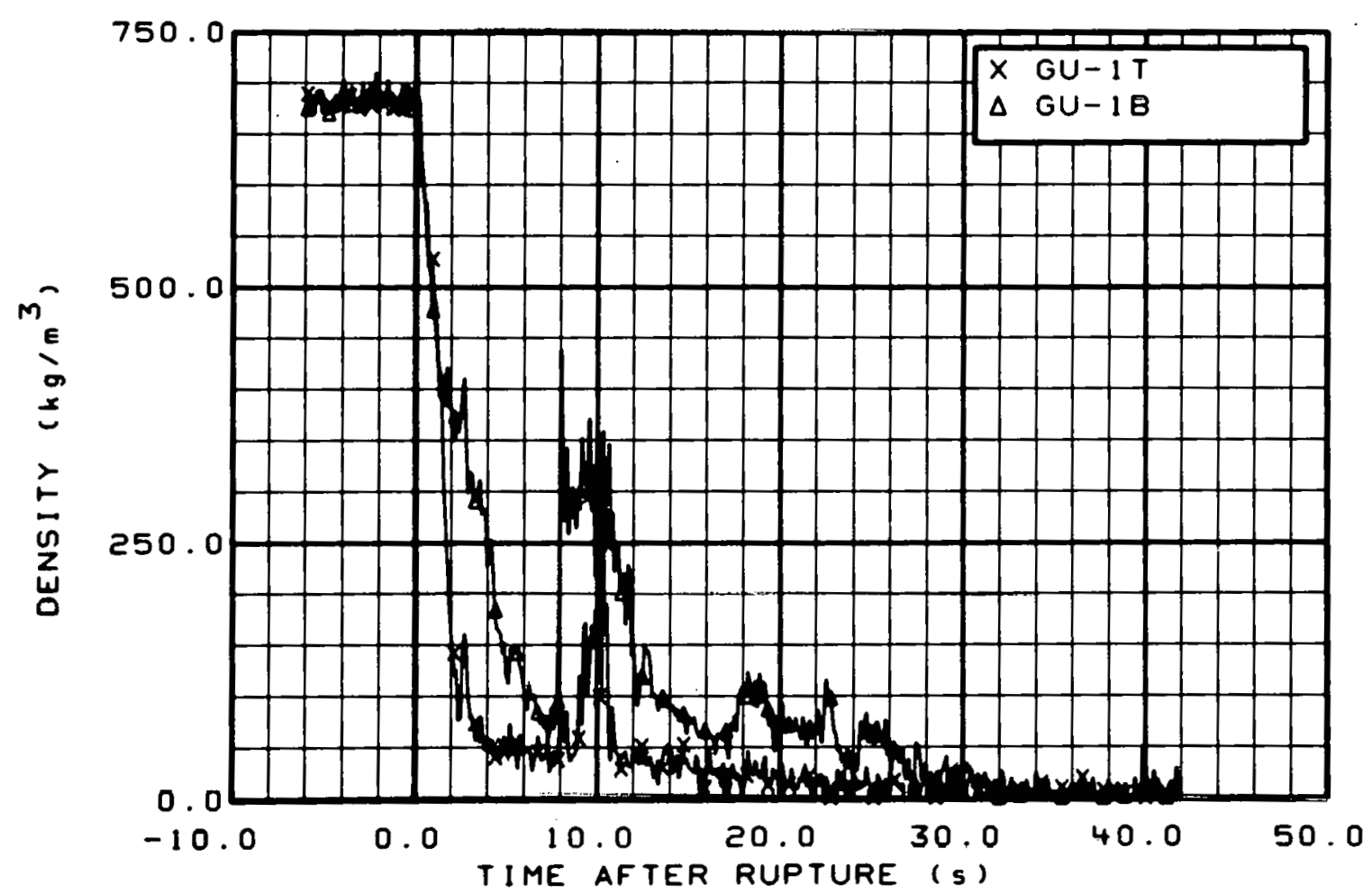

Fig. 292 Density in intact loop (GU-1T and GU-7B), from -6 to $42 \mathrm{~s}$. 


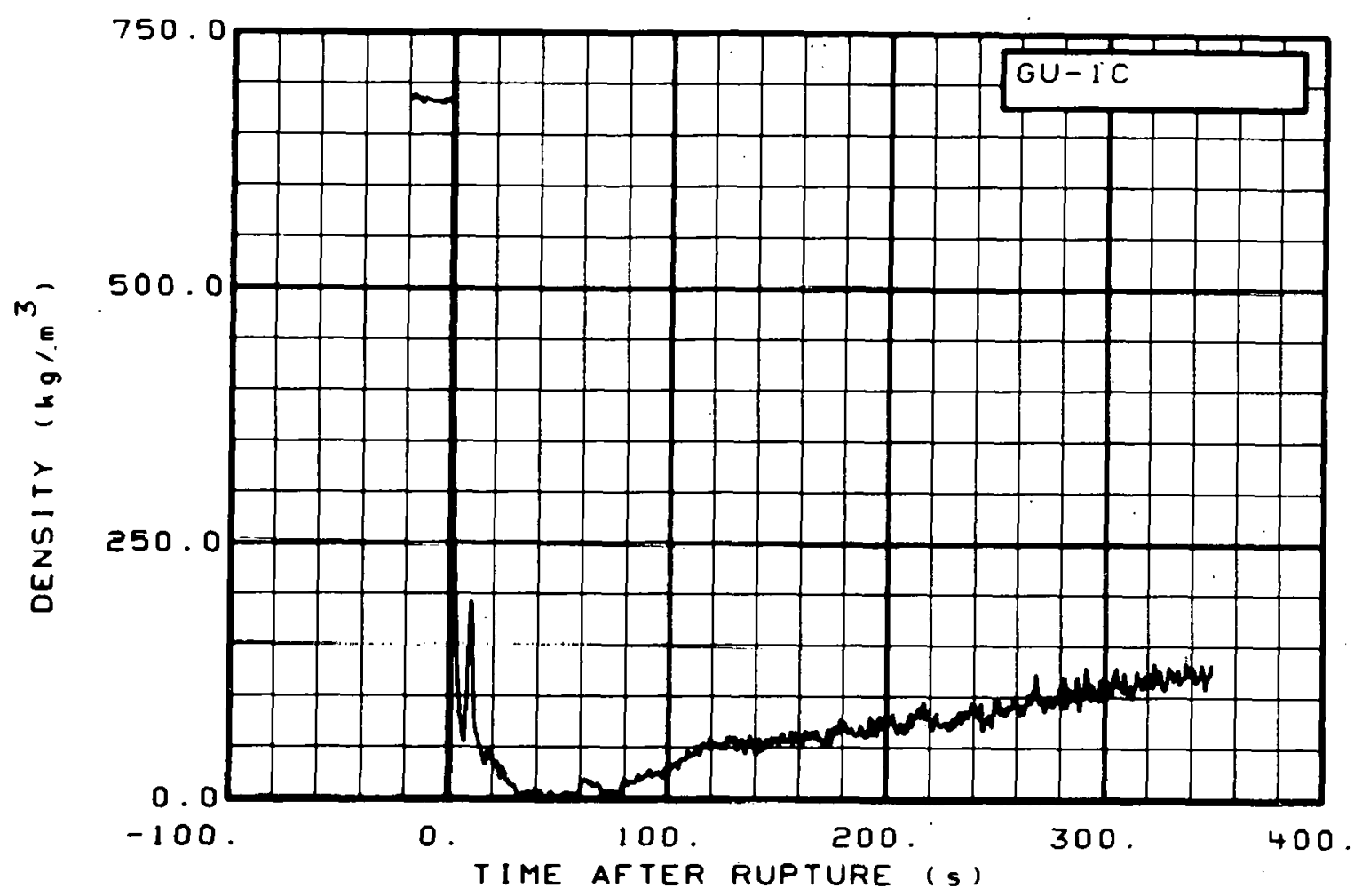

Fig. 293 Density in intact loop (GU-TC), from -20 to $350 \mathrm{~s}$.

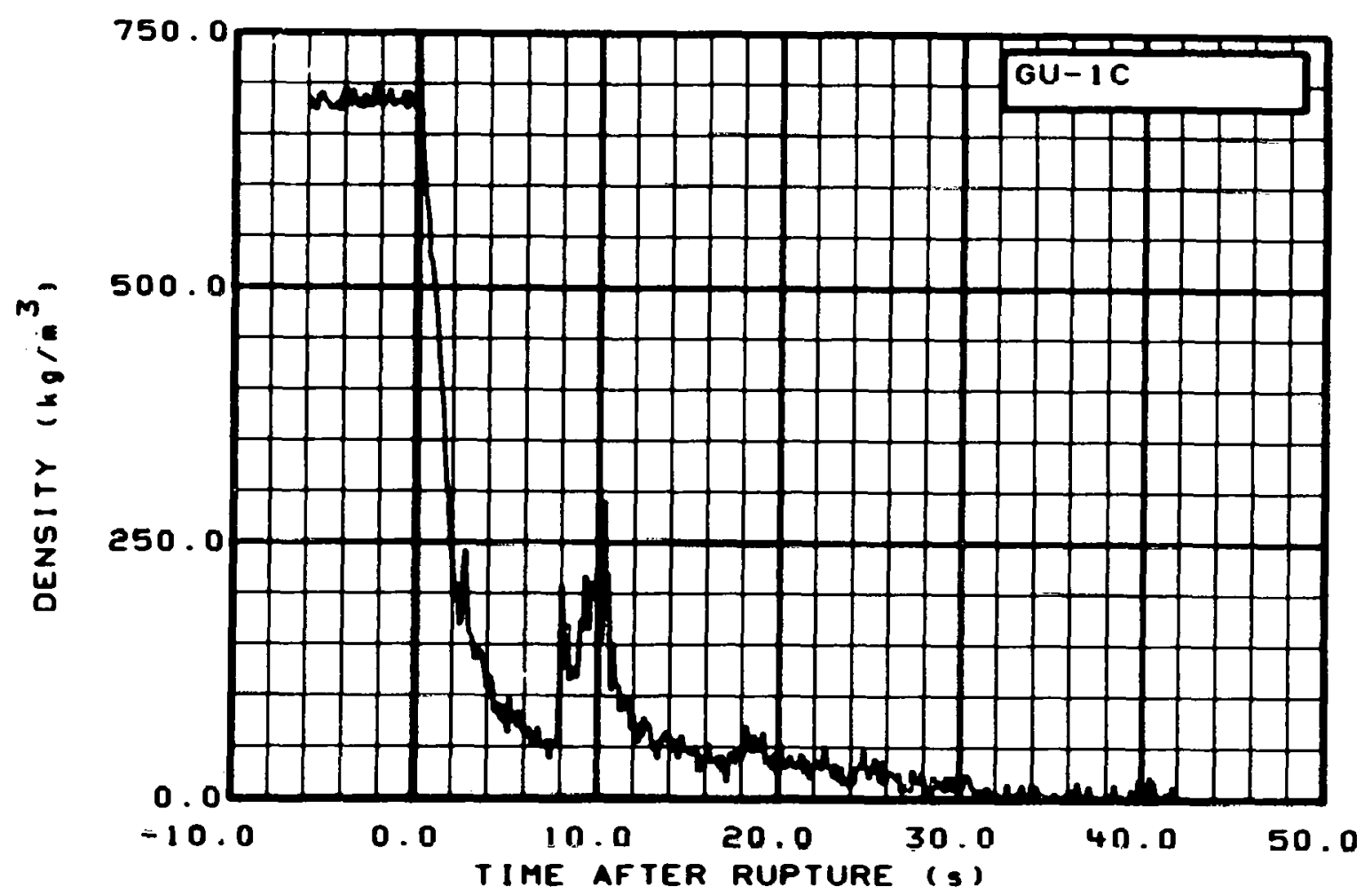

Fig. 294 Density in intact loop (GU-1C), from -6 to $42 \mathrm{~s}$. 


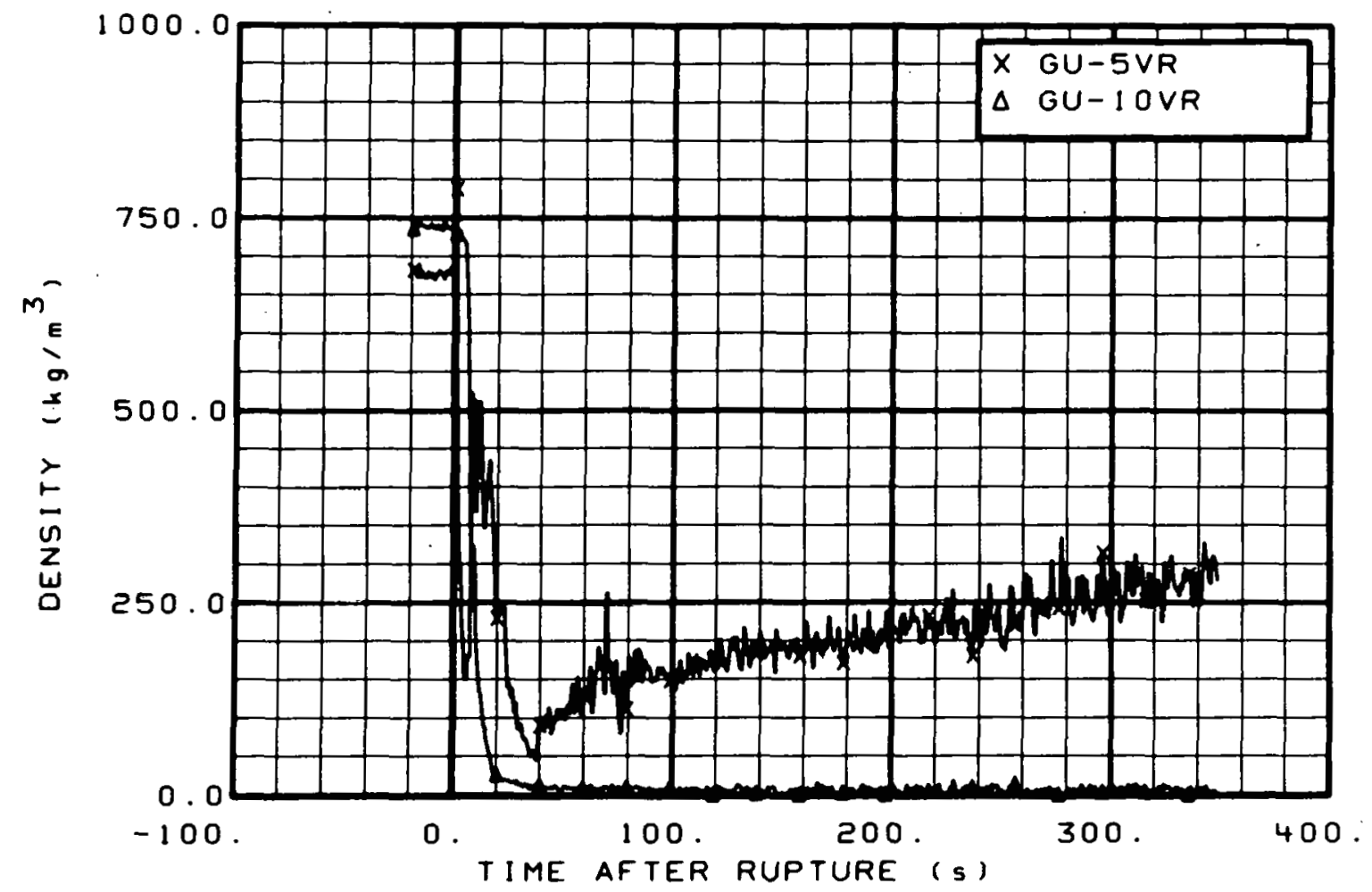

Fig. 295 Density in intact loop (GU-5VR and GU-10VR), from -20 to $350 \mathrm{~s}$.

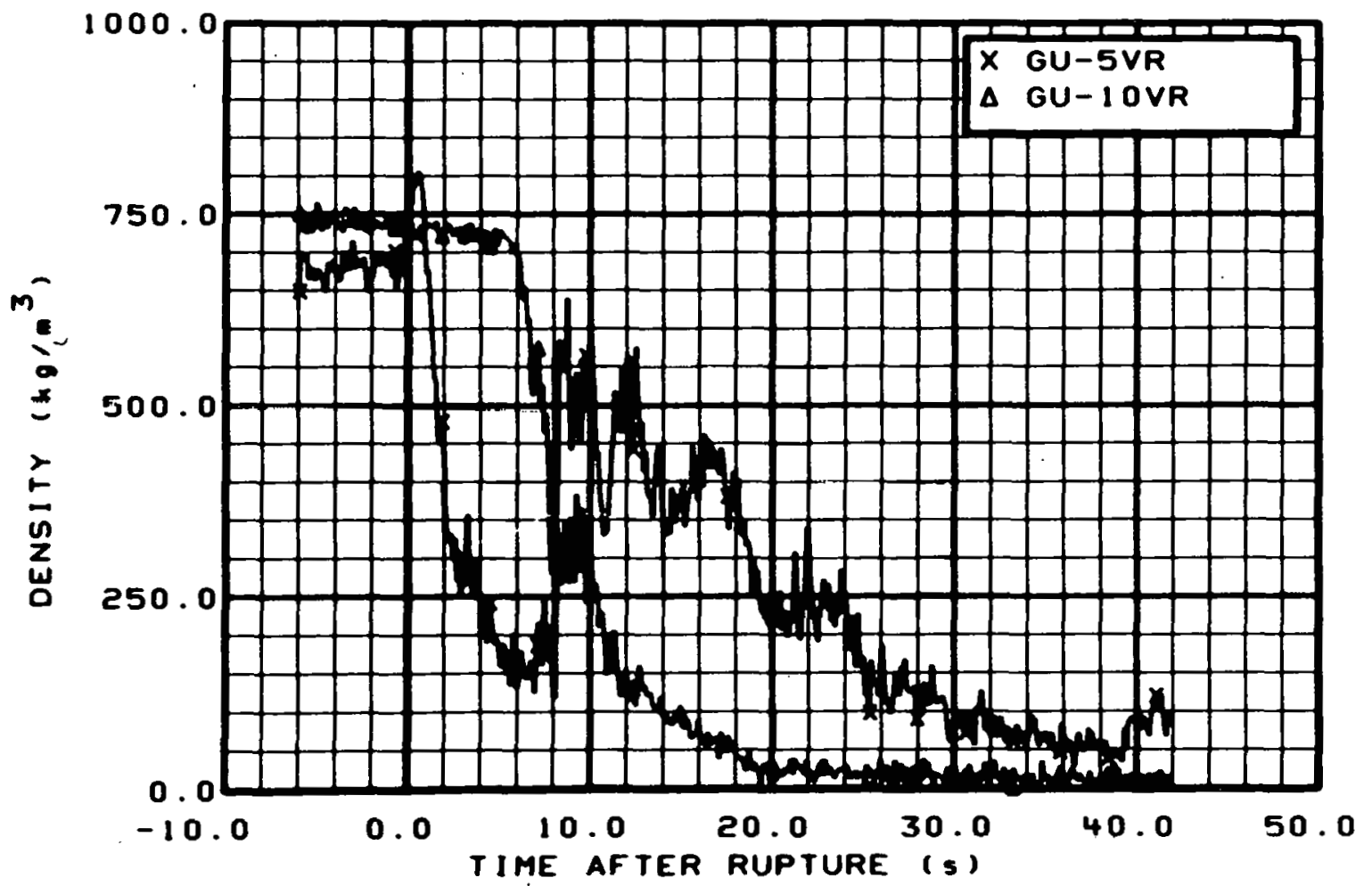

Fig. 296 Density in intact 10op (GU-5VR and GU-10VR), from -6 to $42 \mathrm{~s}$. 


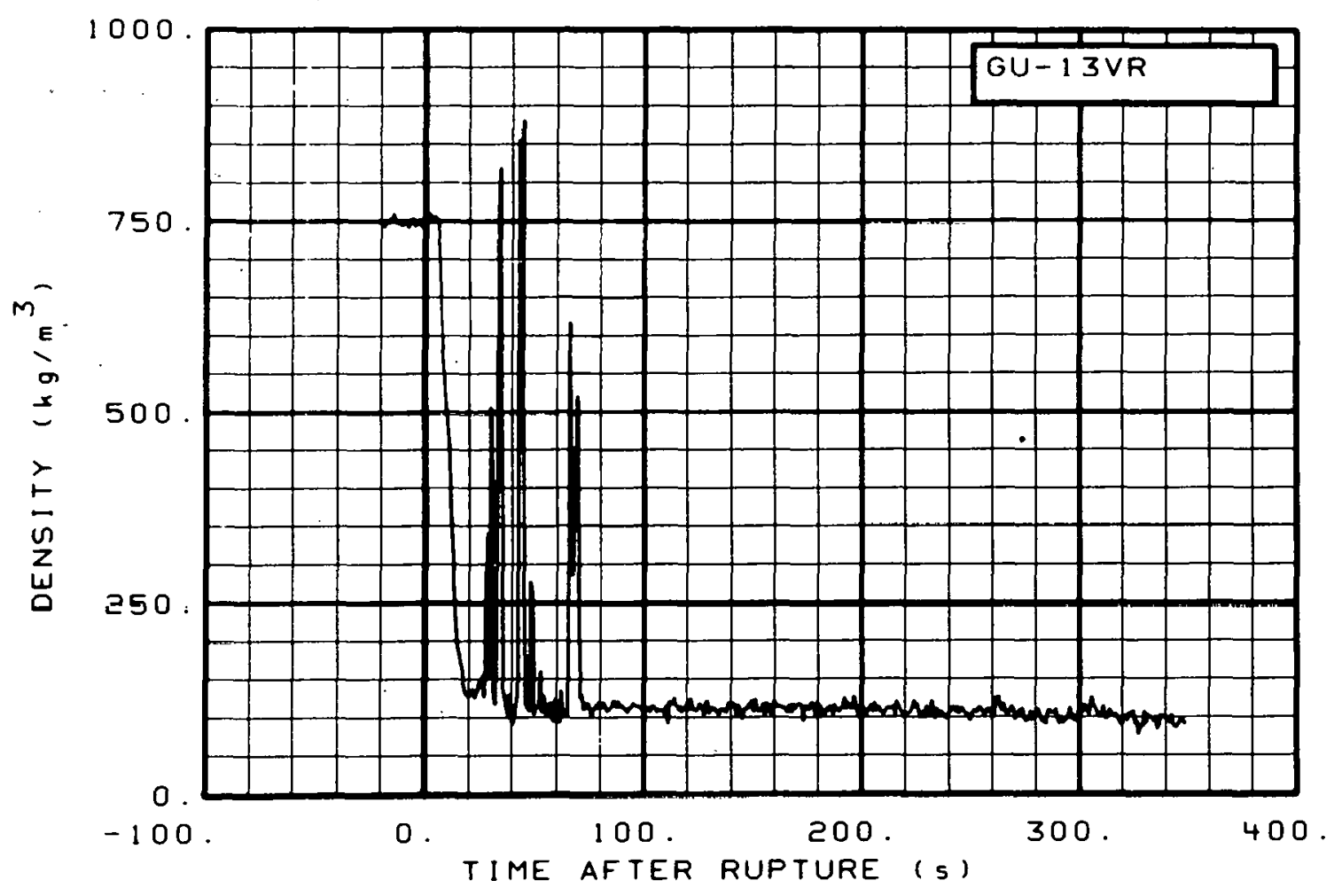

Fig. 297 Density in intact loop (GU-13VR), from -20 to $350 \mathrm{~s}$.

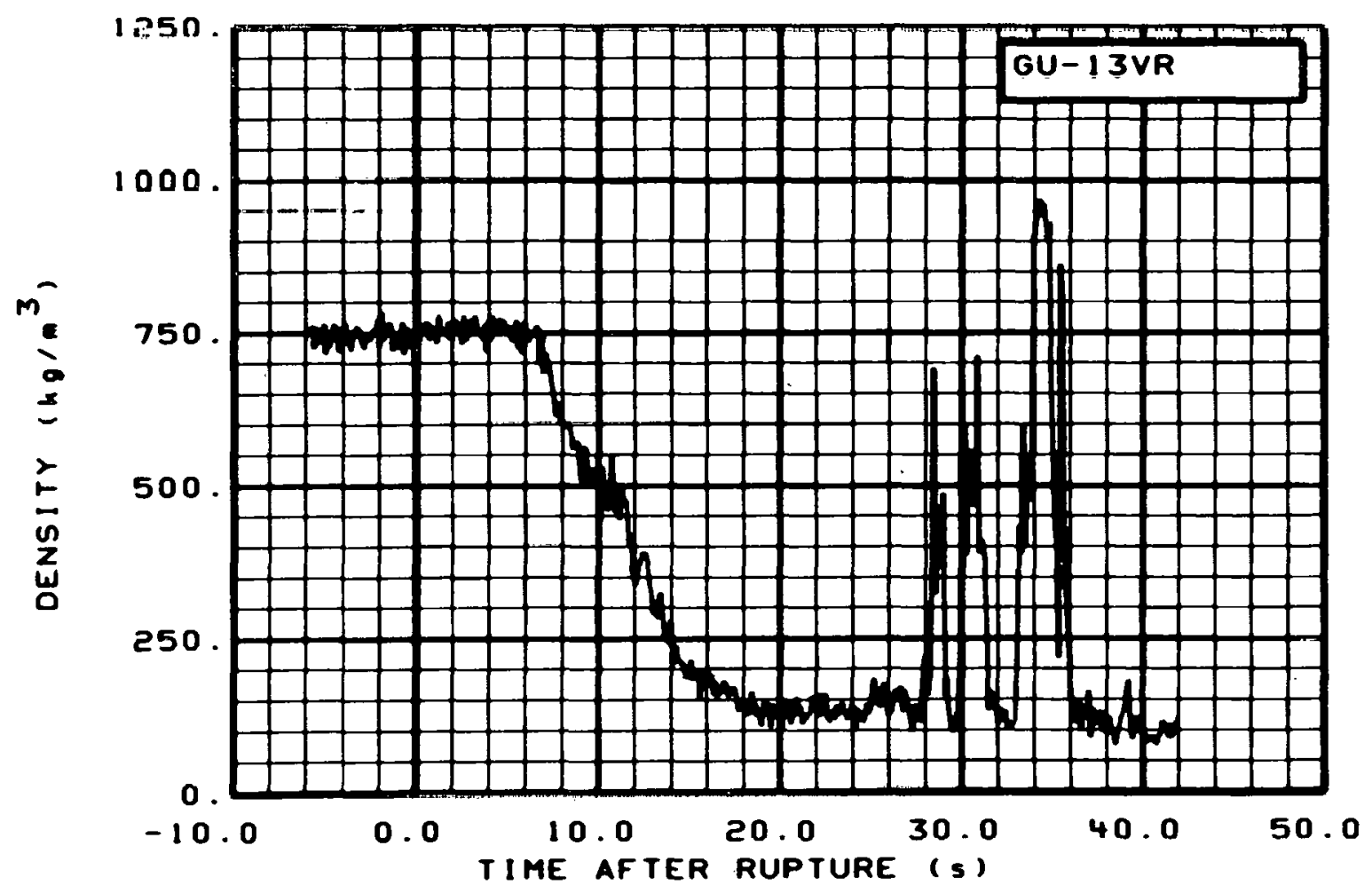

Fig. 298 Density in intact loop (GU-13VR), from -6 to $42 \mathrm{~s}$. 


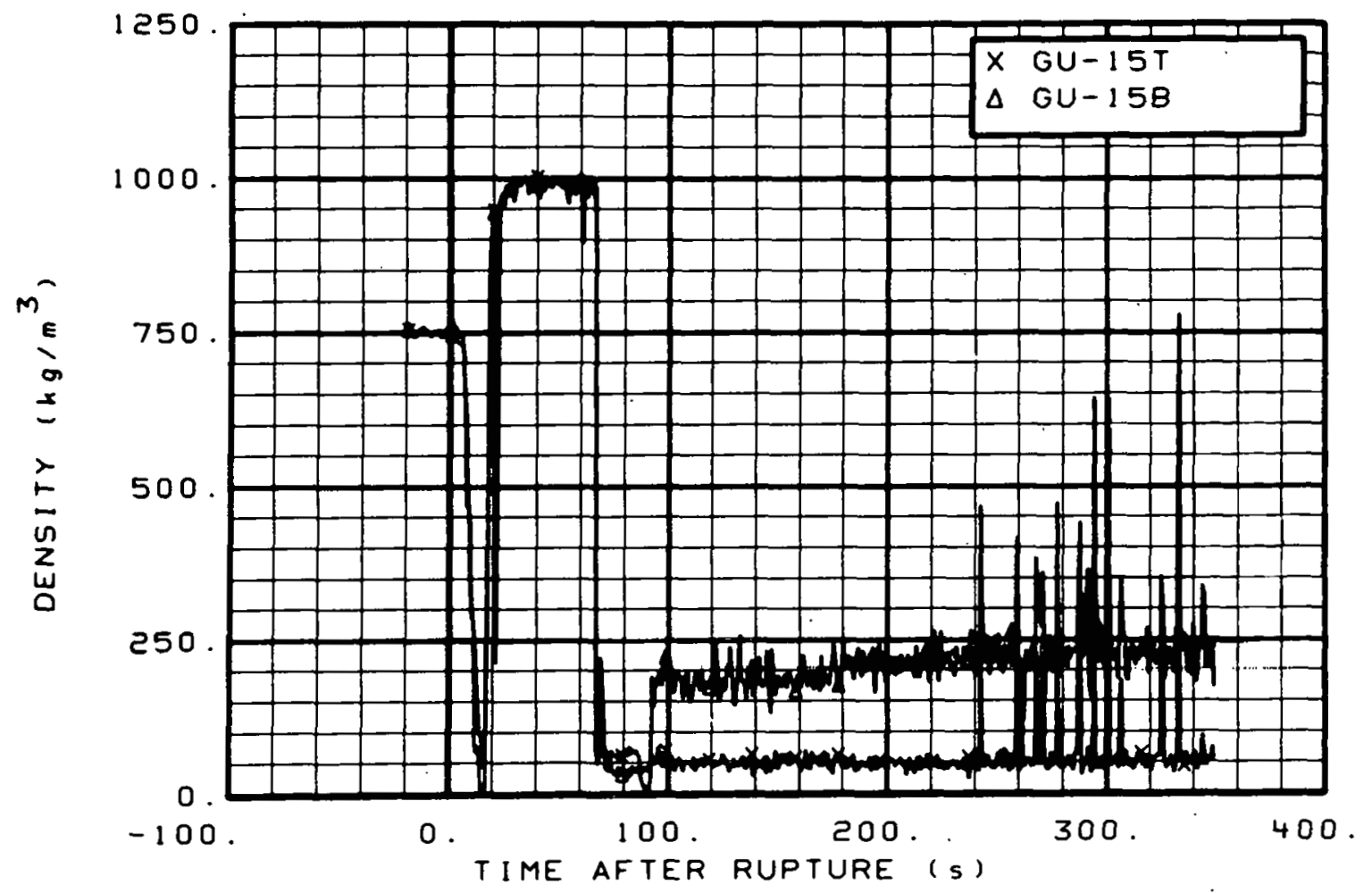

Fig. 299 Density in intact loop (GU-15T and GU-15B), from -20 to $350 \mathrm{~s}$.

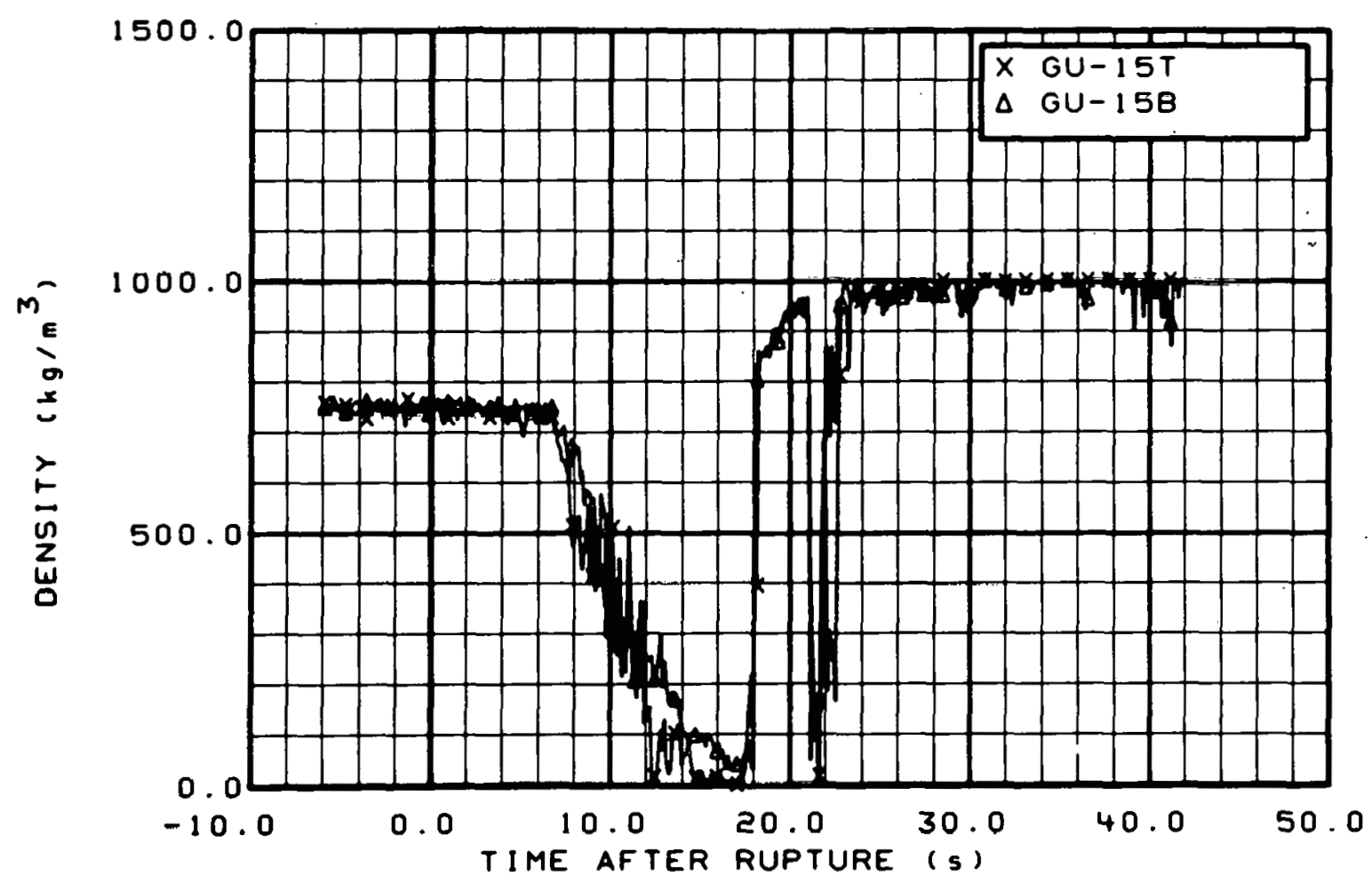

Fig. 300 Density in intact loop (GU-15T and GU-15B), from -6 to $42 \mathrm{~s}$. 


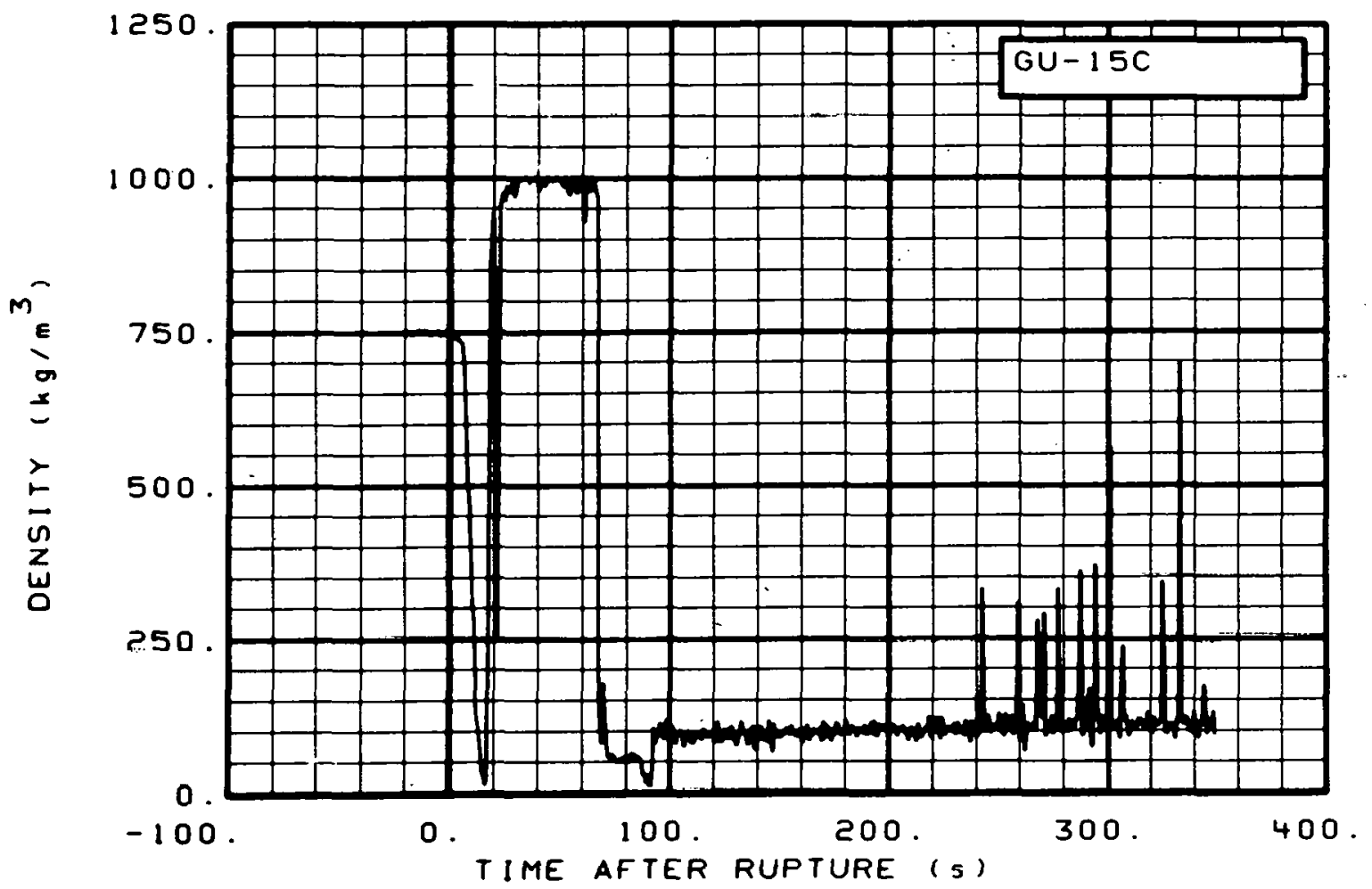

Fig. 301 Density in intact loop (GU-15C), from -20 to $350 \mathrm{~s}$.

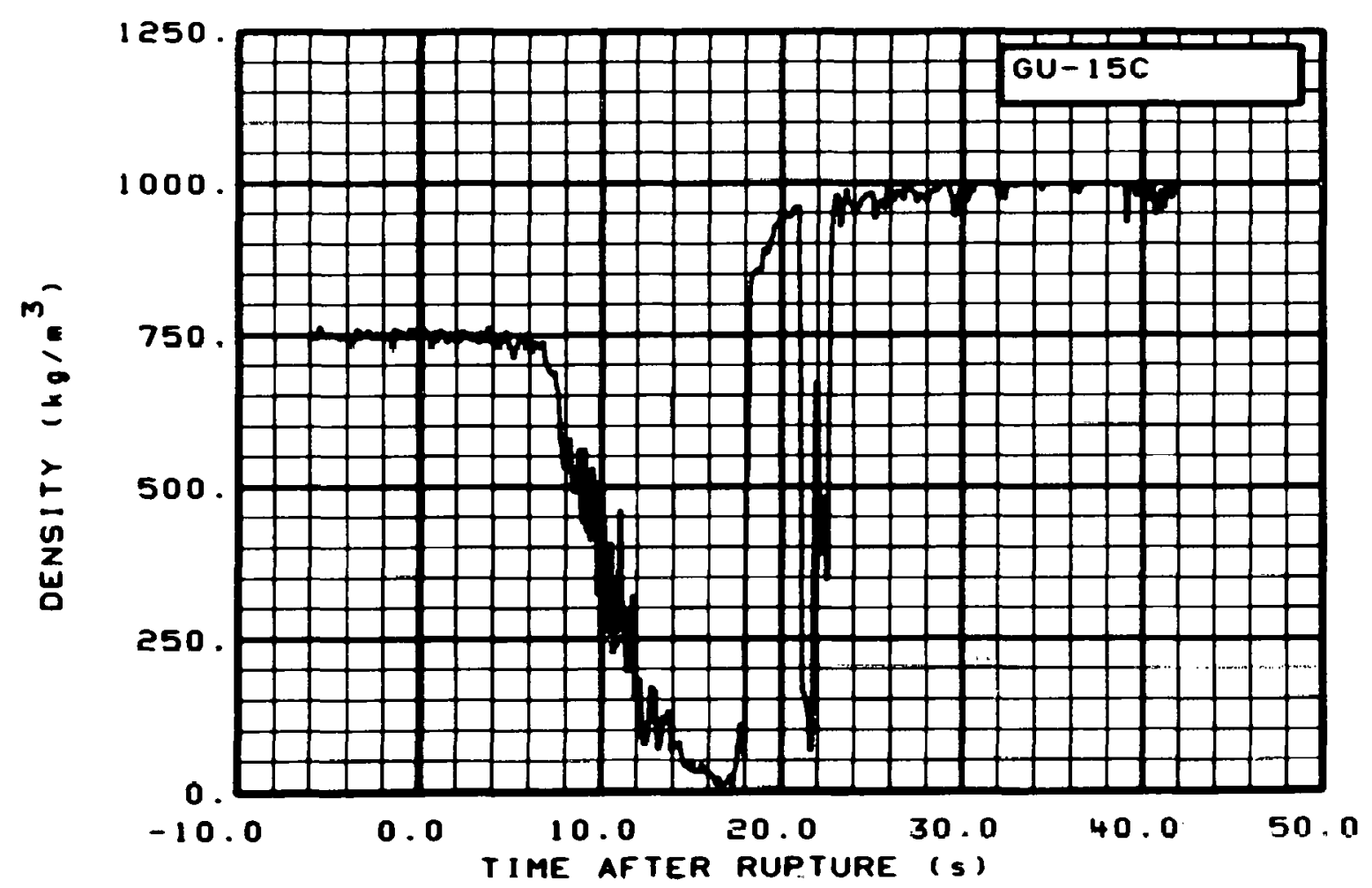

Fig. 302 Density in intact loop (GU-15C), from -6 to $42 \mathrm{~s}$. 


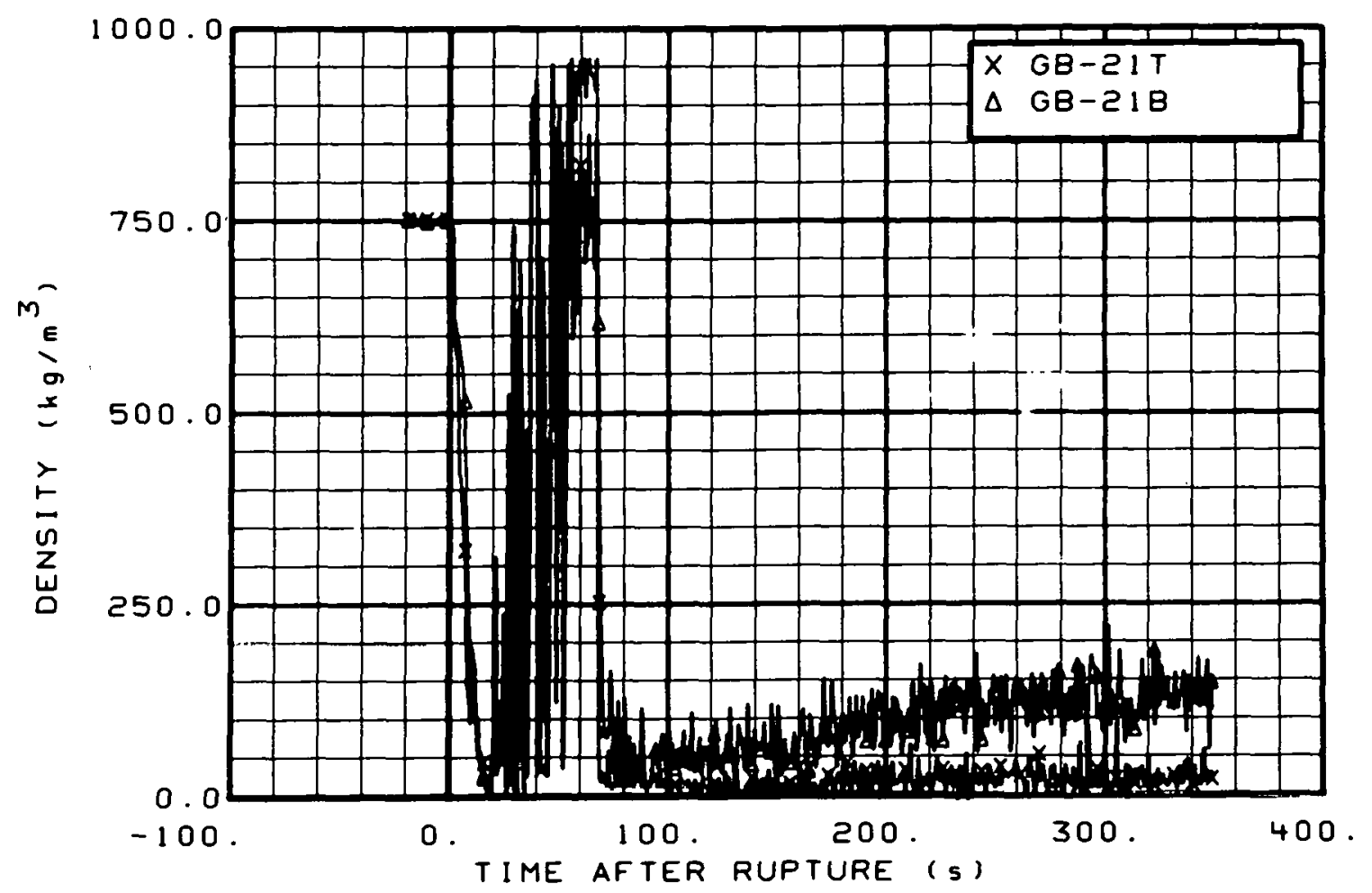

Fig. 303 Density in broken loop (GB-21T and GB-21B), from -20 to $350 \mathrm{~s}$.

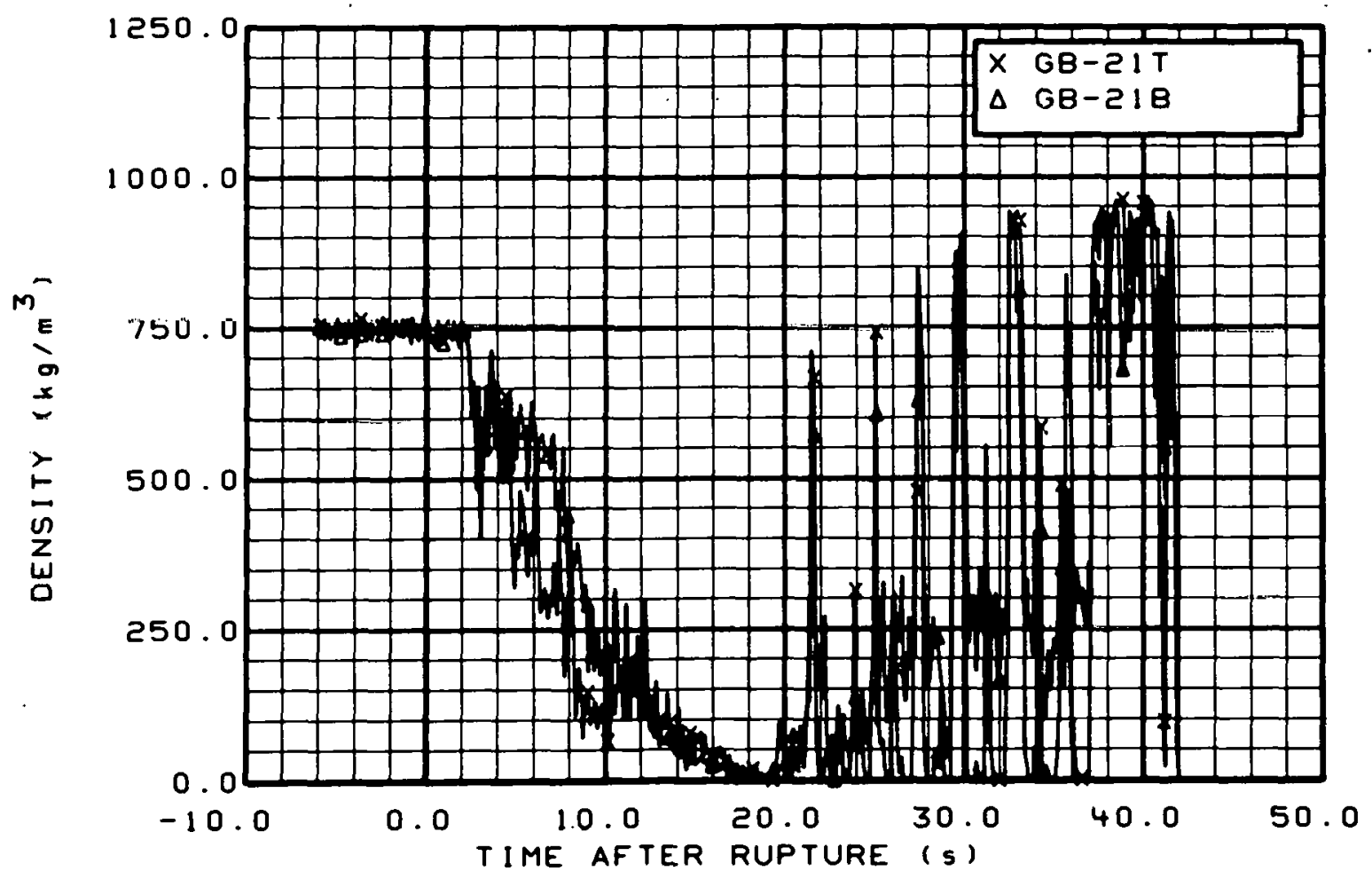

Fig. 304 Density in broken loop (GB-21T and GB-21B), from -6 to $42 \mathrm{~s}$. 


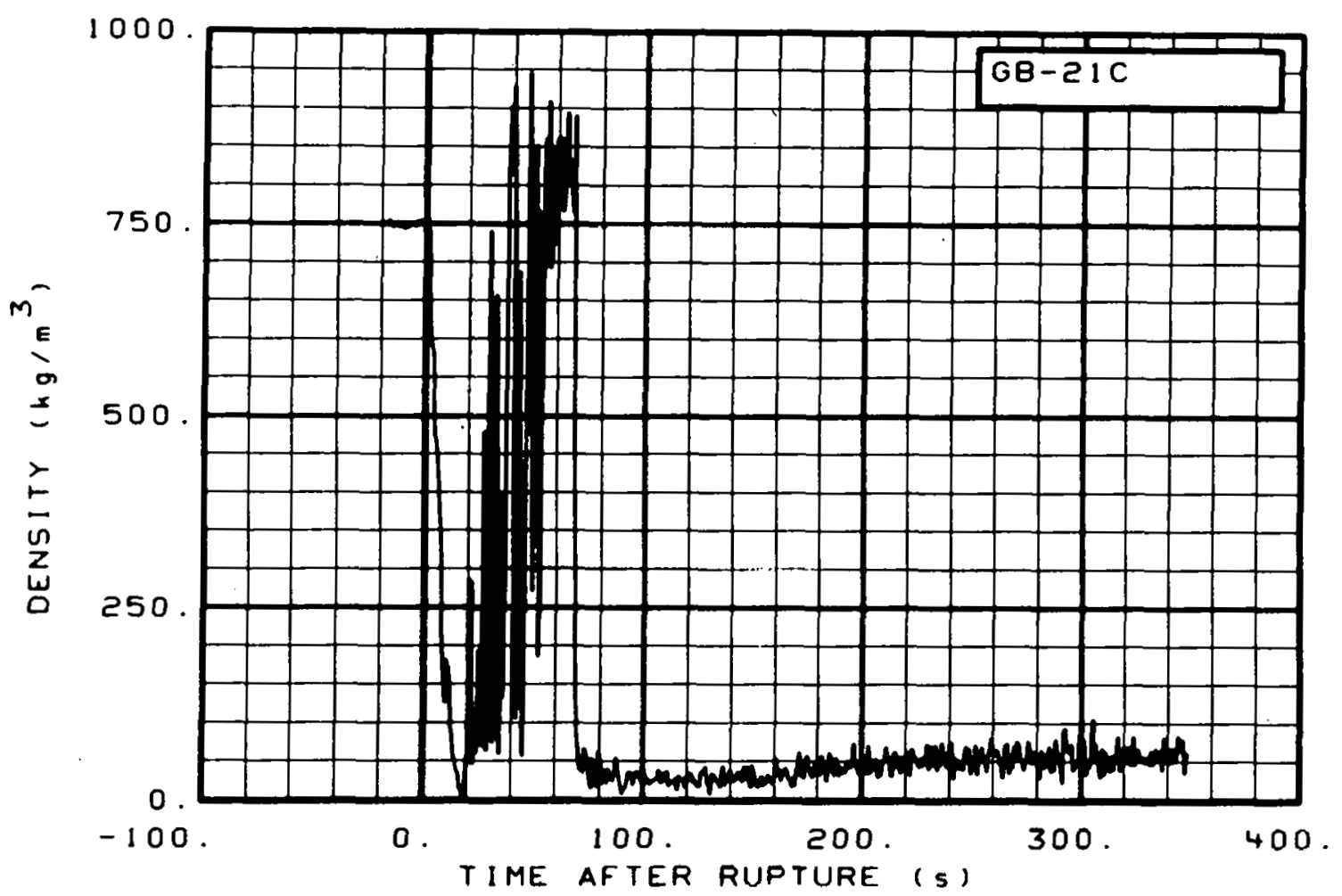

Fig. 305 Density in broken loop (GB-21C), from -20 to $350 \mathrm{~s}$.

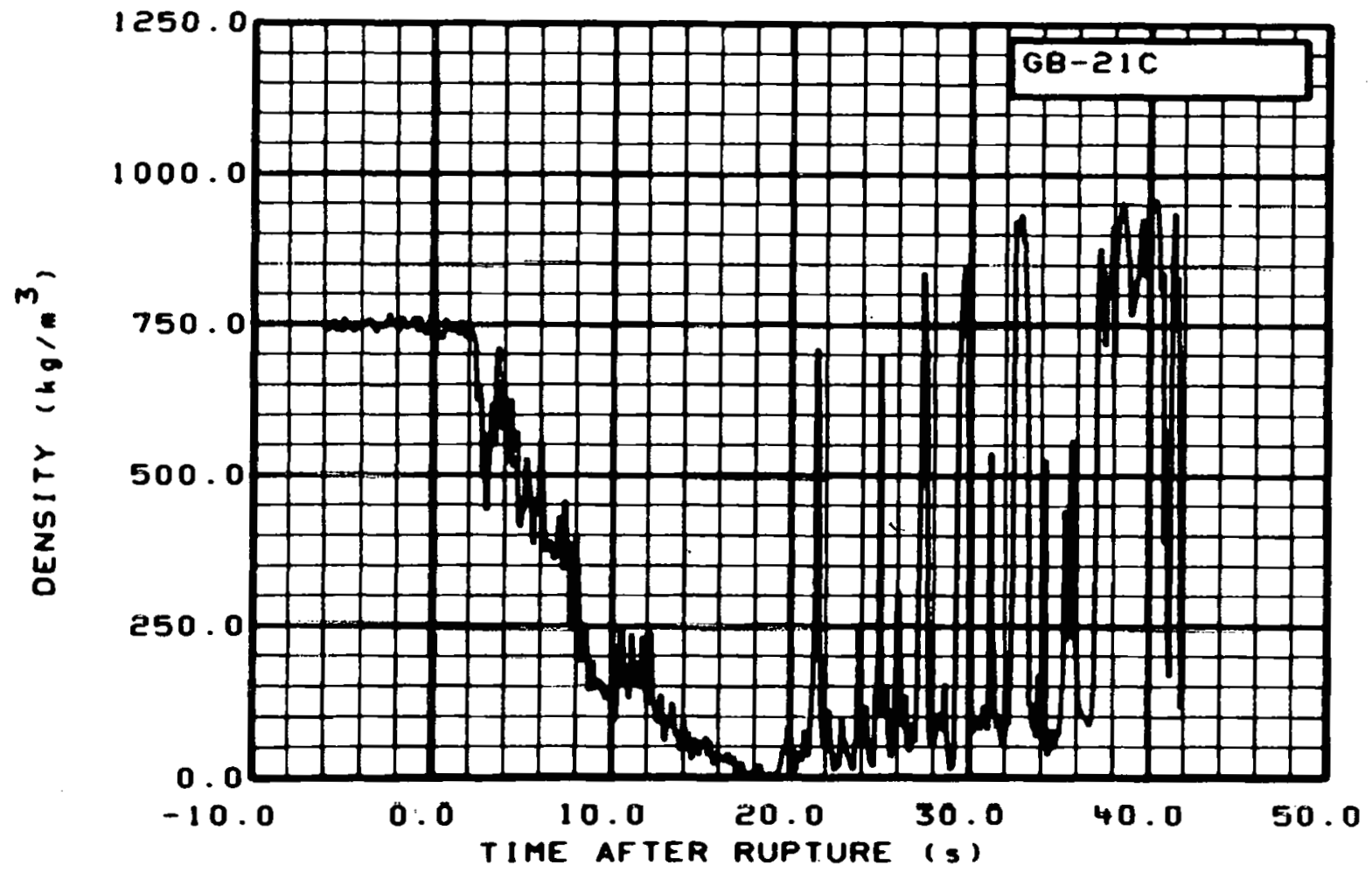

Fig. 306 Density in broken loop (GB-2lC), from -6 to $42 \mathrm{~s}$. 


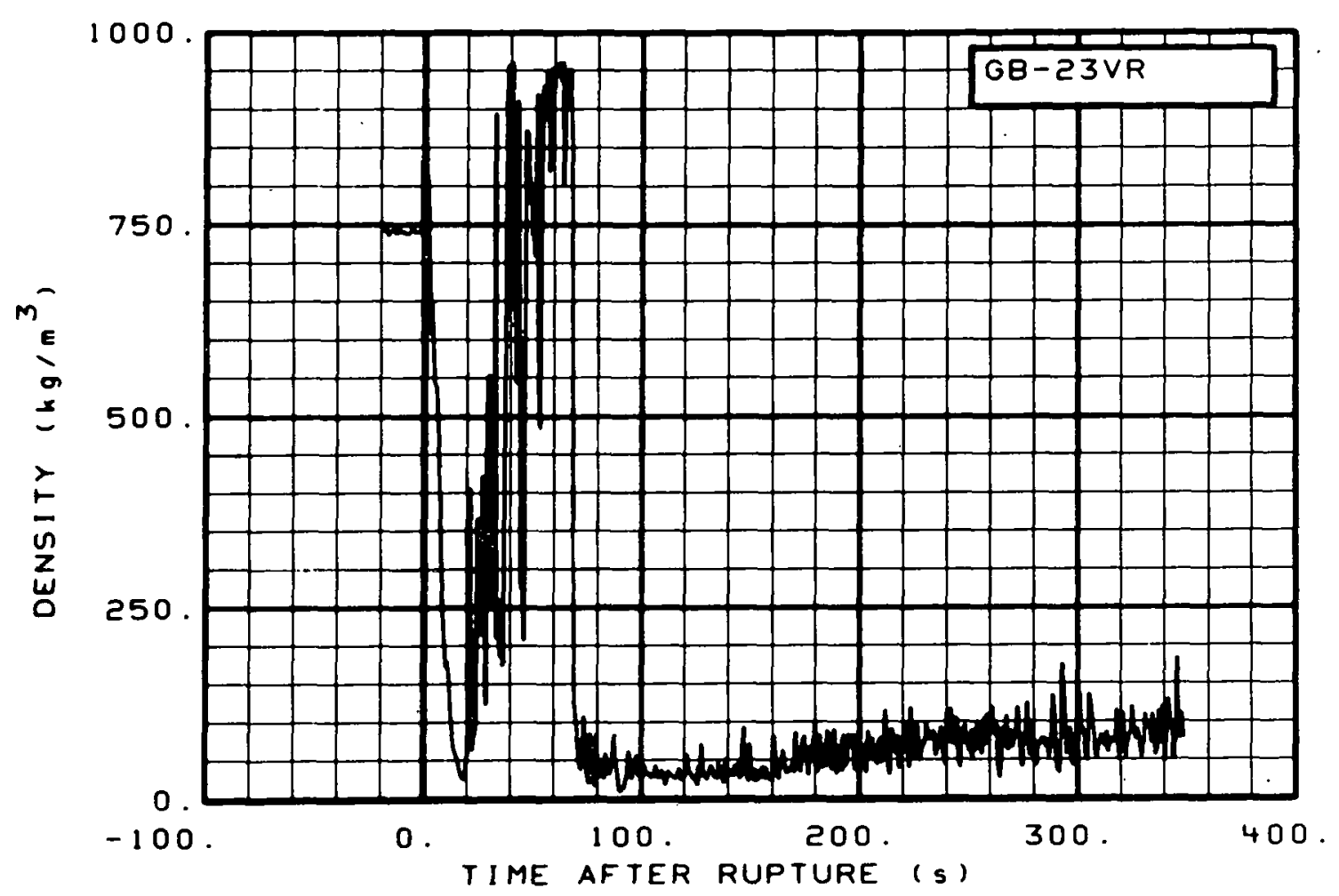

Fig. 307 Density in broken loop (GB-23VR), from -20 to $350 \mathrm{~s}$.

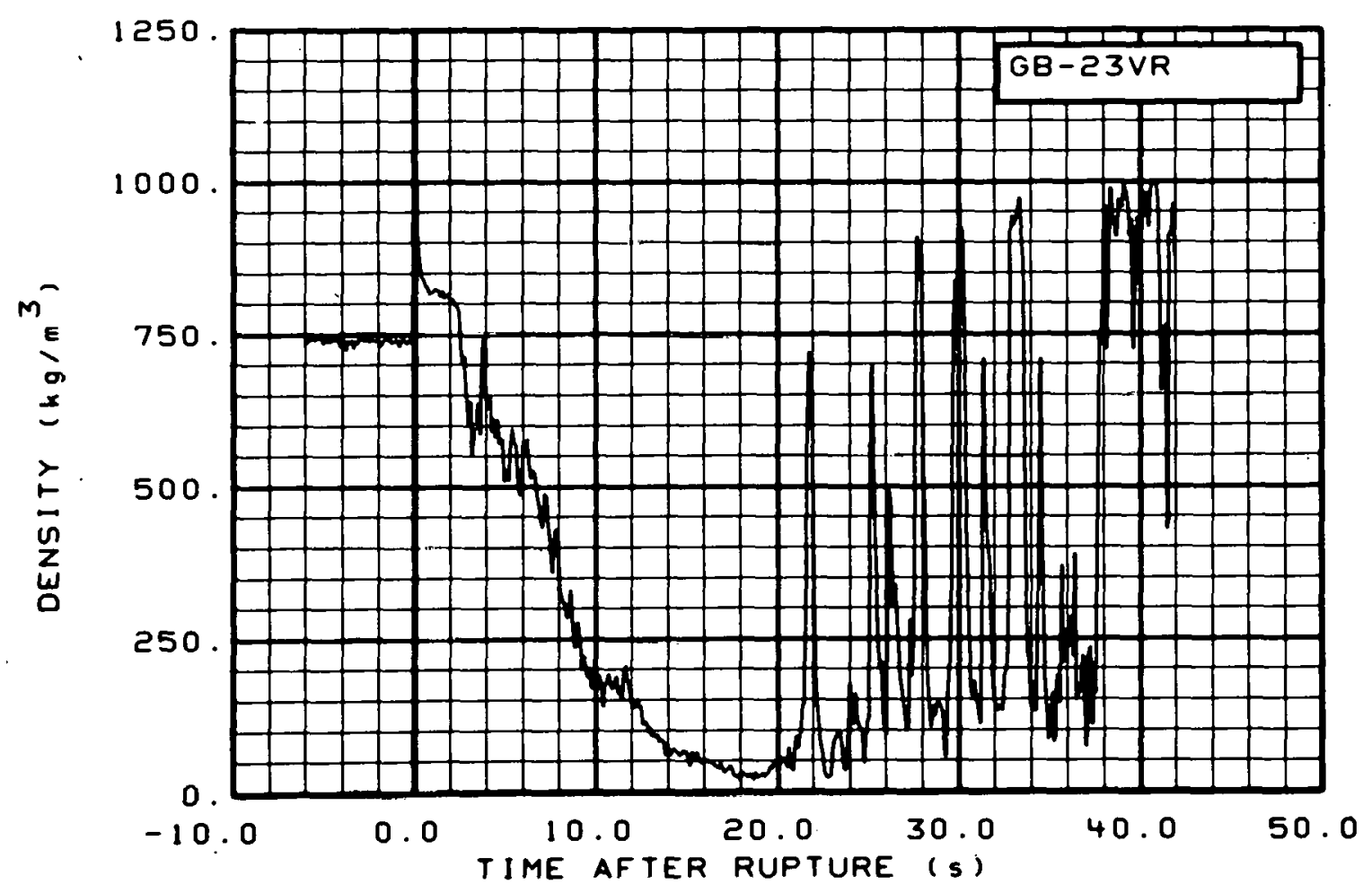

Fig. 308 Density in broken loop (GB-23VR), from -6 to $42 \mathrm{~s}$. 


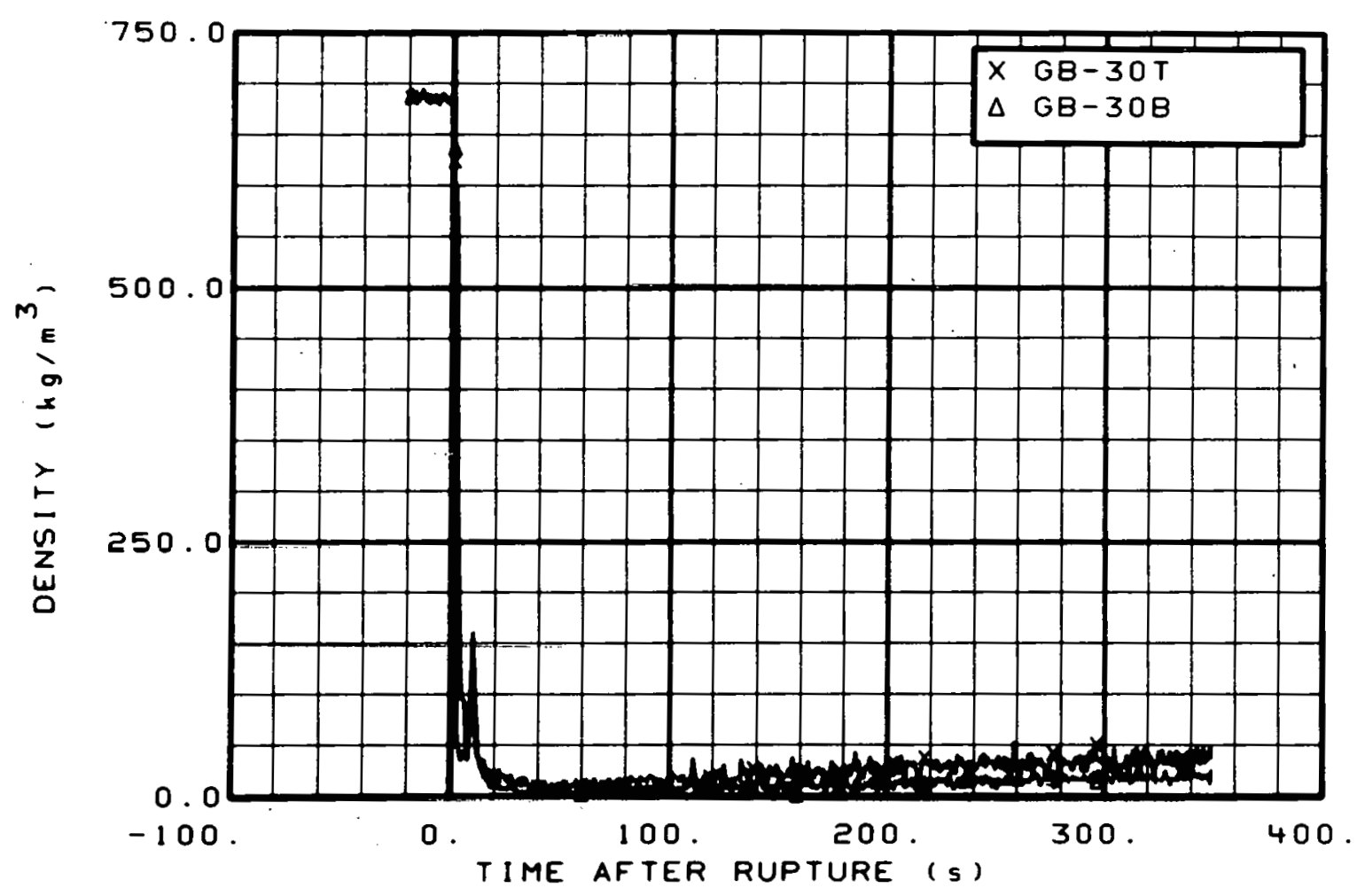

Fig. 309 Density in broken loop (GB-3UI and GB-3UB), from -20 to $350 \mathrm{~s}$.

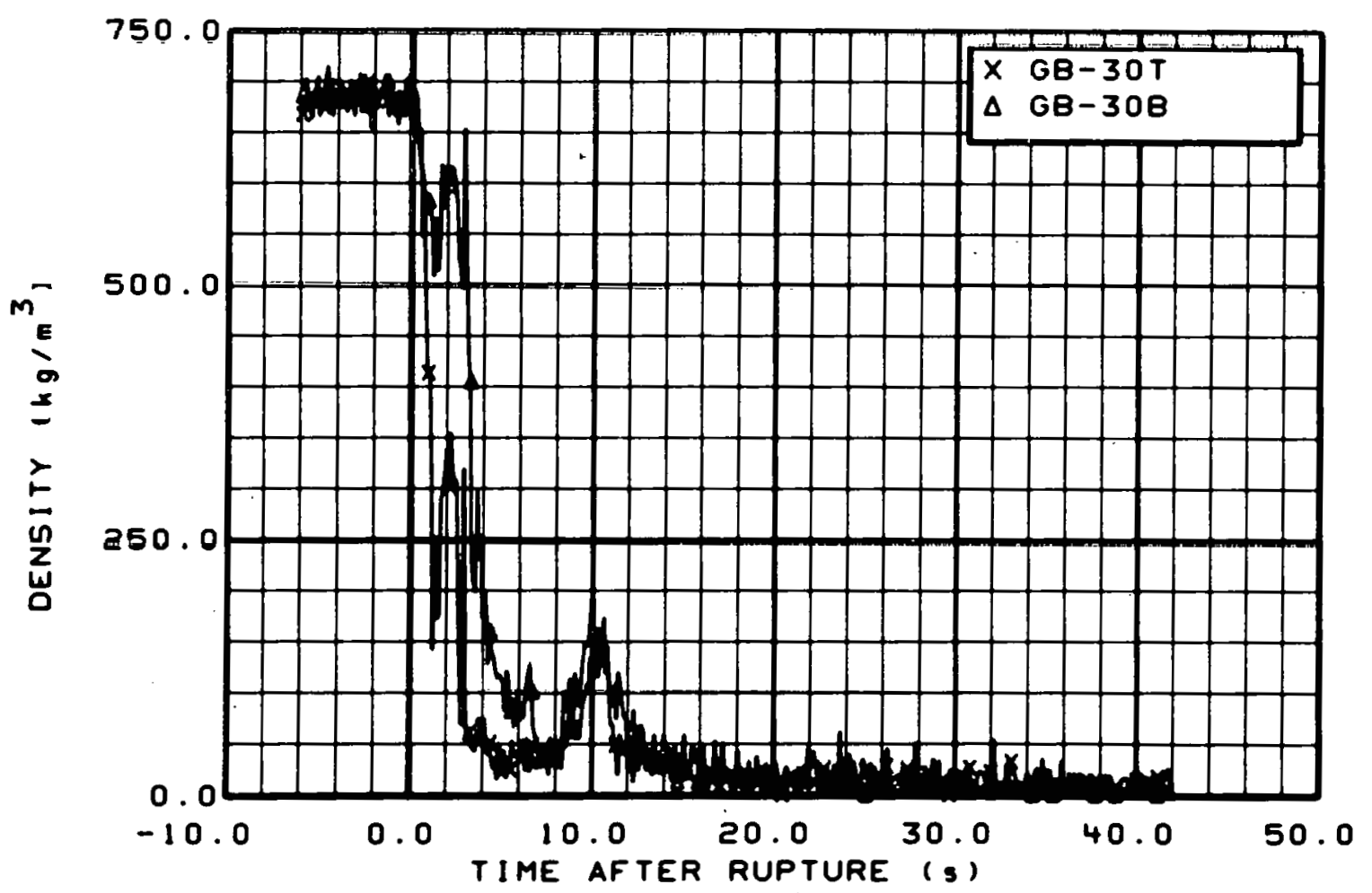

Fig. 310 Density in broken 10op (GB-30T and GB-30B), from -6 to $42 \mathrm{~s}$. 


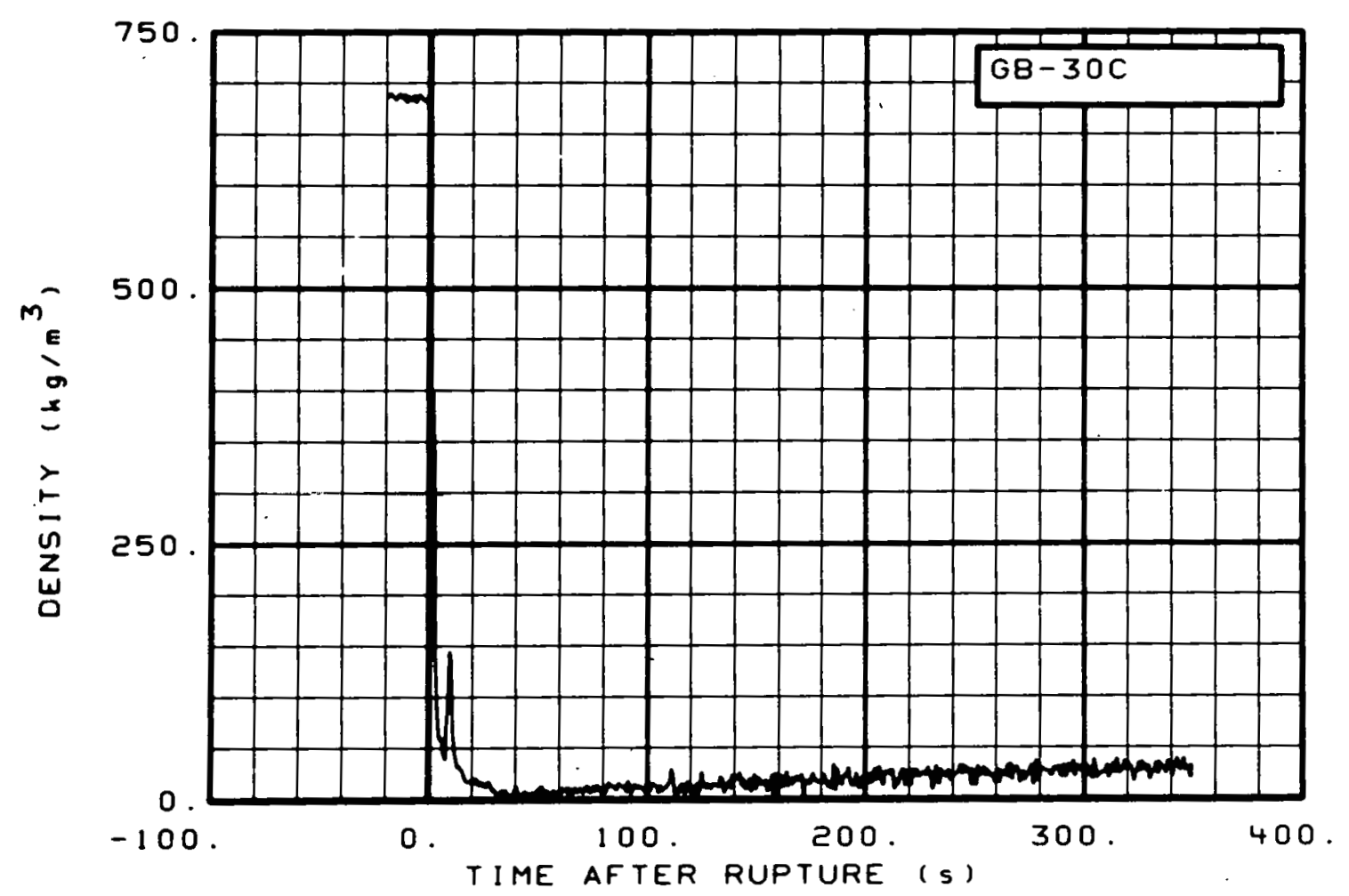

Fig. 311 Density in broken loop (GB-30C), from -20 to $350 \mathrm{~s}$.

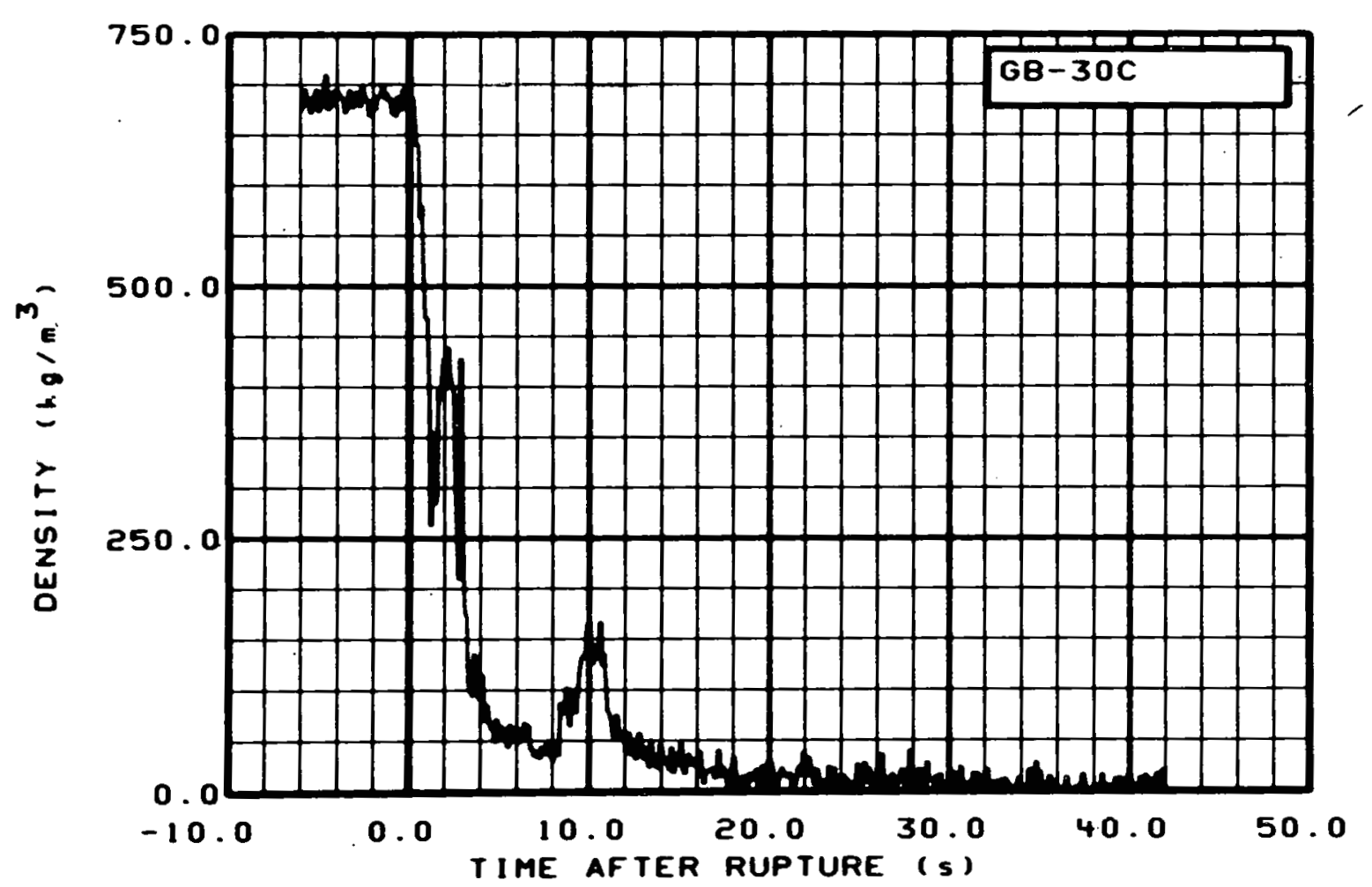

Fig. 312 Density in broken loop (GB-30C), from -6 to $42 \mathrm{~s}$. 


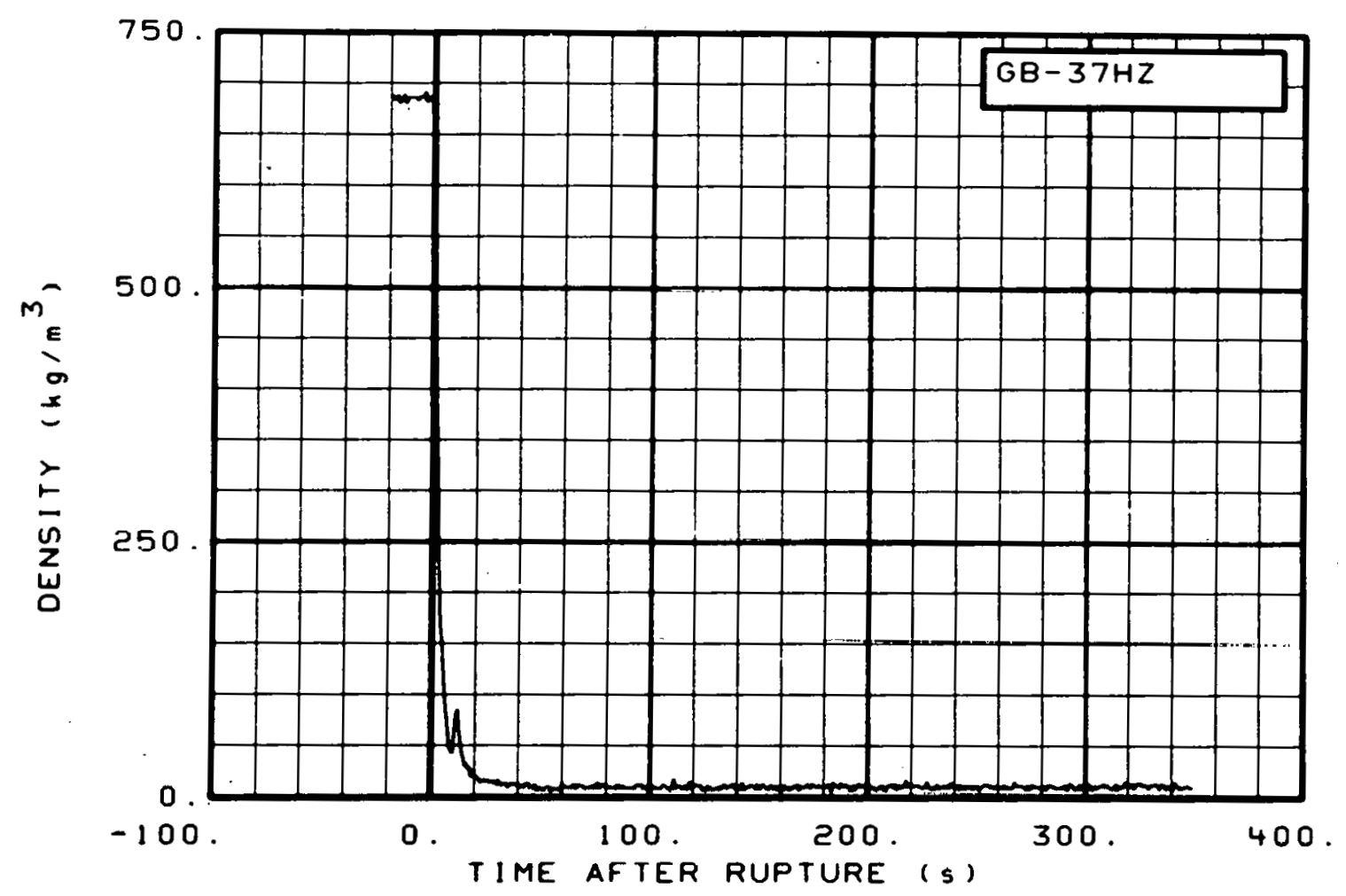

Fig. 313 Density in broken loop (GB-37HZ), from -20 to $350 \mathrm{~s}$.

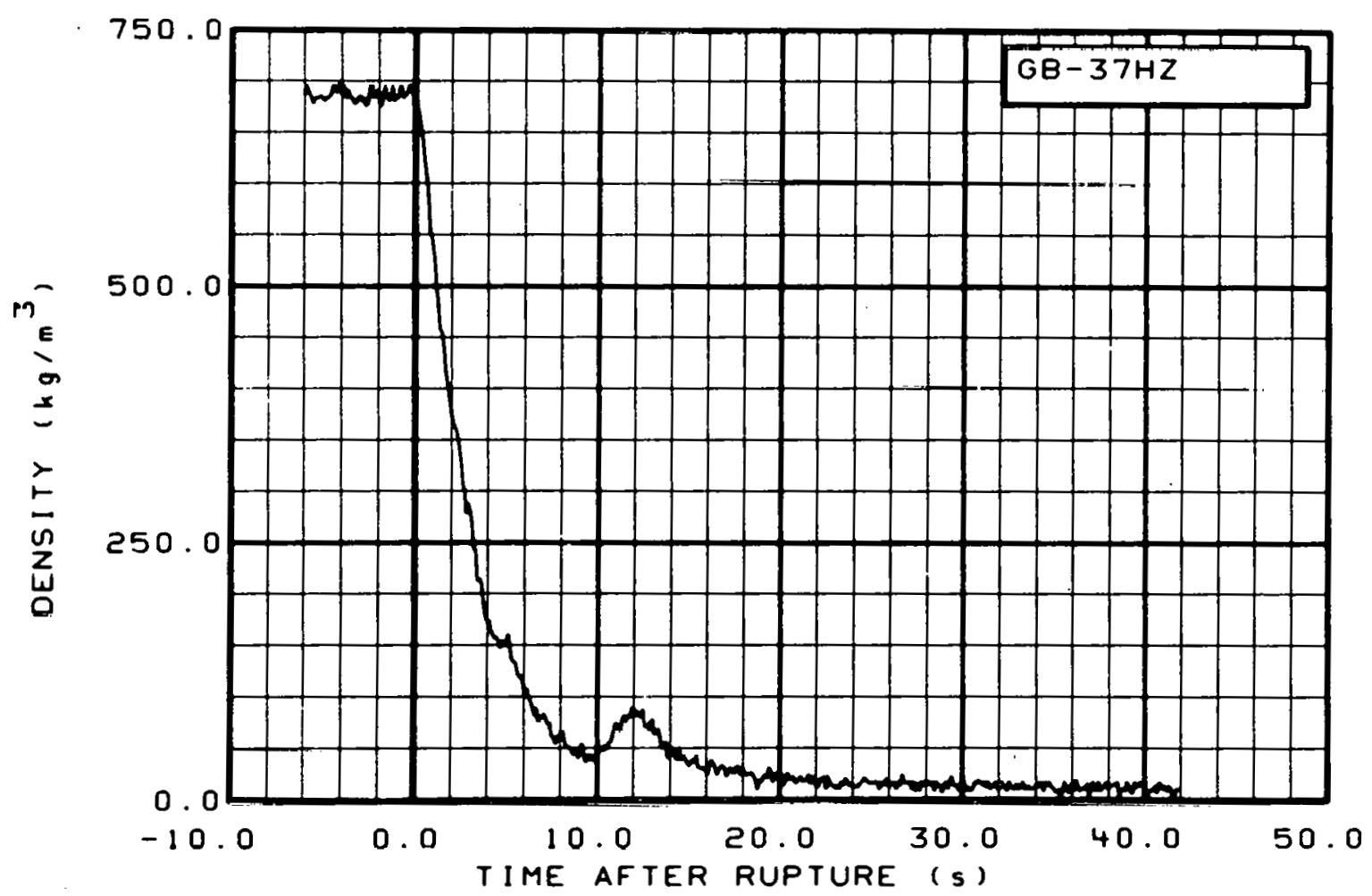

Fig. 314 Density in broken loop (GB-37HZ), from -6 to $42 \mathrm{~s}$. 


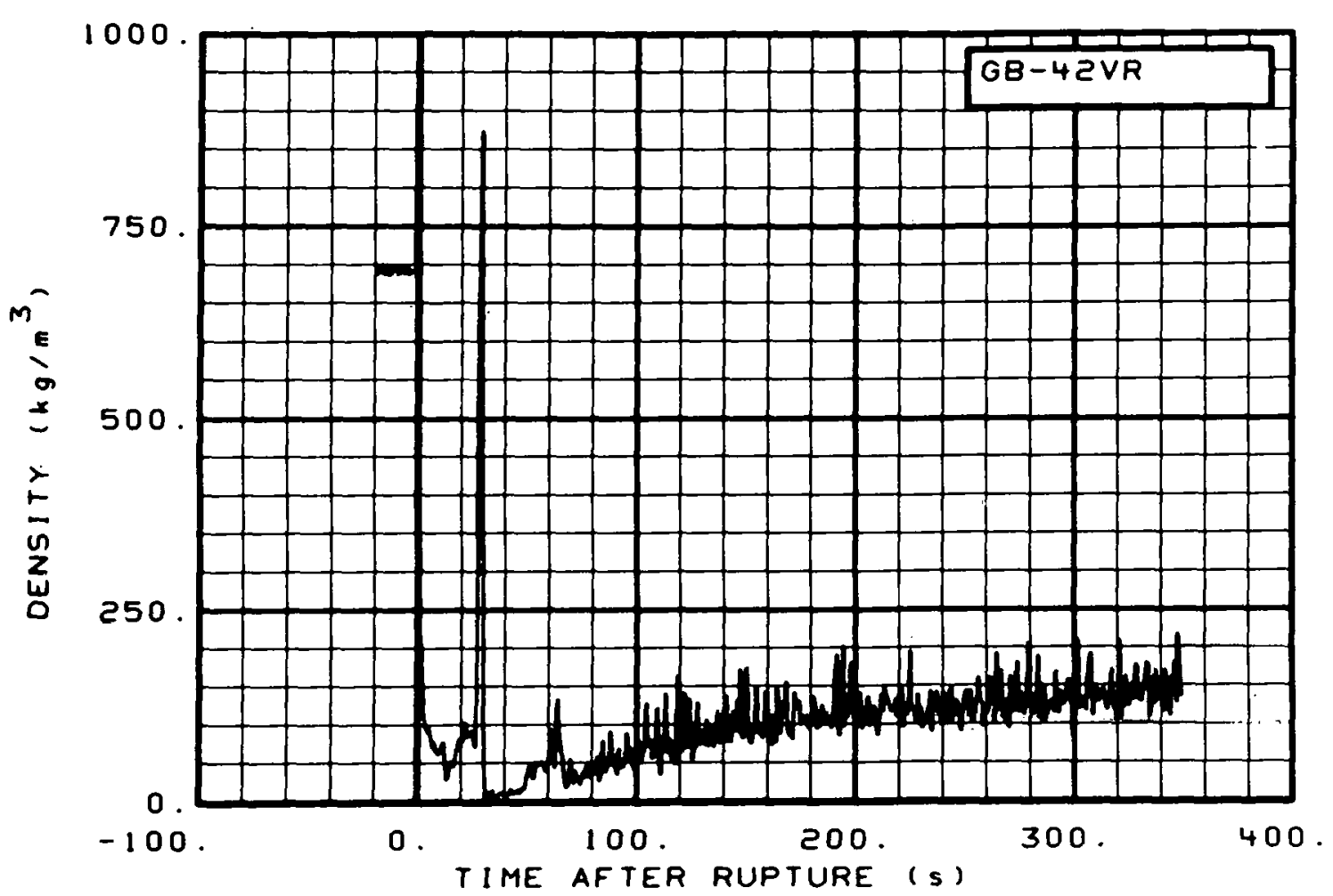

Fig. 315 Density in broken loop (GB-42VR), from -20 to $350 \mathrm{~s}$.

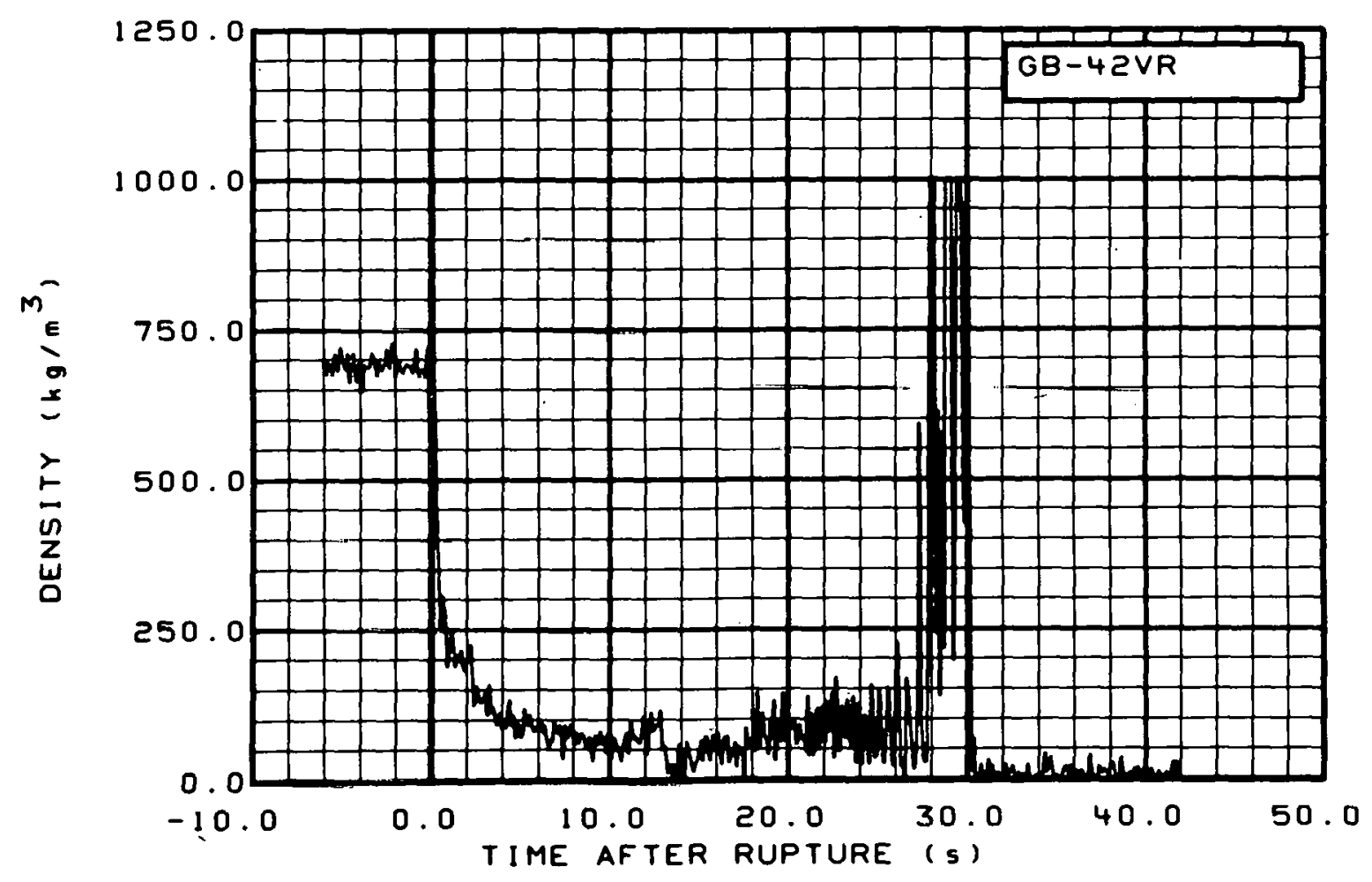

Fig. 316 Density in broken loop (GB-42VR), from -6 to $42 \mathrm{~s}$. 


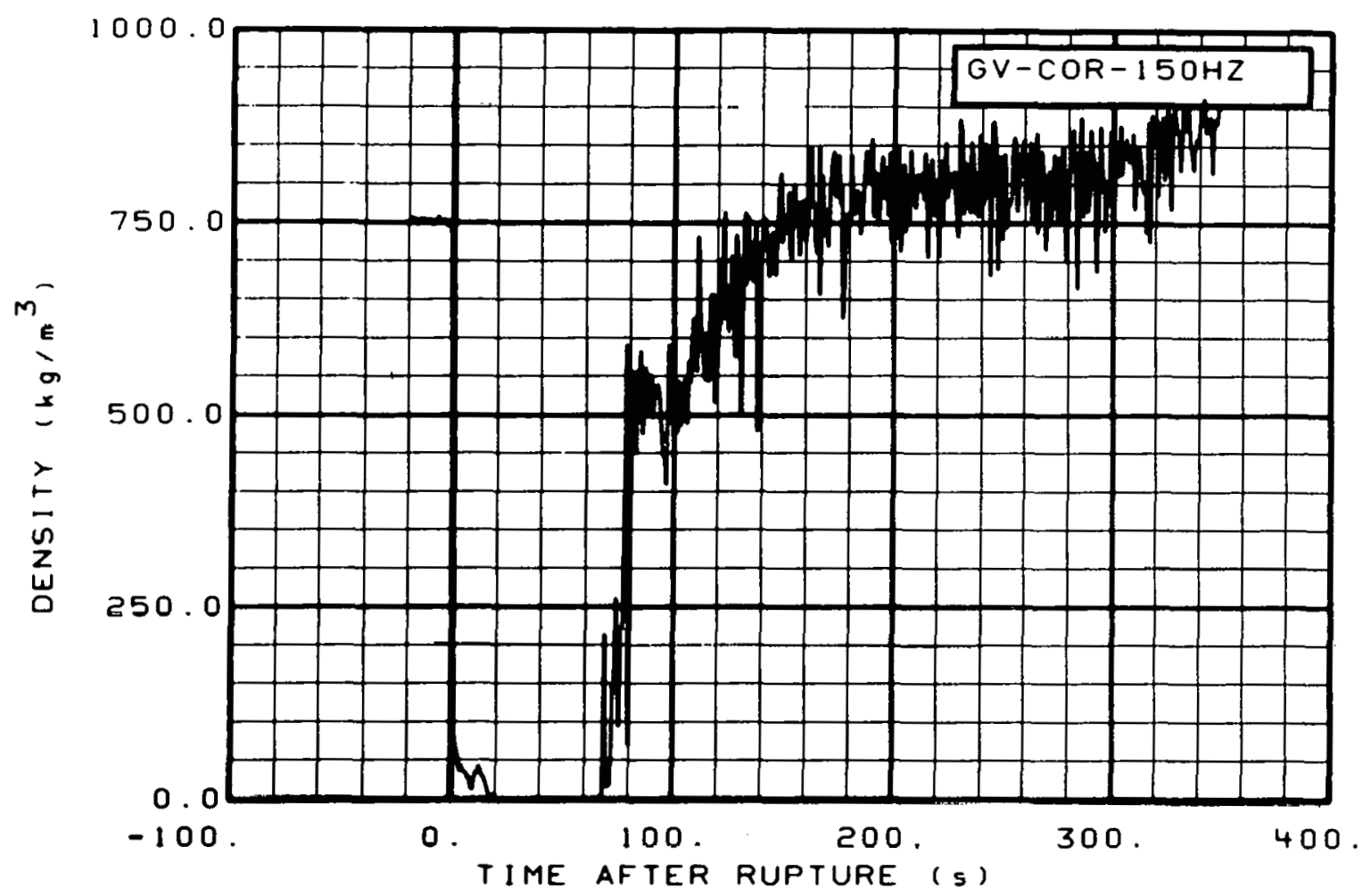

Fig. 317 Density in vessel (GV-COR-150HZ), from -20 to $350 \mathrm{~s}$.

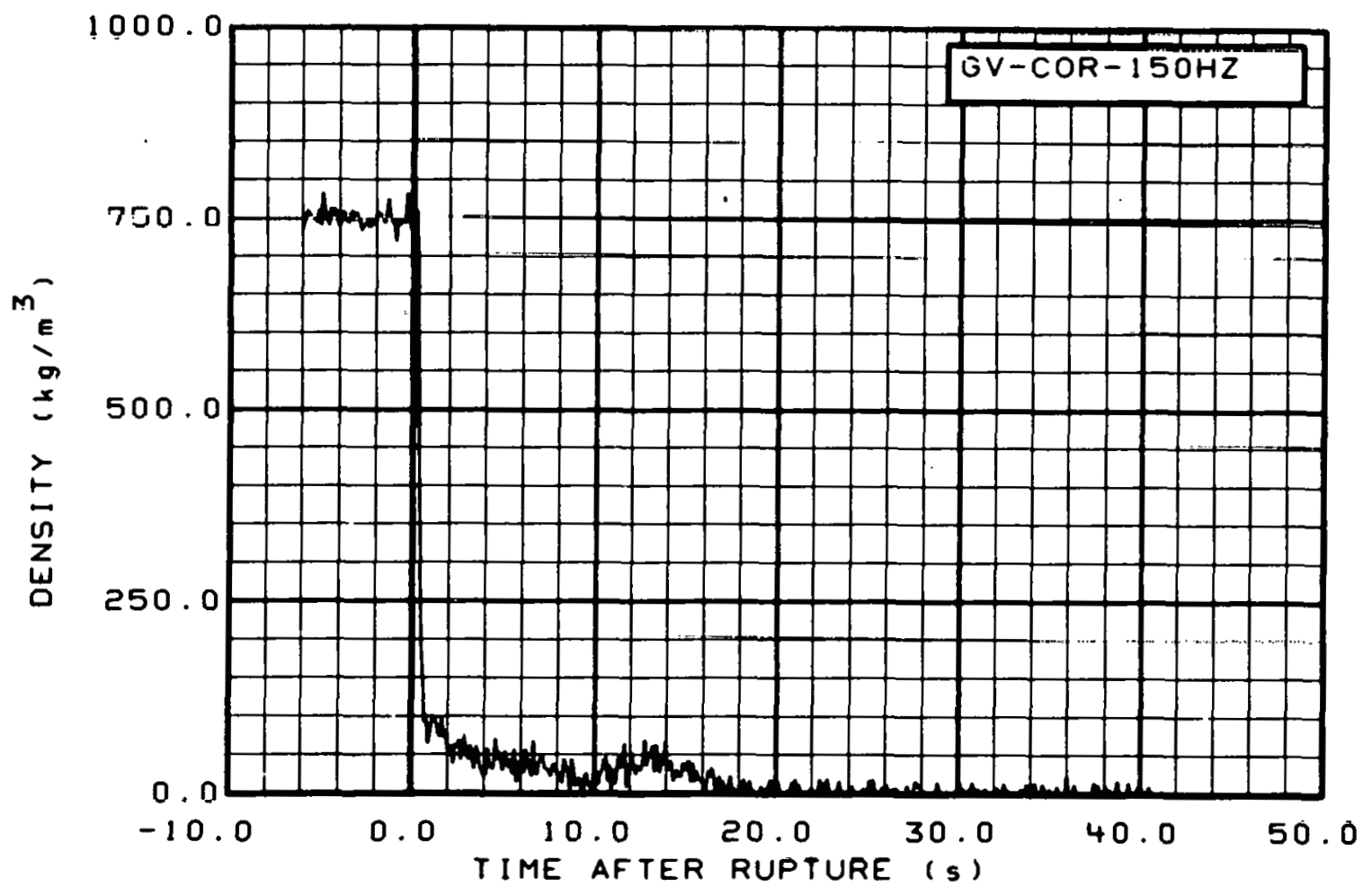

Fig. 318 Density in vessel (GV-COR-150HZ), from -6 to $42 \mathrm{~s}$. 


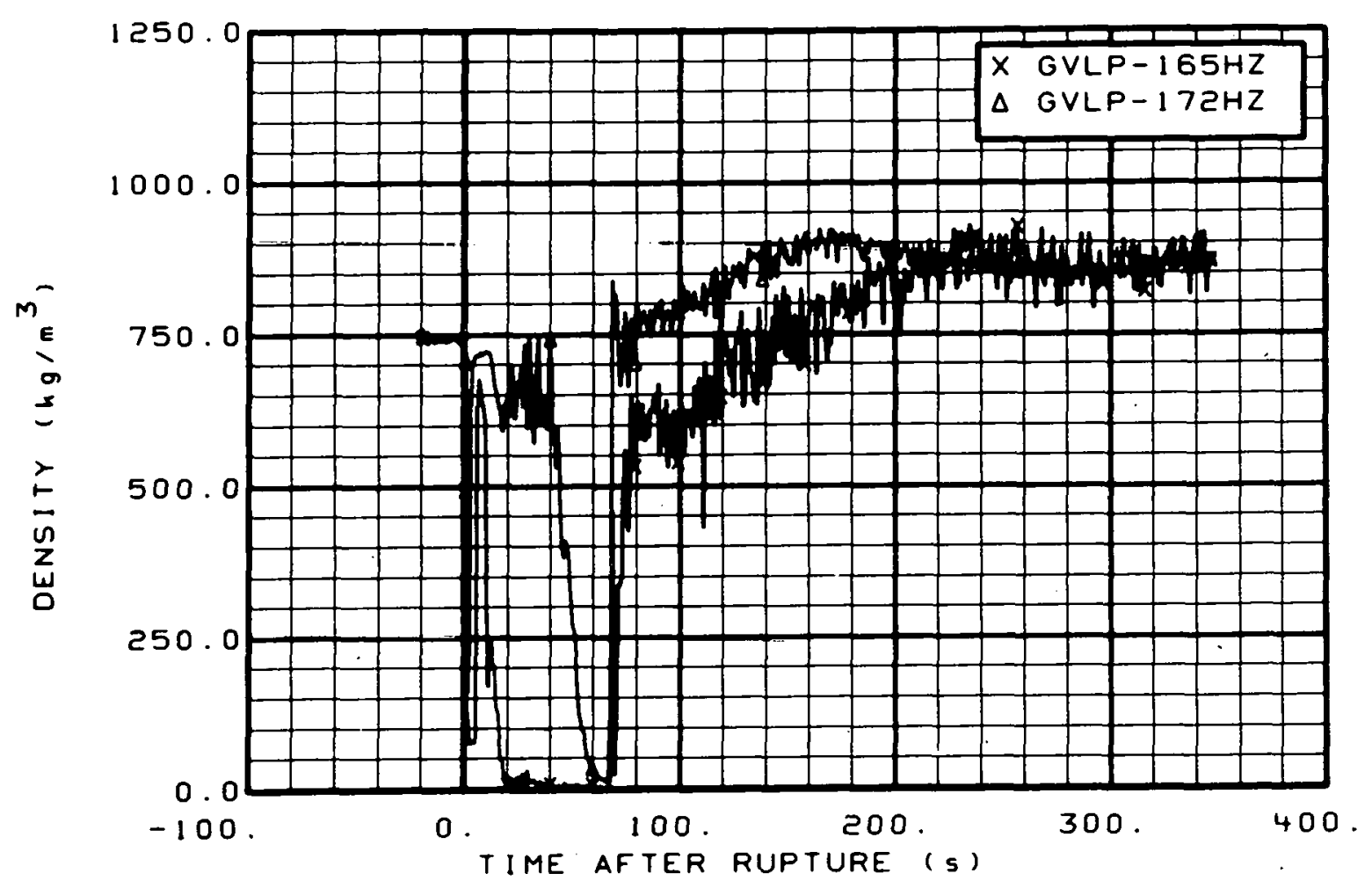

Fig. 319 Density in vessel (GVLP-165HZ and GVLP-172HZ), from -20 to $350 \mathrm{~s}$.

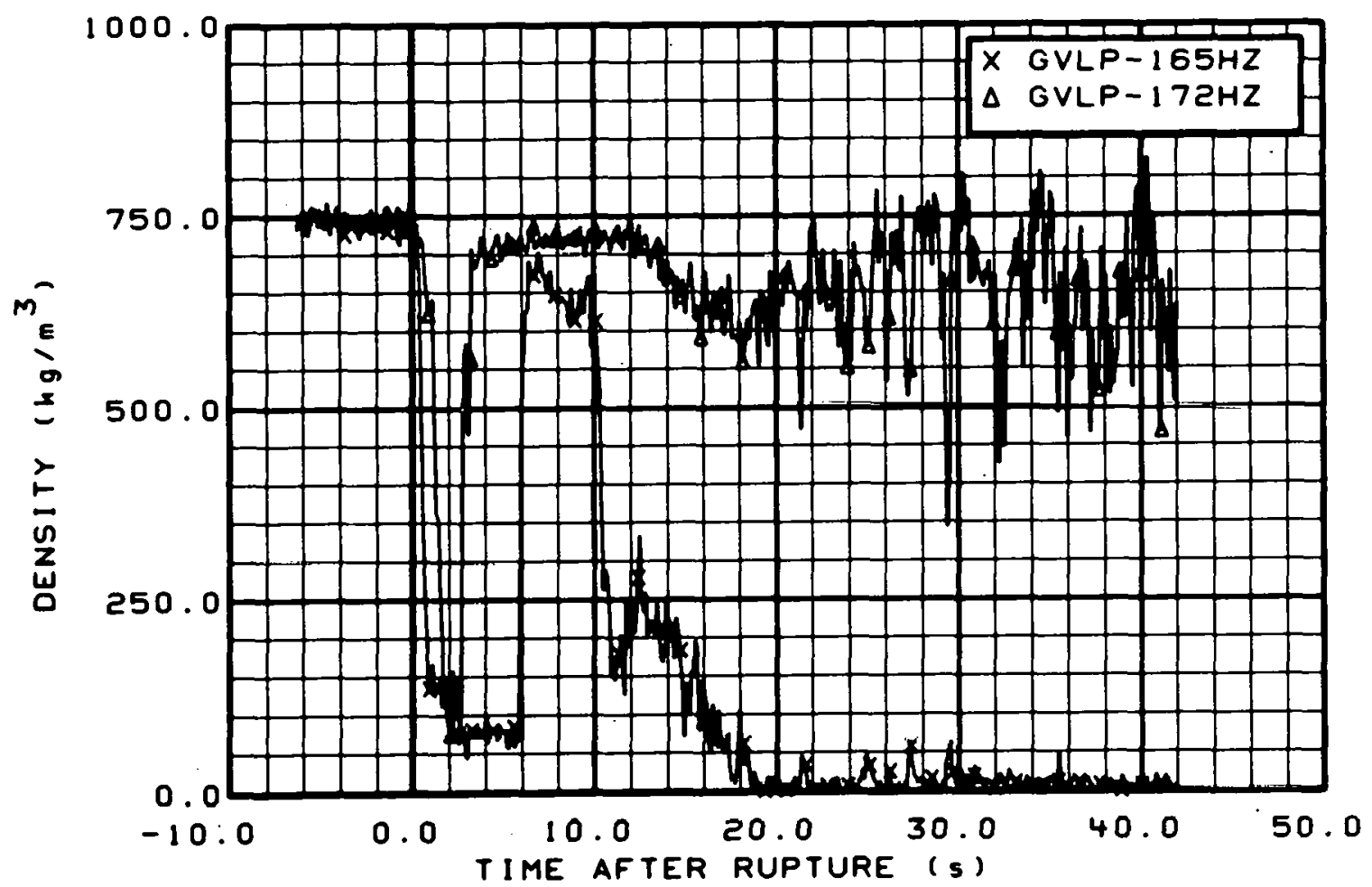

Fig. 320 Density in vessel (GVLP-165HZ and GVLP-172HZ), from -6 to $42 \mathrm{~s}$. 


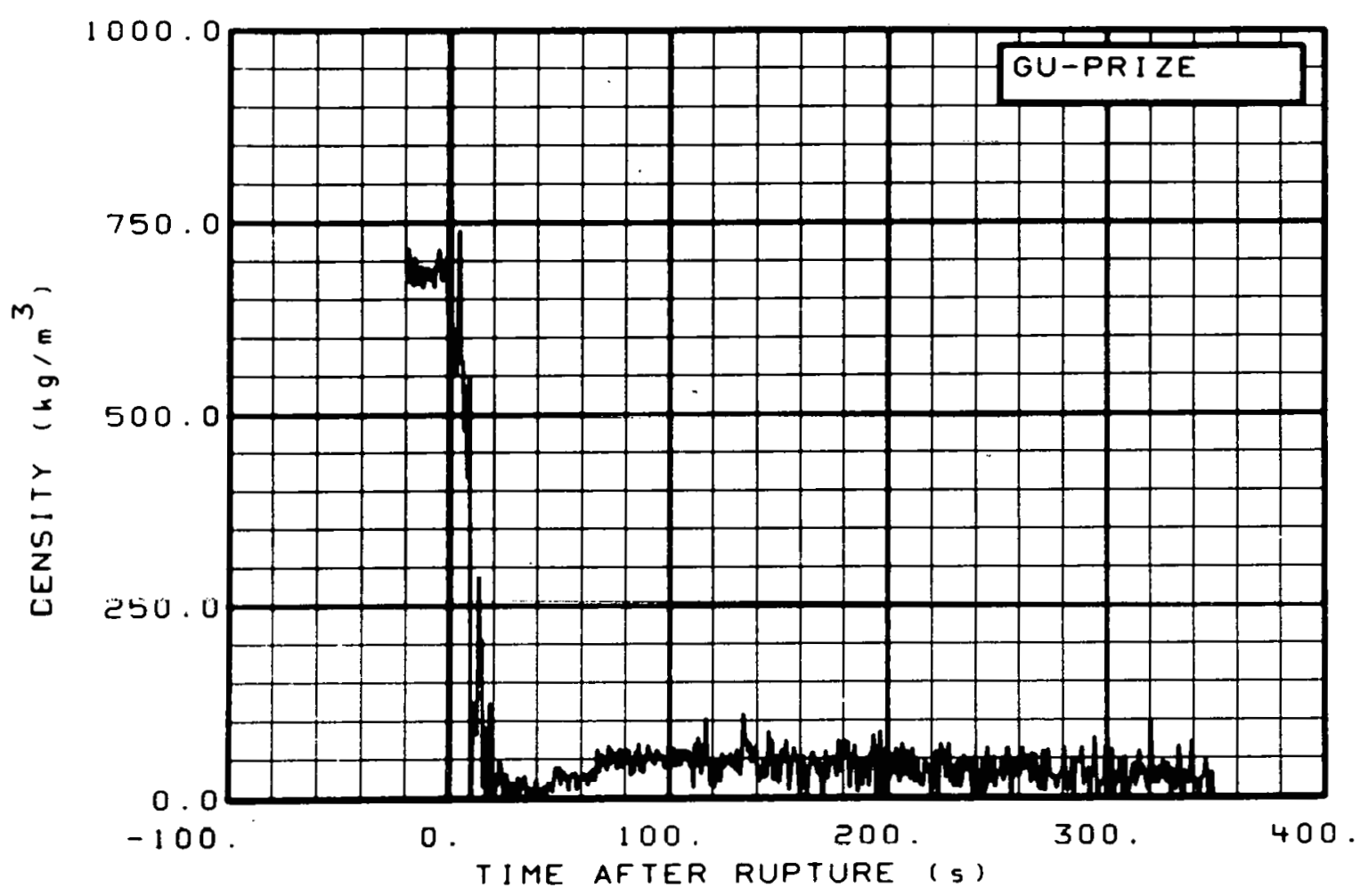

Fig. 321 Density in pressurizer (GU-PRIZE), from -20 to $350 \mathrm{~s}$.

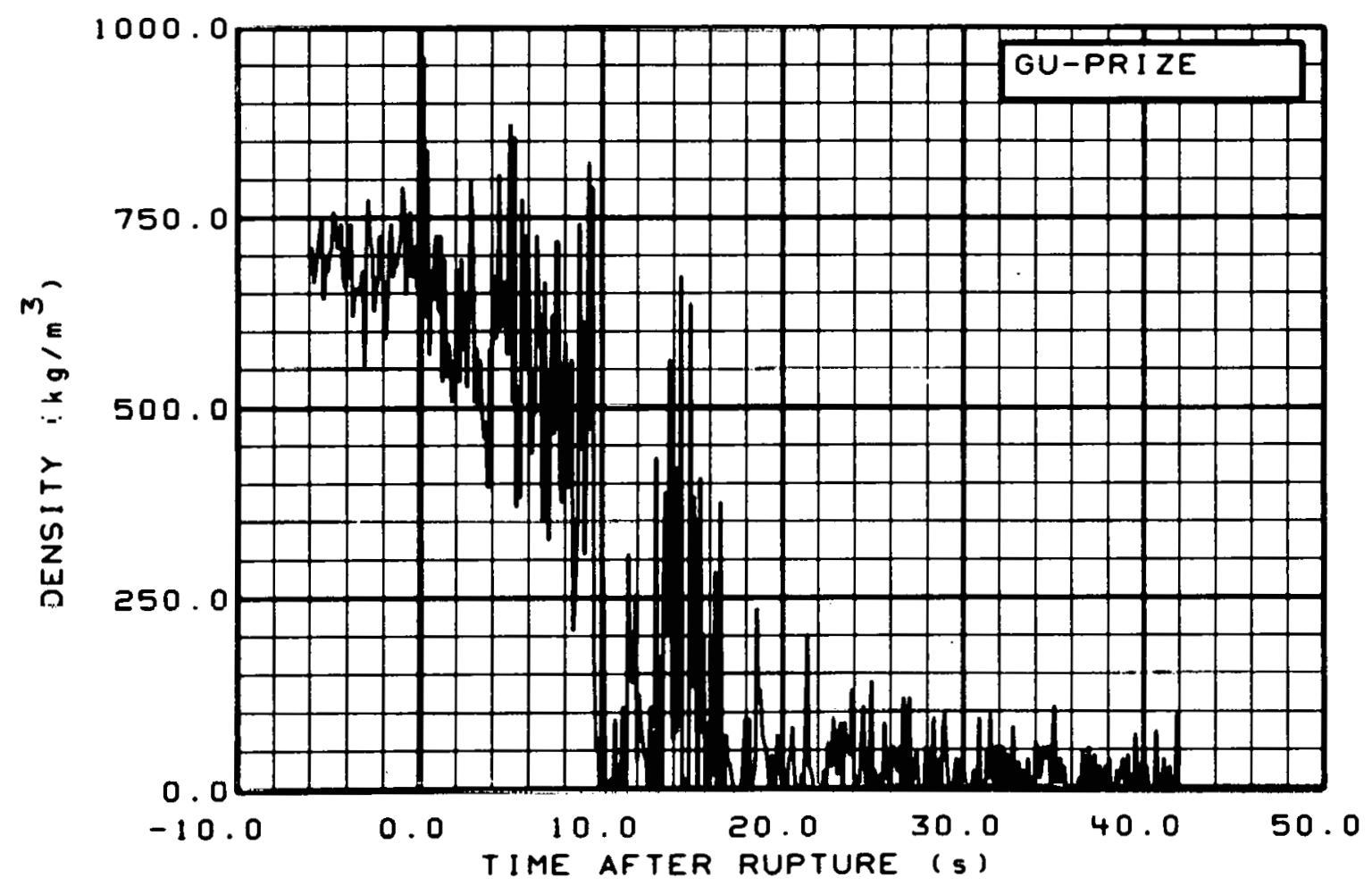

Fig. 322 Density in pressurizer (GU-PRIZE), from -6 to $42 \mathrm{~s}$. 


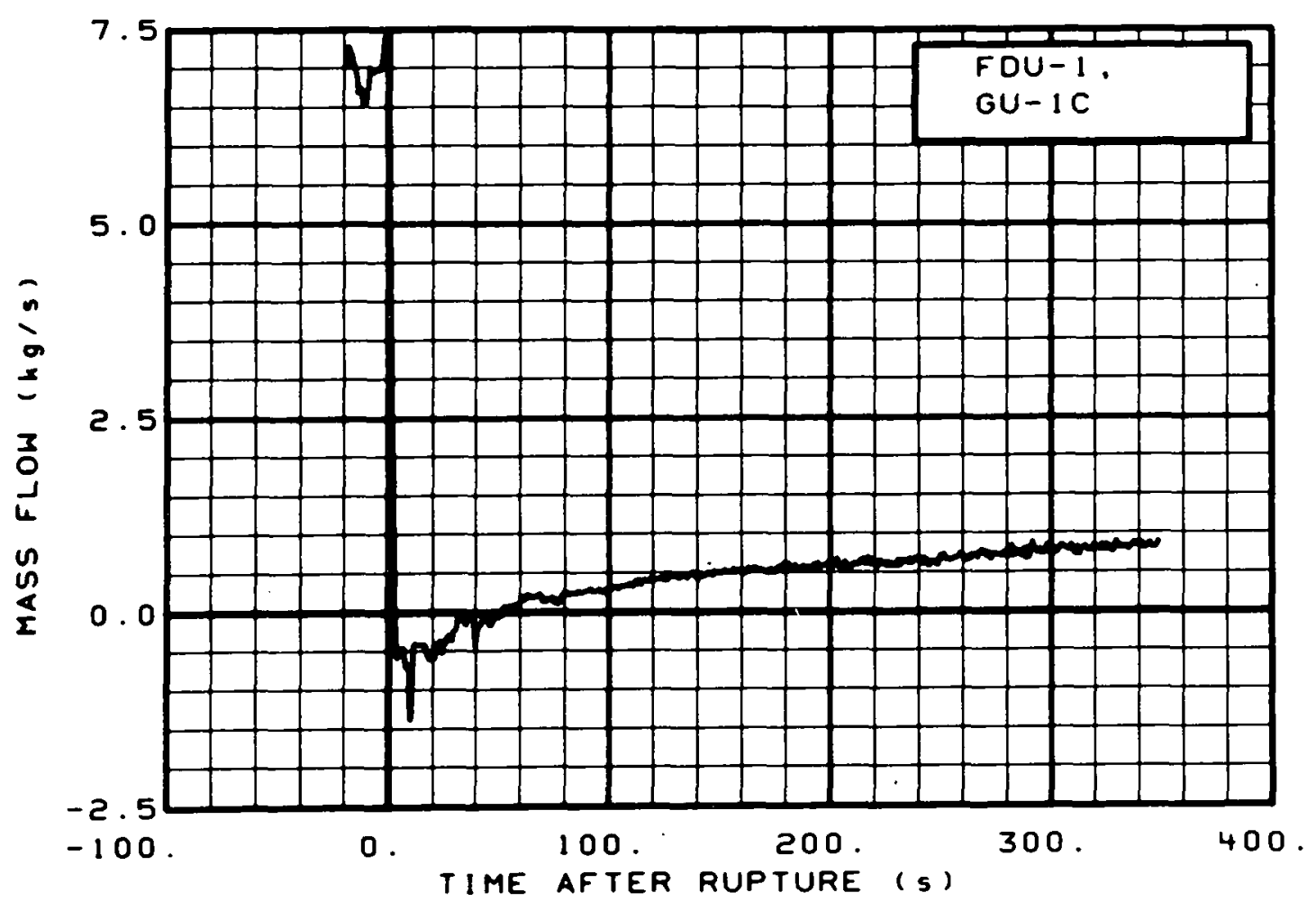

Fig. 323 Mass flow in intact loop (FDU-1, GU-1C), from -20 to $350 \mathrm{~s}$.

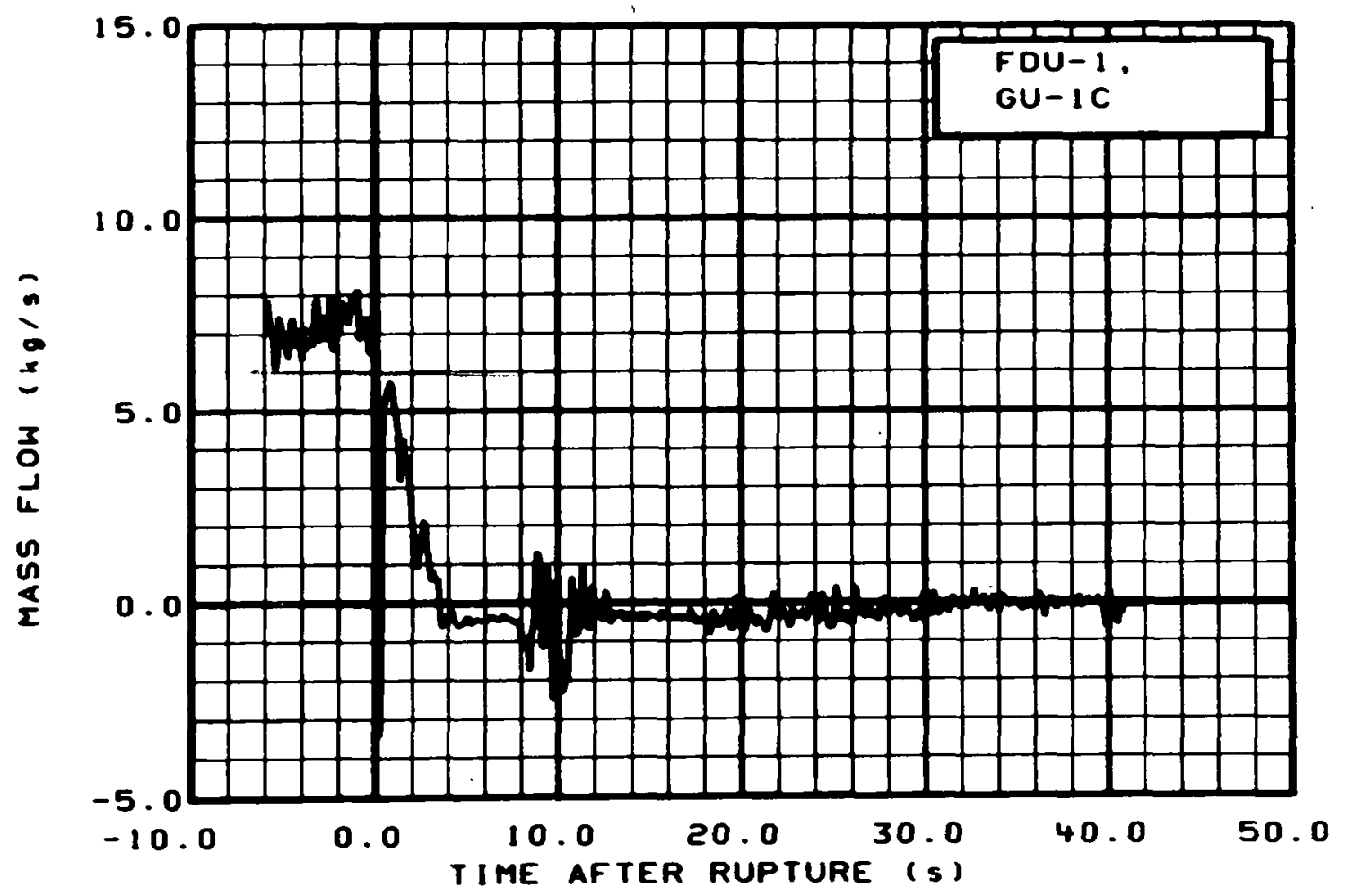

Fig. 324 Mass flow in intact loop (FDU-1, GU-1C), from -6 to $42 \mathrm{~s}$. 


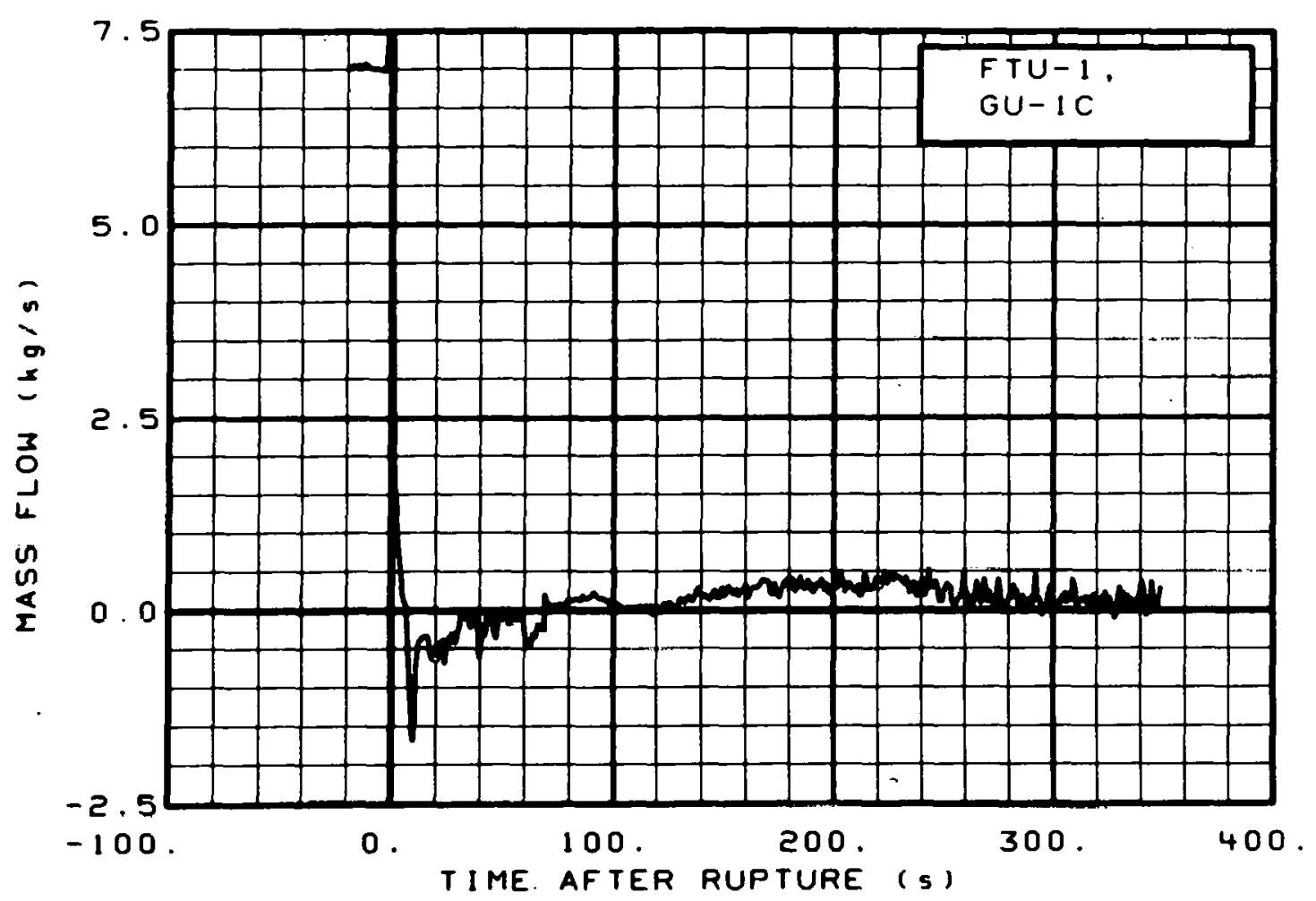

Fig. 325 Mass flow in intact loop (FTU-1, GU-1C), from -20 to $350 \mathrm{~s}$.

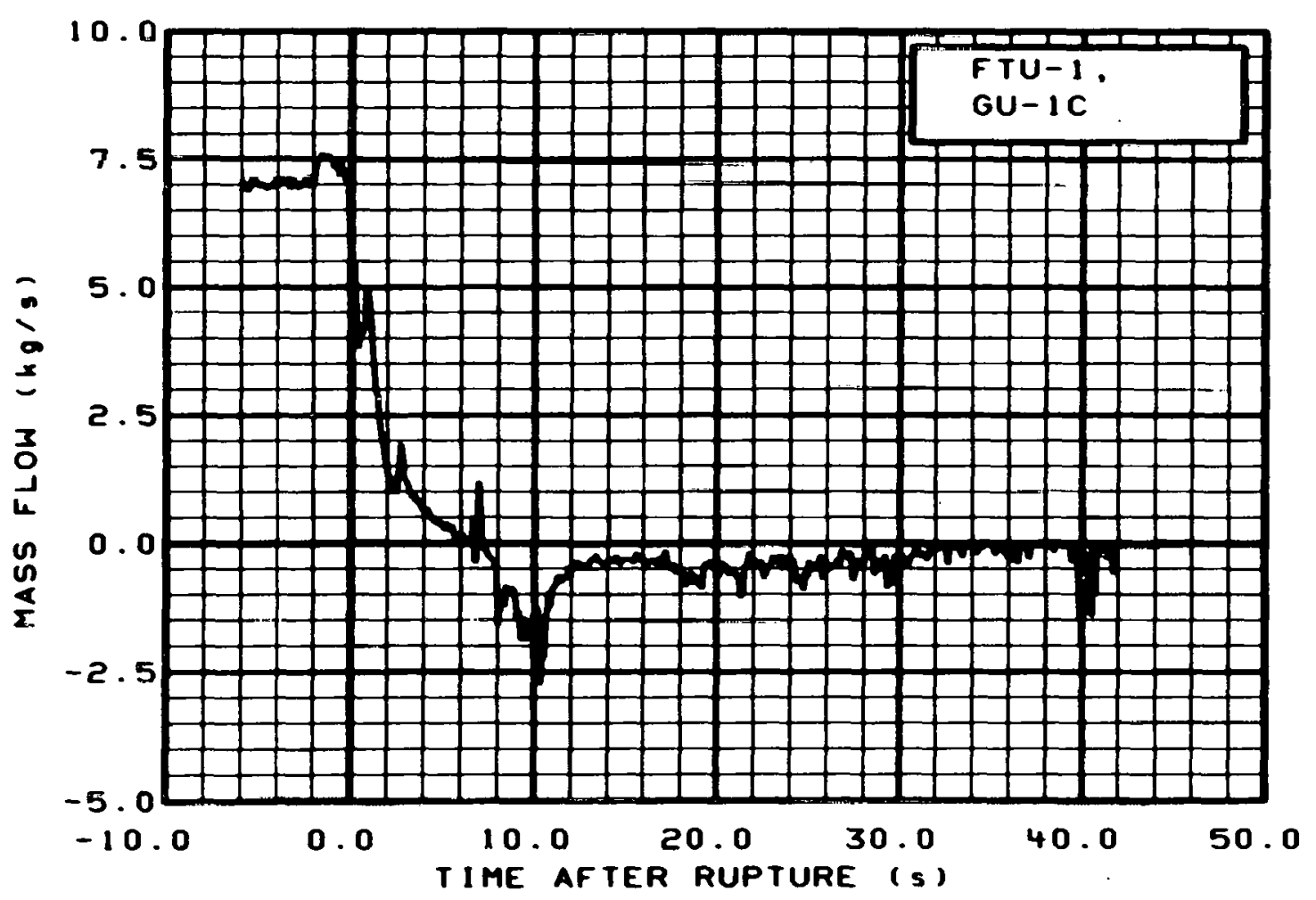

Fig. 326 Mass flow in intact loop (FTU-1, GU-1C), from -6 to $42 \mathrm{~s}$. 


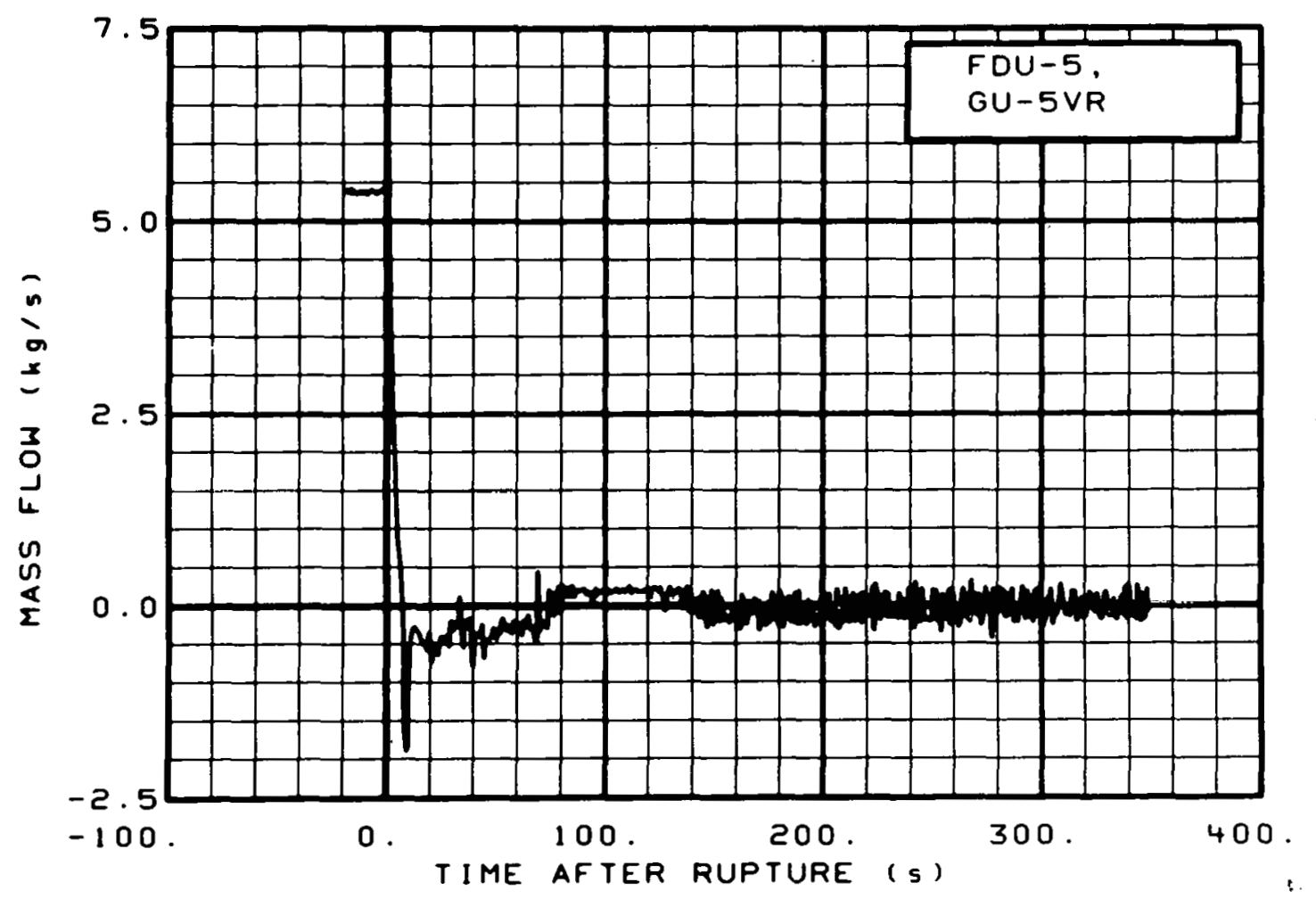

Fig. 327 Mass flow in intact loop (FDU-5, GU-5VR), from -20 to $350 \mathrm{~s}$.

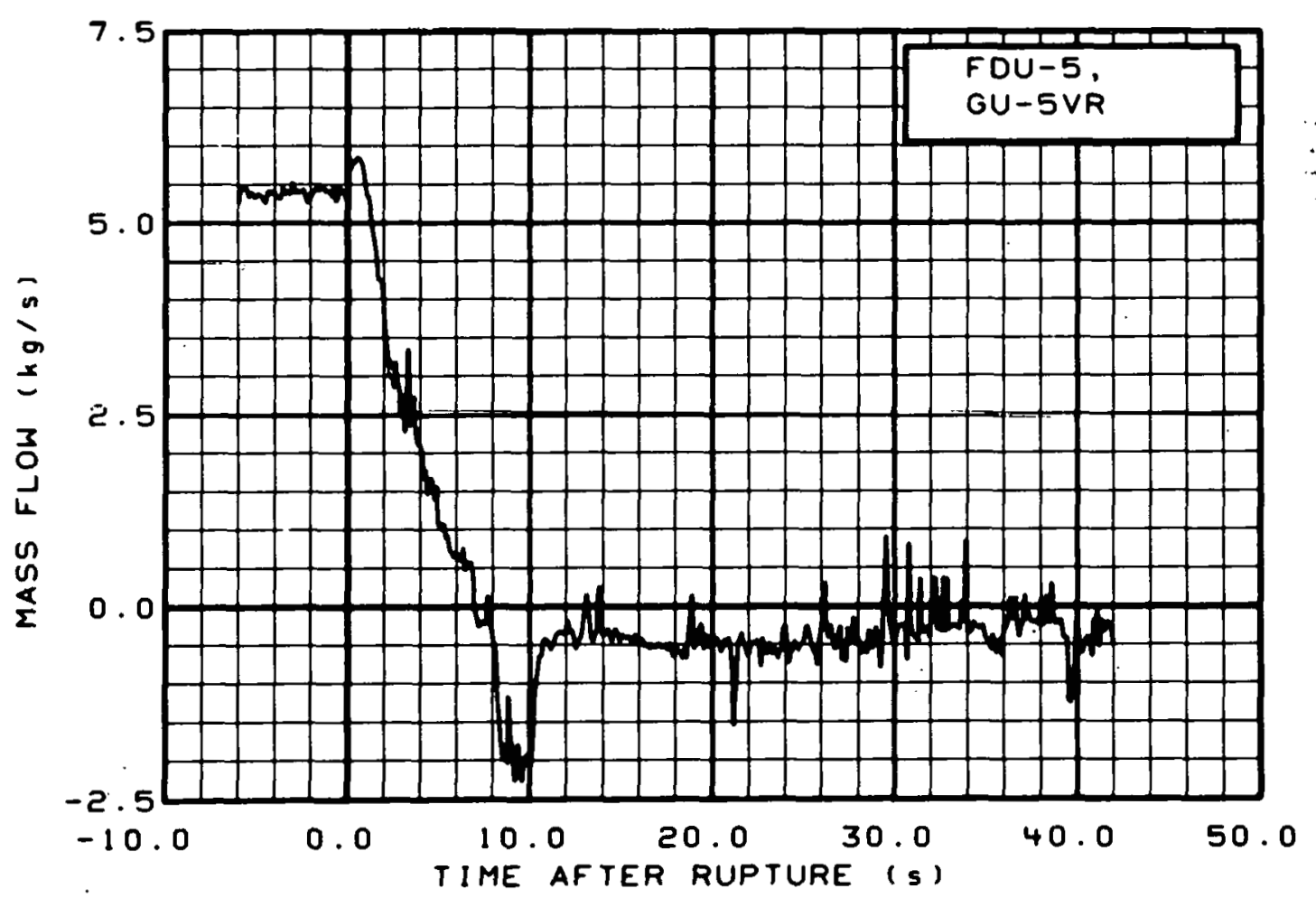

Fig. 328 Mass flow in intact loop (FDU-5, GU-5VR), from -6 to $42 \mathrm{~s}$. 


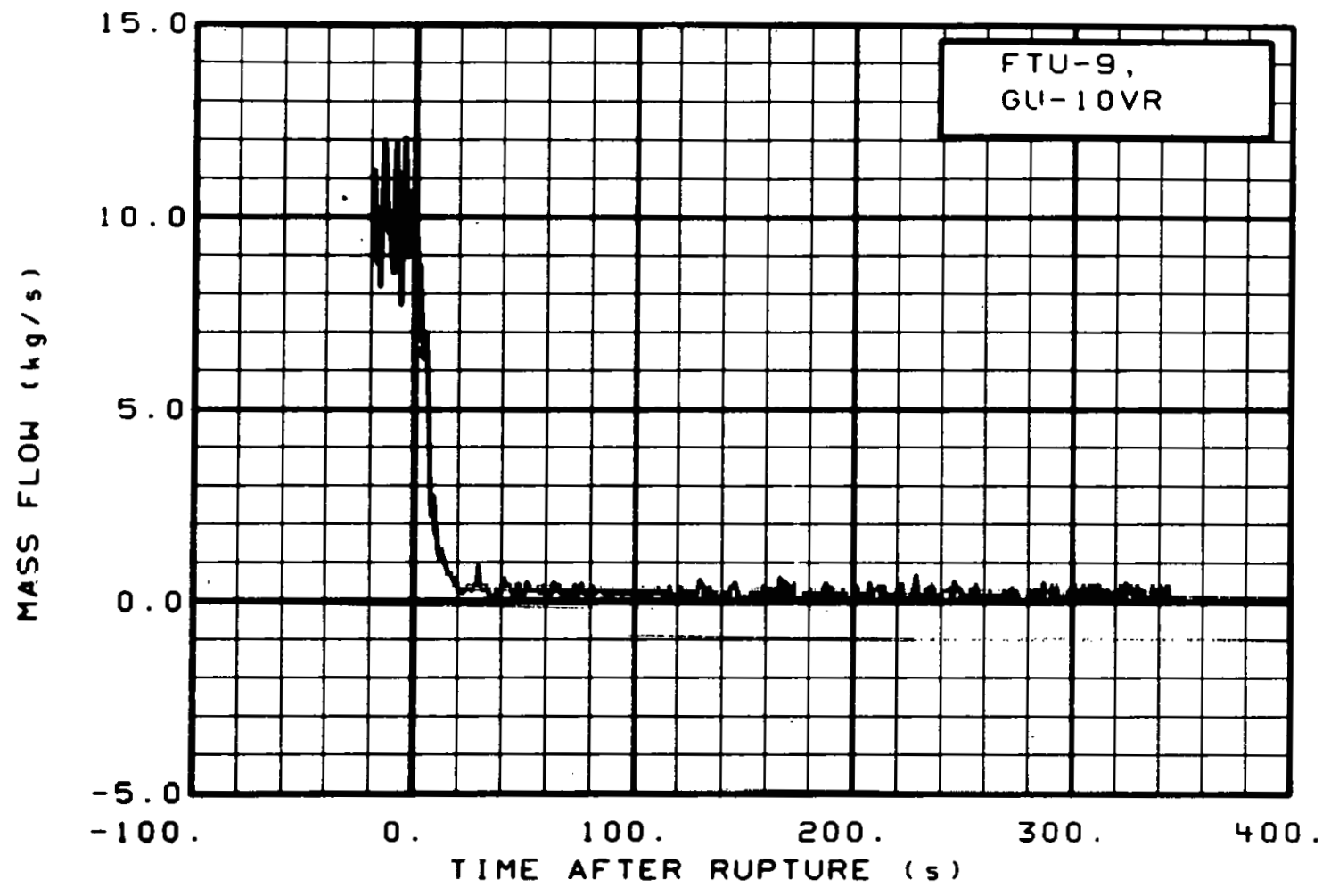

Fig. 329 Mass flow in intact loop (FTU-9, GU-10VR), from -20 to $350 \mathrm{~s}$.

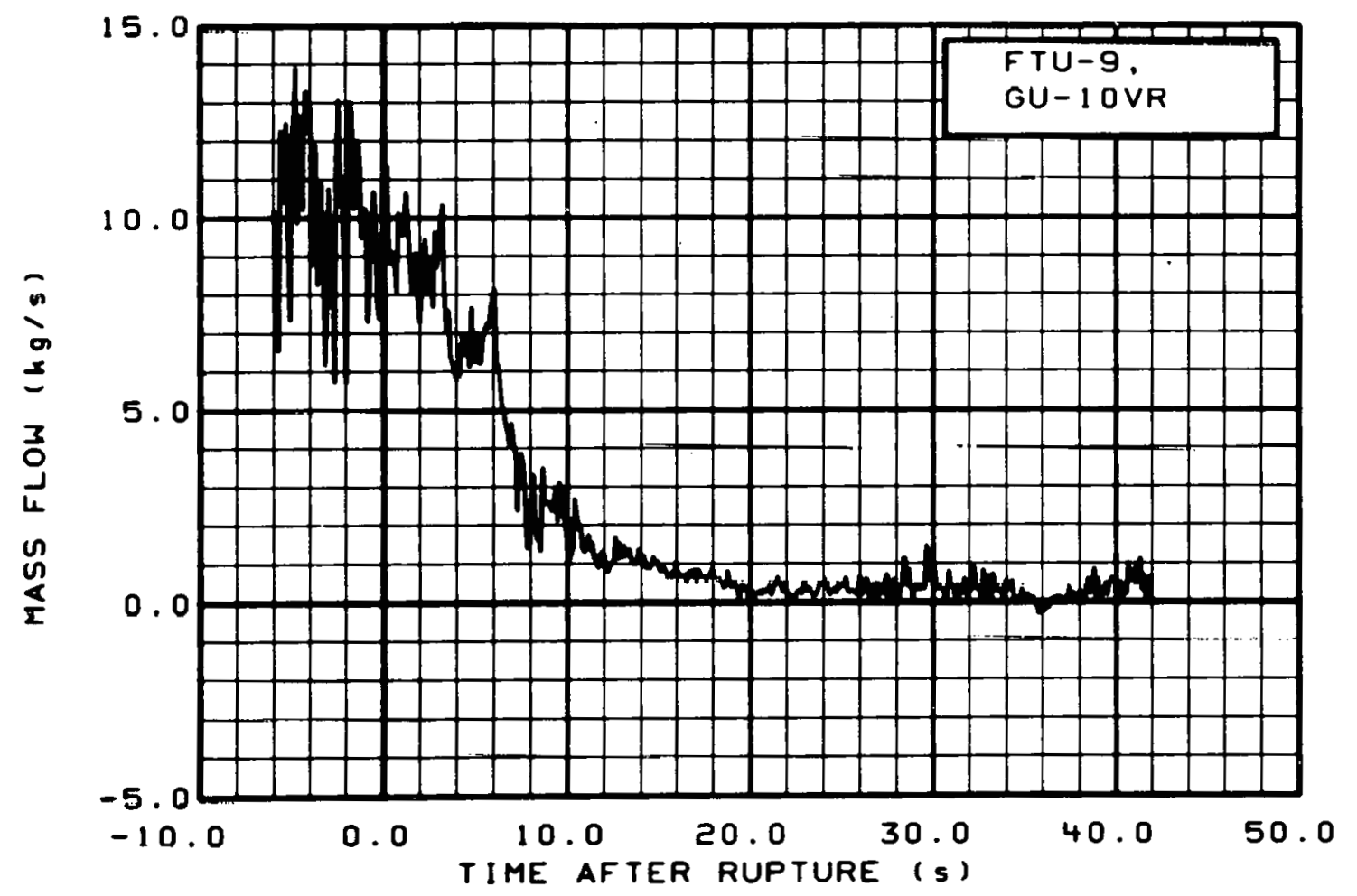

Fig. 330 Mass flow in intact loop (FTU-9, GU-70VR), from -6 to $42 \mathrm{~s}$. 


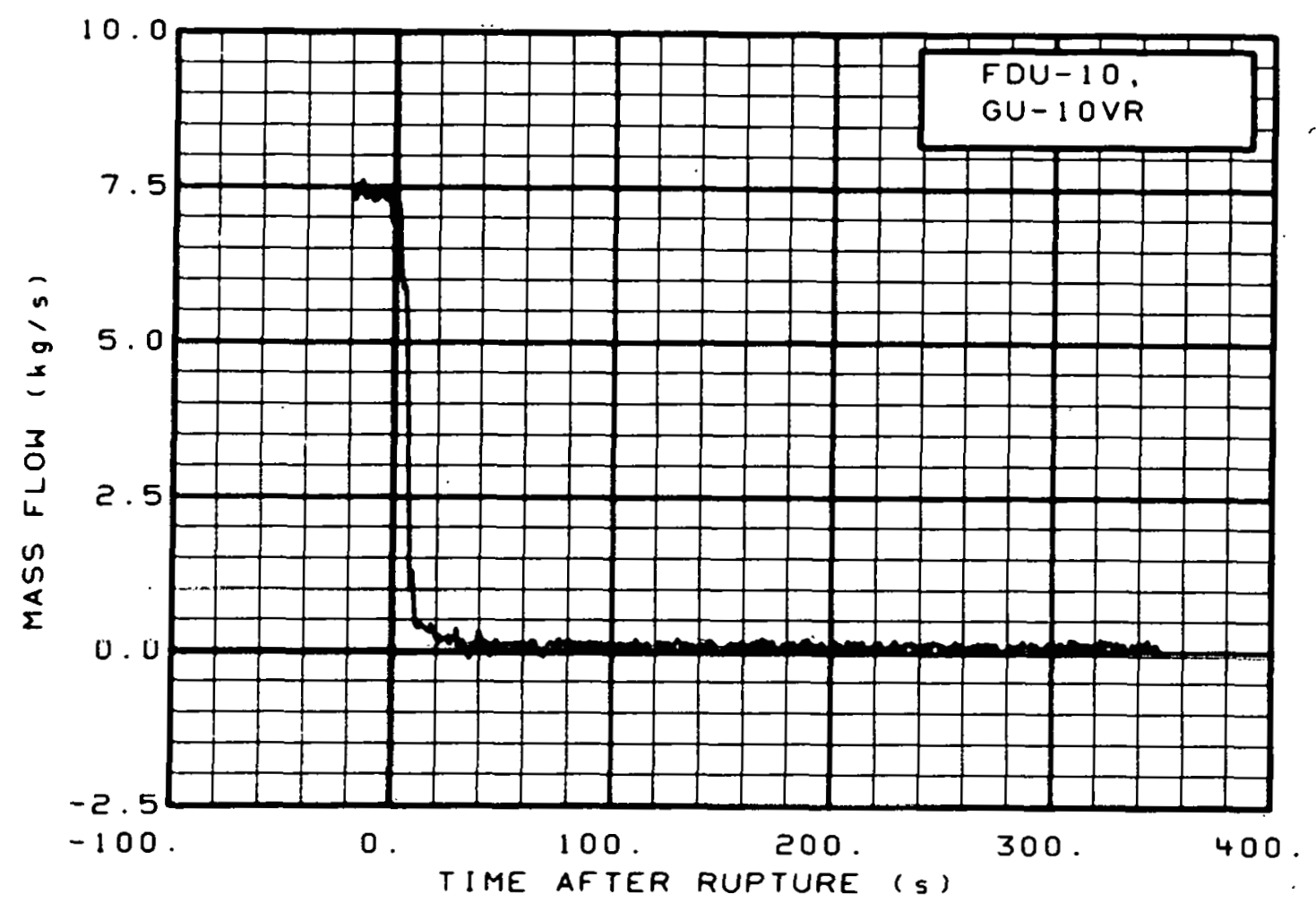

Fig. 331 Mass flow in intact loop (FDU-10, GU-10VR), from -20 to $350 \mathrm{~s}$.

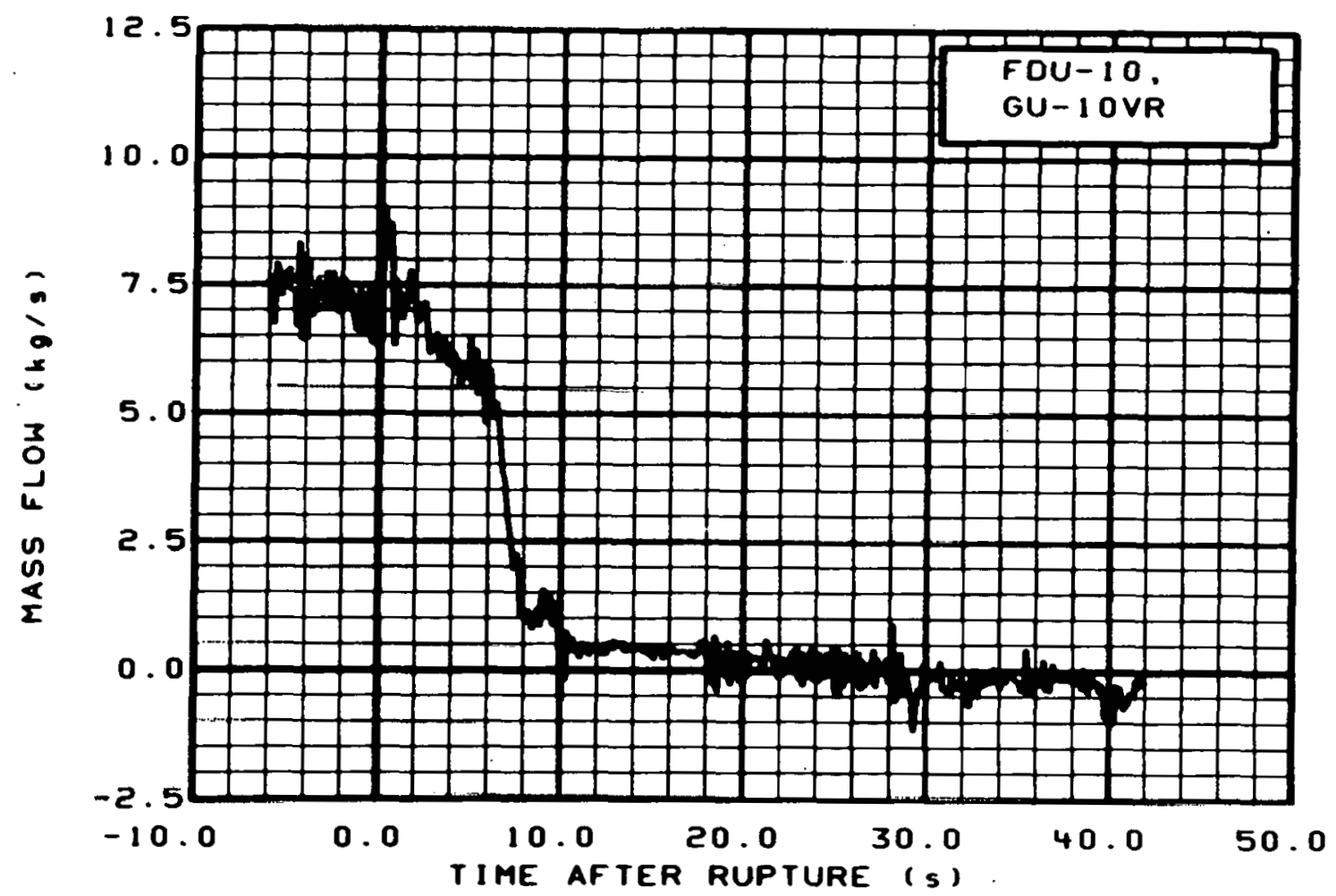

Fig. 332 Mass flow in intact loop (FDU-10, GU-10VR), from -6 to $42 \mathrm{~s}$. 


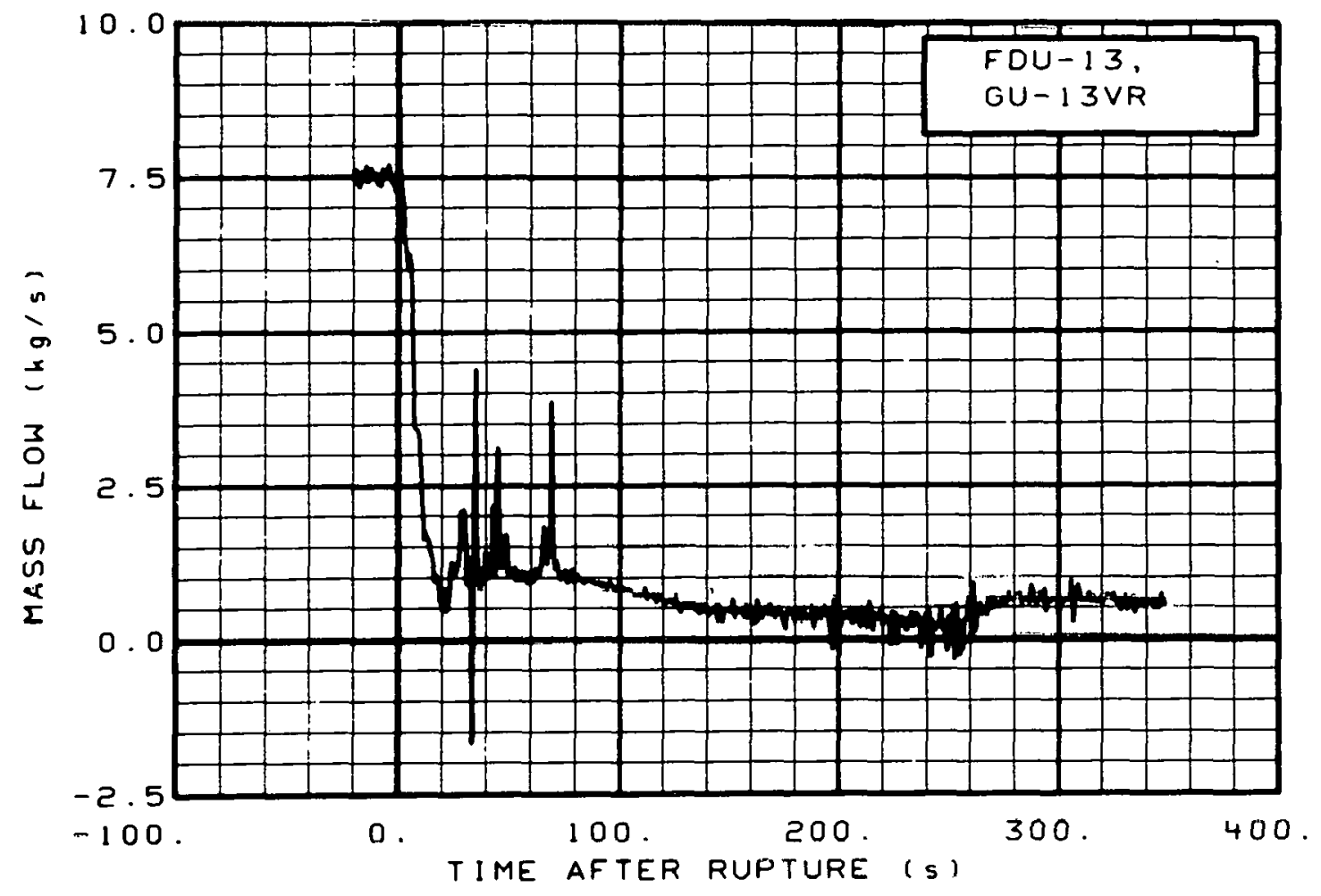

Fig. 333 Mass flow in intact loop (FDU-13, GU-13VR), from -20 to $350 \mathrm{~s}$.

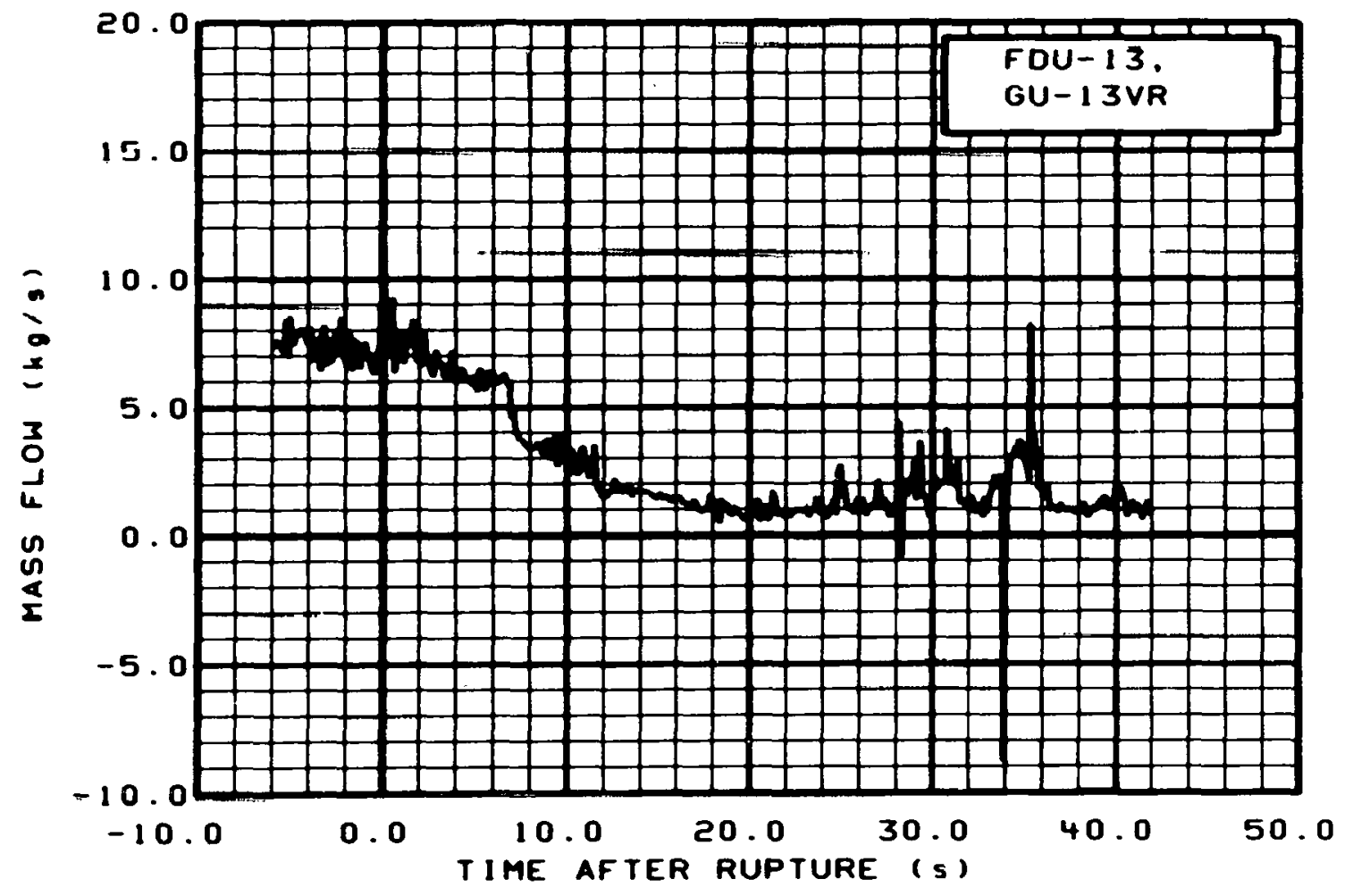

Fig. 334 Mass flow in intact loop (FDU-13, GU-13VR), from -6 to $42 \mathrm{~s}$. 


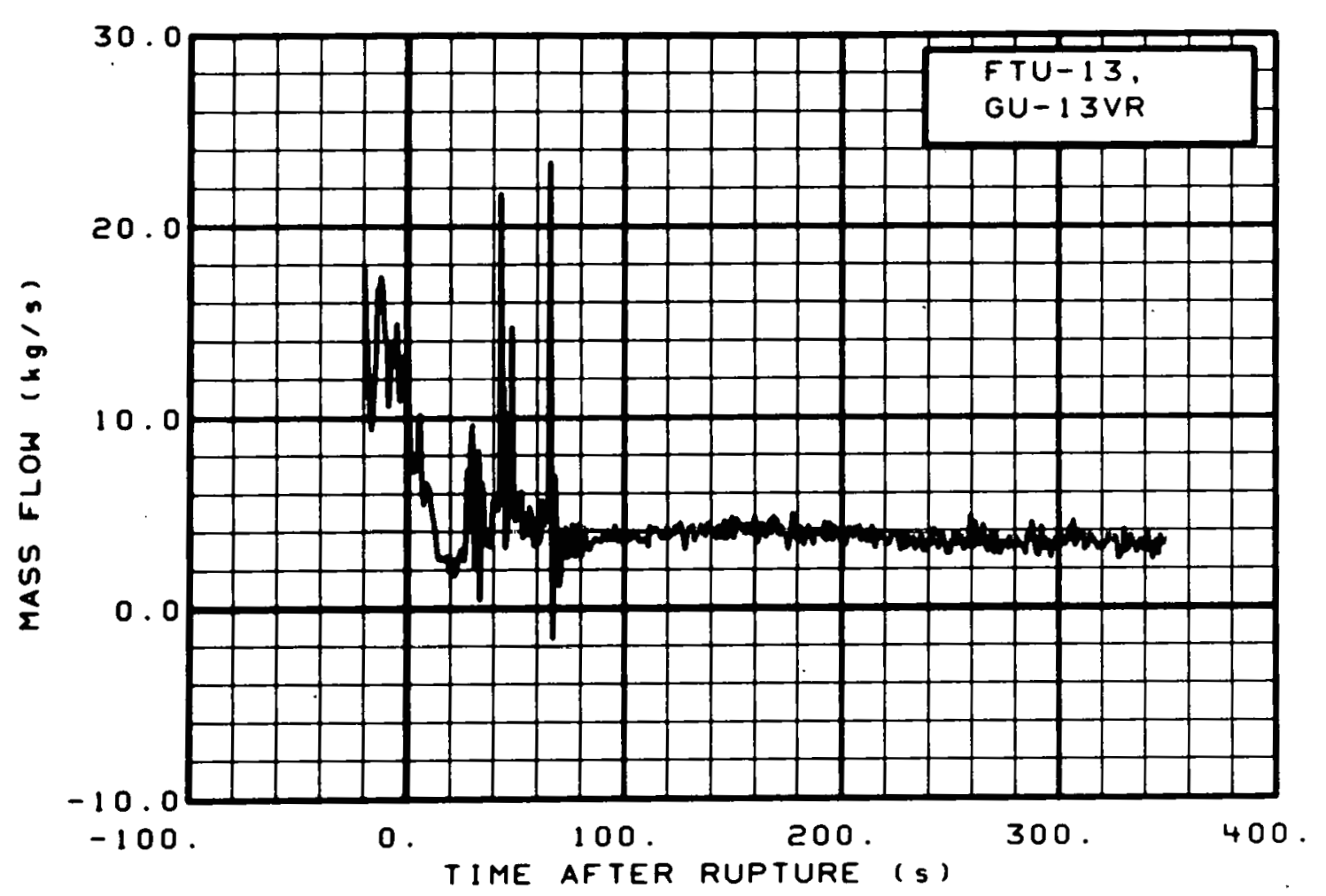

Fig. 335 Mass flow in intact 100p (FTU-13, GU-13VR), from -20 to $350 \mathrm{~s}$.

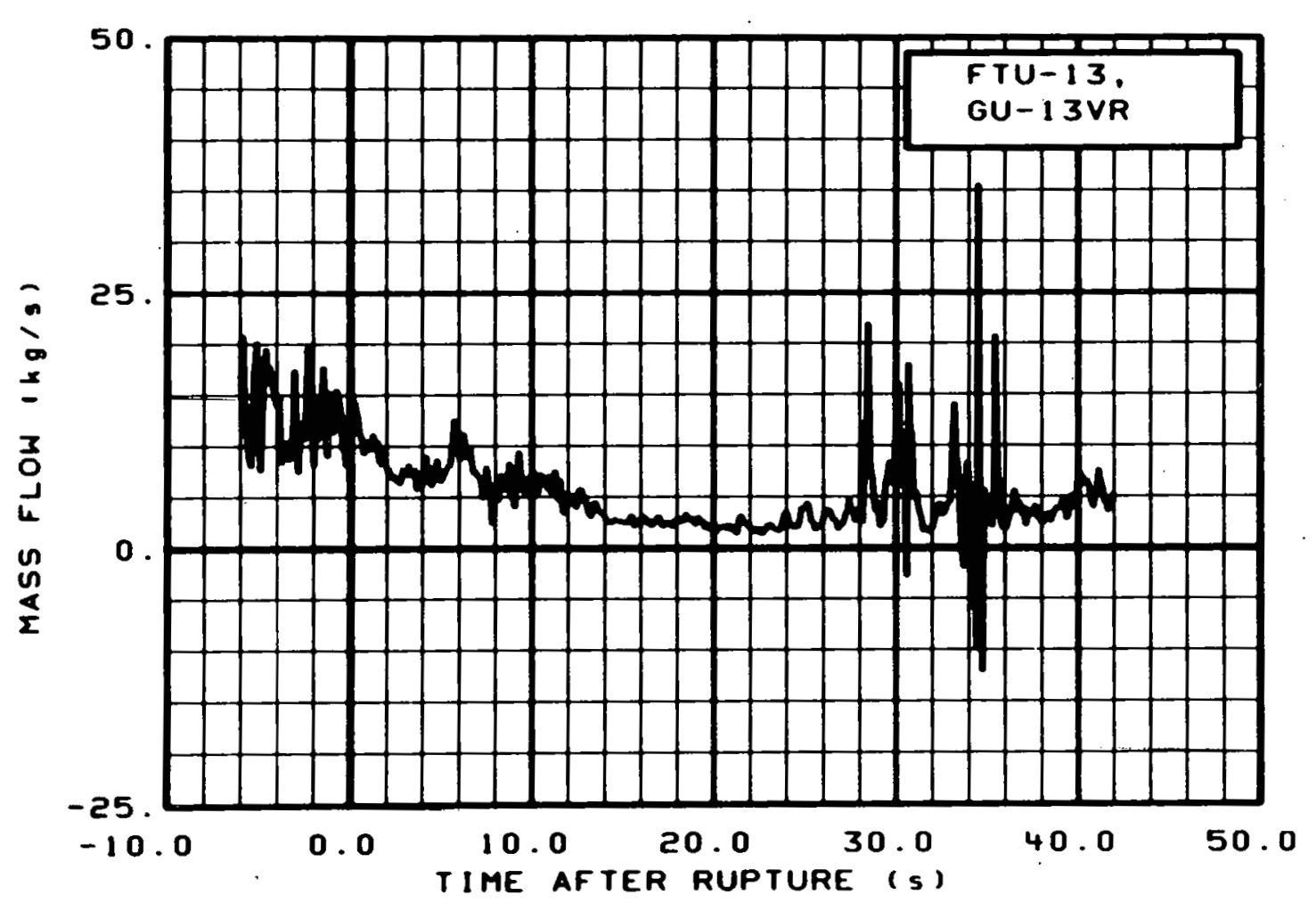

Fig. 336 Mass flow in intact loop (FTU-13, GU-13VR), from -6 to $42 \mathrm{~s}$. 


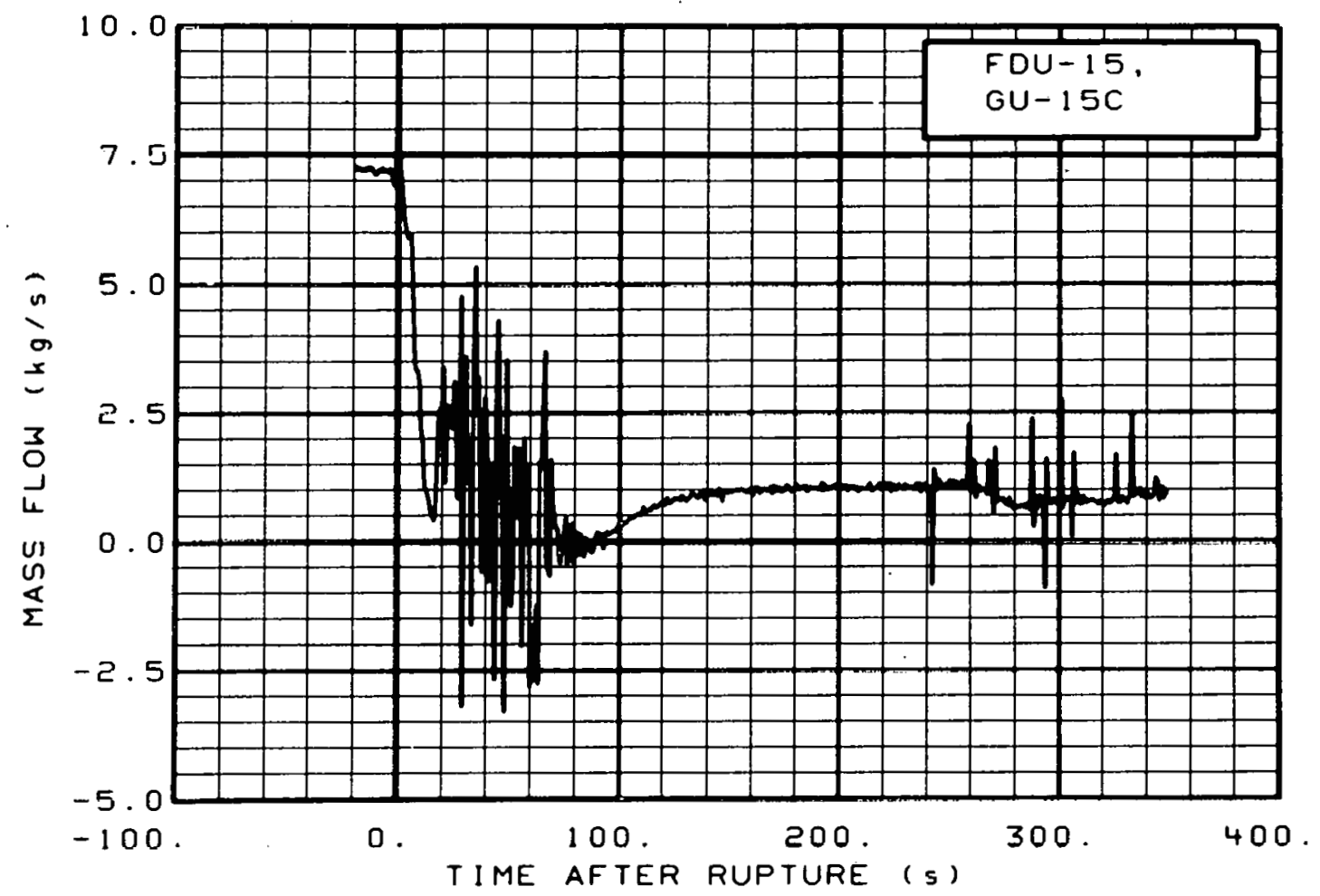

Fig. 337 Mass flow in intact loop (FDU-15, GU-15C), from -20 to $350 \mathrm{~s}$.

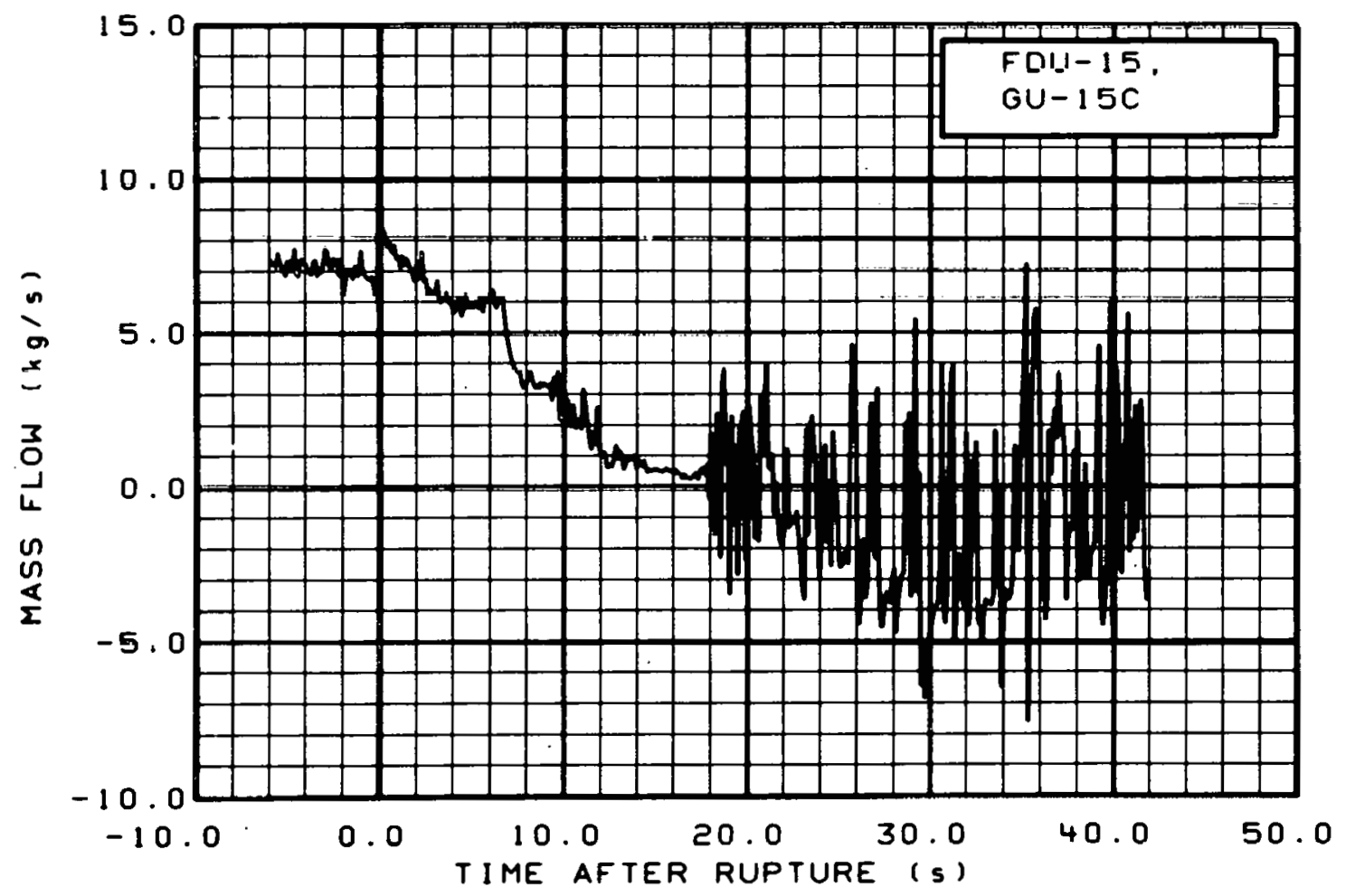

Fig. 338 Mass flow in intact loop (FDU-15, GU-15C), from -6 to $42 \mathrm{~s}$. 


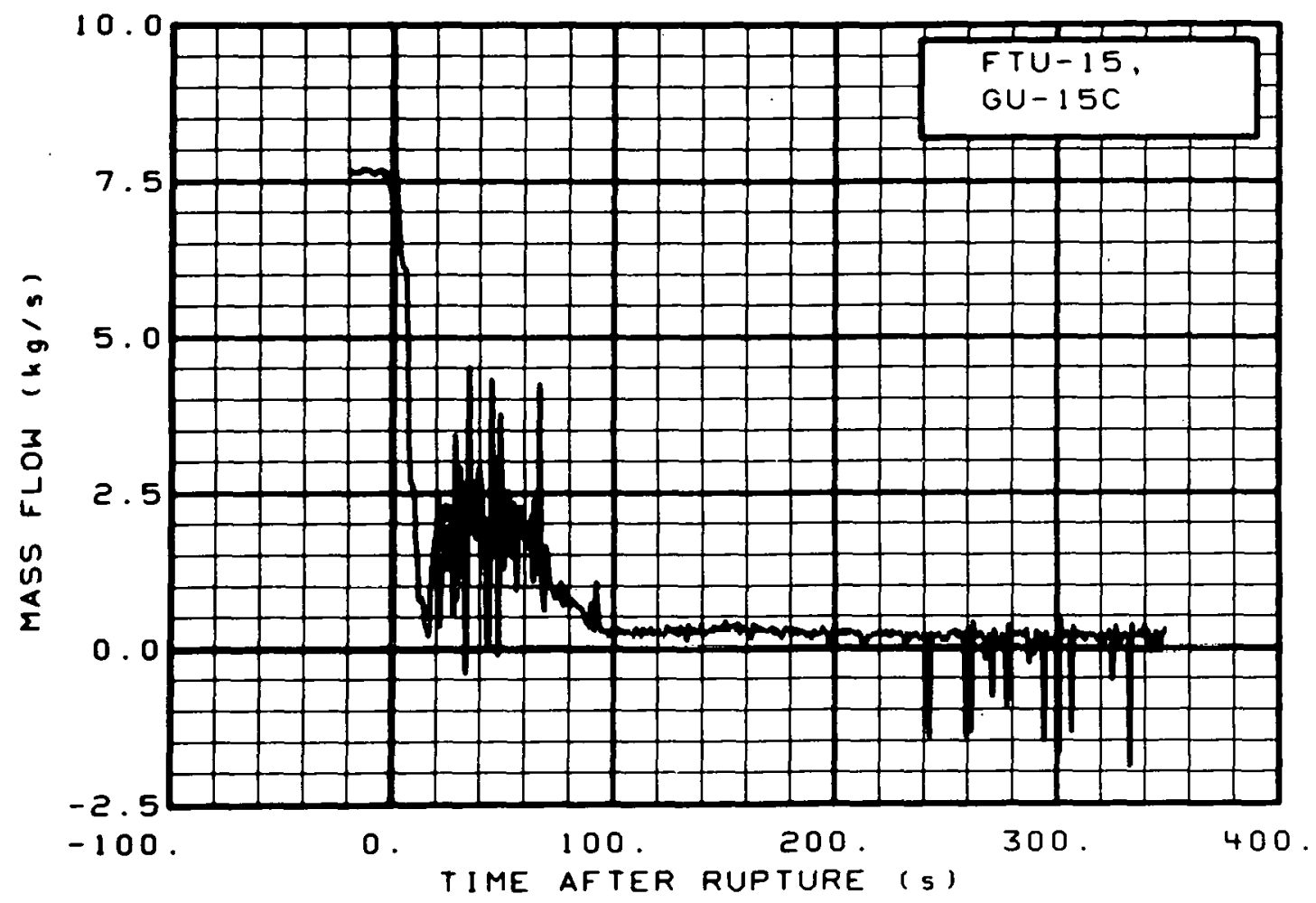

Fig. 339 Mass flow in intact loop (FTU-15, GU-15C), from -20 to $350 \mathrm{~s}$.

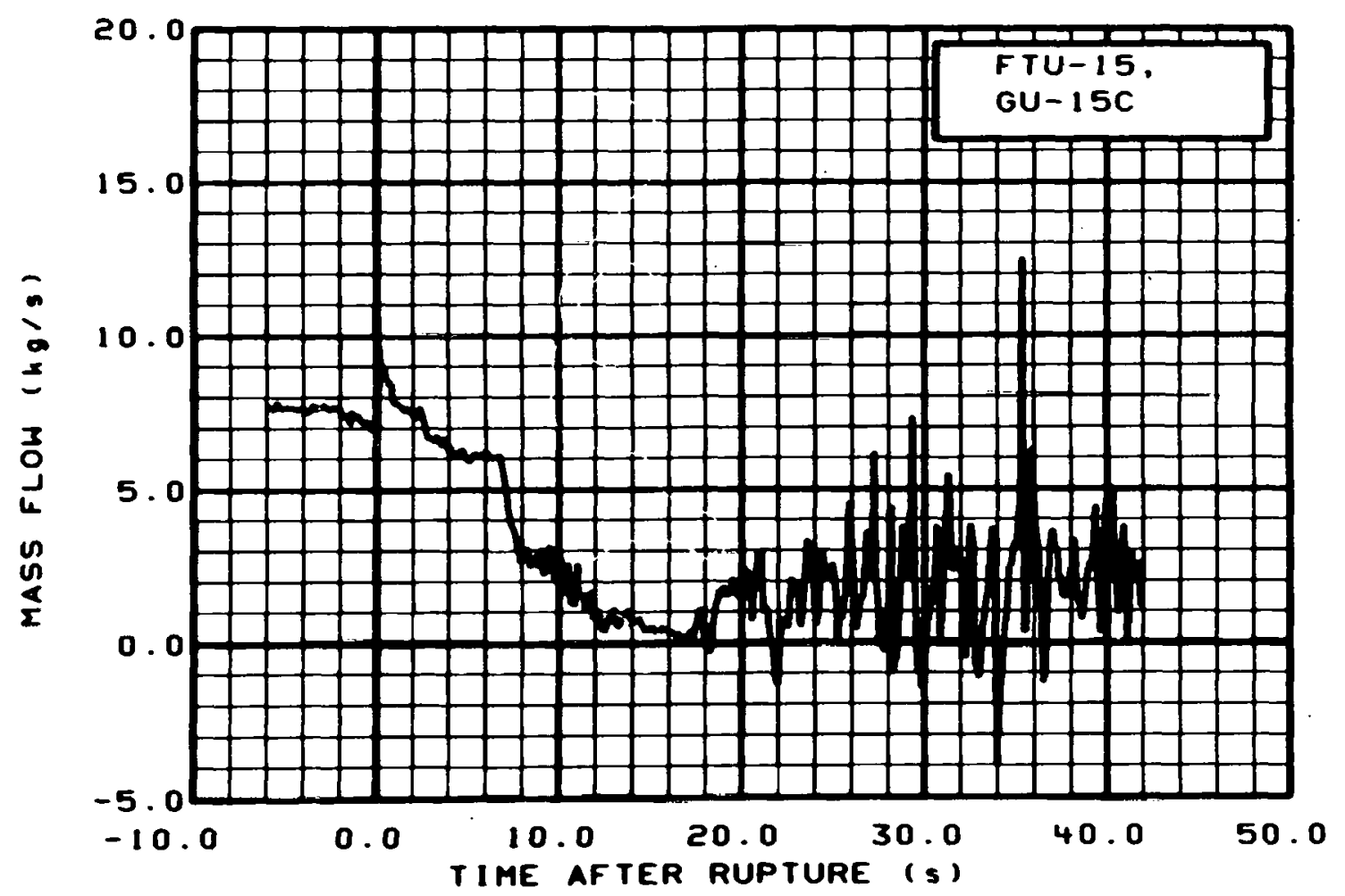

Fig. 340 Màss flow in intact loop (FTU-15, GU-15C), from -6 to $42 \mathrm{~s}$. 


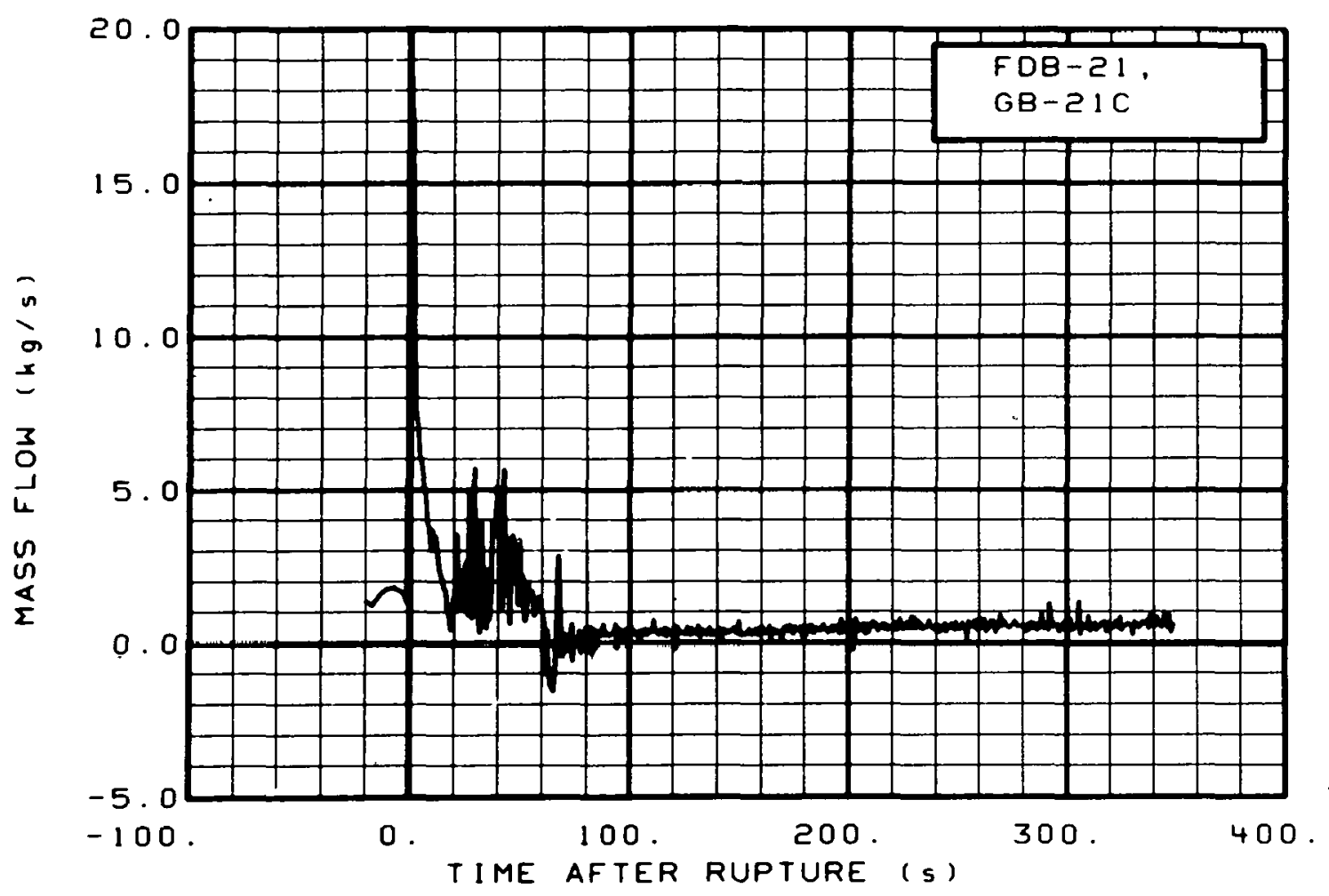

Fig. 341 Mass flow in broken loop (FDB-21, GB-21C), from -20 to $350 \mathrm{~s}$.

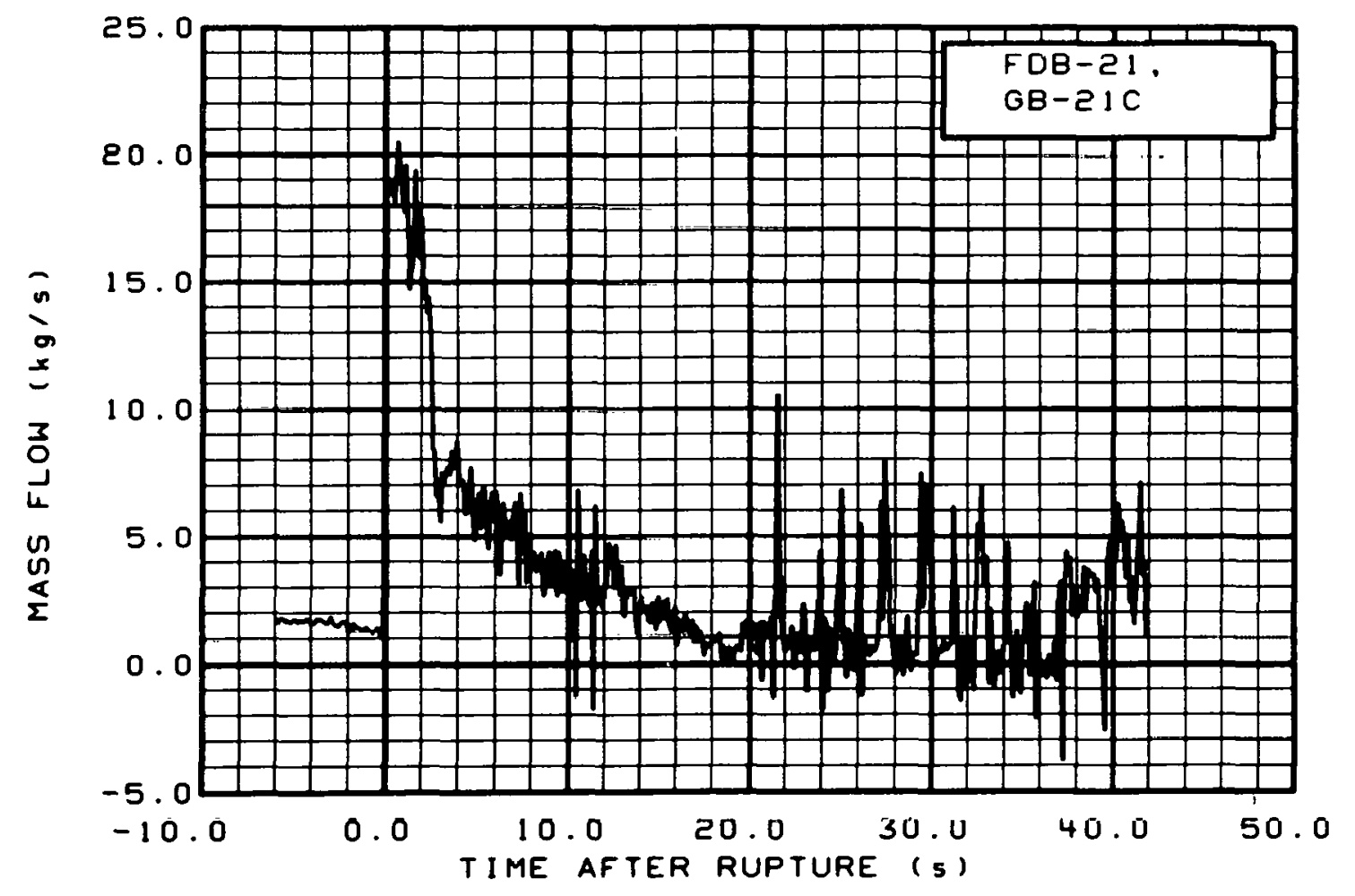

Fig. 342 Mass flow in broken loop (FDB-21, GB-21C), from -6 to $42 \mathrm{~s}$. 


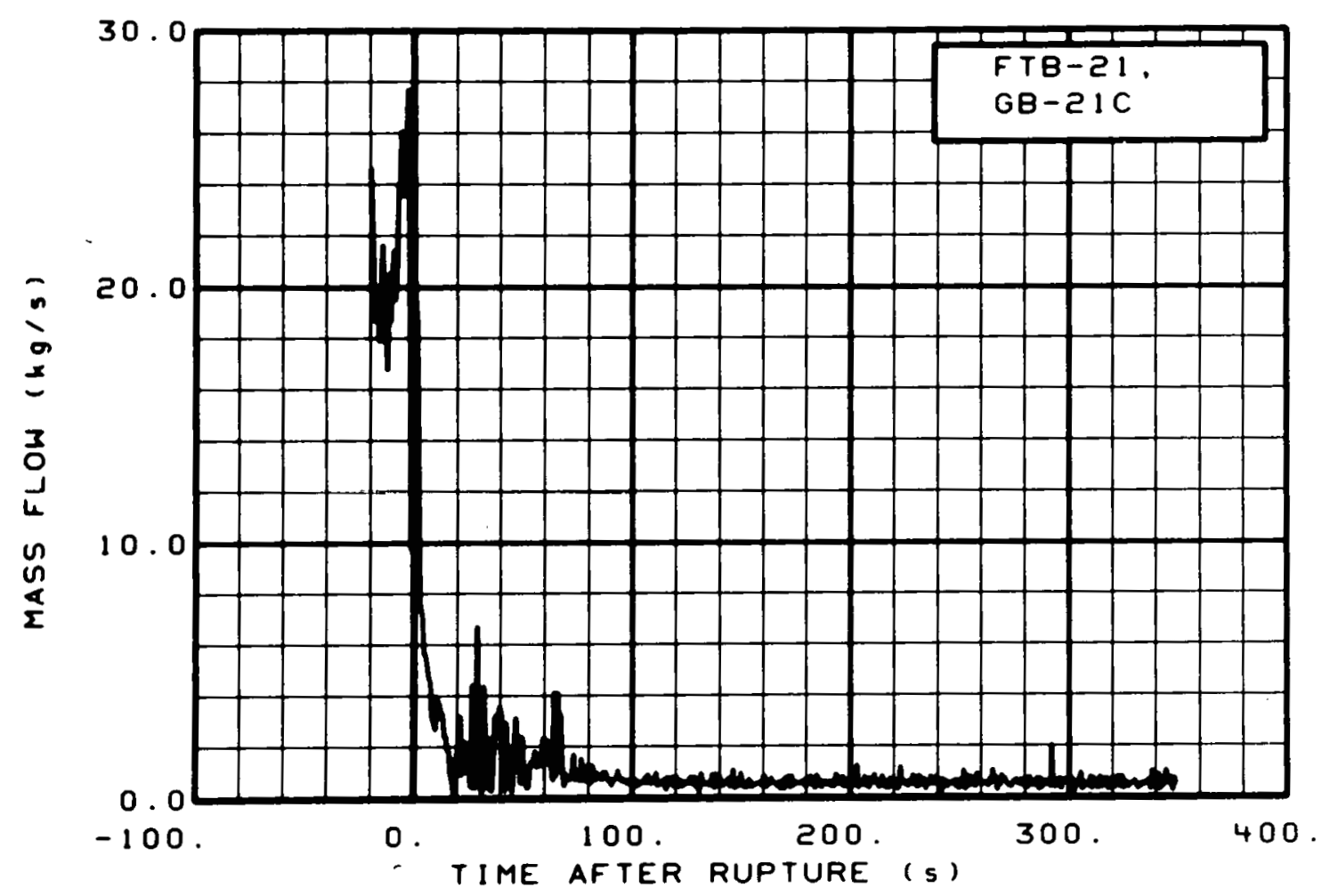

Fig. 343 Mass flow in broken loop (FTB-21, GB-21C), from -20 to $350: \mathrm{s}$.

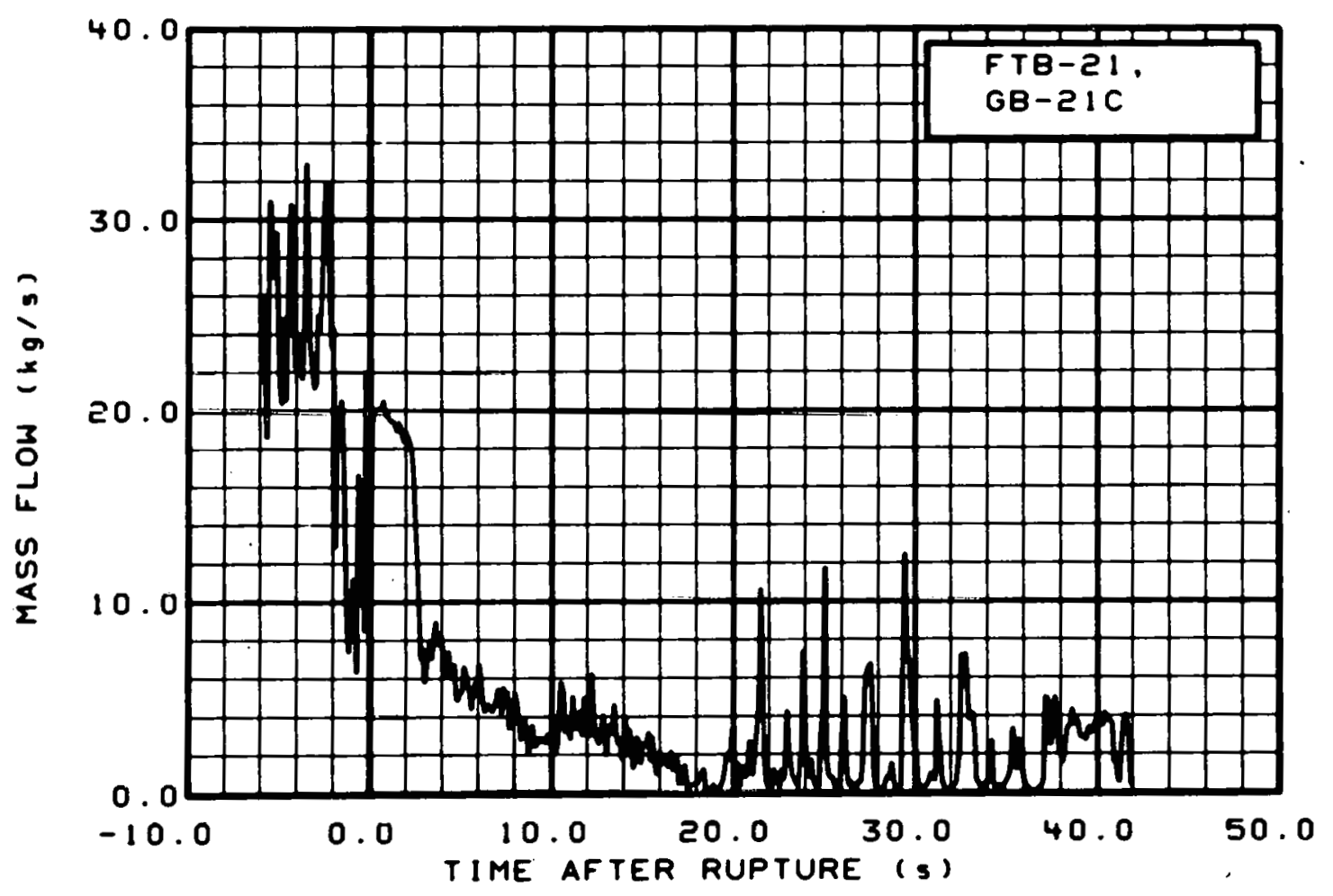

Fig. 344 Mass flow in broken loop (FTB-21, GB-21C), from -6 to $42 \mathrm{~s}$. 


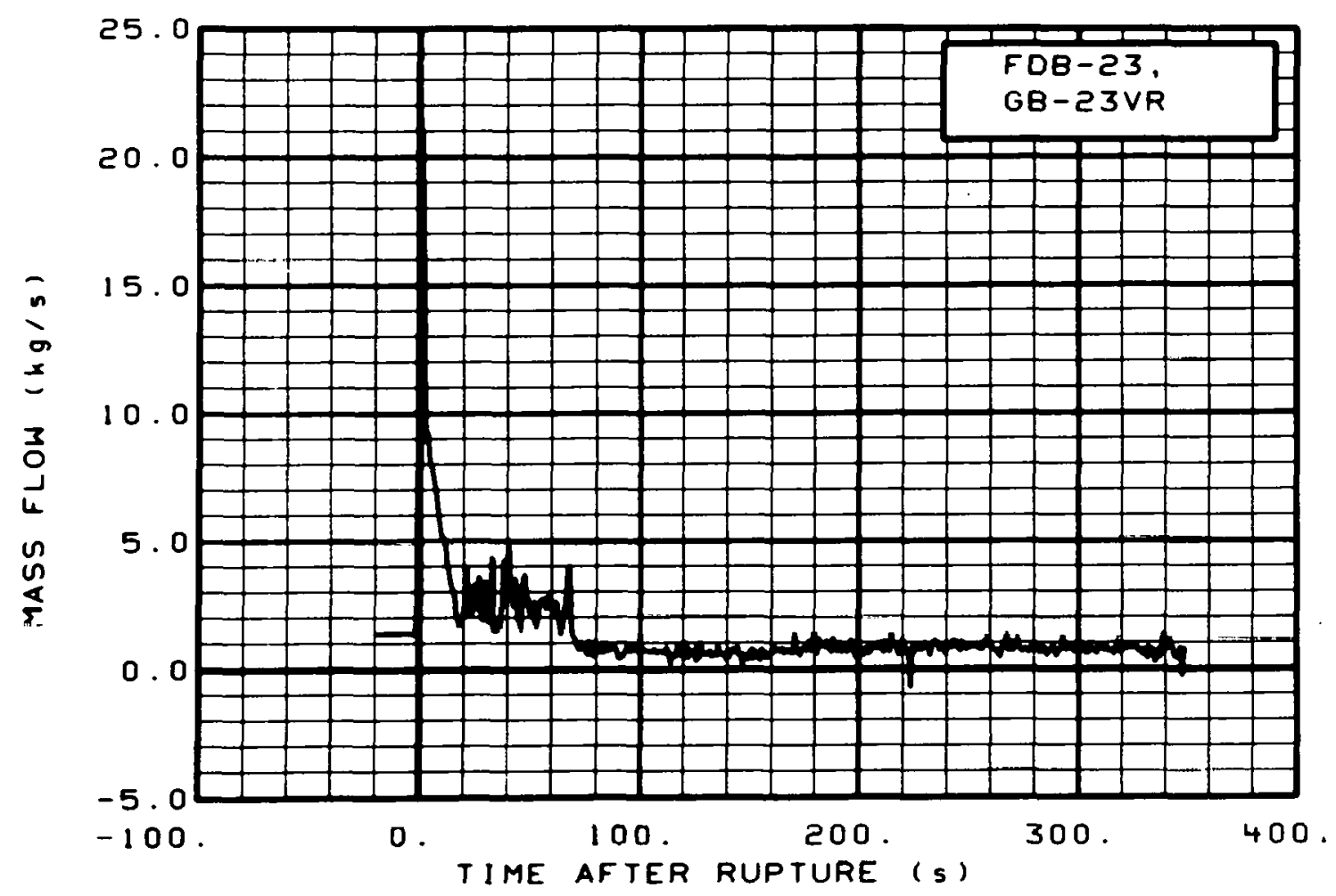

Fig. 345 Mass flow in broken loop (FDB-23, GB-23VR), from -20 to $350 \mathrm{~s}$.

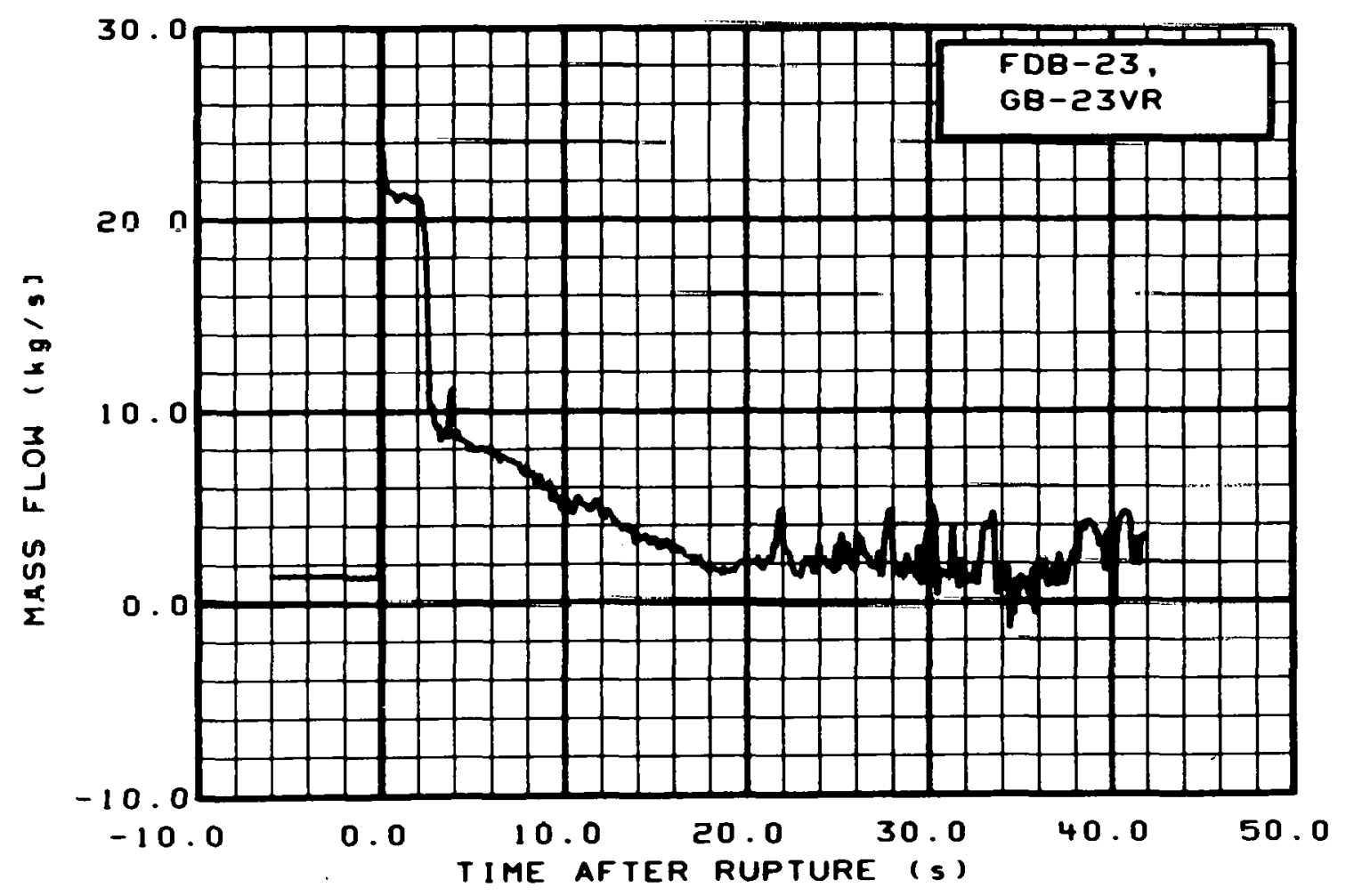

Fig. 346 Mass flow in broken loop (FDB-23, GB-23VR), from -6 to $42 \mathrm{~s}$. 


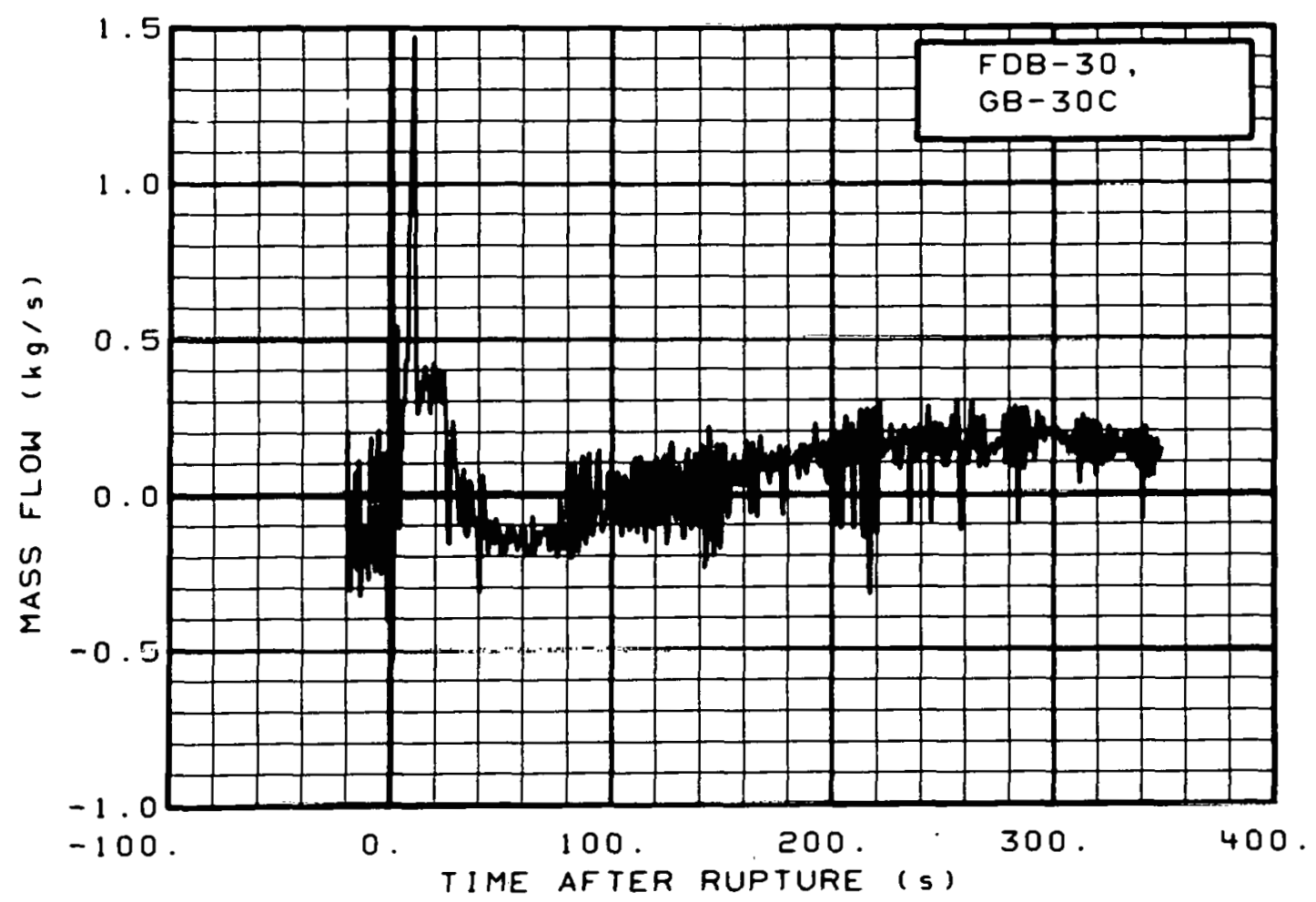

Fig. 347 Mass flow in broken loop (FDB-30, GB-30C), from -20 to $350 \mathrm{~s}$.

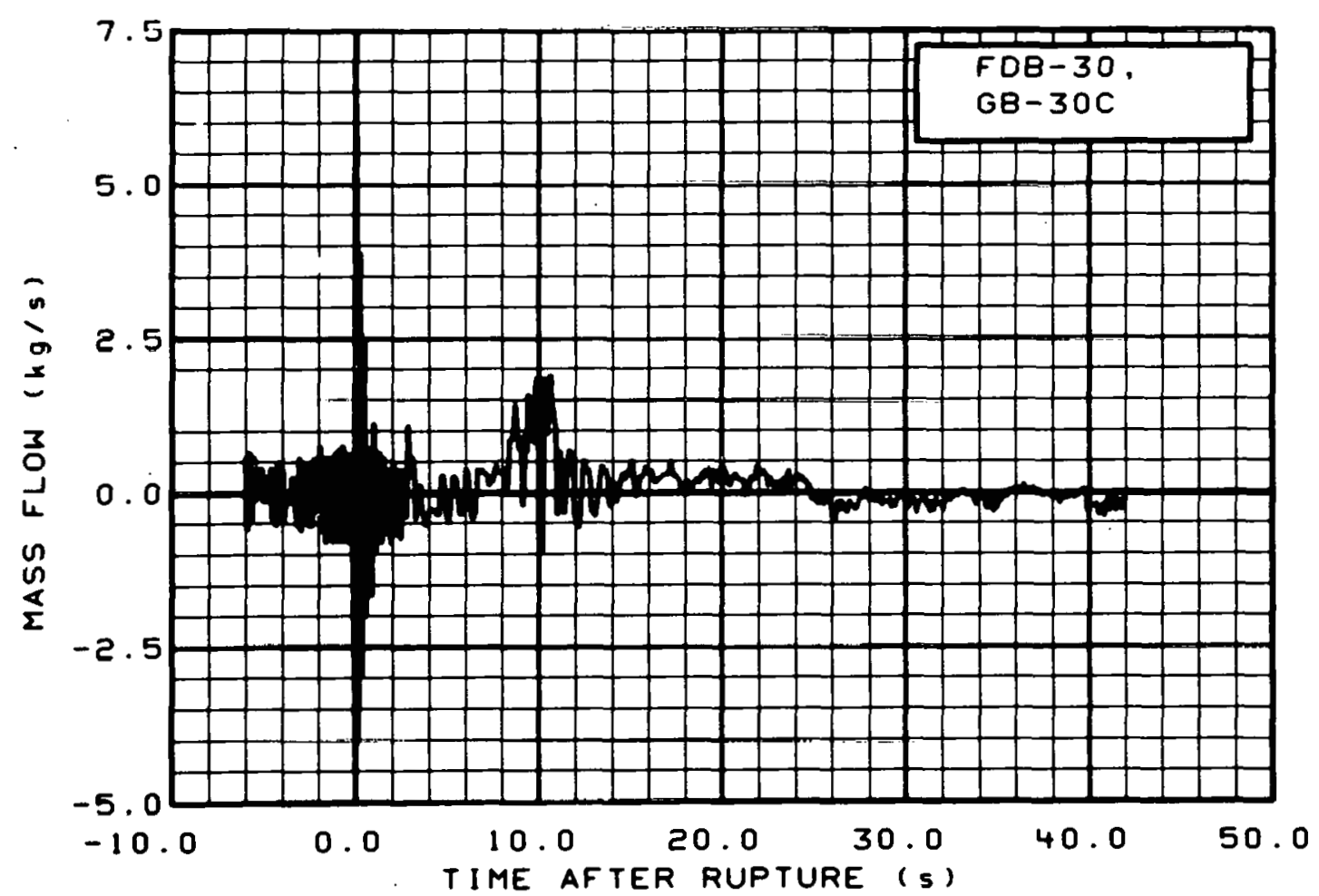

Fig. 348 Mass flow in broken loop (FDB-30, GB-30C), from -6 to $42 \mathrm{~s}$. 


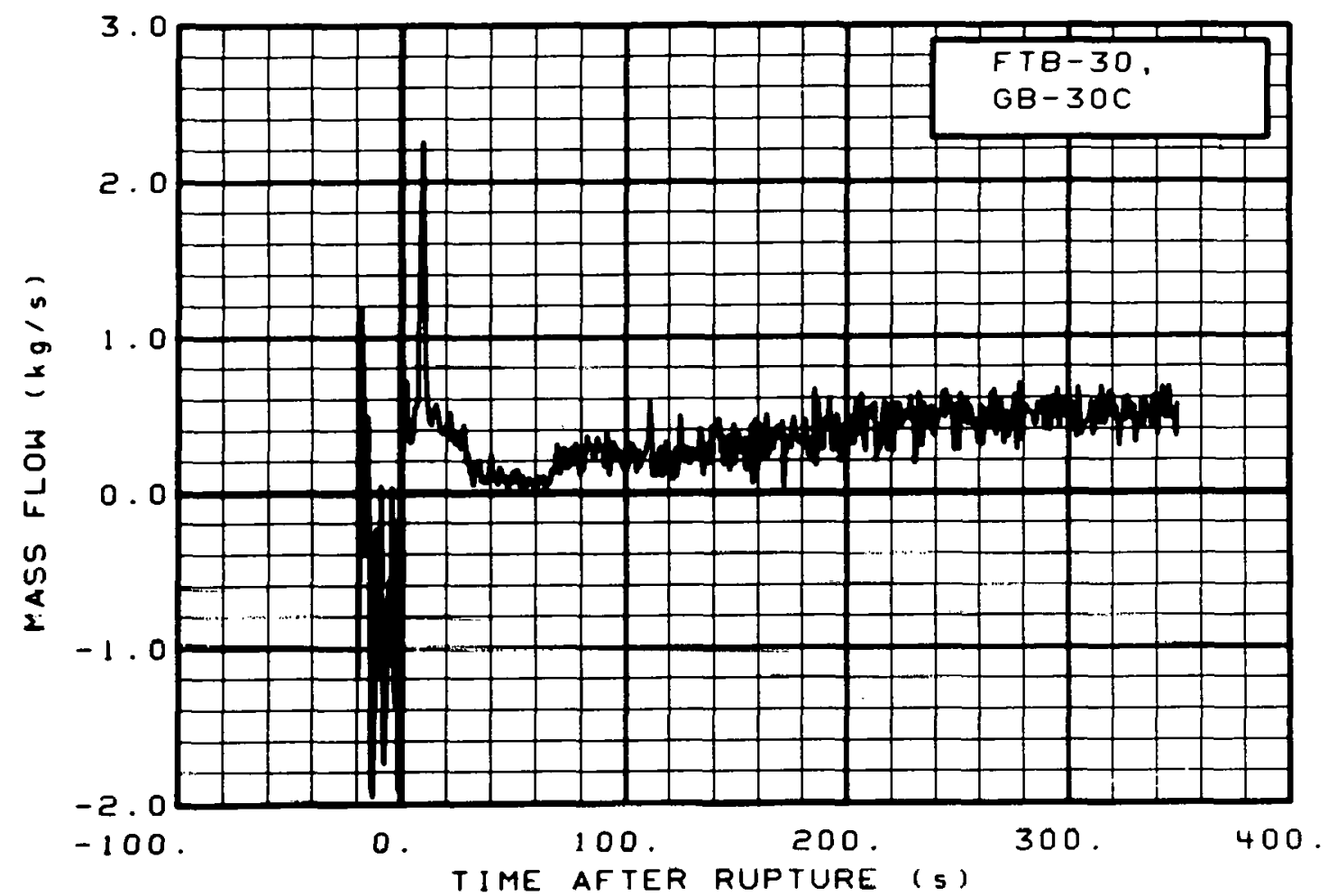

Fig. 349 Mass flow in broken loop (FTB-30, GB-30C), frum -20 to $350 \mathrm{~s}$.

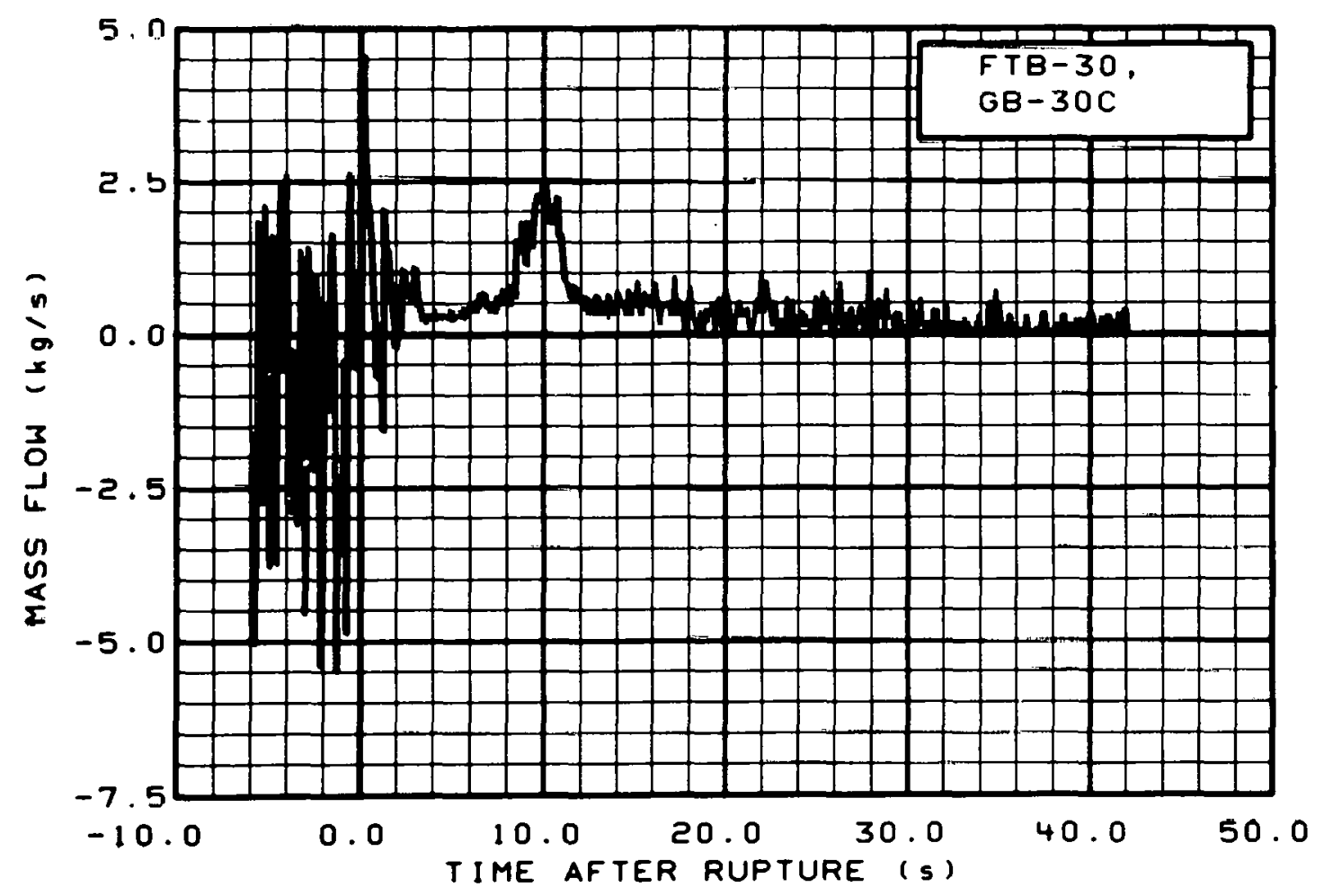

Fig. 350 Mass flow in broken loop (FTB-30, GB-30C), from -6 to $42 \mathrm{~s}$. 


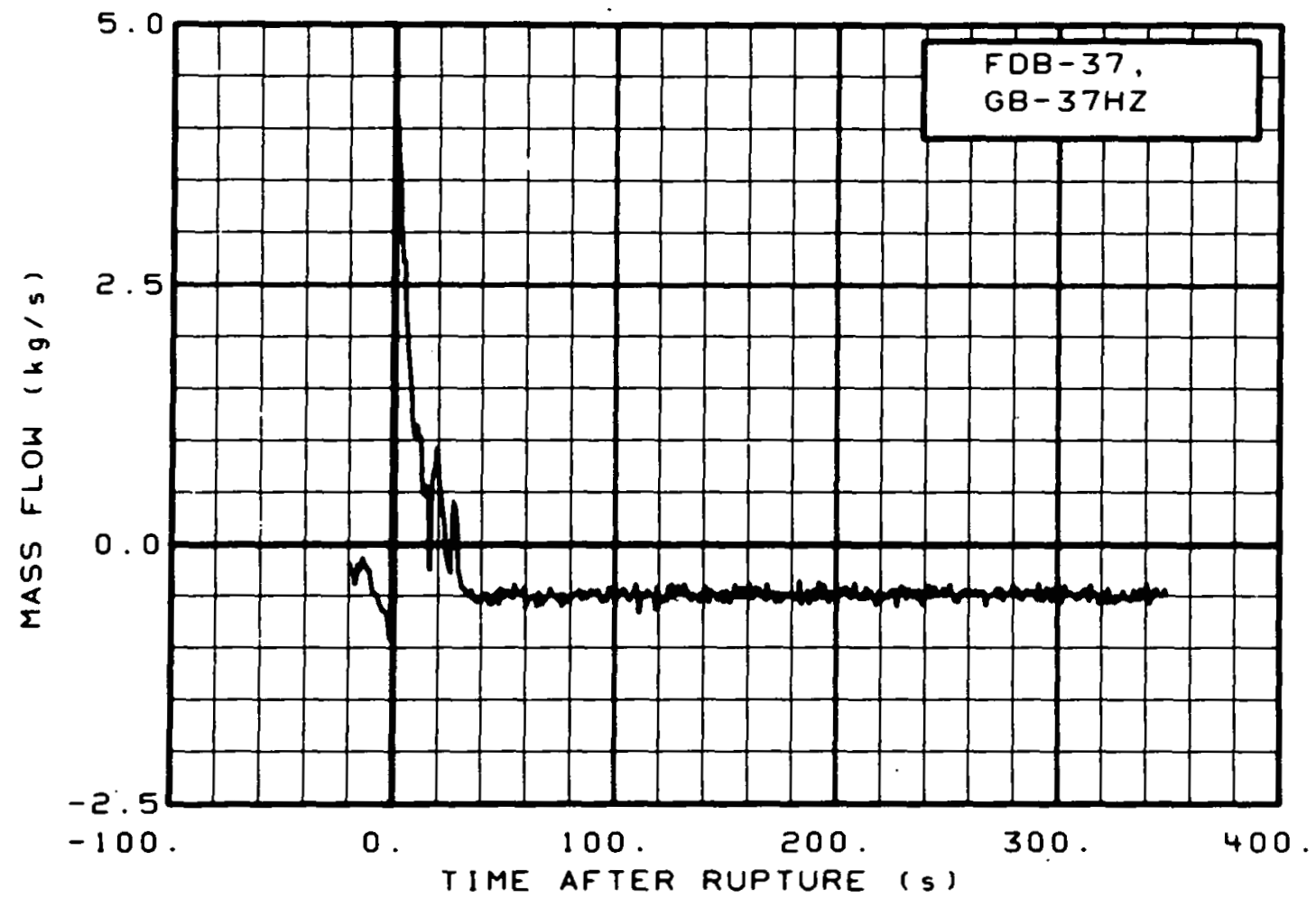

Fig. 351 Mass flow in broken loop (FDB-37, GB-37HZ), from -20 to $350 \mathrm{~s}$.

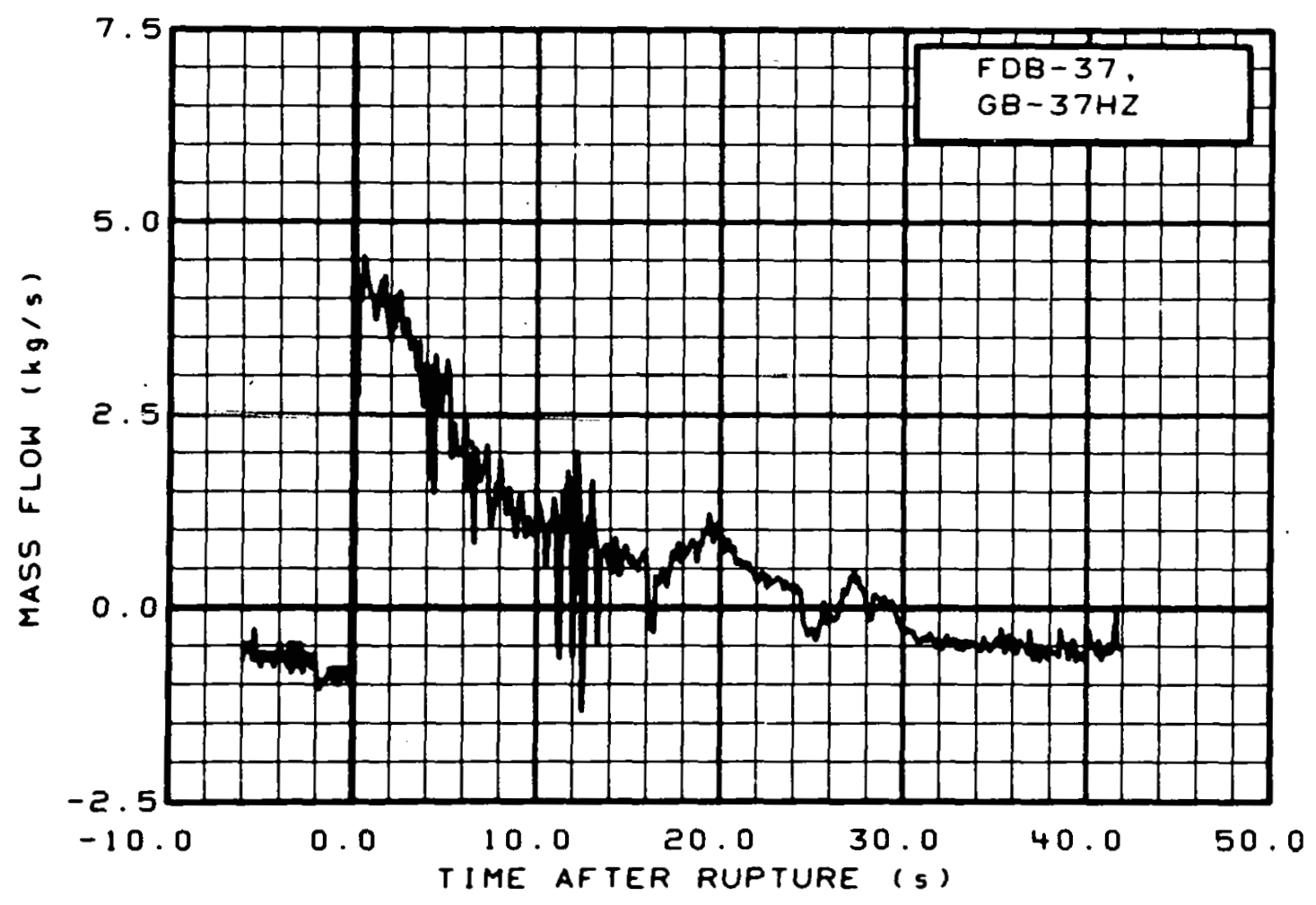

Fig. 352 Mass flow in broken loop (FDB-37, GB-37HZ), from -6 to $42 \mathrm{~s}$. 


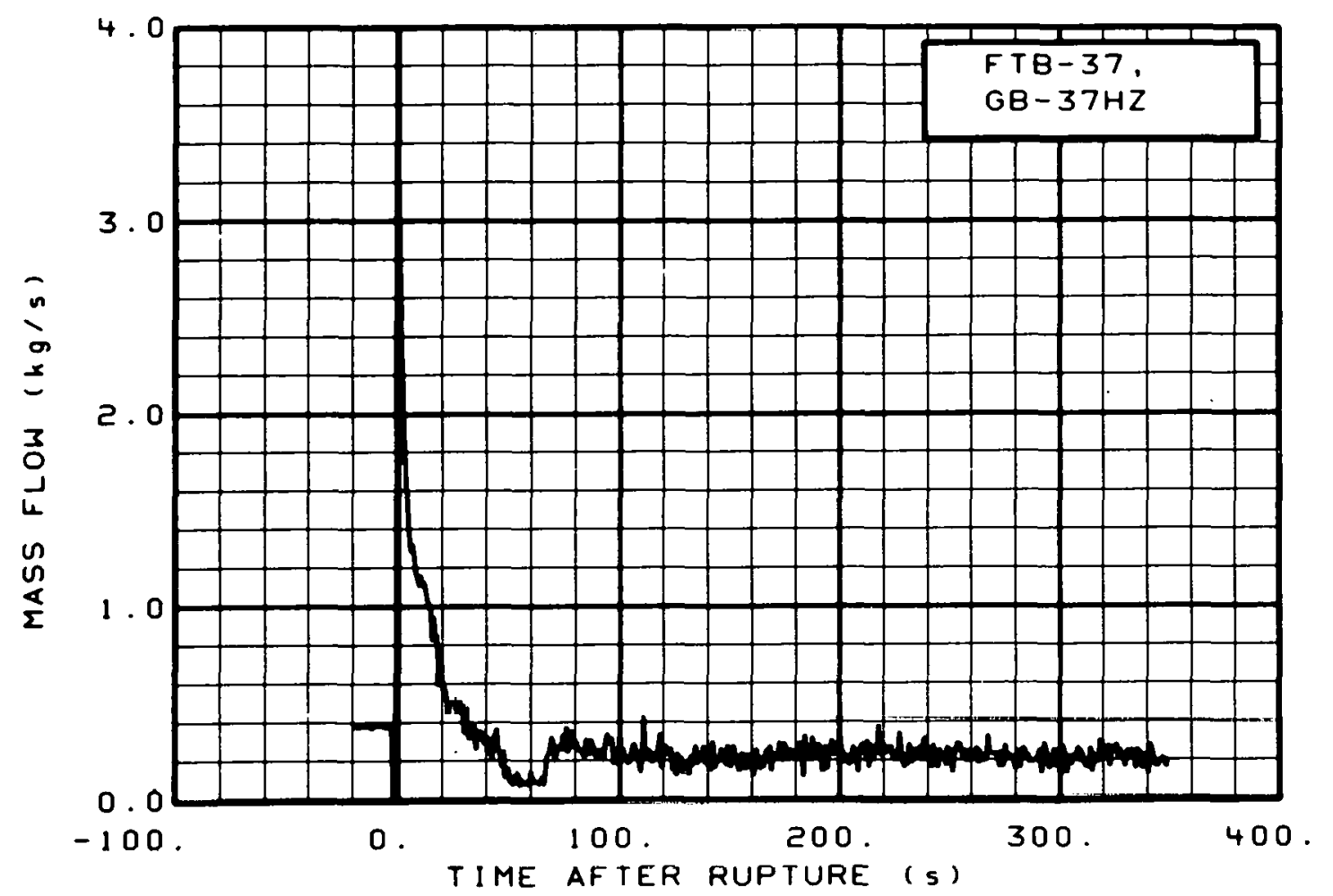

Fig. 353 Mass flow in broken loop (FTB-37, GB-37HZ), from -20 to $350 \mathrm{~s}$.

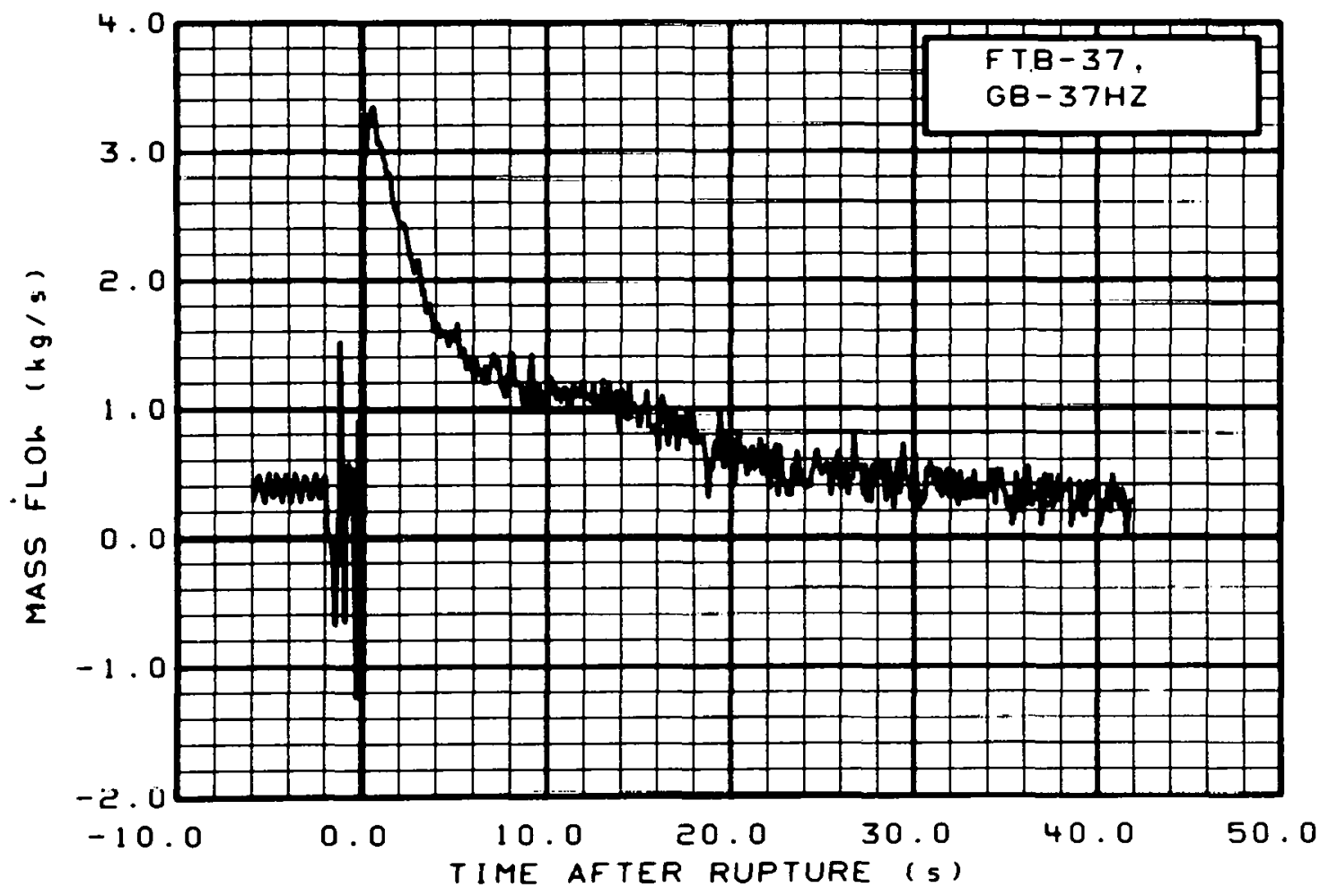

Fig. 354 Mass flow in broken loop (FTB-37, GB-37HZ), from -6 to $42 \mathrm{~s}$. 


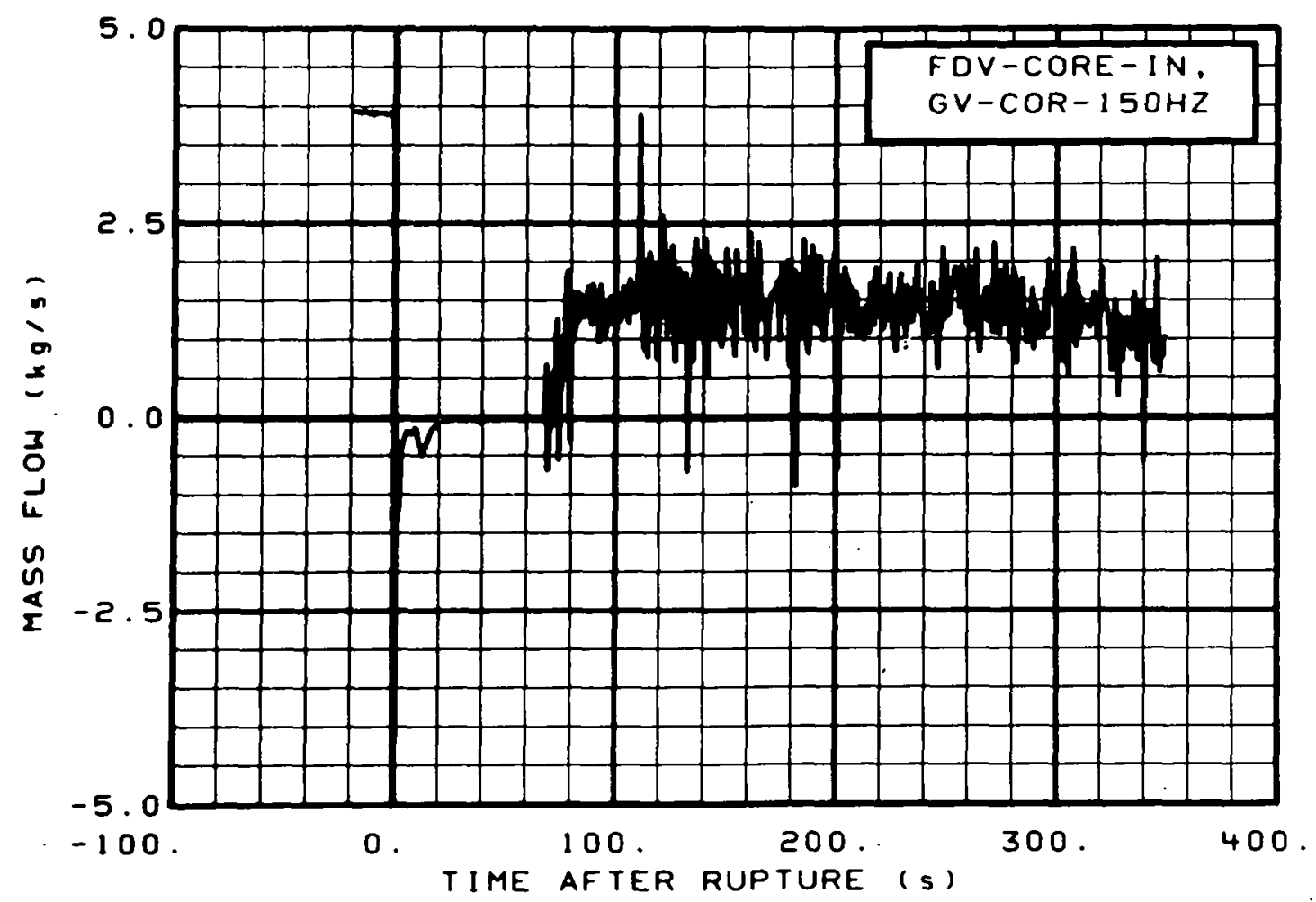

Fig. 355 Mass flow in vessel (FDV-CORE-IN, GV-COR-150HZ), from -20 to $350 \mathrm{~s}$.

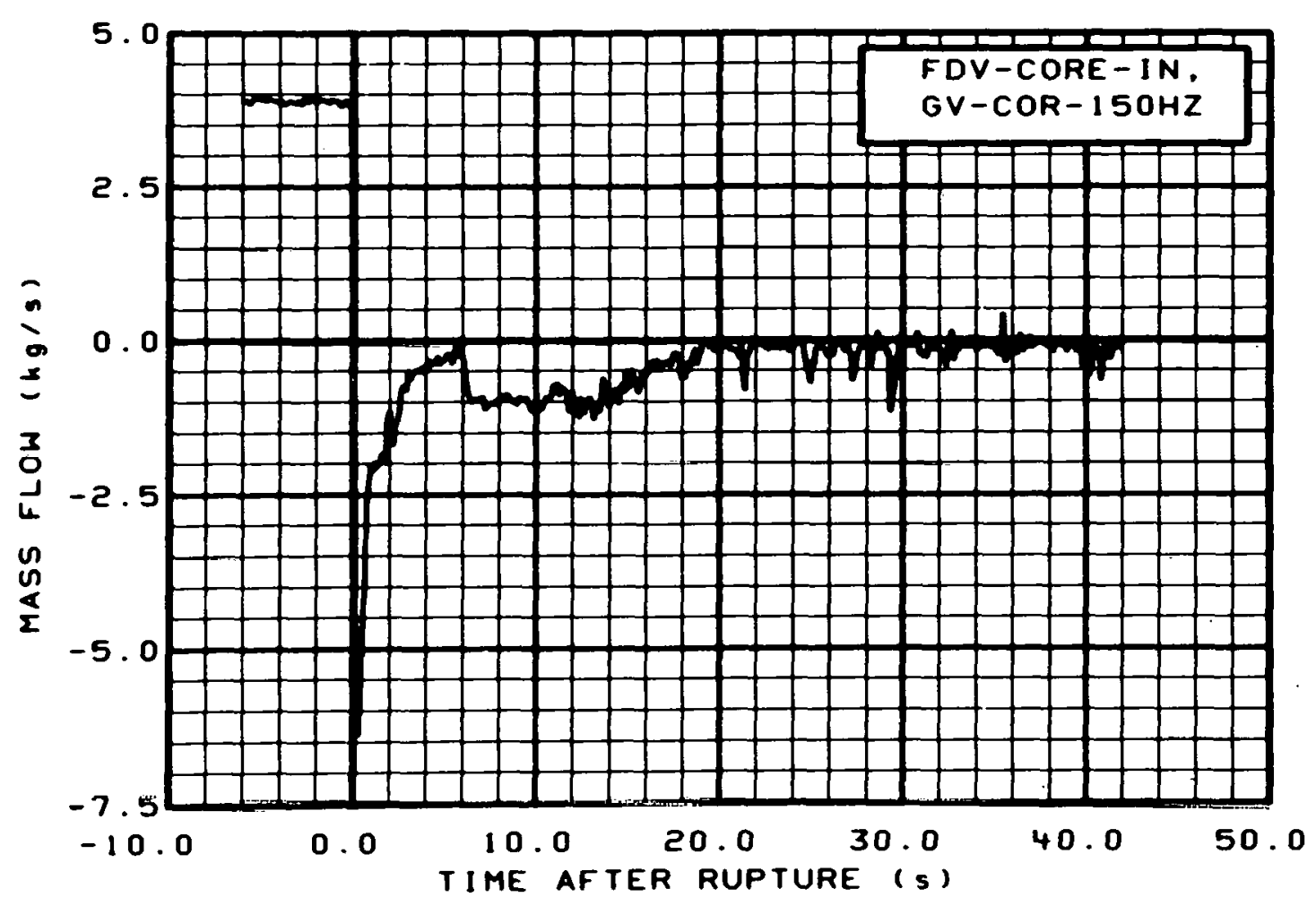

Fig. 356 Mass flow in vessel (FDV-CORE-IN, GV-COR-150HZ), from -6 to $42 \mathrm{~s}$. 


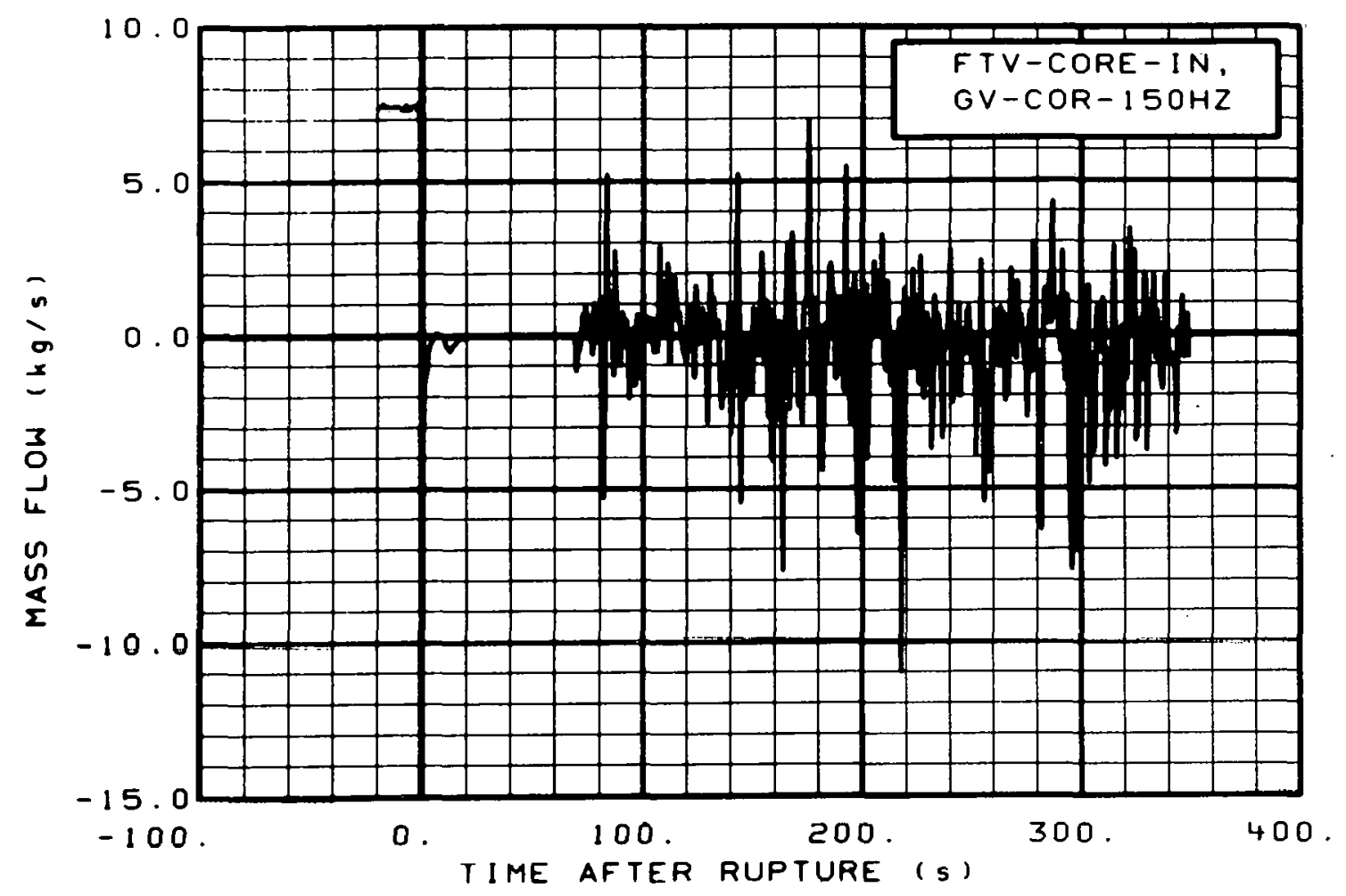

Fig. 357 Mass flow in vessel (FTV-CORE-IN, GV-COR-150HZ), from -20 to $350 \mathrm{~s}$.

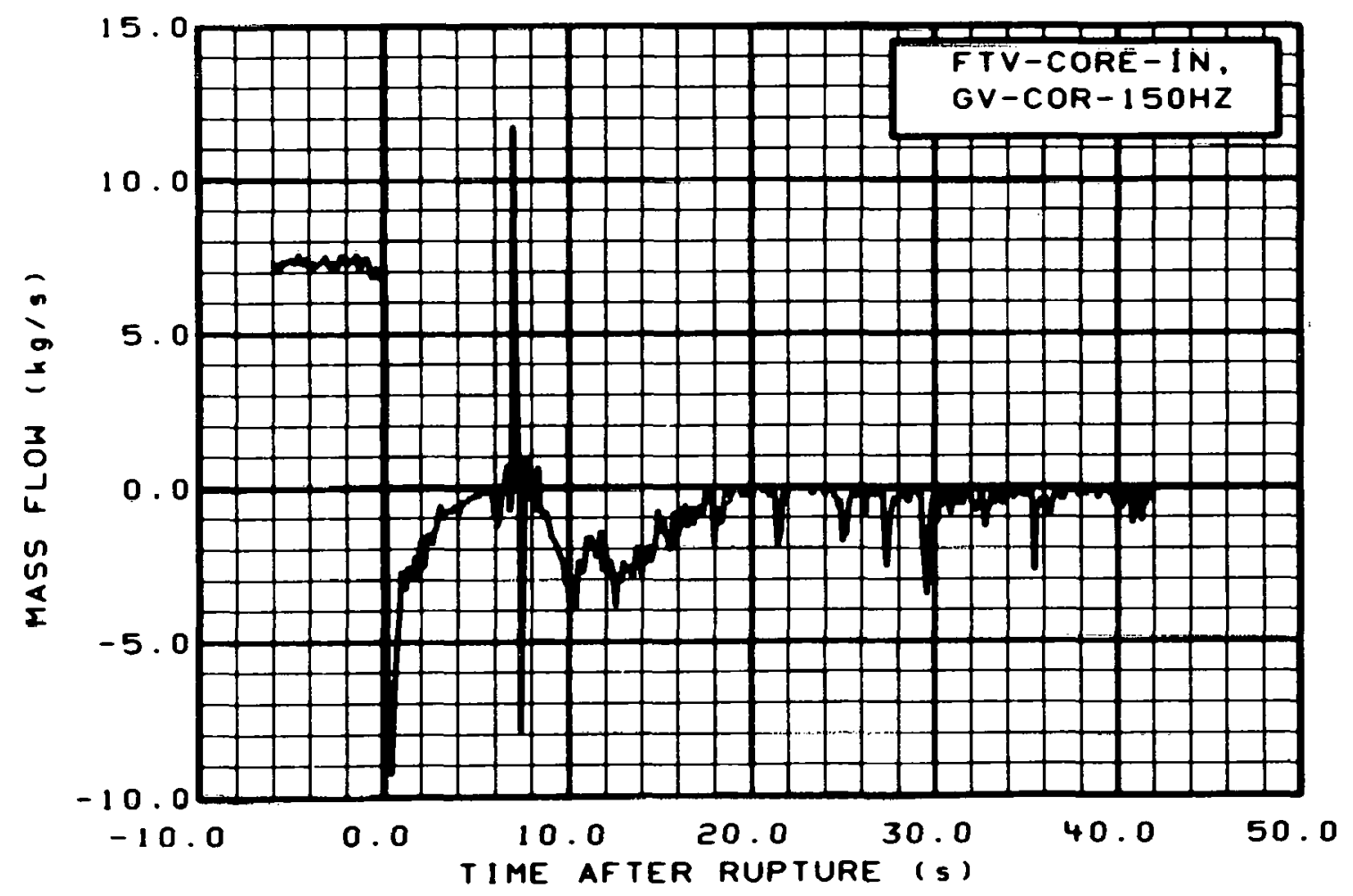

Fig. 358 Mass flow in vessel (FTV-CORE-IN, GV-COR-150HZ), from -6 to $42 \mathrm{~s}$. 


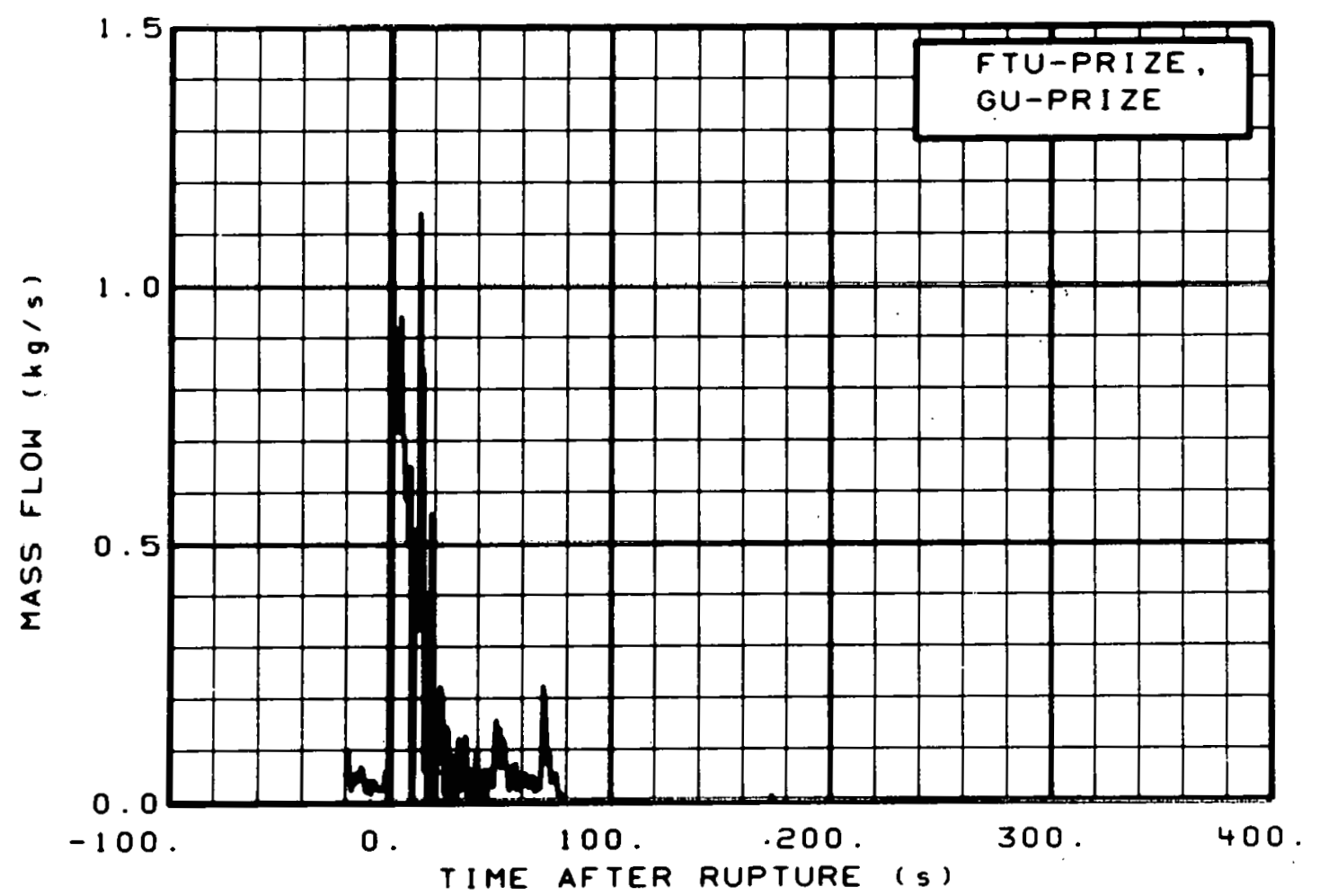

Fig. 359 Mass flow in pressurizer (FTU-PRIZE, GU-PRIZE), from -20 to $350 \mathrm{~s}$.

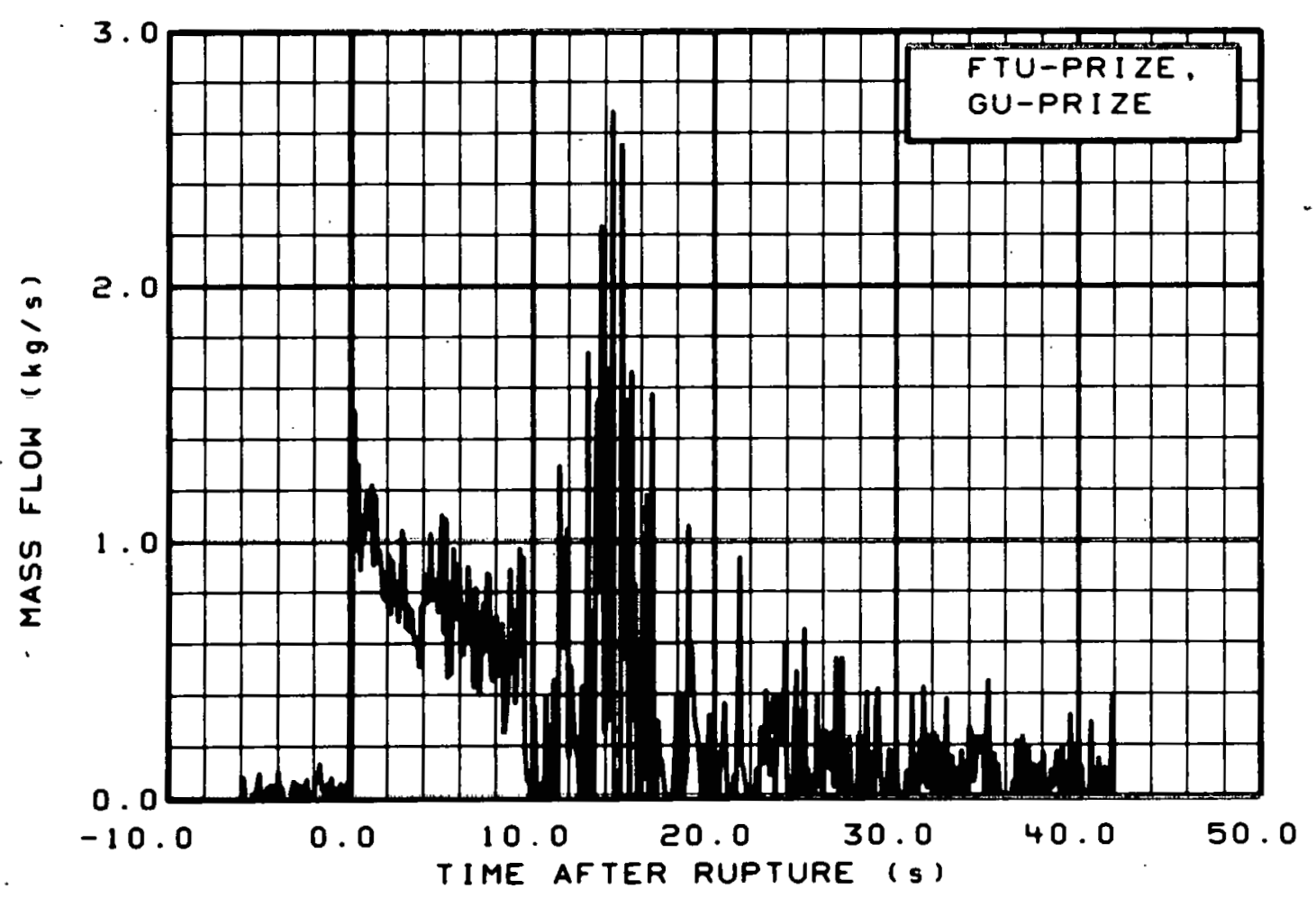

Fig. 360 Mass flow in pressurizer (FTU-PRIZE, GU-PRIZE), from -6 to $42 \mathrm{~s}$. 


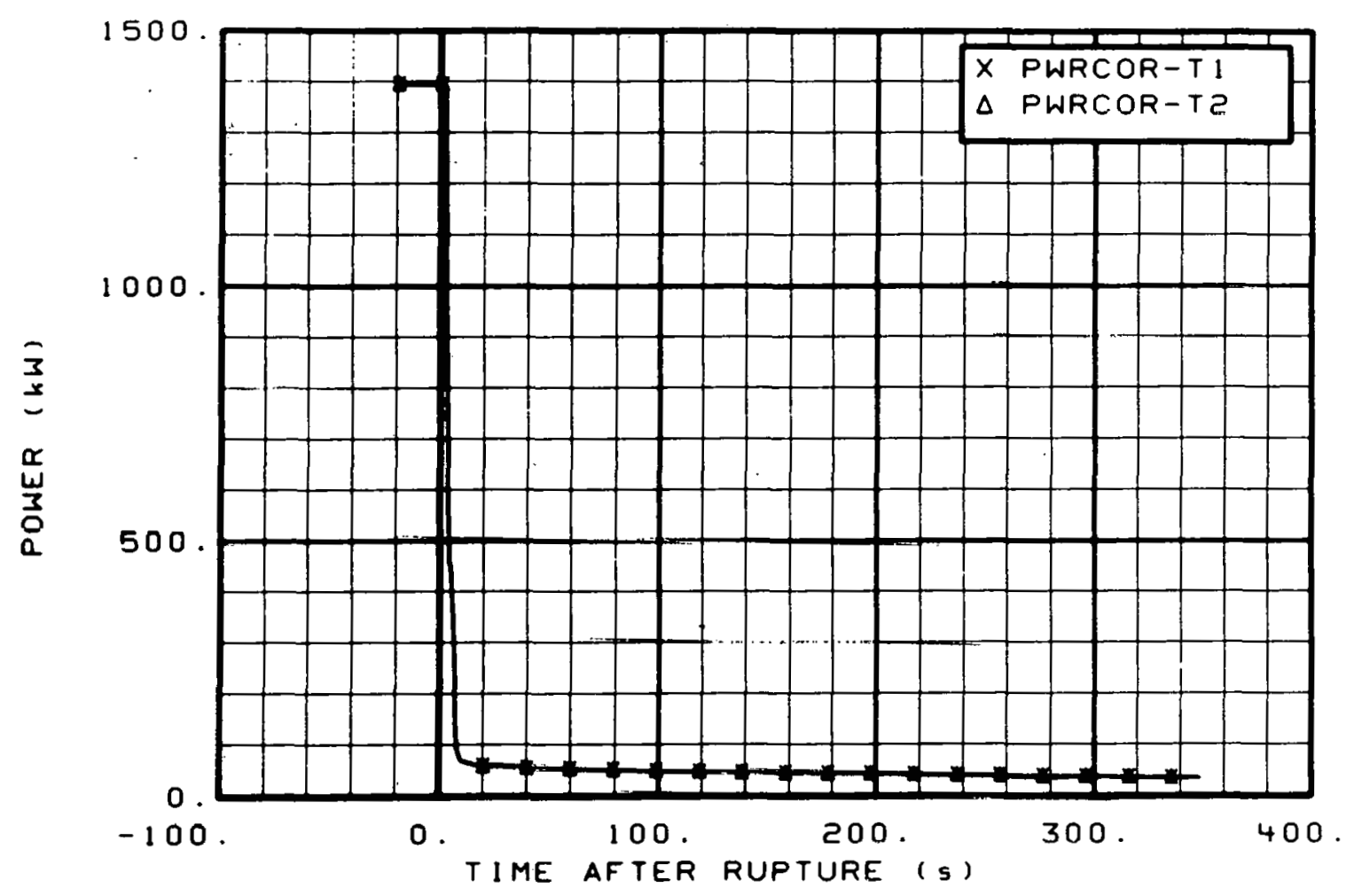

Fig. 361 Core heater rod total power (PWRCOR T-1 and PWRCOR T-2), from -20 to $350 \mathrm{~s}$.

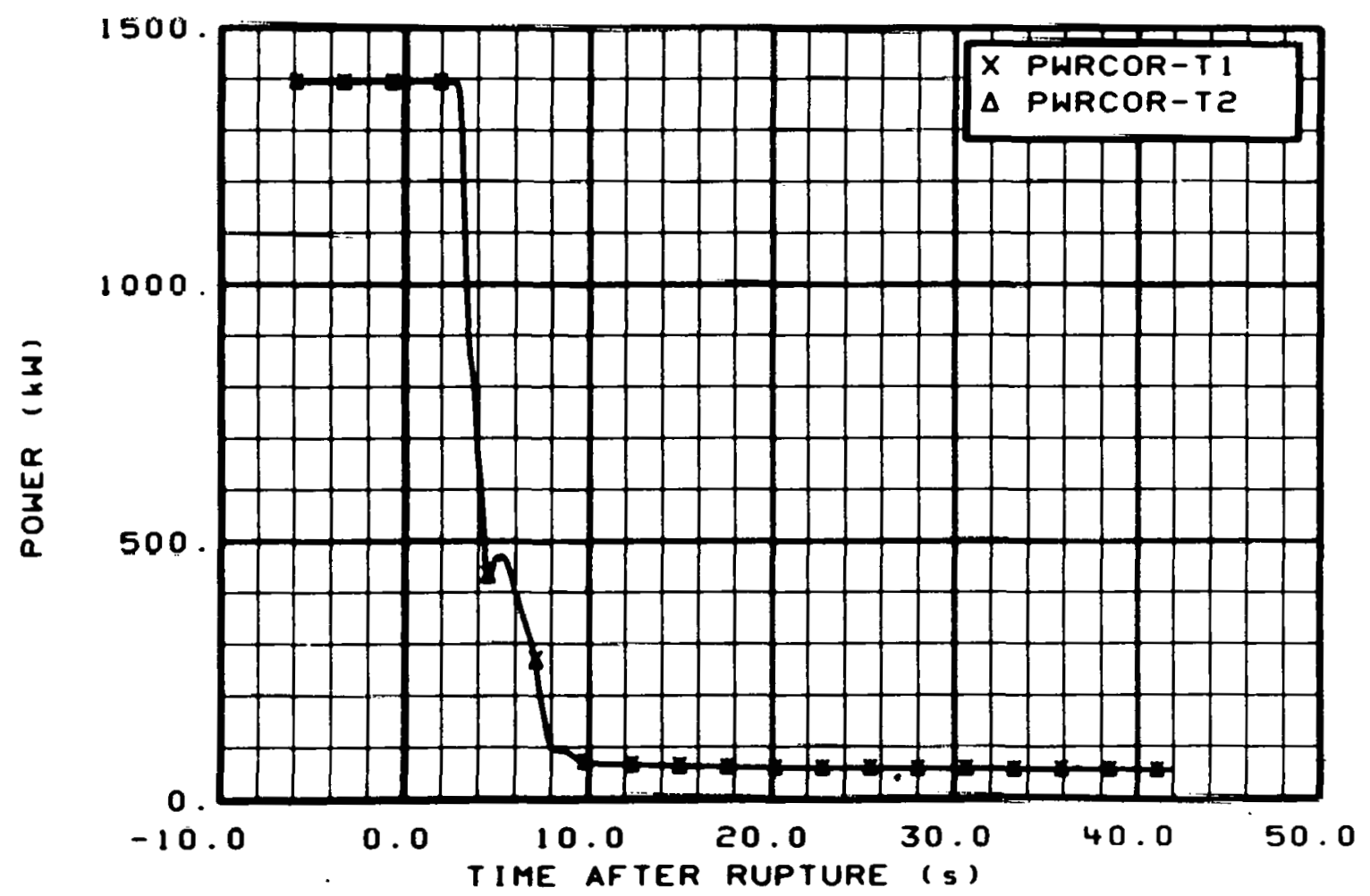

Fig. 362 Core heater rod total power (PWRCOR T-1 and PWRCOR T-2), from -6 to $42 \mathrm{~s}$. 


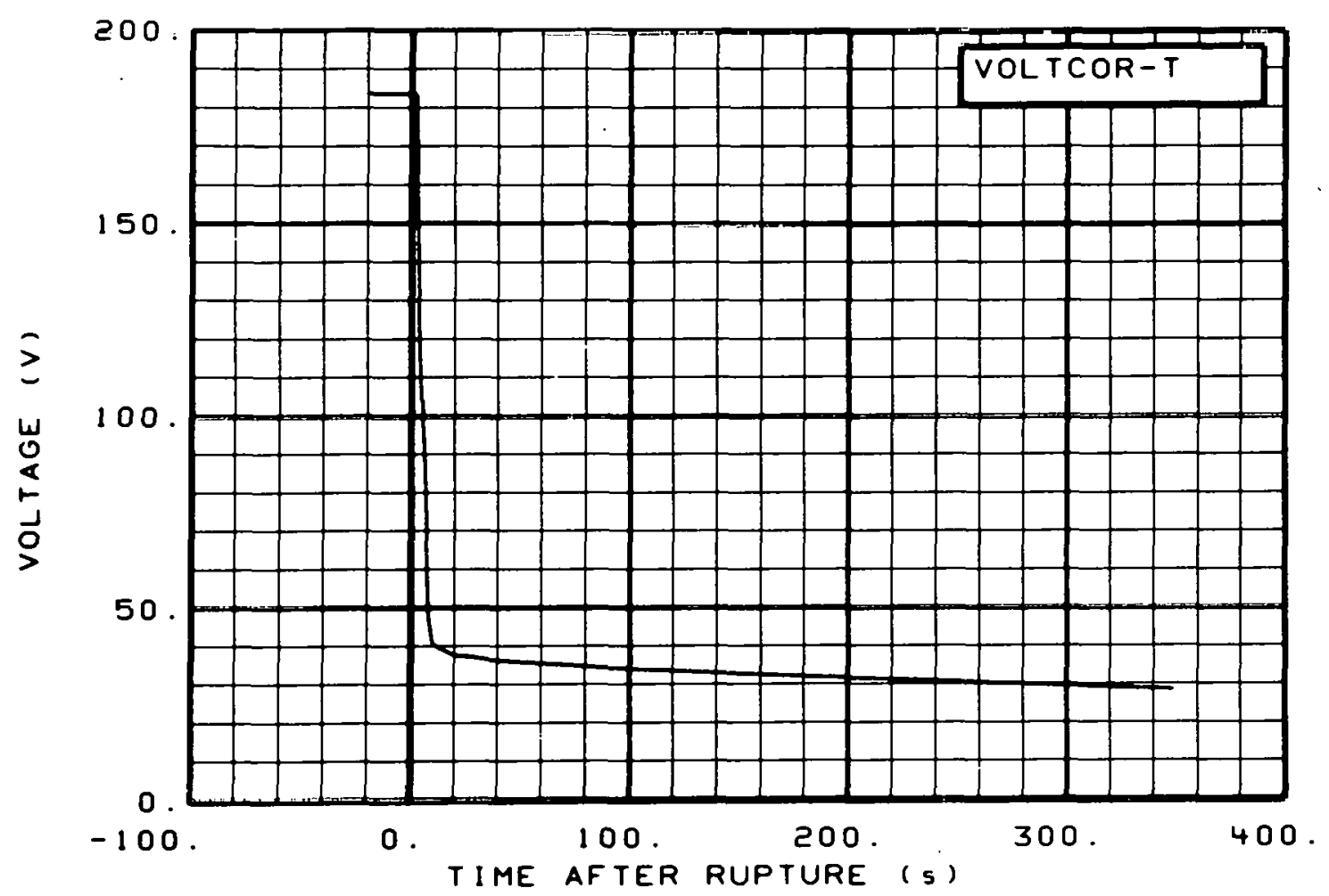

Fig. 363 Core heater voltage (VOLTCOR-T), from -20 to $350 \mathrm{~s}$.

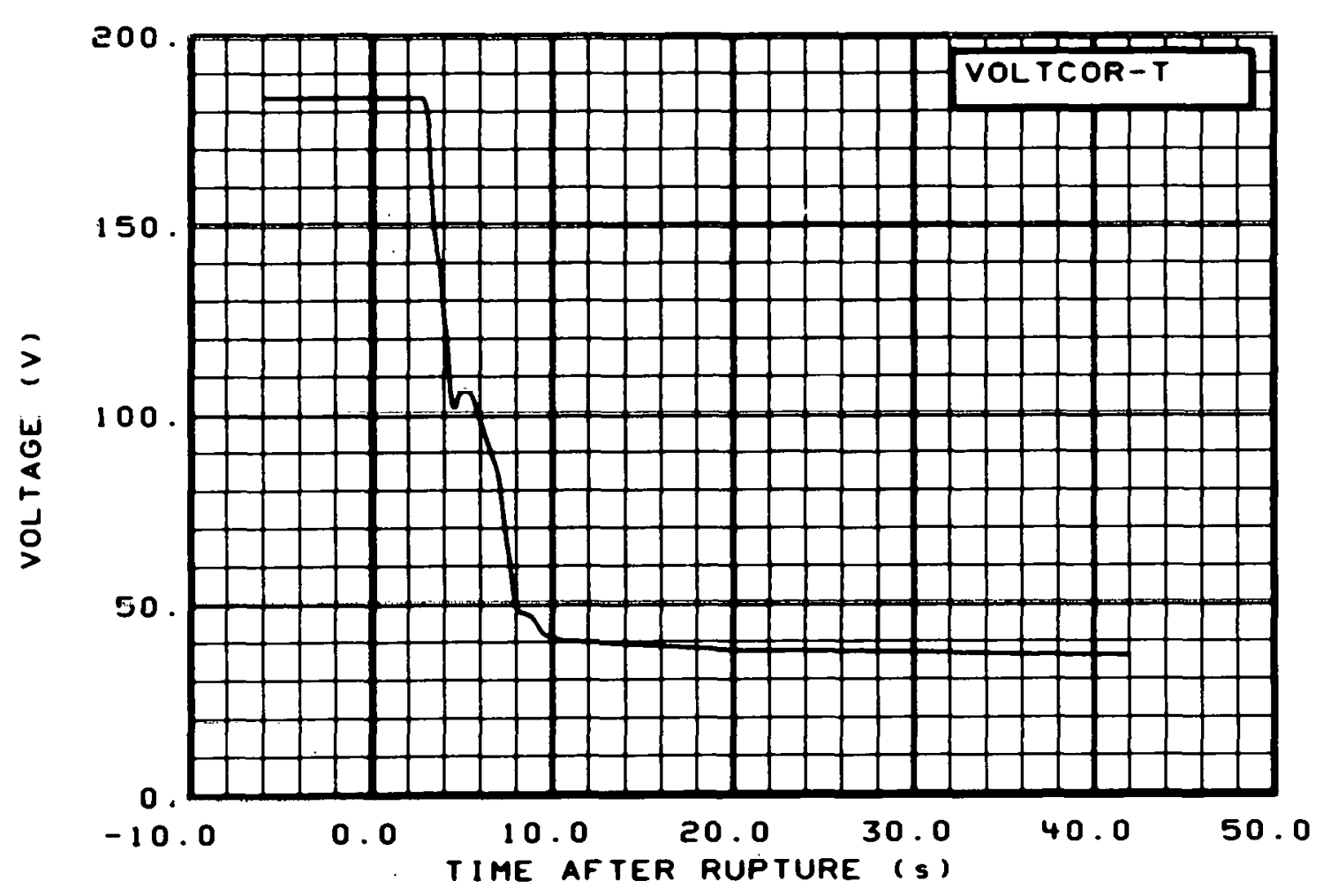

Fig. 364 Core heater voltage (VOLTCOR-T), from -6 to $42 \mathrm{~s}$. 


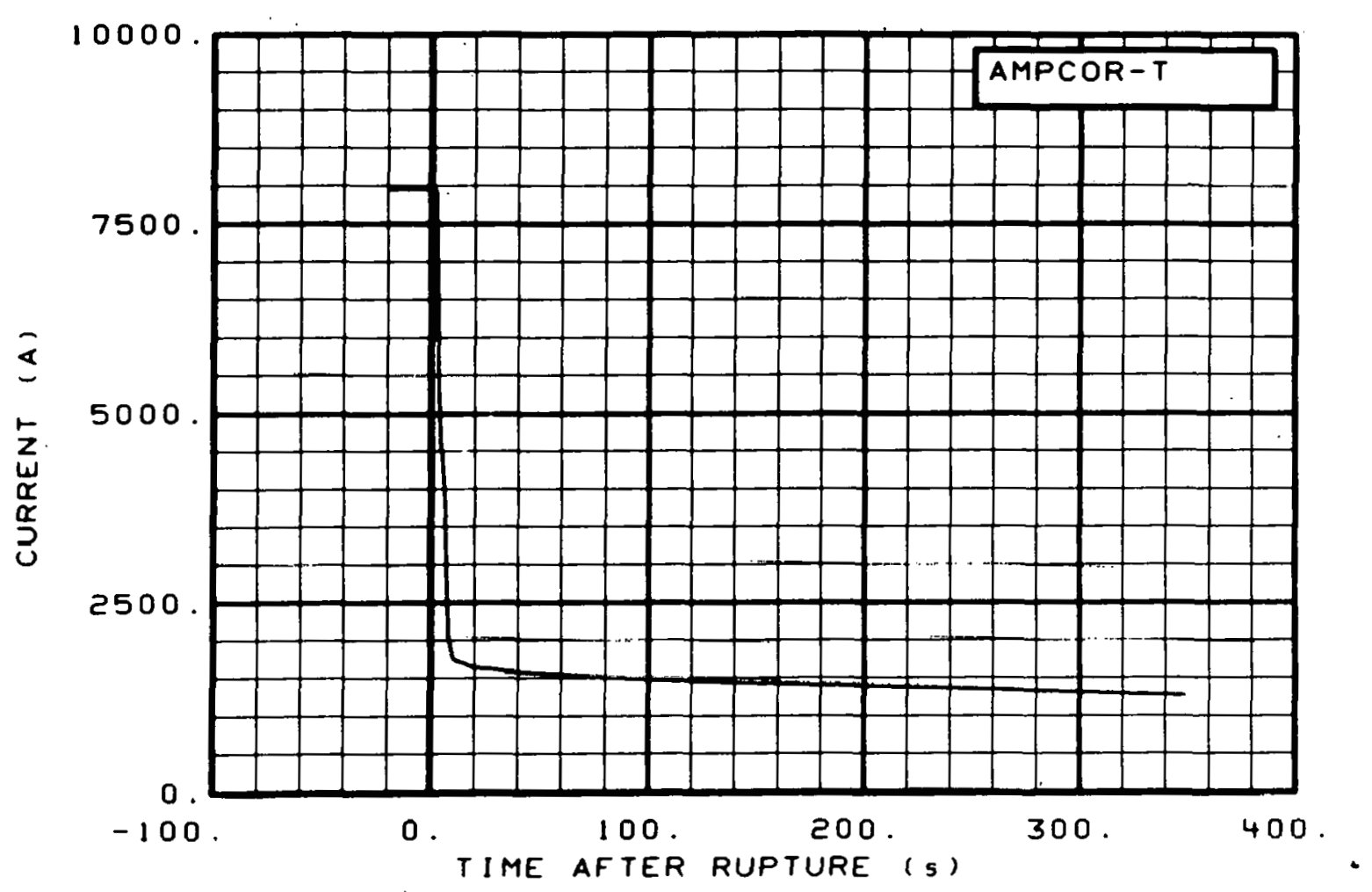

Fig. 365 Core heater total current (AMPCOR-T), from -20 to $350 \mathrm{~s}$.

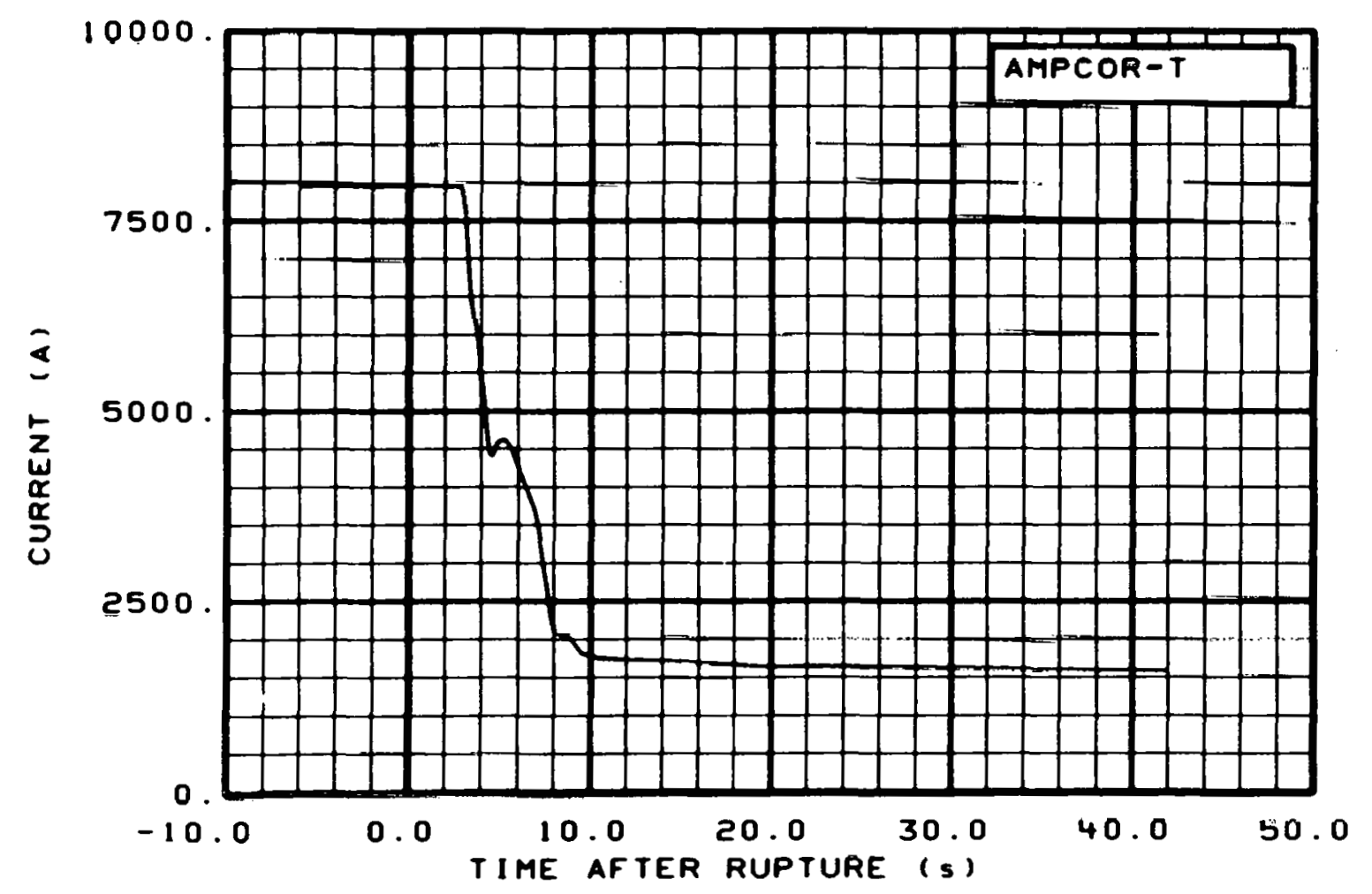

Fig. 366 Core heater total current (AMPCOR-T), from -6 to $42 \mathrm{~s}$. 


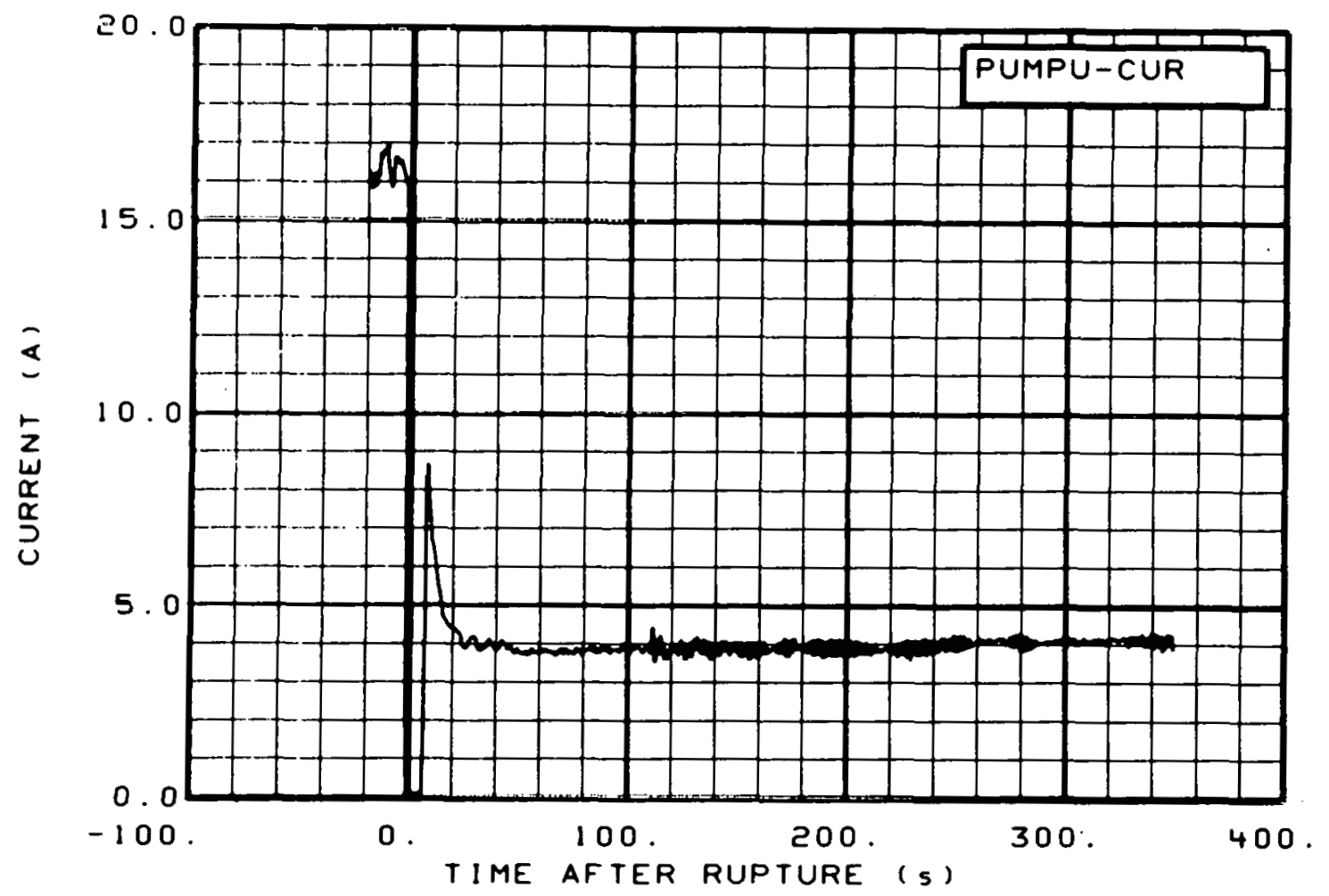

Fig. 367 Primary pump current (PUMPU-CUR), from -20 to $350 \mathrm{~s}$.

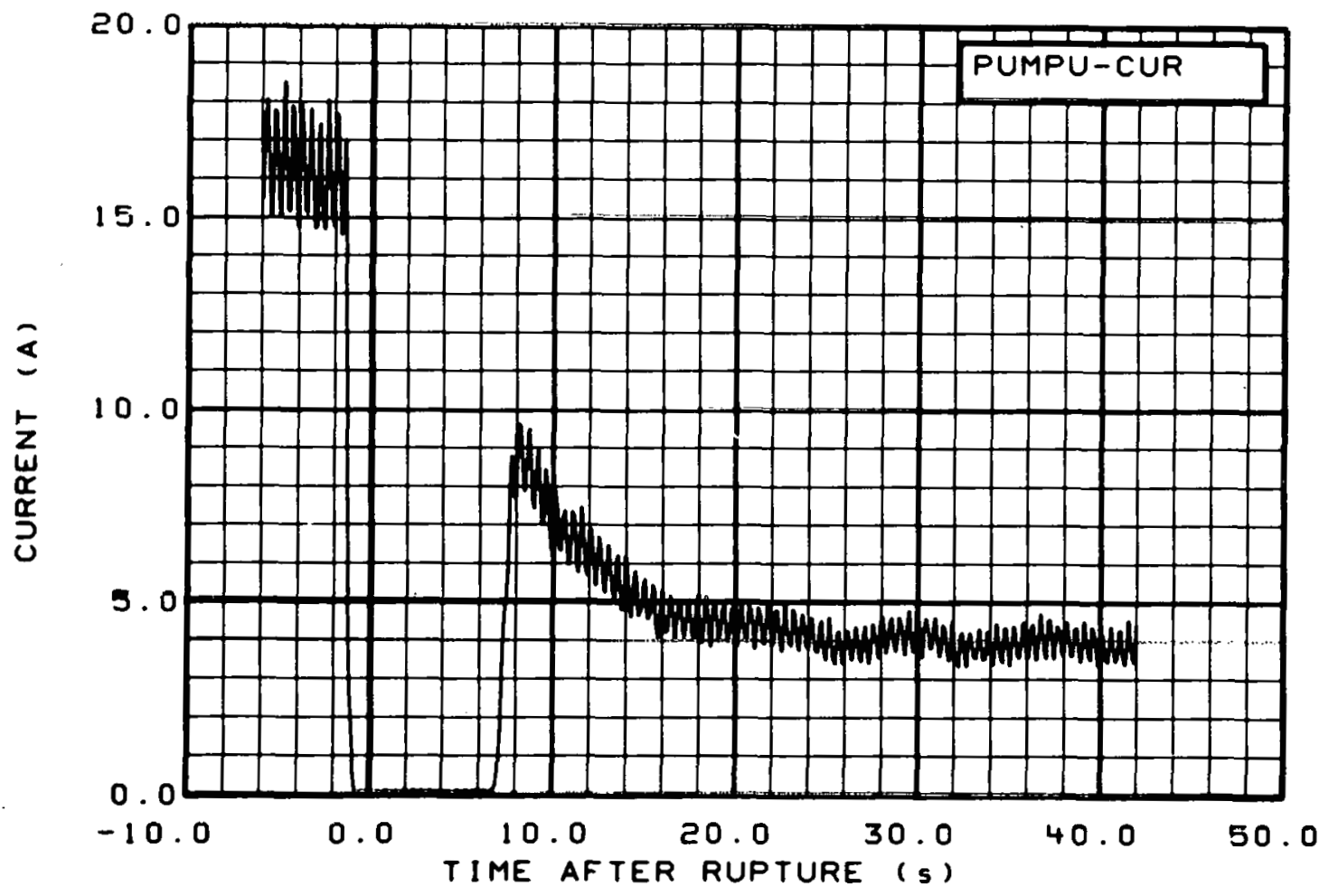

Fig. 368 Primary pump current (PUMPU-CUR), from -6 to $42 \mathrm{~s}$. 


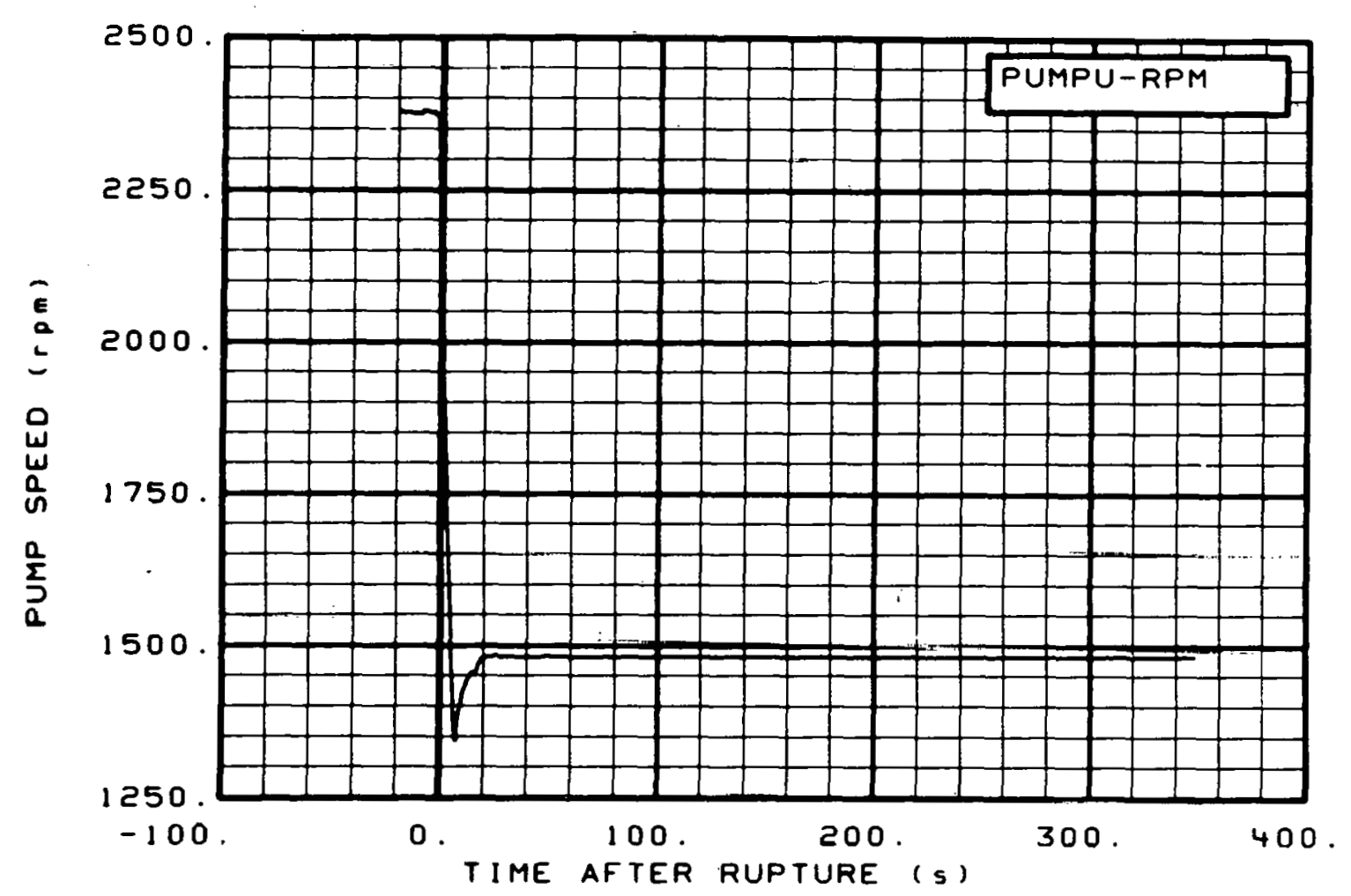

Fig. 369 Primary pump speed (PUMPU-RPM), from -20 to $350 \mathrm{~s}$.

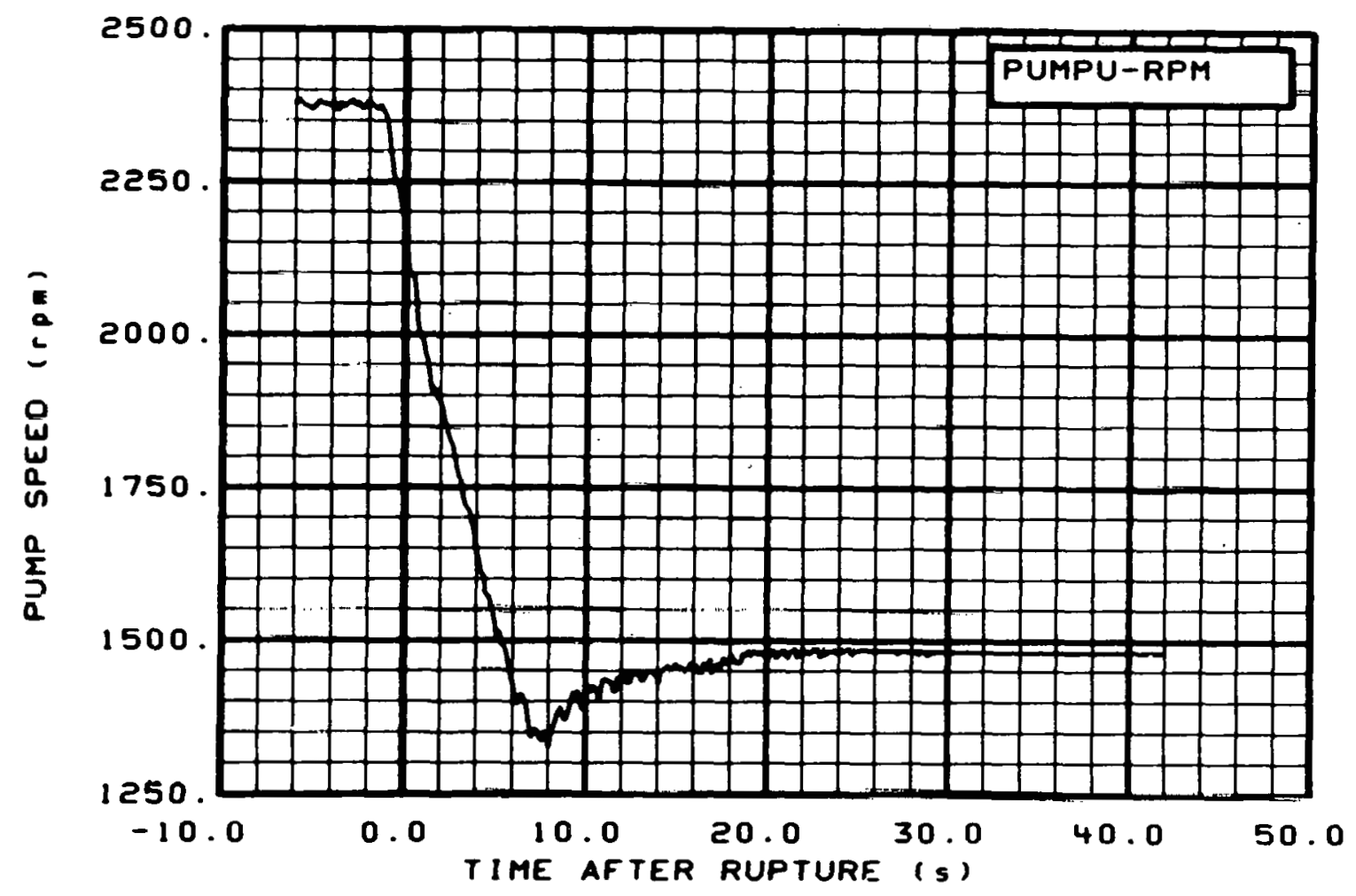

Fig. 370 Primary pump speed (PUMPU-RPM), from -6 to $42 \mathrm{~s}$. 


\section{REFERENCE}

1. E. M. Feldman and D. J. Olson, Semiscale Mod-1 Program and System Description for the Blowdown Heat Transfer Tests (Test Series 2), ANCR-1230 (August 1975). 


\section{THIS PAGE \\ WAS INTENTIONALLY \\ LEFT BLANK}


APPENDIX A

DATA ACQUISITION SYSTEM CAPABILITIES 
THIS PAGE

\section{WAS INTENTIONALLY LEFT BLANK}




\section{APPENDIX A \\ DATA ACQUISITION SYSTEM CAPABILITIES}

The Semiscale Mod-1 system provides for the acquisition, processing, and presentation of test data. Detectors, signal conditioners, signal processors, and recording and display equipment comprise the test data system. The data obtained are principally recorded on an on-line digital system. Selected data channels are also recorded on an analog system.

The on-line digital system is called the digital data acquisition and processing system (DDAPS). The DDAPS has dual and single speed capabilities with identical storage and data output limitations. The dual speed mode is used to extend the recording time when obtaining high frequency data.

From each of up to 240 data channels, the test data system stores 20 blocks of data. Each block of data contains 920 words (each word is the abscissa and ordinate of a data point) of digital information. These 920 words represent a fixed storage display.

The maximum measured throughput rate for the system is 24000 words per second. This throughput rate can be reduced in increments of 100 words per second. The throughput rate, the number of data channels recorded, and the fixed display of 920 words per block determine the time base for displaying the data.

After the data have been stored, data reduction can be made for presentation and analysis purposes. Because of hardware limitations and aesthetic considerations of data presentation, only certain time bases are used when the data are reduced. For data displayed from -20 to $300 \mathrm{~s}$, the recorded data are made to occupy a $320 \mathrm{~s}$ span. This yields a time base of $16 \mathrm{~s}$, which is the $320 \mathrm{~s}$ span divided by the 20 blocks of recorded data.

Generally, 920 words from a given data channel are displayed in the nominal time base of $16 \mathrm{~s}$. Integral ( 1 to 20) multiples of $16 \mathrm{~s}$ may be used as variations on the nominal time base. Because the output is fixed at 920 words, data compression is made by averaging adjacent data points to give the desired compression. 


\section{THIS PAGE}

WAS INTENTIONALLY

LEFT BLANK 
APPENDIX B

POSTTEST ADJUSTMENTS TO DATA FROM SEMISCALE MOD-1 TEST S-28-2 
THIS PAGE

WAS INTENTIONALLY

LEFT BLANK 


\section{APPENDIX B \\ POSTTEST ADJUSTMENTS TO DATA FROM SEMISCALE MOD-1 TEST S-28-2}

Many of the transducers used in the Semiscale Mod-1 system exhibit significant sensitivity to one or more spurious inputs. Strain gage bridge circuits used in pressure transducers, differential pressure transducers, and drag discs are sensitive to changes in ambient _temperature. Differential pressure cells are also sensitive to changes in system pressure. Photomultiplier tubes used as gamma ray detectors in the density transducers are sensitive to temperature changes, as well as to random variations in the locations of the radiation sources. Core power measurements depend on a calibrated resistor, whose resistance changes in value as a function of time and power level as it heats up.

Although the errors introduced into the data by spurious secondary inputs generally do not exceed the specified error ranges of the transducers, significant improvement in measurement accuracy can be achieved if the secondary sensitivity can be identified and removed. In the case of the drag discs, corrections are absolutely. necessary because the signal due to temperature fluctuations can exceed that due to flow by several hundred percent. Since the exact values of the spurious inputs to which different transducers might be sensitive cannot often be easily predicted and are sometimes inconvenient to measure, secondary effects have been accounted for by correcting the data after the test rather than by using elaborate real time programs in the data acquisition system computer. The methods and results of the posttest data correction analysis for Test S-28-2 are presented in the following paragraphs and tables.

\section{PRESSURE MEASUREMENTS}

Corrections to pressure transducer measurements in the main system loop are based on data taken from the standard reference (Heise) gauge at Spool 4, taken $15 \mathrm{~s}$ before initiation of blowdown and at $300 \mathrm{~s}$ after initiation of blowdown. The pressure readings are adjusted to account for pressure variations around the main loop, using the readings. of nearby differential pressure cells. A linear correction is then applied to the pressure data to match the data to the calculated reference data at the two specified time points.

Correction of the steam generator secondary pressure (PU-SGSD) and intact and broken loop emergency core coolant (ECC) accumulator pressure (PU-ACCl and PB-ACC2) measurements are done in the same manner as for the main loop pressures using Heise gauges installed expressly for this purpose. The data from the pressure transducer for the pressure suppression system (P-PSS) are corrected to match the process instrumentation at preblowdown conditions.

Pressure measurement corrections are performed using the data acquisition system (DAS) computer using the following equation: 


$$
F^{\prime}(t)=c_{0}+c_{1} \quad[F(t)]
$$

where

$$
\begin{aligned}
& F^{\prime}(t)=\quad \text { corrected data } \\
& F(t)=\text { raw data } \\
& C_{O}=\text { offset } \\
& C_{1}=\text { scaling friclor. }
\end{aligned}
$$

The values of the offset and: scaling factor are given in Table B-I.

TABLE B-I

CONSTANTS FOR PRESSURE MEASUREMENT

CORRECTIONS (TEST S-28-2)

\begin{tabular}{lcc}
\hline $\begin{array}{c}\text { Detector } \\
\text { Identification }\end{array}$ & \multicolumn{1}{c}{$C_{0}$} & \multicolumn{1}{c}{$C_{1}$} \\
\cline { 2 - 3 } PB-23 & -1.6 & 0.9856 \\
PU-SGSD & -0.6 & 1.0234 \\
PU-PRIZE & -6.2 & 0.9864 \\
PV-LP-166 & 3.5 & 0.9798 \\
\hline
\end{tabular}

\section{DIFFERENTLAL PRESSURE MEASUREMENTS}

Pressure sensitivity in the differential pressure cells in the main system loop is determined from the pretest system pressure check. Digital data are recorded for all measurements at ambient temperature, with no system flow, at pressures of ambient, 1380, $3450,6900,10350,13800$, and $155.00 \mathrm{kPa}$. The output of the differential pressure cells is plotted against system pressure, with the resulting plots used to describe the pressure response of the transducers.

The response of the differential pressure cells due to ambient temperature is determined from a digital data scan taken at $533 \mathrm{~K}$ and $12150 \mathrm{kPa}$ with no system flow. The measured transducer outputs are corrected for pressure and compared with the values calculated due only to the density difference between the water inside the loop ( $533 \mathrm{~K}$ ). and outside the loop in the sense lines ( 300 to $311 \mathrm{~K}$ ). 
The difference between the measured pressure corrected value and the calculated value is the thermal drift. After the data scan at $533 \mathrm{~K}$ is made, no more opportunities exist to obtain data with the pump stopped and the system full of fluid; therefore, for lack of later data, the thermal drift calculated from the $533 \mathrm{~K}$ data is assumed to be constant throughout the test.

For some differential pressure measurements, the data scan at $533 \mathrm{~K}$ cannot be used as a reference for thermal drift, so other references are used. The reading from the steam generator discharge venturi (DPU-SG-DISC) is shifted to read zero after flow is stopped. For these detectors, and those having nonlinear pressure sensitivities, the corrections are performed according to the following equations:

$$
F^{\prime}(t)=K F(t)+c_{1} \text { for } t<t_{1} \text { or when no } t_{i} \text { are listed }
$$

for time points $t$, where $t_{1} \leqslant t \leqslant t_{n}$

$$
F^{\prime}(t)=K F(t)+c_{i}+\frac{t-t_{i}}{t_{i+1}-t_{i}}\left(c_{i+1}-c_{i}\right) \text { for } t_{i} \leq t \leq t_{i+1}
$$

where $i$ takes on values 1 to $n-1$

$$
F^{\prime}(t)=K F(t)+C_{n} \text { for } t>t_{n}
$$

where

$$
\begin{array}{lll}
\mathbf{t} & = & \text { time } \\
\mathrm{F}^{\prime}(\mathrm{t}) & = & \text { corrected data } \\
\mathrm{F}(\mathrm{t}) & = & \text { raw data } \\
\mathrm{K} & = & \text { scaling factor } \\
\mathrm{C}_{\mathrm{i}} \text { and } \mathrm{t}_{\mathrm{i}}= & \text { corrections and time points. }
\end{array}
$$

The values of the constants are given in Table B-II. 


\begin{tabular}{|c|c|c|c|c|c|c|c|}
\hline $\begin{array}{c}\text { Detector } \\
\text { Identification } \\
\end{array}$ & $K$ & $c_{1}$ & $t_{1}$ & $\mathrm{C}_{2}$ & $t_{2}$ & $c_{3}$ & $t_{3}$ \\
\hline DPU-7-10 & 1 & 0.689 & 0 & 0.648 & 0.01 & 0.545 & 30.0 \\
\hline DPU-12-10 & 1 & -7.129 & 0 & -5.750 & 0.01 & -1.269 & 30.0 \\
\hline DPB-23-CNI & 1 & 115.141 & 0 & 97.905 & 0.01 & 22.063 & 30.0 \\
\hline DPB-42-HN1 & -1 & 0 & & & & & \\
\hline DPV $-166+10$ & 1 & -83.385 & & & & & \\
\hline
\end{tabular}

\section{MOMENTUM FLUX MEASUREMENTS}

The temperature sensitivity of drag discs is determined from pretest warmup data taken at 366 and $533 \mathrm{~K}$ with no system flow. The temperature sensitivity is removed before the data are converted to momentum flux. The temperature of each transducer in taken from the signal of a nearby fluid or metal temperature thermocouple. Slight corrections for errors in setting the transducer output to zero at ambient conditions are also made at this time. Corrections are made using the following equation:

$$
F^{\prime}(t)=F(t)+C_{0}-T_{1} T(t)-P_{1} P(t)
$$

where

$$
\begin{aligned}
& F^{\prime}(t)=\text { corrected data } \\
& F(t)=\text { raw data } \\
& T_{i}=\text { temperature sensitivity } \\
& T(t)=\quad \begin{array}{l}
\text { temperature data from the transducer used for tempera- } \\
C_{0}=
\end{array}
\end{aligned}
$$




$$
\begin{aligned}
& \mathrm{P}_{\mathrm{i}}=\text { pressure sensitivity } \\
& \mathrm{P}(\mathrm{t})=\begin{array}{l}
\text { pressure data from the indicated transducer used for } \\
\text { pressure correction sensitivity. }
\end{array}
\end{aligned}
$$

Values of the constants are given in Table B-III.

\section{TABLE B-III}

\begin{tabular}{|c|c|c|c|c|c|}
\hline $\begin{array}{c}\text { Detector } \\
\text { Identification }\end{array}$ & $\mathrm{C}_{0}$ & $T_{1}$ & $T(t)^{[a]}$ & $P_{1}$ & $P(t)^{[c]}$ \\
\hline FDU-1 & 0.270 & 0.000364 & RBU -2 & -0.0000063 & $P V-U P+10$ \\
\hline FDU-5 & 0 & -0.000169 & TFU-6 & 0.0000107 & $P V-U P+10$ \\
\hline FDU-10 & 0 & 0.000148 & TFU-10 & 0.0000047 & $P U-13$ \\
\hline FDU-13 & 0.120 & 0.000862 & RBU-14A & & \\
\hline FDU- 15 & 0 & -0.001235 & RBU-14A & -0.000 019 & $P U-13$ \\
\hline FDB-21 & 0 & -0.000643 & TFB-20 & -0.0000189 & $P B-21$ \\
\hline FDB-23 & 0 & -0.000841 & TFB-23 & & \\
\hline $\mathrm{FDB}-30^{[\mathrm{b}]}$ & -0.055 & -0.000821 & TFB-30 & $0.000008 \quad 1$ & $P V-U P+10$ \\
\hline$F D B-37^{[b]}$ & 0.170 & 0.000810 & TFB-37 & 0.0000003 & PB -42 \\
\hline FDB -42 & 0.129 & 0.000981 & TFB -42 & -0.0000087 & PB -42 \\
\hline FDV-CORE-IN & 0 & $-0.000 \quad 024$ & TFV-CORE-IN & & \\
\hline \multicolumn{6}{|c|}{$\begin{array}{l}\text { [a] } T(t) \text { is the temperature data used for temperature sensitivity } \\
\text { correction. The symbols listed identify the thermocouples from } \\
\text { which the data are obtained. }\end{array}$} \\
\hline \multicolumn{6}{|c|}{$\begin{array}{l}\text { [b] Temperature sensitivity corrections were applied as usual; however, } \\
\text { because FDB- } 30 \text { and FDB-37 are mounted horizontally, during blowdown } \\
\text { they were partially filled with subcooled water which affected the } \\
\text { temperature sensitivity. Therefore, the temperature sensitivity } \\
\text { correction is more uncertain than that applied to other temperature } \\
\text { sensitivity corrections. }\end{array}$} \\
\hline
\end{tabular}

CONSTANTS FOR MOMENTUM FLUX

MEASUREMENT CORRECTIONS (TEST S-28-2) 


\section{DENSITY MEASUREMENTS}

Density calculations are based on the voltage output of the photomultiplier tubes in the gamma-attenuation densitometer assemblies. The equation used for converting voltage to density is as follows:

$$
\rho=(1 / C) \ln \{D /[A F(t)+B]\}
$$

where

$$
\begin{aligned}
& \rho=\text { the density in } \mathrm{kg} / \mathrm{m}^{3} \\
& \mathrm{C}=\text { a conscanl based in the length of the gamma beam path } \\
& \mathrm{D}=\text { a theoretical voltage for zero attenuation inside the vessel } \\
& \mathrm{A}=\text { an amplification factor } \\
& \mathrm{B}=\text { a biasing factor } \\
& \mathrm{F}(\mathrm{t})=\text { the transducer voltage output. }
\end{aligned}
$$

Constants A and B are adiusted to match the final data to density values calculated from measured pressure and temperature values at the preblowdown and postdrain conditions, effectively giving the data an in-place calibration. The values of the constants for various transducers are given in Table B-IV.

The density measurements GVLP-172HZ and GV-COR-150HZ use amplifiers which precalculate the logarithm function, and hence have a simpler conversion formula:

$$
\begin{aligned}
& \text { GVLP-172HZ } \rho=-1.76 F(t)-69.4 \\
& \text { GV-COR-150HZ } \rho=-3.2 \quad F(t)-126.6 \text {. }
\end{aligned}
$$




\section{TABLE B-IV}

\section{CONSTANTS FOR DENSITY MEASUREMENT \\ CONVERSIONS TO ENGINEERING UNITS (TEST S-28-2)}

\begin{tabular}{llllll}
\hline \multicolumn{1}{c}{$\begin{array}{c}\text { Detector } \\
\text { Identification }\end{array}$} & \multicolumn{1}{c}{$\mathrm{A}$} & $\mathrm{B}$ & \multicolumn{1}{c}{$\mathrm{C}$} & $\mathrm{D}$ \\
\cline { 2 - 3 } GU-1T & 1.086 & & -0.281 & 0.0057 & 6.21 \\
GU-1B & 1.033 & 0.009 & 0.008 & 6.99 \\
GU-5VR & 1.395 & -1.883 & 0.0095 & 5.46 \\
GU-10VR & 1.097 & -0.432 & 0.0095 & 6.84 \\
GU-13VR & 1.560 & -1.784 & 0.0095 & 3.54 \\
GU-15T & 1.162 & -0.756 & 0.0057 & 6.34 \\
GU-15B & 1.060 & -0.163 & 0.008 & 6.42 \\
GB-21T & 1.105 & -0.391 & 0.0057 & 5.98 \\
GB-21B & 1.056 & -0.150 & 0.008 & 7.04 \\
GB-23VR & 1.234 & -1.327 & 0.006 & 7.06 \\
GB-30T & 1.172 & -0.703 & 0.0057 & 5.48 \\
GB-30B & 1.075 & -0.257 & 0.008 & 6.58 \\
GB-37HZ & 1.841 & -3.345 & 0.0095 & 4.34 \\
GB-42VR & 1.318 & -1.748 & 0.006 & 6.55 \\
GVLP-165HZ & 1.085 & -0.207 & 0.0095 & 3.09 \\
GU-PRIZE & 1.38 & -0.303 & 0.0095 & 1.08 \\
\hline & & & & \\
\hline
\end{tabular}

Some density measurements are obtained using a two-beam gamma densitometer which operates on the same basic principle of gamma attenuation as does the single-beam gamma densitometer. Each beam originates from the same gamma source and is allowed to pass through separate portions of the piping cross-sectional flow area to obtain an average density measurement in that particular region. The geometrical relationship of the gamma beam path through the piping and geometrically related variables used for processing of data from a two-beam gamma densitometer are shown in Figure B-1. 


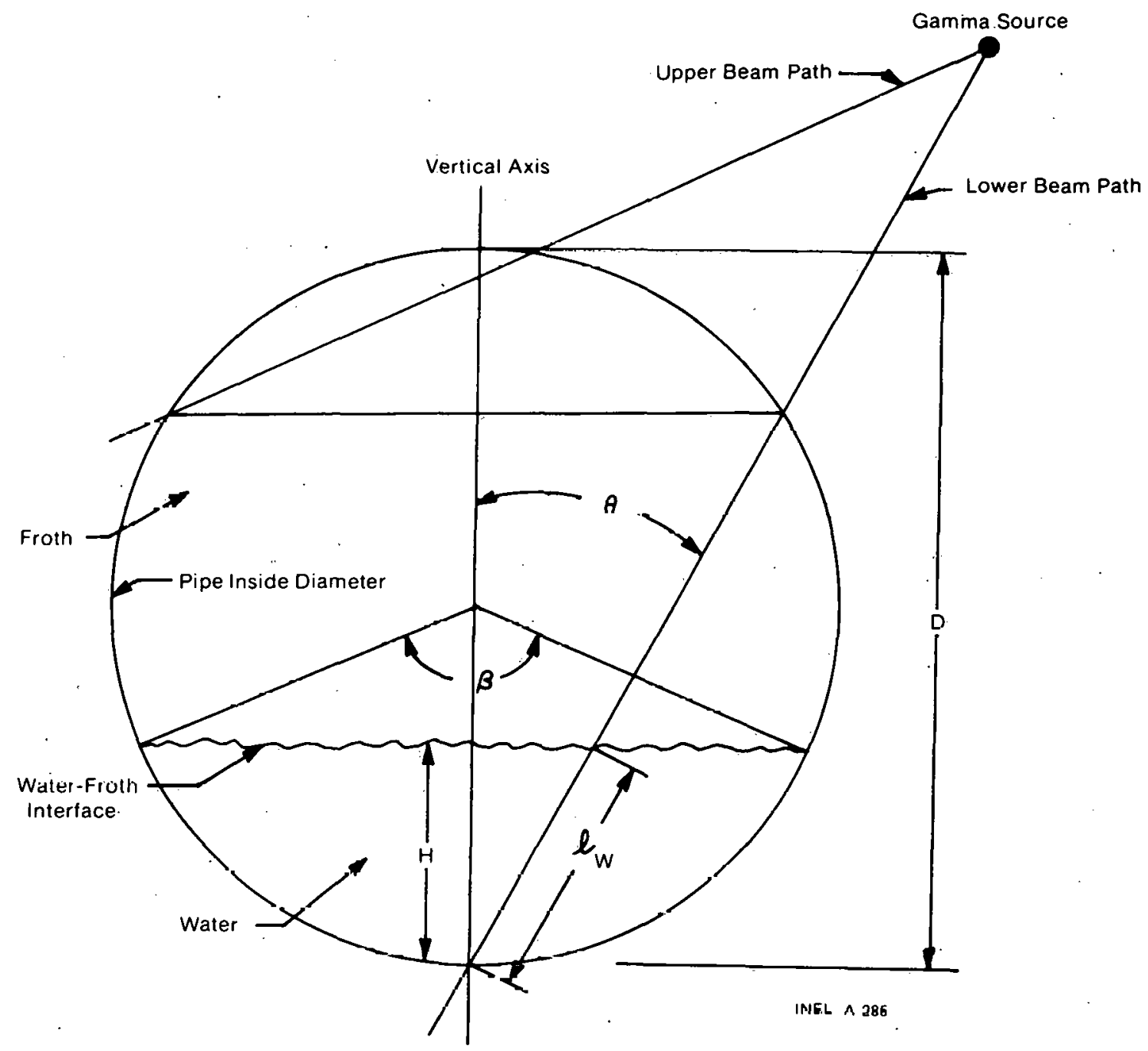

Fig. B-1 Geometry used for processing of density data obtained from two-beam gamma densitometers.

The average density measured by each individual gamma beam is obtained using the same equation as is used for the single-beam gamma densitometers. Values for the constants for the single-beam density measurements obtained with the two-beam gamma densitometers are presented in Table A-IV along with the constants for single-beam gamma densitometers.

In the Semiscale Mod-1 system, two-beam gamma densitometers provide added information which allows the calculation of a better average density than that obtained from a single beam. A mathematical model is used for processing the two-beam data to obtain the improved average density information. The processing method used is based on a froth-water model coupled with information from the two individual gamma beams and related beam path and piping cross-sectional geometry. The resulting information is recorded and reported under the density measurement identification ending with a " $\mathrm{C}$ ", for example, GB-21C. 
The use of the froth-water model for obtaining average density from a two-beam gamma densitometer is based on observations indicating that flow regimes in the Semiscale Mod-1 system can be modeled by a layer of water on the bottom of the pipe with a degree of froth on the surface. For homogeneous flow conditions such as all froth or all liquid the model remains valid. At any point in time slug flow is also modeled. The froth-water model does not model annular or inverted annular flows very well. However, these flows are not expected to exist for significant portions of a Semiscale Mod-1 system blowdown in horizontal piping. Density gradients from the top to the bottom of the pipe may exist showing no distinct location change from water to froth. This flow is neither totally homogeneous nor stratified, but the froth-water model does provide an adequate approximation of the average density characteristic of this flow pattern.

The average density obtained by using the gamma beam geometry shown in Figure B-1 and by applying the froth-water model is given by

$$
\bar{\rho}=\alpha_{f} \rho_{1}+\left(1-\alpha_{f}\right) \rho_{w} \mathrm{~kg} / \mathrm{m}^{3}
$$

where

$$
\begin{aligned}
& \bar{\rho}=\text { average cross-sectional density } \\
& \rho 1=\begin{array}{l}
\text { average density measured by the upper gamma beam } \\
\text { (measures the froth density) }
\end{array} \\
& \rho_{\mathrm{W}}=\text { density of liquid water (at local system conditions) } \\
& \alpha_{\mathrm{f}}=1+(1 / 2 \pi)(\sin \beta-\beta)=\text { froth fraction. }
\end{aligned}
$$

The angle which $\beta$ represents is shown in Figure B-1. Values for $\beta$ are obtained as follows:

$$
B=2 \cos ^{-1}(1-2 h)
$$

where

$$
\mathrm{h}=\frac{\mathrm{H}}{\mathrm{D}}=\cos ^{2} \theta \frac{\rho_{2}-\rho_{1}}{\rho_{\mathrm{W}}-\rho_{1}}
$$

where
$\mathrm{H}=\ell_{\mathrm{w}} \cos \theta\left(\ell_{\mathrm{w}}\right.$ and $\theta$ are defined in Figure A-1 $)$
$\mathrm{D}=$ piping inside diameter
$\rho_{2}=$ the average density mcasurcd by the lower gamma beam. 
Average density is not calculated using the two-beam froth-water model when the angle $\theta$ is not favorable due to system hardware restrictions in positioning the source. The froth-water model requires separate density sampling in both the upper portions of the piping cross section. 
APPENDIX C

SELECTED DATA WITH ESTIMATED TOTAL ERROR BANDS FROM SEMISCALE MOD-1 TEST S-28-2 


\section{THIS PAGE}

\section{WAS INTENTIONALLY \\ LEFT BLANK}




\section{APPENDIX C \\ SELECTED DATA WITH ESTIMATED TOTAL ERROR \\ BANDS FROM SEMISCALE MOD-1 TEST S-28-2}

Analysis has been performed on selected data from Test S-28-2 to provide a guide to the uncertainty associated with data measurements in the Semiscale Mod-1 system. The end result of the analysis is presented as error bands about the measured data which represent a $95 \%$ confidence level.

The error bands are obtained by combining uncertainties obtained from analysis of the data itself (random error) and engineering analysis of the measurement system (engineering error). The procedure by which error bands were established for the data presented in this appendix is described in the following paragraphs.

The data trace under analysis was empirically fitted with a linear difference equation, which was subject to a white noise input at each sampling time point. The objective of the empirical fitting procedure was to characterize the white noise, which was taken to represent the random error. The procedures for fitting the difference equation are discussed in depth in Reference C-1. A data trace was often segmented and different equations were fitted to each segment with statistical correlations between successive observations accounted for by the fitting procedure. The white noise input was assumed to arise from a normally distributed population. The standard deviation of the white noise, as found during the fitting procedures, was taken as an estimate of the random error standard deviation and is shown in Table C-I. The data traces of the error band analysis are shown in Figures C-1 through $\mathrm{C}-40$.

TABLE C-I

RANDOM ERROR VARIANCE

\begin{tabular}{|c|c|c|c|c|}
\hline Measurement & $\begin{array}{c}\text { Random Error } \\
\text { Variance } \\
\sigma_{\mathrm{R}} \\
\end{array}$ & $\begin{array}{l}\text { Period of } \\
\text { Application } \\
\text { (s) }\end{array}$ & Figure & Comments \\
\hline TFB-23 & $\begin{array}{r}11.892 \\
3.386 \\
0.611\end{array}$ & $\begin{aligned} 0 & \text { to } 81 \\
81 & \text { to } 89 \\
89 & \text { to } 350\end{aligned}$ & $B-1$ & \\
\hline TFV-ANN-35A & $\begin{array}{r}15.754 \\
0.344\end{array}$ & $\begin{array}{r}0 \text { to } 82 \\
82 \text { to } 350\end{array}$ & $B-2$ & $\begin{array}{l}\text { The error bands should be } \\
\text { smaller than what is rep- } \\
\text { resented from } 82 \text { to } \\
89 \mathrm{~s}[\mathrm{a}] \text {. }\end{array}$ \\
\hline
\end{tabular}


TABLE C-I (continued)

\begin{tabular}{|c|c|c|c|c|}
\hline Measurement & $\begin{array}{c}\text { Random Error } \\
\text { Variance } \\
\sigma_{\mathrm{R}} \\
\end{array}$ & $\begin{array}{l}\text { Period of } \\
\text { Application } \\
\text { (s) } \\
\end{array}$ & Figure & Comments \\
\hline $\begin{array}{r}\text { TFV-LP-7 } \\
.\end{array}$ & $\begin{array}{r}61.998 \\
33.203 \\
45.333 \\
0.243\end{array}$ & $\begin{aligned} & 0 \text { to } 40 \\
& 40 \text { to } 62 \\
& 62 \text { to } 70 \\
& 70 \text { to } 350\end{aligned}$ & $B-3$ & . \\
\hline TFU-PRIZE & $\begin{array}{l}4.594 \\
2.283 \\
0.179\end{array}$ & $\begin{aligned} 0 & \text { to } 29 \\
29 & \text { to } 67 \\
67 & \text { to } 350\end{aligned}$ & $B-4$ & \\
\hline TMU-1T16 & 0.259 & 0 to 350 & $B-5$ & \\
\hline TMV-CI - 70A & $\begin{array}{l}0.561 \\
5.599 \\
1.582\end{array}$ & $\begin{array}{l}0 \text { to } 62 \\
62 \text { to } 91 \\
91 \text { to } 350\end{array}$ & $B-6$ & 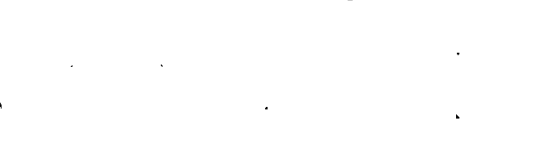 \\
\hline TH-E4-09 & $\begin{array}{r}2.981 \\
19.010 \\
2.670 \\
0.237\end{array}$ & $\begin{array}{r}0 \text { to } 70 \\
70 \text { to } 116 \\
116 \text { to } 129 \\
129 \text { to } 350\end{array}$ & $B-7$ & $\begin{array}{l}\text { The error bands should be } \\
\text { smaller than what is rep- } \\
\text { resented from } 69 \text { to } \\
77 \mathrm{~s}[\mathrm{a}] \text {. }\end{array}$ \\
\hline TH-E $4-27$ & $\begin{array}{r}12.772 \\
1.404 \\
12.813 \\
30.993 \\
0.247\end{array}$ & 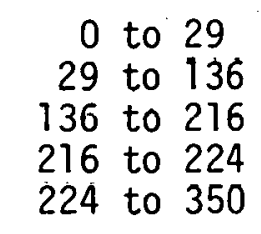 & $B-8$ & $\begin{array}{l}\text { The error bands should be } \\
\text { smaller than what is rep- } \\
\text { resented from } 136 \text { to } \\
142 \mathrm{~s} \text { [a]. }\end{array}$ \\
\hline TH-E4-55 & $\begin{array}{r}1.665 \\
0.546 \\
19.940 \\
11.900\end{array}$ & $\begin{array}{r}0 \text { to } 173 \\
173 \text { to } 341 \\
341 \text { to } 348 \\
348 \text { to } 350\end{array}$ & $B-9$ & \\
\hline PU-13 & $\begin{array}{r}10.527 \\
.1 .003\end{array}$ & $\begin{aligned} 0 & \text { to } 40 \\
40 & \text { to } 350\end{aligned}$ & $B-10$ & \\
\hline $\mathrm{PB}-23$ & $\begin{array}{r}10.677 \\
1.188\end{array}$ & 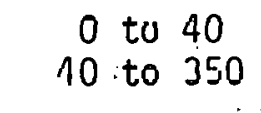 & $B-11$ & . \\
\hline DPU-7-10 & $\begin{array}{l}0.135 \\
0.037\end{array}$ & $\begin{array}{rll}0 & \text { to } & 70 \\
70 & \text { to } & 350\end{array}$ & $B-12$ & \\
\hline DPU-12-10 & $\begin{array}{l}0.750 \\
0.315 \\
0.292 \\
0.092\end{array}$ & $\begin{aligned} & 0 \text { to } \\
& 8 \text { to } 29 \\
& 29 \text { to } 70 \\
& 70 \text { to } 350\end{aligned}$ & $B-13$ & - \\
\hline
\end{tabular}


TABLE C-I (continued)

\begin{tabular}{|c|c|c|c|c|}
\hline Measurement & $\begin{array}{c}\text { Random Error } \\
\text { Variance } \\
{ }^{\sigma} \mathrm{R} \\
\end{array}$ & $\begin{array}{l}\text { Period of } \\
\text { Application } \\
\text { (s) } \\
\end{array}$ & Figure & Comments \\
\hline DPU-12-15 & $\begin{array}{l}0.281 \\
0.049\end{array}$ & 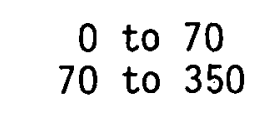 & $B-14$ & \\
\hline FTU-1 & $\begin{array}{r}108.207 \\
121.187 \\
15.958 \\
13.666\end{array}$ & $\begin{aligned} 0 & \text { to } 53 \\
53 & \text { to } 71 \\
71 & \text { to } 101 \\
101 & \text { to } 350\end{aligned}$ & $B-15$ & \\
\hline FTU-15 & $\begin{array}{l}33.714 \\
16.581 \\
32.959 \\
39.171 \\
59.839 \\
10.549 \\
28.467\end{array}$ & $\begin{array}{r}0 \text { to } 18 \\
18 \text { to } 59 \\
59 \text { to } 72 \\
72 \text { to } 80 \\
80 \text { to } 94 \\
94 \text { to } 235 \\
235 \text { to } 350\end{array}$ & $B-16$ & \\
\hline FTU-HPIS & $\begin{array}{l}0.380 \\
0.185\end{array}$ & $\begin{array}{c}0 \text { to } 70 \\
70 \text { to } 350\end{array}$ & $B-17$ & \\
\hline FTU-LPIS & $\begin{array}{l}0.198 \\
0.961 \\
0.520 \\
0.009 \\
0.012\end{array}$ & $\begin{array}{r}0 \text { to } 20 \\
20 \text { to } 27 \\
27 \text { to } 67 \\
67 \text { to } 341 \\
341 \text { to } 350\end{array}$ & $B-18$ & \\
\hline FTU-ACC 1 & 1.966 & 0 to 65 & $B-19$ & \\
\hline FTV $-40 A$ & $\begin{array}{l}8.463 \\
1.392\end{array}$ & $\begin{aligned} 0 & \text { to } \\
75 & \text { to } 350\end{aligned}$ & $B-20$ & \\
\hline FTV-40M & $\begin{array}{l}7.620 \\
0.746\end{array}$ & $\begin{aligned} 0 & \text { to } \\
80 & \text { to } 350\end{aligned}$ & $B-21$ & $\begin{array}{l}\text { The error bands should be } \\
\text { smaller than what is rep- } \\
\text { resented from } 80 \text { to } \\
87 \mathrm{~s} \text { [a]. }\end{array}$ \\
\hline GU-1T & $\begin{array}{l}2.166 \\
0.229 \\
0.310\end{array}$ & $\begin{array}{l}0 \text { to } 11 \\
11 \text { to } 29 \\
29 \text { to } 350\end{array}$ & $B-22$ & \\
\hline GU-1B & $\begin{array}{l}3.116 \\
0.558 \\
0.746 \\
1.369\end{array}$ & $\begin{array}{r}0 \text { to } 12 \\
12 \text { to } 120 \\
120 \text { to } 220 \\
220 \text { to } 350\end{array}$ & $B-23$ & \\
\hline
\end{tabular}


TABLE C-I (continued)

\begin{tabular}{|c|c|c|c|c|}
\hline Measurement & $\begin{array}{c}\text { Random Error } \\
\text { Variance } \\
\sigma_{R} \\
\end{array}$ & $\begin{array}{l}\text { Period of } \\
\text { Application } \\
\text { (s) }\end{array}$ & Figure & Comments \\
\hline GU-1C & $\begin{array}{l}1.439 \\
0.369 \\
0.338 \\
0.594\end{array}$ & 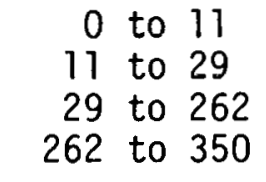 & $B-24$ & . \\
\hline GU-10VR & $\begin{array}{l}1.626 \\
0.239\end{array}$ & 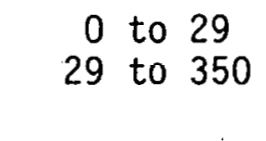 & $B-25$ & $\begin{array}{l}\text { The error bands are smal1- } \\
\text { er than what is represent- } \\
\text { ed from } 29 \text { tol } 37 \mathrm{a}[\mathrm{a} \text {. }\end{array}$ \\
\hline GU-15T & $\begin{array}{r}3.878 \\
12.586 \\
6.133 \\
11.401 \\
0.603 \\
5.319\end{array}$ & $\begin{array}{r}0 \text { to } 7 \\
7 \text { to } 18 \\
18 \text { to } 60 \\
60 \text { to } 67 \\
67 \text { to } 235 \\
235 \text { to } 350\end{array}$ & $B-26$ & \\
\hline$G U-15 B$ & $\begin{array}{r}2.696 \\
10.632 \\
1.415 \\
10.971 \\
0.629 \\
2.586 \\
1.362 \\
5.689\end{array}$ & $\begin{aligned} & 0 \text { to } \\
& 7 \text { to } 22 \\
& 22 \text { to } 59 \\
& 59 \text { to } 72 \\
& 72 \text { to } 86 \\
& 86 \text { to } 94 \\
& 94 \text { to } 235 \\
& 235 \text { to } 350\end{aligned}$ & $B-27$ & . \\
\hline$G U-15 C$ & $\begin{array}{r}3.018 \\
9.552 \\
0.862 \\
10.208 \\
0.938 \\
0.547 \\
5.287\end{array}$ & $\begin{aligned} 0 & \text { to } \\
7 & \text { to } 23 \\
23 & \text { to } 60 \\
60 & \text { to } 72 \\
72 & \text { to } 101 \\
101 & \text { to } 235 \\
235 & \text { to } 350\end{aligned}$ & $B-28$ & . \\
\hline GB-7.3VR & $\begin{array}{r}10.845 \\
8.193 \\
1.735\end{array}$ & $\begin{array}{l}0 \text { to } 61 \\
61 \text { to } 69 \\
69 \text { to } 350\end{array}$ & $B-2 \dot{y}$ & \\
\hline GV-COR-150HZ & $\begin{array}{l}1.888 \\
7.688 \\
9.120 \\
3.303 \\
1.651\end{array}$ & $\begin{array}{r}0 \text { to } 62 \\
62 \text { to } 73 \\
73 \text { to } 80 \\
80 \text { to } 304 \\
304 \text { to } 350\end{array}$ & $B-30$ & . \\
\hline
\end{tabular}


TABLE C-I (continued)

\begin{tabular}{|c|c|c|c|c|}
\hline Measurement & $\begin{array}{c}\text { Random Error } \\
\text { Variance } \\
\quad \sigma_{R} \\
\end{array}$ & $\begin{array}{l}\text { Period of } \\
\text { Application } \\
\text { (s) } \\
\end{array}$ & $\underline{\text { Figure }}$ & Comments \\
\hline GVLP-165HZ & $\begin{array}{l}7.183 \\
0.363 \\
9.250 \\
2.195\end{array}$ & $\begin{array}{l}0 \text { to } 18 \\
18 \text { to } 61 \\
61 \text { to } 69 \\
69 \text { to } 350\end{array}$ & $B-31$ & . \\
\hline GVLP-172HZ & $\begin{array}{r}4.660 \\
2.758 \\
10.663 \\
1.571 \\
0.477\end{array}$ & $\begin{aligned} & 0 \text { to } 37 \\
& 37 \text { to } 61 \\
& 61 \text { to } 69 \\
& 69 \text { to } 180 \\
& 180 \text { to } 350\end{aligned}$ & $B-32$ & $\begin{array}{l}\text { The error bands are smal1- } \\
\text { er than what is represent- } \\
\text { ed from } 55 \text { to } 62 \mathrm{~s} \text { [a]. }\end{array}$ \\
\hline $\begin{array}{l}\text { FDU-1, } \\
\text { GU-1C }\end{array}$ & $\begin{array}{l}0.858 \\
0.192 \\
0.092\end{array}$ & $\begin{array}{l}0 \text { to } 11 \\
11 \text { to } 41 \\
41 \text { to } 350\end{array}$ & $B-33$ & . \\
\hline $\begin{array}{l}\text { FTU-1, } \\
\text { GU-1C }\end{array}$ & $\begin{array}{l}1.212 \\
0.362 \\
0.123 \\
0.249\end{array}$ & $\begin{aligned} 0 & \text { to } \\
7 & \text { to } 70 \\
70 & \text { to } 247 \\
247 & \text { to } 350\end{aligned}$ & $B-34$ & \\
\hline $\begin{array}{l}\text { FDU-5, } \\
\text { GU-5VR }\end{array}$ & $\begin{array}{l}1.028 \\
0.373 \\
0.373 \\
0.151 \\
0.339\end{array}$ & $\begin{array}{r}0 \text { to } 10 \\
10 \text { to } 40 \\
40 \text { to } 75 \\
75 \text { to } 129 \\
129 \text { to } 350\end{array}$ & $B-35$ & \\
\hline $\begin{array}{l}\text { FTU-13, } \\
\text { GU-13VR }\end{array}$ & $\begin{array}{l}0.889 \\
2.075 \\
0.510 \\
1.500 \\
0.526 \\
0.271\end{array}$ & $\begin{array}{r}0 \text { to } 26 \\
26 \text { to } 46 \\
46 \text { to } 62 \\
62 \text { to } 70 \\
70 \text { to } 755 \\
155 \text { to } 350\end{array}$ & $B-36$ & \\
\hline $\begin{array}{l}\text { FTU-15, } \\
\text { GU-15C }\end{array}$ & $\begin{array}{l}2.443 \\
1.147 \\
0.112 \\
1.129\end{array}$ & $\begin{aligned} & 0 \text { to } 50 \\
& 50 \text { to } 94 \\
& 94 \text { to } 235 \\
& 235 \text { to } 350\end{aligned}$ & $B-37$ & \\
\hline $\begin{array}{l}\text { FDB-21, } \\
\text { GB-21C }\end{array}$ & $\begin{array}{l}3.690 \\
3.029 \\
0.408\end{array}$ & $\begin{array}{l}0 \text { to } 7 \\
7 \text { to } 68 \\
68 \text { to } 350\end{array}$ & $B-38$ & \\
\hline $\begin{array}{l}\text { FTB-21, } \\
\text { GB-21C }\end{array}$ & $\begin{array}{l}4.225 \\
3.004 \\
0.444\end{array}$ & $\begin{aligned} 0 & \text { to } \\
7 & \text { to } 68 \\
68 & \text { to } 350\end{aligned}$ & $B-39$ & . \\
\hline
\end{tabular}


IABLE C-I (continued)

\begin{tabular}{|c|c|c|c|c|}
\hline Measurement & $\begin{array}{c}\text { Random Error } \\
\text { Variance } \\
\sigma_{\mathrm{R}}\end{array}$ & $\begin{array}{l}\text { Period of } \\
\text { Appl ication } \\
\text { (s) }\end{array}$ & Figure & Comments \\
\hline $\begin{array}{l}\text { FTV-CORE-IN, } \\
\text { GV-COR-15OHZ }\end{array}$ & $\begin{array}{l}0.389 \\
0.094 \\
5.055\end{array}$ & $\begin{array}{l}0 \text { to } 7 \\
7 \text { to } 61 \\
61 \text { to } 350\end{array}$ & $B-40$ & $\begin{array}{l}\text { The error bands are smail- } \\
\text { er than what is represent- } \\
\text { ed from } 61 \text { to } 69 \mathrm{~s}[\mathrm{a}] \text {. }\end{array}$ \\
\hline
\end{tabular}

[a] Due to present software limitations, error bands are uncertain wherever segments with different size crror bands are merged together.

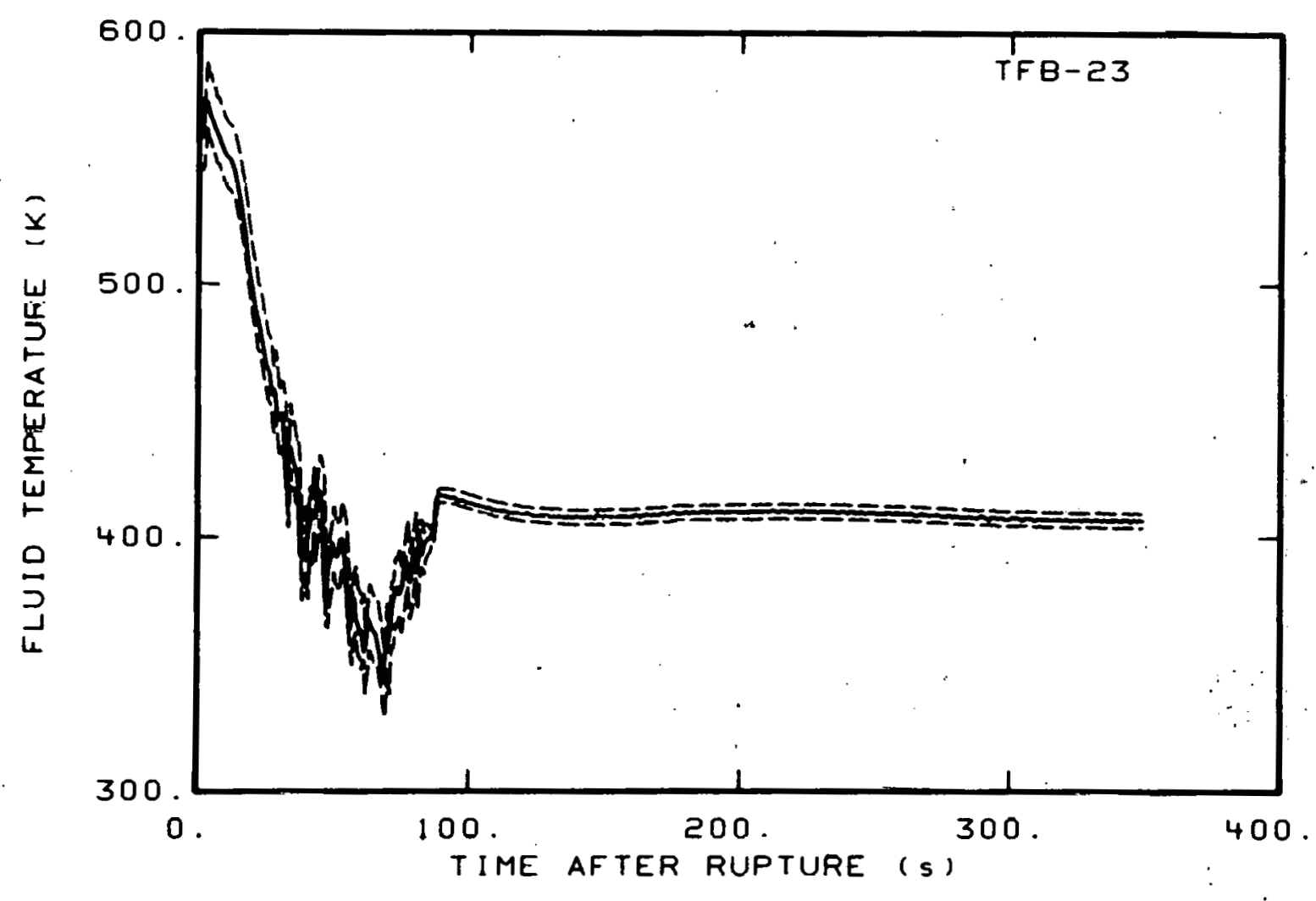

Fig. C-1 Fluid temperature in broken loup (TFB-23). 


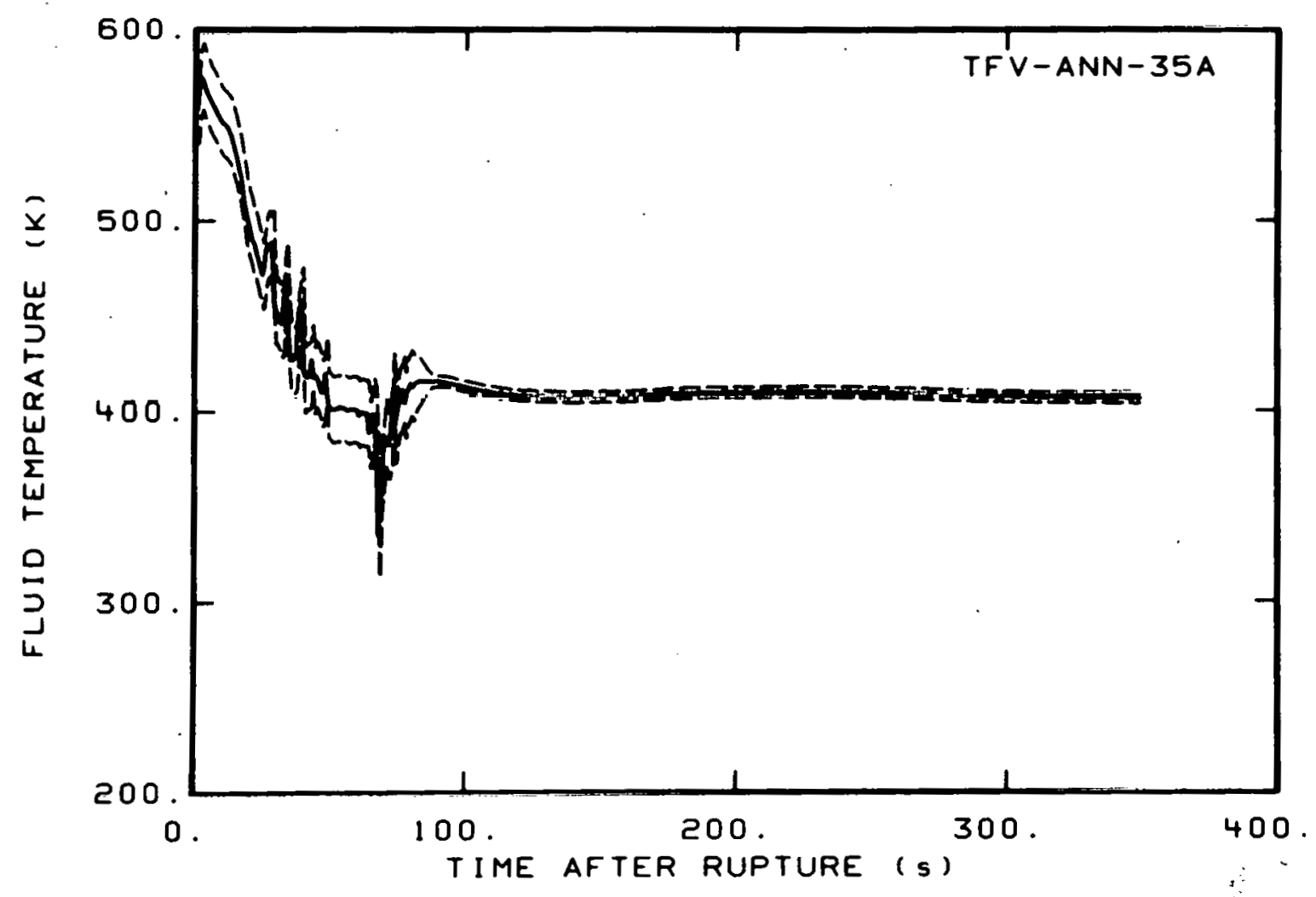

Fig. C-2 Fluid temperature in downcomer annulus (TFV-ANN-35A).

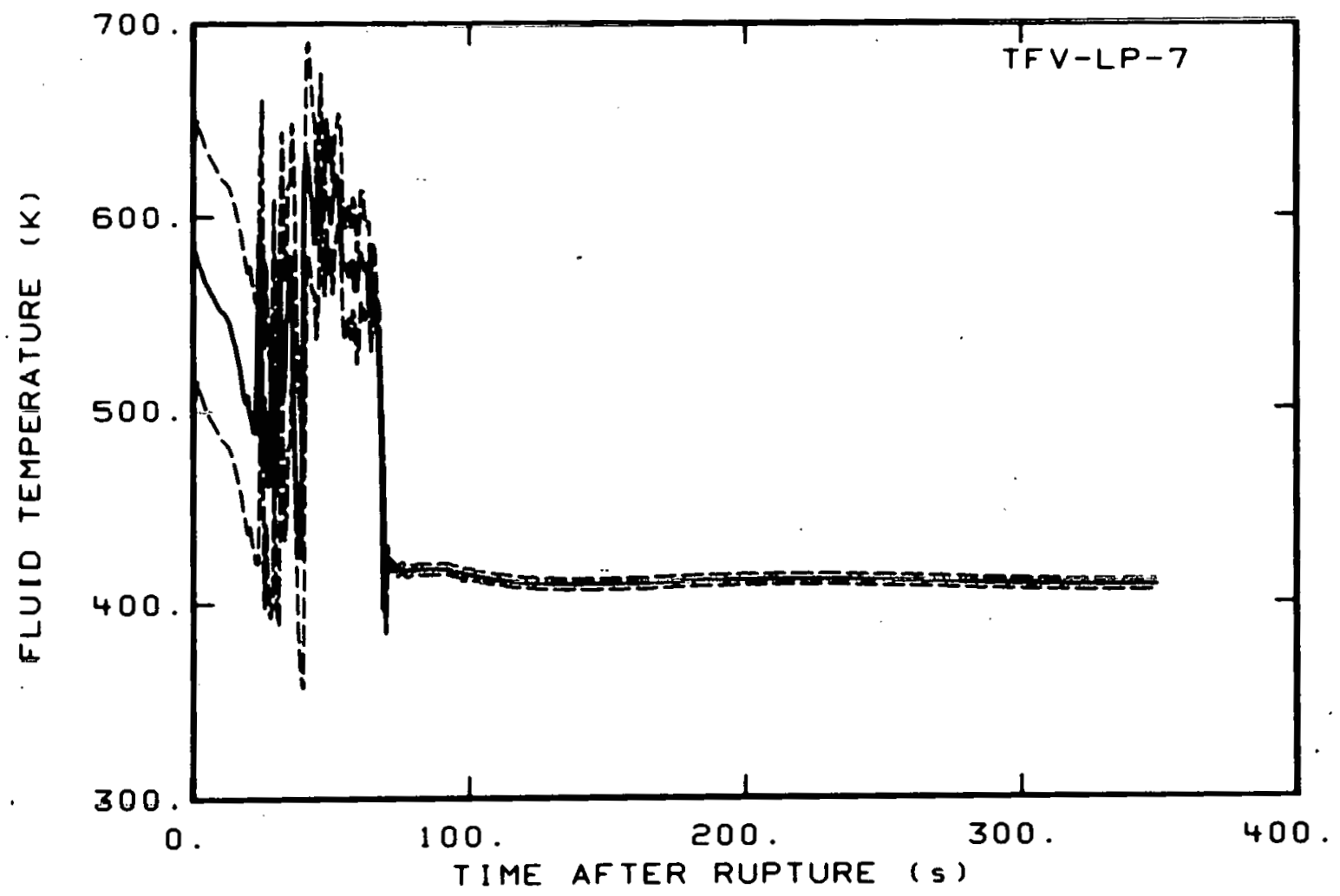

Fig. C-3 Fluid temperature in lower plenum (TFV-LP-8). 


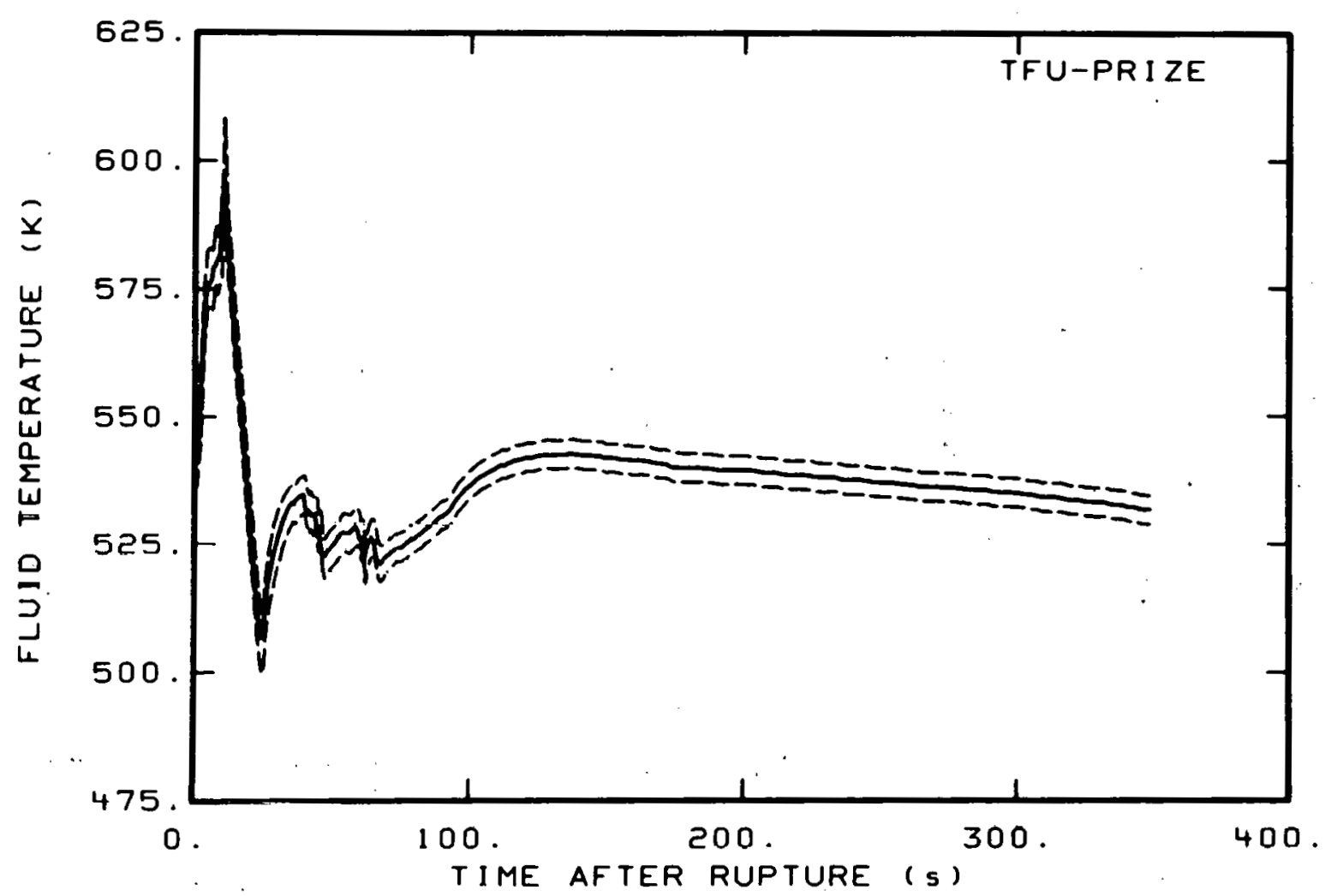

Fig. C-4 Fluid temperature in pressurizer surge line (TFU-PRIZE).

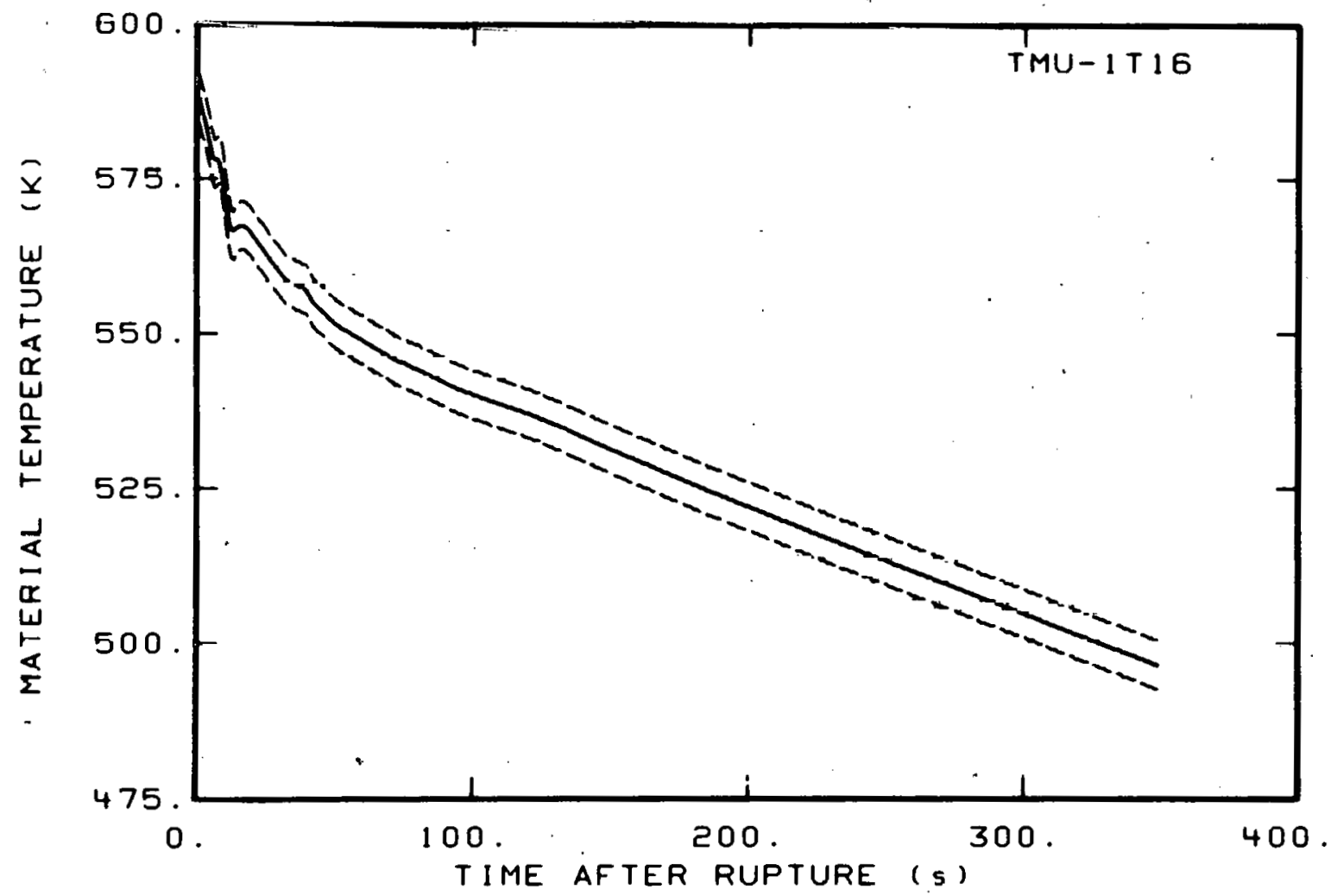

Fig. C-5 Material temperature in intact loop (TMU-1T16). 


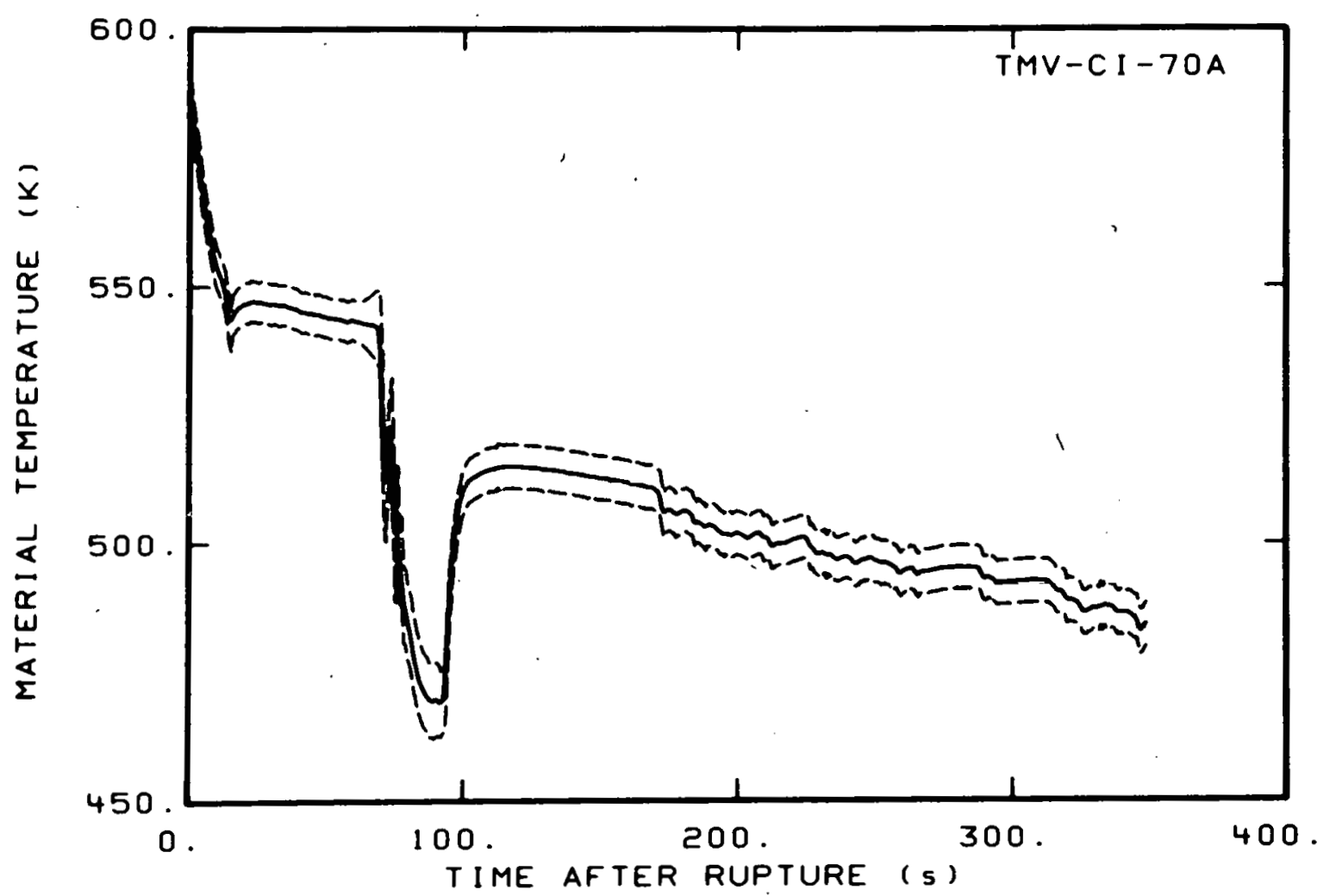

Fig. C-6 Material temperature in vessel filler (TMV-CI-70A).

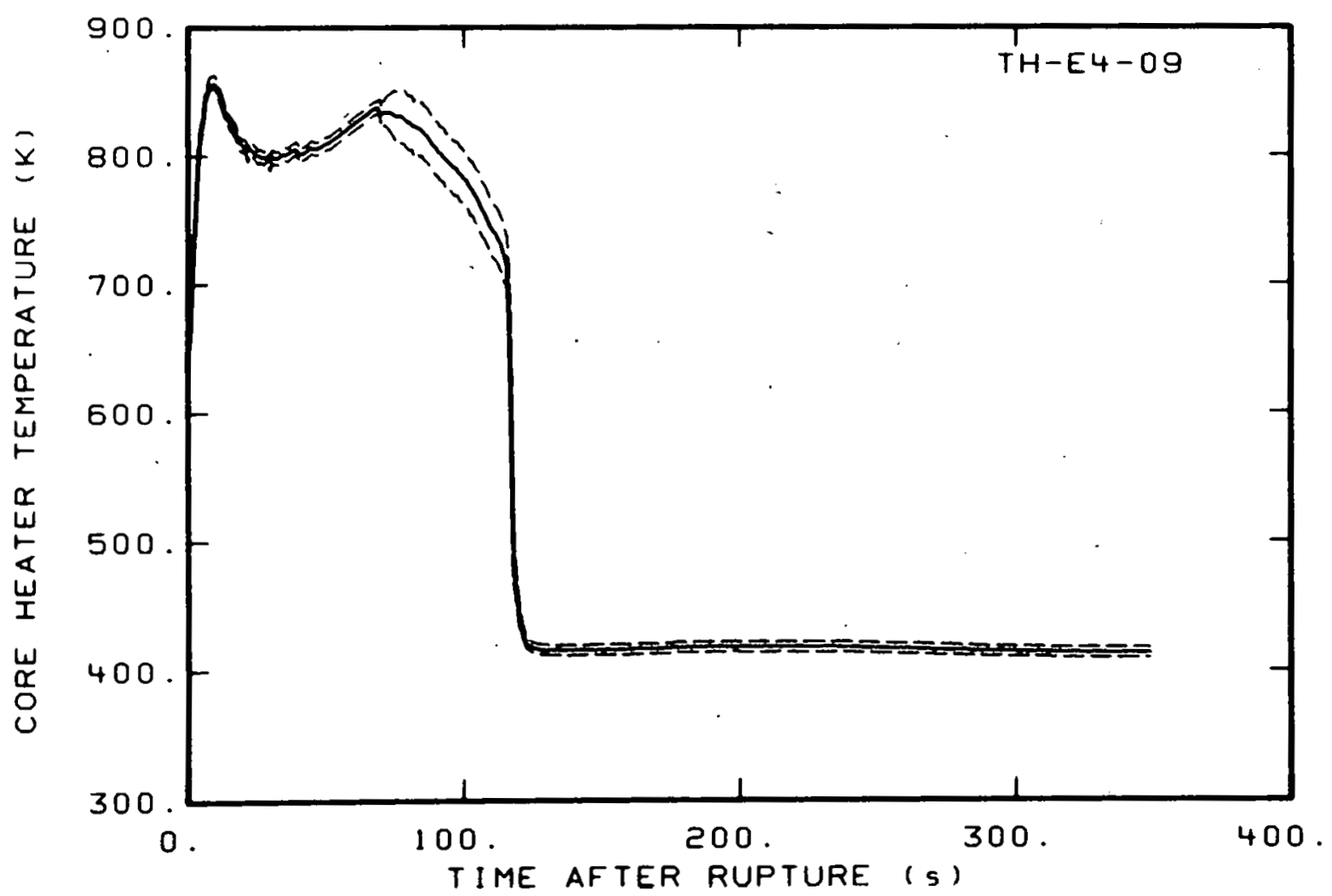

Fig. C-7 Core heater temperature, Rod E-4 (TH-E4-09). 


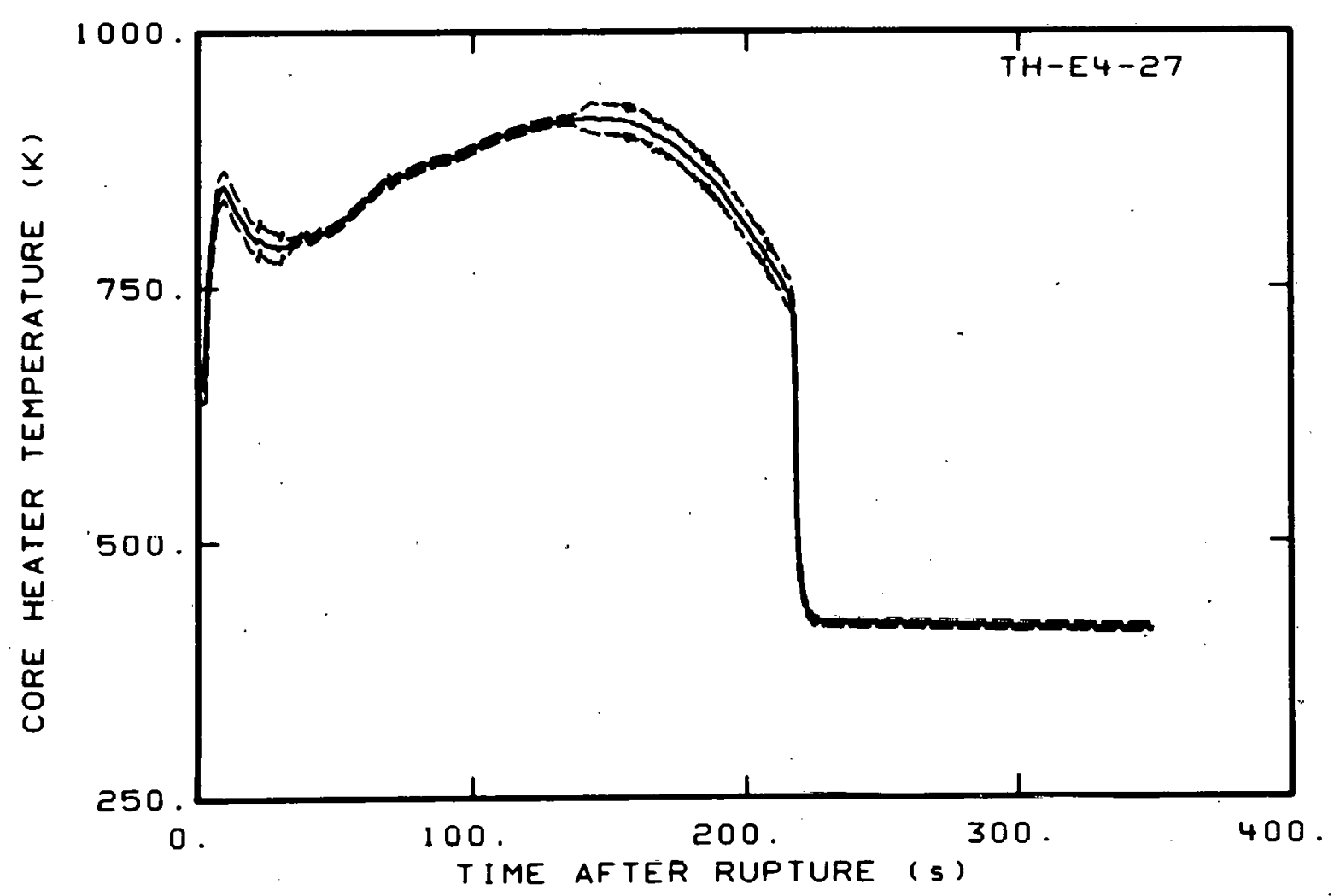

Fig. C-8 Core heater temperature, Rod E-4 (TH-E4-27).

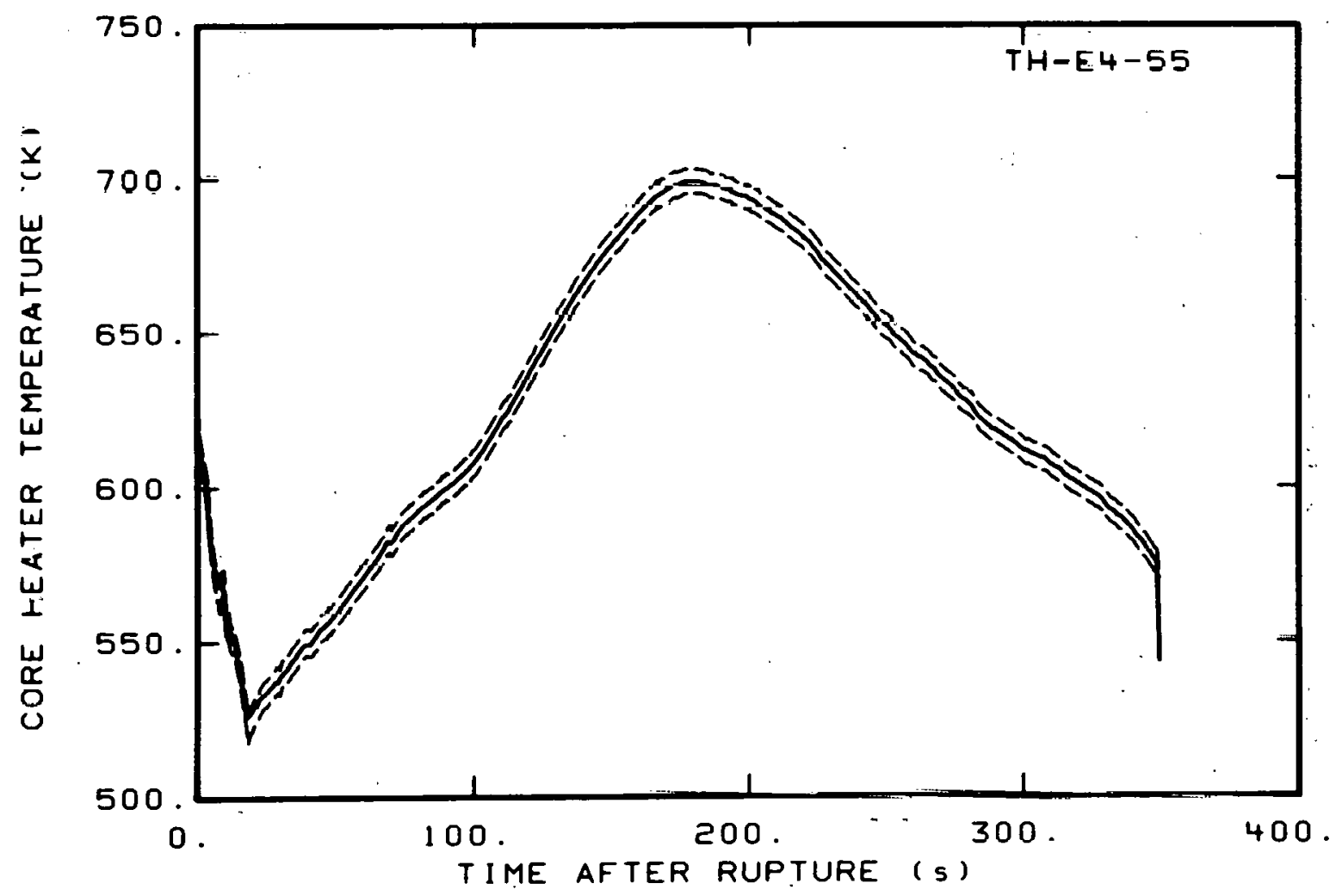

Fig. C-9 Core heater temperature, Rod E-4 (TH-E4-55). 


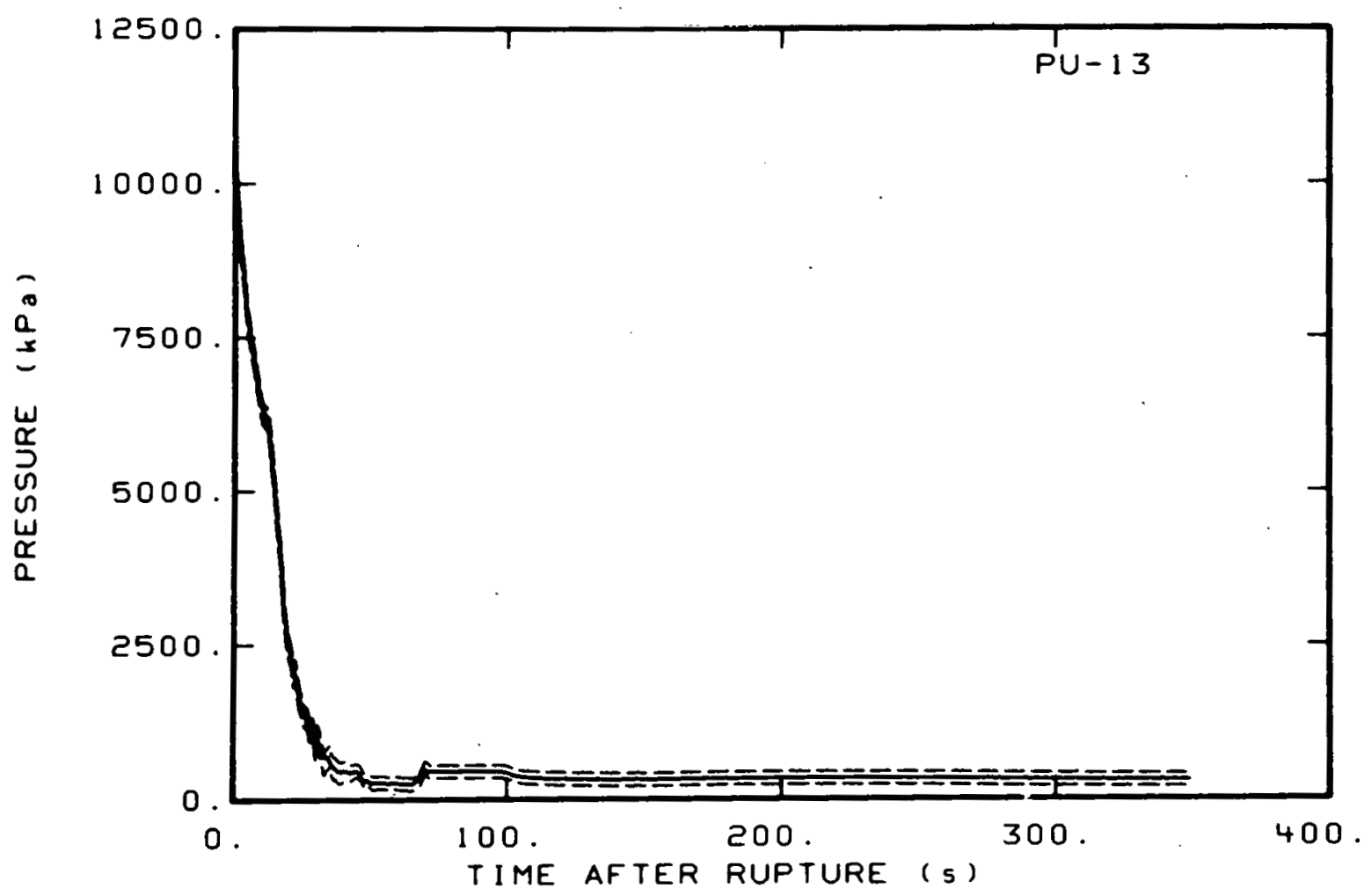

Fig. C-10 Pressure in intact 10op, Spool 13 (PU-13).

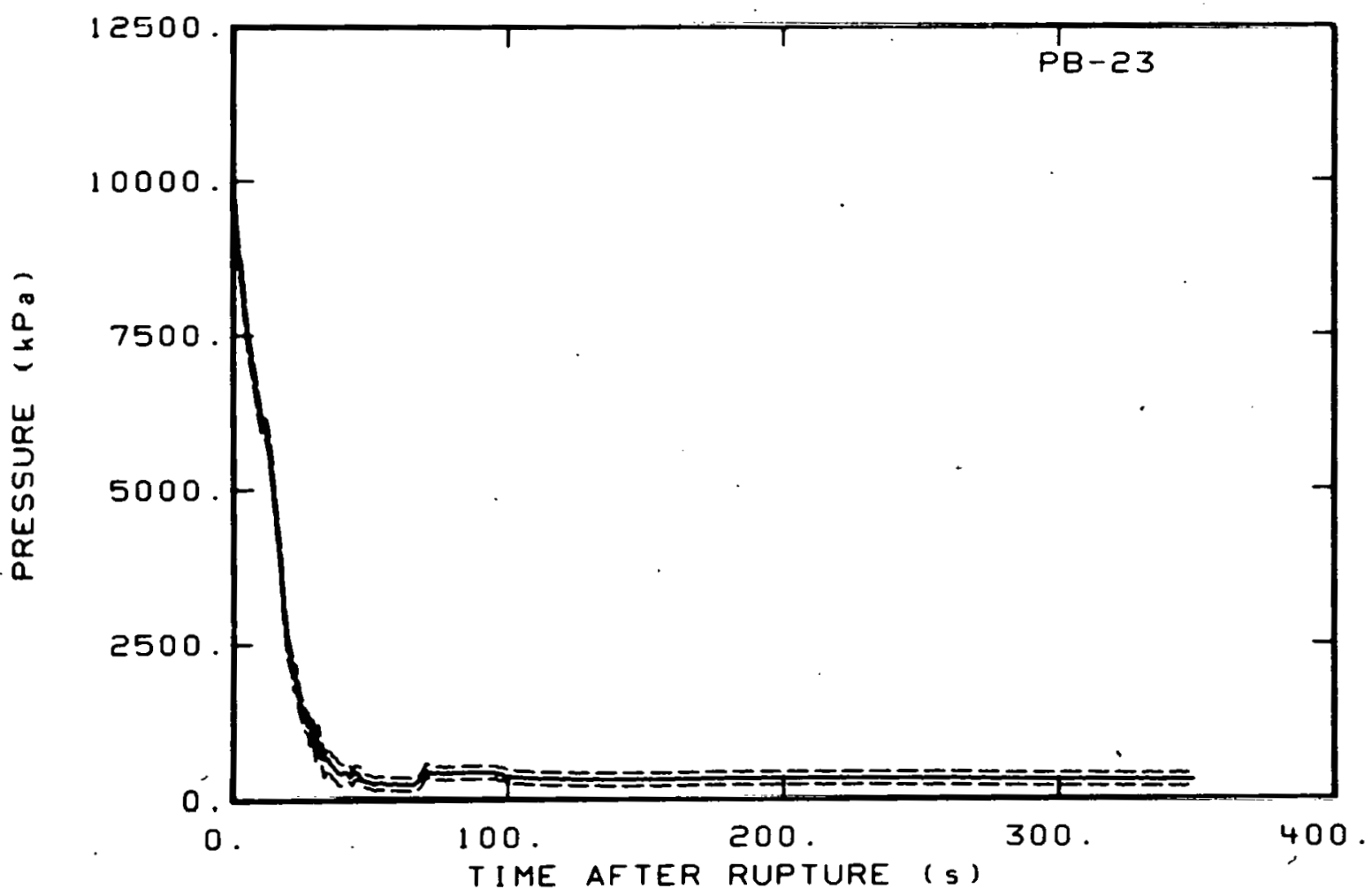

Fig. C-11 Pressure in broken loop, Spool 23 (PB-23). 


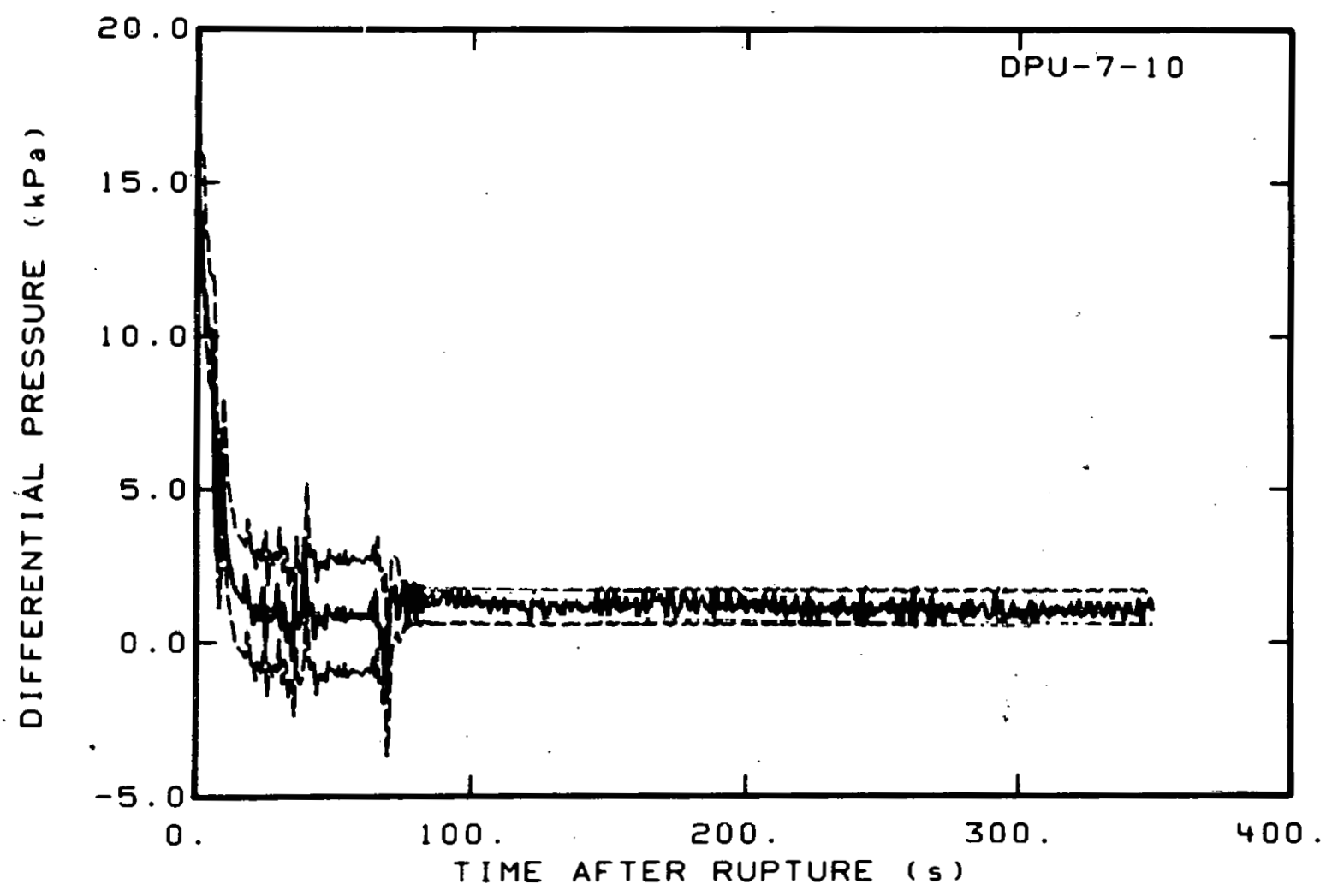

Fig. C-12 Differential pressure in intact loop (DPU-7-10).

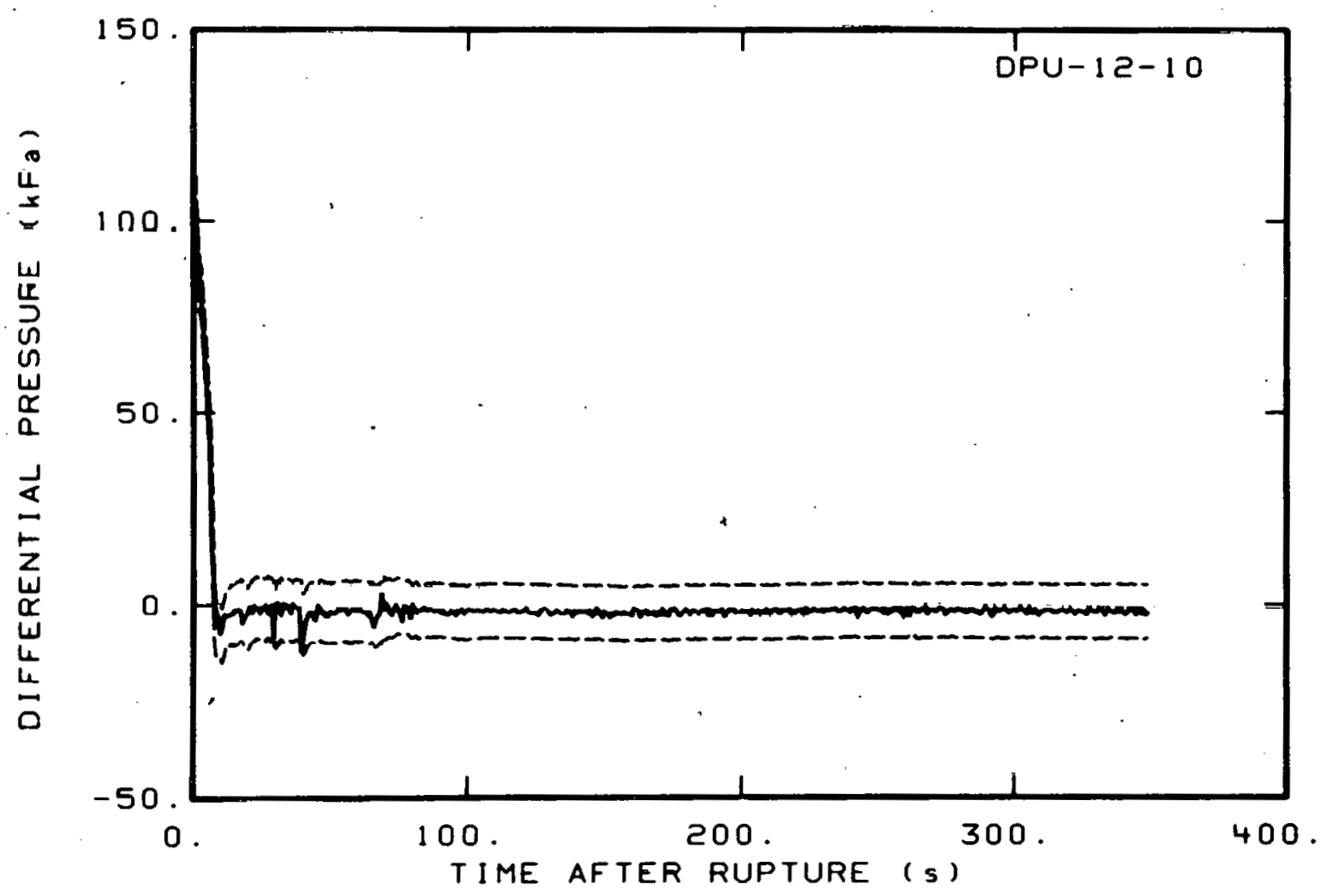

Fig. C-13 Differential pressure in intact loop (DPU-12-10). 


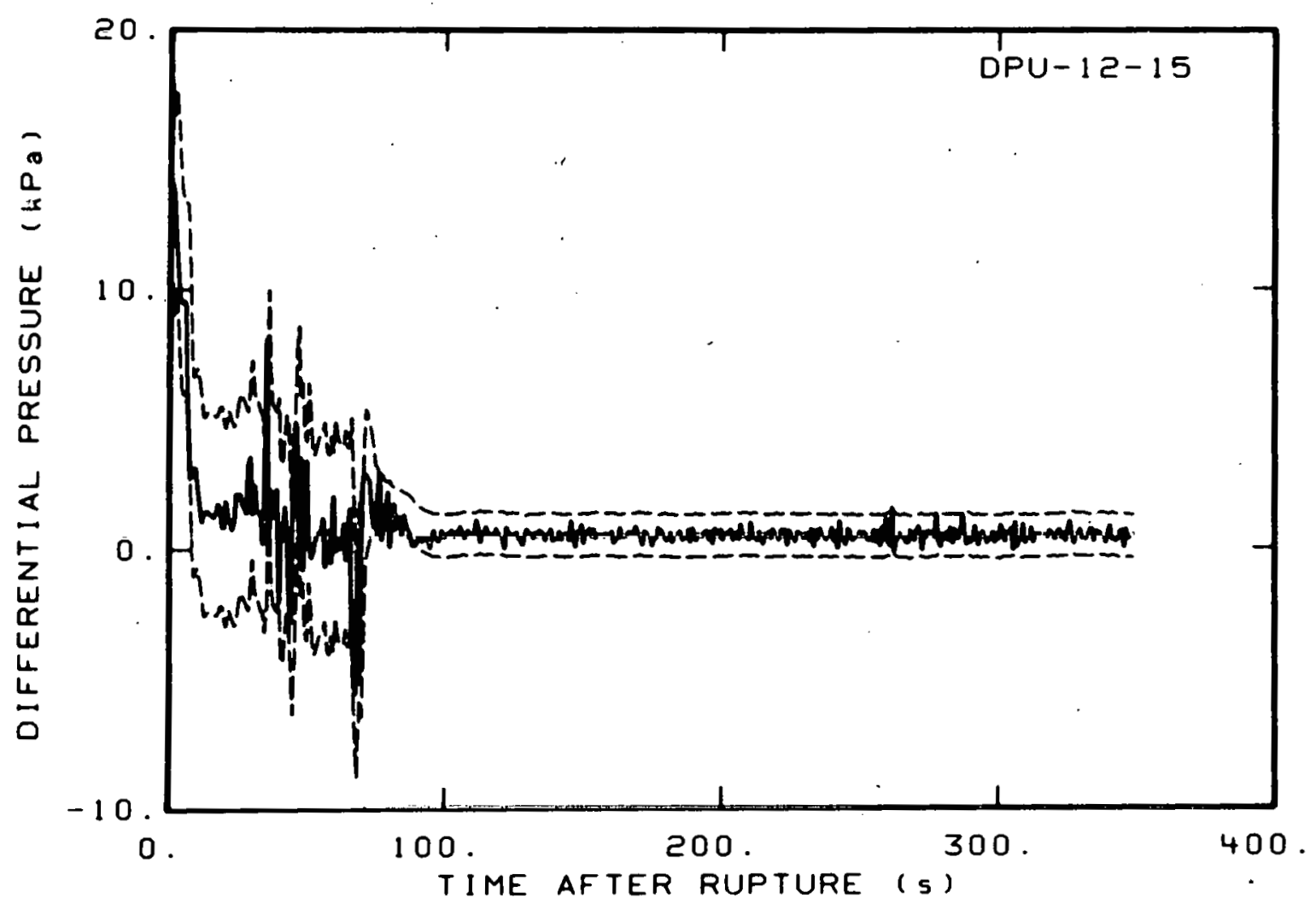

Fig. C-14 Differential pressure in intact loop (DPU-12-15).

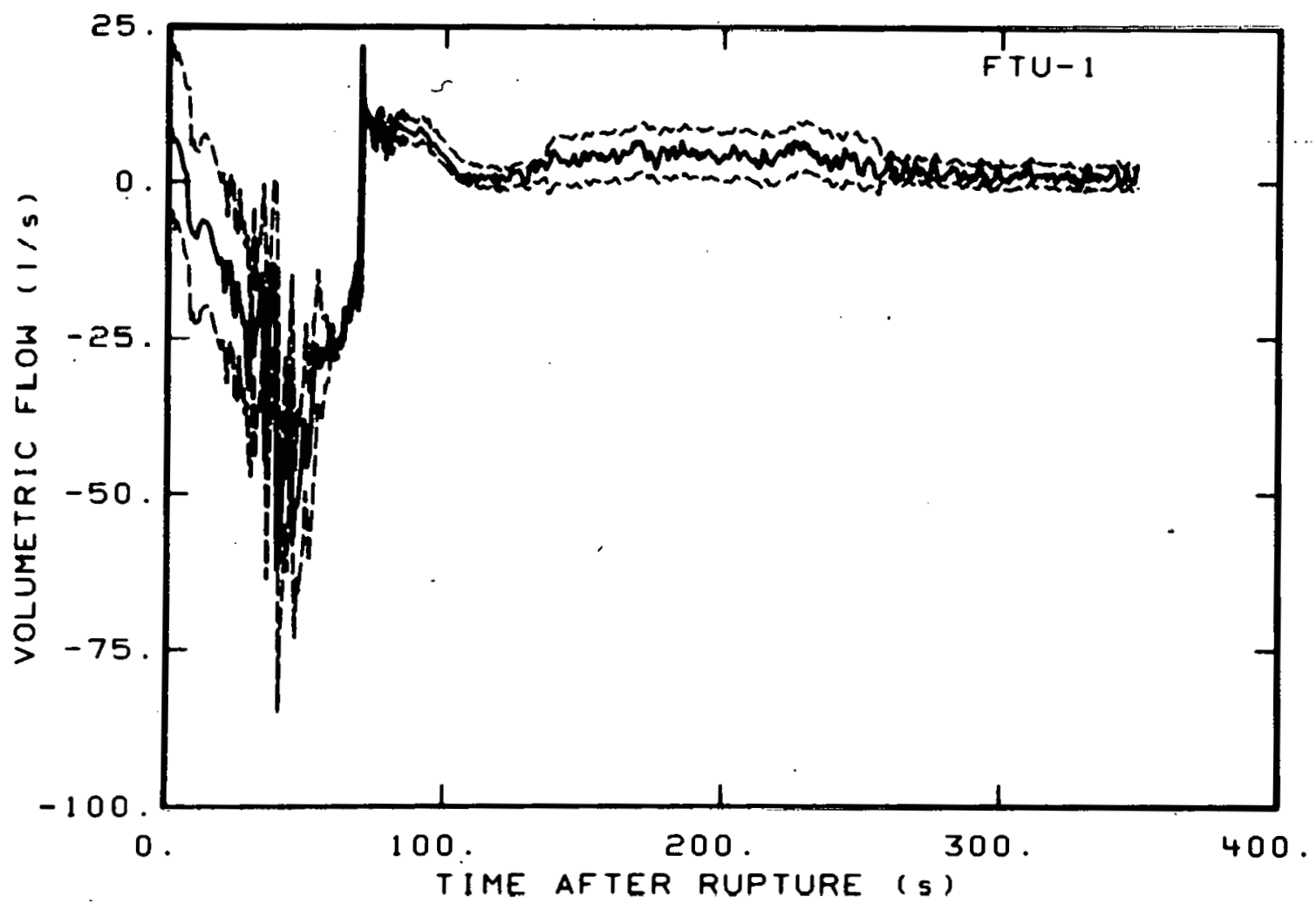

Fig. C-15 Volumetric flow in intact 10op (FTU-1). 


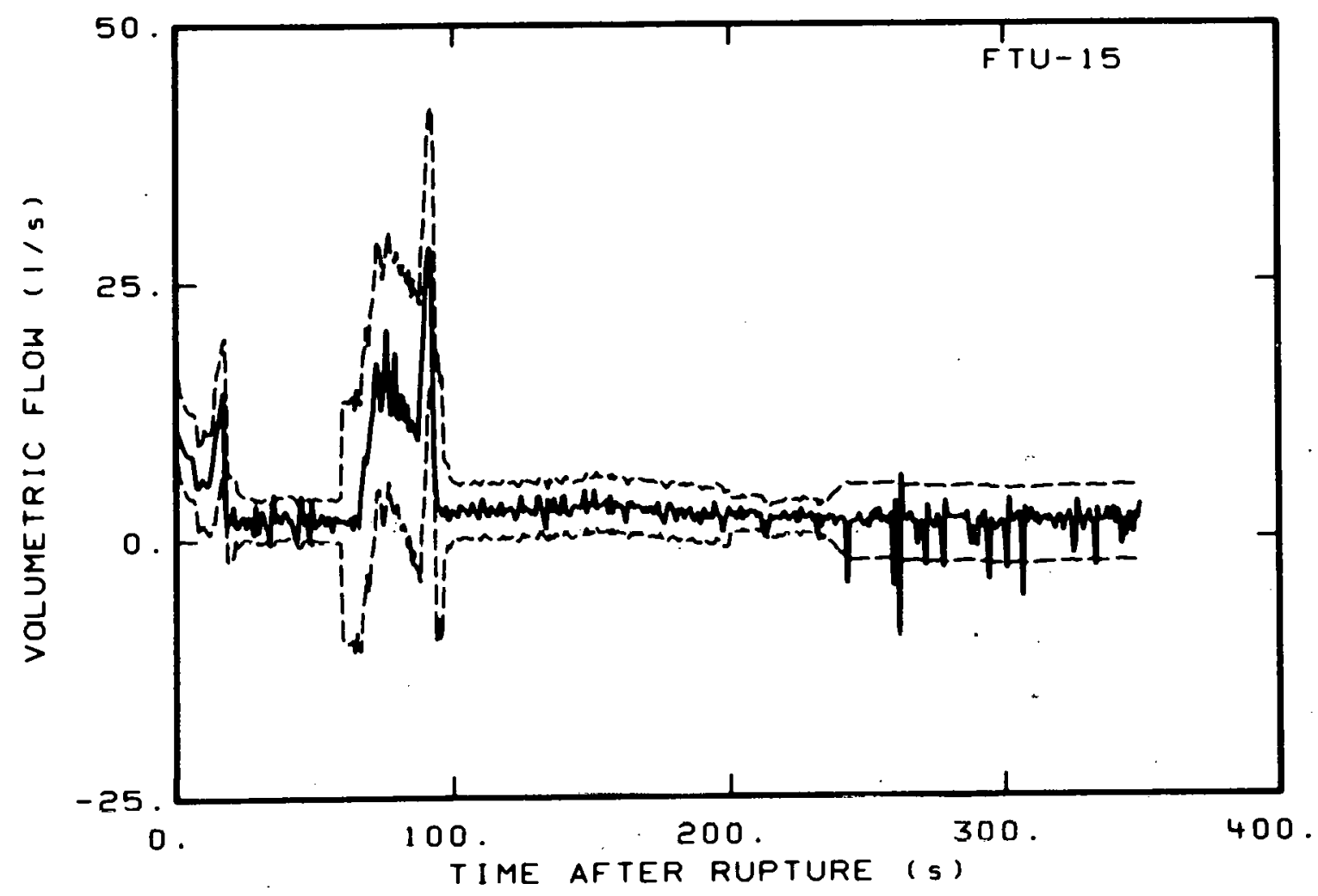

Fig. C-16 Volumetric flow in intact loop (FTU-15).

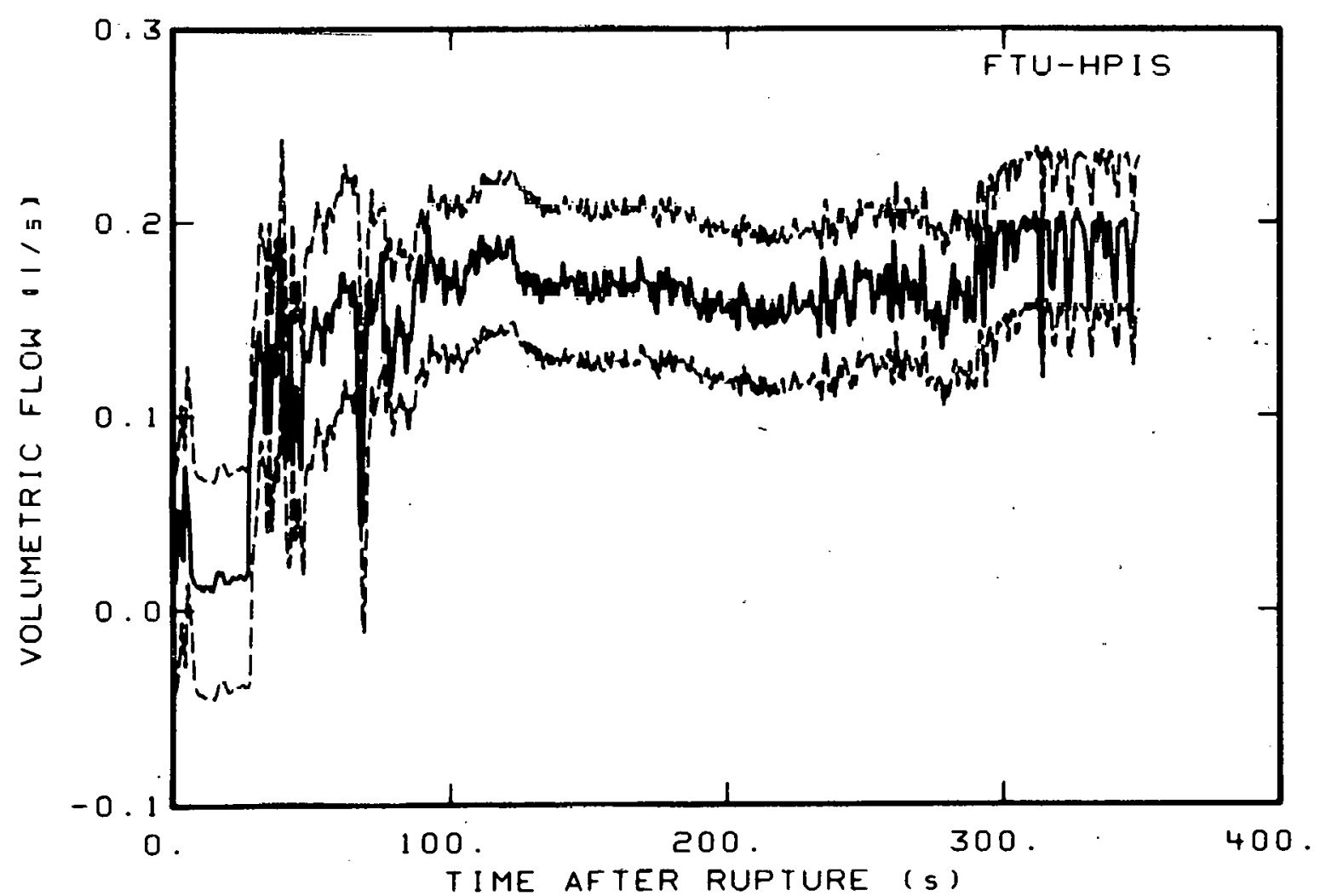

Fig. $\mathrm{C}-17$ Volumetric flow in intact loop high pressure injection line (FTU-HPIS). 


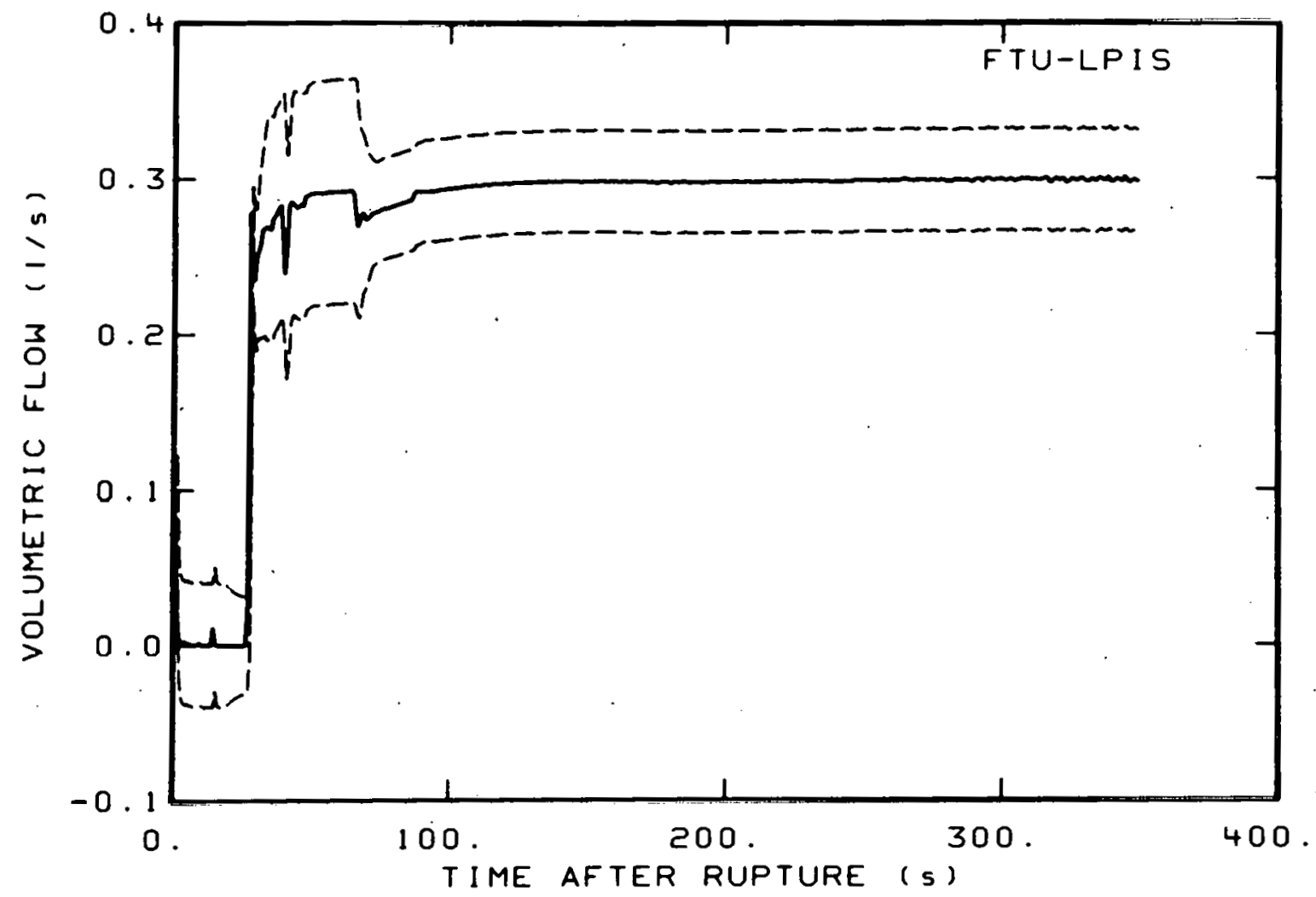

Fig. C-18 Volumetric flow in intact loop low pressure injection line (FTU-LPIS).

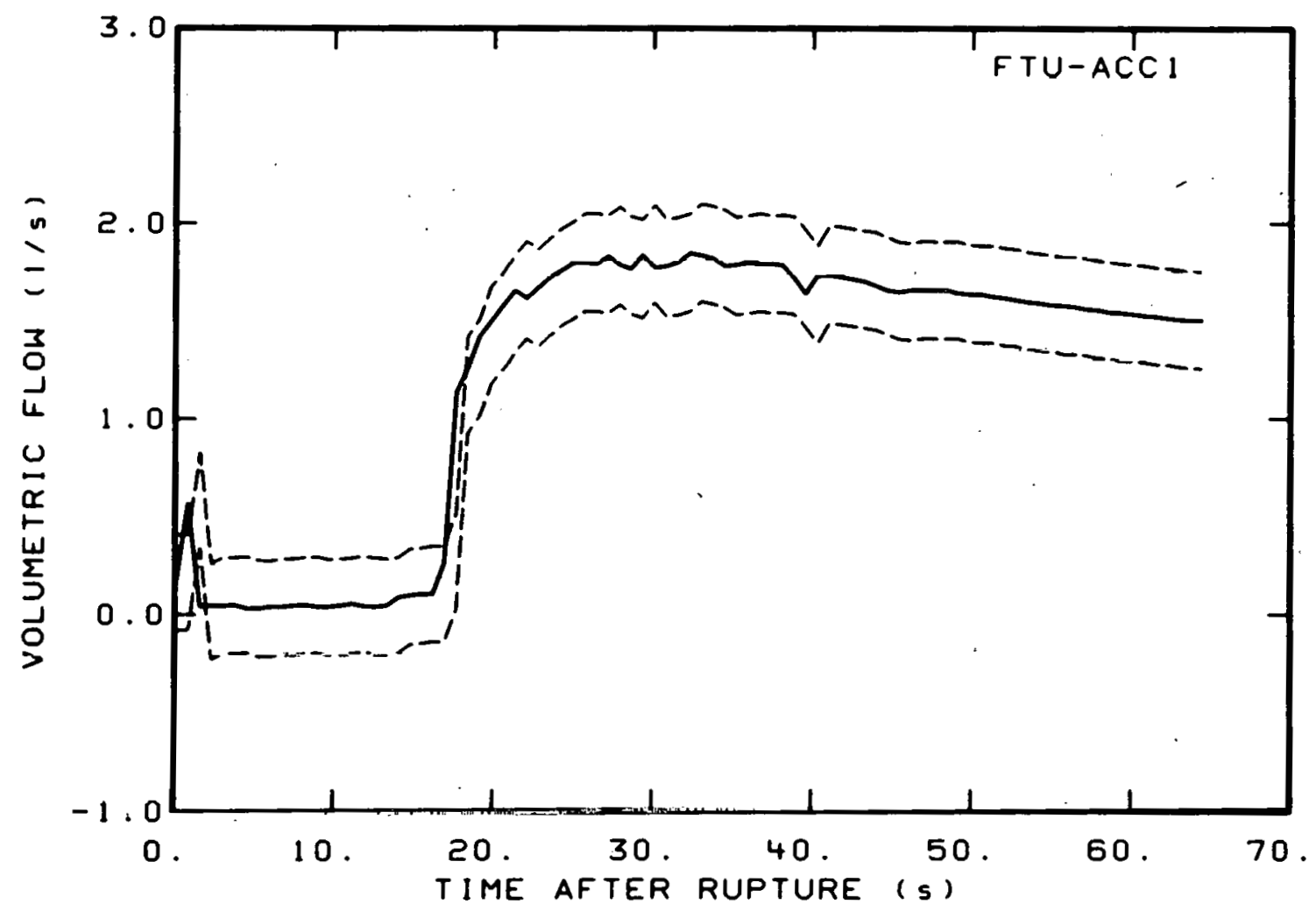

Fig. C-19 Volumetric flow in intact loop accumulator discharge. Tine (FTU-ACCT). 


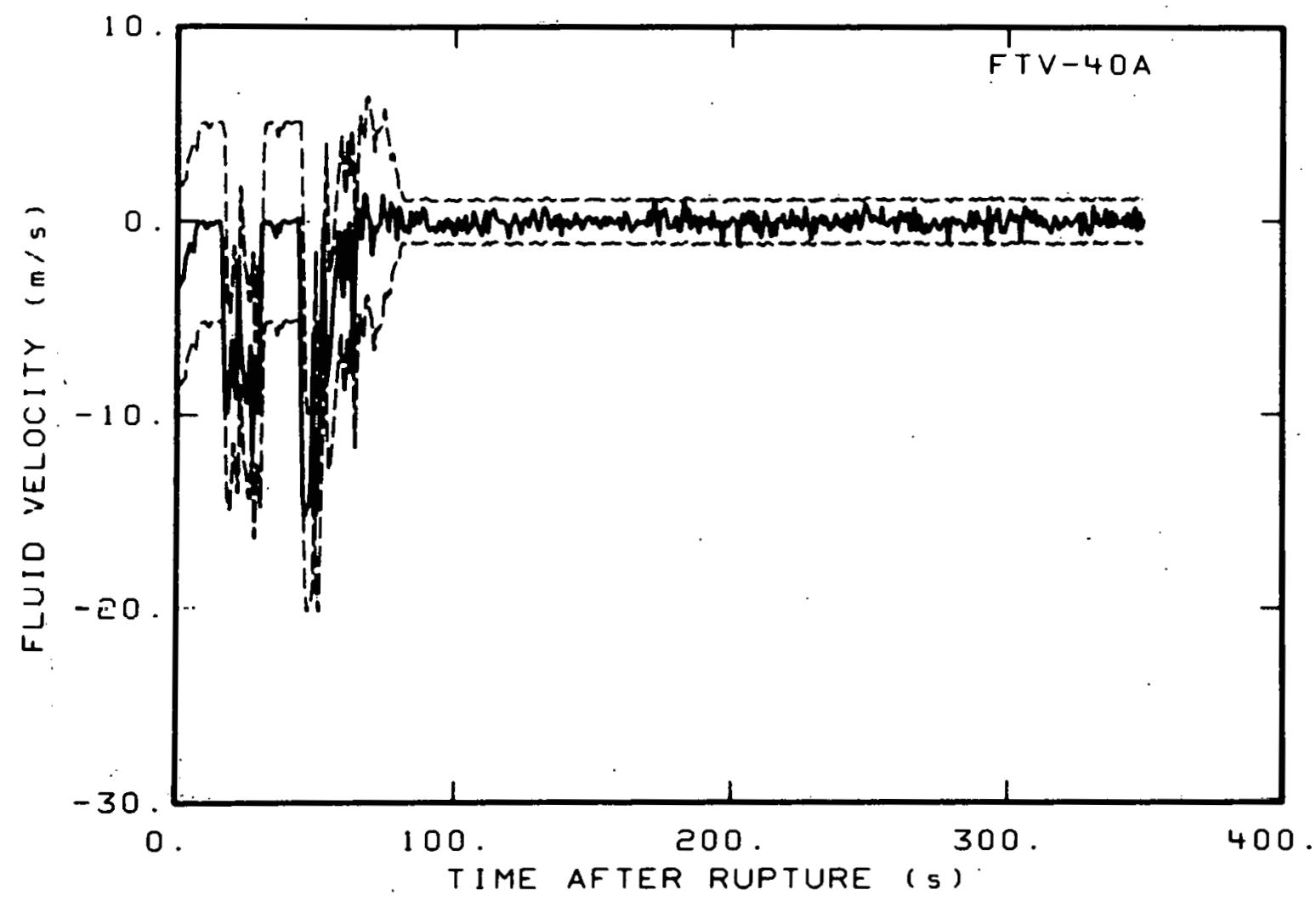

Fig. C-20 Fluid velocity in vessel (FTV-40A).

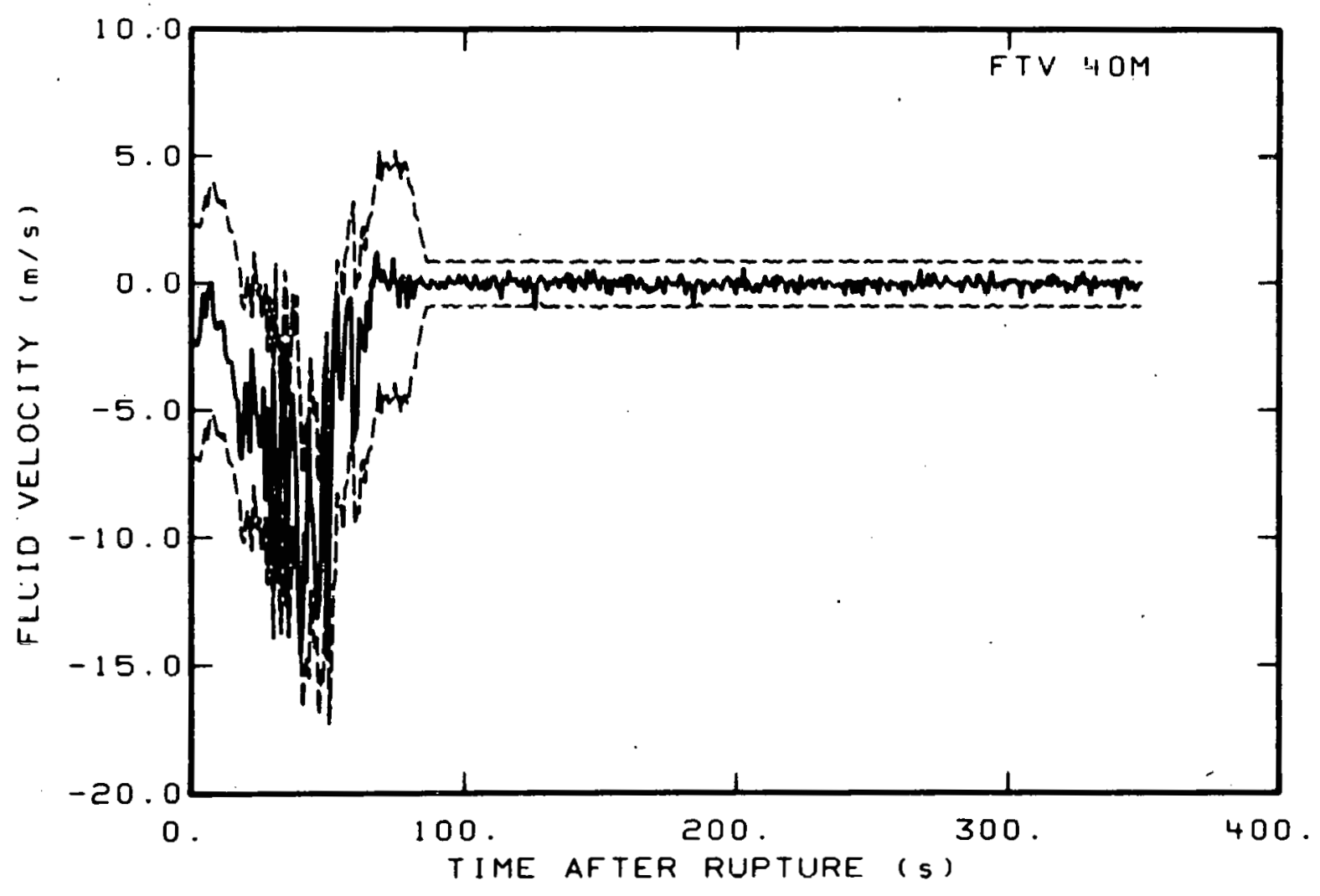

Fig. C-21 Fluid velocity in vessel (FTV-40M). 


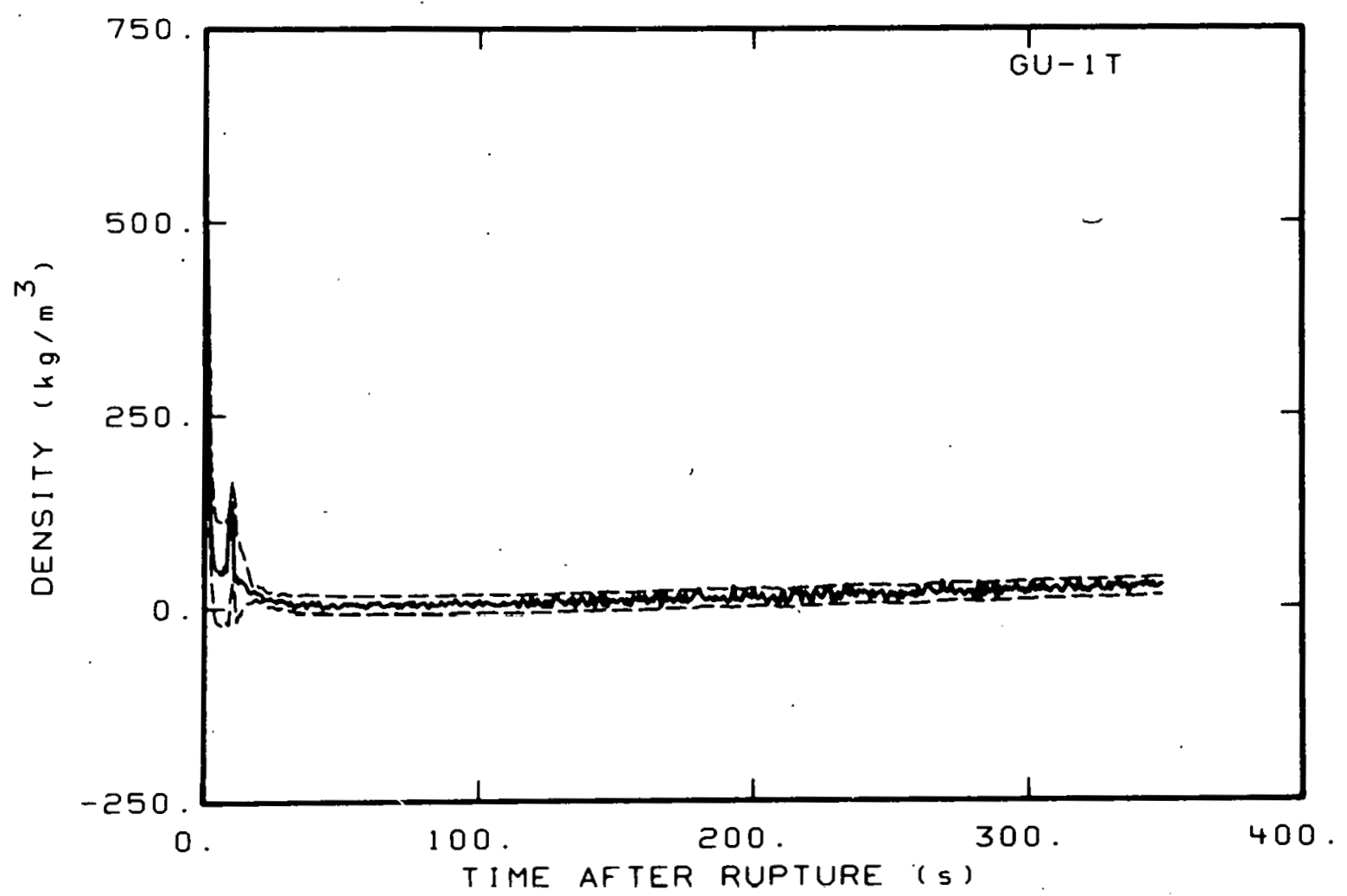

Fig. C-22 Density in intact loop (GU-IT).

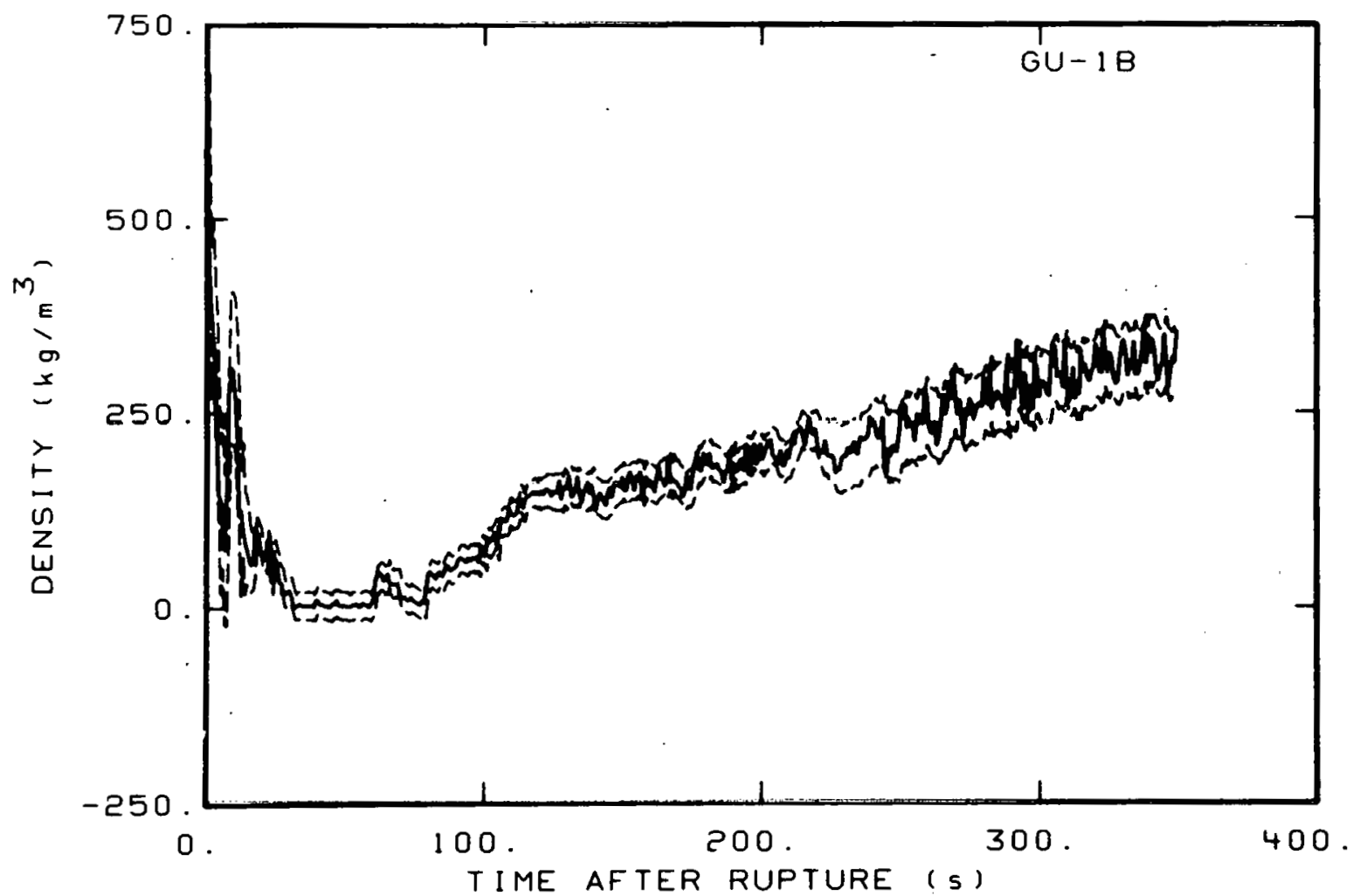

Fig. C-23 Density in intact loop (GU-1B). 


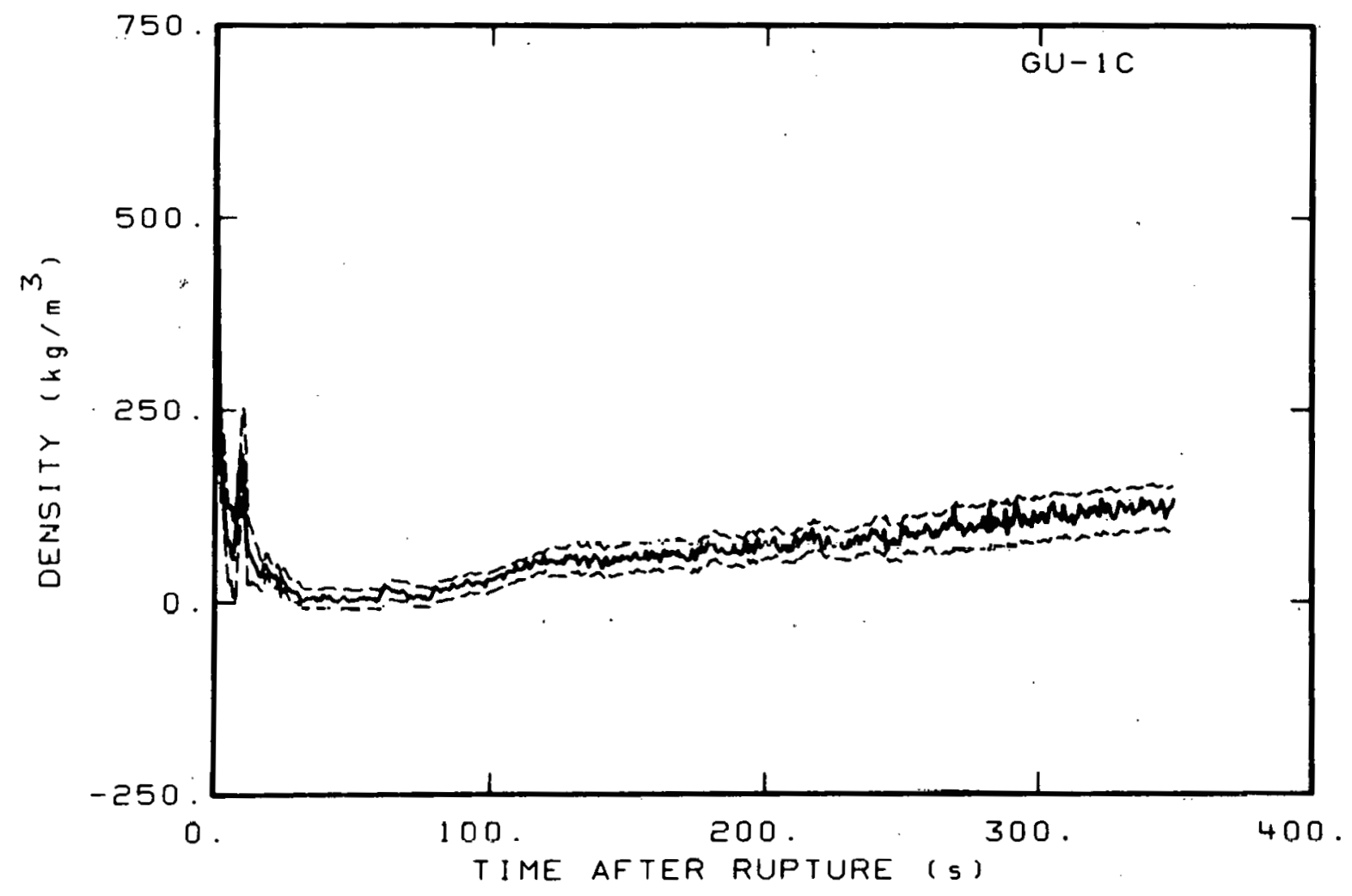

Fig. C-24 Density in intact loop (GU-1C).

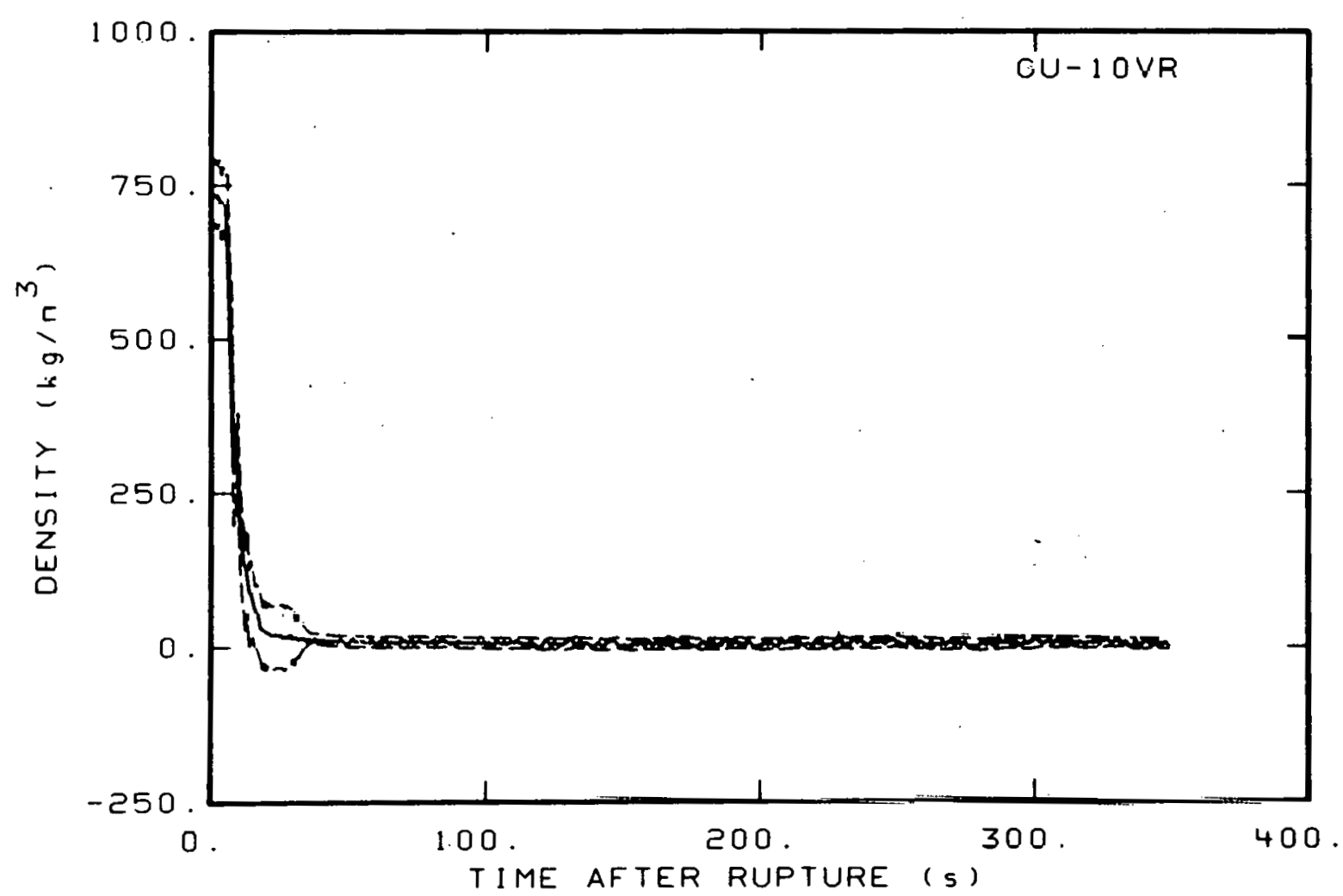

Fig. C-25 Density in intact loop (GU-10VR). 


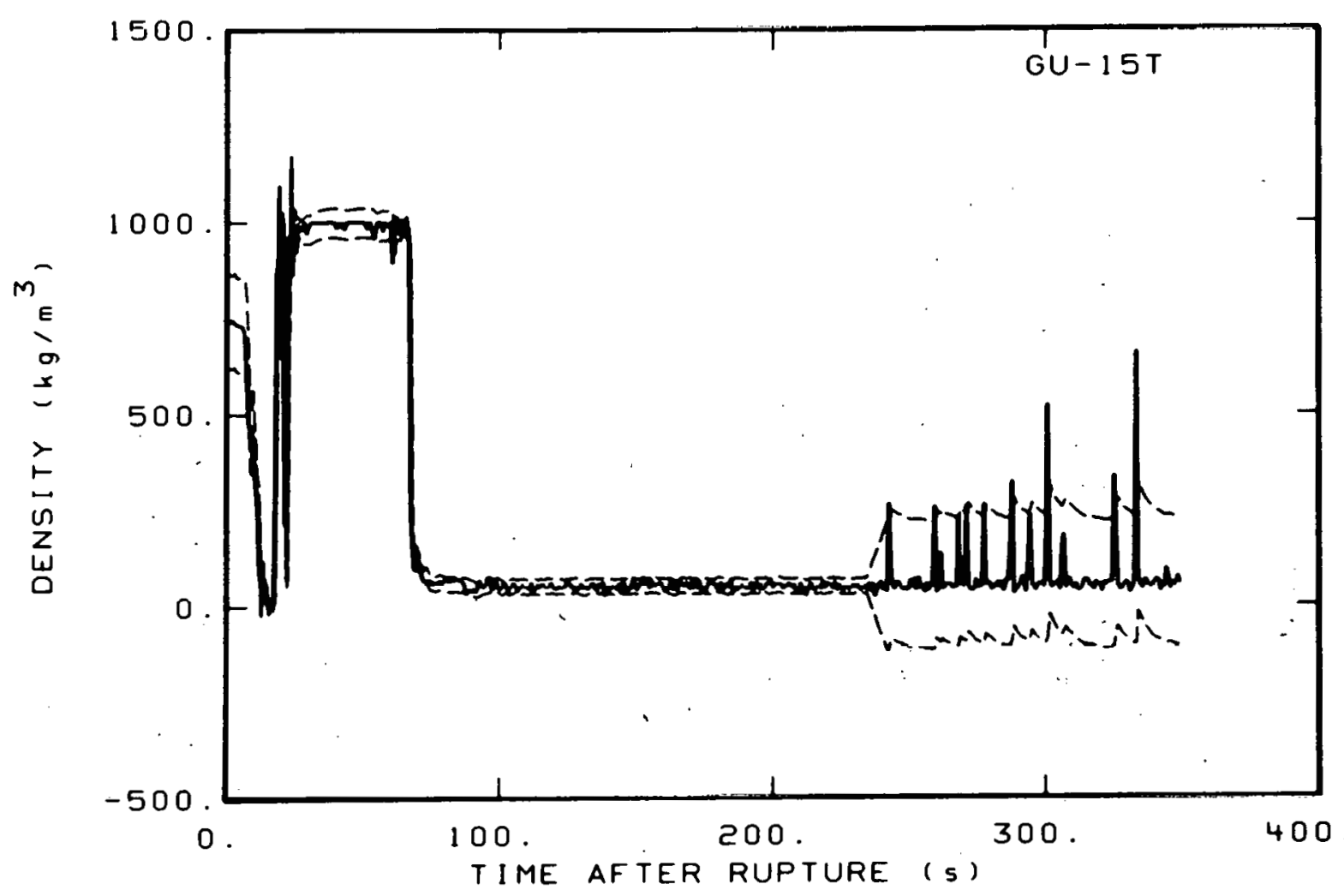

Fig. C-26 Density in intact loop (GU-15T).

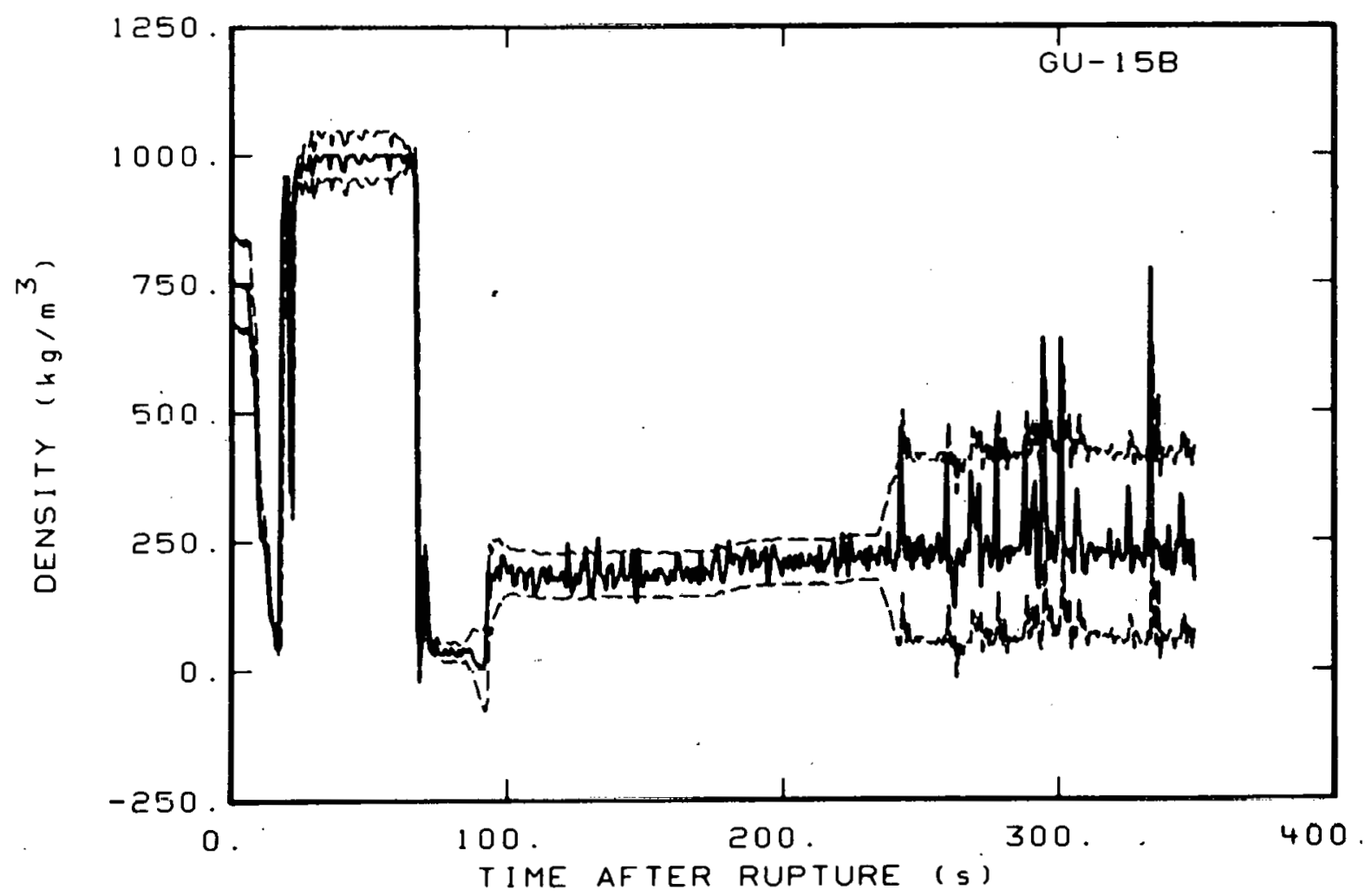

Fig. C-27 Density in intact loop (GU-15B). 


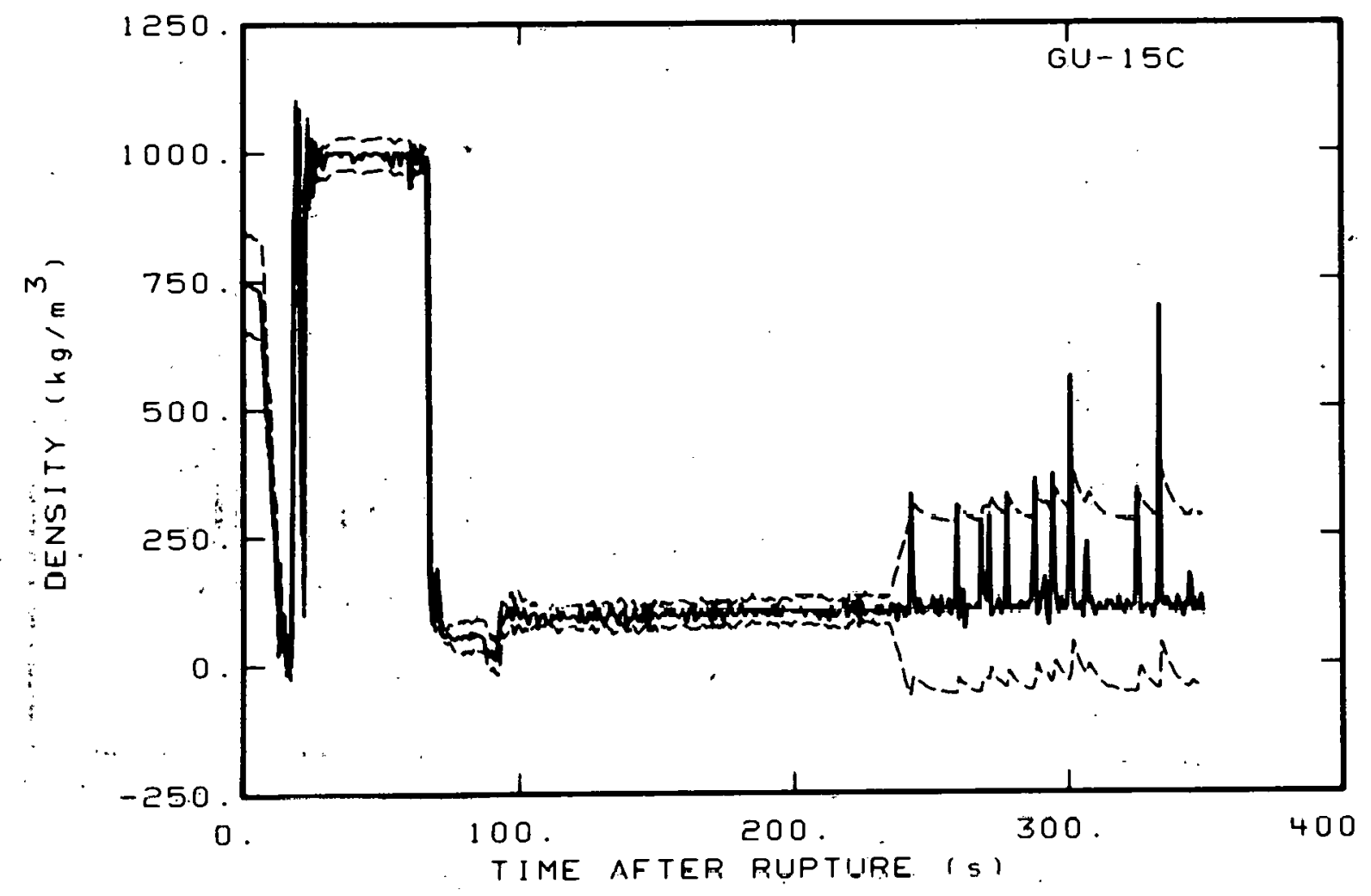

Fig. C-28 Density in intact 100p (GU-15C).

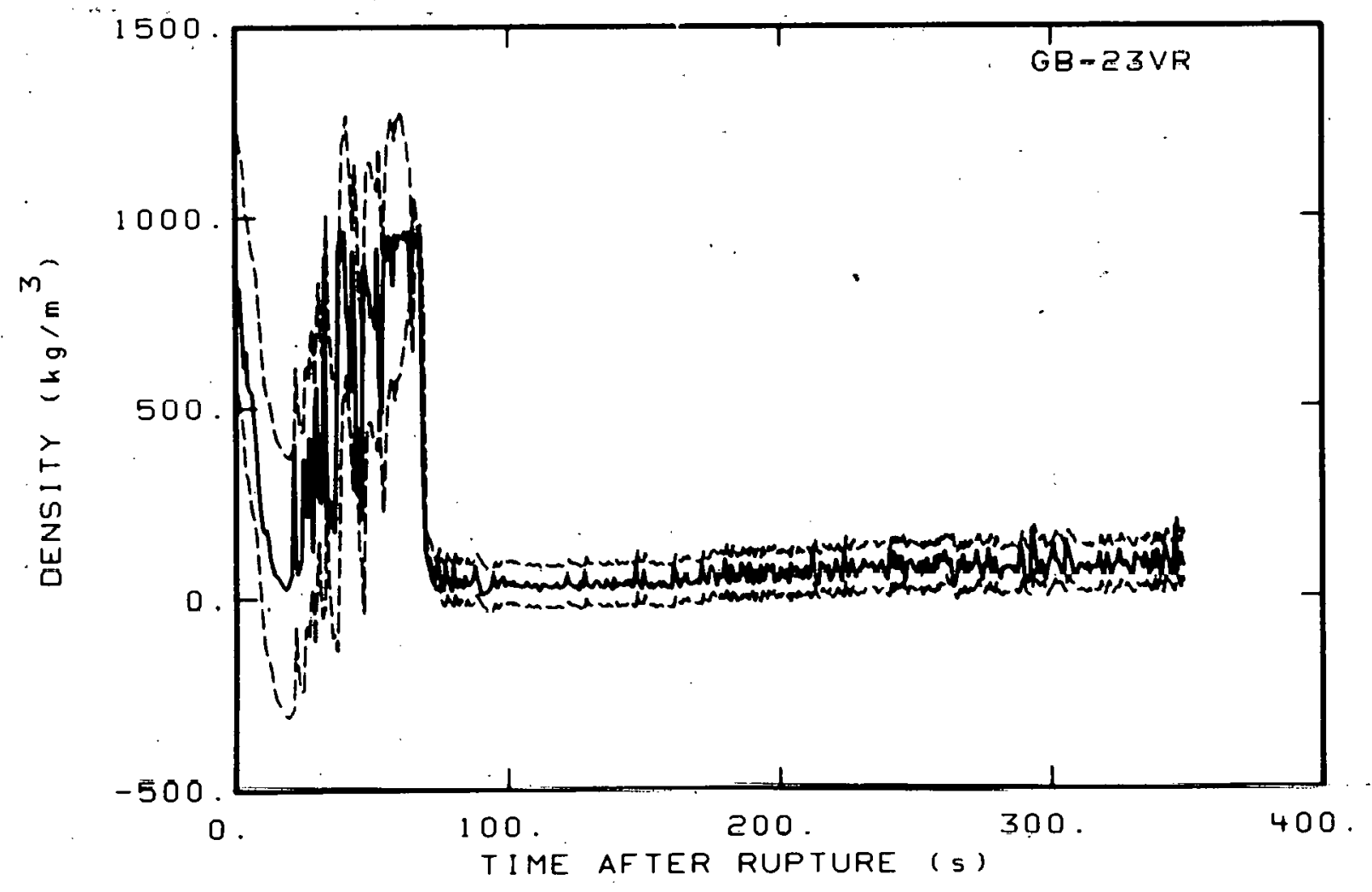

Fig. C-29 Density in broken loop (GB-23VR). 


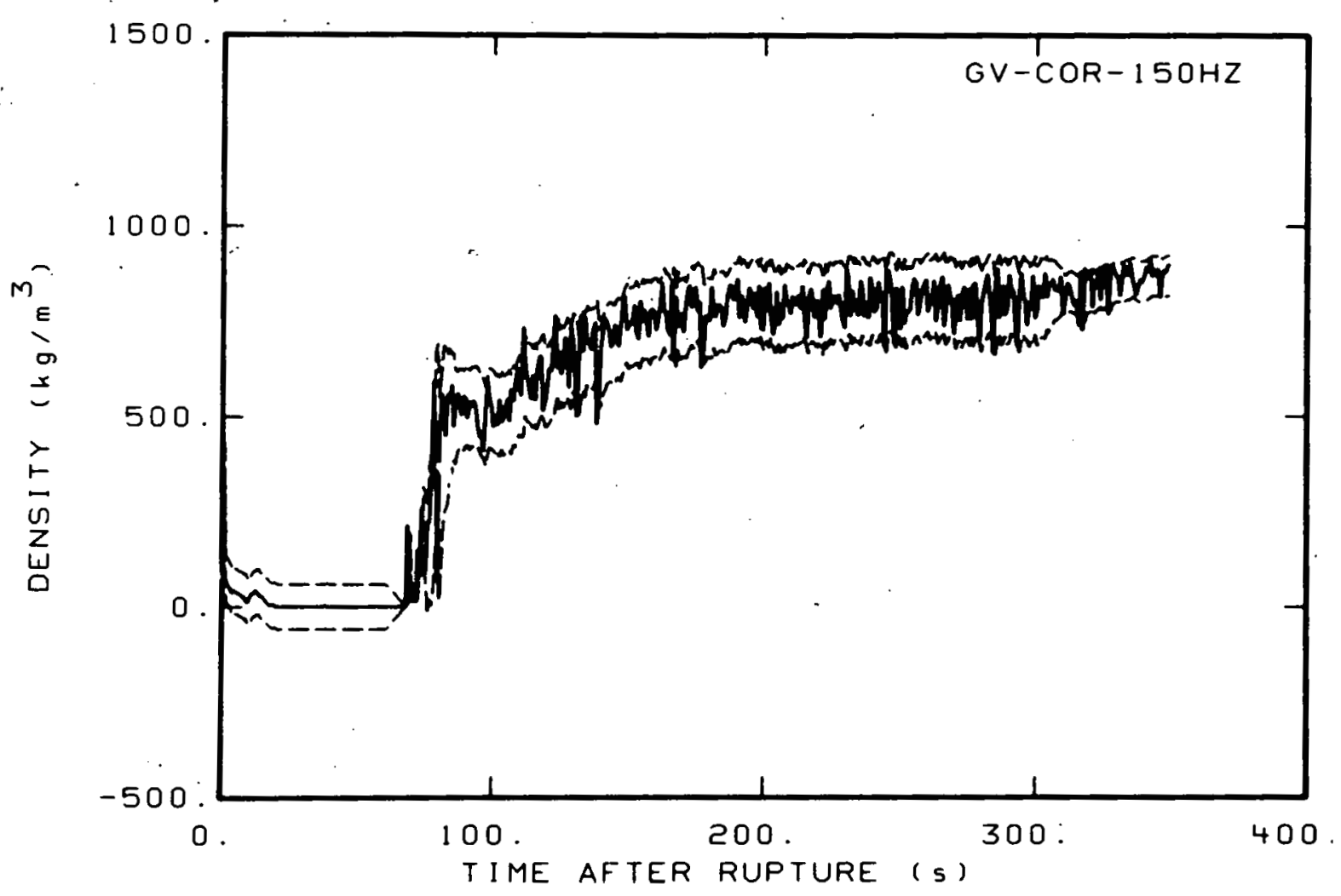

Fig. C-30 Density in vessel (GV-COR-150HZ).

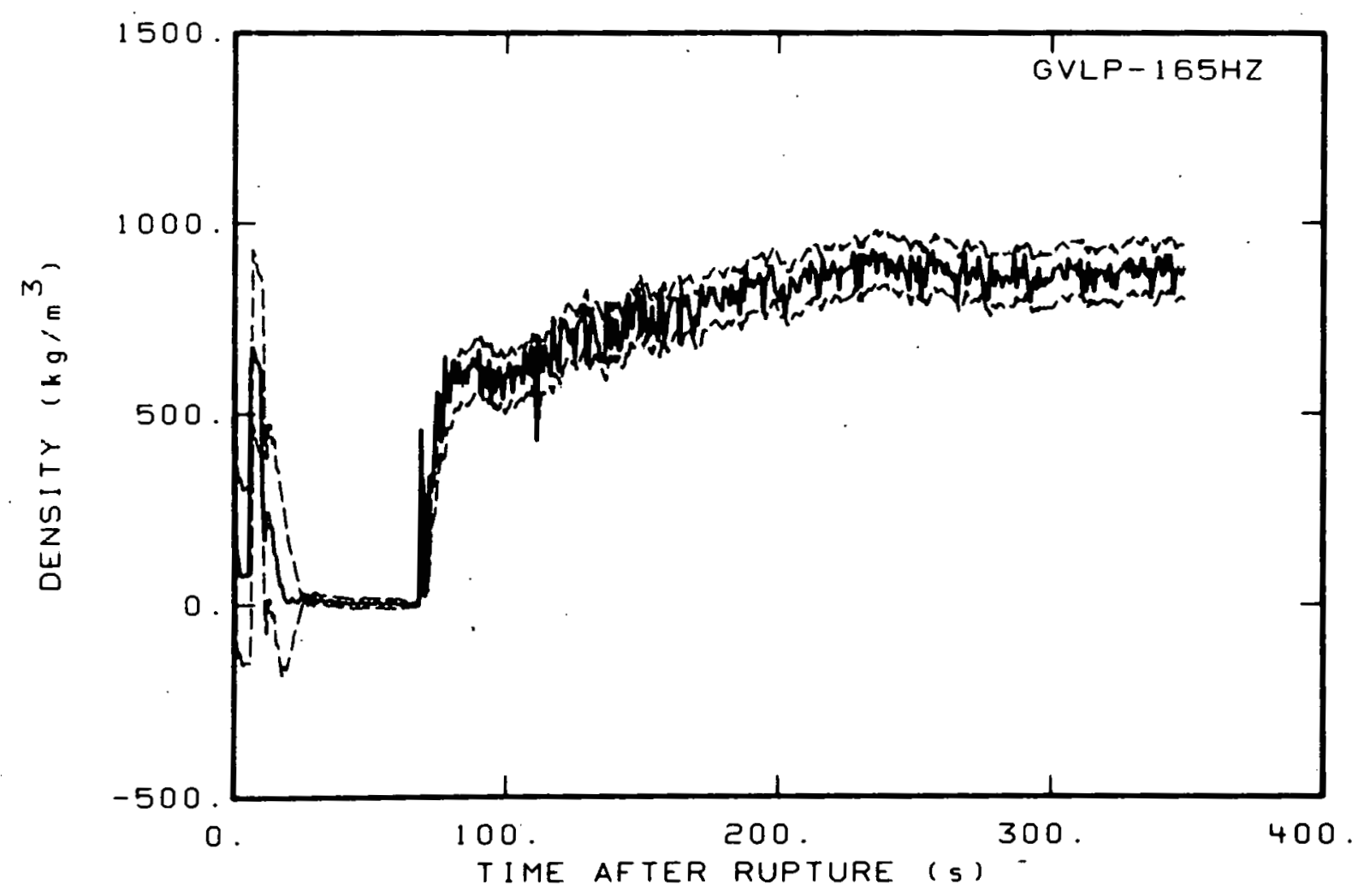

Fig. C-31 Density in vessel (GVLP-16,5HZ). 


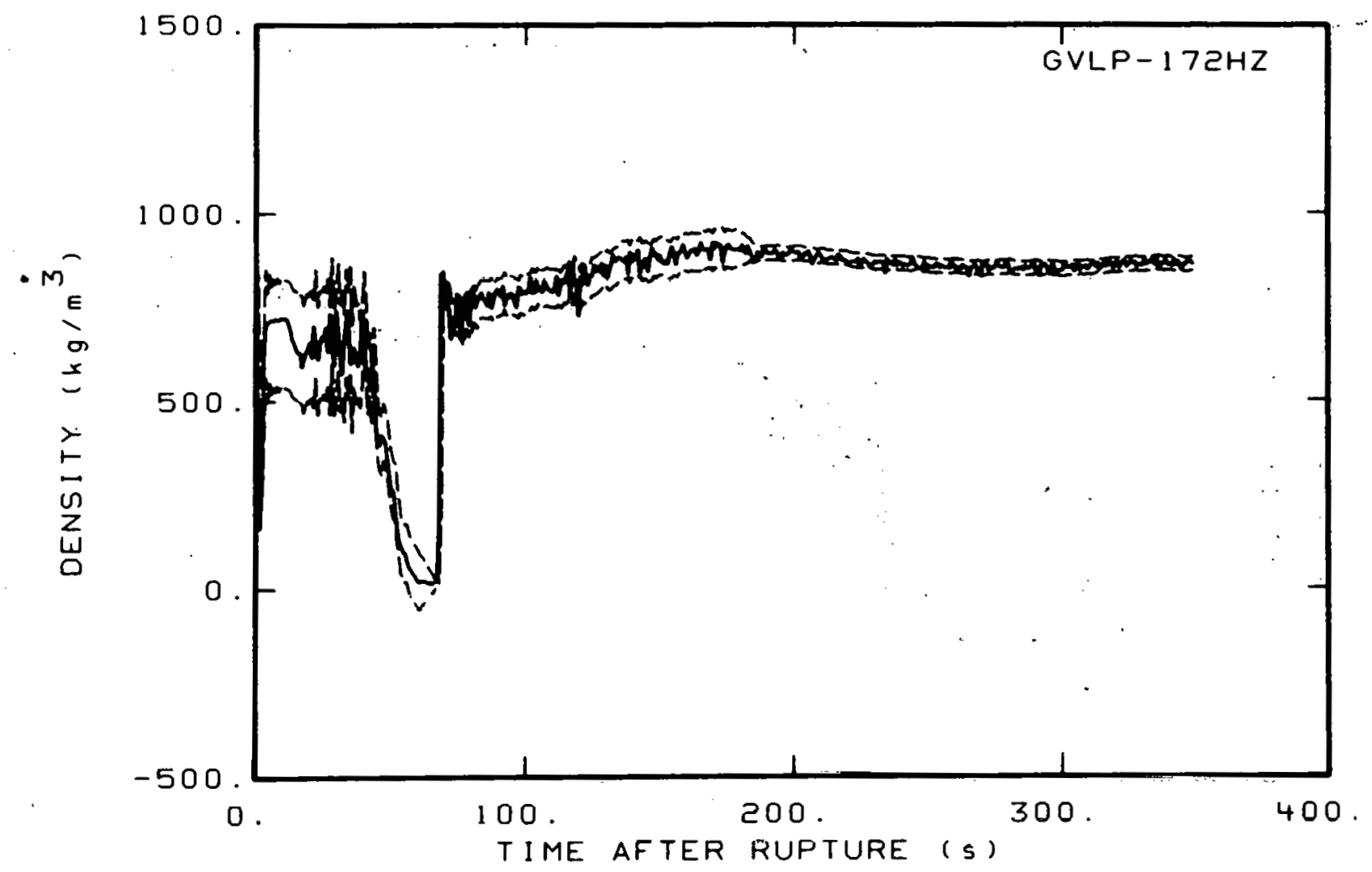

Fig. C-32 Density in vessel (GVLP-172HZ).

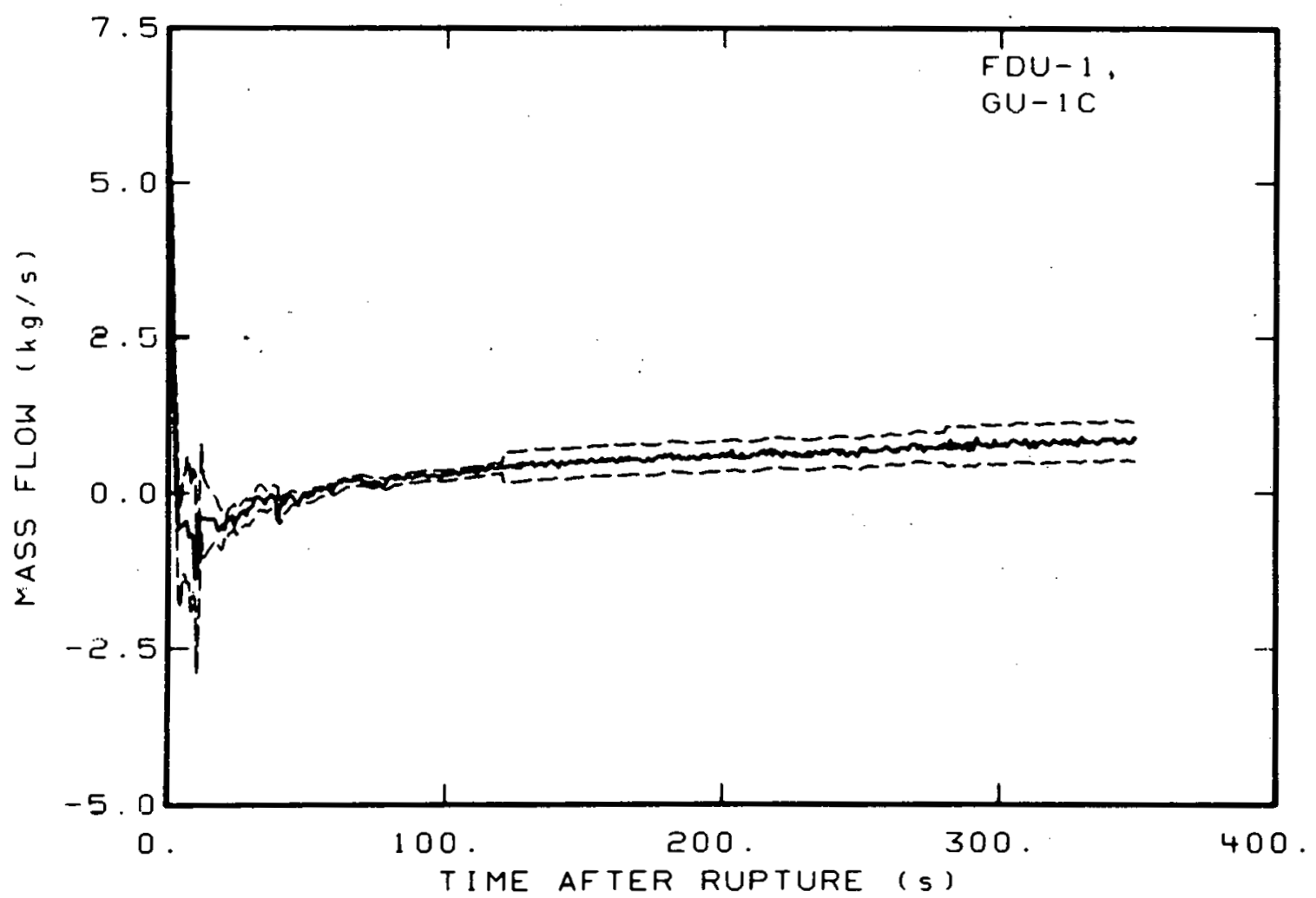

Fig. C-33 Mass flow in intact loop (FDU-1, GU-1C). 


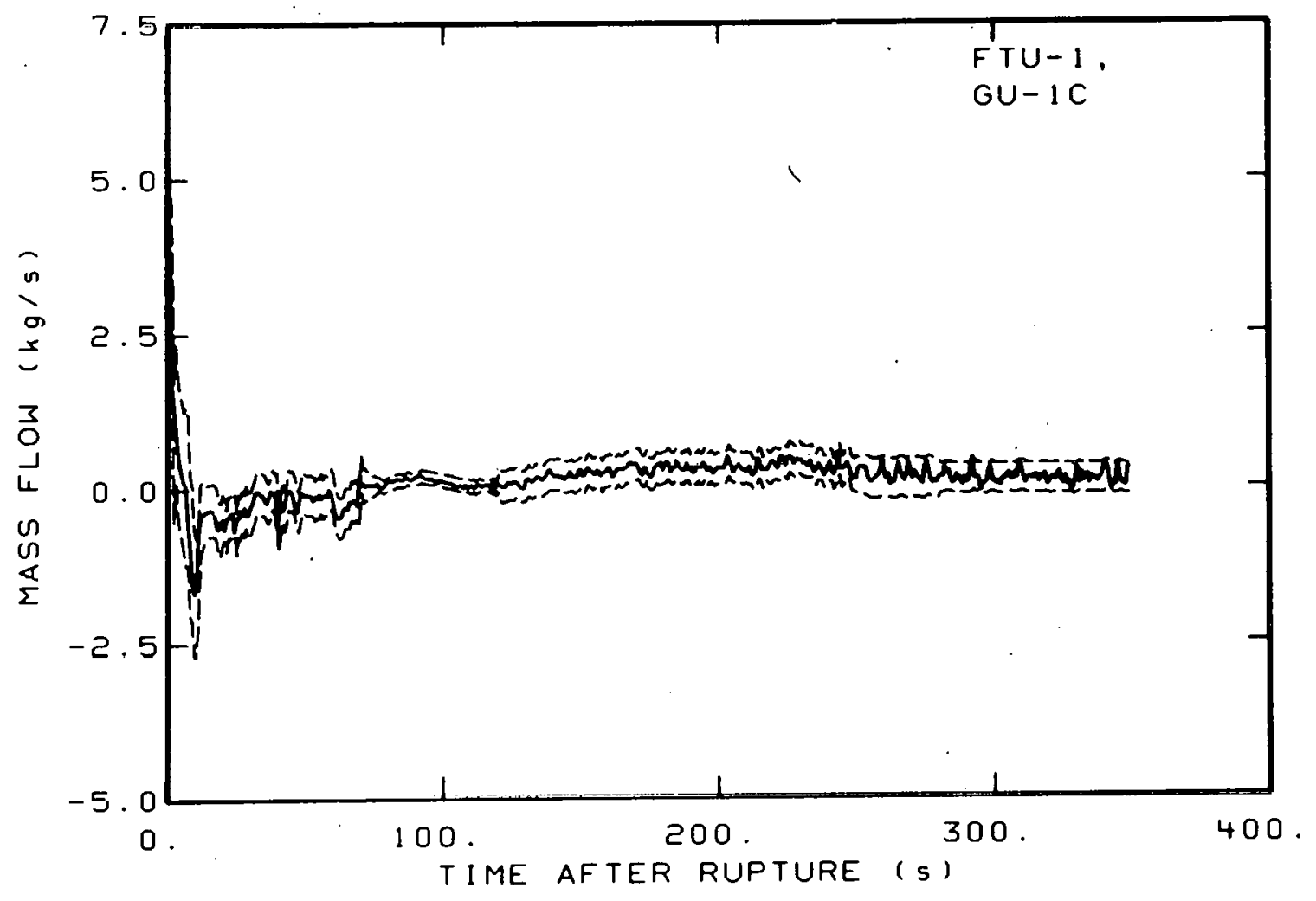

Fig. C-34 Mass flow in intact loop (FTU-1, GU-1C).

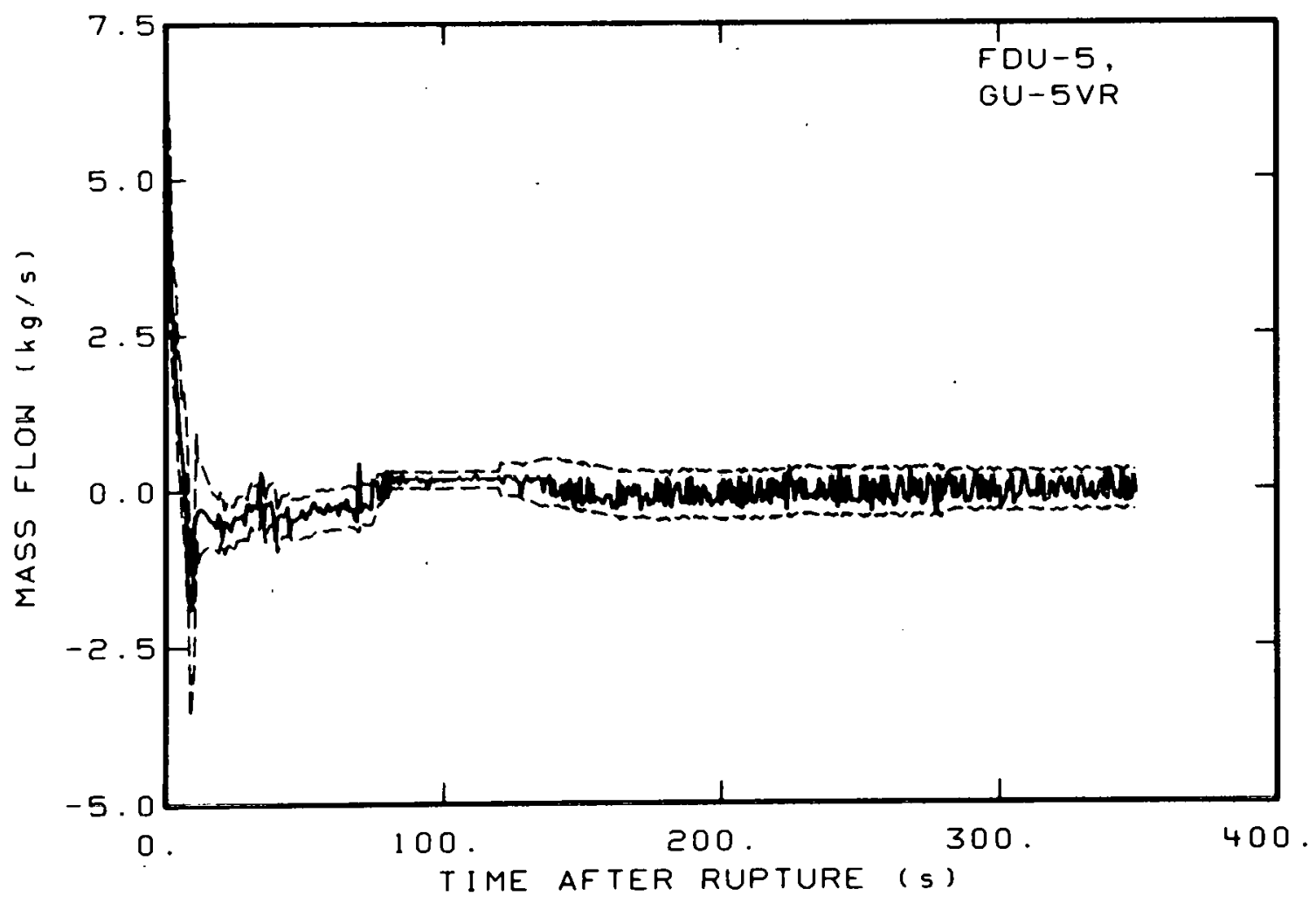

Fig. C-35 Mass flow in intact loop (FDU-5, GU-5VR). 


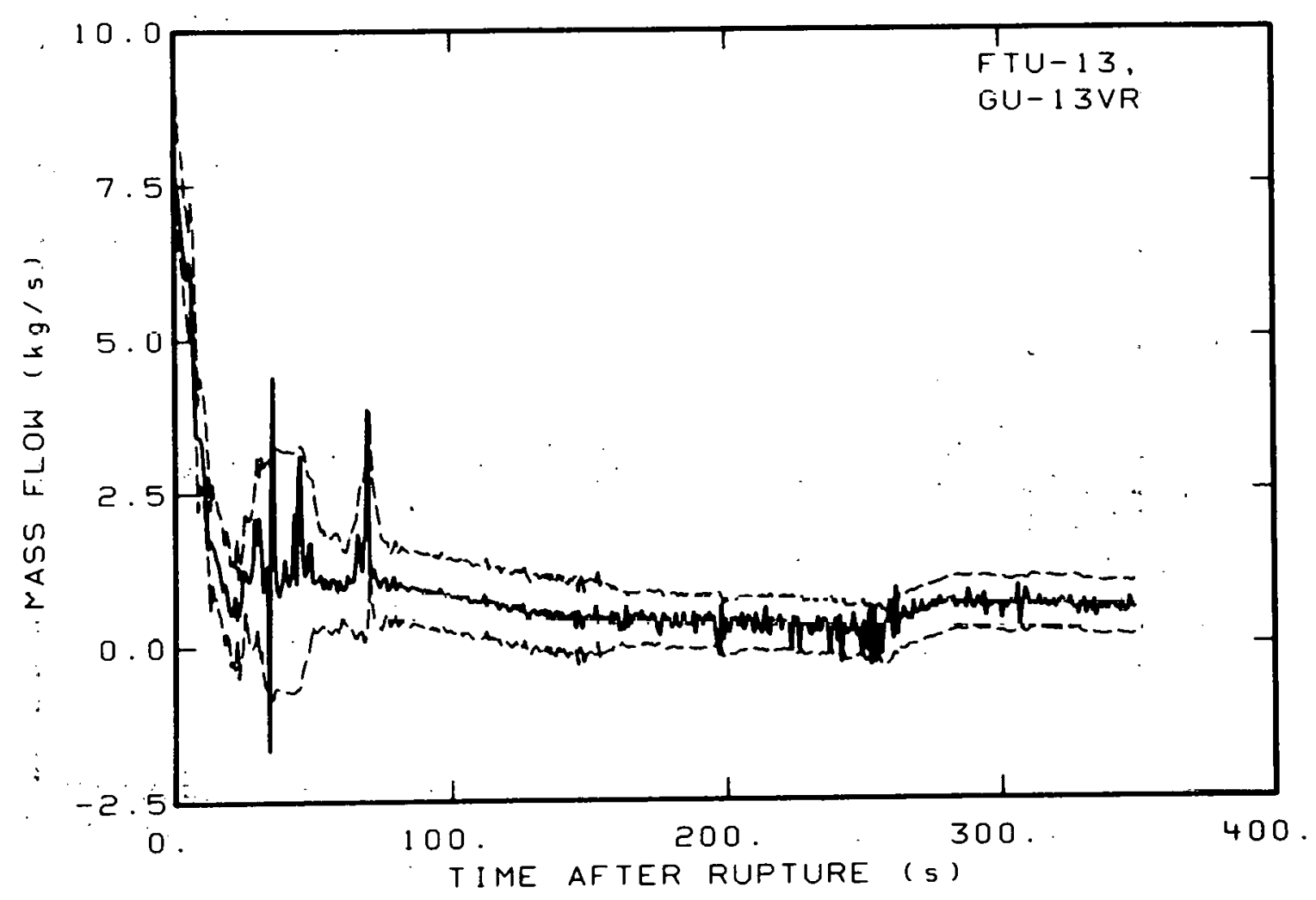

Fig. C-36 Mass flow in intact loop (FTU-13, GU-13VR).

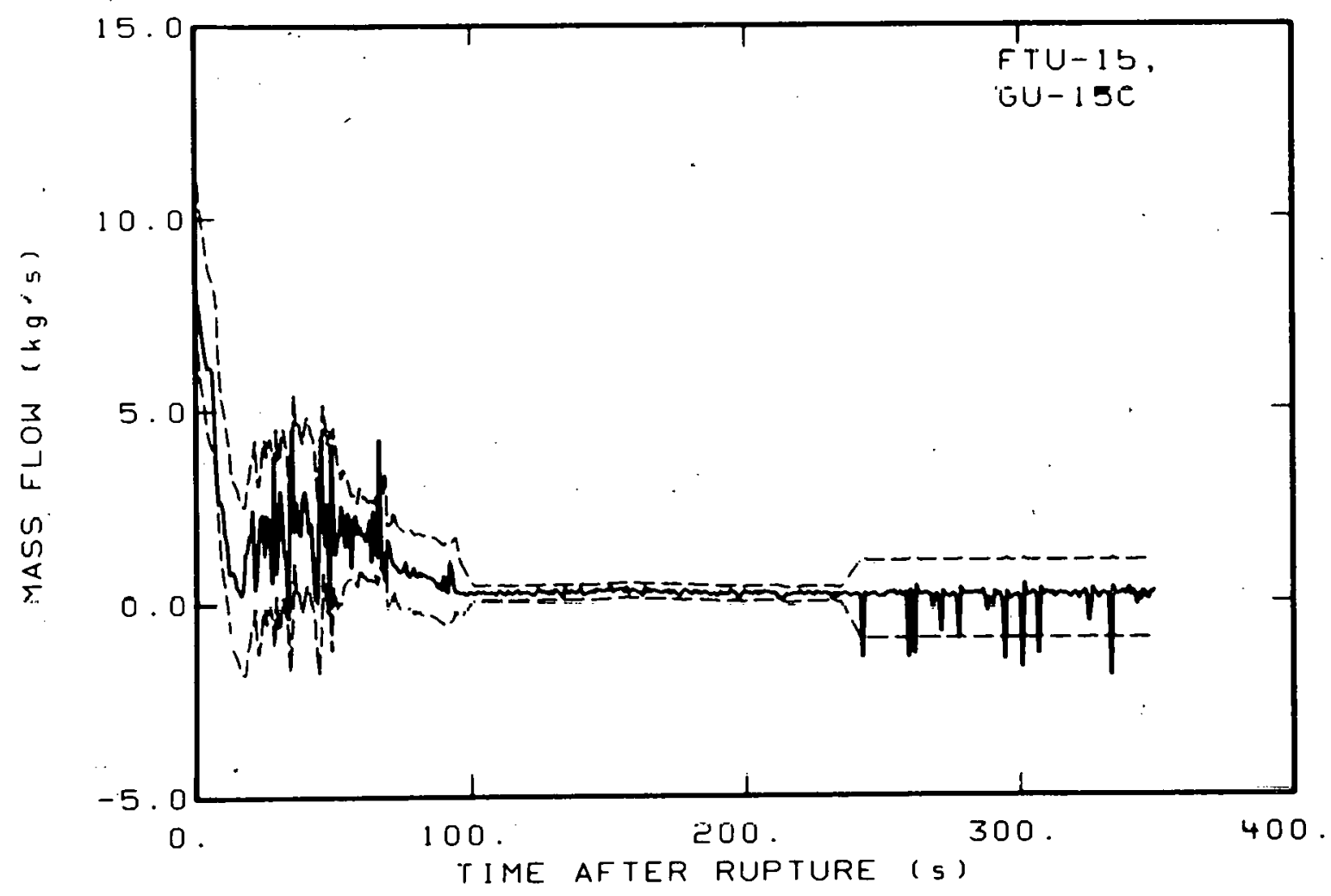

Fig. C-37 Mass flow in intact loop (FTU-15, GU-15C). 


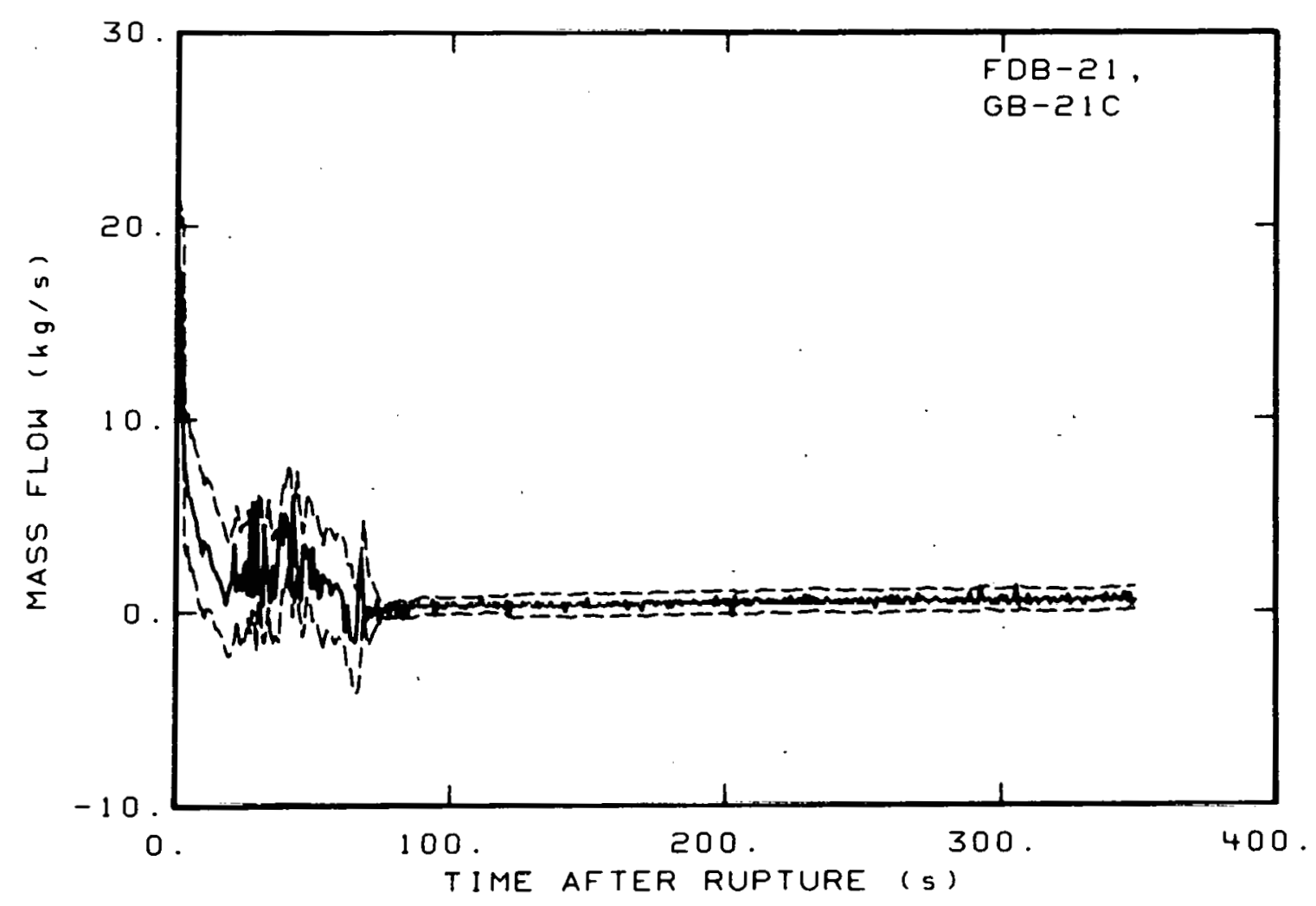

Fig. C-38 Mass flow in broken loop (FDB-21, GB-21C).

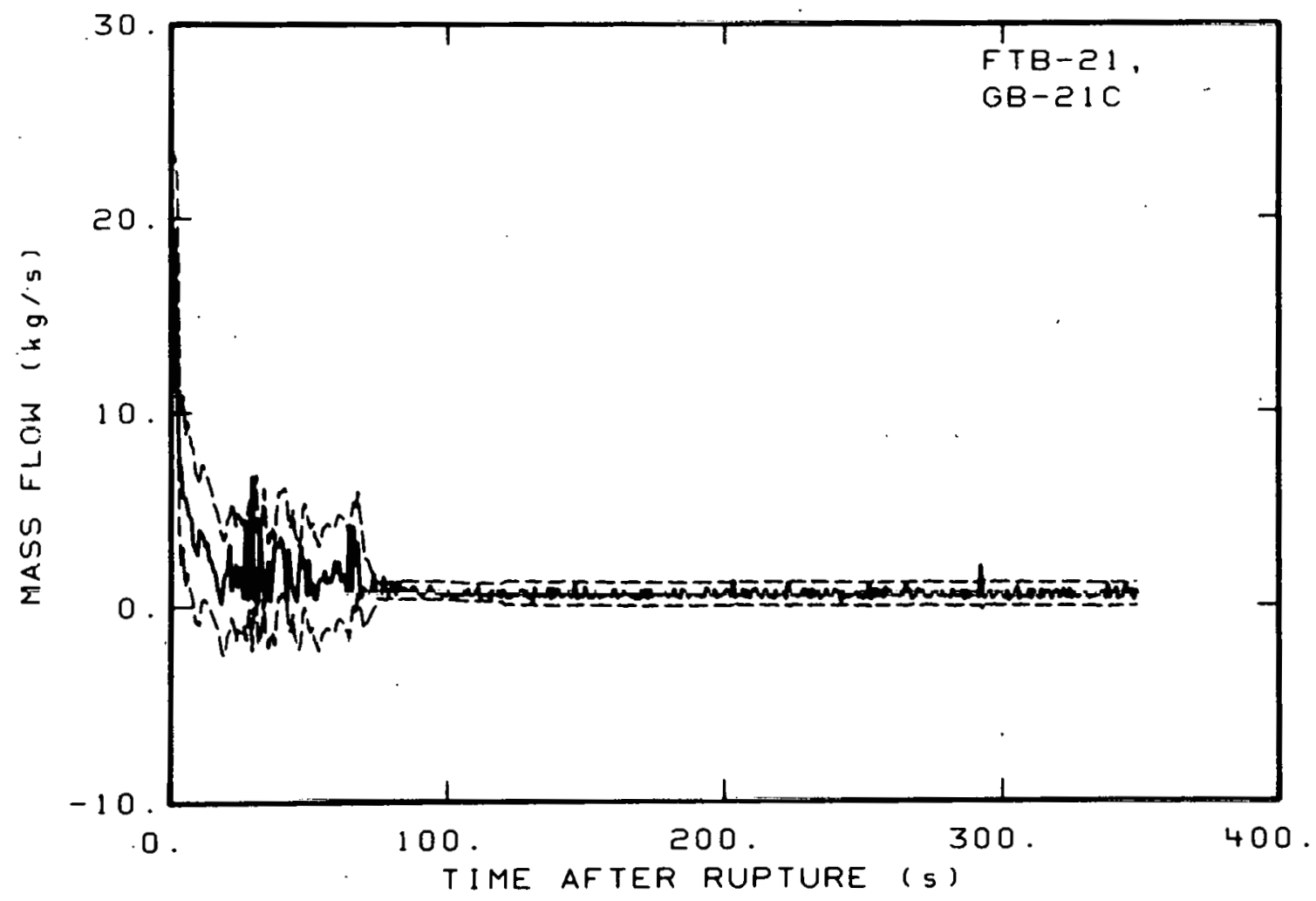

Fig. C-39 Mass flow in broken loup (FTB-21, GB-21C). 


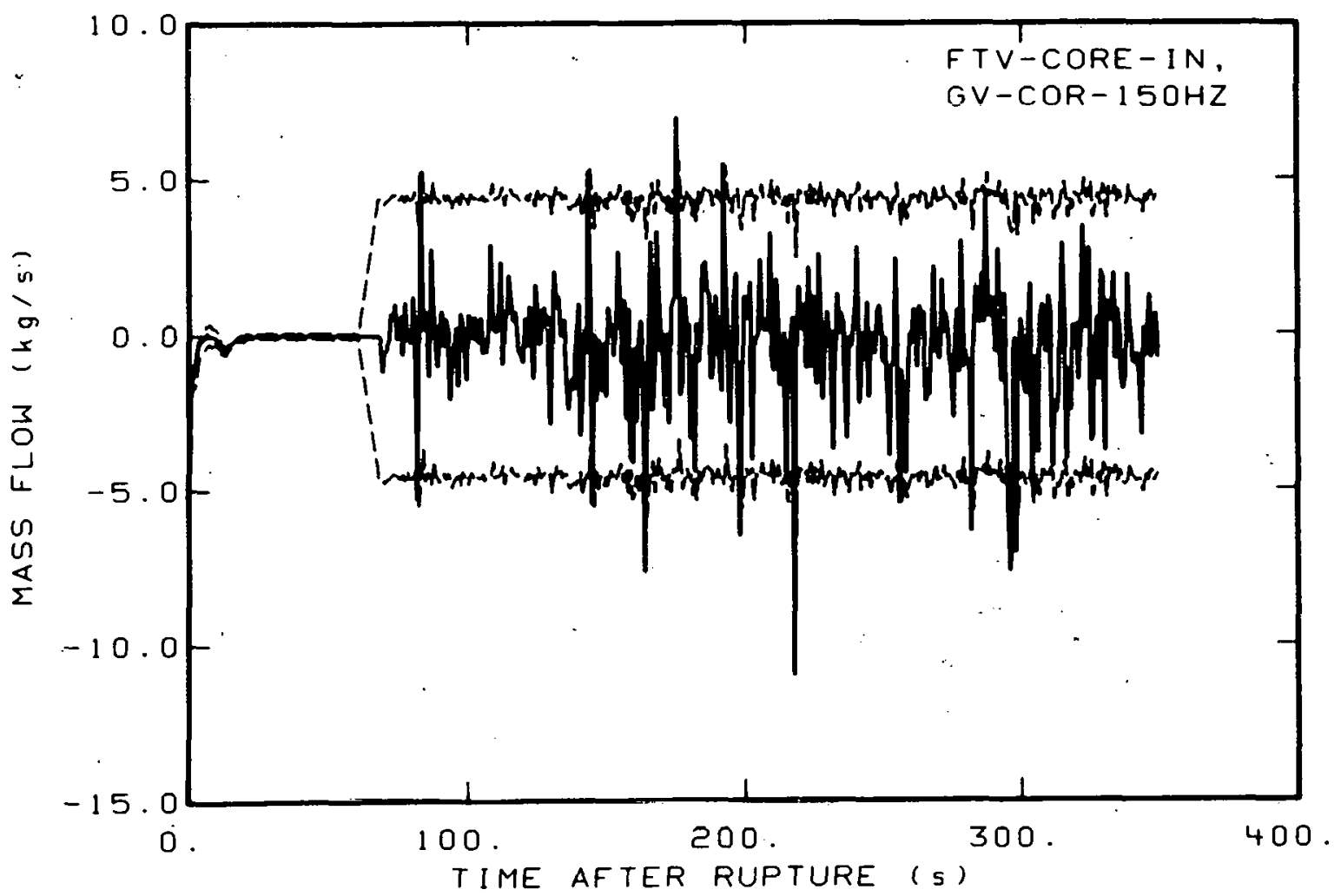

Fig. C-40 Mass flow in vessel (FTV-CORE-IN, GV-COR-150HZ).

Other errors in the data exist because of such factors as variability in installation procedures and techniques, calibration errors, variability in materials, and temperature and pressure sensitivities. These errors and the procedures for estimating them are discussed in Reference C-2. They are referred to as engineering errors and the estimates are largely subjective. Because of the continuing effort to improve the accuracy of the measured data, such as through the use of better transducers, better signal conditioning and processing equipment, and better calibration and installation techniques, the engineering errors for data from most of the transducer systems have changed from those published in Reference C-2. Table C-II provides a summary of engineering error values obtained from current analysis techniques as applied to the data presented herein.

In addition to the normal hardware and installation related sources of engineering error, a significant measurement uncertainty results when the current transducer systems are subjected to separated two-phase flow regimes during the course of the blowdown transient. Accordingly, for those data affected (fluid density, momentum flux, volumetric flow, and mass flow), which are presented in this appendix, a morc extensive assessment was conducted for additional engineering error due to flow regime effects. Table C-III identifies the data analyzed and the period in the blowdown process for which flow regime errors were included as a part of the total engineering error. The time of occurrence of separated two-phase flow and the resulting effect on the uncertainty of the data were evaluated by considering, on an individual basis, each detector output with reference to indications by other auxiliary measurements. 
TABLE C-I I

GENERAL MEASUREMENT ENGINEERING ERROR SOURCES AND ERROR VALUES (TEST S-28-2)

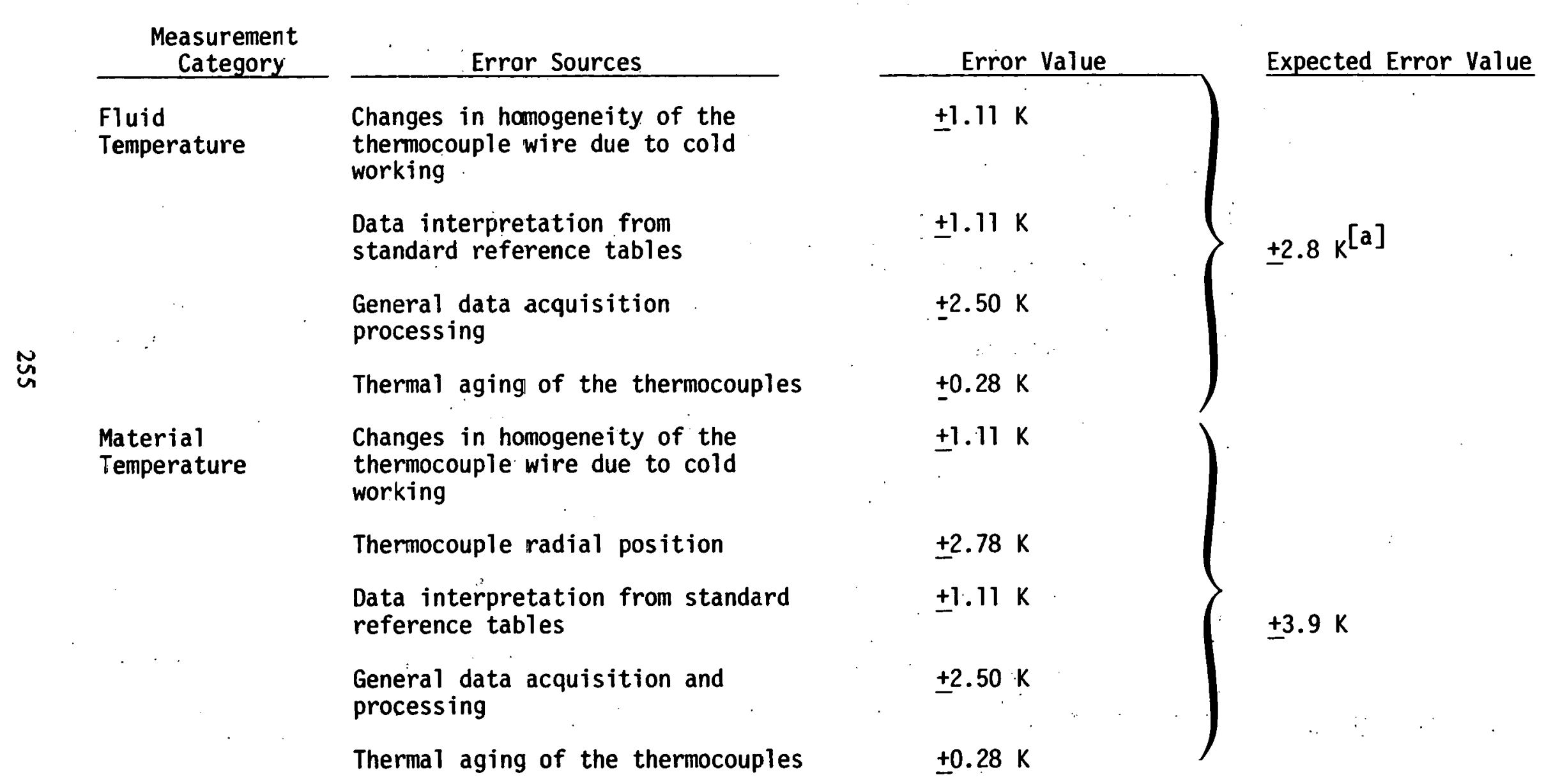




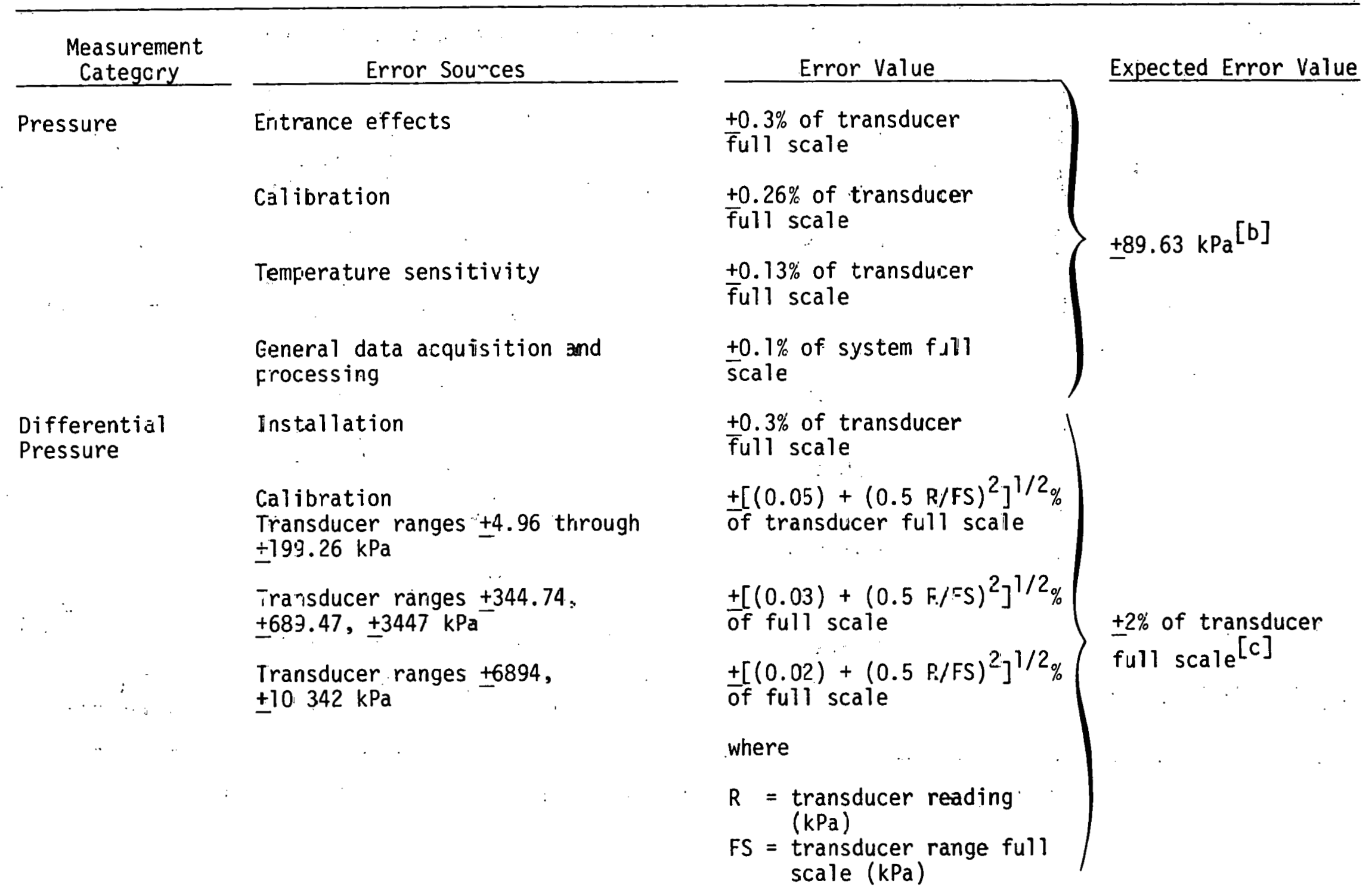


TABLE C-II (continued)

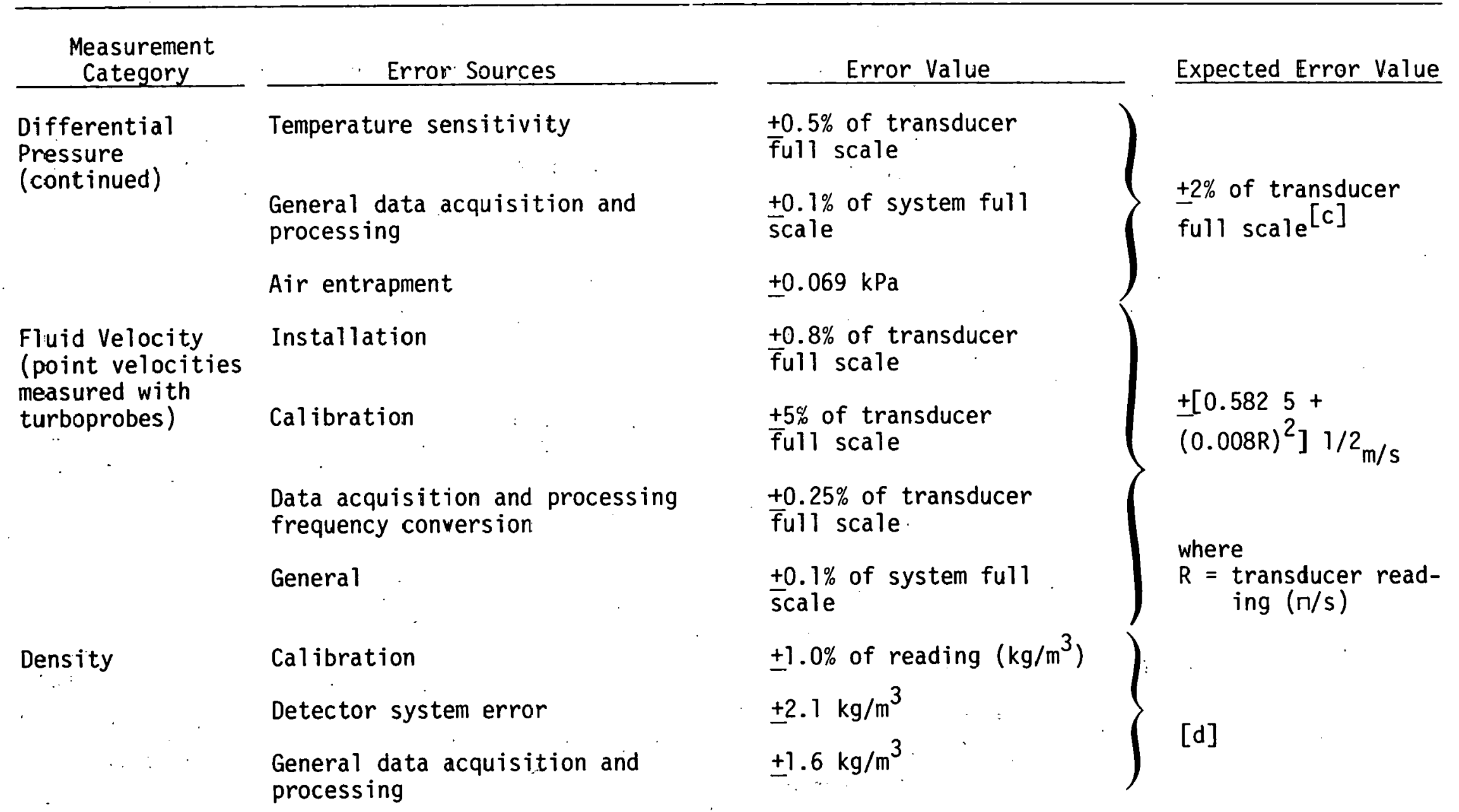


TABLE C-II (continued)

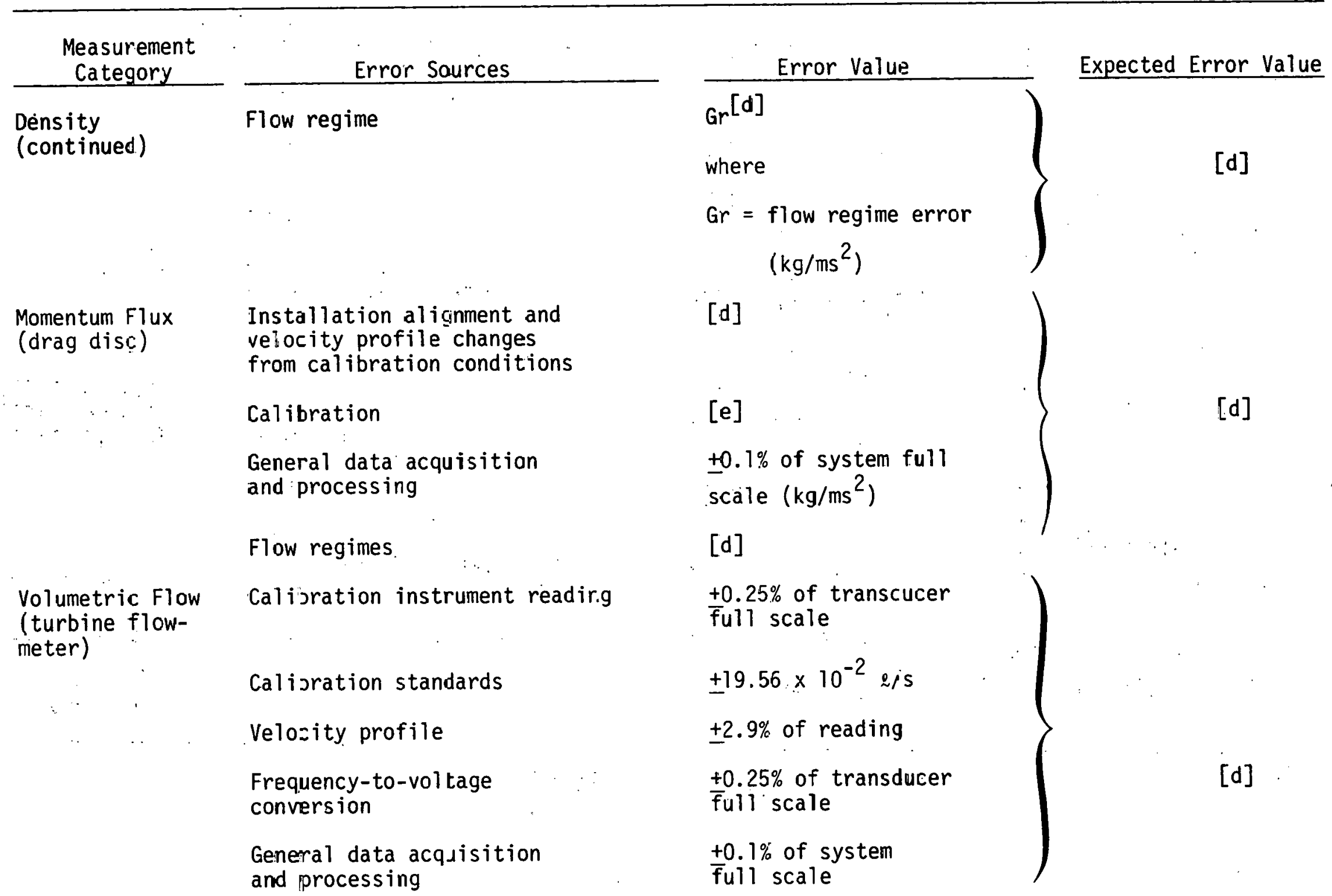


TABLE C-II (continued)

\begin{tabular}{|c|c|c|c|}
\hline $\begin{array}{c}\text { Measurement } \\
\text { Category }\end{array}$ & Error Sources & Error Value & Expected Error Value \\
\hline \multirow{2}{*}{$\begin{array}{l}\text { 'olumetric Flow } \\
\text { (turbine flow- } \\
\text { meter) (continued) }\end{array}$} & Dead bands & $\begin{array}{l}+5 \% \text { of transducer } \\
\text { full scale }\end{array}$ & [d] \\
\hline & Flow regimes & {$[d]$} & \\
\hline $\begin{array}{l}\text { Mass Flow Rate } \\
\text { (from volumetric } \\
\text { flow and density } \\
\text { data) }\end{array}$ & $\begin{array}{l}\text { Combined results from } \\
\text { individual error sources } \\
\text { for volumetric flow and density } \\
\text { data }[\mathrm{f}]\end{array}$ & [d] & [d] \\
\hline $\begin{array}{l}\text { Mass Flow Rate } \\
\text { (from momentum } \\
\text { flux and density } \\
\text { data) }\end{array}$ & $\begin{array}{l}\text { Combined results from } \\
\text { individual error sources for } \\
\text { momentum flux and density data }[f]\end{array}$ & [d] & [d] \\
\hline
\end{tabular}

[a] This value is no longer valid after thermocouple dryout occurs.

[b] Value for transducers with $20684 \mathrm{kPa}$ full-scale ranges.

[c] Value is based on observed system performance. It is more conservative than that obtained from the statistical summation of the identified engineering errors.

[d] Error value is time and flow regime dependent.

[e] Dependent on transducer full-scale range reading.

[f] The general nethod for combining volumetric flow or momentum flux with density data to obtain mass flow rate and the resulting errors in the data are explained in Reference $\mathrm{C}-2$. 
TABLE C-III

TIME PERIODS WHEN FLOW REGIME ERRORS WERE APPLIED (TEST S-28-2)

\begin{tabular}{|c|c|c|}
\hline $\begin{array}{c}\text { Transducer } \\
\text { Identification }\end{array}$ & $\begin{array}{c}\text { Time During Which } \\
\text { Flow Regime Errors } \\
\text { Were Appl ied } \\
(\mathrm{s}) \\
\end{array}$ & Figure Number \\
\hline GU-1C & 2 to 40 & $C-24$ \\
\hline GU-10VR & 10 to 25 & $C-25$ \\
\hline GU-15C & $\begin{array}{l}13 \text { to } 20 \\
\text { and } \\
57 \text { to } 100\end{array}$ & $C-28$ \\
\hline GB-23VR & $\begin{array}{c}3 \text { to } 15 \\
\text { and } \\
58 \text { to } 300\end{array}$ & $C-29$ \\
\hline FDU-1, GU-IC & 2 to 40 & $C-33$ \\
\hline FDU-5, GU-5VR & $\begin{array}{r}3 \text { to } 10 \\
\text { and } \\
27 \text { to } 44\end{array}$ & $C-35$ \\
\hline FTU-13, GU-13VR & 11 to 33 & $c-36$ \\
\hline FTII-15, GU-15C & $\begin{array}{l}13 \text { to } 20 \\
\text { and } \\
57 \text { to } 100\end{array}$ & r. -.37 \\
\hline FTB-21, GB-21C & $\begin{array}{l}3 \text { to } 1.5 \\
\text { and } \\
58 \text { to } 300\end{array}$ & c. -39 \\
\hline
\end{tabular}

The gamma densitometer density measurement data are affected by two-phase separated flow regimes. The resulting transducer output is a measurement of the average attenuation of the gamma beam through the measured medium. The beam attenuation, in turn, is interpreted through physical relationship to be a measure of the average density along the beam path. When stratified type flow was considered present, the gamma beam attenuation was considered to be a result of a liquid layer and steam at system conditions. With this assumption and the system geometry, a void fraction was calculated and a new "effective" average density was calculated. The difference between the average density based on the assumption of homogeneous conditions and the average density for stratified conditions was considered to be the error. 
Momentum flux measurement uncertainties for two-phase flow regimes present the most difficult engineering evaluation problems. The drag target and arm location, degree of flow stratification, transducer temperature sensitivity, and slip ratios all combine to produce possible flow regime errors ranging from a small fraction of the transducer output value to multiples of it. Therefore, the error values were obtained, where possible, through use of the observed discrepancies between the momentum flux and turbine flowmeter data in combination with system pressure measurements and the analysis of system fluid density measurements.

The flow regime errors of the turbine flowmeter were estimated by calculating a void fraction and the cross-sectional liquid and steam flow areas for stratified flow. This calculation was accomplished using methods similar to those used to calculate the average density for stratified flows. A simple model was used to equate the forces on the turbine with the ascumption of a known void fraction, stratified flow, known component densities, and slip ratio greater than unity. This process provided phase velucities. Witlı the phase densities, velocities, and void fraction, a volumetric flow rate could be calculated. The difference between this value and the measured value was considered to be the error.

The overall standard deviation of a data point is taken as the root mean of the sum of the random error variation and the total engineering error variance; that is,

$$
\sigma_{0}=\sigma_{R}^{2}+\sigma_{E}^{2}
$$

where

$$
\begin{aligned}
& \sigma_{\mathrm{O}}=\text { overall standard deviation of a data point } \\
& \sigma_{\mathrm{R}}=\text { random error standard deviatinn } \\
& \sigma_{\mathrm{E}}=\text { engineering error standard deviation. }
\end{aligned}
$$

The error bands for the data are computed about the value given by the fitted difference equation $y_{i}$ at time point, $i$; that is,

$$
\text { error band }=y_{i} \pm 1.96 \sigma_{0}
$$

With due regard to the fact that $o_{E}$ has been estimated subjectively, the error band may bc interpreted as an appruxinuate $95 \%$ confidence interval within which any true value of the measured variable is consistent with the data.

On certain occasions, the symmetrical error band given by Equation (C-2) is not appropriate. On those occasions, asymmetrical error hands were computed. (That is, with the width being greater on one side of $y_{i}$ than on the other.) 
Finally, the original data trace, along with its error band from Equation.(C-2), was input to a computer plot package. The resulting plot contained the actual data trace surrounded by an error band derived both from random error and engineering error considerations. The indicated error bands after thermocouple dryout occurred for the fluid temperature measurements should be ignored. Error bands indicated during periods when detector or data acquisition system saturation occurred should also be ignored. Error bands for these segments of the data were not obtained and bands only appear because of limitations in the plotting package.

\section{REFERENCES}

C-1. G. E. P. Box and B. M. Jenkins, Time Series Analysis - Forecasting and Control, San Francisco: Holden-Day, 1970.

C-2. E. M. Feldman and S. A. Naff, Error Analysis for 1-1/2-Loop Semiscale System Isothermal Test Data, ANCR-1188 (May 1975). 
Internal Distribution

1 - Chicago Patent Group 9800 South Cass Avenue

Argonne, Illinois, 60439

2 - CA Benson Idaho Operations Office-ERDA

Idaho Falls, ID 83401

3 - RJ Beers, ID

4 - PE Litteneker, ID

5 - RE Tiller, ID

6 - RE Wood, ID

7 - HP Pearson, Supervisor Technical Information

8-17 - INEL Technical Library

18-37 - Authors

38-93 - Special Internal

External Distribution

94-95 - Saul Levine, Director Office of Nuclear Regulatory Research, NRC Washington, DC 20555

96-400 - Distribution under NRC-2; Water Reactor Safety Research Systems Engineering 